\title{
Thermodynamic Properties of Minerals and
}

\section{Related Substances at 298.15 K and 1 Bar}

(10 $0^{5}$ Pascals) Pressure and at Higher Temperatures

U.S. GEOLOGICAL SURVEY BULLETIN 2131 


\section{AVAILABILITY OF BOOKS AND MAPS OF THE U.S. GEOLOGICAL SURVEY}

Instructions on ordering publications of the U.S. Geological Survey, along with prices of the last offerings, are given in the currentyear issues of the monthly catalog "New Publications of the U.S. Geological Survey." Prices of available U.S. Geological Survey publications released prior to the current year are listed in the most recent annual "Price and Availability List." Publications that may be listed in various U.S. Geological Survey catalogs (see back inside cover) but not listed in the most recent annual "Price and Availability List" may be no longer available.

Order U.S. Geological Survey publications by mail or over the counter from the offices given below.

\section{BY MAIL}

\section{Books}

Professional Papers, Bulletins, Water-Supply Papers, Techniques of Water-Resources Investigations, Circulars, publications of general interest (such as leaflets, pamphlets, booklets), single copies of Earthquakes \& Volcanoes, Preliminary Determination of Epicenters, and some miscellaneous reports, including some of the foregoing series that have gone out of print at the Superintendent of Documents, are obtainable by mail from

\section{U.S. Geological Survey, Information Services \\ Box 25286, Federal Center, Denver, CO 80225}

Subscriptions to periodicals (Earthquakes \& Volcanoes and Preliminary Determination of Epicenters) can be obtained ONLY from the

\section{Superintendent of Documents \\ Government Printing Office \\ Washington, DC 20402} Documents.)

(Check or money order must be payable to Superintendent of

Maps

For maps, address mail orders to

U.S. Geological Survey, Information Services

Box 25286, Federal Center, Denver, CO 80225

Residents of Alaska may order maps from

U.S. Geological Survey, Earth Science Information Center 101 Twelfth Ave. - Box 12

Fairbanks, AK 99701

\section{OVER THE COUNTER}

\section{Books and Maps}

Books and maps of the U.S. Geological Survey are available over the counter at the following U.S. Geological Survey Earth Science Information Centers (ESIC), all of which are authorized agents of the Superintendent of Documents:

- ANCHORAGE, Alaska-Rm. 101, 4230 University Dr.

- LAKEWOOD, Colorado-Federal Center, Bldg. 810

- MENLO PARK, California-Bldg. 3, Rm. 3128, 345 Middlefield Rd.

- RESTON, Virginia-USGS National Center, Rm. 1C402, 12201 Sunrise Valley Dr.

- SALT LAKE CITY, Utah-Federal Bldg., Rm. 8105, 125 South State St.

- SPOKANE, Washington-U.S. Post Office Bldg., Rm. 135, West 904 Riverside Ave.

- WASHINGTON, D.C.-Main Interior Bldg., Rm. 2650, 18th and C Sts., NW.

\section{Maps Only}

Maps may be purchased over the counter at the following U.S. Geological Survey offices:

- FAIRBANKS, Alaska-New Federal Bldg., 101 Twelfth Ave.

- ROLLA, Missouri-1400 Independence Rd.

- STENNIS SPACE CENTER, Mississippi-Bldg. 3101 


\section{Thermodynamic Properties of Minerals and Related Substances at 298.15 K and 1 Bar (10 Pascals) Pressure and at Higher Temperatures}

By Richard A. Robie and Bruce S. Hemingway

U. S. GEOLOGICAL SURVEY BULLETIN 2131

A summary of the thermodynamic data for minerals at $298.15 \mathrm{~K}$ together with calculated values for the functions $\mathrm{C}_{\mathrm{p}, \mathrm{T}}^{\circ}, \Delta_{\mathrm{f}} \mathrm{H}_{\mathrm{T}}^{\circ}, \Delta_{\mathrm{f}} \mathrm{G}_{\mathrm{T}}^{\circ}, \mathrm{S}_{\mathrm{T}}^{\circ},\left(\mathrm{H}_{\mathrm{T}}^{\circ}-\mathrm{H}_{298}^{\circ}\right) \mathrm{T}^{-1}$, and $\left(\mathrm{G}_{\mathrm{T}}^{\circ}-\mathrm{H}_{298}^{\circ}\right) \mathrm{T}^{-1}$ at temperatures up to $1800 \mathrm{~K}$

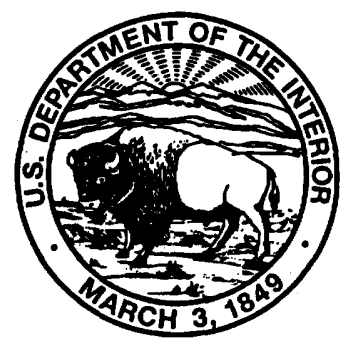




\title{
U.S. DEPARTMENT OF THE INTERIOR BRUCE BABBITT, Secretary
}

\author{
U.S. GEOLOGICAL SURVEY \\ GORDON P. EATON, Director
}

For sale by U.S. Geological Survey, Information Services

Box 25286, Federal Center, Denver, CO 80225

Any use of trade, product, or firm names in this publication is for descriptive purposes only and does not imply endorsement by the U.S. Government.

Published in the Eastern Region, Reston, Va.

Manuscript approved for publication March 23, 1995.

\section{Library of Congress Cataloging in Publication Data}

Robie, Richard A., 1928-

Thermodynamic properties of minerals and related substances at $298.15 \mathrm{~K}$ and $\mathrm{I}$ bar $(10$ Pascals) pressure and at higher temperatures / by Richard A. Robie and Bruce S.

Hemingway.

p. cm.- (Geological Survey bulletin ; 2131)

Includes bibliographical references and indexes.

Supt. of Docs. no. : I 19.3:B2131

1. Minerals-Tables. 2. Thermodynamics-Tables. I. Hemingway, Bruce S. II. Title. III.

Series: U.S. Geological Survey bulletin ; 2131.

QE75.B9 no. 2131

[QE366.8]

$557.3 \mathrm{~s}-\mathrm{dc} 20$

[549'. 12] 


\section{CONTENTS}

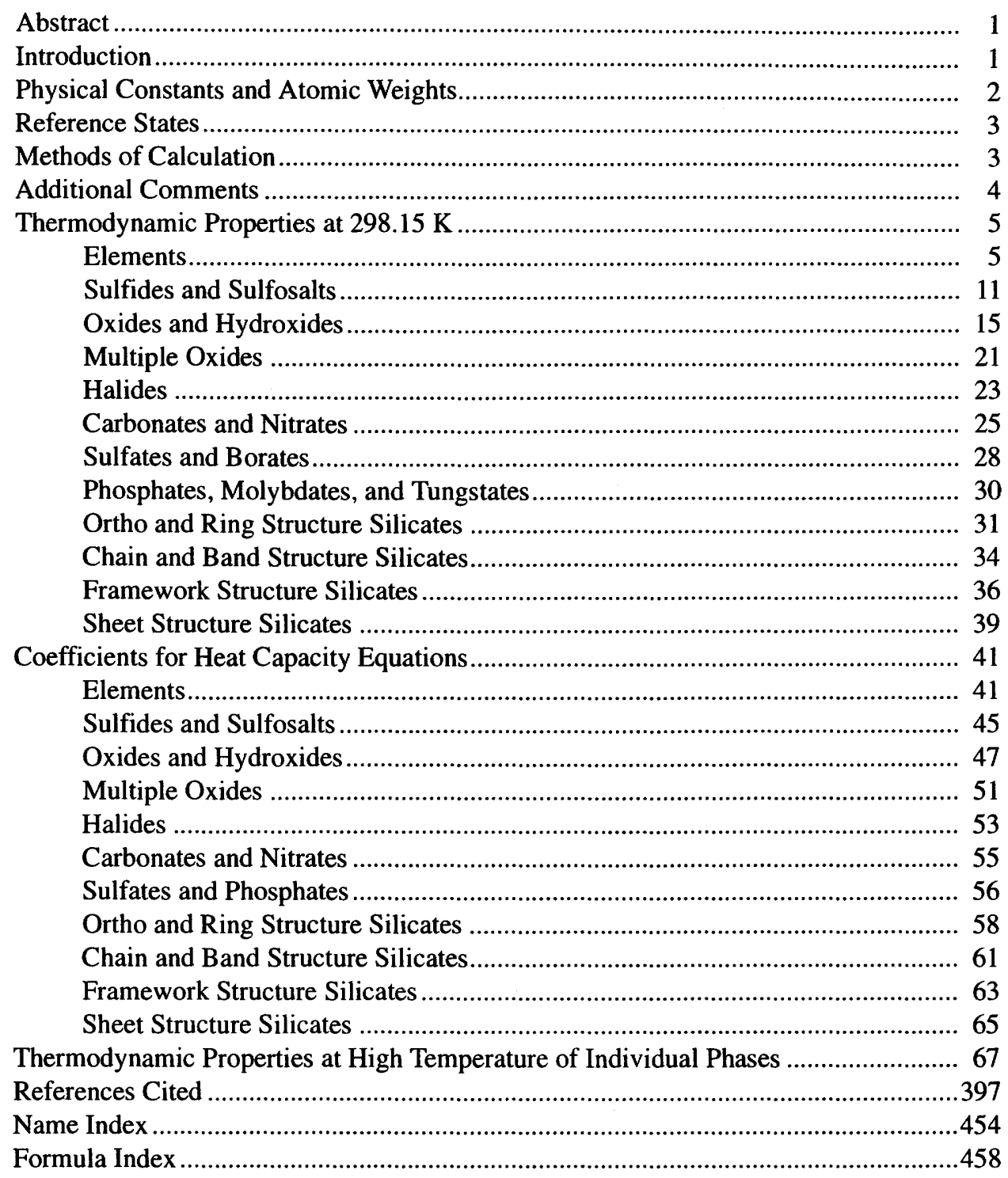

\section{TABLE}

1. Atomic weights of the elements for 1985 . 


\section{SYMBOLS, CONSTANTS, AND CONVERSION FACTORS}

$\mathrm{T}$ Temperature in Kelvins.

$\mathrm{T}_{\mathrm{tm}}$ Temperature of transition in Kelvins.

$\mathrm{K}$ Kelvin, the unit of temperature. It is the fraction $1 / 273.16$ of the thermodynamic temperature of the triple point of water.

J Joule, the unit of energy (or work). One joule $=1 / 4.1840$ thermochemical calories or $10 \mathrm{~cm}^{3} \cdot$ bar.

mol Mole, the amount of substance of a system that contains as many elementary entities as there are atoms in 0.012 kilograms of carbon 12. It is identical with the gfw (gram formula weight).

p Pressure in bars. One bar $=10^{5}$ Pascals or $0.1 \mathrm{MPa}$. The standard atmosphere is equal to 101325 Pascals. The kilogram ${ }^{\circ} \mathrm{cm}^{-2}$ is equal to 98065.5 Pascals.

- Superscript indicates that the substance is in its standard state, 1 bar ( $10^{5}$ Pascals) for a condensed phase.

$\mathrm{H}_{\mathrm{T}}^{\circ}-\mathrm{H}_{298}^{\circ}$ Enthalpy at temperature $\mathrm{T}$ relative to $298.15 \mathrm{~K}^{\circ}$ in J०mol ${ }^{-1}$, also called the heat content.

$\left(\mathrm{H}_{\mathrm{T}}^{\circ}-\mathrm{H}_{298}^{\circ}\right) \mathrm{T}^{-1}$ Enthalpy function in $\mathrm{J} \cdot \mathrm{mol}^{-1} \cdot \mathrm{K}^{-1}$ (See note below).

$\mathrm{S}_{\mathrm{T}}^{\circ}$ Entropy at temperature $\mathrm{T}$, in $\mathrm{J} \cdot \mathrm{mol}^{-1} \cdot \mathrm{K}^{-1}$.

$\left(\mathrm{G}_{\mathrm{T}}^{\circ}-\mathrm{H}_{298}^{\circ}\right) \mathrm{T}^{-1}$ Gibbs energy function in $\mathrm{J}^{\circ} \mathrm{mol}^{-1} \cdot \mathrm{K}^{-1}$.

$\mathrm{C}_{\mathrm{p}}^{\circ}$ Heat capacity at constant pressure in $\mathrm{J} \cdot \mathrm{mol}^{-1} \cdot \mathrm{K}^{-1}$.

$V_{298}^{\circ}$ Volume of 1 mol of a substance at 1 bar pressure and at $298.15 \mathrm{~K}$, in cm $\mathrm{cm}^{3}$, or J•bar ${ }^{-1}$.

$\Delta_{\text {fus }} \mathrm{H}^{\circ} \quad$ Enthalpy of melting at 1 bar pressure in J०mol ${ }^{-1}$.

$\Delta_{\mathrm{trn}} \mathrm{H}^{\circ} \quad$ Enthalpy of transition at 1 bar pressure in $\mathrm{J}^{\circ} \mathrm{mol}^{-1}$.

$\Delta_{\text {vap }} \mathrm{H}^{\circ}$ Enthalpy of vaporization to the ideal gas at 1 bar pressure at the normal boiling point in $\mathrm{J} \cdot \mathrm{mol}^{-1}$.

$\Delta_{\mathrm{f}} \mathrm{H}^{\circ}$ Enthalpy of formation from the elements in their reference states in J•mol ${ }^{-1}$.

$\Delta_{\mathrm{f}} \mathrm{G}^{\circ}$ Gibbs energy of formation from the elements in their reference states in $\mathrm{J}^{\circ} \mathrm{mol}^{-1}$.

$\mathrm{K}_{\mathrm{f}}$ Equilibrium constant of formation.

R Gas constant, 8.31451 $\pm 0.00007 \mathrm{~J} \cdot \mathrm{K}^{-1} \cdot \mathrm{mol}^{-1}$.

F Faraday constant, $96,485.309 \pm 0.029 \mathrm{~J} \cdot \mathrm{V}^{-1} \cdot \mathrm{mol}^{-1}$.

$\mathrm{N}_{\mathrm{A}}$ Avogadro constant, $(6.0221367 \pm 0.0000036) \times 10^{23} \mathrm{~mol}^{-1}$.

log Common logarithm, base 10.

In Natural logarithm, base e $=\mathbf{2 . 7 1 8 2 8}$...

atm Standard atmosphere.

$m=1$ The standard state for an electrolyte in aqueous solution is the hypothetical ideal solution at unit activity and with the ions at infinite dilution.

When multiple units appear in the denominator of the symbol (for example, enthalpy function), the SI system does not recommend a particular order. For example, the units for entropy are given as $J$ $\mathrm{mol}^{-1} \mathrm{~K}^{-1}$ in table 2.11 and as $\mathrm{J} \mathrm{K}^{-1} \mathrm{~mol}^{-1}$ in table 3.5 of the Greenbook (278). 


\title{
Thermodynamic Properties of Minerals and Related Substances at $298.15 \mathrm{~K}$ and $1 \mathrm{Bar}\left(10^{5}\right.$ Pascals) Pressure and at Higher Temperatures
}

\author{
By Richard A. Robie and Bruce S. Hemingway
}

\begin{abstract}
Selected values for the entropy $\left(\mathrm{S}^{\circ}\right)$, molar volume $\left(V^{\circ}\right)$, and for the enthalpy and Gibbs energy of formation $\left(\Delta_{\mathrm{f}} \mathrm{H}^{\circ}\right.$ and $\left.\Delta_{\mathrm{f}} \mathrm{G}^{\circ}\right)$ are given for 50 reference elements, 55 sulfides, 93 oxides and hydroxides, 141 silicates, and 154 other minerals and related substances at $298.15 \mathrm{~K}$. For those minerals for which high-temperature heat-capacity or heatcontent data are also available $\left(\mathrm{H}_{\mathrm{T}}^{\circ}-\mathrm{H}_{298}^{\circ}\right) \mathrm{T}^{-1}, \mathrm{~S}_{\mathrm{T}}^{\circ},\left(\mathrm{G}_{\mathrm{T}}^{\circ}\right.$ $\left.\mathrm{H}_{298}^{\circ}\right) \mathrm{T}^{-1}, \mathrm{C}_{\mathrm{p}}^{\circ}, \Delta_{\mathrm{f}} \mathrm{H}_{\mathrm{T}}^{\circ}, \Delta_{\mathrm{f}} \mathrm{G}_{\mathrm{T}}^{\circ}$ and $\log \mathrm{K}_{\mathrm{f}, \mathrm{T}}$ are tabulated at $100 \mathrm{~K}$ intervals for temperatures up to $1800 \mathrm{~K}$.
\end{abstract}

\section{INTRODUCTION}

In the 17 years since the predecessor to this bulletin was published, an enormous body of new thermochemical and equilibrium measurements for minerals has been published, personal computers have become almost universally available, and a number of "internally consistent thermodynamic property databases" (ICTDB) have been published $(37,67,117,149,150,159,187,188,374)$. (In this section, references are indicated by numbers in parentheses.) This report is an updated revision and expansion of U.S. Geological Survey (USGS) Bulletin 1452 (361) and entirely supersedes it. As an example of growth of the thermodynamic properties of mineral database, USGS Bulletin 1452 contained data for 58 silicate minerals whereas in the current set of tables this number has been increased to 141 .

The purpose of these tables is to present a critical summary of the available thermodynamic data for minerals and related substances in a convenient form for the use of earth scientists. To make the tables as useful as possible, we have tried (1) to include enough auxiliary data so that a single set of tables would suffice for most calculations, (2) to ensure internal consistency, and (3) to provide the means for rapid revision and expansion as new data become available.

The tables in this compilation are divided into three sections. In the first section, values are given for the entropy $\left(S_{298}^{\circ}\right)$, molar volume $\left(V_{298}^{\circ}\right)$, enthalpy of formation
$\left(\Delta_{\mathrm{f}} \mathrm{H}_{298}^{\circ}\right)$, Gibbs energy of formation $\left(\Delta_{\mathrm{f}} \mathrm{G}_{298}^{\circ}\right)$, and the logarithm of the equilibrium constant of formation $\left(\log \mathrm{K}_{\mathrm{f}, 298}\right)$ for the reference elements, minerals, and other substances of geological interest (properties at $298.15 \mathrm{~K}$ and $1 \mathrm{bar}$ ). In the second section, coefficients for the polynomial used to represent the heat capacity at high temperature are tabulated together with the temperature limits of applicability. In the third section, values are given for the thermodynamic properties at $100 \mathrm{~K}$ intervals for temperatures up to $1800 \mathrm{~K}$. The data are arranged in order of their conventional mineralogical groups. Within each group (for example, the oxides) the listing is by alphabetical order of the chemical symbol of the principal cation.

The data have been taken from the original literature, recent critical evaluations, or have been evaluated by the present authors and estimated uncertainties have been assigned to the thermodynamic properties at $298.15 \mathrm{~K}$. The primary sources of data are indicated numerically in the thermodynamic table of properties at $298.15 \mathrm{~K}$, as " $\mathrm{S}$ " for the reference to the data for the entropy, " $\mathrm{H} / \mathrm{G}$ " for the reference to the formation properties, and " $\mathrm{C}$ " to references to measured heat capacities above $298.15 \mathrm{~K}$. The references are listed in complete form following the tables. The entropy and heat-capacity values tabulated are based almost exclusively on calorimetric measurements whereas the values for the enthalpy or Gibbs energy of formation were obtained from both equilibrium and calorimetric measurements. The values for the thermodynamic properties of the reference elements have been taken from the JANAF tables (67), the CODATA (85) recommended values, or in the case of calcium, from measurement at the USGS (360). For those silicate glasses for which $S_{T}^{\circ}-S_{\delta}^{\circ}$ has been determined calorimetrically and for which the enthalpy of fusion of the crystalline equivalent could be evaluated, the residual entropy (that is, $S_{0}^{\circ}$ ) of the glass has been evaluated and added to the calorimetric entropy to obtain the correct entropy for use in thermodynamic calculations. Glasses and other phases for which $S_{T}^{\circ}-S_{0}^{\circ}$ has been measured but for which the residual entropy has not been evaluated are indicated by a " + " sign and the user must be aware that the 
value tabulated does not include the configurational entropy.

Although a number of different forms of equations for the representation of the heat capacity at high temperatures have been proposed over the past 10 years, for example Berman (37), Fei and Saxena (104), Richet and Fiquet (342), we have retained the form adopted in the previous version of these tables,

$$
\mathrm{C}_{\mathrm{p}}=\mathrm{A}_{1}+\mathrm{A}_{2} \cdot \mathrm{T}+\mathrm{A}_{3} \cdot \mathrm{T}^{-2}+\mathrm{A}_{4} \cdot \mathrm{T}^{-0.5}+\mathrm{A}_{5} \cdot \mathrm{T}^{2}
$$

This equation can not be extrapolated beyond its upper limit. Inasmuch as most users will have access to a personal computer, the conversion from one form of equation to an alternative form is possible and is left up to the individual user's preference. The entropies listed in the table of coefficients for the $\mathrm{C}_{\mathrm{p}}^{\circ}$ polynomials corresponds to the temperature at the lower limit of the temperature range for the polynomial. This is usually $298.15 \mathrm{~K}$.

For several phases, the existence of multiple transitions at high temperature makes it impractical to provide a simple polynomial expression for the heat capacity. For such phases, for example $\mathrm{Fe}, \mathrm{Cu}_{1.9} \mathrm{~S}$, and several pyrrhotites, we have listed only the heat capacity at $298.15 \mathrm{~K}$.

For these phases, one must go to the high-temperature table for the particular phase (if tabulated) to get the heat capacity (and entropy) above room temperature.

Internally consistent thermodynamic property data sets for silicate minerals have been published by Haas et al. (149), Holland and Powell $(187,188)$, Berman (37), Halbach and Chatterjee (150), and Saxena and Chatterjee (374) among others. Inasmuch as most of the authors relied on the same sources of calorimetric and equilibrium data to derive their adopted values, it is surprising that significant differences in their listed values for such important phases as grossular, anorthite, phlogopite and muscovite exist. For example, between the most frequently cited ICTDB, those of Berman (37) and Holland and Powell (188), the values of $\Delta_{\mathrm{f}} \mathrm{G}_{298}^{\circ}$ calculated from their listed values of $S_{298}^{\circ}$ and $\Delta_{\mathrm{f}} \mathrm{H}_{298}^{\circ}$ differ by $5.7,3.7$ and $3.7 \mathrm{~kJ} \cdot \mathrm{mol}^{-1}$ for grossular, anorthite, and muscovite, respectively, and for analbite and muscovite the tabulated entropies differ by 4.4 and 9.1 $\mathrm{J} \cdot \mathrm{mol}^{-1} \cdot \mathrm{K}^{-1}$, respectively. The point is, of course, that internal consistency does not imply that the derived values are accurate.

The reason for these discrepancies is most likely in the assumptions that the individual compiler makes concerning the state of internal $\mathrm{Al} / \mathrm{Si}$ order. For a 1:3 Al/Si compound such as the alkali feldspars or micas the complete random mixing of the $3 \mathrm{Si}$ and $1 \mathrm{Al}$ over the 4 tetrahedral sites leads to a contribution to the entropy of $18.7 \mathrm{~J} \cdot \mathrm{mol}^{-1} \cdot \mathrm{K}^{-1}$. However, if the aluminum-avoidance model is valid, the configurational entropy will be considerably smaller and of the order of 13 to $14 \mathrm{~J} \cdot \mathrm{mol}^{-1} \cdot \mathrm{K}^{-1}$. The aluminum-avoidance model has not been calculated exactly and only approxi- mate values are available. The values for the configurational entropies that have been derived from phase equilibrium studies by Holland and Powell (188) and by Berman (37) are 12.6 and $17.0 \mathrm{~J} \cdot \mathrm{mol}^{-1} \cdot \mathrm{K}^{-1}$ for analbite and 16.0 and $15.1 \mathrm{~J} \cdot \mathrm{mol}^{-1} \cdot \mathrm{K}^{-1}$ for sanidine, respectively. It appears that these calculated values for the entropy of disorder do not unequivocally support the aluminum-avoidance model but rather tend to yield a configurational entropy that is greater than that estimated by the aluminum-avoidance value, but definitely less than the fully random model. Any value of $\Delta S$ greater than that calculated from aluminumavoidance would presumably mean that this model was inapplicable at high temperatures; for example, at temperatures greater than $1300 \mathrm{~K}$ for analbite the disordering entropy would approach $18.7 \mathrm{~J} \cdot \mathrm{mol}^{-1} \cdot \mathrm{K}^{-1}$. Moreover Goldsmith and Jenkins (123) have shown that the $\mathrm{Al} / \mathrm{Si}$ disorder in $\mathrm{NaAlSi}_{3} \mathrm{O}_{8}$ is a continuous function of the temperature and that it occurs over a range of several hundred degrees $(500-1050 \mathrm{~K})$ and occurs without change in symmetry.

As a consequence of these points the question arises whether one can extract meaningful (unique) values for the entropy of the disordered phase of an alumino-silicate from phase equilibrium studies without having additional information on the Al/Si distribution. For this reason, we have adopted the fully random model for tabulating the hightemperature properties for the $\mathrm{Al} / \mathrm{Si}$ disordered feldspars and micas. For an extended discussion of the contribution to the entropy of silicate minerals arising from $\mathrm{Al} / \mathrm{Si}$ or magnetic spin disorder, see Ulbrich and Waldbaum (413).

The differences in the derived values for the thermodynamic properties of phases in the CASH system is due in part to the existence of two calorimetric values for the entropy of grossular which differ from one another by 4.6 $\mathrm{J} \cdot \mathrm{mol}^{-1} \cdot \mathrm{K}^{-1}$. The value of Westrum et al. (438) was determined on a natural sample and involved a substantial correction for impurities. The value given by Haselton and Westrum (157) was derived from measurements on a synthetic sample. The heat capacity of the synthetic sample used by Haselton and Westrum (157) was later remeasured at the USGS by the present authors (359), who found an entropy $\mathrm{S}^{\circ}\left(\mathrm{Ca}_{3} \mathrm{Al}_{2} \mathrm{Si}_{3} \mathrm{O}_{12}, 298.15 \mathrm{~K}\right)$ of $259.9 \mathrm{~J} \cdot \mathrm{mol}^{-1} \cdot \mathrm{K}^{-1}$ which agrees with the Haselton and Westrum value to within $0.2 \mathrm{~J} \cdot \mathrm{mol}^{-1} \cdot \mathrm{K}^{-1}(0.08$ percent $)$. For this reason we have accepted the Haselton-Westrum value for the entropy of grossular in evaluating the thermodynamic properties of phases in the CASH system.

\section{PHYSICAL CONSTANTS AND ATOMIC WEIGHTS}

The symbols and constants used to prepare these tables are listed in the table of contents. The units adopted for reporting the thermodynamic properties are those of the International System of Units (SI). A formal description of 
the SI system is given by Goldman and Bell (122). The recommended symbols and terminology used are those from the International Union of Pure and Applied Chemistry (IUPAC), "Quantities, Units and Symbols in Physical Chemistry", by Mills et al. (278) and commonly called the "Greenbook". The Greenbook is also a convenient source for the CODATA values of the fundamental physical constants, the 1985 atomic weights of the elements, and contains a detailed description of the SI system of units.

Values for the gas constant (R), the Faraday constant (F), and the Avogadro constant (A) used in the calculations are those adopted by the CODATA Task Group from the least squares evaluation of Cohen and Taylor (75). For convenience, we also give values for the atomic weights for 1985 (scale $C^{12}=12.000$, Mills et al. (278)) in alphabetical order by their chemical symbol in table 1 .

\section{REFERENCE STATES}

The reference pressure for the standard state adopted for these tables is 1 bar, that is $10^{5}$ Pascals. For a gaseous phase, $\mathrm{C}_{\mathrm{p}}^{\circ},\left(\mathrm{H}_{\mathrm{T}}^{\circ}-\mathrm{H}_{298}^{\circ}\right) \mathrm{T}^{-1}$, and $\Delta_{\mathrm{f}} \mathrm{H}^{\circ}$ are independent of pressure, but the numerical values of $\mathrm{S}^{\circ},\left(\mathrm{G}_{\mathrm{T}}^{\circ}-\mathrm{H}_{298}^{\circ}\right) \mathrm{T}^{-1}$, $\Delta G^{\circ}$ depend upon the choice of pressure. The change in pressure from $\mathrm{p}=1 \mathrm{~atm}(1.0135 \mathrm{bar})$ to $1 \mathrm{bar}$ yields $\Delta S=$ $0.11 \mathrm{~J} \cdot \mathrm{mol}^{-1} \cdot \mathrm{K}^{-1}$. For a condensed phase (liquid or solid), it can be shown that the effect of changing the reference pressure from $1 \mathrm{~atm}$ to $1 \mathrm{bar}$ has only a trivial effect upon all the thermodynamic properties tabulated in this report except for $\Delta_{\mathrm{f}} \mathrm{G}^{\circ}$, where a change will occur if one of the reference elements (usually oxygen) is a gas.

The standard states for the condensed elements are the most stable form at 1 bar pressure and the stated temperature. For gaseous elements, the standard state is the ideal gas at 1 bar pressure. Data are listed for the elements in their standard reference states, and for a few in nonstandard states; for example, $\mathrm{S}^{\mathbf{2}}$ gas and the diamond form of carbon. The reference state adopted for an element above its normal boiling or sublimation temperature is not necessarily its equilibrium state. Thus, at equilibrium, liquid sulfur boils at 1 bar pressure and approximately $716.9 \mathrm{~K}$ to a gas composed of $S, S_{2}, S_{4}, S_{6}, S_{8}$, etc., but for our reference table, we have assumed the gas phase to be only $S_{2}$. For the reference state table for sulfur we have followed the JANAF (67) tables procedure using $882.12 \mathrm{~K}$ as the fictive boiling temperature at which liquid sulfur is in equilibrium with $S_{2}$ gas at 1 bar pressure. Because of the convenience of using diatomic sulfur gas in calculations of sulfide mineral equilibria we have included a second set of high-temperature tables for which the reference state for calculating $\Delta_{\mathrm{f}} \mathrm{G}^{\circ}$, and $\Delta_{\mathrm{f}} \mathrm{H}^{\circ}$ and $\log \mathrm{K}_{\mathrm{f}}$ for the sulfide phase is $\mathrm{S}_{2}$ gas. The user is cautioned not to intermix values from the two different reference states in their calculations. In other ambiguous cases, we have chosen either the dominant species in the gas or that species for which the best thermodynamic data are available as the reference state.

The reference states for $\Delta_{\mathrm{f}} \mathrm{H}^{\circ}, \Delta_{\mathrm{f}} \mathrm{G}^{\circ}$, and $\log \mathrm{K}_{\mathrm{f}}$ of the compounds are the elements in their standard states at 1 bar pressure and the stated temperature.

\section{METHODS OF CALCULATION}

Having chosen what we believe are the currently "best available" values for $\mathrm{H}_{\mathrm{T}}^{\circ}-\mathrm{H}_{298}^{\circ}$, or $\mathrm{C}_{\mathrm{p}}^{\circ}(\mathrm{T}), \mathrm{S}_{298}^{\circ}$ and either $\Delta_{\mathrm{f}} \mathrm{H}_{298}^{\circ}$ or $\Delta_{\mathrm{f}} \mathrm{G}_{298}^{\circ}$, we have calculated the Gibbs energy function, and the enthalpy, Gibbs energy, and the equilibrium constant of formation at $100 \mathrm{~K}$ intervals using the following relations:

$$
\begin{gathered}
\left(\mathrm{G}_{\mathrm{T}}^{\circ}-\mathrm{H}_{298}^{\circ}\right) \mathrm{T}^{-1}=\left(\mathrm{H}_{\mathrm{T}}^{\circ}-\mathrm{H}_{298}^{\circ}\right) \mathrm{T}^{-1}-\mathrm{S}_{\mathrm{T}}^{\circ} \\
\Delta_{\mathrm{f}} \mathrm{H}_{\mathrm{T}}^{\circ}=\Delta_{\mathrm{f}} \mathrm{H}_{298}^{\circ}+\Delta\left(\mathrm{H}_{\mathrm{T}}^{\circ}-\mathrm{H}_{298}^{\circ}\right) \\
\Delta_{\mathrm{f}} \mathrm{G}_{\mathrm{T}}^{\circ}=\Delta_{\mathrm{f}} \mathrm{H}_{298}^{\circ}+\mathrm{T} \Delta\left[\left(\mathrm{G}_{\mathrm{T}}^{\circ}-\mathrm{H}_{298}^{\circ}\right) \mathrm{T}^{-1}\right]
\end{gathered}
$$

and

$$
\log \mathrm{K}_{\mathrm{f}, \mathrm{T}}=\Delta_{\mathrm{f}} \mathrm{G}_{\mathrm{T}}^{\circ}(2.30258 \mathrm{RT})^{-1}=-\Delta_{\mathrm{f}} \mathrm{G}_{298}^{\circ}(19.145 \mathrm{~T})^{-1}
$$

These values together with $\mathrm{C}_{\mathrm{p}}^{\circ}, \mathrm{S}_{\mathrm{T}}^{\circ}$ and $\left(\mathrm{H}_{\mathrm{T}}^{\circ}-\mathrm{H}_{298}^{\circ}\right) \mathrm{T}^{-1}$ are tabulated in the third section.

Transitions in heat capacity are treated in the standard manner: heat capacity curves are fit to the experimental values above and below the transition; values calculated from an extrapolation of the equation to the transition temperature are compared to the experimental values; and differences are summed to give an enthalpy of transition that is applied at the transition temperature.

The uncertainties assigned to the properties apply only to the values at $298.15 \mathrm{~K}$. The principal source has been the original report of the experimental data. By convention, the uncertainty interval reported for calorimetric measurements is twice the standard deviation of the mean; that is,

$$
\delta=2\left[\Sigma\left(x_{i}-x_{m}\right)^{2} / n(n-1)\right]^{1 / 2}
$$

where $x_{i}$ is the value for an individual measurement, $x_{m}$ is the arithmetic mean of all the measurements, and $n$ is the number of measurements.

For generating the high-temperature tables, the input data supplied to the computer are the identifying name of the substance, the entropy, the enthalpy of formation at $298.15 \mathrm{~K}$, the number of atoms of each element in the chemical formula, and the coefficients for the heat capacity equation. A commercial spreadsheet is used to compute the formula weight of the compound and values for $\mathrm{S}_{\mathrm{T}}^{\circ}, \Delta_{\mathrm{f}} \mathrm{H}_{\mathrm{T}}$, $\Delta_{\mathrm{f}} \mathrm{G}_{\mathrm{T}}^{\circ}, \log \mathrm{K}_{\mathrm{f}, \mathrm{T}}, \mathrm{C}_{\mathrm{p}}^{\circ}, \quad\left(\mathrm{H}_{\mathrm{T}}^{\circ}-\mathrm{H}_{298}^{\circ}\right) \mathrm{T}^{-1}$, and $\left(\mathrm{G}_{\mathrm{T}}^{\circ}-\mathrm{H}_{298}^{\circ}\right) \mathrm{T}^{-1}$, at $100 \mathrm{~K}$ intervals up to $1800 \mathrm{~K}$. Auxiliary data such as the melting and boiling points and enthalpies of melting and vaporization are also included. In addition, coefficients 
from a regression performed on the calculated values of $\Delta_{\mathrm{f}} \mathrm{G}_{\mathrm{T}}^{\circ}$ are included. The equation adopted is:

$$
\Delta_{\mathrm{f}} \mathrm{G}_{\mathrm{T}}^{\circ}=\mathrm{A}+\mathrm{BT}+\mathrm{CT}^{-2}
$$

The coefficients for the equations are listed, in scientific notation, at the bottom of the page for the individual hightemperature tables. The equations are given only for those minerals for which the regression yielded a fit of better than 1 percent.

\section{ADDITIONAL COMMENTS}

Small numbers of some groups are presented with other groups for convenience and economy. Examples include tellurides, selenides and arsenides that are included with sulfides and sulfosalts, and methane, cohenite, and ammonia that are included with the elements.

The data available in this publication are also available on diskette for a nominal fee through the Generic Mineral Technology Center for Pyrometallurgy at the University of Missouri at Rolla as a part of the FREED database. FREED is a thermodynamic database program for storing, retrieving, editing, and manipulating thermodynamic data on a computer. FREED was developed with support from the U.S. Department of Interior's Mineral Institute Program, administered by the U.S. Bureau of Mines, and the U.S. Department of Energy through the Westinghouse Savannah River Plant.

Table 1. Atomic weights of the elements for 1985 .

\begin{tabular}{|c|c|c|c|c|c|}
\hline Element & Symbol & Atomic Weight & Element & Symbol & Atomic Weight \\
\hline $\begin{array}{l}\text { Actinium } \\
\text { Silver } \\
\text { Aluminum } \\
\text { Americium } \\
\text { Argon } \\
\text { Arsenic } \\
\text { Astatine }\end{array}$ & $\begin{array}{l}\text { Ac } \\
\text { Ag } \\
\text { Al } \\
\text { Am } \\
\text { Ar } \\
\text { As } \\
\text { At } \\
\text { Au } \\
\text { B } \\
\text { Ba }\end{array}$ & $\begin{array}{l}(227) \\
107.8682 \\
26.98154 \\
(243) \\
39.948 \\
74.92159 \\
(210) \\
196.96654 \\
10.811 \\
137.327\end{array}$ & 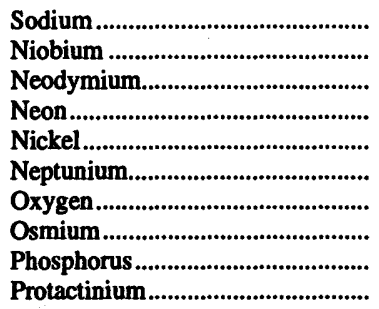 & $\begin{array}{l}\mathrm{Na} \\
\mathrm{Nb} \\
\mathrm{Nd} \\
\mathrm{Ne} \\
\mathrm{Ni} \\
\mathrm{Np} \\
\mathrm{O} \\
\mathrm{Os} \\
\mathrm{P} \\
\mathrm{Pa}\end{array}$ & $\begin{array}{c}22.98977 \\
92.90638 \\
144.24 \\
20.1797 \\
58.69 \\
(237) \\
15.9994 \\
190.2 \\
30.97376 \\
231.03588\end{array}$ \\
\hline 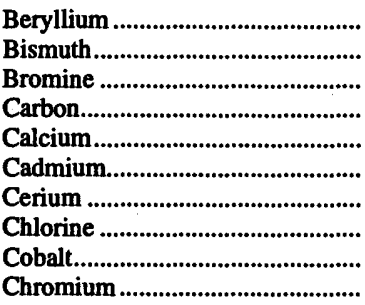 & $\begin{array}{l}\mathrm{Be} \\
\mathrm{Bi} \\
\mathrm{Br} \\
\mathrm{C} \\
\mathrm{Ca} \\
\mathrm{Cd} \\
\mathrm{Ce} \\
\mathrm{Cl} \\
\mathrm{Co} \\
\mathrm{Cr}\end{array}$ & $\begin{array}{c}9.01218 \\
208.98037 \\
79.904 \\
12.011 \\
40.078 \\
112.411 \\
140.115 \\
35.4527 \\
58.9332 \\
51.9961\end{array}$ & 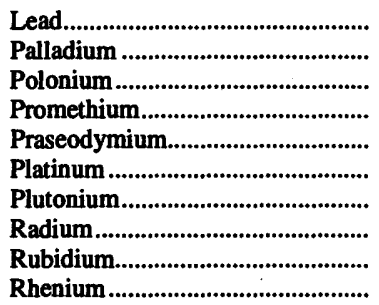 & $\begin{array}{l}\text { Pb } \\
\text { Pd } \\
\text { Po } \\
\text { Pm } \\
\text { Pr } \\
\text { Pt } \\
\text { Pu } \\
\text { Ra } \\
\text { Rb } \\
\text { Re }\end{array}$ & $\begin{array}{l}207.2 \\
106.42 \\
(209) \\
(145) \\
140.90765 \\
195.08 \\
(244) \\
(266) \\
85.4678 \\
186.207\end{array}$ \\
\hline 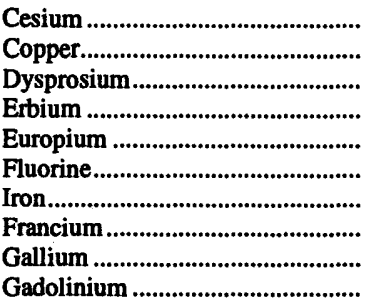 & $\begin{array}{l}\text { Cs } \\
\text { Cu } \\
\text { Dy } \\
\text { Er } \\
\text { Eu } \\
\text { F } \\
\text { Fe } \\
\text { Fr } \\
\text { Ga } \\
\text { Gd }\end{array}$ & $\begin{array}{l}132.90543 \\
63.546 \\
162.50 \\
167.26 \\
151.965 \\
18.99840 \\
55.847 \\
(223) \\
69.723 \\
157.25\end{array}$ & $\begin{array}{l}\text { Rhodium } \\
\text { Radon } \\
\text { Suthenium. } \\
\text { Antimony } \\
\text { Scandium } \\
\text { Selenium } \\
\text { Silicon }\end{array}$ & $\begin{array}{l}\text { Rh } \\
\text { Rn } \\
\text { Ru } \\
\text { S } \\
\text { Sb } \\
\text { Sc } \\
\text { Se } \\
\text { Si } \\
\text { Sm } \\
\text { Sn }\end{array}$ & $\begin{array}{l}102.90550 \\
(222) \\
101.07 \\
32.066 \\
121.75 \\
44.95591 \\
78.96 \\
28.0855 \\
150.36 \\
118.710\end{array}$ \\
\hline 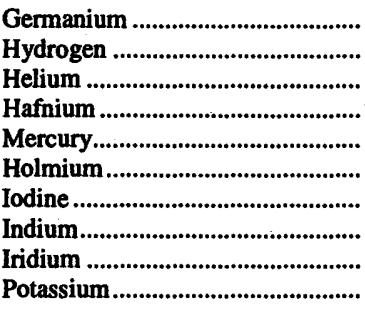 & $\begin{array}{l}\mathrm{Ge} \\
\mathrm{H} \\
\mathrm{He} \\
\mathrm{Hf} \\
\mathrm{Hg} \\
\mathrm{Ho} \\
\mathrm{I} \\
\text { In } \\
\mathrm{Ir} \\
\mathrm{K}\end{array}$ & $\begin{array}{l}72.61 \\
1.00794 \\
4.00260 \\
178.49 \\
200.59 \\
164.93032 \\
126.90447 \\
114.82 \\
192.22 \\
39.0983\end{array}$ & 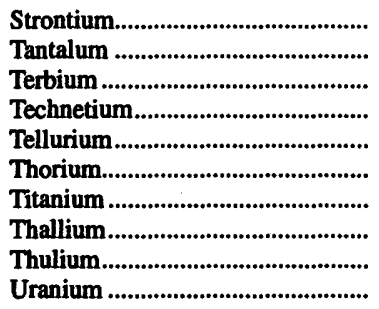 & $\begin{array}{l}\text { Sr } \\
\text { Ta } \\
\text { Tb } \\
\text { Tc } \\
\text { Te } \\
\text { Th } \\
\text { Ti } \\
\text { Tl } \\
\text { Tm } \\
\text { U }\end{array}$ & $\begin{array}{l}87.62 \\
180.9479 \\
158.92534 \\
(98) \\
127.60 \\
232.0381 \\
47.88 \\
204.3833 \\
168.93421 \\
238.0289\end{array}$ \\
\hline $\begin{array}{l}\text { Krypton } \\
\text { Lanthanum } \\
\text { Lithium } \\
\text { Lutetium } \\
\text { Magnesium } \\
\text { Manganese } \\
\text { Molybdenum } \\
\text { Nitrogen }\end{array}$ & $\begin{array}{l}\mathrm{Kr} \\
\mathrm{La} \\
\mathrm{Li} \\
\mathrm{Lu} \\
\mathrm{Mg} \\
\mathrm{Mn} \\
\mathrm{Mo} \\
\mathrm{N}\end{array}$ & $\begin{array}{l}83.80 \\
138.9055 \\
6.941 \\
174.967 \\
24.3050 \\
54.93805 \\
95.94 \\
14.00674\end{array}$ & 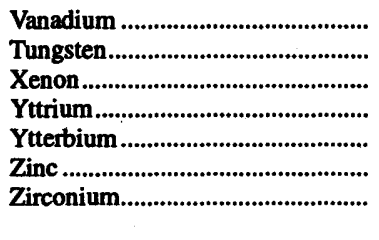 & $\begin{array}{l}\mathbf{V} \\
\mathbf{W} \\
\mathbf{X e} \\
\mathbf{Y} \\
\mathbf{Y b} \\
\mathbf{Z n} \\
\mathbf{Z r}\end{array}$ & $\begin{array}{l}50.9415 \\
183.85 \\
131.29 \\
88.90585 \\
173.04 \\
65.39 \\
91.224\end{array}$ \\
\hline
\end{tabular}


THERMOOYMAMIC PROPERTIES OF ELEMENTS IN THEIR REFERENCE STATE AT 298.15 K

\begin{tabular}{|c|c|c|c|c|c|c|c|c|c|}
\hline MAME AND FORMULA & $\begin{array}{l}\text { UEIGHT } \\
\text { g }\end{array}$ & $\begin{array}{c}\text { ENTROPY } \\
\mathrm{s}^{\circ} \\
\mathrm{J} \cdot \mathrm{mol} \mathrm{l}^{-1} \cdot \mathrm{K}^{-1}\end{array}$ & $\begin{array}{c}v^{0} \\
c m^{3}\end{array}$ & $\begin{array}{c}\text { ENTHALPY } \\
\qquad H^{\circ} \\
\mathrm{kJ} \cdot \mathrm{mol}^{-1}\end{array}$ & $\begin{array}{c}\text { FREE } \\
\text { ENERGY } \\
\mathrm{Ar}_{\mathrm{G}}^{\circ} \\
\mathrm{kJ} \cdot \mathrm{mol}^{-1}\end{array}$ & $\operatorname{LOG}(K)$ & RE & FEREN & ICES \\
\hline $\begin{array}{l}\text { SILVER (REFERENCE STATE) } \\
\text { Ag }\end{array}$ & 107.868 & $\begin{array}{r}42.55 \\
0.21\end{array}$ & $\begin{array}{r}10.272 \\
0.002\end{array}$ & 0.0 & 0.0 & 0.00 & 85 & & 85 \\
\hline $\begin{array}{l}\text { Ag }^{+} \text {(AQUEOUS IOW) } \\
\text { STD. STATE, } m=1\end{array}$ & 107.868 & $\begin{array}{r}73.45 \\
0.40\end{array}$ & & $\begin{array}{r}105.8 \\
0.1\end{array}$ & $\begin{array}{r}77.1 \\
0.1\end{array}$ & $\begin{array}{r}-13.50 \\
0.02\end{array}$ & 85 & 85 & \\
\hline $\begin{array}{l}\text { ALUMINUN (REFERENCE STATE) } \\
\text { Al }\end{array}$ & 26.982 & $\begin{array}{r}28.30 \\
0.08\end{array}$ & $\begin{array}{l}9.999 \\
0.001\end{array}$ & 0.0 & 0.0 & 0.00 & $\begin{array}{l}67 \\
92\end{array}$ & & $\begin{array}{l}92 \\
67\end{array}$ \\
\hline $\begin{array}{l}\text { Al }^{3+} \text { (AQUEOUS ION) } \\
\text { STD. STATE, }=1\end{array}$ & 26.982 & $\begin{array}{r}-332 \\
10\end{array}$ & & $\begin{array}{r}-538.4 \\
1.5\end{array}$ & $\begin{array}{r}-489.4 \\
1.4\end{array}$ & $\begin{array}{r}86.11 \\
0.79\end{array}$ & $\begin{array}{r}85 \\
170\end{array}$ & $\begin{array}{r}85 \\
330\end{array}$ & \\
\hline $\begin{array}{l}\text { ARSENIC (REFERENCE STATE) } \\
\text { As }\end{array}$ & 74.922 & $\begin{array}{r}35.69 \\
0.84\end{array}$ & $\begin{array}{r}12.963 \\
0.020\end{array}$ & 0.0 & 0.0 & 0.00 & 361 & & 361 \\
\hline $\begin{array}{l}\text { GOLD (REFERENCE STATE) } \\
\text { AU }\end{array}$ & 196.967 & $\begin{array}{r}47.49 \\
0.21\end{array}$ & $\begin{array}{r}10.215 \\
0.002\end{array}$ & 0.0 & 0.0 & 0.00 & 361 & & \\
\hline $\begin{array}{l}\text { BORON (REFERENCE STATE) } \\
\text { B }\end{array}$ & 10.811 & $\begin{array}{l}5.83 \\
0.08\end{array}$ & $\begin{array}{l}4.386 \\
0.007\end{array}$ & 0.0 & 0.0 & 0.00 & $\begin{array}{l}67 \\
85\end{array}$ & & $\begin{array}{l}67 \\
85\end{array}$ \\
\hline $\begin{array}{l}\text { BARIUM (REFERENCE STATE) } \\
\text { Ba }\end{array}$ & 137.327 & $\begin{array}{r}62.42 \\
0.84\end{array}$ & $\begin{array}{r}38.21 \\
0.02\end{array}$ & 0.0 & 0.0 & 0.00 & 361 & & 361 \\
\hline $\begin{array}{l}\mathrm{Ba}^{2+} \text { (AQUEOUS ION) } \\
\text { STD. STATE, }=1\end{array}$ & 137.327 & $\begin{array}{l}8.40 \\
0.50\end{array}$ & & $\begin{array}{r}-532.5 \\
1.0\end{array}$ & $\begin{array}{r}-555.4 \\
1.0\end{array}$ & $\begin{array}{r}97.30 \\
0.18\end{array}$ & 54 & 54 & \\
\hline $\begin{array}{l}\text { BERYLLIUN (REFERENCE STATE) } \\
\text { Be }\end{array}$ & 9.012 & $\begin{array}{l}9.50 \\
0.08\end{array}$ & $\begin{array}{l}4.880 \\
0.002\end{array}$ & 0.0 & 0.0 & 0.00 & $\begin{array}{l}85 \\
67\end{array}$ & & $\begin{array}{l}85 \\
67\end{array}$ \\
\hline $\begin{array}{l}\text { BISINTH (REFERENCE STATE) } \\
\text { Bi }\end{array}$ & 208.980 & $\begin{array}{r}56.74 \\
0.42\end{array}$ & $\begin{array}{r}21.31 \\
0.01\end{array}$ & 0.0 & 0.0 & 0.00 & 361 & & $\begin{array}{l}361 \\
127\end{array}$ \\
\hline $\begin{array}{l}\text { BROMINE (REFERENCE STATE) } \\
\mathrm{Br}_{2} \text { (LICUID) }\end{array}$ & 159.808 & $\begin{array}{r}152.20 \\
0.30\end{array}$ & $\begin{array}{r}54.58 \\
0.20\end{array}$ & 0.0 & 0.0 & 0.00 & 85 & & 67 \\
\hline $\begin{array}{l}\text { BROMINE (IDEAL GAS) } \\
\mathrm{Br}_{2}\end{array}$ & 159.808 & $\begin{array}{r}245.47 \\
0.05\end{array}$ & $\begin{array}{r}24789.7 \\
0.2\end{array}$ & $\begin{array}{r}30.9 \\
0.1\end{array}$ & $\begin{array}{l}3.1 \\
0.3\end{array}$ & $\begin{array}{r}-0.55 \\
0.05\end{array}$ & 85 & 85 & 67 \\
\hline $\begin{array}{l}\text { Br- (ACUEOUS ION) } \\
\text { STD. STATE, } m=1\end{array}$ & 79.904 & $\begin{array}{r}82.55 \\
0.20\end{array}$ & & $\begin{array}{r}-121.4 \\
0.2\end{array}$ & $\begin{array}{r}-103.8 \\
0.2\end{array}$ & $\begin{array}{r}18.18 \\
0.04\end{array}$ & 85 & 85 & \\
\hline $\begin{array}{l}\text { GRAPHITE (REFEREMCE STATE) } \\
\text { C }\end{array}$ & 12.011 & $\begin{array}{l}5.74 \\
0.10\end{array}$ & $\begin{array}{l}5.298 \\
0.001\end{array}$ & 0.0 & 0.0 & 0.00 & 85 & & 85 \\
\hline DIAMOAD & 12.011 & 2.38 & 3.417 & 1.9 & 2.9 & -0.51 & 419 & 419 & 419 \\
\hline C & & 0.20 & 0.001 & 0.0 & 0.1 & 0.02 & & & \\
\hline
\end{tabular}


THERMOOYMAMIC PROPERTIES OF ELEMENTS IN THEIR REFERENCE STATE AT $298.15 \mathrm{~K}$

\begin{tabular}{|c|c|c|c|c|c|c|c|c|c|}
\hline MAME AND FORMULA & $\begin{array}{l}\text { WEIGHT } \\
\text { g }\end{array}$ & $\begin{array}{c}\text { ENTROPY } \\
\mathrm{s}^{\circ} \\
\mathrm{J} \cdot \mathrm{mol}^{-1} \cdot \mathrm{K}^{\prime}\end{array}$ & $\begin{array}{l}\text { VOLUNE } \\
\mathrm{ve}^{3} \\
\mathrm{~cm}^{3}\end{array}$ & $\begin{array}{c}\text { ENTHALPY } \\
\mathrm{AJ}_{\mathrm{f}} \cdot \mathrm{mol}^{\circ}\end{array}$ & $\begin{array}{c}\text { FREE } \\
\text { ENERGY } \\
\Delta_{\mathbf{H}^{\circ}} \\
\mathrm{kJ} \cdot \mathrm{mol}^{-1}\end{array}$ & $\operatorname{LOG}(K)$ & RE & FERENC & CES \\
\hline $\begin{array}{l}\text { CALCIUM (REFERENCE STATE) } \\
\text { Ca }\end{array}$ & 40.078 & $\begin{array}{r}42.90 \\
0.10\end{array}$ & $\begin{array}{r}26.19 \\
0.04\end{array}$ & 0.0 & 0.0 & 0.00 & 360 & & 117 \\
\hline $\begin{array}{l}\text { Ca }^{2+} \text { (AQUEOUS ION) } \\
\text { STD. STATE, } m=1\end{array}$ & 40.078 & $\begin{array}{r}-56.2 \\
1.0\end{array}$ & & $\begin{array}{r}-543.0 \\
1.0\end{array}$ & -553.6 & 96.99 & 85 & $\begin{array}{r}85 \\
419\end{array}$ & \\
\hline $\begin{array}{l}\text { CADMIUM (REFERENCE STATE) } \\
\text { Cd }\end{array}$ & 112.411 & $\begin{array}{r}51.80 \\
0.15\end{array}$ & $\begin{array}{r}13.005 \\
0.003\end{array}$ & 0.0 & 0.0 & 0.00 & 85 & & 85 \\
\hline $\begin{array}{l}\text { Ca }{ }^{2+} \text { (AQUEOUS IOW) } \\
\text { STD. STATE, } m=1\end{array}$ & 112.411 & $\begin{array}{r}-72.8 \\
1.5\end{array}$ & & $\begin{array}{r}-75.9 \\
0.6\end{array}$ & $\begin{array}{r}-77.6 \\
0.6\end{array}$ & $\begin{array}{r}-13.59 \\
0.11\end{array}$ & 85 & 85 & \\
\hline $\begin{array}{l}\text { CERIUN (REFERENCE STATE) } \\
\text { Ce }\end{array}$ & 140.115 & $\begin{array}{r}72.0 \\
4.0\end{array}$ & $\begin{array}{r}20.77 \\
0.02\end{array}$ & 0.0 & 0.0 & 0.00 & 419 & & 361 \\
\hline $\begin{array}{l}\text { CHLORIME (REFERENCE STATE) } \\
\mathrm{Cl}_{2} \text { (IDEAL GAS) }\end{array}$ & 70.905 & $\begin{array}{r}223.08 \\
0.01\end{array}$ & $\begin{array}{r}24789.7 \\
0.2\end{array}$ & 0.0 & 0.0 & 0.00 & $\begin{array}{l}67 \\
85\end{array}$ & & $\begin{array}{l}67 \\
85\end{array}$ \\
\hline $\begin{array}{l}\mathrm{Cl}^{-} \text {(AQUEOUS IOW) } \\
\text { STD. STATE, } m=1\end{array}$ & 35.453 & $\begin{array}{r}56.60 \\
0.20\end{array}$ & & $\begin{array}{r}-167.1 \\
0.1\end{array}$ & $\begin{array}{r}-131.2 \\
0.1\end{array}$ & 22.99 & 85 & 85 & \\
\hline $\begin{array}{l}\text { COBALT (REFERENCE STATE) } \\
\text { CO }\end{array}$ & 58.933 & $\begin{array}{r}30.04 \\
0.42\end{array}$ & $\begin{array}{l}6.670 \\
0.002\end{array}$ & 0.0 & 0.0 & 0.00 & 361 & & 361 \\
\hline $\begin{array}{l}\text { CHROMIUH (REFERENCE STATE) } \\
\mathrm{Cr}\end{array}$ & 51.996 & $\begin{array}{r}23.62 \\
0.21\end{array}$ & $\begin{array}{l}7.231 \\
0.001\end{array}$ & 0.0 & 0.0 & 0.00 & 67 & & 67 \\
\hline $\begin{array}{l}\text { CESIUN (REFERENCE STATE) } \\
\text { CS }\end{array}$ & 132.905 & $\begin{array}{r}85.23 \\
0.40\end{array}$ & $\begin{array}{r}69.73 \\
0.40\end{array}$ & 0.0 & 0.0 & 0.00 & 85 & & \\
\hline $\begin{array}{l}\text { CS }^{+} \text {(AQUEOUS ION) } \\
\text { STD. STATE, } m=1\end{array}$ & 132.905 & $\begin{array}{r}132.1 \\
0.5\end{array}$ & & $\begin{array}{r}-258.0 \\
0.5\end{array}$ & $\begin{array}{r}-291.5 \\
1.0\end{array}$ & $\begin{array}{r}51.07 \\
0.18\end{array}$ & 85 & 85 & \\
\hline $\begin{array}{l}\text { COPPER (REFERENCE STATE) } \\
\text { Cu }\end{array}$ & 63.546 & $\begin{array}{r}33.14 \\
0.03\end{array}$ & $\begin{array}{l}7.113 \\
0.003\end{array}$ & 0.0 & 0.0 & 0.00 & 365 & & $\begin{array}{r}443 \\
67\end{array}$ \\
\hline $\begin{array}{l}\mathrm{Cu}^{+} \text {(AQUEOUS IOW) } \\
\text { STD. STATE, } m=1\end{array}$ & 63.546 & $\begin{array}{r}40.60 \\
0.40\end{array}$ & & $\begin{array}{r}71.7 \\
0.1\end{array}$ & $\begin{array}{r}50.0 \\
0.1\end{array}$ & $\begin{array}{r}-8.76 \\
0.02\end{array}$ & 419 & 419 & \\
\hline $\begin{array}{l}\mathrm{Cu}^{2+} \text { (AQUEOUS IOW) } \\
\text { STD. STATE, } m=1\end{array}$ & 63.546 & $\begin{array}{r}-98.0 \\
4.0\end{array}$ & & $\begin{array}{r}64.9 \\
1.0\end{array}$ & $\begin{array}{r}65.1 \\
0.1\end{array}$ & $\begin{array}{r}-11.40 \\
0.02\end{array}$ & 85 & 85 & \\
\hline $\begin{array}{l}\text { FLUORINE (REFEREMCE STATE) } \\
F_{2} \text { (IDEAL GAS) }\end{array}$ & 37.997 & $\begin{array}{r}202.79 \\
0.02\end{array}$ & $\begin{array}{r}24789.7 \\
0.2\end{array}$ & 0.0 & 0.0 & 0.00 & $\begin{array}{l}67 \\
85\end{array}$ & & $\begin{array}{l}67 \\
85\end{array}$ \\
\hline $\begin{array}{l}F^{-} \quad \text { (NQUEOUS ION) } \\
\text { STD. STATE, } m=1\end{array}$ & 18.998 & $\begin{array}{r}-13.8 \\
0.8\end{array}$ & & $\begin{array}{r}-335.4 \\
0.7\end{array}$ & $\begin{array}{r}-281.5 \\
0.7\end{array}$ & $\begin{array}{r}49.32 \\
0.12\end{array}$ & 85 & 85 & \\
\hline
\end{tabular}


THERMOOYMANIC PROPERTIES OF ELEMENTS IN THEIR REFERENCE STATE AT $298.15 \mathrm{~K}$

\begin{tabular}{|c|c|c|c|c|c|c|c|c|c|}
\hline MAME AMO FORMULA & $\begin{array}{l}\text { MEIGHT } \\
g\end{array}$ & $\begin{array}{c}\text { ENTROPY } \\
\mathbf{S}^{\circ} \\
\mathrm{J} \cdot \mathrm{mol}^{-1} \cdot \mathrm{K}^{\prime}\end{array}$ & $\begin{array}{l}\text { VOLUNE } \\
v^{0} \\
\mathrm{~cm}^{3}\end{array}$ & $\begin{array}{l}\text { ENTHALPY } \\
\Delta_{1}^{\Delta_{1} H^{\bullet}} \\
k J \cdot \operatorname{mol}^{-1}\end{array}$ & $\begin{array}{c}\text { FREE } \\
\text { ENERGY } \\
\Delta, 6^{\circ} \\
k J \cdot \mathbf{m o l}^{-1}\end{array}$ & $\operatorname{LOG}(K)$ & RE & FEREMC & CES \\
\hline $\begin{array}{l}\text { IRON (REFERENCE STATE) } \\
\text { Fe }\end{array}$ & 55.847 & $\begin{array}{r}27.09 \\
0.13\end{array}$ & $\begin{array}{l}7.092 \\
0.004\end{array}$ & 0.0 & 0.0 & 0.00 & $\begin{array}{l}90 \\
67\end{array}$ & & $\begin{array}{l}90 \\
67\end{array}$ \\
\hline $\begin{array}{l}\text { Fe }^{2+} \text { (AQUECUS IOW) } \\
\text { STD. STATE, } m=1\end{array}$ & 55.847 & $\begin{array}{r}-107.1 \\
2.0\end{array}$ & & $\begin{array}{r}-91.1 \\
3.0\end{array}$ & $\begin{array}{r}-90.0 \\
2.0\end{array}$ & $\begin{array}{r}15.77 \\
0.35\end{array}$ & 411 & 411 & \\
\hline $\begin{array}{l}\text { Fe }^{3+} \quad \text { (ACUEOUS IOW) } \\
\text { STO. STATE, } m=1\end{array}$ & 55.847 & $\begin{array}{r}-280.0 \\
13.0\end{array}$ & & $\begin{array}{r}-49.9 \\
5.0\end{array}$ & $\begin{array}{r}-16.7 \\
2.0\end{array}$ & $\begin{array}{l}2.93 \\
0.35\end{array}$ & 411 & 411 & \\
\hline $\begin{array}{l}\text { GERMANIUN (REFERENCE STATE) } \\
\text { Ge }\end{array}$ & 72.61 & $\begin{array}{r}31.09 \\
0.15\end{array}$ & $\begin{array}{r}13.630 \\
0.005\end{array}$ & 0.0 & 0.0 & 0.00 & 85 & & 361 \\
\hline $\begin{array}{l}\text { HYDROGEN (REFERENCE STATE) } \\
\mathrm{H}_{2} \text { (IDEAL GAS) }\end{array}$ & 2.016 & $\begin{array}{r}130.68 \\
0.02\end{array}$ & $\begin{array}{r}24789.7 \\
0.2\end{array}$ & 0.0 & 0.0 & 0.00 & $\begin{array}{l}67 \\
85\end{array}$ & & $\begin{array}{l}67 \\
85\end{array}$ \\
\hline $\begin{array}{l}n^{+} \quad \text { (AQUEOUS IOW) } \\
\text { STD. STATE, } m=1\end{array}$ & 1.008 & 0.00 & 0 & 0.0 & 0.0 & 0.00 & & & \\
\hline $\begin{array}{l}\text { MERCURY (REFERENCE STATE) } \\
\text { Hg (LIQUID) }\end{array}$ & 200.59 & $\begin{array}{r}75.90 \\
0.12\end{array}$ & $\begin{array}{r}14.822 \\
0.002\end{array}$ & 0.0 & 0.0 & 0.00 & 85 & & 361 \\
\hline $\begin{array}{l}\mathrm{Hg}^{2+} \text { (AQUEOUS IOW) } \\
\text { STD. STATE, } m=1\end{array}$ & 200.59 & $\begin{array}{r}-36.2 \\
0.8\end{array}$ & & $\begin{array}{r}170.2 \\
0.2\end{array}$ & $\begin{array}{r}163.5 \\
0.2\end{array}$ & $\begin{array}{r}-28.64 \\
0.04\end{array}$ & 85 & 85 & \\
\hline $\begin{array}{l}\mathrm{Hg}_{2}^{2+} \text { (ACUEOUS ION) } \\
\text { STD.STATE, } m=1\end{array}$ & 401.18 & $\begin{array}{r}65.74 \\
0.80\end{array}$ & & $\begin{array}{r}166.9 \\
0.5\end{array}$ & $\begin{array}{r}153.6 \\
0.5\end{array}$ & $\begin{array}{r}-26.91 \\
0.09\end{array}$ & 85 & 85 & \\
\hline $\begin{array}{l}\text { ICOINE (REFERENCE STATE) } \\
I_{2}\end{array}$ & 253.809 & $\begin{array}{r}116.14 \\
0.30\end{array}$ & $\begin{array}{r}51.29 \\
0.06\end{array}$ & 0.0 & 0.0 & 0.00 & 85 & 67 & 67 \\
\hline $\begin{array}{l}\text { ICOINE (IDEAL GAS) } \\
I_{2}\end{array}$ & 253.809 & $\begin{array}{r}260.69 \\
0.02\end{array}$ & $\begin{array}{r}24789.7 \\
0.2\end{array}$ & $\begin{array}{r}62.4 \\
0.1\end{array}$ & $\begin{array}{r}19.3 \\
0.1\end{array}$ & $\begin{array}{r}-3.39 \\
0.01\end{array}$ & 67 & & 67 \\
\hline $\begin{array}{l}\text { (AQUECUS ION) } \\
\text { STD. STATE, } m=1\end{array}$ & 126.904 & $\begin{array}{r}106.45 \\
0.30\end{array}$ & & $\begin{array}{r}-56.8 \\
0.1\end{array}$ & $\begin{array}{r}-51.7 \\
0.1\end{array}$ & $\begin{array}{l}9.06 \\
0.02\end{array}$ & 85 & 85 & \\
\hline $\begin{array}{l}\text { POTASSIUA (REFEREMCE STATE) } \\
K\end{array}$ & 39.098 & $\begin{array}{r}64.67 \\
0.20\end{array}$ & $\begin{array}{r}45.36 \\
0.09\end{array}$ & 0.0 & 0.0 & 0.00 & $\begin{array}{l}85 \\
67\end{array}$ & & $\begin{array}{l}85 \\
67\end{array}$ \\
\hline $\begin{array}{l}K^{+} \quad \text { (AQUEOUS IOW) } \\
\text { STD. STATE, } m=1\end{array}$ & 39.098 & $\begin{array}{r}101.20 \\
0.20\end{array}$ & & $\begin{array}{r}-252.1 \\
0.1\end{array}$ & $\begin{array}{r}-282.5 \\
0.1\end{array}$ & $\begin{array}{r}49.48 \\
0.01\end{array}$ & 85 & 85 & \\
\hline $\begin{array}{l}\text { LITHIUM (REFERENCE STATE) } \\
\text { Li }\end{array}$ & 6.941 & $\begin{array}{r}29.09 \\
0.20\end{array}$ & $\begin{array}{r}13.017 \\
0.007\end{array}$ & 0.0 & 0.0 & 0.00 & 67 & & $\begin{array}{l}67 \\
85\end{array}$ \\
\hline $\begin{array}{l}\mathrm{Li}^{+} \text {(AQUEOUS IOW) } \\
\text { STD. STATE, } m=1\end{array}$ & 6.941 & $\begin{array}{r}12.24 \\
0.15\end{array}$ & & $\begin{array}{r}-278.5 \\
0.1\end{array}$ & $\begin{array}{r}-292.9 \\
0.1\end{array}$ & $\begin{array}{r}51.31 \\
0.01\end{array}$ & 85 & 85 & \\
\hline
\end{tabular}


THERMOOYNAMIC PROPERTIES OF ELEMENTS IN THEIR REFERENCE STATE AT $298.15 \mathrm{~K}$

\begin{tabular}{|c|c|c|c|c|c|c|c|c|c|}
\hline MAME AMD FORHULA & MEIGHT & ENTROPY & VOLUANE & ENTHALPY & FREE & LOG(K) & & FEREMC & \\
\hline & 9 & $\mathrm{~J} \cdot \mathrm{mol}^{-1} \cdot \mathrm{K}^{-1}$ & $\mathrm{~cm}^{3}$ & $k J \cdot m^{-1}$ & $\mathrm{~kJ} \cdot \mathrm{mol}^{-1}$ & & $\mathbf{s}$ & $H / G$ & C \\
\hline $\begin{array}{l}\text { MAGNESIUH (REFERENCE STATE) } \\
\text { Mg }\end{array}$ & 24.305 & $\begin{array}{r}32.67 \\
0.10\end{array}$ & $\begin{array}{r}13.996 \\
0.007\end{array}$ & 0.0 & 0.0 & 0.00 & $\begin{array}{l}85 \\
67\end{array}$ & & 85 \\
\hline $\begin{array}{l}\text { Mg } \\
\text { STD. STATE, } m=1\end{array}$ & 24.305 & $\begin{array}{r}-137.0 \\
4.0\end{array}$ & & $\begin{array}{r}-467.0 \\
0.6\end{array}$ & $\begin{array}{r}-455.4 \\
0.6\end{array}$ & $\begin{array}{r}79.78 \\
0.11\end{array}$ & 85 & 85 & \\
\hline $\begin{array}{l}\text { MWGGANESE (REFERENCE STATE) } \\
\text { in }\end{array}$ & 54.938 & $\begin{array}{r}32.01 \\
0.08\end{array}$ & $\begin{array}{l}7.354 \\
0.007\end{array}$ & 0.0 & 0.0 & 0.00 & 91 & & 91 \\
\hline $\begin{array}{l}\text { Mn } \\
\text { STD. STATE, } m=1\end{array}$ & 54.938 & $\begin{array}{r}-73.60 \\
1.00\end{array}$ & & $\begin{array}{r}-220.8 \\
0.5\end{array}$ & $\begin{array}{r}-228.1 \\
0.5\end{array}$ & $\begin{array}{r}39.96 \\
0.09\end{array}$ & 419 & 419 & \\
\hline $\begin{array}{l}\text { MOLYBDENUM (REFERENCE STATE) } \\
\text { Mo }\end{array}$ & 95.94 & $\begin{array}{r}28.66 \\
0.21\end{array}$ & $\begin{array}{l}9.387 \\
0.005\end{array}$ & 0.0 & 0.0 & 0.00 & 91 & & 91 \\
\hline $\begin{array}{l}\text { NITROGEN (REFERENCE STATE) } \\
\mathrm{N}_{2}\end{array}$ & 28.013 & $\begin{array}{r}191.61 \\
0.02\end{array}$ & $\begin{array}{r}24789.7 \\
0.2\end{array}$ & 0.0 & 0.0 & 0.00 & $\begin{array}{l}67 \\
85\end{array}$ & & $\begin{array}{l}67 \\
85\end{array}$ \\
\hline $\begin{array}{l}\text { SODIUN (REFERENCE STATE) } \\
\text { Ma }\end{array}$ & 22.990 & $\begin{array}{r}51.46 \\
0.20\end{array}$ & $\begin{array}{r}23.81 \\
0.01\end{array}$ & 0.0 & 0.0 & 0.00 & $\begin{array}{l}67 \\
85\end{array}$ & & $\begin{array}{l}67 \\
85\end{array}$ \\
\hline $\begin{array}{l}\text { Ma+ (AQUEOUS IOW) } \\
\text { STD. STATE, } m=1\end{array}$ & 22.990 & $\begin{array}{r}58.45 \\
0.15\end{array}$ & & $\begin{array}{r}-240.3 \\
0.1\end{array}$ & $\begin{array}{r}-261.5 \\
0.1\end{array}$ & $\begin{array}{r}45.81 \\
0.01\end{array}$ & 85 & 85 & \\
\hline $\begin{array}{l}\text { MICKEL (REFERENCE STATE) } \\
\text { Mi }\end{array}$ & 58.69 & $\begin{array}{r}29.87 \\
0.08\end{array}$ & $\begin{array}{l}6.588 \\
0.003\end{array}$ & 0.0 & 0.0 & 0.00 & 67 & & 67 \\
\hline $\begin{array}{l}M i^{2+} \text { (AQUEOUS IOW) } \\
\text { STD. STATE, } m=1\end{array}$ & 58.69 & $\begin{array}{r}-128.9 \\
2.0\end{array}$ & & $\begin{array}{r}-54.0 \\
0.9\end{array}$ & $\begin{array}{r}-45.6 \\
0.9\end{array}$ & $\begin{array}{l}7.99 \\
0.15\end{array}$ & 419 & 419 & \\
\hline $\begin{array}{l}\text { OXYGEN (REFERENCE STATE) } \\
\mathrm{O}_{2} \text { (IDEAL GAS) }\end{array}$ & 31.999 & $\begin{array}{r}205.15 \\
0.02\end{array}$ & $\begin{array}{r}24789.7 \\
0.2\end{array}$ & 0.0 & 0.0 & 0.00 & $\begin{array}{l}67 \\
85\end{array}$ & & $\begin{array}{l}67 \\
85\end{array}$ \\
\hline $\begin{array}{l}\text { PHOSPHORUS (REFERENCE STATE) } \\
\begin{array}{l}\text { P (WHITE) }\end{array}\end{array}$ & 30.974 & $\begin{array}{r}41.09 \\
0.25\end{array}$ & $\begin{array}{r}17.3 \\
0.3\end{array}$ & 0.0 & 0.0 & 0.00 & 85 & & 85 \\
\hline $\begin{array}{l}\text { LEAD (REFERENCE STATE) } \\
\text { Pb }\end{array}$ & 207.2 & $\begin{array}{r}64.8 \\
0.5\end{array}$ & $\begin{array}{r}18.267 \\
0.006\end{array}$ & 0.0 & 0.0 & 0.00 & 67 & & 67 \\
\hline $\begin{array}{l}\mathrm{Pb}^{2+} \text { (AQUEOUS IOW) } \\
\text { STD. STATE, } m=1\end{array}$ & 207.2 & $\begin{array}{r}18.5 \\
1.0\end{array}$ & & $\begin{array}{l}0.9 \\
0.3\end{array}$ & $\begin{array}{r}-24.2 \\
0.2\end{array}$ & $\begin{array}{l}4.24 \\
0.04\end{array}$ & 85 & 85 & \\
\hline $\begin{array}{l}\text { PLATIMUN (REFERENCE STATE) } \\
\text { Pt }\end{array}$ & 195.08 & $\begin{array}{r}41.63 \\
0.21\end{array}$ & $\begin{array}{l}9.091 \\
0.004\end{array}$ & 0.0 & 0.0 & 0.00 & 361 & & 361 \\
\hline $\begin{array}{l}\text { SULFUR (REFERENCE STATE) } \\
\text { S ORTHORHOMBIC }\end{array}$ & 32.066 & $\begin{array}{r}32.05 \\
0.05\end{array}$ & $\begin{array}{r}15.511 \\
0.005\end{array}$ & 0.0 & 0.0 & 0.00 & $\begin{array}{l}67 \\
85\end{array}$ & & 67 \\
\hline
\end{tabular}


THERMOOYNAMIC PROPERTIES OF ELEMENTS IN THEIR REFERENCE STATE AT $298.15 \mathrm{~K}$

\begin{tabular}{|c|c|c|c|c|c|c|c|c|c|}
\hline \multirow[t]{2}{*}{ MANE AND FORIULA } & \multirow{2}{*}{$\begin{array}{l}\text { HEIGHT } \\
\text { g }\end{array}$} & \multirow{2}{*}{$\begin{array}{c}\text { ENTROPY } \\
S^{\circ} \\
J \cdot \mathrm{mol}^{-1} \cdot \mathrm{K}^{-1}\end{array}$} & \multirow{2}{*}{$\begin{array}{l}\text { VOLUME } \\
\qquad \begin{array}{c}v^{\circ} \\
\mathrm{cm}^{3}\end{array}\end{array}$} & \multirow{2}{*}{$\begin{array}{l}\text { ENTHALPY } \\
\qquad \begin{array}{c}A_{\mathbf{f}} \mathrm{H}^{0} \\
\mathrm{~kJ} \cdot \mathrm{mol}^{-1}\end{array}\end{array}$} & \multirow{2}{*}{$\begin{array}{c}\text { FREE } \\
\text { ENERGY } \\
\text { ArG } \\
\text { kd.mol }\end{array}$} & \multirow[t]{2}{*}{$\operatorname{LOO}(K)$} & \multicolumn{3}{|c|}{ REFERENCES } \\
\hline & & & & & & & $\mathbf{s}$ & H/G & C \\
\hline SULFUR & 32.066 & 33.03 & & 0.3 & 0.0 & 0.00 & 67 & 419 & 67 \\
\hline S MONOCLINIC & & 0.05 & & 0.1 & & & & & \\
\hline$S^{2-}$ (AQUEOUS ION) & 32.066 & -14.6 & & 33.1 & 85.8 & -15.03 & 419 & 419 & \\
\hline STD. STATE, $=1$ & & 1.0 & & 1.0 & 1.0 & 0.18 & & & \\
\hline DIATOHIC SULFUR & 64.132 & 228.17 & 24789.7 & 128.6 & 79.7 & -13.96 & 67 & 67 & 67 \\
\hline$S_{2}$ (IDEAL GAS) & & 0.02 & 0.2 & 0.3 & 0.3 & 0.05 & & & \\
\hline ANTIMONY (REFERENCE STATE) & 121.75 & 45.52 & 18.178 & 0.0 & 0.0 & 0.00 & 361 & & 361 \\
\hline sb & & 0.21 & 0.009 & & & & & & \\
\hline $\begin{array}{l}\text { SELENIUN (REFERENCE STATE) } \\
\text { SE }\end{array}$ & 78.96 & $\begin{array}{r}42.27 \\
0.05\end{array}$ & $\begin{array}{r}16.42 \\
0.01\end{array}$ & 0.0 & 0.0 & 0.00 & 62 & & 126 \\
\hline SILICON (REFERENCE STATE) & 28.086 & 18.81 & 12.056 & 0.0 & 0.0 & 0.00 & 67 & & 67 \\
\hline si & & 0.08 & 0.002 & & & & 85 & & \\
\hline $\mathrm{H}_{4} \mathrm{SiO}_{4}^{\circ}$ (ACUEOUS ION) & 96.115 & 180.0 & & -1460.0 & -1307.5 & 229.12 & & 179 & 422 \\
\hline STD. STATE, $m=1$ & & 4.2 & & 1.7 & 2.1 & 0.37 & & 285 & 416 \\
\hline TIN (REFERENCE STATE) & 118.710 & 51.18 & 16.29 & 0.0 & 0.0 & 0.00 & 85 & & 85 \\
\hline Sn (HHITE, TET.) & & 0.08 & 0.01 & & & & & & \\
\hline STRONTIUN (REFERENCE STATE) & 87.62 & 55.69 & 33.92 & 0.0 & 0.0 & 0.00 & 39 & & 67 \\
\hline Sr & & 0.20 & 0.02 & & & & & & \\
\hline $\mathrm{Sr}^{2+}$ (AQUEOUS ION) & 87.62 & -31.5 & & -550.9 & -563.8 & 98.78 & 55 & 55 & \\
\hline STD. STATE, $m=1$ & & 2.0 & & 0.5 & 0.8 & 0.14 & & & \\
\hline TELLURIUM (REFERENCE STATE) & 127.60 & 49.71 & 20.48 & 0.0 & 0.0 & 0.00 & 361 & & 361 \\
\hline Te & & 0.20 & 0.01 & & & & & & \\
\hline THORIUN (REFERENCE STATE) & 232.038 & 51.83 & 19.79 & 0.0 & 0.0 & 0.00 & 319 & & 319 \\
\hline Th & & 0.50 & 0.01 & & & & & & \\
\hline TITANIUN (REFERENCE STATE) & 47.88 & 30.76 & 10.63 & 0.0 & 0.0 & 0.00 & 67 & & 67 \\
\hline $\mathbf{T i}$ & & 0.10 & 0.01 & & & & & & \\
\hline URANIUN (REFERENCE STATE) & 238.029 & 50.2 & 12.50 & 0.0 & 0.0 & 0.00 & 85 & & 85 \\
\hline $\mathbf{u}$ & & 0.2 & 0.02 & & & & & & \\
\hline VAMADIUM (REFERENCE STATE) & 50.942 & 28.94 & 8.350 & 0.0 & 0.0 & 0.00 & 67 & & 67 \\
\hline $\mathbf{v}$ & & 0.42 & 0.004 & & & & & & \\
\hline TUNGSTEN (REFERENCE STATE) & 183.85 & 32.65 & 9.545 & 0.0 & 0.0 & 0.00 & 67 & & 67 \\
\hline $\mathbf{u}$ & & 0.10 & 0.004 & & & & & & \\
\hline
\end{tabular}


THERMOOYMAMIC PROPERTIES OF ELEMENTS IN THEIR REFERENCE STATE AT $298.15 \mathrm{~K}$

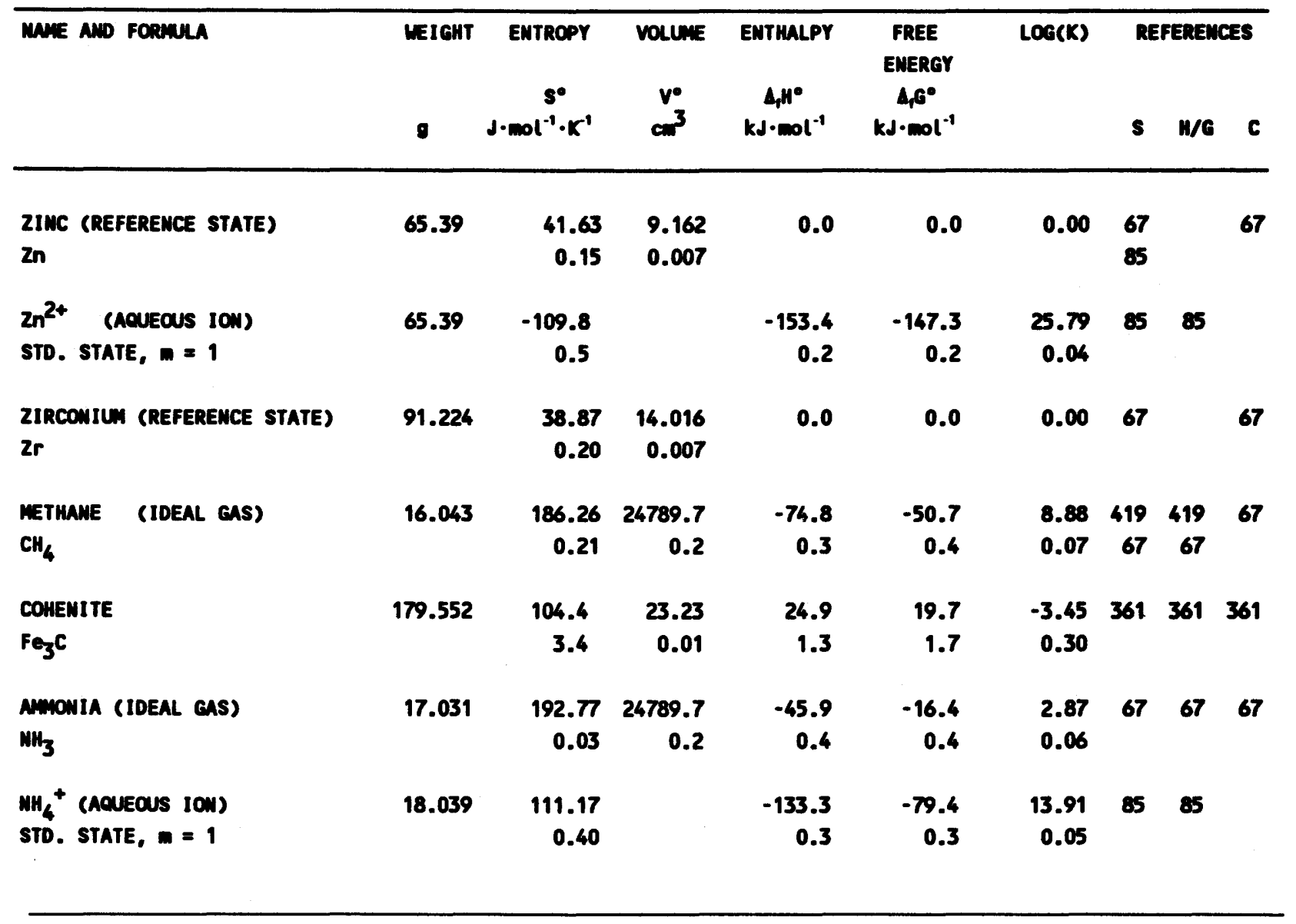


THERMODYMAMIC PROPERTIES OF SULFIDE MINERALS AT $298.15 \mathrm{~K}$

\begin{tabular}{|c|c|c|c|c|c|c|c|c|c|}
\hline MAME AND FORMULA & $\begin{array}{l}\text { MEIENT } \\
\text { g }\end{array}$ & $\begin{array}{c}\text { ENTROPY } \\
S^{\circ} \\
J \cdot \mathrm{mol}^{-1} \cdot \mathrm{K}^{-1}\end{array}$ & $\begin{array}{l}\text { VOLune } \\
v^{3} \\
\mathrm{~cm}^{3}\end{array}$ & $\begin{array}{l}\text { ENTHALPY } \\
\Delta_{\mathbf{H}^{\prime}} \mathrm{H}^{\bullet} \\
\mathrm{kJ} \cdot \mathrm{mol}^{-1}\end{array}$ & $\begin{array}{c}\text { FREE } \\
\text { ENERGY } \\
\Delta_{\mathbf{p}^{\circ}} \\
\mathrm{kJ} \cdot \mathrm{mol}^{-1}\end{array}$ & $L O G(K)$ & RE & FERENC & ICES \\
\hline ACANTHITE (ARGENTITE) & 247.802 & 142.9 & 34.19 & -32.0 & -39.7 & 6.96 & 161 & 121 & 141 \\
\hline $\mathrm{Ag}_{2} \mathrm{~S}$ & & 0.3 & 0.04 & 1.0 & 1.0 & 0.18 & & 52 & 306 \\
\hline REALGAR & 106.988 & 63.5 & 29.80 & -30.9 & -29.6 & 5.19 & 429 & 419 & 270 \\
\hline Ass & & 0.6 & 0.24 & 5.0 & 5.0 & 0.88 & & 270 & \\
\hline ORPIMENT & 246.041 & 163.6 & 70.51 & -91.6 & -90.4 & 15.84 & 372 & 50 & 210 \\
\hline $\mathrm{As}_{2} \mathrm{~S}_{3}$ & & 1.6 & 0.25 & 4.2 & 4.2 & 0.74 & & & \\
\hline BISWUTHINITE & 514.159 & 200.4 & 75.52 & -135.2 & -132.4 & 23.20 & 372 & 52 & \\
\hline$B i_{2} S_{3}$ & & 3.3 & 0.04 & 2.4 & 2.6 & 0.46 & & & \\
\hline TELLUROBISNTTHITE & 800.761 & 261.1 & 101.85 & -78.7 & -78.2 & 13.71 & 201 & 279 & \\
\hline $\mathrm{Bi}_{2} \mathrm{~T}_{3}$ & & 5.0 & 0.09 & 2.1 & 2.6 & 0.46 & & & \\
\hline OLDHAMITE & 72.144 & 56.7 & 27.72 & -474.9 & -469.5 & 82.25 & 67 & 67 & \\
\hline Cas & & 1.3 & 0.09 & 2.1 & 2.1 & 0.37 & & 279 & \\
\hline GREEMOCKITE & 144.477 & 72.2 & 29.93 & -149.6 & -146.1 & 25.60 & 38 & 3 & 38 \\
\hline $\cos$ & & 0.3 & 0.02 & 1.3 & 1.3 & 0.23 & & & \\
\hline CATtIERITE & 123.065 & 74.8 & 25.52 & -150.9 & -145.1 & 25.42 & 399 & 60 & 326 \\
\hline $\cos _{2}$ & & 0.2 & 0.01 & 4.9 & 4.9 & 0.86 & & 376 & \\
\hline LIMMAEITE & 305.064 & 176.0 & 62.55 & -347.5 & -334.9 & 58.66 & 399 & 60 & \\
\hline $\cos _{3} S_{4}$ & & 0.4 & 0.02 & 7.3 & 7.3 & 1.28 & & 98 & \\
\hline COVELLITE & 95.612 & 67.4 & 20.42 & -54.6 & -55.3 & 9.69 & 109 & 337 & 109 \\
\hline cus & & 0.1 & 0.02 & 0.3 & 0.3 & 0.05 & 442 & & 462 \\
\hline AMILITE & 143.272 & 107.5 & 25.79 & -73.3 & -78.5 & 13.76 & 133 & 337 & 133 \\
\hline $\mathrm{Cu}_{1.75^{\mathrm{S}}}$ & & 0.2 & 0.02 & 0.3 & 0.3 & 0.05 & & 140 & \\
\hline DIGENITE & 146.449 & 109.6 & 25.63 & -73.0 & -78.3 & 13.72 & 133 & 337 & 133 \\
\hline $\mathrm{Cu}_{1.80^{\mathrm{S}}}$ & & 0.2 & 0.05 & 0.5 & 0.5 & 0.09 & & & \\
\hline DJURLEITE & 155.981 & 114.8 & 26.89 & -78.8 & -84.2 & 14.75 & 394 & 337 & 394 \\
\hline $\mathrm{Cu}_{1.95^{\mathrm{S}}}$ & & 0.2 & 0.02 & 0.4 & 0.4 & 0.07 & & & \\
\hline CHALCOCITE & 159.158 & 116.2 & 27.48 & -83.9 & -89.2 & 15.62 & 142 & 369 & 109 \\
\hline $\mathrm{Cu}_{2} \mathrm{~s}$ & & 0.2 & 0.02 & 1.1 & 1.1 & 0.19 & 109 & 48 & 142 \\
\hline CHALCOPYRITE & 183.525 & 124.9 & 43.92 & -194.9 & -195.1 & 34.18 & 371 & 369 & 325 \\
\hline Cufes $_{2}$ & & 0.2 & 0.02 & 1.6 & 1.6 & 0.28 & & 371 & 369 \\
\hline BORNITE & 501.841 & 398.5 & 98.73 & -371.6 & -394.7 & 69.32 & 369 & 369 & 325 \\
\hline $\mathrm{Cu}_{5} \mathrm{FeS}_{4}$ & & 0.8 & 0.05 & 2.1 & 2.1 & 0.37 & & & 369 \\
\hline
\end{tabular}


THERMCOYMAMIC PROPERTIES OF SULFIDE MINERALS AT $298.15 \mathrm{~K}$

\begin{tabular}{|c|c|c|c|c|c|c|c|c|c|}
\hline MAME AND FORIULA & $\begin{array}{l}\text { MEIGHT } \\
\text { g }\end{array}$ & $\begin{array}{c}\text { ENTROPY } \\
\mathrm{s}^{\bullet} \\
\mathrm{J} \cdot \mathrm{mol}^{-1} \cdot \mathrm{K}^{\prime}\end{array}$ & $\begin{array}{l}\text { VOLUME } \\
\text { vo } \\
\mathrm{cm}^{3}\end{array}$ & $\begin{array}{l}\text { ENTHALPY } \\
\Delta_{\mathbf{H}} \mathrm{H}^{\circ} \\
\mathrm{kJ} \cdot \mathrm{mol}^{-1}\end{array}$ & $\begin{array}{c}\text { FREE } \\
\text { EMERGY } \\
\begin{array}{c}\Delta, 6^{\circ} \\
\mathrm{kJ} \cdot \mathrm{mol}^{-1}\end{array}\end{array}$ & $\operatorname{LOO}(K)$ & $\mathbf{s}$ & FEREMC & |CES \\
\hline $\begin{array}{l}\text { CUBANITE } \\
\text { CuFe }_{2} s_{3}\end{array}$ & 271.438 & $\begin{array}{r}205.0 \\
0.8\end{array}$ & $\begin{array}{r}67.44 \\
0.06\end{array}$ & & & & 388 & & 280 \\
\hline $\begin{array}{l}\text { MUKUNDAMITE } \\
\mathrm{Cu}_{5.5} \mathrm{FeS}_{6.5}\end{array}$ & 613.779 & & $\begin{array}{r}278.0 \\
0.5\end{array}$ & & $\begin{array}{r}-564.5 \\
3.4\end{array}$ & $\begin{array}{r}98.90 \\
0.59\end{array}$ & & 369 & \\
\hline $\begin{array}{l}\text { TROILITE } \\
\text { FeS }\end{array}$ & 87.913 & $\begin{array}{r}60.3 \\
0.2\end{array}$ & $\begin{array}{r}18.20 \\
0.03\end{array}$ & $\begin{array}{r}-101.0 \\
1.5\end{array}$ & $\begin{array}{r}-101.3 \\
1.5\end{array}$ & $\begin{array}{r}17.75 \\
0.26\end{array}$ & $\begin{array}{l}143 \\
347\end{array}$ & $\begin{array}{r}69 \\
3\end{array}$ & $\begin{array}{r}79 \\
131\end{array}$ \\
\hline $\begin{array}{l}\text { PYRRHOTITE } \\
\text { Fe. } 875^{S}\end{array}$ & 80.932 & $\begin{array}{r}60.7 \\
0.2\end{array}$ & $\begin{array}{r}17.49 \\
0.05\end{array}$ & $\begin{array}{r}-97.5 \\
2.0\end{array}$ & $\begin{array}{r}-98.9 \\
2.0\end{array}$ & $\begin{array}{r}17.33 \\
0.35\end{array}$ & 143 & 131 & 131 \\
\hline $\begin{array}{l}\text { PYRRHOTITE } \\
\text { Fe } .90^{5}\end{array}$ & 82.328 & $\begin{array}{r}63.2 \\
0.1\end{array}$ & $\begin{array}{r}16.88 \\
0.10\end{array}$ & $\begin{array}{r}-97.6 \\
2.0\end{array}$ & $\begin{array}{r}-99.6 \\
2.0\end{array}$ & $\begin{array}{r}14.45 \\
0.35\end{array}$ & 135 & 375 & 135 \\
\hline $\begin{array}{l}\text { PYRITE } \\
\text { fes }_{2}\end{array}$ & 119.979 & $\begin{array}{r}52.9 \\
0.1\end{array}$ & $\begin{array}{r}23.94 \\
0.01\end{array}$ & $\begin{array}{r}-171.5 \\
1.7\end{array}$ & $\begin{array}{r}-160.1 \\
1.7\end{array}$ & $\begin{array}{r}28.05 \\
0.30\end{array}$ & 138 & $\begin{array}{r}409 \\
67\end{array}$ & 79 \\
\hline $\begin{array}{l}\text { MARCASITE } \\
\text { FeS }_{2}\end{array}$ & 119.979 & $\begin{array}{r}53.9 \\
0.1\end{array}$ & $\begin{array}{r}24.58 \\
0.02\end{array}$ & $\begin{array}{r}-169.5 \\
2.1\end{array}$ & $\begin{array}{r}-158.4 \\
2.1\end{array}$ & $\begin{array}{r}27.75 \\
0.37\end{array}$ & 139 & 139 & 139 \\
\hline $\begin{array}{l}\text { LOELLINGITE } \\
\text { FeAs }_{2}\end{array}$ & 205.690 & $\begin{array}{r}80.1 \\
0.7\end{array}$ & $\begin{array}{r}27.51 \\
0.02\end{array}$ & $\begin{array}{r}-57.8 \\
5.0\end{array}$ & $\begin{array}{r}-52.3 \\
5.0\end{array}$ & $\begin{array}{l}9.17 \\
0.88\end{array}$ & 332 & 25 & \\
\hline $\begin{array}{l}\text { FERROSELITE } \\
\text { FeSe }_{2}\end{array}$ & 213.767 & $\begin{array}{r}86.9 \\
0.3\end{array}$ & $\begin{array}{r}29.96 \\
0.05\end{array}$ & & & & 138 & & \\
\hline $\begin{array}{l}\text { FROHBERGITE } \\
\text { FeTe }_{2}\end{array}$ & 311.047 & $\begin{array}{r}100.2 \\
0.2\end{array}$ & $\begin{array}{r}38.43 \\
0.05\end{array}$ & $\begin{array}{r}-72.4 \\
4.2\end{array}$ & $\begin{array}{r}-64.6 \\
4.2\end{array}$ & $\begin{array}{r}11.31 \\
0.74\end{array}$ & 437 & 279 & \\
\hline $\begin{array}{l}\text { HYDROGEN SULFIDE (IDEAL GAS) } \\
\mathrm{H}_{2} \mathrm{~S}\end{array}$ & 34.082 & $\begin{array}{r}205.8 \\
0.2\end{array}$ & $\begin{array}{r}24789.70 \\
0.20\end{array}$ & $\begin{array}{r}-20.6 \\
0.6\end{array}$ & $\begin{array}{r}-33.4 \\
0.6\end{array}$ & $\begin{array}{l}5.86 \\
0.11\end{array}$ & $\begin{array}{l}85 \\
67\end{array}$ & $\begin{array}{l}85 \\
67\end{array}$ & $\begin{array}{l}85 \\
67\end{array}$ \\
\hline $\begin{array}{l}\text { HS }^{-} \text {(AQUEOUS IOW) } \\
\text { STD. STATE, } m=1\end{array}$ & 33.074 & $\begin{array}{r}67.0 \\
0.9\end{array}$ & & $\begin{array}{r}16.3 \\
0.2\end{array}$ & $\begin{array}{r}44.8 \\
0.3\end{array}$ & $\begin{array}{r}-7.86 \\
0.05\end{array}$ & 85 & 85 & \\
\hline $\begin{array}{l}\text { CIMMABAR } \\
\text { HgS }\end{array}$ & 232.656 & $\begin{array}{r}82.5 \\
2.1\end{array}$ & $\begin{array}{r}28.42 \\
0.02\end{array}$ & $\begin{array}{r}-54.3 \\
2.1\end{array}$ & $\begin{array}{r}-40.7 \\
2.7\end{array}$ & $\begin{array}{l}7.13 \\
0.47\end{array}$ & 233 & $\begin{array}{l}326 \\
279\end{array}$ & \\
\hline $\begin{array}{l}\text { METACI MMABAR } \\
\text { MgS }\end{array}$ & 232.656 & $\begin{array}{r}96.2 \\
4.2\end{array}$ & $\begin{array}{r}30.17 \\
0.02\end{array}$ & $\begin{array}{r}-46.7 \\
1.5\end{array}$ & $\begin{array}{r}-43.3 \\
0.8\end{array}$ & $\begin{array}{l}7.59 \\
0.15\end{array}$ & 217 & 120 & \\
\hline $\begin{array}{l}\text { MININGERITE } \\
\text { MgS }\end{array}$ & 56.371 & $\begin{array}{r}50.3 \\
0.4\end{array}$ & $\begin{array}{r}21.17 \\
0.02\end{array}$ & $\begin{array}{r}-345.7 \\
4.2\end{array}$ & $\begin{array}{r}-341.4 \\
4.2\end{array}$ & $\begin{array}{r}59.81 \\
0.74\end{array}$ & 67 & 67 & 67 \\
\hline $\begin{array}{l}\text { ALABAND ITE } \\
\text { mins }\end{array}$ & 87.004 & $\begin{array}{r}80.3 \\
0.8\end{array}$ & $\begin{array}{r}21.46 \\
0.01\end{array}$ & $\begin{array}{r}-213.9 \\
0.8\end{array}$ & $\begin{array}{r}-218.7 \\
0.9\end{array}$ & $\begin{array}{r}38.31 \\
0.17\end{array}$ & 279 & 375 & $\begin{array}{r}79 \\
203\end{array}$ \\
\hline
\end{tabular}


THERMOOYMAMIC PROPERTIES OF SULFIDE MIMERALS AT $298.15 \mathrm{~K}$

\begin{tabular}{|c|c|c|c|c|c|c|c|c|c|}
\hline \multirow[t]{2}{*}{ MAME AND FORIULA } & \multirow{2}{*}{$\begin{array}{l}\text { MEIGHT } \\
g\end{array}$} & \multirow{2}{*}{$\begin{array}{c}\text { ENTROPY } \\
\mathrm{S}^{\circ} \\
\mathrm{J} \cdot \mathrm{mol}^{-1} \cdot \mathrm{K}^{\prime}\end{array}$} & \multirow{2}{*}{$\begin{array}{l}\text { VOLUAE } \\
v^{\circ} \\
\mathrm{cm}^{3}\end{array}$} & \multirow{2}{*}{$\begin{array}{l}\text { ENTHALPY } \\
\mathrm{AJ}_{\mathrm{f}} \mathrm{H}^{\circ} \\
\mathrm{mol}^{-1}\end{array}$} & \multirow{2}{*}{$\begin{array}{c}\text { FREE } \\
\text { ENERGY } \\
\triangle \mathrm{A}^{\circ} \\
\mathrm{kJ} \cdot \mathrm{mol}^{-1}\end{array}$} & \multirow[t]{2}{*}{$\operatorname{LOC}(K)$} & \multicolumn{3}{|c|}{ REFEREMCES } \\
\hline & & & & & & & $\mathbf{s}$ & H/G & c \\
\hline MUEERITE & 119.070 & 99.9 & 34.20 & -223.8 & -224.6 & 39.35 & 441 & 279 & \\
\hline $\mathrm{MnS}_{2}$ & & 0.1 & 0.01 & 10.0 & 10.0 & 1.75 & & & \\
\hline MOLYBDENITE & 160.072 & 62.6 & 32.02 & -271.8 & -262.8 & 46.04 & 275 & 305 & 114 \\
\hline $\operatorname{Mos}_{2}$ & & 0.2 & 0.02 & 4.9 & 4.9 & 0.86 & & & \\
\hline MILLERITE & 90.756 & 53.0 & 16.89 & -91.0 & -63.9 & 14.97 & 429 & 58 & 271 \\
\hline Nis & & 0.4 & 0.01 & $\mathbf{3 . 0}$ & 3.0 & 0.53 & & 271 & 98 \\
\hline HEAZLEWOODITE & 240.202 & 133.2 & 40.95 & -216.3 & -210.2 & 36.83 & 395 & 58 & 107 \\
\hline$M i_{3} s_{2}$ & & 0.3 & 0.02 & 3.0 & 3.0 & 0.53 & & 67 & 276 \\
\hline PEMTLAMDITE & 771.944 & & 155.40 & -837.4 & & & & 59 & \\
\hline 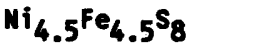 & & & 0.20 & 14.6 & & & & & \\
\hline VIOLARITE & 301.775 & & 63.80 & -378 & & & & 59 & \\
\hline (Ni $\left..7^{\mathrm{Fe}} .3_{3}\right)_{3} \mathrm{~S}_{4}$ & & & 0.30 & 11.8 & & & & & \\
\hline GALEMA & 239.266 & 91.7 & 31.49 & -98.3 & -96.8 & 16.96 & 67 & 397 & 67 \\
\hline Pbs & & 0.7 & 0.01 & 2.0 & 2.0 & 0.35 & 326 & 245 & 326 \\
\hline CLAUSTHALITE & 286.160 & 102.5 & 34.61 & -100.1 & -98.7 & 17.30 & 279 & 279 & \\
\hline Pbse & & 2.1 & 0.01 & 2.3 & 2.1 & 0.37 & & & \\
\hline altaite & 334.800 & 110.0 & 40.60 & -70.7 & -69.4 & 12.15 & 217 & 419 & \\
\hline PbTe & & 2.1 & 0.01 & 1.4 & 1.5 & 0.27 & & & \\
\hline COOPERITE & 227.146 & 55.1 & 22.15 & -82.4 & -76.9 & 13.46 & 136 & 136 & \\
\hline Pts & & 0.1 & 0.01 & 3.4 & 3.4 & 0.59 & & & \\
\hline STIBNITE & 339.698 & 182.0 & 73.41 & -151.4 & -149.9 & 26.26 & 234 & 382 & 211 \\
\hline $\mathrm{Sb}_{2} \mathrm{~s}_{\mathbf{3}}$ & & 3.3 & 0.04 & 2.3 & 2.1 & 0.37 & & 53 & 51 \\
\hline HERZENBERGITE & 150.776 & 77.0 & 29.01 & -106.5 & -104.6 & 18.33 & 229 & 347 & 315 \\
\hline Sns & & 0.8 & 0.02 & 1.5 & 1.5 & 0.26 & & & \\
\hline BERNDTITE & 182.842 & 87.5 & 40.96 & -149.8 & -141.5 & 24.79 & 229 & 326 & 315 \\
\hline $\mathrm{SnS}_{2}$ & & 0.2 & 0.02 & 5.0 & 5.0 & 0.88 & & & \\
\hline TUNGSTENITE & 247.982 & 67.8 & 32.07 & -241.6 & -233.0 & 40.82 & 304 & 303 & 304 \\
\hline $\mathrm{ws}_{2}$ & & 0.3 & 0.02 & 2.5 & 2.5 & 0.44 & & 304 & \\
\hline SPHALERITE & 97.456 & 58.7 & 23.83 & -204.1 & -199.6 & 34.97 & 398 & 378 & 324 \\
\hline Zns & & 0.2 & 0.01 & 1.5 & 1.5 & 0.26 & & & \\
\hline WRTZITE & 97.456 & 58.8 & 23.85 & -203.8 & -199.3 & 34.92 & 398 & 338 & 324 \\
\hline Zns & & 0.2 & 0.01 & 1.5 & 1.5 & 0.26 & & & \\
\hline
\end{tabular}


THERMOOYMAMIC PROPERTIES OF SULFIDE MINERALS AT $298.15 \mathrm{~K}$

\begin{tabular}{|c|c|c|c|c|c|c|c|c|c|}
\hline \multirow[t]{2}{*}{ MAME AND FORMULA } & \multirow{2}{*}{$\begin{array}{l}\text { MEIGHT } \\
\text { g }\end{array}$} & \multirow{2}{*}{$\begin{array}{c}\text { ENTROPY } \\
\mathrm{S}^{\circ} \\
\mathrm{J} \cdot \mathrm{mol}^{-1} \cdot \mathrm{K}^{1}\end{array}$} & \multirow{2}{*}{$\begin{array}{l}\text { VOLUME } \\
\text { vo } \\
\text { cmon }^{3}\end{array}$} & \multirow{2}{*}{$\begin{array}{l}\text { ENTHALPY } \\
\begin{array}{l}\mathrm{AH}_{\mathrm{H}} \mathrm{H}^{\circ} \\
\mathrm{kJ} \cdot \mathrm{mol}^{-1}\end{array}\end{array}$} & \multirow{2}{*}{$\begin{array}{c}\text { FREE } \\
\text { EMERGY } \\
\Delta 6^{\circ} \\
\mathrm{kJ} \cdot \mathrm{mol}^{-1}\end{array}$} & \multirow[t]{2}{*}{$\operatorname{LOG}(K)$} & \multicolumn{3}{|c|}{ REFERENCES } \\
\hline & & & & & & & $\mathbf{s}$ & H/6 & c \\
\hline PROUSTITE & 494.724 & 303.7 & 88.42 & & & & 146 & & \\
\hline $\mathrm{Ag}_{3} \mathrm{AsS}_{3}$ & & 0.5 & 0.02 & & & & & & \\
\hline SMITHITE & 246.922 & 150.5 & 50.12 & & & & 146 & & \\
\hline $\mathrm{AgAsS}_{2}$ & & 0.2 & 0.30 & & & & & & \\
\hline ENARGITE & 393.824 & 257.6 & 88.24 & & & & 381 & & \\
\hline $\mathrm{Cu}_{3} \mathrm{AsS}_{4}$ & & 0.6 & 0.12 & & & & & & \\
\hline ARSENOPYRITE & 166.263 & 68.8 & 26.42 & & & & 331 & & \\
\hline FeAs $1.08 s^{5} 0.92$ & & 0.4 & 0.07 & & & & & & \\
\hline LIVINGSTONITE & 944.118 & 470.5 & 192.90 & -373.2 & -360.2 & 63.10 & 145 & 454 & \\
\hline $\mathrm{HgSb}_{4} \mathrm{~s}_{8}$ & & 1.5 & & 6.5 & 6.5 & 1.14 & & & \\
\hline BERTHIERITE & 427.611 & 245.0 & 92.22 & -256.2 & -255.8 & 44.82 & 97 & 382 & 382 \\
\hline $\mathrm{FeSb}_{2} \mathrm{~S}_{4}$ & & 1.0 & 0.20 & 6.5 & 6.5 & 1.14 & & & \\
\hline CHALCOSTIBITE & 249.428 & 149.2 & 50.06 & -130.8 & -132.7 & 23.25 & 382 & 382 & 382 \\
\hline $\mathrm{Cusbs}_{2}$ & & 3.8 & 0.10 & 4.4 & 4.2 & 0.74 & & & \\
\hline Fe-TENMANTITE & 1463.698 & 1024.2 & 332.10 & & & & 380 & & \\
\hline $\mathrm{Cu}_{10} \mathrm{Fe}_{2} \mathrm{As}_{4} \mathrm{~S}_{13}$ & & & & & & & & & \\
\hline Fe-TETRAHEDRITE & 1651.012 & 1061.0 & 336.70 & & & & 380 & & \\
\hline $\mathrm{Cu}_{10} \mathrm{Fe}_{2} \mathrm{Sb}_{4} \mathrm{~S}_{13}$ & & & & & & & & & \\
\hline Zn-TENAANTITE & 1482.784 & 1025.6 & 332.10 & & & & 380 & & \\
\hline $\mathrm{Cu}_{10^{2} n_{2} \mathrm{As}_{4} s_{13}}$ & & & & & & & & & \\
\hline Zn-TETRAHEDRITE & 1670.098 & 1062.4 & 336.70 & & & & 380 & & \\
\hline $\mathrm{Cu}_{10} \mathrm{zn}_{2} \mathrm{Sb}_{4} \mathrm{~s}_{13}$ & & & & & & & & & \\
\hline PYRARGYRI TE & 541.553 & & 92.56 & -131.5 & & & & 51 & \\
\hline $\mathrm{Ag}_{3} \mathrm{SbS}_{3}$ & & & 0.04 & 3 & & & & & \\
\hline MIARGYRITE & 293.750 & & 52.03 & -95.8 & & & & 51 & \\
\hline$a-\mathrm{AgSbS}_{2}$ & & & 0.09 & 2.6 & & & & & \\
\hline MATILDITE & 380.980 & & 54.44 & -165.3 & & & & 52 & \\
\hline$B-A g B i S_{2}$ & & & 0.10 & 8 & & & & & \\
\hline
\end{tabular}


THERMOOYMAMIC PROPERTIES OF OXIDE AND HYDROXIDE MIMERALS AT $298.15 \mathrm{~K}$

\begin{tabular}{|c|c|c|c|c|c|c|c|c|c|}
\hline \multirow[t]{2}{*}{ MAME AMD FORAULA } & \multirow{2}{*}{$\begin{array}{l}\text { MEIGHT } \\
\text { g }\end{array}$} & \multirow{2}{*}{$\begin{array}{c}\text { ENTROPY } \\
\mathrm{s}^{\bullet} \\
\mathrm{J} \cdot \mathrm{mol}^{-1} \cdot \mathrm{K}^{\prime}\end{array}$} & \multirow{2}{*}{$\begin{array}{l}\text { VOLUME } \\
\text { ve } \\
\mathrm{cm}^{3}\end{array}$} & \multirow{2}{*}{$\begin{array}{l}\text { ENTHALPY } \\
\Delta_{\mathbf{H}} \mathrm{H}^{\circ} \\
\mathrm{kJ} \cdot \mathrm{mol}^{-1}\end{array}$} & \multirow{2}{*}{$\begin{array}{l}\text { FREE } \\
\text { EMERGY } \\
\text { HrG }^{\circ} \\
\mathrm{kJ}^{\circ}=\mathrm{mol}^{-1}\end{array}$} & \multirow[t]{2}{*}{$\operatorname{LOG}(K)$} & \multicolumn{3}{|c|}{ REFEREMCES } \\
\hline & & & & & & & $\mathbf{s}$ & W/G & c \\
\hline CORUNDUN & 101.961 & 50.9 & 25.58 & -1675.7 & -1582.3 & 277.20 & 93 & 268 & 94 \\
\hline $\mathrm{Al}_{2} \mathrm{O}_{3}$ & & 0.1 & 0.01 & 1.3 & 1.3 & 0.23 & 94 & 85 & 57 \\
\hline BOEMMITE & 59.988 & 37.2 & 19.54 & -996.4 & -918.4 & 160.90 & 176 & 176 & \\
\hline AlO(OH) & & 0.1 & 0.03 & 2.2 & 2.2 & 0.39 & & 12 & \\
\hline DIASPORE & 59.988 & 35.3 & 17.76 & -1001.3 & .922 .7 & 161.53 & 334 & 12 & \\
\hline$A(O(O H)$ & & 0.2 & 0.03 & 2.2 & . $\quad 2.1$ & 0.88 & 230 & 176 & \\
\hline GIBBSITE & 78.004 & 68.4 & 31.96 & -1293.1 & -1154.9 & 202.33 & 178 & 171 & 178 \\
\hline $\mathrm{Al}(\mathrm{OH})_{3}$ & & 0.1 & 0.02 & 1.2 & 1.2 & 0.21 & & 212 & \\
\hline ARSEMOLITE & 197.841 & 107.4 & 51.12 & -657.0 & .576 .0 & 100.90 & 61 & 419 & \\
\hline $\mathrm{As}_{2} \mathrm{O}_{3}$ & & 0.1 & 0.07 & 1.7 & 1.9 & 0.33 & & & \\
\hline CLAUDETITE & 197.841 & 113.3 & 47.26 & -654.8 & .575 .6 & 100.83 & 61 & & \\
\hline $\mathrm{As}_{2} \mathrm{O}_{3}$ & & 0.1 & 0.03 & 1.7 & 1.1 & 0.18 & & & \\
\hline DIBORON TRIOXIDE & 69.620 & 54.0 & 27.26 & -1273.5 & -1194.4 & 209.26 & 85 & 85 & 67 \\
\hline $\mathrm{B}_{2} \mathrm{O}_{3}$ & & 0.3 & 0.06 & 1.4 & 1.4 & 0.25 & & 67 & \\
\hline BARIUM MONOXIDE & 153.326 & 72.1 & 25.59 & -548.1 & .520 .4 & 91.17 & 85 & $\pi$ & 85 \\
\hline 880 & & 0.4 & 0.01 & 2.1 & 2.1 & 0.37 & & 111 & 262 \\
\hline BROMELLITE & 25.012 & 13.8 & 8.31 & -609.4 & .580 .1 & 101.63 & 85 & 85 & 67 \\
\hline BeO & & 0.1 & 0.03 & 2.5 & 2.5 & 0.44 & & & 418 \\
\hline BISNITE & 465.959 & 151.5 & 49.73 & -573.9 & -493.5 & 86.45 & 419 & 419 & 319 \\
\hline $\mathrm{Bi}_{2} \mathrm{O}_{3}$ & & 2.1 & 0.06 & 1.3 & 1.5 & 0.26 & & 269 & \\
\hline CARBON MONOXIDE & 28.010 & 197.3 & 24789.70 & -110.5 & -137.1 & 26.01 . & 67 & 67 & 67 \\
\hline CO (IDEAL GAS) & & 0.0 & 0.20 & 0.2 & 0.2 & 0.03 & 117 & 117 & 117 \\
\hline CARBOW DIOXIDE & 44.010 & 213.8 & 24789.70 & -393.5 & -394.4 & 69.09 & 67 & 67 & 67 \\
\hline $\mathrm{CO}_{2}$ (IDEAL GAS) & & 0.0 & 0.20 & 0.1 & 0.2 & 0.03 & 117 & 117 & 117 \\
\hline $\mathrm{CO}_{3}{ }^{--}$(AQUEOUS IOW) & 60.009 & -50.0 & & -675.2 & -527.0 & 92.33 & 85 & 85 & \\
\hline STD. STATE, $m=1$ & & 1.0 & & 0.1 & 0.3 & 0.05 & 36 & 36 & \\
\hline $\mathrm{HCO}_{3}^{-}$(AQUEOUS IOW) & 61.017 & 98.4 & & -689.9 & -586.8 & 102.81 & 85 & 85 & \\
\hline STD. STATE, $m=1$ & & 0.5 & & 0.2 & $\quad 0.3$ & 0.04 & 36 & 36 & \\
\hline $\mathrm{H}_{2} \mathrm{CO}_{3}$ (UN-IONIZED) & 62.025 & 184.7 & & -699.7 & -623.2 & 109.17 & 419 & 419 & \\
\hline STD. STATE, $m=1$ & & 0.9 & & 0.1 & 0.1 & 0.02 & & & \\
\hline LIME & 56.077 & 38.1 & 16.76 & -635.1 & -603.1 & 105.66 & 67 & 67 & 67 \\
\hline caro & & 0.4 & 0.01 & 0.9 & 0.9 & 0.16 & 117 & 117 & 117 \\
\hline
\end{tabular}


THERMOOYMAMIC PROPERTIES OF OXIDE AMD MYDROXIDE MINERALS AT $298.15 \mathrm{~K}$

\begin{tabular}{|c|c|c|c|c|c|c|c|c|c|}
\hline MAME AND FORINLA & $\begin{array}{l}\text { MEIGHT } \\
g\end{array}$ & $\begin{array}{c}\text { ENTROPY } \\
\mathbf{s}^{\bullet} \\
J \cdot \mathrm{mol}^{-1} \cdot \boldsymbol{K}^{\prime}\end{array}$ & $\begin{array}{l}\text { Volune } \\
\text { ve } \\
\mathrm{cm}^{3}\end{array}$ & $\begin{array}{l}\text { ENTHALPY } \\
\mathrm{Ad}_{\mathbf{A}} \mathrm{H}^{\circ} \\
\mathrm{kJ} \cdot \mathrm{mol}^{-1}\end{array}$ & $\begin{array}{c}\text { FREE } \\
\text { ENERGY } \\
\triangle 6^{\circ} \\
\mathrm{kJ} \cdot \mathrm{mol}^{-1}\end{array}$ & $\operatorname{LOG}(K)$ & REI & FEREW & ICES \\
\hline PORTLANDITE & 74.093 & 83.4 & 33.06 & -986.1 & -898.0 & 157.33 & 67 & 67 & 67 \\
\hline $\mathrm{Ca}(\mathrm{OH})_{2}$ & & 0.4 & 0.02 & 1.3 & 1.3 & 0.23 & 158 & 158 & 117 \\
\hline MONTEPONITE & 128.400 & 54.8 & 15.59 & -258.4 & -228.7 & 40.07 & 85 & 85 & \\
\hline $\operatorname{col}$ & & 1.5 & 0.01 & 0.4 & 0.6 & 0.11 & & 267 & \\
\hline CERIANITE & 172.114 & 62.3 & 23.85 & -1088.7 & -1025.4 & 179.64 & 436 & 14 & 227 \\
\hline $\mathrm{CeO}_{2}$ & & 0.1 & 0.03 & 1.5 & 1.9 & 0.34 & & & \\
\hline COBALT MONOXIDE & 74.933 & 52.8 & 11.64 & -237.9 & -214.1 & 37.52 & 287 & 308 & 319 \\
\hline $\mathrm{CoO}$ & & 0.3 & 0.02 & 1.3 & 1.3 & 0.23 & & 45 & 125 \\
\hline TRICOBALT TETRAOXIDE & 240.797 & 109.3 & 39.77 & -918.8 & -802.2 & 140.54 & 222 & 319 & 319 \\
\hline $\mathrm{CO}_{3} \mathrm{O}_{4}$ & & 0.3 & 0.02 & 2.0 & 2.0 & 0.35 & & 307 & \\
\hline ESKOLAITE & 151.990 & 81.2 & 29.09 & -1134.7 & -1053.1 & 184.49 & 67 & 67 & 67 \\
\hline $\mathrm{Cr}_{2} \mathrm{O}_{3}$ & & 1.3 & 0.03 & 8.4 & 8.4 & 1.47 & & 267 & \\
\hline DICESIUM MONOXIDE & 281.810 & 146.9 & 59.62 & -346.0 & -308.4 & 54.02 & 112 & 384 & 112 \\
\hline $\mathrm{Cs}_{2} \mathrm{O}$ & & .0 .4 & 0.07 & 1.2 & 1.2 & 0.21 & & & \\
\hline TENORITE & 79.545 & 42.6 & 12.22 & -156.1 & -128.3 & 22.48 & 67 & 67 & 272 \\
\hline cus & & 0.2 & 0.03 & 2.0 & 2.0 & 0.35 & & 272 & \\
\hline CUPRITE & 143.091 & 92.4 & 23.64 & -170.6 & -147.8 & 25.89 & 67 & 190 & 272 \\
\hline $\mathrm{Cu}_{2} \mathrm{O}$ & & 0.3 & 0.02 & 0.1 & 0.1 & 0.02 & 310 & 272 & 67 \\
\hline WSTITE & 68.887 & 56.6 & 12.04 & -266.3 & -244.9 & 42.91 & 67 & 198 & 83 \\
\hline Fe. $.947^{\circ}$ & & 0.4 & 0.04 & 0.8 & 0.8 & 0.14 & 132 & 148 & 67 \\
\hline FERROUS OXIDE (STOICHIOMETRIC) & 71.846 & 60.6 & 12.00 & -272.0 & -251.4 & 44.05 & 67 & 67 & 67 \\
\hline Feo & & 1.7 & 0.05 & 2.1 & 2.2 & 0.38 & 132 & & \\
\hline HEMATITE & 159.692 & 87.4 & 30.27 & -826.2 & -744.4 & 130.41 & 137 & 161 & 83 \\
\hline $\mathrm{Fe}_{2} \mathrm{O}_{3}$ & & 0.2 & 0.01 & 1.3 & 1.3 & 0.23 & 67 & 310 & 130 \\
\hline MGNETITE & 231.539 & 146.1 & 44.52 & -1115.7 & -1012.7 & 177.42 & 440 & 161 & 161 \\
\hline $\mathrm{Fe}_{3} \mathrm{O}_{4}$ & & 0.4 & 0.01 & 2.1 & 2.1 & 0.37 & 22 & 310 & 134 \\
\hline COETHITE & 88.854 & 60.4 & 20.82 & -562.6 & -491.8 & 86.16 & 235 & 221 & \\
\hline FeO(OH) & & 0.6 & 0.04 & 2.1 & 2.1 & 0.12 & & 18 & \\
\hline MATER & 18.015 & 70.0 & 18.07 & -285.8 & -237.1 & 41.55 & 85 & 85 & 117 \\
\hline $\mathrm{H}_{2} \mathrm{O}$ (LIQUID) & & 0.1 & 0.00 & 0.1 & 0.1 & 0.01 & & & 67 \\
\hline $\begin{array}{l}\text { OH' (AQUEOUS ION) } \\
\text { STD. STATE, } m=1\end{array}$ & 17.007 & $\begin{array}{r}-10.7 \\
0.2\end{array}$ & & $\begin{array}{r}-230.0 \\
0.1\end{array}$ & $\begin{array}{r}-157.3 \\
0.1\end{array}$ & $\begin{array}{r}27.56 \\
0.02\end{array}$ & 85 & 85 & \\
\hline
\end{tabular}


THERMOOYMAMIC PROPERTIES OF OXIDE AND HYDROXIDE MIMERALS AT $298.15 \mathrm{~K}$

\begin{tabular}{|c|c|c|c|c|c|c|c|c|c|}
\hline \multirow[t]{2}{*}{ MAME AMD FORMULA } & \multirow{2}{*}{$\begin{array}{l}\text { VEIGHT } \\
g\end{array}$} & \multirow{2}{*}{$\begin{array}{c}\text { ENTROPY } \\
\mathrm{S}^{\circ} \\
\mathrm{J} \cdot \mathrm{mol}^{-1} \cdot \mathrm{K}^{-1}\end{array}$} & \multirow{2}{*}{$\begin{array}{l}\text { VOLUME } \\
v^{\circ} \\
\mathrm{cm}^{3}\end{array}$} & \multirow{2}{*}{$\begin{array}{l}\text { ENTHALPY } \\
\mathrm{AJ}_{\mathrm{f}} \mathrm{H}^{\circ} \\
\mathrm{mol} \mathrm{l}^{-1}\end{array}$} & \multirow{2}{*}{$\begin{array}{c}\text { FREE } \\
\text { ENERGY } \\
\triangle \mathrm{B}^{\circ} \\
\mathrm{kJ} \cdot \mathrm{mol}^{-1}\end{array}$} & \multirow[t]{2}{*}{$L 00(K)$} & \multicolumn{3}{|c|}{ REFERENCES } \\
\hline & & & & & & & $\mathbf{s}$ & H/G & C \\
\hline STEAM & 18.015 & 188.8 & 24789.70 & -241.8 & -228.6 & 40.05 & 85 & 85 & 67 \\
\hline $\mathrm{H}_{2} \mathrm{O}$ (IDEAL GAS) & & 0.0 & 0.20 & 0.0 & 0.1 & 0.02 & & & 117 \\
\hline HAFNIUM DIOXIDE & 210.489 & 59.3 & 20.82 & -1117.6 & -1061.1 & 185.90 & 319 & 256 & 319 \\
\hline $\mathrm{HFO}_{2}$ & & 0.4 & 0.01 & 1.3 & 1.3 & 0.23 & & 319 & \\
\hline MONTROYDITE & 216.589 & 70.3 & 19.32 & -90.8 & -58.5 & 10.25 & 85 & 85 & 67 \\
\hline HoO & & 0.3 & 0.02 & 0.1 & 0.1 & 0.01 & & 415 & \\
\hline DIPOTASSIUN MONOXIDE & 94.196 & 94.1 & 40.38 & -363.2 & -322.1 & 56.43 & 67 & 67 & 67 \\
\hline $\mathrm{K}_{2} \mathrm{O}$ & & 6.3 & 0.20 & 2.1 & 2.8 & 0.49 & & & \\
\hline POTASSIUM HYDROXIDE & 56.106 & 78.9 & 27.45 & -424.7 & -378.9 & 66.39 & 67 & 67 & 67 \\
\hline KOH & & 0.8 & 0.02 & 0.6 & 0.6 & 0.11 & & 254 & \\
\hline DILITHIUN MONOXIDE & 29.881 & 37.6 & 14.76 & -597.9 & -561.2 & 98.31 & 319 & 209 & 319 \\
\hline $\mathrm{Li}_{2} \mathrm{O}$ & & 0.3 & 0.01 & 2.1 & 2.1 & 0.37 & 419 & 67 & \\
\hline PERICLASE & 40.304 & 26.9 & 11.25 & -601.6 & -569.3 & 99.74 & 85 & 85 & 418 \\
\hline MeO & & 0.2 & 0.00 & 0.3 & 0.3 & 0.05 & 67 & 321 & 117 \\
\hline BRUCITE & 58.320 & 63.2 & 24.63 & -924.5 & -833.5 & 146.02 & 419 & 619 & 228 \\
\hline $\mathrm{Mg}(\mathrm{OH})_{2}$ & & 0.1 & 0.07 & 0.4 & 0.4 & 0.07 & & 193 & \\
\hline MANGAMOSITE & 70.937 & 59.7 & 13.22 & -385.2 & -362.9 & 63.58 & 319 & 419 & 391 \\
\hline Mno & & 0.4 & 0.00 & 0.5 & 0.5 & 0.09 & & & 319 \\
\hline PYROLUSITE & 86.937 & 52.8 & 16.61 & -520.0 & -465.0 & 81.47 & 357 & 357 & 357 \\
\hline $\mathrm{NnO}_{2}$ & & 0.1 & 0.02 & 0.7 & 0.7 & 0.12 & & & \\
\hline BIXBYITE & 157.874 & 113.7 & 31.37 & -959.0 & -882.1 & 154.53 & 357 & 357 & 357 \\
\hline $\mathrm{mn}_{2} \mathrm{O}_{3}$ & & 0.2 & 0.05 & 1.0 & 1.0 & 0.18 & & & \\
\hline MAUSMANNITE & 228.812 & 164.1 & 46.95 & -1384.5 & -1282.5 & 224.68 & 357 & 357 & 357 \\
\hline $\mathrm{Mn}_{3} \mathrm{O}_{4}$ & & 0.2 & 0.06 & 1.4 & 1.4 & 0.25 & & & \\
\hline BRAUNITE & 604.645 & 416.4 & 125.08 & -4260.0 & -3944.7 & 691.08 & 367 & 367 & 367 \\
\hline $\mathrm{In}_{7} \mathrm{SiO}_{12}$ & & 0.8 & 0.05 & 4.0 & 4.0 & 0.70 & & & \\
\hline MOLYEDITE & 143.938 & 77.7 & 30.56 & -745.2 & -668.1 & 117.04 & 85 & 85 & 85 \\
\hline $\mathrm{HoO}_{3}$ & & 0.4 & 0.04 & 0.4 & 0.4 & 0.07 & & & 200 \\
\hline MITROGEN DIOXIDE & 46.006 & 240.1 & 24789.70 & 33.1 & 51.2 & -8.96 & 67 & 67 & 67 \\
\hline $\mathrm{MO}_{2}$ (IDEAL GAS) & & 0.1 & 0.20 & 0.4 & 0.4 & 0.07 & & & \\
\hline $\begin{array}{l}\mathrm{MO}_{3}^{-} \text {(AQUEOUS IOW) } \\
\text { STD. STATE, } m=1\end{array}$ & 62.005 & $\begin{array}{r}146.7 \\
0.4\end{array}$ & & $\begin{array}{r}-206.9 \\
0.4\end{array}$ & $\begin{array}{r}-110.8 \\
0.4\end{array}$ & $\begin{array}{r}19.42 \\
0.07\end{array}$ & 85 & 85 & \\
\hline
\end{tabular}


THERMCOYMAMIC PROPERTIES OF OXIDE AND hYDROXIDE MINERALS AT $298.15 \mathrm{~K}$

\begin{tabular}{|c|c|c|c|c|c|c|c|c|c|}
\hline MAME AND FORHULA & $\begin{array}{l}\text { WEIGHT } \\
\text { g }\end{array}$ & $\begin{array}{c}\text { ENTROPY } \\
\mathrm{S}^{\circ} \\
J \cdot \mathrm{mol}^{-1} \cdot K^{\prime}\end{array}$ & $\begin{array}{l}\text { VOLUME } \\
v^{0} \\
\mathrm{~cm}^{3}\end{array}$ & $\begin{array}{l}\text { ENTHALPY } \\
\mathrm{Ad}_{\mathrm{B}} \mathrm{H}^{\circ} \\
\mathrm{kJ} \cdot \mathrm{mol}^{-1}\end{array}$ & $\begin{array}{c}\text { FREE } \\
\text { ENERGY } \\
\triangle \mathrm{CG}^{\circ} \\
\mathrm{kJ} \cdot \mathrm{mol}^{-1}\end{array}$ & $\operatorname{LOG}(K)$ & RE & FEREM & MCES \\
\hline DISODIUN MONOXIDE & 61.979 & 75.3 & 25.88 & -414.8 & -376.0 & 65.87 & 67 & 302 & 67 \\
\hline $\mathrm{Ma}_{2} \mathrm{O}$ & & 0.8 & 0.08 & 0.3 & 0.4 & 0.07 & & 419 & 115 \\
\hline SODIUN MYDROXIDE & 39.997 & 64.4 & 18.78 & -425.8 & -379.6 & 66.50 & 67 & 67 & 67 \\
\hline MeOH & & 0.8 & 0.06 & 0.1 & 0.3 & 0.05 & & 419 & \\
\hline BUNSENITE & 74.689 & 38.0 & 10.97 & -239.3 & -211.1 & 36.99 & 271 & 161 & 161 \\
\hline Mio & & 0.2 & 0.02 & 0.4 & 0.4 & 0.07 & 419 & 191 & 319 \\
\hline PHOSPHORUS PENTOXIDE & 141.945 & 114.4 & 59.40 & -1504.9 & -1361.6 & 238.54 & 319 & 319 & \\
\hline $\mathrm{P}_{2} \mathrm{O}_{5}$ & & 0.4 & 0.20 & 0.5 & 0.5 & 0.10 & 67 & 67 & \\
\hline $\mathrm{PO}_{4}{ }^{---}$(ACUEOUS IOW) & 94.971 & -222.0 & & -1259.6 & -1001.6 & 175.47 & 419 & 419 & \\
\hline STO. STATE, $m=1$ & & 4.2 & & 0.9 & 0.9 & 0.15 & & & \\
\hline LITHAREE (RED) & 223.199 & 66.5 & 23.91 & -219.0 & -188.9 & 33.10 & 72 & 419 & 319 \\
\hline Pbo & & 0.2 & 0.05 & 0.8 & 0.8 & 0.14 & 419 & & \\
\hline MASSICOT (YELLOW) & 223.199 & 68.7 & 23.15 & -217.3 & -187.9 & 32.92 & 419 & 15 & 319 \\
\hline Pto & & 0.2 & 0.03 & 0.3 & 0.3 & 0.06 & & 419 & \\
\hline PLATTMERITE & 239.199 & 71.8 & 25.01 & -277.4 & -218.3 & 38.25 & 319 & 419 & 319 \\
\hline $\mathrm{PbO}_{2}$ & & 0.4 & 0.01 & 2.9 & 2.9 & 0.51 & & & \\
\hline MINIUM & 685.598 & 212.0 & 76.81 & -718.7 & -601.6 & 105.40 & 67 & 67 & 67 \\
\hline $\mathrm{Pb}_{3} \mathrm{O}_{4}$ & & 6.7 & 0.09 & 6.3 & 6.6 & 1.16 & & 419 & \\
\hline SULFUR DIOXIDE & 64.065 & 248.2 & 24789.70 & -296.8 & -300.1 & 52.57 & 85 & 85 & 67 \\
\hline $\mathrm{SO}_{2}$ (IDEAL GAS) & & 0.1 & 0.20 & 0.2 & 0.2 & 0.04 & & & 117 \\
\hline SULFUR TRIOXIDE & 80.064 & 256.8 & 24789.70 & -395.7 & -371.0 & 64.99 & 419 & 419 & 67 \\
\hline $\mathrm{SO}_{3}$ (IDEAL GAS) & & 0.8 & 0.20 & 0.7 & 0.7 & 0.12 & 67 & 67 & \\
\hline $\mathrm{SO}_{3}{ }^{--}$(AQUEOUS ION) & 80.064 & -29.0 & & -635.5 & -486.5 & 85.23 & 419 & 419 & \\
\hline STD. STATE, $m=1$ & & 4.2 & & 0.9 & 0.9 & 0.15 & & & \\
\hline $\mathrm{SO}_{4}{ }^{--}$(ACUEOUS IOW) & 96.064 & 18.5 & & -909.3 & -744.0 & 130.34 & 85 & 85 & \\
\hline STD. STATE, $m=1$ & & 0.4 & & 0.4 & 0.4 & 0.07 & & & \\
\hline VALENTINITE & 291.498 & 123.0 & 50.01 & -708.6 & -626.4 & 109.75 & 319 & 419 & \\
\hline $\mathrm{Sb}_{2} \mathrm{O}_{3}$ & & 2.5 & 0.05 & 2.9 & 3.0 & 0.53 & & & \\
\hline SILICON MONOXIDE & 44.085 & 211.6 & 24789.70 & -100.4 & -127.3 & 22.30 & 67 & 67 & 67 \\
\hline SiO (IDEAL GAS) & & 0.8 & 0.20 & 8.4 & 8.4 & 1.47 & & & \\
\hline CunRtz & 60.084 & 41.5 & 22.69 & -910.7 & -856.3 & 150.01 & 435 & 444 & 160 \\
\hline $\mathrm{SiO}_{2}$ & & 0.1 & 0.00 & 1.0 & 1.0 & 0.18 & 317 & 419 & 341 \\
\hline
\end{tabular}


THERMOOYMAMIC PROPERTIES OF OXIDE AMD HYOROXIDE MIMERALS AT $298.15 \mathrm{~K}$

\begin{tabular}{|c|c|c|c|c|c|c|c|c|c|}
\hline MNE AND FORMULA & $\begin{array}{l}\text { IEIGHT } \\
9\end{array}$ & $\begin{array}{c}\text { ENTROPY } \\
S^{\bullet} \\
J \cdot m^{-1} \cdot K^{\prime}\end{array}$ & $\begin{array}{l}\text { VOLUME } \\
\qquad \begin{array}{l}\text { vo }^{\circ} \\
c^{3}\end{array}\end{array}$ & $\begin{array}{l}\text { ENTHALPY } \\
\qquad \begin{array}{c}\Delta_{\text {H }} \\
\mathrm{kJ} \cdot \mathrm{mol}^{\circ}\end{array}\end{array}$ & $\begin{array}{l}\text { FREE } \\
\text { ENERGY } \\
\text { A.G } \\
\text { kJ·mol-1 }\end{array}$ & $\log (K)$ & $\mathbf{s}$ & FEREN & CES \\
\hline $\begin{array}{l}\mathrm{H}_{4} \mathrm{SiO}_{4} \text { (UN-IONIZED) } \\
\text { STD. STATE, }=1=1\end{array}$ & 96.115 & $\begin{array}{r}180.0 \\
4.2\end{array}$ & & $\begin{array}{r}-1460.0 \\
1.7\end{array}$ & $\begin{array}{r}-1307.8 \\
2.1\end{array}$ & $\begin{array}{r}229.12 \\
0.37\end{array}$ & & $\begin{array}{l}179 \\
285\end{array}$ & $\begin{array}{l}422 \\
414\end{array}$ \\
\hline $\begin{array}{l}\text { CRISTOBALITE } \\
\mathrm{SiO}_{2}\end{array}$ & 60.084 & $\begin{array}{r}43.4 \\
0.1\end{array}$ & $\begin{array}{r}25.74 \\
0.03\end{array}$ & $\begin{array}{r}-908.4 \\
2.1\end{array}$ & $\begin{array}{r}-854.6 \\
2.1\end{array}$ & $\begin{array}{r}149.71 \\
0.37\end{array}$ & $\begin{array}{l}435 \\
404\end{array}$ & $\begin{array}{r}67 \\
341\end{array}$ & $\begin{array}{l}286 \\
341\end{array}$ \\
\hline $\begin{array}{l}\text { TRIDYMITE } \\
\mathrm{siO}_{2}\end{array}$ & 60.084 & $\begin{array}{r}43.9 \\
0.4\end{array}$ & $\begin{array}{r}26.53 \\
0.20\end{array}$ & $\begin{array}{r}-907.5 \\
2.4\end{array}$ & $\begin{array}{r}-853.8 \\
2.4\end{array}$ & $\begin{array}{r}149.58 \\
0.43\end{array}$ & 361 & & $\begin{array}{l}286 \\
404\end{array}$ \\
\hline $\begin{array}{l}\text { COESITE } \\
\mathrm{SiO}_{2}\end{array}$ & 60.084 & $\begin{array}{r}38.5 \\
1.0\end{array}$ & $\begin{array}{r}20.64 \\
0.01\end{array}$ & $\begin{array}{r}-907.8 \\
2.1\end{array}$ & $\begin{array}{r}-852.5 \\
2.1\end{array}$ & $\begin{array}{r}149.35 \\
0.37\end{array}$ & $\begin{array}{r}6 \\
189\end{array}$ & 6 & $\begin{array}{l}361 \\
423\end{array}$ \\
\hline $\begin{array}{l}\text { STISHOVITE } \\
\mathrm{SiO}_{2}\end{array}$ & 60.084 & $\begin{array}{r}27.8 \\
0.4\end{array}$ & $\begin{array}{r}14.01 \\
0.01\end{array}$ & $\begin{array}{r}-861.3 \\
2.1\end{array}$ & $\begin{array}{r}-802.8 \\
2.1\end{array}$ & $\begin{array}{r}140.65 \\
0.37\end{array}$ & $\begin{array}{r}6 \\
189\end{array}$ & 189 & 361 \\
\hline $\begin{array}{l}\text { SILICALITE } \\
\mathrm{SiO}_{2}\end{array}$ & 60.084 & $\begin{array}{r}46.3 \\
0.2\end{array}$ & $\begin{array}{r}33.29 \\
0.5\end{array}$ & $\begin{array}{r}-905.2 \\
0.8\end{array}$ & $\begin{array}{r}-852.2 \\
0.8\end{array}$ & $\begin{array}{r}149.30 \\
0.14\end{array}$ & 213 & 213 & \\
\hline $\begin{array}{l}\text { SILICA GLASS } \\
\mathrm{SiO}_{2}\end{array}$ & 60.084 & $\begin{array}{r}48.5 \\
1.0\end{array}$ & $\begin{array}{r}27.27 \\
0.10\end{array}$ & $\begin{array}{r}-901.6 \\
2.1\end{array}$ & $\begin{array}{r}-849.3 \\
2.1\end{array}$ & $\begin{array}{r}148.79 \\
0.37\end{array}$ & $\begin{array}{l}341 \\
435\end{array}$ & $\begin{array}{l}341 \\
446\end{array}$ & 341 \\
\hline $\begin{array}{l}\text { CASSITERITE } \\
\mathrm{SnO}_{2}\end{array}$ & 150.709 & $\begin{array}{r}49.0 \\
0.2\end{array}$ & $\begin{array}{r}21.55 \\
0.03\end{array}$ & $\begin{array}{r}-577.6 \\
0.2\end{array}$ & $\begin{array}{r}-515.8 \\
0.2\end{array}$ & $\begin{array}{r}90.36 \\
0.04\end{array}$ & 449 & $\begin{array}{l}85 \\
16\end{array}$ & 85 \\
\hline $\begin{array}{l}\text { STRONTIUN OXIDE } \\
\text { SrO }\end{array}$ & 103.619 & $\begin{array}{r}55.5 \\
0.4\end{array}$ & $\begin{array}{r}20.69 \\
0.01\end{array}$ & $\begin{array}{r}-591.3 \\
1.0\end{array}$ & $\begin{array}{r}-560.7 \\
1.0\end{array}$ & $\begin{array}{r}98.22 \\
0.18\end{array}$ & 67 & $\begin{array}{l}46 \\
7\end{array}$ & $\begin{array}{r}67 \\
262\end{array}$ \\
\hline $\begin{array}{l}\text { TELLURITE } \\
\text { TeO }_{2}\end{array}$ & 159.599 & $\begin{array}{r}70.4 \\
0.3\end{array}$ & $\begin{array}{r}27.75 \\
0.02\end{array}$ & $\begin{array}{r}-319.7 \\
3.0\end{array}$ & $\begin{array}{r}-264.8 \\
3.1\end{array}$ & $\begin{array}{r}46.39 \\
0.54\end{array}$ & 333 & $\begin{array}{r}419 \\
70\end{array}$ & 319 \\
\hline $\begin{array}{l}\text { THORIAMITE } \\
\mathrm{ThO}_{2}\end{array}$ & 264.037 & $\begin{array}{r}65.2 \\
0.2\end{array}$ & $\begin{array}{r}26.37 \\
0.01\end{array}$ & $\begin{array}{r}-1226.4 \\
3.5\end{array}$ & $\begin{array}{r}-1169.2 \\
3.5\end{array}$ & $\begin{array}{r}204.84 \\
0.61\end{array}$ & 419 & 419 & $\begin{array}{l}417 \\
389\end{array}$ \\
\hline $\begin{array}{l}\text { RUTILE } \\
\mathrm{TiO}_{2}\end{array}$ & 79.879 & $\begin{array}{r}50.6 \\
0.6\end{array}$ & $\begin{array}{r}18.82 \\
0.01\end{array}$ & $\begin{array}{r}-944.0 \\
0.8\end{array}$ & $\begin{array}{r}-888.8 \\
1.0\end{array}$ & $\begin{array}{r}155.70 \\
0.14\end{array}$ & $\begin{array}{l}85 \\
67\end{array}$ & $\begin{array}{r}85 \\
294\end{array}$ & $\begin{array}{l}67 \\
85\end{array}$ \\
\hline $\begin{array}{l}\text { AMATASE } \\
\mathrm{TiO}_{2}\end{array}$ & 79.879 & $\begin{array}{r}49.9 \\
0.3\end{array}$ & $\begin{array}{r}20.52 \\
0.03\end{array}$ & $\begin{array}{r}-938.7 \\
2.1\end{array}$ & $\begin{array}{r}-883.2 \\
2.1\end{array}$ & $\begin{array}{r}154.74 \\
0.37\end{array}$ & 67 & $\begin{array}{r}67 \\
419\end{array}$ & 294 \\
\hline $\begin{array}{l}\text { DITITANIUA TRIOXIDE } \\
\mathrm{Ti}_{2} \mathrm{O}_{3}\end{array}$ & 143.758 & $\begin{array}{r}7.3 \\
1.0\end{array}$ & $\begin{array}{r}31.43 \\
0.02\end{array}$ & $\begin{array}{r}-1520.9 \\
8.4\end{array}$ & $\begin{array}{r}-1433.9 \\
8.4\end{array}$ & $\begin{array}{r}251.21 \\
1.47\end{array}$ & 67 & 67 & $\begin{array}{r}67 \\
294\end{array}$ \\
\hline $\begin{array}{l}\text { URANIMITE } \\
\mathrm{UO}_{2}\end{array}$ & 270.028 & $\begin{array}{r}7.0 \\
0.2\end{array}$ & $\begin{array}{r}24.62 \\
0.01\end{array}$ & $\begin{array}{r}-1084.9 \\
1.0\end{array}$ & $\begin{array}{r}-1031.7 \\
1.0\end{array}$ & $\begin{array}{r}180.75 \\
0.18\end{array}$ & $\begin{array}{l}199 \\
248\end{array}$ & $\begin{array}{l}419 \\
248\end{array}$ & $\begin{array}{l}113 \\
128\end{array}$ \\
\hline $\begin{array}{l}\text { KARELIANITE } \\
V_{2} O_{3}\end{array}$ & 149.881 & $\begin{array}{r}98.1 \\
1.3\end{array}$ & $\begin{array}{r}29.85 \\
0.03\end{array}$ & $\begin{array}{r}-1218.8 \\
6.3\end{array}$ & $\begin{array}{r}-1139.0 \\
6.3\end{array}$ & $\begin{array}{r}199.55 \\
1.11\end{array}$ & 67 & 67 & 76 \\
\hline
\end{tabular}


THERMODYMAMIC PROPERTIES OF OXIDE AND HYOROXIDE MINERALS AT $298.15 \mathrm{~K}$

\begin{tabular}{|c|c|c|c|c|c|c|c|c|c|}
\hline \multirow[t]{2}{*}{ MAME ANO FORNULA } & \multirow{2}{*}{$\begin{array}{l}\text { MEIGHT } \\
\text { g }\end{array}$} & \multirow{2}{*}{$\begin{array}{c}\text { ENTROPY } \\
\mathrm{s}^{\bullet} \\
\mathrm{J} \cdot \mathrm{mol}^{-1} \cdot \mathrm{K}^{-1}\end{array}$} & \multirow{2}{*}{$\begin{array}{l}\text { VOLUME } \\
v^{0} \\
\mathrm{~cm}^{3}\end{array}$} & \multirow{2}{*}{$\begin{array}{l}\text { ENTHALPY } \\
\qquad \begin{array}{c}\Delta_{H} H^{\circ} \\
k J \cdot \mathrm{mol}^{-1}\end{array}\end{array}$} & \multirow{2}{*}{$\begin{array}{c}\text { FREE } \\
\text { ENERGY } \\
\Delta \mathrm{G}^{\circ} \\
\mathrm{kJ} \cdot \mathrm{mol}^{-1}\end{array}$} & \multirow[t]{2}{*}{$\operatorname{LOG}(K)$} & \multicolumn{3}{|c|}{ REFERENCES } \\
\hline & & & & & & & $\mathbf{s}$ & $H / G$ & c \\
\hline TUNGSTEN DIOXIDE & 215.849 & 50.6 & 20.03 & -589.7 & -533.9 & 93.53 & 67 & 67 & 67 \\
\hline$\omega_{2}$ & & 0.3 & 0.05 & 0.9 & 0.9 & 0.16 & & & \\
\hline TUNGSTEN TRIOXIDE & 231.848 & 75.9 & 31.61 & -842.9 & -764.1 & 133.86 & 67 & 67 & 236 \\
\hline $\mathrm{WO}_{3}$ & & 1.3 & 0.10 & 0.8 & 0.9 & 0.16 & & & \\
\hline ZINCITE & 81.389 & 43.2 & 14.34 & -350.5 & -320.4 & 56.12 & 352 & 85 & 352 \\
\hline $2 n 0$ & & 0.1 & 0.01 & 0.3 & 0.3 & 0.05 & & & 319 \\
\hline BADDELEYITE & 123.223 & 50.4 & 21.15 & -1100.6 & -1042.9 & 182.70 & 319 & 196 & 82 \\
\hline $\mathrm{ZrO}_{2}$ & & 0.3 & 0.01 & 1.7 & 1.7 & 0.30 & 419 & 419 & \\
\hline
\end{tabular}


THERMOOYMAMIC PROPERTIES OF MULTIPLE OXIDE MINERALS AT $298.15 \mathrm{~K}$

\begin{tabular}{|c|c|c|c|c|c|c|c|c|c|}
\hline MAME AMD FORINLA & $\begin{array}{l}\text { MEIGHT } \\
\text { g }\end{array}$ & $\begin{array}{c}\text { ENTROPY } \\
\mathrm{S}^{\bullet} \\
\mathrm{J} \cdot \mathrm{mol}^{-1} \cdot \mathrm{K}^{\prime}\end{array}$ & $\begin{array}{l}\text { VOLUNE } \\
\qquad \begin{array}{l}v^{\circ} \\
\mathrm{cm}^{3}\end{array}\end{array}$ & $\begin{array}{l}\text { ENTHALPY } \\
\Delta_{\Delta^{\prime}} H^{\bullet} \\
\mathrm{kJ} \cdot \mathrm{mol}^{-1}\end{array}$ & $\begin{array}{c}\text { FREE } \\
\text { ENERGY } \\
\triangle \mathrm{Ar}^{\circ} \\
\mathrm{kJ} \cdot \mathrm{mol}^{-1}\end{array}$ & $L O G(K)$ & RE & FEREW & ICES \\
\hline $\begin{array}{l}\text { TIALITE } \\
\mathrm{Al}_{2} \mathrm{TIO}_{5}\end{array}$ & 181.840 & $\begin{array}{r}109.6 \\
0.8\end{array}$ & $\begin{array}{r}48.75 \\
0.05\end{array}$ & & & & 361 & & 43 \\
\hline $\begin{array}{l}\text { CHRYSOBERYL } \\
\text { BeAl }_{2} \mathrm{O}_{4}\end{array}$ & 126.973 & $\begin{array}{r}66.3 \\
0.1\end{array}$ & $\begin{array}{r}34.32 \\
0.02\end{array}$ & $\begin{array}{r}-2298.5 \\
2.8\end{array}$ & $\begin{array}{r}-2176.2 \\
2.8\end{array}$ & $\begin{array}{r}381.3 \\
0.5\end{array}$ & $\begin{array}{l}116 \\
164\end{array}$ & 164 & 164 \\
\hline $\begin{array}{l}\text { CALCIUM FERRITE } \\
\mathrm{CaFe}_{2} \mathrm{O}_{4}\end{array}$ & 215.770 & $\begin{array}{r}145.4 \\
0.8\end{array}$ & $\begin{array}{r}44.98 \\
0.05\end{array}$ & $\begin{array}{r}-1520.3 \\
0.2\end{array}$ & $\begin{array}{r}-1412.4 \\
0.2\end{array}$ & $\begin{array}{r}247.4 \\
0.0\end{array}$ & 419 & 419 & 42 \\
\hline $\begin{array}{l}\text { DICALCIUM FERRITE } \\
\mathrm{Ca}_{2} \mathrm{Fe}_{2} \mathrm{O}_{5}\end{array}$ & 271.847 & $\begin{array}{r}188.8 \\
1.3\end{array}$ & $\begin{array}{r}67.18 \\
0.10\end{array}$ & $\begin{array}{r}-2138.3 \\
0.9\end{array}$ & $\begin{array}{r}-1999.9 \\
0.9\end{array}$ & $\begin{array}{r}350.4 \\
0.2\end{array}$ & 419 & 419 & 42 \\
\hline $\begin{array}{l}\text { PEROVSKITE } \\
\text { CaTiO }\end{array}$ & 135.956 & $\begin{array}{r}93.6 \\
0.4\end{array}$ & $\begin{array}{r}33.63 \\
0.01\end{array}$ & $\begin{array}{r}-1660.6 \\
1.7\end{array}$ & $\begin{array}{r}-1574.8 \\
1.8\end{array}$ & $\begin{array}{r}275.9 \\
0.3\end{array}$ & 419 & 419 & 295 \\
\hline $\begin{array}{l}\text { HERCYNITE } \\
\text { FeAl } 2 \mathrm{O}_{4}\end{array}$ & 173.808 & $\begin{array}{r}117.0 \\
3.0\end{array}$ & $\begin{array}{r}40.75 \\
0.05\end{array}$ & $\begin{array}{r}-1950.5 \\
8.5\end{array}$ & $\begin{array}{r}-1838.1 \\
10.0\end{array}$ & $\begin{array}{r}322.0 \\
1.8\end{array}$ & $\begin{array}{l}373 \\
188\end{array}$ & 188 & 450 \\
\hline $\begin{array}{l}\text { CHROMITE } \\
\text { FeCr }_{2} \mathrm{O}_{4}\end{array}$ & 223.837 & $\begin{array}{r}146.0 \\
1.7\end{array}$ & $\begin{array}{r}44.01 \\
0.10\end{array}$ & $\begin{array}{r}-1445.5 \\
5.0\end{array}$ & $\begin{array}{r}-1344.5 \\
6.0\end{array}$ & $\begin{array}{r}235.5 \\
1.1\end{array}$ & 361 & 373 & 292 \\
\hline $\begin{array}{l}\text { ILMENITE } \\
\text { FeTiO }_{3}\end{array}$ & 151.725 & $\begin{array}{r}108.9 \\
0.3\end{array}$ & $\begin{array}{r}31.69 \\
0.08\end{array}$ & $\begin{array}{r}-1232.0 \\
2.5\end{array}$ & $\begin{array}{r}-1155.5 \\
2.5\end{array}$ & $\begin{array}{r}202.4 \\
0.4\end{array}$ & 11 & 11 & $\begin{array}{r}11 \\
295\end{array}$ \\
\hline $\begin{array}{l}\text { ULVOSPINEL } \\
\mathrm{Fe}_{2} \mathrm{TiO}_{4}\end{array}$ & 223.572 & $\begin{array}{c}180.40 \\
2.5\end{array}$ & $\begin{array}{r}46.82 \\
0.05\end{array}$ & $\begin{array}{r}-1493.8 \\
2.0\end{array}$ & $\begin{array}{r}-1399.9 \\
2.1\end{array}$ & $\begin{array}{r}245.3 \\
0.4\end{array}$ & $\begin{array}{l}216 \\
312\end{array}$ & 216 & 43 \\
\hline $\begin{array}{l}\text { PSEUDOBROOKITE } \\
\mathrm{Fe}_{2} \mathrm{TiO}_{5}\end{array}$ & 239.571 & $\begin{array}{r}156.5 \\
1.3\end{array}$ & $\begin{array}{r}54.53 \\
0.05\end{array}$ & & & & & & 43 \\
\hline $\begin{array}{l}\text { SPIMEL } \\
\mathrm{MgAl}_{2} \mathrm{O}_{4}\end{array}$ & 142.266 & $\begin{array}{r}88.7 \\
4.0\end{array}$ & $\begin{array}{r}39.71 \\
0.03\end{array}$ & $\begin{array}{r}-2299.1 \\
2.0\end{array}$ & $\begin{array}{r}-2176.6 \\
2.3\end{array}$ & $\begin{array}{r}381.3 \\
0.4\end{array}$ & $\begin{array}{l}67 \\
37\end{array}$ & $\begin{array}{l}67 \\
64\end{array}$ & $\begin{array}{r}44 \\
386\end{array}$ \\
\hline $\begin{array}{l}\text { MMGMESIOCHROMITE } \\
\mathrm{MgCr}_{2} \mathrm{O}_{4}\end{array}$ & 192.295 & $\begin{array}{r}106.0 \\
0.8\end{array}$ & $\begin{array}{r}43.56 \\
0.05\end{array}$ & $\begin{array}{r}-1783.6 \\
0.9\end{array}$ & $\begin{array}{r}-1669.1 \\
0.9\end{array}$ & $\begin{array}{r}292.4 \\
0.1\end{array}$ & 373 & 373 & 292 \\
\hline $\begin{array}{l}\text { MAGESIOFERRITE } \\
{\text { MgFe } 2 \mathrm{O}_{4}}\end{array}$ & 199.997 & $\begin{array}{r}121.8 \\
2.0\end{array}$ & $\begin{array}{r}44.57 \\
0.05\end{array}$ & $\begin{array}{r}-1441.5 \\
3.0\end{array}$ & $\begin{array}{r}-1329.6 \\
3.1\end{array}$ & $\begin{array}{r}232.9 \\
0.5\end{array}$ & 290 & $\begin{array}{l}373 \\
249\end{array}$ & 42 \\
\hline $\begin{array}{l}\text { GEIKIELITE } \\
\mathrm{MgTiO}_{3}\end{array}$ & 120.183 & $\begin{array}{r}74.6 \\
0.2\end{array}$ & $\begin{array}{r}30.86 \\
0.07\end{array}$ & $\begin{array}{r}-1572.8 \\
1.2\end{array}$ & $\begin{array}{r}-1484.4 \\
1.2\end{array}$ & $\begin{array}{r}260.1 \\
0.2\end{array}$ & 352 & 425 & $\begin{array}{l}352 \\
295\end{array}$ \\
\hline $\begin{array}{l}\text { PYROPHANITE } \\
\text { minion }\end{array}$ & 150.816 & $\begin{array}{r}104.9 \\
0.2\end{array}$ & $\begin{array}{r}32.77 \\
0.05\end{array}$ & $\begin{array}{r}-1360.1 \\
4.0\end{array}$ & $\begin{array}{r}-1280.9 \\
4.0\end{array}$ & $\begin{array}{r}224.4 \\
0.7\end{array}$ & 393 & $\begin{array}{l}103 \\
102\end{array}$ & $\begin{array}{l}103 \\
102\end{array}$ \\
\hline $\begin{array}{l}\text { TREVORITE } \\
\text { NiFe }_{2} \mathrm{O}_{4}\end{array}$ & 234.382 & $\begin{array}{r}140.9 \\
5.0\end{array}$ & $\begin{array}{r}43.65 \\
0.05\end{array}$ & $\begin{array}{r}-1070.5 \\
2.0\end{array}$ & $\begin{array}{r}-965.1 \\
2.5\end{array}$ & $\begin{array}{r}169.1 \\
0.4\end{array}$ & 450 & $\begin{array}{l}271 \\
290\end{array}$ & 271 \\
\hline
\end{tabular}


THERMOOYMAIC PROPERTIES OF MULTIPLE OXIDE MIMERALS AT 298.15 K

\begin{tabular}{|c|c|c|c|c|c|c|c|c|c|}
\hline \multirow[t]{2}{*}{ MAME AMO FORRULA } & \multirow{2}{*}{$\begin{array}{l}\text { MEIGNT } \\
\text { g }\end{array}$} & \multirow{2}{*}{$\begin{array}{c}\text { ENTROPY } \\
\mathbf{S}^{\circ} \\
\mathrm{J} \cdot \mathrm{mol}^{-1} \cdot \mathrm{K}^{\prime}\end{array}$} & \multirow{2}{*}{$\begin{array}{l}\text { VOLumE } \\
\text { ve } \\
\mathrm{cm}^{3}\end{array}$} & \multirow{2}{*}{$\begin{array}{l}\text { ENTHALPY } \\
\begin{array}{l}\Delta H^{\circ} \\
\mathrm{kJ} \cdot \mathrm{mol}^{-1}\end{array}\end{array}$} & \multirow{2}{*}{$\begin{array}{c}\text { FREE } \\
\text { ENERGY } \\
\Delta_{r} G^{\circ} \\
k J \cdot \text { mol l}^{-1}\end{array}$} & \multirow[t]{2}{*}{$\operatorname{LOG}(K)$} & \multicolumn{3}{|c|}{ REFERENCES } \\
\hline & & & & & & & $\mathbf{s}$ & $H / G$ & C \\
\hline FRANKLINITE & 241.082 & 150.7 & 44.94 & -1188.1 & -1082.1 & 189.6 & 439 & 290 & 21 \\
\hline $\mathrm{ZnFe}_{2} \mathrm{O}_{4}$ & & 0.3 & 0.05 & 4.0 & 4.0 & 0.7 & & & \\
\hline HETAEROLITE & 239.264 & 149.7 & 45.57 & -1337.0 & -1227.8 & 215.1 & 71 & 290 & \\
\hline $2 \mathrm{nln}_{2} \mathrm{O}_{4}$ & & 0.5 & 0.05 & 4.0 & 4.0 & 0.7 & & & \\
\hline ZINC TITAMIUN SPINEL & 242.658 & 143.1 & 45.58 & -1652.1 & -1538.4 & 269.5 & 406 & 290 & 43 \\
\hline $2 n_{2} \mathrm{TiO}_{4}$ & & $\mathbf{3 . 0}$ & 0.02 & 2.0 & 2.0 & 0.4 & & & \\
\hline
\end{tabular}


TMERMCOYNAMIC PROPERTIES OF MALIDE MIMERALS AT $298.15 \mathrm{~K}$

\begin{tabular}{|c|c|c|c|c|c|c|c|c|c|}
\hline MANE ANO FORMULA & WEIGHT & $\begin{array}{c}\text { ENTROPY } \\
\mathrm{S}^{\circ} \\
J \cdot \mathrm{mol}^{-1} \cdot \mathrm{K}^{\prime}\end{array}$ & $\begin{array}{l}\text { VOLUME } \\
\text { ve } \\
\mathrm{cm}^{3}\end{array}$ & $\begin{array}{l}\text { ENTHALPY } \\
\mathrm{AJ}_{\mathrm{f}} \mathrm{H}^{\bullet} \\
\mathrm{kJ} \cdot \mathrm{mol}^{-1}\end{array}$ & $\begin{array}{c}\text { FREE } \\
\text { ENERGY } \\
\triangle \mathrm{A}^{\circ} \\
\mathrm{kJ} \cdot \mathrm{mol}^{-1}\end{array}$ & $\operatorname{LOG}(K)$ & REI & FEREM & MCES \\
\hline BROMARGYRITE & 187.772 & 107.1 & 28.99 & -100.4 & -97.0 & 16.99 & 419 & 419 & 361 \\
\hline $\mathrm{AgBr}$ & & 0.4 & 0.01 & 0.2 & 0.2 & 0.03 & & 320 & 318 \\
\hline POTASSIUM BROMIDE & 119.002 & 95.9 & 43.28 & -393.8 & -380.4 & 66.65 & 419 & 419 & 67 \\
\hline $\mathrm{KBr}$ & & 0.2 & 0.01 & 0.2 & 0.2 & 0.03 & & & 320 \\
\hline CHLORARGYRITE & 143.321 & 96.2 & 25.73 & -127.1 & -109.8 & 19.24 & 85 & 85 & 361 \\
\hline AoCl & & 0.2 & 0.01 & 0.1 & 0.1 & 0.02 & & 320 & 318 \\
\hline HYDROPHILITE & 110.983 & 104.6 & 50.75 & -795.8 & -747.7 & 130.99 & 67 & 67 & 320 \\
\hline $\mathrm{CaCl}_{2}$ & & 1.3 & 0.01 & 0.7 & 0.8 & 0.14 & & & 284 \\
\hline MNTOKITE & 98.999 & 86.2 & 23.92 & -137.2 & -119.9 & 21.01 & 419 & 419 & \\
\hline cucl & & 2.0 & 0.04 & 10.0 & 10.0 & 1.75 & & & \\
\hline LAURENCITE & 126.752 & 118.0 & 39.46 & -341.7 & -302.2 & 52.95 & 67 & 67 & 284 \\
\hline $\mathrm{FeCl}_{2}$ & & 0.4 & 0.21 & 0.4 & 0.4 & 0.07 & & 250 & 67 \\
\hline MOLYSITE & 162.205 & 142.3 & 57.86 & -399.5 & -334.0 & 58.51 & 67 & 250 & 284 \\
\hline $\mathrm{FeCl}_{3}$ & & 0.4 & 0.10 & 0.4 & 0.4 & 0.07 & & 419 & \\
\hline HYDROGEN CHLORIDE & 36.461 & 186.9 & 24789.70 & -92.3 & .95 .3 & 16.70 & 85 & 85 & 67 \\
\hline HCL (IDEAL GAS) & & 0.0 & 0.20 & 0.1 & 0.1 & 0.02 & 67 & 67 & 85 \\
\hline CALOMEL & 236.043 & 95.8 & 32.94 & -132.7 & -105.2 & 18.43 & 85 & 85 & \\
\hline $\mathrm{MgCl}$ & & 0.4 & 0.08 & 0.2 & 0.2 & 0.04 & & & \\
\hline SYLVITE & 74.551 & 82.6 & 37.52 & -436.5 & -408.6 & 71.58 & 117 & 117 & 117 \\
\hline KCl & & 0.2 & 0.00 & 0.2 & 0.2 & 0.04 & 85 & 85 & 67 \\
\hline CHLOROMUENESITE & 95.210 & 89.6 & 40.81 & -641.3 & -591.8 & 103.68 & 67 & 320 & 284 \\
\hline $\mathrm{MgCl}_{2}$ & & 0.8 & 0.10 & 0.7 & 0.7 & 0.13 & & 85 & \\
\hline SCACCHITE & 125.844 & 118.2 & 42.11 & -481.3 & -440.5 & 77.17 & $\mathbf{7 3}$ & 250 & 284 \\
\hline $\mathrm{MnCl}_{2}$ & & 0.2 & 0.17 & 0.8 & 0.8 & 0.16 & & 419 & \\
\hline SALAMWONIAC & 53.491 & 94.6 & 35.06 & -314.4 & -202.9 & 35.55 & 419 & 619 & 67 \\
\hline $\mathrm{NH}_{4} \mathrm{Cl}$ & & 0.4 & 0.05 & 0.3 & 0.3 & 0.05 & 67 & & \\
\hline MALITE & 58.442 & 72.1 & 27.02 & -411.3 & -384.2 & 67.30 & 419 & 419 & 67 \\
\hline $\mathrm{MaCl}$ & & 0.2 & 0.00 & 0.1 & 0.1 & 0.02 & & & 320 \\
\hline MICKELOUS CHLORIDE & 129.595 & 98.2 & 36.70 & -304.9 & -258.8 & 45.33 & 67 & 67 & 80 \\
\hline $\mathrm{NiCl}_{2}$ & & 0.2 & 0.07 & 2.0 & 2.0 & 0.35 & & 419 & \\
\hline Cotunnite & 278.105 & 136.0 & 47.09 & -359.4 & -316.1 & 55.03 & 67 & 67 & 67 \\
\hline $\mathrm{PbCl}_{2}$ & & 2.1 & 0.05 & 0.3 & 0.7 & 0.12 & & 419 & 320 \\
\hline
\end{tabular}


THERMOOYNAMIC PROPERTIES OF HALIDE MINERALS AT $298.15 \mathrm{~K}$

\begin{tabular}{|c|c|c|c|c|c|c|c|c|c|}
\hline \multirow[t]{2}{*}{ MAME AMD FORIULA } & \multirow[t]{2}{*}{ WEIGHT } & \multirow{2}{*}{$\begin{array}{c}\text { ENTROPY } \\
\mathrm{S}^{\circ} \\
\mathrm{J} \cdot \mathrm{mol}^{-1} \cdot \mathrm{K}^{\prime}\end{array}$} & \multirow{2}{*}{$\begin{array}{l}\text { VOLUNE } \\
\qquad \begin{array}{l}V^{\circ} \\
\mathrm{cm}^{3}\end{array}\end{array}$} & \multirow{2}{*}{$\begin{array}{l}\text { ENTHALPY } \\
\qquad \begin{array}{c}A_{\text {H }} \mathrm{H}^{\circ} \\
\mathrm{kJ} \cdot \mathrm{mol}^{-1}\end{array}\end{array}$} & \multirow{2}{*}{$\begin{array}{c}\text { FREE } \\
\text { ENERGY } \\
4 G^{\circ} \\
\text { kJ.mol }\end{array}$} & \multirow[t]{2}{*}{$\operatorname{LOG}(K)$} & \multicolumn{3}{|c|}{ REFERENCES } \\
\hline & & & & & & & $\mathbf{s}$ & $H / G$ & C \\
\hline FLUORITE & 78.075 & 68.9 & 24.54 & -1228.0 & -1175.3 & 205.90 & 117 & 117 & 293 \\
\hline $\mathrm{CaF}_{2}$ & & 0.3 & 0.01 & 2.0 & 2.0 & 0.35 & & 67 & 117 \\
\hline HYOROEEN FLUORIDE & 20.006 & 173.8 & 24789.70 & -273.3 & -275.4 & 48.25 & 85 & 85 & 67 \\
\hline HF (IDEAL EAS) & & 0.0 & 0.20 & 0.7 & 0.7 & 0.12 & 67 & & \\
\hline SELlaite & 62.302 & 57.2 & 19.61 & -1124.2 & -1071.1 & 187.64 & 117 & 117 & 117 \\
\hline $\mathrm{MgF}_{2}$ & & 0.5 & 0.01 & 1.2 & 1.2 & 0.21 & & & \\
\hline VILLIAUMITE & 41.988 & 51.5 & 14.98 & -573.6 & -543.4 & 95.20 & 419 & 419 & 300 \\
\hline NaF & & 0.1 & 0.01 & 0.7 & 0.7 & 0.12 & & & 67 \\
\hline CRYOLITE & 209.941 & 238.5 & 70.81 & -3316.8 & -3152.1 & 552.22 & 10 & 10 & 10 \\
\hline $\mathrm{Na}_{3} \mathrm{AlF} / 6$ & & 0.5 & 0.20 & 6.0 & 6.0 & 1.05 & & 301 & 300 \\
\hline CHIOLITE & 461.871 & 515.3 & 154.08 & -7546.3 & -7174.7 & 1256.95 & 400 & 400 & 400 \\
\hline $\mathrm{Na}_{5} \mathrm{Al} \mathbf{3}^{\mathrm{F}} 14$ & & 0.8 & 0.10 & 20.0 & 20.0 & 3.50 & & & \\
\hline IODARGYRITE & 234.773 & 115.5 & 41.30 & -61.8 & -66.2 & 11.60 & 419 & 419 & 320 \\
\hline AgI & & 1.7 & 0.04 & 1.7 & 1.8 & 0.31 & & & \\
\hline MARSHITE & 190.450 & 96.6 & 33.35 & -67.8 & -69.4 & 12.16 & 320 & 320 & 108 \\
\hline CuI & & & 0.02 & 5.0 & 5.0 & 0.88 & & & \\
\hline COCCINITE & 454.399 & 180.0 & 71.84 & -105.4 & -101.7 & 17.82 & 419 & 419 & 67 \\
\hline $\mathrm{HgI}_{2}$ & & 6.3 & 0.10 & 1.7 & 2.6 & 0.45 & 67 & 67 & \\
\hline
\end{tabular}


THERHODYMNIC PROPERTIES OF CARBONATE AND MITRATE MINERALS AT $298.15 \mathrm{~K}$

\begin{tabular}{|c|c|c|c|c|c|c|c|c|c|}
\hline MUNE AND FORMULA & $\begin{array}{l}\text { MEIGHT } \\
\text { g }\end{array}$ & $\begin{array}{c}\text { ENTROPY } \\
S^{\circ} \\
J \cdot \mathrm{mol}^{-1} \cdot K^{-1}\end{array}$ & $\begin{array}{l}\text { VOLunE } \\
\text { vo } \\
\mathrm{cm}^{3}\end{array}$ & $\begin{array}{c}\text { ENTHALPY } \\
\Delta_{\mathrm{r}} \mathrm{H}^{\circ} \\
\mathrm{kJ} \cdot \mathrm{mol}^{-1}\end{array}$ & $\begin{array}{c}\text { FREE } \\
\text { EMERGY } \\
\triangle \mathrm{AG}^{\circ} \\
\mathrm{kJ} \cdot \mathrm{mol}^{-1}\end{array}$ & LOG(K) & RE & FEREM & ICES \\
\hline MITHERITE & 197.336 & 112.1 & 45.81 & -1210.9 & -1132.2 & 198.35 & 54 & 1 & 262 \\
\hline $\mathrm{BaCO}_{3}$ & & 2.1 & 0.06 & 2.2 & 2.2 & 0.39 & & & \\
\hline ARACONITE & 100.087 & 88.0 & 34.15 & -1207.4 & -1127.4 & 197.51 & 392 & 336 & \\
\hline $\mathrm{CaCO}_{3}$ & & 0.2 & 0.05 & 1.4 & 1.5 & 0.26 & & & \\
\hline CALCITE & 100.087 & 91.7 & 36.93 & -1207.4 & -1128.5 & 197.70 & 392 & 246 & 202 \\
\hline $\mathrm{CaCO}_{3}$ & & 0.2 & 0.01 & 1.3 & 1.4 & 0.24 & & 336 & \\
\hline VATERITE & 100.087 & & 37.63 & & -1125.5 & 197.19 & & 336 & \\
\hline $\mathrm{CaCO}_{3}$ & & & 0.40 & & 1.5 & 0.26 & & 412 & \\
\hline MONOHYDROCALCITE & 118.102 & 131.1 & 48.70 & -1498.3 & -1361.6 & 238.54 & 197 & 197 & \\
\hline $\mathrm{CaCO}_{3} \cdot \mathrm{H}_{2} \mathrm{O}$ & & $\mathbf{3 . 0}$ & 0.40 & 1.2 & 1.1 & 0.20 & & & \\
\hline IKAITE & 208.179 & 370.0 & & -2954.1 & -2540.9 & 445.15 & 273 & 273 & 273 \\
\hline $\mathrm{CaCO}_{3} \cdot 6 \mathrm{H}_{2} \mathrm{O}$ & • & 5.0 & & 5.0 & 5.0 & 0.88 & & & \\
\hline DOLOMITE & 184.401 & 155.2 & 64.34 & -2324.5 & -2161.3 & 378.64 & 396 & 175 & 257 \\
\hline $\mathrm{Callo}\left(\mathrm{CO}_{3}\right)_{2}$ & & 0.3 & 0.03 & 1.5 & 1.7 & 0.29 & & & \\
\hline MUNTITE & 353.030 & 299.5 & 122.58 & -4529.6 & -4203.1 & 736.34 & 168 & 169 & \\
\hline $\mathrm{CeNH}_{3}\left(\mathrm{CO}_{3}\right)_{4}$ & & 0.9 & 0.10 & 1.6 & 1.6 & 0.29 & & 253 & \\
\hline OtAVIte & 172.419 & 92.5 & 34.30 & -750.6 & -669.4 & 117.28 & 63 & 419 & \\
\hline $\mathrm{CoCO}_{3}$ & & 5.5 & 0.02 & 2.5 & 2.6 & 0.46 & & 63 & \\
\hline MLACHITE & 221.116 & 166.3 & 54.86 & -1054.0 & -890.2 & 155.96 & 241 & 339 & \\
\hline $\mathrm{Cu}_{2}\left(\mathrm{CO}_{3}\right)(\mathrm{OH})_{2}$ & & 2.5 & 0.08 & 2.1 & 2.2 & 0.39 & & & \\
\hline AZURITE & 344.671 & 254.4 & 91.01 & -1632.2 & -1391.4 & 243.75 & 241 & 419 & \\
\hline $\mathrm{Cu}_{3}\left(\mathrm{OH}_{2}\right)_{2}\left(\mathrm{CO}_{3}\right)_{2}$ & & 3.8 & 0.13 & 2.0 & 2.2 & 0.39 & & & \\
\hline SIDERITE & 115.856 & 95.5 & 29.38 & -755.9 & -682.8 & 119.63 & 351 & 351 & 351 \\
\hline $\mathrm{FeCO}_{3}$ & & 0.2 & 0.01 & 5.5 & 5.5 & 0.96 & & & \\
\hline MGNESITE & 84.314 & 65.1 & 28.02 & -1113.3 & -1029.5 & 180.36 & 178 & 347 & 359 \\
\hline $\mathrm{MgCO}_{3}$ & & 0.1 & 0.01 & 1.3 & 1.4 & 0.24 & & & \\
\hline MESQUEHONITE & 138.360 & 195.6 & 75.47 & -1977.3 & -1723.8 & 301.99 & 353 & 354 & \\
\hline $\mathrm{MgCO}_{3} \cdot 3 \mathrm{H}_{2} \mathrm{O}$ & & 0.6 & 0.05 & 0.3 & 0.5 & 0.09 & & & \\
\hline HYDROMAGNESITE & 467.638 & 503.7 & 211.10 & -6514.9 & -5864.2 & 1027.37 & 353 & 354 & \\
\hline $4 \mathrm{HgCO}_{3} \cdot \mathrm{Mg}(\mathrm{OH})_{2} \cdot 4 \mathrm{H}_{2} \mathrm{O}$ & & 1.6 & 0.10 & 1.1 & 1.1 & 0.19 & & & \\
\hline ARTIMITE & 196.680 & 232.9 & 96.43 & -2920.6 & -2568.4 & 449.95 & 168 & 169 & \\
\hline $\mathrm{Mg}_{2}(\mathrm{OH})_{2} \mathrm{CO}_{3} \cdot 3 \mathrm{H}_{2} \mathrm{O}$ & & 0.7 & 0.10 & 0.7 & 0.8 & 0.13 & & & \\
\hline
\end{tabular}


THERMOOYMAMIC PROPERTIES OF CARBONATE AND MITRATE MINERALS AT $298.15 \mathrm{~K}$

\begin{tabular}{|c|c|c|c|c|c|c|c|c|c|}
\hline MNEE AND FORALLA & $\begin{array}{l}\text { MEIGHT } \\
\bullet\end{array}$ & $\begin{array}{c}\text { EWTROPY } \\
\mathbf{S}^{\circ} \\
\mathrm{J} \cdot \mathrm{mol}^{-1} \cdot \mathrm{K}^{\prime}\end{array}$ & $\begin{array}{l}\text { Volune } \\
\qquad \begin{array}{c}\text { ve }^{3} \\
c^{3}\end{array}\end{array}$ & $\begin{array}{c}\text { EUTHALPY } \\
\begin{array}{c}\Delta H^{\circ} \\
\mathrm{kJ} \cdot \mathrm{mol}^{-1}\end{array}\end{array}$ & $\begin{array}{c}\text { FREE } \\
\text { ENERGY } \\
\text { A,G } \\
\mathbf{k J} \cdot \mathrm{mol}^{-1}\end{array}$ & $\operatorname{LOG}(K)$ & $\mathbf{s}$ & FEREN & CES \\
\hline RHODOCHROSITE & 114.947 & 98.0 & 31.07 & -892.9 & -819.1 & 143.50 & 351 & 351 & 283 \\
\hline $\mathrm{NinCO}_{3}$ & & 0.1 & 0.01 & 0.5 & 0.6 & 0.10 & & & \\
\hline MAMCOLITE & 84.007 & 102.1 & 38.08 & -949.0 & -851.2 & 149.12 & 36 & 36 & \\
\hline $\mathrm{NaHCO}_{3}$ & & 1.7 & 0.25 & 0.2 & 0.6 & 0.10 & & & \\
\hline THERMONATRITE & 124.004 & 168.1 & 54.99 & -1429.7 & -1286.1 & 225.32 & 424 & 36 & 129 \\
\hline $\mathrm{Na}_{2} \mathrm{CO}_{3} \cdot \mathrm{H}_{2} \mathrm{O}$ & & 0.8 & 0.27 & 0.4 & 0.5 & 0.09 & 36 & & \\
\hline Trown & 226.026 & & 107.00 & -2682.1 & & & & 416 & \\
\hline $\mathrm{Na}_{2} \mathrm{CO}_{3} \cdot \mathrm{NaHCO}_{3} \cdot 2 \mathrm{H}_{2} \mathrm{O}$ & & & 0.50 & 0.4 & & & & & \\
\hline UEGSCHEIDERITE & 358.010 & & 153.30 & -3984.0 & & & & 416 & \\
\hline $\mathrm{Ne}_{2} \mathrm{CO}_{3} \cdot 3 \mathrm{NaHCO}_{3}$ & & & 0.80 & 0.8 & & & & & \\
\hline SODIUN CARBOMATE & 105.989 & 135.0 & 41.60 & -1129.2 & -1045.3 & 183.13 & 424 & 36 & \\
\hline $\mathrm{Ne}_{2} \mathrm{CO}_{3}$ & & 0.6 & & 0.3 & 0.4 & 0.06 & 36 & & \\
\hline DAMSONITE & 143.995 & 132.0 & 59.30 & -1964.0 & -1786.0 & 312.89 & 110 & 110 & 110 \\
\hline $\operatorname{MaA}\left(\mathrm{CO}_{3}(\mathrm{OH})_{2}\right.$ & & 0.5 & 0.30 & 2.9 & 3.0 & 0.52 & & & \\
\hline GASPEITE & 118.699 & 85.4 & 27.05 & & & & 255 & & \\
\hline $\mathrm{NiCO}_{3}$ & & 2.0 & 0.02 & & & & & & \\
\hline CERUSSITE & 267.209 & 131.0 & 40.59 & -699.2 & -625.5 & 109.58 & 217 & 419 & \\
\hline $\mathrm{PbCO}_{3}$ & & 3.4 & 0.06 & 1.2 & 1.6 & 0.27 & & 4 & \\
\hline STRONTIANITE & 147.629 & 97.1 & 39.01 & -1218.7 & -1137.6 & 199.29 & 55 & 55 & 262 \\
\hline $\mathrm{SrCO}_{3}$ & & 1.7 & 0.06 & 1.5 & 1.5 & 0.26 & & & \\
\hline SNITHSONITE & 125.399 & 81.2 & 28.28 & -817.0 & -735.3 & 128.82 & 352 & 153 & 153 \\
\hline $\mathrm{ZnCO}_{3}$ & & 0.2 & 0.01 & 3.1 & 3.1 & 0.54 & & & \\
\hline MITROBARITE & 261.337 & 213.8 & 80.58 & -992.1 & -796.6 & 139.55 & 419 & 419 & 361 \\
\hline $\mathrm{Ba}\left(\mathrm{NO}_{3}\right)_{2}$ & & 0.9 & 2.10 & 2.5 & 0.4 & 0.08 & & & \\
\hline CALCIUN MITRATE & 164.088 & 193.3 & 66.09 & -938.4 & -742.6 & 130.10 & 419 & 419 & 361 \\
\hline $\mathrm{Ca}\left(\mathrm{NO}_{3}\right)_{2}$ & & 0.4 & 0.03 & 1.5 & 1.8 & 0.31 & & & \\
\hline MITER & 101.103 & 133.1 & 48.04 & -494.5 & -394.6 & 69.13 & 419 & 419 & 361 \\
\hline $\mathrm{KMO}_{3}$ & & 0.7 & 0.06 & 0.4 & 0.5 & 0.08 & & & \\
\hline MGEESIUN MITRATE & 148.315 & 164.0 & 62.93 & -790.6 & -589.2 & 103.22 & 419 & 419 & 361 \\
\hline $\mathrm{Mg}\left(\mathrm{NO}_{3}\right)_{2}$ & & 1.6 & 0.03 & 1.3 & 1.4 & 0.25 & & & \\
\hline AMNONIA-MITER & 80.043 & 151.1 & 46.49 & -365.6 & -183.8 & 32.20 & 419 & 419 & 361 \\
\hline $\mathrm{mH}_{4} \mathrm{NO}_{3}$ & & 0.2 & 0.10 & 0.8 & 0.9 & 0.15 & & & \\
\hline
\end{tabular}


THERMOOYMAIC PROPERTIES OF CARBONATE ND NITRATE MINERALS AT $298.15 \mathrm{~K}$

\begin{tabular}{|c|c|c|c|c|c|c|c|c|c|}
\hline \multirow[t]{2}{*}{ MANE AND FORRULA } & \multirow[t]{2}{*}{ MEIGMT } & \multirow{2}{*}{$\begin{array}{c}\text { ENTROPY } \\
\mathrm{S}^{\bullet} \\
\mathrm{J} \cdot \mathrm{mol}^{-1} \cdot \mathrm{K}^{-1}\end{array}$} & \multirow{2}{*}{$\begin{array}{l}\text { VOLunE } \\
\text { ve } \\
\mathrm{cm}^{3}\end{array}$} & \multirow{2}{*}{$\begin{array}{l}\text { ENTMALPY } \\
\Delta_{H^{\circ}} \\
\mathrm{kJ} \cdot \mathrm{mol}^{-1}\end{array}$} & \multirow{2}{*}{$\begin{array}{c}\text { FREE } \\
\text { ENERGY } \\
\triangle \mathrm{G}^{\circ} \\
\mathrm{kJ} \cdot \mathrm{mol}^{-1}\end{array}$} & \multirow[t]{2}{*}{$\operatorname{LOG}(K)$} & \multicolumn{3}{|c|}{ REFERENCES } \\
\hline & & & & & & & $\mathbf{s}$ & H/G & C \\
\hline SOOA-MITER & 84.995 & 116.5 & 37.60 & -468.0 & -367.1 & 64.32 & 419 & 419 & 361 \\
\hline $\mathrm{NaNO}_{3}$ & & 0.7 & 0.02 & 0.4 & 0.4 & 0.07 & & & \\
\hline STRONTIUN MITRATE & 211.630 & 194.6 & 70.93 & -978.2 & -779.0 & 136.47 & 419 & 419 & 361 \\
\hline $\operatorname{Sr}\left(\mathrm{NO}_{3}\right)_{2}$ & & 0.5 & 0.04 & 1.0 & 1.3 & 0.23 & & & \\
\hline
\end{tabular}


THERMOOYMAMIC PROPERTIES OF SULFATE AND BORATE MINERALS AT 298.15 K

\begin{tabular}{|c|c|c|c|c|c|c|c|c|c|}
\hline \multirow[t]{2}{*}{ MAME AND FORINULA } & \multirow{2}{*}{$\begin{array}{l}\text { WEIGHT } \\
\text { g }\end{array}$} & \multirow{2}{*}{$\begin{array}{c}\text { EWTROPY } \\
\mathbf{s}^{0} \\
J \cdot \mathrm{mol}^{-1} \cdot \boldsymbol{K}^{-1}\end{array}$} & \multirow{2}{*}{$\begin{array}{l}\text { VOLUME } \\
v^{0} \\
\mathrm{~cm}^{3}\end{array}$} & \multirow{2}{*}{$\begin{array}{l}\text { ENTHALPY } \\
\Delta_{\mathbb{P}^{\prime}} \mathrm{H}^{\circ} \\
\mathrm{kJ} \cdot \mathrm{mol}^{-1}\end{array}$} & \multirow{2}{*}{$\begin{array}{c}\text { FREE } \\
\text { ENERGY } \\
\triangle \mathrm{AG}^{\circ} \\
\mathrm{kJ} \cdot \mathrm{mol}^{-1}\end{array}$} & \multirow[t]{2}{*}{$\operatorname{LOG}(K)$} & \multicolumn{3}{|c|}{ REFERENCES } \\
\hline & & & & & & & $\mathbf{s}$ & $H / G$ & c \\
\hline ALUMIMUM SULFATE & 342.154 & 239.3 & 73.41 & -3441.8 & -3100.6 & 543.20 & 88 & 88 & 88 \\
\hline $\mathrm{Al}_{2}\left(\mathrm{SO}_{4}\right)_{3}$ & & 1.2 & 0.20 & 1.8 & 1.9 & 0.33 & & & \\
\hline BARITE & 233.391 & 132.2 & 52.10 & -1473.6 & -1362.5 & 238.70 & 88 & 88 & 88 \\
\hline $\mathrm{BaSO}_{4}$ & & 0.8 & 0.06 & 1.0 & 1.3 & 0.23 & & 419 & \\
\hline ANHYDRITE & 136.142 & 107.4 & 46.01 & -1434.4 & -1321.8 & 231.57 & 368 & 88 & 368 \\
\hline $\mathrm{CaSO}_{4}$ & & 0.2 & 0.01 & 4.2 & 4.3 & 0.75 & & 419 & \\
\hline GYPSUM & 172.172 & 193.8 & 74.69 & -2023.0 & -1797.0 & 314.82 & 368 & 88 & \\
\hline $\mathrm{CaSO}_{4} \cdot 2 \mathrm{H}_{2} \mathrm{O}$ & & 0.3 & 0.22 & 4.3 & 4.4 & 0.77 & 88 & & \\
\hline CHALCOCYANITE & 159.610 & 109.5 & 40.88 & -771.4 & -662.3 & 116.03 & 106 & 4 & 106 \\
\hline $\mathrm{CuSO}_{4}$ & & 0.6 & 0.03 & 1.3 & 1.4 & 0.25 & 87 & 419 & \\
\hline CHALCANTHITE & 249.686 & 301.2 & 108.97 & -2279.7 & -1880.0 & 329.36 & 87 & 87 & \\
\hline $\mathrm{CuSO}_{4} \cdot 5 \mathrm{H}_{2} \mathrm{O}$ & & 0.6 & 0.22 & 3.4 & 3.6 & 0.63 & & 419 & \\
\hline BROCHANTITE & 452.292 & & 113.60 & & -1818.0 & 318.49 & & 26 & \\
\hline $\mathrm{Cu}_{4}\left(\mathrm{SO}_{4}\right)(\mathrm{OH})_{6}$ & & & 0.20 & & 2.5 & 0.44 & & 419 & \\
\hline FERRIC SULFATE & 399.885 & 282.8 & 130.80 & -2581.9 & -2254.4 & 394.96 & 328 & 19 & 328 \\
\hline $\mathrm{Fe}_{2}\left(\mathrm{SO}_{4}\right)_{3}$ & & 0.9 & 0.20 & 2.9 & $\mathbf{3 . 0}$ & 0.52 & & & \\
\hline SZOMOLMOKITE & 169.926 & & 55.90 & -1264.3 & & & 87 & 2 & \\
\hline $\mathrm{FeSO}_{4} \cdot \mathrm{H}_{2} \mathrm{O}$ & & & 0.40 & 0.5 & & & & 87 & \\
\hline MELANTERITE & 278.018 & 409.2 & 146.50 & -3014.3 & -2509.5 & 439.64 & 87 & 2 & \\
\hline $\mathrm{FeSO}_{4} \cdot 7 \mathrm{H}_{2} \mathrm{O}$ & & 1.3 & 0.30 & 0.6 & 1.3 & 0.23 & & 87 & \\
\hline SULFURIC ACID (LIQUID) & 98.080 & 156.9 & 53.57 & -814.0 & -690.0 & 120.88 & 419 & 419 & \\
\hline $\mathrm{H}_{2} \mathrm{SO}_{4}$ & & 0.2 & 0.07 & 0.4 & 0.5 & 0.08 & & 124 & \\
\hline ARCANITE & 174.260 & 175.6 & 65.50 & -1437.7 & -1319.6 & 231.18 & 67 & 67 & 387 \\
\hline $\mathrm{K}_{2} \mathrm{SO}_{4}$ & & 0.4 & 0.07 & 0.5 & 0.5 & 0.09 & & & 67 \\
\hline K-AL SULFATE & 258.207 & 204.6 & 92.33 & -2470.9 & -2240.4 & 392.50 & 419 & 419 & 217 \\
\hline $\mathrm{KAl}\left(\mathrm{SO}_{4}\right)_{2}$ & & 1.3 & 0.08 & 1.3 & 1.4 & 0.24 & & & \\
\hline ALUNITE & 414.214 & 321.0 & 293.60 & -5176.5 & -4663.5 & 817.00 & 452 & 452 & 219 \\
\hline $\mathrm{KAl}_{3}(\mathrm{OH})_{6}\left(\mathrm{SO}_{4}\right)_{2}$ & & 5.0 & 0.40 & 2.4 & 2.4 & 0.42 & & 219 & \\
\hline LANGBEIMITE & 414.997 & 389.3 & 146.95 & -4071.0 & -3733.4 & 654.05 & 368 & 419 & 368 \\
\hline $\mathrm{K}_{2} \mathrm{Mg}_{2}\left(\mathrm{SO}_{4}\right)_{3}$ & & 1.0 & 0.04 & 3.0 & 3.0 & 0.52 & 40 & & \\
\hline MAGNESIUM SULFATE & 120.369 & 91.4 & & -1284.9 & -1170.5 & 205.07 & 67 & 247 & 67 \\
\hline $\mathrm{MgSO}_{4}$ & & 0.8 & & 0.6 & 0.8 & 0.14 & & 88 & \\
\hline
\end{tabular}


THERMOOYMAMIC PROPERTIES OF SULFATE AND BORATE MIMERALS AT $298.15 \mathrm{~K}$

\begin{tabular}{|c|c|c|c|c|c|c|c|c|c|}
\hline \multirow[t]{2}{*}{ MAME AMD FORMULA } & \multirow{2}{*}{$\begin{array}{l}\text { WEIGHT } \\
g\end{array}$} & \multirow{2}{*}{$\begin{array}{c}\text { ENTROPY } \\
\mathbf{S}^{\circ} \\
\mathrm{J} \cdot \mathrm{mol}^{-1} \cdot \mathrm{K}^{\prime}\end{array}$} & \multirow{2}{*}{$\begin{array}{l}\text { VOLUME } \\
\qquad \begin{array}{l}v^{0} \\
\mathrm{~cm}^{3}\end{array}\end{array}$} & \multirow{2}{*}{$\begin{array}{l}\text { ENTHALPY } \\
\qquad \begin{array}{c}\Delta, H^{\bullet} \\
k J \cdot \mathrm{mol}^{-1}\end{array}\end{array}$} & \multirow{2}{*}{$\begin{array}{c}\text { FREE } \\
\text { ENERGY } \\
\Delta, G^{\circ} \\
k J \cdot \text { mol l}^{-1}\end{array}$} & \multirow[t]{2}{*}{$\operatorname{LOG}(K)$} & \multicolumn{3}{|c|}{ REFERENCES } \\
\hline & & & & & & & $\mathbf{s}$ & H/G & c \\
\hline EPSONITE & 246.476 & 372.0 & 146.80 & -3388.7 & -2871.2 & 503.01 & 419 & 419 & 88 \\
\hline $\mathrm{MgSO}_{4} \cdot \mathrm{ZH}_{2} \mathrm{O}$ & & 4.0 & 0.20 & 0.1 & 0.9 & 0.15 & & 88 & \\
\hline muMganese sULfate & 151.002 & 127.0 & 43.62 & -1065.7 & -962.1 & 168.56 & 450 & 87 & 87 \\
\hline $\mathrm{MnSO}_{4}$ & & 1.5 & 0.04 & 1.1 & 1.5 & 0.26 & & 377 & \\
\hline mascagnite & 132.141 & 220.5 & 74.68 & -1182.7 & -903.5 & 158.29 & 419 & 88 & 387 \\
\hline$\left(\mathrm{NH}_{4}\right)_{2} \mathrm{SO}_{4}$ & & 1.3 & 0.09 & 1.3 & 1.6 & 0.28 & 88 & 419 & 219 \\
\hline THEMARDITE & 142.043 & 149.6 & 53.33 & -1387.8 & -1269.8 & 222.46 & 88 & 88 & 81 \\
\hline $\mathrm{Na}_{2} \mathrm{SO}_{4}$ & & 0.1 & 0.06 & 0.4 & 0.4 & 0.07 & 419 & 419 & \\
\hline MIRABILITE & 322.196 & 591.9 & 219.80 & -4327.3 & -3645.8 & 638.71 & 47 & 47 & \\
\hline $\mathrm{Ma}_{2} \mathrm{SO}_{4} \cdot \mathrm{1OH}_{2} \mathrm{O}$ & & 0.6 & 0.40 & 4.0 & 3.4 & 0.59 & & 81 & \\
\hline MICKELOUS SULFATE & 154.754 & 101.3 & 38.57 & -873.2 & -762.7 & 133.61 & 401 & 401 & 401 \\
\hline $\mathrm{NiSO}_{4}$ & & 0.3 & 0.03 & 1.0 & 1.0 & & & 4 & \\
\hline RETGERSITE (ALPHA, GREEN) & 262.845 & 334.5 & 126.60 & -2683.4 & -2225.1 & 389.82 & 87 & 87 & \\
\hline $\mathrm{MiSO}_{4} \cdot 6 \mathrm{H}_{7} \mathrm{O}$ & & 0.4 & 0.20 & 0.5 & 0.5 & 0.09 & & 419 & \\
\hline MOREMOSITE & 280.861 & 378.9 & 143.80 & -2976.5 & -2461.9 & 431.30 & 87 & 87 & \\
\hline $\mathrm{NiSO}_{4} \cdot 7 \mathrm{H}_{2} \mathrm{O}$ & & 0.4 & 0.50 & 0.5 & 0.5 & 0.09 & & 419 & \\
\hline AMGLESITE & 303.264 & 148.5 & 47.95 & -920.0 & -813.1 & 142.44 & 85 & 85 & 88 \\
\hline $\mathrm{PbSO}_{4}$ & & 0.6 & 0.06 & 0.4 & 0.5 & 0.09 & & & \\
\hline CELESTITE & 183.684 & 117.0 & 46.25 & -1453.2 & -1339.6 & 234.69 & 419 & 419 & \\
\hline $\mathrm{SrSO}_{4}$ & & 4.2 & 0.06 & 4.2 & 4.4 & 0.77 & & 129 & \\
\hline ZINKOSITE & 161.454 & 110.5 & 41.57 & -980.1 & -868.7 & 152.20 & 87 & 87 & 87 \\
\hline $\mathrm{ZnSO}_{4}$ & & 1.3 & 0.07 & 0.8 & 0.9 & 0.16 & & 4 & 192 \\
\hline BIANCHITE & 269.545 & 363.6 & 130.20 & -2777.8 & -2324.6 & 407.26 & 87 & 87 & \\
\hline $\mathrm{ZnSO}_{4} \cdot 6 \mathrm{H}_{2} \mathrm{O}$ & & 1.3 & 0.50 & 1.0 & 1.0 & 0.18 & & 419 & \\
\hline COSLARITE & 287.561 & 388.7 & 145.80 & -3077.5 & -2562.3 & 448.89 & 87 & 87 & \\
\hline $\mathrm{ZnSO}_{4} \cdot 7 \mathrm{H}_{2} \mathrm{O}$ & & 1.3 & 0.10 & 0.1 & 0.1 & 0.02 & & 419 & \\
\hline BORAX & 381.372 & 586.0 & 222.70 & -6288.6 & -5516.2 & 966.39 & 419 & 419 & \\
\hline $\mathrm{Na}_{2}{ }^{\mathrm{B}} 4^{\mathrm{O}} 7^{-} \cdot{ }^{10 \mathrm{H}_{2} \mathrm{O}}$ & & 2.3 & 0.20 & 8.5 & 8.5 & 1.49 & & & \\
\hline
\end{tabular}


THERMCOYMAMIC PROPERTIES OF PHOSPHATE, MOLYBDATE ANO TUMGSTATE MINERALS AT $298.15 \mathrm{~K}$

\begin{tabular}{|c|c|c|c|c|c|c|c|c|c|}
\hline MUE AND Fonevila & $\begin{array}{l}\text { VEIGHT } \\
g\end{array}$ & $\begin{array}{c}\text { ENTROPY } \\
S^{\bullet} \\
J \cdot \mathrm{mol}^{-1} \cdot \mathrm{K}^{-1}\end{array}$ & $\begin{array}{l}\text { volune } \\
v^{0} \\
\mathrm{con}^{3}\end{array}$ & $\begin{array}{l}\text { ENTHALPY } \\
\begin{array}{l}\Delta \mathrm{HH}^{\circ} \\
\mathrm{kJ} \cdot \mathrm{mol}^{-1}\end{array}\end{array}$ & $\begin{array}{c}\text { FREE } \\
\text { ENERGY } \\
\triangle \sigma^{\circ} \\
\mathrm{kJ} \cdot \mathrm{mol}^{-1}\end{array}$ & LOC(K) & REI & FERENC & CES \\
\hline BERLINITE & 121.953 & 90.8 & 46.58 & -1733.8 & -1617.9 & 283.44 & 419 & 419 & 181 \\
\hline $\mathrm{AlPO}_{4}$ & & 0.2 & 0.10 & 5.0 & 5.0 & 0.88 & & 181 & \\
\hline HIITLOCKITE & 310.177 & 236.0 & 97.62 & -4120.8 & -3883.6 & 680.38 & 419 & 419 & 361 \\
\hline $\mathrm{Co}_{3}\left(\mathrm{PO}_{4}\right)_{2}$ & & 0.8 & 0.09 & 5.0 & 5.0 & 0.88 & & & \\
\hline FLUORAPATITE & 504.302 & 387.9 & 157.56 & -6872.0 & -6489.7 & 1136.95 & 419 & 419 & 99 \\
\hline $\mathrm{Ca}_{5}\left(\mathrm{PO}_{4}\right)_{3} \mathrm{~F}$ & & 1.7 & 0.12 & 5.0 & 5.0 & 0.88 & & & \\
\hline HYDROXYAPATITE & 502.311 & 390.4 & 159.60 & -6738.5 & -6337.1 & 1110.22 & 419 & 419 & 99 \\
\hline $\mathrm{Ce}_{5}\left(\mathrm{PO}_{4}\right)_{3} \mathrm{OH}$ & & 1.7 & 0.20 & 5.0 & 5.0 & 0.88 & & & \\
\hline STRENGITE & 186.849 & 171.3 & 64.50 & -1888.2 & -1657.5 & 290.37 & 100 & 419 & 100 \\
\hline $\mathrm{FePO}_{4} \cdot 2 \mathrm{H}_{2} \mathrm{O}$ & & 1.3 & 0.30 & 0.9 & 1.0 & 0.18 & & & \\
\hline PONELLITE & 200.016 & 122.6 & 47.00 & -1541.4 & -1434.7 & 251.34 & 431 & 419 & \\
\hline $\mathrm{CaNOO}_{4}$ & & 0.8 & 0.09 & 0.9 & 0.9 & 0.15 & & 12 & \\
\hline WLFENITE & 367.138 & 166.1 & 53.86 & -1051.9 & -951.2 & 166.65 & 320 & 419 & \\
\hline $\mathrm{PbHOO}_{4}$ & & 2.1 & 0.10 & 0.9 & 1.2 & 0.20 & & 89 & \\
\hline SCHEELITE & 287.926 & 126.4 & 47.05 & -1645.2 & -1538.0 & 269.51 & 232 & 419 & \\
\hline $\mathrm{CanOH}_{4}$ & & 0.8 & 0.09 & 0.9 & 0.9 & 0.16 & & 17 & \\
\hline FERBERITE & 303.695 & 131.8 & 40.38 & -1154.8 & -1054.0 & 184.64 & 430 & 419 & \\
\hline $\mathrm{FeLO}_{4}$ & & 1.7 & 0.05 & 8.5 & 8.5 & 1.49 & 265 & 7 & \\
\hline WUEBNERITE & 302.786 & 132.5 & 41.89 & -1337.6 & -1235.4 & 216.44 & 304 & 7 & \\
\hline $\mathrm{MnwO}_{4}$ & & 0.2 & 0.06 & 7.0 & 7.1 & 1.24 & & & \\
\hline STOLZITE & 455.048 & 168.2 & 54.10 & -1127.2 & -1026.0 & 179.74 & 428 & 89 & \\
\hline $\mathrm{PblN}_{4}$ & & 2.1 & 0.06 & 2.0 & 2.0 & 0.35 & & & \\
\hline SAMMARTIMITE & 313.238 & 119.3 & 39.79 & -1232.6 & -1123.7 & 196.86 & 260 & 266 & 260 \\
\hline $2 n \mathbf{n O}_{4}$ & & 0.3 & 0.04 & 1.3 & 1.4 & 0.25 & & & 266 \\
\hline
\end{tabular}


THERMOOYMAMIC PROPERTIES OF ORTHO AND RING STRUCTURE SILICATE MINERALS AT $298.15 \mathrm{~K}$

\begin{tabular}{|c|c|c|c|c|c|c|c|c|c|}
\hline \multirow[t]{2}{*}{ MANE AMD FORIULA } & \multirow{2}{*}{$\begin{array}{l}\text { MEIGHT } \\
\text { g }\end{array}$} & \multirow{2}{*}{$\begin{array}{c}\text { ENTROPY } \\
\mathrm{s}^{\bullet} \\
\mathrm{J} \cdot \mathrm{mol}^{-1} \cdot \mathrm{K}^{1}\end{array}$} & \multirow{2}{*}{$\begin{array}{l}\text { VOLUME } \\
\text { vo } \\
\mathrm{cm}^{3}\end{array}$} & \multirow{2}{*}{$\begin{array}{c}\text { ENTHALPY } \\
\Delta_{\mathbf{H}^{\circ}} \\
\mathrm{kJ} \cdot \mathrm{mol}^{-1}\end{array}$} & \multirow{2}{*}{$\begin{array}{c}\text { FREE } \\
\text { ENERGY } \\
\Delta_{\mathbf{F}^{\circ}} \\
\mathrm{kJ} \cdot \mathrm{mol}^{-1}\end{array}$} & \multirow[t]{2}{*}{ LOG(K) } & \multicolumn{3}{|c|}{ REFERENCES } \\
\hline & & & & & & & $\mathbf{s}$ & H/G & c \\
\hline TOPAZ & 184.043 & 105.4 & 51.53 & -3084.5 & -2910.6 & 509.9 & 24 & 23 & 24 \\
\hline $\mathrm{Al}_{2} \mathrm{SiO}_{4} \mathrm{~F}_{2}$ & & 0.2 & 0.03 & 4.7 & 4.7 & 0.8 & & 24 & \\
\hline KYAMITE & 162.046 & 82.8 & 44.15 & -2593.8 & -2443.1 & 428.0 & 355 & 177 & 177 \\
\hline $\mathrm{Al}_{2} \mathrm{SiO}_{5}$ & & 0.5 & 0.05 & 2.0 & 2.0 & 0.4 & 177 & 407 & 322 \\
\hline ANDALUSITE & 162.046 & 91.4 & 51.52 & -2589.9 & -2441.8 & 427.8 & 355 & 177 & 177 \\
\hline $\mathrm{Al}_{2} \mathrm{SiO}_{5}$ & & 0.5 & 0.05 & 2.0 & 2.0 & 0.4 & 177 & 407 & 322 \\
\hline SILLIMANITE & 162.046 & 95.4 & 49.86 & -2586.1 & -2439.1 & 427.3 & 355 & 177 & 177 \\
\hline $\mathrm{Al}_{2} \mathrm{SiO}_{5}$ & & 0.5 & 0.05 & 2.0 & 2.0 & 0.4 & 17 & 407 & 322 \\
\hline MLLITE & 426.052 & 275.0 & 134.55 & -6819.2 & -6441.8 & 1128.6 & 67 & 67 & 67 \\
\hline $\mathrm{Al}_{6} \mathrm{Si}_{2} \mathrm{O}_{13}$ & & 5.0 & 0.07 & 10.0 & 10.0 & 1.8 & 329 & 86 & 329 \\
\hline DUMORTIERITE & 565.938 & 334.9 & 168.4 & -9109.0 & -8568.2 & 1501.1 & 163 & 163 & 163 \\
\hline 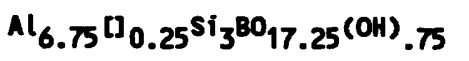 & & 5.0 & 0.4 & 20.0 & 20.0 & 3.5 & & & \\
\hline EUCLASE & 145.084 & 89.1 & 46.86 & -2532.9 & -2370.2 & 415.2 & 164 & 164 & 164 \\
\hline $\operatorname{BeAlsio} 4(\mathrm{OH})$ & & 0.4 & 0.06 & 3.0 & 3.0 & 0.5 & & 238 & \\
\hline PHENAKITE & 110.107 & 63.4 & 37.18 & -2143.1 & -2028.4 & 355.4 & 164 & 164 & 164 \\
\hline $\mathrm{Be}_{2} \mathrm{SiO}_{4}$ & & 0.3 & 0.02 & 4.0 & 4.0 & 0.7 & & 379 & \\
\hline BERYL & 537.502 & 346.7 & 203.3 & -9006.6 & -8500.5 & 1489.2 & 164 & 164 & 164 \\
\hline $\operatorname{Be}_{3} A l_{2}\left(S i_{6} O_{18}\right)$ & & 4.7 & 0.1 & 7.0 & 6.4 & 1.1 & & 238 & \\
\hline BERTRAMDITE & 238.230 & 172.1 & 91.8 & -4580.5 & -4295.1 & 752.5 & 164 & 164 & 164 \\
\hline $\mathrm{Be}_{4} \mathrm{Si}_{2} \mathrm{O}_{7}(\mathrm{OH})_{2}$ & & 0.8 & 0.1 & 5.5 & 5.5 & 1.0 & & 238 & \\
\hline EPIDOTE & 483.223 & 328.9 & 138.1 & & & & 147 & & 239 \\
\hline $\mathrm{Ce}_{2} \mathrm{Al}_{2} \mathrm{FeSi}_{3} \mathrm{O}_{12}$ (OH) & & 2.5 & 0.6 & & & & & & 243 \\
\hline LAUSOWITE & 314.238 & 230.0 & 101.32 & -4869.0 & -4512.9 & 790.6 & 335 & 68 & 335 \\
\hline $\mathrm{CaAl}_{2}\left[\mathrm{Si}_{2} \mathrm{O}_{7}(\mathrm{OH})_{2}\right] \mathrm{H}_{2} \mathrm{O}$ & & 2.1 & 0.12 & 2.1 & 2.1 & 0.4 & 68 & & \\
\hline GEHLENITE & 274.200 & 210.1 & 90.15 & -3985.0 & -3785.5 & 663.2 & 172 & 66 & 323 \\
\hline $\mathrm{Ca}_{2} \mathrm{Al}_{2} \mathrm{SiO}_{7}$ & & 0.6 & 0.02 & 5.0 & 5.0 & 0.9 & 420 & & \\
\hline ZOISITE & 454.357 & 295.9 & 136.5 & -6901.1 & -6504.5 & 1139.5 & 335 & 239 & 335 \\
\hline $\mathrm{Ca}_{2} \mathrm{Al}_{3} \mathrm{Si}_{3} \mathrm{O}_{12}(\mathrm{OH})$ & & 0.6 & 0.4 & 3.3 & 3.3 & 0.6 & & & \\
\hline GROSSULAR & 450.446 & 260.1 & 125.28 & -6640.0 & -6278.5 & 1099.9 & 157 & 68 & 258 \\
\hline $\mathrm{Co}_{3} \mathrm{Al}_{2} \mathrm{Si}_{3} \mathrm{O}_{12}$ & & 0.5 & 0.05 & 3.2 & 3.2 & 0.6 & 251 & 237 & 438 \\
\hline GLASS & 450.446 & $329.2+$ & 158.8 & & & & 345 & & \\
\hline $\mathrm{Co}_{3} \mathrm{Al}{ }_{2} \mathrm{Si}_{3} \mathrm{O}_{12}$ & & & 0.3 & & & & & & \\
\hline
\end{tabular}


THERMOOYMAMIC PROPERTIES OF ORTHO AND RING STRUCTURE SILICATE MINERALS AT 298.15 K

\begin{tabular}{|c|c|c|c|c|c|c|c|c|c|}
\hline MAME AMD FORMULA & WEIGHT & $\begin{array}{c}\text { ENTROPY } \\
S^{\circ} \\
J \cdot \mathrm{mol}^{-1} \cdot \mathrm{K}^{\prime}\end{array}$ & $\begin{array}{c}v^{0} \\
c m^{3}\end{array}$ & $\begin{array}{c}\text { ENTHALPY } \\
\Delta_{\mathrm{f}} \mathrm{H}^{\circ} \\
\mathrm{kJ} \cdot \mathrm{mol}^{-1}\end{array}$ & $\begin{array}{l}\text { FREE } \\
\text { ENERGY } \\
\text { ArG }^{\circ} \\
\mathrm{kJ} \cdot \mathrm{mol}^{-1}\end{array}$ & $\operatorname{LOG}(K)$ & RE & FEREN & ICES \\
\hline DATOLITE & 159.979 & 110.2 & 53.32 & -2477.8 & -2318.1 & 406.1 & 448 & 383 & 274 \\
\hline $\mathrm{CaB}\left(\mathrm{SiO}_{4}\right)(\mathrm{OH})$ & & 0.6 & 0.05 & 2.3 & 2.3 & 0.4 & & & 5 \\
\hline ILVAITE & 408.793 & 292.3 & 101.07 & -3692.8 & -3437.0 & 602.1 & 349 & 349 & 349 \\
\hline $\mathrm{CaFe}_{2} \mathrm{Fe}\left(\mathrm{Si}_{2} \mathrm{O}_{7}\right) \mathrm{O}(\mathrm{OH})$ & & 0.6 & 0.10 & 5.0 & 5.0 & 0.9 & & & \\
\hline AMDRNDITE & 508.177 & 316.4 & 132.04 & -5771.0 & -5427.0 & 950.8 & 348 & 281 & 348 \\
\hline $\mathrm{Ce}_{3} \mathrm{Fe}_{2} \mathrm{Si}_{3} \mathrm{O}_{12}$ & & 2.0 & 0.05 & 5.9 & 5.9 & 1.0 & & 242 & 244 \\
\hline MONTICELLITE & 156.466 & 108.1 & 51.3 & -2251.0 & -2132.8 & 373.6 & 385 & 49 & 385 \\
\hline $\mathrm{CengSiO}_{4}$ & & 0.3 & 0.1 & 3.0 & 3.1 & 0.5 & & 385 & \\
\hline AKERMANITE & 272.628 & 212.5 & 92.54 & -3864.8 & -3667.5 & 642.5 & 165 & 165 & 165 \\
\hline $\mathrm{Ca}_{2} \mathrm{MgSi}_{2} \mathrm{O}_{7}$ & & 0.4 & 0.02 & 2.0 & 2.0 & 0.4 & 427 & 49 & 323 \\
\hline MERWINITE & 328.705 & 253.1 & 98.5 & -4536.2 & -4307.7 & 754.7 & 427 & 49 & 323 \\
\hline $\mathrm{Ce}_{3} \mathrm{Mg}\left(\mathrm{SiO}_{4}\right)_{2}$ & & 2.1 & 0.1 & 3.0 & 3.0 & 0.5 & & & \\
\hline TITANITE (SPHENE) & 196.041 & 129.2 & 55.74 & -2596.6 & -2454.6 & 430.0 & 406 & 37 & 361 \\
\hline CaTiSiO $_{5}$ & & 0.8 & 0.07 & $\mathbf{3 . 0}$ & 3.2 & 0.6 & & 188 & \\
\hline LARNITE & 172.239 & 127.6 & 51.60 & -2306.7 & -2191.2 & 383.9 & 225 & 149 & 84 \\
\hline$a-\mathrm{Ca}_{2} \mathrm{SiO}_{4}$ & & 0.8 & 0.30 & 1.5 & 1.5 & 0.3 & & & 149 \\
\hline CALCIO-OLIVIME & 172.239 & 120.5 & 58.01 & -2316.5 & -2198.9 & 385.2 & 149 & 149 & 149 \\
\hline$\gamma-\mathrm{Ca}_{2} \mathrm{SiO}_{4}$ & & 0.8 & 0.04 & 2.5 & 2.5 & 0.4 & 405 & & 84 \\
\hline MATURITE (ALITE) & 228.317 & 168.6 & 72.74 & -2933.1 & -2786.5 & 488.2 & 149 & 410 & 149 \\
\hline $\mathrm{Ca}_{3} \mathrm{SiO}_{5}$ & & 0.3 & 0.05 & 2.5 & 2.5 & 0.4 & 405 & & \\
\hline RANKINITE & 288.401 & 210.6 & 96.51 & -3949.0 & -3748.1 & 656.6 & 149 & 410 & 149 \\
\hline $\mathrm{Ce}_{3} \mathrm{Si}_{2} \mathrm{O}_{7}$ & & 2.9 & 0.05 & 10.0 & 10.0 & 1.8 & & & \\
\hline ROSENHAHNITE & 366.500 & 281.8 & 126.46 & -5198.1 & -4882.1 & 855.3 & 68 & 68 & 68 \\
\hline $\mathrm{Ce}_{3} \mathrm{Si}_{3} \mathrm{O}_{8}(\mathrm{OH})_{2}$ & & $\mathbf{3 . 0}$ & 0.05 & 3.0 & 3.0 & 0.5 & & & \\
\hline SPURRITE & 444.565 & 331.0 & 146.97 & -5840.2 & -5525.6 & 968.0 & 450 & 188 & 450 \\
\hline $\mathrm{Ca}_{5}\left(\mathrm{SiO}_{4}\right)_{2} \mathrm{CO}_{3}$ & & 2.0 & 0.30 & 5.7 & 5.9 & 1.0 & 188 & 410 & \\
\hline TILLEYITE & 488.575 & 394.0 & 170.5 & -6372.2 & -6013.5 & 1053.5 & 450 & 188 & 450 \\
\hline $\mathrm{Ce}_{5} \mathrm{Si}_{2} \mathrm{O}_{7}\left(\mathrm{CO}_{3}\right)_{2}$ & & 4.0 & 0.3 & 5.7 & 6.0 & 1.1 & & 410 & \\
\hline COBALT-OLIVINE & 209.950 & 142.6 & 44.49 & -1412.0 & -1308.7 & 229.3 & 364 & 215 & 363 \\
\hline $\mathrm{Co}_{2} \mathrm{SiO}_{4}$ & & 0.2 & 0.01 & 2.0 & 2.0 & 0.3 & & 308 & \\
\hline FAYALITE & 203.77 & 151.0 & 46.31 & -1478.2 & -1379.1 & 241.6 & 350 & 350 & 350 \\
\hline $\mathrm{Fe}_{2} \mathrm{SiO}_{4}$ & & 0.2 & 0.01 & 1.3 & 1.3 & 0.2 & & 309 & 314 \\
\hline
\end{tabular}


THERMCOYMAHIC PROPERTIES OF ORTHO AND RING STRUCTURE SILICATE MINERALS AT $298.15 \mathrm{~K}$

\begin{tabular}{|c|c|c|c|c|c|c|c|c|c|}
\hline \multirow[t]{2}{*}{ MANE ANO FORRULA } & \multirow{2}{*}{$\begin{array}{l}\text { MEIGHT } \\
\text { g }\end{array}$} & \multirow{2}{*}{$\begin{array}{c}\text { ENTROPY } \\
\mathrm{S}^{\circ} \\
J \cdot \mathrm{mol}^{-1} \cdot \mathrm{K}^{\prime}\end{array}$} & \multirow{2}{*}{$\begin{array}{l}\text { VOLUME } \\
\qquad \begin{array}{l}\mathrm{v}^{0} \\
\mathrm{~cm}^{3}\end{array}\end{array}$} & \multirow{2}{*}{$\begin{array}{l}\text { ENTHALPY } \\
\Delta_{1} H^{\circ} \\
\mathrm{kJ} \cdot \mathrm{mol}^{-1}\end{array}$} & \multirow{2}{*}{$\begin{array}{c}\text { FREE } \\
\text { ENERGY } \\
\Delta_{r} G^{\circ} \\
\mathrm{kJ} \cdot \mathrm{mol}^{-1}\end{array}$} & \multirow[t]{2}{*}{$\operatorname{LOG}(K)$} & \multicolumn{3}{|c|}{ REFERENCES } \\
\hline & & & & & & & $\mathbf{s}$ & H/G & C \\
\hline ALMUDINE & 497.753 & 342.6 & 115.32 & -5264.7 & -4942.0 & 865.8 & 9 & 69 & 447 \\
\hline $\mathrm{Fe}_{3} \mathrm{Al}_{2} \mathrm{Si}_{3} \mathrm{O}_{12}$ & & 1.4 & 0.04 & 3.0 & 3.3 & 0.6 & 277 & 445 & 151 \\
\hline STAUROLITE & 1703.727 & 985.0 & 445.9 & & & & 172 & & 172 \\
\hline $\mathrm{Fe}_{4} \mathrm{Al} \mathrm{l}_{18} \mathrm{Si}_{8} \mathrm{O}_{46}(\mathrm{OH})_{2}$ & & 4.0 & 1.7 & & & & & & \\
\hline OSUMILITE & 1001.468 & & 379.2 & & & & & & 180 \\
\hline 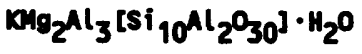 & & & 0.3 & & & & & & \\
\hline CORDIERITE & 584.953 & 407.2 & 233.22 & -9161.5 & -8651.1 & 1515.6 & 427 & 297 & 323 \\
\hline $\operatorname{Mg}_{2} A I_{3}\left(A l s i_{5} 0_{18}\right)$ & & 3.8 & 0.22 & 5.9 & 5.9 & 1.0 & & & \\
\hline SAPPHIRINE & 344.616 & 197.5 & 98.9 & & & & 186 & & \\
\hline $\mathrm{Hg}_{2} \mathrm{Al} \mathbf{4}_{6} \mathrm{O}\left(\mathrm{SiO}_{4}\right)$ & & 2.0 & 0.7 & & & & & & \\
\hline FORSTERITE & 140.693 & 94.1 & 43.65 & -2173.0 & -2053.6 & 359.8 & 364 & 364 & 364 \\
\hline $\mathrm{Mg}_{2} \mathrm{SiO}_{4}$ & & 0.1 & 0.03 & 2.0 & 2.0 & 0.4 & & 240 & 119 \\
\hline PYROPE & 403.127 & 266.3 & 113.12 & -6285.0 & -5934.5 & 1039.7 & 157 & 188 & 453 \\
\hline $\mathrm{Ag}_{3} \mathrm{Al} \mathrm{Si}_{3} \mathrm{O}_{12}$ & & 0.8 & 0.15 & 4.0 & 4.0 & 0.7 & & 298 & \\
\hline GLASS & 403.127 & 346.3 & 146.1 & -6163.0 & -5836.3 & 1022.5 & 402 & 402 & 402 \\
\hline $\mathrm{Ho}_{3} \mathrm{Al} \mathrm{Si}_{3} \mathrm{O}_{12}$ & & 12.0 & 0.2 & 15.0 & 15.0 & 2.6 & & & \\
\hline PYROPE-GROSSULAR s8. & 422.055 & $268.3+$ & 118.35 & & & & 157 & 298 & \\
\hline$\left(\mathrm{Hg}_{1.8} \mathrm{Ca}_{1.2}\right) \mathrm{Al}_{2} \mathrm{Si}_{3} \mathrm{O}_{12}$ & & 0.5 & 0.05 & & & & & & \\
\hline GLASS & 426.787 & $311.8+$ & 152.5 & & & & 345 & & \\
\hline$\left(\mathrm{Hg}_{1.5} \mathrm{Ca}_{1.5}\right) \mathrm{Al}_{2} \mathrm{Si}_{3} \mathrm{O}_{12}$ & & 0.6 & 0.2 & & & & & & \\
\hline TEPHROITE & 201.959 & 155.9 & 48.99 & -1731.5 & -1631.0 & 285.7 & 364 & 364 & 364 \\
\hline $\mathrm{m}_{2} \mathrm{SiO}_{4}$ & & 0.5 & 0.03 & 3.0 & 3.0 & 0.5 & & & \\
\hline LIEBEMBERGITE & 209.463 & 128.1 & 42.57 & -1396.5 & -1288.9 & 225.8 & 363 & 363 & 363 \\
\hline $\mathrm{Ni}_{2} \mathrm{SiO}_{4}$ & & 0.2 & 0.02 & 3.0 & 3.0 & 0.5 & & 308 & \\
\hline $\mathrm{Ni}_{2} \mathrm{SiO}_{4}$-SPIMEL & 209.463 & 124.1 & 39.81 & -1389.7 & -1280.9 & 224.4 & 363 & 363 & 423 \\
\hline $\mathrm{Ni}_{2} \mathrm{SiO}_{4}$ & & 0.4 & 0.01 & 3.5 & 3.5 & 0.6 & & & \\
\hline MILLEMITE & 222.863 & 131.4 & 52.42 & -1636.7 & -1523.1 & 266.8 & 405 & 223 & \\
\hline $2 n_{2} \mathrm{SiO}_{4}$ & & 0.8 & 0.08 & 5.0 & 5.0 & 0.9 & & & \\
\hline ZIRCOW & 183.307 & 84.0 & 39.26 & -2034.2 & -1919.7 & 336.3 & 361 & 101 & 361 \\
\hline $\mathrm{ZrSiO}_{4}$ & & 1.3 & 0.07 & 3.1 & 3.1 & 0.5 & & 379 & \\
\hline
\end{tabular}


THERMOOYMAMIC PROPERTIES OF CHAin AND BAND STRUCTURE SILICATE MIMERALS AT $298.15 \mathrm{~K}$

\begin{tabular}{|c|c|c|c|c|c|c|c|c|c|}
\hline MAME ANO FORMULA & $\begin{array}{l}\text { WEIGHT } \\
g\end{array}$ & $\begin{array}{c}\text { ENTROPY } \\
\mathbf{s}^{\bullet} \\
\mathrm{J} \cdot \mathrm{mol}^{-1} \cdot \mathrm{K}^{\prime}\end{array}$ & $\begin{array}{l}\text { VOLUANE } \\
\qquad \begin{array}{l}V^{0} \\
\mathrm{~cm}^{3}\end{array}\end{array}$ & $\begin{array}{l}\text { ENTHALPY } \\
\mathrm{AJ}_{\mathrm{B}} \mathrm{H}^{\bullet} \\
\mathrm{mol}^{-1}\end{array}$ & 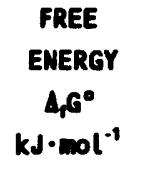 & $\operatorname{LOG}(K)$ & RE & FEREMC & ICES \\
\hline WOLLASTONITE & 116.162 & 81.7 & 39.90 & -1634.8 & -1549.0 & 271.4 & 259 & 348 & 389 \\
\hline $\mathrm{CaSiO}_{3}$ & & 0.1 & 0.03 & 1.4 & 1.4 & 0.2 & 257 & 149 & 344 \\
\hline PSELDONOLLASTONITE & 116.162 & 87.2 & 40.08 & -1627.6 & -1543.5 & 270.4 & 149 & 344 & 344 \\
\hline $\mathrm{CaSiO}_{3}$ & & 0.9 & 0.14 & 1.4 & 1.4 & 0.2 & 344 & 149 & 149 \\
\hline GLASS & 116.162 & 94.8 & 40.13 & -1608.7 & -1526.8 & 267.5 & 344 & 344 & 344 \\
\hline $\mathrm{CaSiO}_{3}$ & & 3.0 & 0.15 & 3.0 & 3.0 & 0.5 & & & \\
\hline Ca-Al pYroxene & 218.123 & 141.0 & 63.57 & -3306.3 & -3129.6 & 548.3 & 154 & 68 & 154 \\
\hline $\mathrm{CaAl}_{2} \mathrm{SiO}_{6}$ & & 2.0 & 0.01 & 2.5 & 2.6 & 0.5 & & 65 & 403 \\
\hline GLASS & 218.123 & $155.3+$ & 78.13 & & & & 154 & & \\
\hline $\mathrm{Canl}_{2} \mathrm{SiO}_{6}$ & & 0.3 & 0.08 & & & & & & \\
\hline FERROBUSTAMITE & 248.092 & 180.5 & 73.00 & & & & 156 & & 156 \\
\hline Cafesi $_{2} \mathrm{O}_{6}$ & & 0.3 & 0.02 & & & & & & \\
\hline HEDENBERGITE & 248.092 & 174.2 & 67.95 & -2839.9 & -2676.3 & 468.9 & 156 & 281 & 156 \\
\hline CaFesi $_{2}{ }_{6} 6$ & & 0.3 & 0.01 & $\mathbf{3 . 0}$ & 3.0 & 0.5 & & 156 & 29 \\
\hline GLASS & 248.092 & $185.7+$ & & & & & 156 & & \\
\hline $\operatorname{CaFeSi}_{2} \mathrm{O}_{6}$ & & 0.4 & & & & & & & \\
\hline DIOPSIDE & 216.550 & 142.7 & 66.09 & -3201.5 & -3026.8 & 530.3 & 259 & 165 & 257 \\
\hline $\operatorname{CalgSi}_{2} \mathrm{O}_{6}$ & & 0.3 & 0.10 & 2.0 & 2.0 & 0.4 & & & \\
\hline GLASS & 216.550 & 166.0 & 76.09 & -3156.1 & -2988.4 & 523.5 & 343 & 340 & 343 \\
\hline $\mathrm{CaMgSi}_{2} \mathrm{O}_{6}$ & & 3.0 & 0.20 & 5.0 & 5.1 & 0.9 & & & 340 \\
\hline FERROSILITE & 131.931 & 94.6 & 33.00 & -1195.2 & -1118.0 & 195.9 & 41 & 41 & 188 \\
\hline $\mathrm{FeSiO}_{3}$ & & 0.3 & 0.02 & 3.0 & 3.0 & 0.5 & & 291 & 423 \\
\hline Q-SPODUMENE & 186.090 & 129.3 & 58.37 & -3053.5 & -2880.2 & 504.6 & 327 & 171 & 327 \\
\hline LiAlsi ${ }_{2} \mathrm{O}_{6}$ & & 0.8 & 0.02 & 2.8 & 3.0 & 0.5 & & 20 & 28 \\
\hline B-SPCOUNENE & 186.090 & 154.4 & 78.25 & -3025.3 & -2859.5 & 501.0 & 327 & 171 & 327 \\
\hline LiAlsi ${ }_{2} \mathrm{O}_{6}$ & & 1.2 & 0.04 & 2.8 & 3.0 & 0.5 & & 20 & \\
\hline EMSTATITE & 100.389 & 66.3 & 31.31 & -1545.6 & -1458.3 & 255.5 & 259 & 37 & 257 \\
\hline $\mathrm{MgSiO}_{3}$ & & 0.1 & 0.02 & 1.5 & 1.6 & 0.3 & & 408 & 188 \\
\hline GLASS & 100.389 & 74.1 & 36.60 & -1542.5 & -1457.5 & 255.3 & 345 & 345 & \\
\hline $\mathrm{MgSiO}_{3}$ & & 0.2 & 0.25 & 2.0 & 2.0 & 0.4 & & & \\
\hline CLINOENSTATITE & 100.389 & 67.9 & 31.28 & -1545.0 & -1458.1 & 255.5 & 361 & 296 & 361 \\
\hline $\mathrm{MgSiO}_{3}$ & & 0.4 & 0.02 & 1.5 & 1.6 & 0.3 & & 311 & \\
\hline
\end{tabular}


THERMODYMAMIC PROPERTIES OF CHAIN AND BAND STRUCTURE SILICATE MINERALS AT $298.15 \mathrm{~K}$

\begin{tabular}{|c|c|c|c|c|c|c|c|c|c|}
\hline \multirow[t]{2}{*}{ MAME AND FORHULA } & \multirow{2}{*}{$\begin{array}{l}\text { WEIGHT } \\
\text { g }\end{array}$} & \multirow{2}{*}{$\begin{array}{c}\text { ENTROPY } \\
\mathrm{S}^{\circ} \\
\mathrm{J} \cdot \mathrm{mol}^{-1} \cdot \mathrm{K}^{-1}\end{array}$} & \multirow{2}{*}{$\begin{array}{l}\text { VOLUME } \\
\qquad \begin{array}{l}\mathrm{v}^{0} \\
\mathrm{~cm}^{3}\end{array}\end{array}$} & \multirow{2}{*}{$\begin{array}{c}\text { ENTHALPY } \\
\mathrm{A}_{1} \mathrm{H}^{\circ} \\
\mathrm{kJ} \cdot \mathrm{mol}^{-1}\end{array}$} & \multirow{2}{*}{$\begin{array}{c}\text { FREE } \\
\text { ENERGY } \\
\Delta_{\mathrm{r}} \mathrm{G}^{\circ} \\
\mathrm{kJ} \cdot \mathrm{mol}^{-1}\end{array}$} & \multirow[t]{2}{*}{$\operatorname{LOG}(K)$} & \multicolumn{3}{|c|}{ REFEREMCES } \\
\hline & & & & & & & $\mathbf{s}$ & $H / G$ & c \\
\hline $\mathrm{MgSiO}_{3}$-PEROVSKITE & 100.389 & 63.6 & 24.50 & -1445.1 & -1357.0 & 237.7 & 105 & 105 & \\
\hline $\mathrm{MgSiO}_{3}$ & & 3.0 & 0.05 & 5.0 & 5.0 & 0.9 & & & \\
\hline $\mathrm{MgSiO}_{3}$-ILMENITE & 100.389 & 60.4 & 26.36 & -1486.6 & -1397.5 & 244.8 & 105 & 13 & 13 \\
\hline $\mathrm{MgSiO}_{3}$ & & 3.0 & 0.02 & 5.0 & 5.0 & 0.9 & & 288 & \\
\hline HYPERSTHEME & 105.120 & 69.0 & 31.53 & & & & 367 & & \\
\hline$\left(\mathrm{Mg} .85 \mathrm{Fe}_{.15}\right)^{\mathrm{SiO}_{3}}$ & & 0.2 & 0.05 & & & & & & \\
\hline RHCDONITE & 131.022 & 100.5 & 34.94 & -1321.6 & -1244.7 & 218.0 & 367 & 367 & 390 \\
\hline $\mathrm{MnSiO}_{3}$ & & 1.0 & 0.05 & 2.0 & 2.2 & 0.4 & & 289 & 224 \\
\hline PYROXMANGITE & 131.022 & 99.4 & 34.72 & -1322.3 & -1245.0 & 218.1 & 367 & 367 & \\
\hline Mnsio $_{3}$ & & 2.0 & 0.02 & 2.0 & 2.4 & 0.4 & & 32 & \\
\hline JADEITE & 202.139 & 133.5 & 60.40 & -3029.3 & -2850.6 & 499.4 & 220 & 166 & 220 \\
\hline $\operatorname{MaAlsi}{ }_{2} \mathrm{O}_{6}$ & & 1.3 & 0.10 & 3.6 & 4.0 & 0.7 & & 184 & \\
\hline GLASS & 202.139 & 170.5 & & & & & 345 & & \\
\hline $\operatorname{MaAlsi} \mathrm{O}_{6}$ & & 0.4 & & & & & & & \\
\hline ACMITE & 231.004 & 170.6 & 64.60 & -2584.5 & -2417.2 & 423.5 & 248 & 31 & 31 \\
\hline NaFeSi $_{2} \mathrm{O}_{6}$ & & 0.8 & 0.11 & 4.0 & 4.2 & 0.7 & 33 & 188 & \\
\hline TREMOL ITE & 812.366 & 548.9 & 272.90 & -12303.0 & -11574.6 & 2027.8 & 370 & 205 & 257 \\
\hline $\mathrm{Ca}_{2} \mathrm{Mg}_{5} \mathrm{Si}_{8} \mathrm{O}_{22}(\mathrm{OH})_{2}$ & & 1.3 & 0.73 & 7.0 & 7.0 & 1.2 & & 426 & \\
\hline GRUNERITE & 1001.614 & 725.0 & 278.7 & -9623.0 & -8964.8 & 1570.6 & 264 & 264 & 252 \\
\hline $\mathrm{Fe}_{7} \mathrm{Si}_{8} \mathrm{O}_{22}(\mathrm{OH})_{2}$ & & 7.0 & 0.5 & 10.0 & 10.2 & 1.8 & 252 & 8 & 34 \\
\hline ANTHOPHYLLITE & 780.820 & 534.5 & 265.4 & -12070.0 & -11343.4 & 1987.3 & 162 & 162 & 162 \\
\hline $\mathrm{Mg}_{7} \mathrm{Si}_{8} \mathrm{O}_{22}(\mathrm{OH})_{2}$ & & 3.5 & 0.4 & $\mathbf{8 . 0}$ & 8.5 & 1.5 & & 70 & \\
\hline RIEBECKITE & 935.900 & 691.0 & 274.7 & & & & 450 & & \\
\hline $\mathrm{Me}_{2} \mathrm{Fe}_{3} \mathrm{Fe}_{2} \mathrm{Si}_{8} \mathrm{O}_{22}(\mathrm{OH})_{2}$ & & 6.0 & 0.9 & & & & & & \\
\hline GLAUCOPHANE & 783.543 & 541.2 & 262.1 & -11964.0 & -11230.8 & 1967.6 & 362 & 188 & 185 \\
\hline $\mathrm{Na}_{2} \mathrm{Mg}_{3} \mathrm{Al}{ }_{2} \mathrm{Si}_{8} \mathrm{O}_{22}(\mathrm{OH})_{2}$ & & 3.0 & 0.2 & 9.0 & 10.0 & 1.8 & & & 118 \\
\hline PARGASITE & 835.825 & 582.0 & 272.0 & -12719.8 & -11981.5 & 2099.1 & 433 & 188 & \\
\hline $\mathrm{NaCa}_{2} \mathrm{Mg}_{4} \mathrm{Al}\left(\mathrm{Al}_{2} \mathrm{Si}_{6}\right)_{22}(\mathrm{OH})_{2}$ & & 4.0 & 0.2 & 22.0 & 19.0 & 3.3 & 186 & 433 & \\
\hline FLUOR-PARGASI TE & 839.807 & 583.0 & 270.6 & -12800.5 & -12102.2 & 2120.2 & 450 & 434 & \\
\hline $\mathrm{NaCa}_{2} \mathrm{Mg}_{4} \mathrm{Al}\left(\mathrm{Al}_{2} \mathrm{Si}_{6}\right) \mathrm{O}_{22} \mathrm{~F}_{2}$ & & 5.0 & 0.2 & 14.0 & 14.0 & 2.5 & 434 & & \\
\hline
\end{tabular}


THERMOOYMAMIC PROPERTIES OF FRAMEWORK STRUCTURE SILICATE MINERALS AT $298.15 \mathrm{~K}$

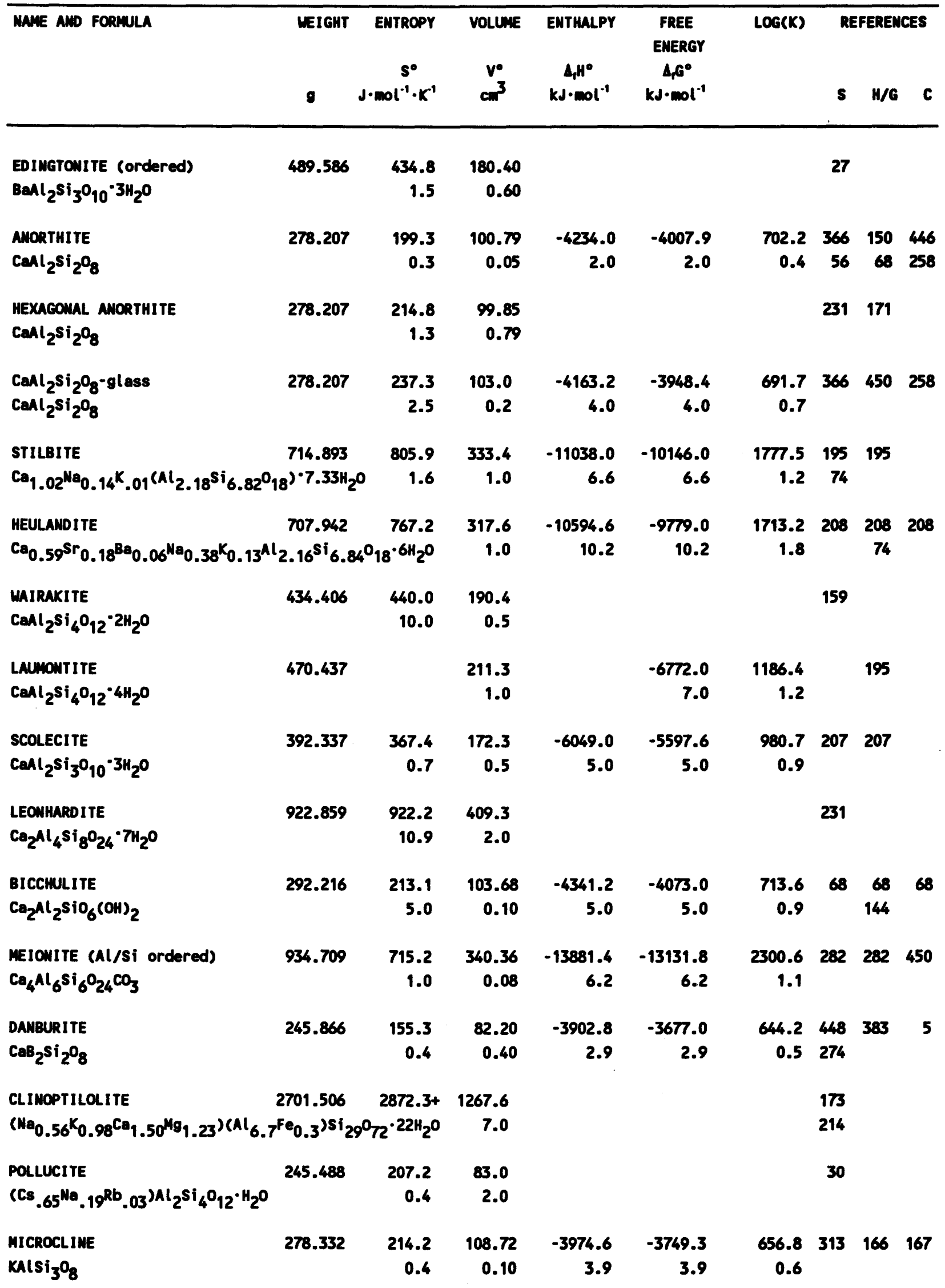


THERMOOYMAMIC PROPERTIES OF FRAMELORK STRUCTURE SILICATE MINERALS AT $298.15 \mathrm{~K}$

\begin{tabular}{|c|c|c|c|c|c|c|c|c|c|}
\hline \multirow[t]{2}{*}{ MANE AMD FORMULA } & \multirow{2}{*}{$\begin{array}{l}\text { LEIGKT } \\
\text { e }\end{array}$} & \multirow{2}{*}{$\begin{array}{c}\text { ENTROPY } \\
S^{\circ} \\
d \cdot \operatorname{mol}^{-1} \cdot K^{\prime}\end{array}$} & \multirow{2}{*}{$\begin{array}{l}\text { VOLUnE } \\
\qquad \begin{array}{c}v^{\circ} \\
\mathrm{cm}^{3}\end{array}\end{array}$} & \multirow{2}{*}{$\begin{array}{l}\text { ENTHALPY } \\
\qquad \begin{array}{c}\Delta_{\mathrm{p}} \mathrm{H}^{\bullet} \\
\mathrm{kJ} \cdot \mathrm{mol}^{-1}\end{array}\end{array}$} & \multirow{2}{*}{$\begin{array}{c}\text { FREE } \\
\text { ENERGY } \\
A_{\mathbf{r}} \mathrm{G}^{\circ} \\
\mathbf{k J} \cdot \mathbf{m o l}^{-1}\end{array}$} & \multirow[t]{2}{*}{$\operatorname{LOG}(K)$} & \multicolumn{3}{|c|}{ REFERENCES } \\
\hline & & & & & & & $\mathbf{s}$ & $M / 6$ & C \\
\hline SANIDINE & 278.332 & 232.8 & 109.05 & -3965.6 & -3745.8 & 656.2 & 313 & 166 & 167 \\
\hline $\mathrm{Kalsi} \mathbf{3}_{8}$ & & 0.5 & 0.10 & 4.1 & 4.1 & 0.7 & 155 & 194 & \\
\hline GLASS & 278.332 & 261.6 & 116.50 & -3920.8 & -3709.6 & 649.9 & 366 & 166 & 258 \\
\hline $\mathrm{KAlsi} \mathbf{3}_{\mathbf{8}}$ & & 1.8 & 1.00 & 4.2 & 4.2 & 0.6 & & 421 & \\
\hline KALIOPHILLITE (KALSILITE) & 158.163 & 133.3 & 59.89 & -2124.7 & -2008.8 & 351.9 & 220 & 188 & 317 \\
\hline $\mathrm{KAlsiO}_{4}$ & & 1.2 & 0.05 & 3.1 & 3.1 & 0.5 & & & \\
\hline LEUCITE & 218.247 & 200.2 & 88.27 & -3037.8 & -2875.1 & 503.7 & 220 & 188 & 317 \\
\hline $\mathrm{kAlsi} \mathrm{i}_{6}$ & & 1.7 & 0.05 & 2.7 & 2.7 & 0.5 & & & 263 \\
\hline EUCRYPTITE & 126.006 & 103.8 & 53.63 & -2123.3 & -2009.2 & 352.0 & 327 & 171 & 327 \\
\hline $\operatorname{LiAlSiO}_{4}$ & & 0.8 & 0.08 & 2.0 & 2.0 & 0.4 & & 20 & \\
\hline PETALITE & 306.259 & 233.2 & 128.40 & -4886.5 & -4610.7 & 807.8 & 180 & 180 & 180 \\
\hline $\operatorname{LiAlsi}{ }_{4} \mathrm{O}_{10}$ & & 0.6 & 0.50 & 6.3 & 6.3 & 1.1 & 35 & 28 & 35 \\
\hline ALBITE & 262.223 & 207.4 & 100.07 & -3935.0 & -3711.6 & 650.2 & 313 & 166 & 167 \\
\hline NaAlsi ${ }_{3} \mathrm{O}_{8}$ & & 0.4 & 0.13 & 2.6 & 2.6 & 0.5 & 123 & 171 & 220 \\
\hline AMALBITE & 262.223 & 225.6 & 100.43 & -3923.6 & -3705.6 & 649.2 & 313 & 166 & 167 \\
\hline $\operatorname{MaAlsi}{ }_{3} \mathrm{O}_{8}$ & & 0.4 & 0.09 & 2.6 & 2.6 & 0.5 & 155 & 171 & \\
\hline GLASS & 262.223 & 251.9 & 110.10 & -3875.5 & -3665.3 & 642.1 & 366 & 421 & 258 \\
\hline $\operatorname{Manlsi}{ }_{3} \mathrm{O}_{8}$ & & 1.5 & 0.20 & 3.7 & 3.7 & 0.6 & & & \\
\hline MA-K FELDSPAR SS. & 264.639 & 231.3 & 102.18 & -3932.1 & -3715.2 & 650.9 & 155 & 155 & \\
\hline$\left(\mathrm{Na}_{0.85} \mathrm{~K}_{0.15}\right)^{\mathrm{Alsi} \mathrm{I}_{3} \mathrm{O}_{8}}$ & & 0.4 & 0.10 & 4.0 & 4.0 & 0.7 & & & \\
\hline MA-K FELDSPAR SS. & 269.472 & 237.0 & 105.22 & -3945.1 & -3728.7 & 653.2 & 155 & 155 & \\
\hline$\left(\mathrm{Na}_{0.55^{-K_{0}}}{ }_{0.45}\right) \mathrm{Alsi}_{3} \mathrm{O}_{8}$ & & 0.4 & 0.10 & 4.0 & 4.0 & 0.7 & & & \\
\hline MA-K FELDSPAR SS. & 274.304 & 237.4 & 107.37 & -3954.5 & -3737.1 & 654.7 & 155 & 155 & \\
\hline$\left(\mathrm{Na}_{0.25} \mathrm{~K}_{0.75}\right) \mathrm{Alsi} \mathrm{i}_{3} \mathrm{O}_{8}$ & & 0.4 & 0.10 & 4.0 & 4.0 & 0.7 & & & \\
\hline MEPHELIME & 142.054 & 124.4 & 54.19 & -2090.4 & -1975.8 & 346.1 & 220 & 188 & 220 \\
\hline $\mathrm{NaAlSiO}_{4}$ & & 1.3 & 0.06 & 3.9 & 3.9 & 0.7 & & & 183 \\
\hline CARNEGIEITE & 142.054 & 118.7 & 56.03 & -2104.3 & -1988.0 & 348.3 & 346 & 450 & 220 \\
\hline $\mathrm{NaAlSiO}_{4}$ & & 0.3 & 0.02 & 4.0 & 4.0 & 0.7 & & & \\
\hline GLASS & 142.054 & 134.5 & 56.86 & -2089.0 & -1977.4 & 346.4 & 346 & 346 & 346 \\
\hline $\mathrm{NaAlsiO}_{4}$ & & 0.5 & 0.06 & 4.0 & 4.0 & 0.7 & & & \\
\hline AWALCIME & 220.154 & 227.7 & 97.4 & -3310.1 & -3090.0 & 541.3 & 206 & 206 & 206 \\
\hline $\operatorname{MaAlsi} \mathrm{O}_{6} \cdot \mathrm{H}_{2} \mathrm{O}$ & & 0.3 & 0.2 & $\mathbf{3 . 3}$ & 3.3 & 0.6 & & & \\
\hline
\end{tabular}


TMERHODYMAIC PROPERTIES OF FRAMELORK STRUCTURE SILICATE MINERALS AT $298.15 \mathrm{~K}$

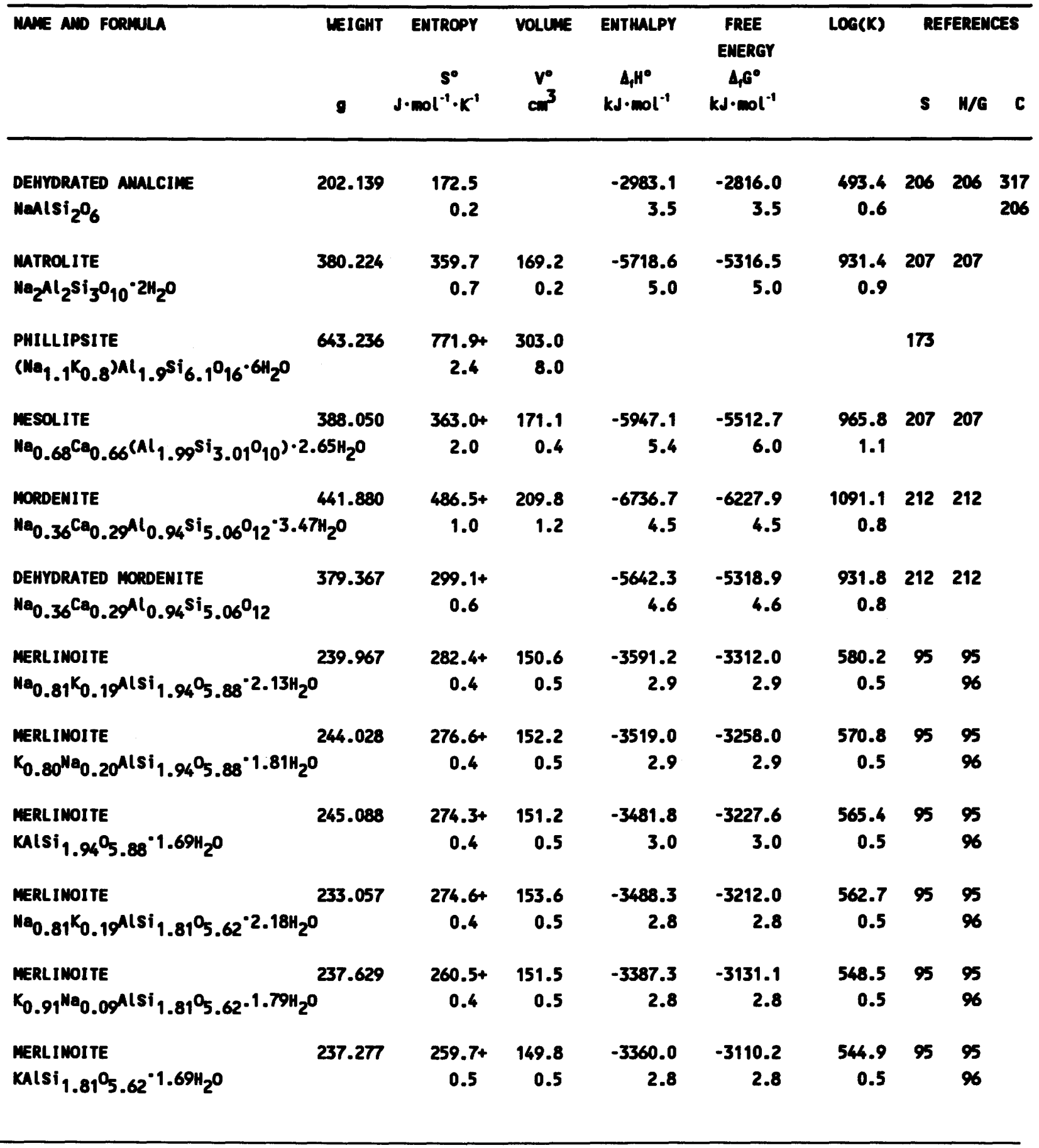


THERMOOYMAMIC PROPERTIES OF SHEET STRUCTURE SILICATE MIMERALS AT $298.15 \mathrm{~K}$

\begin{tabular}{|c|c|c|c|c|c|c|c|c|c|}
\hline MANE AND FORMULA & MEIGHT & $\begin{array}{c}\text { ENTROPY } \\
S^{\bullet} \\
J \cdot m \mathrm{ll}^{-1} \cdot \mathrm{K}^{\prime}\end{array}$ & $\begin{array}{l}\text { VOLUNE } \\
\qquad \begin{array}{c}v^{\circ} \\
c^{3}\end{array}\end{array}$ & $\begin{array}{l}\text { ENTHALPY } \\
\qquad \begin{array}{l}A_{1} H^{\circ} \\
\mathrm{kJ} \cdot \mathrm{mol}^{-1}\end{array}\end{array}$ & $\begin{array}{c}\text { FREE } \\
\text { ENERGY } \\
A_{\text {f }}{ }^{\circ} \\
\text { kJ-mol }\end{array}$ & $\operatorname{LOG}(K)$ & RE & FEREM & ICES \\
\hline DICKITE & 258.160 & 197.1 & 98.56 & -4118.5 & -3796.0 & 665.0 & 230 & 171 & 149 \\
\hline $\mathrm{Al}_{2} \mathrm{Si}_{2} \mathrm{O}_{5}(\mathrm{OH})_{4}$ & & 1.3 & 0.04 & 2.0 & 2.1 & 0.4 & & 149 & \\
\hline KAOLINITE & 258.160 & 200.4 & 99.34 & -6119.0 & -3797.5 & 665.3 & 358 & 179 & 179 \\
\hline$A l_{2} \mathrm{Si}_{2} \mathrm{O}_{5}(\mathrm{OH})_{4}$ & & 0.5 & 0.01 & 1.5 & 1.5 & 0.3 & 198 & 68 & \\
\hline HALLOYSITE & 258.160 & 203.0 & & -4101.5 & -3780.7 & 662.4 & 230 & 171 & \\
\hline $\mathrm{Al}_{2} \mathrm{Si}_{2} \mathrm{O}_{5}(\mathrm{OH})_{4}$ & & 1.3 & & 10.0 & 10.0 & 1.8 & & & \\
\hline PYROPHYLLITE & 360.314 & 239.4 & 128.10 & -5640.0 & -5266.2 & 922.6 & 365 & 258 & 258 \\
\hline $\mathrm{Al}_{2} \mathrm{Si}_{4} \mathrm{O}_{10}(\mathrm{OH})_{2}$ & & 0.4 & 0.08 & 1.5 & 1.5 & 0.3 & & 68 & \\
\hline ILLITE & 1553.665 & 1104.2 & 577.00 & & & & 365 & & \\
\hline$K_{3}\left(A l_{7}-M g\right)\left(A l_{2} \mathrm{Si}_{14}\right) \mathrm{O}_{40}(\mathrm{OH})_{8}$ & & 2.5 & 10.00 & & & & & & \\
\hline MARGARITE & 398.184 & 263.6 & 129.63 & -6244.0 & -5858.9 & 1026.4 & 335 & 68 & 335 \\
\hline $\left.\mathrm{CaAl}_{2} \mathrm{CAl}_{2} \mathrm{Si}_{2} \mathrm{O}_{10}\right](\mathrm{OH})_{2}$ & & 0.3 & 0.06 & 2.6 & 2.6 & 0.5 & & & \\
\hline PREHNITE & 412.384 & 292.8 & 141.10 & -6202.6 & -5824.7 & 1020.4 & 335 & 68 & 335 \\
\hline $\mathrm{Ca}_{2} \mathrm{Al}$ Calsi ${ }_{3} \mathrm{O}_{10} \mathrm{I}(\mathrm{OH})_{2}$ & & 0.7 & 0.10 & 2.0 & 2.0 & 0.4 & & & \\
\hline MUscovIte (Al/si disordered) & 398.308 & 306.4 & 140.81 & -5974.8 & -5598.8 & 980.9 & 365 & 166 & 258 \\
\hline $\mathrm{KAl}_{2}\left[\mathrm{Alsi} \mathbf{3}^{\mathrm{O}_{10}}\right](\mathrm{OH})_{2}$ & & 0.6 & 0.10 & 4.9 & 4.9 & 0.9 & 432 & 152 & 316 \\
\hline mSSCOVITE (Al/si ordered) & 398.308 & 287.7 & 140.81 & -5990.0 & -5608.4 & 982.6 & 365 & 166 & 258 \\
\hline 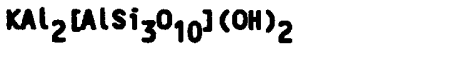 & & 0.6 & 0.10 & 4.9 & 4.9 & 0.9 & 432 & 152 & 316 \\
\hline ANMITE & 511.886 & 415.0 & 154.30 & -5149.3 & -4798.3 & 840.6 & 186 & 188 & 188 \\
\hline $\mathrm{KFe}_{3}\left[\mathrm{Alsi} \mathrm{O}_{10}\right](\mathrm{OH})_{2}$ & & 10.0 & 0.50 & 4.0 & 4.0 & 0.7 & 174 & & \\
\hline PHLOCOPITE (AL/si disordered) & 417.260 & 334.6 & 149.65 & -6226.0 & -5846.0 & 1024.2 & 356 & 356 & 356 \\
\hline $\mathrm{KH}_{3}\left[\mathrm{Alsi} \mathrm{O}_{10}\right](\mathrm{OH})_{2}$ & & 1.0 & 0.10 & 6.0 & 6.0 & 1.0 & & & \\
\hline PHLOCOPITE (Al/si ordered) & 417.260 & 315.9 & 149.65 & -6246.0 & -5860.5 & 1026.7 & 356 & 356 & 356 \\
\hline $\mathrm{OH}_{3} \mathrm{LA}\left(\mathrm{Si}_{3} \mathrm{O}_{10} \mathrm{~J}(\mathrm{OH})_{2}\right.$ & & 1.0 & 0.10 & 6.0 & 6.0 & 01.0 & & & \\
\hline FLUORPHLOCOPITE (AI/Si disorder & .0421 .242 & 336.3 & 146.52 & -6355.5 & -6015.7 & 1053.9 & 218 & 171 & 218 \\
\hline $\mathrm{NHg}_{3}\left[\mathrm{LISi} \mathrm{S}_{3} \mathrm{O} \mathrm{F}_{2}\right.$ & & 2.1 & 0.10 & 4.0 & 4.2 & 0.7 & & 434 & \\
\hline FLUORPHLOCOPITE (AI/Si ordered) & 421.242 & 317.6 & 146.52 & -6375.5 & -6030.1 & 1056.4 & 218 & 356 & 218 \\
\hline $\mathrm{MHO}_{3}\left[\mathrm{Alsi} \mathrm{O}_{10}\right] \mathrm{F}_{2}$ & & 2.1 & 0.10 & 4.0 & 4.2 & 0.7 & & & \\
\hline TALC & 379.266 & 260.8 & 136.20 & -5900.0 & -5520.2 & 967.1 & 370 & 162 & 257 \\
\hline $\mathrm{ng}_{3} \mathrm{Si}_{4} \mathrm{O}_{10}(\mathrm{OH})_{2}$ & & 0.6 & 0.20 & 2.0 & 2.1 & 0.4 & & & \\
\hline CHRYSOTILE (ANTICORITE) & 277.112 & 221.3 & 107.50 & -4360.0 & -4032.4 & 706.4 & 226 & 171 & 226 \\
\hline $\mathrm{Mg}_{3} \mathrm{Si}_{2} \mathrm{O}_{5}(\mathrm{OH})_{4}$ & & 0.8 & 0.40 & $\mathbf{3 . 0}$ & 3.1 & 0.5 & & & \\
\hline
\end{tabular}


THERMODYMAMIC PROPERTIES OF SHEET STRUCTURE SILICATE MINERALS AT $298.15 \mathrm{~K}$

\begin{tabular}{|c|c|c|c|c|c|c|c|c|c|}
\hline \multirow[t]{2}{*}{ MAME AMD FORMULA } & \multirow{2}{*}{$\begin{array}{l}\text { MEIGHT } \\
\text { g }\end{array}$} & \multirow{2}{*}{$\begin{array}{c}\text { ENTROPY } \\
\mathrm{s}^{\bullet} \\
\mathrm{J} \cdot \mathrm{mol}^{-1} \cdot \mathrm{K}^{1}\end{array}$} & \multirow{2}{*}{$\begin{array}{l}\text { VOLUNE } \\
\qquad \begin{array}{l}V^{0} \\
\mathrm{~cm}^{3}\end{array}\end{array}$} & \multirow{2}{*}{$\begin{array}{l}\text { ENTHALPY } \\
\qquad \begin{array}{l}\Delta_{1} H^{0} \\
\mathrm{~kJ} \cdot \mathrm{mol}^{-1}\end{array}\end{array}$} & \multirow{2}{*}{$\begin{array}{c}\text { FREE } \\
\text { ENERGY } \\
\Delta_{,} 6^{\circ} \\
k J \cdot \mathrm{mol}^{-1}\end{array}$} & \multirow[t]{2}{*}{$\operatorname{LOG}(K)$} & \multicolumn{3}{|c|}{ REFERENCES } \\
\hline & & & & & & & $\mathbf{s}$ & H/G & C \\
\hline CLIMOCHLORE & 555.797 & 421.0 & 211.00 & -8919.0 & -8255.8 & 1646.3 & 180 & 182 & 182 \\
\hline $\mathrm{Mg}_{5} \mathrm{Al}\left(\mathrm{AlSi} \mathrm{S}_{3} \mathrm{O}_{10}\right)(\mathrm{OH})_{8}$ & & 15.0 & 0.50 & 20.0 & 20.0 & 3.5 & & 204 & \\
\hline PARAGOMITE (Al/Si disordered) & 382.200 & 295.8 & 132.1 & -5933.0 & -5555.7 & 973.3 & 356 & 356 & 356 \\
\hline $\mathrm{MeAl}_{2} \mathrm{CA}\left(\mathrm{Si}_{3} \mathrm{O}_{10} \mathrm{~J}(\mathrm{OH})_{2}\right.$ & & 0.9 & 0.1 & 3.8 & 3.9 & 0.7 & & & \\
\hline PARAGONITE (AL/Si ordered) & 382.200 & 277.1 & 132.1 & -5949.3 & -5568.5 & 975.6 & 356 & 356 & 356 \\
\hline $\mathrm{MeAl}_{2}\left[\mathrm{Alsi} \mathbf{S i}_{3} \mathrm{O}_{10}\right](\mathrm{OH})_{2}$ & & 0.9 & 0.1 & 3.8 & 3.9 & 0.7 & & 37 & \\
\hline
\end{tabular}


COEFFICIENTS FOR HEAT CAPACITY EQUATIONS FOR SELECTED ELEMENTS

\begin{tabular}{|c|c|c|c|c|c|c|c|c|c|}
\hline MAME AND FORMULA & $\begin{array}{c}\text { EUTROPY } \\
J \cdot \mathrm{mol}^{-1} \cdot K^{\prime}\end{array}$ & $\mathbf{A 1}$ & $\begin{array}{r}12 \\
\text { (T) }\end{array}$ & $\begin{array}{r}A^{3} \\
\left(T^{-2}\right)\end{array}$ & $\left(T^{-0.5}\right)$ & $\begin{array}{r}15 \\
\left(T^{2}\right)\end{array}$ & $\begin{array}{c}T \text { rence } \\
K\end{array}$ & $\mathbf{T}_{\mathrm{tm}}$ & $\begin{array}{c}A_{\tan } H^{\bullet} \\
k J \cdot \operatorname{mol}^{-1}\end{array}$ \\
\hline SILVER & 42.55 & $1.126 E+01$ & $1.169 E-02$ & $-2.124 E+05$ & $2.253 E+02$ & & 298 & 1234.9 & 11.95 \\
\hline Ag (crystal) & 0.21 & & & & & & 1235 & & \\
\hline ALUMINUN & 28.30 & 4.132E+01 & $-1.988 E-02$ & $-1.265 E+05$ & $-1.816 E+02$ & 1.93E-05 & 298 & 933.5 & 10.70 \\
\hline Al (crystal) & 0.08 & & & & & & 933.5 & & \\
\hline ARSENIC & 35.69 & $2.0845 E+01$ & $6.3265 E-03$ & $-9.8777 E+04$ & $5.2398 E+01$ & & 298 & & \\
\hline As & 0.84 & & & & & & 875 & & \\
\hline COLD & 47.49 & 4.197E+01 & $-1.770 E-02$ & & $-2.161 E+02$ & $1.123 E-05$ & 298 & 1337.3 & 12.36 \\
\hline Au (crystal) & 0.21 & & & & & & 1337.3 & & \\
\hline BORON & 5.83 & $4.758 E+01$ & $-5.22 \pi E-03$ & $-5.903 E+04$ & $-5.899 E+02$ & $1.372 E-06$ & 298 & & \\
\hline B (crystal) & 0.08 & & & & & & 2350 & & \\
\hline BERYLLIUM & 9.50 & $1.8735 E+01$ & $9.293 E-03$ & $-4.502 E+05$ & & & 298 & & \\
\hline Be (crystal) & 0.08 & & & & & & 1550 & & \\
\hline BISIUTH & 56.74 & $2.6852 E+01$ & $-1.7289 E-02$ & & & 4.1802E-05 & 298 & & \\
\hline Bi & 0.42 & & & & & & 544.52 & & \\
\hline BRONINE & 249.34 & $4.211 E+01$ & $-2.470 E-03$ & $-3.021 E+04$ & $-8.739 E+01$ & 9.370E-07 & 332.5 & & \\
\hline $\mathrm{Br}_{2}$ (ideal gas) & 0.05 & & & & & & 2200 & & \\
\hline GRAPHITE & 5.74 & $6.086 E+01$ & $-1.024 E-02$ & $7.139 E+05$ & $-9.922 E+02$ & $1.66 \%-06$ & 298 & & \\
\hline C (crystal) & 0.10 & & & & & & 2500 & & \\
\hline DIAMOND & 2.38 & $9.845 E+01$ & $-3.655 E-02$ & $1.217 E+06$ & $-1.659 \varepsilon+03$ & $1.098 E-05$ & 298 & & \\
\hline C (crystal) & 0.20 & & & & & & 1800 & & \\
\hline CALCIUN & 42.90 & $-2.659 E+01$ & $5.236 E-02$ & $-4.877 E+05$ & $7.442 E+02$ & $-9.772 E-06$ & 298 & & \\
\hline Ca (a-crystal) & 0.10 & & , & & & & 716 & & \\
\hline CADHIUM & 51.80 & & $-3.8115 E-02$ & $-1.3402 E+05$ & $3.0044 E+02$ & $-1.4336 E-05$ & 298 & & \\
\hline Cd (crystal) & 0.15 & & & & & & 594.18 & & \\
\hline CERIUM & 72.00 & 1.4017E+01 & 1.9292E-02 & $-1.0802 E+05$ & $1.4469 E+02$ & & 298 & & \\
\hline Ce (crystal) & 4.00 & & & & & & 999 & & \\
\hline CHLORIME & 223.08 & 4.591E+01 & $-3.699 E-03$ & $-4.094 E+04$ & $-1.811 E+02$ & $1.009 \varepsilon-06$ & 298 & & \\
\hline $\mathrm{Cl}_{2}$ (ideal gas) & 0.02 & & & & & & 2500 & & \\
\hline COBALT & 30.04 & & $4.1844 \mathrm{E}-02$ & $-1.5646 E+05$ & $2.6875 E+02$ & $-1.6498 E-05$ & 298 & & \\
\hline Co (erystal) & 0.42 & & & & & & 700 & & \\
\hline CHROMIUH & 23.62 & $-1.625 E+01$ & $2.293 E-02$ & $-1.222 E+06$ & 8.032E+02 & $1.232 E-06$ & 298 & & \\
\hline $\mathrm{Cr}$ (cr retal) & 0.21 & & & & & & 2100 & & \\
\hline
\end{tabular}




\begin{tabular}{|c|c|c|c|c|c|c|c|c|c|}
\hline MAME AND FORMULA & $\begin{array}{l}\text { ENTROPY } \\
\mathrm{J} \cdot \mathrm{mol}^{-1} \cdot \mathrm{K}^{1}\end{array}$ & $A 1$ & $\begin{array}{l}12 \\
\text { (T) }\end{array}$ & $\begin{array}{r}13 \\
\left(r^{-2}\right)\end{array}$ & $\begin{array}{c}1.4 \\
\left(T^{-0.5}\right)\end{array}$ & $\begin{array}{r}15 \\
\left(T^{2}\right)\end{array}$ & $\begin{array}{c}T \text { range } \\
K\end{array}$ & $\underset{\mathbf{T}}{\mathbf{T}_{\mathrm{tm}}}$ & $\begin{array}{c}\Delta_{\mathrm{tm}} \mathrm{H}^{\circ} \\
\mathrm{kJ} \cdot \mathrm{mol}^{-1}\end{array}$ \\
\hline COPPER & 33.14 & $6.084 E+01$ & $-2.875 E-02$ & $3.331 E+05$ & $-5.671 E+02$ & $1.420 E-05$ & 298 & 1357.8 & 13.14 \\
\hline Cu (crystal) & 0.03 & & & & & & 1357.8 & & \\
\hline COPPER & 83.95 & $3.280 E+01$ & & & & & 1357.8 & & \\
\hline Cu (liquid) & 0.50 & & & & & & 2500 & & \\
\hline FLUORINE & 202.79 & $4.05 \pi E+01$ & $1.792 E-03$ & $-1.004 E+05$ & $-1.489 E+02$ & $-4.836 E-07$ & 298 & & \\
\hline$F_{2}$ (ideal gas) & 0.02 & & & & & & 2500 & & \\
\hline GERMANIUM & 31.09 & $2.4033 E+01$ & 3.049E-03 & $-1.5714 E+05$ & $0.84921 E+00$ & $-2.5 E-08$ & 298 & & \\
\hline Ge (crystal) & 0.15 & & & & & & 1211.4 & & \\
\hline HYDROGEN & 130.68 & $4.783 E+00$ & $1.335 E-02$ & $-5.617 E+05$ & $4.583 E+02$ & $-1.825 E-06$ & 298 & & \\
\hline$H_{2}$ (ideal gas) & 0.02 & & & & & & 2500 & & \\
\hline MERCURY & 75.90 & $1.6815 E+01$ & $3.623 E-03$ & $-7.956 E+04$ & $1.860 E+02$ & $2.168 E-06$ & 298 & & 59.2 \\
\hline Hg (liquid) & 0.12 & & & & & & 629 & & \\
\hline MERCURY & 174.97 & $2.079 E+01$ & & & & & 298 & 629 & \\
\hline Hg (ideal gas) & 0.02 & & & & & & 1800 & & \\
\hline IODINE & 116.14 & $1.3122 E+01$ & $9.111 E-03$ & $-1.0131 E+06$ & $5.3419 E+02$ & $-1.5 E-07$ & 457.7 & & \\
\hline$I_{2}$ (ideal gas) & 0.30 & & & & & & 1800 & & \\
\hline MAGNESIUN & 32.67 & & 4.1345E-02 & $-2.7217 E+05$ & $3.1329 E+02$ & $-1.3745 E-05$ & 298 & 923 & 8.48 \\
\hline Mg (crystal) & 0.10 & & & & & & 923 & & \\
\hline MAGMESIUM & 73.23 & $3.431 E+01$ & & & & & 923 & & \\
\hline Mg (liquid) & 0.15 & & & & & & 1366 & & \\
\hline MGEESIUM & 148.65 & $2.079 E+01$ & & & & & 298 & & \\
\hline Mg (ideal gas) & 0.02 & & & & & & 2000 & & \\
\hline MANGANESE & 32.01 & & $4.1345 E-02$ & $-2.7217 E+05$ & $3.1329 E+02$ & $-1.3745 E-05$ & 298 & & \\
\hline Mn (a-crystal) & 0.08 & & & & & & 980 & & \\
\hline MOLYBDENUN & 28.66 & $4.167 E+01$ & $-9.165 E-03$ & $1.082 E+05$ & $-2.882 E+02$ & 4.904E-06 & 298 & & \\
\hline Mo (crystal) & 0.21 & & & & & & 2500 & & \\
\hline NITROGEN & 191.61 & $2.479 E+01$ & $9.648 E-03$ & $1.459 E+05$ & & $-2.003 E-06$ & 298 & & \\
\hline$N_{2}$ (ideal gas) & 0.02 & & & & & & 2500 & & \\
\hline sooriun & 64.92 & $3.7482 E+01$ & $-1.9183 E-02$ & & & $1.0644 E-05$ & 371 & & \\
\hline Ma (liquid) & 0.20 & & & & & & 1170.5 & & \\
\hline soolun & 153.72 & $2.079 E+01$ & & & & & 298 & & \\
\hline Ma (ideal gas) & 0.02 & & & & & & 2500 & & \\
\hline
\end{tabular}


COEFFICIENTS FOR HEAT CAPACITY EQUATIONS FOR SELECTED ELEMENTS

\begin{tabular}{|c|c|c|c|c|c|c|c|c|c|}
\hline MAME AMD FORMULA & $\begin{array}{l}\text { ENTROPY } \\
\mathrm{J} \cdot \mathrm{mol}^{-1} \cdot \mathrm{K}^{1}\end{array}$ & A1 & $\begin{array}{l}A 2 \\
(T)\end{array}$ & $\begin{array}{r}A 3 \\
\left(T^{-2}\right)\end{array}$ & $\begin{array}{r}1.4 \\
\left(T^{-0.5}\right)\end{array}$ & $\begin{array}{r}15 \\
\left(T^{2}\right)\end{array}$ & $\begin{array}{c}\text { T ranoe } \\
K\end{array}$ & $\stackrel{T_{\mathrm{tm}}}{K}$ & $\begin{array}{l}\mathrm{Atm}_{\mathrm{mm}} \mathrm{H}^{\circ} \\
\mathrm{kJ} \cdot \mathrm{mol}^{-1}\end{array}$ \\
\hline $\begin{array}{l}\text { NICKEL } \\
\text { Ni (crystal) }\end{array}$ & $\begin{array}{r}29.87 \\
0.08\end{array}$ & $1.5121 E+02$ & $-2.049 E-01$ & & $-1.3746 E+03$ & $1.742 E-04$ & $\begin{array}{l}298 \\
600\end{array}$ & & \\
\hline OXYGEN & 205.15 & $5.658 E+01$ & $-5.255 E-03$ & $6.856 E+05$ & $-5.780 E+02$ & $1.113 E-06$ & 298 & & \\
\hline $\mathrm{O}_{2}$ (ideal gas) & 0.02 & & & & & & 2500 & & \\
\hline PHOSPHORUS & 42.58 & $2.632 E+01$ & & & & & 317.3 & & \\
\hline$P$ (liquid) & 0.25 & & & & & & 1000 & & \\
\hline $\begin{array}{l}\text { LEAD } \\
\text { Pb (crystal) }\end{array}$ & $\begin{array}{r}64.80 \\
0.30\end{array}$ & $2.266 E+01$ & $1.0089 E-01$ & $-8.717 E+04$ & $4.0666 E+01$ & & $\begin{array}{r}298 \\
600.6\end{array}$ & 600.6 & 4.81 \\
\hline $\begin{array}{l}\text { PLATINUM } \\
\text { Pt (crystel) }\end{array}$ & $\begin{array}{r}41.63 \\
0.20\end{array}$ & $2.0046 E+01$ & $7.415 E-03$ & $-1.298 E+05$ & $8.738 E+01$ & $-4.798 E-07$ & $\begin{array}{r}298 \\
1800\end{array}$ & & \\
\hline $\begin{array}{l}\text { SULFUR } \\
\text { S (ortho-crystal) }\end{array}$ & $\begin{array}{r}32.05 \\
0.05\end{array}$ & $2.270 E+01$ & & & & & 298 & & \\
\hline SULFUR & 228.17 & $3.751 E+01$ & 2.173E-03 & $-1.645 E+05$ & $-6.575 E+01$ & $-7.275 E-08$ & 298 & & \\
\hline$S_{2}$ (ideal gas) & 0.02 & & & & & & 2500 & & \\
\hline $\begin{array}{l}\text { ANTIMONY } \\
\text { Sb (crystal) }\end{array}$ & $\begin{array}{r}45.52 \\
0.21\end{array}$ & $5.2603 E+01$ & $-3.2904 E-02$ & $1.2617 E+05$ & $-3.6512 E+02$ & $2.4691 E-05$ & $\begin{array}{l}298 \\
904\end{array}$ & & \\
\hline $\begin{array}{l}\text { SILICON } \\
\text { si (crystal) }\end{array}$ & $\begin{array}{r}18.81 \\
0.08\end{array}$ & $2.431 E+01$ & 3.003E-03 & $-3.185 E+05$ & $-2.831 E+01$ & $2.330 E-07$ & $\begin{array}{r}298 \\
1685\end{array}$ & 1685 & 50.02 \\
\hline SILICON & 91.56 & $2.720 E+01$ & & & & & 1685 & & \\
\hline si (liquid) & 0.5 & & & & & & 2500 & & \\
\hline $\begin{array}{l}\text { TIN } \\
\text { Sn (crystal) }\end{array}$ & $\begin{array}{r}51.18 \\
0.08\end{array}$ & $-6.8705 E+01$ & $7.2961 E-02$ & $-1.2938 E+06$ & $1.53013 E+03$ & & $\begin{array}{r}298 \\
505.1\end{array}$ & & \\
\hline $\begin{array}{l}\text { STRONTIUH } \\
\text { Sr (crystal) }\end{array}$ & $\begin{array}{r}55.69 \\
0.03\end{array}$ & $2.3369 E+01$ & $1.066 E-02$ & $2.233 E+04$ & & & $\begin{array}{l}298 \\
820\end{array}$ & & \\
\hline $\begin{array}{l}\text { TELLURIUM } \\
\text { Te (crystal) }\end{array}$ & $\begin{array}{r}49.71 \\
0.20\end{array}$ & $1.9124 E+01$ & 2.2061E-02 & & & & $\begin{array}{l}298 \\
723\end{array}$ & & \\
\hline $\begin{array}{l}\text { Thorium } \\
\text { TH (crystal) }\end{array}$ & $\begin{array}{r}51.83 \\
0.50\end{array}$ & $2.27467 E+01$ & $9.338 E-03$ & $-3.00023 E+03$ & $1.27357 E+01$ & $-9.09 E-08$ & $\begin{array}{r}298 \\
1650\end{array}$ & & \\
\hline $\begin{array}{l}\text { TITANTIU⿴囗 } \\
\text { Ti (a-crystal) }\end{array}$ & $\begin{array}{r}30.76 \\
0.10\end{array}$ & $2.01595 E+02$ & $-1.4675 E-01$ & $1.97328 E+06$ & $-2.7691 E+03$ & $6.28 E-05$ & $\begin{array}{r}298 \\
1166\end{array}$ & & \\
\hline $\begin{array}{l}\text { URANIUn } \\
\text { U (crystal) }\end{array}$ & $\begin{array}{r}50.20 \\
0.20\end{array}$ & $3.2944 E+01$ & $-7.800 E-03$ & & $-9.500 E+01$ & $2.860 E-05$ & $\begin{array}{l}296 \\
962\end{array}$ & & \\
\hline
\end{tabular}




\begin{tabular}{|c|c|c|c|c|c|c|c|c|c|}
\hline MAME AND FORIULLA & $\begin{array}{l}\text { ENTROPY } \\
J \cdot n o l^{-1} \cdot K^{-1}\end{array}$ & $A 1$ & $\begin{array}{r}A 2 \\
(T)\end{array}$ & $\begin{array}{r}A 3 \\
\left(T^{-2}\right)\end{array}$ & $\begin{array}{c}14 \\
\left(T^{-0.5}\right)\end{array}$ & $\begin{array}{r}A 5 \\
\left(T^{2}\right)\end{array}$ & $\begin{array}{c}T \text { range } \\
K\end{array}$ & $\underset{K}{T_{t m}}$ & $\begin{array}{c}A_{\mathrm{tm}} \mathrm{H}^{0} \\
\mathrm{~kJ} \cdot \mathrm{mol}^{-1}\end{array}$ \\
\hline VANADIUA & 28.94 & $3.440 E+00$ & $1.334 E-02$ & $-6.971 E+05$ & $4.369 E+02$ & $2.020 E-07$ & 298 & & \\
\hline$V$ (crystal) & 0.42 & & & & & & 2200 & & \\
\hline TUNGSTEN & 32.65 & $3.814 E+01$ & $-5.430 E-03$ & $2.183 E+05$ & $-2.568 E+02$ & $2.552 E-06$ & 298 & & \\
\hline W (crystal) & 0.10 & & & & & & 2500 & & \\
\hline ZINC & 41.63 & $2.909 E+01$ & $-3.507 E-03$ & & $-6.366 E+01$ & $1.161 E-05$ & 298 & 692.7 & 7.30 \\
\hline Zn (crystal) & 0.15 & & & & & & 692.7 & & \\
\hline ZINC & 75.16 & $3.138 E+01$ & & & & & 692.7 & & \\
\hline Zn (liquid) & 0.30 & & & & & & 1180.2 & & \\
\hline ZINC & 160.99 & 2.079E+01 & & & & & 1180.2 & & \\
\hline Zn (ideal gas) & 0.03 & & & & & & 2500 & & \\
\hline ZIRCONIUN & 38.87 & $-2.585 E+01$ & $3.6755 E-02$ & $-9.032 E+05$ & $8.778 E+02$ & $-6.585 E-06$ & 298 & 1135 & 4.02 \\
\hline Zr (a-crystal) & 0.20 & & & & & & 1135 & & \\
\hline ZIRCONIUN & 79.48 & $2.1943 E+01$ & $-6.37 E-03$ & $-1.1452 E+06$ & $2.7991 E+02$ & $4.81 E-06$ & 1135 & & \\
\hline Zr ( $\beta$-crystal) & & & & & & & 2000 & & \\
\hline METHANE & 186.26 & $1.194 E+02$ & $2.055 E-02$ & $2.814 E+06$ & $-2.090 E+03$ & $-5.000 E-06$ & 298 & & \\
\hline $\mathrm{CH}_{4}$ & 0.04 & & & & & & 1800 & & \\
\hline COHENITE & 104.40 & 1.073E+02 & $1.253 E-02$ & & & & 298 & & \\
\hline $\mathrm{Fe}_{3} \mathrm{C}$ & 3.40 & & & & & & 1800 & & \\
\hline AMnONIA & 192.77 & 5.139E+01 & $2.660 E-02$ & $7.584 E+05$ & $-5.480 E+02$ & $-4.900 E-06$ & 298 & & \\
\hline $\mathrm{NH}_{3}$ & 0.03 & & & & & & 1800 & & \\
\hline
\end{tabular}


COEFFICIEMTS FOR HEAT CAPACITY EQUATIONS FOR SULFIDE AND SULFOSALT MIMERALS

\begin{tabular}{|c|c|c|c|c|c|c|c|c|c|}
\hline MAME AMD FORIULA & $\begin{array}{l}\text { ENTROPY } \\
J \cdot \mathrm{mol}^{-1} \cdot K^{-1}\end{array}$ & A1. & $\begin{array}{l}A 2 \\
(T)\end{array}$ & $\begin{array}{r}13 \\
\left(T^{-2}\right)\end{array}$ & $\begin{array}{c}.14 \\
\left(T^{-0.5}\right)\end{array}$ & $\begin{array}{r}15 \\
\left(T^{2}\right)\end{array}$ & $\begin{array}{c}T \text { renge } \\
K\end{array}$ & $\begin{array}{r}T_{\mathrm{tm}} \\
K\end{array}$ & $\begin{array}{c}\mathrm{AJ}_{\mathrm{cm}} \mathrm{H}^{0} \\
\mathrm{kol}\end{array}$ \\
\hline ACANTHITE (ARGENTITE) & 142.9 & $7.531 E+01$ & & & & & 298 & & \\
\hline $\mathrm{Ag}_{2} \mathrm{~S}$ & 0.3 & & & & & & & & \\
\hline REALGAR & 63.5 & $4.702 E+01$ & & & & & 298 & & \\
\hline AsS & 0.6 & & & & & & & & \\
\hline GREENOCKI TE & 72.2 & $3.538 E+01$ & $1.503 E-02$ & $-5.333 E+05$ & $2.357 E+02$ & $-2.400 E-06$ & 298 & & \\
\hline $\cos$ & 0.3 & & & & & & 1100 & & \\
\hline CATTIERITE & 74.8 & $6.582 E+01$ & $3.032 E-02$ & $-5.870 E+05$ & & & 298 & & \\
\hline $\cos _{2}$ & 0.2 & & & & & & 1000 & & \\
\hline COVELLITE & 67.4 & $4.3049 E+01$ & $2.023 E-02$ & $-1.39938 E-05$ & 4.35838E-01 & & 298 & & \\
\hline Cus & 0.2 & & & & & & 780 & & \\
\hline CHALCOCITE & 116.2 & $7.684 E+01$ & & & & & 298 & & \\
\hline $\mathrm{Cu}_{2} \mathrm{~S}$ & 0.1 & & & & & & & & \\
\hline CHALCOPYRITE & 124.9 & $-5.8753 E+02$ & 3.7073E-01 & $-1.4721 E+07$ & $1.275 E+04$ & & 298 & & \\
\hline $\mathrm{CuFeS}_{2}$ & 0.2 & & & & & & 820 & & \\
\hline ISS (intermediate ss) & 250.5 & $-1.01845 E+03$ & $1.358 E+00$ & & & & 820 & & \\
\hline cufes $_{2}$ & & & & & & & 930 & & \\
\hline BORNITE & 398.5 & $2.429 E+02$ & & & & & 298 & & \\
\hline $\mathrm{Cu}_{5} \mathrm{FeS}_{4}$ & 0.8 & & & & & & & & \\
\hline TROILITE & 60.3 & $5.049 E+01$ & & & & & 298 & & \\
\hline Fes & 0.2 & & & & & & & & \\
\hline PYRRHOTITE & 60.7 & $4.988 E+01$ & & & & & 298 & & \\
\hline Fe. $875^{S}$ & 0.2 & & & & & & & & \\
\hline PYRRHOTITE & & $5.084 E+01$ & & & & & 298 & & \\
\hline $\mathrm{Fe} .89^{\mathrm{S}}$ & & & & & & & & & \\
\hline PYRRHOTITE & 63.2 & $5.123 E+01$ & & & & & 298 & & \\
\hline Fe. $.90^{\mathrm{S}}$ & 0.1 & & & & & & & & \\
\hline PYRRHOTITE & & $5.095 E+01$ & & & & & 298 & & \\
\hline Fe $.98^{S}$ & & & & & & & & & \\
\hline PYRITE & 52.9 & $-2.032 E+01$ & $5.030 E-02$ & $-3.200 E+06$ & $1.787 E+03$ & & 298 & & \\
\hline $\mathrm{FeS}_{2}$ & 0.1 & & & & & & 1015 & & \\
\hline mARCASITE & 53.9 & $3.832 E+02$ & $-3.173 E-01$ & $1.676 E+06$ & $-4.488 E+03$ & $1.676 E-04$ & 298 & & \\
\hline $\mathrm{FeS}_{2}$ & 0.1 & & & & & & 700 & & \\
\hline
\end{tabular}


COEFFICIENTS FOR MEAT CAPACITY EQUATIONS FOR SULFIDE AND SULFOSALT MIMERALS

\begin{tabular}{|c|c|c|c|c|c|c|c|c|c|}
\hline NAME AND FORIULA & $\begin{array}{c}\text { ENTROPY } \\
\mathrm{J} \cdot \mathrm{mol}^{-1} \cdot \mathrm{K}^{-1}\end{array}$ & $A 1$ & $\begin{array}{l}A 2 \\
\text { (T) }\end{array}$ & $\begin{array}{r}A 3 \\
\left(T^{-2}\right)\end{array}$ & $\left(T^{-0.5}\right)$ & $\begin{array}{r}A 5 \\
\left(T^{2}\right)\end{array}$ & $\begin{array}{c}T \text { rence } \\
K\end{array}$ & $T_{\operatorname{tin}}$ & $\begin{array}{c}\Delta_{\mathrm{tm}} \mathrm{H}^{\bullet} \\
\mathrm{kJ} \cdot \mathrm{mol}^{-1}\end{array}$ \\
\hline HYDROGEN SULFIDE & 205.8 & $2.636 E+01$ & $2.650 E-02$ & $2.660 E+05$ & $-4.356 E+01$ & $-6.024 E-06$ & 298 & & \\
\hline $\mathrm{H}_{2} \mathrm{~S}$ (IDEAL GAS) & 0.2 & & & & & & 1800 & & \\
\hline Mns & 0.8 & & & & & & 1800 & & \\
\hline MOLYBDENITE & 62.6 & $1.045 E+02$ & $-4.812 E-03$ & $-6.291 E+03$ & $-6.817 E+02$ & & 298 & & \\
\hline $\mathrm{MoS}_{2}$ & 0.2 & & & & & & 1200 & & \\
\hline MILLERITE & 53.0 & $4.711 E+01$ & & & & & 298 & & \\
\hline Nis & 0.4 & & & & & & & & \\
\hline HEAZLEUOODITE & 133.2 & $1.057 E+03$ & $-8.988 E-01$ & $8.139 E+06$ & $-1.388 E+04$ & $4.660 E-04$ & 298 & & \\
\hline $\mathrm{Ni}_{3} \mathrm{~S}_{2}$ & 0.3 & & & & & & 840 & & \\
\hline GALENA & 91.7 & $4.460 E+01$ & $1.640 E-02$ & & & & 298 & & \\
\hline Pbs & 0.7 & & & & & & 900 & & \\
\hline STIBNITE & 182.0 & $1.705 E+02$ & $-4.860 E-03$ & $7.539 E+05$ & $-9.978 E+02$ & & 298 & & \\
\hline $\mathrm{Sb}_{2} \mathrm{~s}_{3}$ & 3.3 & & & & & & 829 & & \\
\hline HERZENBERGITE & 77.0 & $-1.559 E+02$ & $1.219 E-01$ & $-3.548 E+06$ & $3.604 E+03$ & & 298 & & \\
\hline Sns & 0.8 & & & & & & 875 & & \\
\hline HERZENBERGITE & 135.1 & $2.672 E+01$ & $3.035 E-02$ & & & & 875 & & \\
\hline Sns & 1.0 & & & & & & 1153 & & \\
\hline $\mathrm{Sns}_{2}$ & 0.2 & & & & & & 1000 & & \\
\hline TUNGSTENITE & 67.8 & $7.633 E+01$ & $5.561 E-04$ & $-1.137 E+06$ & & $1.408 \varepsilon-06$ & 298 & & \\
\hline $\mathrm{HS}_{2}$ & 0.3 & & & & & & 1500 & & \\
\hline SPHALERITE & 58.7 & $6.151 E+01$ & $7.631 E-04$ & $-7.963 E+04$ & $-2.604 E+02$ & & 29 & & \\
\hline Zns & 0.8 & & & & & & 1300 & & \\
\hline WURTZITE & 58.8 & 4. 191E+01 & 7.619E-03 & $-6.603 E+05$ & $1.577 E+02$ & & 298 & & \\
\hline Zns & 0.2 & & & & & & 1300 & & \\
\hline BERTHIERITE & 245.0 & $1.000 E+01$ & 7.930E-02 & $-6.416 E+06$ & $3.691 E+03$ & & 298 & & \\
\hline $\mathrm{FeSb}_{2} \mathrm{~S}_{4}$ & 1.0 & & & & & & 836 & & \\
\hline CHALCOSTIBITE & 149.2 & $8.810 E+01$ & 4.040E-02 & & & & 298 & & \\
\hline Cusbs $_{2}$ & 3.8 & & & & & & 826 & & \\
\hline
\end{tabular}


COEFFICIENTS FOR HEAT CAPACITY EQUATIONS FOR OXIDE AMD HYDROXIDE MIMERALS

\begin{tabular}{|c|c|c|c|c|c|c|c|c|c|}
\hline MAME AND FORIMLLA & $\begin{array}{l}\text { ENTROPY } \\
J \cdot \mathrm{mol}^{-1} \cdot \mathrm{K}^{\prime}\end{array}$ & A1 & $\begin{array}{l}12 \\
(T)\end{array}$ & $\begin{array}{r}13 \\
\left(T^{-2}\right)\end{array}$ & $\begin{array}{r}14 \\
\left(T^{-0.5}\right)\end{array}$ & $\begin{array}{l}A 5 \\
\left(T^{2}\right)\end{array}$ & $\begin{array}{c}T \text { ranoe } \\
K\end{array}$ & $\underset{K}{T_{\text {tm }}}$ & $\begin{array}{l}\Delta_{\mathrm{tm}} \mathrm{H}^{0} \\
\mathrm{~kJ} \cdot \mathrm{mol}^{-1}\end{array}$ \\
\hline CORUNDUN & 50.9 & $1.612 E+02$ & $-1.352 E-03$ & $-1.815 E+06$ & $-1.059 E+03$ & 5.381E-07 & 298 & 2345 & \\
\hline $\mathrm{Al}_{2} \mathrm{O}_{3}$ & 0.1 & & & & & & 2250 & & \\
\hline BOEHMITE & 37.2 & $2.057 E+02$ & $-3.492 E-02$ & $1.027 E+06$ & $-2.635 E+03$ & & 298 & & \\
\hline$A(O(O H)$ & 0.1 & & & & & & 600 & & \\
\hline DIASPORE & 35.3 & $5.333 E+01$ & & & & & 298 & & \\
\hline$A L O(O H)$ & 0.2 & & & & & & & & \\
\hline GIBBSITE & 68.4 & $9.170 E+01$ & & & & & 298 & & \\
\hline $\mathrm{Al}(\mathrm{OH})_{3}$ & 0.1 & & & & & & & & \\
\hline DIBORON TRIOXIDE & 54.0 & $1.84 \pi E+02$ & $7.108 E-03$ & $6.441 E+05$ & $-2.270 E+03$ & & 298 & & \\
\hline $\mathrm{B}_{2} \mathrm{O}_{3}$ & 0.3 & & & & & & 723 & & \\
\hline DIBORON TRIOXIDE & 160.9 & $1.297 E+02$ & & & & & 723 & & \\
\hline $\mathrm{B}_{2} \mathrm{O}_{3}$ (liquid) & 0.5 & & & & & & 2200 & & \\
\hline BARIUN MONOXIDE & 72.1 & $5.722 E+01$ & $5.370 E-03$ & $-1.669 E+05$ & $-1.668 E+02$ & & 298 & & \\
\hline $\mathrm{BaO}$ & 0.4 & & & & & & 1800 & & \\
\hline BROMELLITE & 13.8 & 8.274E+01 & $-7.301 E-03$ & $-4.158 E+05$ & $-8.718 E+02$ & $1.845 E-06$ & 298 & & \\
\hline Beo & 0.2 & & & & & & 2000 & & \\
\hline BISMITE & 151.5 & $1.036 E+02$ & 3.336E-02 & & & & 298 & & \\
\hline $\mathrm{Bi}_{2} \mathrm{O}_{3}$ & 2.1 & & & & & & 800 & & \\
\hline CARBON MONOXIDE & 197.3 & $3.976 E+01$ & $3.188 E-03$ & $5.292 E+05$ & $-3.013 E+02$ & $-8.078 E-07$ & 298 & & \\
\hline CO (ideal gas) & 0.0 & & & & & & 2500 & & \\
\hline CARBON DIOXIDE & 213.8 & $8.811 E+01$ & $-2.698 E-03$ & $7.232 E+05$ & $-1.007 E+03$ & & 298 & & \\
\hline $\mathrm{CO}_{2}$ (ideal gas) & 0 & & & & & & 2200 & & \\
\hline LIME & 38.1 & $5.185 E+01$ & 2.444E-03 & $-9.340 E+05$ & & & 298 & & \\
\hline $\mathrm{CaO}$ & 0.4 & & & & & & 2500 & & \\
\hline PORTLANDITE & 83.4 & $1.867 E+02$ & $-2.191 E-02$ & & $-1.600 E+03$ & & 298 & & \\
\hline $\mathrm{Ca}(\mathrm{OH})_{2}$ & 0.4 & & & & & & 700 & & \\
\hline CERIANITE & 62.3 & $8.029 E+01$ & $5.699 \varepsilon-03$ & $-7.294 E+05$ & $-2.099 E+02$ & & 298 & & \\
\hline $\mathrm{CeO}_{2}$ & 0.1 & & & & & & 1800 & & \\
\hline COBALT MONOXIDE & 52.8 & $-3.047 E+01$ & $2.946 E-02$ & $-4.166 E+06$ & $1.932 E+03$ & & 298 & & \\
\hline $\mathrm{CoO}$ & 0.3 & & & & & & 1800 & & \\
\hline TRICOBALT TETROXIDE & 109.3 & $1.316 E+02$ & $6.602 E-02$ & $-2.480 E+06$ & & & 298 & & \\
\hline $\mathrm{CO}_{3} \mathrm{O}_{4}$ & 0.3 & & & & & & 1000 & & \\
\hline
\end{tabular}




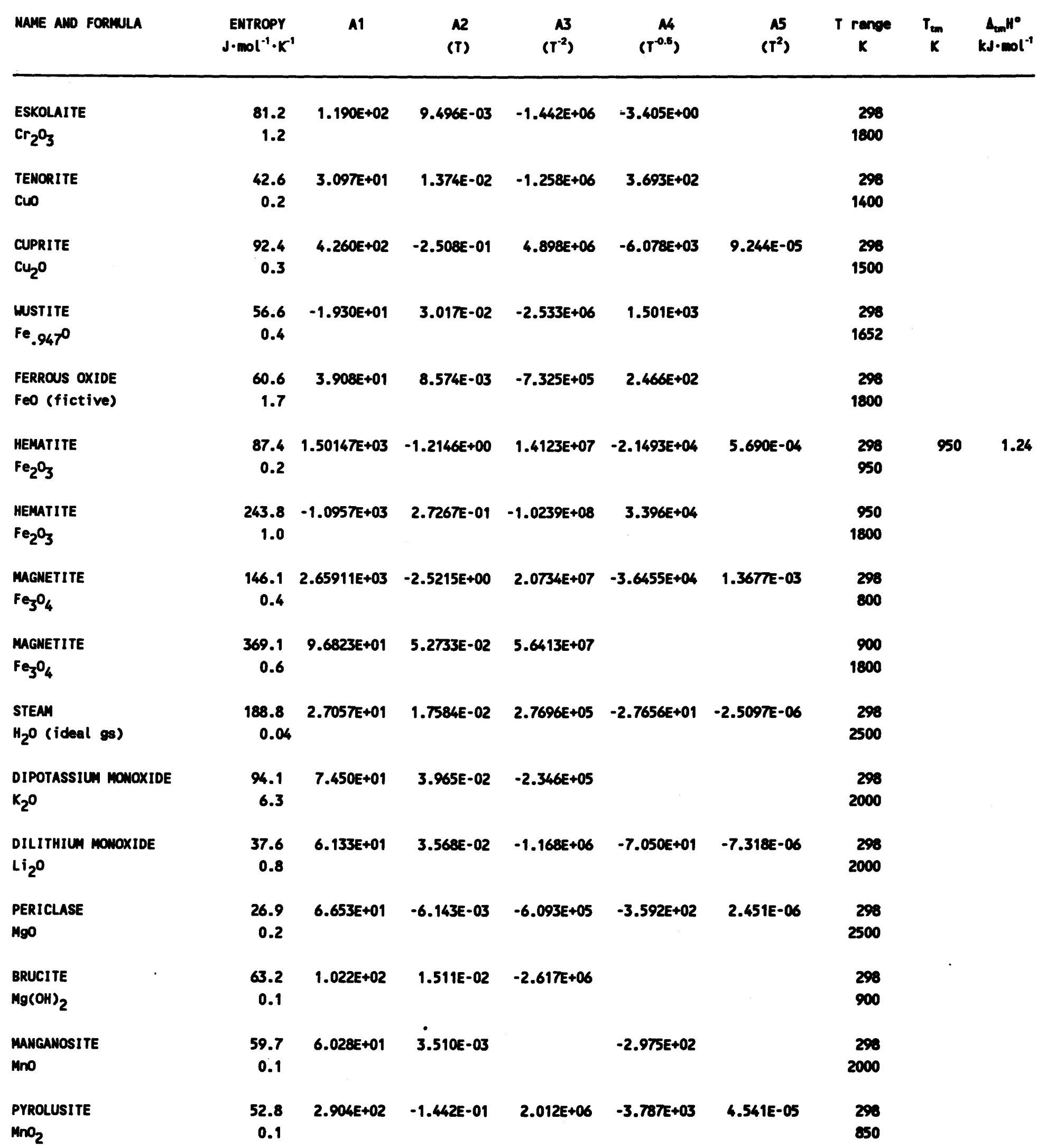


COEFFICIENTS FOR HEAT CAPACITY EQUATIONS FOR OXIDE AMD HYDROXIDE MIMERALS

\begin{tabular}{|c|c|c|c|c|c|c|c|c|c|}
\hline WAME AND FORINLA & $\begin{array}{l}\text { ENTROPY } \\
J \cdot \mathrm{mol}^{-1} \cdot \mathrm{K}^{1}\end{array}$ & A1 & $\begin{array}{l}A 2 \\
(T)\end{array}$ & $\begin{array}{r}\mathbf{A}^{3} \\
\left(T^{-2}\right)\end{array}$ & $\begin{array}{c}1.4 \\
\left(T^{-0.5}\right)\end{array}$ & $\begin{array}{r}15 \\
\left(T^{2}\right)\end{array}$ & $\begin{array}{c}T \text { rence } \\
K\end{array}$ & $\begin{array}{r}T_{\mathrm{tm}} \\
\mathrm{K}\end{array}$ & $\begin{array}{l}\mathrm{AJm}_{\mathrm{cm}} \mathrm{H}^{\bullet} \\
\mathrm{mol}^{-1}\end{array}$ \\
\hline BIXBYITE & 113.7 & $1.624 E+02$ & $1.211 E-02$ & $1.046 E+06$ & $-1.317 E+03$ & $3.462 E-06$ & 325 & & \\
\hline $\mathrm{Mn}_{2} \mathrm{O}_{3}$ & 0.2 & & & & & & 1400 & & \\
\hline HAUSMANMITE & 164.1 & $-7.432 E+00$ & $9.487 E-02$ & $-6.712 E+06$ & $3.396 E+03$ & & 298 & & \\
\hline $\mathrm{Mn}_{3} \mathrm{O}_{4}$ & 0.3 & & & & & & 1400 & & \\
\hline BRAUNITE & 416.4 & $4.301 E+02$ & $1.110 E-01$ & $-7.325 E+06$ & & & 298 & & \\
\hline $\mathrm{An}_{7} \mathrm{SiO}_{12}$ & 0.8 & & & & & & 1500 & & \\
\hline MOLYBDITE & 77.7 & $6.433 E+00$ & $6.278 E-02$ & $-2.460 E+06$ & $1.337 E+03$ & & 298 & 1074 & \\
\hline $\mathrm{MoO}_{3}$ & 0.4 & & & & & & 1074 & & \\
\hline MITROGEN DIOXIDE & 240.1 & $1.018 E+02$ & $-1.121 E-02$ & $1.218 E+06$ & $-1.300 E+03$ & $1.461 E-06$ & 298 & & \\
\hline $\mathrm{NO}_{2}$ & 0.1 & & & & & & 2500 & & \\
\hline DISCDIUN MONOXIDE & 75.3 & $1.1397 E+02$ & 7.4857E-03 & & $-8.1335 E+02$ & & 298 & & \\
\hline $\mathrm{Na}_{2} \mathrm{O}$ & 0.8 & & & & & & 1000 & & \\
\hline BUNSENITE & 38.0 & 4.1107E+03 & $-5.3024 E+00$ & $2.43067 E+07$ & $-5.3039 E+04$ & $3.5206 E-03$ & 298 & & \\
\hline Nio & 0.2 & & & & & & 519 & & \\
\hline BUNSENITE & 67.7 & $-8.776 E+00$ & 4.223E-02 & $3.607 E+06$ & $7.873 E+02$ & $-7.526 E-06$ & 519 & & \\
\hline Nio & 0.5 & & & & & & 1800 & & \\
\hline LITHARGE & 66.5 & $5.102 E+01$ & 1.027E-02 & $-7.387 E+05$ & & & 298 & & \\
\hline Pbo & 0.2 & & & & & & 1000 & & \\
\hline PLAtTMerite & 71.8 & $7.312 E+01$ & $7.484 E-03$ & $-1.261 E+06$ & & & 298 & & \\
\hline $\mathrm{PbO}_{2}$ & 0.4 & & & & & & 1200 & & \\
\hline MINIUM & 212.0 & $1.779 E+02$ & $3.326 E-02$ & $-2.926 E+06$ & & & 298 & & \\
\hline $\mathrm{Pb}_{3} \mathrm{O}_{4}$ & 6.7 & & & & & & 1800 & & \\
\hline SULFUR DIOXIDE & 248.2 & $9.890 E+01$ & $-1.161 E-02$ & $8.465 E+05$ & $-1.127 E+03$ & $1.788 E-06$ & 298 & & \\
\hline $\mathrm{SO}_{2}$ (IDEAL GAS) & 0.1 & & & & & & 2500 & & \\
\hline SULFUR TRIOXIDE & 256.8 & $1.478 E+02$ & $-1.898 E-02$ & $1.079 E+06$ & $-1.794 E+03$ & $2.763 E-06$ & 298 & & \\
\hline $\mathrm{SO}_{3}$ (IDEAL GAS) & 0.8 & & & & & & 2500 & & \\
\hline SILICON MOMOXIDE & 211.6 & $5.686 E+01$ & $-5.616 E-03$ & $4.922 E+05$ & $-5.335 E+02$ & $8.5943 E-07$ & 298 & & \\
\hline SIO (IDEAL GAS) & 0.8 & & & & & & 2500 & & \\
\hline QUARTZ & 41.5 & $8.1145 E+01$ & $1.828 E-02$ & $-1.810 E+05$ & $-6.985 E+02$ & $5.406 E-06$ & 298 & & \\
\hline $\mathrm{SiO}_{2}$ & 0.1 & & & & & & 844 & & \\
\hline QUARTZ & 104.6 & $5.796 E+01$ & $9.330 E-03$ & $1.835 E+06$ & & & 844 & & \\
\hline $\mathrm{SiO}_{2}$ & 0.2 & & & & & & 1700 & & \\
\hline
\end{tabular}


COEFFICIENTS FOR HEAT CAPACITY EQUATIONS FOR OXIDE AND HYDROXIDE MIMERALS

\begin{tabular}{|c|c|c|c|c|c|c|c|c|c|}
\hline MAME AMD FORMULA & $\begin{array}{l}\text { ENTROPY } \\
\mathrm{J} \cdot \mathrm{mol}^{-1} \cdot \mathrm{K}^{\prime}\end{array}$ & A1 & $\begin{array}{l}A 2 \\
(T)\end{array}$ & $\begin{array}{r}A^{3} \\
\left(T^{-2}\right)\end{array}$ & $\begin{array}{c}-14 \\
\left(T^{-0.5}\right)\end{array}$ & $\begin{array}{r}A 5 \\
\left(T^{2}\right)\end{array}$ & $\begin{array}{c}T \text { renge } \\
K\end{array}$ & $\begin{array}{c}T_{t m} \\
K\end{array}$ & $\begin{array}{l}\mathrm{A}_{\mathrm{mm}} \mathrm{H}^{\circ} \\
\mathrm{kJ} \cdot \mathrm{mol}^{-1}\end{array}$ \\
\hline CRISTOBALITE & 43.4 & $-4.160 E+03$ & $2.548 E+00$ & $-6.286 E+07$ & $7.168 E+04$ & & 298 & & \\
\hline $\mathrm{SiO}_{2}$ & 0.1 & & & & & & 523 & & \\
\hline CRISTOBALITE & 76.3 & $7.275 E+01$ & $1.300 E-03$ & $-4.132 E+06$ & & & 523 & & \\
\hline $\mathrm{SiO}_{2}$ & 1.0 & & & & & & 1800 & & \\
\hline COESITE & 38.5 & $2.331 E+02$ & $-7.777 E-02$ & $2.604 E+06$ & $-3.375 E+03$ & $1.924 E-05$ & 298 & & \\
\hline $\mathrm{SiO}_{2}$ & 1.0 & & & & & & 1800 & & \\
\hline STISHOVITE & 27.8 & $1.474 E+02$ & $-4.027 E-02$ & $-2.834 E+05$ & $-1.559 E+03$ & $1.203 E-05$ & 298 & & \\
\hline $\mathrm{SiO}_{2}$ & 0.4 & & & & & & 1800 & & \\
\hline SILICA GLASS & 48.5 & $7.464 E+01$ & $-7.259 E-03$ & $-3.114 E+06$ & & 5.570E-06 & 298 & & \\
\hline $\mathrm{SiO}_{2}$ & 1.0 & & & & & & 1700 & & \\
\hline CASSITERITE & 49.0 & $7.604 E+01$ & 7.364E-03 & $-2.224 E+06$ & & & 298 & & \\
\hline $\mathrm{SnO}_{2}$ & 0.1 & & & & & & 1500 & & \\
\hline STRONTIUN MONOXIDE & 55.5 & $5.365 E+01$ & $5.982 E-03$ & $-3.666 E+05$ & $-1.019 E+02$ & & 298 & & \\
\hline Sro & 0.4 & & & & & & 2500 & & \\
\hline THORIANITE & 65.2 & $7.138 E+01$ & $7.556 E-03$ & $-1.053 E+06$ & & & 298 & & \\
\hline $\mathrm{ThO}_{2}$ & 0.2 & & & & & & 1200 & & \\
\hline RUTILE & 50.6 & $8.462 E+01$ & $5.990 E-04$ & $-1.101 E+06$ & $-2.957 E+02$ & & 298 & & \\
\hline $\mathrm{TiO}_{2}$ & 0.2 & & & & & & 2100 & & \\
\hline AMATASE & 49.9 & $4.396 E+01$ & $1.374 E-02$ & $-2.595 E+06$ & $6.294 E+02$ & & 298 & & \\
\hline $\mathrm{TiO}_{2}$ & 0.3 & & & & & & 1300 & & \\
\hline URANINITE & $\pi .0$ & $5.845 E+01$ & $1.606 E-02$ & $-1.867 E+06$ & $3.689 E+02$ & & 298 & & \\
\hline $\mathrm{wO}_{2}$ & 0.2 & & & & & & 1800 & & \\
\hline KARELIANITE & 98.1 & $5.799 E+01$ & $4.120 E-02$ & $-3.872 E+06$ & $1.351 E+03$ & & 298 & & \\
\hline $\mathrm{V}_{2} \mathrm{O}_{3}$ & 1.3 & & & & & & 1800 & & \\
\hline TUNGSTEN DIOXIDE & 50.6 & $6.638 E+01$ & $1.326 E-02$ & $-1.294 E+06$ & & & 298 & & \\
\hline $\mathrm{wO}_{2}$ & 0.3 & & & & & & 2500 & & \\
\hline ZINCITE & 43.2 & $4.350 E+01$ & $7.658 E-03$ & $-7.573 E+05$ & $5.456 E+01$ & & 298 & & \\
\hline $2 n o$ & 0.1 & & & & & & 1800 & & \\
\hline BADDELEYITE & 50.4 & $1.073 E+02$ & $-5.011 E-03$ & $-2.203 E+05$ & $-8.141 E+02$ & & 298 & 1478 & 8.08 \\
\hline $\mathrm{ZrO}_{2}$ & 0.3 & & & & & & 1478 & & \\
\hline BADDELEYITE & 167.1 & $7.448 E+01$ & & & & & 1478 & & \\
\hline $2 \mathrm{rO}_{2}$ & 1.5 & & & & & & 2000 & & \\
\hline
\end{tabular}


COEFFICIENTS FOR HEAT CAPACITY EQUATIONS FOR MLITIPLE OXIDE MINERALS

\begin{tabular}{|c|c|c|c|c|c|c|c|c|c|}
\hline MAME AND FORNULA & $\begin{array}{l}\text { ENTROPY } \\
J \cdot \mathrm{mol}^{-1} \cdot \mathrm{K}^{\prime}\end{array}$ & A1 & $\begin{array}{l}A 2 \\
(T)\end{array}$ & $\begin{array}{r}A 3 \\
\left(T^{-2}\right)\end{array}$ & $\begin{array}{c}\stackrel{N 4}{(-0.5}) \\
\left(T^{-1}\right)\end{array}$ & $\begin{array}{r}15 \\
\left(T^{2}\right)\end{array}$ & $\begin{array}{c}T \text { renge } \\
K\end{array}$ & $\begin{array}{r}T_{\text {tm }} \\
K\end{array}$ & $\begin{array}{l}\mathrm{A}_{\mathrm{m}} \mathrm{H}^{\bullet} \\
\mathrm{kJ} \cdot \mathrm{mol}^{-1}\end{array}$ \\
\hline TIALITE & 109.6 & 2.111E+02 & 1.461E-02 & $-3.625 E+06$ & $-6.610 E+02$ & & 298 & & \\
\hline $\mathrm{Al}_{2} \mathrm{TiO}_{5}$ & 0.8 & & & & & & 1800 & & \\
\hline CHRYSOBERYL & 66.3 & $3.627 E+02$ & $-8.353 E-02$ & $-6.798 E+04$ & $-4.034 E+03$ & $2.248 E-05$ & 298 & & \\
\hline $\mathrm{BeAl}_{2} \mathrm{O}_{4}$ & 0.1 & & & & & & 1800 & & \\
\hline CALCIUN FERRITE & 145.4 & $9.588 E+01$ & $4.666 E-02$ & $-3.360 E+06$ & $1.410 E+03$ & & 298 & & \\
\hline $\mathrm{CaFe}_{2} \mathrm{O}_{4}$ & 0.8 & & & & & & 1510 & & \\
\hline DICALCIUN FERRITE & 188.8 & $2.223 E+02$ & $9.728 E-03$ & $-5.661 E+06$ & $5.420 E+02$ & & 298 & & \\
\hline $\mathrm{Ce}_{2} \mathrm{Fe}_{2} \mathrm{O}_{5}$ & 1.3 & & & & & & 1750 & & \\
\hline PEROVSKITE & 93.6 & $1.250 E+01$ & $4.516 E-02$ & $-6.302 E+06$ & $2.462 E+03$ & & 298 & & \\
\hline $\mathrm{CaTiO}_{3}$ & 0.4 & & & & & & 1530 & & \\
\hline MERCYNITE & 116.0 & $2.247 E+02$ & $4.480 E-03$ & $-1.581 E+06$ & $-1.370 E+03$ & & 298 & & \\
\hline $\mathrm{FeAl}_{2} \mathrm{O}_{4}$ & $\mathbf{3 . 0}$ & & & & & & 1200 & & \\
\hline CHRONITE & 146.0 & $3.018 E+02$ & $-4.157 E-02$ & $4.877 E+05$ & $-2.803 E+03$ & 1.147E-05 & 298 & & \\
\hline $\mathrm{FeCr}_{2} \mathrm{O}_{4}$ & 1.7 & & & & & & 1800 & & \\
\hline ILMENITE & 108.9 & $2.627 E+02$ & $-7.999 E-02$ & $3.827 E+05$ & $-2.538 E+03$ & $3.388 E-05$ & 298 & & \\
\hline $\mathrm{FeTiO}_{3}$ & 0.3 & & & & & & 1000 & & \\
\hline ULVOSPINEL & 168.9 & $-1.026 E+02$ & $1.425 E-01$ & $-9.145 E+06$ & $5.271 E+03$ & & 298 & & \\
\hline $\mathrm{Fe}_{2} \mathrm{TiO}_{4}$ & 2.5 & & & & & & 1800 & & \\
\hline PSELDOBROOKITE & 156.5 & $2.136 E+02$ & $1.625 E-02$ & $-2.345 E+06$ & $-4.795 E+02$ & & 298 & & \\
\hline $\mathrm{Fe}^{2} \mathrm{TiO}_{5}$ & 1.3 & & & & & & 1800 & & \\
\hline SPINEL & 84.5 & $2.229 \varepsilon+02$ & 6.127E-03 & $-1.686 E+06$ & $-1.551 E+03$ & & 298 & & \\
\hline $\mathrm{MgAl}_{2} \mathrm{O}_{4}$ & 0.04 & & & & & & 1800 & & \\
\hline MMGNESIOCHROMITE & 106.0 & $1.961 E+02$ & 5.398E-03 & $-3.126 E+06$ & $-6.169 E+02$ & & 298 & & \\
\hline $\mathrm{MgCr}_{2} \mathrm{O}_{4}$ & 0.8 & & & & & & 1800 & & \\
\hline MGEESIOFERRITE & 123.8 & $1.782 E+02$ & $1.275 E-02$ & & & & 665 & & \\
\hline $\mathrm{MgFe}_{2} \mathrm{O}_{4}$ & 0.8 & & & & & & 1230 & & \\
\hline MAGESIOFERRITE & 379.2 & $1.066 E+02$ & $5.716 E-02$ & & & & 1230 & & \\
\hline $\mathrm{MgFe}_{2} \mathrm{O}_{4}$ & 3.0 & & & & & & 1800 & & \\
\hline GEIKIELITE & 74.6 & $2.225 E+02$ & $-5.274 E-02$ & $-6.092 E+05$ & $-1.8746 E+03$ & 1.878E-05 & 298 & & \\
\hline $\mathrm{MgTiO}_{3}$ & 0.2 & & & & & & 1800 & & \\
\hline PYROPHANITE & 104.9 & 1.217E+02 & $9.288 E-03$ & $-2.188 E+06$ & & & 298 & & \\
\hline $\mathrm{MnTiO}_{3}$ & 0.3 & & & & & & 1600 & & \\
\hline
\end{tabular}


COEFFICIENTS FOR HEAT CAPACITY EQUATIONS FOR MULTIPLE OXIDE MINERALS

\begin{tabular}{|c|c|c|c|c|c|c|c|c|c|}
\hline MANE AND FOAHULA & $\begin{array}{c}\text { ENTROPY } \\
J \cdot \mathrm{wl}^{-1} \cdot K^{\prime}\end{array}$ & $\mathbf{A 1}$ & $\begin{array}{l}12 \\
(T)\end{array}$ & $\begin{array}{r}A^{3} \\
\left(T^{-2}\right)\end{array}$ & $\begin{array}{r}1.44 \\
\left(T^{-0.5}\right)\end{array}$ & $\begin{array}{r}15 \\
\left(T^{2}\right)\end{array}$ & $\begin{array}{c}T \text { renge } \\
K\end{array}$ & $T_{\text {tm }}$ & $\begin{array}{c}\Delta_{\tan } H^{\circ} \\
k J \cdot \operatorname{mol}^{-1}\end{array}$ \\
\hline TREVORITE & 165.0 & $9.559 E+01$ & $1.705 E-01$ & $3.9927 E+04$ & & & 298 & & \\
\hline $\mathrm{NiFe}_{2} \mathrm{O}_{4}$ & $\mathbf{3 . 0}$ & & & & & & 1000 & & \\
\hline FRANKLIMITE & 150.7 & $1.995 E+02$ & $3.045 E-02$ & $-1.352 E+06$ & & & 298 & & \\
\hline $\mathrm{ZnFe}_{2} \mathrm{O}_{4}$ & 0.3 & & & & & & 600 & & \\
\hline ZIMC TITAMIUN SPIMEL & 143.1 & $2.613 E+02$ & $-5.138 E-03$ & $-4.317 E+05$ & $-2.095 E+03$ & & 298 & & \\
\hline $2 n_{2} T^{T i O}$ & 0.3 & & & & & & 1800 & & \\
\hline
\end{tabular}


COEFFICIENTS FOR HEAT CAPACITY EQUATIONS FOR MALIDE MIMERALS

\begin{tabular}{|c|c|c|c|c|c|c|c|c|c|}
\hline MAME AND FORINLA & $\begin{array}{l}\text { ENTROPY } \\
J \cdot \mathrm{mol}^{-1} \cdot \mathrm{K}^{\prime}\end{array}$ & A1 & $\begin{array}{l}12 \\
(T)\end{array}$ & $\begin{array}{r}A 3 \\
\left(T^{-2}\right)\end{array}$ & $\begin{array}{r}1.4 \\
\left(T^{-0.5}\right)\end{array}$ & $\begin{array}{r}15 \\
\left(T^{2}\right)\end{array}$ & $\begin{array}{c}T \text { renge } \\
K\end{array}$ & $\underset{k}{T_{\mathrm{min}}}$ & $\mathrm{kJ}_{\mathrm{dm} \cdot \mathrm{mol}^{-1}}^{\mathrm{H}^{-1}}$ \\
\hline BROMARGYRITE & 107.1 & $3.317 E+01$ & 6.443E-02 & & & & 298 & & \\
\hline AgBr & 0.4 & & & & & & 703 & & \\
\hline POTASSIUN BROMIDE & 95.9 & $-4.392 E+01$ & 5.855E-02 & $-1.839 E+06$ & $1.717 E+03$ & & 298 & & \\
\hline $\mathrm{KBr}$ & 0.2 & & & & & & 1000 & & \\
\hline CHLORARGYRITE & 96.2 & 5.996E+01 & $7.620 E-03$ & $-1.017 E+06$ & & & 298 & & \\
\hline $\mathrm{AgCl}$ & 0.2 & & & & & & 728 & & \\
\hline HYDROPHILITE & 104.6 & $-4.725 E+00$ & $4.504 E-02$ & $-2.037 E+06$ & $1.503 E+03$ & & 298 & 1045 & 28.54 \\
\hline $\mathrm{CaCl}_{2}$ & 1.3 & & & & & & 1045 & & \\
\hline HYDROPHILITE & 230.2 & $1.025 E+02$ & & & & & 1045 & & \\
\hline $\mathrm{CaCl}_{2}$ (liquid) & 5.0 & & & & & & 1800 & & \\
\hline LAWRENCITE & 118.0 & $-1.123 E+02$ & $8.951 E-02$ & $-5.115 E+06$ & $3.790 E+03$ & & 298 & & \\
\hline $\mathrm{FeCl}_{2}$ & 0.4 & & & & & & 950 & & \\
\hline MOLYSITE & 142.3 & $-2.56 E+02$ & $1.1755 E+00$ & $7.89 E+06$ & & $-9.738 E-04$ & 298 & & \\
\hline $\mathrm{FeCl}_{3}$ & 0.4 & & & & & & 577 & & \\
\hline HYDROGEN CHLORIDE & 186.9 & $1.738 E+01$ & $1.165 E-02$ & $-7.597 E+03$ & $1.477 E+02$ & $-2.052 E-06$ & 298 & & \\
\hline HCl (ideal gas) & 0.03 & & & & & & 2500 & & \\
\hline SYLVITE & 82.6 & $-2.452 E+01$ & $4.852 E-02$ & $-1.605 E+06$ & $1.371 E+03$ & & 298 & & \\
\hline KCl & 0.2 & & & & & & 1043 & & \\
\hline CHLOROMAGNESITE & 89.6 & $7.690 E+01$ & $8.496 E-03$ & $-7.463 E+05$ & & & 298 & & \\
\hline $\mathrm{MgCl}_{2}$ & 0.8 & & & & & & 987 & & \\
\hline SCACCHITE & 118.2 & $7.626 E+01$ & 1.191E-02 & $-6.048 E+05$ & & & 298 & & \\
\hline $\mathrm{AnCl}_{2}$ & 0.2 & & & & & & 923 & & \\
\hline SALAMHOWIAC & 95.0 & $8.410 E+01$ & & & & & 298 & & \\
\hline $\mathrm{NH}_{4} \mathrm{Cl}$ & 0.4 & & & & & & & & \\
\hline HALITE & 72.1 & $4.515 E+01$ & $1.797 E-02$ & & & & 298 & & \\
\hline $\mathrm{NaCl}$ & 0.2 & & & & & & 1074 & & \\
\hline HALITE & 170.3 & $7.2008 E+01$ & $-3.2228 E-03$ & & & & 1074 & & \\
\hline MaCl (liquid) & 2.2 & & & & & & 1791 & & \\
\hline NICKELOUS CHLORIDE & 97.7 & $-5.078 E+01$ & $5.748 E-02$ & $-4.064 E+06$ & $2.608 E+03$ & & 298 & & \\
\hline $\mathrm{NiCl}_{2}$ & 0.2 & & & & & & 1303 & & \\
\hline COTUNNITE & 136.0 & $1.229 E+02$ & & & $-8.585 E+02$ & & 298 & & \\
\hline $\mathrm{PbCl}_{2}$ & 2.1 & & & & & & 768 & & \\
\hline
\end{tabular}


COEFFICIENTS FOR MEAT CAPACITY EOUATIONS FOR MALIDE MIMERALS

\begin{tabular}{|c|c|c|c|c|c|c|c|c|c|}
\hline NAME AMD FORMULA & $\begin{array}{c}\text { ENTROPY } \\
J \cdot \text { mol }^{-1} \cdot K^{\prime}\end{array}$ & $A 1$ & $\begin{array}{l}A 2 \\
(T)\end{array}$ & $\begin{array}{r}A 3 \\
\left(T^{-2}\right)\end{array}$ & $\begin{array}{r}1.4 \\
\left(T^{-0.5}\right)\end{array}$ & $\begin{array}{r}A 5 \\
\left(T^{2}\right)\end{array}$ & $\begin{array}{c}T \text { rance } \\
K\end{array}$ & $\mathbf{T}_{\mathrm{tm}}$ & $\begin{array}{c}\Delta_{\mathrm{tm}} \mathrm{H}^{\bullet} \\
\mathrm{kJ} \cdot \mathrm{mol}^{-1}\end{array}$ \\
\hline FLUORITE & 68.9 & $2.033 E+03$ & $-1.436 E+00$ & $2.988 E+07$ & $-3.312 E+04$ & $5.040 E-04$ & 298 & & \\
\hline $\mathrm{CaF}_{2}$ & 0.3 & & & & & & 1400 & & \\
\hline HYDROCEN FLUORIDE & 173.8 & $6.845 E+00$ & $1.301 E-02$ & $-4.108 E+05$ & 4.007E+02 & $-1.911 E-06$ & 298 & & \\
\hline HF (ideal gas) & 0.04 & & & & & & 2500 & & \\
\hline Sellaite & 57.3 & $9.474 E+01$ & $1.595 E-04$ & $-7.328 E+05$ & $-4.322 E+02$ & & 298 & 1536 & 58.16 \\
\hline $\mathrm{MgF}_{2}$ & 0.4 & & & & & & 1536 & & \\
\hline VILLIAUMITE & 51.5 & $-2.612 E+00$ & $3.347 E-02$ & $-1.402 E+06$ & $9.542 E+02$ & & 298 & 1269 & 33.14 \\
\hline NaF & 0.1 & & & & & & 1269 & & \\
\hline CRYOLITE & 238.5 & $4.04227 E+03$ & $-3.8582 E+00$ & $3.4071 E+07$ & $-5.5876 E+04$ & 2.004E-03 & 298 & 836 & 8.20 \\
\hline $\mathrm{Na}_{3} \mathrm{AlF} / 6$ & 0.5 & & & & & & 836 & & \\
\hline CRYOLITE & 512.4 & $2.3669 E+02$ & 4.7986E-02 & & & & 836 & 1153 & 1.8 \\
\hline $\mathrm{Na}_{3} \mathrm{AlF} / 6$ & 2.5 & & & & & & 1153 & & \\
\hline CRYOLITE & 605.5 & $3.0041 E+02$ & & & & & 1153 & 1290 & 113.75 \\
\hline $\mathrm{Na}_{3} \mathrm{Alf} \mathrm{F}_{6}$ & 2.5 & & & & & & 1290 & & \\
\hline CHIOLITE & 515.3 & $5.099 E+02$ & 8.768E-02 & $-7.301 E+06$ & & & 298 & 1010 & 80.83 \\
\hline $\mathrm{Na}_{5} \mathrm{Al}_{3} \mathrm{~F}_{14}$ & 0.8 & & & & & & 1010 & & \\
\hline IODARGYRI TE & 115.5 & $2.4351 E+01$ & $1.0083 E-02$ & & & & 298 & & \\
\hline AgI & 1.7 & & & & & & 423 & & \\
\hline MARSHITE & 96.6 & $-1.6941 E+03$ & $1.0046 E+00$ & $-2.8543 E+07$ & $3.0558 E+04$ & & 298 & & \\
\hline Cul & 0.5 & & & & & & 643 & & \\
\hline
\end{tabular}


COEFFICIENTS FOR HEAT CAPACITY EQUATIOWS FOR CARBOWATE AND MITRATE MIMERALS

\begin{tabular}{|c|c|c|c|c|c|c|c|c|c|}
\hline MAME AND FORINLA & $\begin{array}{l}\text { ENTROPY } \\
J \cdot \mathrm{mol}^{-1} \cdot \mathrm{K}^{\prime}\end{array}$ & A1 & $\begin{array}{r}A 2 \\
(T)\end{array}$ & $\begin{array}{r}A 3 \\
\left(r^{-2}\right)\end{array}$ & $\begin{array}{c}14 \\
\left(T^{-0.5}\right)\end{array}$ & $\begin{array}{r}15 \\
\left(T^{2}\right)\end{array}$ & $\begin{array}{c}T \text { renge } \\
K\end{array}$ & $\underset{K}{T_{\text {tim }}}$ & 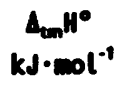 \\
\hline WITHERITE & 112.1 & $-9.936 E+01$ & $1.258 E-01$ & $-6.274 E+06$ & $3.760 E+03$ & & 298 & & \\
\hline $\mathrm{BaCO}_{3}$ & 2.1 & & & & & & 1079 & & \\
\hline ARAGONITE & 88.0 & $8.153 E+01$ & 4.567E-02 & $-1.141 E+06$ & & & 298 & & \\
\hline $\mathrm{CaCO}_{3}$ & 0.2 & & & & & & 1000 & & \\
\hline CALCITE & 91.7 & 9.972E+01 & $2.692 E-02$ & $-2.158 E+06$ & & & 298 & & \\
\hline $\mathrm{CaCO}_{3}$ & 0.2 & & & & & & 1200 & & \\
\hline DOLONITE & 155.2 & $5.479 E+02$ & $-1.676 E-01$ & $2.840 E+06$ & $-6.548 E+03$ & 7.708E-05 & 298 & & \\
\hline $\mathrm{CaMg}\left(\mathrm{CO}_{3}\right)_{2}$ & 0.3 & & & & & & 1100 & & \\
\hline SIDERITE & 95.5 & $2.574 E+02$ & $-4.620 E-02$ & $1.523 E+06$ & $-3.082 E+03$ & & 298 & & \\
\hline $\mathrm{FeCO}_{3}$ & 0.2 & & & & & & 600 & & \\
\hline MGENESITE & 65.1 & $8.112 E+01$ & 5.225E-02 & $-1.832 E+06$ & & & 298 & & \\
\hline $\mathrm{AgCO}_{3}$ & 0.1 & & & & & & 1000 & & \\
\hline RHODOCHROSITE & 98.0 & $1.497 E+02$ & $1.876 E-02$ & $1.417 E+05$ & $-1.3142 E+03$ & & 298 & & \\
\hline $\mathrm{AnCO}_{3}$ & 0.1 & & & & & & 600 & & \\
\hline THERMONATRITE & 168.1 & $7.240 E+01$ & 2.607E-01 & $-1.258 E+05$ & & & 298 & & \\
\hline $\mathrm{Na}_{2} \mathrm{CO}_{3} \cdot \mathrm{H}_{2} \mathrm{O}$ & 0.8 & & & & & & 380 & & \\
\hline DAUSONITE & 132.0 & $3.441 E+01$ & $3.347 E-04$ & $7.464 E+02$ & & & 298 & & \\
\hline $\mathrm{NaAl}\left(\mathrm{CO}_{3}(\mathrm{OH})_{2}\right.$ & 0.5 & & & & & & 500 & & \\
\hline STRONTIANITE & 97.1 & $-1.618 E+02$ & $1.2795 E-01$ & $-9.018 E+06$ & $5.294 E+03$ & & 298 & 1197 & 18.82 \\
\hline $\mathrm{SrCO}_{3}$ & 1.7 & & & & & & 1197 & & \\
\hline SMITHSONITE & 81.2 & $1.4838 E+02$ & $2.835 E-02$ & $4.796 E+05$ & $-1.419 E+03$ & & 298 & & \\
\hline $\mathrm{ZnCO}_{3}$ & 0.2 & & & & & & 1200 & & \\
\hline NITROBARITE & 213.8 & $1.255 E+02$ & 1.497E-01 & $-1.670 E+06$ & & & 298 & & \\
\hline $\mathrm{Ba}\left(\mathrm{NO}_{3}\right)_{2}$ & 0.8 & & & & & & 800 & & \\
\hline CALCIUM MITRATE & 193.3 & $7.815 E+01$ & $1.734 E-01$ & $-2.778 E+06$ & $8.722 E+02$ & & 298 & & \\
\hline $\mathrm{Ca}\left(\mathrm{NO}_{3}\right)_{2}$ & 0.4 & & & & & & 800 & & \\
\hline MAGNESIUM MITRATE & 164.0 & $5.8417 E+01$ & 2.7292E-01 & $1.8949 E+05$ & & & 298 & & \\
\hline $\mathrm{Mg}\left(\mathrm{NO}_{3}\right)_{2}$ & 0.8 & & & & & & 600 & & \\
\hline SOOA MITER & 116.5 & $2.570 E+01$ & $2.237 E-01$ & & $1.161 E+01$ & & 298 & & \\
\hline $\mathrm{NaNO}_{3}$ & 0.7 & & & & & & 549 & & \\
\hline STROWTIUN MITRATE & 194.6 & $1.3389 E+03$ & $-3.79 E-01$ & $2.5757 E+07$ & $-2.3582 E+04$ & & 298 & & \\
\hline $\mathrm{Sr}\left(\mathrm{NO}_{3}\right)_{2}$ & 0.5 & & & & & & 900 & & \\
\hline
\end{tabular}




\begin{tabular}{|c|c|c|c|c|c|c|c|c|c|}
\hline MAME AMD FORMULA & $\begin{array}{c}\text { ENTROPY } \\
J \cdot \mathrm{nol}^{-1} \cdot K^{-1}\end{array}$ & $A 1$ & $\begin{array}{l}A 2 \\
(T)\end{array}$ & $\begin{array}{r}A 3 \\
\left(T^{-2}\right)\end{array}$ & $\begin{array}{c}N_{4} \\
\left(T^{-0.5}\right)\end{array}$ & $\begin{array}{r}A 5 \\
\left(T^{2}\right)\end{array}$ & $\begin{array}{c}T \text { range } \\
K\end{array}$ & $T_{\mathrm{tm}}$ & $\begin{array}{c}\Delta_{2 m} H^{\bullet} \\
k J \cdot \operatorname{mol}^{-1}\end{array}$ \\
\hline ALUMIMUN SULFATE & 239.3 & $7.877 \mathrm{E}+02$ & $-9.899 E-02$ & & $-8.615 E+03$ & & 298 & & \\
\hline $\mathrm{Al}_{2}\left(\mathrm{SO}_{4}\right)_{3}$ & 1.2 & & & & & & 1100 & & \\
\hline BARITE & 132.2 & $1.412 E+02$ & & & $-3.507 E+06$ & & 298 & & \\
\hline $\mathrm{BaSO}_{4}$ & 0.8 & & & & & & 1300 & & \\
\hline ANHYDRITE & 107.4 & $3.728 E+02$ & $-1.574 E-01$ & $1.695 E+06$ & $-4.3308 E+03$ & $7.99 E-05$ & 298 . & & \\
\hline $\mathrm{CaSO}_{4}$ & 0.2 & & & & & & 1000 & & \\
\hline CHALCOCYANITE & 109.5 & $1.615 E+02$ & 4.796E-02 & $-3.192 E+06$ & & & 298 & & \\
\hline $\mathrm{CuSO}_{4}$ & 0.6 & & & & & & 1200 & & \\
\hline FERRIC SULFATE & 282.8 & $2.553 E+02$ & 2.269E-01 & $-4.260 E+06$ & & & 298 & & \\
\hline $\mathrm{Fe}_{2}\left(\mathrm{SO}_{4}\right)_{3}$ & 0.8 & & & & & & 800 & & \\
\hline ARCANITE & 175.6 & $1.2037 E+02$ & 9.958E-02 & $-1.782 E+06$ & & & 298 & & \\
\hline$a-K_{2} \mathrm{SO}_{4}$ & 0.4 & & & & & & 856 & & \\
\hline ARCANITE & 360.9 & $-8.445 E+02$ & $6.845 E-01$ & $3.499 E+08$ & & & 856 & & \\
\hline $\mathrm{B}-\mathrm{K}_{2} \mathrm{SO}_{4}$ & 1.0 & & & & & & 1342 & & \\
\hline K-AI SULFATE & 204.6 & $2.370 E+02$ & $7.828 E-02$ & $-5.988 E+06$ & & & 298 & & \\
\hline $\operatorname{Kal}\left(\mathrm{SO}_{4}\right)_{2}$ & 1.3 & & & & & & 1000 & & \\
\hline ALUNITE & 321.0 & $5.145 E+03$ & 1.13ד万E-01 & $-1.5635 E+07$ & & & 298 & & \\
\hline $\mathrm{Kal}_{3}(\mathrm{OH})_{6}\left(\mathrm{SO}_{4}\right)_{2}$ & $\mathbf{5 . 0}$ & & & & & & 700 & & \\
\hline LANGBEINITE & 389.3 & $5.359 E+02$ & $1.101 E-01$ & $-1.020 E+06$ & $-4.040 E+03$ & $-4.909 E-05$ & 298 & & \\
\hline $\mathrm{K}_{2} \mathrm{Nig}_{2}\left(\mathrm{SO}_{4}\right)_{3}$ & 0.8 & & & & & & 1000 & & \\
\hline MAGNESIUN SULFATE & 91.4 & $1.056 E+02$ & 4.717E-02 & $-2.129 E+06$ & & & 298 & & \\
\hline $\mathrm{MgSO}_{4}$ & 0.8 & & & & & & 1400 & & \\
\hline maNGANOUS SULfate & 127.0 & $1.203 E+02$ & 3.999E-02 & $-2.818 E+06$ & $\cdot$ & & 298 & & \\
\hline $\mathrm{MnSO}_{4}$ & 2.0 & & & & & & 1000 & & \\
\hline MASCAGNITE & 220.5 & $1.0436 E+02$ & $2.788 E-01$ & & & & 298 & & \\
\hline$\left(\mathrm{NH}_{4}\right)_{2} \mathrm{SO}_{4}$ & 1.3 & & & & & & 600 & & \\
\hline THENARDITE & 239.7 & $1.2193 E+02$ & 8.141E-02 & & & & 514 & & \\
\hline $\mathrm{Na}_{2} \mathrm{SO}_{4}$ (hexagonal) & 1.0 & & & & & & 1155 & & \\
\hline THEMARDITE & 424.6 & $2.011 E+02$ & $-3.941 E-03$ & & & & 1155 & & \\
\hline $\mathrm{Na}_{2} \mathrm{SO}_{4}$ (liquid) & 2.0 & & & & & & 1800 & & \\
\hline MICKELOUS SULFATE & 101.3 & $1.240 E+02$ & $3.062 E-02$ & $-3.150 E+06$ & & & 298 & & \\
\hline $\mathrm{NiSO}_{4}$ & 0.2 & & & & & & 1200 & & \\
\hline
\end{tabular}


COEFFICIENTS FOR MEAT CAPACITY EQUATIONS FOR SULFATE ANO PHOSPHATE MIMERALS

\begin{tabular}{|c|c|c|c|c|c|c|c|c|c|}
\hline MAME AND FORINLA & $\begin{array}{l}\text { EMTROPY } \\
J \cdot \mathrm{mol}^{-1} \cdot \mathrm{K}^{\prime}\end{array}$ & A1 & $\begin{array}{l}A 2 \\
(T)\end{array}$ & $\begin{array}{r}13 \\
\left(T^{-2}\right)\end{array}$ & $\begin{array}{c}1.4 \\
\left(T^{-0.5}\right)\end{array}$ & $\begin{array}{r}A 5 \\
\left(T^{2}\right)\end{array}$ & $\begin{array}{c}T \text { rence } \\
K\end{array}$ & $T_{\mathrm{tm}}$ & $\begin{array}{c}\Delta_{\mathrm{mm}} \mathrm{H}^{\bullet} \\
\mathrm{kJ} \cdot \mathrm{mol}^{-1}\end{array}$ \\
\hline NGLLSITE & 148.5 & $4.683 E+01$ & $1.278 E-01$ & $1.724 E+06$ & & & 298 & & \\
\hline $\mathrm{PbSO}_{4}$ & 0.6 & & & & & & 1100 & & \\
\hline ZINKOSITE & 110.5 & $4.019 E+01$ & $1.243 E-01$ & $2.389 E+06$ & & & 298 & & \\
\hline $\mathrm{ZnSO}_{4}$ & 1.3 & & & & & & 1100 & & \\
\hline BERLINITE & 90.8 & $1.9833 E+03$ & $-1.64948 E+00$ & $2.0607 E+07$ & $-2.93193 E+04$ & $7.72 E-04$ & 298 & & \\
\hline $\mathrm{AlPO}_{4}$ & 0.2 & & & & & & 830 & & \\
\hline WHITLOCKITE & 236.0 & $1.929 E+02$ & $1.742 E-01$ & $-1.174 E+06$ & & & 298 & & \\
\hline $\mathrm{Co}_{3}\left(\mathrm{PO}_{4}\right)_{2}$ & 0.8 & & & & & & 1373 & & \\
\hline FLUORAPATITE & 387.9 & $7.543 E+02$ & $-3.026 E-02$ & $-9.084 E+05$ & $-6.201 E+03$ & & 298 & & \\
\hline $\mathrm{Ca}_{5}\left(\mathrm{PO}_{4}\right)_{3}(\mathrm{~F})$ & 1.7 & & & & & & 1600 & & \\
\hline MYDROXYAPATITE & 390.4 & $3.878 E+02$ & $1.186 E-01$ & $-1.270 E+07$ & $1.811 E+03$ & & 298 & & \\
\hline $\mathrm{Co}_{5}\left(\mathrm{PO}_{4}\right)_{3}(\mathrm{OH})$ & 1.7 & & & & & & 1500 & & \\
\hline
\end{tabular}




\begin{tabular}{|c|c|c|c|c|c|c|c|c|c|}
\hline MNE AND FORUULA & $\begin{array}{l}\text { ENTROPY } \\
\cdot \mathrm{mol}^{-1} \cdot K^{-1}\end{array}$ & $\mathbf{A 1}$ & $\begin{array}{l}12 \\
(T)\end{array}$ & $\begin{array}{r}13 \\
\left(T^{-2}\right)\end{array}$ & $\left(T^{-0.5}\right)$ & $\begin{array}{r}15 \\
\left(T^{2}\right)\end{array}$ & $\begin{array}{c}T \text { range } \\
K\end{array}$ & $\mathbf{T}_{\mathrm{tm}}$ & $\begin{array}{c}\Delta_{\min } H^{\circ} \\
k J \cdot \operatorname{mol}^{-1}\end{array}$ \\
\hline TOPAZ & 105.4 & 4.714E+02 & $-8.165 E-02$ & $1.270 E+06$ & $-5.486 E+03$ & & 298 & & \\
\hline $\mathrm{Al}_{2} \mathrm{SiO}_{4} \mathrm{~F}_{2}$ & 0.2 & & & & & & 1000 & & \\
\hline KYANITE & 82.8 & $2.794 E+02$ & $-7.124 E-03$ & $-2.056 E+06$ & $-2.289 E+03$ & & 298 & & \\
\hline $\mathrm{Al}_{2} \mathrm{SiO}_{5}$ & 0.5 & & & & & & 2000 & & \\
\hline ANDALUSITE & 91.4 & $2.77306 E+02$ & $-6.588 E-03$ & $-1.9141 E+06$ & $-2.2656 E+03$ & & 298 & & \\
\hline $\mathrm{Al}_{2} \mathrm{SiO}_{5}$ & 0.1 & & & & & & 2000 & & \\
\hline SILLIMANITE & 95.4 & $2.8019 E+02$ & $-6.900 E-03$ & $-1.376 E+06$ & $-2.399 E+03$ & & 298 & & \\
\hline $\mathrm{Al}_{2} \mathrm{SiO}_{5}$ & 0.6 & & & & & & 2000 & & \\
\hline MULLITE & 275.0 & $7.546 E+02$ & $-2.943 E-02$ & $-3.454 E+06$ & $-6.576 E+03$ & & 298 & & \\
\hline $\mathrm{Al}_{6} \mathrm{Si}_{2} \mathrm{O}_{13}$ & 5.0 & & & & & & 1800 & & \\
\hline DUMORTIERITE & 334.9 & $1.43871 E+03$ & $-2.427 E-01$ & $3.860 E+06$ & $-1.682 E+04$ & 4.884E-05 & 298 & & \\
\hline${ }^{A} l_{6.75}{ }^{\mathrm{d}} .25^{\mathrm{Si}} \mathrm{B}^{\mathrm{BO}} 17.25^{(\mathrm{OH})} .75$ & $5 \quad 5.0$ & & & & & & 1800 & & \\
\hline PHENAKITE & 63.4 & $4.2849 E+02$ & $-9.958 E-02$ & $2.083 E+06$ & $-5.6705 E+03$ & 1.989E-05 & 298 & & \\
\hline $\mathrm{Be}_{2} \mathrm{SiO}_{4}$ & 0.3 & & & & & & 1800 & & \\
\hline BERYL & 346.7 & $1.625 E+03$ & $-4.252 E-01$ & $6.825 E+06$ & $-2.018 E+04$ & $1.203 E-04$ & 298 & & \\
\hline $\mathrm{Be}_{3} A \mathrm{I}_{2}\left(\mathrm{Si}_{6} \mathrm{O}_{18}\right)$ & 4.7 & & & & & & 1800 & & \\
\hline BERTRANDITE & 172.1 & $8.253 E+02$ & $-9.965 E-02$ & $3.662 E+06$ & $-1.057 E+04$ & & 298 & & \\
\hline $\mathrm{Be}_{4} \mathrm{Si}_{2} \mathrm{O}_{7}(\mathrm{OH})_{2}$ & 0.8 & & & & & & 1400 & & \\
\hline EUCLASE & 89.1 & 5.329E+02 & $-1.507 E-01$ & $2.198 E+06$ & $-6.726 E+03$ & $4.122 E-05$ & 298 & & \\
\hline $\mathrm{BeAlSiO}_{4}$ (OH) & 0.4 & & & & & & 1800 & & \\
\hline EPIDOTE & 328.9 & $3.676 E+02$ & & & & & 298 & & \\
\hline $\mathrm{Ca}_{2} \mathrm{Al}_{2} \mathrm{Fe}_{2} \mathrm{Si}_{3} \mathrm{O}_{12}(\mathrm{OH})$ & 2.5 & & & & & & & & \\
\hline LAUSONITE & 230.0 & $2.7732 E+02$ & $2.341 E-01$ & $-6.389 E+06$ & & & 298 & & \\
\hline $\mathrm{CaAl}_{2} \mathrm{Si}_{2} \mathrm{O}_{7}(\mathrm{OH})_{2} \mathrm{H}_{2} \mathrm{O}$ & 0.4 & & & & & & 600 & & \\
\hline GEHLENITE & 210.1 & $4.057 E+02$ & $-7.099 E-03$ & $-1.188 E+06$ & $-3.174 E+03$ & & 298 & & \\
\hline $\mathrm{Ca}_{2} \mathrm{Al}_{2} \mathrm{SiO}_{7}$ & 0.6 & & & & & & 1800 & & \\
\hline ZOISITE & 295.9 & $1.134 E+03$ & $-4.523 E-01$ & & $-1.157 E+04$ & 2.391E-04 & 298 & & \\
\hline $\mathrm{Ca}_{2} \mathrm{Al}_{3} \mathrm{Si}_{3} \mathrm{O}_{12}(\mathrm{OH})$ & 0.3 & & & & & & 750 & & \\
\hline GROSSULAR & 260.1 & $1.5293 E+03$ & $-6.990 E-01$ & $7.443 E+06$ & $-1.894 E+04$ & $2.530 E-04$ & 298 & & \\
\hline $\mathrm{Ca}_{3} \mathrm{Al} \mathrm{I}_{2} \mathrm{Si}_{3} \mathrm{O}_{12}$ & 0.5 & & & & & & 1200 & & \\
\hline GLASS & 329.9+ & $3.392 E+02$ & & & & & 298 & & \\
\hline $\mathrm{Co}_{3} \mathrm{Al} \mathrm{I}_{2} \mathrm{Si}_{3} \mathrm{O}_{12}$ & 0.7 & & & & & & & & \\
\hline
\end{tabular}


COEFFICIENTS FOR HEAT CAPACITY EQUATIONS FOR ORTHO AND RING STRUCTURE SILICATE MIMERALS

\begin{tabular}{|c|c|c|c|c|c|c|c|c|c|}
\hline MAME AND FORIULLA & $\begin{array}{l}\text { EUTROPY } \\
J \cdot \mathrm{mol}^{-1} \cdot K^{\prime}\end{array}$ & A1 & $\begin{array}{r}A 2 \\
\text { (T) }\end{array}$ & $\begin{array}{r}\mathbf{R}^{3} \\
\left(T^{-2}\right)\end{array}$ & $\left(T^{-0.5}\right)$ & $\begin{array}{r}A 5 \\
\left(T^{2}\right)\end{array}$ & $\begin{array}{c}\text { T range } \\
K\end{array}$ & $\begin{array}{r}\mathbf{T}_{\mathrm{tm}} \\
\mathbf{K}\end{array}$ & $\begin{array}{c}\mathbf{A}_{\mathrm{tm}} \mathrm{H}^{\bullet} \\
\mathrm{kJ} \cdot \mathrm{mol}^{-1}\end{array}$ \\
\hline DATOLITE & 110.2 & $1.9446 E+02$ & $1.8447 E-01$ & $4.0191 E+05$ & $-2.0305 E+03$ & $-1.01 E-04$ & 298 & & \\
\hline $\mathrm{CaB}\left(\mathrm{SiO}_{4}\right)(\mathrm{OH})$ & 0.6 & & & & & & 1000 & & \\
\hline ILVAITE & 292.3 & $8.569 E+03$ & $-5.2407 E+00$ & $9.6449 E+07$ & $-1.3456 E+05$ & & 290 & & \\
\hline $\mathrm{CaFe}_{3}\left(\mathrm{Si}_{2} \mathrm{O}_{7}\right) \mathrm{O}(\mathrm{OH})$ & 0.6 & & & & & & 376 & & \\
\hline AMDRADITE & 316.4 & $8.092 E+02$ & $-7.025 E-02$ & $-6.789 E+05$ & $-7.403 E+03$ & & 298 & & \\
\hline $\mathrm{Ce}_{3} \mathrm{Fe}_{2} \mathrm{Si}_{3} \mathrm{O}_{12}$ & 2.0 & & & & & & 1000 & & \\
\hline MONTICELLITE & 108.1 & $2.314 E+02$ & $-8.531 E-04$ & $-1.247 E+06$ & $-1.623 E+03$ & $-1.333 E-06$ & 298 & & \\
\hline $\mathrm{CaMgSiO}_{4}$ & 0.3 & & & & & & 1000 & & \\
\hline AKERMANITE & 212.5 & $7.8146 E+01$ & 1.6573E-01 & $-6.7905 E+06$ & $2.8646 E+03$ & $-3.3437 E-05$ & 298 & 357.9 & 0.68 \\
\hline $\mathrm{Ca}_{2} \mathrm{MgSi}_{2} \mathrm{O}_{7}$ & 0.4 & & & & & & 1731 & & \\
\hline MERUINITE & 253.1 & $6.508 E+02$ & $-1.816 E-01$ & & $-6.053 E+03$ & $7.036 E-05$ & 298 & & \\
\hline $\mathrm{Co}_{3} \mathrm{Mg}\left(\mathrm{SiO}_{4}\right)_{2}$ & 2.1 & & & & & & 1600 & & \\
\hline IITANITE (Sphene) & 129.2 & $1.767 E+02$ & $2.385 E-02$ & $-3.991 E+06$ & & & 298 & & \\
\hline CaTiSiO $_{5}$ & 0.8 & & & & & & 1670 & & \\
\hline LARNITE & 127.6 & $2.487 E+02$ & $-8.315 E-04$ & $-9.077 \mathrm{E}+04$ & $-2.052 E+03$ & & 298 & & \\
\hline $\mathrm{a}-\mathrm{Ca}_{2} \mathrm{SiO}_{4}$ & 0.8 & & & & & & 970 & & \\
\hline BREDIGITE & 314.2 & $1.3456 E+02$ & 4.611E-02 & & & & 970 & & \\
\hline $\mathrm{a}^{\prime}-\mathrm{Ca}_{2} \mathrm{SiO}_{4}$ & 1.0 & & & & & & 1710 & & \\
\hline CALCIO-OLIVINE & 120.5 & $1.327 E+02$ & 5.251E-02 & $-1.905 E+06$ & & & 298 & & \\
\hline$\gamma-\mathrm{Ca}_{2} \mathrm{SiO}_{4}$ & 0.8 & & & & & & 1200 & & \\
\hline HATURITE (ALITE) & 168.6 & $3.339 E+02$ & $-4.651 E-03$ & $-6.526 E+04$ & $-2.766 E+03$ & & 298 & & \\
\hline $\mathrm{Ca}_{3} \mathrm{SiO}_{5}$ & 0.3 & & & & & & 1800 & & \\
\hline RANKINITE & 210.6 & $4.732 E+02$ & $-4.20 \pi E-02$ & $3.397 E+05$ & $-4.319 E+03$ & & 298 & & \\
\hline $\mathrm{Ca}_{3} \mathrm{Si}_{2} \mathrm{O}_{7}$ & 2.9 & & & & & & 1400 & & \\
\hline ROSENHAHNITE & 281.8 & $3.650 E+02$ & 5.419E-02 & $-8.161 E+06$ & & & 298 & & \\
\hline $\mathrm{Ca}_{3} \mathrm{Si}_{3} \mathrm{O}_{8}(\mathrm{OH})_{2}$ & 3.0 & & & & & & 1800 & & \\
\hline SPURRITE & 331.0 & 6.141E+02 & $-3.508 E-03$ & $-2.493 E+06$ & $-4.168 E+03$ & & 298 & & \\
\hline $\mathrm{Ca}_{5}\left(\mathrm{SiO}_{4}\right)_{2} \mathrm{CO}_{3}$ & 2.0 & & & & & & 1300 & & \\
\hline TILLEYITE & 394.0 & $7.417 E+02$ & $-5.345 E-03$ & $-1.435 E+06$ & $-5.879 E+03$ & & 298 & & \\
\hline $\mathrm{Ca}_{5} \mathrm{Si}_{2} \mathrm{O}_{7}\left(\mathrm{CO}_{3}\right)_{2}$ & 4.0 & & & & & & 1200 & & \\
\hline COBALT-OLIVINE & 142.6 & $3.0583 E+02$ & $-3.195 E-02$ & $2.693 E+05$ & $-2.865 E+03$ & & 298 & & \\
\hline $\mathrm{CO}_{2} \mathrm{SiO}_{4}$ & 0.2 & & & & & & 1100 & & \\
\hline
\end{tabular}




\begin{tabular}{|c|c|c|c|c|c|c|c|c|c|}
\hline MAME ANO FORMULA & $\begin{array}{l}\text { ENTROPY } \\
\mathrm{J} \cdot \mathrm{mol}^{-1} \cdot \mathrm{K}^{1}\end{array}$ & A1 & $\begin{array}{l}A 2 \\
(T)\end{array}$ & $\begin{array}{r}\mathbf{A 3} \\
\left(T^{-2}\right)\end{array}$ & $\begin{array}{c}14 \\
\left(T^{-0.5}\right)\end{array}$ & $\begin{array}{r}15 \\
\left(T^{2}\right)\end{array}$ & $\begin{array}{c}\text { T rence } \\
K\end{array}$ & $\underset{\mathrm{T}}{\mathrm{T}_{\mathrm{mm}}}$ & $\begin{array}{c}\mathrm{Atm}_{\mathrm{tm}} \mathrm{H}^{\circ} \\
\mathrm{kJ} \cdot \mathrm{mol}^{-1}\end{array}$ \\
\hline FAYALITE & 151.0 & $1.7602 E+02$ & $-8.808 E-03$ & $-3.889 E+06$ & & 2.471E-05 & 298 & 1490 & \\
\hline $\mathrm{Fe}_{2} \mathrm{SiO}_{4}$ & 0.2 & & & & & & 1490 & & \\
\hline ALMANDIME & 342.6 & $8.629 E+02$ & $-8.288 E-02$ & $1.697 E+06$ & $-8.875 E+03$ & & 298 & & \\
\hline $\mathrm{Fe}_{3} \mathrm{Al}_{2} \mathrm{Si}_{3} \mathrm{O}_{12}$ & 1.4 & & & & & & 1200 & & \\
\hline STAUROLITE & 985.0 & $2.798 E+03$ & $7.921 E-02$ & $-9.952 E+06$ & $-2.474 E+04$ & & 298 & & \\
\hline $\mathrm{Fe}_{4} \mathrm{Al}_{18} \mathrm{Si}_{8} \mathrm{O}_{46}(\mathrm{OH})_{2}$ & 4.0 & & & & & & 1000 & & \\
\hline OSUMILITE & & $5.842 E+02$ & $1.276 E+00$ & $-3.967 E+06$ & $-1.556 E+03$ & $-6.497 E-04$ & 298 & & \\
\hline 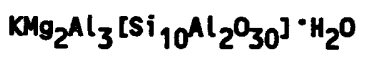 & & & & & & & 1000 & & \\
\hline CORDIERITE & 407.2 & 8.123E+02 & 4.334E-02 & $-8.211 E+06$ & $-5.000 E+03$ & & 298 & & \\
\hline $\mathrm{Mg}_{2} \mathrm{Al}_{3}\left(\mathrm{AlSi}_{5} \mathrm{O}_{18}\right)$ & 3.8 & & & & & & 1700 & & \\
\hline FORSTERITE & 94.1 & 8.736E+01 & 8.717E-02 & $-3.699 E+06$ & $8.436 E+02$ & $-2.237 \mathrm{TE}-05$ & 298 & & \\
\hline $\mathrm{Mg}_{2} \mathrm{SiO}_{4}$ & 0.1 & & & & & & 1800 & & \\
\hline PYROPE & 266.3 & $8.730 E+02$ & $-1.374 E-01$ & $4.500 E+03$ & $-8.794 E+03$ & $3.341 E-05$ & 298 & 1570 & 241.0 \\
\hline $\mathrm{Mg}_{3} \mathrm{Al}{ }_{2} \mathrm{Si}_{3} \mathrm{O}_{12}$ & 0.8 & & & & & & 1570 & & \\
\hline GLASS & 346.3 & $5.138 E+02$ & $7.119 E-02$ & $-5.857 E+05$ & $-2.439 E+03$ & & 298 & & \\
\hline $\mathrm{Mg}_{3} \mathrm{Al}_{2} \mathrm{Si}_{3} \mathrm{O}_{12}$ & 12.0 & & & & & & 1020 & & \\
\hline GLASS & & $6.300 E+02$ & $3.50 E-02$ & & & & 1083 & & \\
\hline $\mathrm{Mg}_{3} \mathrm{Al}_{2} \mathrm{Si}_{3} \mathrm{O}_{12}$ & & & & & & & 1863 & & \\
\hline $\begin{array}{l}\text { PYROPE-GROSSULAR Ss } \\
\left(\mathrm{Mg}_{1.8} \mathrm{Ca}_{1.2}\right) \mathrm{Al}_{2} \mathrm{Si}_{3} \mathrm{O}_{12}\end{array}$ & $\begin{array}{c}268.3+ \\
0.5\end{array}$ & $3.280 E+02$ & & & & & 298 & & \\
\hline $\begin{array}{l}\text { GLASS } \\
\left(\mathrm{Mg}_{1.5} \mathrm{Ca}_{1.5}\right) \mathrm{Al}_{2} \mathrm{Si}_{3} \mathrm{O}_{12}\end{array}$ & $\begin{array}{c}311.8+ \\
0.6\end{array}$ & $3.329 E+02$ & & & & & 298 & & \\
\hline TEPHROITE & 155.9 & $2.613 E+02$ & $-1.378 E-02$ & & $-2.218 E+03$ & & 298 & & \\
\hline $\mathrm{Mn}_{2} \mathrm{SiO}_{4}$ & 0.5 & & & & & & 1600 & & \\
\hline LIEBENBERGITE & 128.1 & $2.897 E+02$ & $-2.402 E-02$ & $1.310 E+05$ & $-2.779 E+03$ & & 298 & & \\
\hline $\mathrm{Mi}_{2} \mathrm{SiO}_{4}$ & 0.2 & & & & & & 1300 & & \\
\hline $\mathrm{Ni}_{2} \mathrm{SiO}_{4}$-SPINEL & 124.1 & $1.654 E+02$ & $1.412 E-02$ & $-4.345 E+06$ & & & 350 & & \\
\hline $\mathrm{Ni}_{2} \mathrm{SiO}_{4}$ & 0.4 & & & & & & 800 & & \\
\hline HILLEMITE & 131.4 & $1.233 E+02$ & & & & & 298 & & \\
\hline $\mathrm{Zn}_{2} \mathrm{SiO}_{4}$ & 0.8 & & & & & & & & \\
\hline ZIRCON & 84.0 & $2.370 E+02$ & $-1.788 E-02$ & $-1.496 E+05$ & $-2.268 E+03$ & & 298 & & \\
\hline $\mathrm{ZrSiO}_{4}$ & 1.3 & & & & & & 1600 & & \\
\hline
\end{tabular}


COEFFICIENTS FOR HEAT CAPACITY EQUATIONS FOR CHAIN STRUCTURE SILICATE MIMERALS

NAME AND FORMULA

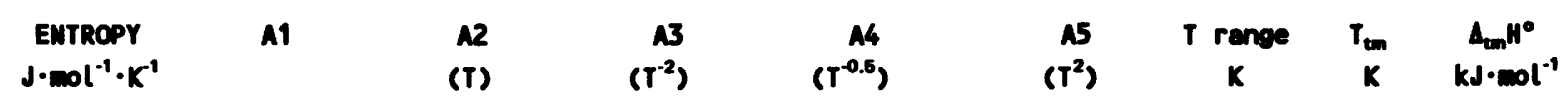

mollastonite $\mathrm{CaSiO}_{3}$

PSELdONOLLASTONITE

$\mathrm{CasiO}_{3}$

GLASS

$\mathrm{CaSiO}_{3}$

Ca-Al pyroxene

$\mathrm{CaAl}_{2} \mathrm{SiO}_{6}$

GLASS

$\mathrm{CaAl}_{2} \mathrm{SiO}_{6}$

FERROBUSTAMITE

CaFeSi $_{2} \mathrm{O}_{6}$

HEDENBERGITE

$\mathrm{CaFeSi}_{2} \mathrm{O}_{6}$

GLASS

CaFesi ${ }_{2} \mathrm{O}_{6}$

DIOPSIDE

$\mathrm{CaMgSi}_{2} \mathrm{O}_{6}$

GLASS

Cangsi ${ }_{2} \mathrm{O}_{6}$

FERROSILITE

$\mathrm{FeSiO}_{3}$

a-SPODUMENE

LiAlsi ${ }_{2} \mathrm{O}_{6}$

8-SPCDUMENE

LiAtsi ${ }_{2} \mathrm{O}_{6}$

ENSTATITE

$\mathrm{MgSiO}_{3}$

GLASS

$\mathrm{MgSiO}_{3}$

ClinOENSTATITE $\mathrm{MgSiO}_{3}$
81.7

0.1

$2.0078 E+02$

$-2.589 E-02$

$-1.579 E+05$

$-1.826 E+03$

7.434E-06

298

1400

$87.2 \quad 1.578 E+02 \quad-1.045 E-03 \quad-6.396 E+05 \quad-1.077 E+03$

0.9

6.396405

298

1821

$94.8 \quad 9.689 E+01 \quad 3.425 E-02 \quad-1.804 E+06$

298

3.0

$4.652 E+02 \quad-7.838 E-02$

$6.729 E+05 \quad-4.941 E+03$

1.934E-05

1821

141.0

2.0

$1.661 E+02$

0.3

180.5

4.038E+02 -4.444E-02

0.3

174.2

3. 1046E+02

1.257E-02 -1.846E+06

$-2.040 E+03$

0.3

$-3.757 E+03$

1.597E-05

298

1600

298

1300

$185.7+$
0.4

142.

0.3

$4.7025 E+02 \quad-9.864 E-02$

$2.454 E+05 \quad-4.823 E+03$

$2.813 E-05$

298

1700

$2 \%$

166.0

$1.041 E+03 \quad-6.358 E-01$

$6.677 E+06-1.351 E+06$

$2.724 E-04$

298

1400

3.0

94.6

$1.243 E+02 \quad 1.454 E-02 \quad-3.378 E+06$

298

0.3

129.3

0.8

$4.212 E+02 \quad-2.401 E-02 \quad 1.910 E+06 \quad-4.776 E+03$

298

1200

154.4

1.2

$3.628 E+02-3.684 E-03$

$-3.435 E+03$

298

1700

66.3

0.1

$3.507 E+02 \quad-1.472 E-01$

$1.769 E+06 \quad-4.296 E+03$

5.826E-05

298

1000

298
$1398 \quad 5.93$

$1821 \quad 57.30$

1821.57 .30




\begin{tabular}{|c|c|c|c|c|c|c|c|c|c|}
\hline WNE AMD FORMLA & $\begin{array}{c}\text { ENTROPY } \\
J \cdot \mathrm{mol}^{-1} \cdot K^{\prime}\end{array}$ & A1 & $\begin{array}{r}12 \\
(T)\end{array}$ & $\begin{array}{r}A 3 \\
\left(T^{-2}\right)\end{array}$ & $\left(T^{-0.5}\right)$ & $\begin{array}{r}15 \\
\left(T^{2}\right)\end{array}$ & $\begin{array}{c}T \text { rance } \\
K\end{array}$ & $\mathbf{T}_{\mathbf{t m}}$ & $\begin{array}{c}\mathrm{A}_{\mathrm{tm}} \mathrm{H}^{\circ} \\
\mathrm{kJ} \cdot \mathrm{mol}^{-1}\end{array}$ \\
\hline $\begin{array}{l}\text { MgsiO } \\
\text { MgSiO }_{3} \text {-ILMENITE }\end{array}$ & $\begin{array}{r}60.4 \\
3.0\end{array}$ & $6.912 E+01$ & 7.330E-02 & $1.037 E+06$ & & & $\begin{array}{l}298 \\
700\end{array}$ & & \\
\hline $\begin{array}{l}\text { HYPERSTHENE } \\
\left(\mathrm{Mg} .85^{\mathrm{Fe}} .15^{)^{3 i O}} 3\right.\end{array}$ & $\begin{array}{c}69.0+ \\
0.2\end{array}$ & $2.079 E+02$ & $-1.489 E-02$ & $1.921 E+05$ & $-2.135 E+03$ & & $\begin{array}{r}298 \\
1000\end{array}$ & & \\
\hline $\begin{array}{l}\text { RHODONITE } \\
\text { Mnsio }\end{array}$ & $\begin{array}{r}100.5 \\
1.0\end{array}$ & $9.904 E+01$ & 1.915E-02 & $-3.041 E+06$ & $2.745 E+02$ & & $\begin{array}{r}298 \\
1500\end{array}$ & & \\
\hline $\begin{array}{l}\text { JADEITE } \\
\text { MaAlsi }{ }_{2}{ }_{6}\end{array}$ & $\begin{array}{r}133.5 \\
1.3\end{array}$ & $3.011 E+02$ & 1.014E-02 & $-2.239 E+06$ & $-2.055 E+03$ & & $\begin{array}{r}298 \\
1300\end{array}$ & & \\
\hline $\begin{array}{l}\text { GLASS } \\
\operatorname{MaAlsi}{ }_{2} \mathrm{O}_{6}\end{array}$ & $\begin{array}{r}170.5 \\
0.4\end{array}$ & $1.663 E+02$ & & & & & 298 & & \\
\hline $\begin{array}{l}\text { ACMITE } \\
\text { NaFesi } 2 \mathrm{O}_{6}\end{array}$ & $\begin{array}{r}170.6 \\
0.8\end{array}$ & $1.994 E+02$ & 6.197E-02 & $-4.267 E+06$ & & & $\begin{array}{r}298 \\
1300\end{array}$ & & \\
\hline $\begin{array}{l}\text { TREMOLITE } \\
\mathrm{Ca}_{2} \mathrm{Hg}_{5} \mathrm{Si}_{8} \mathrm{O}_{22}(\mathrm{OH})_{2}\end{array}$ & $\begin{array}{r}548.9 \\
1.3\end{array}$ & $6.131 E+03$ & $-4.189 E+00$ & $5.139 E+07$ & $-8.566 E+04$ & 1.757E-03 & $\begin{array}{r}298 \\
1100\end{array}$ & & \\
\hline $\begin{array}{l}\text { CRUNERITE } \\
\text { Fe }_{7} \mathrm{Si}_{8} \mathrm{O}_{22}(\mathrm{OH})_{2}\end{array}$ & $\begin{array}{r}725.0 \\
7.0\end{array}$ & $7.555 E+02$ & 3.620E-01 & $-1.621 E+07$ & & & $\begin{array}{l}298 \\
900\end{array}$ & & \\
\hline $\begin{array}{l}\text { ANTHOPHILLITE } \\
\mathrm{Mg}_{7} \mathrm{Si}_{8} \mathrm{O}_{22} \mathrm{CH}_{2}\end{array}$ & $\begin{array}{r}534.5 \\
3.5\end{array}$ & $1.260 E+03$ & $1.888 E-02$ & $-1.164 E+07$ & $-8.127 E+03$ & & $\begin{array}{r}298 \\
1200\end{array}$ & & \\
\hline $\begin{array}{l}\text { RIEBECKITE } \\
\mathrm{Na}_{2} \mathrm{Fe}_{3} \mathrm{Fe}_{2} \mathrm{Si}_{8} \mathrm{O}_{22}(\mathrm{OH})_{2}\end{array}$ & $\begin{array}{r}691.0 \\
6.0\end{array}$ & $7.721 E+02$ & 2.827E-01 & $-1.598 E+07$ & & & $\begin{array}{r}298 \\
1000\end{array}$ & & \\
\hline $\begin{array}{l}\text { GLAUCOPHANE } \\
\mathrm{Ne}_{2} \mathrm{Mg}_{3} \mathrm{Al}{ }_{2} \mathrm{Si}_{8} \mathrm{O}_{22} \mathrm{COH}_{2}\end{array}$ & $\begin{array}{r}541.2 \\
3.0\end{array}$ & $1.718 E+03$ & $-1.211 E-01$ & $7.075 E+06$ & $-1.927 E+04$ & & $\begin{array}{r}298 \\
1200\end{array}$ & & \\
\hline
\end{tabular}


COEFFICIENTS FOR MEAT CAPACITY EQUATIONS FOR FRAMELORK STRUCTURE SILICATE MINERALS

\begin{tabular}{|c|c|c|c|c|c|c|c|c|c|}
\hline NAME AND FORIULLA & $\begin{array}{c}\text { ENTROPY } \\
J \cdot \mathrm{mol}^{-1} \cdot K^{-1}\end{array}$ & A1 & $\begin{array}{c}A 2 \\
(T)\end{array}$ & $\begin{array}{r}{ }^{3} \\
\left(T^{-2}\right)\end{array}$ & $\begin{array}{c}14 \\
\left(T^{-0.5}\right)\end{array}$ & $\begin{array}{r}15 \\
\left(T^{2}\right)\end{array}$ & $\begin{array}{c}T \text { rance } \\
K\end{array}$ & $\mathbf{T}_{\mathrm{tm}}$ & $\begin{array}{c}\Delta_{\mathrm{im}} H^{\circ} \\
\mathrm{kJ} \cdot \mathrm{mol}^{-1}\end{array}$ \\
\hline EDINGTONITE (ordered) & 434.8 & $4.220 E+02$ & & & & & 298 & & \\
\hline $\mathrm{BaAl}_{2} \mathrm{Si}_{3} \mathrm{O}_{10} \cdot 3_{2} \mathrm{O}$ & 1.5 & & & & & & & & \\
\hline ANORTHITE & 199.3 & $5.168 E+02$ & $-9.249 E-02$ & $-1.408 E+06$ & $-4.589 E+03$ & $4.188 E-05$ & 298 & & \\
\hline $\mathrm{CaAl}_{2} \mathrm{Si}_{2} \mathrm{O}_{8}$ & 0.3 & & & & & & 1800 & & \\
\hline $\mathrm{CaAl}{ }_{2} \mathrm{Si}_{2} \mathrm{O}_{8}-\mathrm{glass}$ & 237.3 & $3.752 E+02$ & 3.197E-02 & $-2.815 E+06$ & $-2.459 E+03$ & & 298 & 1830 & 133.0 \\
\hline $\mathrm{CaAl}_{2} \mathrm{Si}_{2} \mathrm{O}_{8}$ & 2.5 & & & & & & 1500 & & 4.0 \\
\hline BICCHULITE & 213.1 & $2.803 E+02$ & 8.204E-02 & $-5.679 E+06$ & & & 298 & & \\
\hline $\mathrm{Ca}_{2} \mathrm{Al}_{2} \mathrm{SiO}_{6}(\mathrm{OH})_{2}$ & $\mathbf{5 . 0}$ & & & & & & 1800 & & \\
\hline MEIONITE (ordered) & 715.2 & $9.103 E+02$ & $1.9435 E-01$ & $-2.300 E+07$ & & & 298 & & \\
\hline $\mathrm{Ca}_{4} \mathrm{Al}_{6} \mathrm{Si}_{6} \mathrm{O}_{24} \mathrm{CO}_{3}$ & 1.0 & & & & & & 1000 & & \\
\hline DANBURITE & 155.3 & $1.488 E+02$ & 2.173E-01 & $-3.280 E+06$ & & & 298 & & \\
\hline $\mathrm{CaB}_{2} \mathrm{Si}_{2} \mathrm{O}_{8}$ & 0.4 & & & & & & 1000 & & \\
\hline POLLUCITE & 207.2 & $1.128 E+02$ & $1.876 E-01$ & $4.556 E+05$ & & & 298 & & \\
\hline$\left(C s .65^{\mathrm{Na}} .19^{\mathrm{Rb}} .03\right) \mathrm{Al}_{2} \mathrm{si}$ & $2^{-\mathrm{H}_{2} \mathrm{O}} \mathrm{O} .4$ & & & & & & 700 & & \\
\hline MICROCLINE & 214.2 & $7.595 E+02$ & $-2.171 E-01$ & $4.746 E+06$ & $-9.527 E+03$ & $6.433 E-05$ & 298 & & \\
\hline $\mathrm{Kalsi} \mathrm{O}_{3}$ & 0.4 & & & & & & 1400 & & \\
\hline SANIDIME & 232.8 & 6.934E+02 & $-1.717 E-01$ & $3.462 E+06$ & $-8.305 E+03$ & 4.919E-05 & 298 & 1500 & 54.0 \\
\hline $\mathrm{Kalsi} \mathrm{O}_{8}$ & 0.5 & & & & & & 1400 & & \\
\hline GLASS & 261.6 & $6.295 E+02$ & $-1.084 E-01$ & $2.496 E+06$ & $-7.210 E+03$ & $-1.928 E-05$ & 298 & & \\
\hline $\mathrm{KAlsi} \mathrm{O}_{8}$ & 1.8 & & & & & & 1300 & & \\
\hline KALIOPHILLITE & 133.3 & $1.889 E+02$ & 5.519E-02 & & $-1.479 E+03$ & & 298 & 810 & 0.5 \\
\hline $\mathrm{KalsiO}_{4}$ & 1.2 & & & & & & 810 & & \\
\hline LEUCITE & 200.2 & $1.4842 E+02$ & $1.343 E-01$ & $-2.165 E+06$ & & & 298 & & \\
\hline $\mathrm{Kalsi} \mathrm{O}_{6}$ & 1.7 & & & & & & 955 & & \\
\hline LEUCITE & 450.1 & $1.9647 E+02$ & $2.7666 \mathrm{E}-02$ & $1.2261 E+07$ & & & 955 & & \\
\hline $\mathrm{kalsi} \mathrm{i}_{2} \mathrm{O}_{6}$ & 2.0 & & & & & & 1800 & & \\
\hline EUCRYPTITE & 103.8 & $2.470 E+02$ & & $-1.290 E+06$ & $-2.058 E+03$ & & 298 & & \\
\hline $\operatorname{LiAlsiO}_{4}$ & 0.8 & & & & & & 1300 & & \\
\hline PETALITE & 233.2 & $8.763 E+02$ & $-2.079 E-01$ & $4.033 E+06$ & $-1.069 E+04$ & 5.301E-05 & 298 & & \\
\hline $\operatorname{LiAlsi}{ }_{4} \mathrm{O}_{10}$ & 0.6 & & & & & & 1300 & & \\
\hline ALBITE & 207.4 & $5.839 E+02$ & $-9.285 E-02$ & $1.678 E+06$ & $-6.424 E+03$ & 2.272E-05 & 298 & & \\
\hline $\operatorname{NaAlSi} \mathrm{O}_{8}$ & 0.4 & & & & & & 1400 & & \\
\hline
\end{tabular}




\begin{tabular}{|c|c|c|c|c|c|c|c|c|c|}
\hline MANE AMD FORMULA & $\begin{array}{c}\text { ENTROPY } \\
J \cdot \mathrm{mol}^{-1} \cdot K^{\prime}\end{array}$ & $\mathbf{A 1}$ & $\begin{array}{r}12 \\
\text { (T) }\end{array}$ & $\begin{array}{r}A 3 \\
\left(T^{-2}\right)\end{array}$ & $\begin{array}{r}M 4 \\
\left(T^{-0.5}\right)\end{array}$ & $\begin{array}{r}A 5 \\
\left(T^{2}\right)\end{array}$ & $\begin{array}{c}T \text { range } \\
K\end{array}$ & $T_{\mathrm{tm}}$ & $\begin{array}{c}A_{\operatorname{tin}} H^{0} \\
k J \cdot m_{0 l}^{-1}\end{array}$ \\
\hline ANALBITE & 225.6 & $6.714 E+02$ & $-1.467 E-01$ & $3.174 E+06$ & $-7.974 E+03$ & $3.659 E-05$ & 298 & 1393 & 64.5 \\
\hline $\mathrm{NaAlsi}{ }_{3} \mathrm{O}_{8}$ & 0.4 & & & & & & 1400 & & \\
\hline GLASS & 251.9 & $9.180 E+02$ & $-3.824 E-01$ & $5.280 E+06$ & $-1.151 E+04$ & $1.474 E-04$ & 298 & & \\
\hline $\mathrm{NaAlsi}{ }_{3} \mathrm{O}_{8}$ & 1.5 & & & & & & 1200 & & \\
\hline MEPHELINE & 124.4 & $2.774 E+01$ & $2.954 E-01$ & & & & 298 & & \\
\hline $\mathrm{NaAlsiO}_{4}$ & 1.3 & & & & & & 457 & & \\
\hline MEPHELIME & & $1.1209 E+02$ & $6.711 E-02$ & & & & 457 & & \\
\hline $\mathrm{NaAlSiO}_{4}$ & & & & & & & 1180 & & \\
\hline MEPHELINE & 338.3 & $1.720 E+02$ & 5.520E-03 & & & & 1180 & & \\
\hline $\mathrm{NaAlSiO}_{4}$ & 2.0 & & & & & & 1525 & & \\
\hline CARNEGIEITE & 118.7 & $1.1613 E+02$ & 8.595E-02 & $-2.000 E+06$ & & & 273 & 966 & 8.5 \\
\hline $\mathrm{NaAlSiO}_{4}$ & 0.3 & & & & & & 966 & & \\
\hline CARNEGIEITE & 311.1 & $1.5232 E+02$ & $2.883 E-02$ & & & & 966 & & \\
\hline $\mathrm{NaAlSiO}_{4}$ & 1.5 & & & & & & 1700 & & \\
\hline GLASS & 134.5 & $3.171 E+02$ & $-4.320 E-02$ & $4.163 E+05$ & $-3.244 E+03$ & & 272 & & \\
\hline $\mathrm{NaAlSiO}_{4}$ & 0.5 & & & & & & 1033 & & \\
\hline MEPHELIME & & $1.121 E+02$ & 6.711E-02 & & & & 467 & & \\
\hline$\left(\mathrm{Na} .78^{K} .22\right)^{\mathrm{AlsiO}} 4$ & & & & & & & 1180 & & \\
\hline ANALCIME & 227.7 & $1.3438 E+02$ & $2.5415 E-01$ & $1.845 E+05$ & & & 298 & & \\
\hline $\operatorname{NaAlsi}{ }_{2} \mathrm{O}_{6} \cdot \mathrm{H}_{2} \mathrm{O}$ & 0.3 & & & & & & 700 & & \\
\hline DEHYDRATED AMALCIME & 172.5 & $1.9211 E+02$ & 8.0735E-02 & $-4.723 E+06$ & & & 298 & & \\
\hline $\operatorname{MaAlsi}{ }_{2} \mathrm{O}_{6}$ & 0.2 & . & & & & & 1000 & & \\
\hline
\end{tabular}


COEFFICIENTS FOR MEAT CAPACITY EQUATIONS FOR SHEET STRUCTURE SILICATES

\begin{tabular}{|c|c|c|c|c|c|c|c|c|c|}
\hline MAME AND FORIULA & $\begin{array}{l}\text { ENTROPY } \\
J \cdot \mathrm{mol}^{-1} \cdot \mathrm{K}^{\prime}\end{array}$ & A1 & $\stackrel{A 2}{(T)}$ & $\left(T^{-2}\right)^{A 3}$ & $\left(T^{-0.5}\right)$ & $\begin{array}{r}A 5 \\
\left(T^{2}\right)\end{array}$ & $\begin{array}{c}T \text { range } \\
K\end{array}$ & $T_{\operatorname{tin}}$ & $\begin{array}{c}A_{\mathrm{tmn}} \mathrm{H}^{\circ} \\
\mathrm{kJ} \cdot \mathrm{mol}^{-1}\end{array}$ \\
\hline DICKITE & 197.1 & 9.084E+02 & $-2.113 E-01$ & $3.804 E+06$ & $-1.120 E+04$ & & 298 & & \\
\hline $\mathrm{Al}_{2} \mathrm{Si}_{2} \mathrm{O}_{5}(\mathrm{OH})_{4}$ & 1.3 & & & & & & 1000 & & \\
\hline KAOLIMITE & 200.4 & $1.4303 E+03$ & $-7.885 E-01$ & $8.334 E+06$ & $-1.852 E+04$ & $3.034 E-04$ & 298 & & \\
\hline $\mathrm{Al}_{2} \mathrm{Si}_{2} \mathrm{O}_{5}(\mathrm{OH})_{4}$ & 0.5 & & & & & & 800 & & \\
\hline HALLOYSITE & 203.0 & $2.463 E+02$ & & & & & 298 & & \\
\hline $\mathrm{Al}_{2} \mathrm{Si}_{2} \mathrm{O}_{5}(\mathrm{OH})_{4}$ & 1.3 & & & & & & & & \\
\hline PYROPHYLLITE & 239.4 & $7.468 E+02$ & $-5.345 E-02$ & & $-7.578 E+03$ & $1.986 E-05$ & 298 & & \\
\hline$A l_{2} \mathrm{Si}_{4} \mathrm{O}_{10}(\mathrm{OH})_{2}$ & 0.4 & & & & & & 800 & & \\
\hline ILLITE & 1104.2 & $1.291 E+03$ & & & & & 298 & & \\
\hline $\mathrm{K}_{3}(\mathrm{Al}-\mathrm{Mg})\left(\mathrm{Al} \mathrm{C}_{2} \mathrm{Si}_{14} \mathrm{OO}_{40}(\mathrm{OH})_{8}\right.$ & 2.5 & & & & & & & & \\
\hline MARGARITE & 263.6 & $8.265 E+02$ & $-5.040 E-01$ & & $-8.7274 E+03$ & & 298 & & \\
\hline $\mathrm{CaAl}_{2}\left[\mathrm{Cl}_{2} \mathrm{Si} \mathrm{O}_{10}\right](\mathrm{OH})_{2}$ & 0.3 & & & & & & 1200 & & \\
\hline PREHNITE & 292.8 & $9.460 E+02$ & $-1.1506 E-01$ & $2.755 E+06$ & $-1.056 E+04$ & & 298 & & \\
\hline $\mathrm{Ca}_{2} \mathrm{Al}$ CAlsi $\left.{ }_{3} \mathrm{O}_{10}\right](\mathrm{OH})_{2}$ & 0.7 & & & & & & 1200 & & \\
\hline mUSCOVITE (disordered) & 306.4 & $9.177 E+02$ & $-8.111 E-02$ & $2.834 E+06$ & $-1.035 E+04$ & & 298 & & \\
\hline $\mathrm{KAl}_{2}\left[\mathrm{LISi}{ }_{3} \mathrm{O}_{10} \mathrm{~J}^{(\mathrm{OH})_{2}}\right.$ & 0.6 & & & & & & 1000 & & \\
\hline muscovite (ordered) & 287.7 & $9.17 T E+02$ & $-8.111 E-02$ & $2.834 E+06$ & $-1.035 E+04$ & & 298 & & \\
\hline $\mathrm{KAl}_{2}\left[\mathrm{AlSi}{ }_{3} \mathrm{O}_{10}\right](\mathrm{OH})_{2}$ & 0.6 & & & & & & 1000 & & \\
\hline ANNITE & 415.0 & $6.366 E+02$ & 8.208E-02 & $-4.860 E+06$ & $-3.731 E+03$ & & 298 & & \\
\hline $\mathrm{KFe}_{3}\left[\mathrm{AlSi} \mathrm{O}_{10}\right](\mathrm{OH})_{2}$ & 10.0 & & & & & & 1000 & & \\
\hline PHLOGOPITE (disordered) & 334.6 & $8.639 E+02$ & $-7.6076 E-02$ & $3.5206 E+05$ & $-8.470 E+03$ & & 298 & & \\
\hline $\mathrm{KMg}_{3}\left[\mathrm{Alsi}{ }_{3} \mathrm{O}_{10}\right](\mathrm{OH})_{2}$ & 1.0 & & & & & & 1000 & & \\
\hline PHLOCOPITE (ordered) & 315.9 & $8.639 E+02$ & $-7.6076 E-02$ & $3.5206 E+05$ & $-8.470 E+03$ & & 298 & & \\
\hline $\mathrm{KMg}_{3}\left[\mathrm{Clsi} \mathrm{S}_{3} \mathrm{O}_{10} \mathrm{CH}_{2}\right.$ & 1.0 & & & & & & 1000 & & \\
\hline FLUORPHLOCOPITE (disordered) & d) 336.3 & $4.9288 E+02$ & 4.910E-02 & $-6.599 E+06$ & $-1.569 E+03$ & & 298 & & \\
\hline $\left.\mathrm{KH}_{3} \mathrm{LAlsi}{ }_{3} \mathrm{O}_{10}\right] \mathrm{F}_{2}$ & 2.1 & & & & & & 1670 & & \\
\hline FLUORPHLOCOPITE (ordered) & 317.6 & $4.9288 E+02$ & 4.910E-02 & $-6.599 E+06$ & $-1.569 E+03$ & & 298 & & \\
\hline $\mathrm{KMg}_{3}\left[\mathrm{CAlsi} \mathrm{S}_{10} \mathrm{O}_{2}\right.$ & 2.1 & & & & & & 1670 & & \\
\hline TALC & 260.8 & $5.654 E+03$ & $-5.272 E+00$ & $4.021 E+07$ & $-7.693 E+04$ & $2.729 E-03$ & 298 & & \\
\hline $\mathrm{Mg}_{3} \mathrm{Si}_{4} \mathrm{O}_{10}(\mathrm{OH})_{2}$ & 0.6 & & & & & & 800 & & \\
\hline CHRYSOTILE & 221.3 & $8.996 E+02$ & $-1.448 E-01$ & $4.500 E+06$ & $-1.093 E+04$ & & 298 & & \\
\hline $\mathrm{Mg}_{3} \mathrm{Si}_{2} \mathrm{O}_{5}(\mathrm{OH})_{4}$ & 0.8 & & & & & & 900 & & \\
\hline
\end{tabular}


COEFFICIENTS FOR HEAT CAPACITY EQUATIONS FOR SHEET STRUCTURE SILICATES

\begin{tabular}{|c|c|c|c|c|c|c|c|c|c|}
\hline MNE ANO FORMULA & $\begin{array}{c}\text { ENTROPY } \\
J \cdot \mathrm{mol}^{-1} \cdot K^{\prime}\end{array}$ & $\mathbf{A 1}$ & $\begin{array}{c}A 2 \\
(T)\end{array}$ & $\stackrel{\left.1 T^{-2}\right)}{(3}$ & $\left(T^{-0.5}\right)$ & $\begin{array}{r}15 \\
\left(T^{2}\right)\end{array}$ & $\begin{array}{c}T \text { range } \\
K\end{array}$ & $T_{\operatorname{tin}}$ & $\begin{array}{l}A_{\lim } H^{\circ} \\
k J \cdot \mathrm{mol}^{-1}\end{array}$ \\
\hline
\end{tabular}

CLIMOCHLORE

$\mathrm{Mg}_{5} \mathrm{Al}\left(\mathrm{Alsi} \mathrm{S}_{3} \mathrm{O}_{10}\right)(\mathrm{OH})_{8}$

PARACONITE (disordered)

$\mathrm{NaAl}_{2}\left[\mathrm{Alsi} \mathrm{O}_{10} \mathrm{O}^{(\mathrm{OH})_{2}}\right.$

PARACONITE (ordered)

$\mathrm{NaAl}_{2} \mathrm{CAlsi}_{3} \mathrm{O}_{10} \mathrm{~J}(\mathrm{OH})_{2}$
421.0 15.0

$295.8 \quad 6.6844 E+02$

0.9

$3.627 E-02 \quad-1.860 E+06 \quad-5.816 E+03$

3.6206E-03

298

800

277.

0.9
298

800

298

800 
Thermodynamic Properties at High Temperature of Individual Phases

Ag: Face-centered cubic crystals 298.15 to melting point $1234.9 \mathrm{~K}$; liquid 1234.9 to 1800 R.

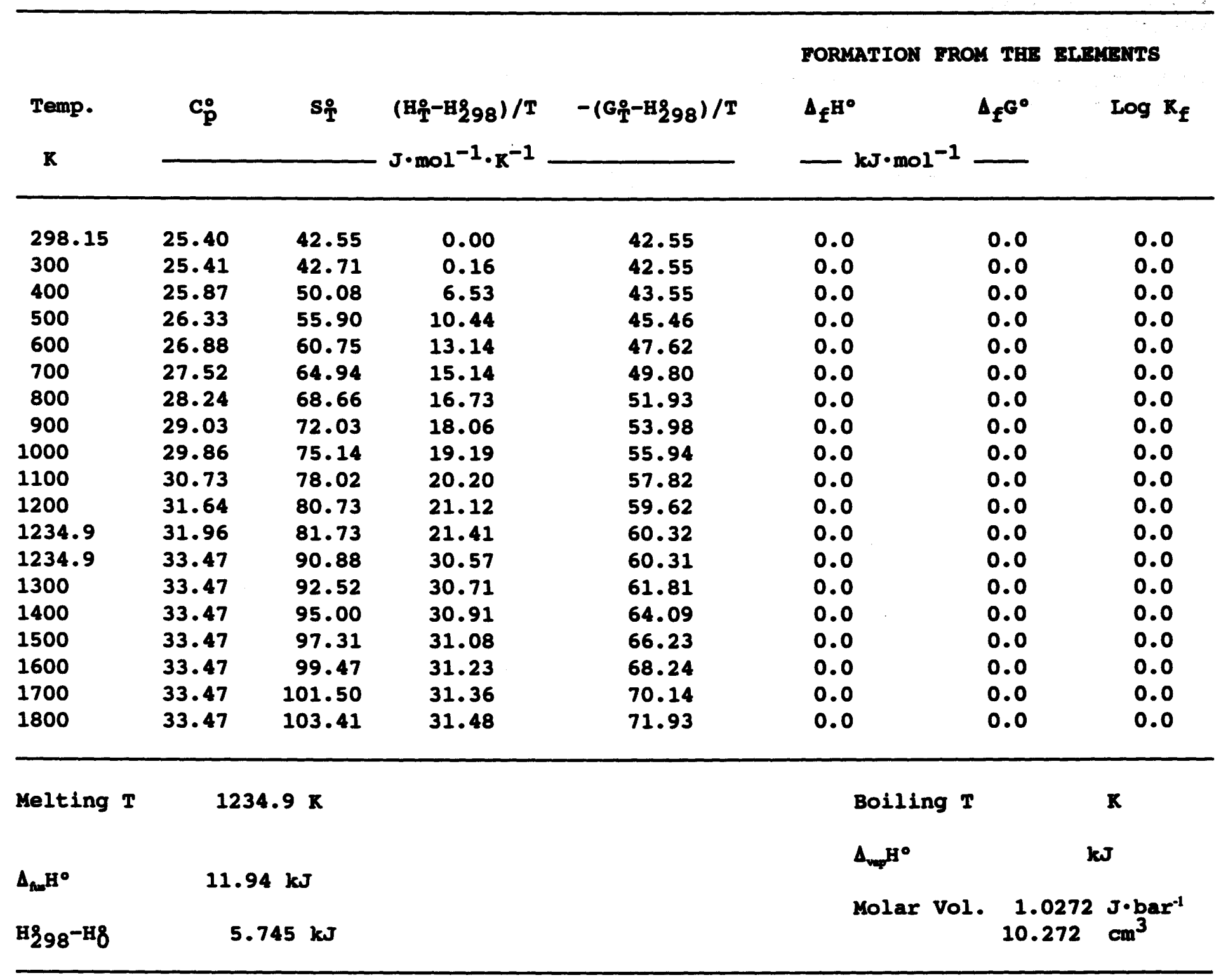


Al: Face-centered cubic crystals 298.15 to melting point $933.5 \mathrm{~K}$. Liquid 933.5 to $1800 \mathrm{~K}$.

\section{FORMATION FROM THE ELEMENTS}

Temp.

$\mathbf{c}_{\mathbf{p}}^{\circ}$

$\mathbf{s}_{\mathrm{T}}$
$\left(\mathrm{H}_{\mathrm{T}}-\mathrm{H}_{298}\right) / \mathrm{T} \quad-\left(\mathrm{G}_{\mathrm{T}}^{\circ}-\mathrm{H}_{298}\right) / \mathrm{T}$

$\Delta_{f} \mathrm{H}^{\circ}$

$\Delta_{F^{G}}$

$\log k_{f}$ 
As: Rhombohedral crystals 298.15 to sublimation point $875 \mathrm{~K}$. Ideal tetratomic gas 875 to $1800 \mathrm{~K}$.

FORMATION PROM THE ELEMENTS

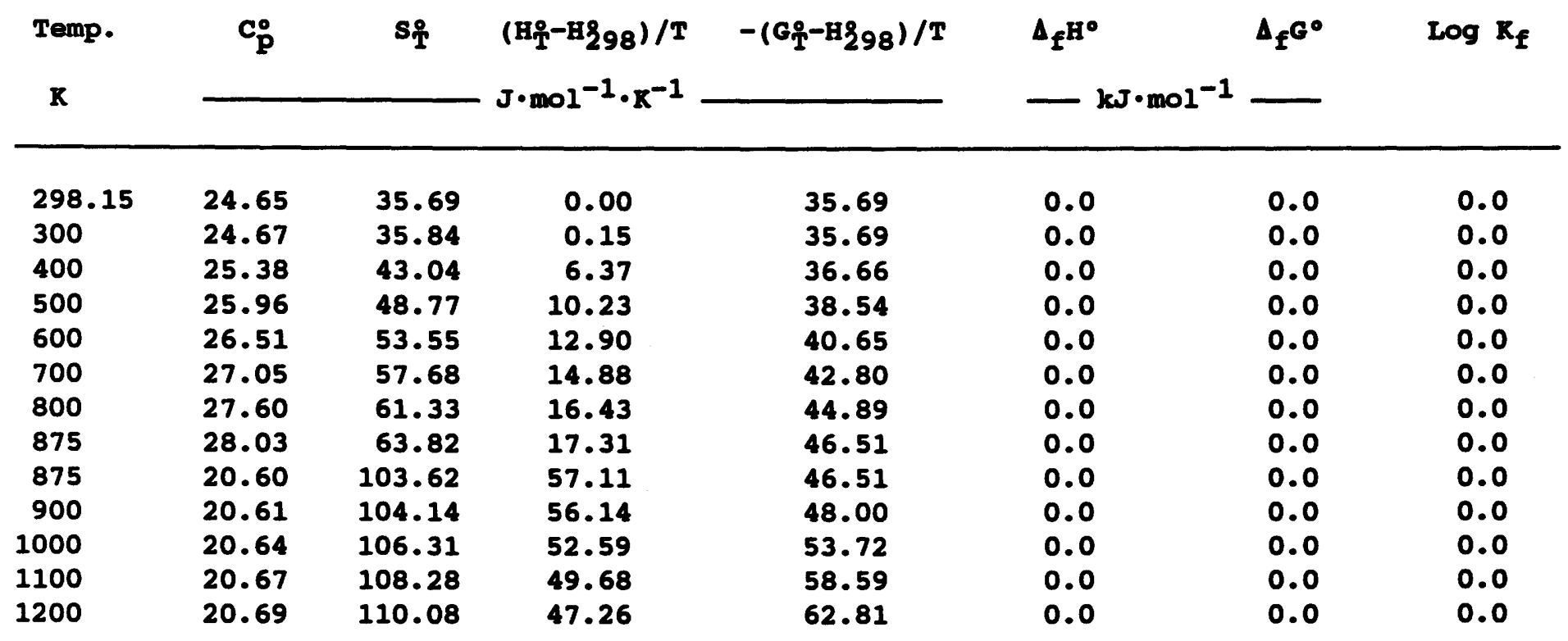

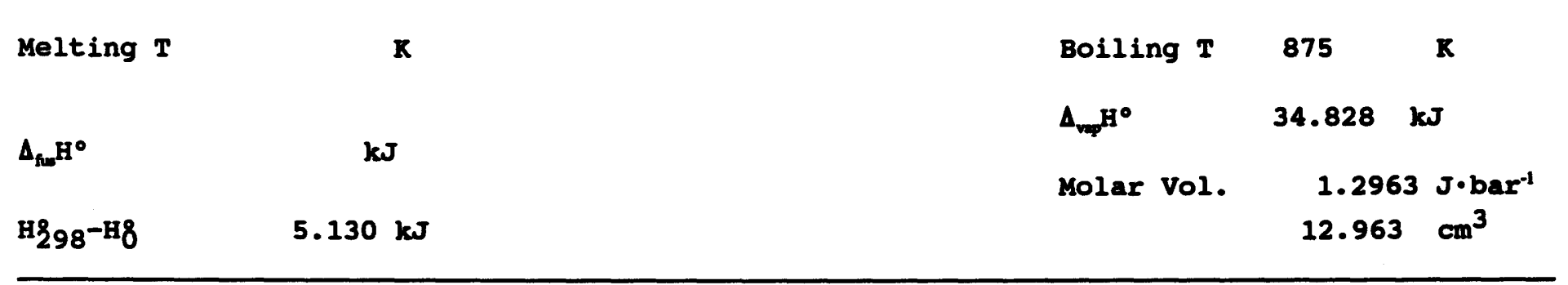


Au: Face-centered cubic crystals 298.15 to melting point $1336.15 \mathrm{~K}$.

\begin{tabular}{|c|c|c|c|c|c|c|c|c|}
\hline \multirow{3}{*}{$\begin{array}{l}\text { Temp. } \\
\mathbf{K}\end{array}$} & \multirow{3}{*}{$\mathbf{c}_{\mathbf{p}}^{\circ}$} & \multirow{3}{*}{$\mathbf{s}_{\mathbf{T}}^{\circ}$} & \multirow{3}{*}{$\begin{array}{l}\left(\mathrm{H}_{\mathrm{T}}^{\mathrm{P}}-\mathrm{H} 298\right) / \mathrm{T} \\
\mathrm{J} \cdot \mathrm{mol}^{-1} \cdot \mathrm{K}^{-1}\end{array}$} & \multirow{3}{*}{-(G足-H298)/T } & \multicolumn{2}{|c|}{ FORMATION FROM THE } & \multicolumn{2}{|c|}{ ELEMENTS } \\
\hline & & & & & \multirow{2}{*}{\multicolumn{2}{|c|}{$\begin{array}{l}\Delta_{\mathbf{f}} \mathrm{H}^{\circ} \\
-\mathrm{kJ} \cdot \mathrm{mol}^{-1}\end{array}$}} & \multirow{2}{*}{\multicolumn{2}{|c|}{$\log R_{f}$}} \\
\hline & & & & & & & & \\
\hline 298.15 & 25.18 & 47.49 & 0.00 & 47.49 & 0.0 & 0.0 & & 0.0 \\
\hline 300 & 25.19 & 47.65 & 0.16 & 47.49 & 0.0 & 0.0 & & 0.0 \\
\hline 400 & 25.88 & 55.00 & 6.51 & 48.49 & 0.0 & 0.0 & & 0.0 \\
\hline 500 & 26.26 & 60.82 & 10.43 & 50.39 & 0.0 & 0.0 & & 0.0 \\
\hline 600 & 26.57 & 65.63 & 13.09 & 52.54 & 0.0 & 0.0 & & 0.0 \\
\hline 700 & 26.91 & 69.76 & 15.04 & 54.71 & 0.0 & 0.0 & & 0.0 \\
\hline 800 & 27.36 & 73.38 & 16.55 & 56.83 & 0.0 & 0.0 & & 0.0 \\
\hline 900 & 27.93 & 76.63 & 17.78 & 58.85 & 0.0 & 0.0 & & 0.0 \\
\hline 1000 & 28.67 & 79.61 & 18.83 & 60.78 & 0.0 & 0.0 & & 0.0 \\
\hline 1100 & 29.57 & 82.38 & 19.77 & 62.62 & 0.0 & 0.0 & & 0.0 \\
\hline 1200 & 30.66 & 85.00 & 20.63 & 64.37 & 0.0 & 0.0 & & 0.0 \\
\hline 1300 & 31.95 & 87.51 & 21.45 & 66.06 & 0.0 & 0.0 & & 0.0 \\
\hline 1336.15 & 32.47 & 88.55 & 21.80 & 66.74 & 0.0 & 0.0 & & 0.0 \\
\hline 1336.15 & 33.54 & 97.94 & 31.20 & 66.74 & 0.0 & 0.0 & & 0.0 \\
\hline 1400 & 32.75 & 99.34 & 31.23 & 68.11 & 0.0 & 0.0 & & 0.0 \\
\hline 1500 & 31.49 & 101.54 & 31.28 & 70.26 & 0.0 & 0.0 & & 0.0 \\
\hline 1600 & 30.81 & 103.54 & 31.25 & 72.29 & 0.0 & 0.0 & & 0.0 \\
\hline 1700 & 30.81 & 105.39 & 31.21 & 74.18 & 0.0 & 0.0 & & 0.0 \\
\hline 1800 & 30.81 & 107.15 & 31.19 & 75.96 & 0.0 & 0.0 & & 0.0 \\
\hline \multirow[t]{2}{*}{ Melting $T$} & \multicolumn{2}{|c|}{$1336.15 \mathrm{~K}$} & & & \multicolumn{2}{|c|}{ Boiling $T$} & \multicolumn{2}{|c|}{$\mathbf{K}$} \\
\hline & \multicolumn{2}{|c|}{$10=5$} & & & \multicolumn{2}{|c|}{$\Delta_{\mathrm{v}} \mathrm{H}^{\circ}$} & \multicolumn{2}{|c|}{$\mathbf{k J}$} \\
\hline \multirow[t]{2}{*}{$\Delta_{\mathrm{hw}} \mathrm{H}^{\circ}$} & & $\mathbf{k J}$ & & & & & & \\
\hline & & & & & & & 1.0215 & $\mathbf{J} \cdot \mathbf{b a r}^{-1}$ \\
\hline H298-H8 & & $.017 \mathrm{~kJ}$ & & & & & .215 & $\mathrm{~cm}^{3}$ \\
\hline
\end{tabular}


B: Rhombohedral crystals 298.15 to melting point $2350 \mathrm{~K}$.

FORMATION FROY THE ELEATENTS

\begin{tabular}{|c|c|c|c|c|c|c|}
\hline Temp. & $\mathbf{c}_{\mathbf{p}}^{\circ}$ & $\mathbf{s}_{\mathbf{T}}^{\circ}$ & $\left(\mathrm{H}_{\mathrm{T}}^{\circ}-\mathrm{H}_{298}\right) / \mathrm{T}$ & $-\left(G_{T}^{\circ}-H_{298}\right) / T$ & $\Delta_{\mathbf{f}^{\mathrm{H}^{\circ}}}$ & $\Delta_{\mathbf{f}} \mathbf{G}^{\circ}$ \\
\hline
\end{tabular}

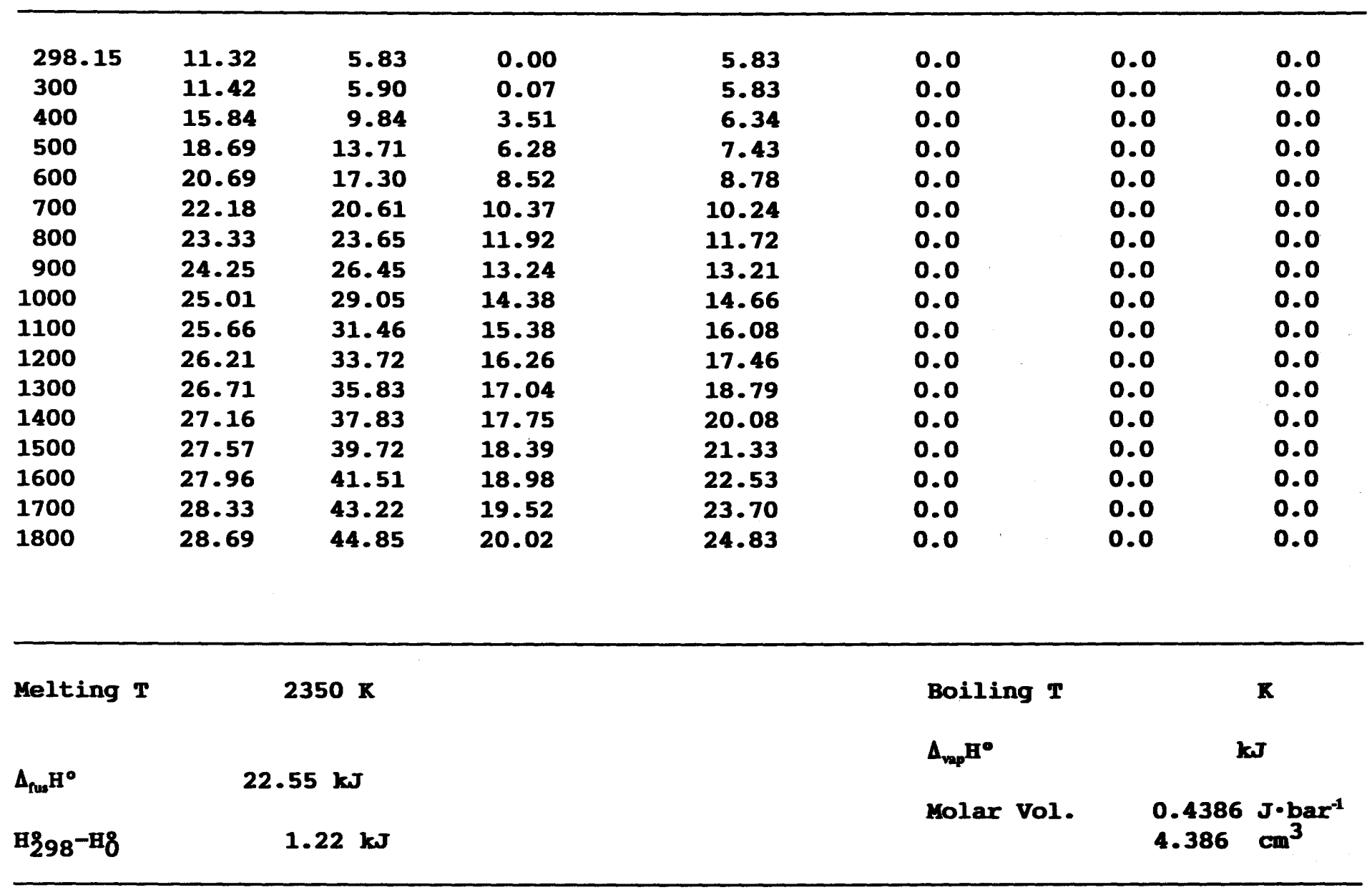


Ba: a-crystals (body-centered cubic) 298.15 to lambda-anomaly in heat capacity at $582 \mathrm{~K}$. $\beta$-crystals 582 to second-order lambda-anomaly in heat capacity at $768.13 \mathrm{~K}$. $\gamma$-crystals 768.13 to melting point $1002 \mathrm{~K}$. Liquid 1002 to boiling point $2169 \mathrm{~K}$.

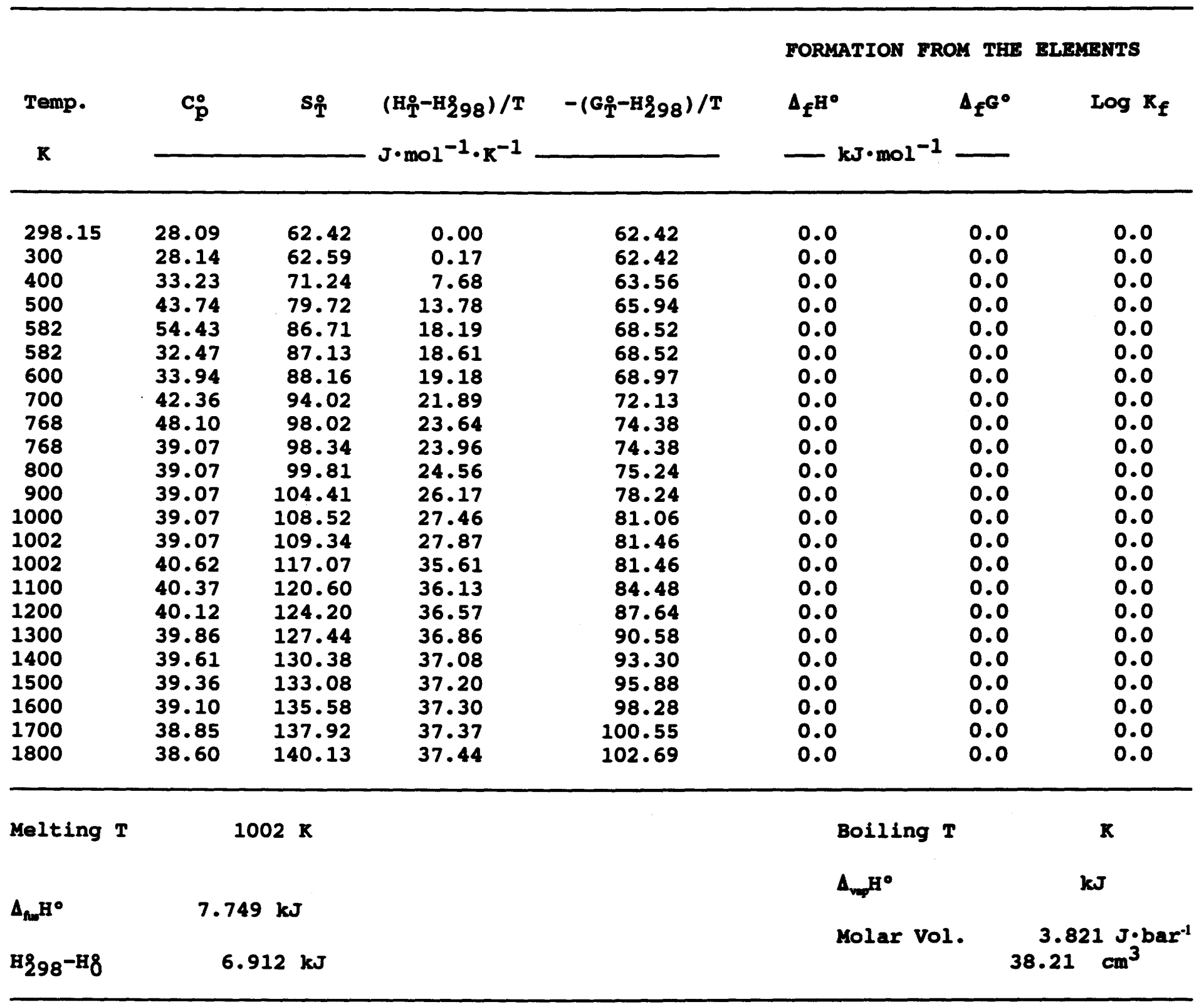


Be: Hexagonal close-packed crystals 298.15 to $1550 \mathrm{~K}$; beta crystals 1550 to melting point $1560 \mathrm{~K}$; liqquid 1560 to $1800 \mathrm{~K}$.

\begin{tabular}{|c|c|c|c|c|c|c|c|}
\hline \multirow{3}{*}{$\begin{array}{l}\text { Temp. } \\
\mathbf{R}\end{array}$} & \multirow{3}{*}{$c_{p}^{0}$} & \multirow{3}{*}{$\mathbf{s}_{\mathrm{T}}$} & \multirow{3}{*}{$\begin{array}{l}\left(\mathrm{H}_{\mathrm{T}}-\mathrm{H}_{298}\right) / \mathrm{T} \\
\mathrm{J} \cdot \mathrm{mol}^{-1} \cdot \mathrm{K}^{-1}\end{array}$} & \multirow{3}{*}{$-\left(G_{T}-\mathrm{H}_{298}\right) / T$} & \multicolumn{2}{|c|}{ FORMATION FROM THE } & \multirow{3}{*}{$\begin{array}{l}\text { ELEMENTS } \\
\qquad \log \mathrm{k}_{\mathbf{f}}\end{array}$} \\
\hline & & & & & $\Delta_{f^{H^{0}}}$ & $\Delta_{f^{G}}^{G^{0}}$ & \\
\hline & & & & & \multicolumn{2}{|c|}{$-\mathrm{kJ} \cdot \mathrm{mol}^{-1}$} & \\
\hline 298.15 & 16.44 & 9.50 & 0.00 & 9.50 & 0.0 & 0.0 & 0.0 \\
\hline 300 & 16.52 & 9.60 & 0.10 & 9.50 & 0.0 & 0.0 & 0.0 \\
\hline 400 & 19.64 & 14.83 & 4.64 & 10.19 & 0.0 & 0.0 & 0.0 \\
\hline 500 & 21.58 & 19.43 & 7.84 & 11.59 & 0.0 & 0.0 & 0.0 \\
\hline 600 & 23.06 & 23.50 & 10.26 & 13.24 & 0.0 & 0.0 & 0.0 \\
\hline 700 & 24.32 & 27.15 & 12.18 & 14.97 & 0.0 & 0.0 & 0.0 \\
\hline 800 & 25.47 & 30.47 & 13.77 & 16.71 & 0.0 & 0.0 & 0.0 \\
\hline 900 & 26.54 & 33.54 & 15.13 & 18.41 & 0.0 & 0.0 & 0.0 \\
\hline 1000 & 27.58 & 36.39 & 16.32 & 20.07 & 0.0 & 0.0 & 0.0 \\
\hline 1100 & 28.59 & 39.06 & 17.39 & 21.67 & 0.0 & 0.0 & 0.0 \\
\hline 1200 & 29.57 & 41.59 & 18.37 & 23.23 & 0.0 & 0.0 & 0.0 \\
\hline 1300 & 30.55 & 44.00 & 19.27 & 24.73 & 0.0 & 0.0 & 0.0 \\
\hline 1400 & 31.52 & 46.30 & 20.11 & 26.19 & 0.0 & 0.0 & 0.0 \\
\hline 1500 & 32.47 & 48.51 & 20.90 & 27.61 & 0.0 & 0.0 & 0.0 \\
\hline 1550 & 32.95 & 49.58 & 21.28 & 28.30 & 0.0 & 0.0 & 0.0 \\
\hline 1550 & 30.00 & 50.94 & 22.64 & 28.30 & 0.0 & 0.0 & 0.0 \\
\hline 1560 & 30.00 & 51.13 & 22.68 & 28.45 & 0.0 & 0.0 & 0.0 \\
\hline 1560 & 30.00 & 59.20 & 30.76 & 28.44 & 0.0 & 0.0 & 0.0 \\
\hline 1600 & 30.00 & 59.96 & 30.74 & 29.22 & 0.0 & 0.0 & 0.0 \\
\hline 1700 & 30.00 & 61.78 & 30.70 & 31.08 & 0.0 & 0.0 & 0.0 \\
\hline 1800 & 30.00 & 63.50 & 30.66 & 32.84 & 0.0 & 0.0 & 0.0 \\
\hline \multirow[t]{2}{*}{ Melting $T$} & \multicolumn{2}{|c|}{$1560 \mathrm{~K}$} & & & \multicolumn{2}{|c|}{ Boiling $T$} & $\mathbf{K}$ \\
\hline & & & & & \multicolumn{2}{|c|}{$\Delta_{\mathrm{v}} \mathrm{H}^{\circ}$} & $\mathbf{k J}$ \\
\hline \multirow[t]{2}{*}{$\Delta_{\mathrm{fu}} \mathrm{H}^{\circ}$} & \multicolumn{2}{|c|}{$12.21 \mathrm{~kJ}$} & & & \multirow{3}{*}{\multicolumn{2}{|c|}{ Molar vol. }} & \\
\hline & & & & & & & $0.488 \mathrm{~J} \cdot \mathrm{bar}^{-1}$ \\
\hline H298-H8 & & $5 \mathrm{~kJ}$ & & & & & $4.88 \mathrm{~cm}^{3}$ \\
\hline
\end{tabular}


Bi: Rhombohedral crystals 298.15 to melting point $544.5 \mathrm{~K}$. Liquid 544.5 to $1800 \mathrm{~K}$.

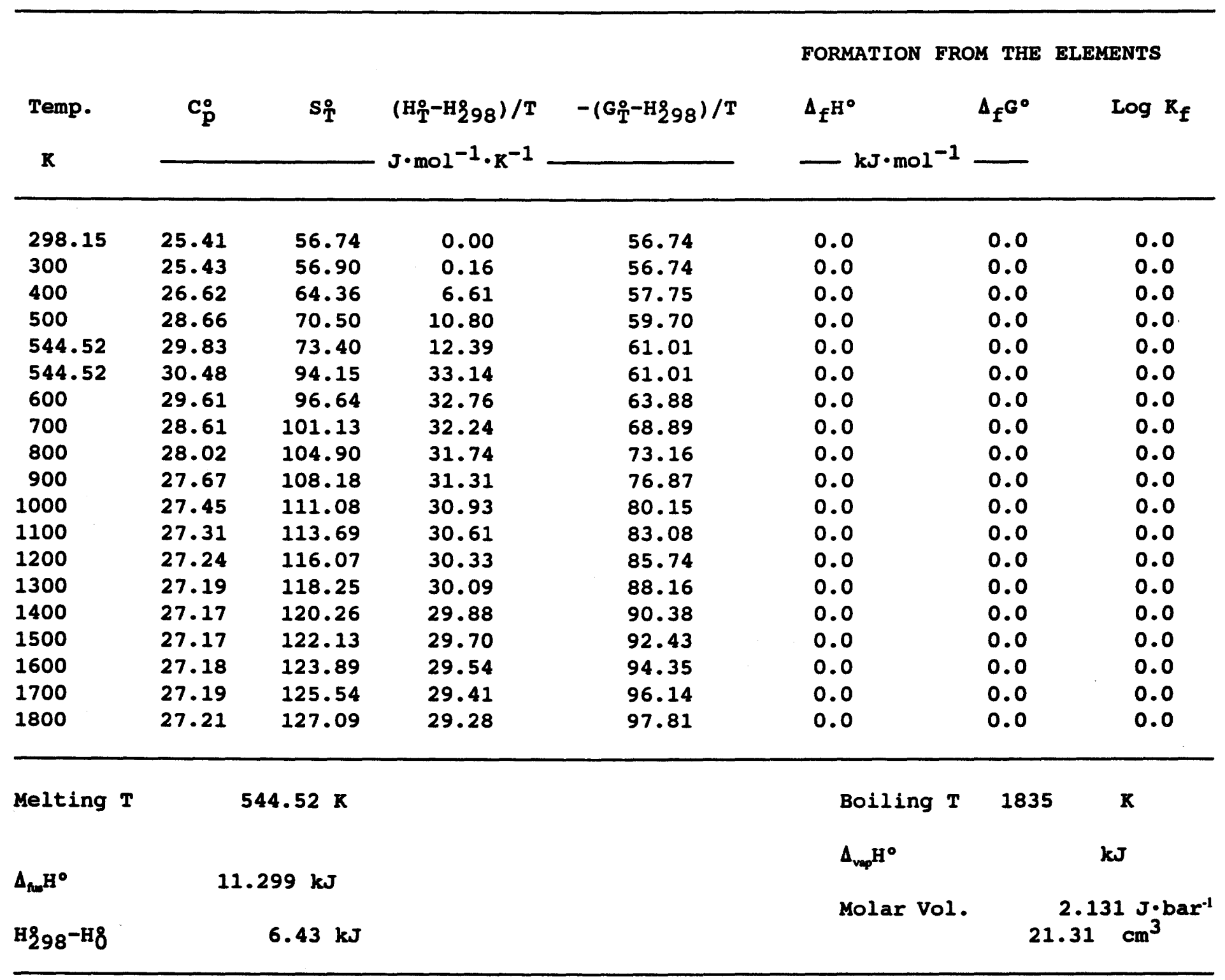


$\mathrm{Br}_{2}$ : Liquid 298.15 to boiling point $332.5 \mathrm{~K}$ i ideal diatomic gas, $\mathrm{P}=1$ bar, 332.5 to $2200 \mathrm{~K}$.

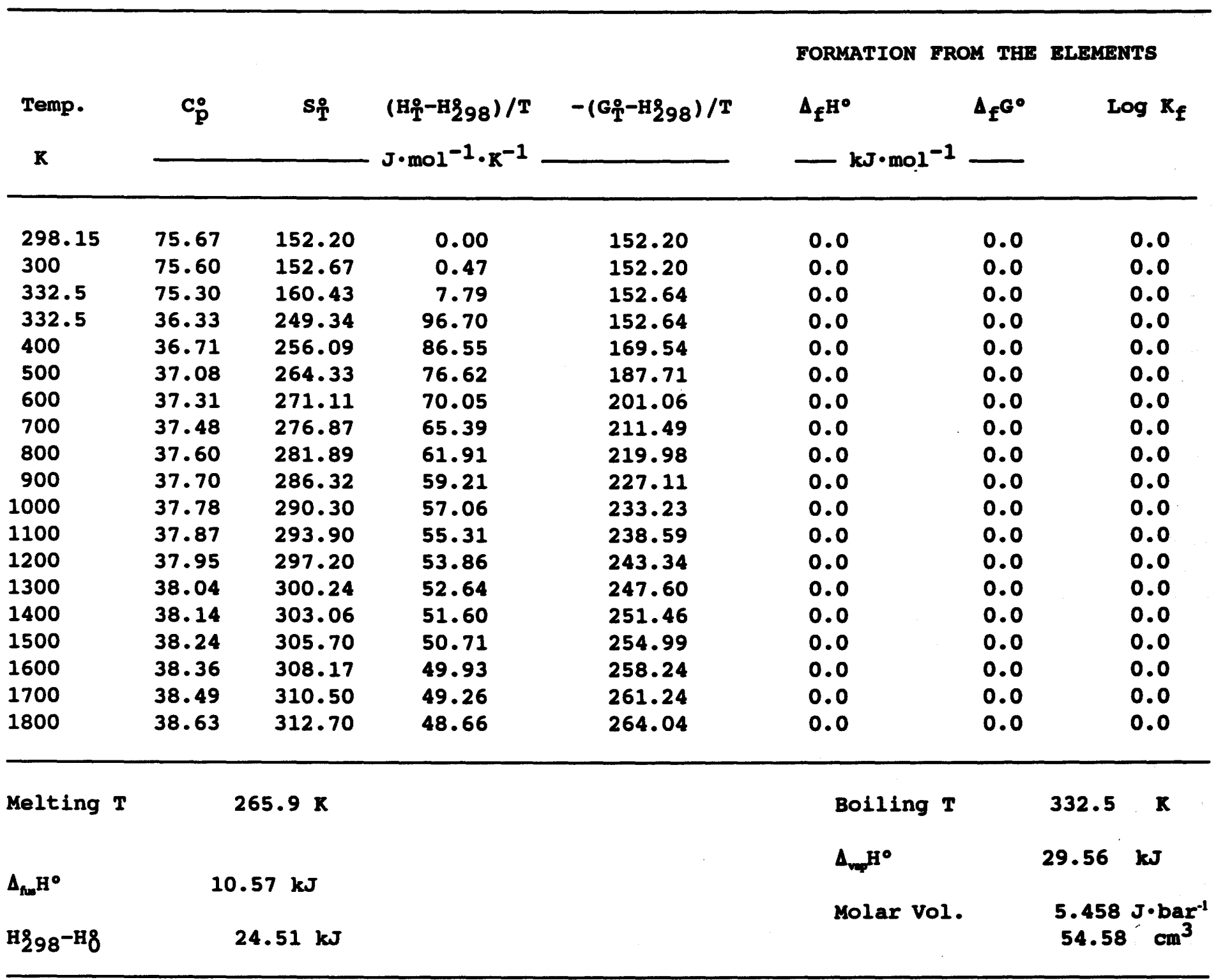


C: Hexagonal crystals 298.15 to $2500 \mathrm{~K}$.

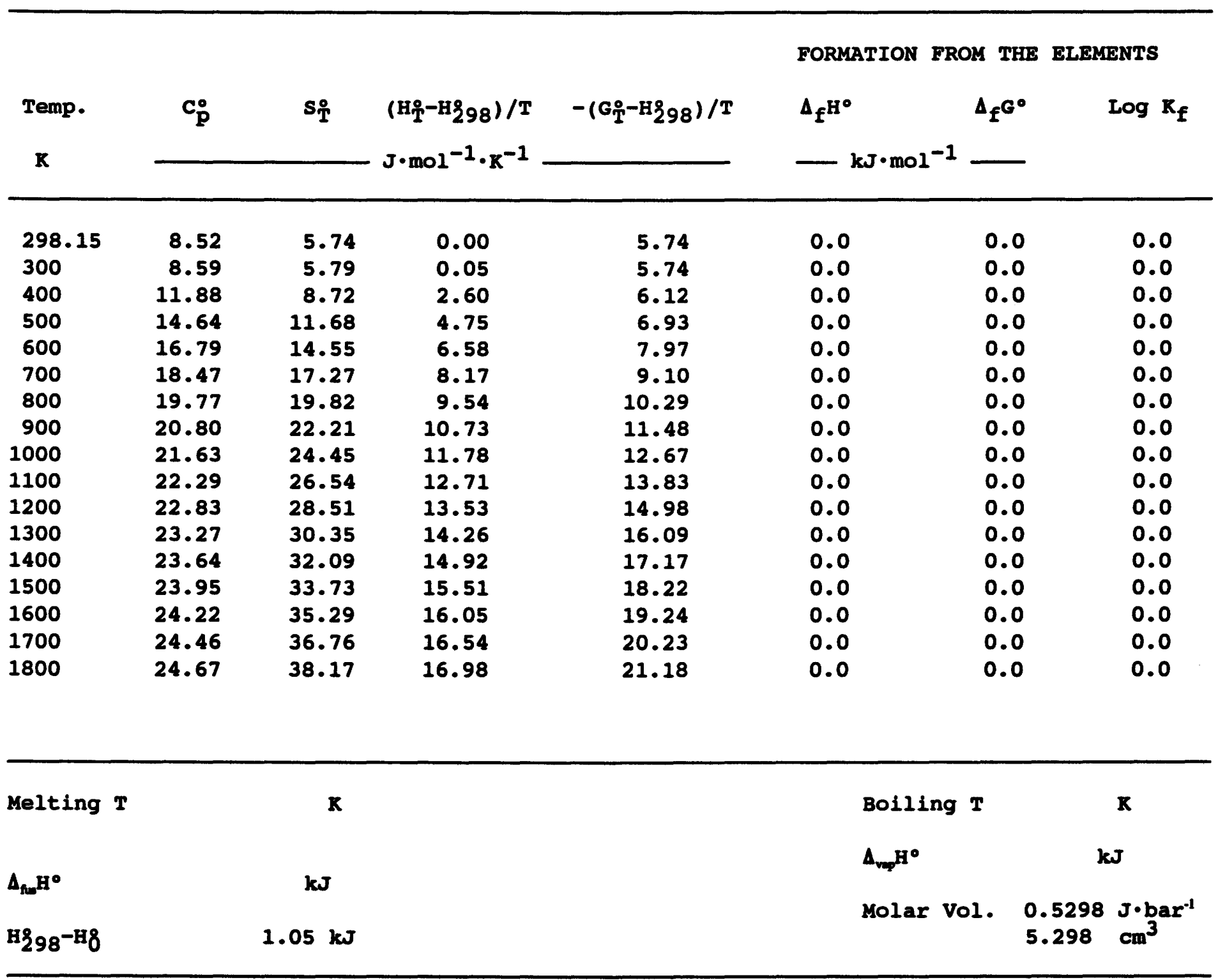


C: Cubic crystals 298.15 to $1600 \mathrm{~K}$.

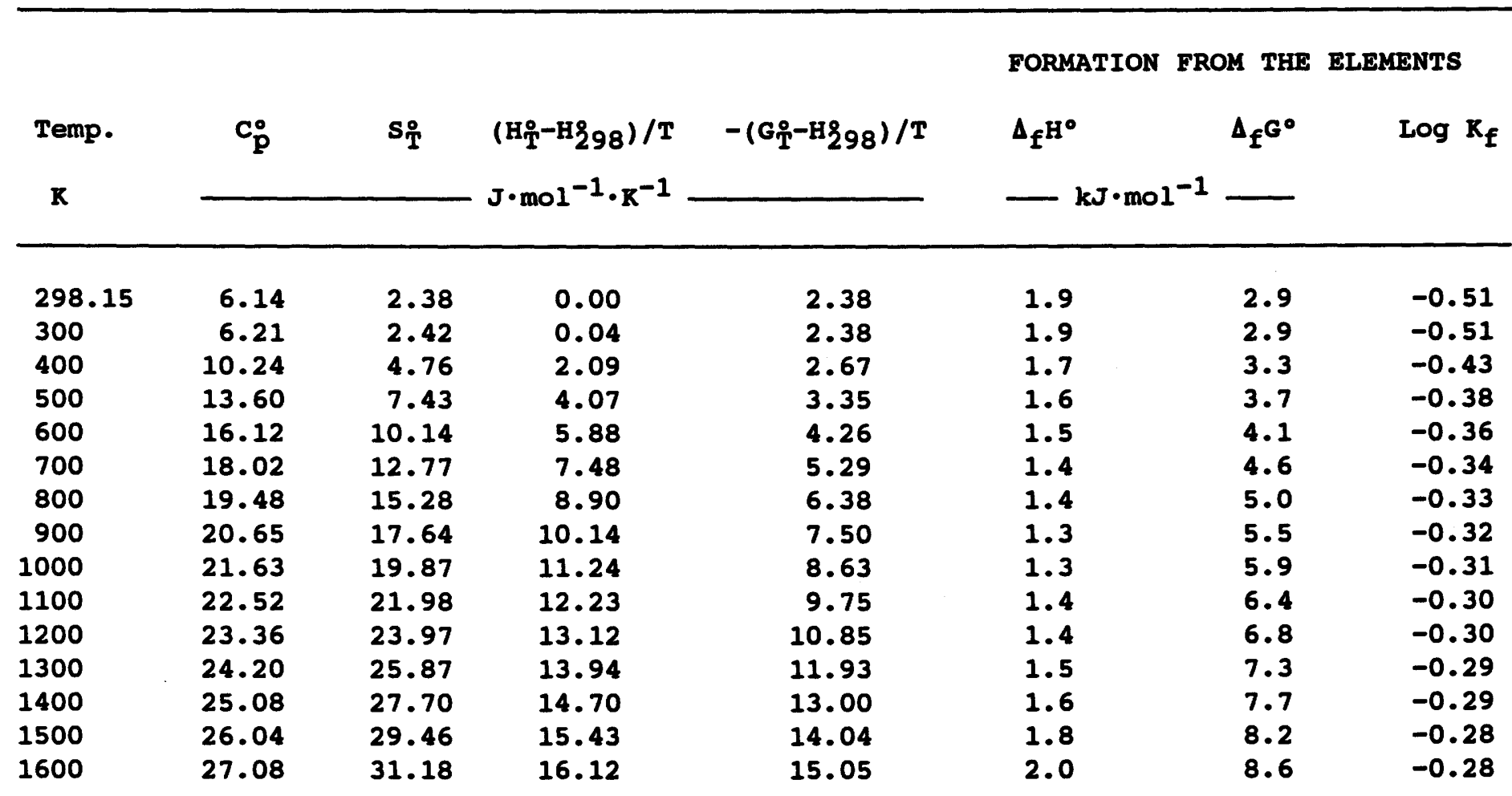

Melting T

$\Delta_{\text {fus }} H^{\circ}$

H298-H8 $\mathbf{k J}$

$\mathbf{K}$

$0.523 \mathrm{~kJ}$
Boiling $\mathbf{T}$

$\Delta_{\mathrm{veg}} \mathrm{H}^{\circ}$

Molar Vol.

\section{$\mathbf{R}$}

$\mathbf{k J}$ 
Ca: a-crystals (face-centered cubic) 298.15 to $716 \mathrm{~K}$. B-crystals (body-centered cubic) 716 to melting point $1115 \mathrm{~K}$. Liquid 1115 to boiling point $1755 \mathrm{~K}$. Ideal monatomic gas, $P$ = 1 bar, 1774 to $1800 \mathrm{~K}$.

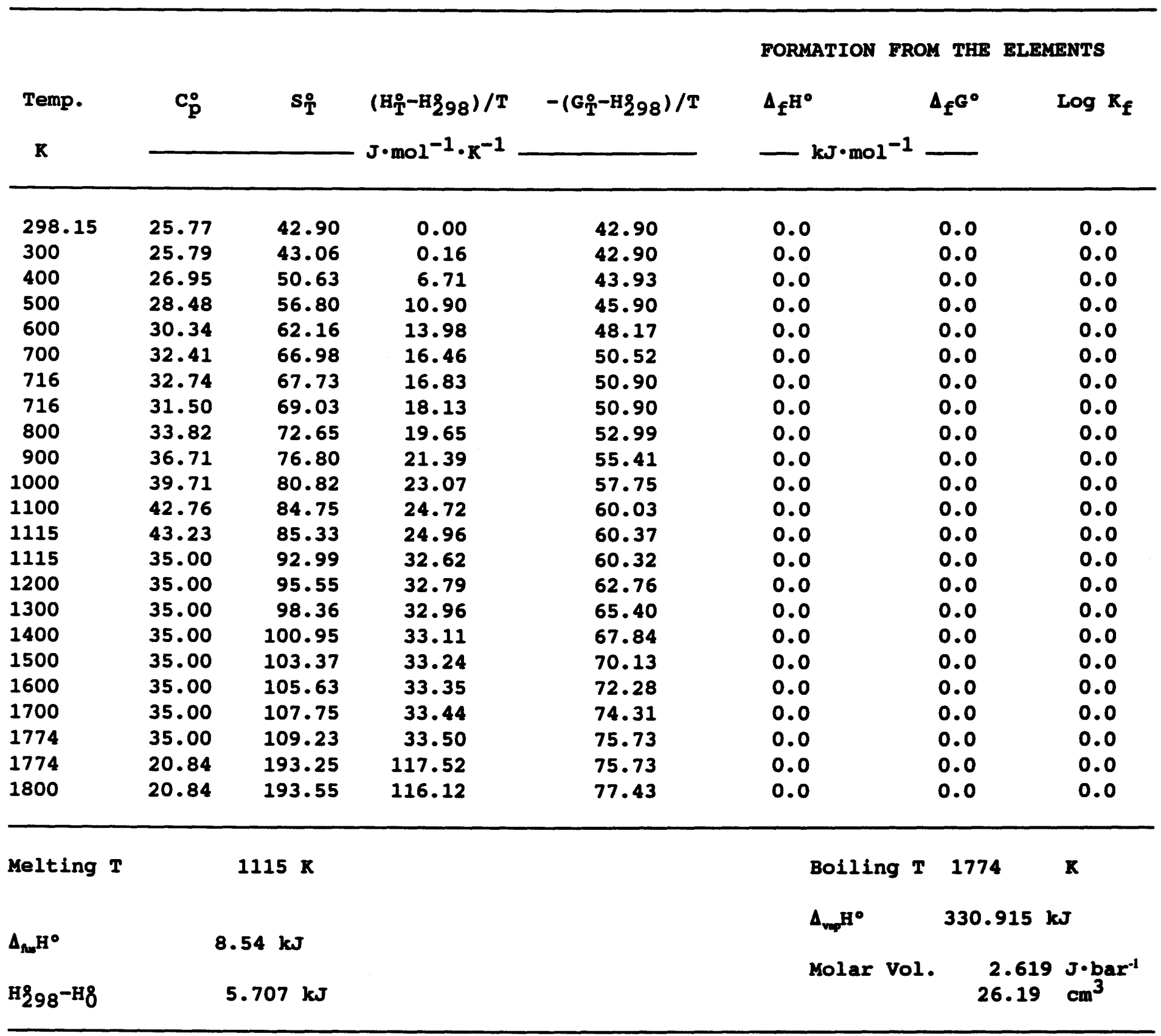


Cd: Hexagonal close packed crystals 298.15 to melting point $594.18 \mathrm{~K}$. Liquid 594.18 to boiling point $1039 \mathrm{~K}$. Ideal monatomic gas 1039 to $1800 \mathrm{~K}$.

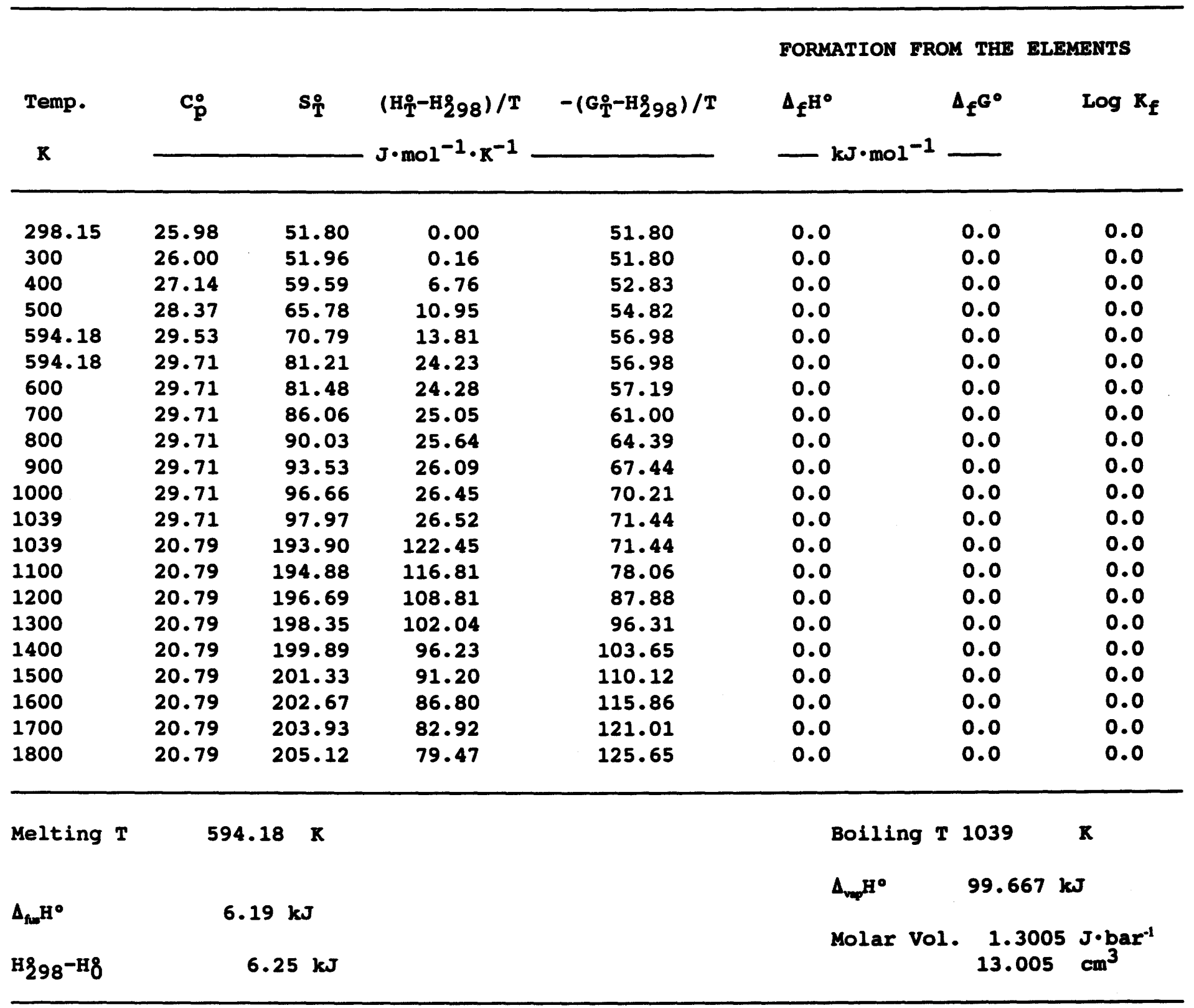


Ce: $\alpha$-crystals (face-centered cubic) 298.15 to $999 \mathrm{~K}$. p-crystals 999 to melting point 1071 R. Iiquid 1071 to $1800 \mathrm{~K}$.

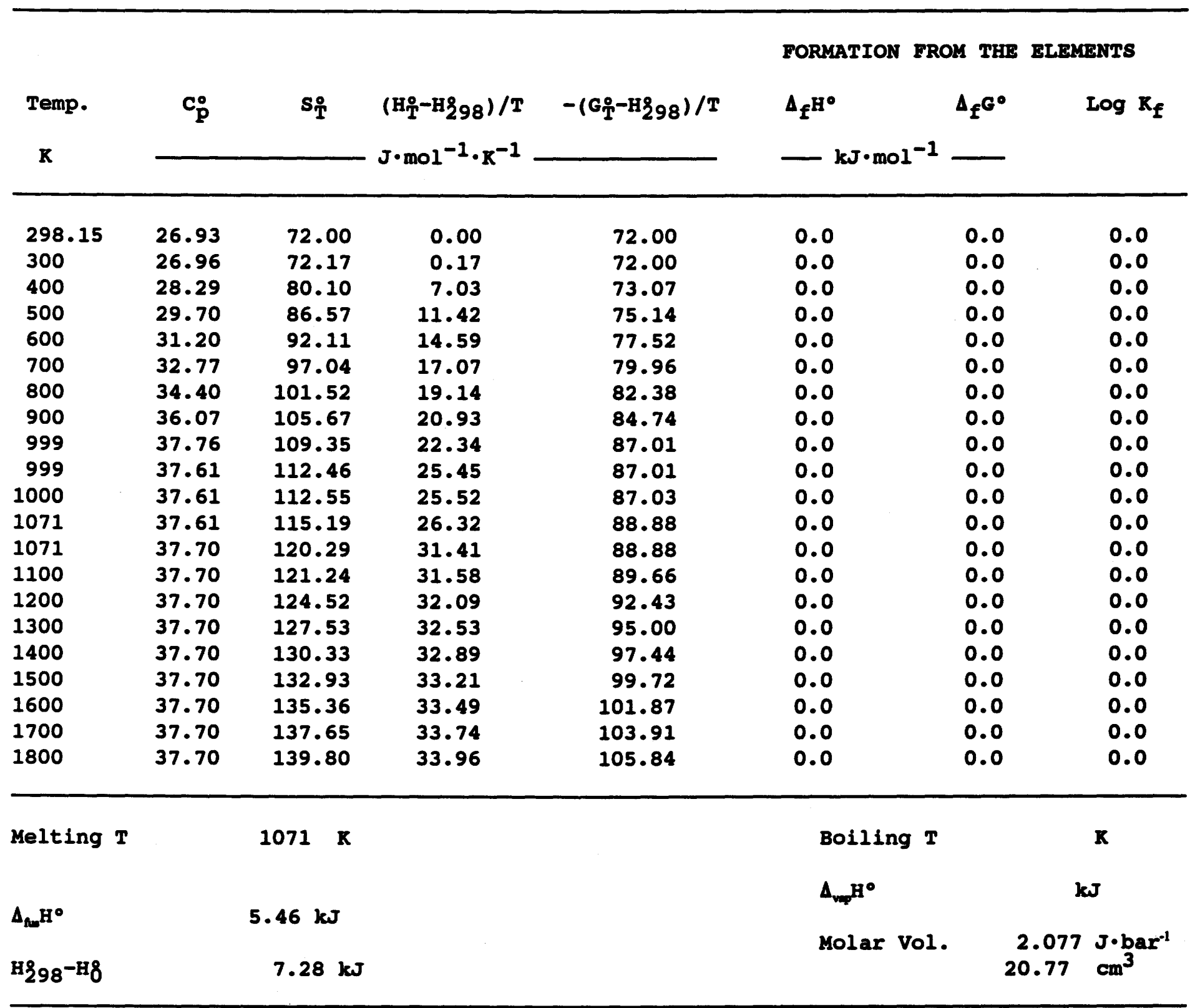




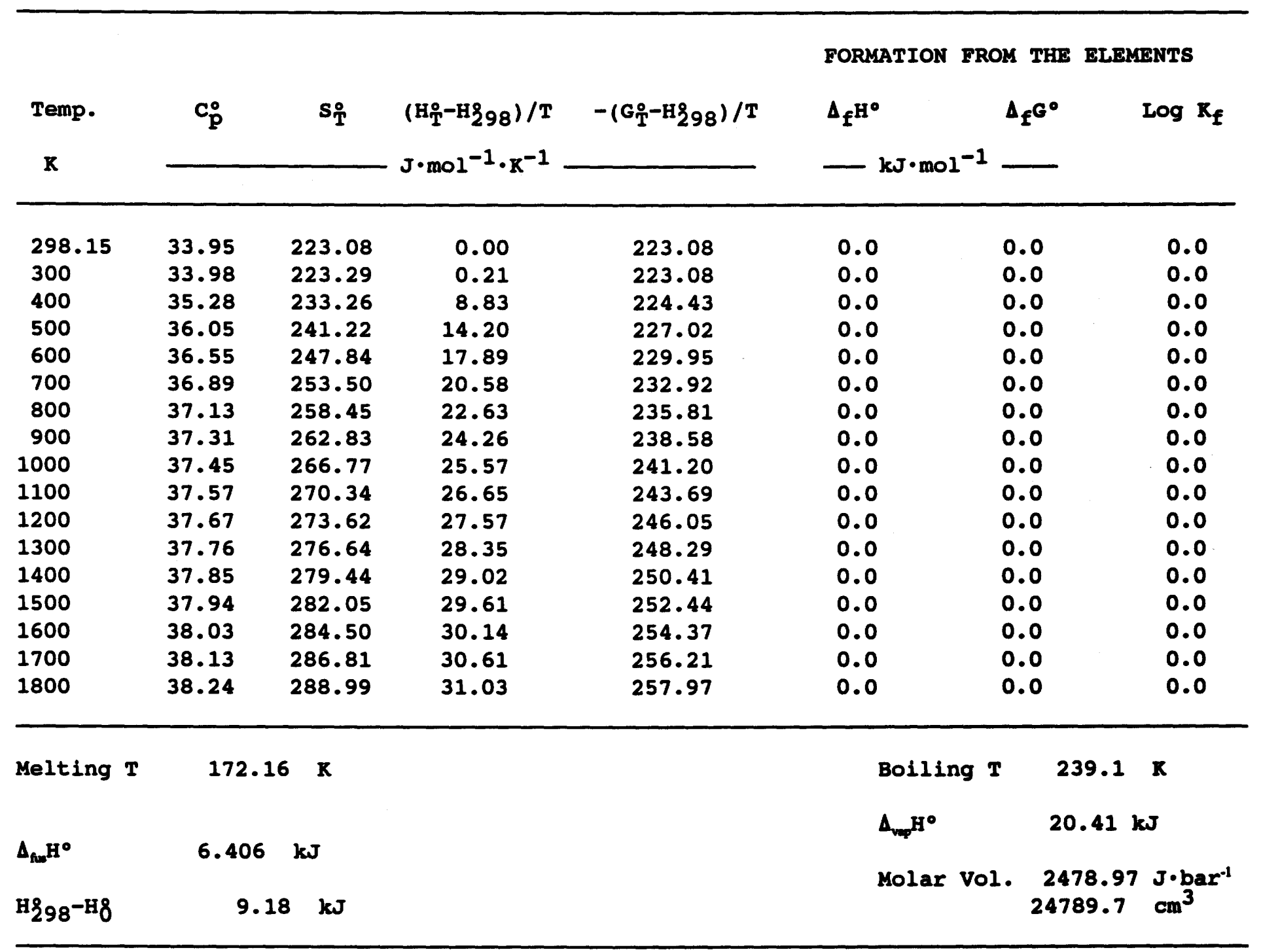


Co: $\alpha$-crystals (hexagonal close packed) 298.15 to $700 \mathrm{~K}$. p-crystals (face-centered cubic) 700 to melting point $1768 \mathrm{~K}$. Liquid 1768 to $1800 \mathrm{~K}$. Curie point at $1394 \mathrm{~K}$.

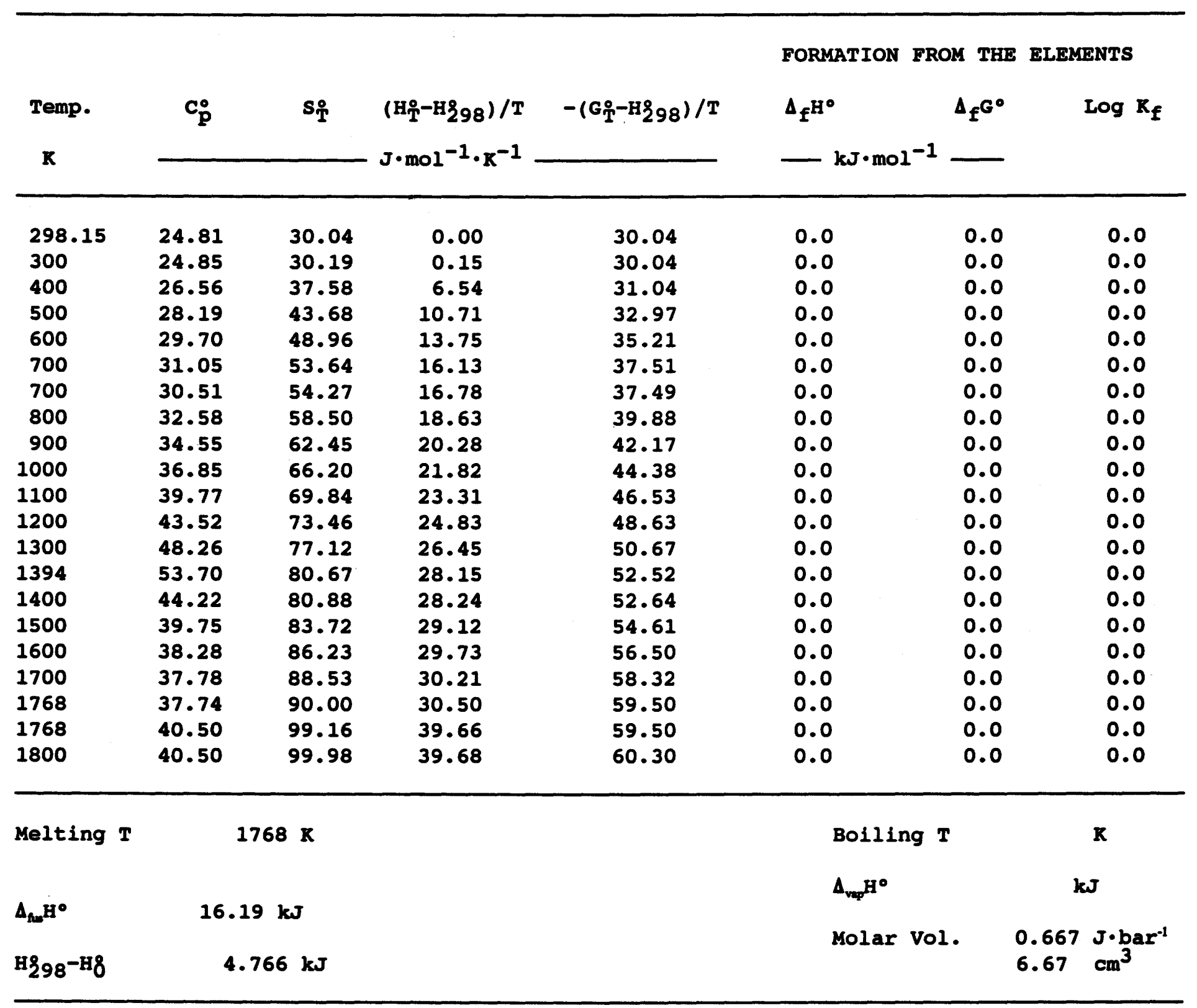


Cr: Body-centered cubic crystals 298.15 to melting point $2130 \mathrm{~K}$. Néel temperature $311.5 \mathrm{~K}$.

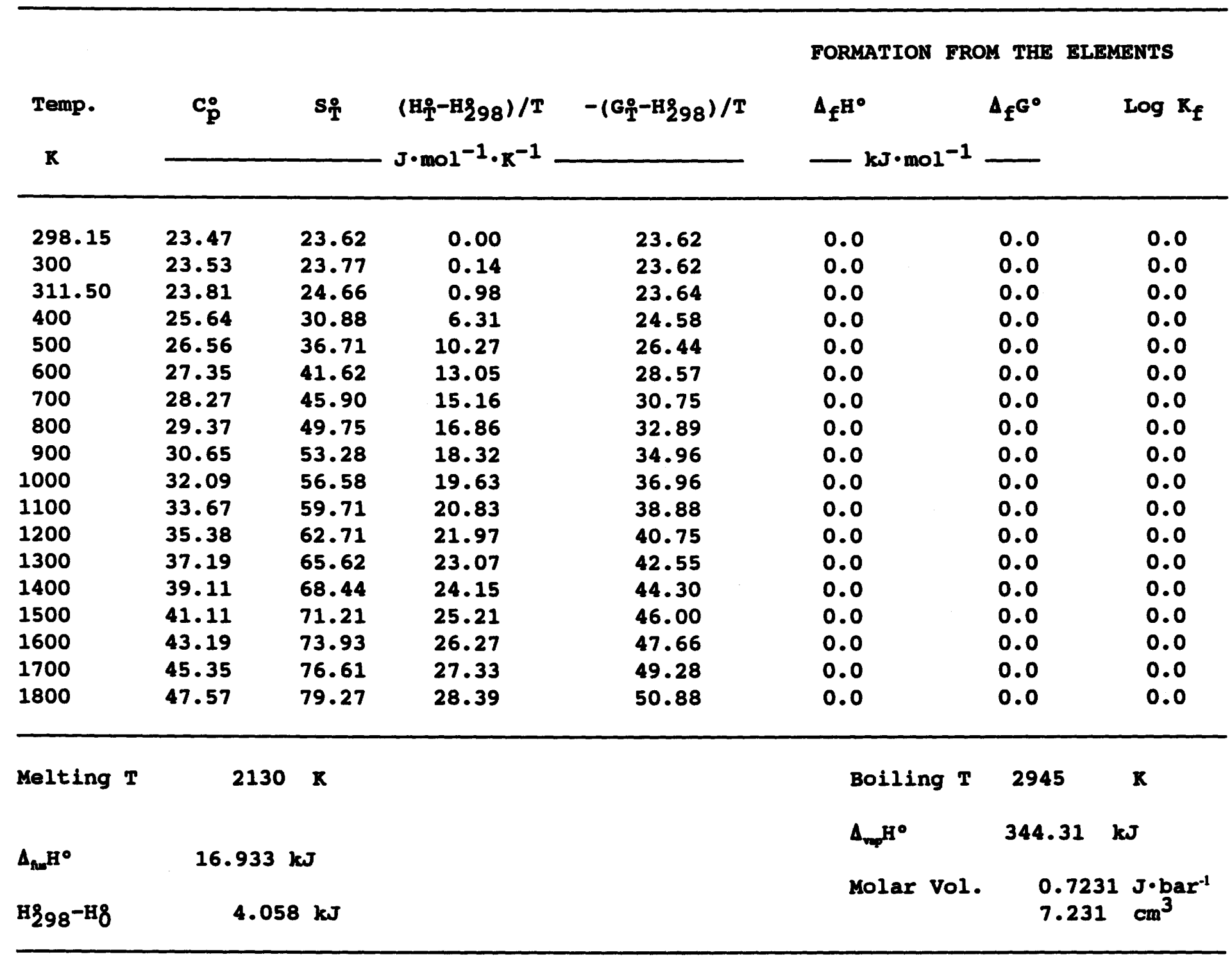


C8: Body-centered cubic crystals 298.15 to melting point $301.55 \mathrm{~K}$. Liquid 301.55 to boiling point $947.96 \mathrm{~K}$. Ideal monatomic gas, $\mathrm{P}=1$ bar, 942 to $1800 \mathrm{~K}$.

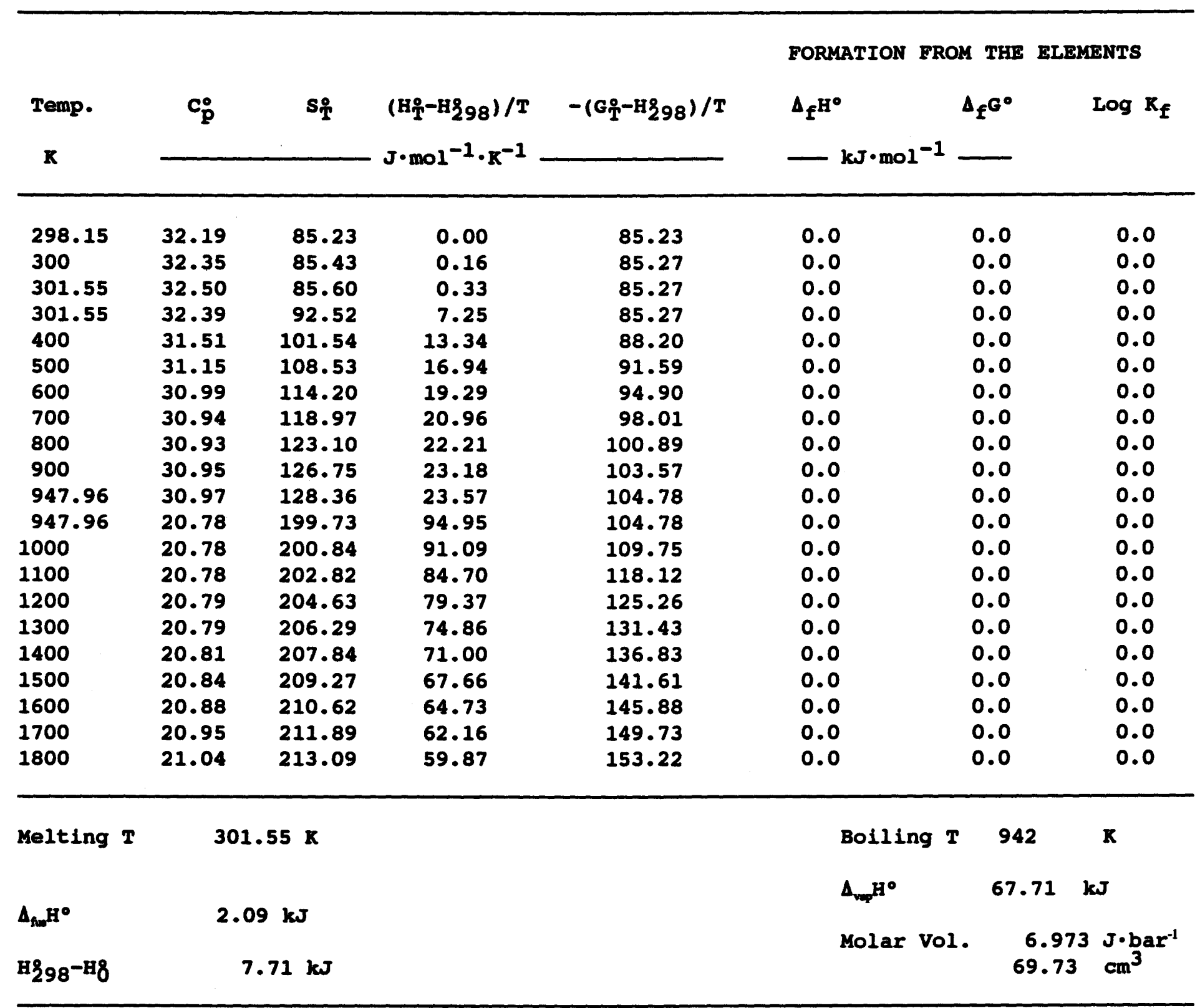


Cu: Face-centered cubic crystals 298.15 to melting point $1357.8 \mathrm{~K}$. Liquid 1357.8 to $1800 \mathrm{~K}$.

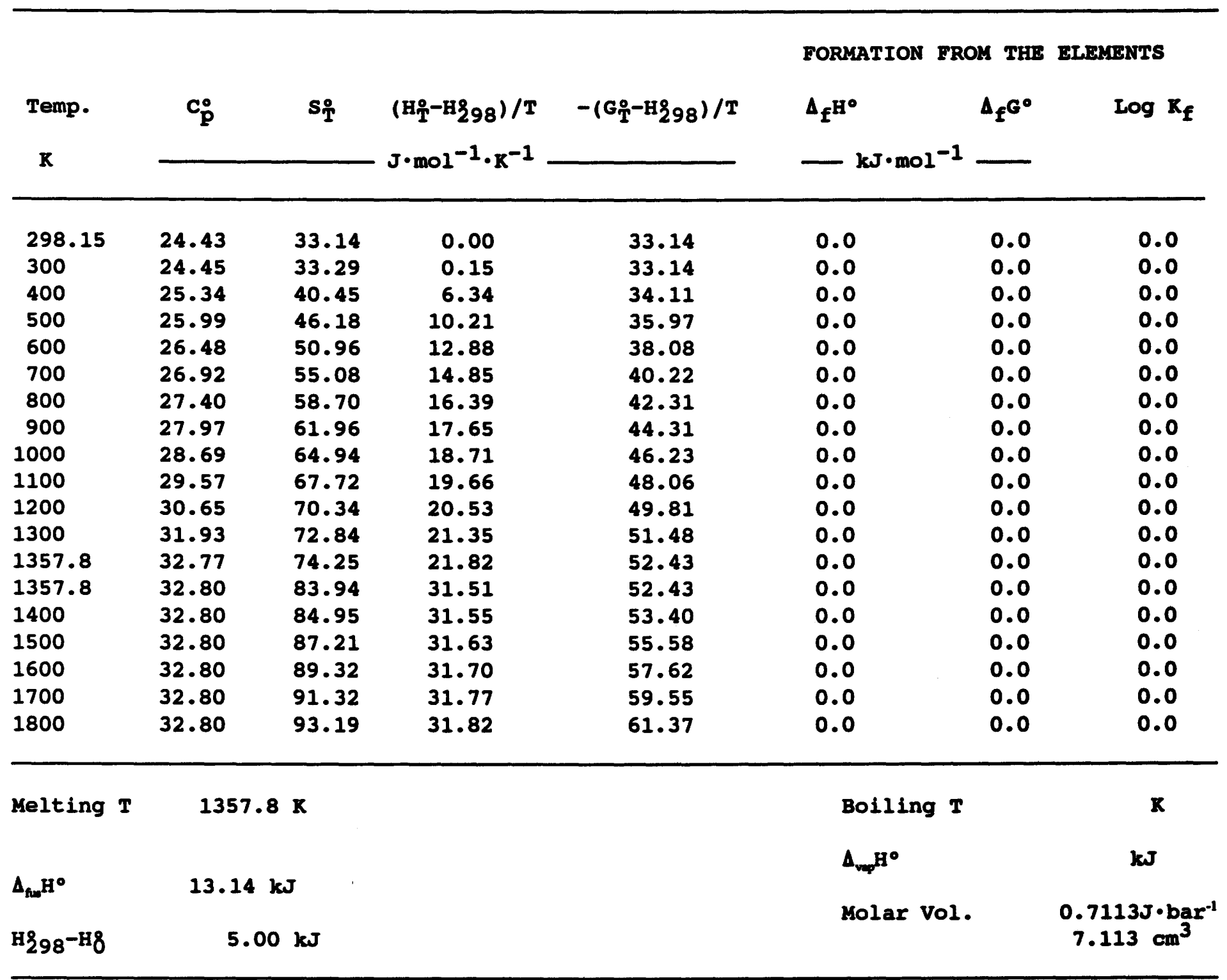


$F_{2}$ : Ideal diatomic gas at $P=1$ bar 298.15 to $2500 \mathrm{~K}$.

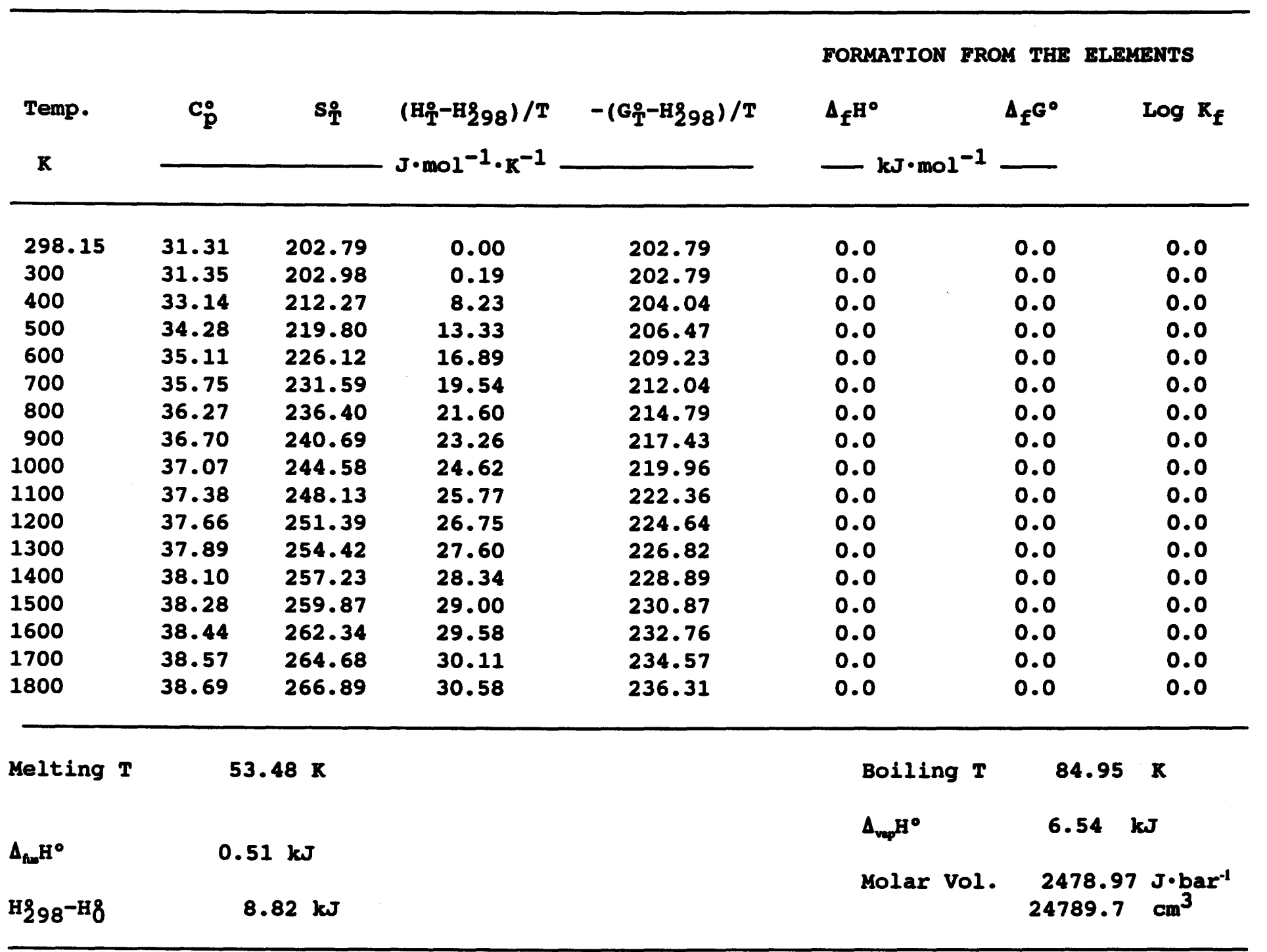


Fe: Body-centered cubic crystals 298.15 to $1185 \mathrm{~K}$. Curie point $1043 \mathrm{~K}$; face-centered cubic crystals 1185 to $1667 \mathrm{~K}$; body-centered cubic crystals 1667 to melting point $1809 \mathrm{~K}$.

\begin{tabular}{|c|c|c|c|c|c|c|c|c|}
\hline \multirow{2}{*}{$\begin{array}{l}\text { Temp. } \\
\mathbf{K}\end{array}$} & \multirow[t]{2}{*}{$\mathbf{c}_{\mathbf{p}}^{\circ}$} & \multirow[t]{2}{*}{$\mathbf{s}_{\text {T }}^{\text {早 }}$} & \multirow{2}{*}{$\begin{array}{l}\left(\mathrm{H}_{\mathrm{T}}-\mathrm{H} 298\right) / \mathrm{T} \\
\mathrm{J} \cdot \mathrm{mol}^{-1} \cdot \mathrm{K}^{-1}\end{array}$} & \multirow[t]{2}{*}{$-\left(G_{T}^{\circ}-H_{298}\right) / T$} & \multicolumn{2}{|c|}{$\begin{array}{l}\text { FORMATION FROM THE } \\
\Delta_{\mathrm{f}^{\mathrm{H}^{\circ}}} \Delta_{\mathrm{f}} \mathrm{G}^{\circ}\end{array}$} & \multicolumn{2}{|c|}{$\begin{array}{l}\text { ELEMENTS } \\
\qquad \log \mathrm{x}_{\mathbf{f}}\end{array}$} \\
\hline & & & & & $-\mathrm{kJ} \cdot \mathrm{mol}^{-1}$ & - & & \\
\hline 298.15 & 25.08 & 27.09 & 0.00 & 27.08 & 0.0 & 0.0 & & 0.0 \\
\hline 300 & 25.13 & 27.24 & 0.16 & 27.08 & 0.0 & 0.0 & & 0.0 \\
\hline 400 & 27.43 & 34.80 & 6.69 & 28.10 & 0.0 & 0.0 & & 0.0 \\
\hline 500 & 29.64 & 41.15 & 11.06 & 30.09 & 0.0 & 0.0 & & 0.0 \\
\hline 600 & 32.01 & 46.76 & 14.35 & 32.41 & 0.0 & 0.0 & & 0.0 \\
\hline 700 & 34.62 & 51.89 & 17.06 & 34.83 & 0.0 & 0.0 & & 0.0 \\
\hline 800 & 37.92 & 56.72 & 19.45 & 37.27 & 0.0 & 0.0 & & 0.0 \\
\hline 900 & 43.16 & 61.46 & 21.76 & 39.70 & 0.0 & 0.0 & & 0.0 \\
\hline 1000 & 54.46 & 66.50 & 24.39 & 42.11 & 0.0 & 0.0 & & 0.0 \\
\hline 1043 & 83.77 & 69.14 & 25.97 & 43.18 & 0.0 & 0.0 & & 0.0 \\
\hline 1100 & 44.35 & 71.91 & 27.31 & 44.60 & 0.0 & 0.0 & & 0.0 \\
\hline 1185 & 40.00 & 75.01 & 28.33 & 46.67 & 0.0 & 0.0 & & 0.0 \\
\hline 1185 & 33.78 & 75.77 & 29.10 & 46.67 & 0.0 & 0.0 & & 0.0 \\
\hline 1200 & 33.90 & 76.19 & 29.16 & 47.04 & 0.0 & 0.0 & & 0.0 \\
\hline 1300 & 34.81 & 78.94 & 29.56 & 49.38 & 0.0 & 0.0 & & 0.0 \\
\hline 1400 & 35.75 & 81.56 & 29.97 & 51.59 & 0.0 & 0.0 & & 0.0 \\
\hline 1500 & 36.69 & 84.06 & 30.38 & 53.67 & 0.0 & 0.0 & & 0.0 \\
\hline 1600 & 37.63 & 86.45 & 30.81 & 55.65 & 0.0 & 0.0 & & 0.0 \\
\hline 1667 & 38.26 & 88.01 & 31.09 & 56.92 & 0.0 & 0.0 & & 0.0 \\
\hline 1667 & 40.40 & 88.52 & 31.60 & 56.92 & 0.0 & 0.0 & & 0.0 \\
\hline 1700 & 41.45 & 89.32 & 31.78 & 57.54 & 0.0 & 0.0 & & 0.0 \\
\hline 1800 & 44.65 & 91.78 & 32.41 & 59.37 & 0.0 & 0.0 & & 0.0 \\
\hline Melting $T$ & & $09 \mathrm{~K}$ & & & Boiling $T$ & & & $\mathbf{K}$ \\
\hline & & & & & $\Delta_{\mathrm{veq}} \mathrm{H}^{\circ}$ & & $\mathbf{k} \mathbf{J}$ & $\mathbf{J}$ \\
\hline$\Delta_{n w} H^{\circ}$ & & $\mathbf{k J}$ & & & & & & \\
\hline $\mathrm{H} 298^{-\mathrm{H}}$ & & $51 \mathrm{~kJ}$ & & & Molar Vol. & & $\begin{array}{l}0.7092 \\
7.092\end{array}$ & $\begin{array}{l}\mathrm{J} \cdot \mathrm{bar}^{-1} \\
\mathrm{~cm}^{3}\end{array}$ \\
\hline
\end{tabular}


Ge: Face-centered cubic crystals (diamond structure) 298.15 to $1211.4 \mathrm{~K}$. Liquid 1211.4 to boiling point $3107 \mathrm{~K}$.

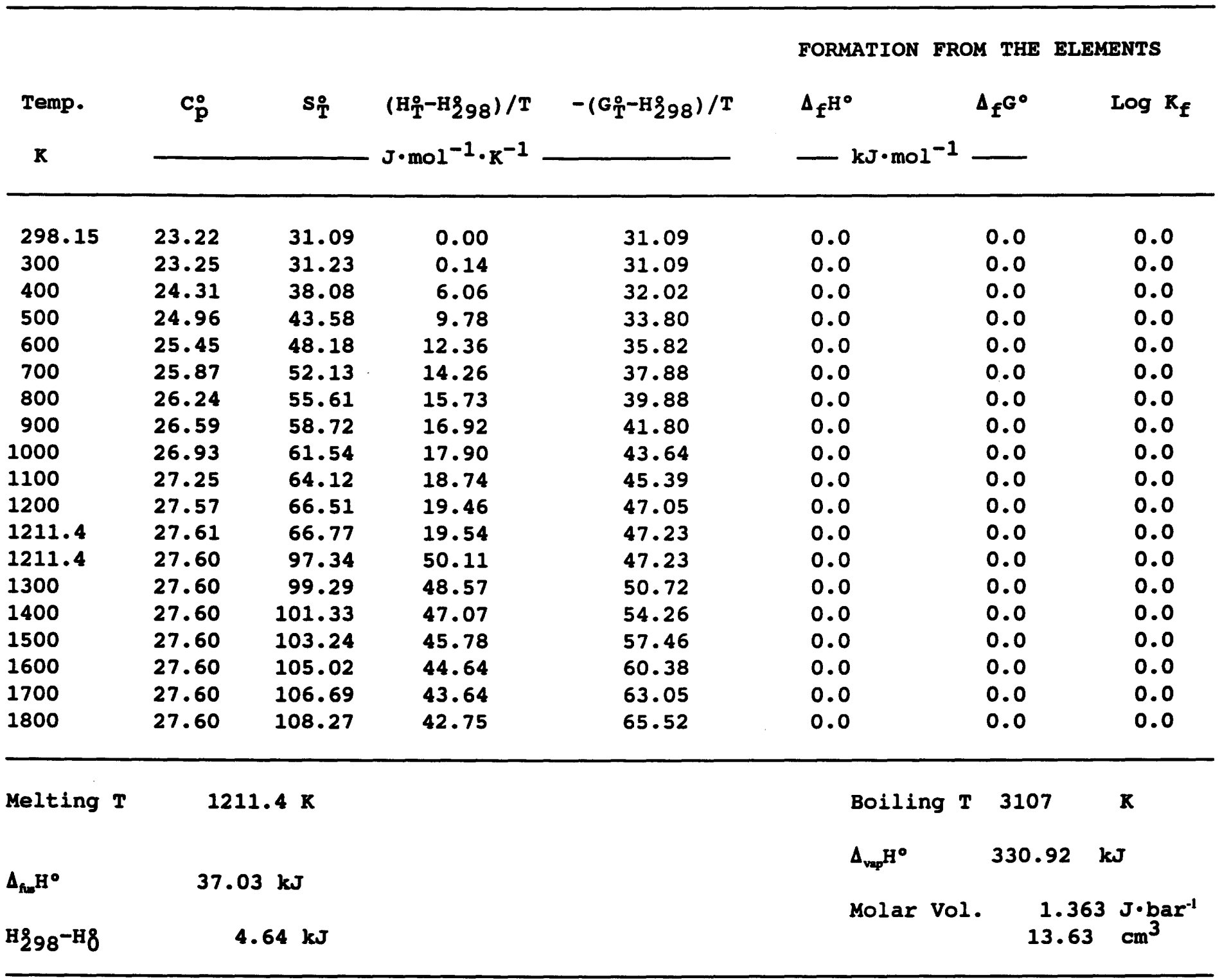




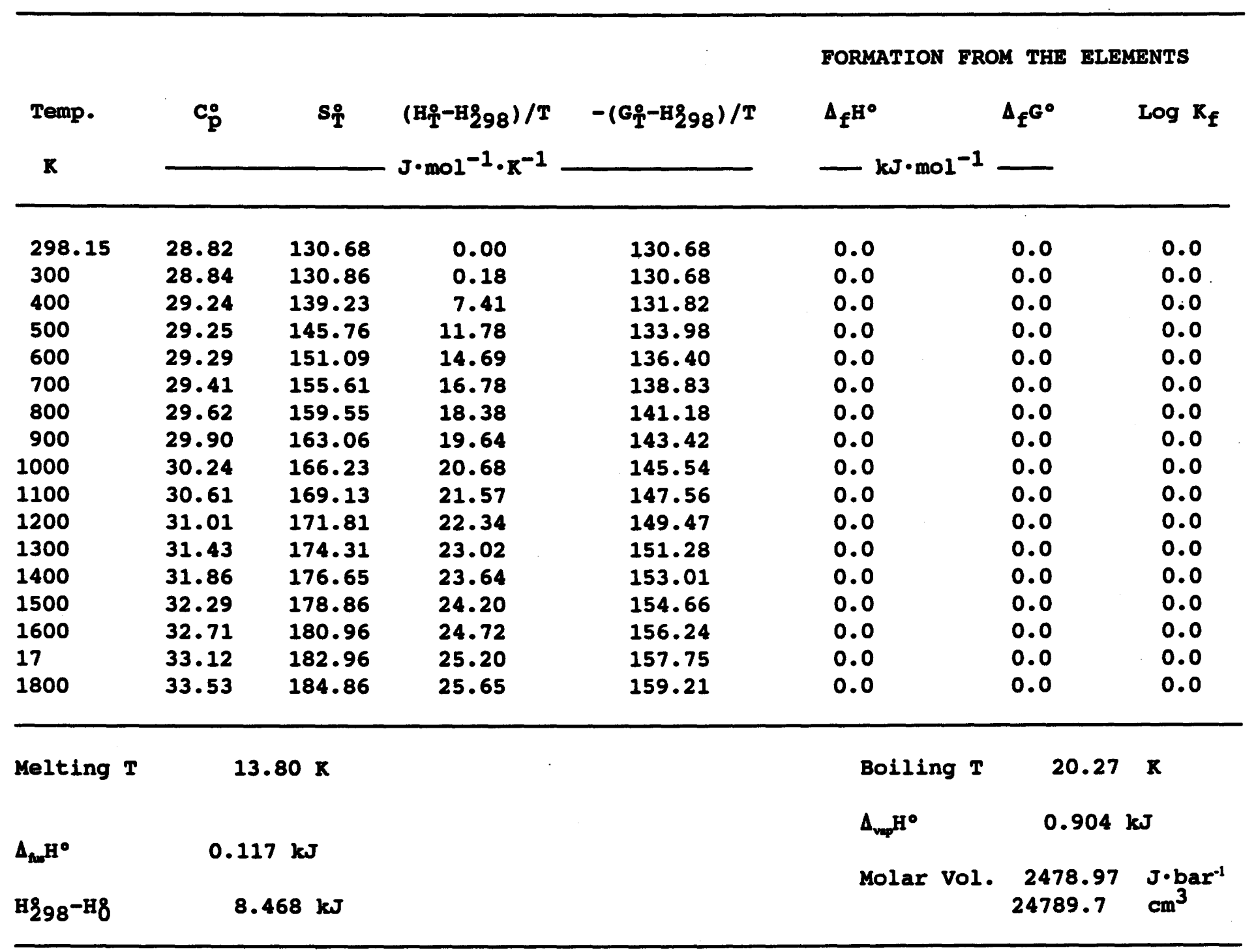


Hg: Iiquid 298.15 to boiling point $629.0 \mathrm{~K}$. Ideal monatonic gas at $\mathrm{P}=1 \mathrm{bar}, 629.0 \mathrm{~K}$.

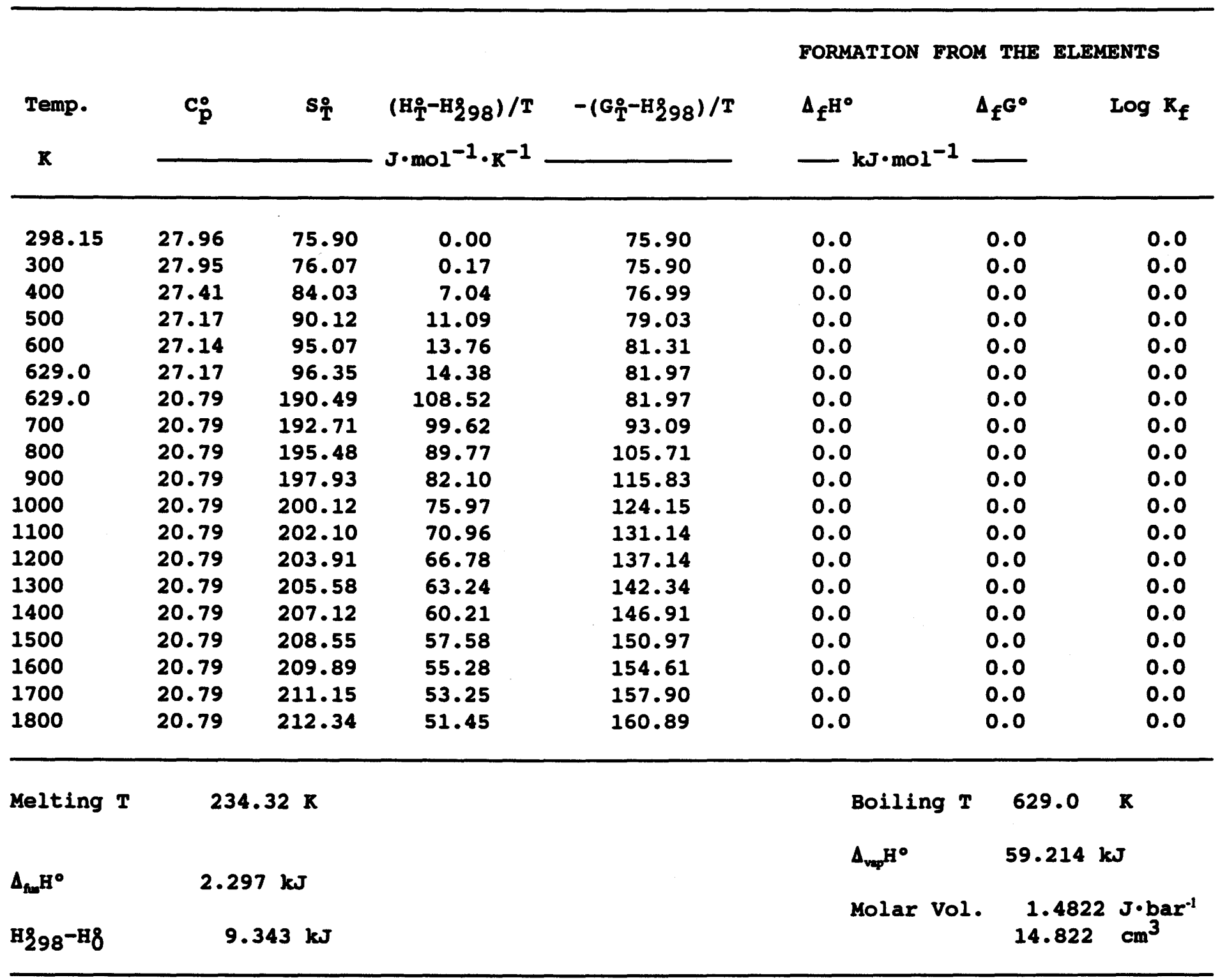


$I_{2}$ Crystals 298.15 to melting point $386.8 \mathrm{~K} ;$ liquid 386.8 to boiling point $457.7 \mathrm{~K}$ ideal diatomic gas, $P=1$ bar, 457.7 to $2200 \mathrm{~K}$.

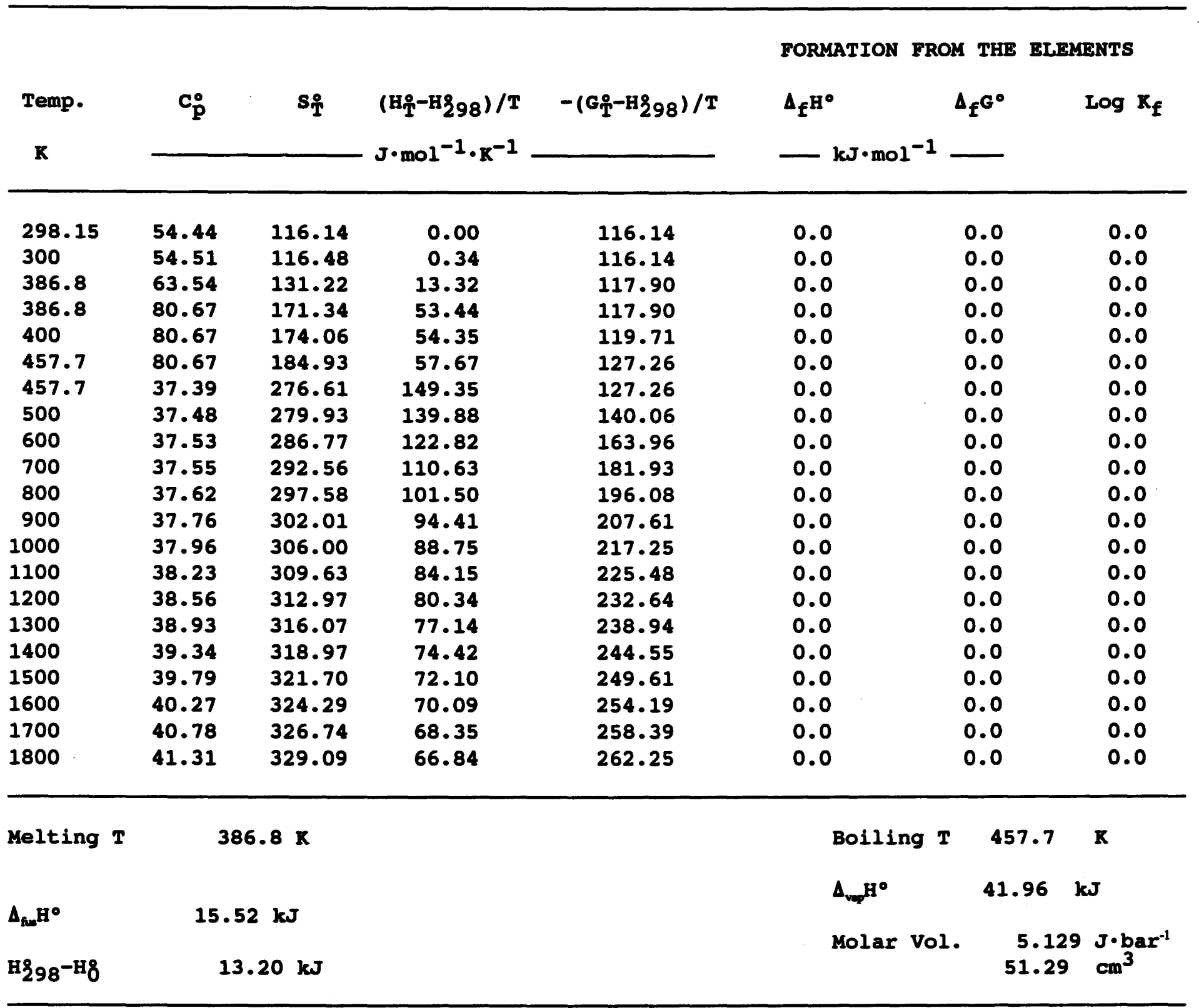


$\mathrm{K}$ : Crystals 298.15 to melting point $336.4 \mathrm{~K}$; liquid 336.4 to boiling point $1039.5 \mathrm{~K}$ ideal monatomic gas, $\mathrm{P}=1$ bar, 1039.5 to $1800 \mathrm{~K}$.

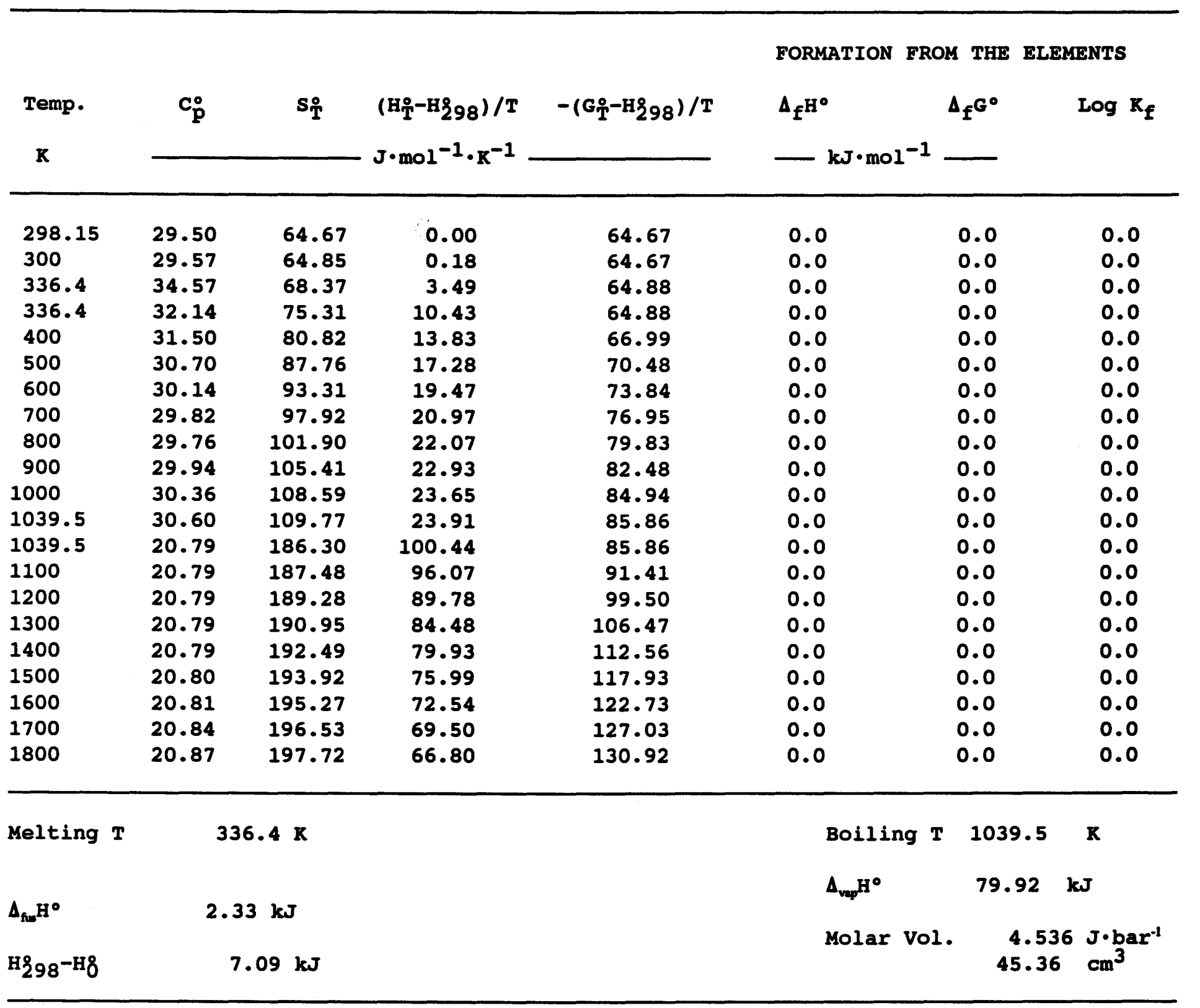


Li: Crystals 298.15 to melting point $453.2 \mathrm{~K}$; liquid $453.7 \mathrm{~K}$ to boiling point $1620 \mathrm{~K}$ ideal monatomic gas, $\mathrm{P}=1$ bar, 1620 to $1800 \mathrm{~K}$.

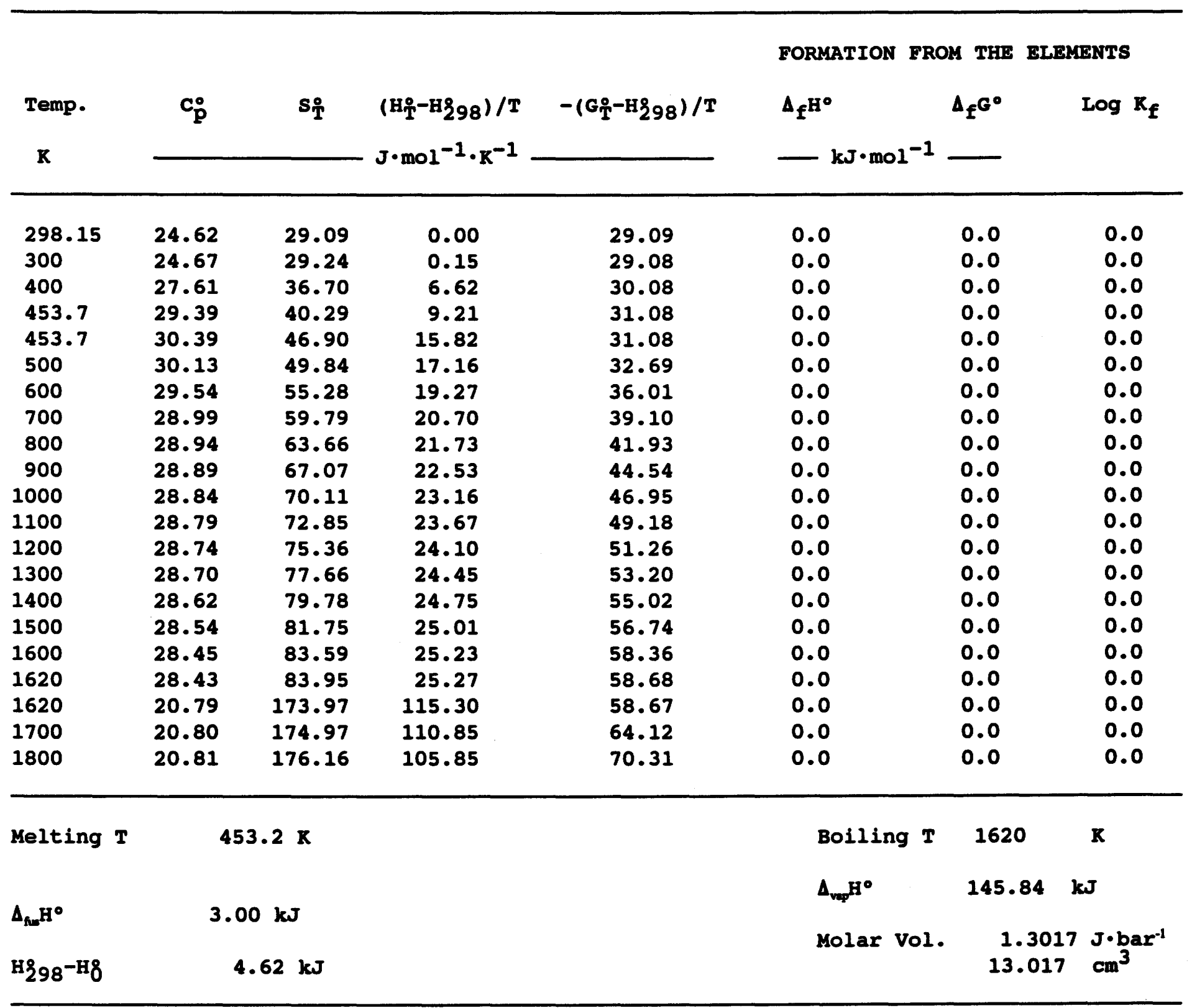


Mg: Hexagonal close-packed crystals 298.15 to melting point $923 \mathrm{~K}$. Liquid 923 to boiling point $1366.1 \mathrm{~K}$. Ideal monotomic gas, $\mathrm{P}=1$ bar, 1366.1 to $1800 \mathrm{~K}$.

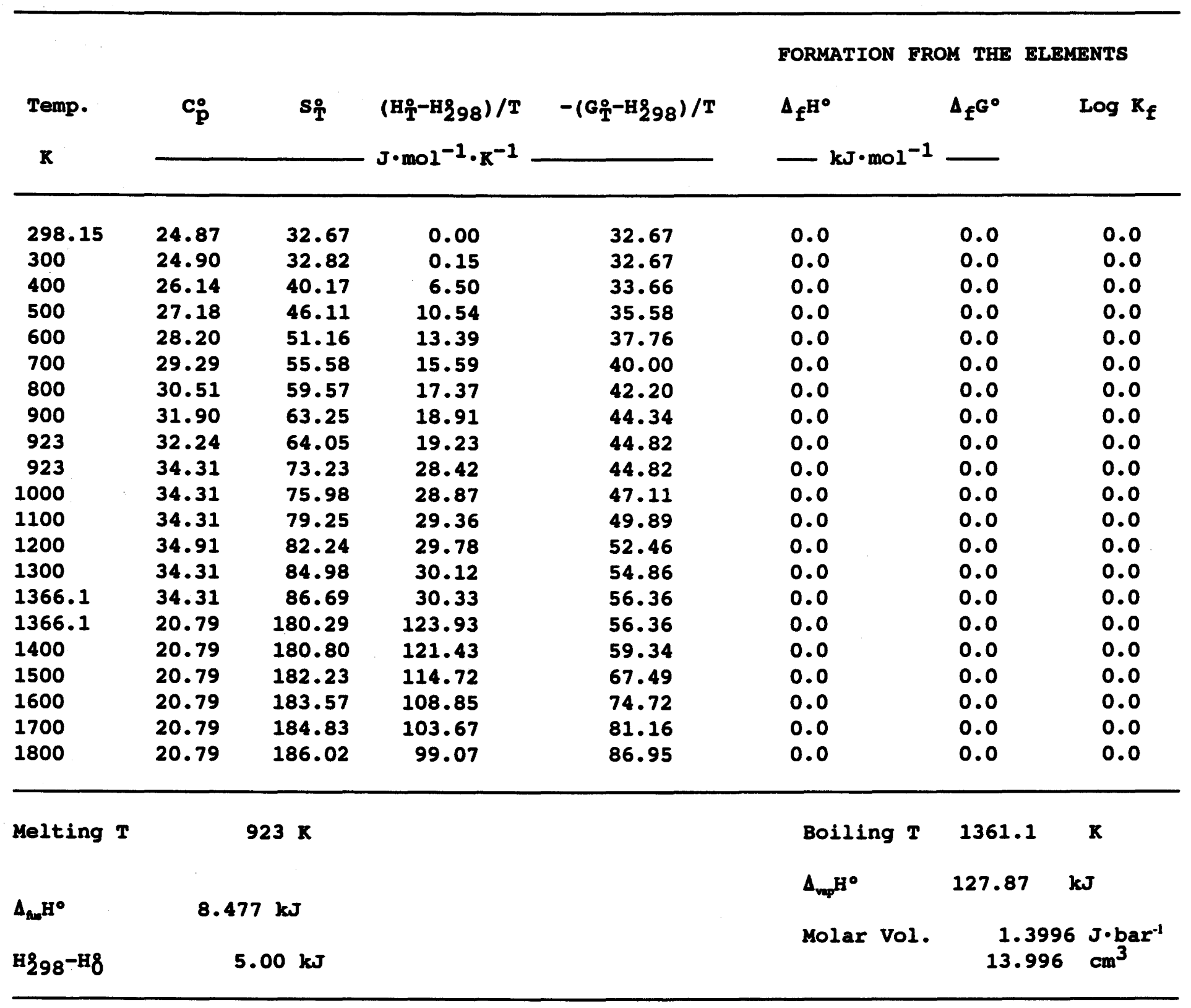


Mn: $\alpha$-crystals 298.15 to $980 \mathrm{~K}$. $\beta$-crystals 980 to $1360 \mathrm{~K}$. $\gamma$-crystals 1360 to $1410 \mathrm{~K}$. $\Delta$ crystals 1410 to melting point $1517 \mathrm{~K}$. Liquid 1517 to boiling point $2335 \mathrm{~K}$.

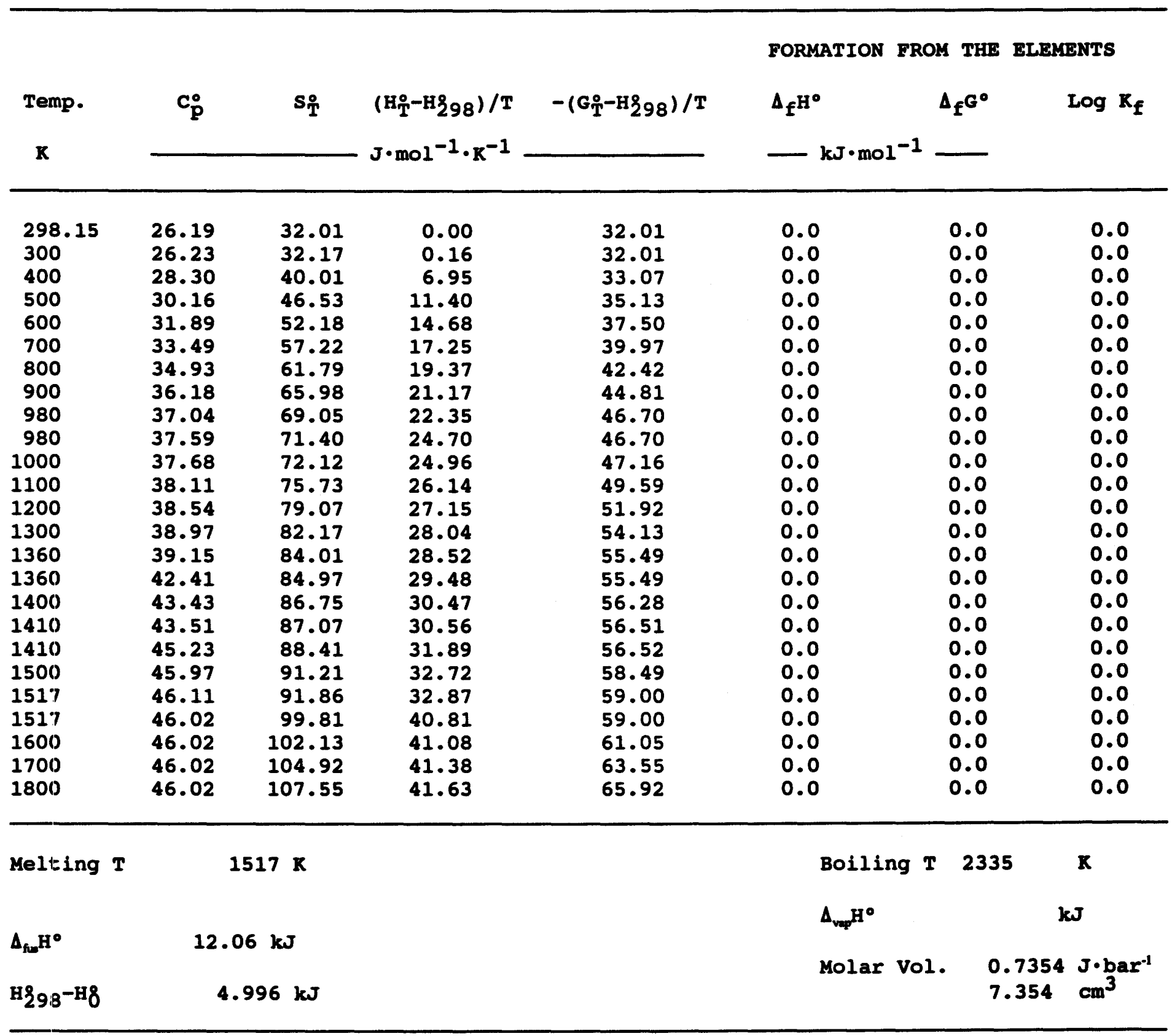


Mo: Body-centered cubic crystals 298.15 to $2500 \mathrm{~K}$.

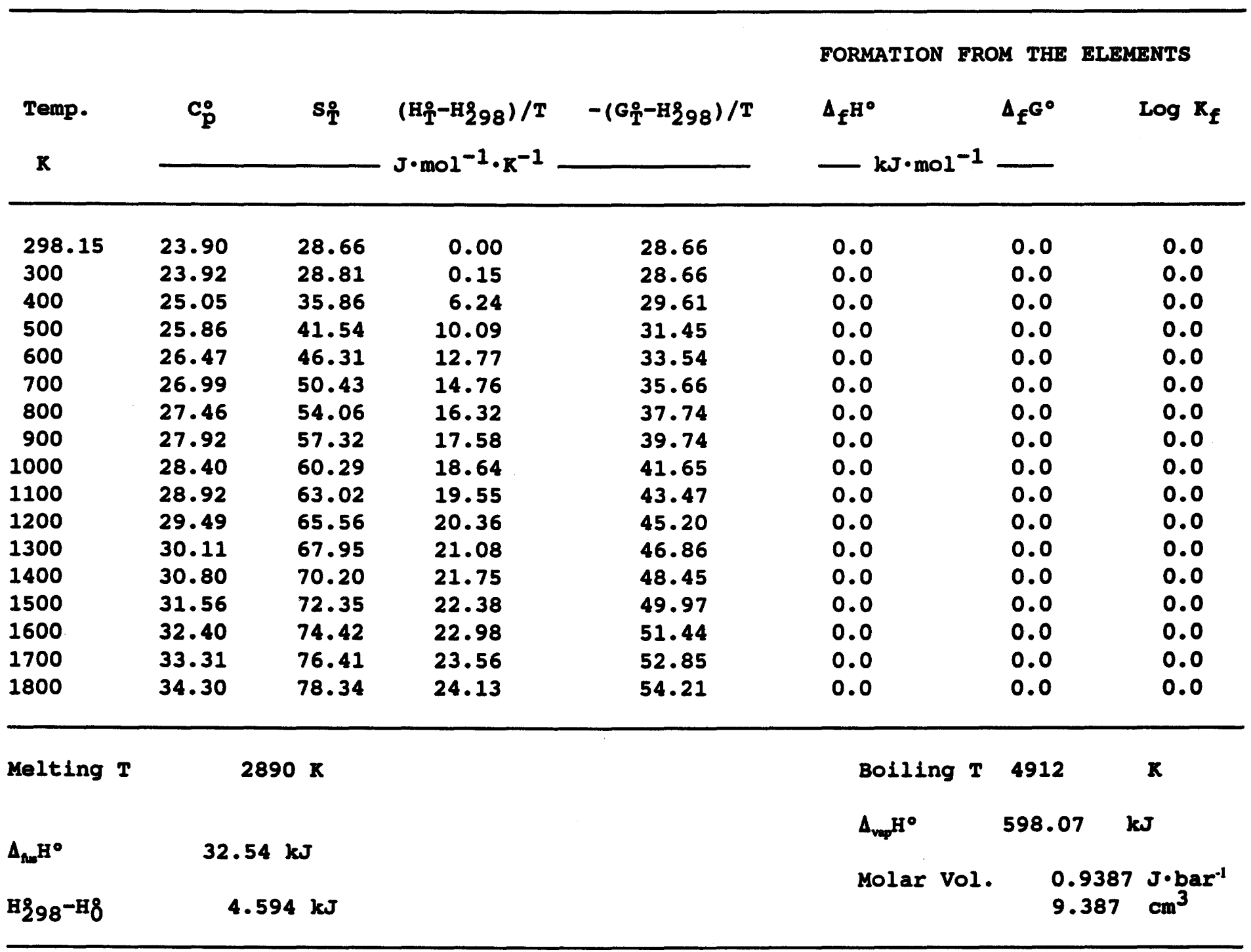


$N_{2}$ : Ideal diatomic gas at $P=1$ bar 298.15 to $2500 \mathrm{~K}$.

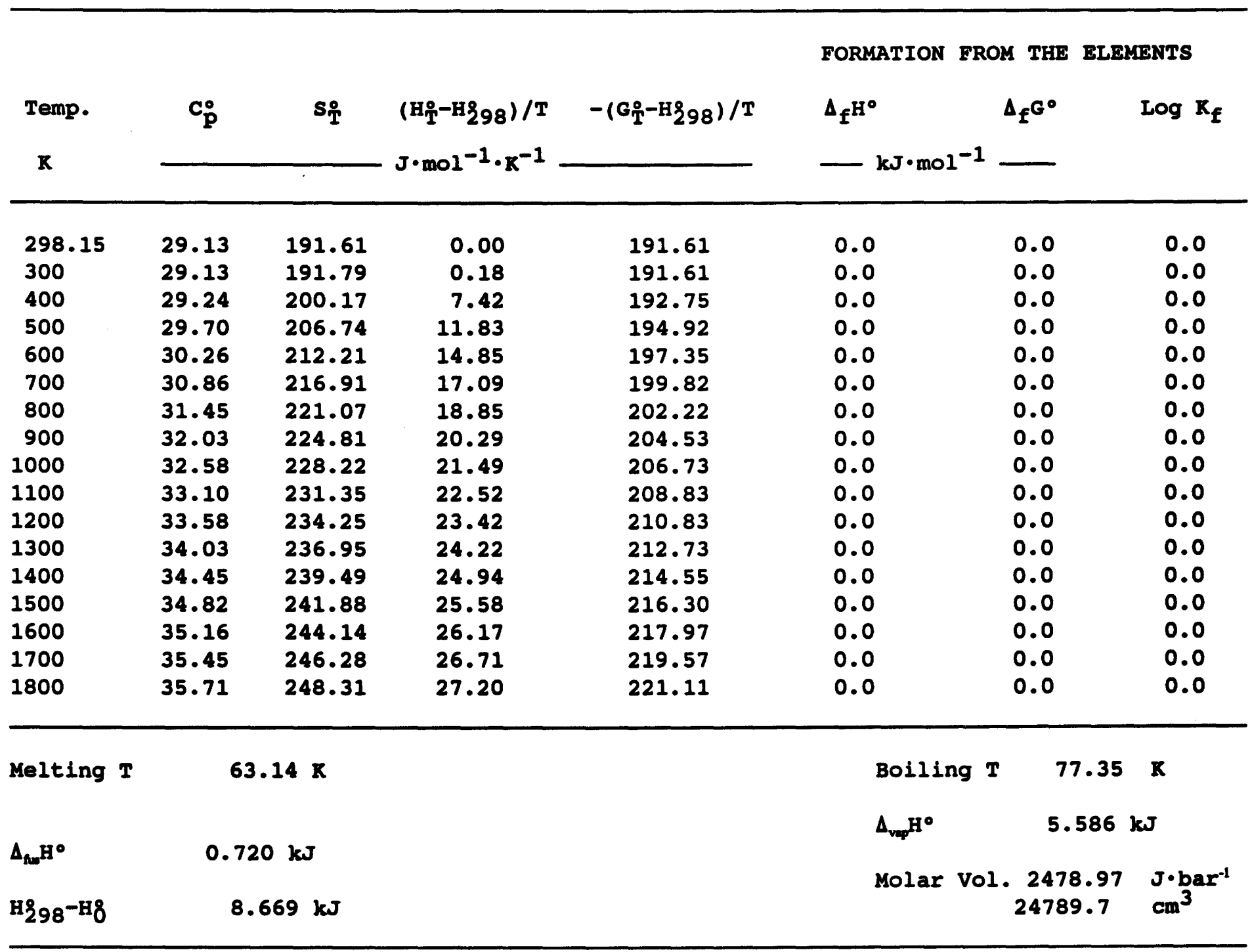


Na: Body-centered cubic crystals 298.15 to melting point $371 \mathrm{~K}$; liquid 371 to fictive boiling point $1170.5 \mathrm{~K}$. Ideal monotomic gas, $\mathrm{P}=1$ bar, 1170.5 to $1800 \mathrm{~K}$.

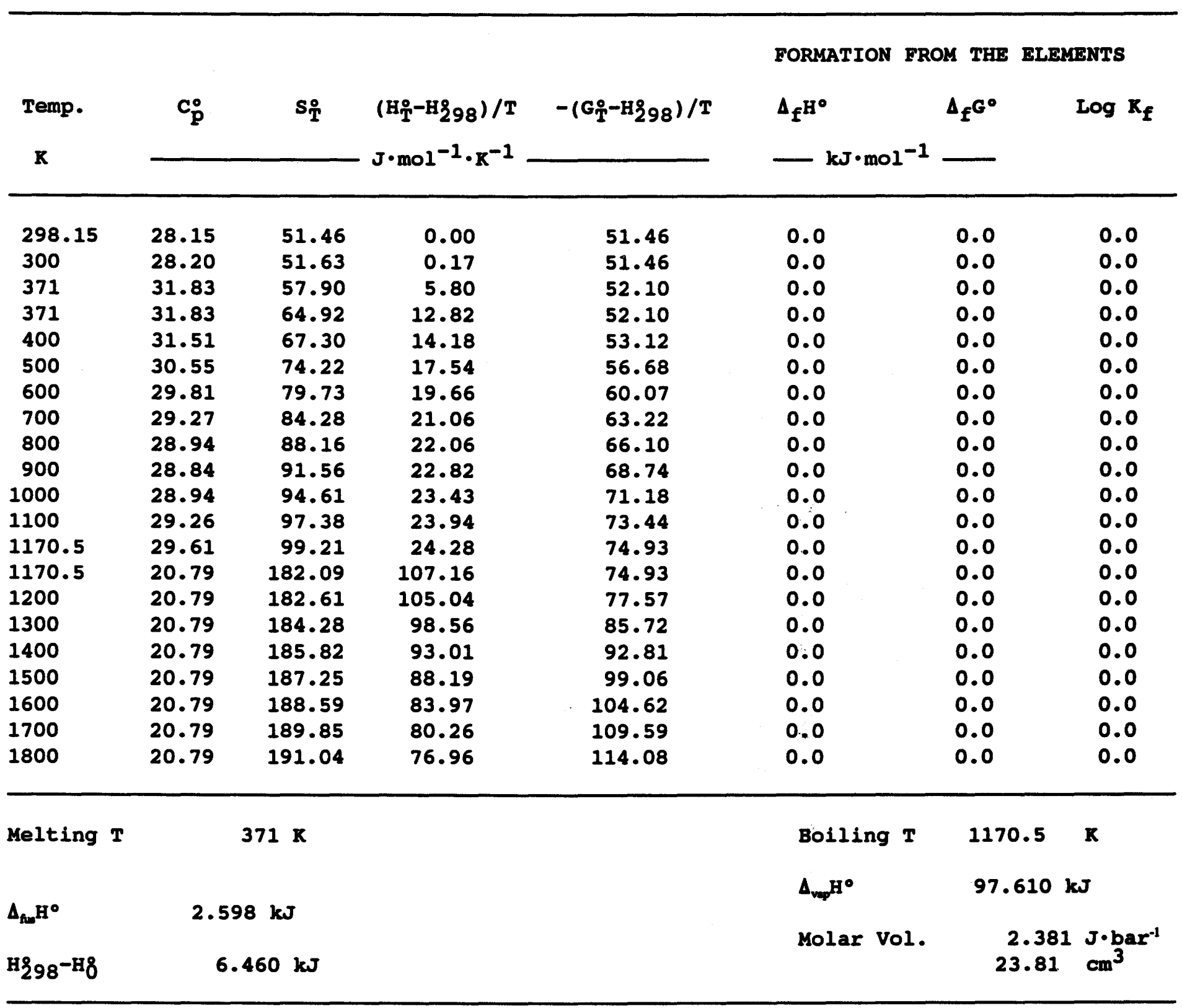


Ni: Face-centered cubic crystals (magnetic) to Curie point $631 \mathrm{~K}$; nonmagnetic 631 to melting point $1728 \mathrm{~K}$; liquid 1728 to $1800 \mathrm{~K}$.

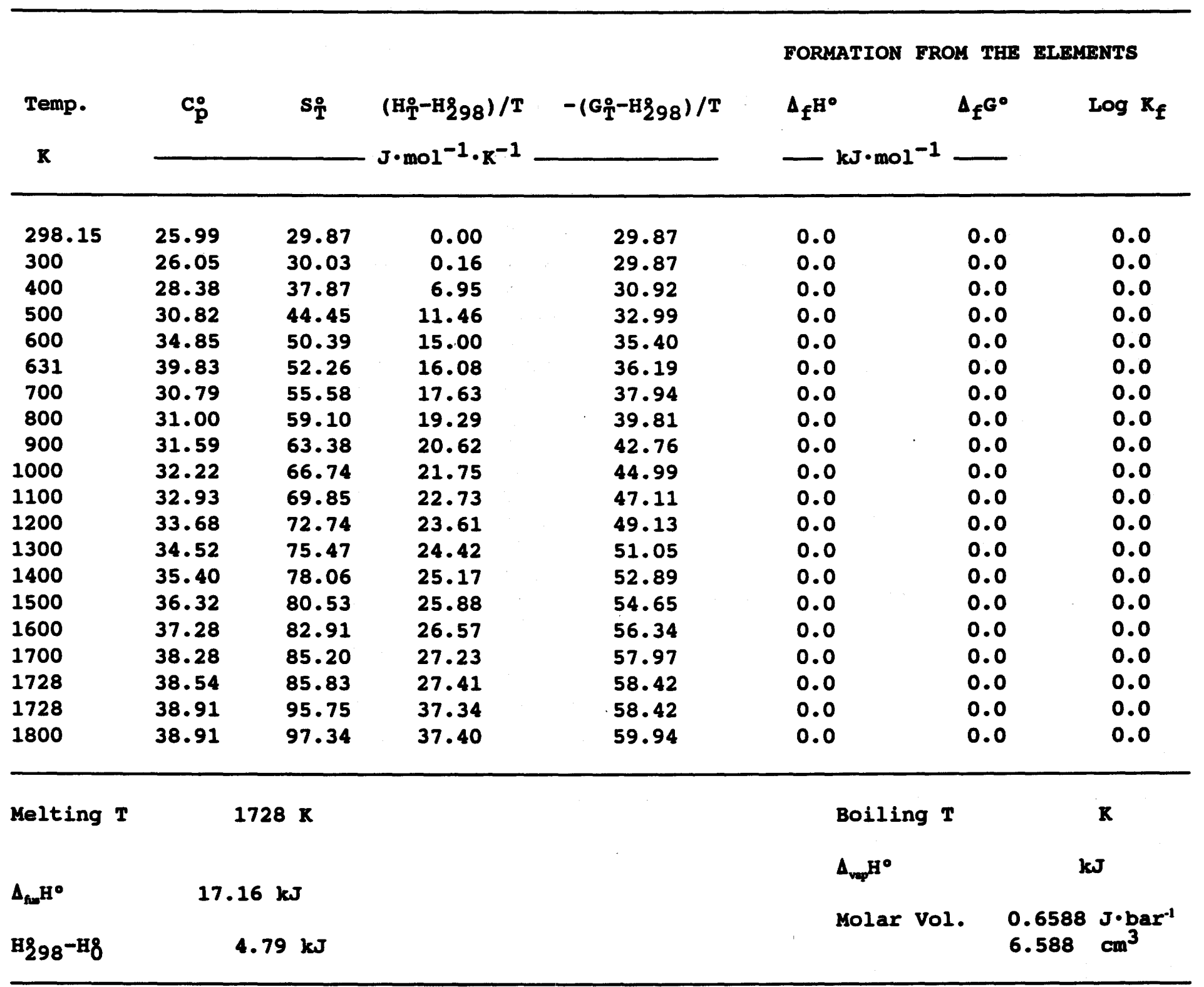


$O_{2}$ : Ideal diatomic gas at $P=1$ bar 298.15 to $2500 \mathrm{~K}$.

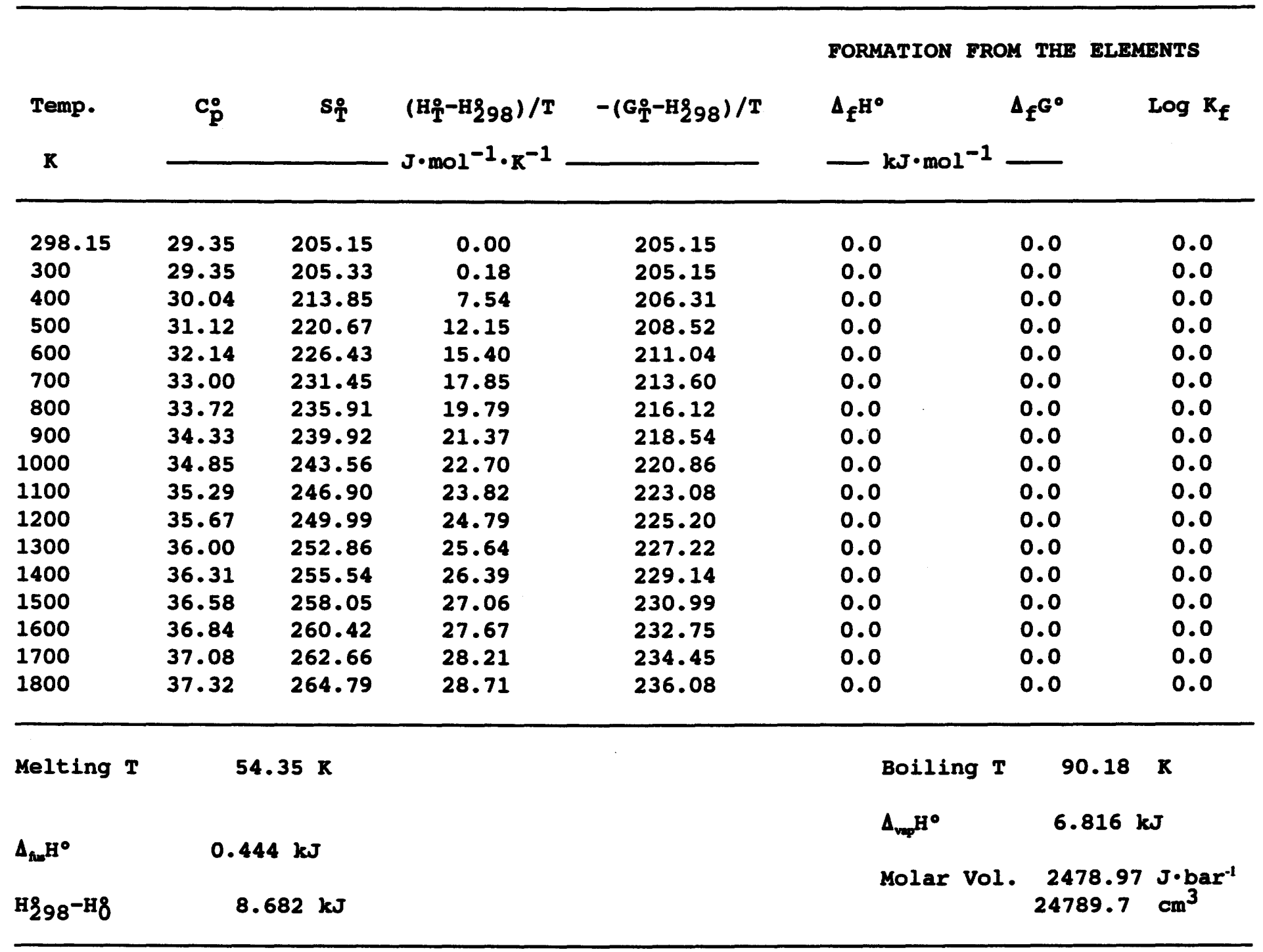


P: Crystals (white, a) 298.15 to melting point $317.3 \mathrm{~K}$. Iiquid 317.3 to sublimation point $1180 \mathrm{~K}$. Ideal diatomic gas 1180 to $1800 \mathrm{~K}$.

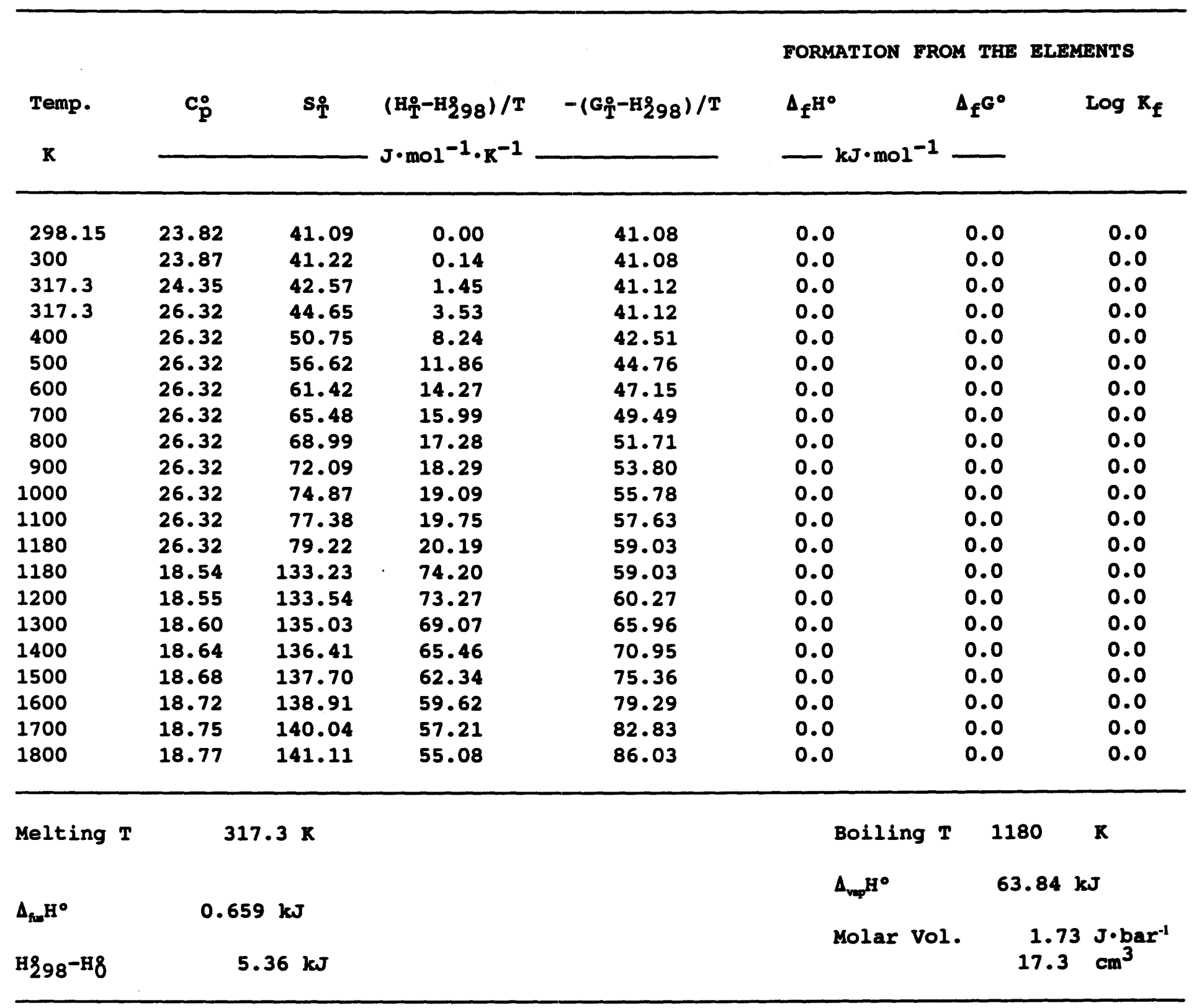


$\mathrm{Pb}$ : Face-centered cubic crystals 298.15 to melting point $600.6 \mathrm{~K}$. Iiquid 600.6 to boiling point $2021 \mathrm{~K}$.

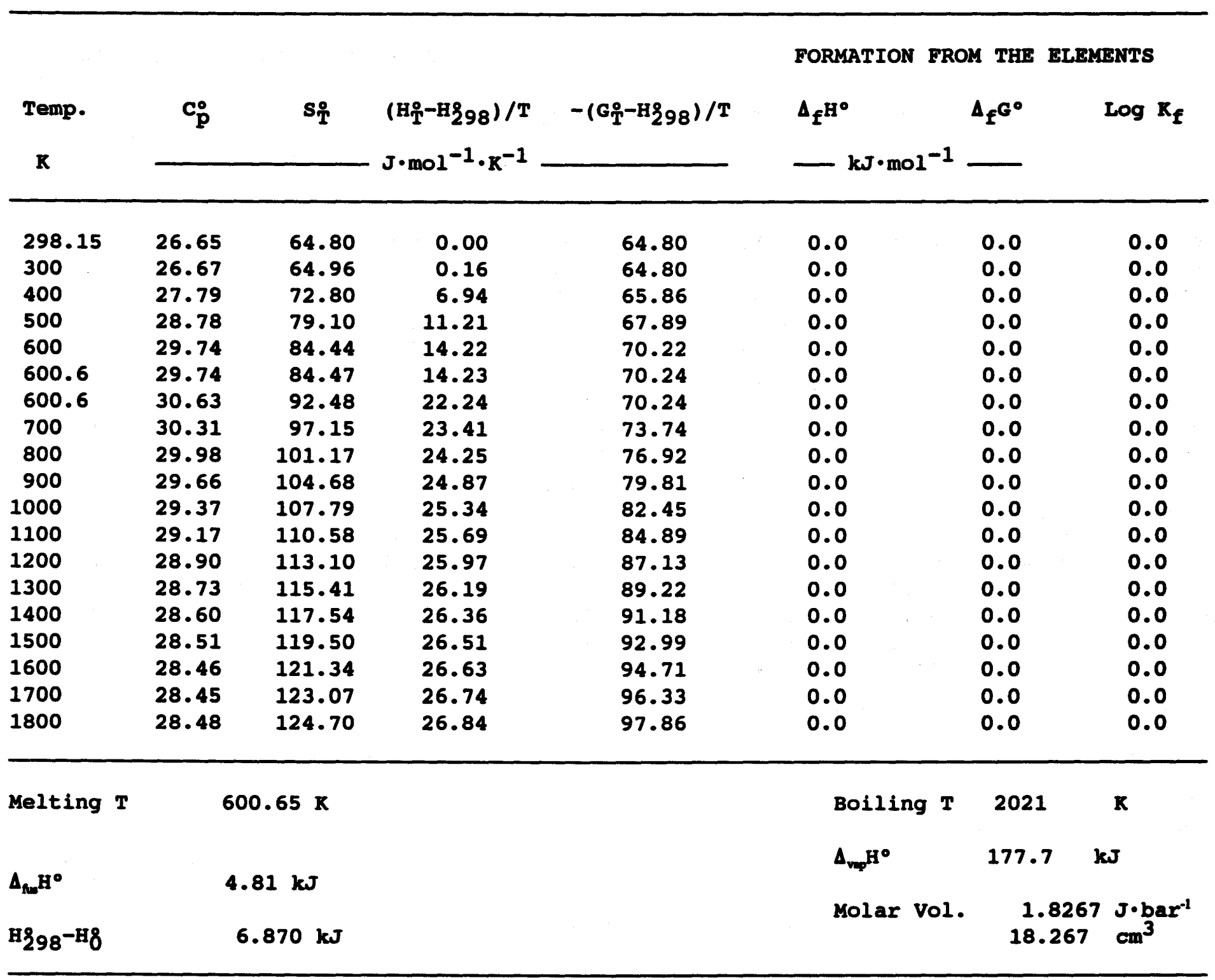


Pt: Face-centered cubic crystals 298.15 to melting point $2042 \mathrm{~K}$.

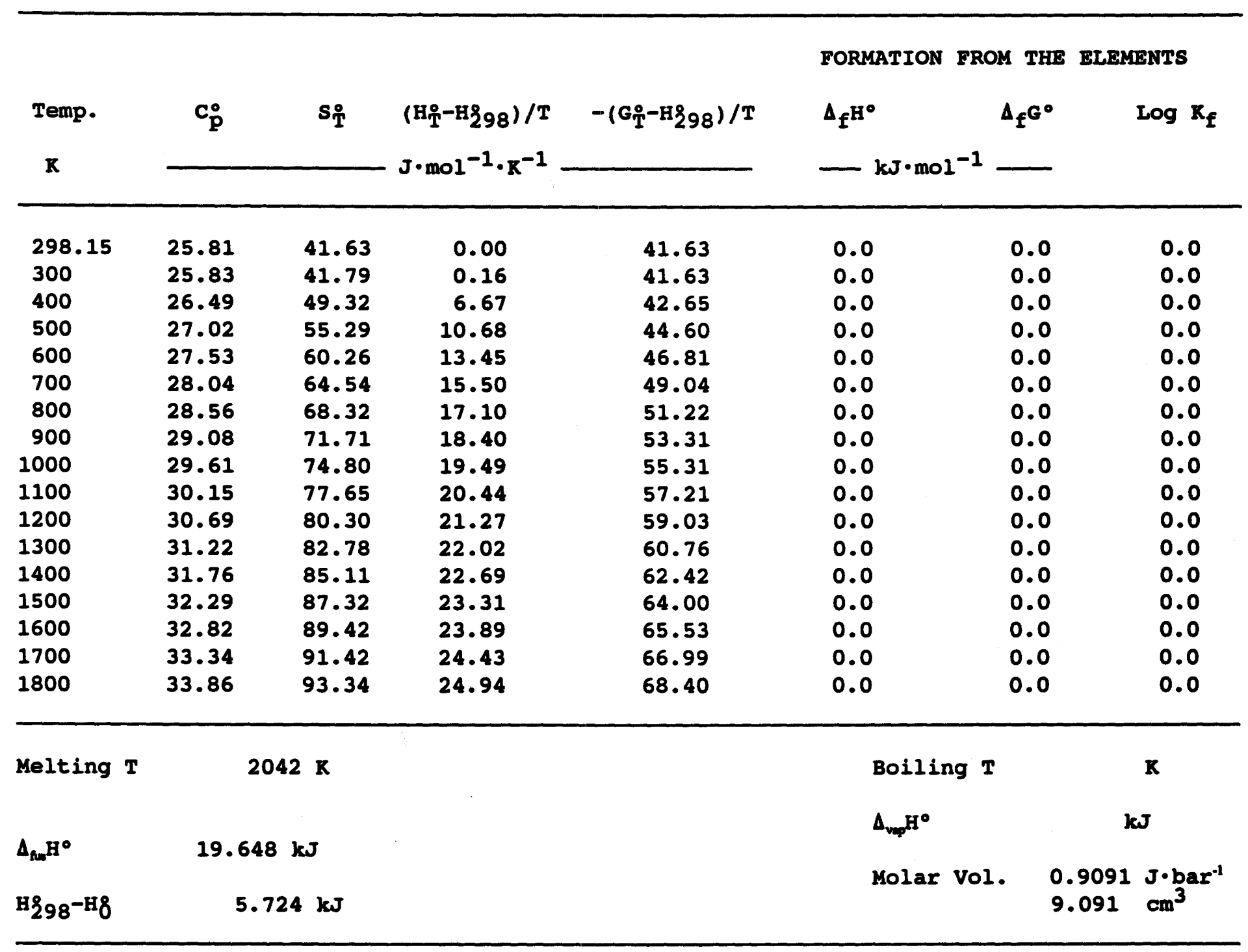


S: Orthorhombic crystals 298.15 to $368.3 \mathrm{~K}$; monoclinic crystals 368.3 to melting point $388.36 \mathrm{~K}$; liquid $388.36 \mathrm{~K}$ to fictive boiling point $882.1 \mathrm{~K}$. Ideal diatomic gas, $P=1$ bar, 882.1 to $1800 \mathrm{~K}$.

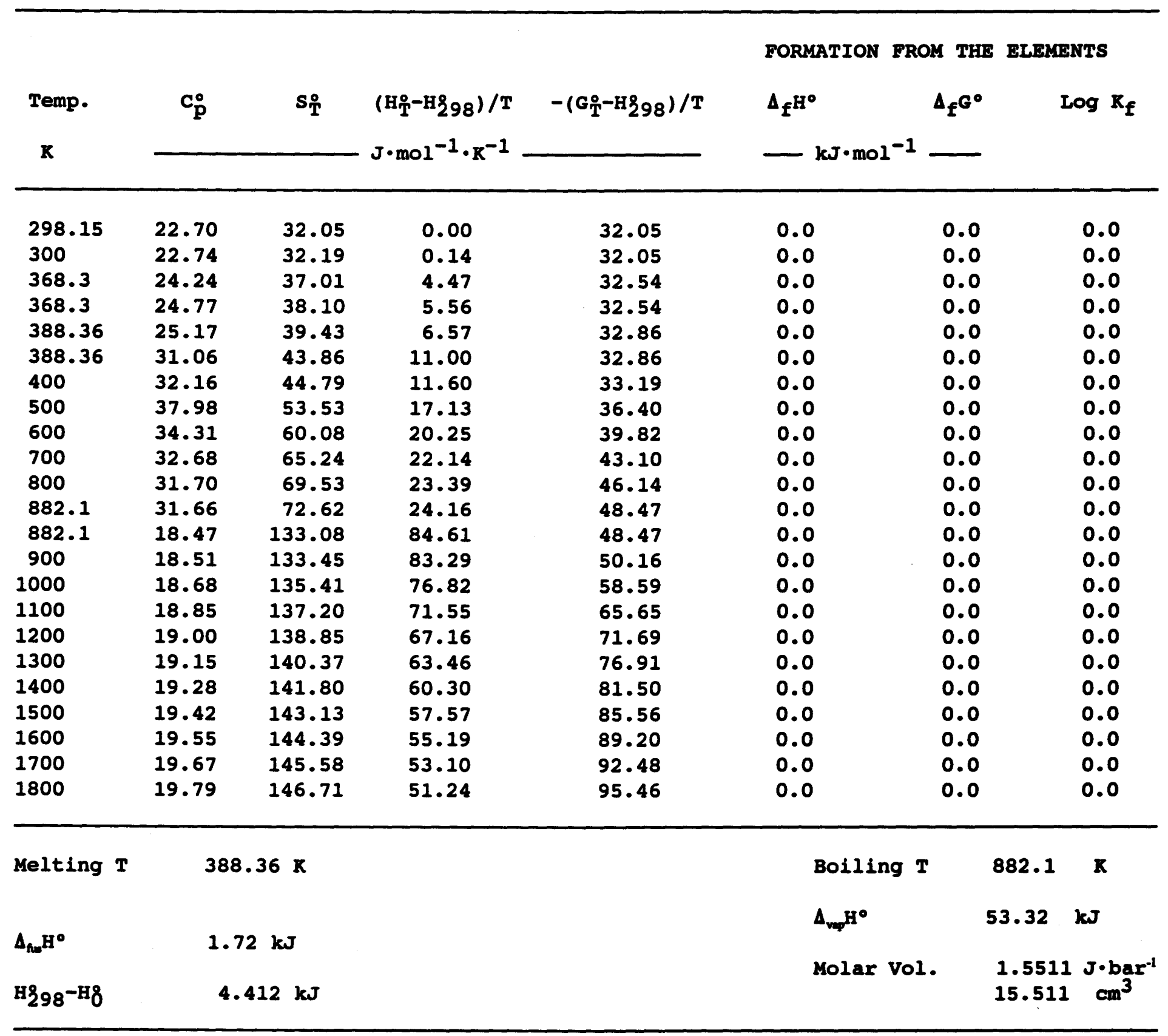


$S_{2}$ : Ideal diatomic gas, $P=1$ bar, 298.15 to $2500 \mathrm{~K}$.

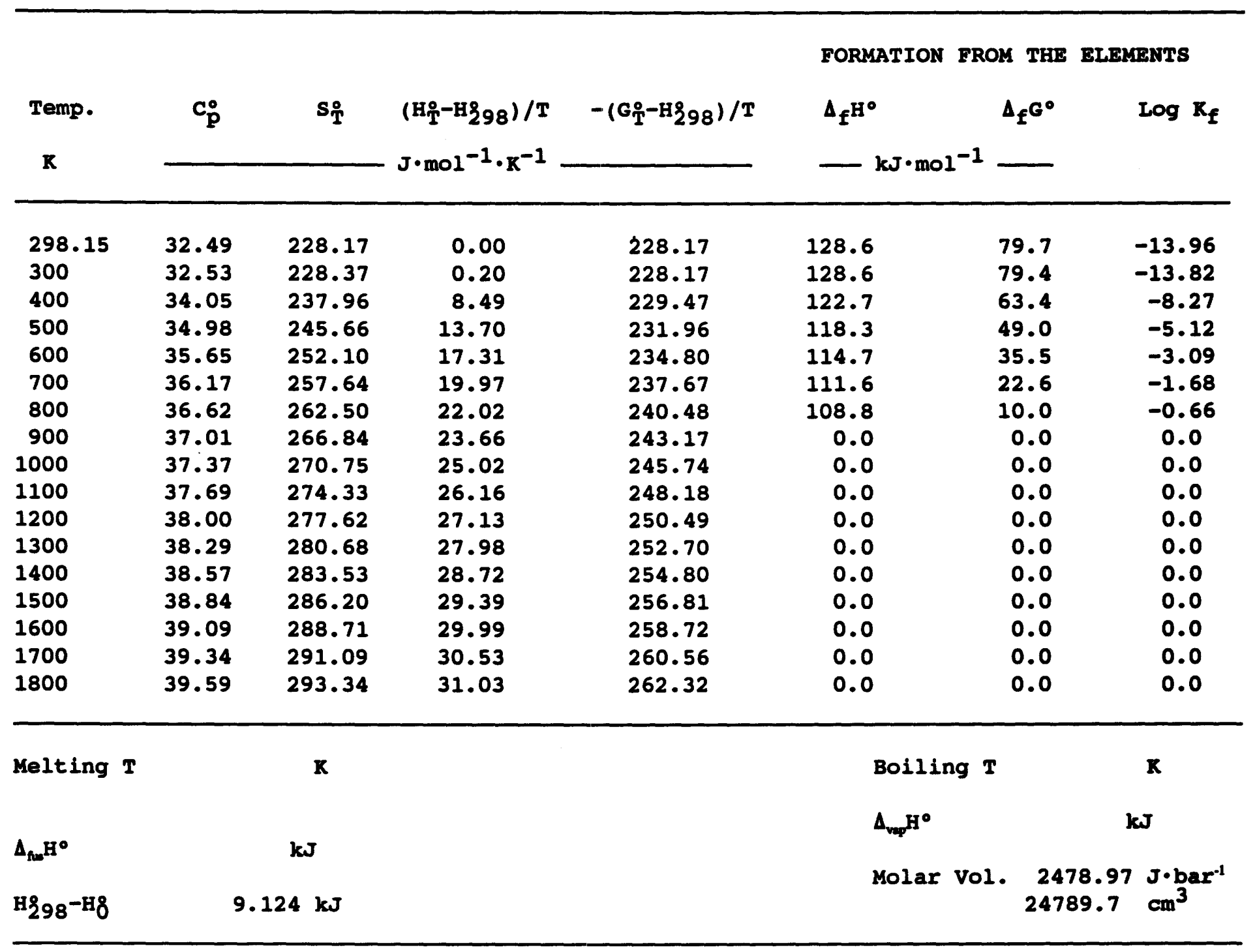


Sb: Rhombohedral crystals 298.15 to melting point $904 \mathrm{~K}$. Liquid 904 to $1800 \mathrm{~K}$.

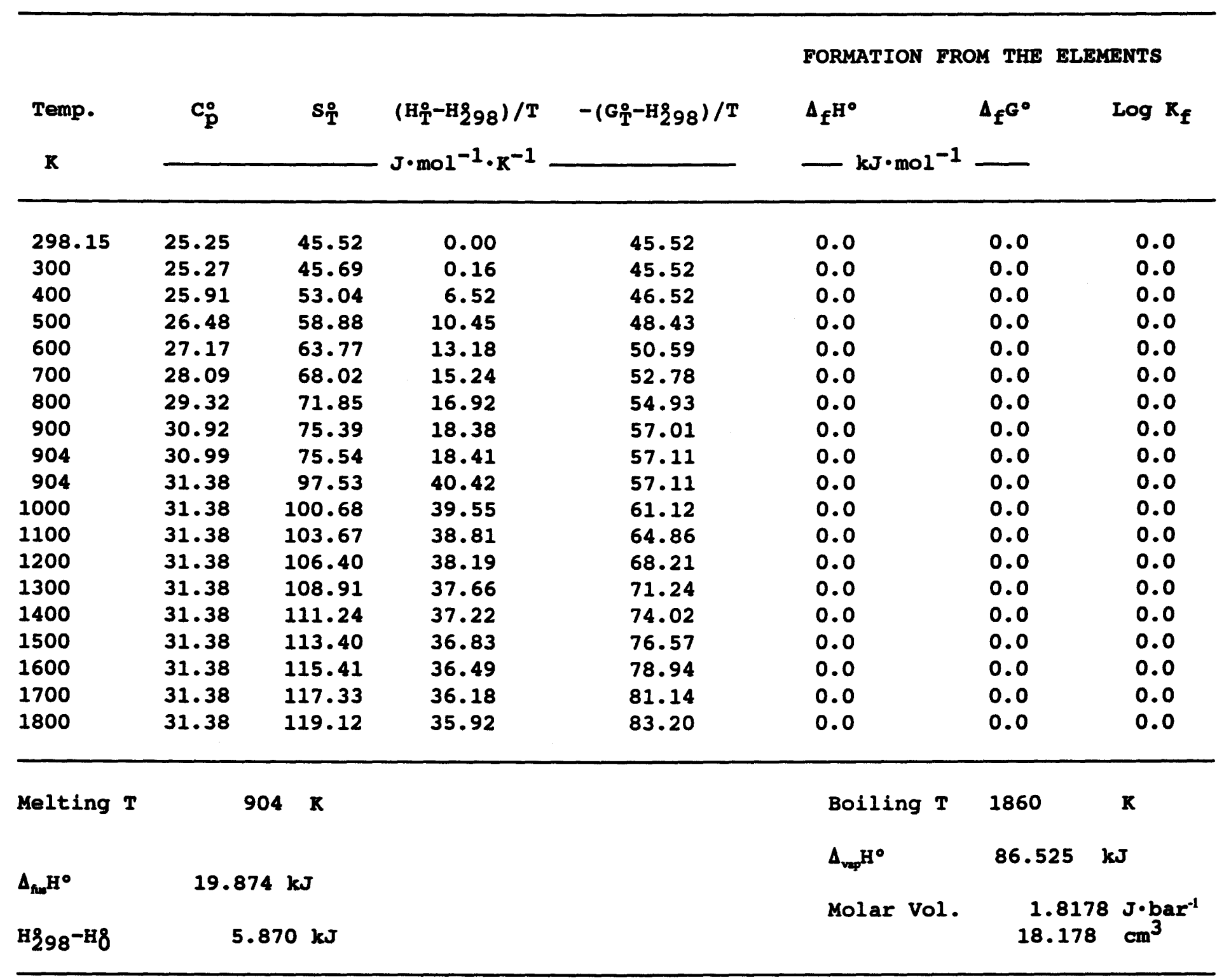


Se: Crystals 298.15 to melting point $494 \mathrm{~K}$. Iiquid 494 to boiling point $957 \mathrm{~K}$. Ideal diatomic gas 957 to $1800 \mathrm{~K}$.

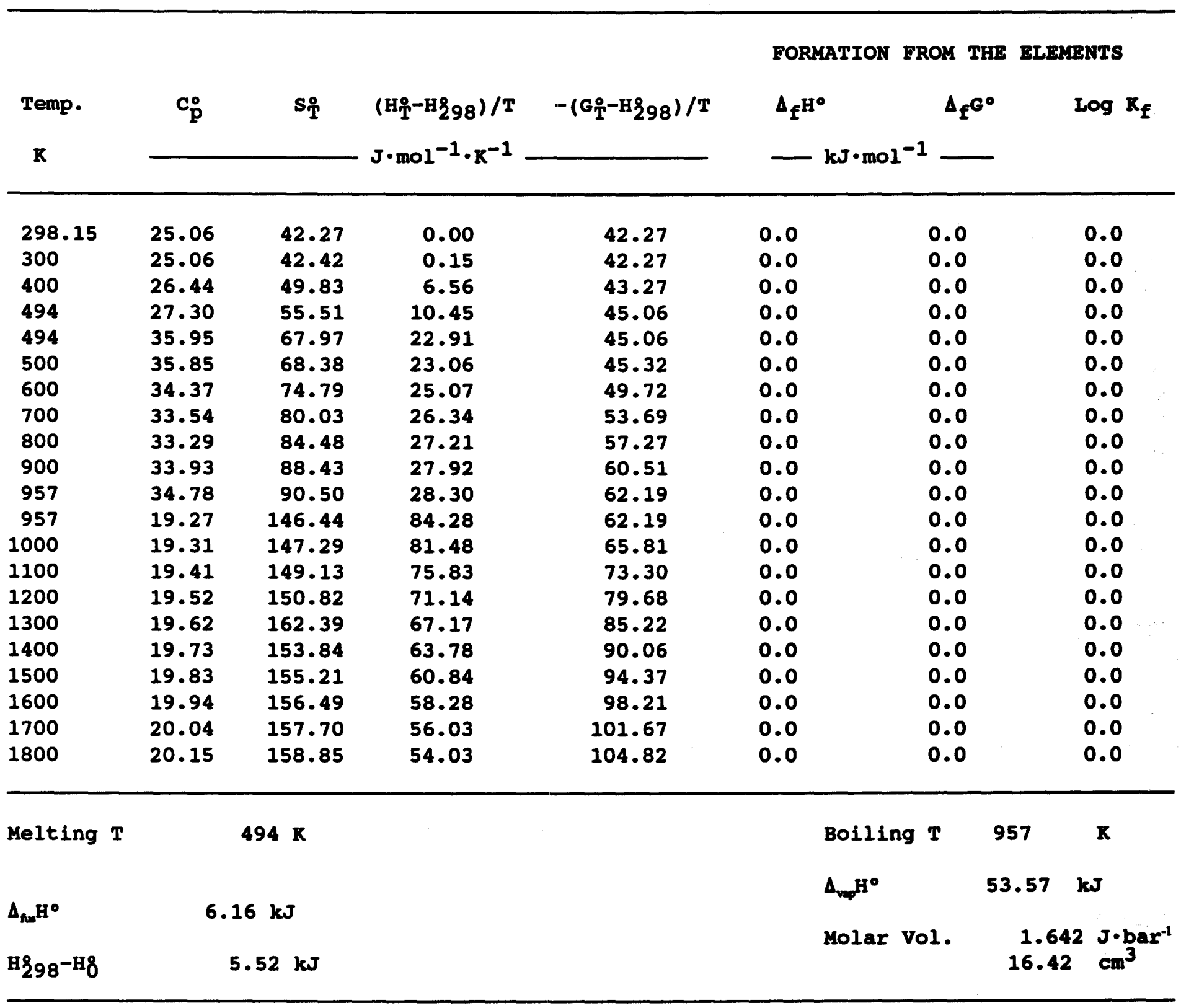


Si: Cubic crystals 298.15 to melting point $1685 \mathrm{~K}$; liquid 1685 to $1800 \mathrm{~K}$.

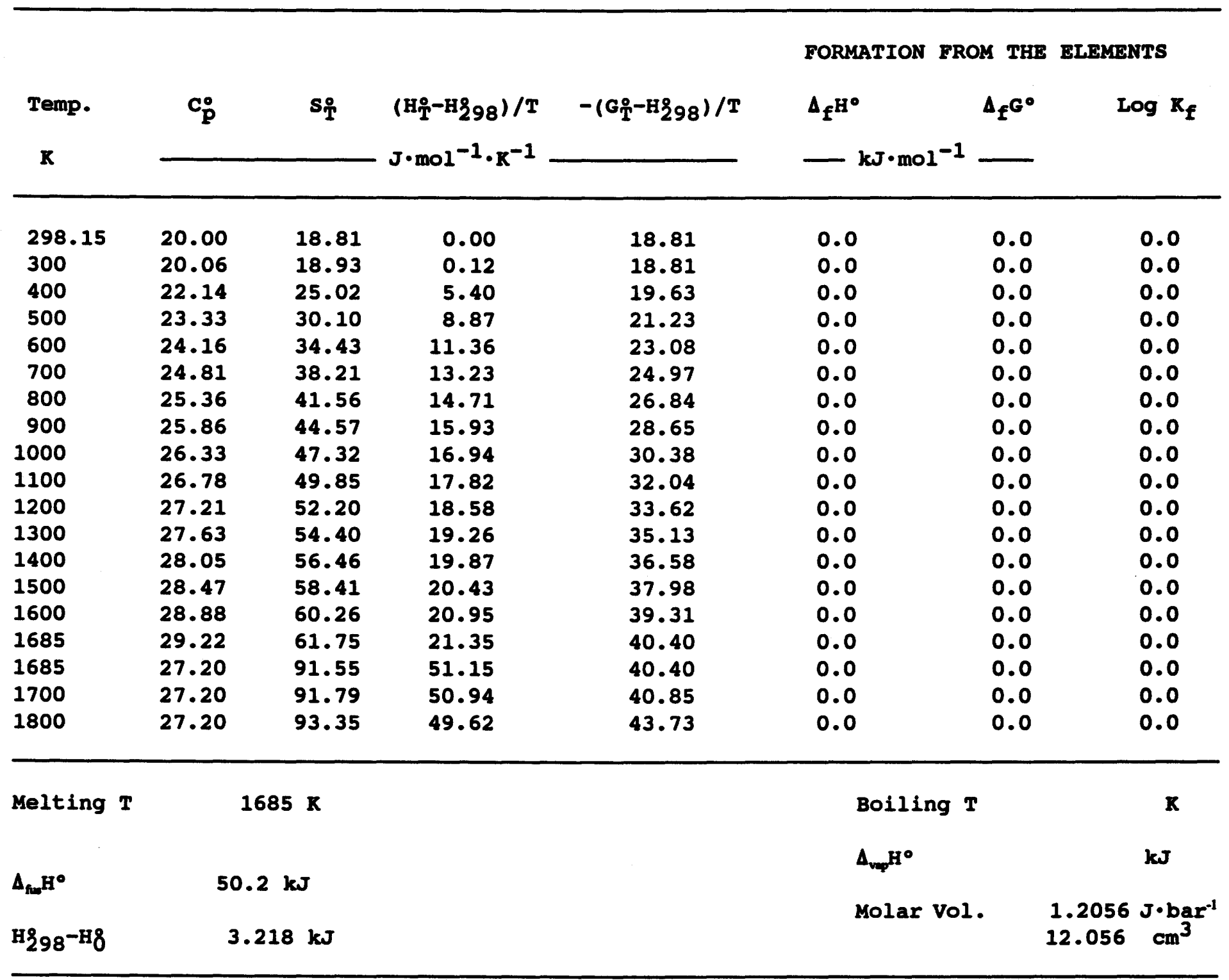


Sn: Crystals 298.15 to melting point 505.1 K. Liquid 501 to $1800 \mathrm{~K}$.

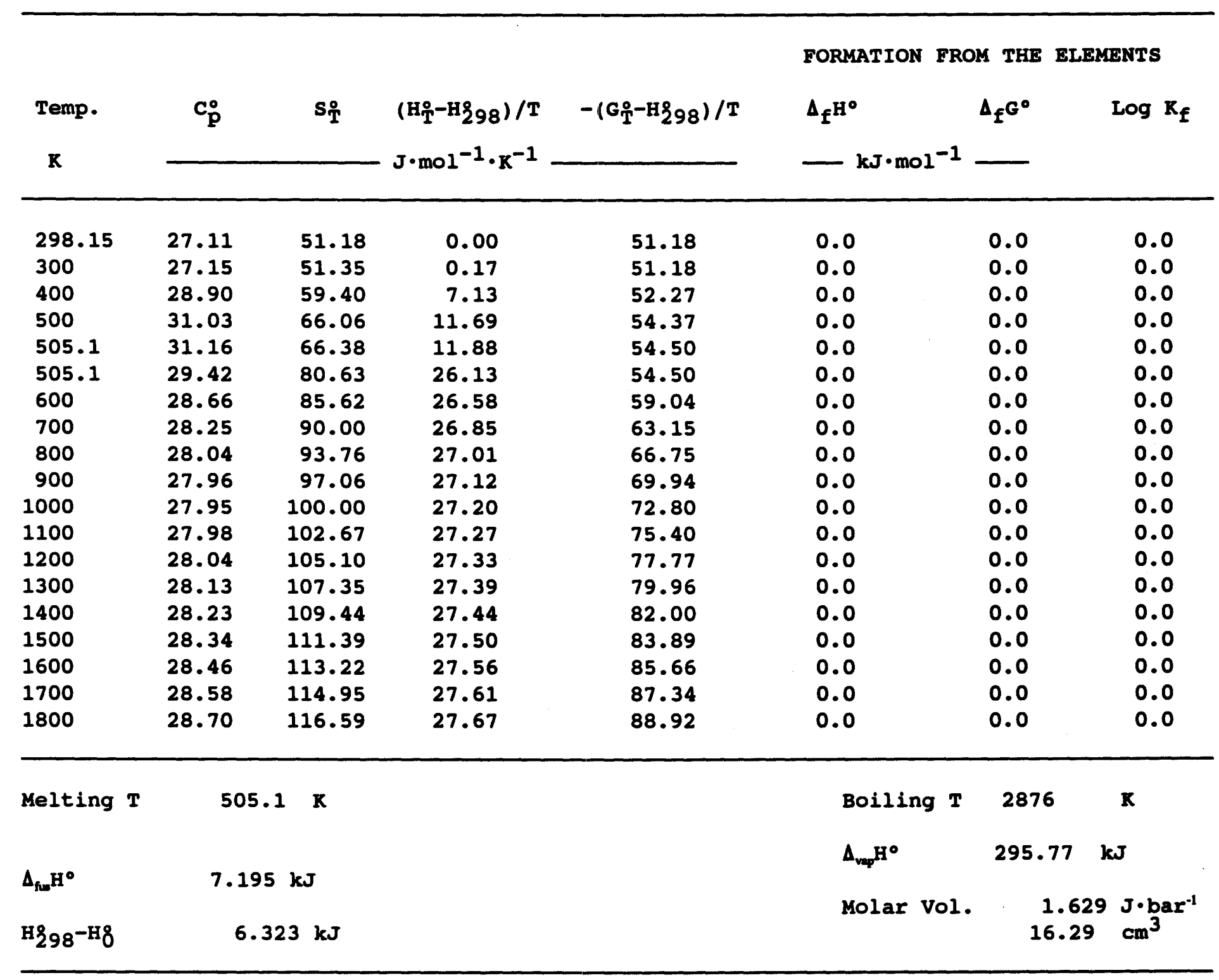


Sr: $\alpha$-crystals (face-centered cubic) 298.15 to $820 \mathrm{~K} ; \gamma$-crystals (body-centere cubic) 820 to melting point $1050 \mathrm{~K}$; liquid 1050 to boiling point $1685.5 \mathrm{~K}$; ideal monatomic gas, $P=1$ bar, 1685.5 to $1800 \mathrm{~K}$.

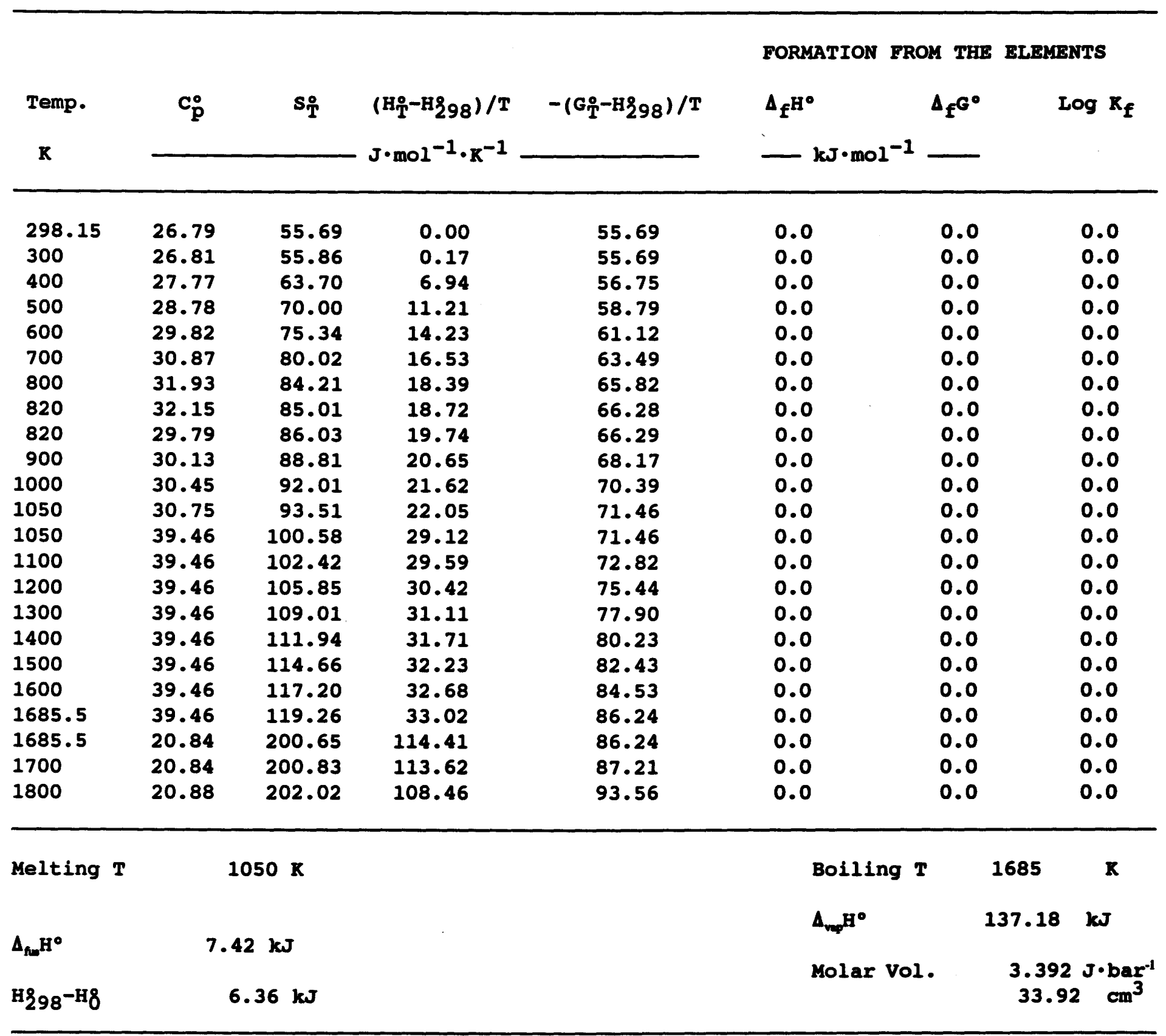


Te: Crystals 298.15 to melting point $723 \mathrm{~K}$. Iiquid 723 to boiling point $1261 \mathrm{~K}$. Ideal diatomic gas 1261 to $1800 \mathrm{~K}$.

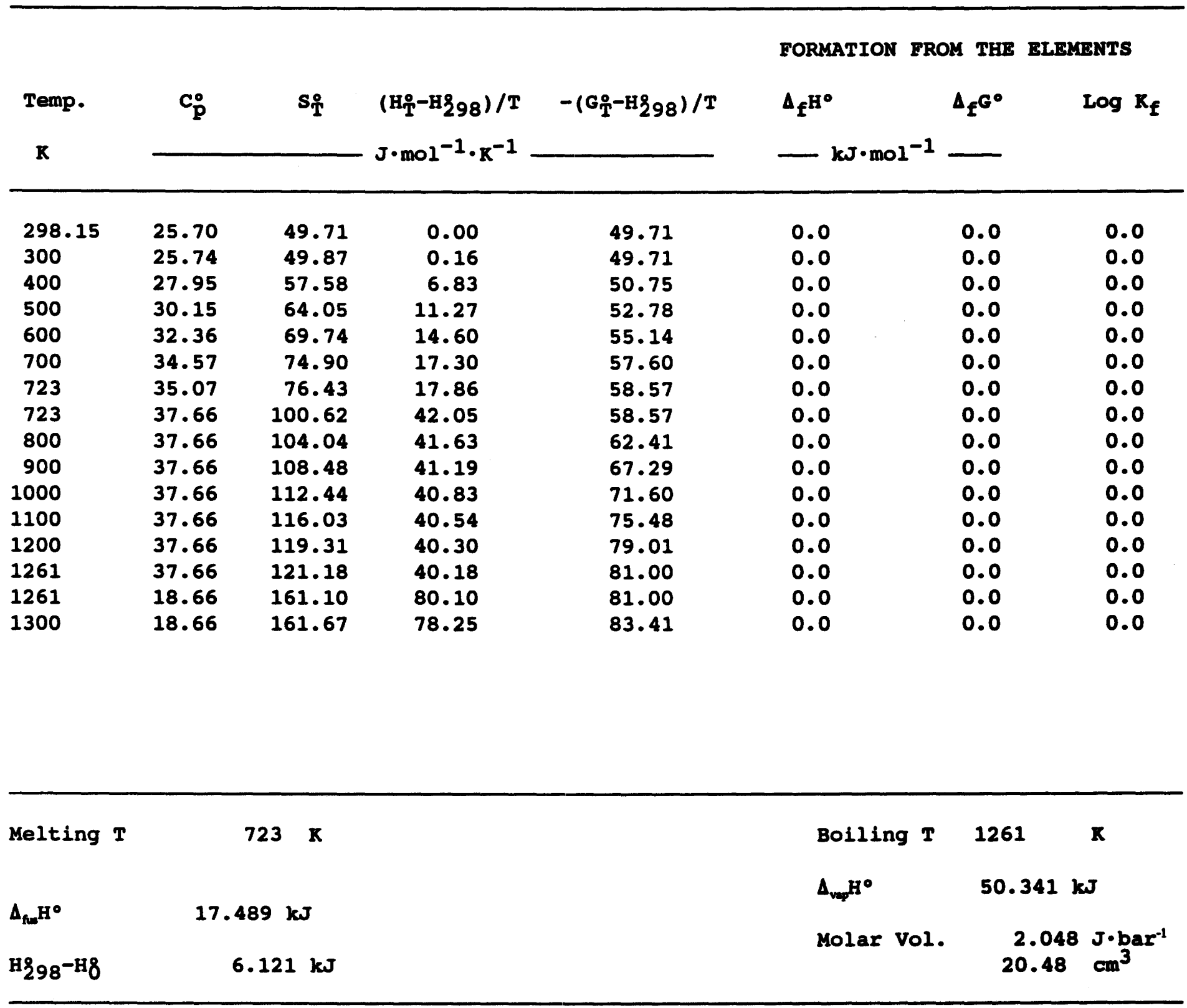


Th: a-crystals (face-centered cubic) 298.15 to $1650 \mathrm{~K}$. B-crystals (body-centered cubic) 1650 to melting point $2023 \mathrm{~K}$.

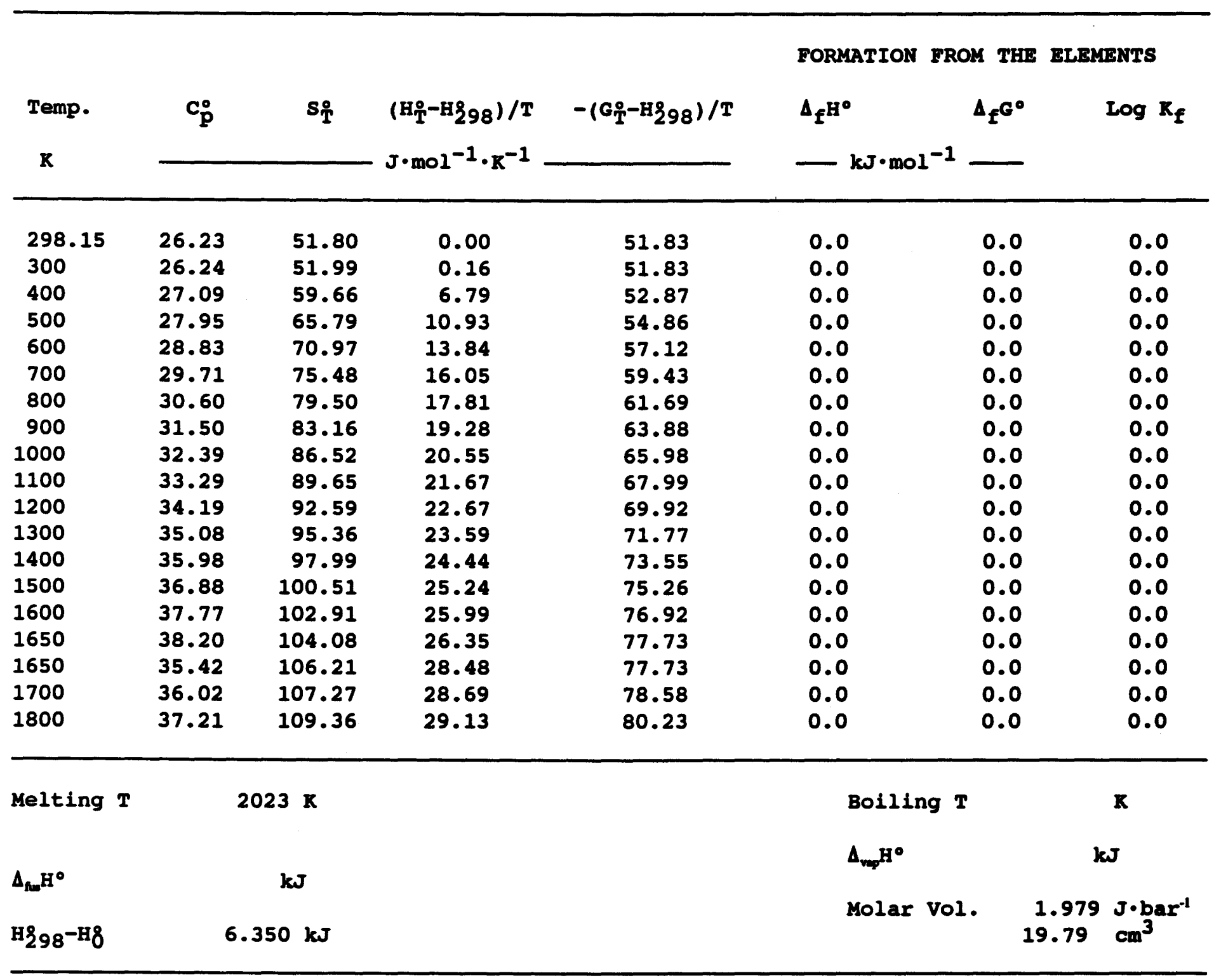


Ti: $\alpha$-crystals (hexagonal close packed) 298.15 to $1166 \mathrm{~K}$. B-crystals (body-centered cubic) 1166 to melting point 1939.

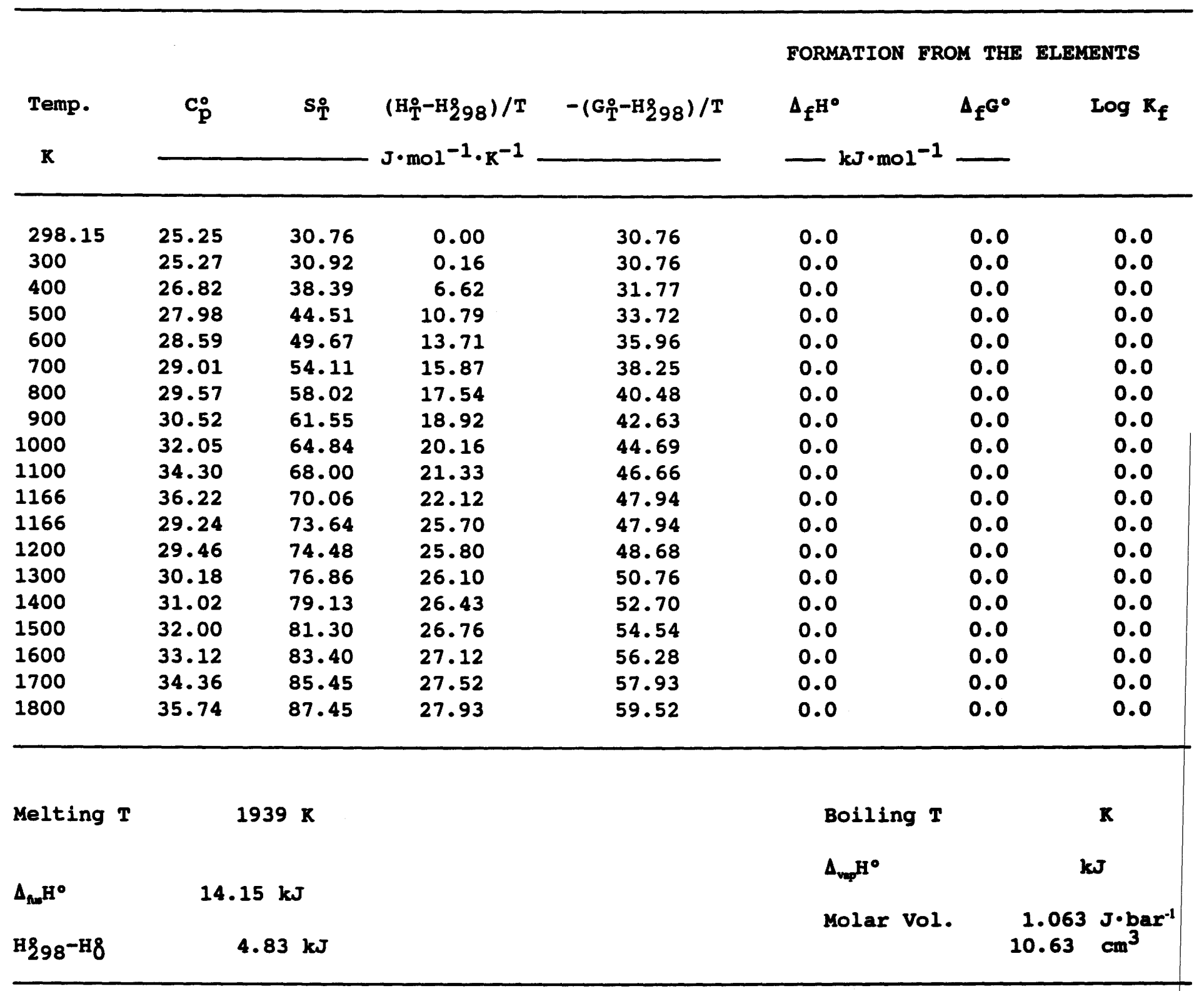


U: $\alpha$-crystals (orthorhombic) 298.15 to $942 \mathrm{~K}$. $\beta$-crystals (tetragonal) 942 to $1049 \mathrm{~K}$. $\gamma$ crystals (body-centered cubic) 1049 to melting point $1408 \mathrm{~K}$. Iiquid 1408 to $1800 \mathrm{~K}$.

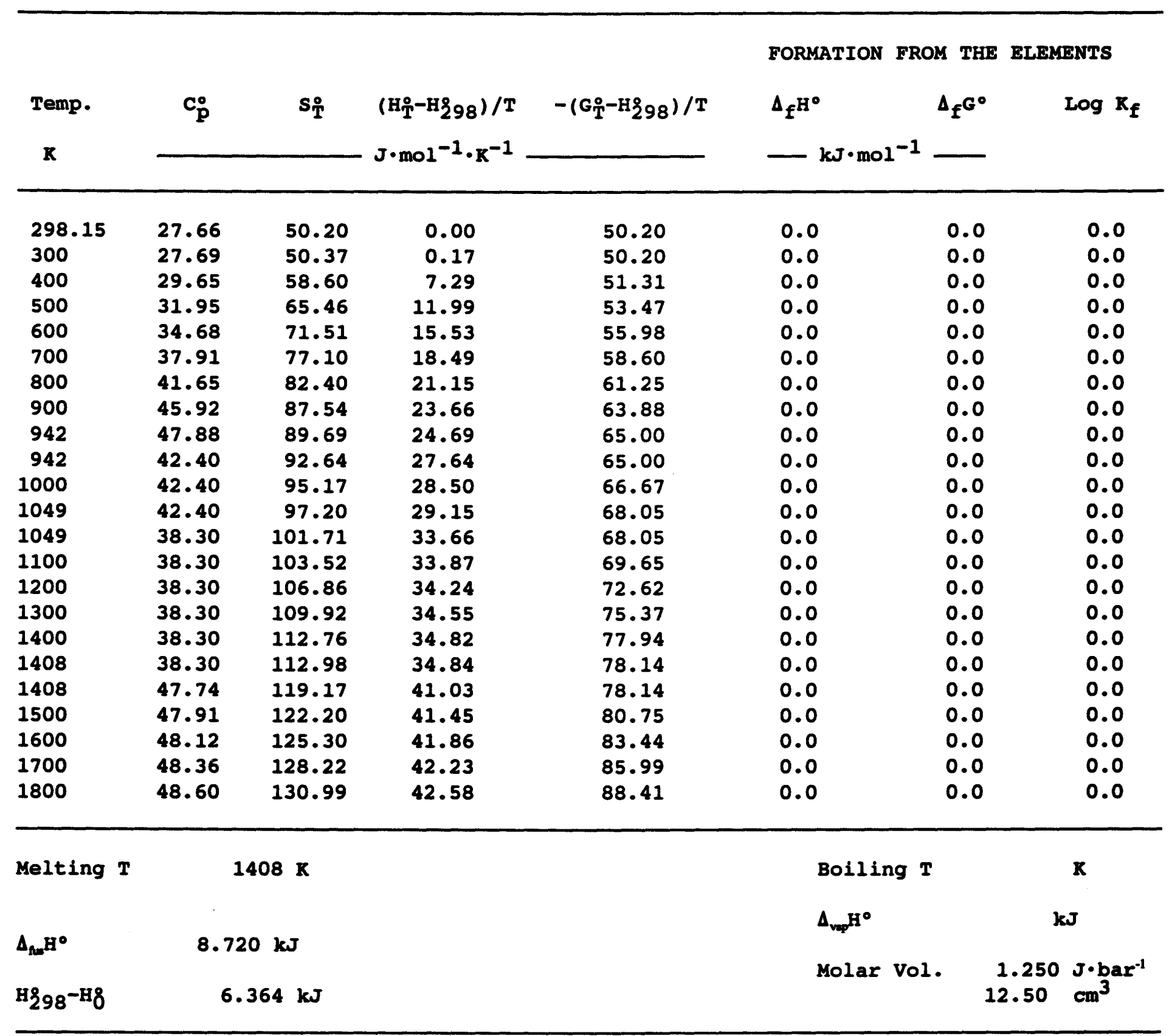


V: Body-centered cubic cryetals 298.15 to $2175 \mathrm{~K}$.

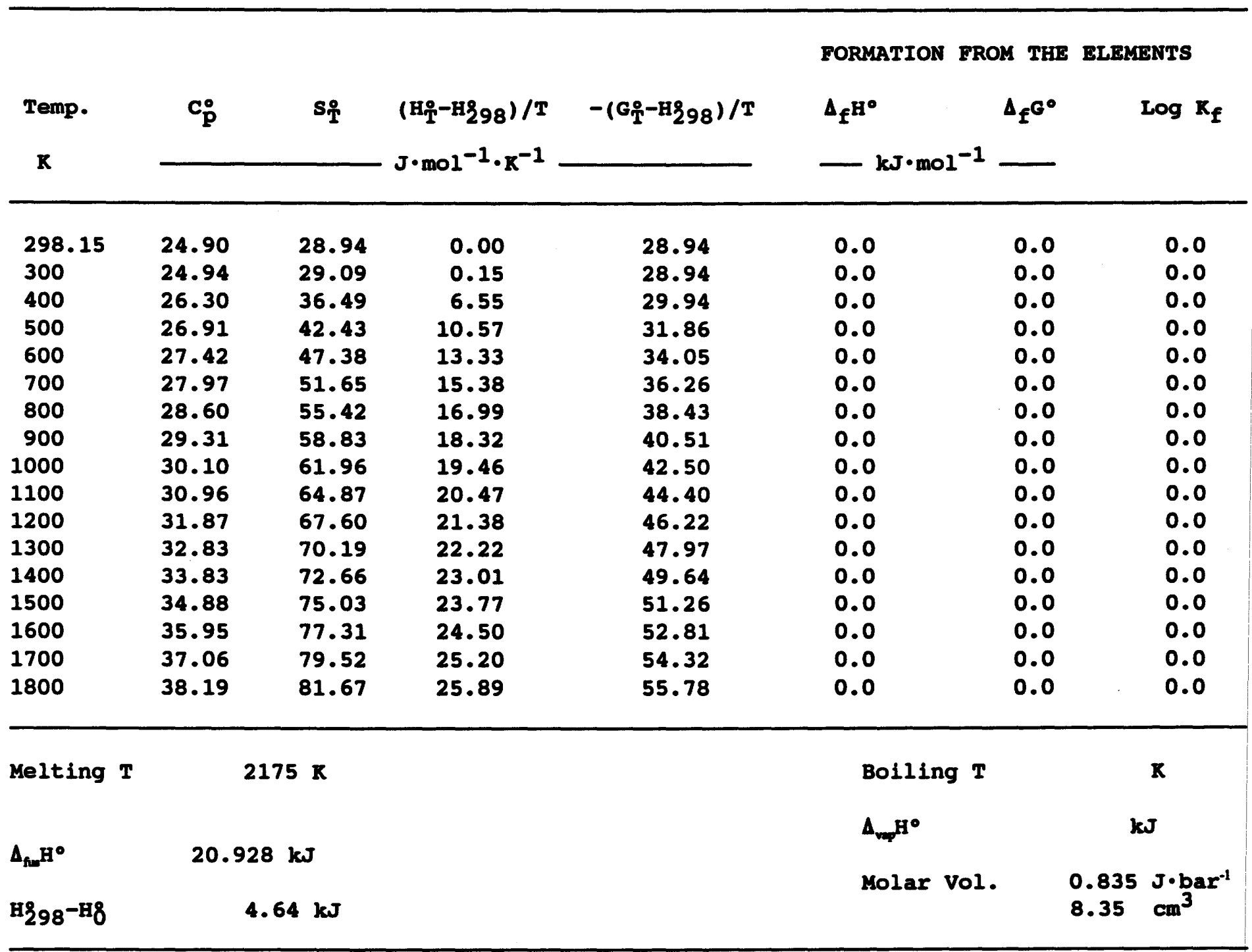


W: Body-centered cubic crystals 298.15 to $2500 \mathrm{~K}$.

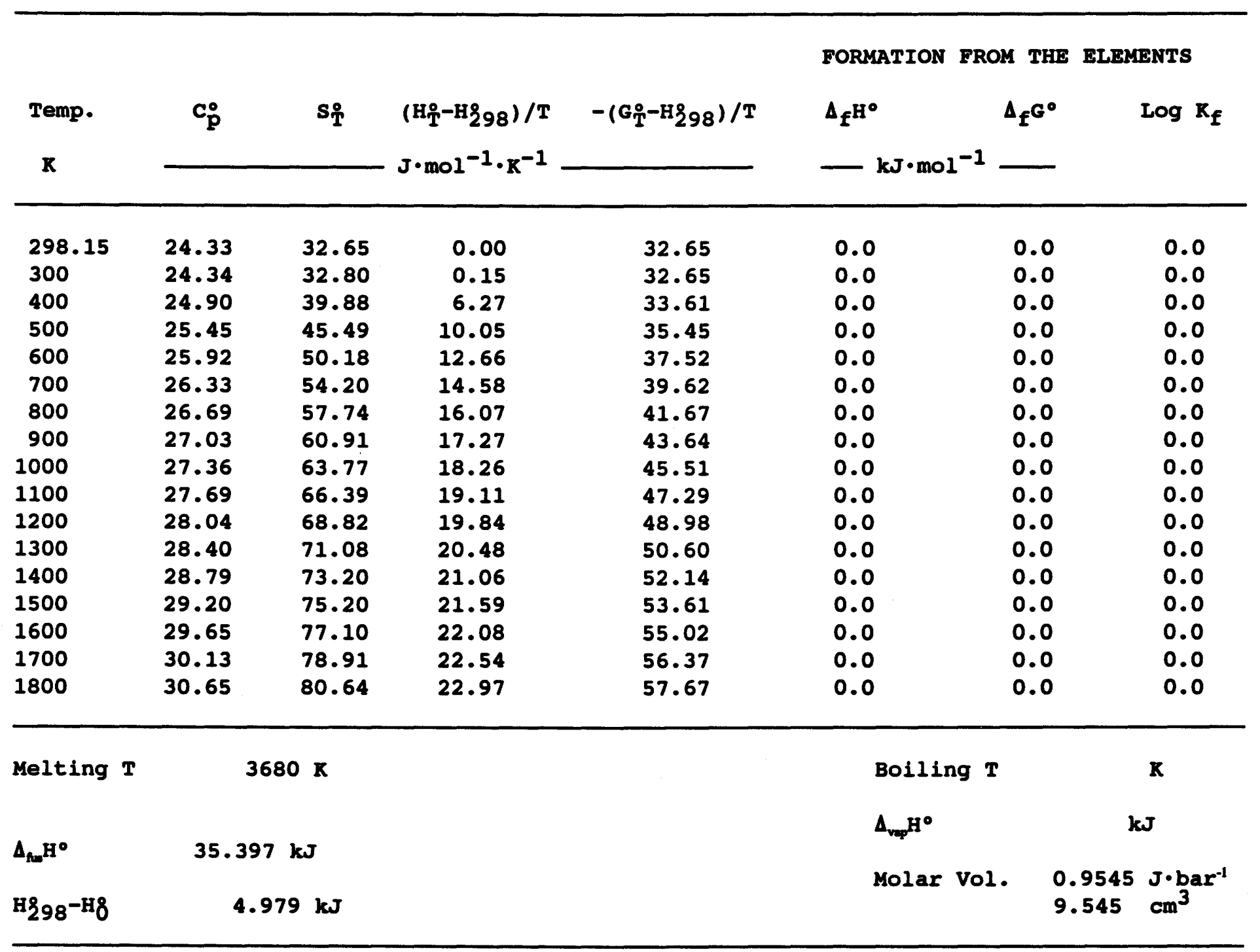


ZINC (REFERENCE STATE)

Zn: Hexagonal close-packed crystals 298.15 to melting point $692.7 \mathrm{~K}$. Liquid 692.7 to boiling point $1180.2 \mathrm{~K}$. Ideal monatomic gas 1180.2 to $2000 \mathrm{~K}$.

\begin{tabular}{|c|c|c|c|c|c|c|c|}
\hline Temp. & $\mathbf{C}_{\mathbf{p}}^{\circ}$ & $\mathbf{s}_{\mathbf{T}}$ & \multirow{2}{*}{$\begin{array}{l}\left(\mathrm{H}_{\mathrm{T}}-\mathrm{H} 298\right) / \mathrm{T} \\
\mathrm{J} \cdot \mathrm{mol}^{-1} \cdot \mathrm{K}^{-1}\end{array}$} & \multirow[t]{2}{*}{$-\left(G_{T}^{\circ}-H_{298}\right) / T$} & \multicolumn{3}{|c|}{$\begin{array}{l}\text { FORMATION FROM THE ELEMENTS } \\
\Delta_{f^{\mathrm{H}^{\circ}}} \Delta_{\mathrm{f}^{\circ}} \quad \text { LOg } \mathrm{K}_{\mathrm{f}}\end{array}$} \\
\hline $\mathbf{K}$ & & & & & $-\mathrm{kJ} \cdot \mathrm{mol}^{-1}$ & - & \\
\hline 298.15 & 25.39 & 41.63 & 0.00 & 41.63 & 0.0 & 0.0 & 0.0 \\
\hline 300 & 25.41 & 41.79 & 0.16 & 41.63 & 0.0 & 0.0 & 0.0 \\
\hline 400 & 26.36 & 49.23 & 6.59 & 42.64 & 0.0 & 0.0 & 0.0 \\
\hline 500 & 27.39 & 55.22 & 10.64 & 44.57 & 0.0 & 0.0 & 0.0 \\
\hline 600 & 28.57 & 60.31 & 13.53 & 46.78 & 0.0 & 0.0 & 0.0 \\
\hline 692.7 & 29.81 & 64.50 & 15.62 & 48.88 & 0.0 & 0.0 & 0.0 \\
\hline 692.7 & 31.38 & 75.07 & 26.19 & 48.88 & 0.0 & 0.0 & 0.0 \\
\hline 700 & 31.38 & 75.40 & 26.24 & 49.16 & 0.0 & 0.0 & 0.0 \\
\hline 800 & 31.38 & 79.59 & 26.89 & 52.70 & 0.0 & 0.0 & 0.0 \\
\hline 900 & 31.38 & 83.29 & 27.39 & 55.90 & 0.0 & 0.0 & 0.0 \\
\hline 1000 & 31.38 & 86.59 & 27.78 & 58.81 & 0.0 & 0.0 & 0.0 \\
\hline 1100 & 31.38 & 89.58 & 28.11 & 61.47 & 0.0 & 0.0 & 0.0 \\
\hline 1180.2 & 31.38 & 91.79 & 28.33 & 63.46 & 0.0 & 0.0 & 0.0 \\
\hline 1180.2 & 20.79 & 189.50 & 126.04 & 63.46 & 0.0 & 0.0 & 0.0 \\
\hline 1200 & 20.79 & 189.84 & 124.29 & 65.55 & 0.0 & 0.0 & 0.0 \\
\hline 1300 & 20.79 & 191.51 & 116.33 & 75.18 & 0.0 & 0.0 & 0.0 \\
\hline 1400 & 20.79 & 193.05 & 109.51 & 83.54 & 0.0 & 0.0 & 0.0 \\
\hline 1500 & 20.79 & 194.48 & 103.59 & 90.89 & 0.0 & 0.0 & 0.0 \\
\hline 1600 & 20.79 & 195.82 & 98.41 & 97.41 & 0.0 & 0.0 & 0.0 \\
\hline 1700 & 20.79 & 197.08 & 93.85 & 103.23 & 0.0 & 0.0 & 0.0 \\
\hline 1800 & 20.79 & 198.27 & 89.79 & 108.48 & 0.0 & 0.0 & 0.0 \\
\hline Melting $\mathrm{T}$ & & $\mathbf{R}$ & & & Boiling $T$ & 1080.2 & $\mathbf{K}$ \\
\hline & & & & & $\Delta_{\mathrm{vep}} \mathrm{H}^{\circ}$ & 115.30 & $\boldsymbol{s}$ \\
\hline$\Delta_{f w} H^{\circ}$ & & $2 \mathrm{~kJ}$ & & & & & \\
\hline $\mathrm{H} 298^{-\mathrm{H}}$ & & $648 \mathrm{~kJ}$ & & & Molar Vol. & $\begin{array}{l}0.9162 \\
9.162\end{array}$ & $\begin{array}{l}J \cdot \mathrm{bar}^{-1} \\
\mathrm{~cm}^{3}\end{array}$ \\
\hline
\end{tabular}


Zr: $\alpha$-crystals (hexagonal close packed) 298.15 to $1135 \mathrm{~K}$. p-crystals (body-centered cubic) 1135 to melting point $2125 \mathrm{~K}$.

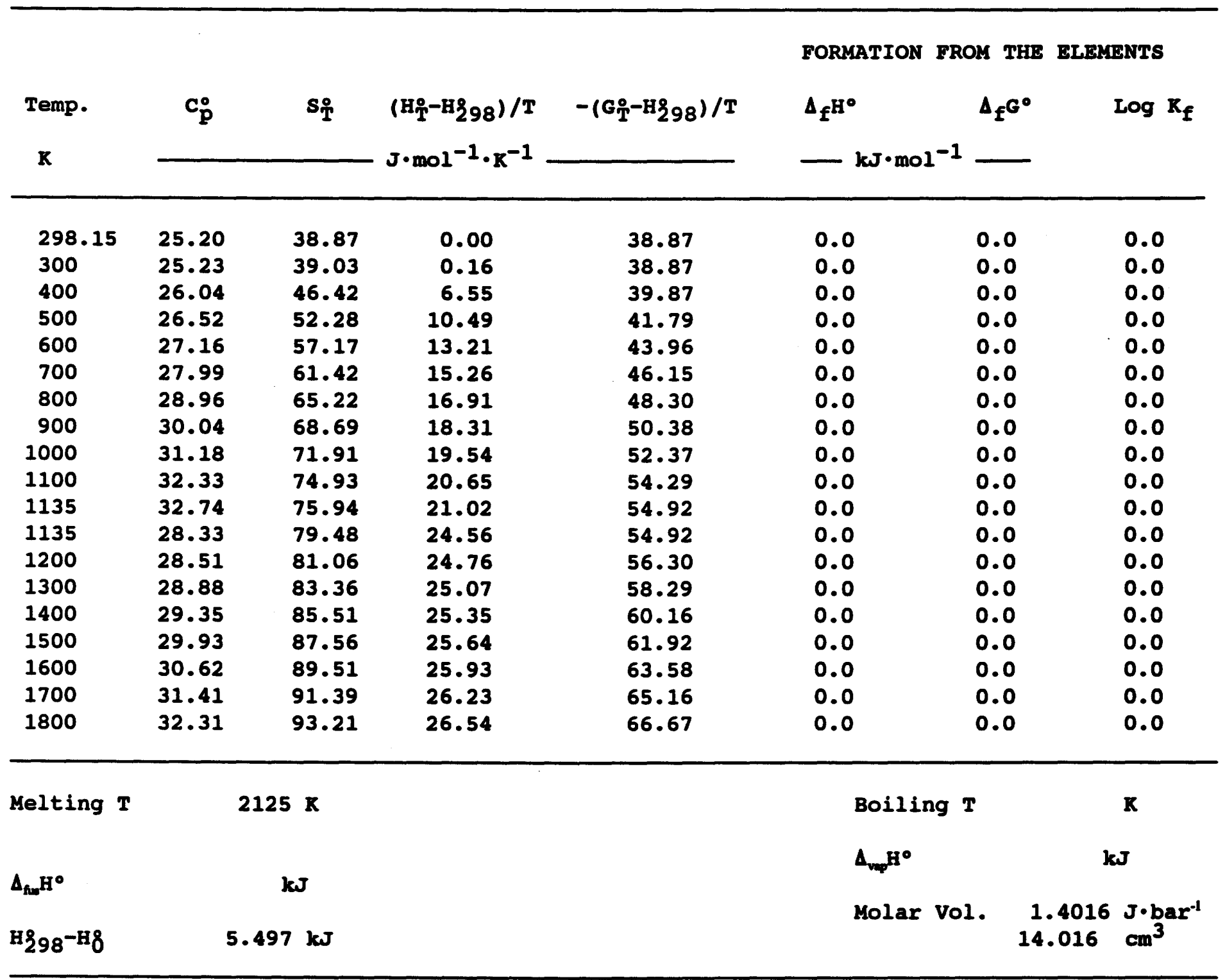


$\mathrm{CH}_{4}$ : Ideal gas, $\mathrm{P}=1$ bar, 298.15 to $1800 \mathrm{~K}$

FORMATION FROM THE ELEMENTS

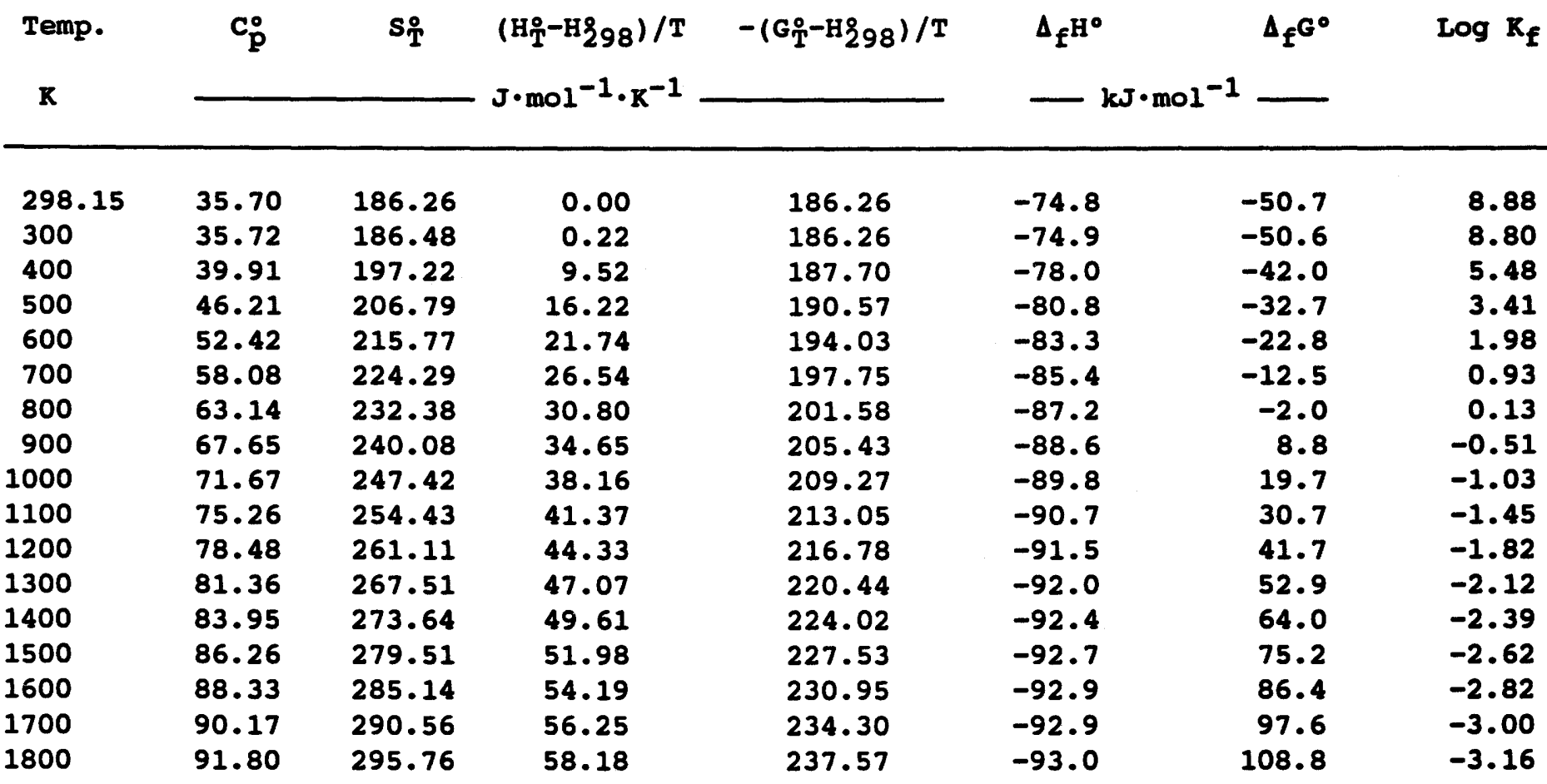

Melting T

$\Delta_{\text {hu }} \mathrm{H}^{\circ}$

H298-H8 $\mathbf{k J}$

$\mathbf{K}$

$10.025 \mathrm{~kJ}$
Boiling $\mathbf{T}$

$\mathbf{R}$

$\Delta_{\mathrm{vq}} \mathrm{H}^{\circ}$

$\mathbf{k J}$

Nolar Vol. $2478.97 \mathrm{~J} \cdot \mathrm{bar}^{-1}$ $24789.7 \mathrm{~cm}^{3}$ 
$\mathrm{Fe}_{3} \mathrm{C}:$ Orthorhombic crystals 298.15 to $1800 \mathrm{~K}$.

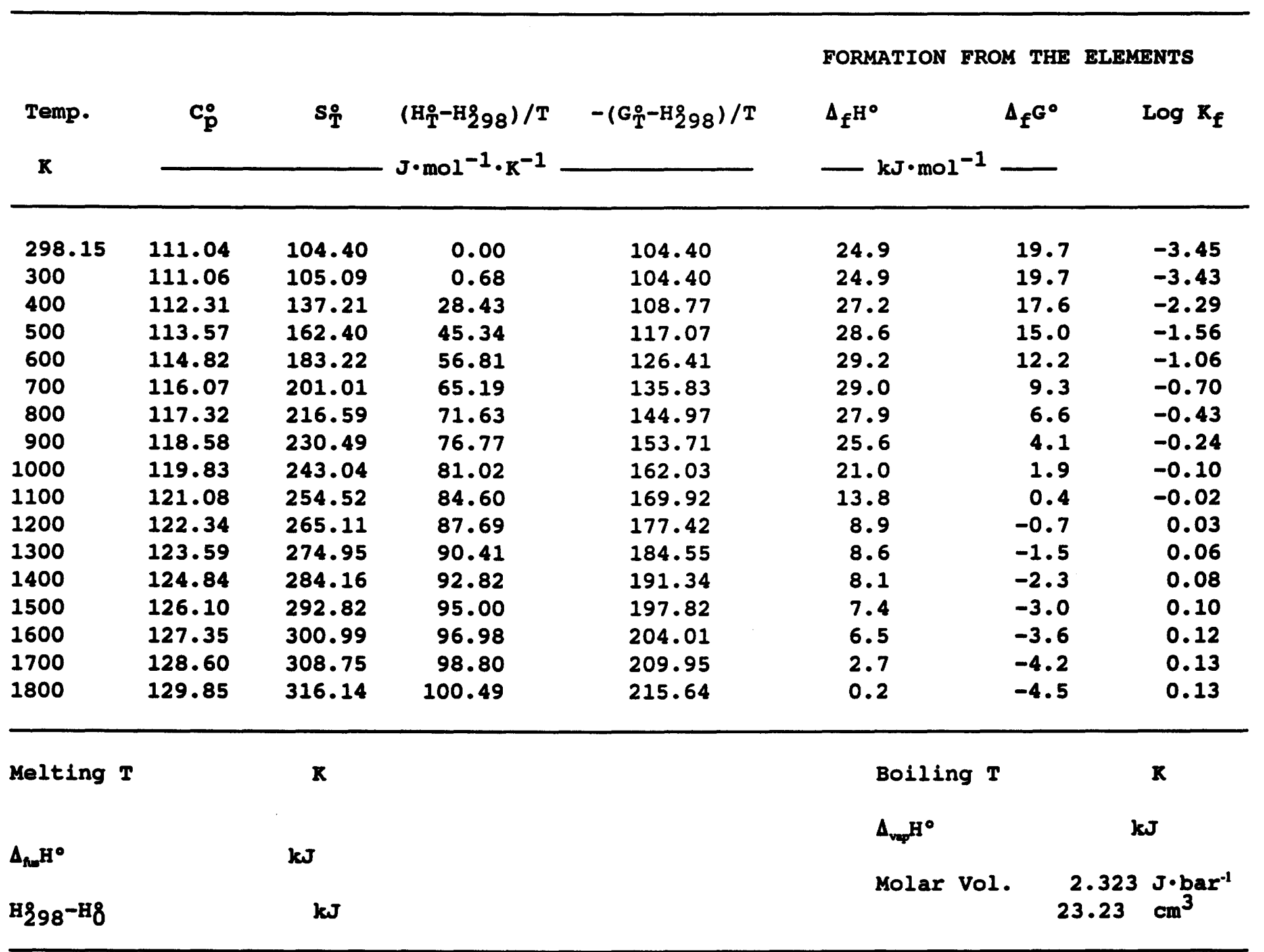


$\mathrm{NH}_{3}$ : Ideal gas, $P=1$ bar, 298.15 to $1800 \mathrm{~K}$.

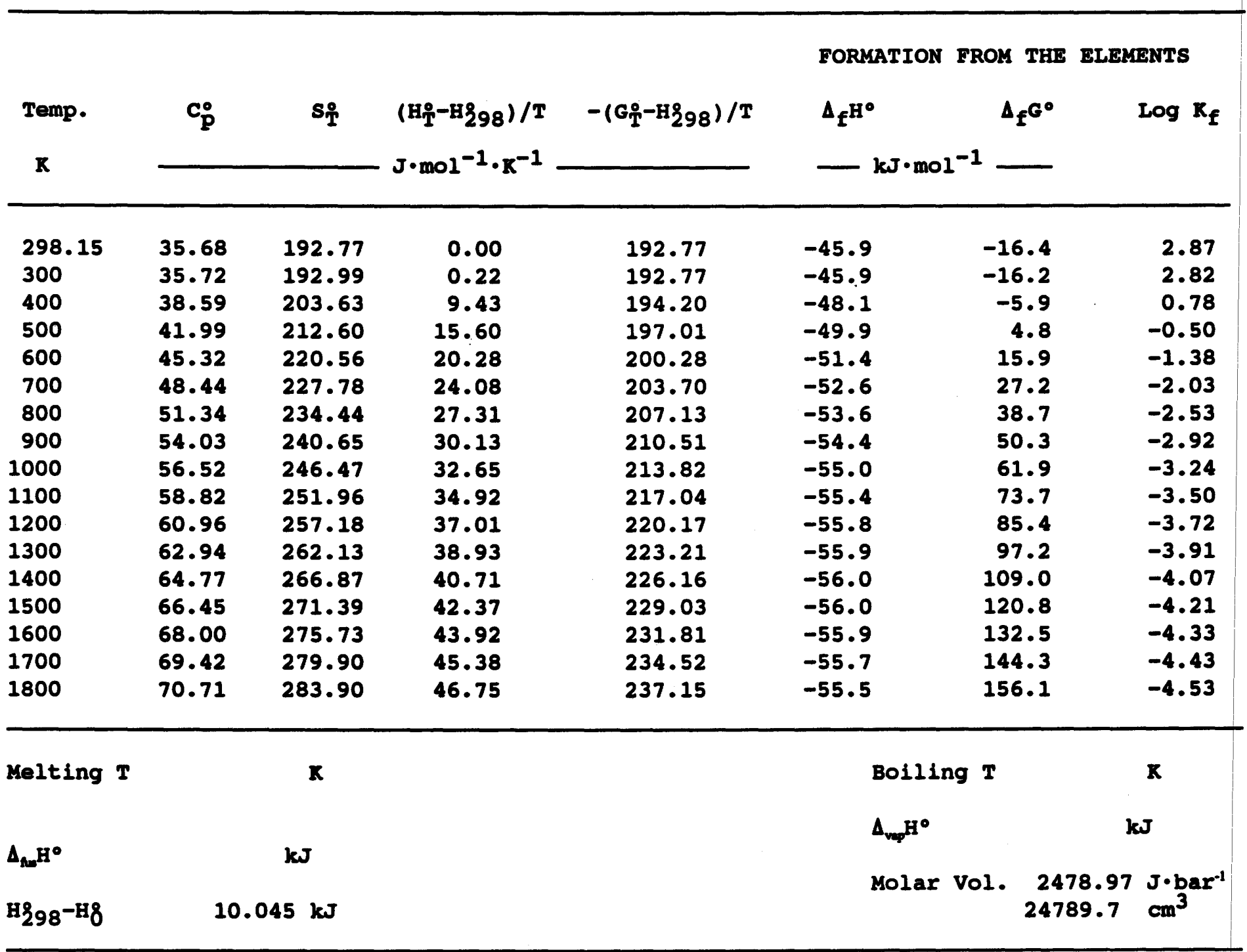


$\mathrm{Ag}_{2}$ S: Monoclinic crystals 298.15 to $451.3 \mathrm{~K}$; cubic crystals (argentite) 451.3 to $865 \mathrm{~K}$. $\gamma$ phase 865 to $1000 \mathrm{~K}$. Reference state for sulfur is orthorhombic sulfur.

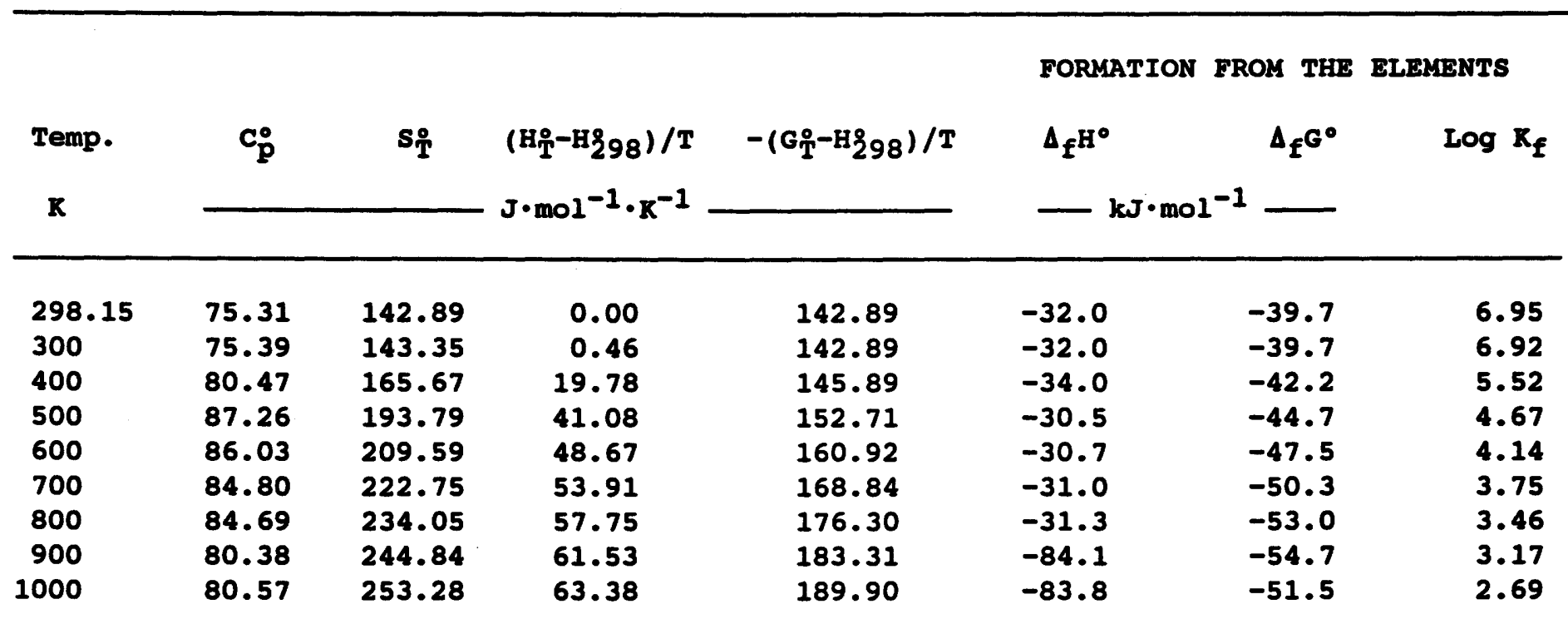

Melting $\mathbf{T}$

$\mathbf{R}$

Boiling $\mathbf{T}$

$\mathbf{R}$

$\Delta_{\text {fue }} \mathrm{H}^{\circ}$

$\mathbf{k J}$

H298-H8

$17.132 \mathrm{~kJ}$
$\Delta_{\mathrm{veq}} \mathrm{H}^{\circ}$

$\mathbf{k J}$

Molar Vol. $\begin{aligned} & 3.419 \mathrm{~J} \cdot \mathrm{bar}^{-1} \\ & 34.19 \mathrm{~cm}^{3}\end{aligned}$ 
Ass: Monoclinic crystals 298.15 to melting point $580 \mathrm{~K}$; liquid 580 to $800 \mathrm{~K}$. Reference state for sulfur is orthorhombic sulfur.

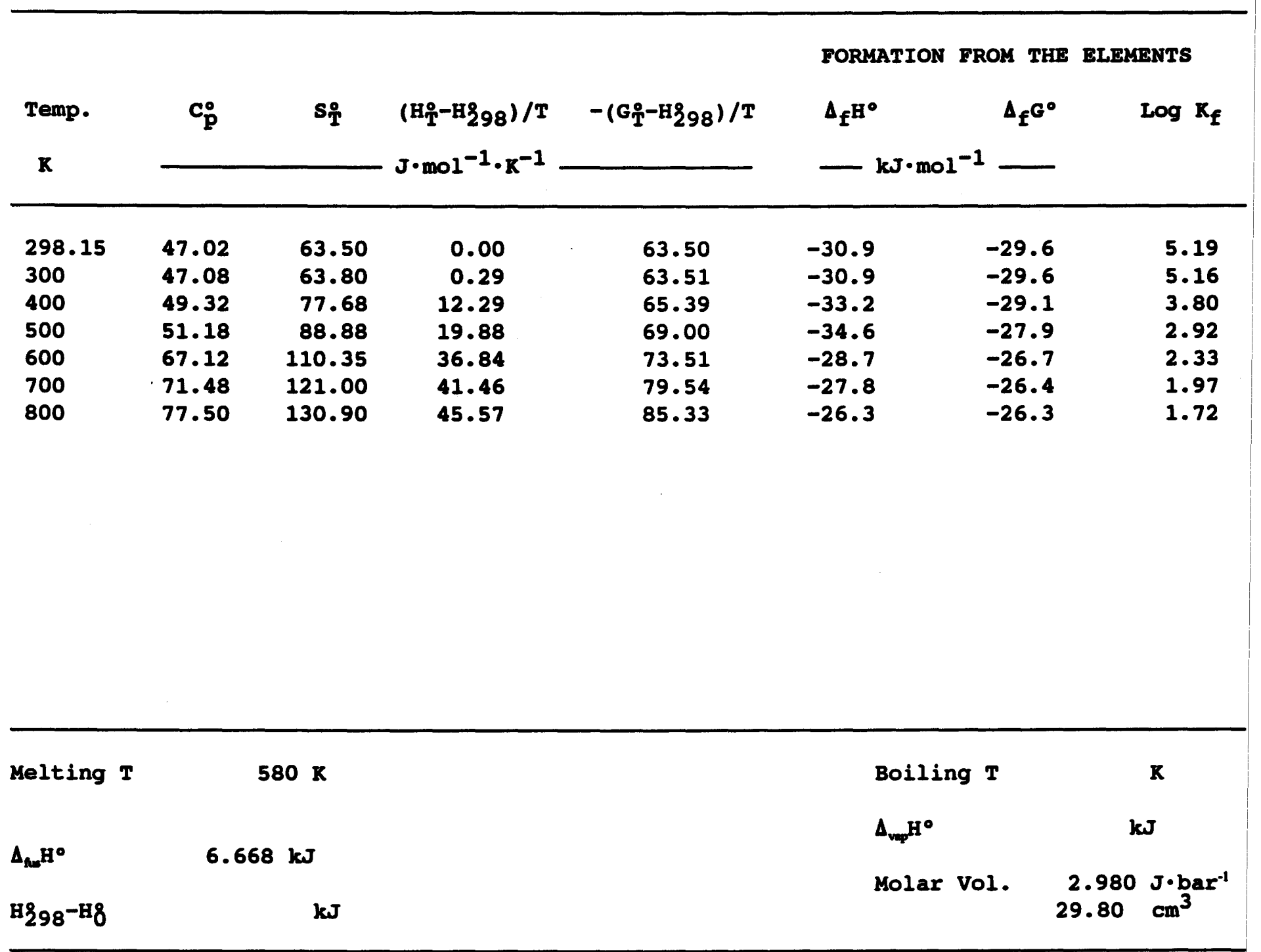


CdS: Cubic crystals 298.15 to $1100 \mathrm{~K}$. Reference state for sulfur is orthorhombic sulfur.

\begin{tabular}{|c|c|c|c|c|c|c|c|}
\hline Temp. & $\mathbf{c}_{\mathbf{p}}^{\circ}$ & $s_{\mathbf{T}}^{\circ}$ & \multirow{2}{*}{$\begin{array}{l}\left(\mathrm{H}_{\mathrm{T}}^{\circ}-\mathrm{H}_{28}\right) / \mathrm{T} \\
\mathrm{J} \cdot \mathrm{mol}^{-1} \cdot \mathrm{K}^{-1}\end{array}$} & \multirow[t]{2}{*}{$-\left(G_{T}^{\circ}-H_{298}\right) / T$} & $\begin{array}{l}\text { FORMATION } \\
\Delta_{f^{H}}\end{array}$ & $\begin{array}{c}\text { FROM THE } \\
\Delta_{f}^{G^{\circ}}\end{array}$ & \multirow[t]{2}{*}{$\begin{array}{l}\text { ELEMENTS } \\
\qquad \operatorname{LOg} k_{f}\end{array}$} \\
\hline $\mathbf{R}$ & & & & & $\ldots \mathbf{k J} \cdot \mathbf{m o l}$ & $1^{-1}$ & \\
\hline 298.15 & 47.30 & 72.18 & 0.00 & 72.18 & -149.6 & -146.1 & 25.60 \\
\hline 300 & 47.36 & 72.47 & 0.29 & 72.18 & -149.6 & -146.1 & 25.44 \\
\hline 400 & 49.46 & 86.42 & 12.35 & 74.07 & -152.0 & -144.8 & 18.91 \\
\hline 500 & 50.70 & 97.60 & 19.90 & 77.69 & -153.7 & -142.8 & 14.92 \\
\hline 600 & 51.68 & 106.93 & 25.12 & 81.81 & -161.2 & -140.5 & 12.23 \\
\hline 700 & 52.55 & 114.96 & 28.98 & 85.99 & -162.3 & -136.9 & 10.22 \\
\hline 800 & 53.37 & 122.03 & 31.98 & 90.06 & -163.2 & -133.2 & 8.70 \\
\hline 900 & 54.16 & 128.37 & 34.40 & 93.97 & -217.1 & -128.4 & 7.45 \\
\hline 1000 & 54.93 & 134.11 & 36.41 & 97.70 & -216.5 & -118.5 & 6.19 \\
\hline 1100 & 55.67 & 139.38 & 38.13 & 101.25 & -314.8 & -102.9 & 4.89 \\
\hline
\end{tabular}

Melting $T$

$\mathbf{K}$

$\Delta_{\text {fux }} \mathrm{H}^{\circ}$

$\mathbf{k J}$

H298-H8
Boiling $\mathbf{T}$

$\Delta_{\mathrm{vep}} \mathrm{H}^{\circ}$

Molar Vol.

\section{K}

$\mathbf{k J}$ 
$\operatorname{Cos}_{2}:$ Cubic crystals 298.15 to $1000 \mathrm{~K}$. Reference state for sulfur is orthorhombic sulfur.

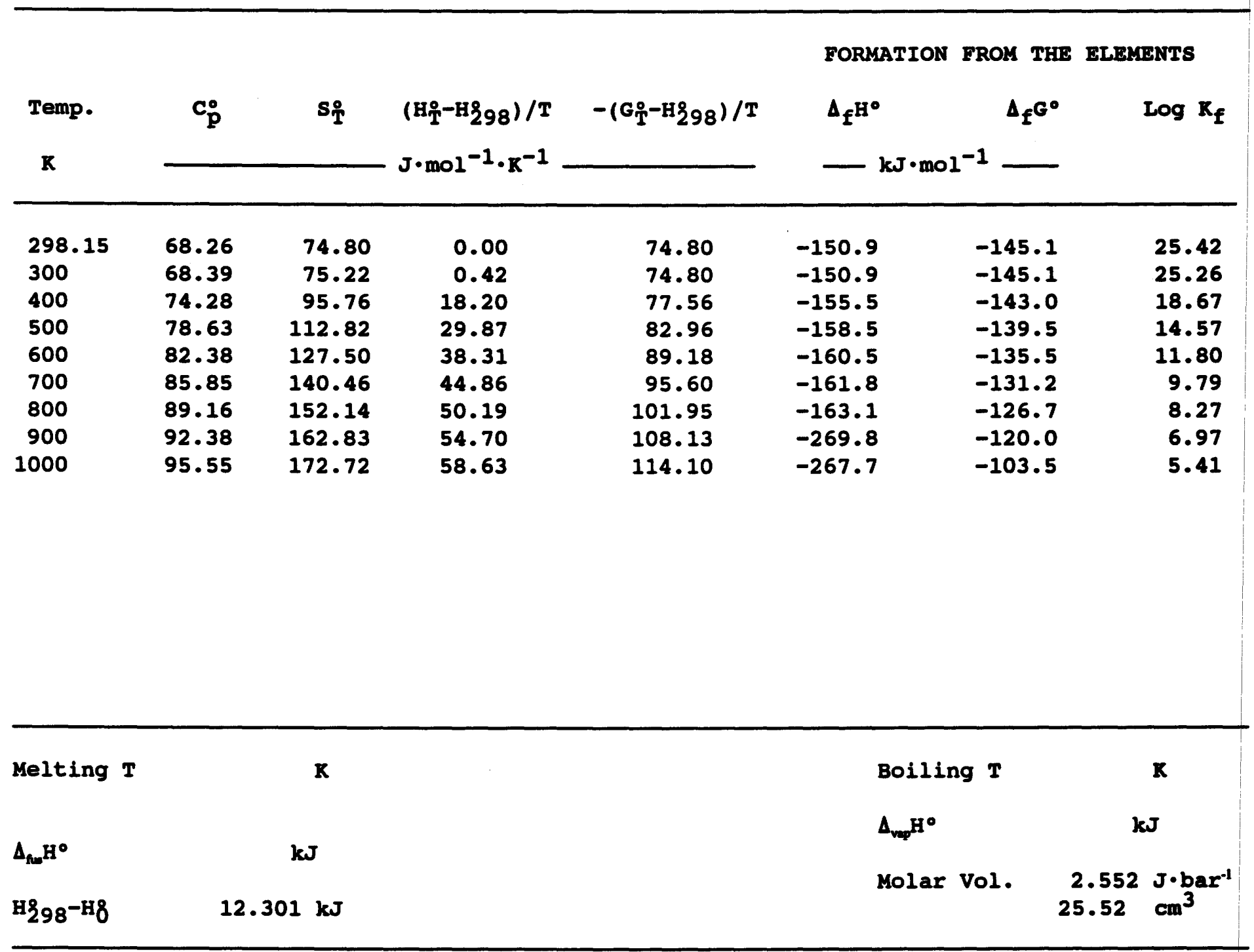


Cus: Hexagonal crystals 298.15 to $800 \mathrm{~K}$. Reference state for sulfur is orthorhombic sulfur.

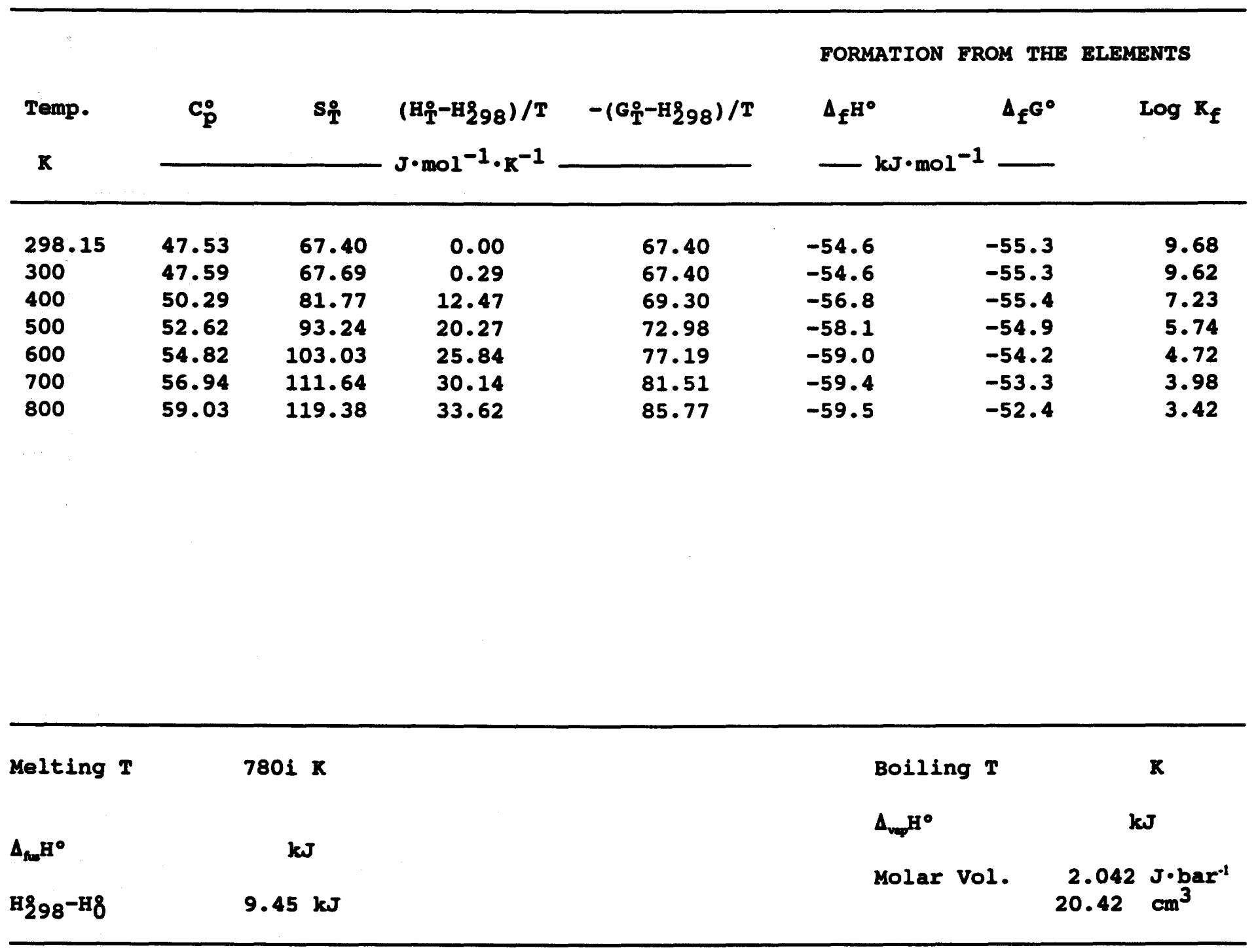


$\mathrm{Cu}_{2} \mathrm{~s}$ : Orthorhombic crystals 298.15 to $376 \mathrm{~K}_{i}$ hexagonal crystals 376 to $710 \mathrm{~K}$; cubic crystals 710 to melting point $1403 \mathrm{~K}$. Reference state for sulfur is orthorhombic sulfur.

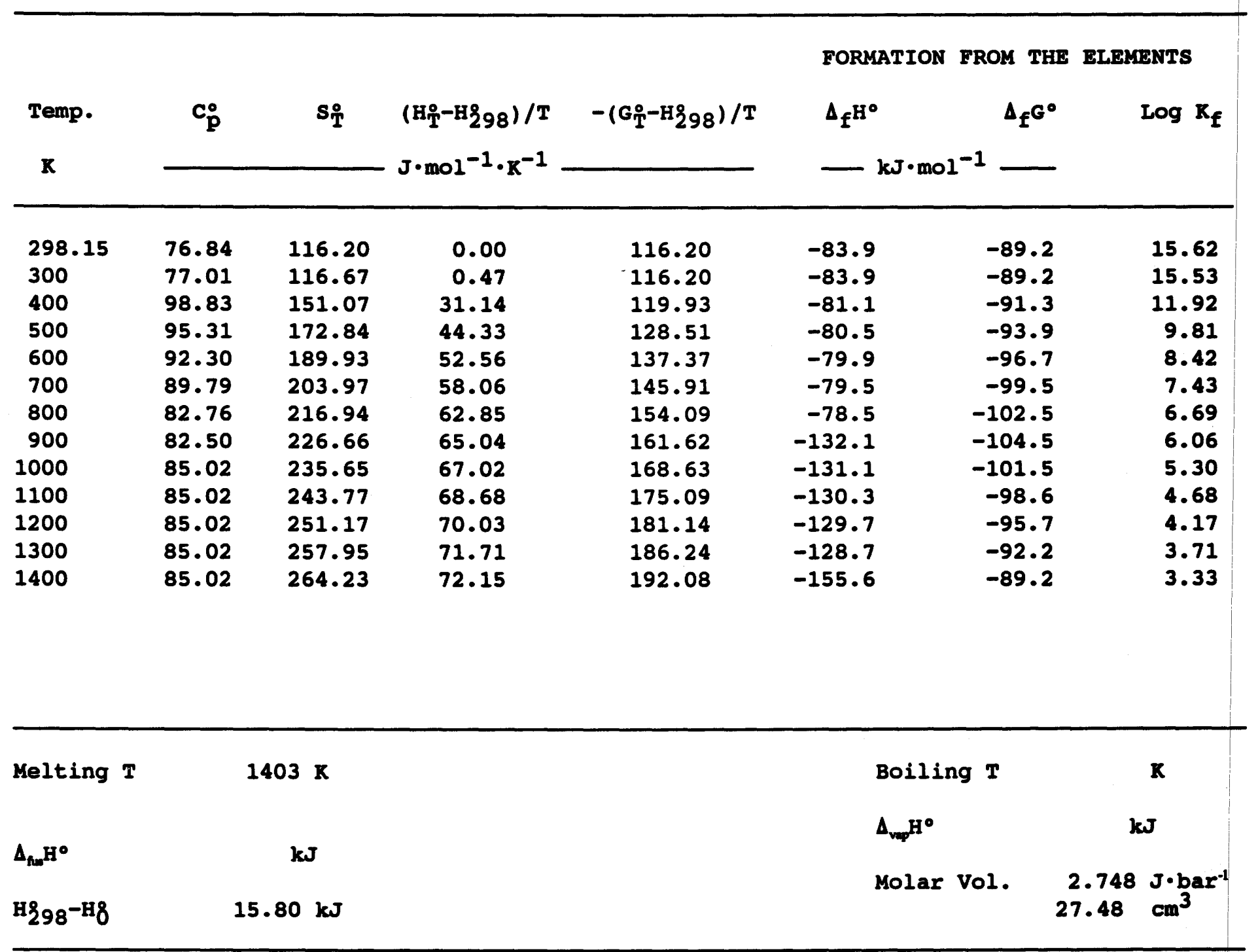


CuFes $_{2}$ : Tetragonal crystals 298.15 to $820 \mathrm{~K}_{i}$ tetragonal crystals 820 to $930 \mathrm{~K}$; cubic crystals 930 to $1200 \mathrm{~K}$. Reference state for sulfur is orthorhombic sulfur. Above $820 \mathrm{~K}$, the phase is called ISs (intermediate solid solution).

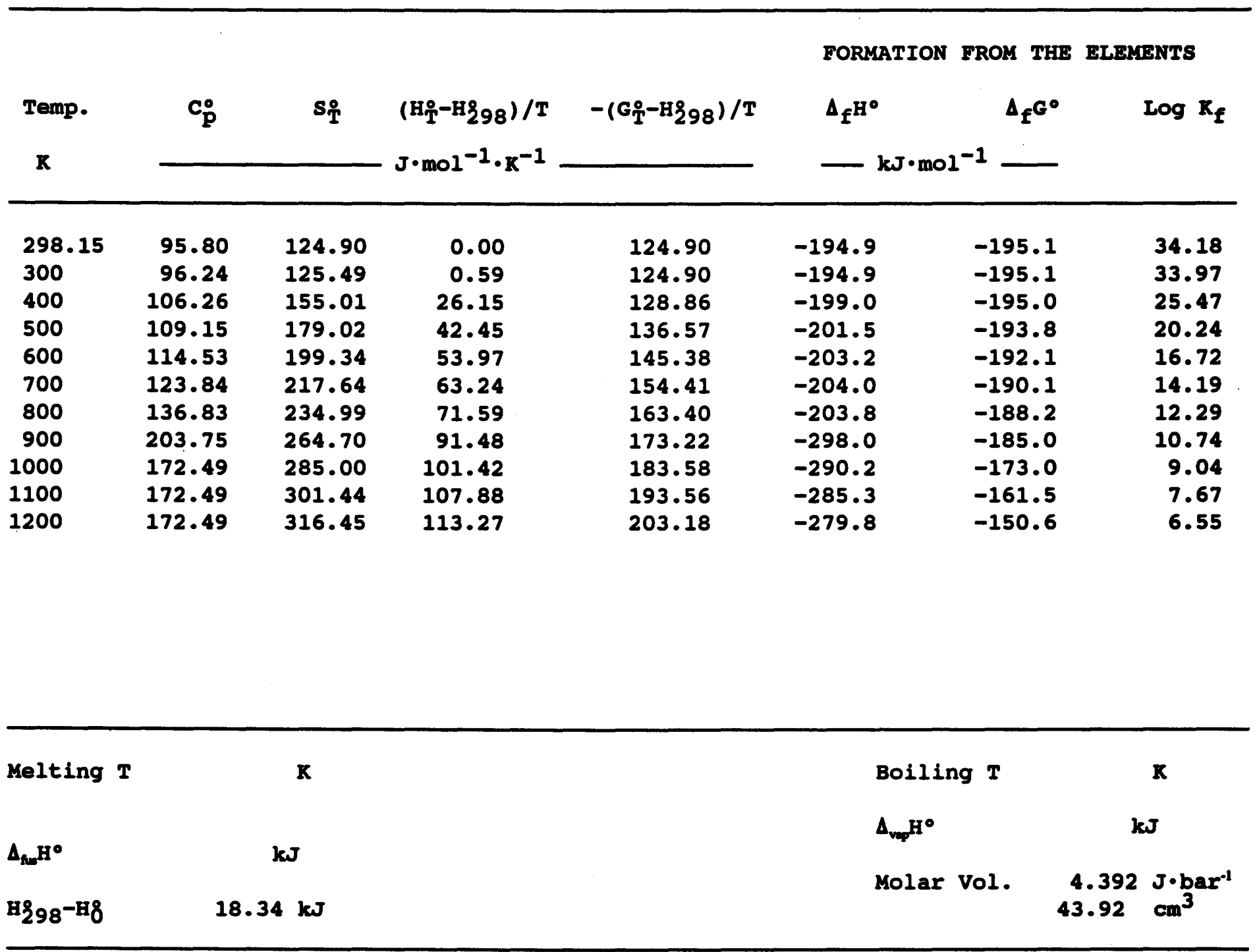




\begin{tabular}{|c|c|c|c|c|c|c|c|c|}
\hline \multirow{2}{*}{$\begin{array}{l}\text { Temp. } \\
\mathbf{K}\end{array}$} & \multirow[b]{2}{*}{$\mathbf{c}_{\mathbf{p}}^{0}$} & \multirow[b]{2}{*}{$\mathbf{s}_{\mathrm{T}}$} & \multirow{2}{*}{$\begin{array}{l}\left(\mathrm{H}_{\mathrm{T}}^{\circ}-\mathrm{H}_{298}\right) / \mathrm{T} \\
\mathrm{J} \cdot \mathrm{mol}^{-1} \cdot \mathrm{K}^{-1}\end{array}$} & \multirow[b]{2}{*}{$-\left(G_{T}^{\circ}-H_{298}\right) / T$} & \multicolumn{2}{|c|}{ FORMATION FROM THE } & \multicolumn{2}{|c|}{ ELEMENTS } \\
\hline & & & & & $\Delta_{f} \mathrm{H}^{0}$ & $\Delta_{f} G^{0}$ & \multicolumn{2}{|r|}{$\log X_{f}$} \\
\hline 298.15 & 242.90 & 398.50 & 0.00 & 398.50 & -371.6 & -394.7 & & 69.15 \\
\hline 300 & 243.20 & 400.00 & 1.50 & 398.50 & -371.6 & -394.8 & & 68.75 \\
\hline 400 & 260.60 & 472.40 & 64.09 & 408.31 & -379.9 & -402.3 & & 52.54 \\
\hline 500 & 369.45 & 549.41 & 121.22 & 428.19 & -376.3 & -407.9 & & 42.62 \\
\hline 600 & 327.89 & 615.63 & 161.33 & 454.30 & -370.6 & -414.9 & & 36.12 \\
\hline 700 & 323.50 & 665.90 & 184.73 & 481.17 & -368.2 & -422.6 & & 31.53 \\
\hline 800 & 325.20 & 709.20 & 202.19 & 507.01 & -365.8 & -430.5 & & 28.11 \\
\hline 900 & 327.30 & 747.53 & 215.89 & 531.64 & -576.1 & -434.4 & & 25.21 \\
\hline 1000 & 332.90 & 782.29 & 227.29 & 555.00 & -569.5 & -419.1 & & 21.89 \\
\hline 1100 & 340.00 & 814.34 & 237.21 & 577.13 & -563.5 & $-404 \cdot 3$ & & 19.20 \\
\hline 1200 & 348.30 & 844.27 & 246.12 & 598.15 & -556.7 & -390.1 & & 16.98 \\
\hline \multirow[t]{2}{*}{ Melting $T$} & \multicolumn{2}{|r|}{$\mathbf{R}$} & & & \multicolumn{2}{|c|}{ Boiling T } & \multicolumn{2}{|r|}{$\mathbf{K}$} \\
\hline & & & & & \multicolumn{2}{|c|}{$\Delta_{\mathrm{vup}} \mathrm{H}^{\circ}$} & \multicolumn{2}{|c|}{$\mathbf{k J}$} \\
\hline$\Delta_{\Lambda N} H^{0}$ & & $\mathbf{k J}$ & & & & & & \\
\hline H $298^{-H} 8$ & & $11 \mathrm{~kJ}$ & & & & Vol. & $\begin{array}{l}9.873 \\
8.73\end{array}$ & $\begin{array}{l}\mathrm{J} \cdot \mathrm{bar}^{-1} \\
\mathrm{~cm}^{3}\end{array}$ \\
\hline
\end{tabular}


Fes: Hexagonal crystals (NiAs structure) 298.15 to a complex transition at $411 \mathrm{~K}$ and a Néel temperature at $590 \mathrm{~K}$; paramagnetic 590 to melting point at $1468 \mathrm{~K}$; liquid 1468 to 1800 K. Reference state for sulfur is orthorhombic sulfur.

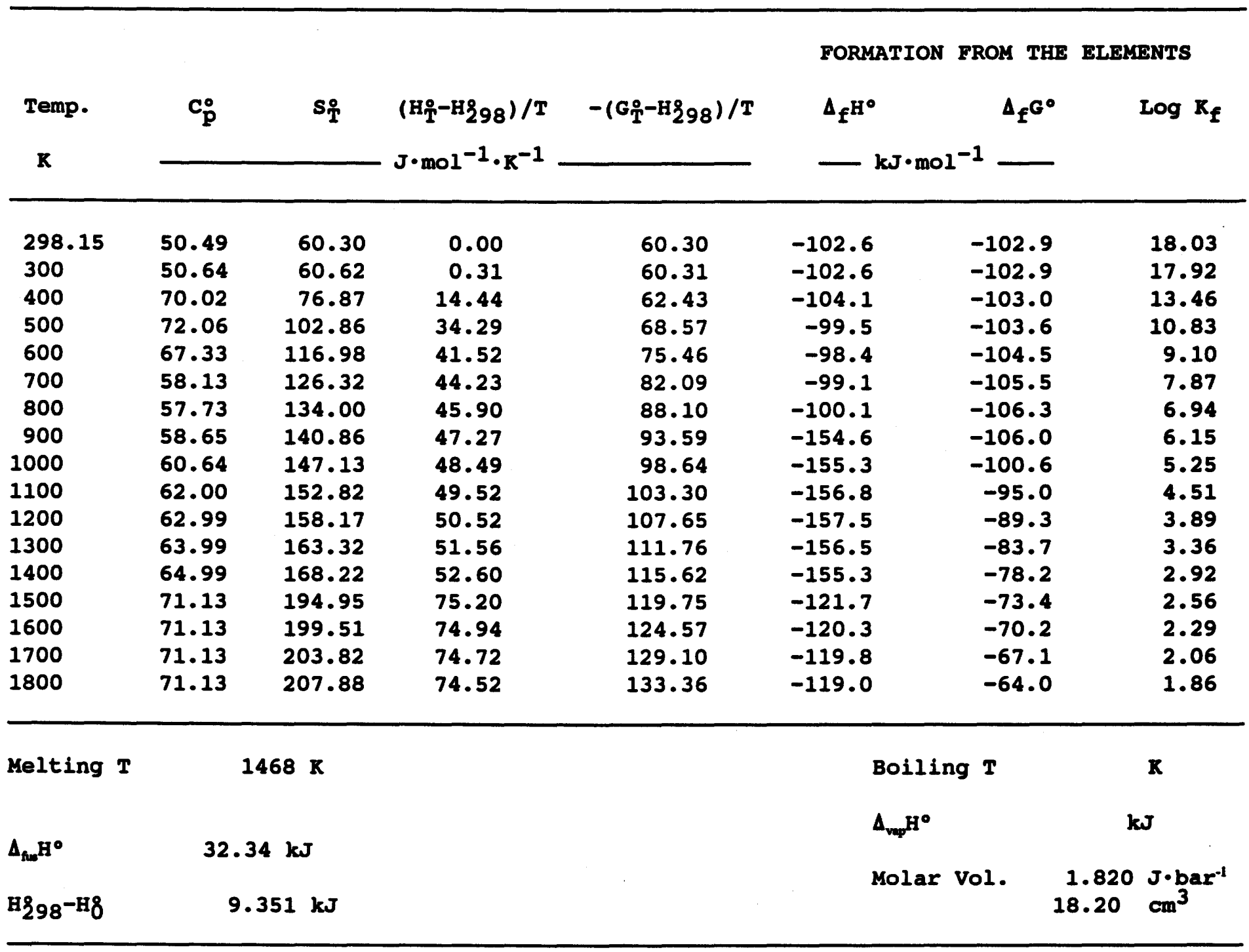


Fe.875s: Monoclinic crystals 298.15 to combined structural and magnetic transition at 590 $K_{i}$ hexagonal crystals (Ni-As-type) 590 to $1000 \mathrm{~K}$. Reference state for sulfur is orthorhombic sulfur.

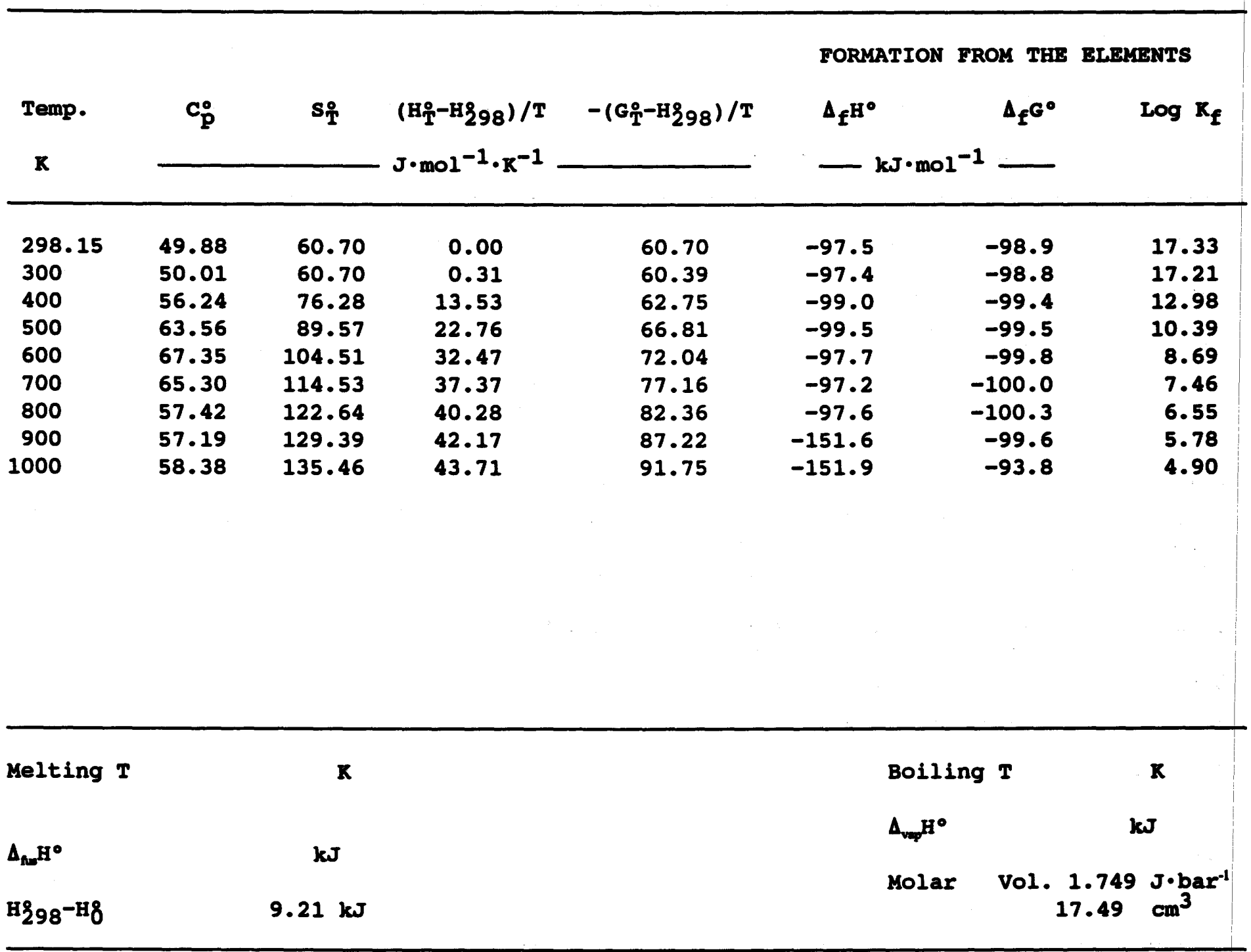


Fes $_{2}$ : Cubic crystals 298.15 to $1000 \mathrm{~K}$. Reference state for sulfur is orthorhombic sulfur.

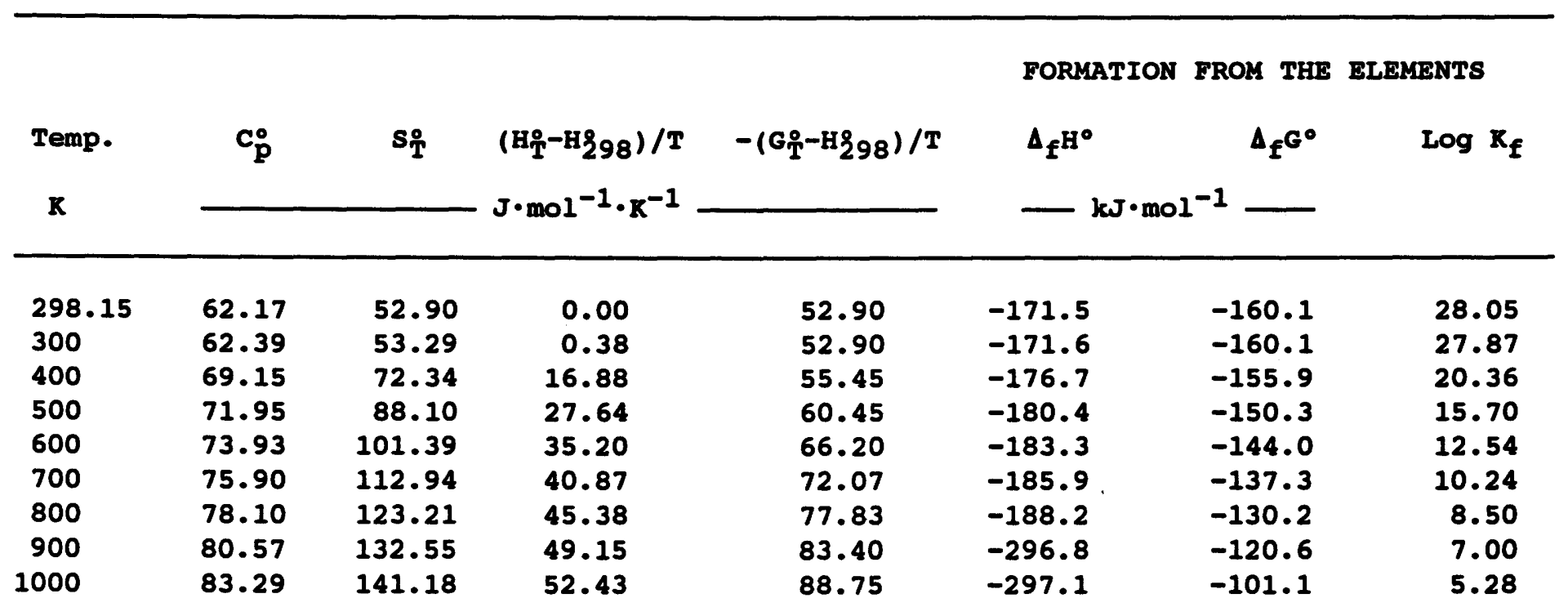

Melting $\mathrm{T} \quad 1015 \mathrm{i} \mathrm{K}$

$\Delta_{\text {fw }} \mathrm{H}^{\circ}$

H298-H8 
Fes $_{2}$ : Orthorhombic crystals 298.15 to $700 \mathrm{~K}$. Reference state for sulfur is orthorhombic sulfur.

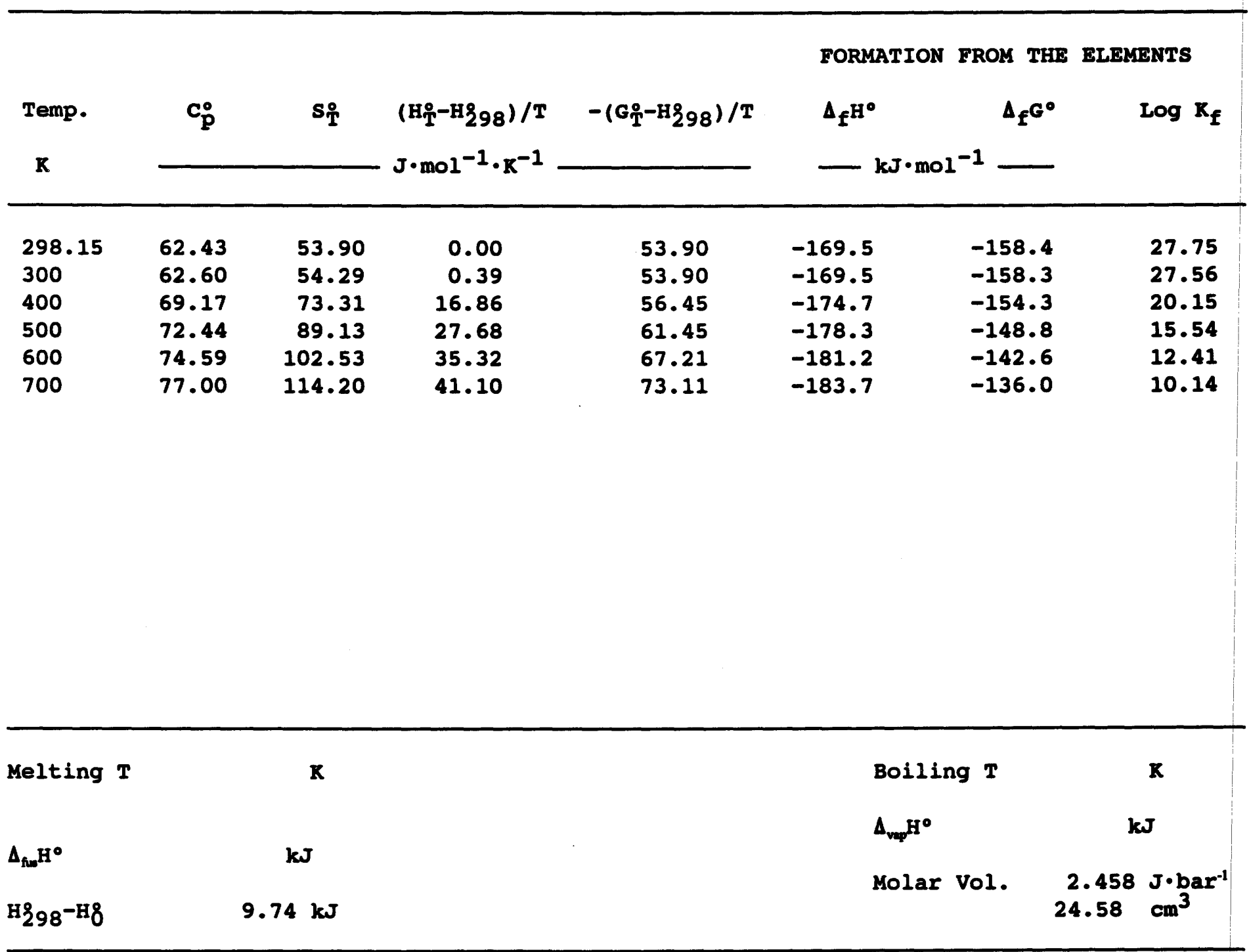


$\mathrm{H}_{2} \mathrm{~s}$ : Ideal gas at $\mathrm{p}=1$ bar, 298.15 to $1800 \mathrm{~K}$. Reference state for sulfur is orthorhombic sulfur.

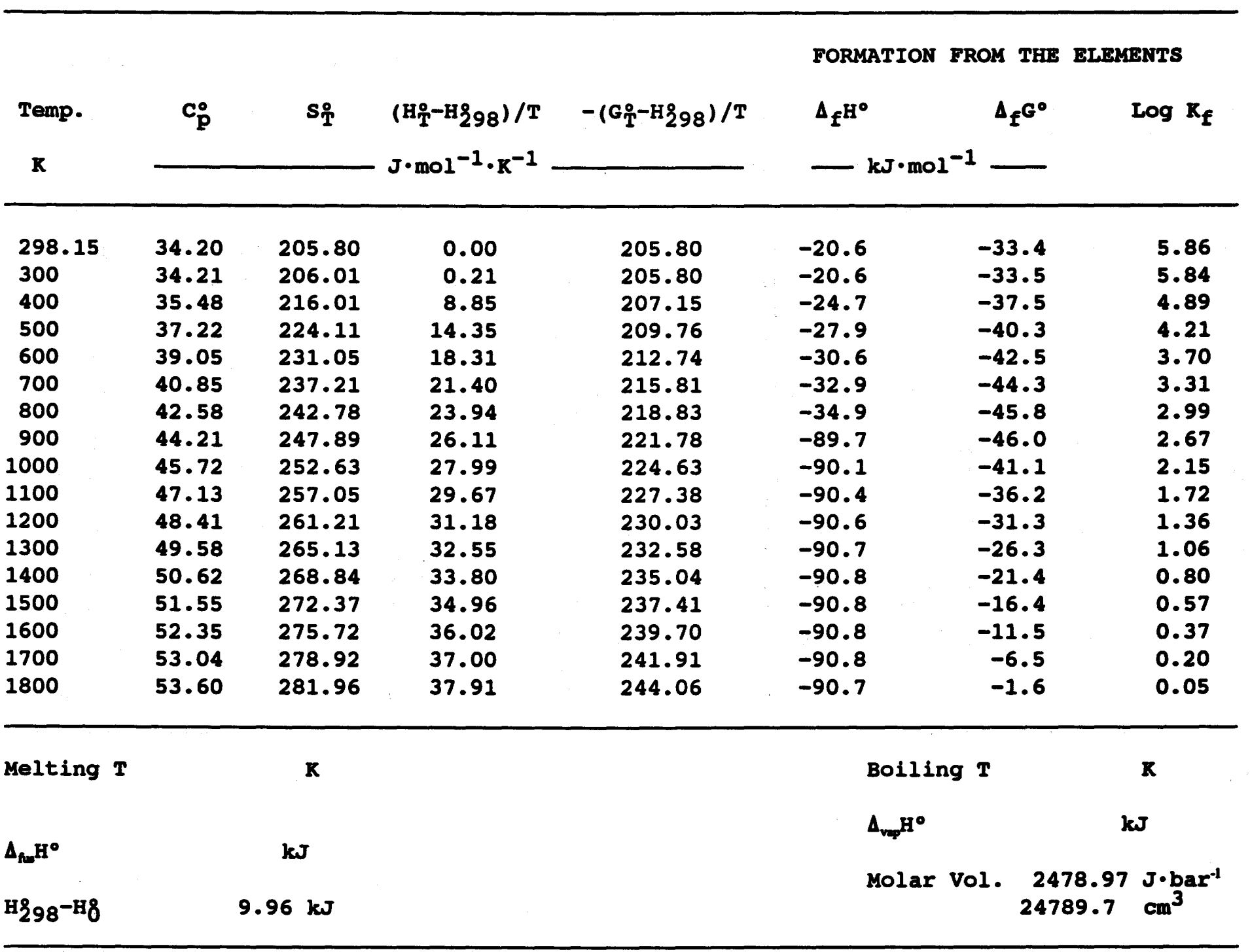


Mns: Cubic crystals 298.15 to $1800 \mathrm{~K}$. Reference state for sulfur is orthorhombic sulfur.

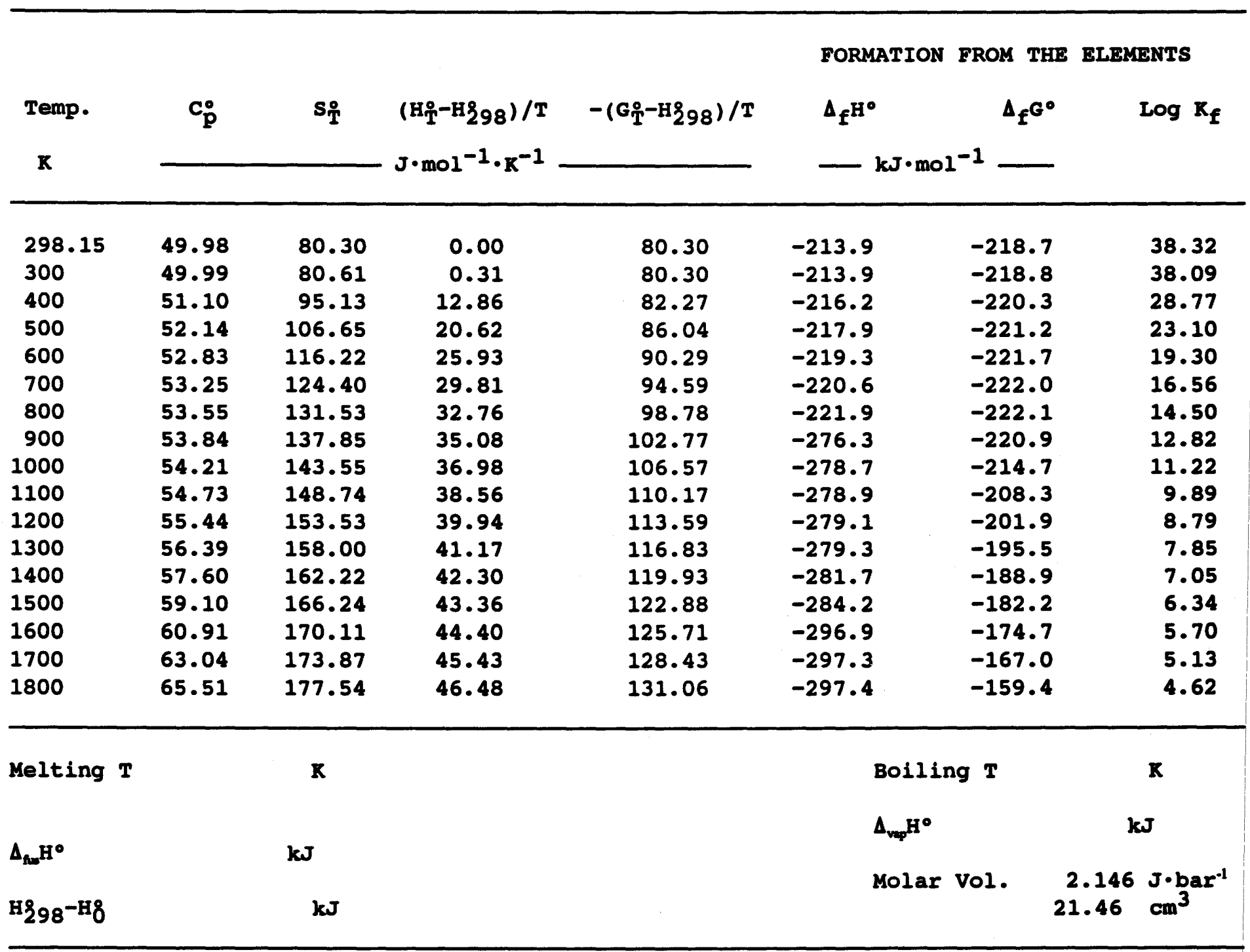


MoS $_{2}$ : Hexagonal crystals 298.15 to $1200 \mathrm{~K}$. Reference state for sulfur is orthorhombic sulfur.

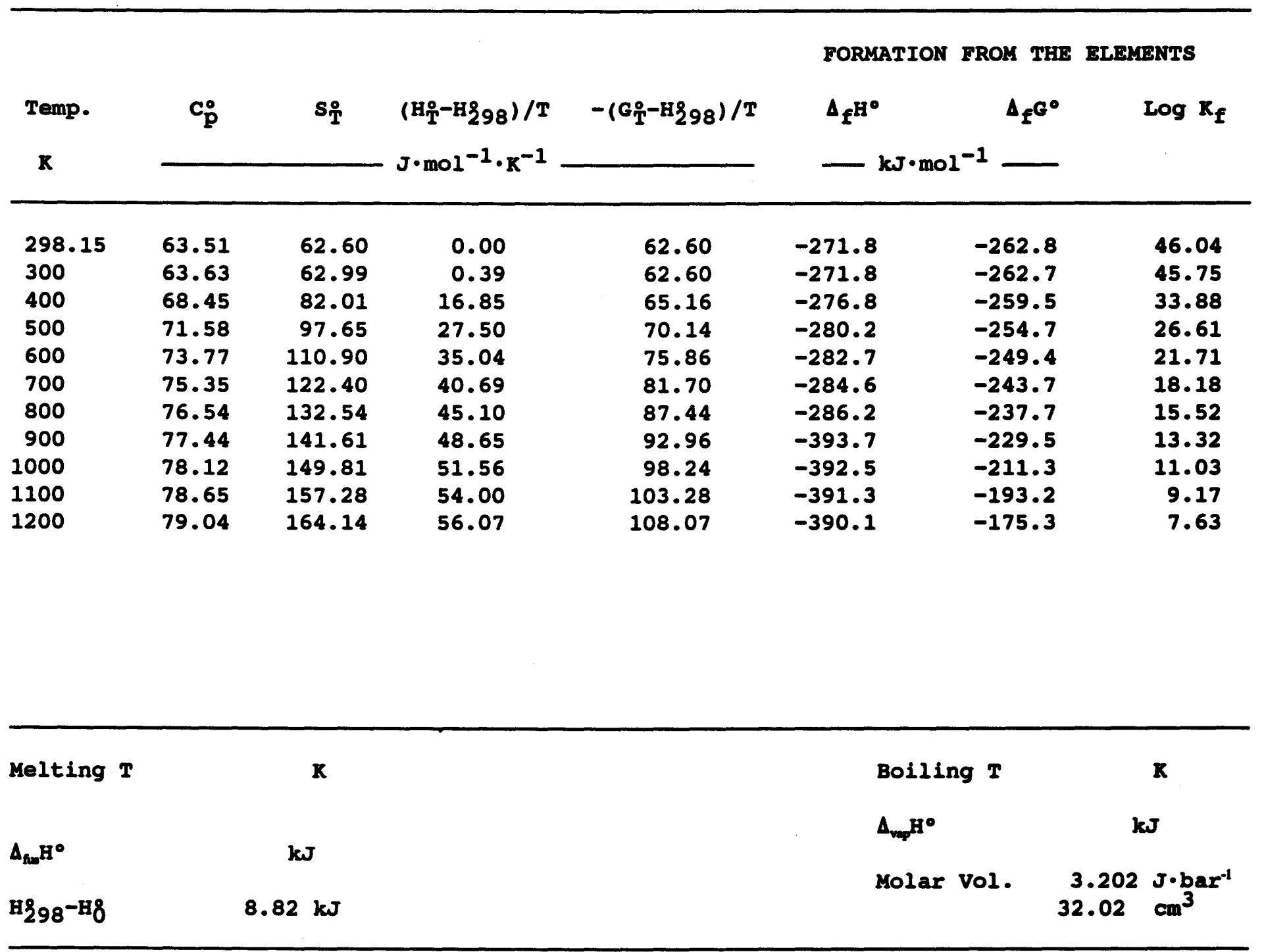


Nis: Rhombohedral crystals 298.15 to $623 \mathrm{~K}$; hexagonal crystals 623 to melting point $1066 \mathrm{~K}$. Reference state for sulfur is orthorhombic sulfur.

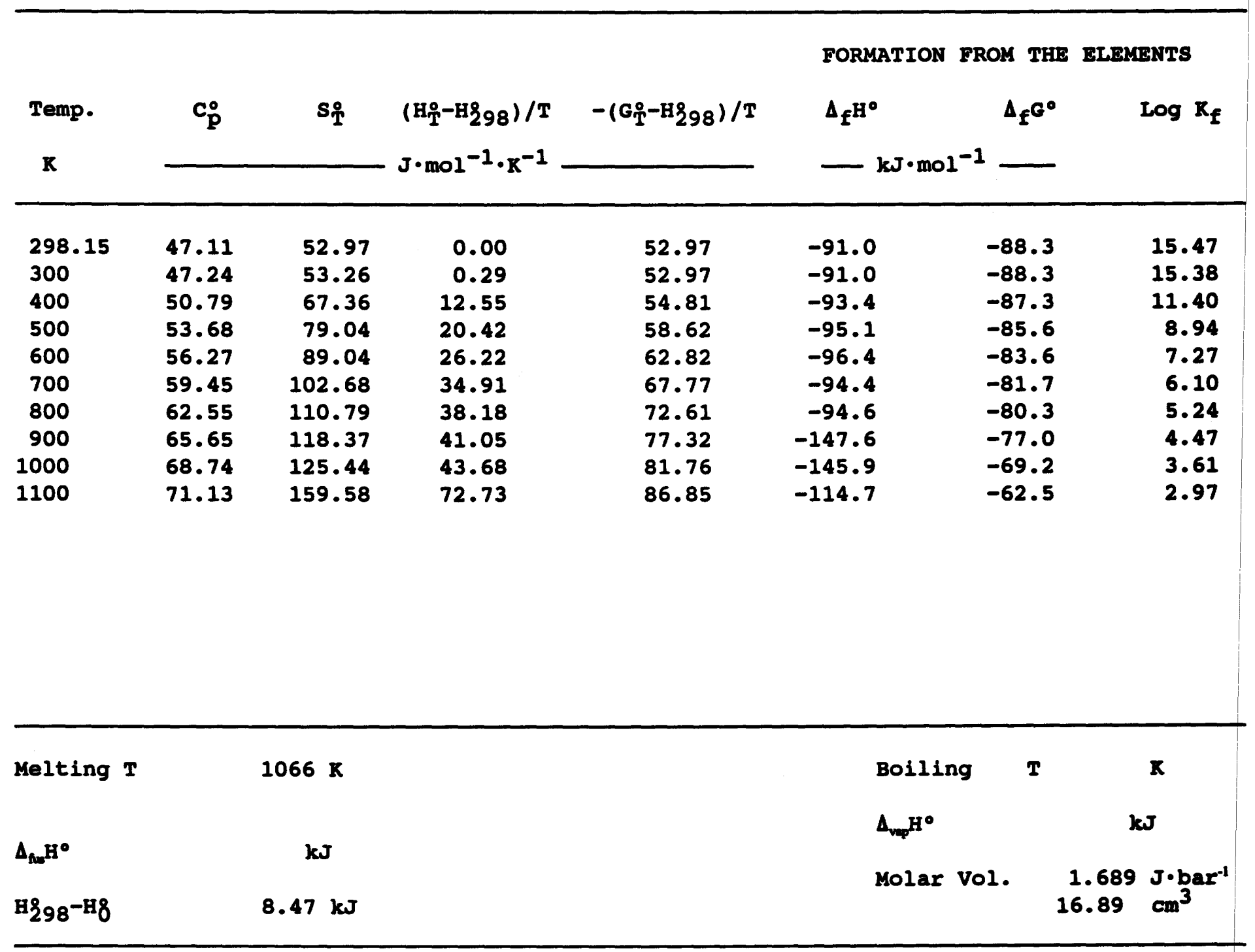


$\mathrm{Ni}_{3} \mathrm{~S}_{2}$ : Hexagonal crystals 298.15 to $834 \mathrm{~K}$; cubic crystals $834 \mathrm{~K}$ to incongruent melting point $1064 \mathrm{~K} ;$ liquid 1064 to $1400 \mathrm{~K}$. Reference state for sulfur is orthorhombic sulfur.

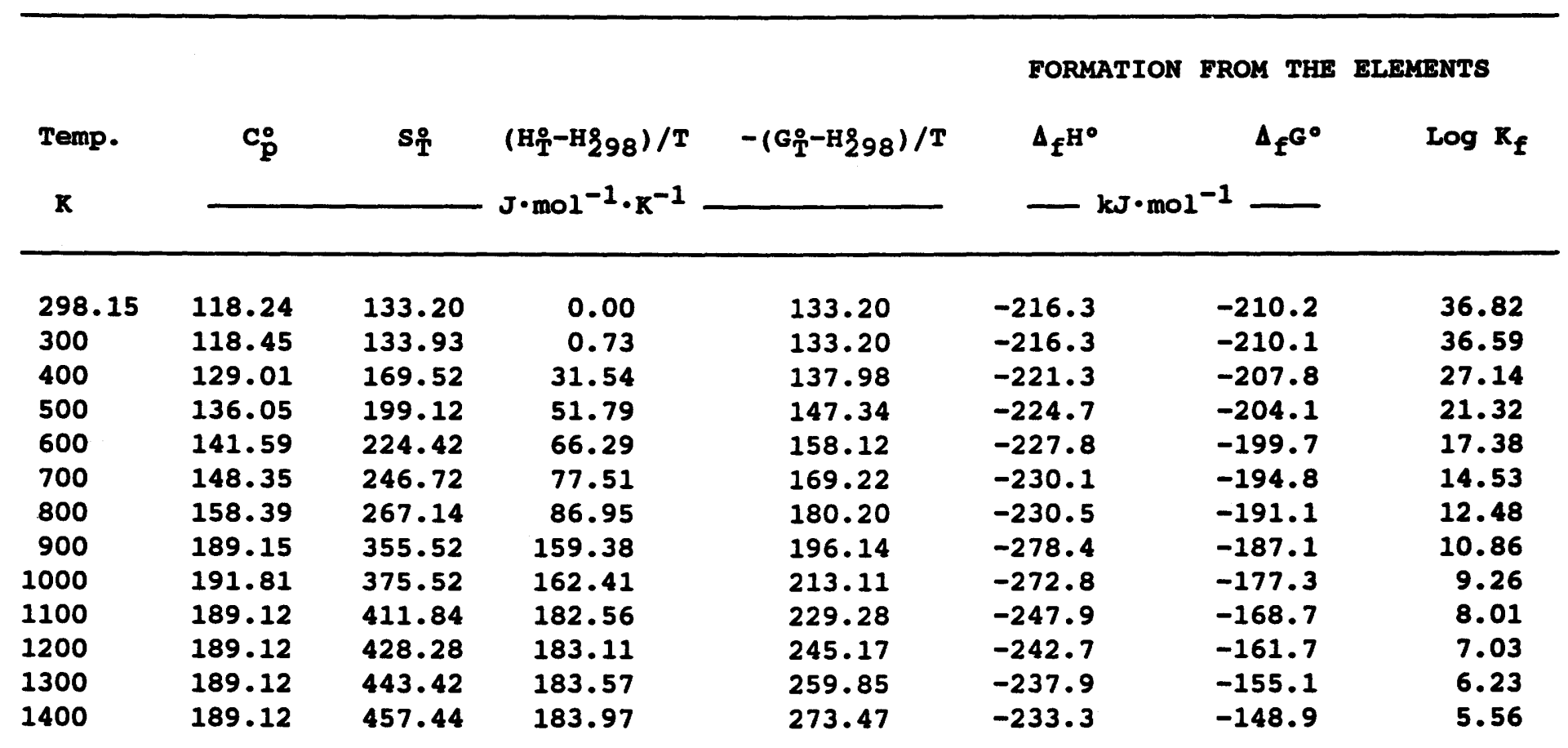

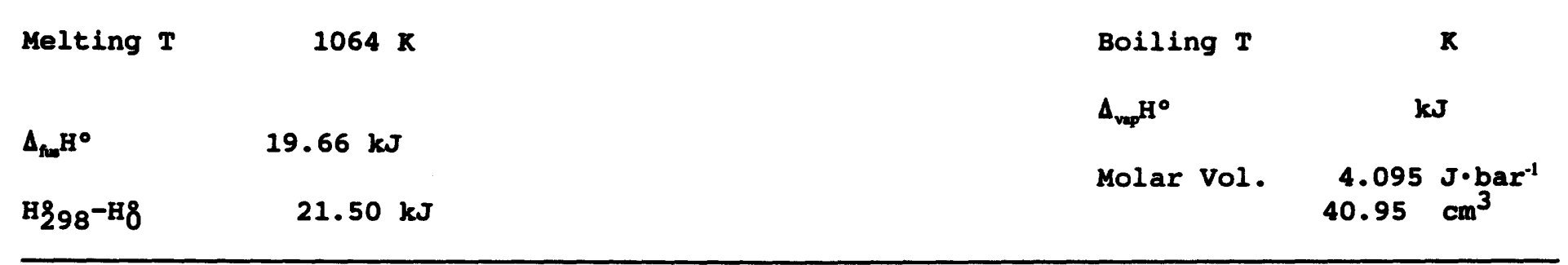


Pbs: Cubic crystals 298.15 to $900 \mathrm{~K}$. Reference state for sulfur is orthorhombic sulfur.

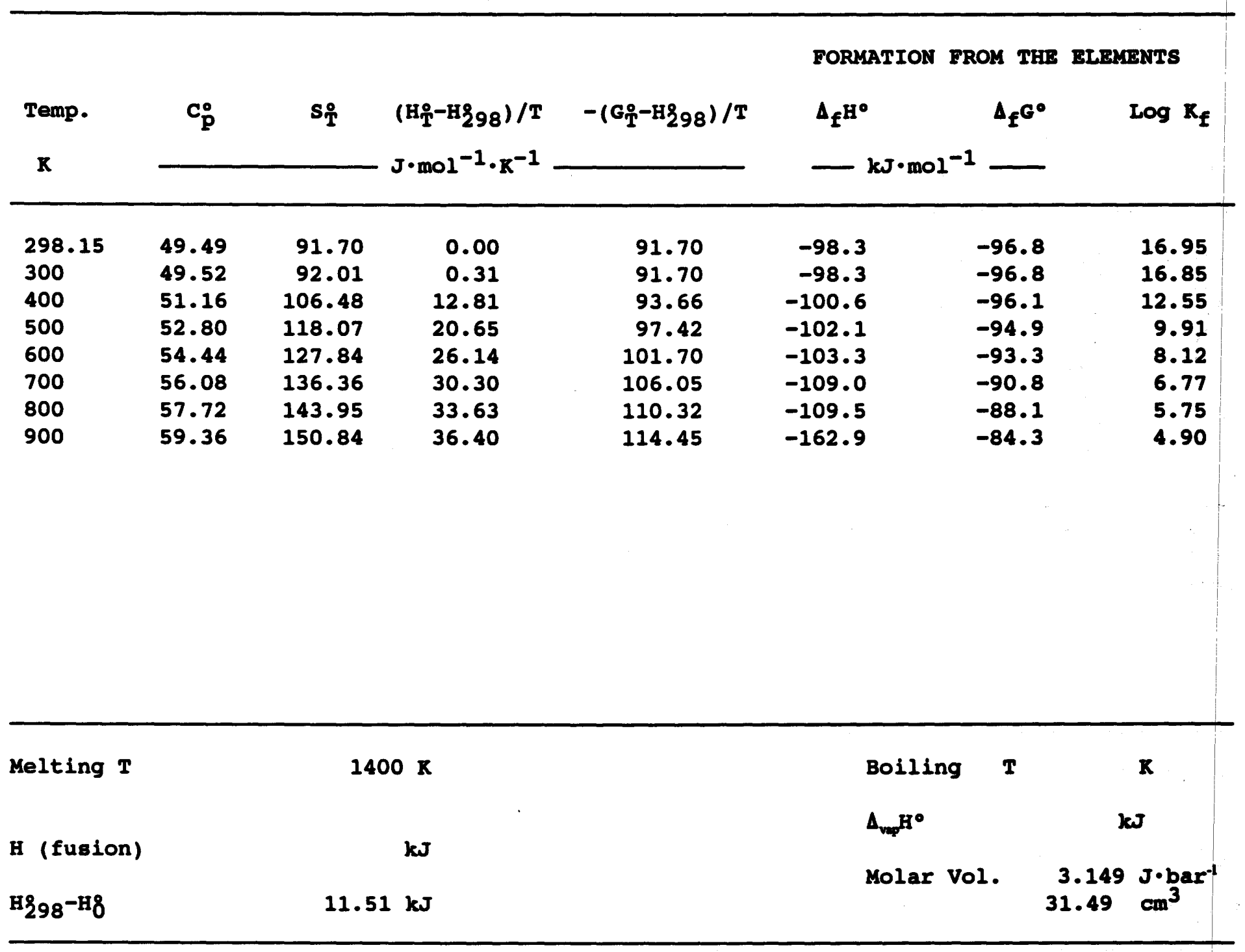


$\mathrm{Sb}_{2} \mathrm{~s}_{3}$ : Orthorhombic crystals 298.15 to $900 \mathrm{~K}$. Reference state for sulfur is orthorhombic sulfur.

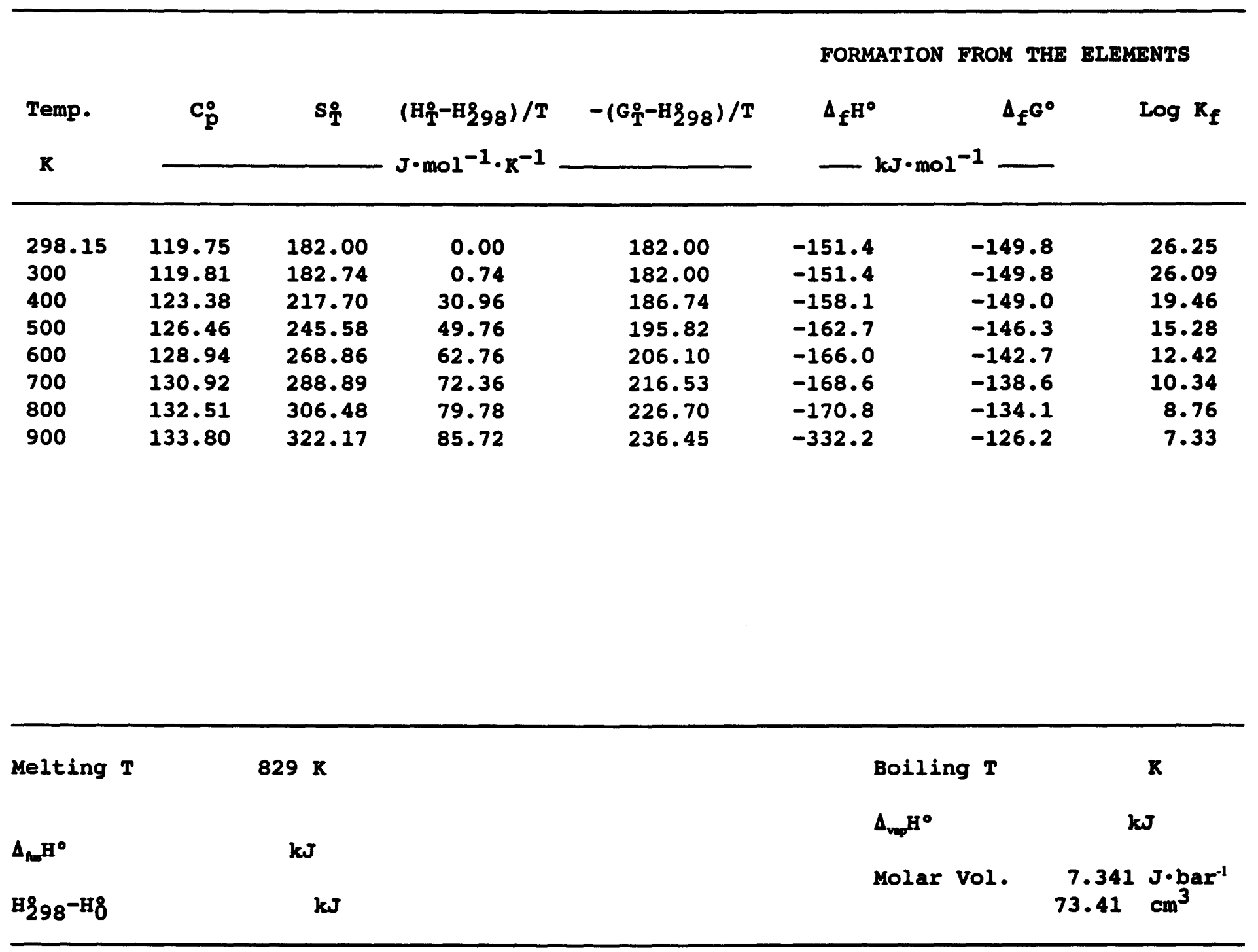


Sns: Orthorhombic crystals 298.15 to $875 \mathrm{~K}$; cubic crystals 875 to melting point $1153 \mathrm{~K}$. Liquid 1153 to $1300 \mathrm{~K}$. Reference state for sulfur is orthorhombic sulfur.

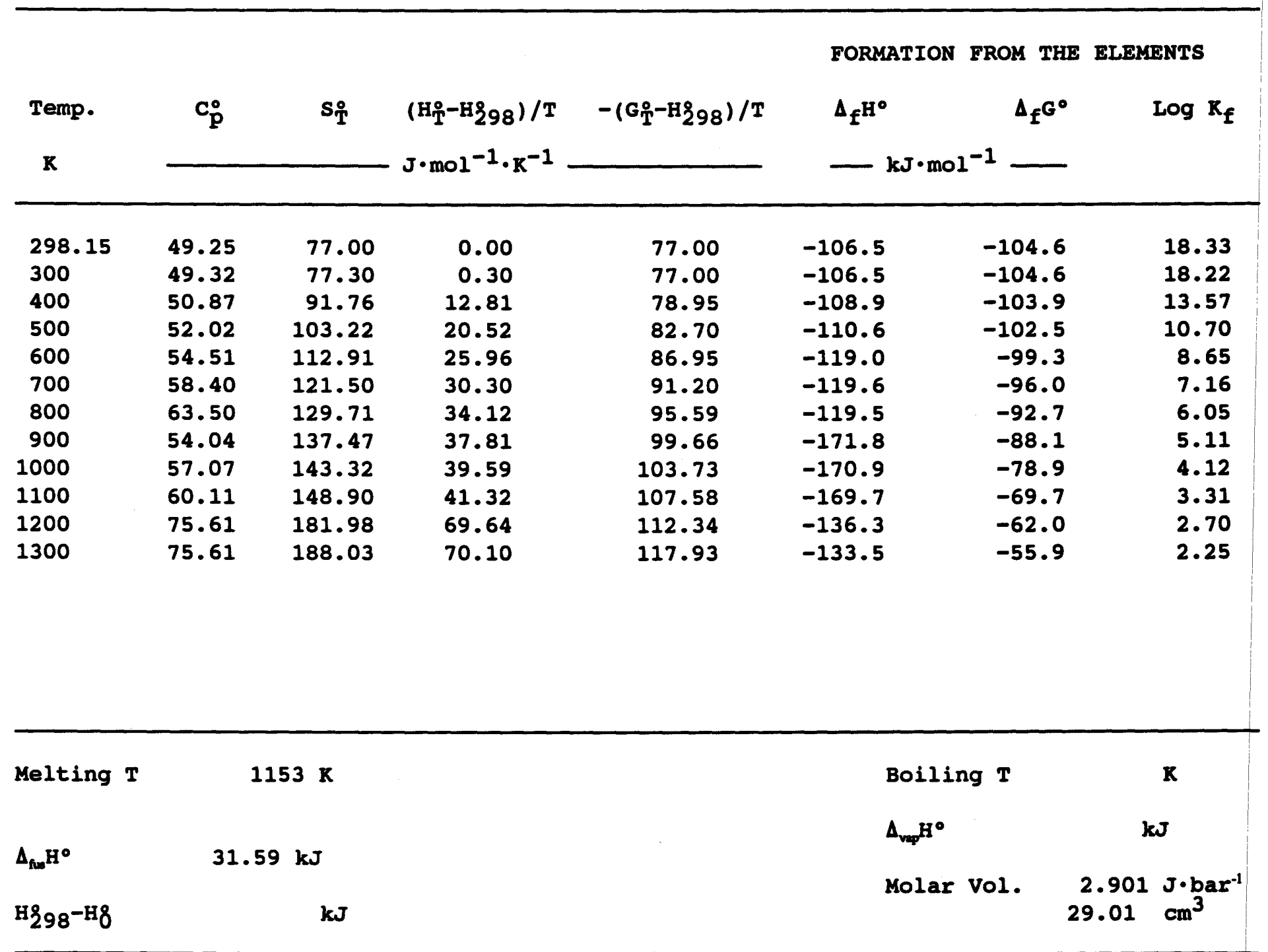


$\mathrm{SnS}_{2}$ : Hexagonal crystal 298.15 to $1000 \mathrm{~K}$. Reference state for sulfur is orthorhombic sulfur.

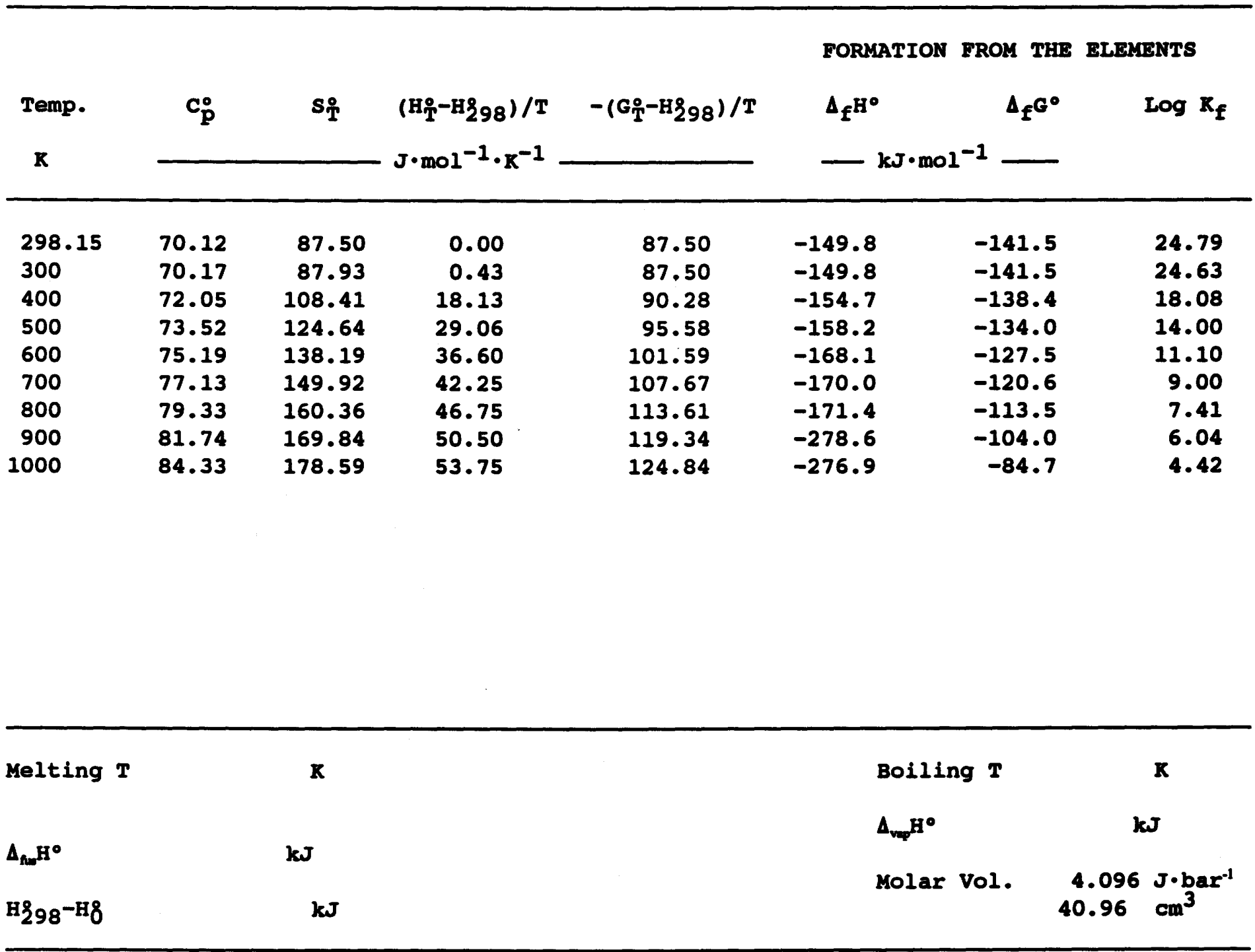


$\mathrm{WS}_{2}$ : Hexagonal crystals 298.15 to $1500 \mathrm{~K}$. Reference state for sulfur is orthorhombic sulfur.

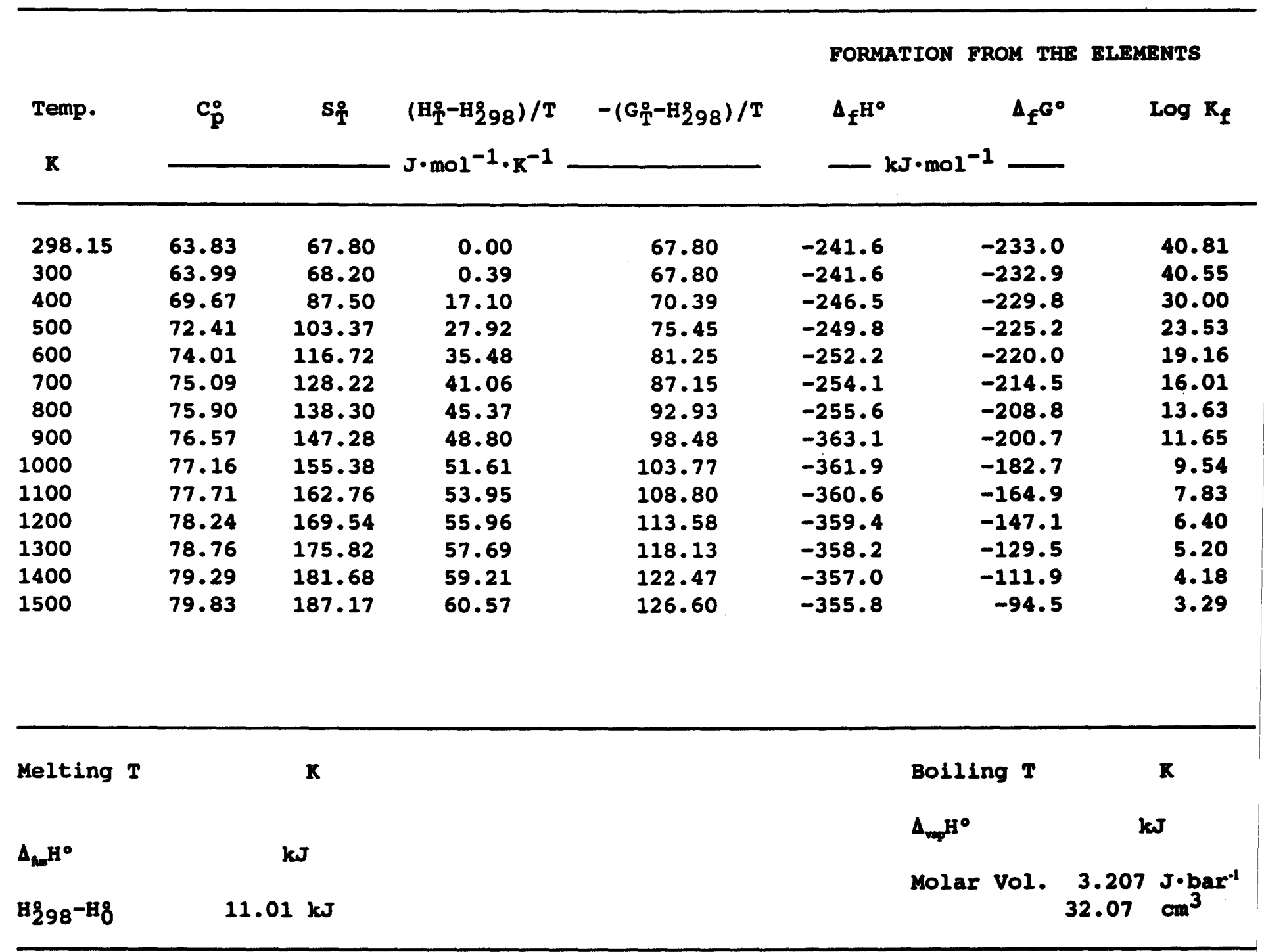


ZnS: Cubic crystals $298.15 \mathrm{~K}$ to $1300 \mathrm{~K}$. Reference state for sulfur is orthorhombic sulfur.

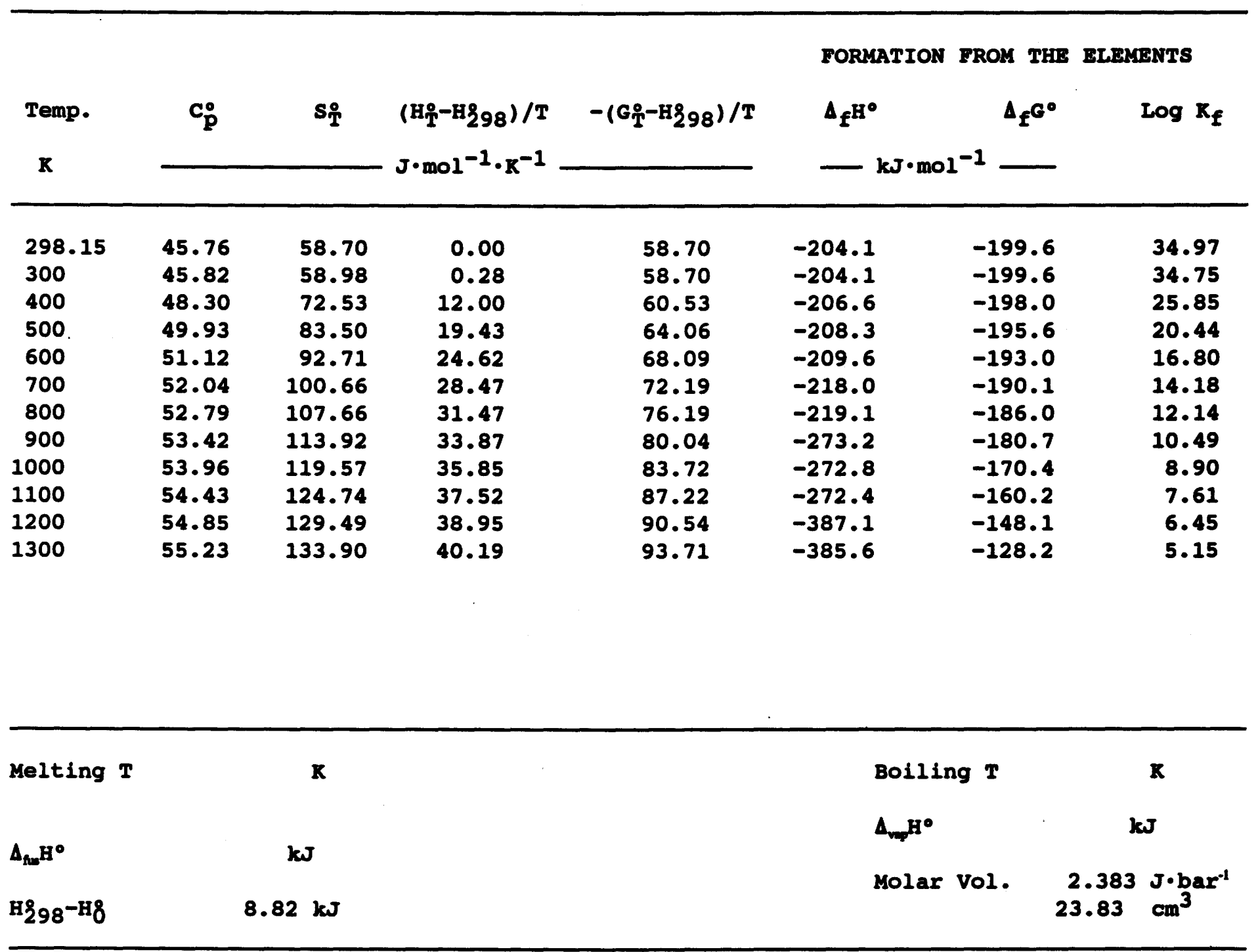


Zns: Hexagonal crystals 298.15 to $1300 \mathrm{~K}$. Reference state for sulfur is orthorhombic sulfur.

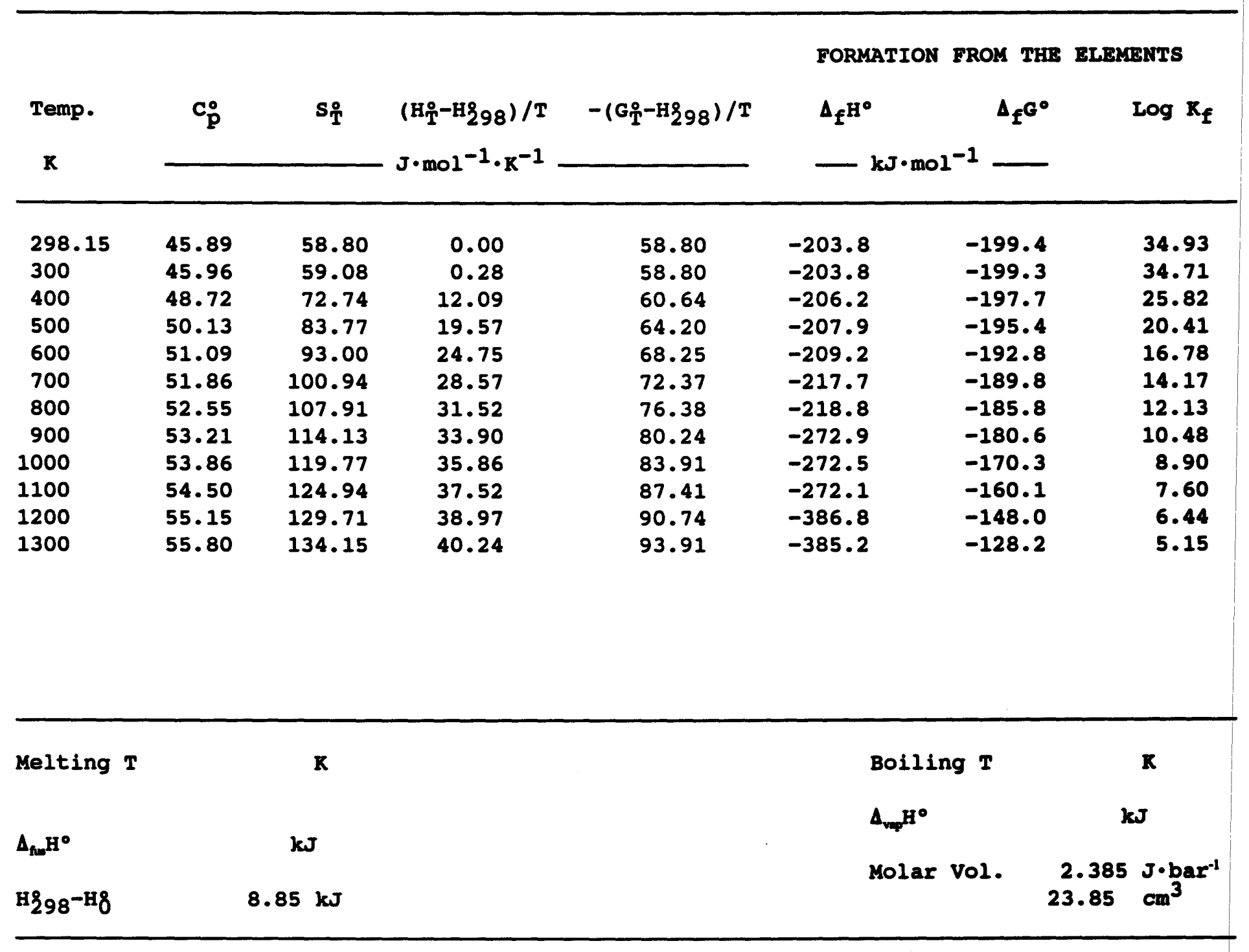


$\mathrm{FeSb}_{2} \mathrm{~s}_{4}$ : Orthorhombic crystals 298.15 to $836 \mathrm{~K}$. Reference state for sulfur is orthorhombic sulfur.

\begin{tabular}{|c|c|c|c|c|c|c|c|}
\hline Temp. & $\mathbf{C}_{\mathbf{p}}^{\circ}$ & $\mathbf{s}_{\mathbf{T}}^{\circ}$ & \multirow{2}{*}{$\begin{array}{l}\left(\mathrm{H}_{\mathrm{T}}^{\circ}-\mathrm{H} 298\right) / \mathrm{T} \\
\mathrm{J} \cdot \mathrm{mol}^{-1} \cdot \mathrm{K}^{-1}\end{array}$} & \multirow[t]{2}{*}{$-\left(G_{T}^{\circ}-H_{298}\right) / T$} & \multirow{2}{*}{\multicolumn{2}{|c|}{$\begin{array}{l}\text { FORMATION FROM THE } \\
\Delta_{\mathrm{f}^{\mathrm{H}^{\circ}}} \Delta_{\mathrm{f}^{\circ}}\end{array}$}} & \multirow[t]{2}{*}{$\begin{array}{l}\text { ELEMENTS } \\
\qquad \operatorname{LOg} K_{f}\end{array}$} \\
\hline $\mathbf{K}$ & & & & & & & \\
\hline 298.15 & 175.23 & 245.00 & 0.00 & 245.00 & -256.2 & -255.8 & 44.81 \\
\hline 300 & 175.60 & 246.09 & 1.08 & 245.00 & -256.2 & -255.8 & 44.54 \\
\hline 400 & 186.17 & 298.40 & 46.33 & 252.06 & -264.1 & -255.4 & 33.36 \\
\hline 500 & 189.05 & 340.31 & 74.64 & 265.67 & -269.1 & -252.8 & 26.40 \\
\hline 600 & 190.44 & 374.90 & 93.83 & 281.07 & -272.9 & -249.1 & 21.69 \\
\hline 700 & 191.92 & 404.37 & 107.73 & 296.63 & -276.0 & -244.9 & 18.27 \\
\hline 800 & 193.91 & 430.12 & 118.37 & 311.74 & -279.0 & -240.2 & 15.68 \\
\hline 900 & 196.48 & 453.10 & 126.91 & 326.20 & -494.4 & -230.9 & 13.40 \\
\hline
\end{tabular}

Melting $\mathbf{T}$

$\Delta_{\text {nNo }} \mathrm{H}^{\circ}$

$\mathbf{k J}$

H298-H8

\section{$\mathbf{R}$}

$\mathbf{k J}$
Boiling $T$

$\mathbf{K}$

$\Delta_{\text {vep }} \mathrm{H}^{\circ}$

$\mathbf{k J}$

Molar Vol. $\quad 9.222 \mathrm{~J} \cdot \mathrm{bar}^{-1}$

$92.22 \mathrm{~cm}^{3}$ 
CuSbs $_{2}$ : Orthorhombic crystals 298.15 to $826 \mathrm{~K}$. Reference state for sulfur is orthorhombic sulfur.

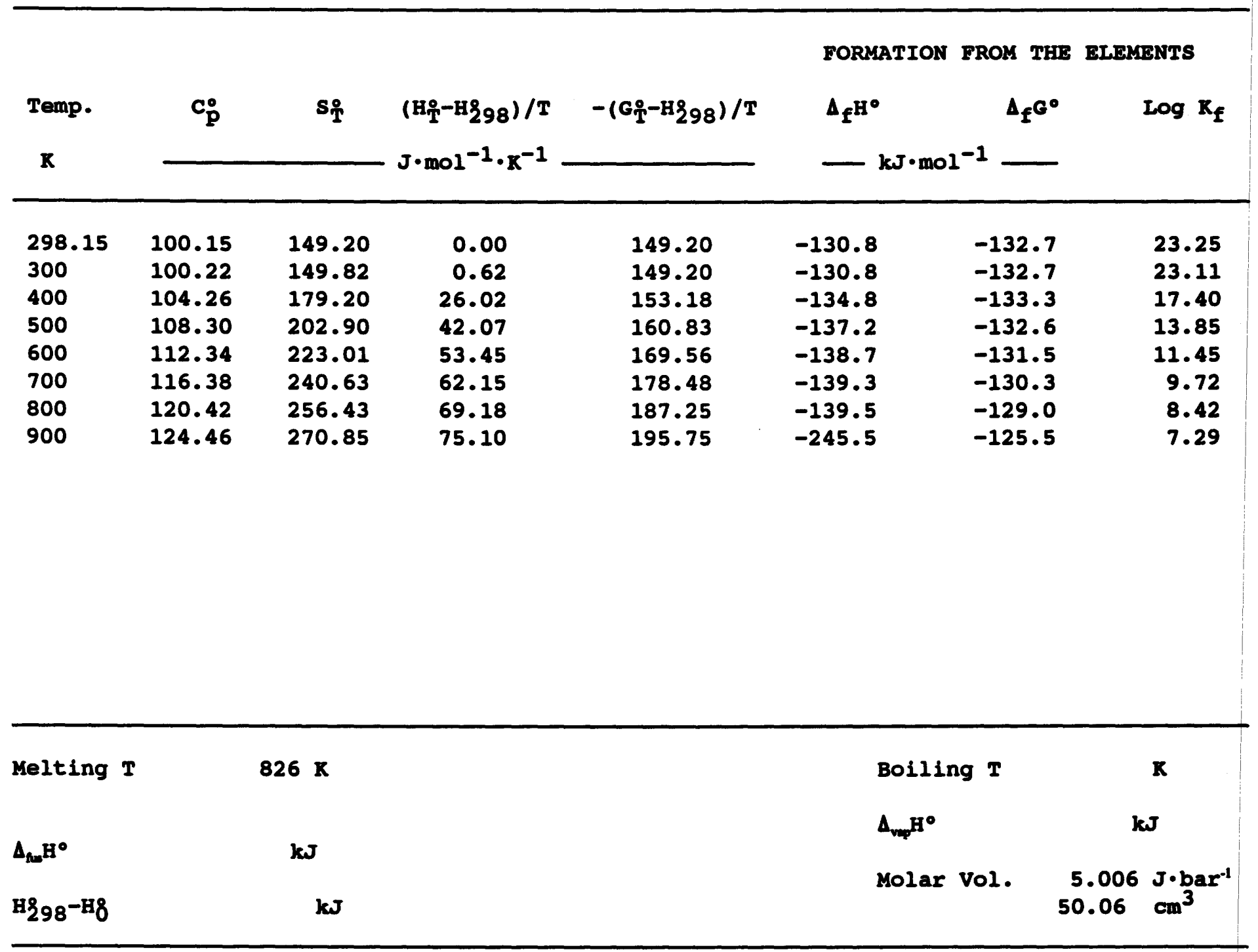


$\mathrm{Ag}_{2}$ S: Monoclinic crystals 298.15 to $451.3 \mathrm{~K}$; cubic crystals (argentite) 451.3 to $865 \mathrm{~K}$. $\gamma$ phase 865 to $1000 \mathrm{~K}$. Reference state for sulfur is ideal $s_{2}$ gas at $p=1$ bar.

\begin{tabular}{|c|c|c|c|c|c|c|c|}
\hline Temp. & $\mathbf{c}_{\mathbf{p}}^{0}$ & $\mathbf{s}_{\mathbf{T}}$ & \multirow{2}{*}{$\begin{array}{l}\left(\mathrm{H}_{\mathrm{T}}^{\circ}-\mathrm{H}_{298}\right) / \mathrm{T} \\
\mathrm{J} \cdot \mathrm{mol}^{-1} \cdot \mathrm{K}^{-1}\end{array}$} & \multirow[t]{2}{*}{$-\left(G_{T}^{\circ}-H_{298}\right) / T$} & $\begin{array}{l}\text { FORMATION } \\
\Delta_{\mathrm{f}} \mathrm{H}^{\circ}\end{array}$ & $\begin{array}{c}\text { FROM THE } \\
\Delta_{f^{G}} G^{\circ}\end{array}$ & \multirow[t]{2}{*}{$\begin{array}{l}\text { ELEMENTS } \\
\qquad \log \mathrm{K}_{\mathbf{f}}\end{array}$} \\
\hline $\mathbf{K}$ & & & & & $-\mathbf{k J} \cdot \mathrm{mol}$ & -1 & \\
\hline 298.15 & 75.31 & 142.89 & 0.00 & 142.89 & -96.3 & -79.5 & 13.93 \\
\hline 300 & 75.39 & 143.35 & 0.46 & 142.89 & -96.3 & -79.4 & 13.83 \\
\hline 400 & 80.47 & 165.67 & 19.78 & 145.89 & -95.3 & -73.9 & 9.65 \\
\hline 500 & 87.26 & 193.79 & 41.08 & 152.71 & -89.6 & -69.2 & 7.23 \\
\hline 600 & 86.03 & 209.59 & 48.67 & 160.92 & -88.1 & -65.3 & 5.68 \\
\hline 700 & 84.80 & 222.75 & 53.91 & 168.84 & -86.7 & -61.6 & 4.60 \\
\hline 800 & 84.69 & 234.05 & 57.75 & 176.30 & -85.7 & -58.1 & 3.79 \\
\hline 900 & 80.38 & 244.84 & 61.53 & 183.31 & -84.1 & -54.7 & 3.17 \\
\hline 1000 & 80.57 & 253.28 & 63.38 & 189.90 & -83.8 & -51.5 & 2.69 \\
\hline
\end{tabular}

Melting $\mathbf{T}$

$\Delta_{\mathrm{fum}} \mathrm{H}^{\circ}$

$\mathbf{k J}$

H298-H8
$\mathbf{K}$

Boiling $\mathbf{T}$

$\Delta_{\text {vep }} \mathbf{H}^{\circ}$

Molar Vol.
$\mathbf{R}$

$\mathbf{k J}$ $34.19 \mathrm{~cm}^{3}$
$A=-8 \cdot 355 E+01$
$B=3.273 E-02$
$C=-5 \cdot 14 E+05$ 


\begin{abstract}
Ass: Monoclinic crystals 298.15 to melting point $580 \mathrm{~K}$; liquid 580 to $800 \mathrm{~K}$.
Reference state for sulfur is ideal $s_{2}$ gas at $p=1$ bar.
\end{abstract}

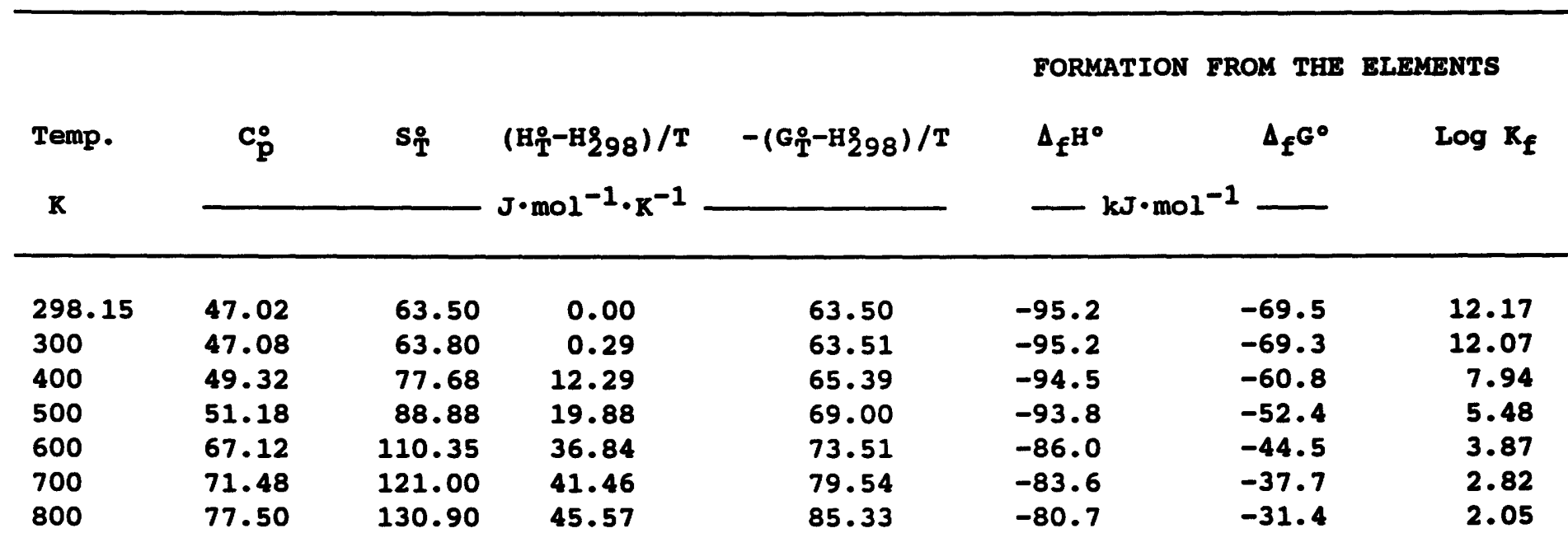

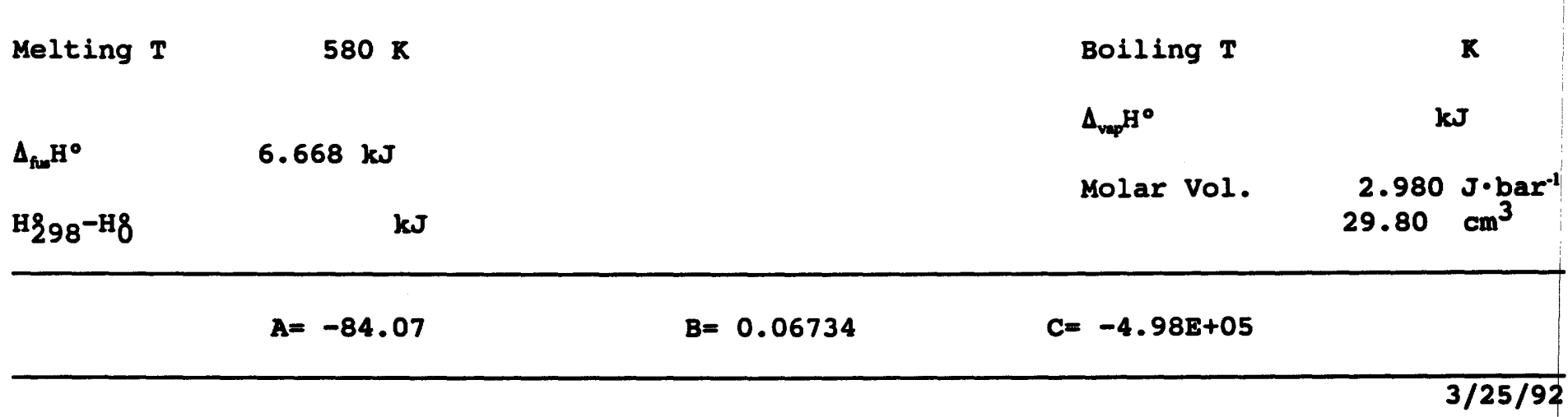


Cds: Cubic crystals 298.15 to $1100 \mathrm{k}$. Reference state for sulfur is ideal $\mathrm{s}_{2}$ gas at $\mathrm{p}=1$ bar.

\begin{tabular}{|c|c|c|c|c|c|c|c|}
\hline \multirow[b]{2}{*}{ Temp. } & \multirow[b]{2}{*}{$\mathbf{C}_{\mathbf{p}}^{\circ}$} & \multirow[b]{2}{*}{$\mathbf{s} \stackrel{\mathbf{T}}{\mathbf{s}}$} & \multirow{3}{*}{$\begin{array}{l}\left(\mathrm{H}_{\mathrm{T}}-\mathrm{H}_{298}\right) / \mathrm{T} \\
\mathrm{J} \cdot \mathrm{mol} \\
-1 \cdot \mathrm{K}^{-1}\end{array}$} & \multirow[b]{2}{*}{$-\left(G_{T}-H_{298}\right) / T$} & \multicolumn{2}{|c|}{ FORMATION FROM THE } & \multirow{2}{*}{$\begin{array}{l}\text { BIEMENTS } \\
\text { Log } k_{f}\end{array}$} \\
\hline & & & & & $\boldsymbol{\Delta}_{\mathrm{f}^{\mathrm{H}^{\circ}}}$ & $\Delta_{f^{G}} G^{0}$ & \\
\hline $\mathbf{K}$ & & & & & $-\mathbf{k J} \cdot \mathbf{m o l}$ & -1 & \\
\hline 298.15 & 47.30 & 72.18 & 0.00 & 72.18 & -213.9 & -186.0 & 32.58 \\
\hline 300 & 47.36 & 72.47 & 0.29 & 72.18 & -213.9 & -185.8 & 32.35 \\
\hline 400 & 49.46 & 86.42 & 12.35 & 74.07 & -213.4 & -176.5 & 23.05 \\
\hline 500 & 50.70 & 97.60 & 19.91 & 77.69 & -212.8 & -167.4 & 17.48 \\
\hline 600 & 51.68 & 106.93 & 25.12 & 81.81 & -218.6 & -158.2 & 13.78 \\
\hline 700 & 52.55 & 114.96 & 28.98 & 85.99 & -218.1 & -148.2 & 11.06 \\
\hline 800 & 53.37 & 122.03 & 31.98 & 90.06 & -217.6 & -138.3 & 9.03 \\
\hline 900 & 54.16 & 128.37 & 34.40 & 93.97 & -217.1 & -128.4 & 7.45 \\
\hline 1000 & 54.93 & 134.11 & 36.41 & 97.70 & -216.5 & $-118 \cdot 5$ & 6.19 \\
\hline 1100 & 55.67 & 139.38 & 38.13 & 101.25 & -314.8 & -102.9 & 4.89 \\
\hline
\end{tabular}

Melting $\mathbf{T}$

$\Delta_{\text {fis }} \mathbf{H}^{\circ}$

H298-H8 $\mathbf{k J}$

$\mathbf{K}$

$8.81 \mathrm{~kJ}$
Boiling $T$

$\Lambda_{\text {vaph }} \mathrm{H}^{\circ}$

Molar Vol.
K

$\mathbf{k J}$
$2.993 \mathrm{~J} \cdot$ bar $^{-1}$ $29.93 \mathrm{~cm}^{3}$ 
$\cos _{2}$ : Cubic crystals 298.15 to $1000 \mathrm{~K}$. Reference state for sulfur is ideal $s_{2}$ gas at $p=$ 1 bar.

\begin{tabular}{|c|c|c|c|c|c|c|c|}
\hline Temp. & $\mathbf{c}_{\mathbf{p}}^{\circ}$ & $\mathbf{s}_{\mathbf{T}}^{\circ}$ & \multirow{2}{*}{$\begin{array}{l}\left(\mathrm{H}_{\mathrm{T}}^{\circ}-\mathrm{H}_{298}\right) / \mathrm{T} \\
\mathrm{J} \cdot \mathrm{mol}^{-1} \cdot \mathrm{K}^{-1}\end{array}$} & \multirow[t]{2}{*}{$-\left(G_{\mathrm{T}}^{\circ}-\mathrm{H}_{298}\right) / \mathrm{T}$} & $\begin{array}{l}\text { FORMATION } \\
\Delta_{\mathrm{f}^{\mathrm{H}}}{ }^{\circ}\end{array}$ & \multirow[t]{2}{*}{$\begin{array}{c}\text { FROM THE } \\
\Delta_{f^{G^{\circ}}}\end{array}$} & \multirow[t]{2}{*}{$\begin{array}{l}\text { ELEMENTS } \\
\qquad \log \mathrm{K}_{\mathrm{f}}\end{array}$} \\
\hline $\mathbf{R}$ & & & & & $-k J \cdot \operatorname{mol}$ & & \\
\hline 298.15 & 68.26 & 74.80 & 0.00 & 74.80 & -279.5 & -224.8 & 39.38 \\
\hline 300 & 68.39 & 75.22 & 0.42 & 74.80 & -279.5 & -224.5 & 39.08 \\
\hline 400 & 74.28 & 95.76 & 18.20 & 77.56 & -278.2 & -206.3 & 26.94 \\
\hline 500 & 78.63 & 112.82 & 29.87 & 82.96 & -276.8 & -188.5 & 19.69 \\
\hline 600 & 82.38 & 127.50 & 38.31 & 89.18 & -275.1 & -171.0 & 14.89 \\
\hline 700 & 85.85 & 140.46 & 44.86 & 95.60 & -273.4 & -153.8 & 11.48 \\
\hline 800 & 89.16 & 152.14 & 50.19 & 101.95 & -271.9 & -136.8 & 8.93 \\
\hline 900 & 92.38 & 162.83 & 54.70 & 108.13 & -269.8 & -120.0 & 6.97 \\
\hline 1000 & 95.55 & 172.72 & 58.63 & 114.10 & -267.7 & -103.5 & 5.41 \\
\hline
\end{tabular}

Melting $\mathbf{T}$

$\mathbf{R}$

$\Delta_{\text {fuw }} \mathrm{H}^{\circ}$

$\mathbf{k J}$

H298-H8

$12.30 \mathrm{~kJ}$
Boiling $\mathbf{T}$

$\Delta_{\mathrm{vep}} \mathrm{H}^{\circ}$

Molar Vol.

$B=1.682 \mathrm{E}-01$

$C=-3.57 \mathrm{E}+05$ 
Cus: Hexagonal crystals 298.15 to $800 \mathrm{k}$. Reference state for sulfur is ideal s, gas at p $=1$ bar.

\begin{tabular}{|c|c|c|c|c|c|c|c|}
\hline \multirow[b]{2}{*}{ Temp. } & \multirow[b]{2}{*}{$\mathbf{c}_{\mathbf{p}}^{\circ}$} & \multirow[b]{2}{*}{$\mathbf{s}_{\mathrm{T}}^{\circ}$} & \multirow{3}{*}{$\begin{array}{l}\left(\mathrm{H}_{\mathrm{T}}^{\circ}-\mathrm{H}_{298}\right) / \mathrm{T} \\
\mathrm{J} \cdot \mathrm{mol}^{-1} \cdot \mathrm{K}^{-1}\end{array}$} & \multirow[b]{2}{*}{$-\left(G_{T}^{\circ}-H_{298}\right) / T$} & \multicolumn{2}{|c|}{ FORMATION FROM THE } & \multirow{2}{*}{ ELEMENTS } \\
\hline & & & & & $\Delta_{f^{H^{0}}}$ & $\Delta_{f} G^{0}$ & \\
\hline $\mathbf{K}$ & & & & & $\ldots \mathbf{k J} \cdot \mathbf{m o l}$ & -1 & \\
\hline 298.15 & 47.53 & 67.40 & 0.00 & 67.40 & -118.9 & $-95 \cdot 1$ & 16.66 \\
\hline 300 & 47.59 & 67.69 & 0.29 & 67.40 & -118.9 & -95.0 & 16.53 \\
\hline 400 & 50.29 & 81.77 & 12.47 & 69.30 & -118.1 & $-87 \cdot 1$ & 11.37 \\
\hline 500 & 52.62 & 93.24 & 20.27 & 72.98 & $-117 \cdot 3$ & $-79 \cdot 4$ & 8.30 \\
\hline 600 & 54.82 & 103.03 & 25.84 & 77.19 & -116.3 & -71.9 & 6.26 \\
\hline 700 & 56.94 & 111.64 & 30.14 & 81.51 & -115.2 & -64.6 & 4.82 \\
\hline 800 & 59.03 & 119.38 & 33.62 & 85.77 & -113.9 & -57.5 & 3.75 \\
\hline
\end{tabular}

\section{Melting $\mathbf{T}$}

780 K

$\Delta_{\text {fin }} \mathrm{H}^{\circ}$

$\mathbf{k J}$

H298-H8
Boiling T

$\Delta_{\text {vep }} \mathrm{H}^{\circ}$

Molar Vol.
K

$\mathbf{k J}$
$B=7.208 E-02$

$$
C=-1 \cdot 60 \mathrm{E}+05
$$
$A=-1 \cdot 148 \mathrm{E}+02$
$B=7.208 E-02$ 
$\mathrm{Cu}_{2}$ s: Orthorhombic crystals 298.15 to $376 \mathrm{~K}$; hexagonal crystals 376 to $710 \mathrm{~K}$; cubic crystals 710 to melting point $1403 \mathrm{~K}$. Reference state for sulfur is ideal $s_{2}$ gas at $p=1 \mathrm{bar}$.

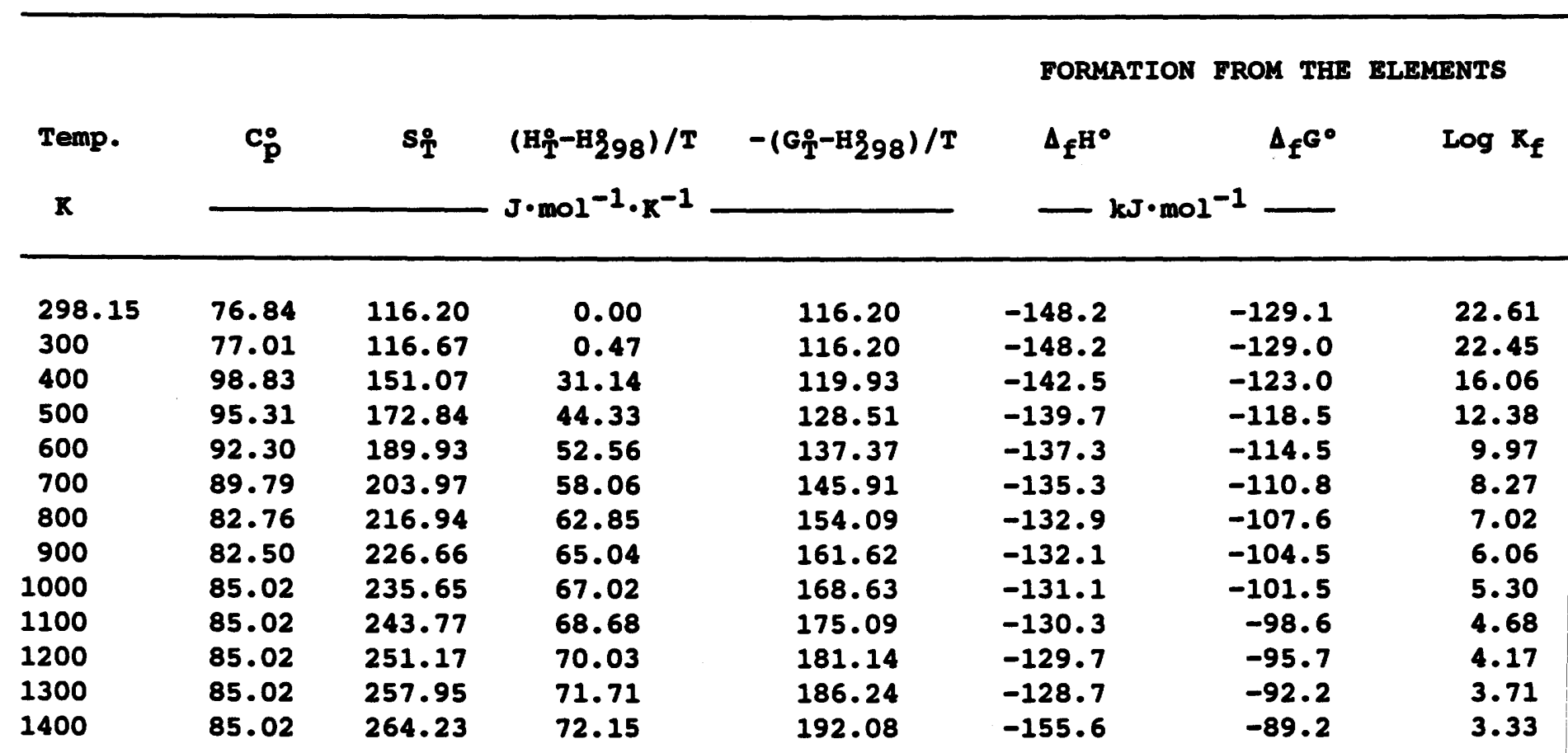

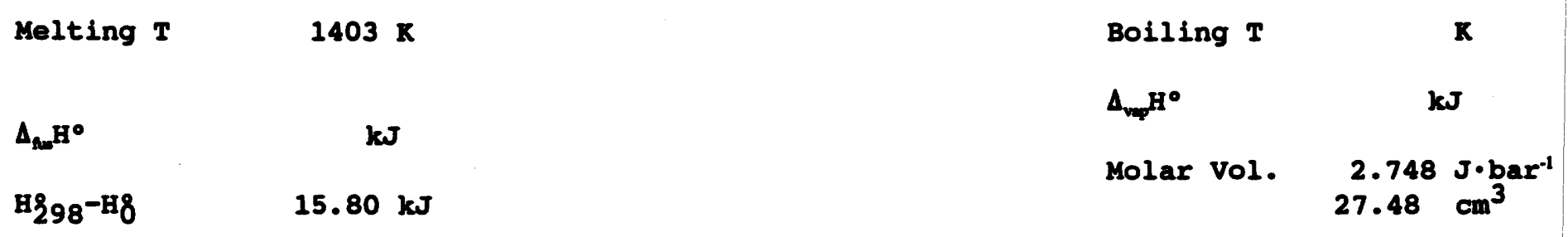


$\mathrm{CuFes}_{2}$ : Tetragonal crystals 298.15 to $820 \mathrm{~K}$; tetragonal crystals 820 to $930 \mathrm{~K}$; cubic crystals 930 to $1200 \mathrm{~K}$. Reference state for sulfur is ideal $s_{2}$ gas at $p=1$ bar. Above $820 \mathrm{~K}$, phase is called ISs (intermediate solid solution).

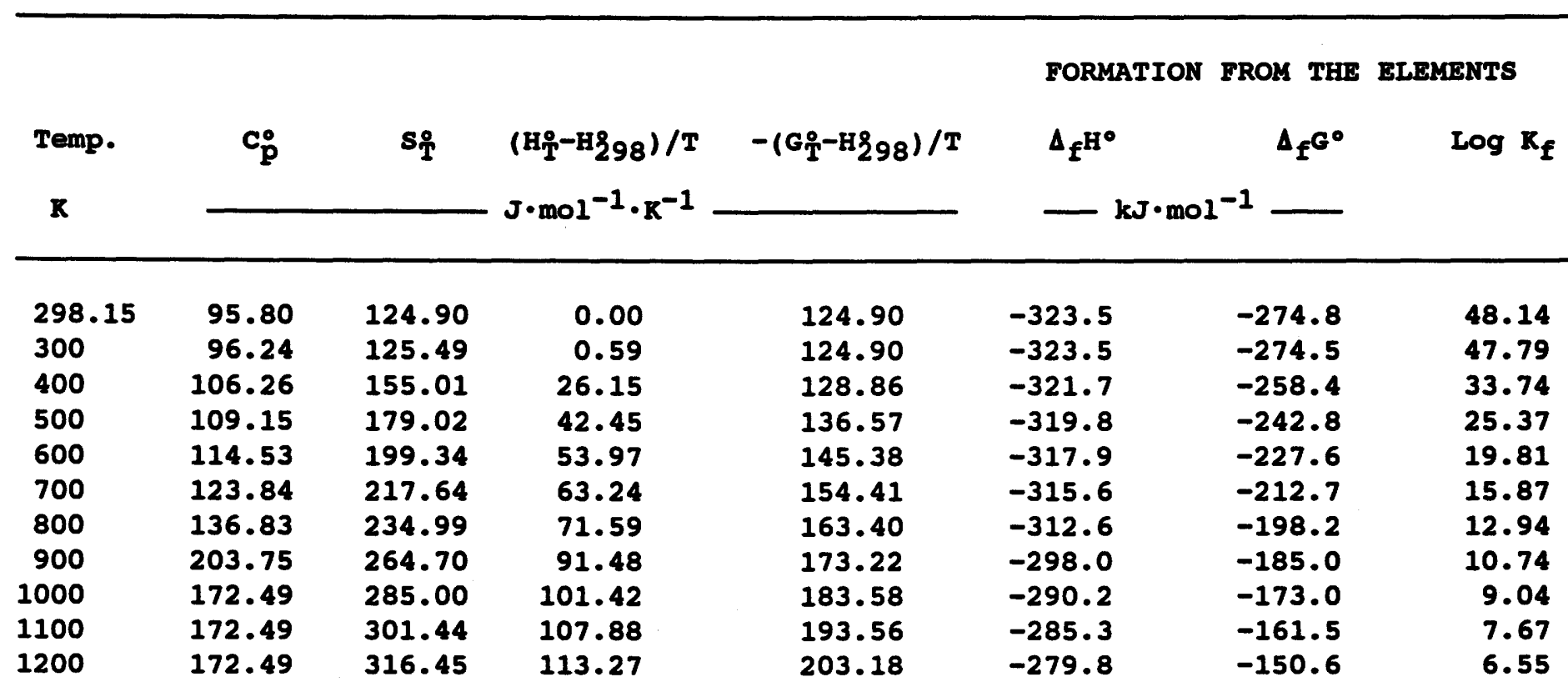

Melting T

$\Delta_{\text {fur }} \mathrm{H}^{\circ}$

H298-H8 $\mathbf{k J}$

$\mathbf{R}$

$18.34 \mathrm{~kJ}$
Boiling T

$\Delta_{\mathrm{vq}} \mathrm{H}^{\circ}$

Molar Vol.
$\mathbf{K}$

$\mathbf{k J}$
47.79

25.37

19.81

15.87

12.94

9.04

7.67 
$\mathrm{Cu}_{5} \mathrm{Fes}_{4}$ : Orthorhombic crystals 298.15 to $470 \mathrm{~K}$; cubic crystals 470 to $535 \mathrm{~K}$; cubic crystals 535 to $1200 \mathrm{~K}$. Reference state for sulfur is ideal $s_{2}$ gas at $p=1$ bar.

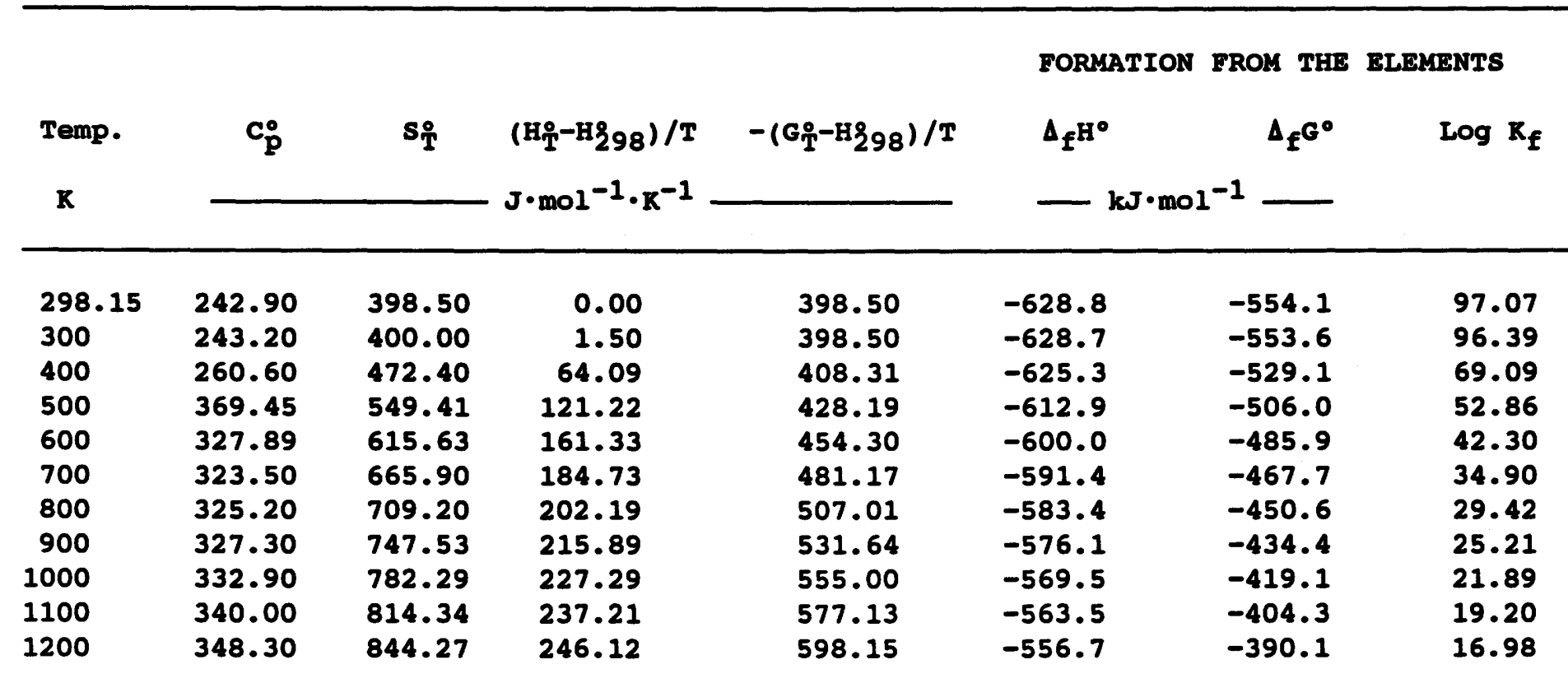

Melting $\mathbf{T}$

$\Delta_{\text {fue }} \mathrm{H}^{\circ}$

н298-н8 $\mathbf{k J}$

$\mathbf{K}$

$53.01 \mathrm{~kJ}$
Boiling T

$\Delta_{\mathrm{veq}} \mathrm{H}^{\circ}$

Molar Vol.
$\mathbf{K}$

$\mathbf{k J}$

$9.873 \mathrm{~J} \cdot \mathrm{bar}^{-1}$ $98.73 \mathrm{~cm}^{3}$
$A=-5 \cdot 714 E+02$
$B=1.542 \mathrm{E}-01$
$C=-2.625 E+06$ 
Fes: Hexagonal crystals (NiAs structure) 298.15 to a complex transition at $411 \mathrm{~K}$ and a Neel temperature at $590 \mathrm{~K}$; paramagnetic 590 to melting point at $1468 \mathrm{~K}$; liquid 1468 to 1800 K. Reference state for sulfur is ideal $s_{2}$ gas at $p=1$ bar.

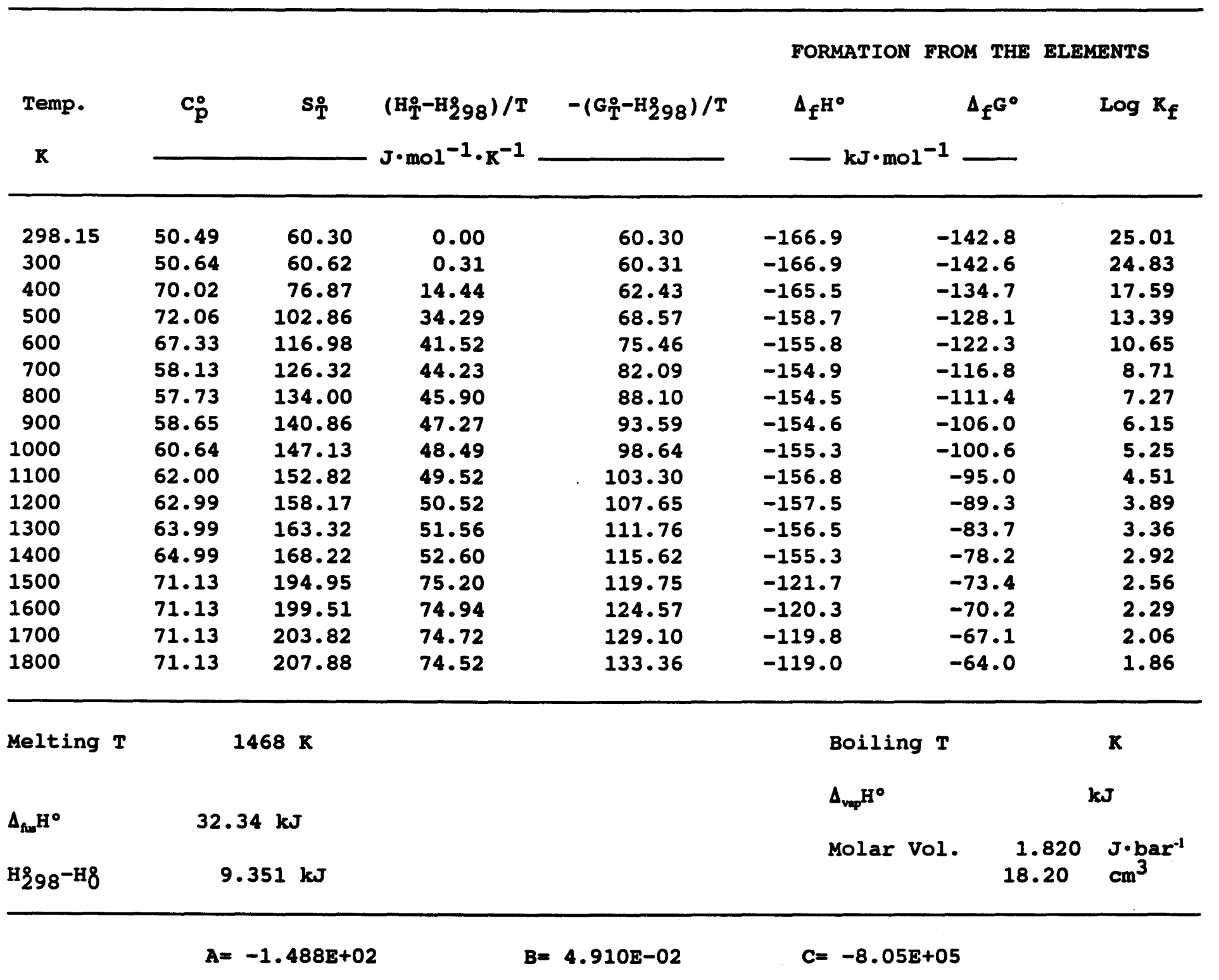


Fe.875s: Monoclinic crystals 298.15 to combined structural and magnetic transition at 590 $K_{i}$ hexagonal crystal (NiAs-type) 590 to $1000 \mathrm{~K}$. Reference state for sulfur is ideal $s_{2}$ gas at $p=1$ bar.

FORMATION FROM THE ELEMENTS

\begin{tabular}{|c|c|c|c|c|c|c|}
\hline Temp. & $\mathbf{c}_{\mathbf{p}}^{\circ}$ & $\mathbf{s}_{\mathbf{T}}$ & $\left(\mathrm{H}_{\mathrm{T}}^{\mathrm{T}}-\mathrm{H}_{298}\right) / \mathrm{T}$ & $-\left(G_{T}-H_{298}\right) / T$ & $\Delta_{f} \mathrm{H}^{\circ}$ & $\Delta_{f} G^{0}$ \\
\hline
\end{tabular}

\begin{tabular}{rrrrrrrr}
\hline 298.15 & 49.88 & 60.70 & 0.00 & 60.70 & -161.8 & -138.8 & 24.31 \\
300 & 50.01 & 60.70 & 0.31 & 60.39 & -161.7 & -138.5 & 24.12 \\
400 & 56.24 & 76.28 & 13.53 & 62.75 & -160.4 & -131.1 & 17.12 \\
500 & 63.56 & 89.57 & 22.76 & 66.81 & -158.6 & -124.0 & 12.95 \\
600 & 67.35 & 104.51 & 32.47 & 72.04 & -155.0 & -117.5 & 10.23 \\
700 & 65.30 & 114.53 & 37.37 & 77.16 & -153.0 & -111.2 & 8.30 \\
800 & 57.42 & 122.64 & 40.28 & 82.36 & -151.9 & -105.4 & 6.88 \\
900 & 57.19 & 129.39 & 42.17 & 87.22 & -151.6 & -99.6 & 5.78 \\
1000 & 58.38 & 135.46 & 43.71 & 91.75 & -151.9 & -93.8 & 4.90
\end{tabular}

Melting $T$

$\Delta_{\text {fun }} \mathbf{H}^{\circ}$

H298-H8 $\mathbf{k J}$

K

$$
9.21 \mathrm{~kJ}
$$

Boiling T

$\Delta_{\mathrm{vep}} \mathrm{H}^{\circ}$

$\mathbf{k J}$ 
Fes $_{2}$ : Cubic crystals 298.15 to $1000 \mathrm{~K}$. Reference state for sulfur is ideal $\mathrm{s}_{2}$ gas at $\mathrm{p}=$ 1 bar.

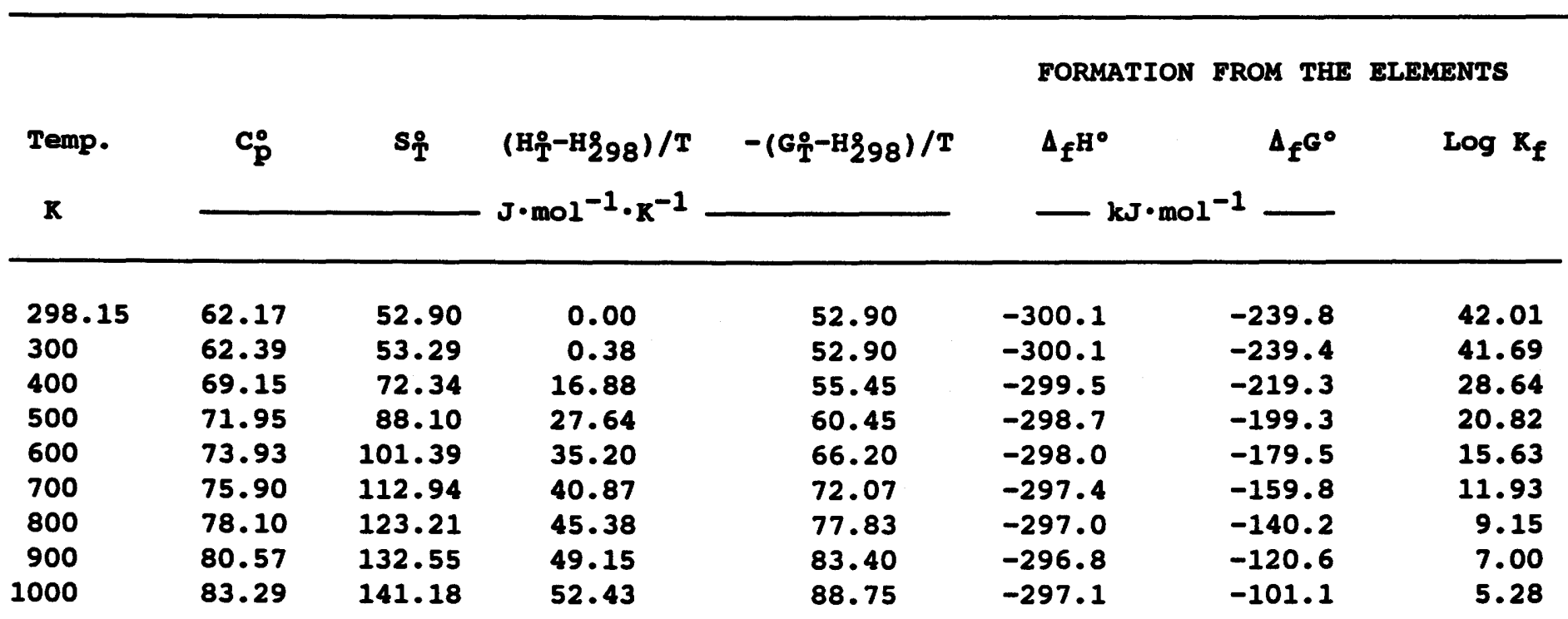

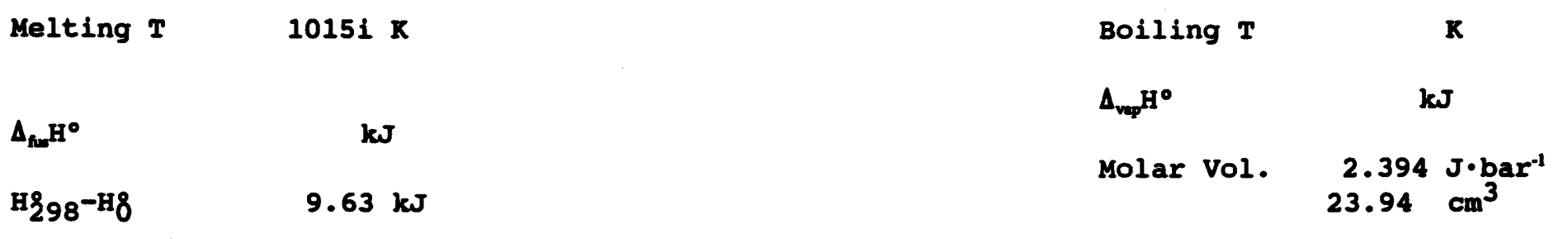

$A=-2.967 \mathrm{E}+02 \quad B=1.958 \mathrm{E}-01 \quad \mathrm{C}=-1.36 \mathrm{E}-05$


Fes $_{2}$ : Orthorhombic crystals 298.15 to $700 \mathrm{~K}$. Reference state for sulfur is ideal $s_{2}$ gas at $\mathbf{p}=1$ bar.

\begin{tabular}{|c|c|c|c|c|c|c|c|}
\hline \multirow{3}{*}{$\begin{array}{l}\text { Temp. } \\
\mathbf{R}\end{array}$} & \multirow{3}{*}{$\mathbf{c}_{\mathbf{p}}^{\circ}$} & \multirow{3}{*}{$\mathbf{s}_{\mathbf{T}}$} & \multirow{3}{*}{$\begin{array}{l}\left(\mathrm{H}_{\mathrm{T}}^{\circ}-\mathrm{H} 298\right) / \mathrm{T} \\
\mathrm{J} \cdot \mathrm{mol}^{-1} \cdot \mathrm{K}^{-1}\end{array}$} & \multirow{3}{*}{$-\left(G_{T}^{\circ}-H_{298}\right) / T$} & \multirow{3}{*}{\multicolumn{2}{|c|}{$\begin{array}{l}\text { FORMATION FROM THE } \\
\Delta_{f^{H^{\circ}}}\end{array}$}} & \multirow{3}{*}{$\begin{array}{l}\text { ELEMENTS } \\
\qquad \operatorname{LOg} k_{f}\end{array}$} \\
\hline & & & & & & & \\
\hline & & & & & & & \\
\hline 298.15 & 62.43 & 53.90 & 0.00 & 53.90 & -298.1 & -238.1 & 41.71 \\
\hline 300 & 62.60 & 54.29 & 0.39 & 53.90 & -298.1 & -237.7 & 41.39 \\
\hline 400 & 69.17 & 73.31 & 16.86 & 56.45 & -297.4 & -217.7 & 28.42 \\
\hline 500 & 72.44 & 89.13 & 27.68 & 61.45 & -296.6 & -197.8 & 20.66 \\
\hline 600 & 74.59 & 102.53 & 35.32 & 67.21 & -295.9 & -178.1 & 15.50 \\
\hline 700 & 77.00 & 114.20 & 41.10 & 73.11 & -295.2 & -158.5 & 11.83 \\
\hline
\end{tabular}

Melting $\mathbf{T}$ 
$\mathrm{H}_{2} \mathrm{~S}$ : Ideal gas at $\mathrm{p}=1 \mathrm{bar}, 298.15$ to $1800 \mathrm{~K}$. Reference state for sulfur is ideal $\mathrm{s}_{2} \mathrm{gas}$ at $p=1$ bar.

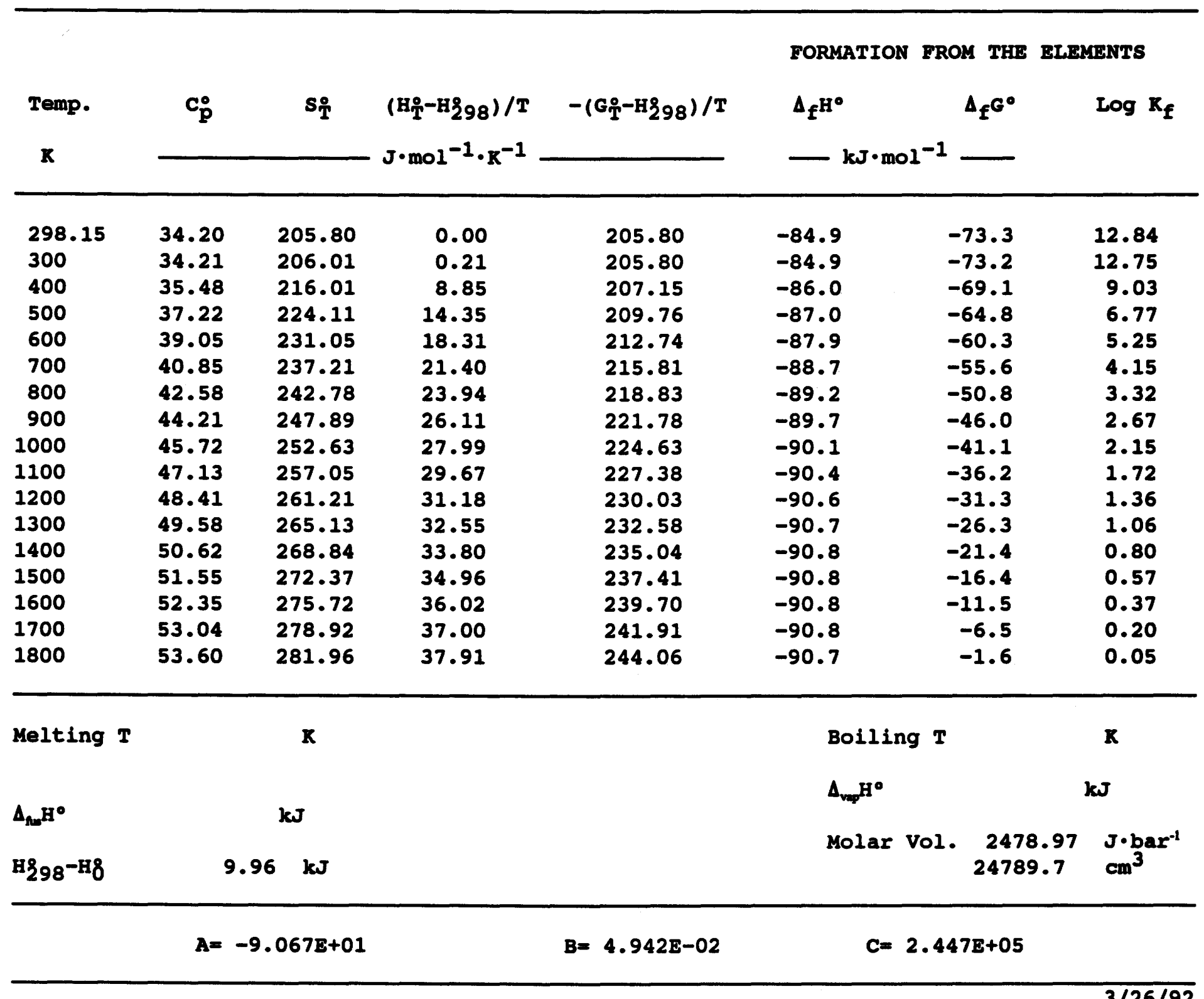


Mns: Cubic crystals 298.15 to $1800 \mathrm{~K}$. Reference state for sulfur is ideal $s_{2}$ gas at $p=1$ bar.

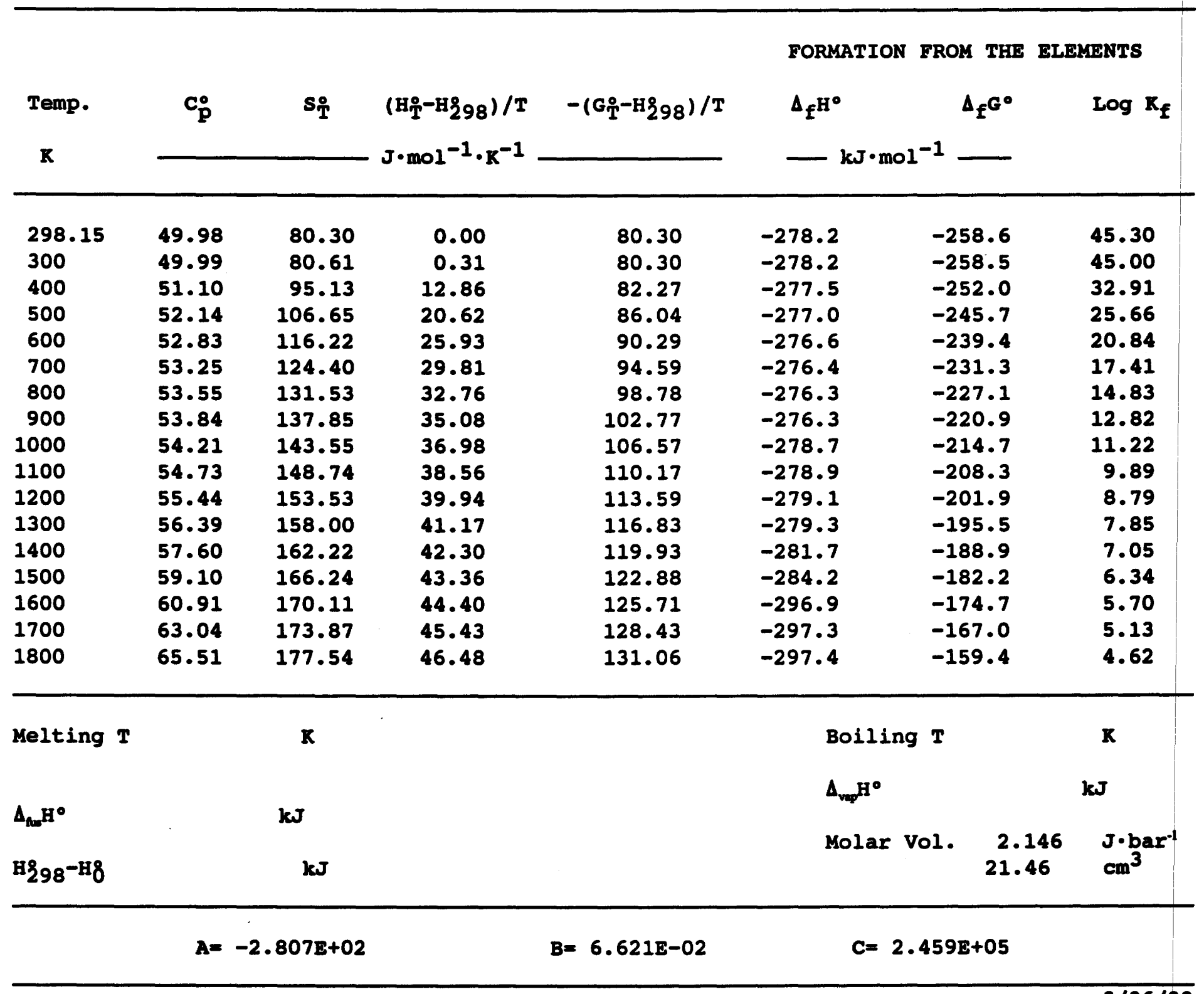


$\mathrm{MoS}_{2}$ : Hexagonal crystals 298.15 to $1200 \mathrm{~K}$. Reference state for sulfur is ideal $\mathrm{s}_{2}$ gas at $p=1$ bar.

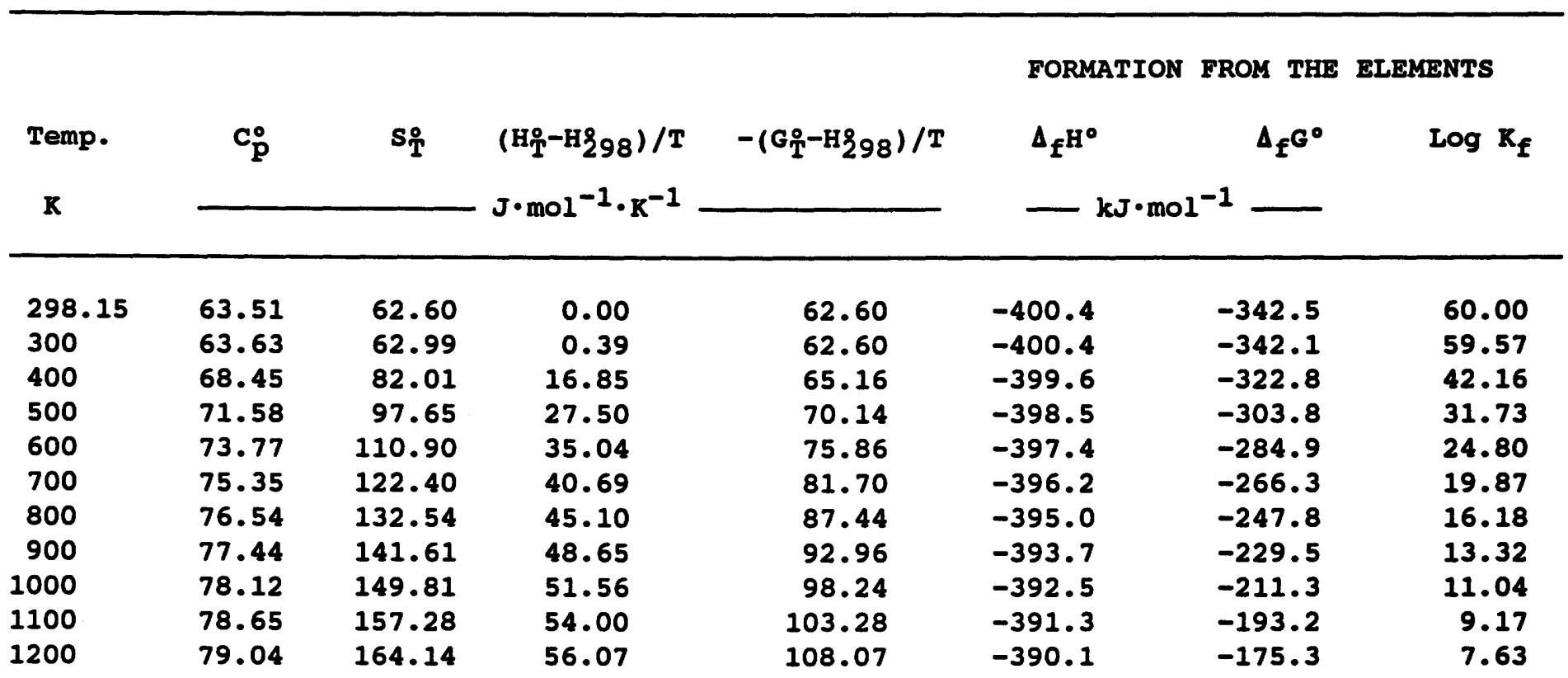

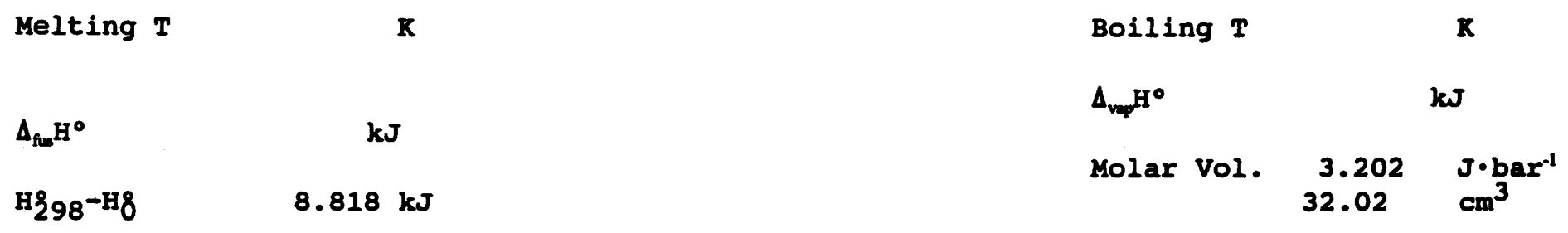
$A=-3.933 E+02$
$B=1.822 \mathrm{E}-01$
$C=-3 \cdot 21 E+05$ 
Nis: Rhombohedral crystals 298.15 to $623 \mathrm{~K}$ i hexagonal crystals 623 to melting point at $1066 \mathrm{~K}$. Reference state for sulfur is ideal $s_{2}$ gas at $p=1$ bar.

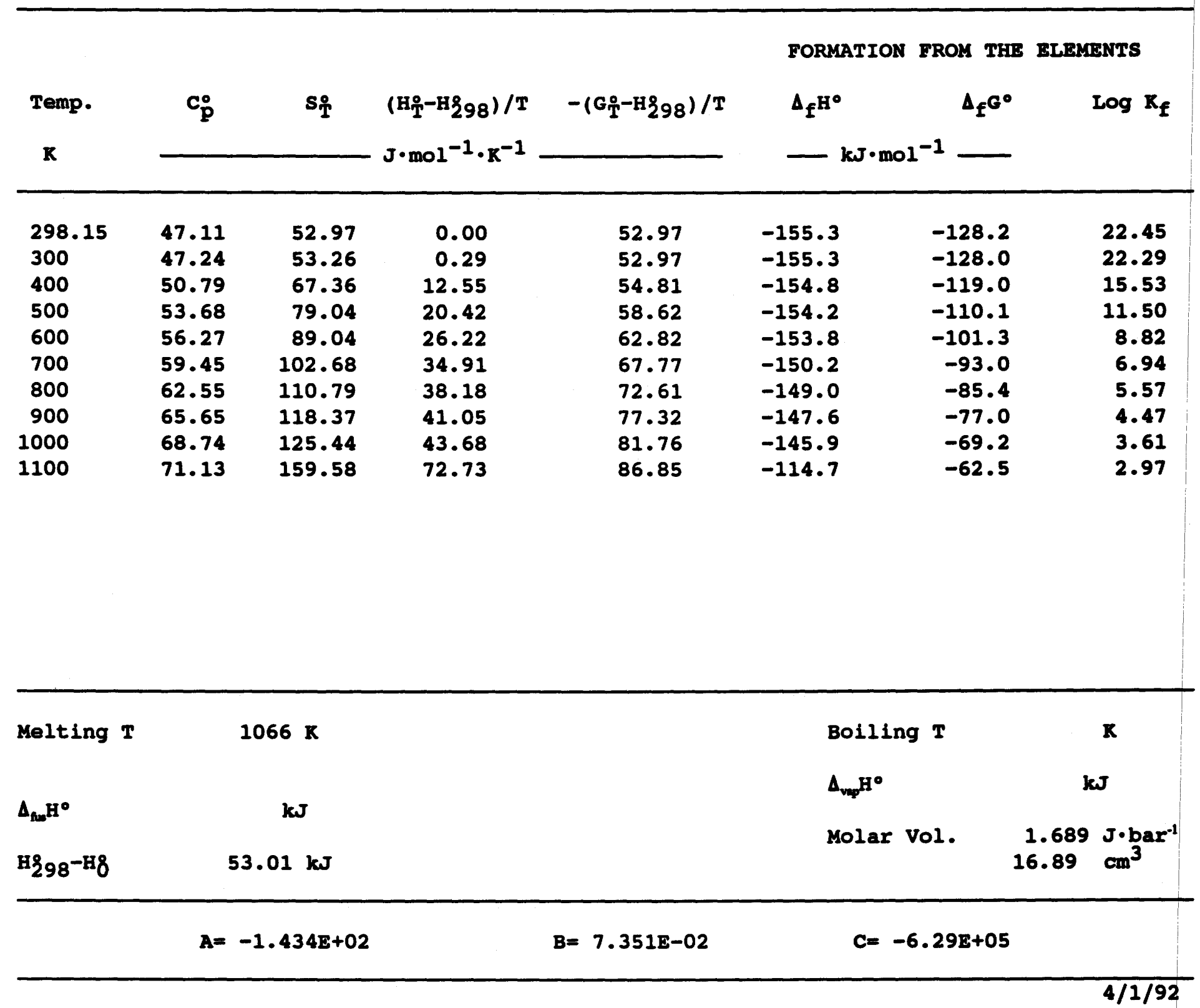


$\mathrm{Ni}_{3} \mathrm{~S}_{2}$ : Hexagonal crystals 298.15 to $834 \mathrm{~K}$; cubic crystals $834 \mathrm{~K}$ to incongruent melting point $1064 \mathrm{~K}$; liquid 1064 to $1400 \mathrm{~K}$. Reference state for sulfur is ideal $\mathrm{s}_{2}$ gas at $p=1$ bar.

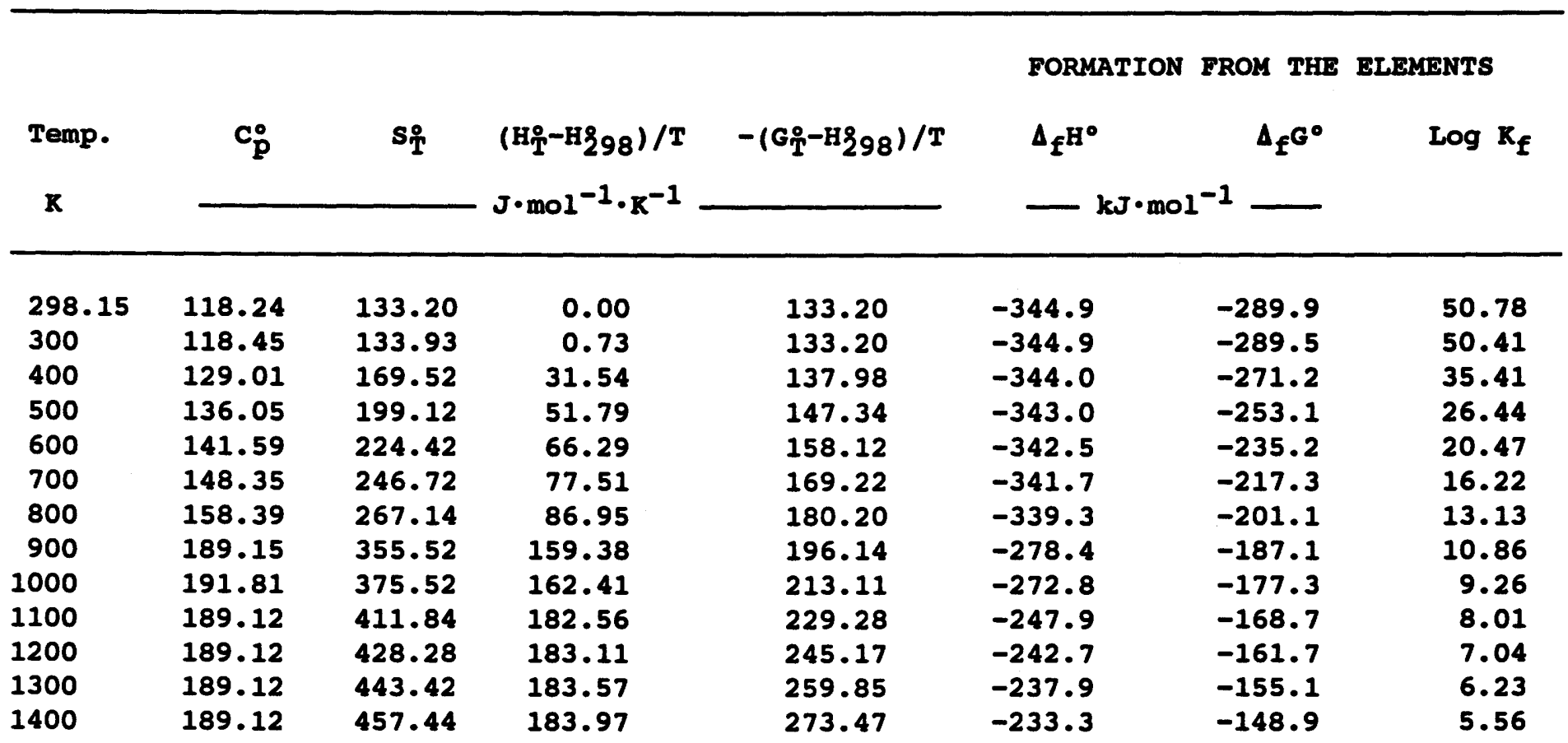

Melting $\mathrm{T} \quad 1064 \mathrm{~K}$

$\Delta_{\text {tur }} \mathrm{H}^{\circ}$

$19.66 \mathrm{~kJ}$

H298-H8
Boiling $T$

$\Delta_{\mathrm{veq}} \mathrm{H}^{\circ}$

Molar Vol.
$\mathbf{K}$

kJ

$4.095 \mathrm{~J} \cdot \mathrm{bar}^{-1}$ $40.95 \mathrm{~cm}^{3}$
$A=-2 \cdot 815 E+02$
$B=1.016 \mathrm{E}-01$
$C=-3.71 E+06$ 
Pbs: Cubic crystals 298.15 to $900 \mathrm{~K}$. Reference state for sulfur is ideal $\mathrm{s}_{2} \mathrm{gas}$ at $\mathrm{p}=1$ bar.

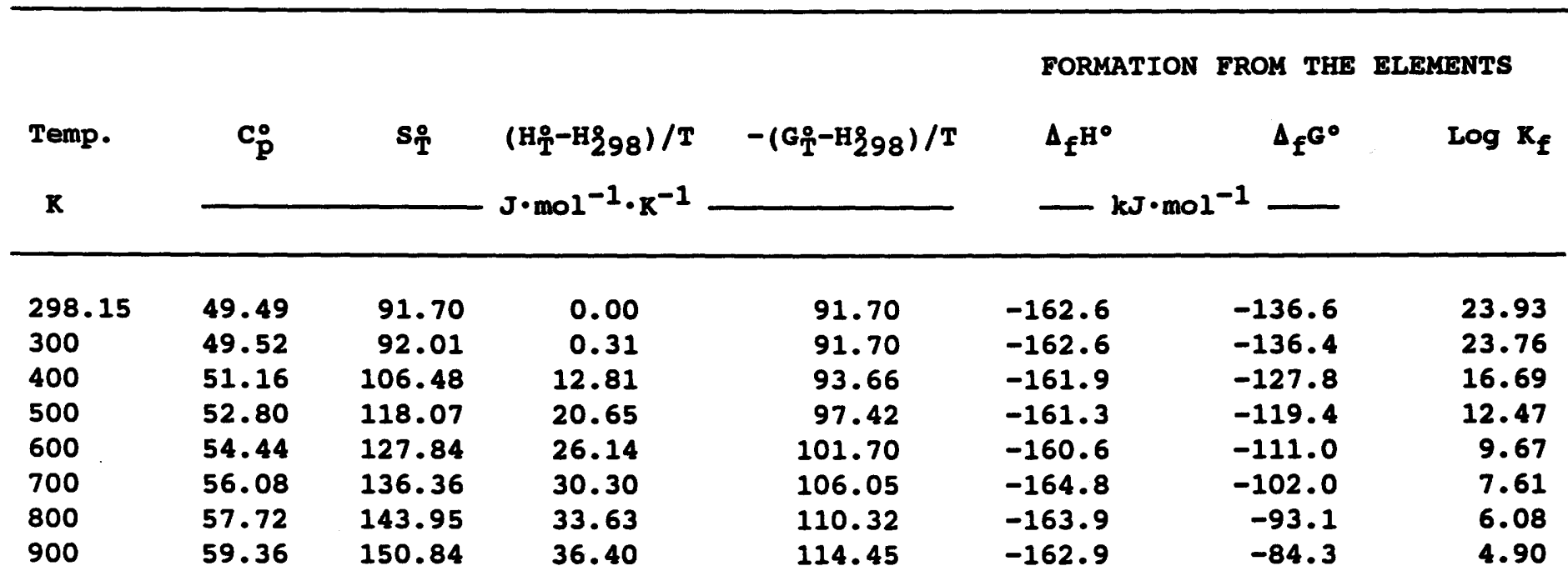

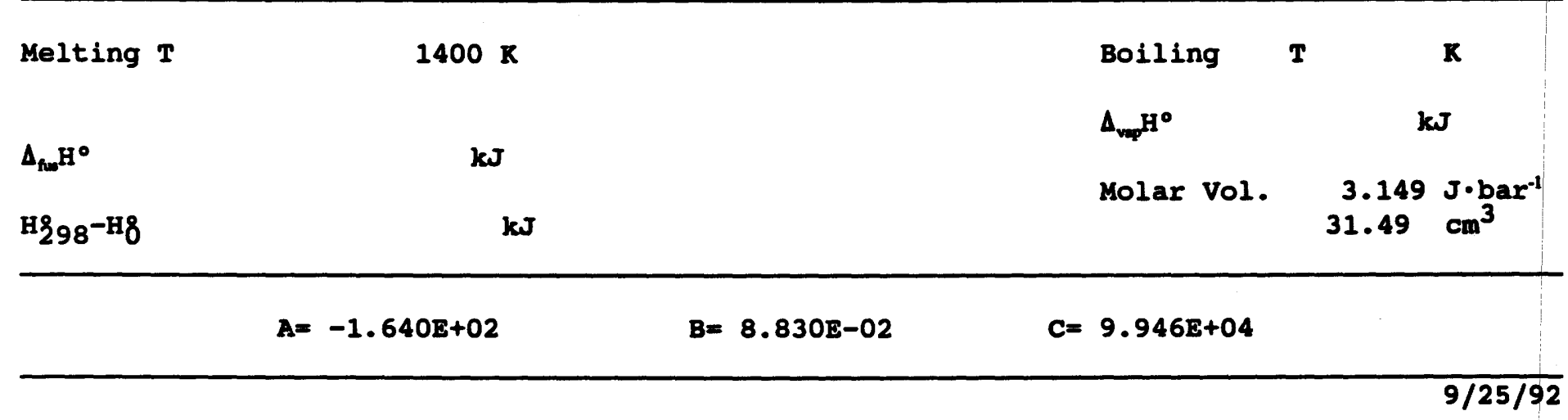


$\mathrm{Sb}_{2} \mathrm{~s}_{3}$ : Orthorhombic crystals 298.15 to $900 \mathrm{~K}$. Reference state for sulfur is ideal $\mathrm{s}_{2}$ gas at $p=1$ bar.

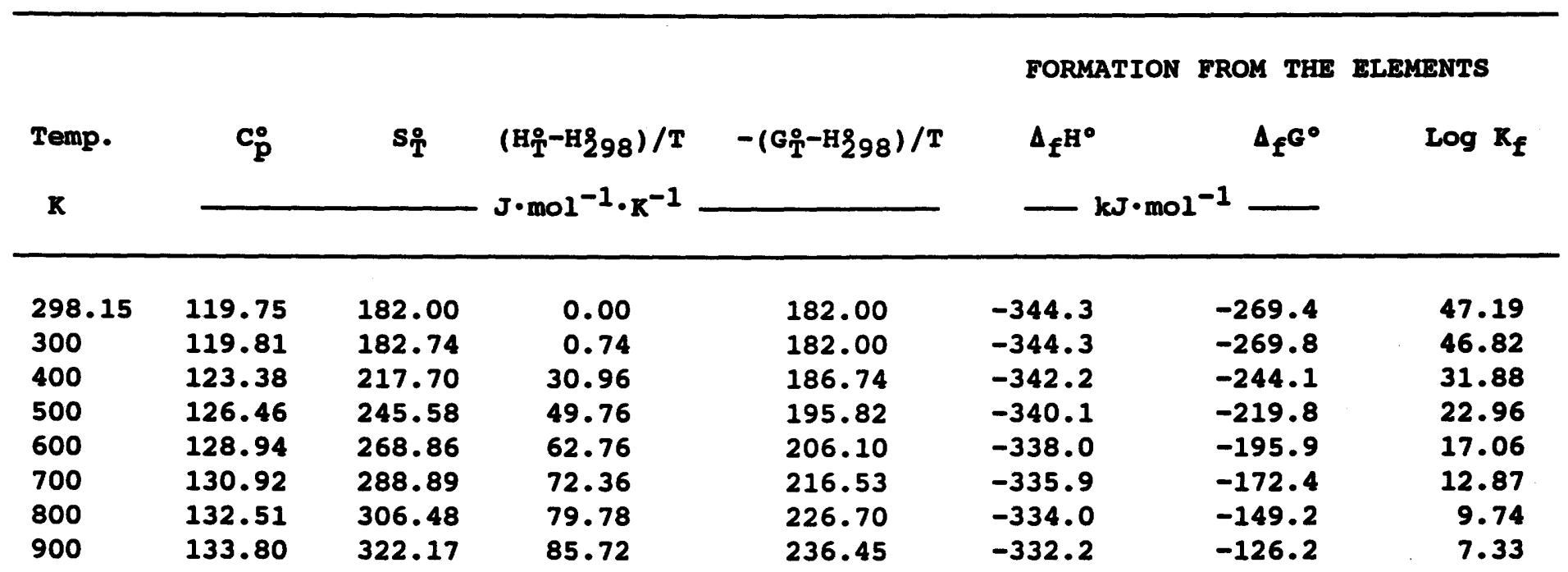

Melting T

$\Delta_{\text {fun }} \mathbf{H}^{\circ}$

$\mathrm{H}_{298^{-\mathrm{H}}}$ $\mathbf{k J}$

\section{$829 \mathrm{~K}$}

$\mathbf{k J}$
Boiling $\mathbf{T}$

$\Delta_{\mathrm{vep}} \mathrm{H}^{\circ}$

Molar vol.
$\mathbf{R}$

$\mathbf{k J}$ $73.41 \mathrm{~cm}^{3}$
$A=-3.338 \mathrm{E}+02$
$B=2.315 \mathrm{E}-01$
$C=-4.19 \mathrm{E}+05$ 
Sns: Orthorhombic crystals 298.15 to $875 \mathrm{~K}$; cubic crystals 875 to melting point $1153 \mathrm{~K}$. Iiquid 1153 to $1300 \mathrm{~K}$. Reference state for sulfur is ideal $s_{2}$ gas at $p=1$ bar.

\begin{tabular}{|c|c|c|c|c|c|c|c|}
\hline \multirow{2}{*}{$\begin{array}{l}\text { Temp. } \\
\mathbf{K}\end{array}$} & \multirow[t]{2}{*}{$\mathbf{c}_{\mathbf{p}}^{\circ}$} & \multirow[t]{2}{*}{$\mathbf{s}_{\mathbf{T}}^{\circ}$} & \multirow{2}{*}{$\begin{array}{l}\left(H_{\mathrm{T}}-\mathrm{H}_{298}\right) / \mathrm{T} \\
\mathrm{J} \cdot \mathrm{mol}^{-1} \cdot \mathrm{K}^{-1}\end{array}$} & \multirow[t]{2}{*}{$-\left(G_{T}^{\circ}-H_{298}\right) / T$} & \multirow{2}{*}{\multicolumn{2}{|c|}{$\begin{array}{l}\text { FORMATION FROM THE } \\
\Delta_{f} \mathrm{H}^{\circ} \\
\Delta_{f} \mathrm{G}^{\circ}\end{array}$}} & \multirow[t]{2}{*}{$\begin{array}{l}\text { ELEMENTS } \\
\qquad \log k_{f}\end{array}$} \\
\hline & & & & & & & \\
\hline 298.15 & 49.25 & 77.00 & 0.00 & 77.00 & -170.8 & -144.5 & 25.31 \\
\hline 300 & 49.32 & 77.30 & 0.30 & 77.00 & -170.8 & -144.3 & 25.13 \\
\hline 400 & 50.89 & 91.76 & 12.81 & 78.96 & -170.2 & -135.6 & 17.71 \\
\hline 500 & 52.03 & 103.22 & 20.52 & 82.70 & -169.8 & -127.0 & 13.26 \\
\hline 600 & 54.52 & 112.91 & 25.96 & 86.95 & -176.4 & -117.1 & 10.19 \\
\hline 700 & 58.41 & 121.50 & 30.30 & 91.20 & -175.4 & -107.3 & 8.00 \\
\hline 800 & 63.50 & 129.71 & 34.12 & 95.59 & -173.9 & -97.7 & 6.38 \\
\hline 900 & 54.04 & 137.47 & 37.81 & 99.66 & -171.8 & -88.1 & 5.11 \\
\hline 1000 & 57.07 & 143.32 & 39.59 & 103.73 & -170.9 & -78.9 & 4.12 \\
\hline 1100 & 60.11 & 148.90 & 41.32 & 107.58 & -169.7 & -69.7 & 3.31 \\
\hline 1200 & 75.61 & 181.98 & 69.64 & 112.34 & -136.3 & -62.0 & 2.70 \\
\hline 1300 & 75.61 & 188.03 & 70.10 & 117.93 & -133.5 & -55.9 & 2.25 \\
\hline
\end{tabular}

Melting T

$\Delta_{\mathrm{fu}} \mathrm{H}^{\circ}$

$31.59 \mathrm{~kJ}$

H298-H8
$1153 \mathrm{~K}$

kJ
Boiling T

$\Delta_{\mathrm{vq}} \mathrm{H}^{\mathrm{O}}$

Molar Vol. 2.901 29.01
$\mathbf{R}$

$\mathbf{k J}$

$\mathrm{J} \cdot \mathrm{bar}^{-1}$
$\mathrm{~cm}^{3}$ 
$\mathrm{SnS}_{2}$ : Hexagonal crystals 298.15 to $1000 \mathrm{~K}$. Reference state for sulfur 18 ideal $\mathrm{S}_{2}$ gas at $p=1$ bar.

\begin{tabular}{|c|c|c|c|c|c|c|c|}
\hline \multirow[b]{2}{*}{ Temp. } & \multirow[b]{2}{*}{$\mathbf{c}_{\mathbf{p}}^{\circ}$} & \multirow[b]{2}{*}{$\mathbf{S}_{\mathrm{T}}^{\circ}$} & \multirow{3}{*}{$\begin{array}{l}\left(\mathrm{H}_{\mathrm{T}}-\mathrm{H}_{298}\right) / \mathrm{T} \\
\mathrm{J} \cdot \mathrm{mol}^{-1} \cdot \mathrm{K}^{-1}\end{array}$} & \multirow[b]{2}{*}{$-\left(G_{T}^{\circ}-H_{298}\right) / T$} & FORMATION & FROY THE & ELEYENTS \\
\hline & & & & & $\Delta_{f^{H^{\circ}}}$ & $\Delta_{f} G^{0}$ & $\log R_{f}$ \\
\hline $\mathbf{K}$ & & & & & $\ldots \mathrm{kJ} \cdot \mathrm{mol}$ & $L^{-1}$ & \\
\hline 298.15 & 70.12 & 87.50 & 0.00 & 87.50 & -278.4 & -221.2 & 38.75 \\
\hline 300 & 70.17 & 87.93 & 0.43 & 87.50 & -278.4 & -220.8 & 38.45 \\
\hline 400 & 72.05 & 108.41 & 18.13 & 90.28 & -277.4 & -201.8 & 26.35 \\
\hline 500 & 73.52 & 124.64 & 29.06 & 95.58 & -276.6 & $-183 \cdot 0$ & 19.12 \\
\hline 600 & 75.19 & 138.19 & 36.60 & 101.59 & -282.8 & -163.1 & 14.19 \\
\hline 700 & 77.13 & 149.92 & 42.25 & 107.67 & -281.6 & -143.2 & 10.69 \\
\hline 800 & 79.33 & 160.36 & 46.75 & 113.61 & -280.2 & -123.5 & 8.06 \\
\hline 900 & 81.74 & 169.84 & 50.50 & 119.34 & -278.6 & -104.0 & 6.04 \\
\hline 1000 & 84.33 & 178.59 & 53.75 & 124.84 & -276.9 & -84.7 & 4.42 \\
\hline
\end{tabular}

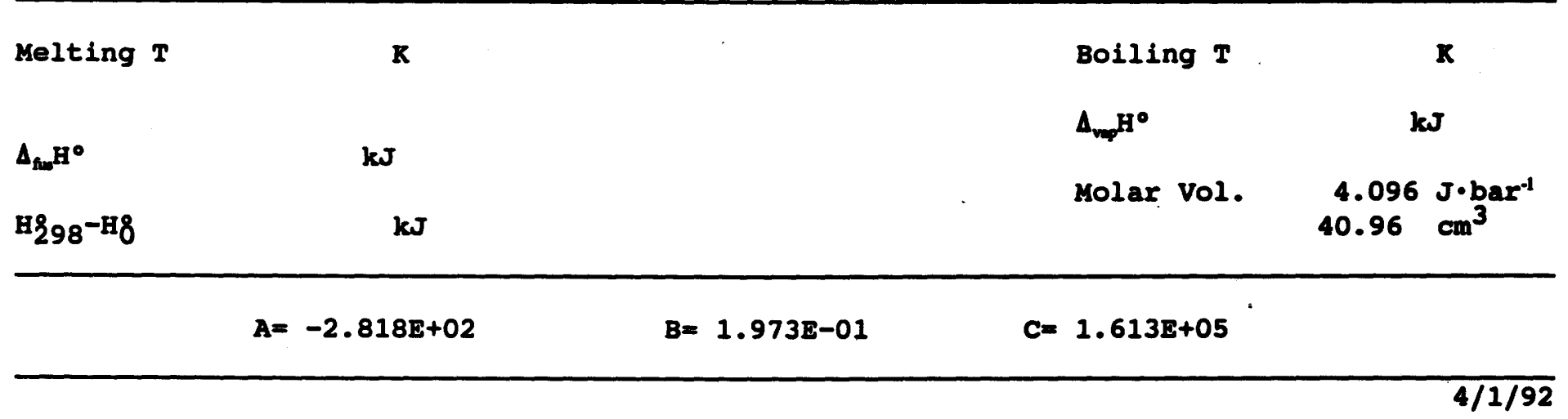


WS $_{2}$ : Hexagonal crystals 298.15 to $1500 \mathrm{~K}$. Reference state for sulfur is ideal $s_{2}$ gas at $p$ $=1$ bar.

\begin{tabular}{|c|c|c|c|c|c|c|c|c|}
\hline \multirow{2}{*}{$\begin{array}{l}\text { Temp. } \\
\mathbf{K}\end{array}$} & \multirow[b]{2}{*}{$\mathbf{c}_{\mathbf{p}}^{\circ}$} & \multirow[b]{2}{*}{$\mathbf{s}_{\mathbf{T}}^{\circ}$} & \multirow{2}{*}{$\begin{array}{l}\left(\mathrm{H}_{\mathrm{T}}^{\circ}-\mathrm{H}_{298}\right) / \mathrm{T} \\
\mathrm{J} \cdot \mathrm{mol}^{-1} \cdot \mathrm{K}^{-1}\end{array}$} & \multirow[b]{2}{*}{$-\left(G_{T}^{\circ}-H_{298}\right) / T$} & \multicolumn{4}{|c|}{ FORMATION FROM THE ELEMENTS } \\
\hline & & & & & $\Delta_{\mathbf{f}} \mathrm{H}^{0}$ & \multicolumn{2}{|c|}{$\Delta_{\mathcal{E}} G^{\circ}$} & $\log K_{f}$ \\
\hline 298.15 & 63.83 & 67.80 & 0.00 & 67.80 & -370.2 & \multicolumn{2}{|c|}{-312.7} & 54.77 \\
\hline 300 & 63.99 & 68.20 & 0.39 & 67.80 & -370.2 & \multicolumn{2}{|c|}{-312.3} & 54.37 \\
\hline 400 & 69.67 & 87.50 & 17.10 & 70.39 & -369.3 & \multicolumn{2}{|c|}{-293.1} & 38.28 \\
\hline 500 & 72.41 & 103.37 & 27.92 & 75.45 & -368.1 & \multicolumn{2}{|c|}{-274.2} & 28.65 \\
\hline 600 & 74.01 & 116.72 & 35.48 & 81.25 & -366.9 & \multicolumn{2}{|c|}{-255.6} & 22.25 \\
\hline 700 & 75.09 & 128.22 & 41.06 & 87.15 & -365.6 & \multicolumn{2}{|c|}{-237.1} & 17.69 \\
\hline 800 & 75.90 & 138.30 & 45.37 & 92.93 & -364.4 & \multicolumn{2}{|c|}{-218.8} & 14.29 \\
\hline 900 & 76.57 & 147.28 & 48.80 & 98.48 & -363.1 & \multicolumn{2}{|c|}{-200.7} & 11.65 \\
\hline 1000 & 77.16 & 155.38 & 51.61 & 103.77 & -361.9 & \multicolumn{2}{|c|}{-182.7} & 9.54 \\
\hline 1100 & 77.71 & 162.76 & 53.95 & 108.80 & -360.6 & \multicolumn{2}{|c|}{-164.9} & 7.83 \\
\hline 1200 & 78.24 & 169.54 & 55.96 & 113.58 & -359.4 & \multicolumn{2}{|c|}{-147.1} & 6.40 \\
\hline 1300 & 78.76 & 175.82 & 57.69 & 118.13 & -358.2 & \multicolumn{2}{|c|}{-129.5} & 5.20 \\
\hline 1400 & 79.29 & 181.68 & 59.21 & 122.47 & -357.0 & \multicolumn{2}{|c|}{-111.9} & 4.18 \\
\hline 1500 & 79.83 & 187.17 & 60.57 & 126.60 & -355.8 & \multicolumn{2}{|c|}{-94.5} & 3.29 \\
\hline \multirow[t]{2}{*}{ Melting $T$} & \multicolumn{2}{|r|}{$\mathbf{K}$} & & & \multicolumn{2}{|c|}{ Boiling $T$} & 239.1 & $\mathbf{K}$ \\
\hline & & & & & \multicolumn{3}{|c|}{$\begin{array}{ll}\Delta_{\text {vpp }} H^{\circ} & 20.41\end{array}$} & $\mathbf{k J}$ \\
\hline \multirow[t]{2}{*}{$\Delta_{\text {hum }} \mathrm{H}^{\circ}$} & \multicolumn{2}{|r|}{$\mathbf{k J}$} & & & \multirow{3}{*}{\multicolumn{2}{|c|}{ Molar Vol. }} & & \\
\hline & \multirow{2}{*}{\multicolumn{2}{|c|}{$11.01 \mathrm{~kJ}$}} & & & & & 3.20 & $J \cdot \mathbf{b a r}^{-1}$ \\
\hline \multirow[t]{2}{*}{ H298-H8 } & & & & $=$ & & & 32.07 & cm3 \\
\hline & $A=$ & $610 \mathrm{E}+02$ & $\mathrm{~B}=$ & $1.782 \mathrm{E}-01$ & $C=-4$ & $E+05$ & & \\
\hline
\end{tabular}


Zns: Cubic crystals to $1300 \mathrm{~K}$. Wurtzite is the stable form of zns above $1300 \mathrm{~K}$. Reference state for sulfur is ideal $s_{2}$ gas at $p=1$ bar.

\begin{tabular}{|c|c|c|c|c|c|c|c|}
\hline \multirow[b]{2}{*}{ Temp. } & \multirow[b]{2}{*}{$\mathbf{c}_{\mathbf{p}}^{\circ}$} & \multirow[b]{2}{*}{ s亩 } & \multirow{3}{*}{$\begin{array}{l}\left(\mathrm{H}_{\mathrm{T}}-\mathrm{H}_{298}\right) / \mathrm{T} \\
\mathrm{J} \cdot \mathrm{mol}^{-1} \cdot \mathrm{K}^{-1}\end{array}$} & \multirow[b]{2}{*}{$-\left(G_{T}^{\circ}-H_{298}\right) / T$} & \multicolumn{2}{|c|}{ FORMATION FROM THE } & \multirow{2}{*}{$\begin{array}{l}\text { ELEMISNTS } \\
\text { Log } \mathrm{K}_{\mathrm{f}}\end{array}$} \\
\hline & & & & & $\Delta_{f^{H^{\circ}}}$ & $\Delta_{\mathbf{f}} \mathbf{G}^{0}$ & \\
\hline $\mathbf{K}$ & & & & & $\ldots \mathrm{kJ} \cdot \mathrm{mol}$ & -1 & \\
\hline 298.15 & 45.76 & 58.70 & 0.00 & 58.70 & -268.4 & -239.5 & 41.95 \\
\hline 300 & 45.82 & 58.98 & 0.28 & 58.70 & -268.4 & -239.3 & 41.66 \\
\hline 400 & 48.30 & 72.53 & 12.00 & 60.53 & -267.9 & -229.7 & 29.99 \\
\hline 500 & 49.93 & 83.50 & 19.43 & 64.06 & -267.4 & -220.2 & 23.00 \\
\hline 600 & 51.12 & 92.71 & 24.62 & 68.09 & -266.9 & -210.7 & 18.35 \\
\hline 700 & 52.04 & 100.66 & 28.47 & 72.19 & -273.8 & -201.3 & 15.02 \\
\hline 800 & 52.79 & 107.66 & 31.47 & 76.19 & -273.5 & -191.0 & 12.47 \\
\hline 900 & $53 \cdot 42$ & 113.92 & 33.87 & 80.04 & -273.2 & -180.7 & 10.49 \\
\hline 1000 & 53.96 & 119.57 & 35.85 & 83.72 & -272.8 & -170.4 & 8.90 \\
\hline 1100 & 54.43 & 124.74 & 37.52 & 87.22 & -272.4 & -160.2 & 7.61 \\
\hline 1200 & 54.85 & 129.49 & 38.95 & 90.54 & -387.1 & -148.1 & 6.45 \\
\hline 1300 & 55.23 & 133.90 & 40.19 & 93.71 & -385.6 & -128.2 & 5.15 \\
\hline
\end{tabular}

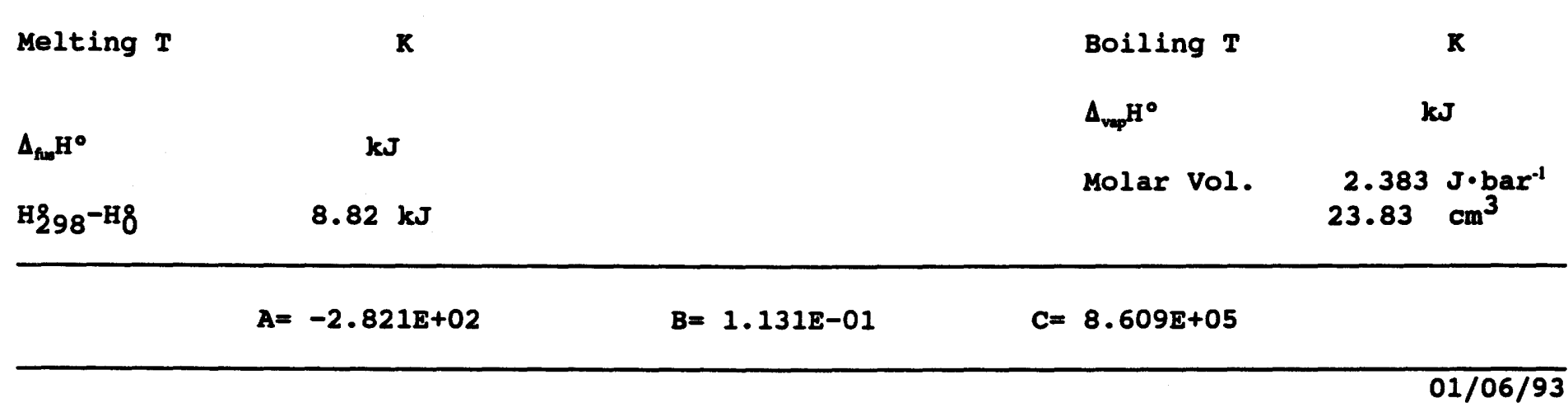


Zns: Hexagonal crystals 298.15 to $1300 \mathrm{~K}$. Reference state for sulfur is ideal $\mathrm{s}_{2}$ gas at $p$ $=1$ bar.

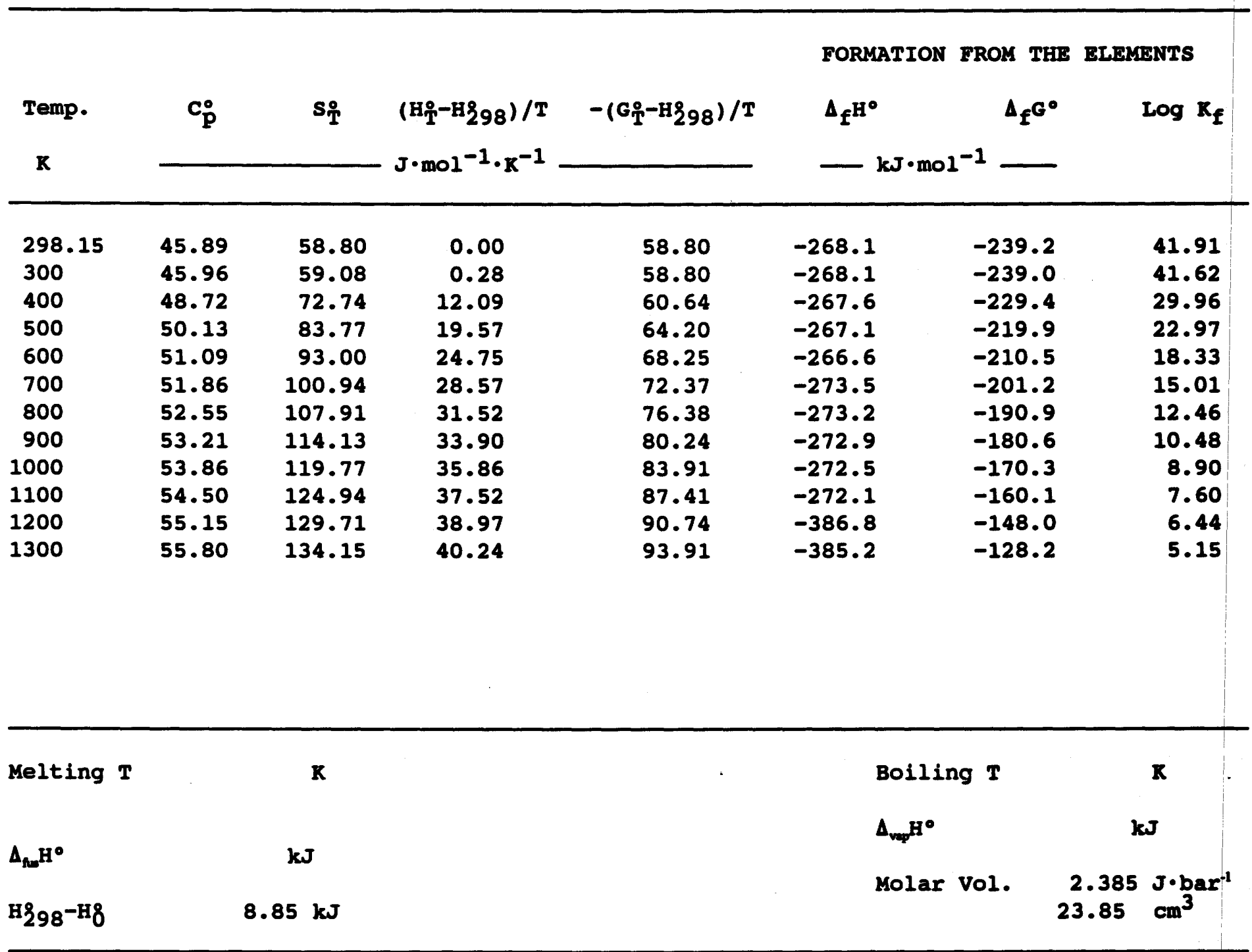


$\mathrm{FeSb}_{2} \mathrm{~s}_{4}$ : Orthorhombic crystals 298.15 to $836 \mathrm{~K}$. Reference state for sulfur is ideal $\mathrm{s}_{2}$ gas at $p=1$ bar.

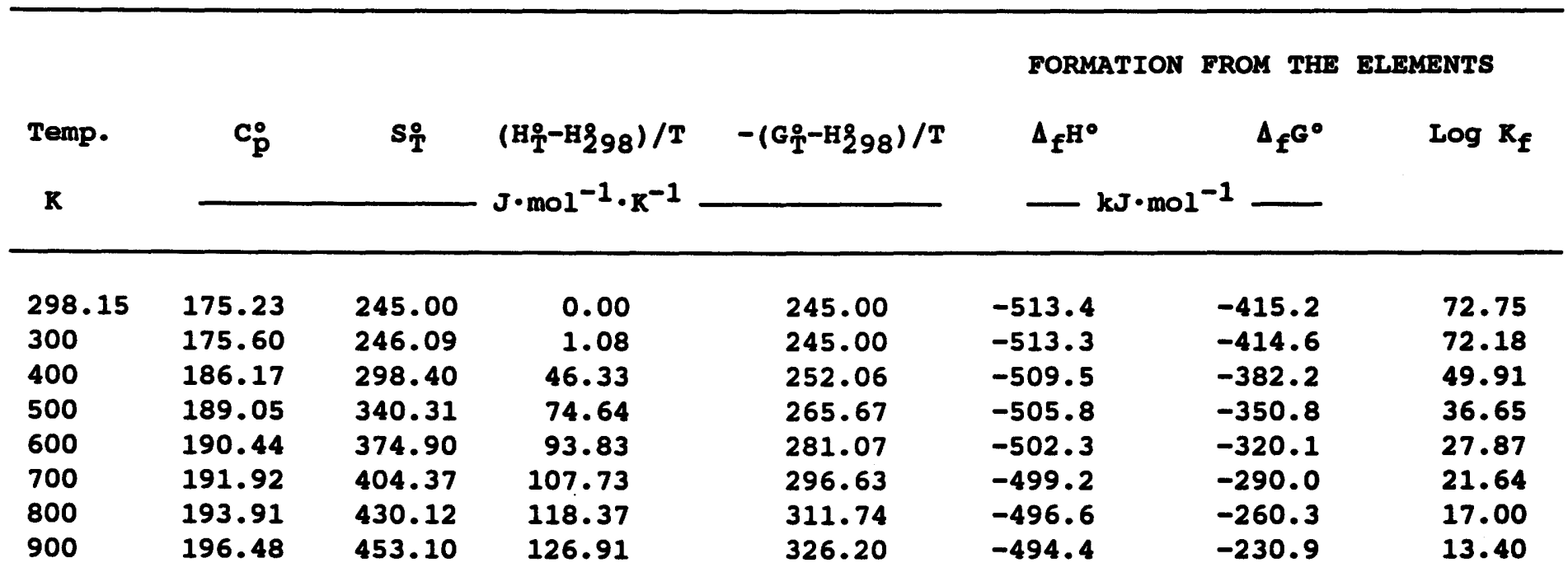

\begin{tabular}{|c|c|c|c|c|}
\hline Melting $T$ & $\mathbf{K}$ & Boiling $\mathbf{T}$ & & $\mathbf{K}$ \\
\hline & & $\Delta_{\mathrm{vep}} \mathrm{H}^{\circ}$ & & $\mathbf{k J}$ \\
\hline $\begin{array}{l}\Delta_{\text {fus }} H^{\circ} \\
H_{298^{-H} 8}\end{array}$ & kJ & Molar vol. & $\begin{array}{l}9.222 \\
92.22\end{array}$ & $\begin{array}{l}J \cdot \text { bar }^{-1} \\
\mathrm{~cm}^{3}\end{array}$ \\
\hline
\end{tabular}
$A=-4.957 \mathrm{E}+02$
$B=2.954 \mathrm{E}-01$
$C=-6.84 \mathrm{E}+05$ 
CuSbs $_{2}$ : Orthorhombic crystals 298.15 to $826 \mathrm{~K}$. Reference state for sulfur is ideal $s_{2}$ gas at $p=1$ bar.

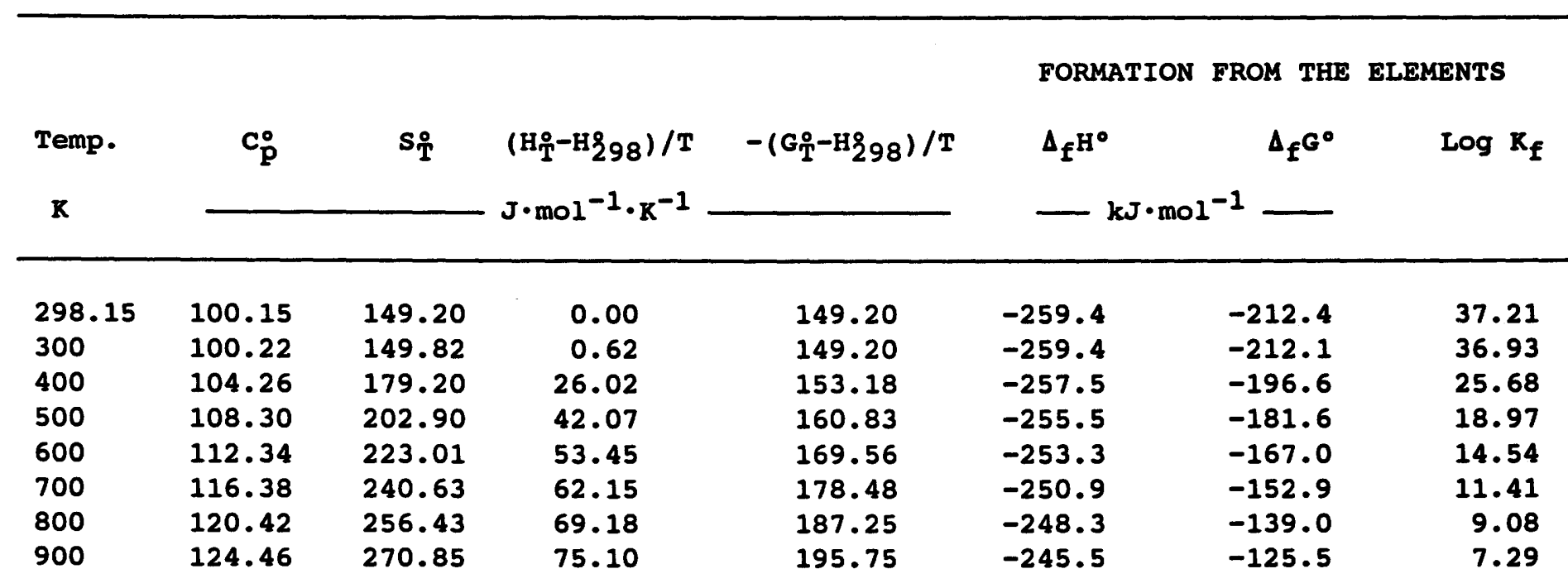

\begin{tabular}{lccc|} 
Melting $T$ & $826 \mathrm{~K}$ & Boiling & $\mathrm{T}$ \\
$\Delta_{\mathrm{fw}} \mathrm{H}^{\circ}$ & $\mathrm{kJ}$ & $\Delta_{\text {vey }} \mathrm{H}^{\circ}$ & $\mathrm{kJ}$ \\
$\mathrm{H} 298^{-\mathrm{H} 8}$ & $\mathrm{~kJ}$ & Molar Vol. & $5.006 \mathrm{~J} \cdot \mathrm{bar}^{-1}$ \\
\hline
\end{tabular}


$\mathrm{Al}_{2} \mathrm{O}_{3}$ : Rhombohedral crystals 298.15 to $1800 \mathrm{~K}$.

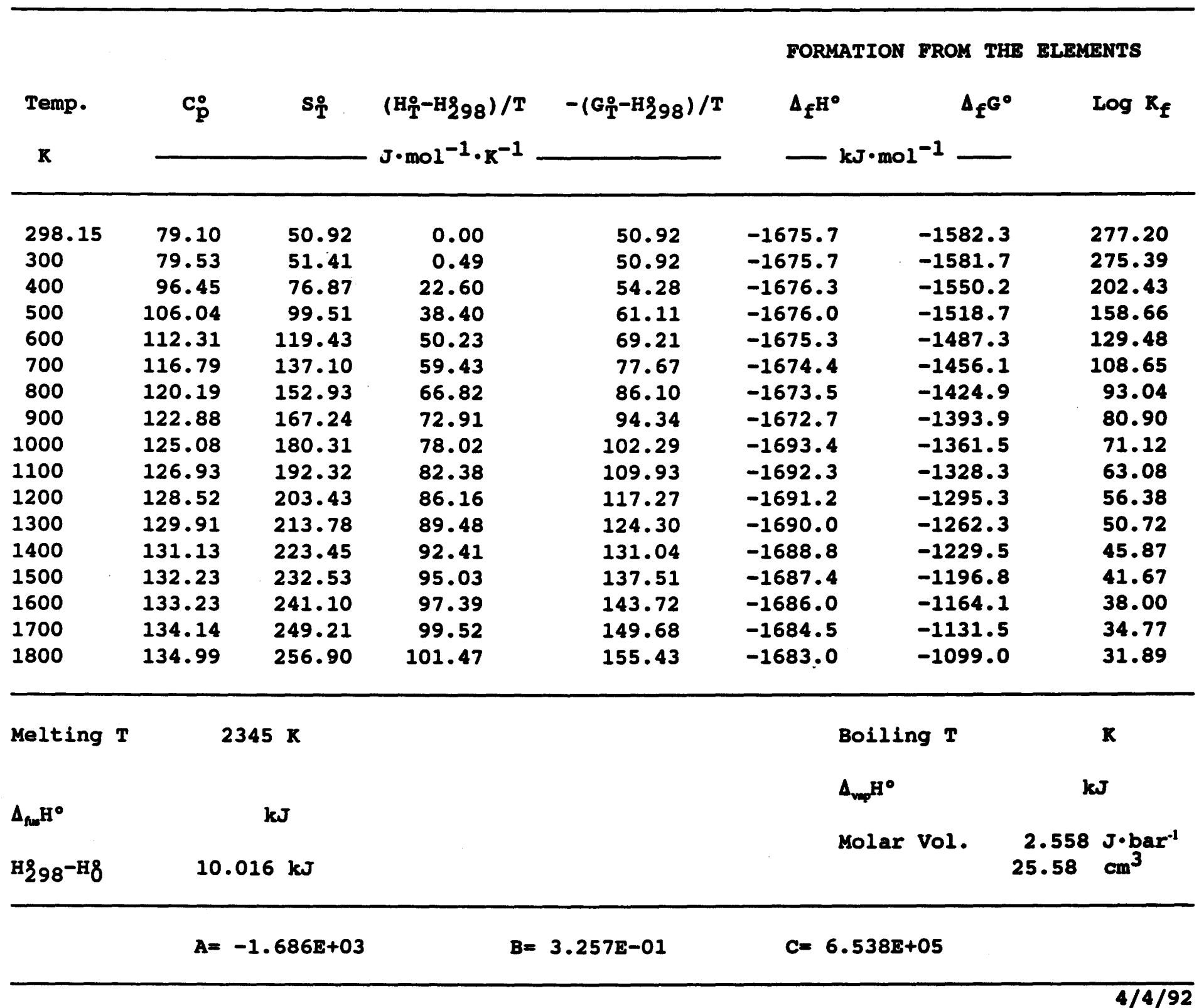


AlO(OH): Orthorhombic crystals 298.15 to $600 \mathrm{~K}$.

\begin{tabular}{|c|c|c|c|c|c|c|c|}
\hline \multirow{3}{*}{$\begin{array}{l}\text { Temp. } \\
\mathbf{K}\end{array}$} & \multirow{3}{*}{$\mathbf{C}_{\mathbf{p}}^{0}$} & \multirow{3}{*}{$\mathbf{s}_{\mathbf{T}}$} & \multirow{3}{*}{$\begin{array}{l}\left(\mathrm{H}_{\mathrm{T}}^{\circ}-\mathrm{H}_{298}\right) / \mathrm{T} \\
\mathrm{J} \cdot \mathrm{mol}^{-1} \cdot \mathrm{K}^{-1}\end{array}$} & \multirow{3}{*}{$-\left(G_{T}^{\circ}-H \xi 98\right) / T$} & \multirow{3}{*}{\multicolumn{2}{|c|}{$\begin{array}{l}\text { FORMATION FROM THE } \\
\Delta_{\mathrm{f}} \mathrm{H}^{\circ}\end{array}$}} & \multirow{3}{*}{$\begin{array}{l}\text { ELEMENTS } \\
\qquad \log \mathbf{K}_{\boldsymbol{f}}\end{array}$} \\
\hline & & & & & & & \\
\hline & & & & & & & \\
\hline 298.15 & 54.24 & 37.20 & 0.00 & 37.20 & -996.4 & -918.4 & 160.90 \\
\hline 300 & 54.50 & 37.54 & 0.34 & 37.20 & -996.4 & -917.9 & 159.82 \\
\hline 400 & 66.40 & 54.95 & 15.46 & 39.49 & -997.3 & -891.6 & 116.43 \\
\hline 500 & 74.51 & 70.70 & 26.51 & 44.19 & -997.3 & -865.2 & 90.38 \\
\hline 600 & 80.03 & 84.80 & 35.00 & 49.80 & -997.0 & -838.8 & 73.02 \\
\hline
\end{tabular}

Melting T

$\boldsymbol{\Lambda}_{\text {fut }} \mathrm{H}^{\circ}$

$\mathbf{k J}$

H298-H8

$8.83 \mathrm{~kJ}$
Boiling T

$\Lambda_{\mathrm{v}} \mathrm{H}^{\circ}$

Molar Vol.
$\mathbf{K}$

$\mathbf{k} \mathbf{J}$
$1.954 \mathrm{~J} \cdot$ bar $^{-1}$ $19.54 \mathrm{~cm}^{3}$ 
$\mathrm{B}_{2} \mathrm{O}_{3}$ : Hexagonal crystals 298.15 to melting point $723 \mathrm{~K}$.

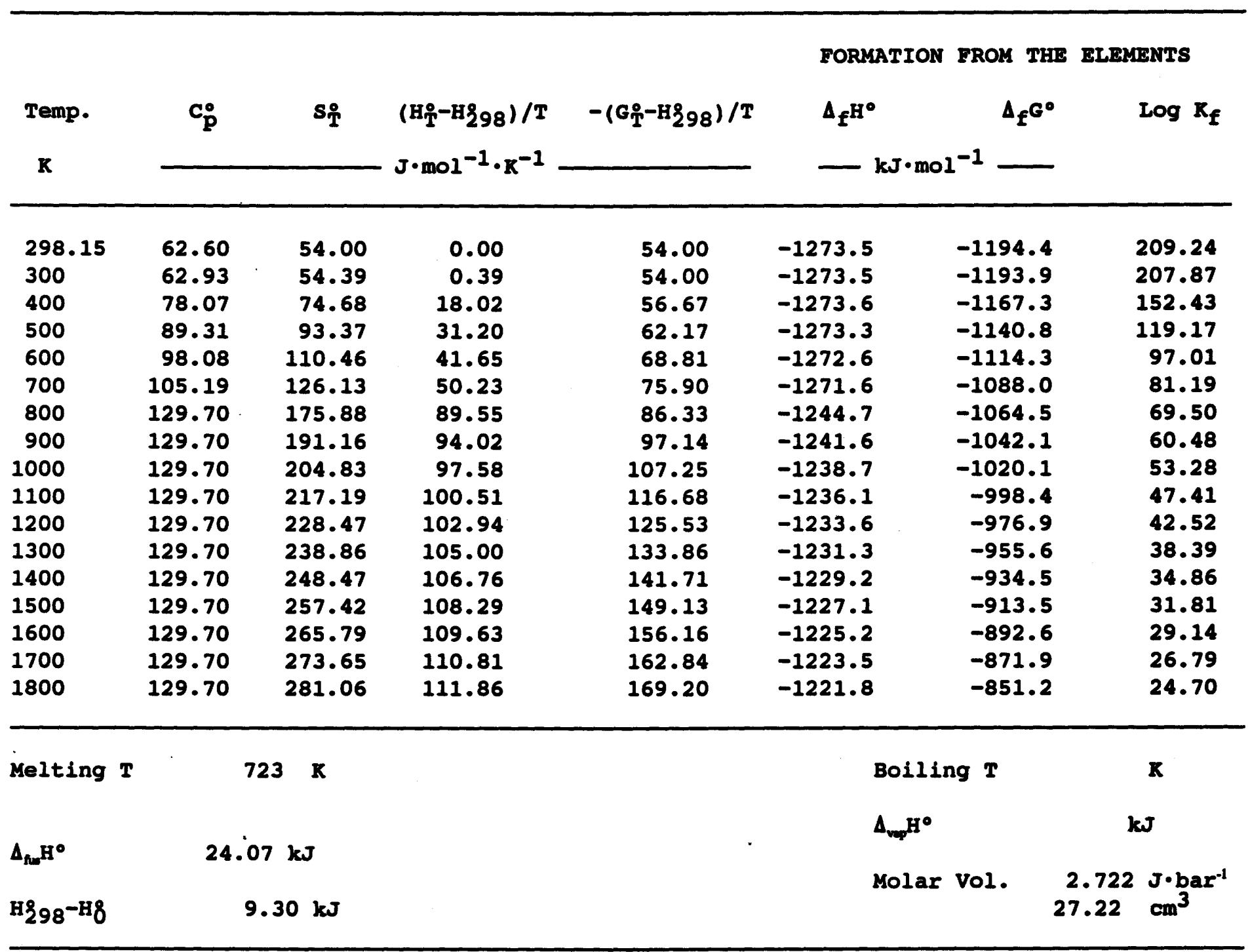


BaO: Cubic crystals 298.15 to $1800 \mathrm{~K}$.

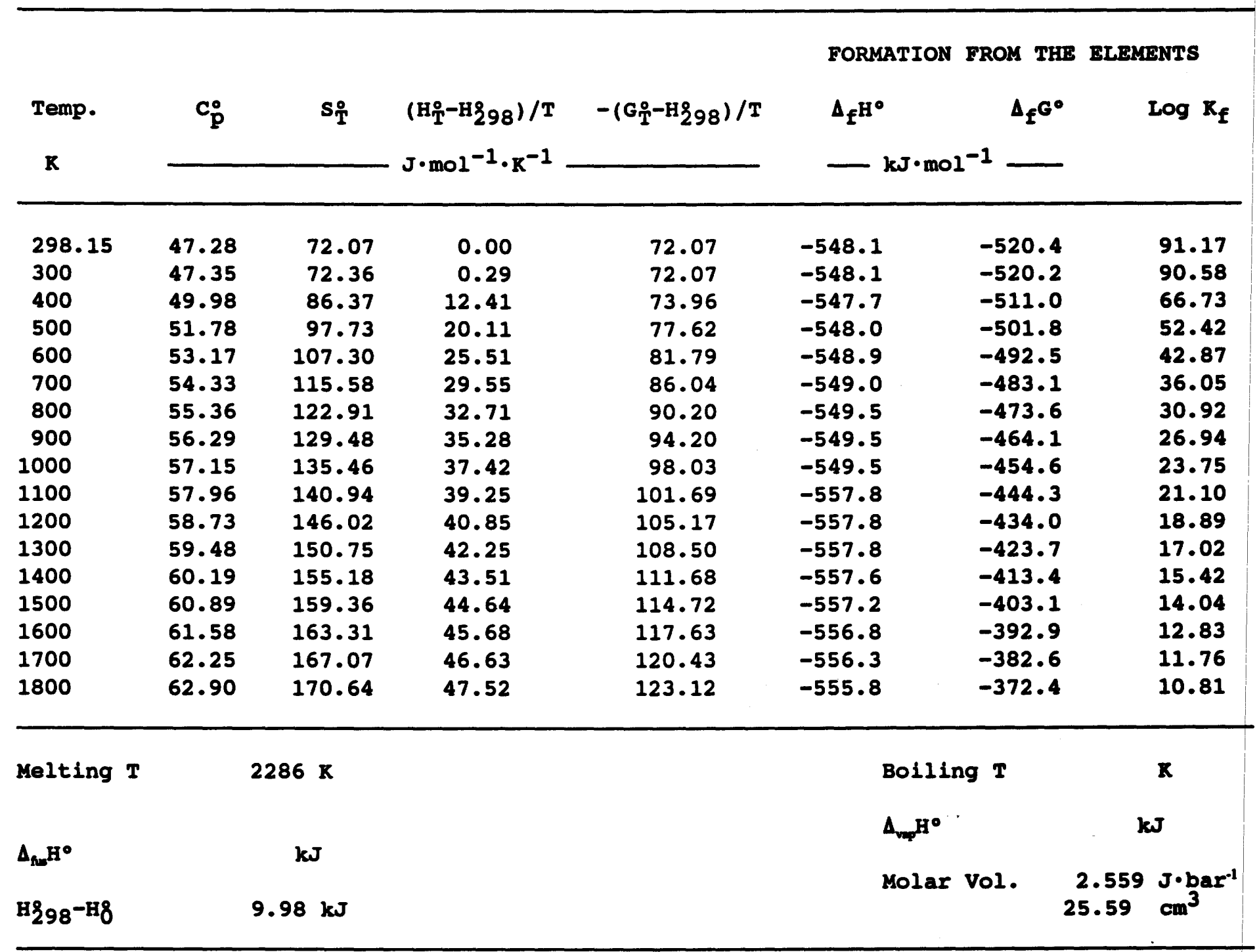

$A=-5 \cdot 518 \mathrm{E}+02$

$B=9.893 E-02$

$C=2.781 \mathrm{E}-01$ 
Beo: Hexagonal crystals 298.15 to $1800 \mathrm{~K}$.

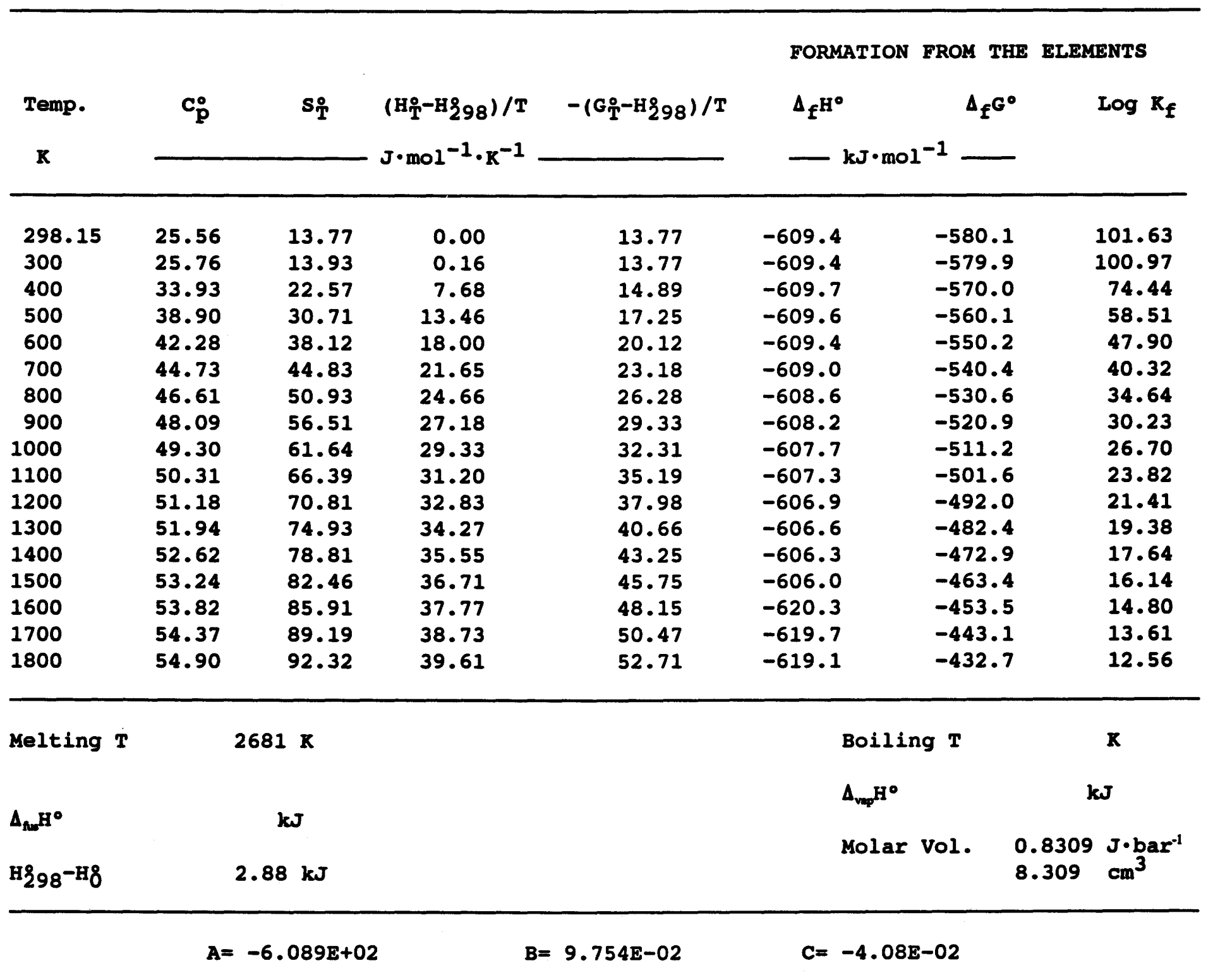


$\mathrm{Bi}_{2} \mathrm{O}_{3}$ : Monoclinic crystals 298.15 to melting point $1098 \mathrm{~K}$.

FORMATION FROM THE ELEMENTS

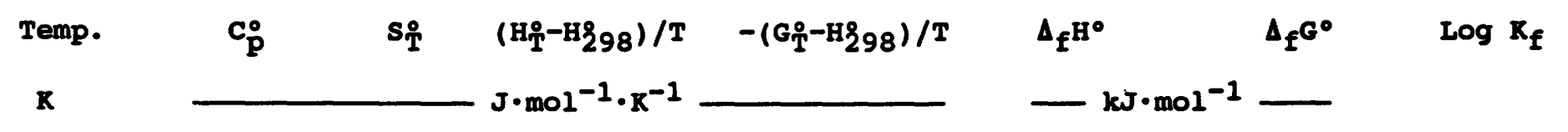

\begin{tabular}{rrrrrrrr}
\hline 298.15 & 113.55 & 151.50 & 0.00 & 151.50 & -573.9 & -493.5 & 86.45 \\
300 & 113.61 & 152.20 & 0.70 & 151.50 & -573.9 & -493.0 & 85.83 \\
400 & 116.94 & 185.34 & 29.34 & 156.00 & -572.0 & -466.3 & 60.89 \\
500 & 120.28 & 211.80 & 47.20 & 164.60 & -570.2 & -440.1 & 45.98 \\
600 & 123.62 & 234.02 & 59.66 & 174.36 & -591.3 & -411.9 & 35.86 \\
700 & 126.95 & 253.33 & 69.03 & 184.30 & -589.5 & -382.2 & 28.52 \\
800 & 130.29 & 270.50 & 76.48 & 194.02 & -587.3 & -352.7 & 23.03 \\
900 & 133.62 & 286.03 & 82.64 & 203.39 & -584.7 & -323.5 & 18.78 \\
1000 & 136.96 & 300.29 & 87.91 & 212.38 & -581.9 & -294.7 & 15.39
\end{tabular}

\begin{tabular}{|c|c|c|c|c|}
\hline Melting $T$ & $1098 \mathrm{~K}$ & Boiling $T$ & & $\mathbf{R}$ \\
\hline & & $\Delta_{\mathrm{vup}} \mathrm{H}^{\circ}$ & & $\mathbf{J}$ \\
\hline $\begin{array}{l}\Delta_{\text {fur }} \mathrm{H}^{\circ} \\
\mathrm{H}_{298^{-H}}{ }^{-\mathrm{H}}\end{array}$ & $\begin{array}{l}\mathbf{k J} \\
\quad \mathbf{k J}\end{array}$ & Molar Vol. & $\begin{array}{c}4.973 \\
49.73\end{array}$ & $\begin{array}{l}\mathrm{J} \cdot \mathrm{bar} \\
\mathrm{cm}^{3}\end{array}$ \\
\hline
\end{tabular}




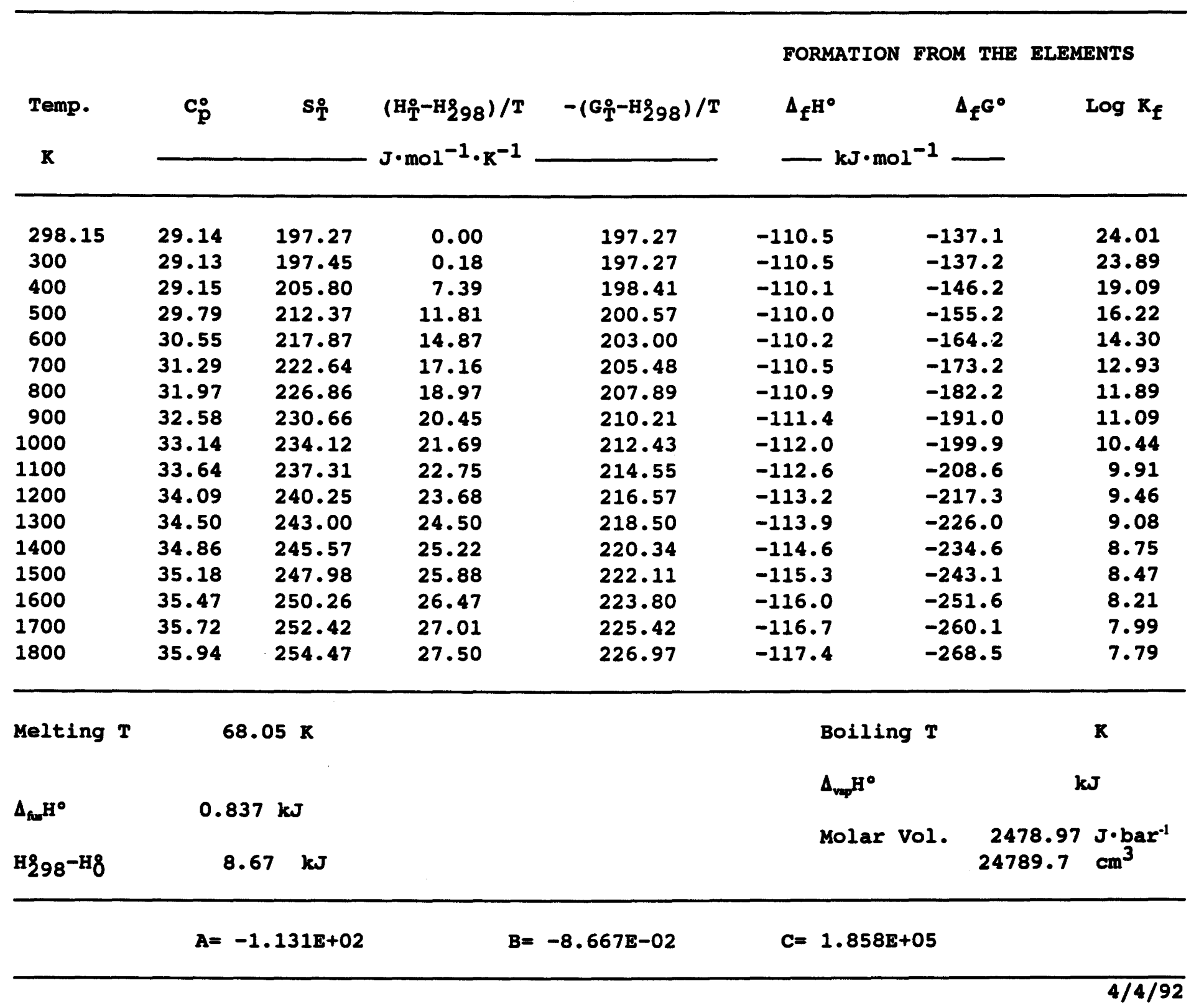


$\mathrm{CO}_{2}$ : Ideal gas at $\mathrm{p}=1$ bar, 298.15 to $2200 \mathrm{~K}$.

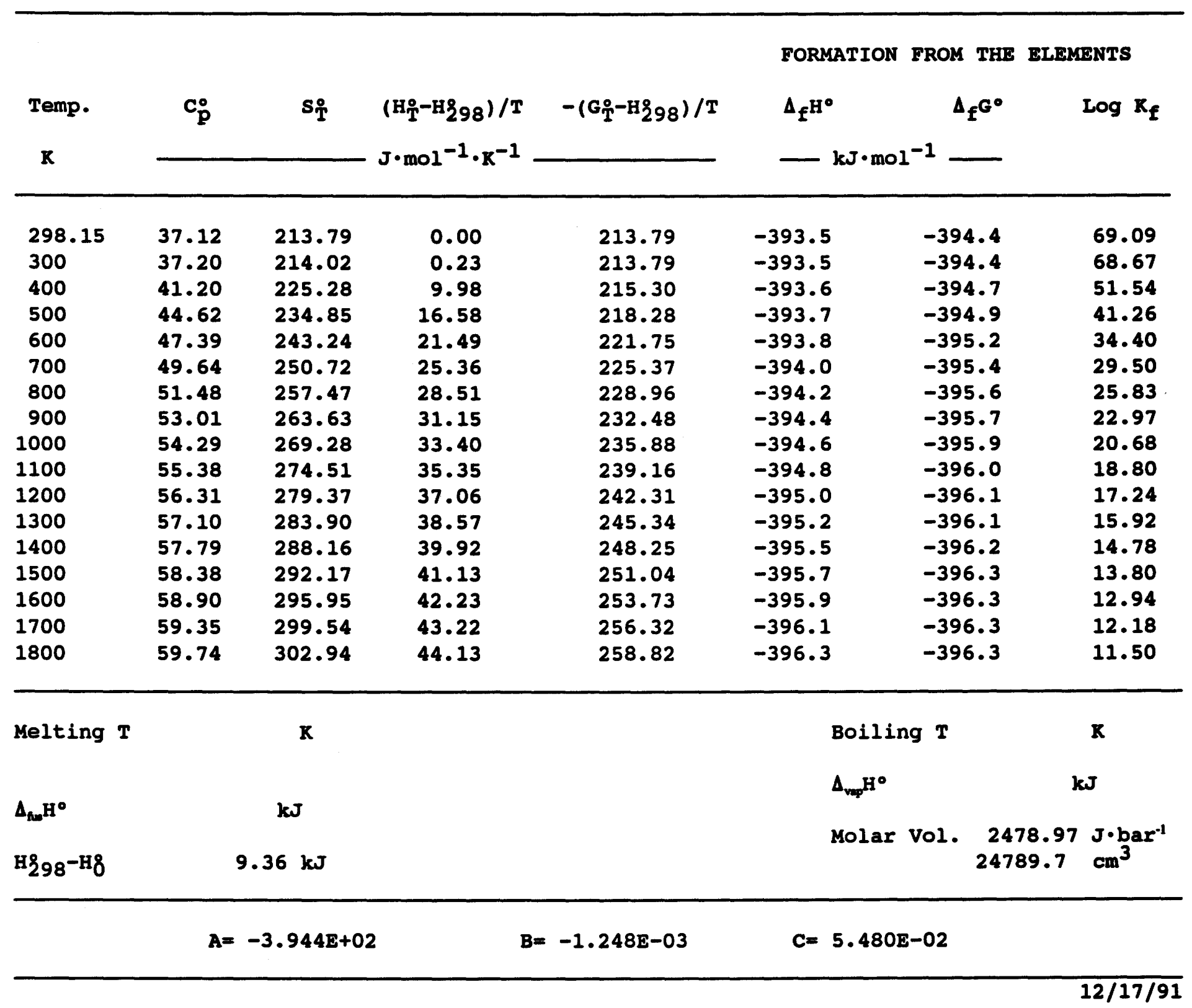


CaO: Cubic crystals 298.15 to $1800 \mathrm{~K}$.

FORMATION FROM THE ELEMENTS

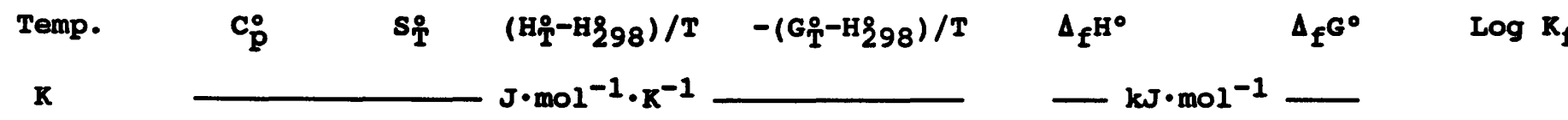

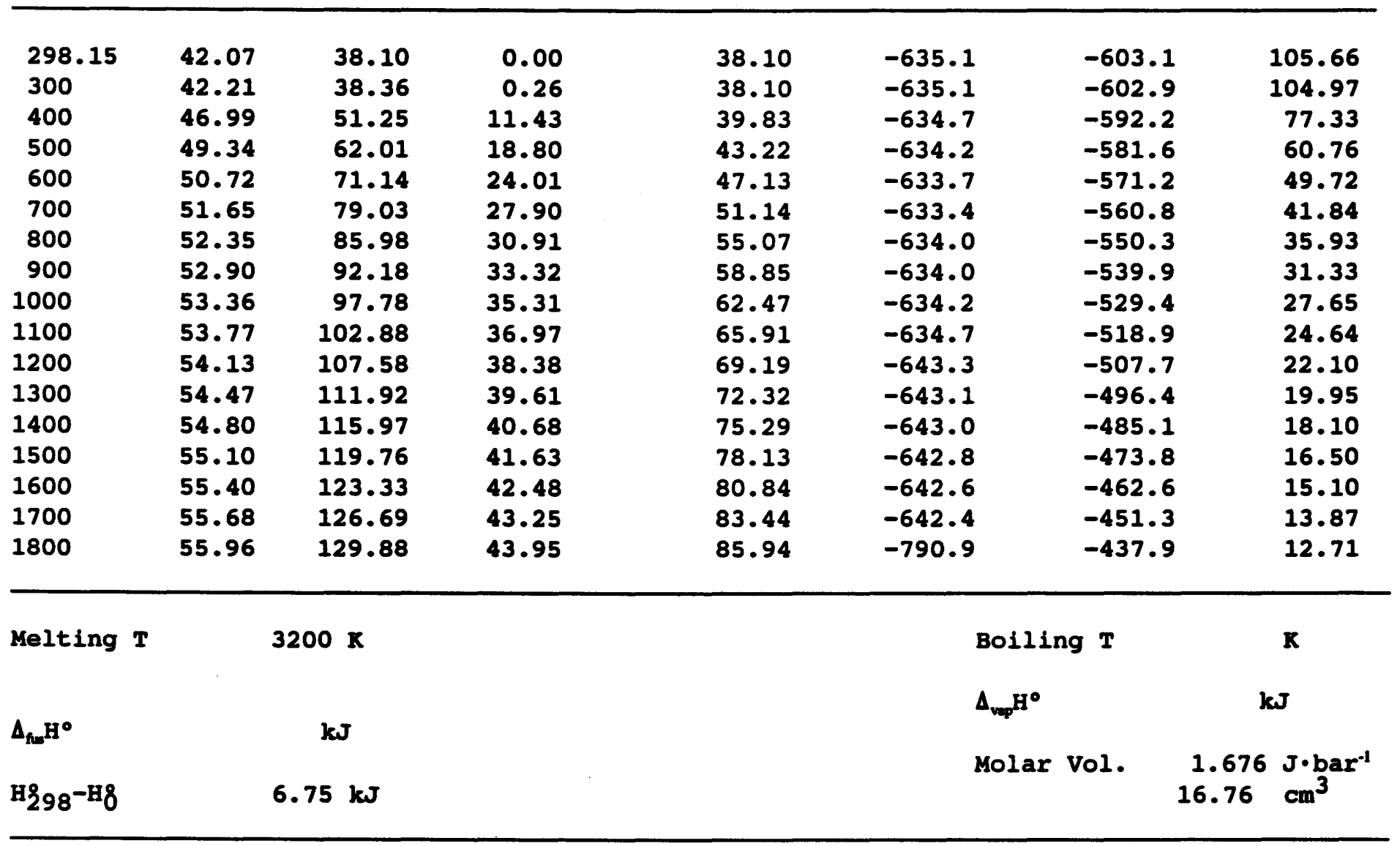


$\mathrm{Ca}(\mathrm{OH})_{2}$ : Hexagonal crystals 298.15 to $700 \mathrm{~K}$.

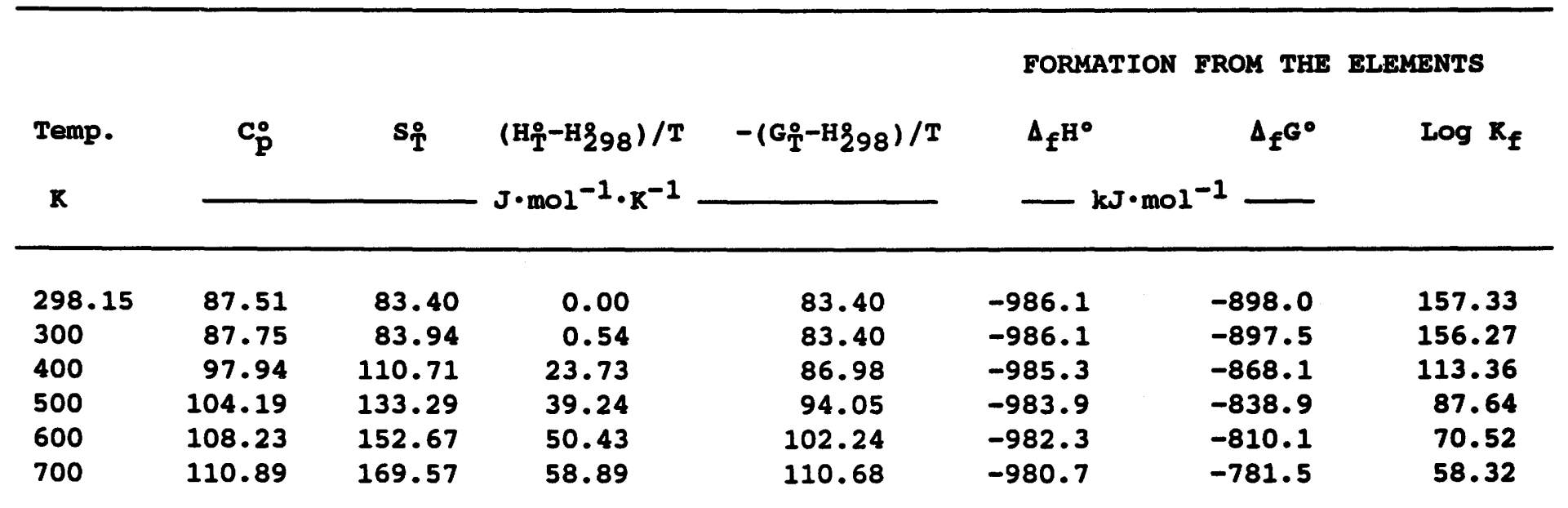

Melting $\mathbf{T}$

R

$\mathbf{k J}$

$\Delta_{\text {fus }} \mathrm{H}^{\circ}$

H298-स8
Boiling $\mathbf{T}$

$\Delta_{\text {vepp }} \mathrm{H}^{\circ}$

Molar Vol.
K

$\mathbf{k J}$

$3.306 \mathrm{~J} \cdot$ bar $^{-1}$ $33.06 \mathrm{~cm}^{3}$
$A=-9.810 E+02$
$B=2.857 E-01$
$C=-1.98 E+05$ 
$\mathrm{CeO}_{2}$ : Cubic crystals 298.15 to $1800 \mathrm{~K}$.

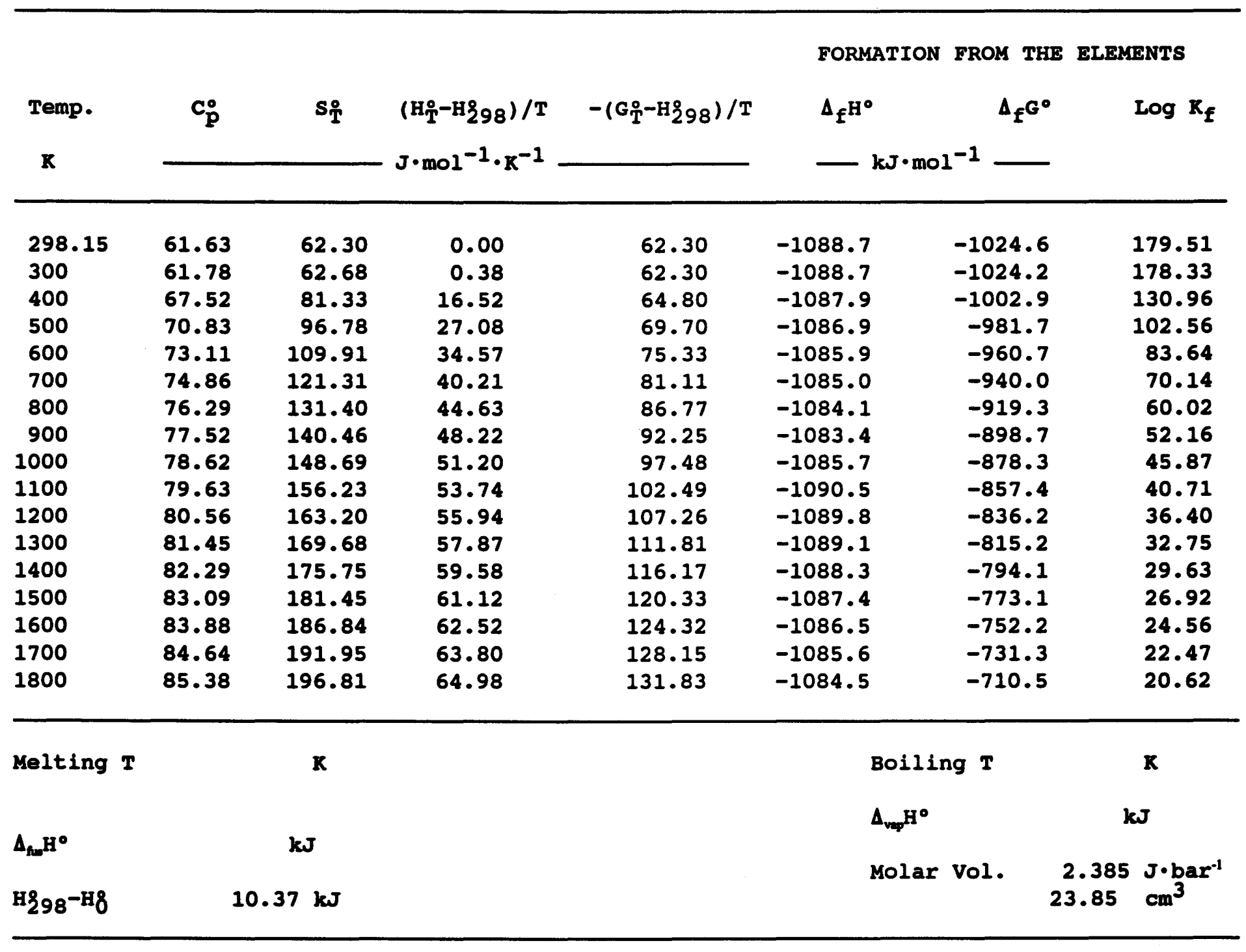
$A=-1.0865 \mathrm{E}+03$
$B=2.088 E-01$
$C=-5.536 \mathrm{E}-02$ 
CoO: Cubic crystals 298.15 to $1800 \mathrm{~K}$.

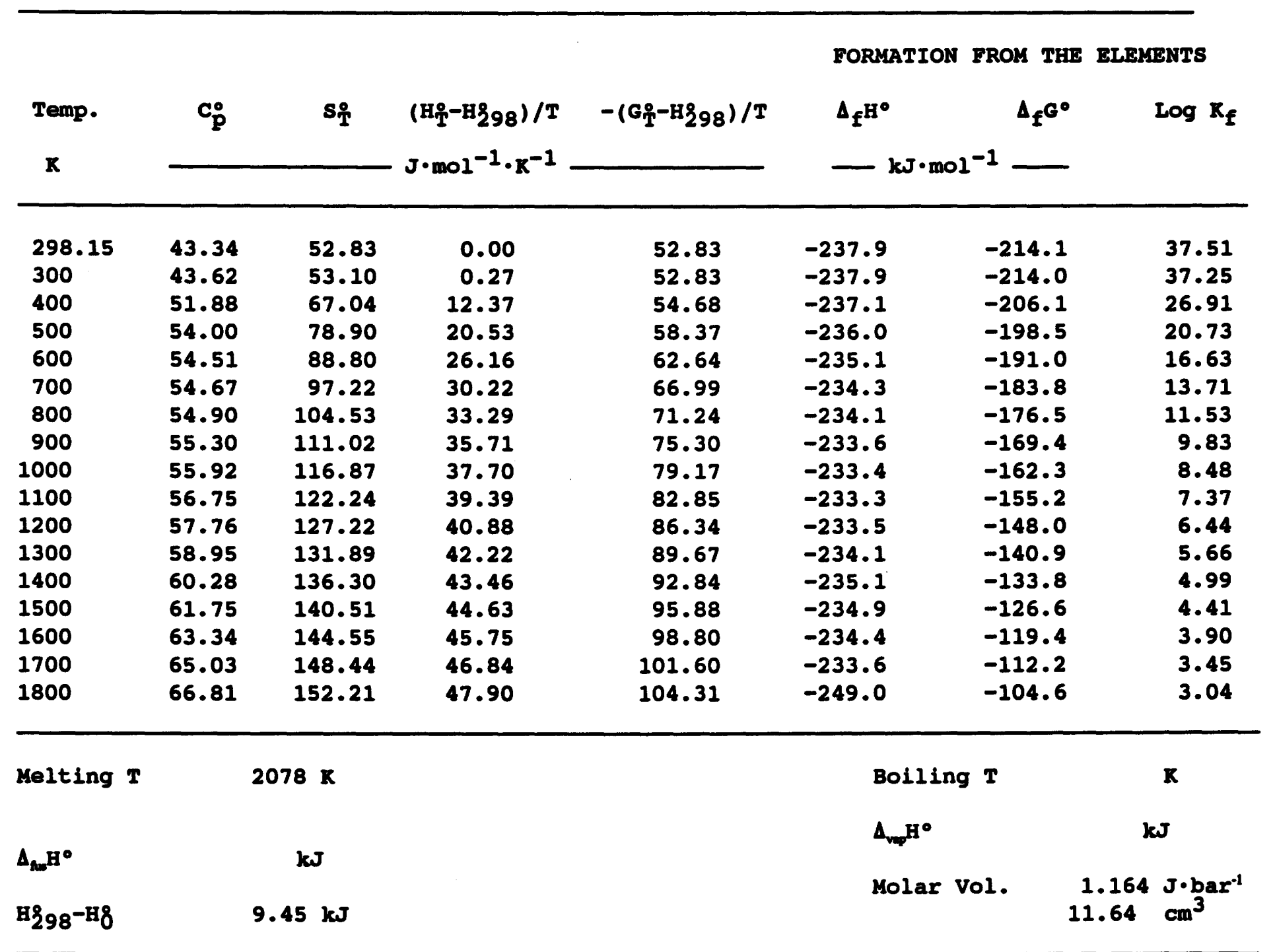
$A=-2 \cdot 336 E+02$
$B=7 \cdot 152 \mathrm{E}-02$
$c=-1.63 \mathrm{E}+05$ 
$\mathrm{CO}_{3} \mathrm{O}_{4}:$ Cubic crystals 298.15 to $1000 \mathrm{~K}$.

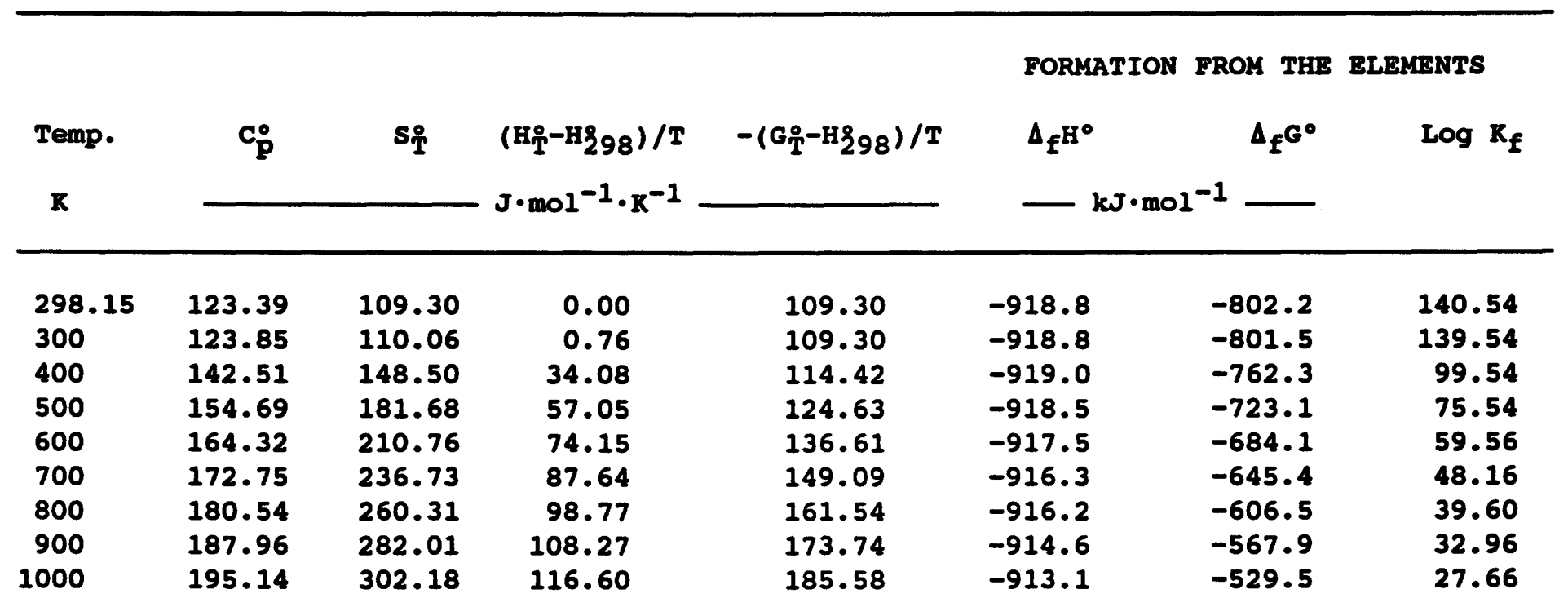

Melting $\mathbf{T}$

$\Delta_{\text {hus }} \mathrm{H}^{\circ}$

H298-H8 $\mathbf{k J}$

$\mathbf{R}$

$18.13 \mathrm{~kJ}$
Boiling T

$\Delta_{\mathrm{veq}} \mathrm{H}^{\circ}$

Molar Vol.
$\mathbf{K}$

$\mathbf{k J}$

$3.977 \mathrm{~J} \cdot \mathrm{bar}^{-1}$ $39.77 \mathrm{~cm}^{3}$
$A=-9.157 E+02$
$B=3.866 E-01$
$C=-1.61 \mathrm{E}+05$ 


\begin{tabular}{|c|c|c|c|c|c|c|c|c|c|}
\hline \multirow{3}{*}{$\begin{array}{l}\text { Temp. } \\
\text { K }\end{array}$} & \multirow{3}{*}{$\mathbf{c}_{\mathbf{p}}^{\circ}$} & \multirow{3}{*}{$\mathbf{s}_{\mathbf{T}}^{\circ}$} & \multirow{3}{*}{$\begin{array}{l}\left(\mathrm{H}_{\mathrm{T}}-\mathrm{H}_{298}\right) / \mathrm{T} \\
\mathrm{J} \cdot \mathrm{mol}^{-1} \cdot \mathrm{K}^{-1}\end{array}$} & \multirow{3}{*}{$-\left(G_{\mathrm{T}}-\mathrm{H}_{298}\right) / \mathrm{T}$} & \multirow{2}{*}{\multicolumn{2}{|c|}{$\begin{array}{l}\text { FORMATION } \\
\Delta_{\mathrm{f}} \mathrm{H}^{\circ}\end{array}$}} & \multirow{2}{*}{$\begin{array}{r}\text { FROM THE } \\
\Delta_{f} G^{\circ}\end{array}$} & \multirow{2}{*}{\multicolumn{2}{|c|}{$\begin{array}{l}\text { ELEMENTS } \\
\qquad \log R_{f}\end{array}$}} \\
\hline & & & & & & & & & \\
\hline & & & & & \multicolumn{3}{|c|}{$\mathrm{kJ} \cdot \mathrm{mol}^{-1}$} & & \\
\hline 298.15 & 105.41 & 81.20 & 0.00 & 81.20 & -1134.7 & & -1053.1 & & 184.49 \\
\hline 300 & 105.63 & 81.85 & 0.65 & 81.20 & -1134.7 & & -1052.6 & & 183.26 \\
\hline 400 & 113.62 & 113.48 & 28.02 & 85.46 & -1133.1 & & -1025.4 & & 133.90 \\
\hline 500 & 117.83 & 139.32 & 45.60 & 93.73 & -1131.3 & & -998.7 & & 104.33 \\
\hline 600 & 120.55 & 161.06 & 57.87 & 103.19 & -1129.5 & & -972.4 & & 84.65 \\
\hline 700 & 122.58 & 179.80 & 66.98 & 112.82 & -1127.8 & & -946.3 & & 70.62 \\
\hline 800 & 124.22 & 196.28 & 74.03 & 122.25 & -1126.2 & & -920.5 & & 60.10 \\
\hline 900 & 125.65 & 211.00 & 79.69 & 131.31 & -1124.8 & & -894.9 & & 51.94 \\
\hline 1000 & 126.95 & 224.30 & 84.35 & 139.95 & -1123.7 & & -869.4 & & 45.41 \\
\hline 1100 & 128.15 & 236.46 & 88.28 & 148.18 & -1122.7 & & -844.1 & & 40.08 \\
\hline 1200 & 129.30 & 247.66 & 91.65 & 156.01 & -1122.1 & & -818.7 & & 35.64 \\
\hline 1300 & 130.40 & 258.05 & 94.59 & 163.46 & -1121.7 & & -793.5 & & 31.88 \\
\hline 1400 & 131.47 & 267.76 & 97.19 & 170.57 & -1121.7 & & -768.2 & & 28.66 \\
\hline 1500 & 132.52 & 276.86 & 99.51 & 177.36 & -1122.0 & & -743.0 & & 25.87 \\
\hline 1600 & 133.55 & 285.45 & 101.60 & 183.85 & -1122.6 & & -717.7 & & 23.43 \\
\hline 1700 & 134.56 & 293.58 & 103.51 & 190.06 & -1123.6 & & -692.4 & & 21.27 \\
\hline 1800 & 135.57 & 301.29 & 105.26 & 196.03 & -1125.0 & & -666.9 & & 19.35 \\
\hline \multirow[t]{2}{*}{ Melting $T$} & \multicolumn{2}{|r|}{$2603 \mathrm{~K}$} & & & \multicolumn{3}{|c|}{ Boiling $\mathbf{T}$} & \multicolumn{2}{|r|}{$\mathbf{K}$} \\
\hline & & & & & \multicolumn{3}{|c|}{$\Delta_{\mathrm{vep}} \mathrm{H}^{\circ}$} & \multicolumn{2}{|c|}{$\mathbf{k J}$} \\
\hline \multirow{2}{*}{$\Delta_{\text {fux }} \mathrm{H}^{\circ}$} & \multicolumn{2}{|r|}{$\mathbf{k J}$} & & & \multirow{2}{*}{\multicolumn{3}{|c|}{ Molar Vol. }} & \multirow{3}{*}{$\begin{array}{c}2.909 \\
29.09\end{array}$} & \multirow{3}{*}{$\begin{array}{l}\mathrm{J} \cdot \mathrm{bar}^{-1} \\
\mathrm{~cm}^{3}\end{array}$} \\
\hline & & & & & & & & & \\
\hline H298-H8 & & $9.45 \mathrm{~kJ}$ & & & & & & & \\
\hline
\end{tabular}


CuO: Monoclinic crystals 298.15 to $1400 \mathrm{~K}$.

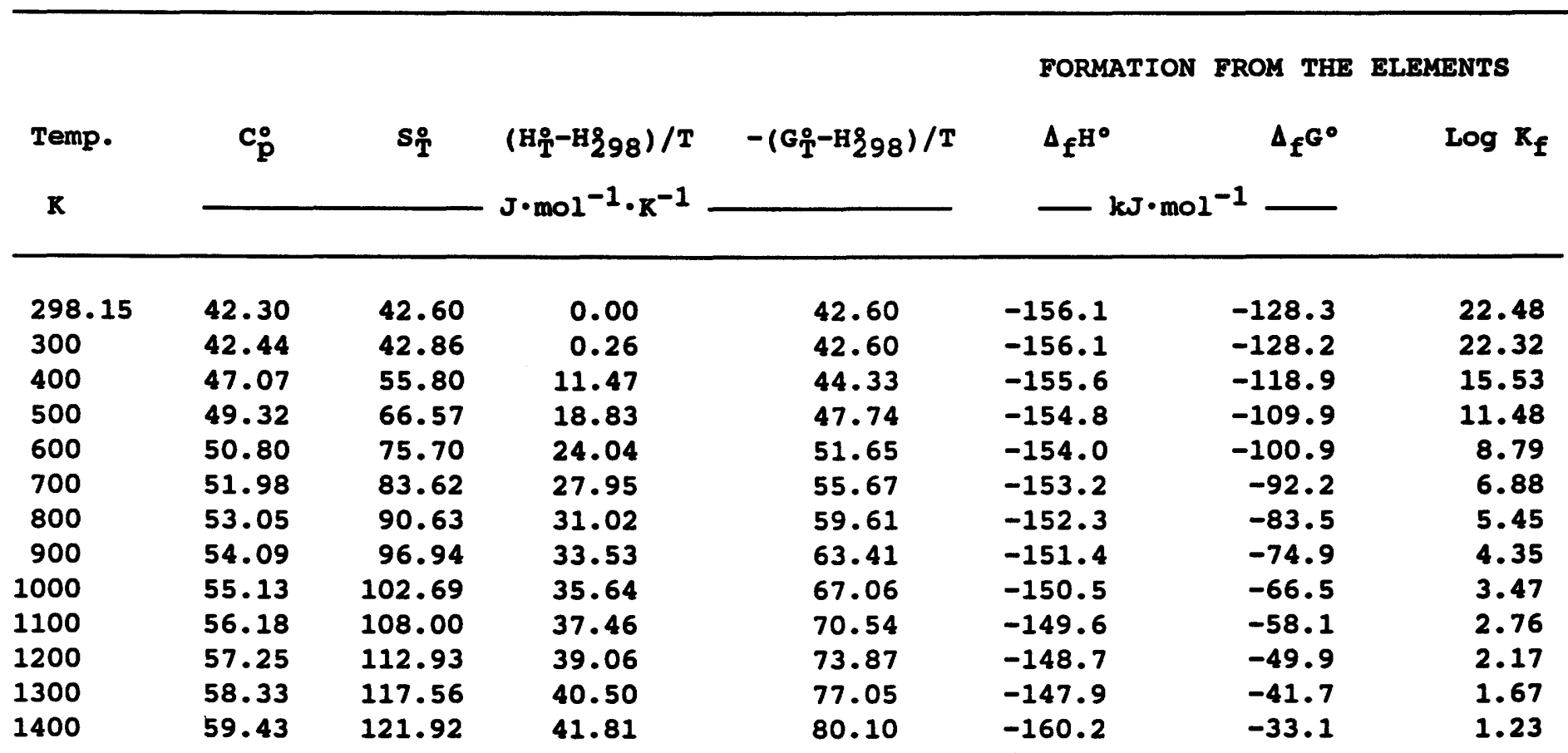

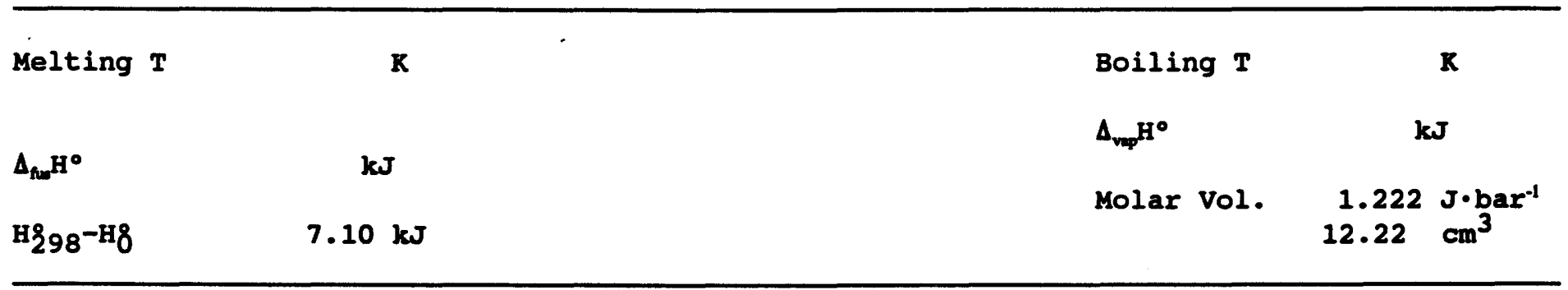


$\mathrm{Cu}_{2}$ O: Cubic crystals 298.15 to $1509 \mathrm{~K}$.

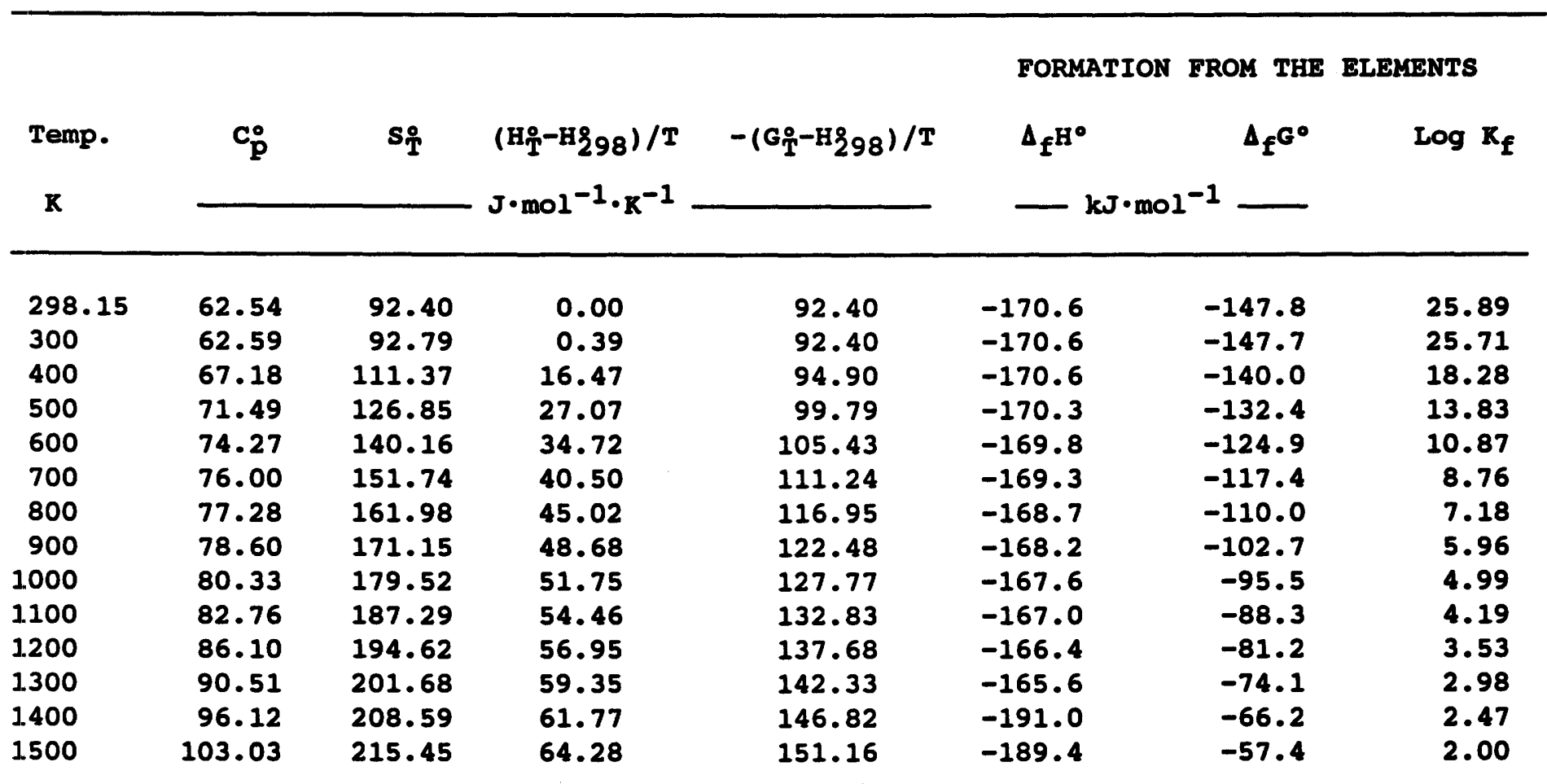

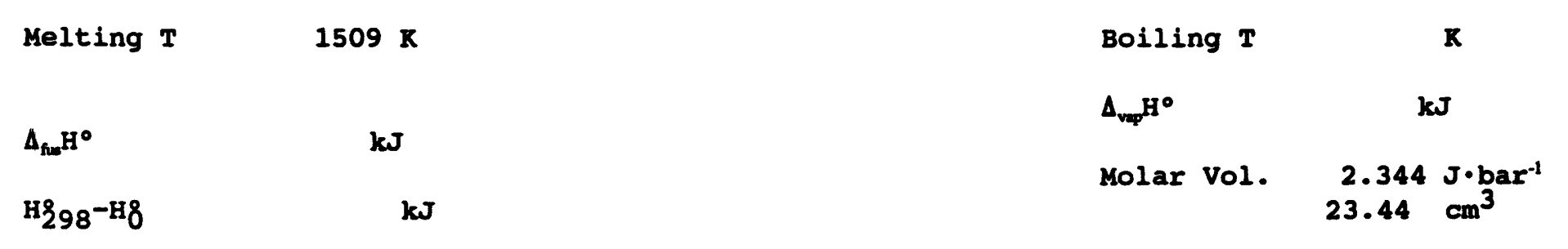
$A=-1 \cdot 690 \mathrm{E}+02$
$B=7.367 \mathrm{E}-02$
$C=-6 \cdot 26 E+04$ 
Fe.9470: Rhombohedral crystals 298.15 to melting point $1652 \mathrm{~K}$.

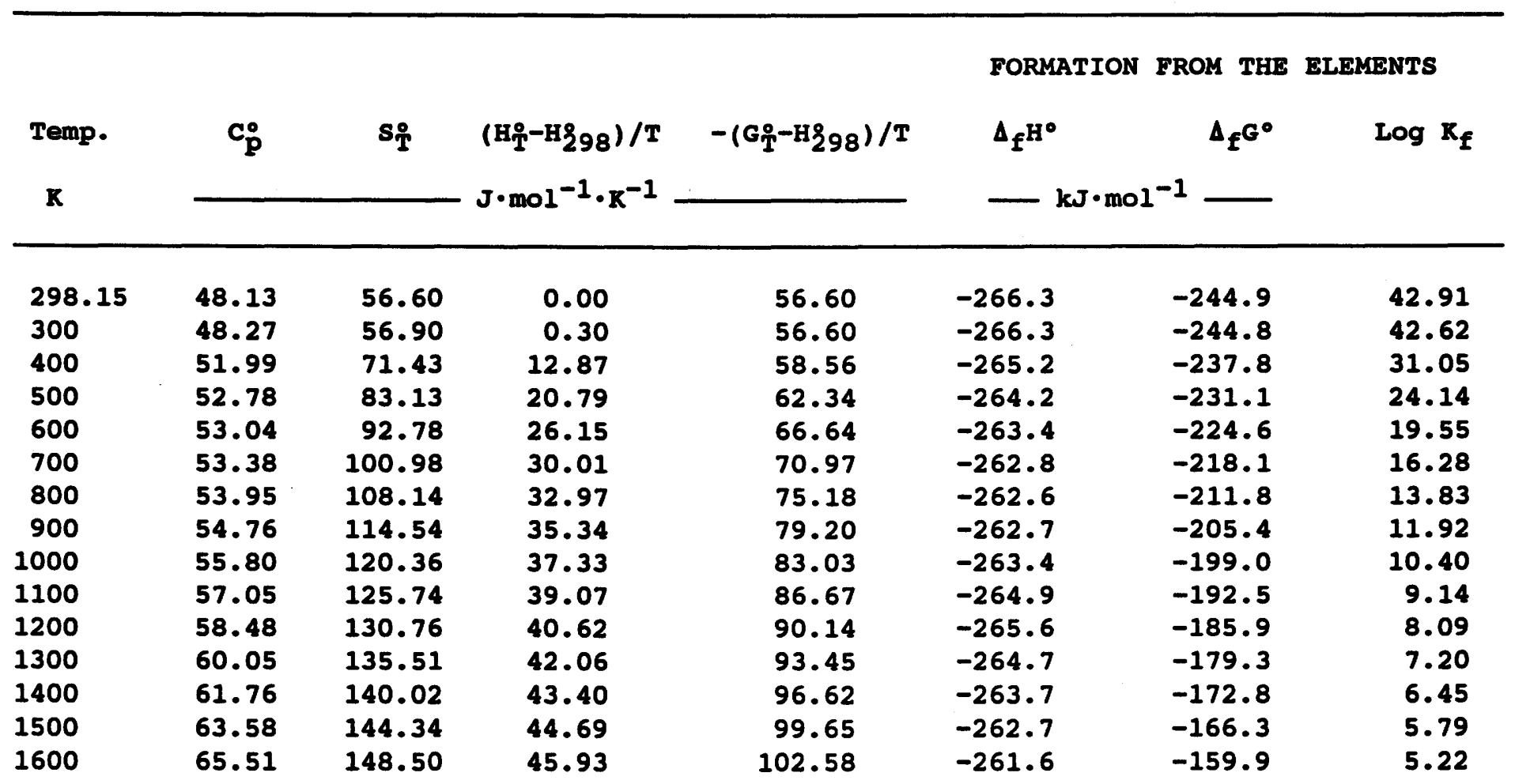

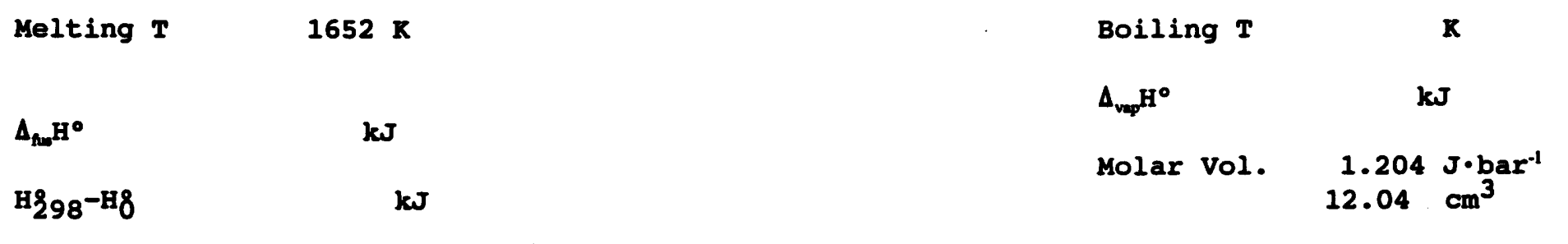


Feo: Cubic crystals 298.15 to $1800 \mathrm{~K}$.

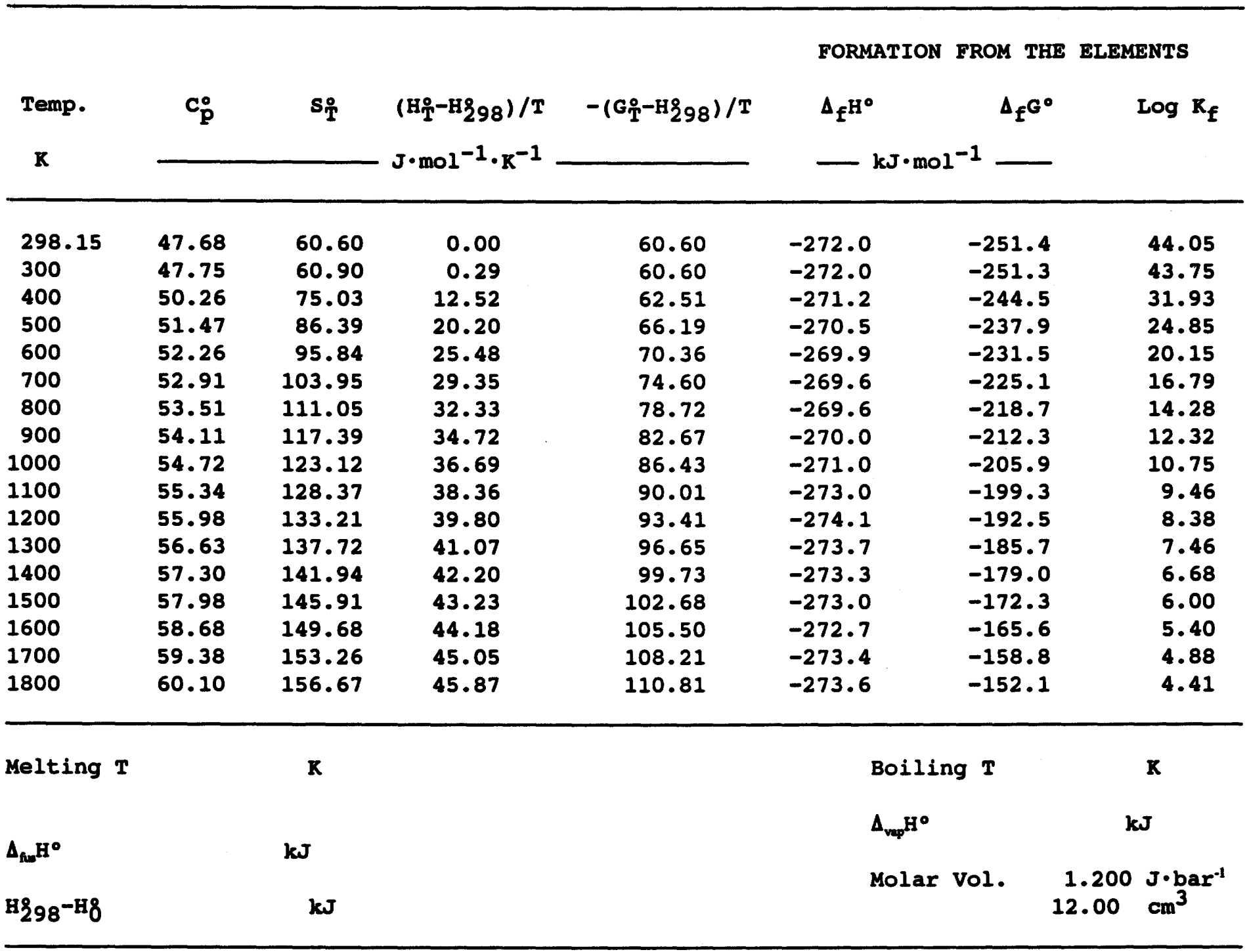


$\mathrm{Fe}_{2} \mathrm{O}_{3}$ : Rhombohedral crystal 298.15 to Neel temperature $950 \mathrm{~K}$. Hexagonal crystals 950 to $1800 \mathrm{~K}$.

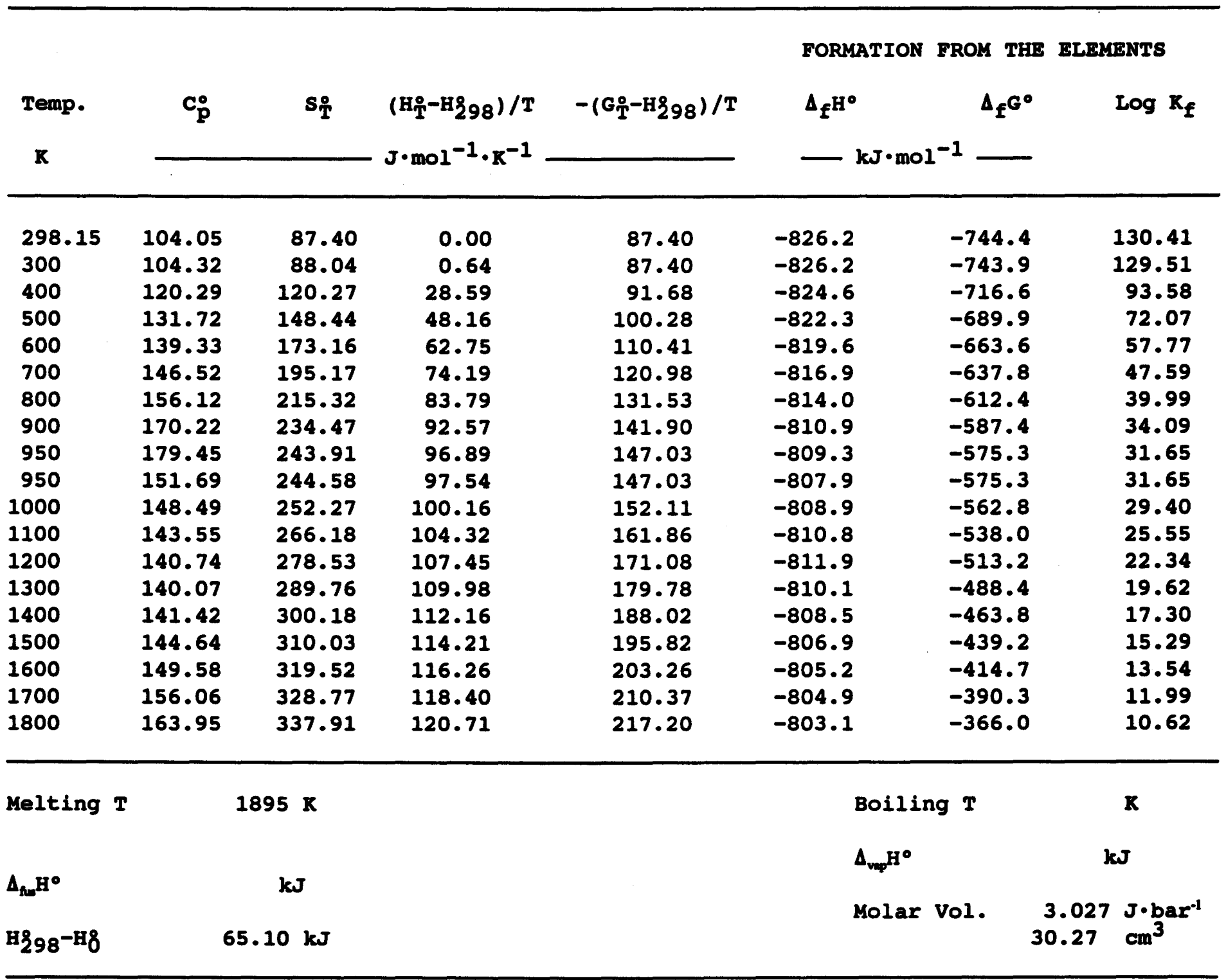
$A=-8.089 E+02$
$B=2.466 E-01$
$C=-8.423 E+05$ 
$\mathrm{Fe}_{3} \mathrm{O}_{4}$ : Cubic (ferrimagnetic) crystals 298.15 to Neél temperature $845.5 \mathrm{~K}$. Cubic crystals (paramagnetic) 845.5 to $1800 \mathrm{~K}$.

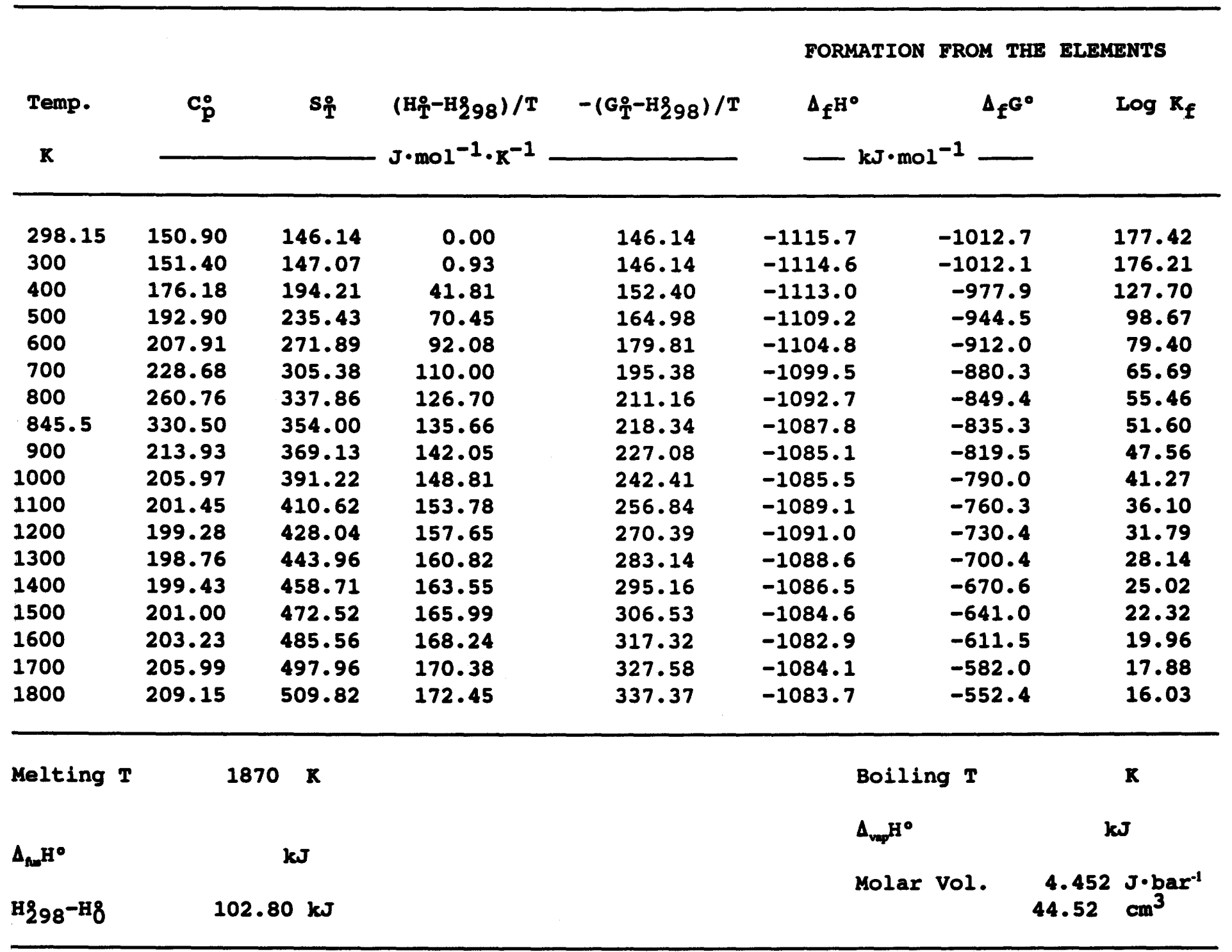


$\mathrm{H}_{2} \mathrm{O}$ : Ideal gas at $\mathrm{p}=1$ bar, 298.15 to $2500 \mathrm{~K}$.

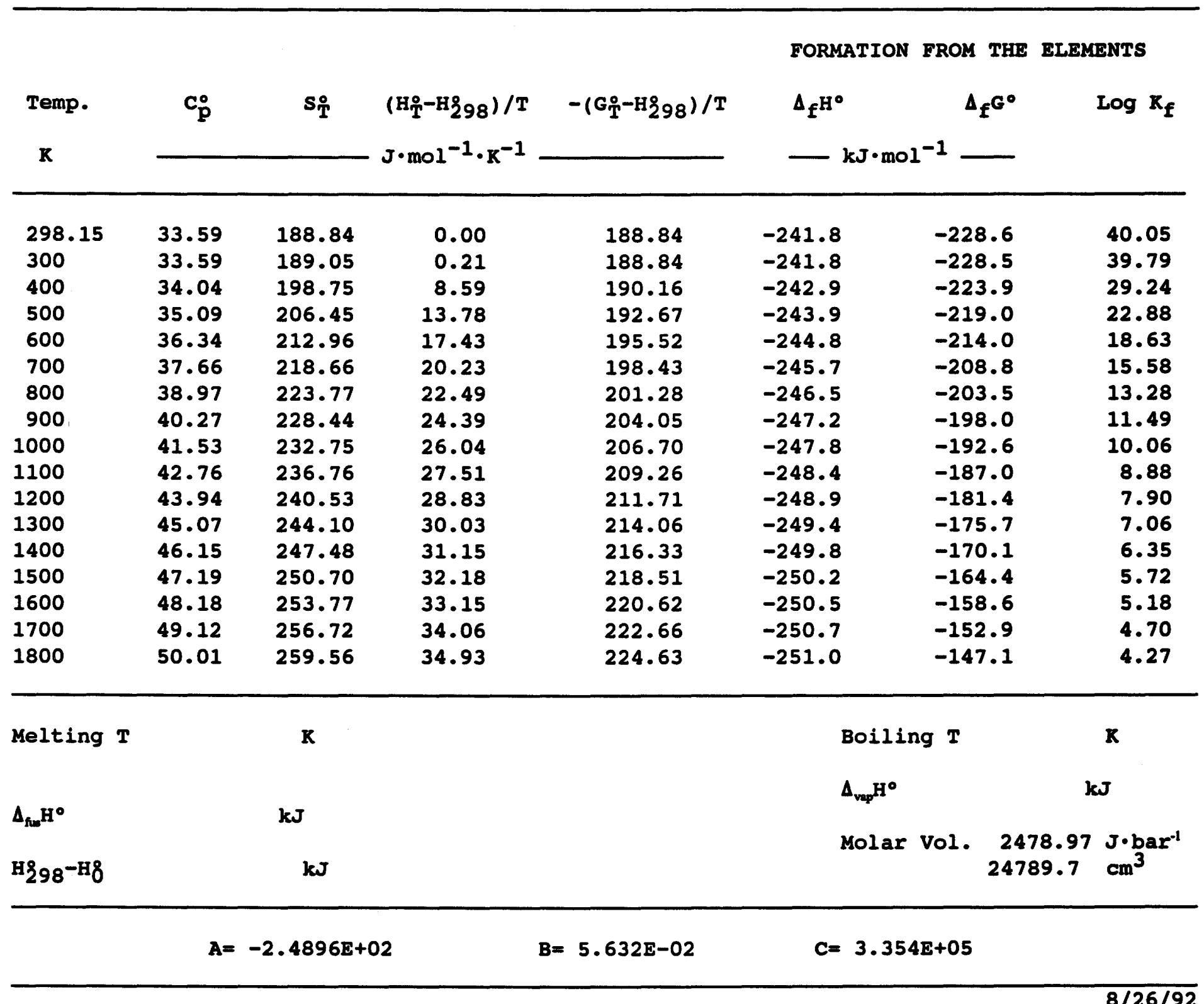


$\mathrm{K}_{2} \mathrm{O}$ : Cubic crystals 298.15 to $1200 \mathrm{~K}$.

\begin{tabular}{|c|c|c|c|c|c|c|c|}
\hline \multirow{3}{*}{$\begin{array}{l}\text { Temp. } \\
\mathbf{K}\end{array}$} & \multirow{3}{*}{$\mathbf{c}_{\mathbf{p}}^{\circ}$} & \multirow{3}{*}{$s_{\text {T }}$} & \multirow{3}{*}{$\begin{array}{l}\left(\mathrm{H}_{\mathrm{T}}^{\circ}-\mathrm{H}_{298}\right) / \mathrm{T} \\
\mathrm{J} \cdot \mathrm{mol}^{-1} \cdot \mathrm{K}^{-1}\end{array}$} & \multirow{3}{*}{$-\left(G_{T}^{\circ}-H_{298}\right) / T$} & \multirow{2}{*}{\multicolumn{2}{|c|}{$\begin{array}{l}\text { FORMATION FROM THE } \\
\Delta_{f^{H^{\circ}}}\end{array}$}} & \multirow{3}{*}{$\begin{array}{l}\text { ELELENTS } \\
\qquad \log k_{f}\end{array}$} \\
\hline & & & & & & & \\
\hline & & & & & $\ldots \mathrm{kJ} \cdot \mathrm{mol}$ & -1 & \\
\hline 298.15 & 83.68 & 94.10 & 0.00 & 94.10 & -363.2 & -322.1 & 56.43 \\
\hline 300 & 83.79 & 94.62 & 0.52 & 94.10 & -363.2 & -321.9 & 56.04 \\
\hline 400 & 88.89 & 119.45 & 21.99 & 97.45 & -367.0 & -307.3 & 40.13 \\
\hline 500 & 93.39 & 139.77 & 35.83 & 103.94 & -365.6 & -292.6 & 30.56 \\
\hline 600 & 97.64 & 157.17 & 45.78 & 111.40 & -363.7 & -278.1 & 24.21 \\
\hline 700 & 101.78 & 172.54 & 53.48 & 119.05 & -361.4 & -264.0 & 19.70 \\
\hline 800 & 105.85 & 186.39 & 59.77 & 126.62 & -358.6 & -250.3 & 16.34 \\
\hline 900 & 109.90 & 199.10 & 65.12 & 133.98 & -355.5 & -237.0 & 13.75 \\
\hline 1000 & 113.92 & 210.88 & 69.80 & 141.08 & -352.0 & -224.0 & 11.70 \\
\hline 1100 & 117.92 & 221.93 & 73.99 & 147.94 & -506.2 & -202.1 & 9.60 \\
\hline 1200 & 121.92 & 232.36 & 77.82 & 154.54 & -500.2 & -174.7 & 7.61 \\
\hline
\end{tabular}

Melting $T$

$\Lambda_{\text {An }} \mathrm{H}^{\circ}$

H $298^{-\mathrm{H}}$
$\mathbf{K}$

$\mathbf{k J}$

kis
Boiling T

$\Delta_{\text {vep }} \mathrm{H}^{\circ}$

Molar vol.
$\mathbf{K}$

$k \boldsymbol{J}$

$4.038 \mathrm{~J} \cdot \mathrm{bar}^{-1}$ $40.38 \mathrm{~cm}^{3}$ 
$\mathrm{Li}_{2}$ O: Cubic crystals 298.15 to melting point $1700 \mathrm{~K}$.

\begin{tabular}{|c|c|c|c|c|c|c|c|}
\hline \multirow{2}{*}{$\begin{array}{l}\text { Temp. } \\
\mathbf{K}\end{array}$} & \multirow[t]{2}{*}{$\mathbf{c}_{\mathbf{p}}^{\circ}$} & \multirow[t]{2}{*}{$\mathbf{s}_{\mathbf{T}}$} & \multirow{2}{*}{$\begin{array}{l}\left(\mathrm{H}_{\mathrm{T}}-\mathrm{H}_{298}\right) / \mathrm{T} \\
\mathrm{J} \cdot \operatorname{mol}^{-1} \cdot \mathrm{K}^{-1}\end{array}$} & \multirow[t]{2}{*}{$-\left(G_{T}^{\circ}-H_{298}\right) / T$} & \multicolumn{2}{|c|}{$\begin{array}{l}\text { FORMATION FROM THE } \\
\Delta_{\mathrm{f}^{\mathrm{H}^{\circ}}}\end{array}$} & \multirow[t]{2}{*}{$\begin{array}{l}\text { ELEMENTS } \\
\qquad \log \mathbf{R}_{\mathbf{f}}\end{array}$} \\
\hline & & & & & $-k$ & $L^{-1}$ & \\
\hline 298.15 & 54.10 & 37.60 & 0.00 & 37.60 & -597.9 & -561.2 & 98.31 \\
\hline 300 & 54.33 & 37.94 & 0.33 & 37.60 & -597.9 & -561.0 & 97.67 \\
\hline 400 & 63.61 & 54.96 & 15.10 & 39.86 & -598.7 & -548.5 & 71.63 \\
\hline 500 & 69.52 & 69.83 & 25.43 & 44.40 & -605.4 & -535.3 & 55.92 \\
\hline 600 & 73.98 & 82.91 & 33.16 & 49.75 & -605.7 & -521.2 & 45.38 \\
\hline 700 & 77.67 & 94.60 & 39.26 & 55.34 & -605.6 & -507.1 & 37.84 \\
\hline 800 & 80.87 & 105.19 & 44.27 & 60.92 & -605.2 & -493.1 & 32.20 \\
\hline 900 & 83.72 & 114.88 & 48.50 & 66.38 & -604.4 & -479.1 & 27.81 \\
\hline 1000 & 86.29 & 123.83 & 52.15 & 71.68 & -603.4 & -465.3 & 24.30 \\
\hline 1100 & 88.63 & 132.17 & 55.36 & 76.81 & -602.2 & -451.5 & 21.44 \\
\hline 1200 & 90.76 & 139.98 & 58.22 & 81.75 & $-600 \cdot 7$ & -437.9 & 19.06 \\
\hline 1300 & 92.70 & 147.32 & 60.80 & 86.51 & -599.1 & -424.4 & 17.05 \\
\hline 1400 & 94.46 & 154.25 & 63.15 & 91.11 & -597.3 & -411.0 & 15.33 \\
\hline 1500 & 96.05 & 160.83 & 65.29 & 95.54 & -595.3 & -397.7 & 13.85 \\
\hline 1600 & 97.47 & 167.07 & 67.25 & 99.82 & -593.2 & -384.6 & 12.56 \\
\hline 1700 & 98.72 & 173.02 & 69.07 & 103.95 & -881.4 & -357.3 & 10.98 \\
\hline
\end{tabular}

Melting $\mathbf{T} \quad 1700 \mathrm{~K}$

$\Delta_{\text {fue }} \mathrm{H}^{\circ}$

$\mathbf{k J}$

H298-H8
Boiling $\mathbf{T}$

$\Delta_{\text {vap }} H^{\circ}$

$\mathbf{k J}$

Molar Vol. $\begin{aligned} & 1.476 \mathrm{~J} \cdot \mathrm{bar}^{-1} \\ & 14.76 \mathrm{~cm}^{3}\end{aligned}$ 
Mgo: Cubic crystals 298.15 to $1800 \mathrm{~K}$.

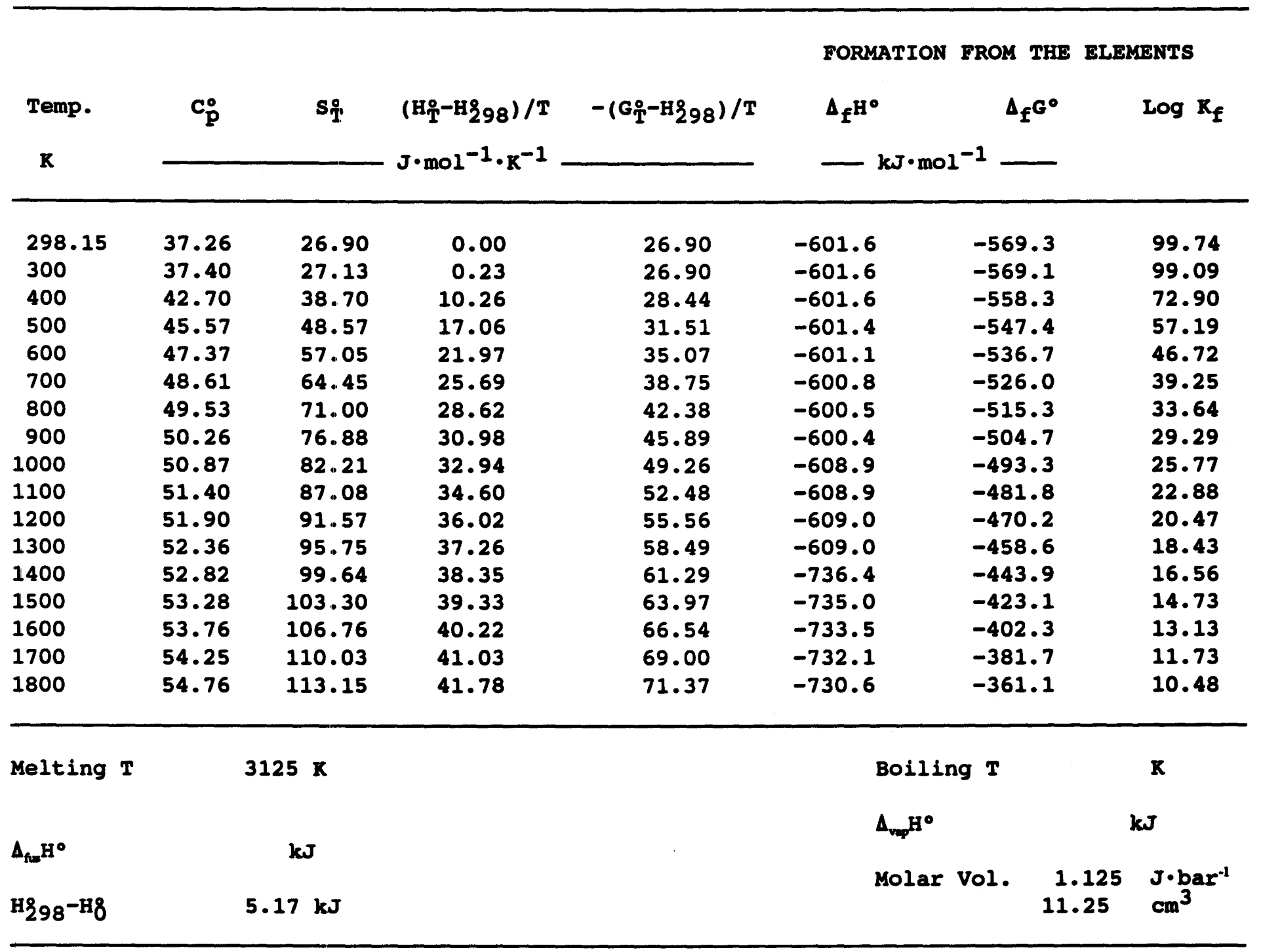

$A=-6 \cdot 373 E+02$

$B=1.449 \mathrm{E}-01$

$C=2 \cdot 512 \mathrm{E}+06$ 
$\mathrm{Mg}(\mathrm{OH})_{2}$ : Hexagonal crystals 298.15 to $900 \mathrm{~K}$.

\begin{tabular}{|c|c|c|c|c|c|c|c|}
\hline Temp. & $\mathbf{C}_{\mathbf{p}}^{\circ}$ & $\mathbf{s}_{\mathrm{T}}$ & \multirow{2}{*}{$\begin{array}{l}\left(\mathrm{H}_{\mathrm{T}}^{\circ}-\mathrm{H} 298\right) / \mathrm{T} \\
\mathrm{J} \cdot \mathrm{mol}^{-1} \cdot \mathrm{K}^{-1}\end{array}$} & \multirow[t]{2}{*}{$-\left(G_{\mathrm{T}}^{\circ}-\mathrm{H}_{298}\right) / \mathrm{T}$} & \multirow{2}{*}{\multicolumn{2}{|c|}{$\begin{array}{l}\text { FORMATION FROM THE } \\
\Delta_{f^{H}} H^{\circ}\end{array}$}} & \multirow[t]{2}{*}{$\begin{array}{l}\text { ELEMENTS } \\
\qquad \log K_{f}\end{array}$} \\
\hline $\mathbf{K}$ & & & & & & & \\
\hline 298.15 & 77.27 & 63.20 & 0.00 & 63.20 & -924.5 & -833.5 & 146.02 \\
\hline 300 & 77.66 & 63.68 & 0.48 & 63.20 & -924.5 & -832.9 & 145.02 \\
\hline 400 & 91.89 & 88.23 & 21.78 & 66.45 & -924.4 & -802.4 & 104.78 \\
\hline 500 & 99.29 & 109.60 & 36.61 & 73.00 & -923.4 & -772.0 & 80.64 \\
\hline 600 & 104.00 & 128.15 & 47.47 & 80.68 & -922.1 & -741.8 & 64.58 \\
\hline 700 & 107.44 & 144.45 & 55.80 & 88.65 & -920.6 & -711.9 & 53.12 \\
\hline 800 & 110.20 & 158.98 & 62.43 & 96.55 & -919.0 & -682.1 & 44.54 \\
\hline 900 & 112.57 & 172.10 & 67.87 & 104.23 & -917.3 & -652.6 & 37.88 \\
\hline
\end{tabular}

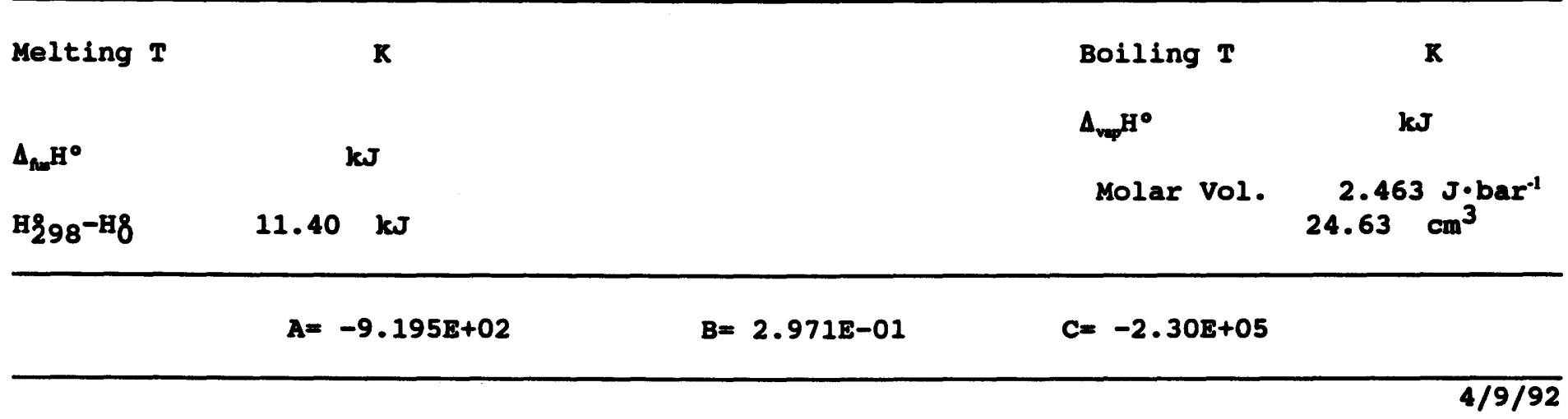


Mno: Cubic crystals 298.15 to $1800 \mathrm{~K}$.

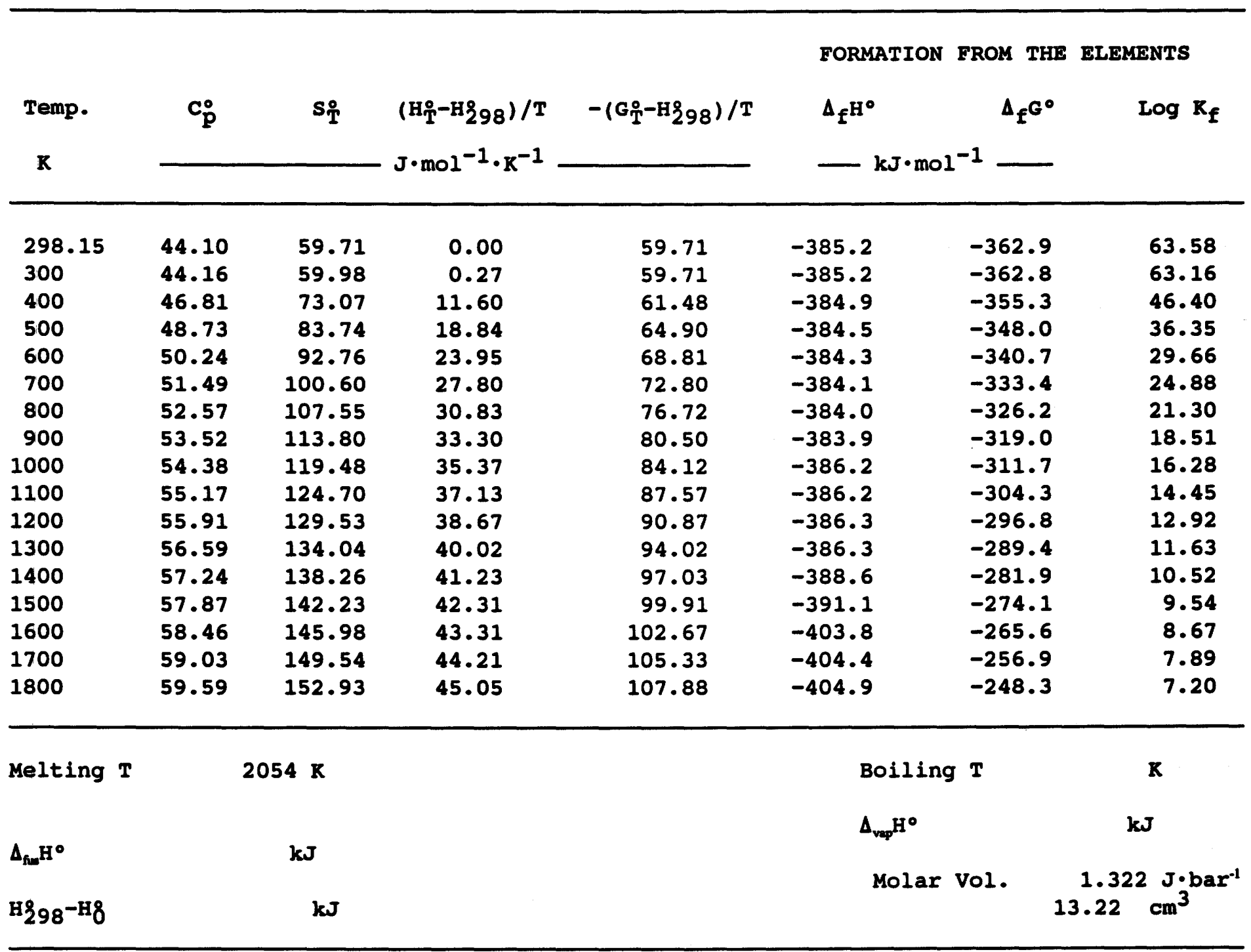
$A=-3 \cdot 880 \mathrm{E}+02$
$B=7.651 E-02$
$C=2.398 E+05$ 
$\mathrm{MnO}_{2}$ : Crystals 298.15 to $850 \mathrm{~K}$.

\begin{tabular}{|c|c|c|c|c|c|c|c|}
\hline Temp. & $\mathbf{c}_{\mathbf{p}}^{\circ}$ & $s_{T}^{\circ}$ & \multirow{2}{*}{$\begin{array}{l}\left(\mathrm{H}_{\mathrm{T}}-\mathrm{H}_{298}\right) / \mathrm{T} \\
\mathrm{J} \cdot \mathrm{mol}^{-1} \cdot \mathrm{K}^{-1}\end{array}$} & \multirow[t]{2}{*}{$-\left(G_{T}^{\circ}-H_{298}\right) / T$} & \multirow{2}{*}{\multicolumn{2}{|c|}{$\begin{array}{l}\text { FORMATION FROM THE } \\
\Delta_{\mathrm{f}^{\mathrm{H}}}{ }^{\circ} \\
-\mathrm{kJ} \cdot \mathrm{mol}^{-1} \mathrm{G}^{\circ}\end{array}$}} & \multirow[t]{2}{*}{$\begin{array}{l}\text { ELEMENTS } \\
\qquad \log \mathbf{K}_{\mathbf{f}}\end{array}$} \\
\hline $\mathbf{K}$ & & & & & & & \\
\hline 298.15 & 54.76 & 52.75 & 0.00 & 52.75 & -520.0 & -465.0 & 81.47 \\
\hline 300 & 54.94 & 53.09 & 0.34 & 52.75 & -520.0 & -464.7 & 80.91 \\
\hline 400 & 63.21 & 70.11 & 15.09 & 55.01 & -519.8 & -446.3 & 58.27 \\
\hline 500 & 68.34 & 84.81 & 25.27 & 59.54 & -519.1 & -427.9 & 44.71 \\
\hline 600 & 71.21 & 97.56 & 32.72 & 64.84 & -518.4 & -409.8 & 35.67 \\
\hline 700 & 72.68 & 108.66 & 38.33 & 70.32 & -517.7 & -391.7 & 29.23 \\
\hline 800 & 73.36 & 118.41 & 42.67 & 75.74 & -517.2 & -373.8 & 24.40 \\
\hline 900 & 73.65 & 127.07 & 46.10 & 80.97 & -516.8 & -355.9 & 20.65 \\
\hline
\end{tabular}

Melting $\mathbf{T}$

$\Delta_{\text {fun }} \mathrm{H}^{\circ}$

H298-H8

\section{$\mathbf{K}$}

$\mathbf{k J}$

$\mathbf{k J}$
Boiling $\mathbf{T}$

$\Delta_{\mathrm{vap}} \mathrm{H}^{\circ}$

Molar Vol.

\section{$\mathbf{R}$}

$\mathbf{k J}$

$1.661 \mathrm{~J} \cdot$ bar $^{-1}$ $16.61 \mathrm{~cm}^{3}$
$B=1.795 E-01$

$C=-1 \cdot 20 E+05$ 
$\mathrm{Mn}_{2} \mathrm{O}_{3}:$ Cubic crystals 298.15 to $1400 \mathrm{~K}$.

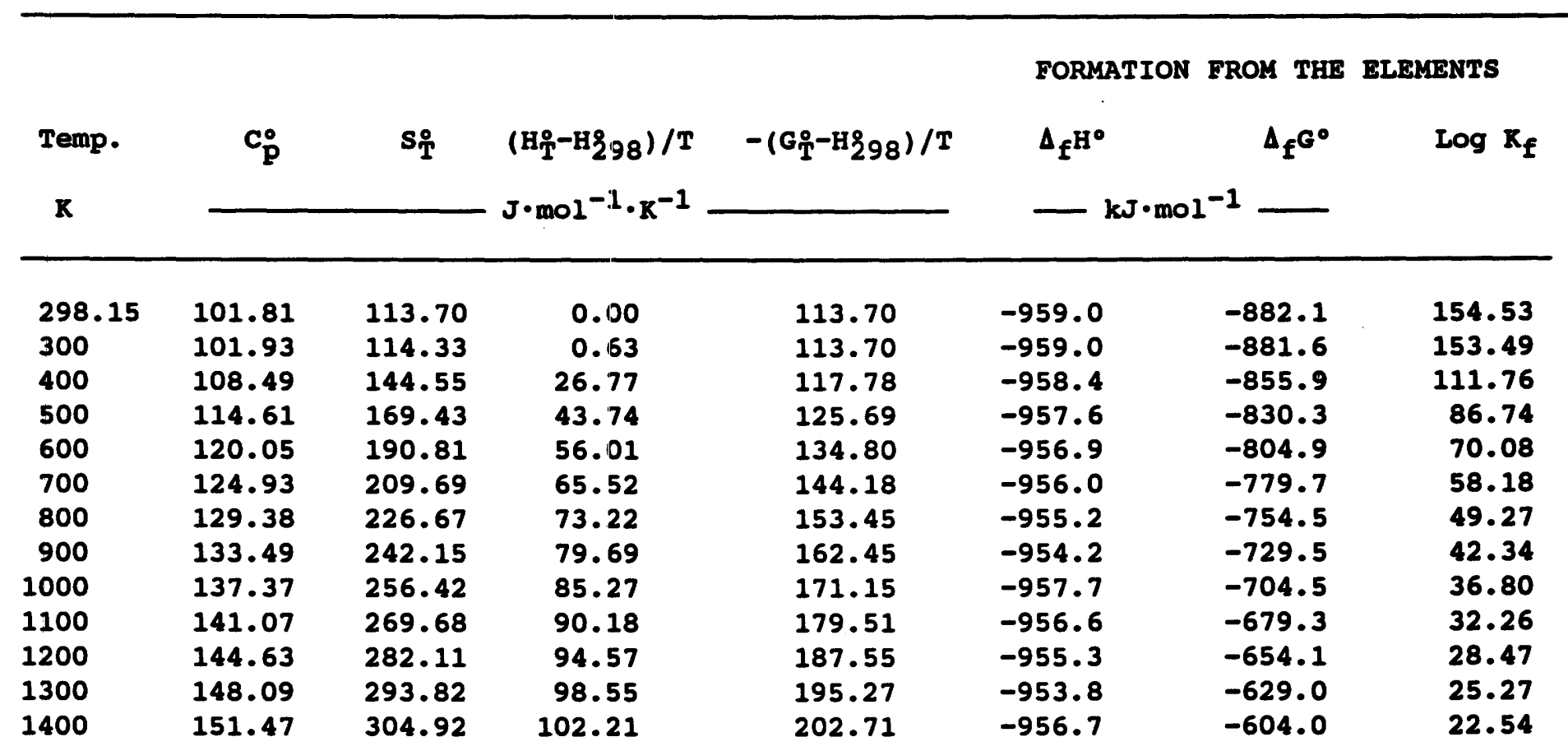

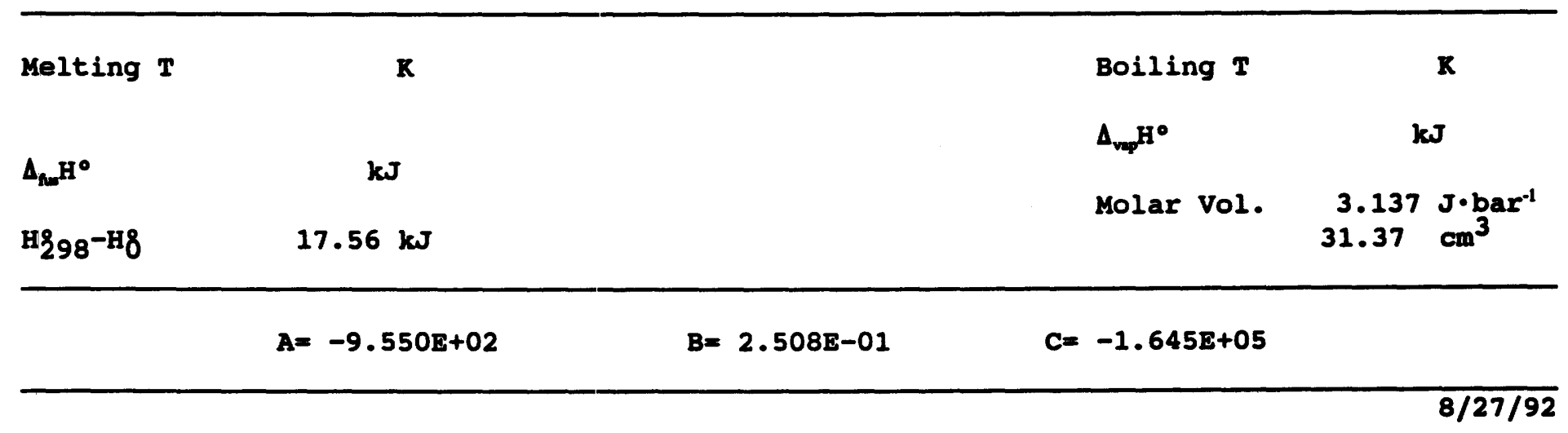


$\mathrm{Mn}_{3} \mathrm{O}_{4}$ : Tetragonal crystals 298.15 to $1400 \mathrm{~K}$.

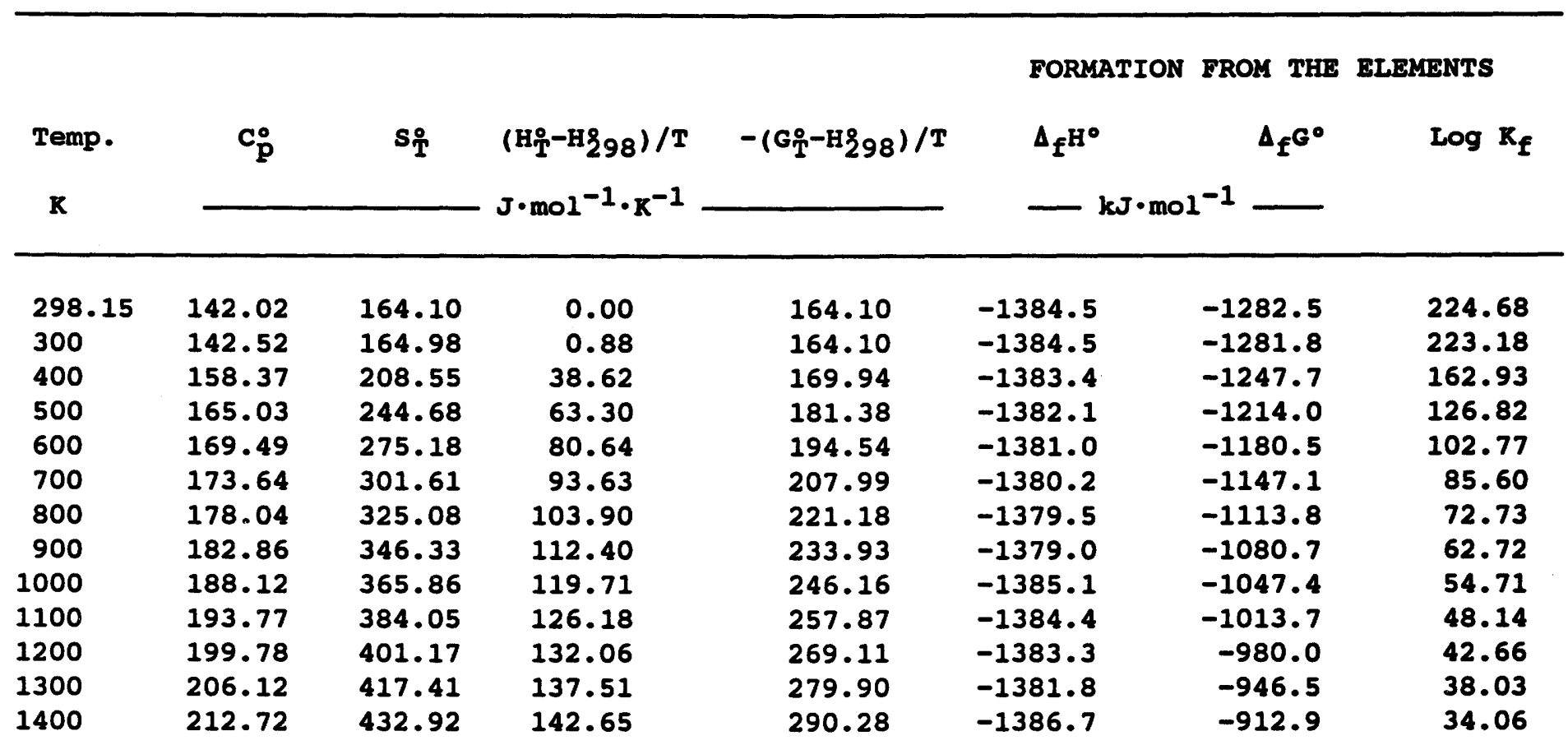

\begin{tabular}{lccc}
\hline Melting $T$ & $\mathrm{~K}$ & Boiling $\mathrm{T}$ & $\mathrm{K}$ \\
$\Delta_{\mathrm{fu}} \mathrm{H}^{\circ}$ & $\mathrm{kJ}$ & $\Delta_{\mathrm{veq}} \mathrm{H}^{\circ}$ & $\mathrm{kJ}$ \\
$\mathrm{H} 298^{-\mathrm{H} 8}$ & $24.78 \mathrm{~kJ}$ & Molar Vol. & $\begin{array}{c}4.695 \mathrm{~J} \cdot \mathrm{bar}^{-1} \\
\mathbf{3}\end{array}$ \\
\hline & $\mathrm{C}=-1.3809 \mathrm{E}+03$ & $\mathrm{Cm}$ & $\mathrm{C}=-9.874 \mathrm{E}+05$ \\
\hline
\end{tabular}


$\mathrm{Mn}_{7} \mathrm{SiO}_{12}$ : Tetragonal crystals 298.15 to $1500 \mathrm{~K}$.

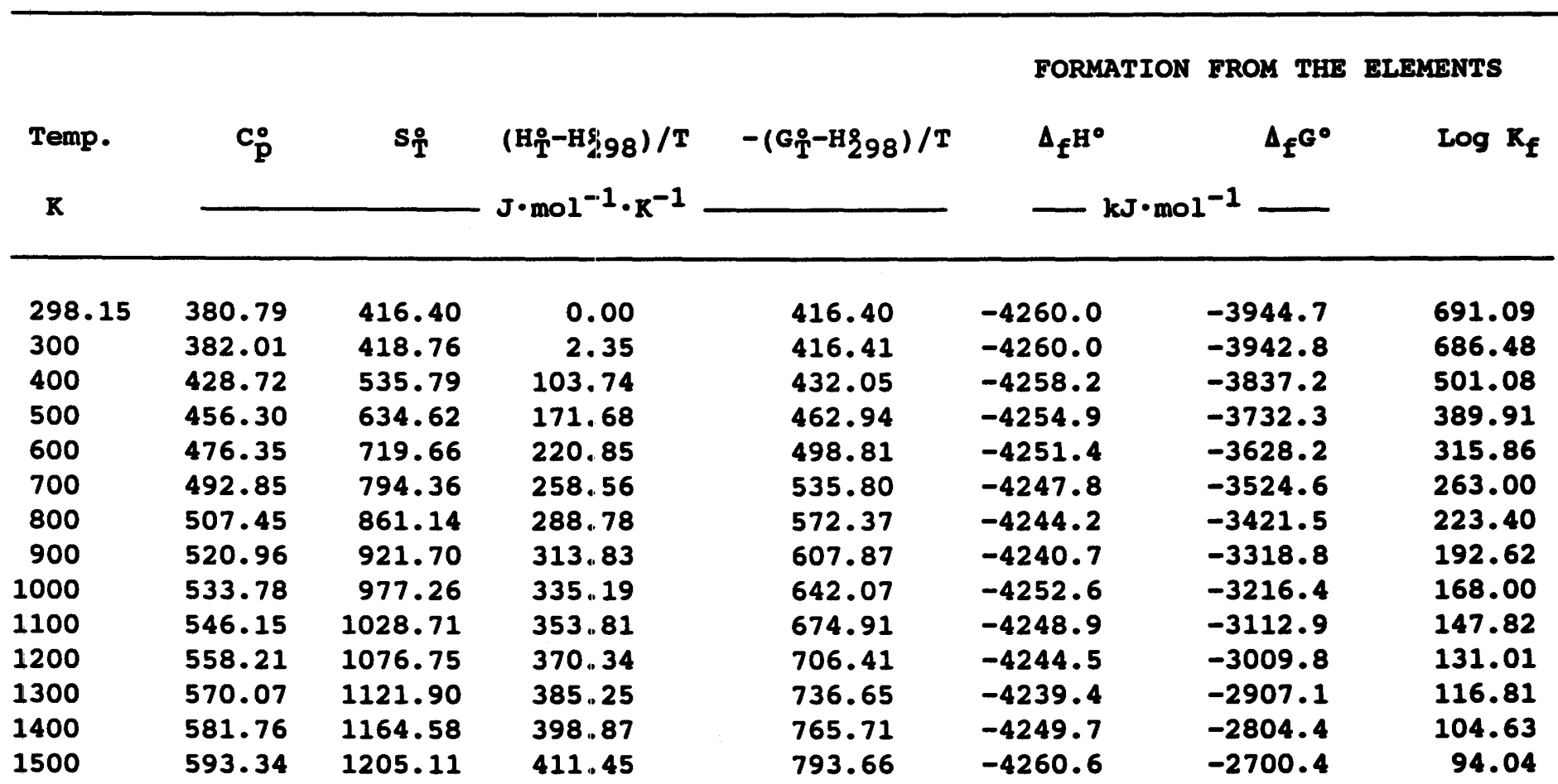

Melting $\mathbf{T}$

$\Delta_{\text {fue }} \mathrm{H}^{\circ}$

H298-H8 $\mathbf{k J}$

$\mathbf{K}$

$65.68 \mathrm{~kJ}$
Boiling T

$\Delta_{\text {vap }} H^{\circ}$

Molar Vol.
$\mathbf{X}$

$\mathbf{k J}$ $12.508 \mathrm{~J} \cdot \mathrm{bar}^{-1}$ $125.08 \mathrm{~cm}^{3}$
$B=1.0286 \mathrm{E}+00$

$C=-6.946 E+05$ 
$\mathrm{MOO}_{3}$ : Orthorhombic crystals 298.15 to melting point $1074 \mathrm{~K}$; liquid 1074 to $1500 \mathrm{~K}$.

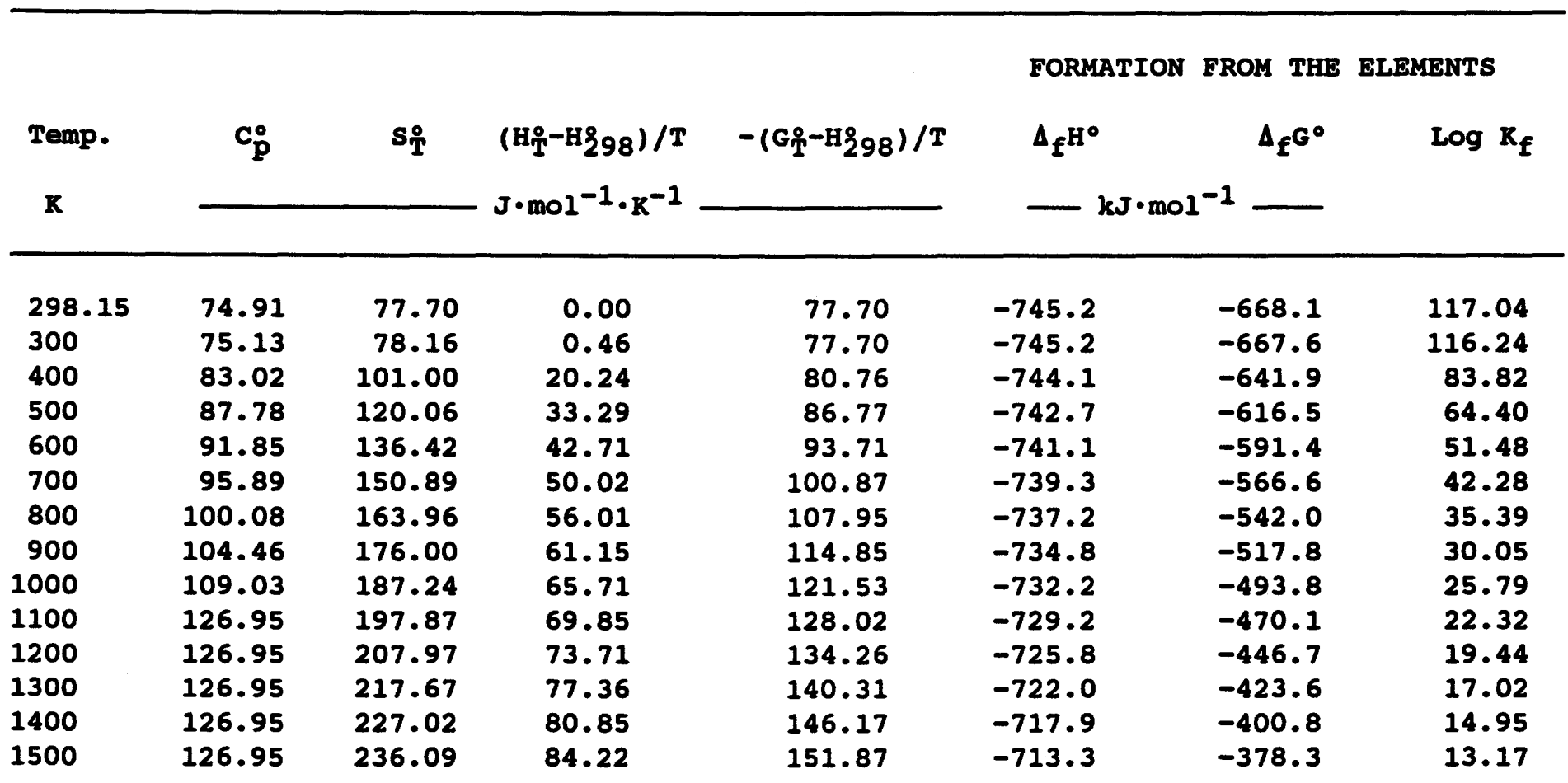

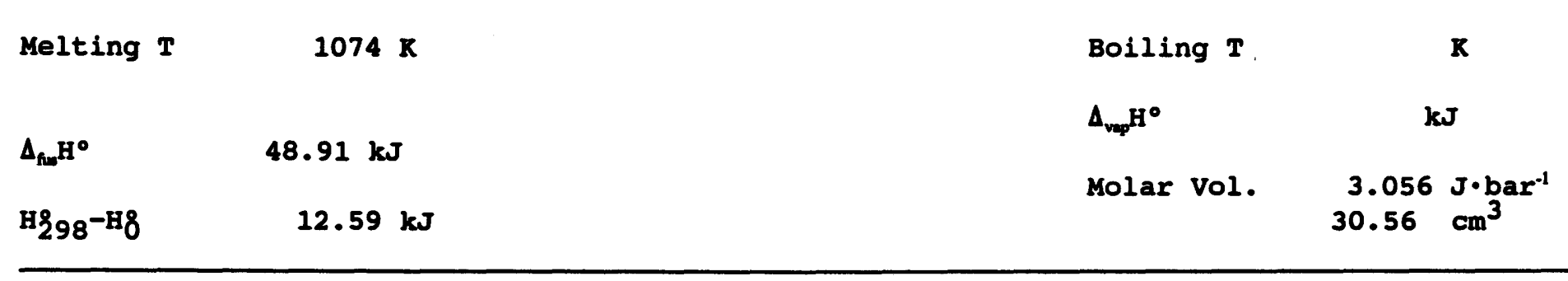
$A=-7.299 \mathrm{E}+02$
$B=2 \cdot 358 E-01$
$C=-8.02 E+05$ 


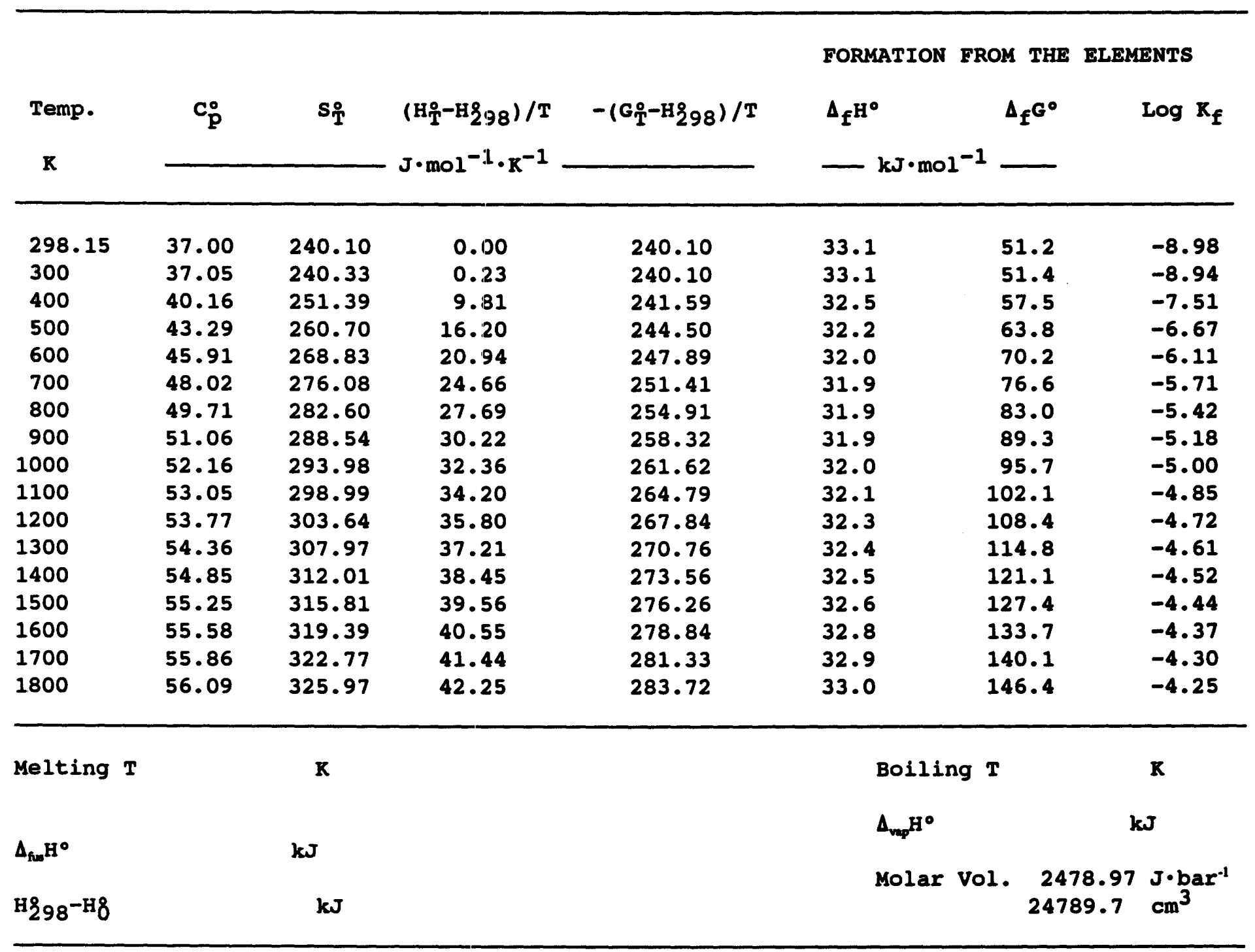


$\mathrm{Na}_{2} \mathrm{O}$ : Cubic crystals 298.15 to melting point $1193 \mathrm{~K}$.

\begin{tabular}{|c|c|c|c|c|c|c|c|}
\hline \multirow{3}{*}{$\begin{array}{l}\text { Temp. } \\
\mathbf{K}\end{array}$} & \multirow{3}{*}{$\mathbf{C}_{\mathbf{p}}^{\circ}$} & \multirow{3}{*}{$s_{\text {T }}^{\circ}$} & \multirow{3}{*}{$\begin{array}{l}\left(\mathrm{H}_{\mathrm{T}}^{\circ}-\mathrm{H} 298\right) / \mathrm{T} \\
\mathrm{J} \cdot \mathrm{mol}^{-1} \cdot \mathrm{K}^{-1}\end{array}$} & \multirow{3}{*}{$-\left(G_{T}^{\circ}-H_{298}\right) / T$} & \multicolumn{2}{|c|}{ FORMATION FROM THE } & \multirow{3}{*}{$\begin{array}{l}\text { ELEMENTS } \\
\qquad \log R_{f}\end{array}$} \\
\hline & & & & & $\Delta_{f^{H^{\circ}}}$ & $\Delta_{f} G^{\circ}$ & \\
\hline & & & & & \multicolumn{2}{|c|}{$\mathrm{kJ} \cdot \mathrm{mol}^{-1}$} & \\
\hline 298.15 & 69.10 & 75.30 & 0.00 & 75.30 & -414.8 & -376.0 & 65.87 \\
\hline 300 & 69.26 & 75.73 & 0.43 & 75.30 & -414.8 & -375.7 & 65.42 \\
\hline 400 & 76.30 & 96.68 & 18.57 & 78.11 & -420.2 & -362.3 & 47.31 \\
\hline 500 & 81.34 & 114.27 & 30.64 & 83.63 & -420.1 & -347.8 & 36.33 \\
\hline 600 & 85.26 & 129.46 & 39.43 & 90.03 & -419.3 & -333.4 & 29.03 \\
\hline 700 & 88.47 & 142.85 & 46.21 & 96.64 & -418.2 & -319.2 & 23.82 \\
\hline 800 & 91.20 & 154.85 & 51.67 & 103.18 & -416.7 & -305.1 & 19.92 \\
\hline 900 & 93.60 & 165.73 & 56.20 & 109.53 & -414.9 & -291.3 & 16.91 \\
\hline 1000 & 95.74 & 175.71 & 60.05 & 115.66 & -413.0 & -277.7 & 14.50 \\
\hline
\end{tabular}

\begin{tabular}{lccc}
\hline Melting $T$ & $1193 \mathrm{~K}$ & Boiling $T$ & $\mathrm{~K}$ \\
$\Delta_{\mathrm{fu}} \mathrm{H}^{\circ}$ & $\mathrm{kJ}$ & $\Delta_{\mathrm{veg}} \mathrm{H}^{\circ}$ & $\mathrm{kJ}$ \\
$\mathrm{H} 298^{-\mathrm{H} 8}$ & $12.40 \mathrm{~kJ}$ & Yolar Vol. & $\begin{array}{c}2.588 \mathrm{~J} \cdot \mathrm{bar}^{-1} \\
25.88 \\
\mathrm{Cm}^{3}\end{array}$ \\
\hline & $\mathrm{A}=-4.180 \mathrm{E}+02$ & $\mathrm{~B}=1.407 \mathrm{E}-01$ & $\mathrm{C}=-5.93 \mathrm{E}+03$ \\
\hline
\end{tabular}


Nio: Rhombohedral (distorted cubic) crystals 298.15 to Nél temperature 519 K. Cubic crystals 519 to $1800 \mathrm{~K}$.

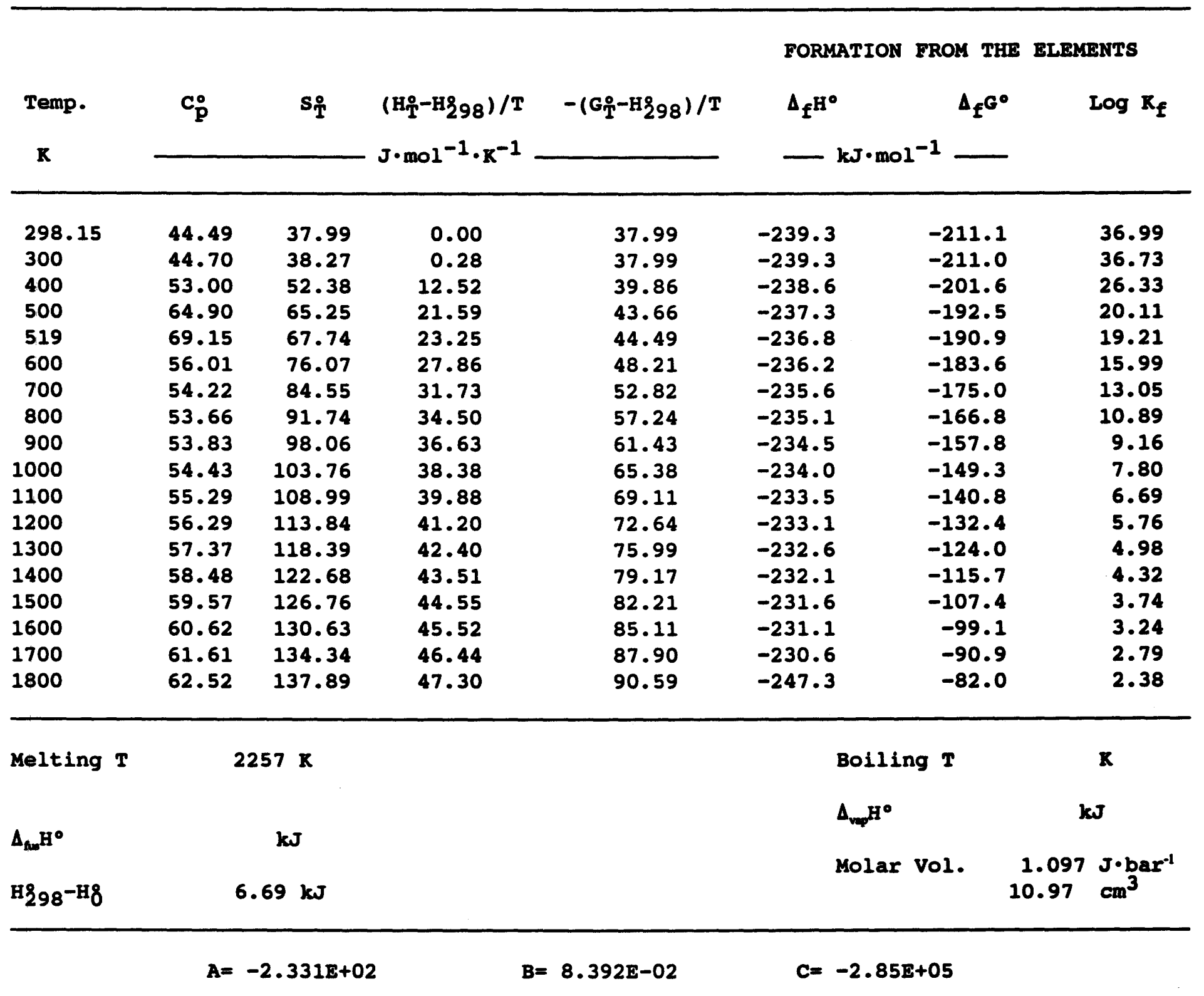


Pbo: Tetragonal crystals (red) 298.15 to $1000 \mathrm{~K}$.

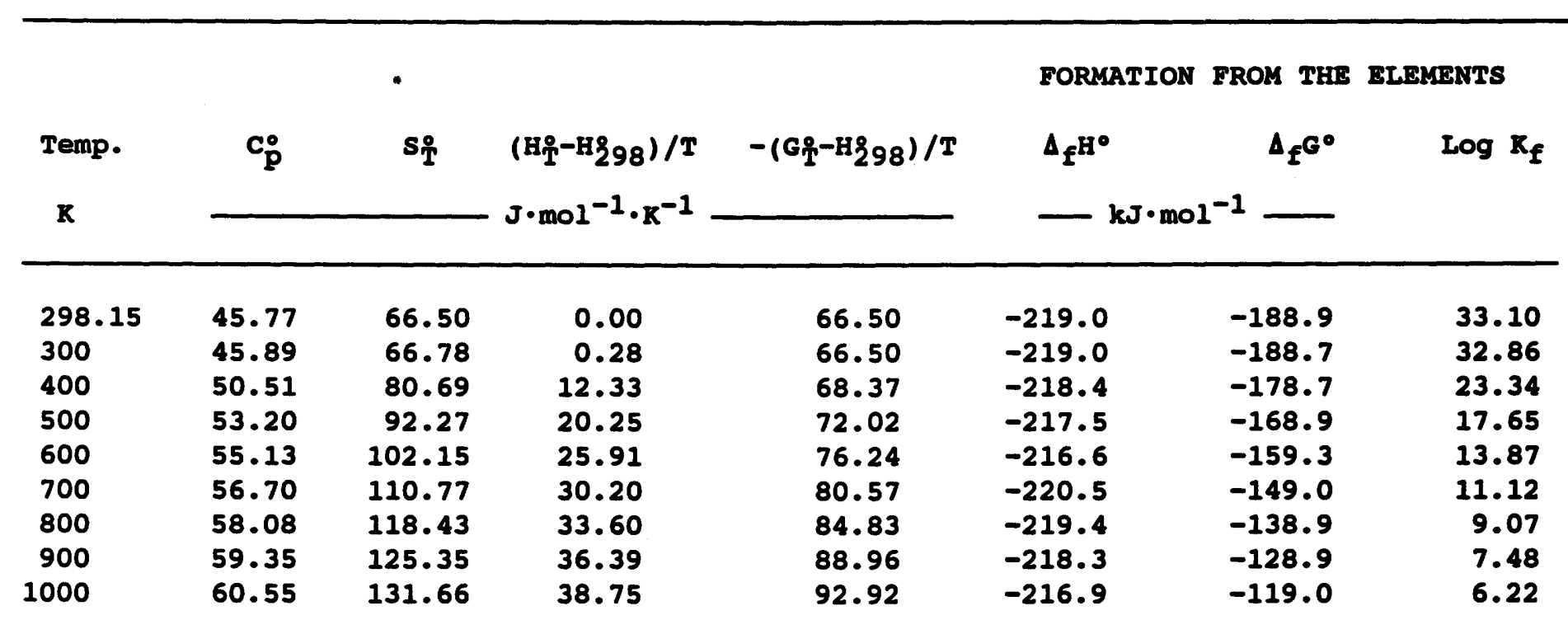

\begin{tabular}{|c|c|c|c|}
\hline Melting $T$ & $\mathbf{K}$ & Boiling $T$ & $\mathbf{K}$ \\
\hline & & $\Delta_{\mathrm{vep}} \mathrm{H}^{\circ}$ & $\mathbf{k J}$ \\
\hline$\Delta_{\text {fue }} \mathrm{H}^{\circ}$ & $\mathbf{k J}$ & Molar vol & 2 391 T.har-1 \\
\hline H298-H8 & $\mathbf{k J}$ & Molar vol. & $23.91 \mathrm{~cm}^{3}$ \\
\hline
\end{tabular}
$A=-2 \cdot 194 \mathrm{E}+02$
$B=1.004 \mathrm{E}-01$
$C=4.877 \mathrm{E}+04$ 
$\mathrm{PbO}_{2}$ : Tetragonal crystals 298.15 to $1200 \mathrm{~K}$.

\begin{tabular}{|c|c|c|c|c|c|c|c|}
\hline \multirow[b]{2}{*}{ Temp. } & \multirow[b]{2}{*}{$\mathbf{c}_{\mathbf{p}}^{\circ}$} & \multirow[b]{2}{*}{$\mathbf{s}_{\mathrm{T}}$} & \multirow{3}{*}{$\begin{array}{l}\left(\mathrm{H}_{\mathrm{T}}-\mathrm{H}_{298}\right) / \mathrm{T} \\
\mathrm{J} \cdot \mathrm{mol}^{-1} \cdot \mathrm{K}^{-1}\end{array}$} & \multirow[b]{2}{*}{$-\left(G_{T}^{\circ}-H_{298}\right) / T$} & \multicolumn{2}{|c|}{ FORMATION FROM THE } & \multirow{2}{*}{$\begin{array}{l}\text { ELEMENTS } \\
\text { Iog } R_{f}\end{array}$} \\
\hline & & & & & $\Delta_{f} H^{\circ}$ & $\Delta_{f} G^{0}$ & \\
\hline $\mathbf{K}$ & & & & & $\ldots \mathrm{kJ} \cdot \mathrm{mol}$ & -1 & \\
\hline 298.15 & 61.17 & 71.80 & 0.00 & 71.80 & -277.4 & $-218 \cdot 3$ & 38.25 \\
\hline 300 & 61.35 & 72.18 & 0.38 & 71.80 & -277.4 & -218.0 & 37.95 \\
\hline 400 & 68.23 & 90.90 & 16.59 & 74.31 & -276.6 & $-198 \cdot 3$ & 25.89 \\
\hline 500 & 71.82 & 106.54 & 27.31 & 79.23 & -275.4 & -178.8 & 18.68 \\
\hline 600 & 74.11 & 119.85 & 34.93 & 84.92 & -274.2 & -159.6 & 13.89 \\
\hline 700 & 75.79 & 131.41 & 40.65 & 90.76 & -277.8 & -139.8 & 10.43 \\
\hline 800 & 77.14 & 141.62 & 45.13 & 96.49 & -276.5 & -120.2 & 7.85 \\
\hline 900 & 78.30 & 150.77 & 48.75 & 102.02 & -275.1 & -100.7 & 5.84 \\
\hline 1000 & 79.34 & 159.08 & 51.76 & 107.32 & -273.7 & -81.4 & 4.25 \\
\hline 1100 & 80.31 & 166.69 & 54.31 & 112.37 & -272.1 & -62.2 & 2.96 \\
\hline 1200 & 81.23 & 173.71 & 56.52 & 117.20 & -270.5 & -43.2 & 1.88 \\
\hline
\end{tabular}

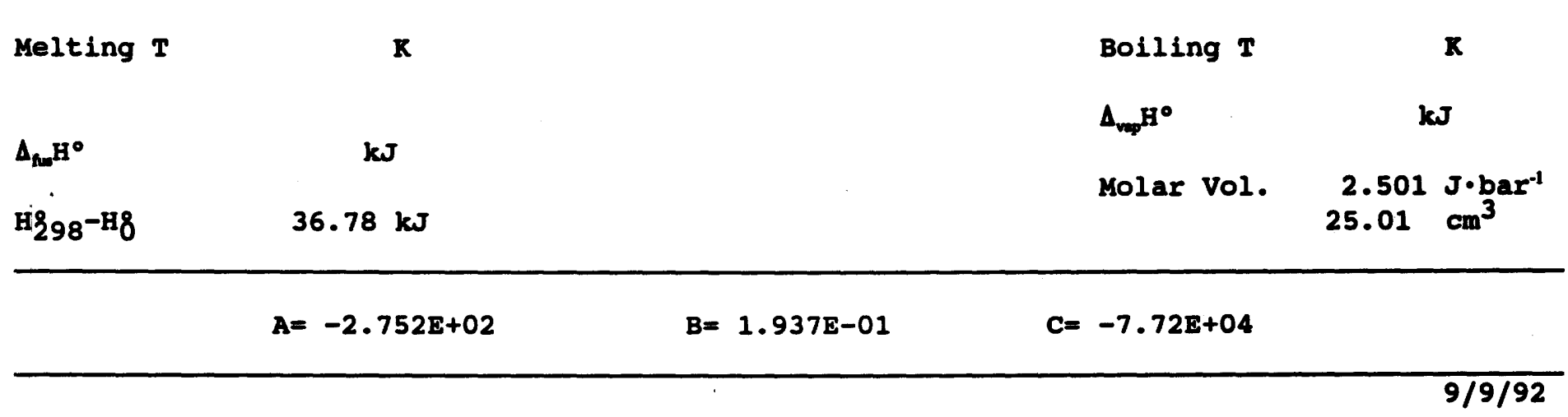


$\mathrm{Pb}_{3} \mathrm{O}_{4}$ : Tetragonal crystals 298.15 to $1800 \mathrm{~K}$.

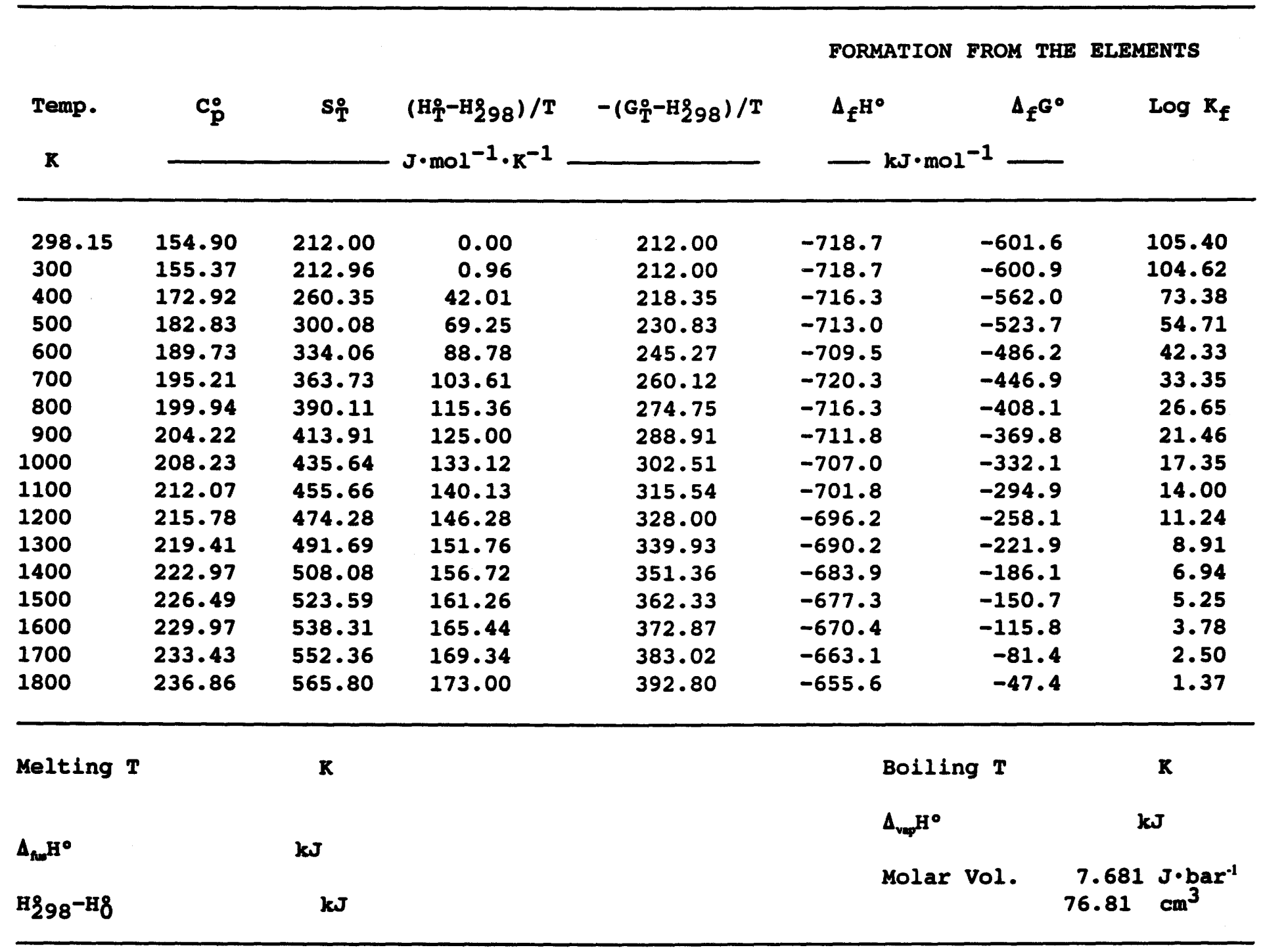


$\mathrm{SO}_{2}$ : Ideal gas at $\mathrm{p}=1$ bar, 298.15 to $2500 \mathrm{~K}$.

FORMATION PROY THE ELEMENTS

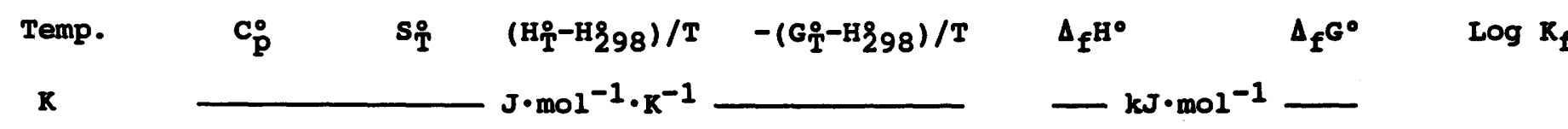

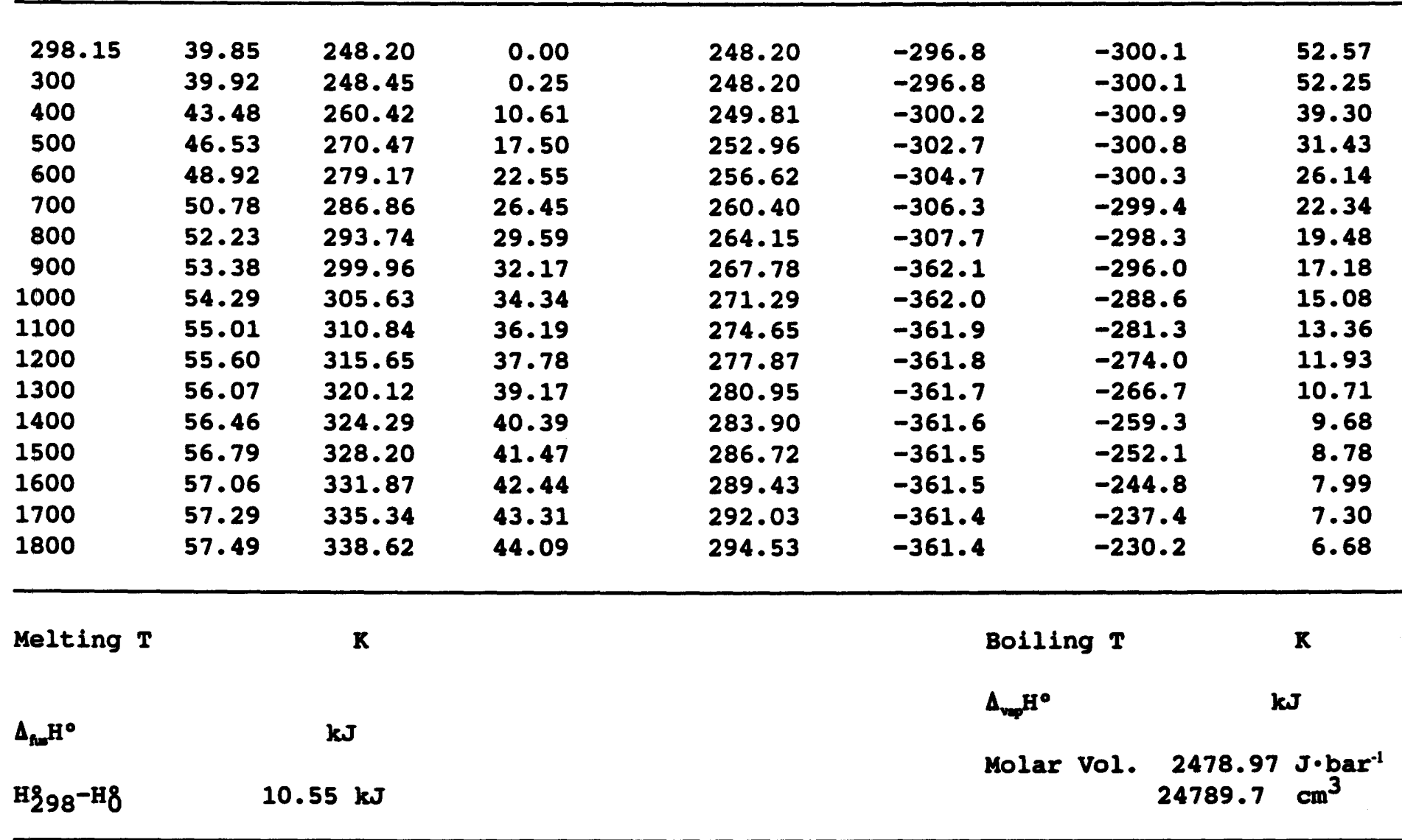
$A=-3.536 \mathrm{E}+02$
$\mathrm{B}=6.628 \mathrm{E}-02$
$C=3.246 \mathrm{E}+06$ 


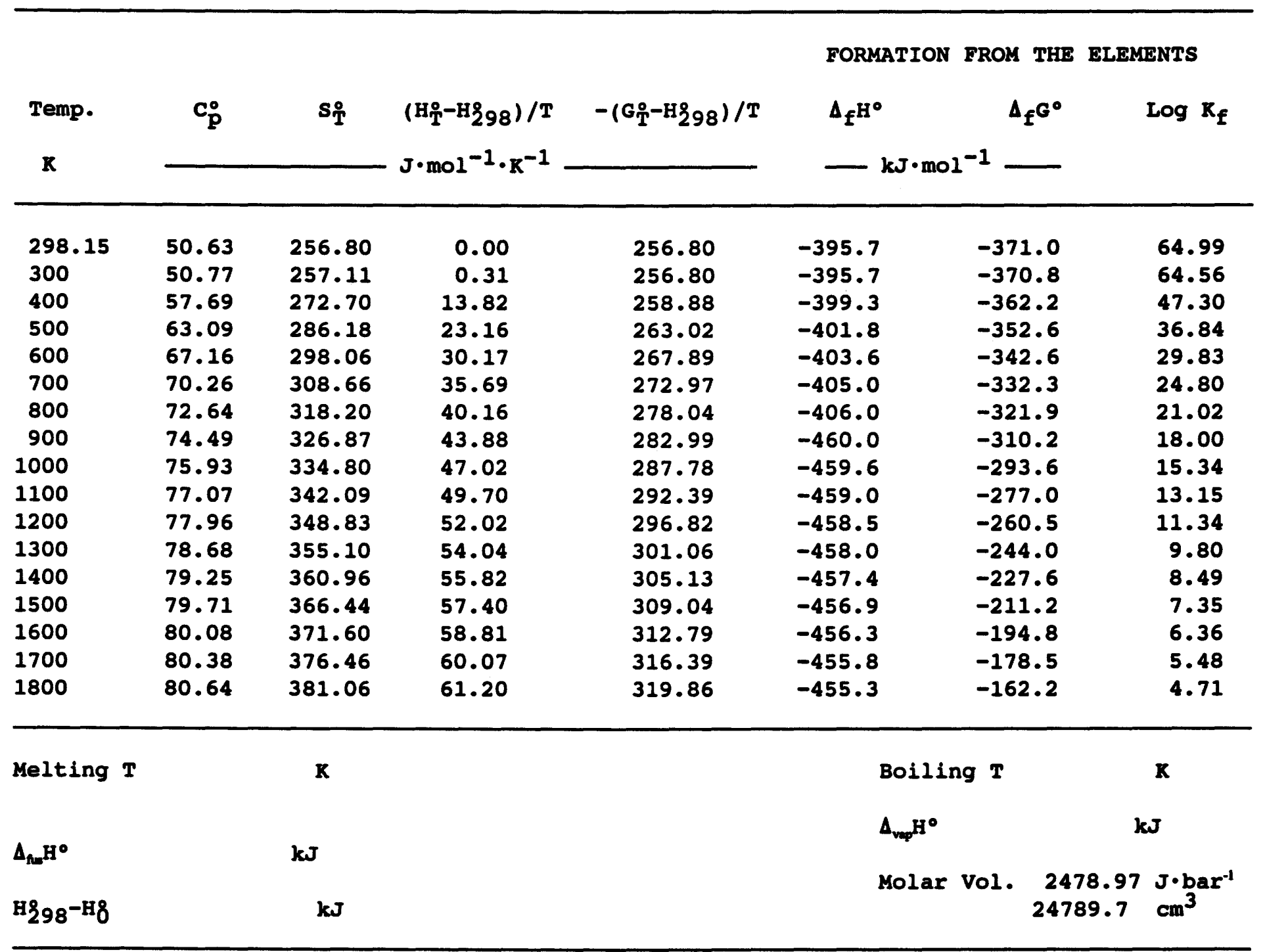


Sio: Ideal gas at $p=1$ bar, 298.15 to $1800 \mathrm{~K}$.

FORMATION FROM THE ELEMENTS

\begin{tabular}{|c|c|c|c|c|}
\hline Temp. & $\mathbf{C}_{\mathbf{p}}^{\circ}$ & $\mathbf{s}_{\mathbf{T}}^{\circ}$ & (H首-H298)/T & $-\left(G_{T}^{\circ}-H_{298}\right) / T$ \\
\hline
\end{tabular}

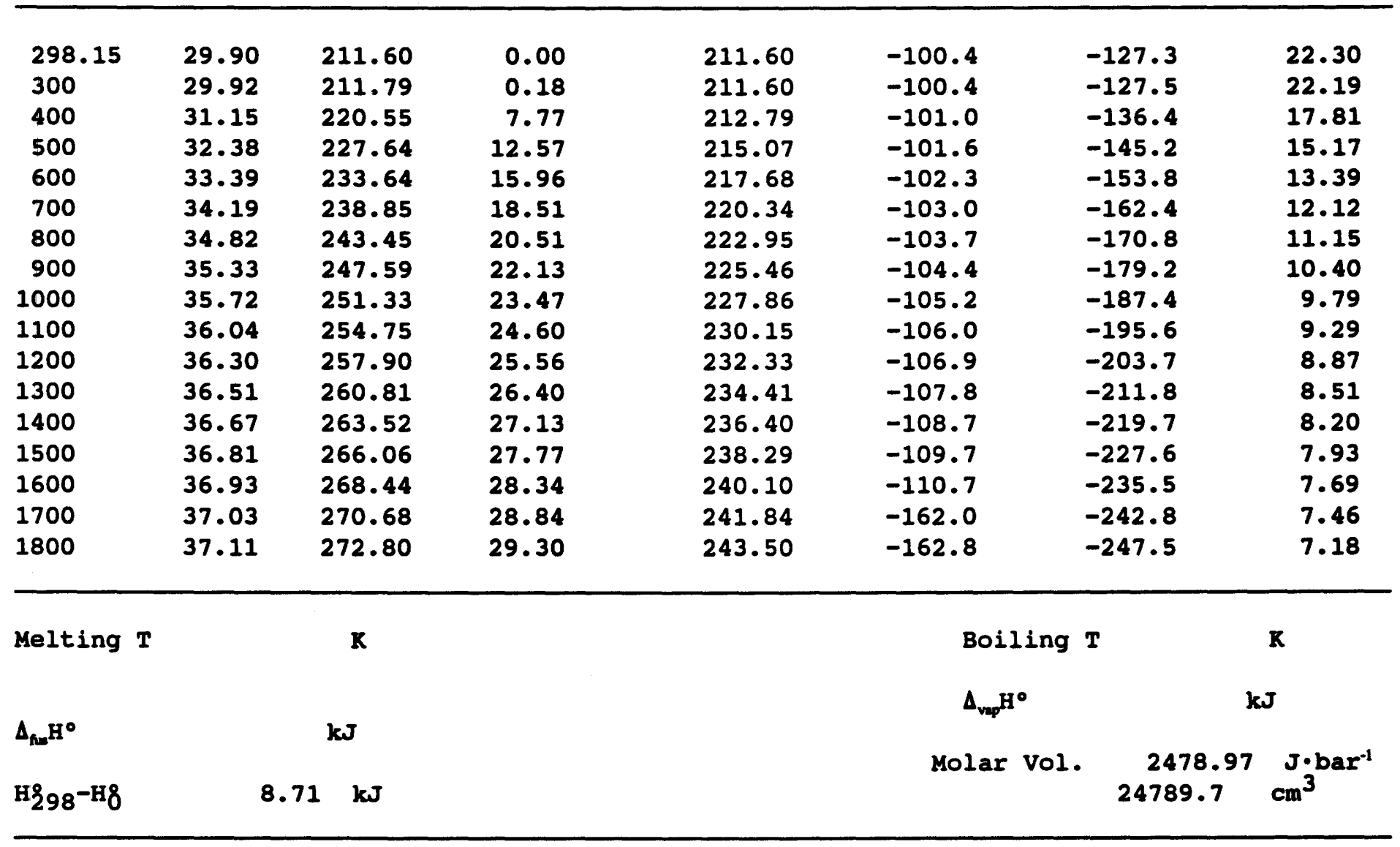

$A=-1.084 E+02 \quad B=-7.908 E-02 \quad C=4.562 E+05$ 
$\mathrm{SiO}_{2}$ : Trigonal crystals 298.15 to $\alpha-\beta$ transition at $844 \mathrm{~K}$. $\beta$-crystals 844 to 1700. The melting point is estimated to be $1700 \mathrm{~K}$. Liquid 1700 to $1800 \mathrm{~K}$.

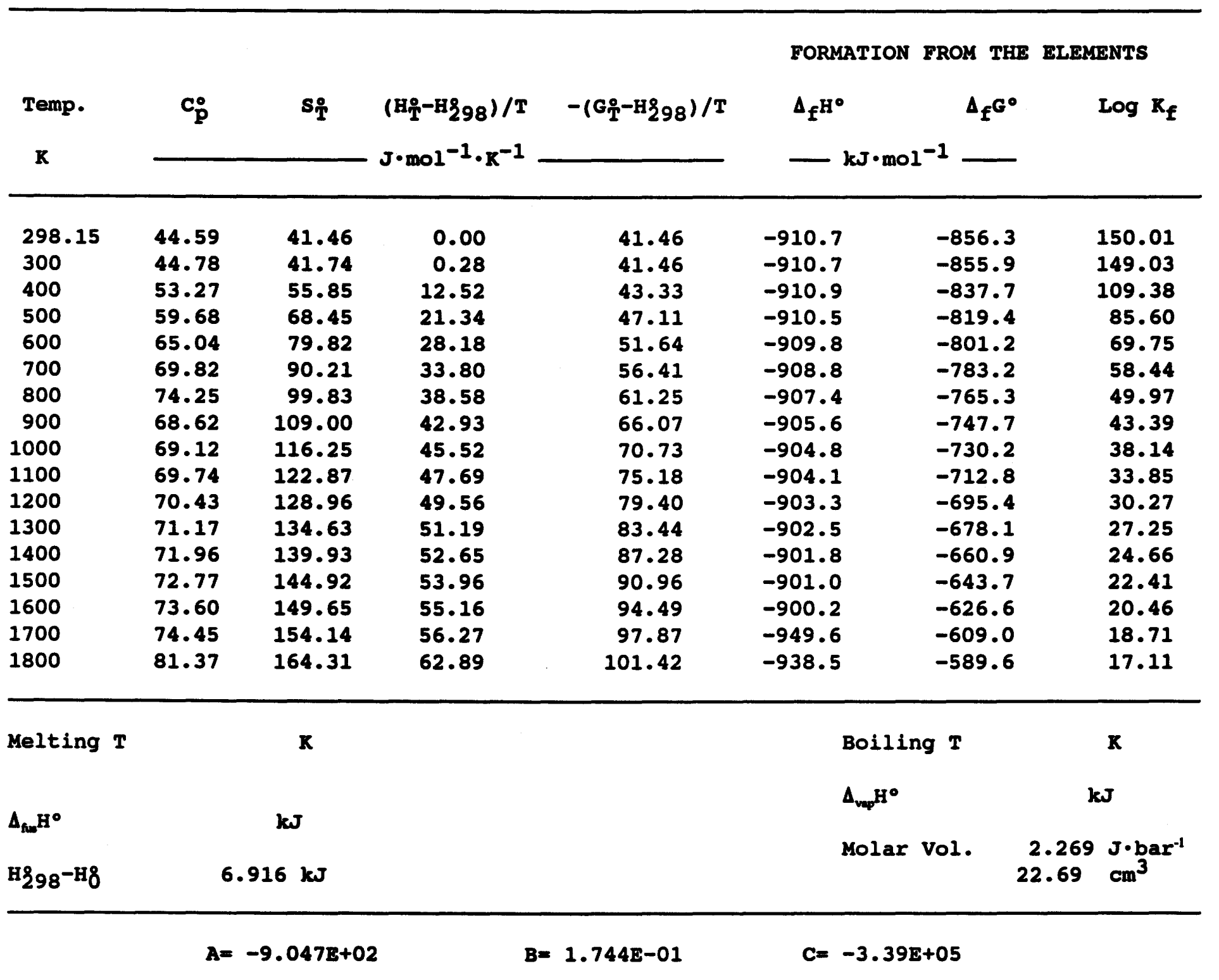




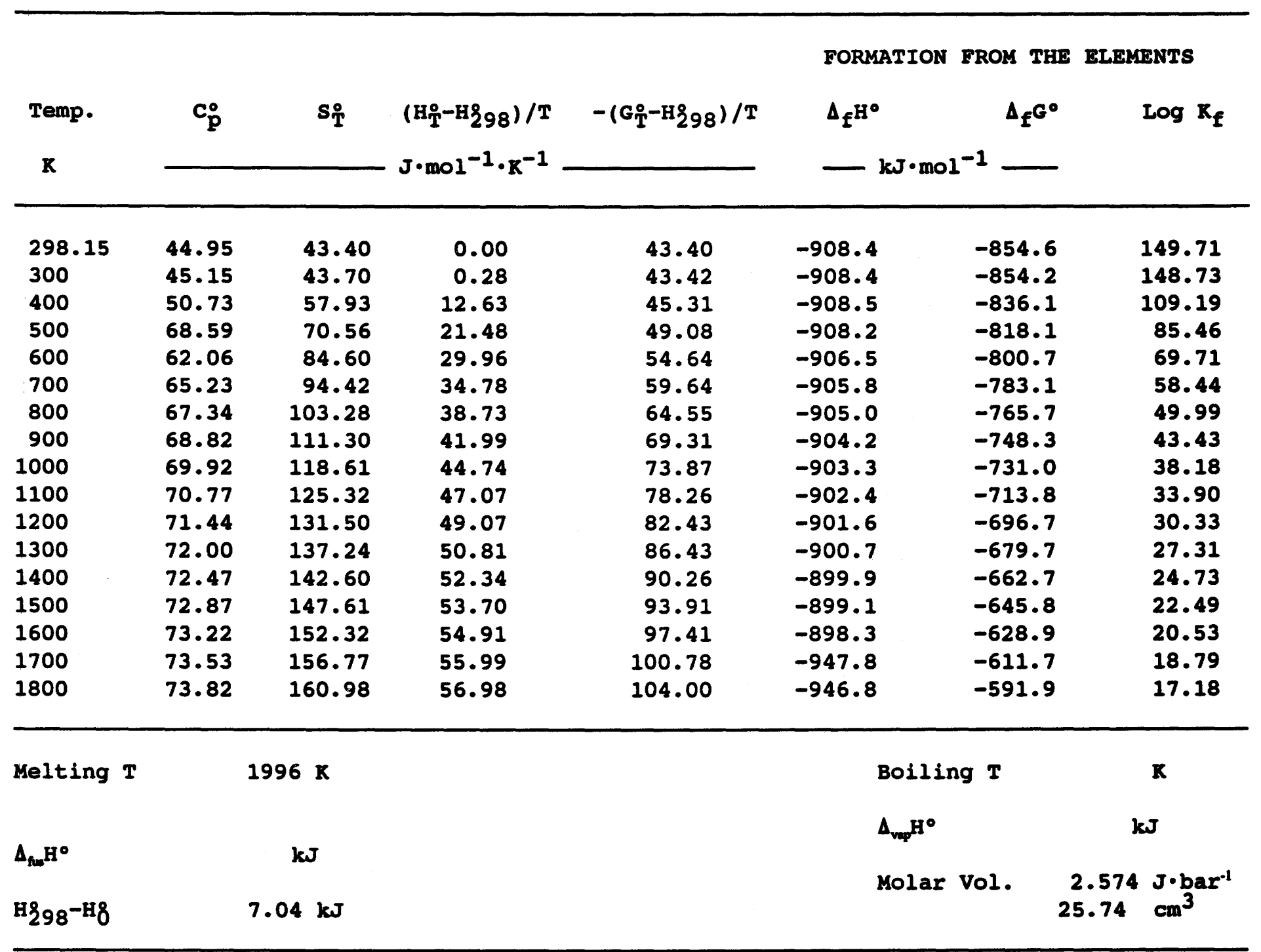

$$
A=-9.029 E+02 \quad B=1.719 E-01 \quad C=-2.62 E+05
$$


$\mathrm{SiO}_{2}$ : Monoclinic crystals 298.15 to $1800 \mathrm{~K}$.

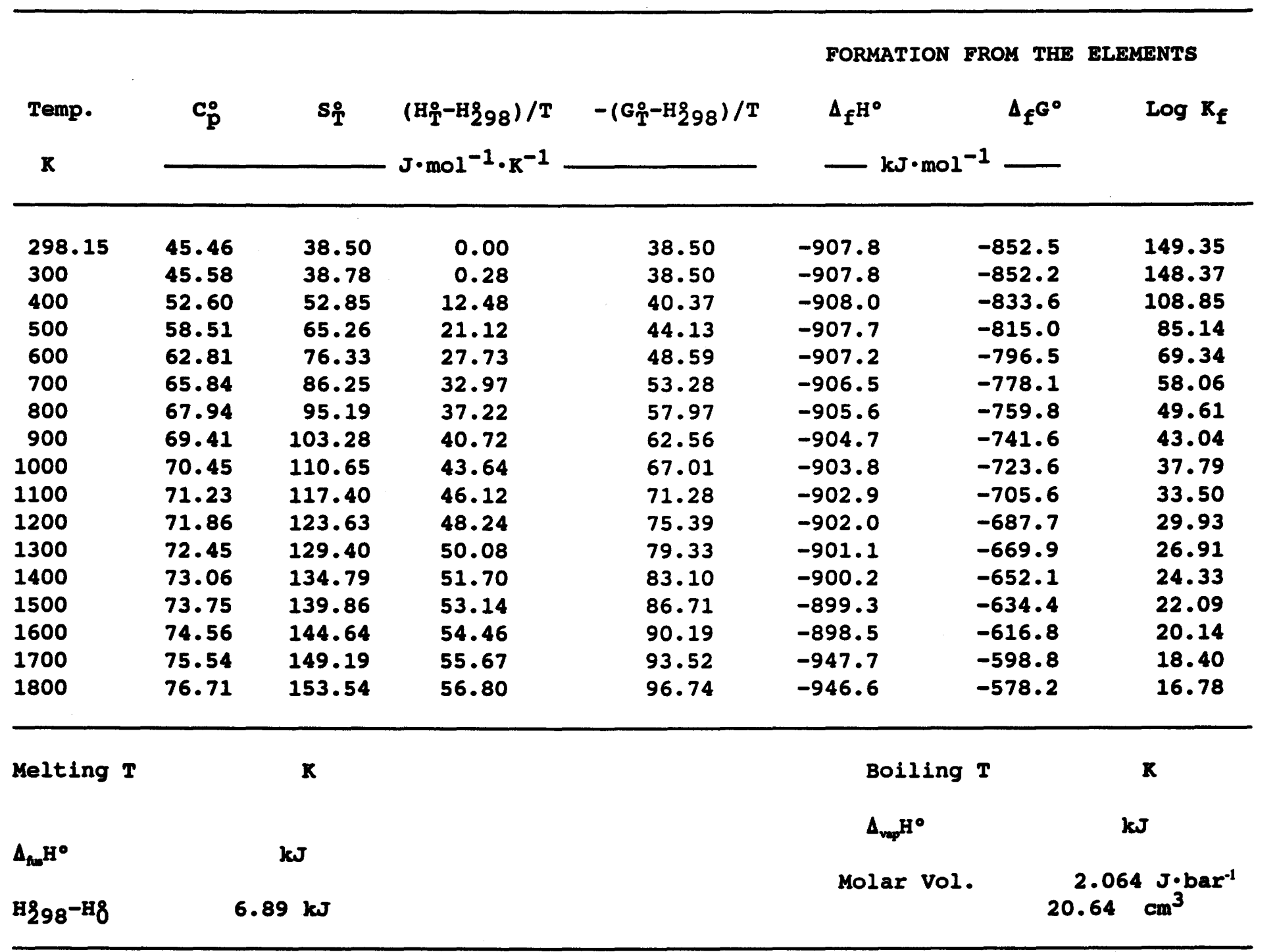
$A=-9.036 \mathrm{E}+02$
$B=1.799 E-01$
$C=-2.39 E+05$ 
$\mathrm{SiO}_{2}$ : Tetragonal crystals 298.15 to $1800 \mathrm{~K}$.

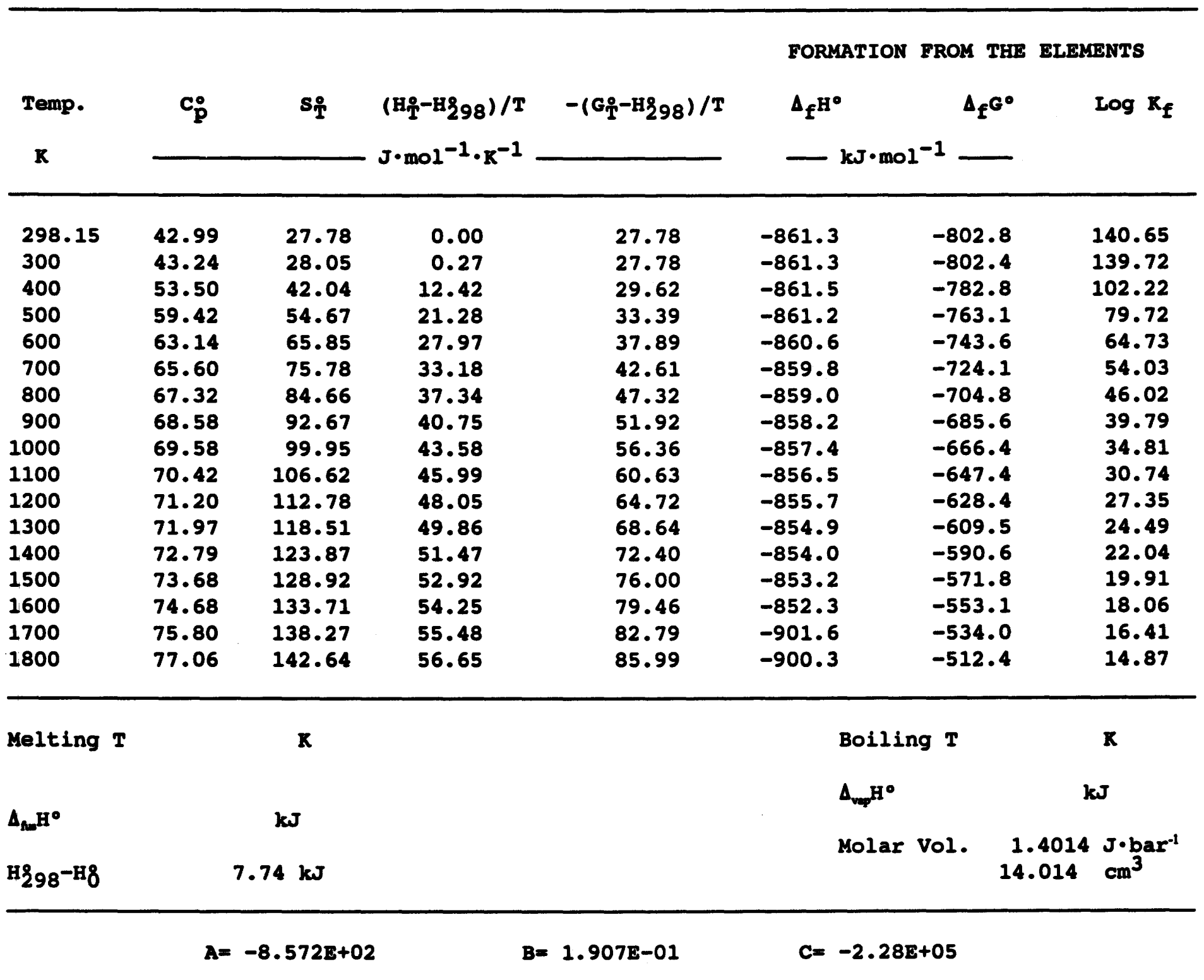


$\mathrm{SiO}_{2}$ : Glass 298.15 to estimated melting point $1700 \mathrm{~K}$.

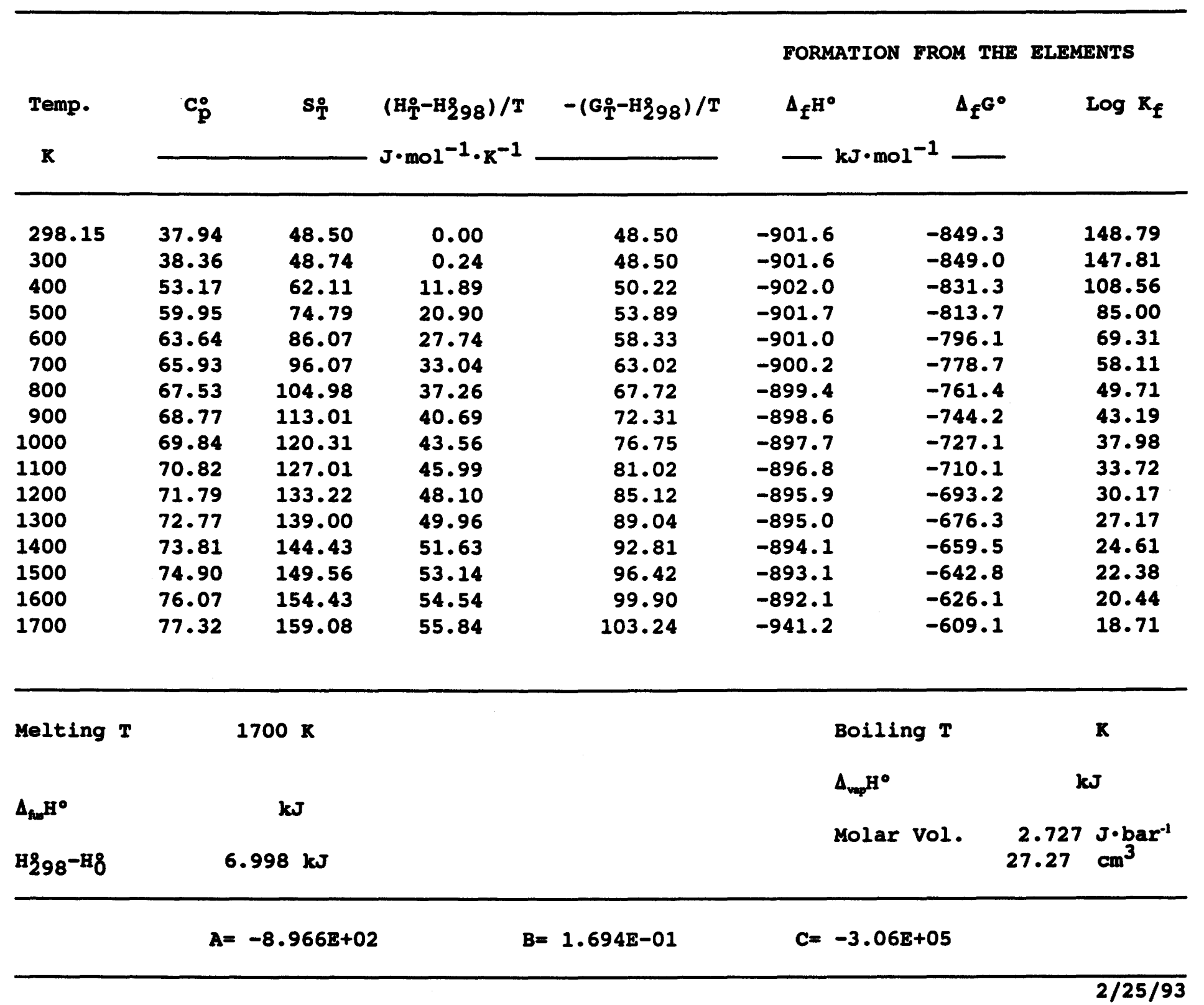


$\mathrm{SnO}_{2}:$ Tetragonal crystals 298.15 to $1903 \mathrm{~K}$.

\begin{tabular}{|c|c|c|c|c|c|c|c|}
\hline \multirow{3}{*}{$\begin{array}{l}\text { Temp. } \\
\mathbf{K}\end{array}$} & \multirow{3}{*}{$\mathbf{c}_{\mathbf{p}}^{\circ}$} & \multirow{3}{*}{$\mathbf{s} \stackrel{9}{\mathbf{T}}$} & \multirow{3}{*}{$\begin{array}{l}\left(\mathrm{H}_{\mathrm{T}}-\mathrm{H}_{298}\right) / \mathrm{T} \\
\mathrm{J} \cdot \mathrm{mol}^{-1} \cdot \mathrm{K}^{-1}\end{array}$} & \multirow{3}{*}{$-\left(G_{T}^{\circ}-H_{298}\right) / T$} & \multicolumn{2}{|c|}{ FORMATION FROM THE } & \multirow{3}{*}{$\begin{array}{l}\text { ELEMENTS } \\
\qquad \log \mathrm{K}_{\mathbf{f}}\end{array}$} \\
\hline & & & & & $\Delta_{f^{H}} H^{\circ}$ & $\Delta_{f^{G}} G^{0}$ & \\
\hline & & & & & \multicolumn{2}{|c|}{$\mathrm{kJ} \cdot \mathrm{mol}^{-1}$} & \\
\hline 298.15 & 53.22 & 49.02 & 0.00 & 49.02 & -577.6 & -515.8 & 90.36 \\
\hline 300 & 53.54 & 49.35 & 0.33 & 49.02 & -577.6 & -515.4 & 89.74 \\
\hline 400 & 65.09 & 66.56 & 15.27 & 51.29 & $-577 \cdot 4$ & -494.7 & 64.60 \\
\hline 500 & 70.83 & 81.76 & 25.86 & 55.90 & -576.6 & -474.1 & 49.53 \\
\hline 600 & 74.28 & 95.00 & 33.66 & 61.34 & -582.6 & -452.3 & 39.38 \\
\hline 700 & 76.66 & 106.64 & 39.64 & 66.99 & $-581 \cdot 1$ & -430.8 & 32.14 \\
\hline 800 & 78.46 & 117.00 & 44.39 & 72.61 & -579.5 & -409.4 & 26.73 \\
\hline 900 & 79.92 & 126.32 & 48.26 & 78.07 & -577.8 & -388.2 & 22.53 \\
\hline 1000 & 81.18 & 134.81 & 51.49 & 83.32 & -576.0 & -367.3 & 19.18 \\
\hline 1100 & 82.30 & 142.60 & 54.24 & 88.36 & -574.1 & -346.5 & 16.45 \\
\hline 1200 & 83.33 & 149.81 & 56.62 & 93.19 & -572.2 & -325.9 & 14.18 \\
\hline 1300 & 84.30 & 156.52 & 58.71 & 97.80 & -570.2 & -305.4 & 12.27 \\
\hline 1400 & 85.21 & 162.80 & 60.57 & 102.22 & -568.2 & -285.1 & 10.64 \\
\hline 1500 & 86.10 & 168.71 & 62.25 & 106.46 & -566.1 & -265.0 & 9.23 \\
\hline
\end{tabular}

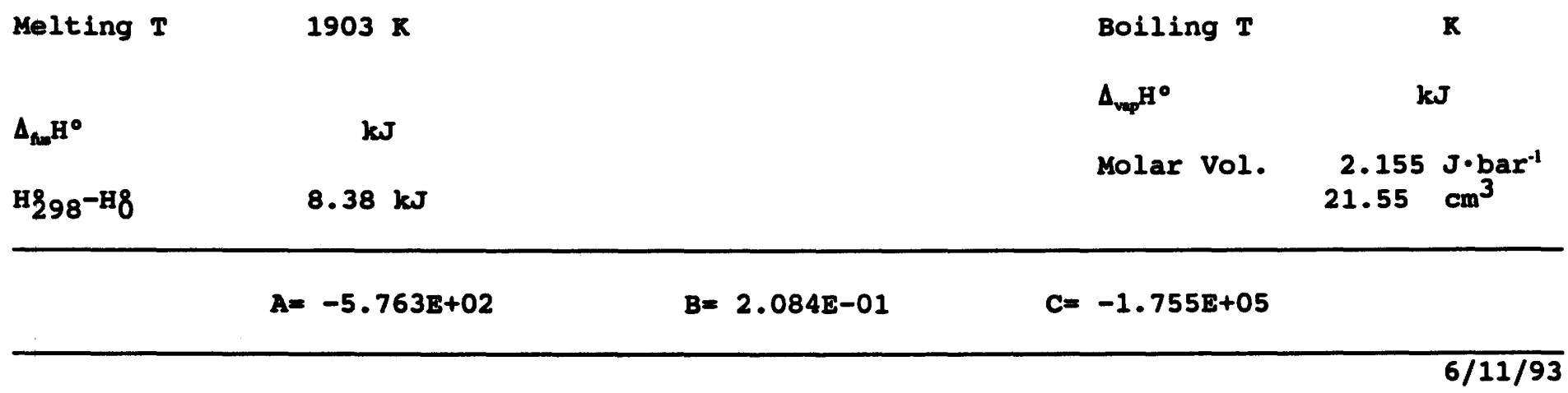


Sro: Cubic crystals 298.15 to $1800 \mathrm{~K}$.

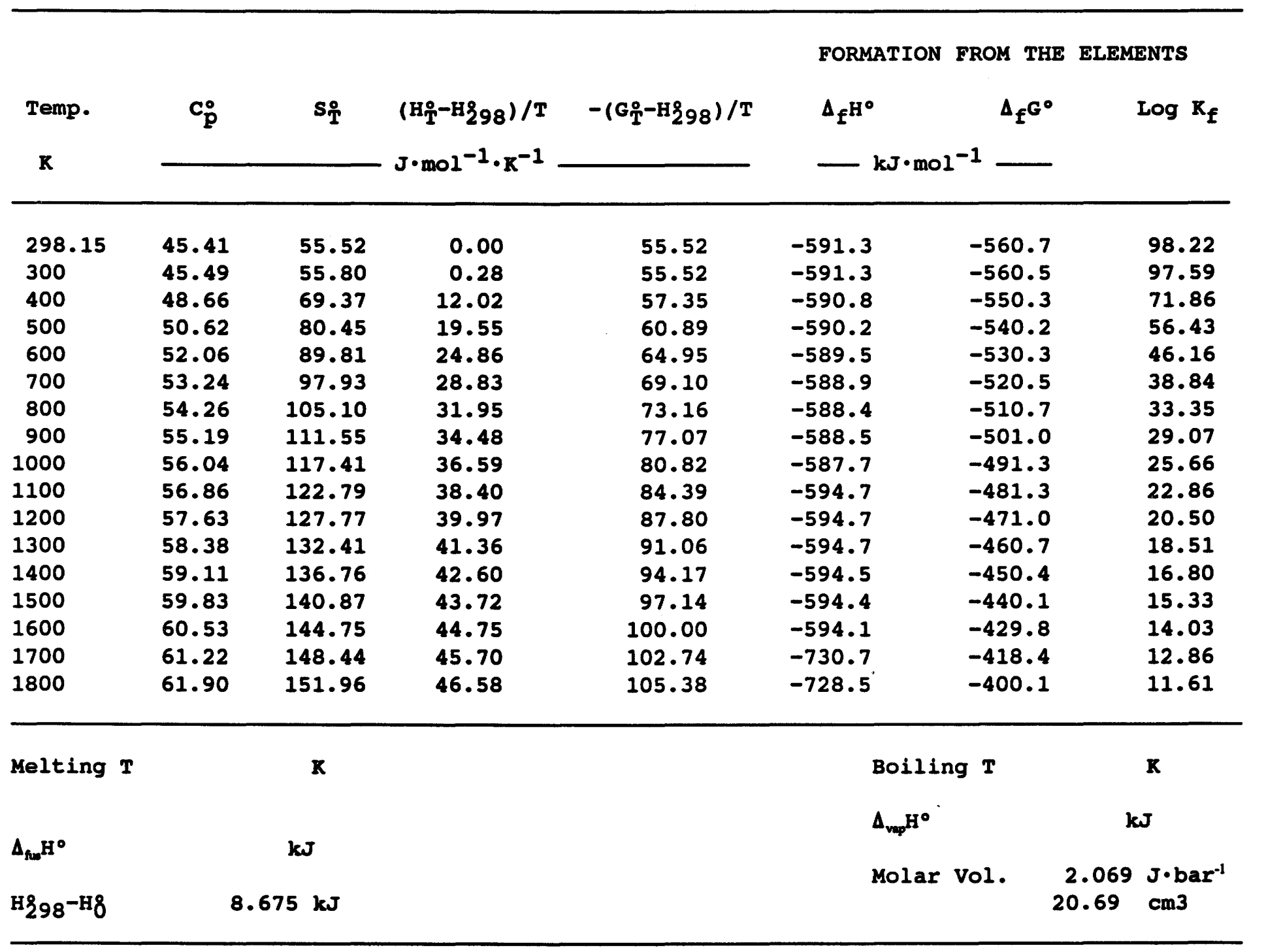
$A=-5 \cdot 956 \mathrm{E}+02$
$\mathrm{B}=1.047 \mathrm{E}-01$
$C=3.940 E+05$ 
$\mathrm{ThO}_{2}$ : Cubic crystals 298.15 to $1200 \mathrm{~K}$.

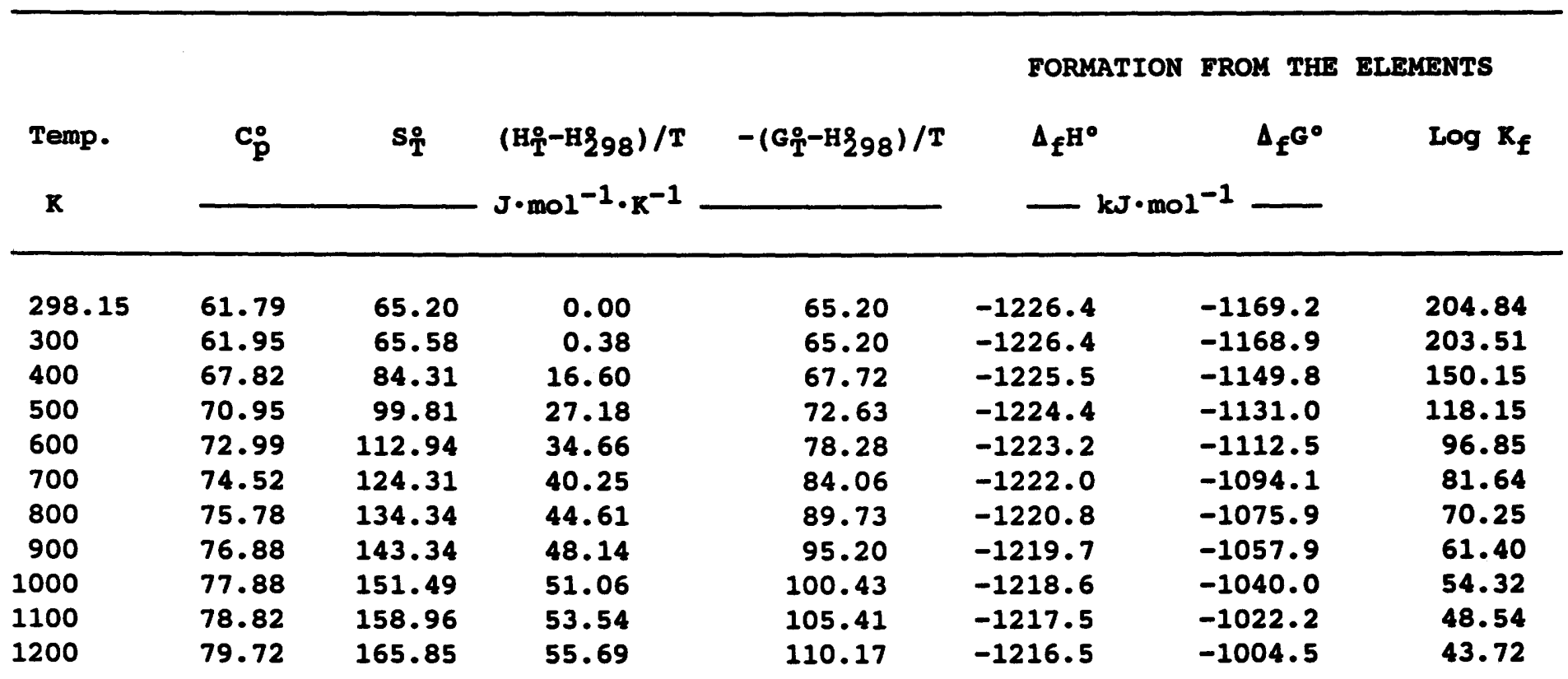

Melting T

$\mathbf{x}$

$\mathbf{k J}$

$\Delta_{\text {fum }} \mathrm{H}^{\circ}$

H298-H8

$10.56 \mathrm{~kJ}$
Boiling T

$\Delta_{\mathrm{ve}} \mathrm{H}^{\circ}$

$k J$

Molar vol. $\quad 2.637 \mathrm{~J} \cdot$ bar $^{-1}$ $26.37 \mathrm{~cm}^{3}$
$A=-1.219 \mathrm{E}+03$
$B=1.794 \mathrm{E}-01$
$C=-3.21 E+05$ 
$\mathrm{TiO}_{2}$ : Tetragonal crystals 298.15 to $1800 \mathrm{~K}$.

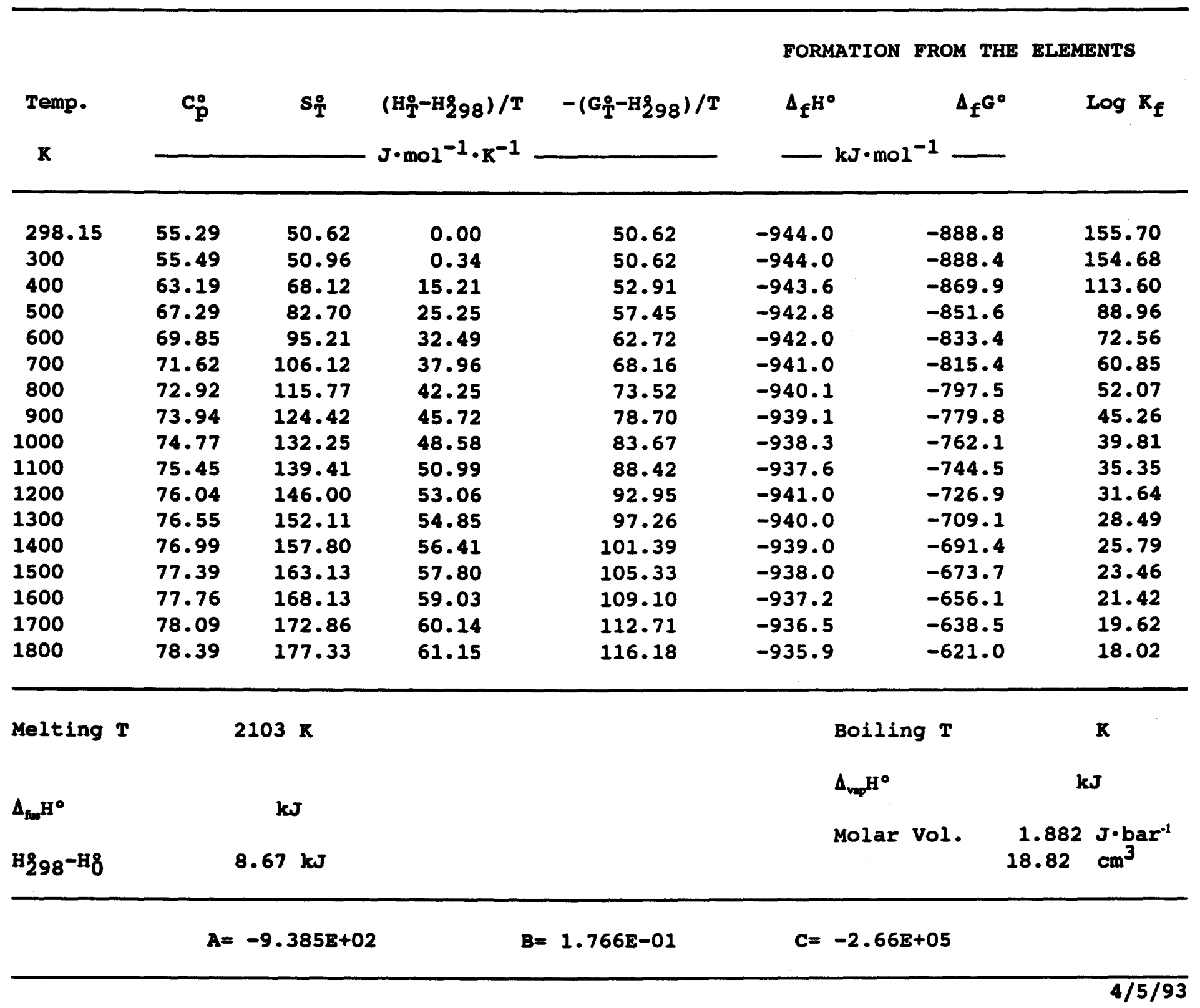


$\mathrm{TiO}_{2}$ : Tetragonal crystals 298.15 to $1300 \mathrm{~K}$.

\begin{tabular}{|c|c|c|c|c|c|c|c|}
\hline \multirow[b]{2}{*}{ Temp. } & \multirow[b]{2}{*}{$\mathbf{c}_{\mathbf{p}}^{\circ}$} & \multirow[b]{2}{*}{$\mathbf{S}_{\mathrm{T}}^{\circ}$} & \multirow{3}{*}{$\begin{array}{l}\left(\mathrm{H}_{\mathrm{T}}^{\circ}-\mathrm{H}_{298}\right) / \mathrm{T} \\
\mathrm{J} \cdot \mathrm{mol}^{-1} \cdot \mathrm{K}^{-1}\end{array}$} & \multirow[b]{2}{*}{$-\left(G_{T}^{\circ}-H_{298}\right) / T$} & \multicolumn{2}{|c|}{ FORMATION FROM THE } & ELEMENTS \\
\hline & & & & & $\Delta_{\mathrm{f}} \mathrm{H}^{\circ}$ & $\Delta_{f} G^{0}$ & $\log R_{f}$ \\
\hline $\mathbf{K}$ & & & & & _ $\mathrm{kJ} \cdot \mathrm{mol}$ & -1 & \\
\hline 298.15 & 55.32 & 49.90 & 0.00 & 49.90 & -938.7 & -883.2 & 154.74 \\
\hline 300 & 55.59 & 50.24 & 0.34 & 49.90 & -938.7 & -882.9 & 153.72 \\
\hline 400 & 64.71 & 67.69 & 15.47 & 52.22 & -938.2 & -864.4 & 112.87 \\
\hline 500 & 68.60 & 82.60 & 25.76 & 56.85 & -937.3 & -846.0 & 88.38 \\
\hline 600 & 70.69 & 95.31 & 33.09 & 62.22 & -936.3 & -827.8 & 72.07 \\
\hline 700 & 72.07 & 106.32 & 38.56 & 67.75 & -935.3 & -809.8 & 60.43 \\
\hline 800 & 73.15 & 116.01 & 42.82 & 73.19 & -934.3 & -792.0 & 51.71 \\
\hline 900 & 74.10 & 124.68 & 46.24 & 78.44 & -933.3 & -774.2 & 44.93 \\
\hline 1000 & 75.01 & 132.54 & 49.07 & 83.46 & -932.5 & -756.6 & 39.52 \\
\hline 1100 & 75.91 & 139.73 & 51.47 & 88.26 & -931.8 & -739.1 & 35.09 \\
\hline 1200 & 76.82 & 146.37 & 53.55 & 92.83 & -935.2 & -721.4 & 31.40 \\
\hline 1300 & 77.74 & 152.56 & 55.37 & 97.19 & -934.0 & -703.7 & 28.27 \\
\hline
\end{tabular}

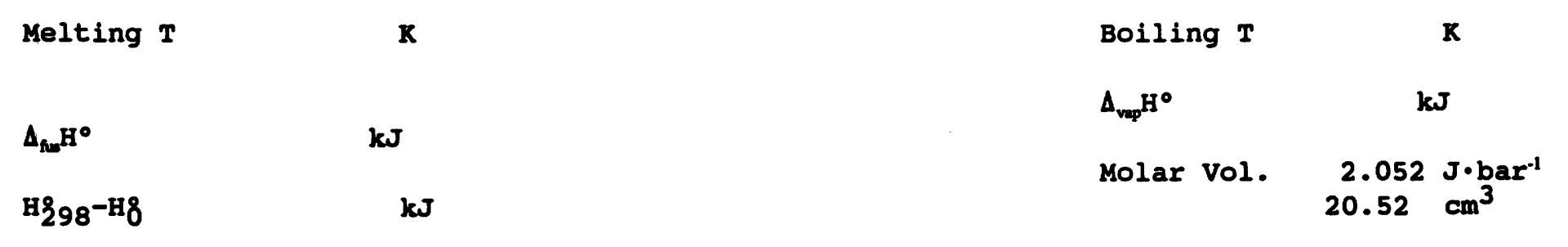
$A=-9 \cdot 331 E+02$
$B=1.767 \mathrm{E}-01$
$C=-2.60 E+05$ 
$\mathrm{UO}_{2}$ : Cubic crystals 298.15 to $1800 \mathrm{~K}$.

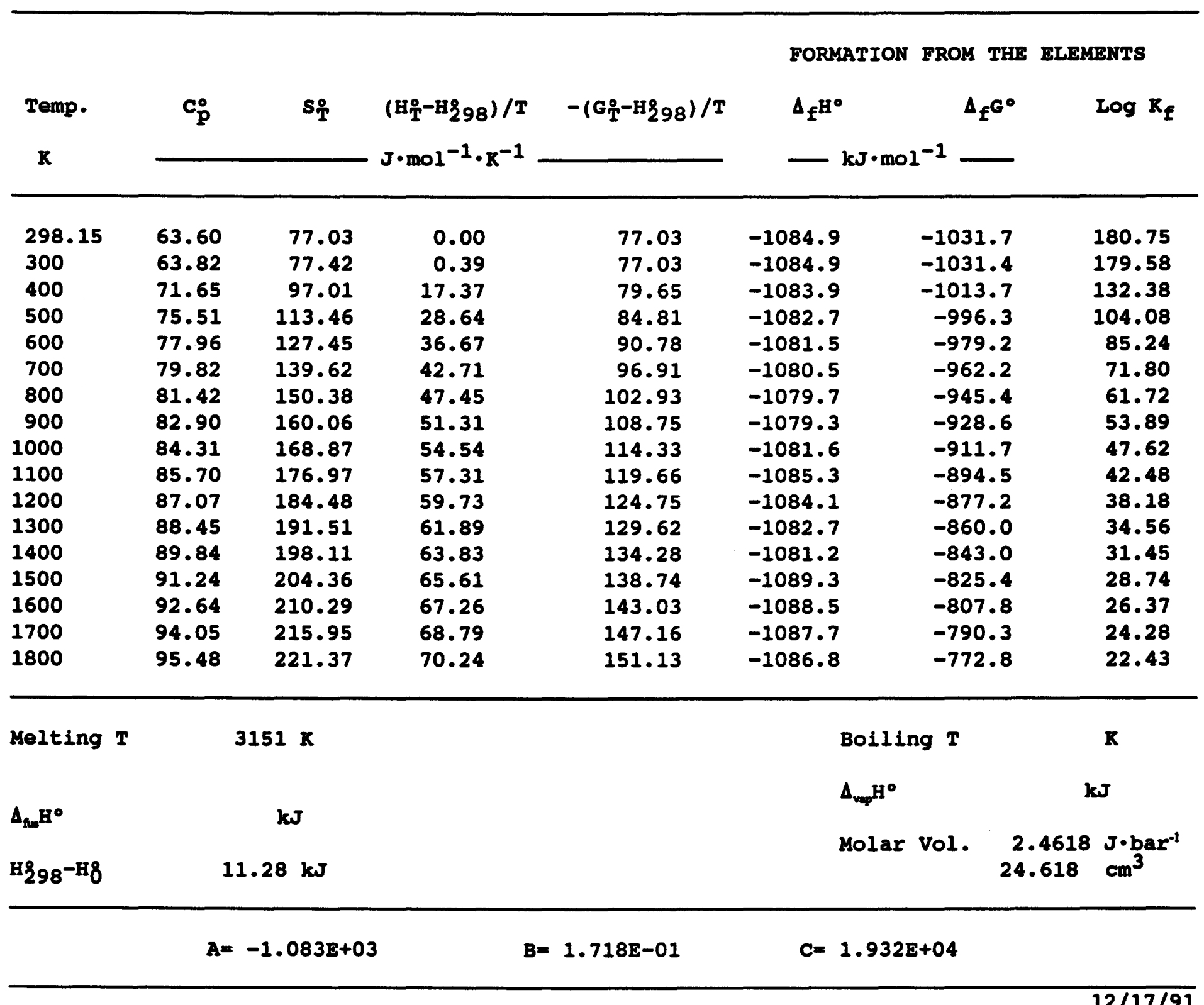


$\mathrm{V}_{2} \mathrm{O}_{3}$ : Rhombohedral crystals 298.15 to $1800 \mathrm{~K}$.

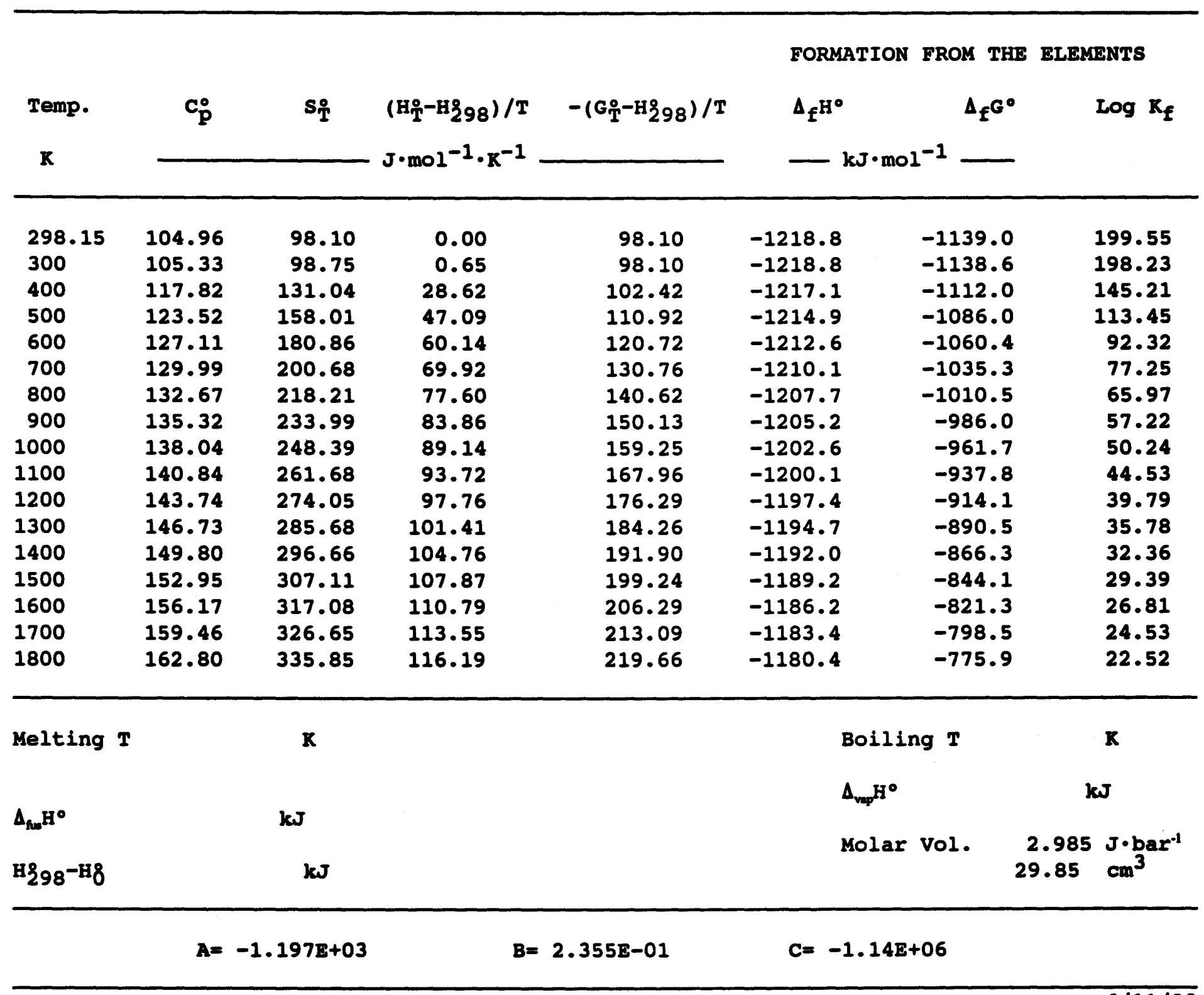


$\mathrm{HO}_{2}$ : Monoclinic crystals 298.15 to $1800 \mathrm{~K}$.

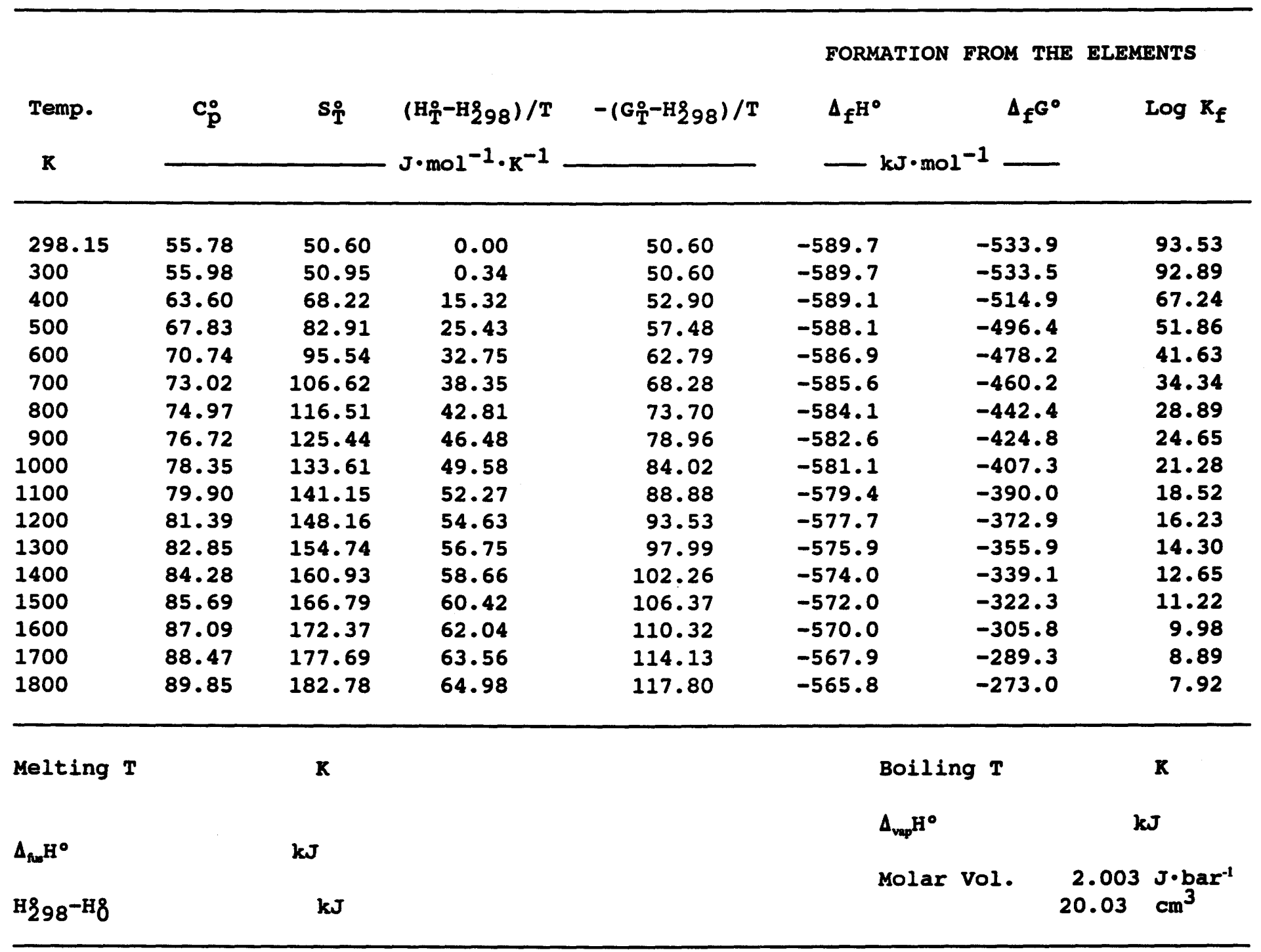
$A=-5 \cdot 776 E+02$
$B=1.7025-01$
$C=-6 \cdot 726 E+05$ 
Zno: Hexagonal crystals 298.15 to $1800 \mathrm{~K}$.

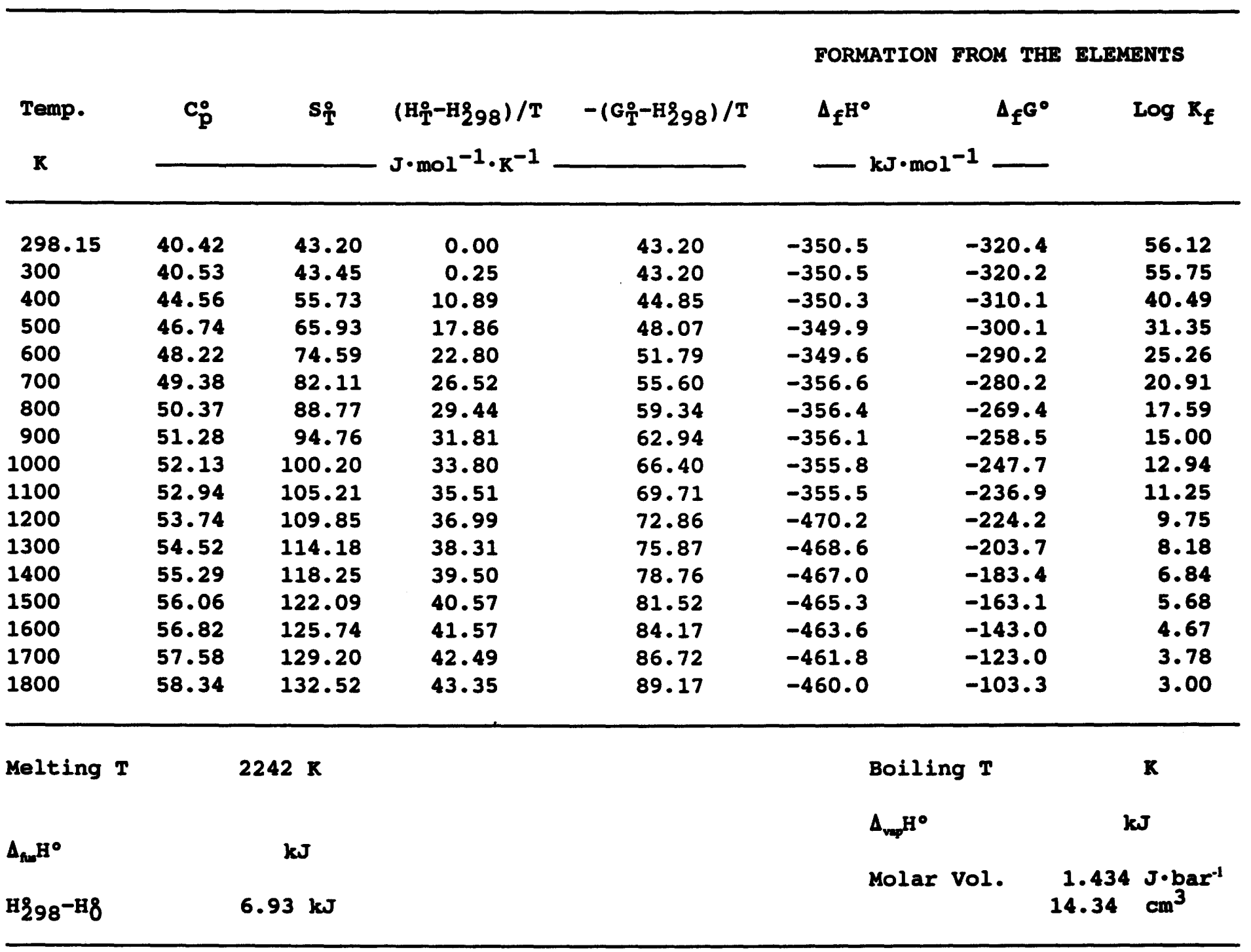


$\mathrm{ZrO}_{2}$ : Monoclinic crystals 298.15 to $1478 \mathrm{~K}$. Tetragonal crystals 1478 to $1800 \mathrm{~K}$.

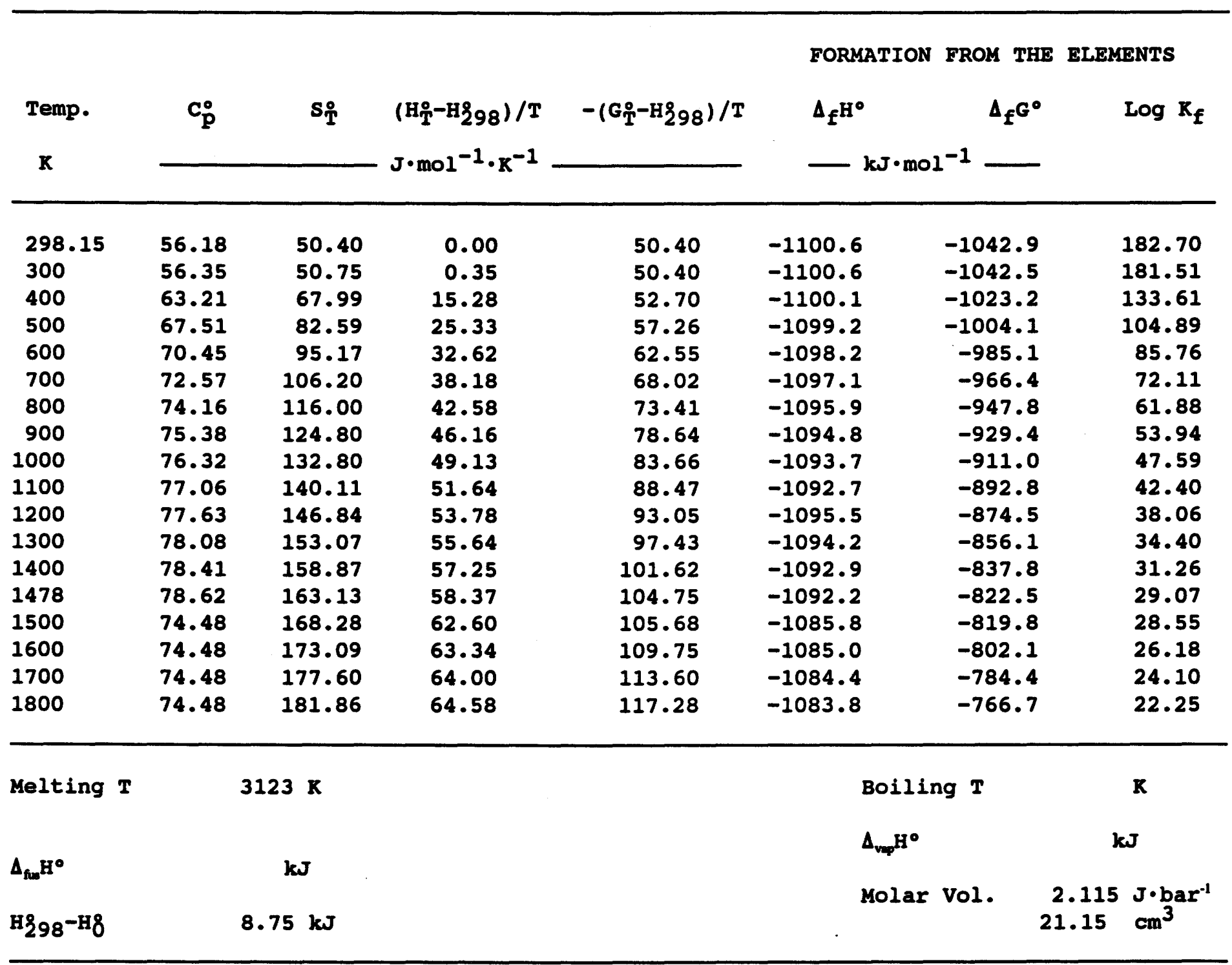


CHRYSOBERYL

Formula wt 126.973

$\mathrm{BeAl}_{2} \mathrm{O}_{4}$ : Orthorhombic crystals 298.15 to $1800 \mathrm{~K}$.

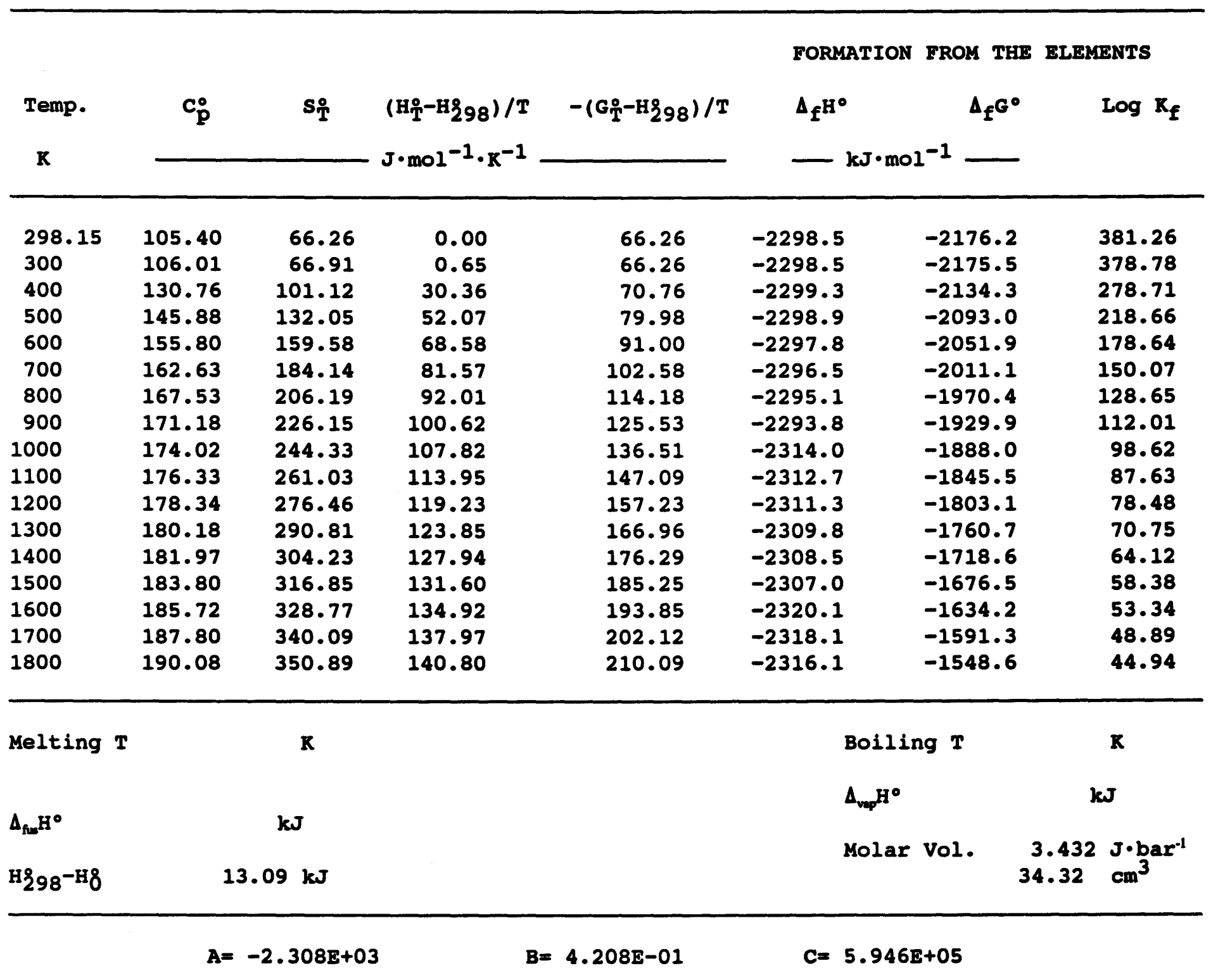


$\mathrm{CaFe}_{2} \mathrm{O}_{4}$ : Orthorhombic crystals 298.15 to melting point $1510 \mathrm{~K}$.

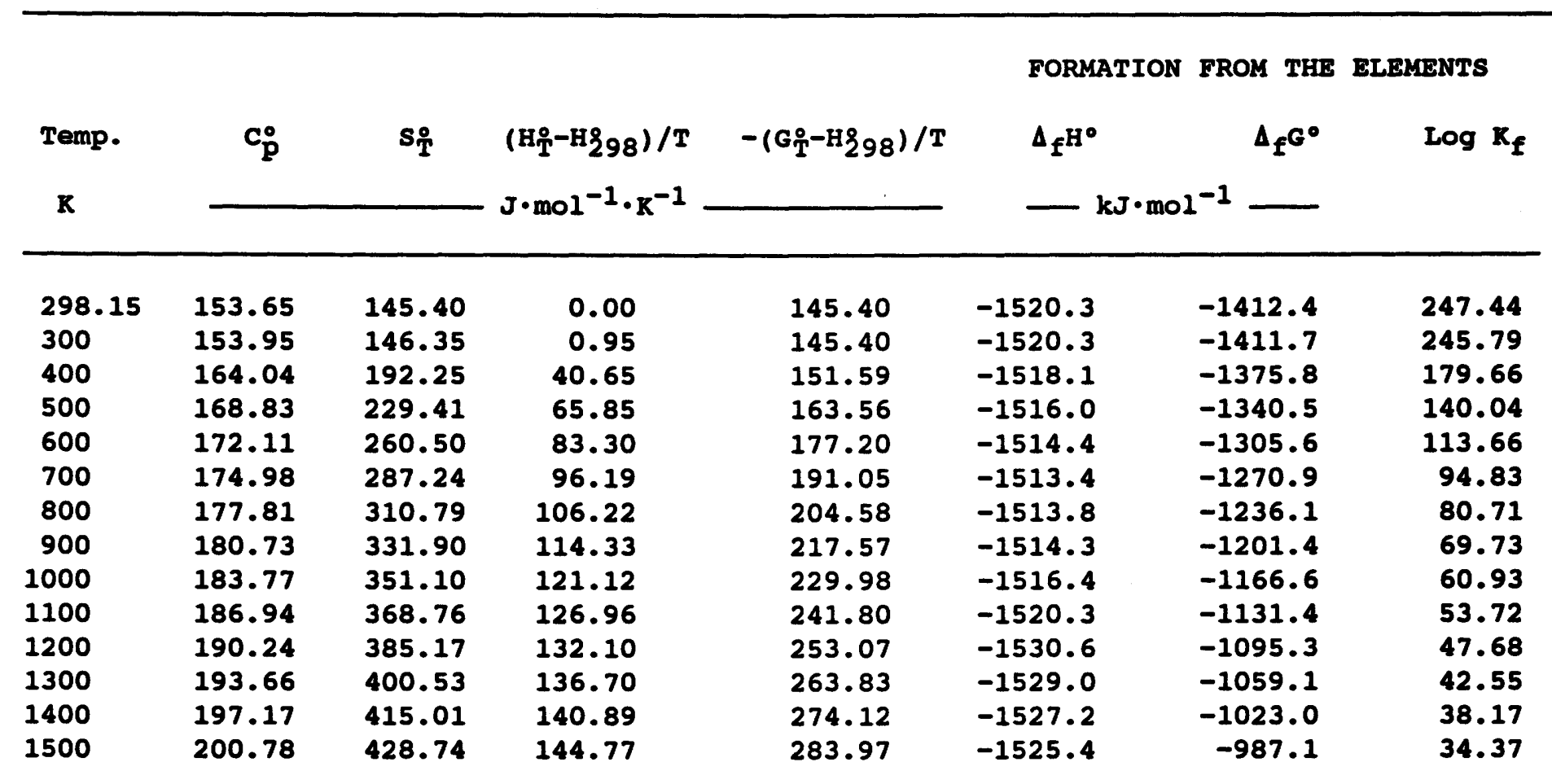

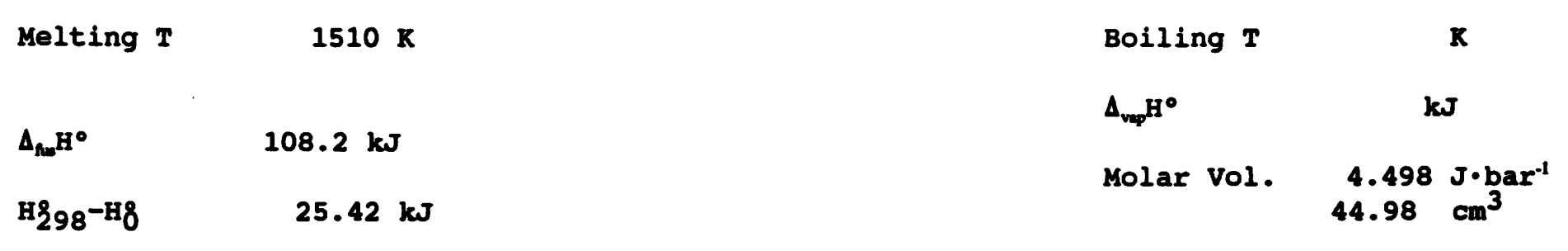
$A=-1.519 \mathrm{E}+03$
$B=3.537 E-01$
$C=1.473 E+05$ 
$\mathrm{Ca}_{2} \mathrm{Fe}_{2} \mathrm{O}_{5}$ : Orthorhombic crystals 298.15 to melting point $1750 \mathrm{~K}$.

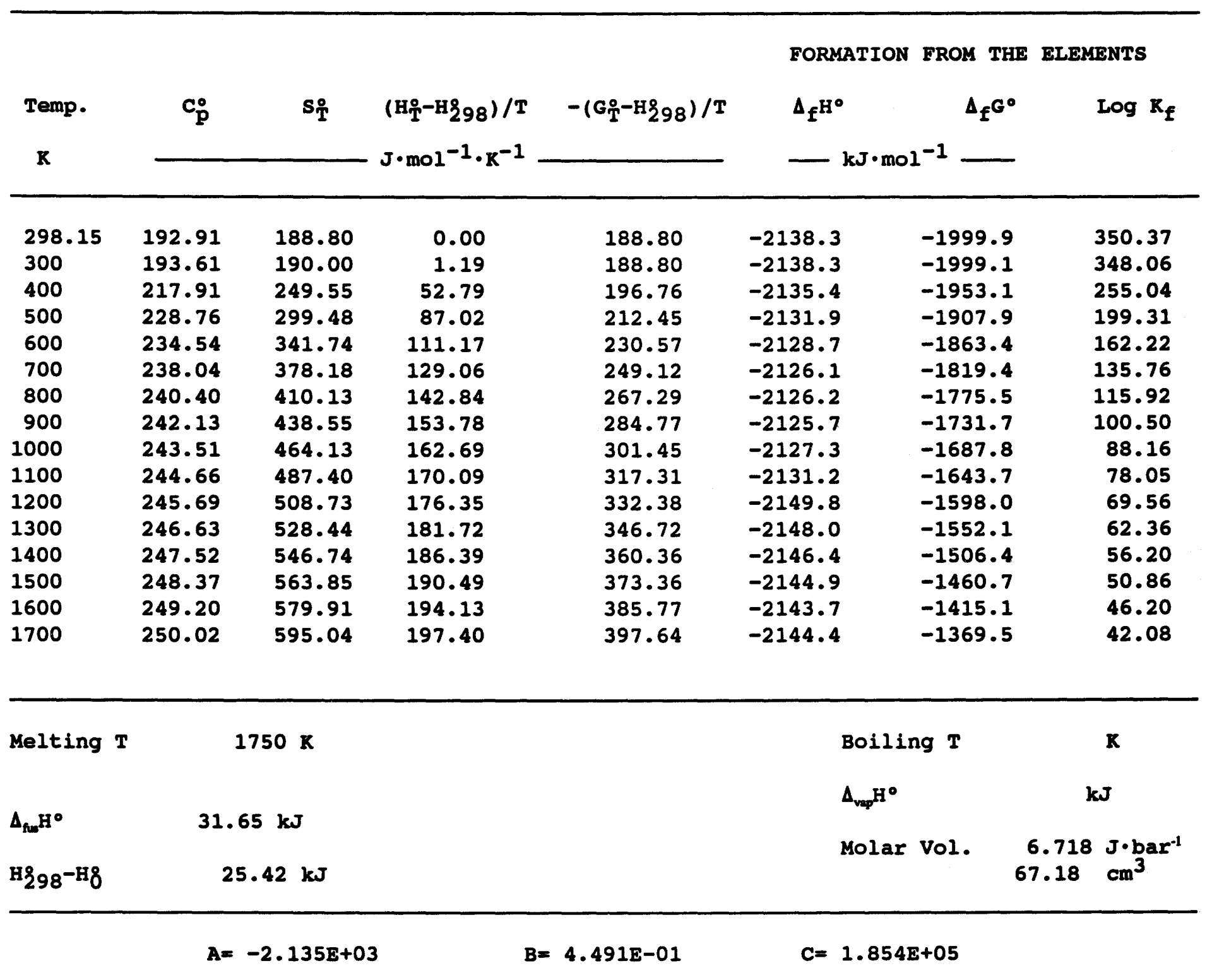


$\mathrm{CaTiO}_{3}$ : Orthorhombic crystals $1530 \mathrm{~K}$; cubic crystals 1530 to $1800 \mathrm{~K}$.

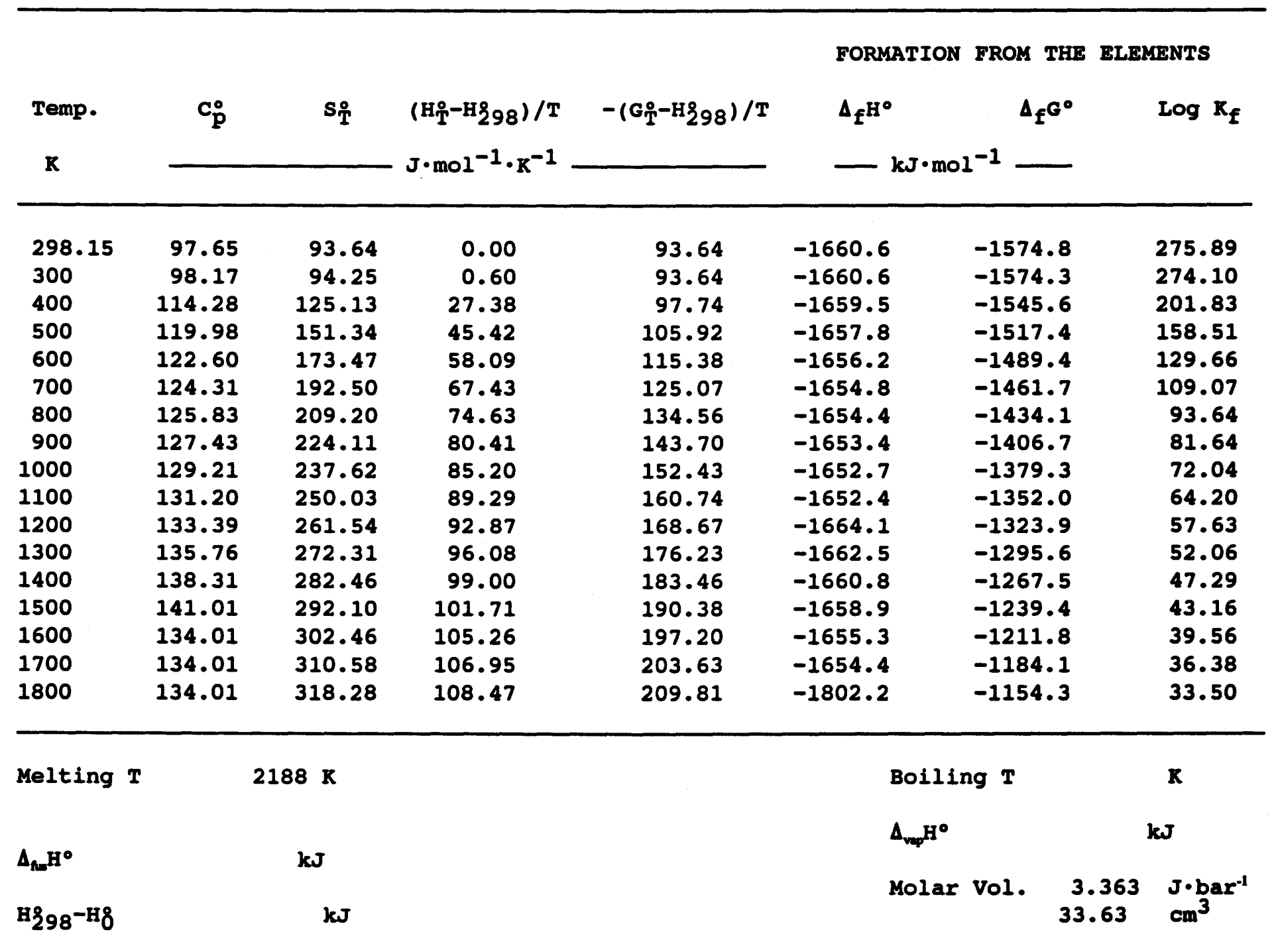


$\mathrm{FeAl}_{2} \mathrm{O}_{4}:$ Cubic crystals 298.15 to $1400 \mathrm{~K}$.

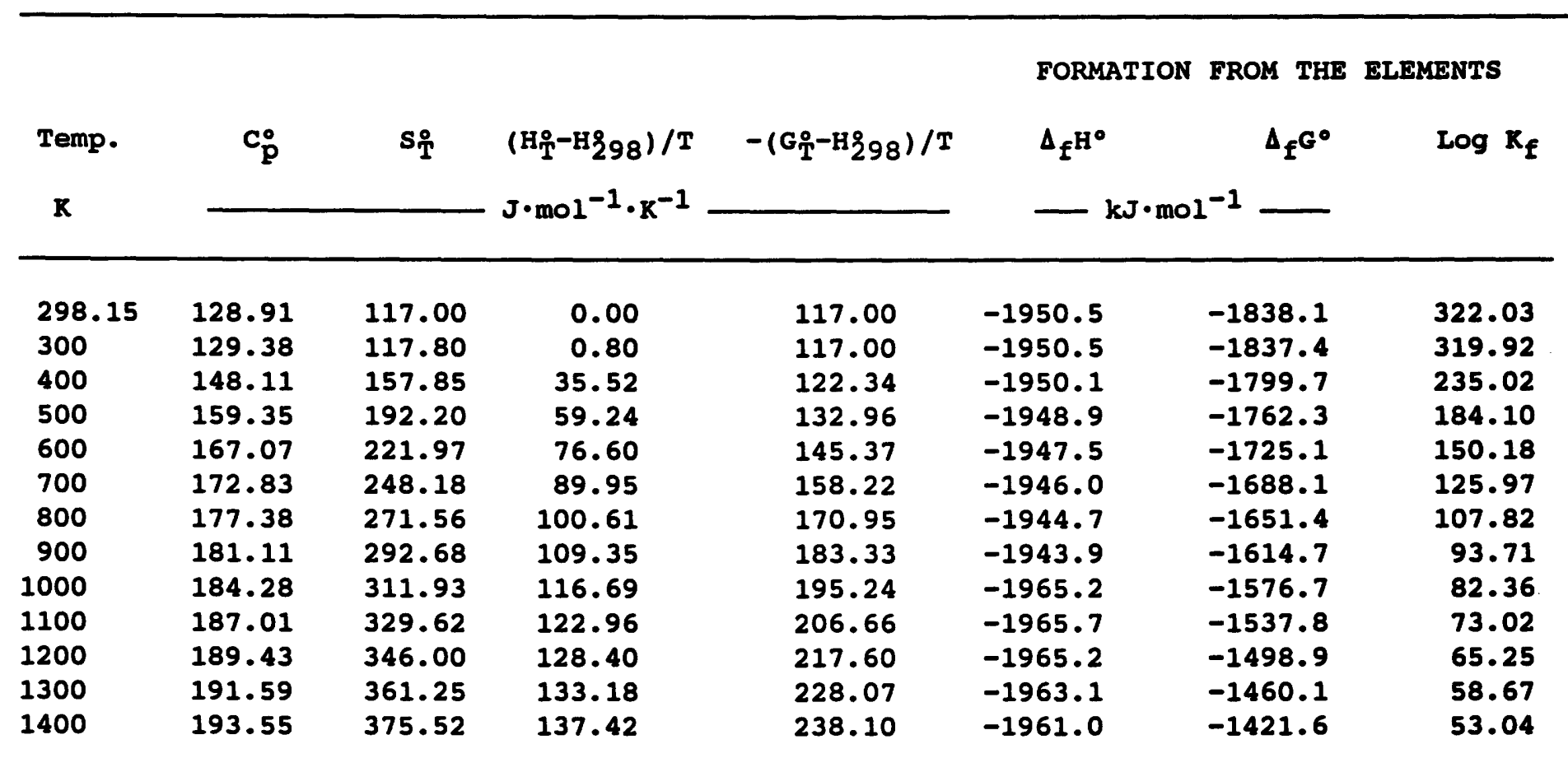

Melting $T$

$\mathbf{K}$

$\Delta_{\text {fur }} \mathrm{H}^{\circ}$

$\mathbf{k J}$

H298-H8

$\mathbf{k J}$
Boiling T

$\mathbf{K}$

$\mathbf{k J}$

Molar Vol.
$4.075 \mathrm{~J} \cdot \mathrm{bar}^{-1}$ $40.75 \mathrm{~cm}^{3}$ 
FeCr ${ }_{2} \mathrm{O}_{4}:$ Cubic crystals 298.15 to $1800 \mathrm{~K}$.

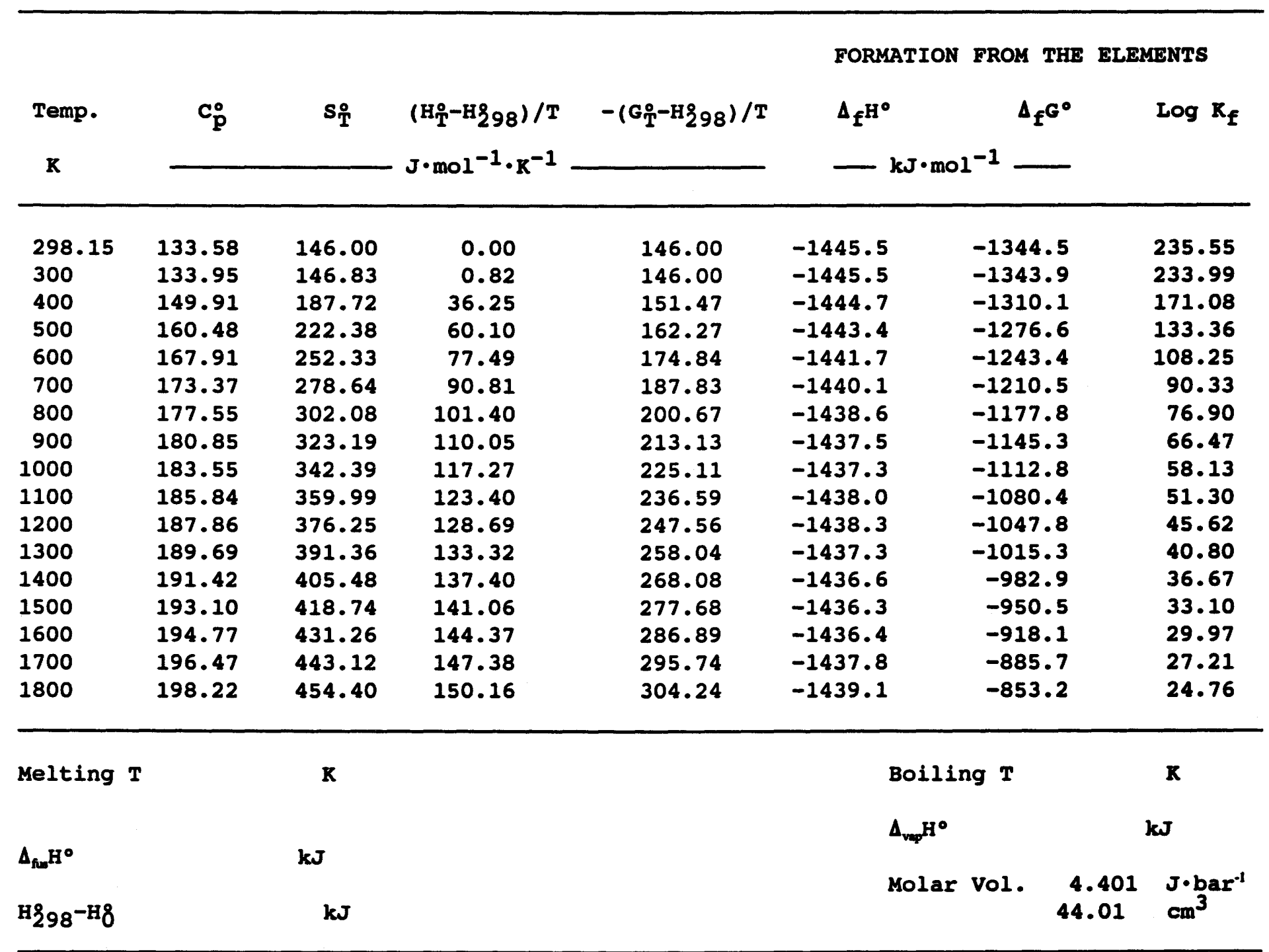
$A=-1 \cdot 437 \mathrm{E}+03$
$\mathrm{B}=3 \cdot 242 \mathrm{E}-01$
$C=-4.01 E+05$ 
FeTiO $_{3}$ : Rhombohedral crystals 298.15 to $1640 \mathrm{~K}$.

FORMATION FROM THE ELEMENTS

\begin{tabular}{|c|c|c|c|c|c|c|c|}
\hline $\begin{array}{l}\text { Temp. } \\
\mathbf{K}\end{array}$ & $\mathbf{c}_{\mathbf{p}}^{0}$ & $\mathbf{s}_{\mathbf{T}}^{\circ}$ & $\begin{array}{l}\left(\mathrm{H}_{\mathrm{T}}-\mathrm{H}_{298}\right) / \mathrm{T} \\
\mathrm{J} \cdot \mathrm{mol}^{-1} \cdot \mathrm{K}^{-1}\end{array}$ & $-\left(G_{T}^{0}-H_{298}\right) / T$ & $\begin{array}{l}\Delta_{\mathbf{E}^{H^{\circ}}} \\
-1\end{array}$ & $1^{-1}$ & $\log K_{f}$ \\
\hline 298.15 & 99.18 & 108.90 & 0.00 & 108.90 & -1232.0 & -1155.0 & 202.43 \\
\hline 300 & 99.47 & 109.51 & 0.61 & 108.90 & -1232.0 & -1155.0 & 201.10 \\
\hline 400 & 111.62 & 139.94 & 26.97 & 112.97 & -1231.1 & -1129.5 & 147.49 \\
\hline 500 & 119.20 & 165.72 & 44.72 & 121.01 & -1229.7 & -1104.2 & 115.35 \\
\hline 600 & 124.35 & 187.94 & 57.58 & 130.36 & -1228.1 & -1079.2 & 93.95 \\
\hline 700 & 128.16 & 207.41 & 67.41 & 140.00 & -1226.6 & -1054.6 & 78.69 \\
\hline 800 & 131.26 & 224.73 & 75.20 & 149.53 & -1225.2 & -1030.1 & 67.26 \\
\hline 900 & 134.02 & 240.35 & 81.58 & 158.77 & -1224.1 & -1005.8 & 58.37 \\
\hline 1000 & 136.71 & 254.61 & 86.96 & 167.65 & -1223.6 & -981.5 & 51.27 \\
\hline 1100 & 139.50 & 267.77 & 91.61 & 176.16 & -1224.1 & -957.3 & 45.46 \\
\hline 1200 & 142.50 & 280.03 & 95.72 & 184.31 & -1227.7 & -933.0 & 40.61 \\
\hline 1300 & 145.81 & 291.57 & 99.45 & 192.12 & -1225.1 & -908.5 & 36.50 \\
\hline 1400 & 149.48 & 302.51 & 102.89 & 199.62 & -1222.3 & -884.2 & 32.99 \\
\hline 1500 & 153.58 & 312.96 & 106.13 & 206.83 & -1219.4 & -860.2 & 29.95 \\
\hline 1600 & 158.15 & 323.01 & 109.23 & 213.78 & -1216.3 & -836.3 & 27.30 \\
\hline
\end{tabular}

Melting $T$

$\mathbf{K}$

Boiling T

$\mathbf{K}$

$\Delta_{\mathrm{Ru}} \mathrm{H}^{\circ}$

$\mathbf{k J}$

$\Delta_{\mathrm{veq}} \mathbf{H}^{\circ}$

$\mathbf{k J}$

H298-H8

$16.992 \mathrm{~kJ}$

Molar Vol.

$3.169 \mathrm{~J} \cdot \mathrm{bar}^{-1}$
$31.69 \mathrm{~cm}^{3}$

$A=-1.224 \mathrm{E}+03$

$B=2.424 \mathrm{E}-01$

$C=-3.82 \mathrm{E}-01$ 
$\mathrm{Fe}_{2} \mathrm{TiO}_{4}$ : Cubic crystals 298.15 to $1800 \mathrm{~K}$.

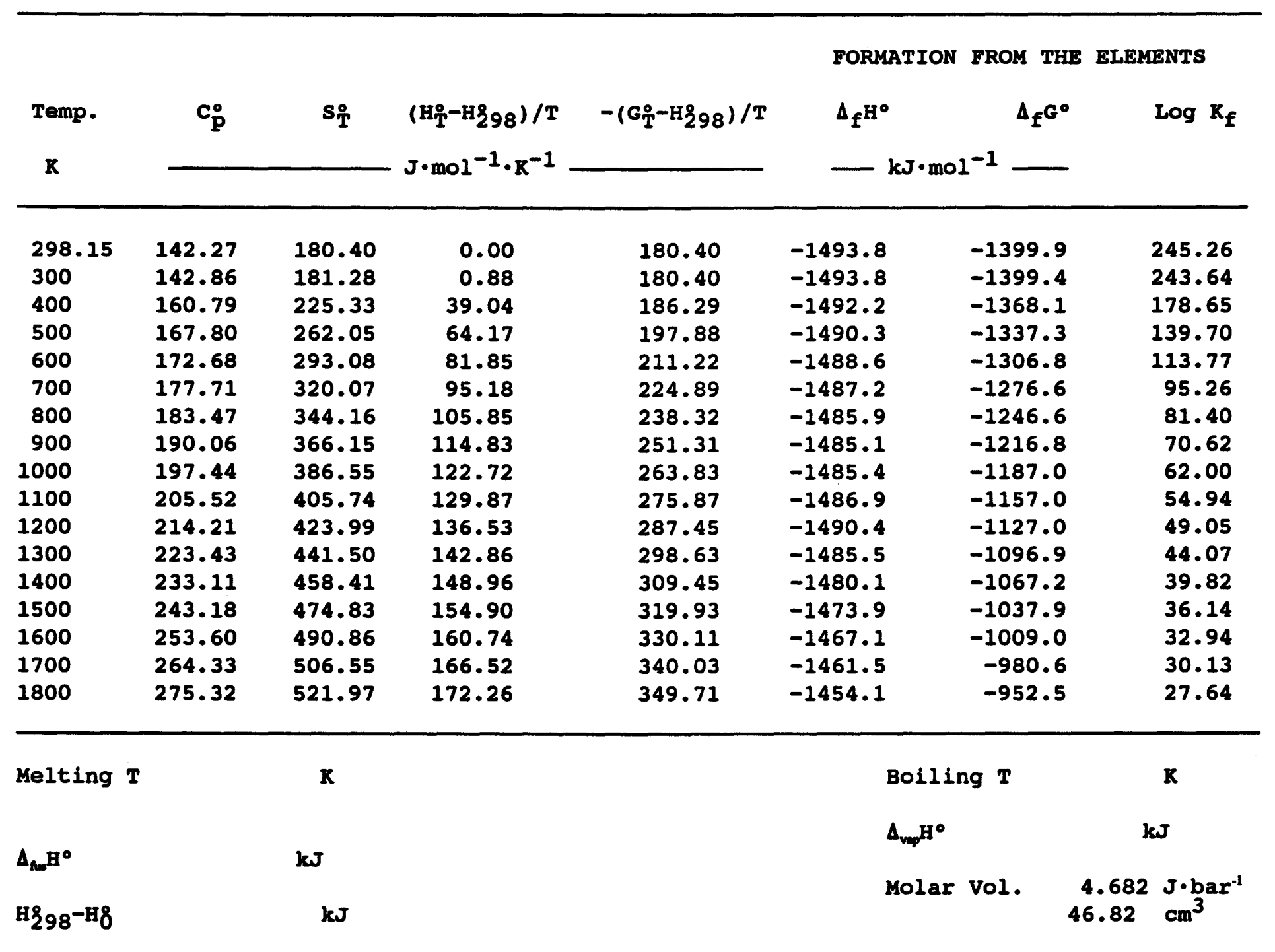


$\mathrm{MgAl}_{2} \mathrm{O}_{4}:$ Cubic crystals 298.15 to $1800 \mathrm{~K}$.

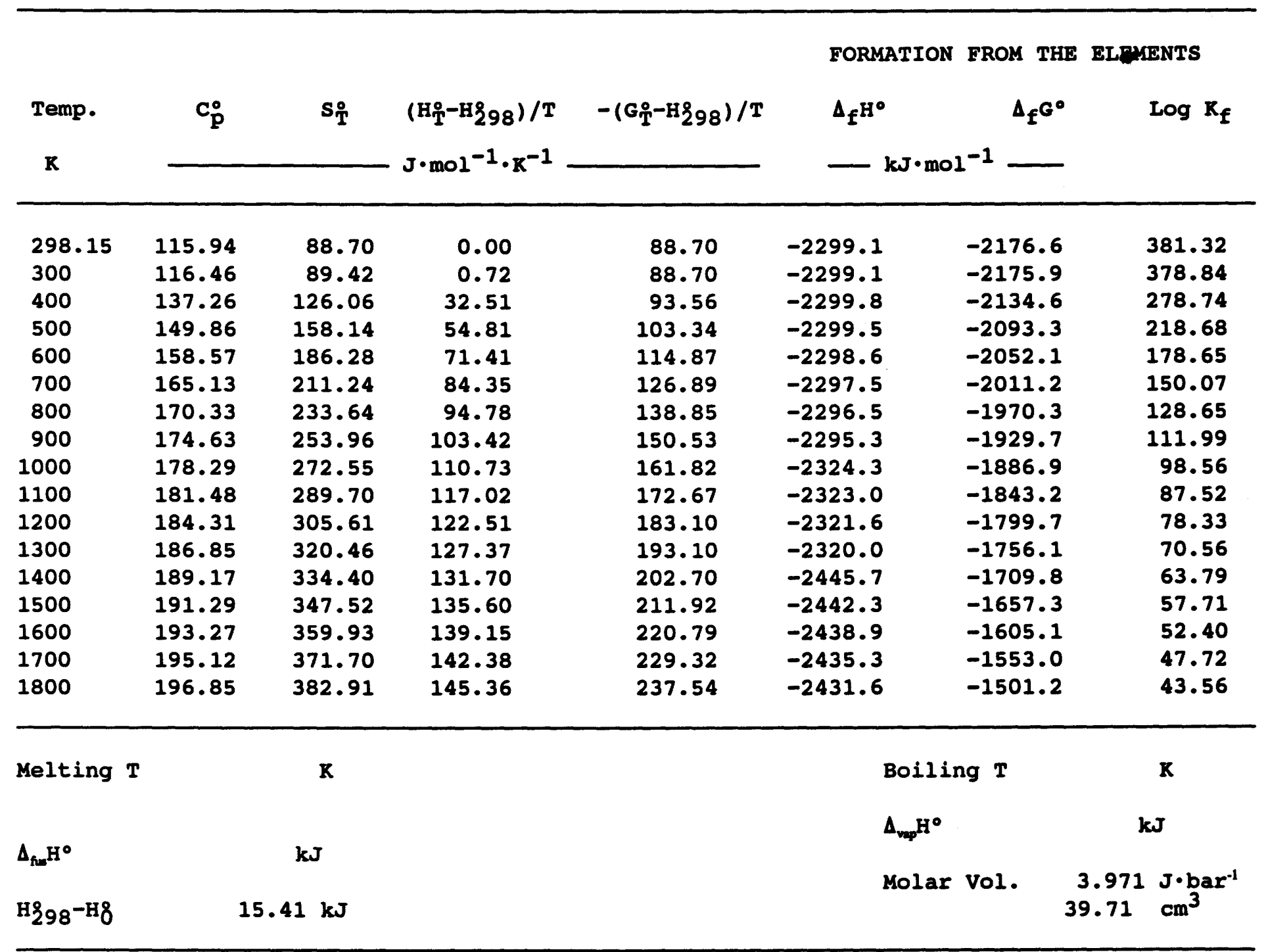

$A=-2.3446 \mathrm{E}+03 \quad \mathrm{~B}=4.598 \mathrm{E}-01 \quad \mathrm{C}=3.114 \mathrm{E}+06$


$\mathrm{MgCr}_{2} \mathrm{O}_{4}:$ Cubic crystals 298.15 to $1800 \mathrm{~K}$.

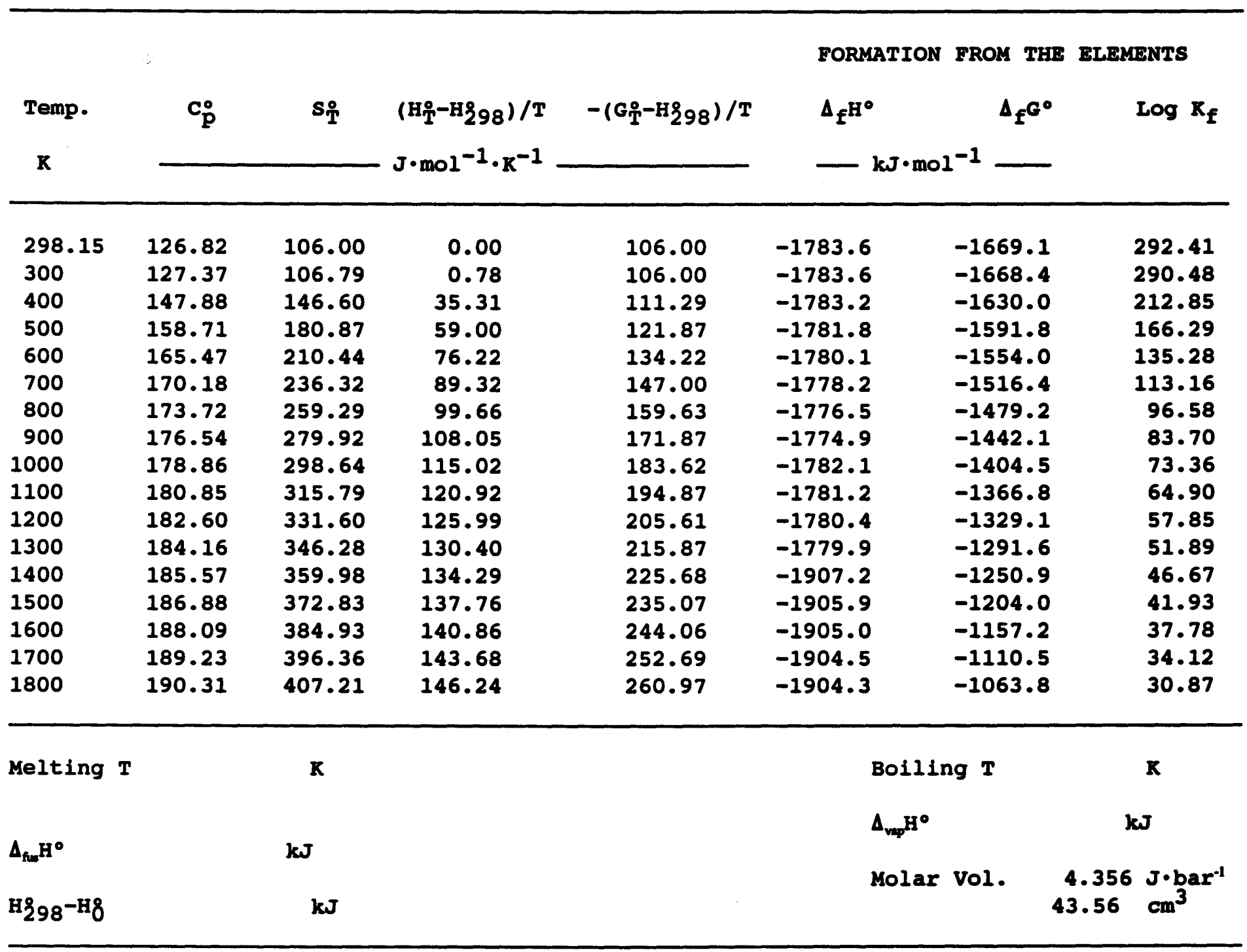
$A=-1.7945 E+03$
$B=3.960 E-01$
$C=1.0556 \mathrm{E}+00$ 
$\mathrm{MgFe}_{2} \mathrm{O}_{4}:$ Cubic $(\alpha)$ crystals 298.15 to $665 \mathrm{~K}$. Cubic ( $\beta$ ) crystals 665 to $1230 \mathrm{~K}$. $\gamma$-crystals 1230 to $1800 \mathrm{~K}$.

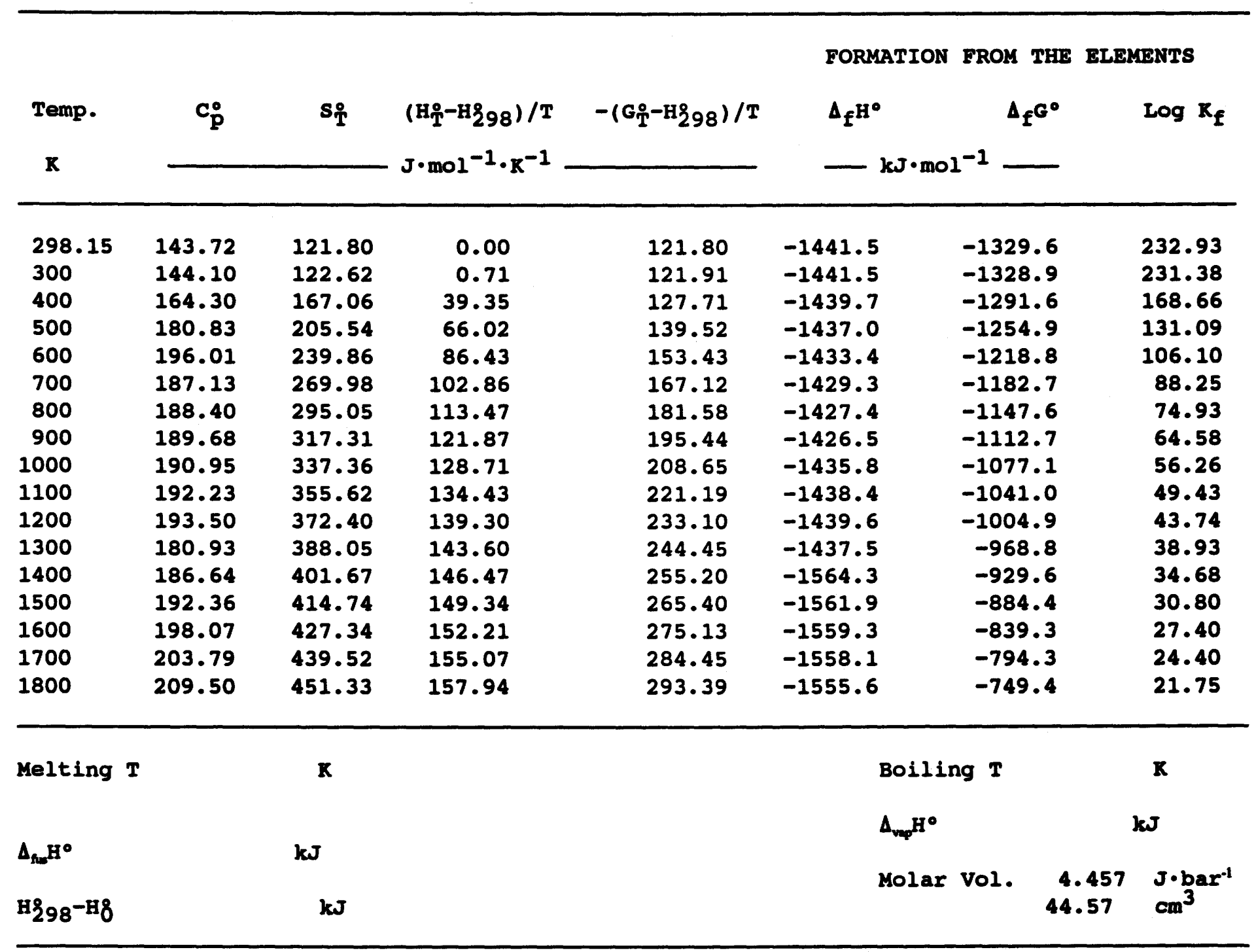


MgTiO $_{3}$ : Rhombohedral crystals 298.15 to $1800 \mathrm{~K}$.

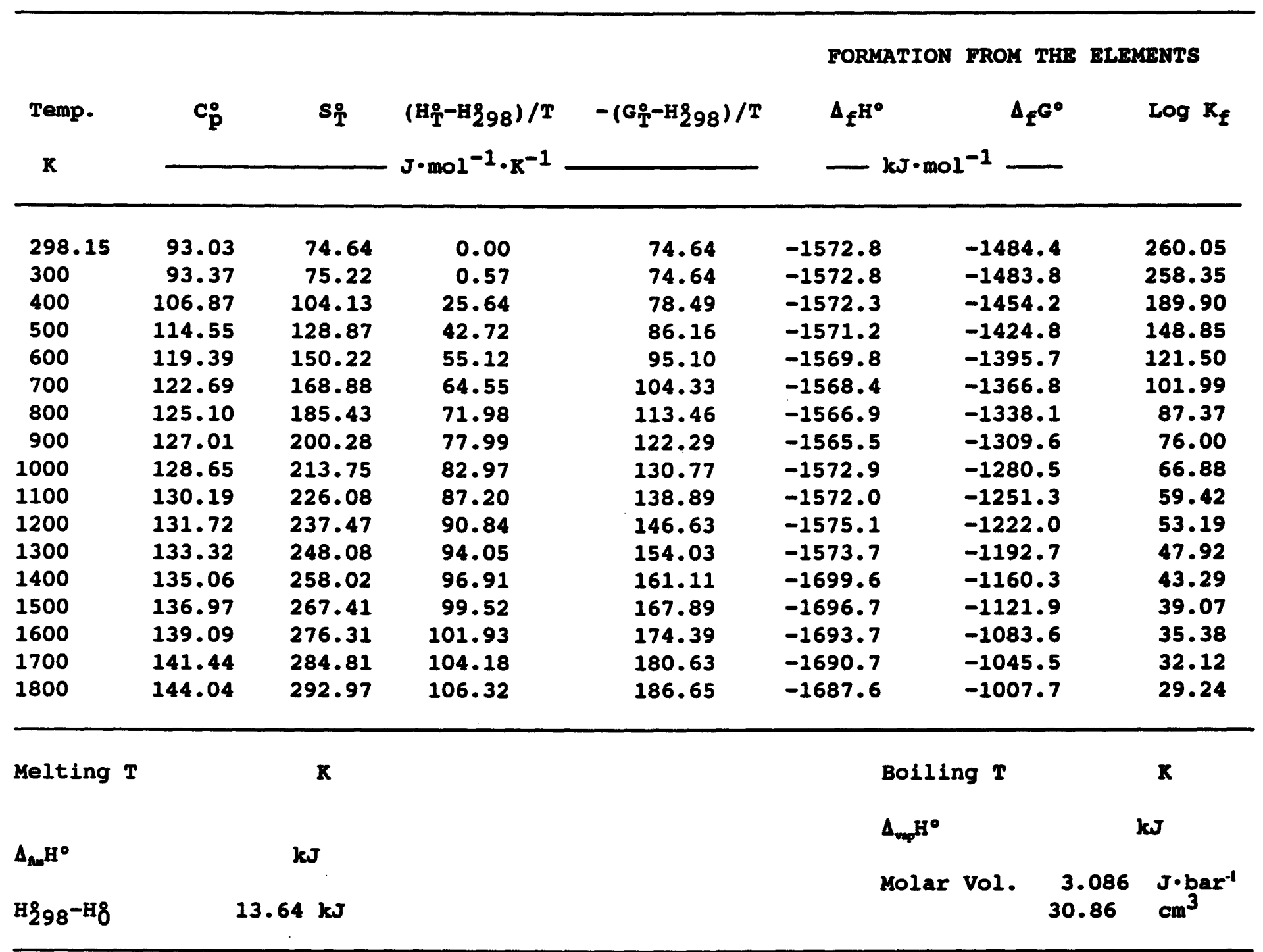


$\mathrm{MnTiO}_{3}:$ Rhombohedral crystals 298.15 to $1600 \mathrm{~K}$.

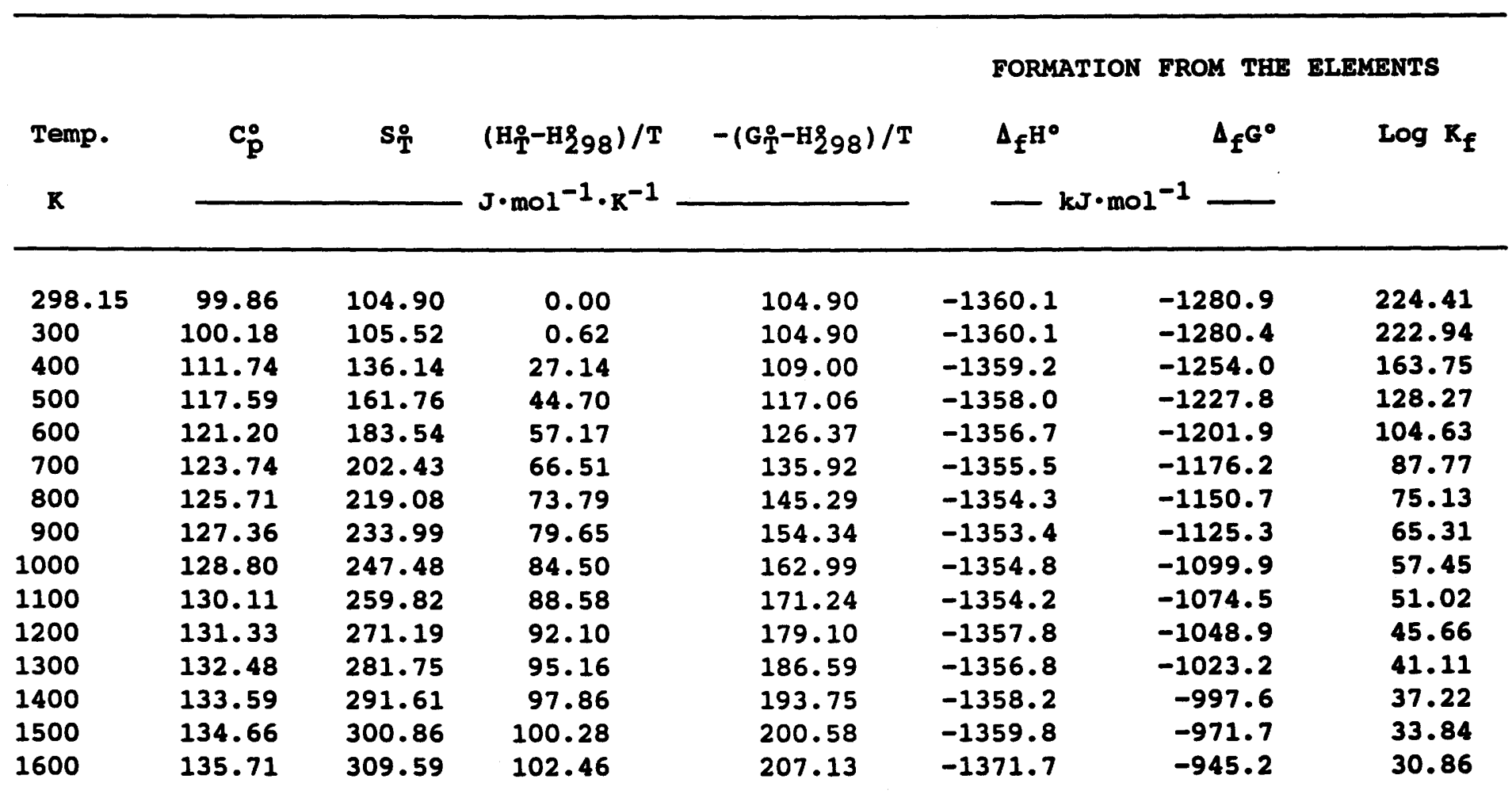

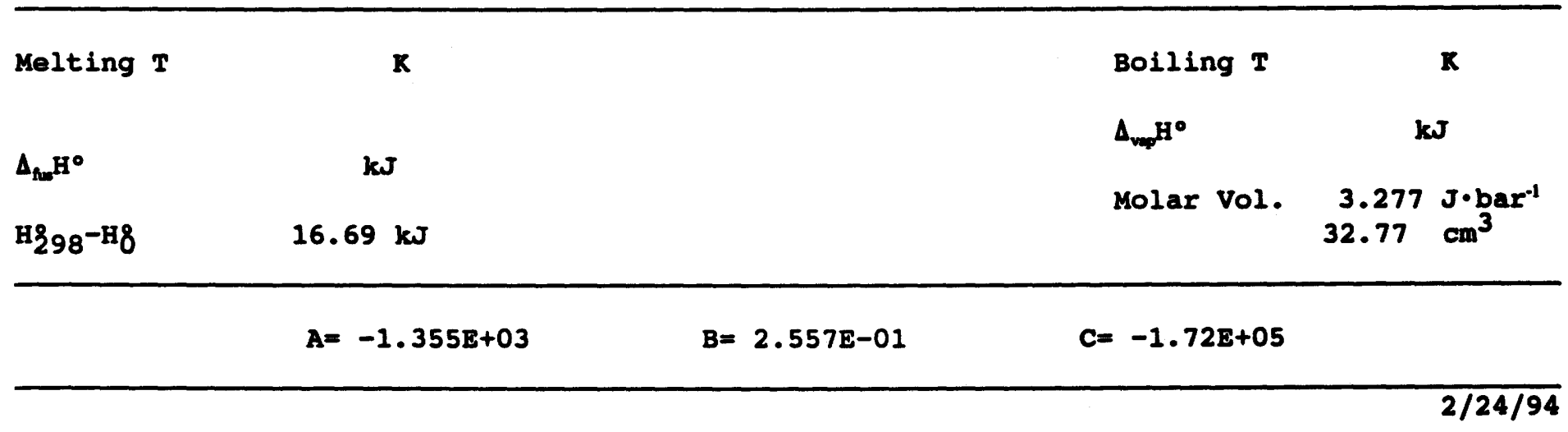


$\mathrm{NiFe}_{2} \mathrm{O}_{4}:$ Cubic crystals 298.15 to $1000 \mathrm{~K}$.

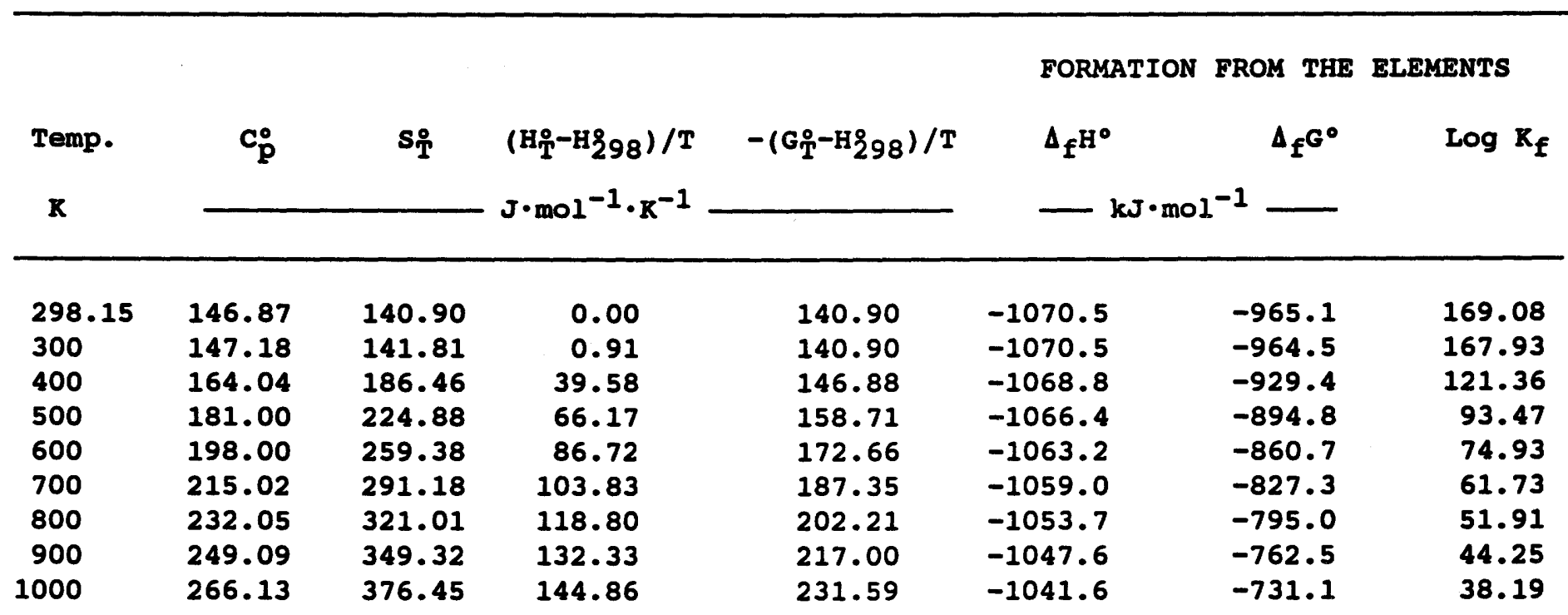

Melting T

$\boldsymbol{A}_{\mathrm{fu}} \mathrm{H}^{\circ}$

H298-H8
$\mathbf{R}$

$\mathbf{k J}$

$\mathbf{k J}$
Boiling T

$\Delta_{\text {vap }} H^{\circ}$

Molar Vol.

\section{$\mathbf{K}$}

$\mathbf{k J}$

$4.365 \mathrm{~J} \cdot \mathrm{bar}^{-1}$ $43.65 \mathrm{~cm}^{3}$ 
$\mathrm{ZnFe}_{2} \mathrm{O}_{4}$ : Tetragonal crystals 298.15 to $600 \mathrm{~K}$.

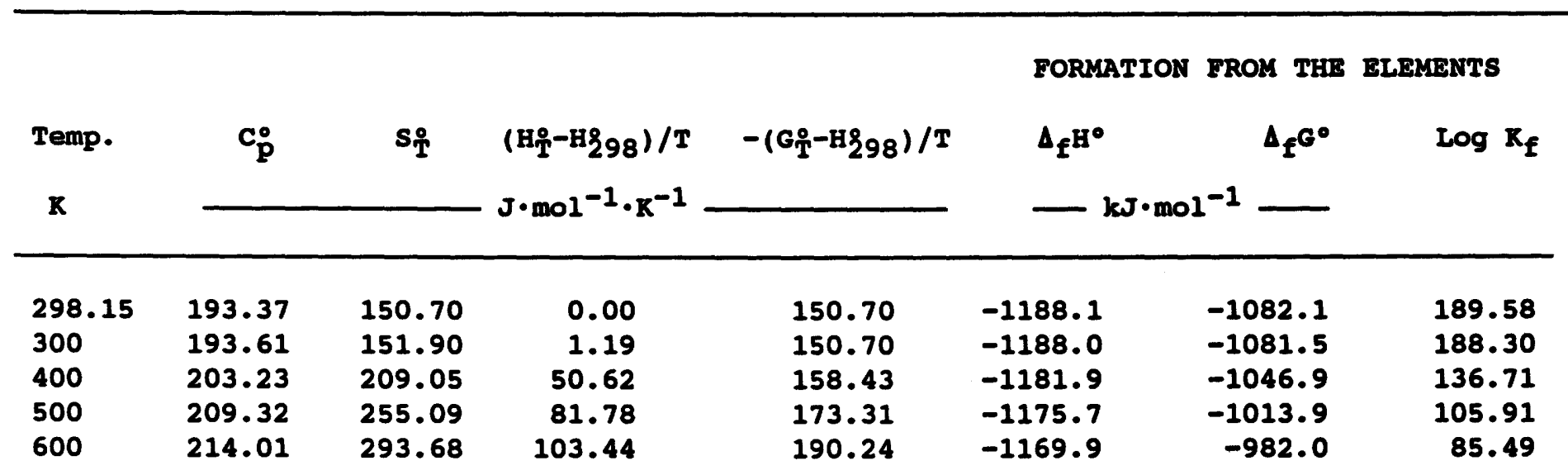

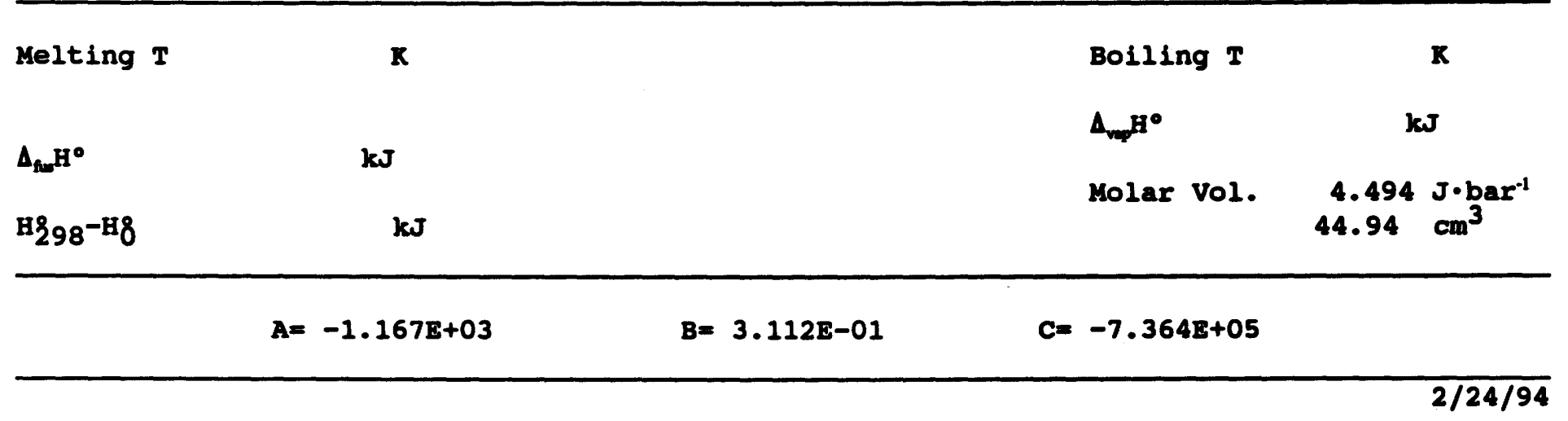


$\mathrm{Zn}_{2} \mathrm{TiO}_{4}$ : Tetragonal crystals 298.15 to $1800 \mathrm{~K}$.

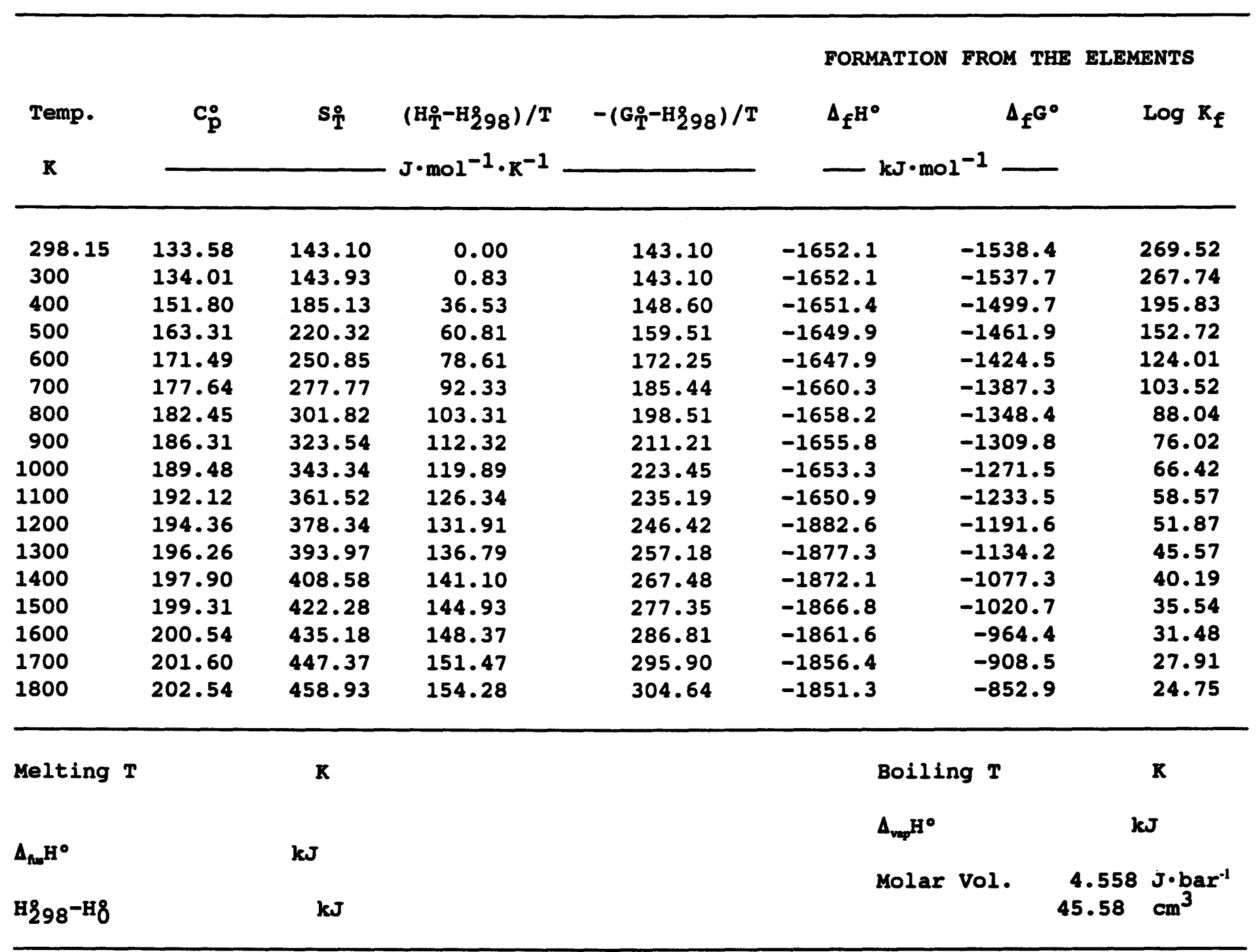
$A=-1.751 \mathrm{E}+03$
$B=4.850 E-01$
$C=6.790 E+06$ 
AgBr: Cubic crystals 298.15 to melting point $703 \mathrm{~K}$. Liquid 703 to $1000 \mathrm{~K}$.

\begin{tabular}{|c|c|c|c|c|c|c|c|}
\hline \multirow{3}{*}{$\begin{array}{l}\text { Temp. } \\
\text { K }\end{array}$} & \multirow{3}{*}{$\mathbf{c}_{\mathbf{p}}^{\circ}$} & \multirow{3}{*}{$\mathbf{s}_{\mathrm{T}}^{0}$} & \multirow{3}{*}{$\begin{array}{l}\left(\mathrm{H}_{\mathrm{T}}^{\circ}-\mathrm{H}_{298}\right) / \mathrm{T} \\
\mathrm{J} \cdot \mathrm{mol}^{-1} \cdot \mathrm{K}^{-1}\end{array}$} & \multirow{3}{*}{$-\left(G_{T}^{\circ}-H_{298}\right) / T$} & \multicolumn{2}{|c|}{ FORMATION FROM THE } & \multirow{3}{*}{$\begin{array}{l}\text { ELEMENTS } \\
\qquad \operatorname{LOg} \mathrm{K}_{\mathrm{f}}\end{array}$} \\
\hline & & & & & \multirow{2}{*}{\multicolumn{2}{|c|}{$-\mathrm{kJ} \cdot \operatorname{mol}^{-1}$}} & \\
\hline & & & & & & & \\
\hline 298.15 & 52.38 & 107.10 & 0.00 & 107.10 & $-100 \cdot 4$ & -97.0 & 16.99 \\
\hline 300 & 52.50 & 107.42 & 0.32 & 107.10 & -100.4 & -96.9 & 16.88 \\
\hline 400 & 58.94 & 123.41 & 14.17 & 109.24 & -114.6 & -92.8 & 12.11 \\
\hline 500 & 65.39 & 137.25 & 23.77 & 113.48 & -112.9 & -87.5 & 9.14 \\
\hline 600 & 71.83 & 149.75 & 31.24 & 118.50 & -110.5 & -82.6 & 7.19 \\
\hline 700 & 78.27 & 161.30 & 37.50 & 123.80 & -107.6 & -78.2 & 5.83 \\
\hline 703 & 78.46 & 162.63 & 37.70 & 124.93 & -107.5 & -78.3 & 5.82 \\
\hline 800 & 62.34 & 182.76 & 52.14 & 130.62 & -96.8 & -75.4 & 4.92 \\
\hline 900 & 62.34 & 190.08 & 53.28 & 136.80 & -95.3 & -72.7 & 4.22 \\
\hline 1000 & 62.34 & 196.65 & 54.18 & 142.47 & -93.9 & -70.3 & 3.67 \\
\hline
\end{tabular}

Melting $\mathbf{T}$

$703 \mathrm{~K}$

$\Delta_{\text {fwe }} \mathrm{H}^{\circ}$

$9.16 \mathrm{~kJ}$

H298-H8

$\mathbf{k J}$
Boiling $T$

$\Delta_{\mathrm{vap}} \mathrm{H}^{\circ}$

$\mathbf{k J}$
Molar Vol. $2.899 \mathrm{~J} \cdot$ bar $^{-1}$ $28.99 \mathrm{~cm}^{3}$
$A=-1.0054 E+02$
$B=3.173 \mathrm{E}-02$
$C=-5.577 \mathrm{E}+05$ 
KBr: Cubic crystale 298.15 to melting point $1007 \mathrm{~K}$. Liquid 1007 to boiling point $1671 \mathrm{~K}$.

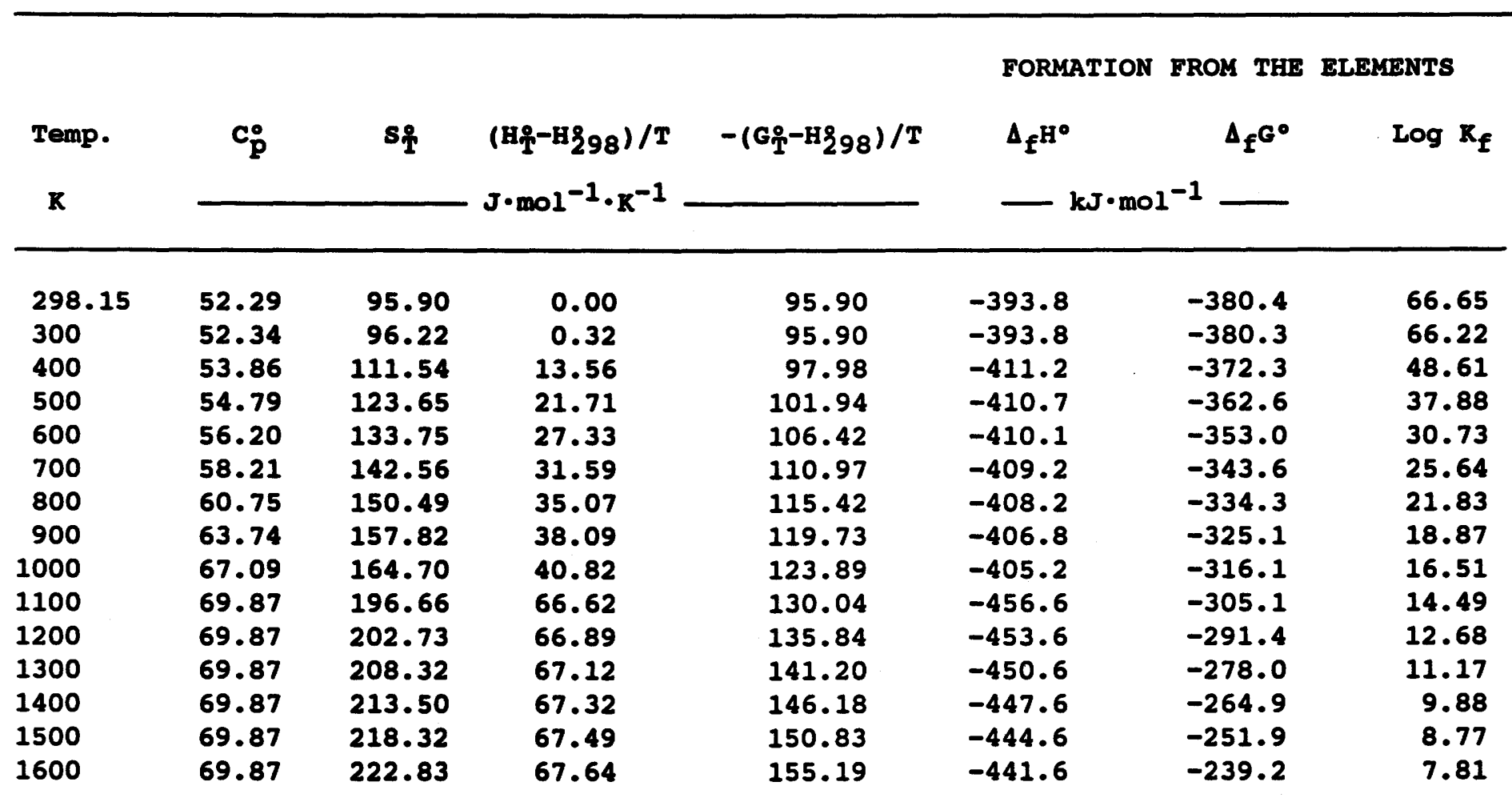

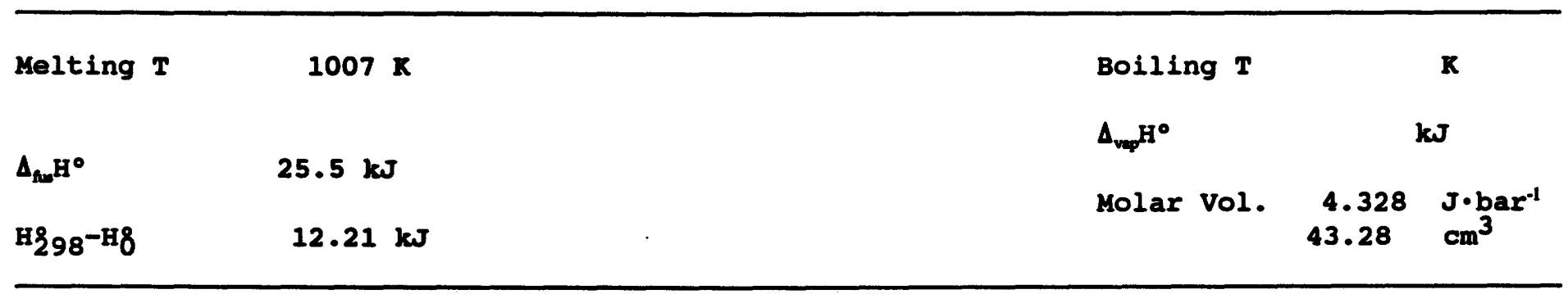

$A=-4.330 \mathrm{E}+02 \quad B=1.198 \mathrm{E}-01 \quad \mathrm{C}=1.642 \mathrm{E}+06$


AgCl: Cubic crystals 298.15 to melting point $728 \mathrm{~K}$; liquid 728 to $1000 \mathrm{~K}$.

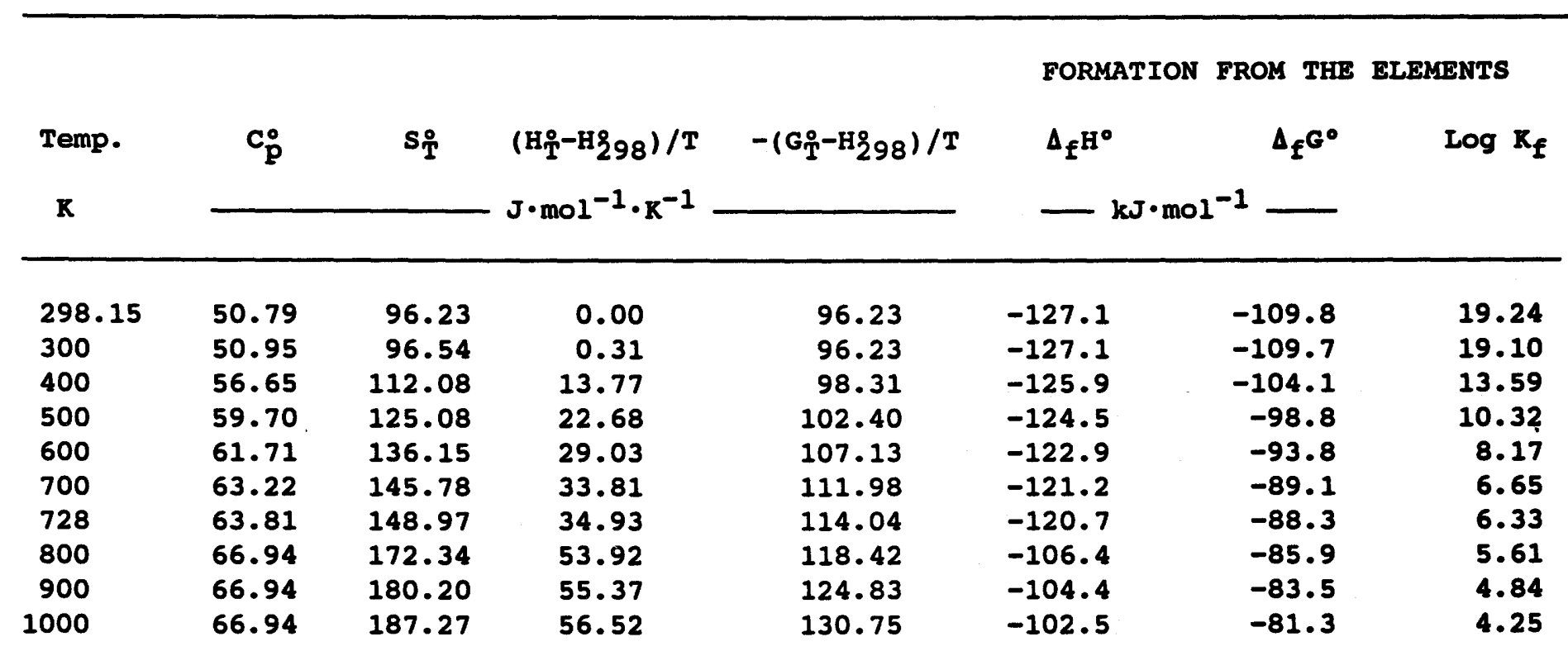

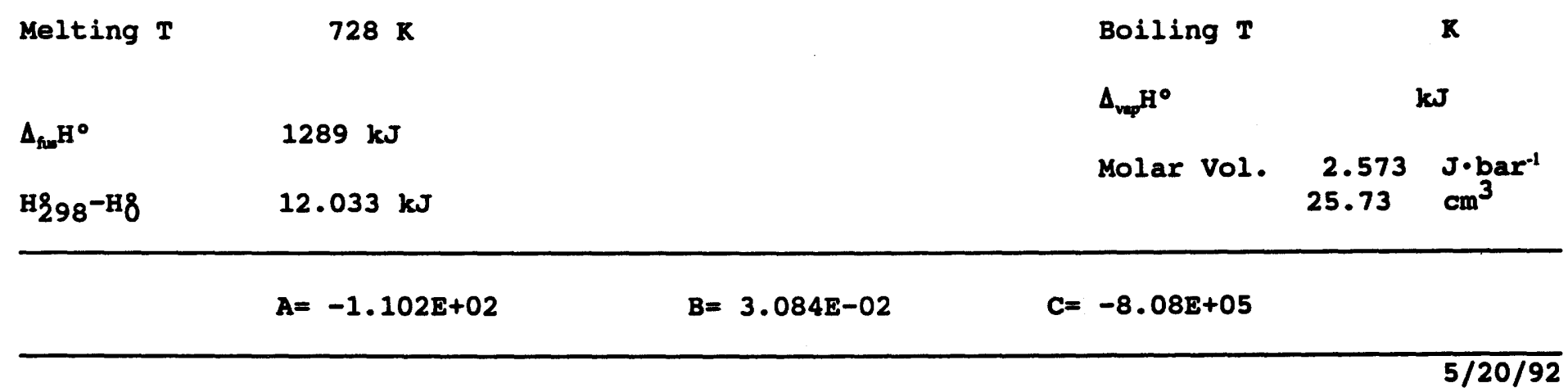


$\mathrm{CaCl}_{2}$ : Orthorhombic crystals 298.15 to $1045 \mathrm{~K}$. Liquid 1045 to $1800 \mathrm{~K}$.

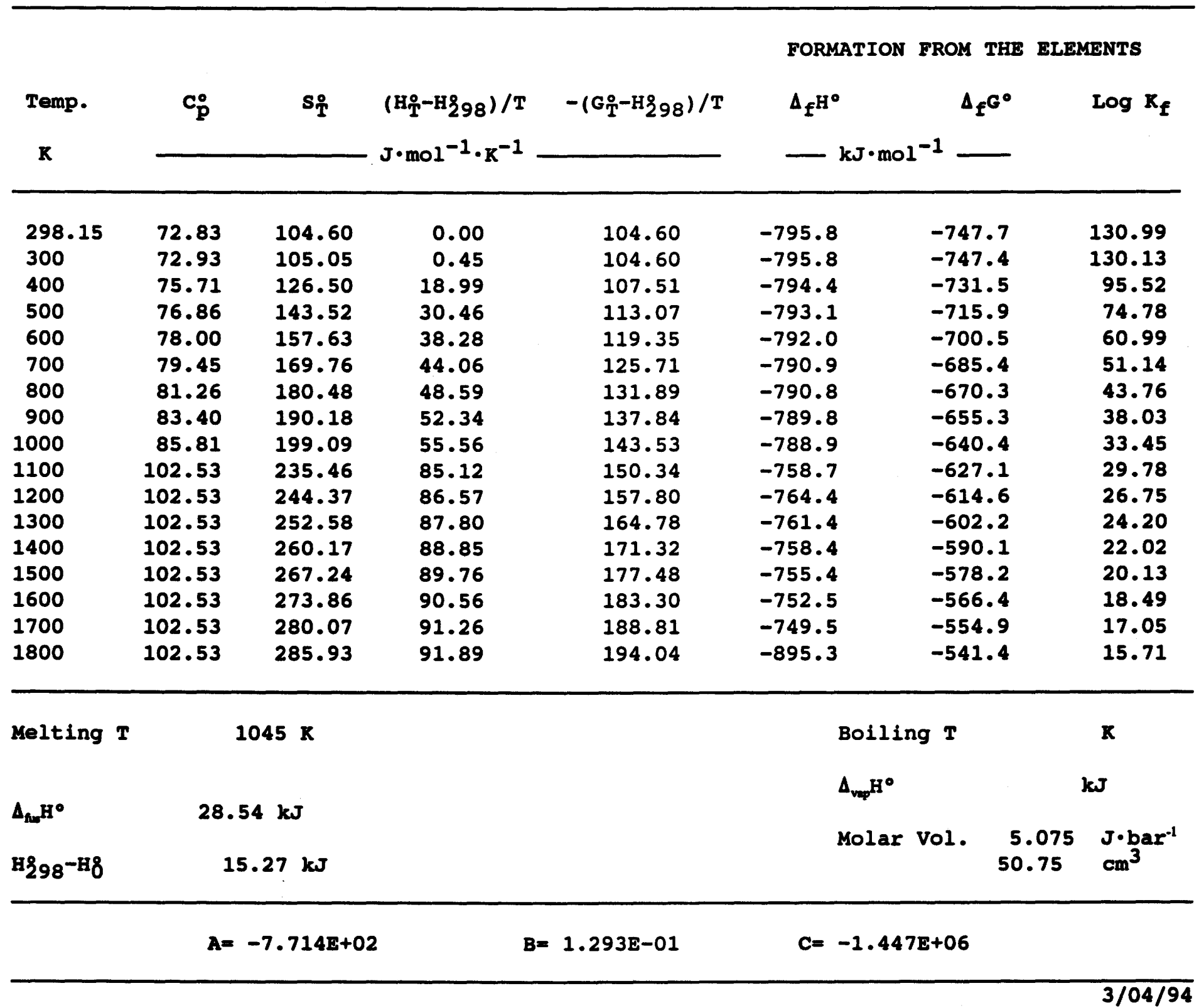


$\mathrm{FeCl}_{2}$ Rhombohedral crystals 298.15 to $950 \mathrm{~K} ;$ liquid 950 to $1300 \mathrm{~K}$.

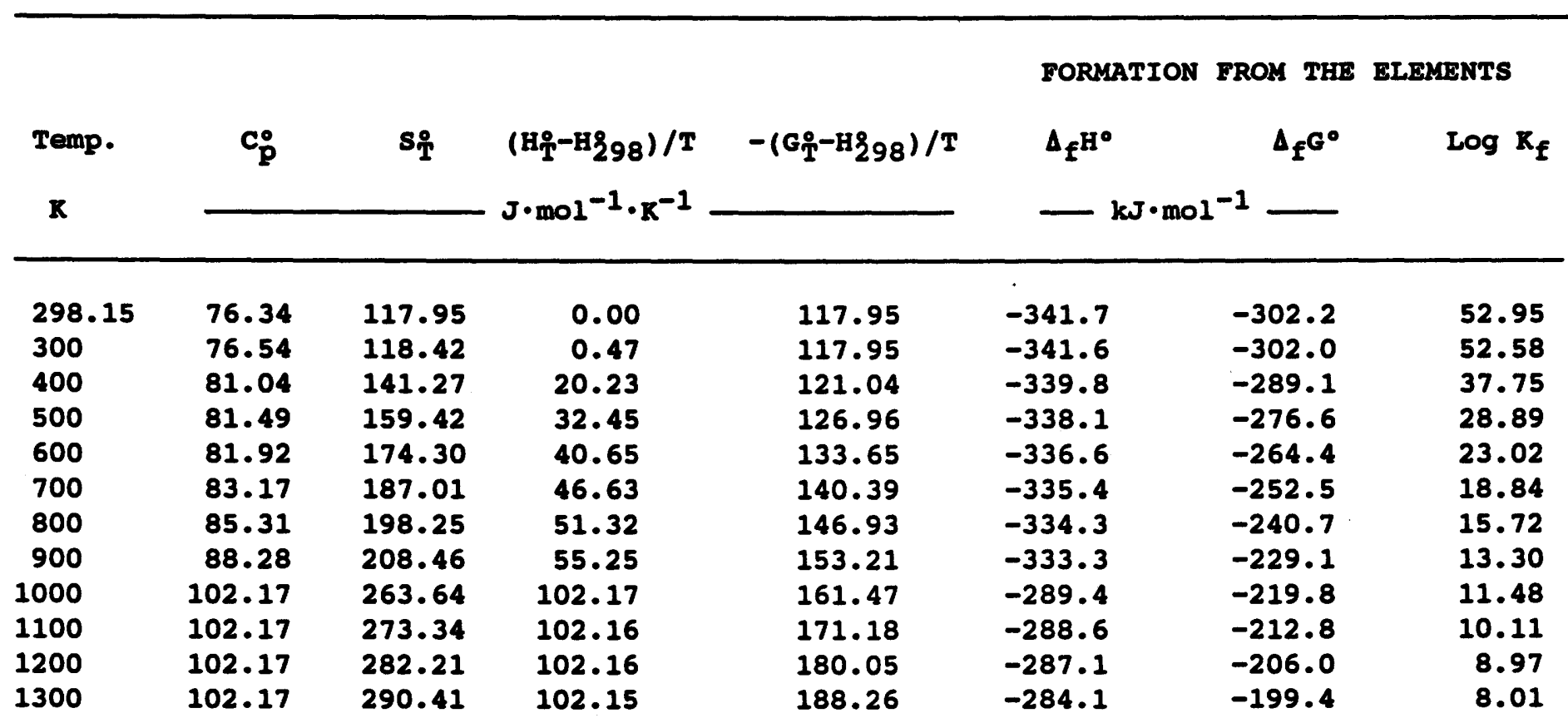

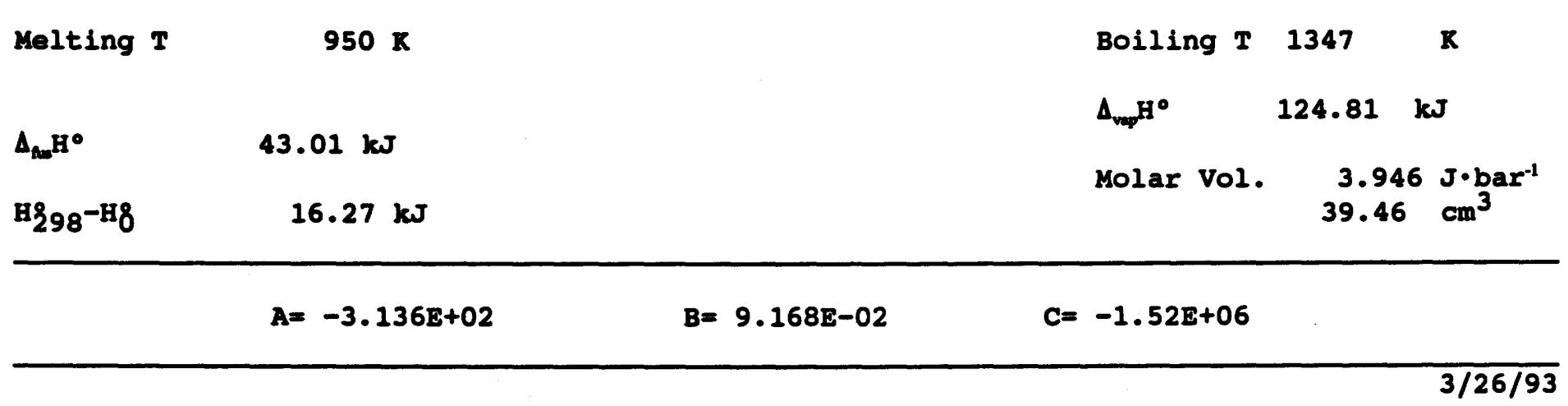


$\mathrm{FeCl}_{3}$ : Crystals 298.15 to melting point $577 \mathrm{~K}$; liquid 577 to boiling point $605 \mathrm{~K}$; Ideal gas (dimer) 605 to $700 \mathrm{~K}$.

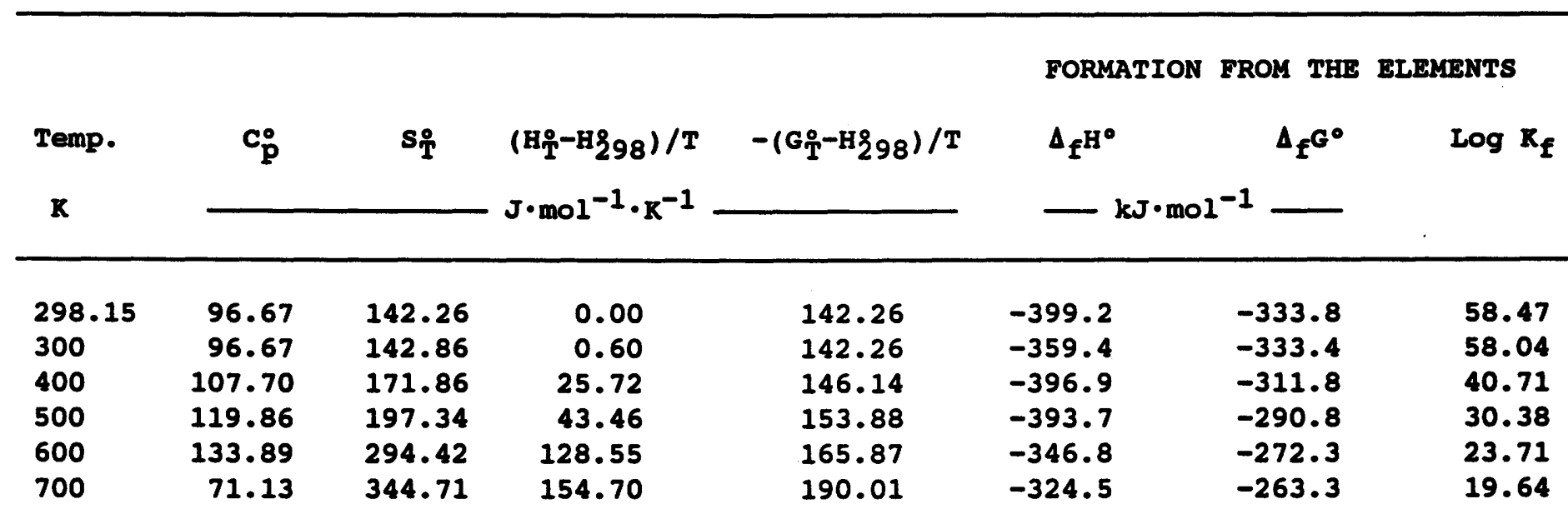

Melting $T$

$577 \mathrm{~K}$

$\Delta_{\text {fur }} \mathrm{H}^{\circ}$

$43.10 \mathrm{~kJ}$

H298-H8
Boiling T $605 \quad R$

$\Delta_{\mathrm{vep}} \mathrm{H}^{\circ} \quad 21.88 \mathrm{~kJ}$

Molar vol. $\quad 5.786 \mathrm{~J} \cdot \mathrm{bar}^{-1}$ $57.86 \mathrm{~cm}^{3}$
$A=-3 \cdot 455 E+02$
$B=1.274 \mathrm{E}-01$
$C=-2 \cdot 375 E+06$ 
HCl: Ideal gas at $\mathrm{p}=1$ bar, 298.15 to $1800 \mathrm{~K}$.

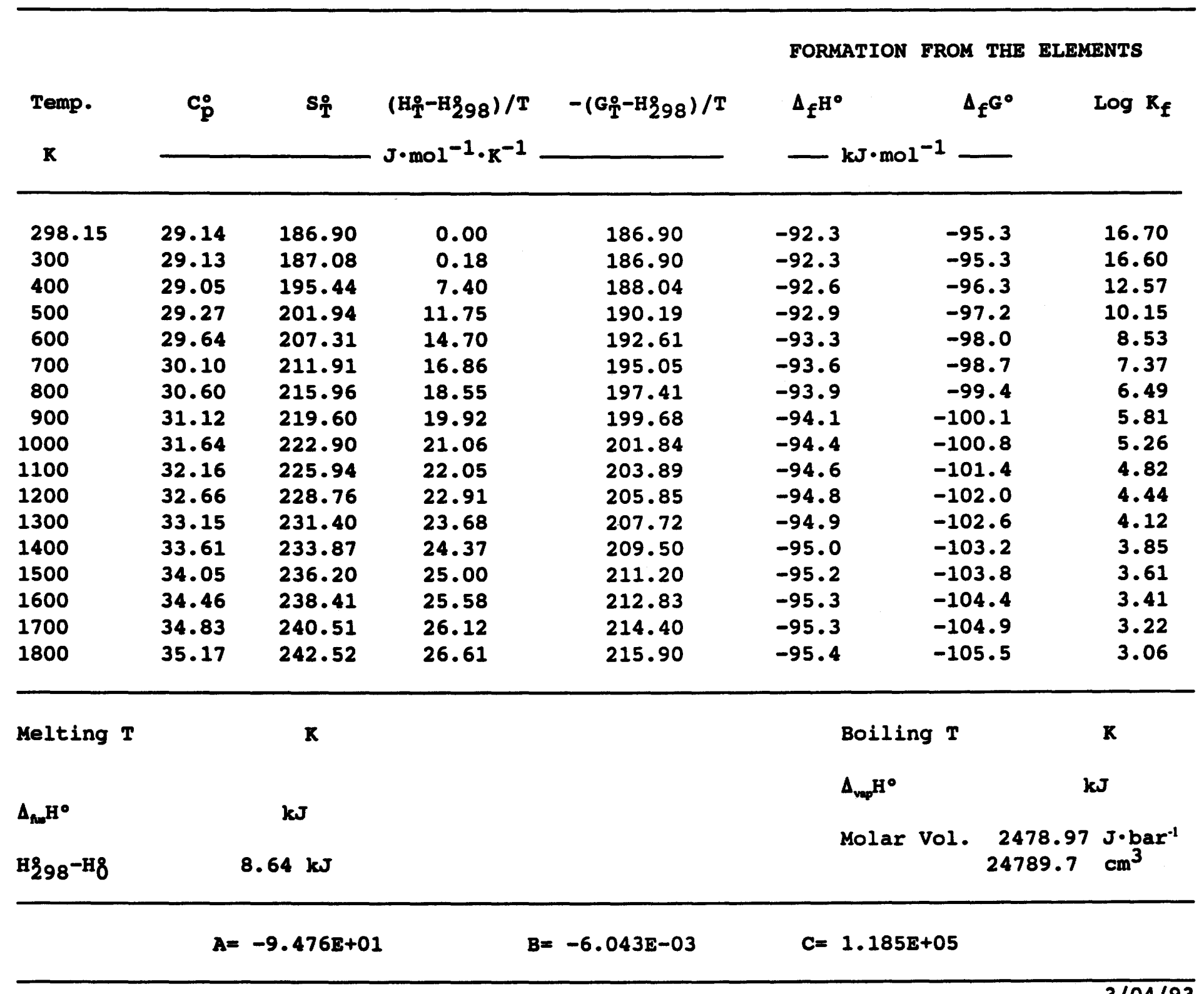


KCl: Cubic crystals 298.15 to $1043 \mathrm{~K}$, melting point; liquid 1043 to fictive boiling point $1475 \mathrm{~K}$.

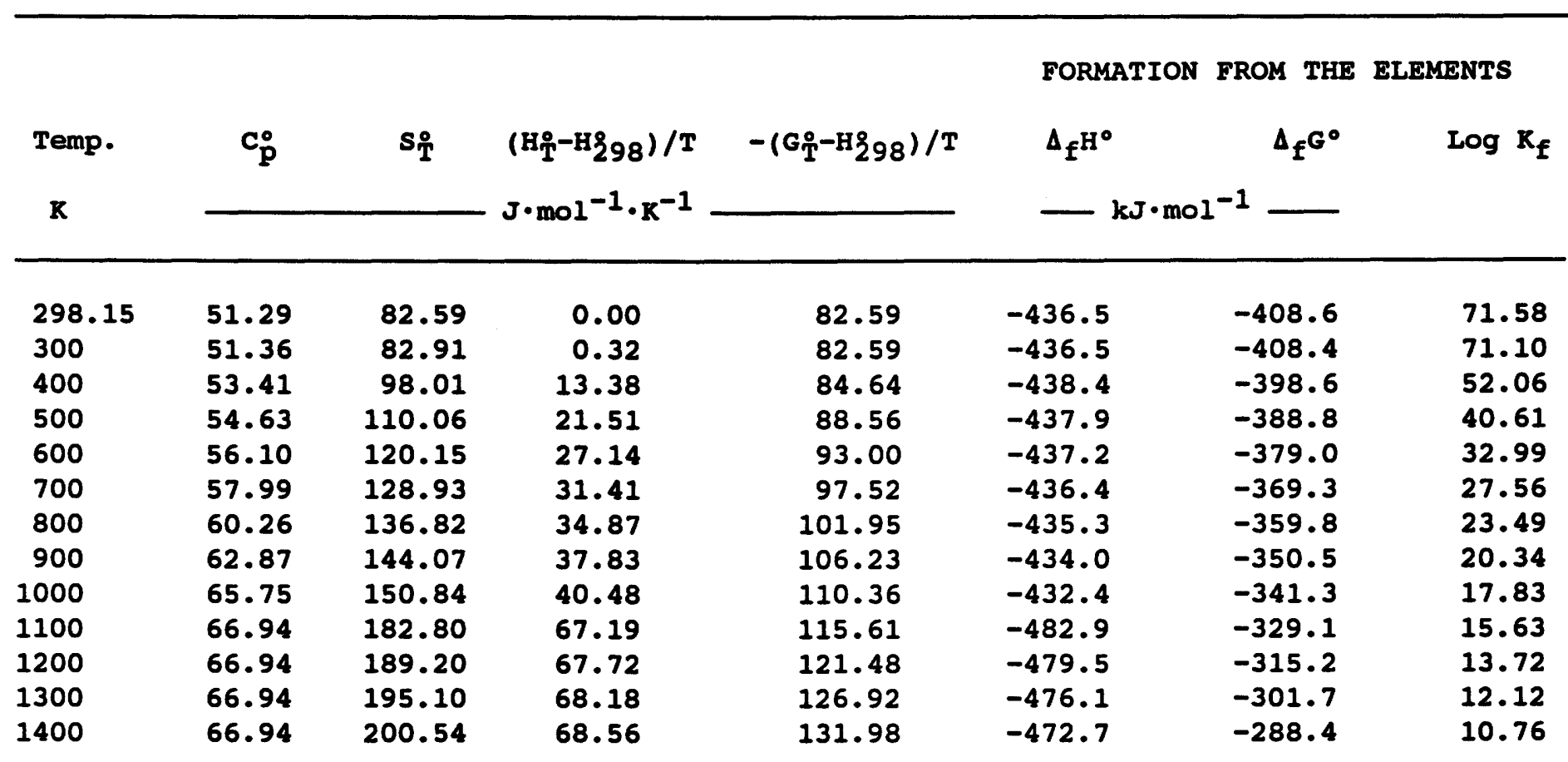

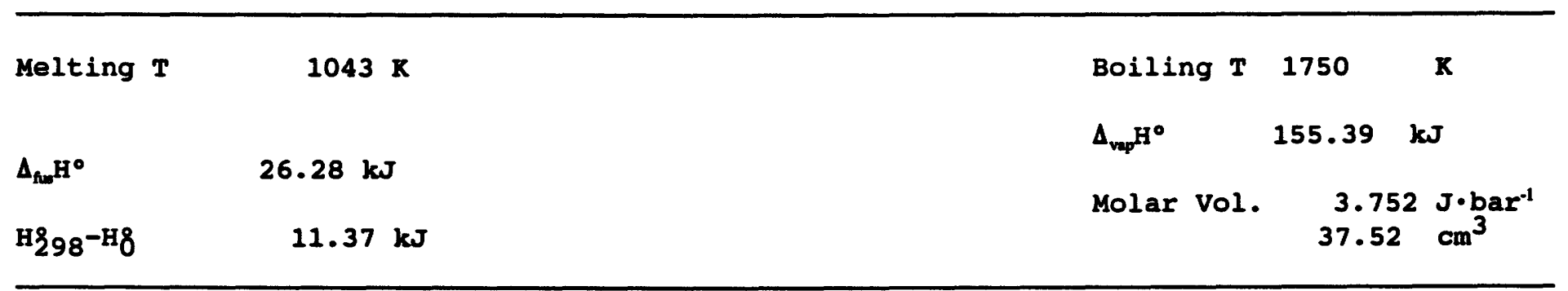
$A=-4 \cdot 584 E+02$
$B=1.207 E-01$
$C=1 \cdot 373 E+06$ 
$\mathrm{MgCl}_{2}$ : Rhombohedral crystals 298.15 to melting point $987 \mathrm{~K}$. Liquid 987 to boiling point $1710 \mathrm{~K}$.

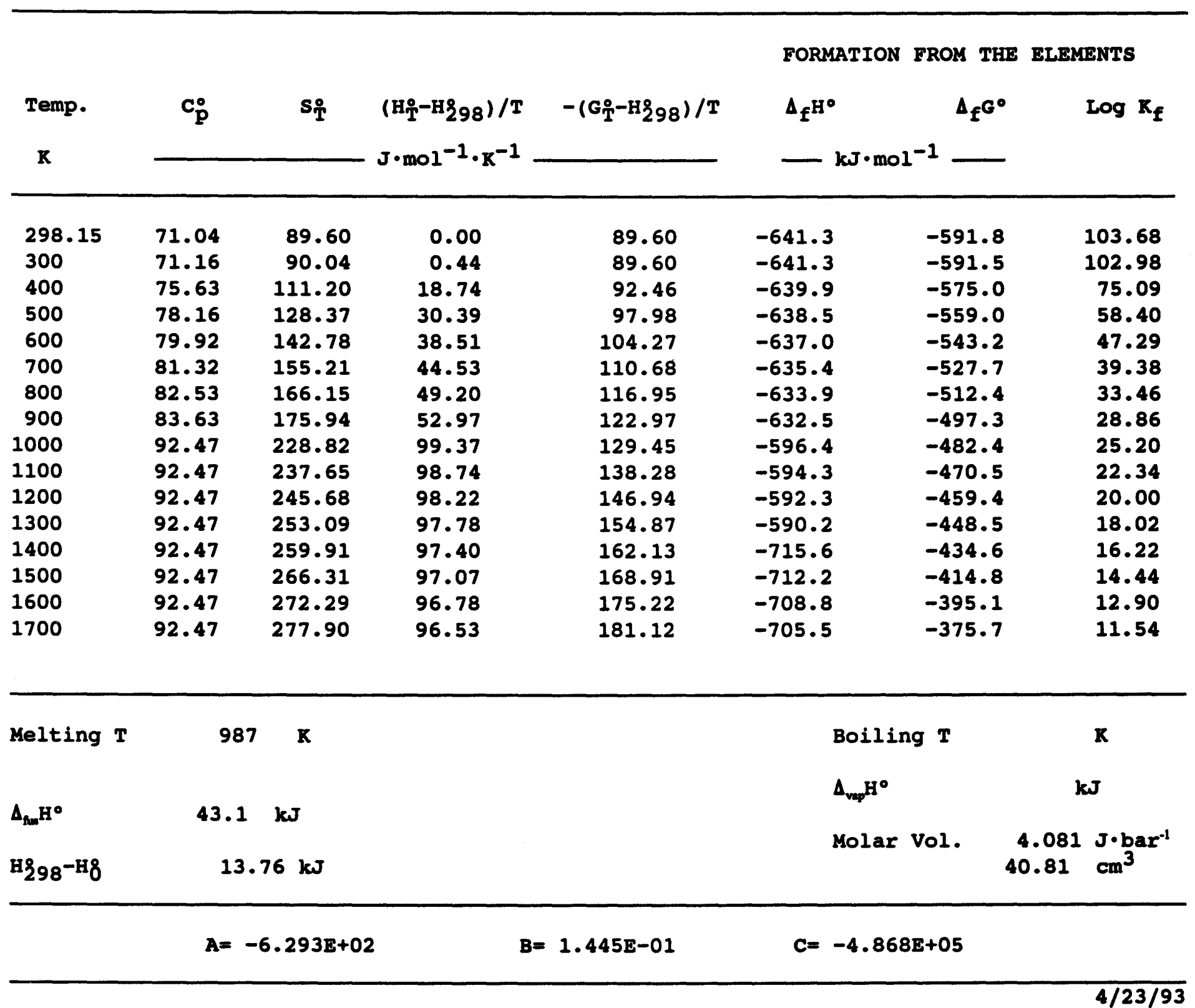


$\mathrm{MnCl}_{2}$ : Rhombohedral crystals 298.15 to $923 \mathrm{~K}$; liquid 923 to $1400 \mathrm{~K}$.

\begin{tabular}{|c|c|c|c|c|c|c|c|}
\hline \multirow{3}{*}{$\begin{array}{l}\text { Temp. } \\
\text { K }\end{array}$} & \multirow{3}{*}{$\mathbf{c}_{\mathbf{p}}^{\circ}$} & \multirow{3}{*}{$\mathbf{s}_{\mathbf{T}}$} & \multirow{3}{*}{$\begin{array}{l}\left(\mathrm{H}_{\mathrm{T}}^{0}-\mathrm{H} 298\right) / \mathrm{T} \\
\mathrm{J} \cdot \mathrm{mol}^{-1} \cdot \mathrm{K}^{-1}\end{array}$} & \multirow{3}{*}{$-\left(G_{T}^{\circ}-H_{298}\right) / T$} & \multirow{2}{*}{\multicolumn{2}{|c|}{$\begin{array}{l}\text { FORMATION FROM THE } \\
\Delta_{f^{H^{\circ}}}\end{array}$}} & \multirow{3}{*}{$\begin{array}{l}\text { ELEMENTS } \\
\qquad \log \mathrm{K}_{\mathbf{f}}\end{array}$} \\
\hline & & & & & & & \\
\hline & & & & & $\ldots \mathbf{k J} \cdot \mathrm{mol}$ & -1 & \\
\hline 298.15 & 73.01 & 118.24 & 0.00 & 118.24 & $-481 \cdot 3$ & -440.5 & 77.17 \\
\hline 300 & 73.11 & 118.69 & 0.45 & 118.24 & $-481 \cdot 3$ & -440.2 & 76.65 \\
\hline 400 & 77.24 & 140.35 & 19.19 & 121.17 & -479.9 & -426.8 & 55.73 \\
\hline 500 & 79.80 & 157.88 & 31.07 & 126.81 & -478.6 & -413.6 & 43.21 \\
\hline 600 & 81.73 & 172.60 & 39.36 & 133.25 & -477.2 & -400.8 & 34.89 \\
\hline 700 & 83.36 & 185.33 & 45.53 & 139.80 & -475.9 & -388.1 & 28.96 \\
\hline 800 & 84.84 & 196.56 & 50.35 & 146.21 & -474.6 & -375.7 & 24.53 \\
\hline 900 & 86.23 & 206.63 & 54.26 & 152.37 & -473.3 & -363.4 & 21.09 \\
\hline 1000 & 94.56 & 257.19 & 95.73 & 161.46 & -436.1 & -354.4 & 18.51 \\
\hline 1100 & 94.56 & 266.19 & 95.62 & 170.57 & -434.2 & -346.3 & 16.44 \\
\hline 1200 & 94.56 & 274.43 & 95.54 & 178.89 & -432.3 & -338.4 & 14.73 \\
\hline 1300 & 94.56 & 282.00 & 95.46 & 186.54 & -430.5 & -330.6 & 13.29 \\
\hline 1400 & 94.56 & 288.99 & 95.40 & 193.59 & -431.0 & -322.9 & 12.05 \\
\hline
\end{tabular}

Melting $\mathbf{T}$

$\Delta_{\mathrm{fum}} \mathrm{H}^{\circ}$

$37.53 \mathrm{~kJ}$

H298-H8
Boiling T

$\Delta_{\mathrm{vpq}} \mathrm{H}^{\circ}$

Molar vol.
$A=-4.505 E+02$
$B=9.437 E-02$
$C=-1.734 E+06$ 
NaCl: Cubic crystals 298.15 to $1073.8 \mathrm{~K}$; liquid 1073.8 to fictive boiling point $1791 \mathrm{~K}$.

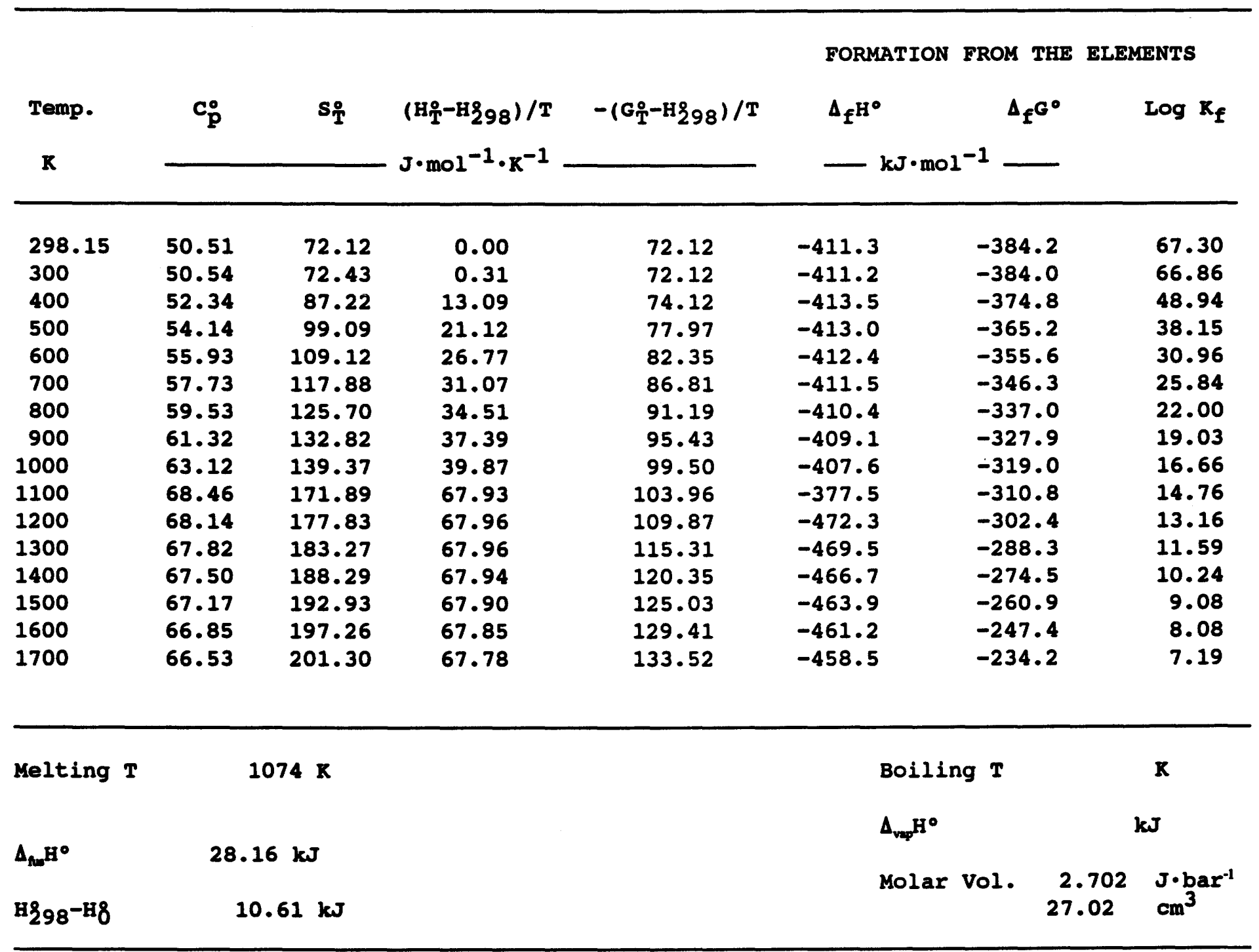


$\mathrm{NiCl}_{2}$ : Rhombohedral crystals 298.15 to melting point.

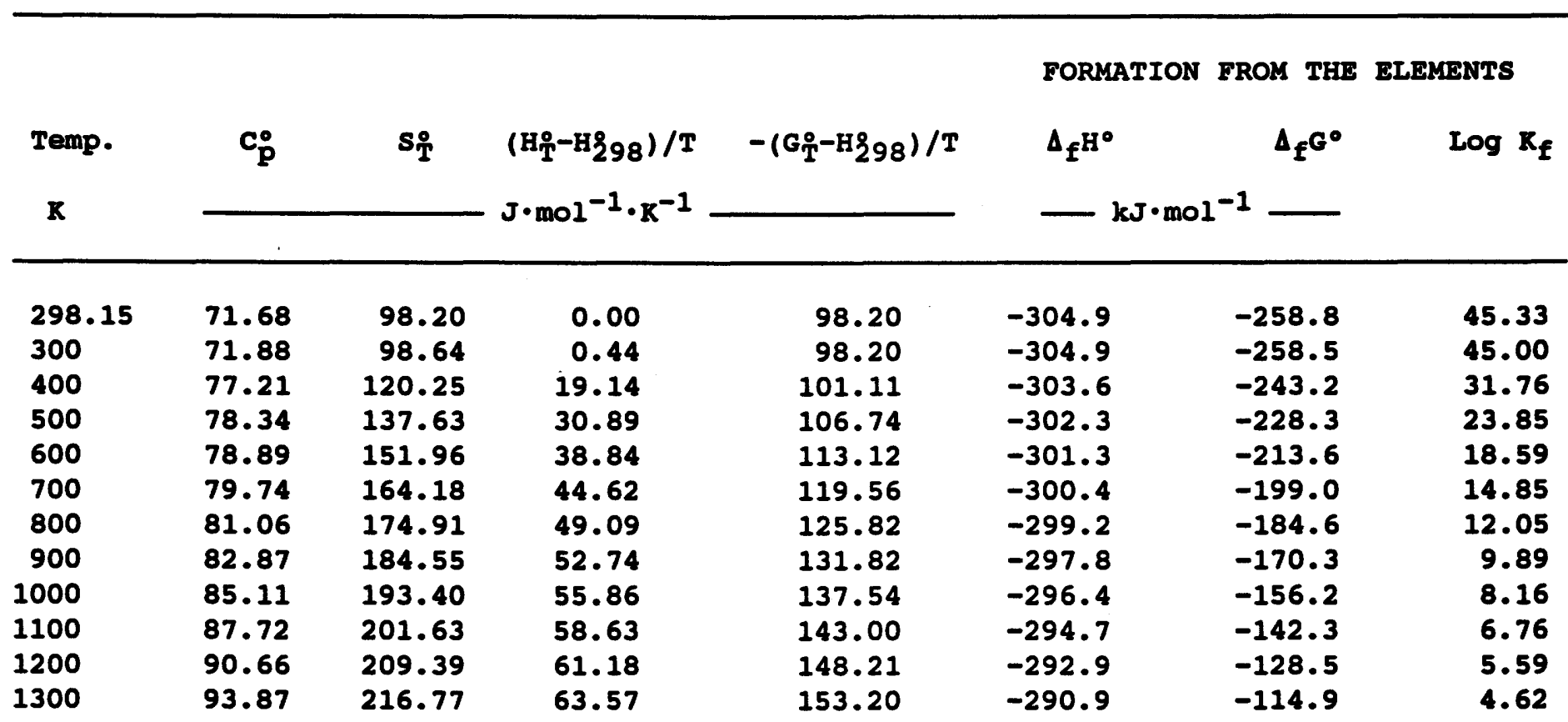

Melting $\mathbf{T}$

$\Delta_{\text {hu }} \mathrm{H}^{\circ}$

H298-H8
$1303 \mathrm{~K}$

$77.17 \mathrm{~kJ}$

$14.43 \mathrm{~kJ}$
Boiling T

$\Delta_{\mathrm{vq}} \mathrm{H}^{\circ}$

Molar Vol.

\section{$\mathbf{R}$}

$\mathbf{k J}$
$A=-2.965 E+02$
$B=1.403 \mathrm{E}-01$
$C=-3.75 E+05$ 
$\mathrm{PbCl}_{2}$ : Orthorhombic crystals 298.15 to $768 \mathrm{~K}$; liquid 768 to $1000 \mathrm{~K}$.

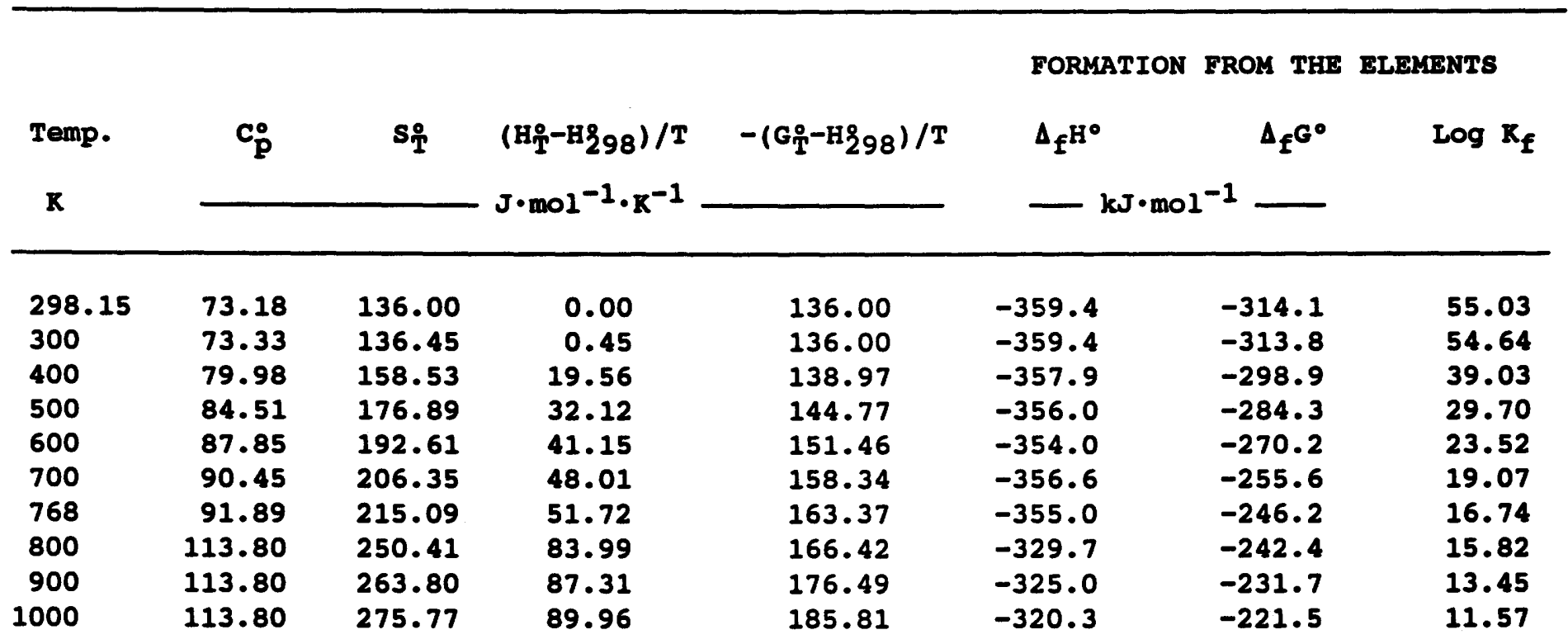

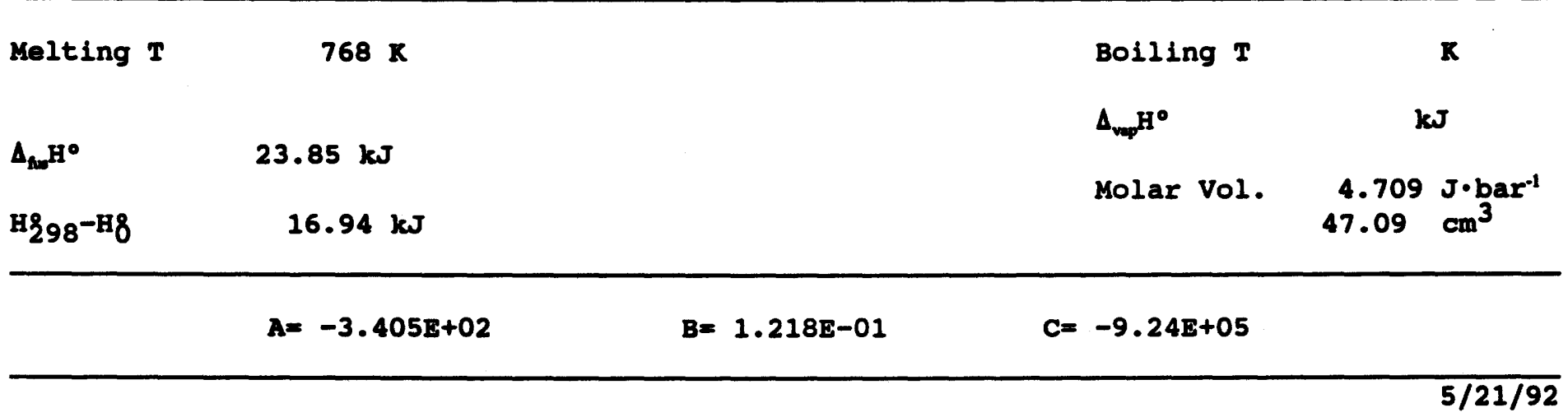


$\mathrm{CaF}_{2}$ : Cubic crystals $298.15 \mathrm{~K}$ to transition at $1424 \mathrm{~K}$.

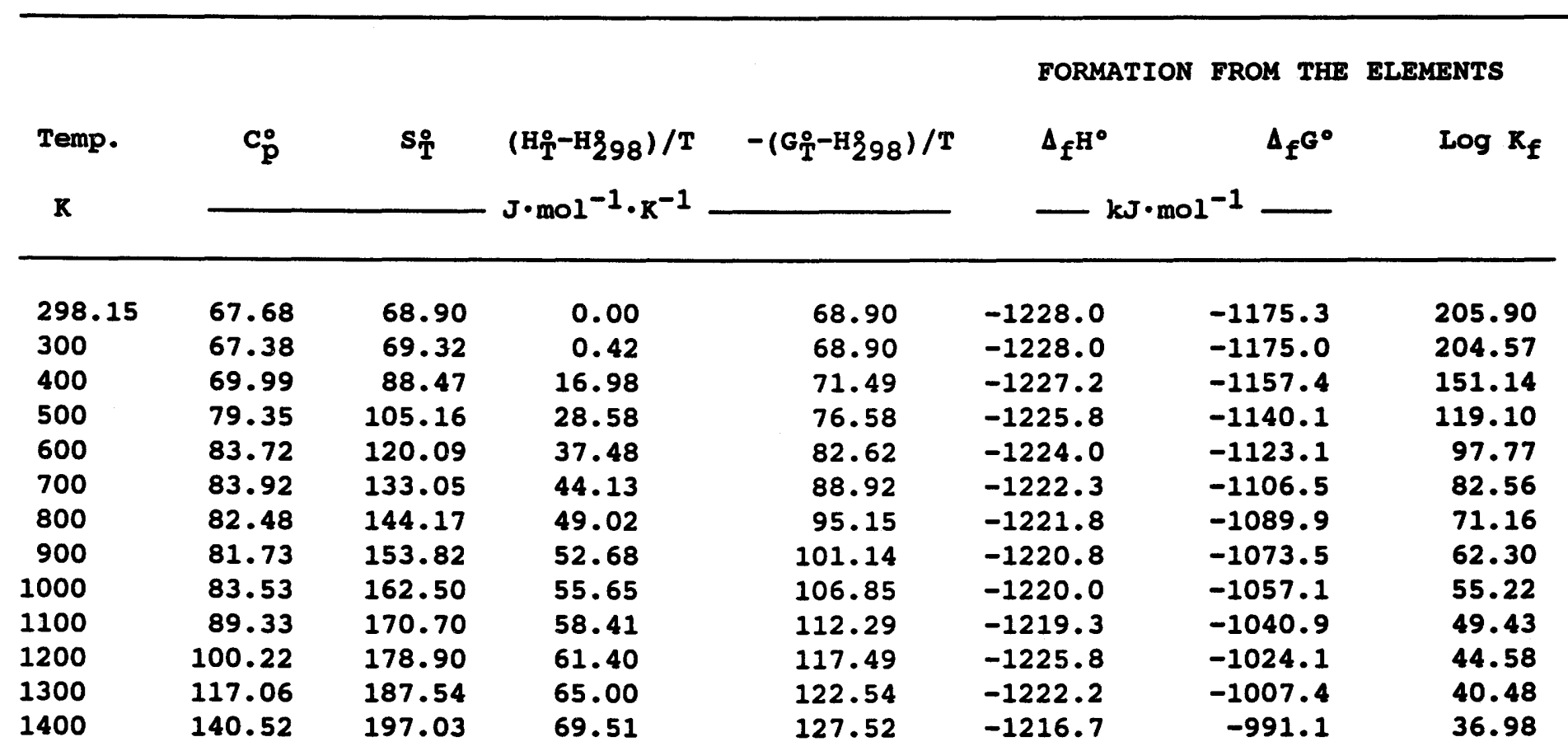

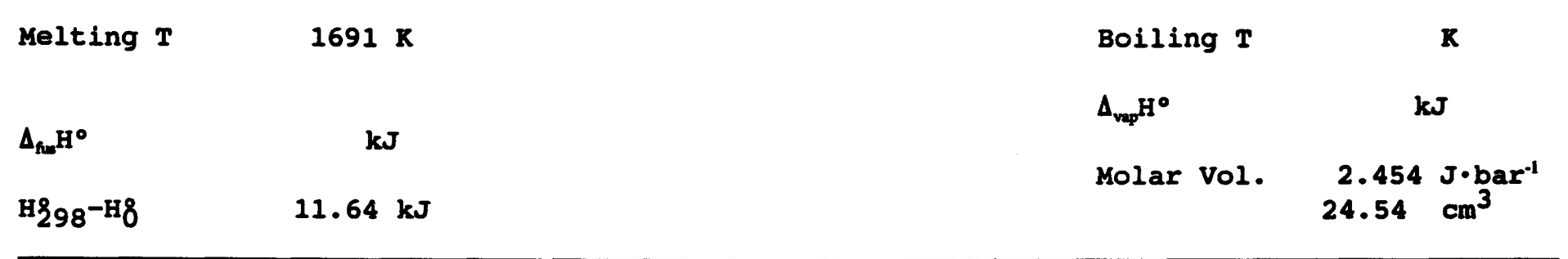
$A=-1.221 \mathrm{E}+03$
$B=1.643 \mathrm{E}-01$
$C=-2.93 E+05$ 


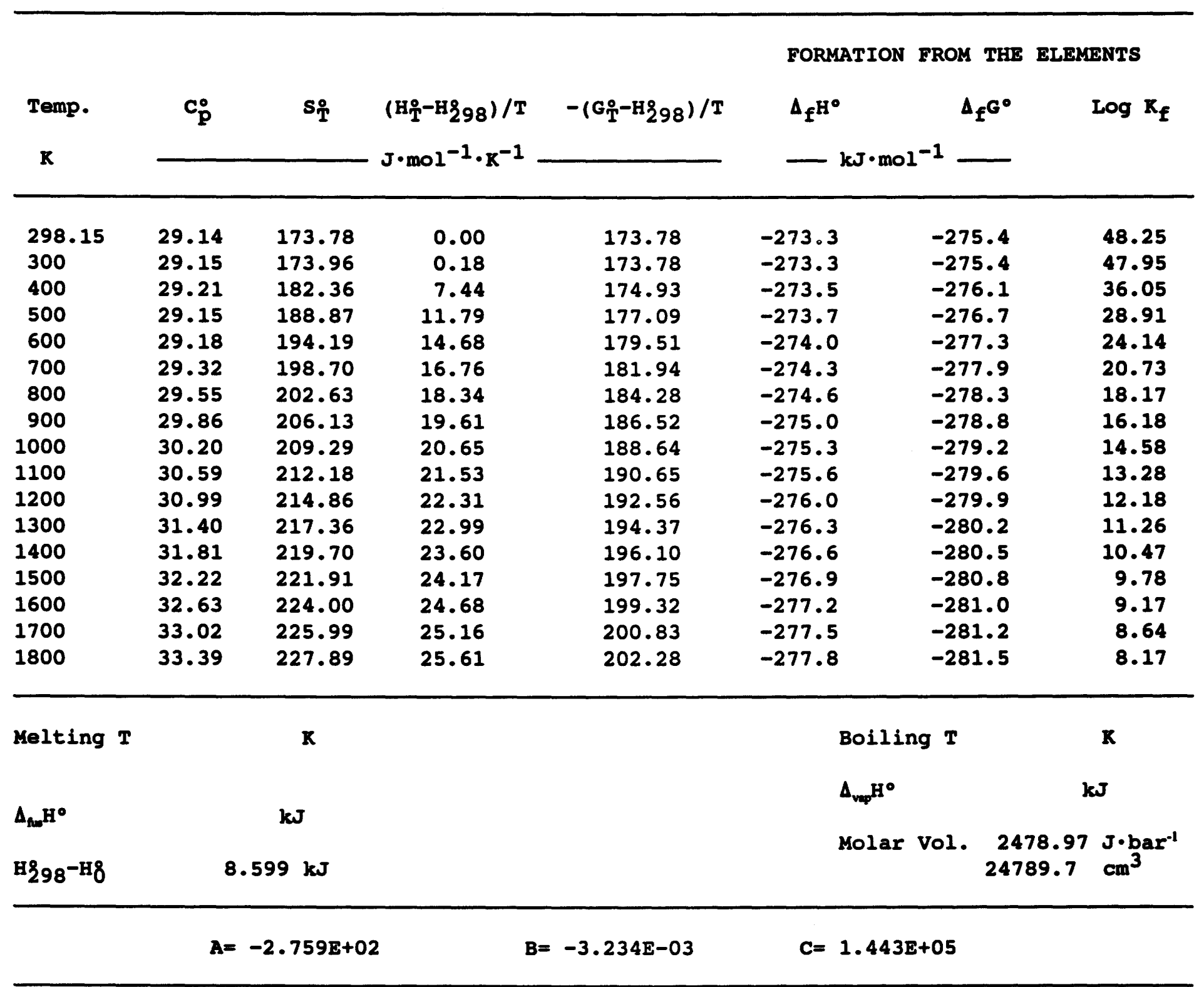


$\mathrm{MgF}_{2}$ : Tetragonal crystals 298.15 to melting point $1536 \mathrm{~K}$. Liquid 1536 to $1800 \mathrm{~K}$.

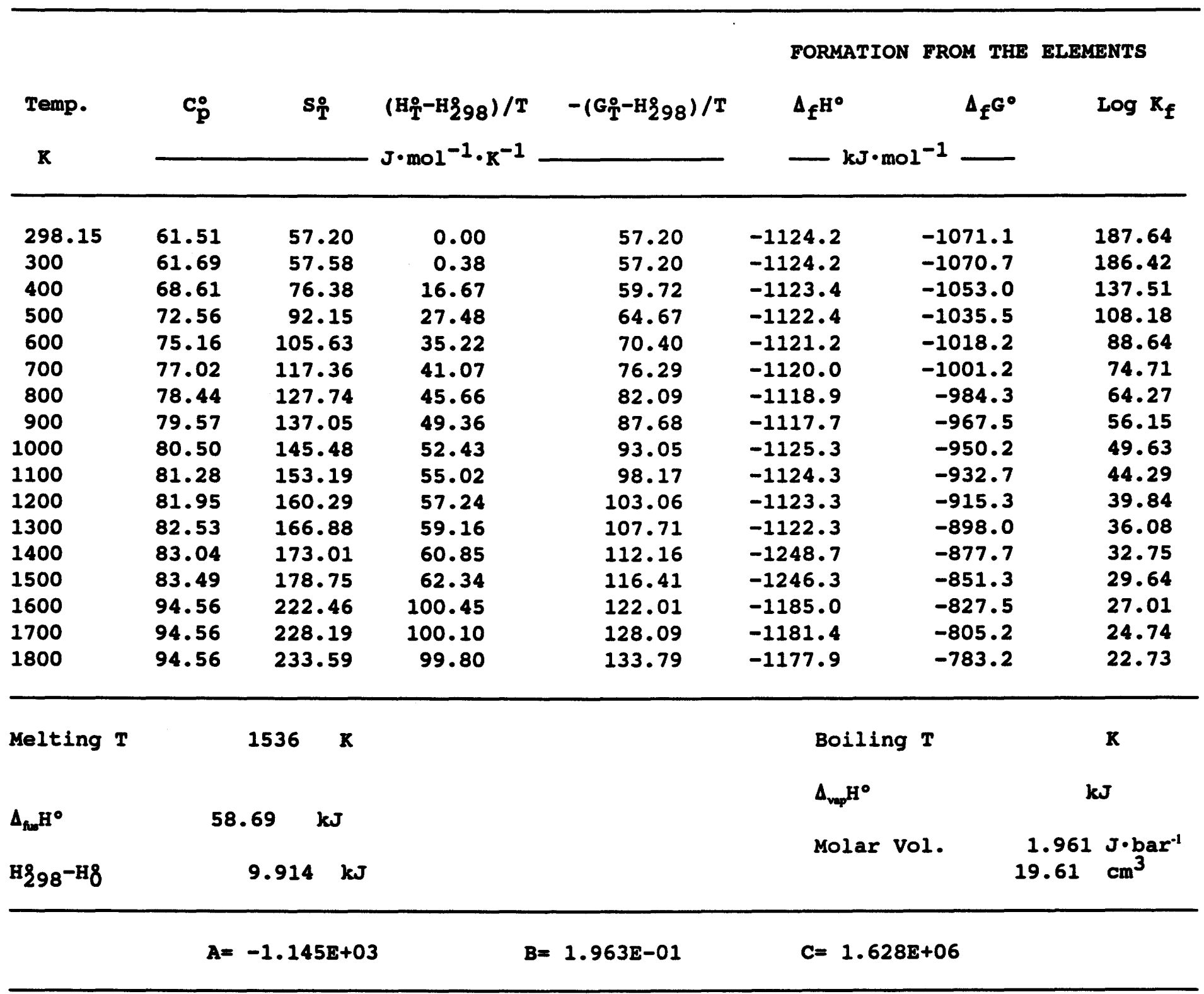


NaF: Cubic crystals 298.15 to melting point $1269 \mathrm{~K}$. Iiquid 1269 to $1800 \mathrm{~K}$.

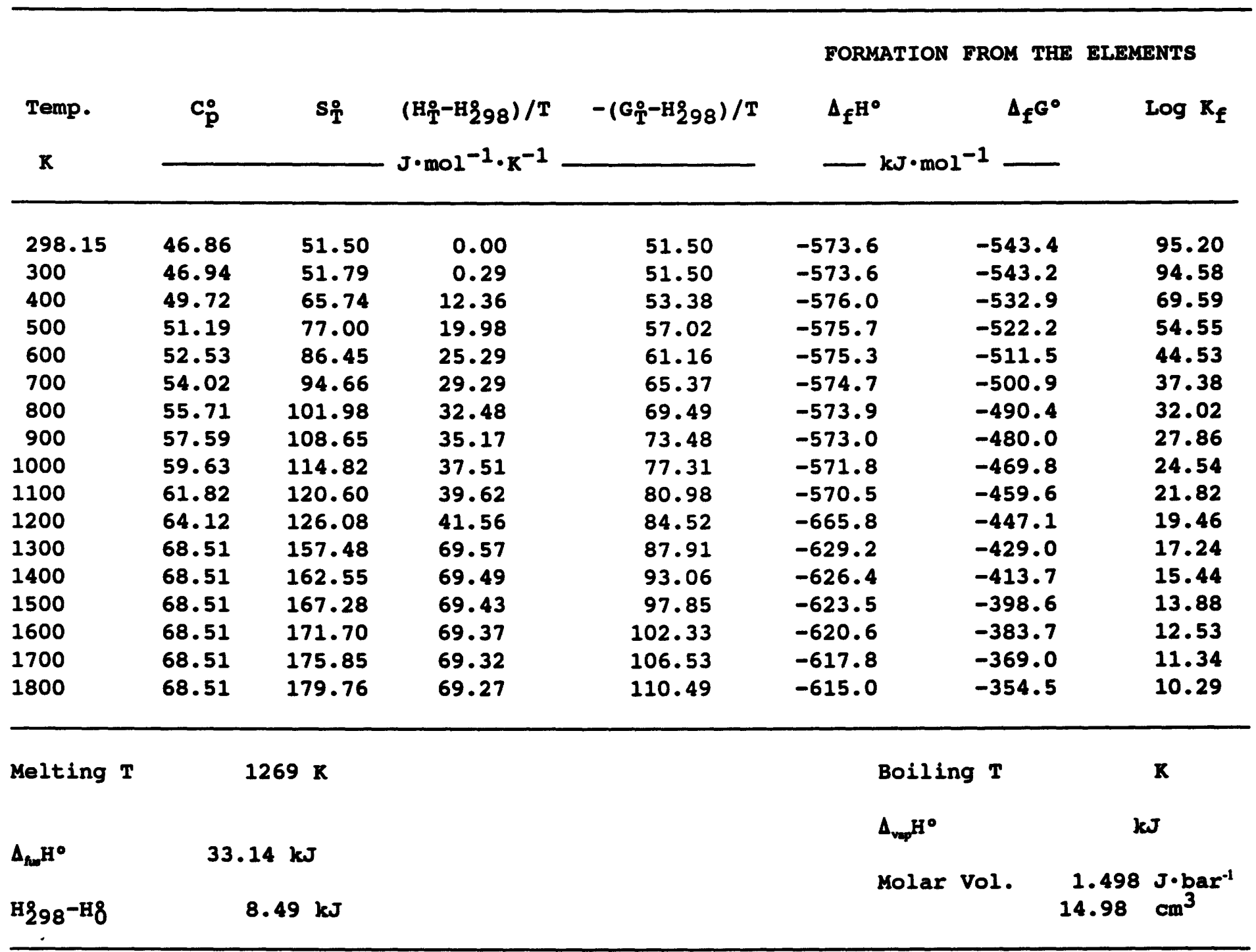




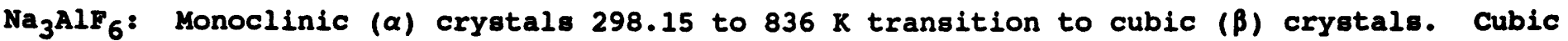
crystals 836 to $1153 \mathrm{~K}$. $\gamma$ crystals 1153 to melting point $1290 \mathrm{~K}$. Liquid 1290 to $1800 \mathrm{~K}$.

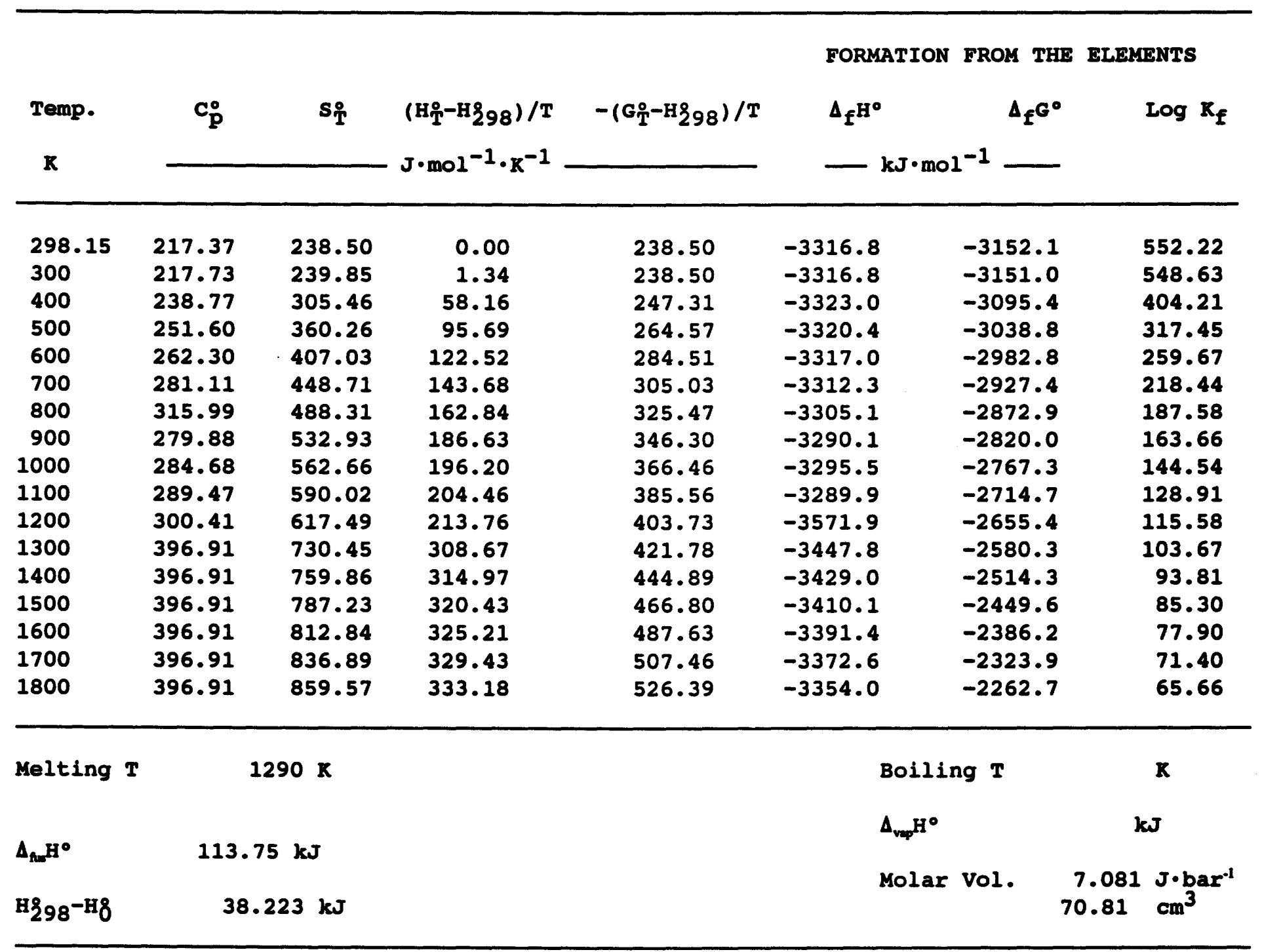

$A=-3.370 \mathrm{E}+03 \quad B=6.097 \mathrm{E}-01 \quad \mathrm{C}=-3.62 \mathrm{E}+06$


$\mathrm{Na}_{5} \mathrm{Al}_{3} \mathrm{~F}_{14}$ : Tetragonal crystals 298.15 to melting point $1010 \mathrm{~K}$.

\begin{tabular}{|c|c|c|c|c|c|c|c|}
\hline \multirow[b]{2}{*}{ Temp. } & \multirow[b]{2}{*}{$\mathbf{c}_{\mathbf{p}}^{\circ}$} & \multirow[b]{2}{*}{$\mathbf{S}_{\mathrm{T}}^{\circ}$} & \multirow{3}{*}{$\begin{array}{l}\left(\mathrm{H}_{\mathrm{T}}-\mathrm{H}_{298}\right) / \mathrm{T} \\
\mathrm{J} \cdot \mathrm{mol}^{-1} \cdot \mathrm{K}^{-1}\end{array}$} & \multirow[b]{2}{*}{$-\left(G_{T}^{\circ}-H_{298}\right) / T$} & \multirow{2}{*}{$\begin{array}{l}\text { FORMATION } \\
\Delta_{f} \mathrm{H}^{\circ}\end{array}$} & \multirow{2}{*}{$\begin{array}{c}\text { FROM THE } \\
\Delta_{\mathrm{F}} \mathbf{G}^{\circ}\end{array}$} & \multirow{2}{*}{$\begin{array}{l}\text { ELEMENTS } \\
\text { Log } \mathrm{K}_{\mathbf{f}}\end{array}$} \\
\hline & & & & & & & \\
\hline $\mathbf{R}$ & & & & & $-\mathbf{k J} \cdot \mathbf{m o}$ & $1^{-1}$ & \\
\hline 298.15 & 453.91 & 515.30 & 0.00 & 515.30 & $-7546 \cdot 3$ & -7174.7 & 1256.95 \\
\hline 300 & 455.08 & 518.11 & 2.80 & 515.31 & -7546.3 & -7172.4 & 1248.79 \\
\hline 400 & 499.34 & 655.82 & 122.04 & 533.78 & -7556.5 & -7047.1 & 920.24 \\
\hline 500 & 524.54 & 770.16 & 200.20 & 569.96 & -7552.3 & -6920.2 & 722.94 \\
\hline 600 & 542.23 & 867.43 & 255.80 & 611.63 & -7546.5 & -6794.3 & 591.49 \\
\hline 700 & 556.38 & 952.11 & 297.76 & 654.35 & -7539.6 & -6669.5 & 497.67 \\
\hline 800 & 568.64 & 1027.22 & 330.87 & 696.35 & -7532.1 & -6545.7 & 427.38 \\
\hline 900 & 579.80 & 1094.85 & 357.91 & 736.94 & -7524.1 & -6422.9 & 372.77 \\
\hline 1000 & 590.28 & 1156.48 & 380.63 & 775.85 & -7547.6 & -6298.8 & 329.01 \\
\hline
\end{tabular}

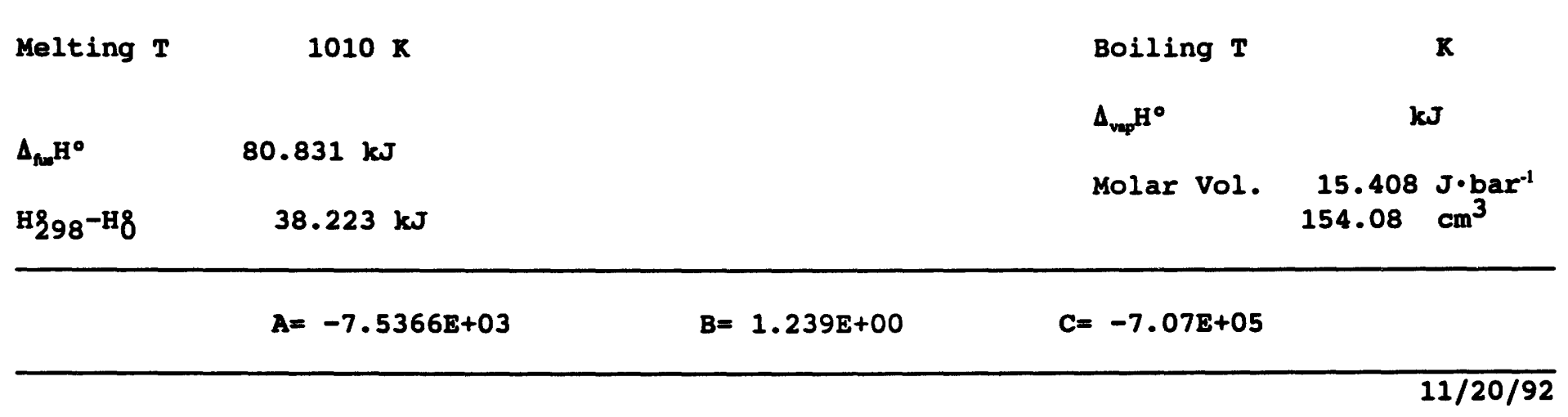


AgI: Hexagonal crystals 298.15 to $423 \mathrm{~K}$; cubic crystals 423 to $831 \mathrm{~K}$.

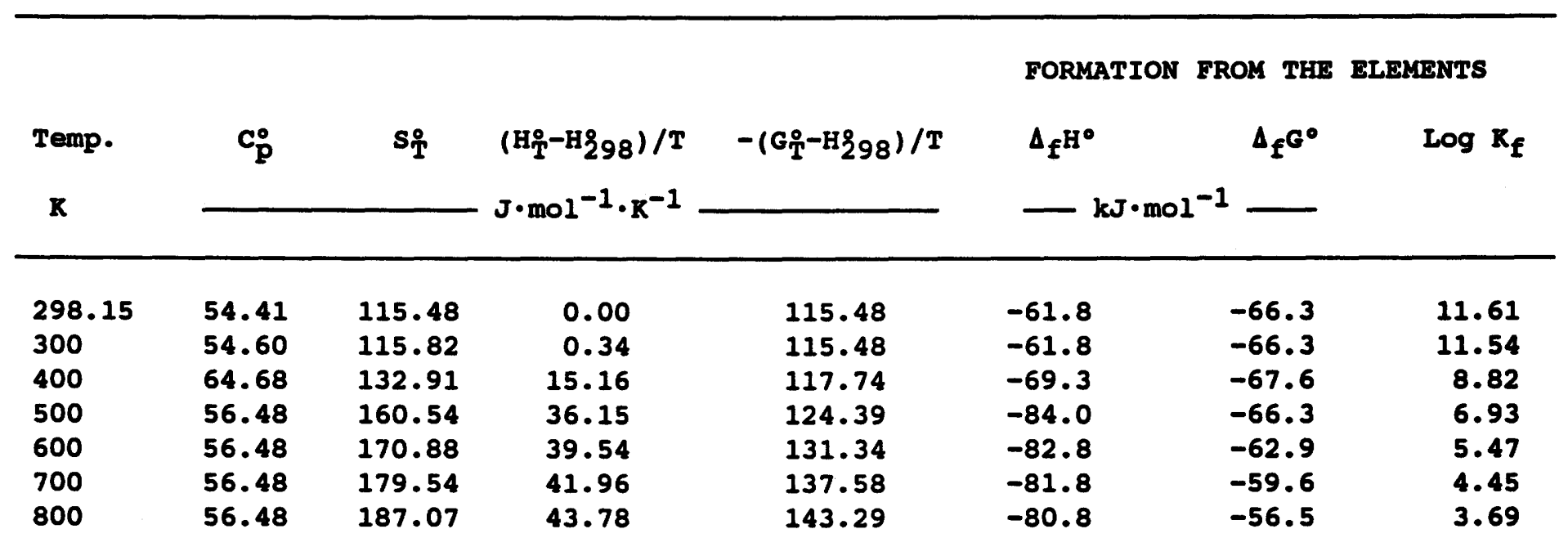

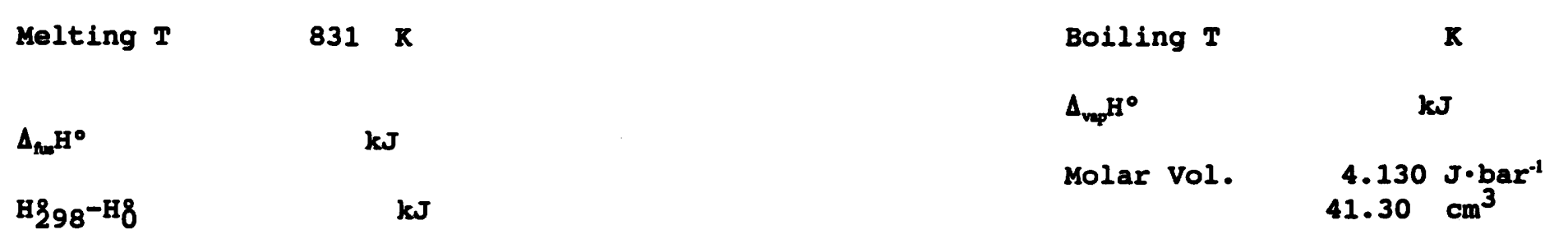
$A=-9 \cdot 280 \mathrm{E}+01$
$B=4 \cdot 399 E-02$
$C=1.196 \mathrm{E}+06$ 
CuI: Cubic crystals 298.15 to transition point at $643 \mathrm{~K}$. There is a second transition at $679 \mathrm{~K}$. CuI melts at $868 \mathrm{~K}$.

\begin{tabular}{|c|c|c|c|c|c|c|c|}
\hline \multirow{3}{*}{$\begin{array}{l}\text { Temp. } \\
\text { K }\end{array}$} & \multirow{3}{*}{$\mathbf{c}_{\mathbf{p}}^{\circ}$} & \multirow{3}{*}{$\mathbf{s}_{\mathrm{T}}$} & \multirow{3}{*}{$\begin{array}{l}\left(\mathrm{H}_{\mathrm{T}}^{\circ}-\mathrm{H} 298\right) / \mathrm{T} \\
\mathrm{J} \cdot \mathrm{mol}^{-1} \cdot \mathrm{K}^{-1}\end{array}$} & \multirow{3}{*}{$-\left(G_{T}^{\circ}-H_{298}\right) / T$} & \multicolumn{2}{|c|}{ FORMATION FROM THE } & \multirow{3}{*}{$\begin{array}{l}\text { ELEMENTS } \\
\qquad \log k_{f}\end{array}$} \\
\hline & & & & & $\Delta_{f} \mathrm{H}^{\circ}$ & $\Delta_{f} G^{0}$ & \\
\hline & & & & & \multicolumn{2}{|c|}{$-\mathrm{kJ} \cdot \mathrm{mol}^{-1}$} & \\
\hline 298.15 & 54.06 & 96.60 & 0.00 & 96.60 & -67.8 & -69.4 & 12.16 \\
\hline 300 & 54.14 & 96.93 & 0.33 & 96.60 & -67.8 & -69.4 & 12.09 \\
\hline 400 & 57.20 & 112.92 & 14.17 & 98.75 & -75.5 & -69.7 & 9.10 \\
\hline 500 & 60.59 & 125.89 & 22.98 & 102.91 & -96.4 & -66.3 & 6.92 \\
\hline 600 & 76.90 & 137.85 & 30.12 & 107.73 & -94.3 & -60.4 & 5.26 \\
\hline 700 & 68.62 & 163.05 & 49.32 & 113.73 & -82.4 & -55.6 & 4.15 \\
\hline 800 & 68.62 & 172.22 & 51.74 & 120.48 & -80.1 & -51.9 & 3.39 \\
\hline 900 & 65.75 & 189.35 & 62.33 & 127.02 & -70.1 & -48.8 & 2.83 \\
\hline 1000 & 63.45 & 196.15 & 62.56 & 133.59 & -68.3 & -46.5 & 2.43 \\
\hline 1100 & 61.43 & 202.11 & 62.55 & 139.56 & -66.9 & -44.4 & 2.11 \\
\hline 1200 & 59.68 & 207.38 & 62.38 & 145.00 & -65.8 & -42.5 & 1.85 \\
\hline 1300 & 58.20 & 212.09 & 62.11 & 149.98 & -65.0 & -40.5 & 1.63 \\
\hline 1400 & 56.99 & 216.36 & 61.79 & 154.57 & -77.6 & -38.3 & 1.43 \\
\hline
\end{tabular}

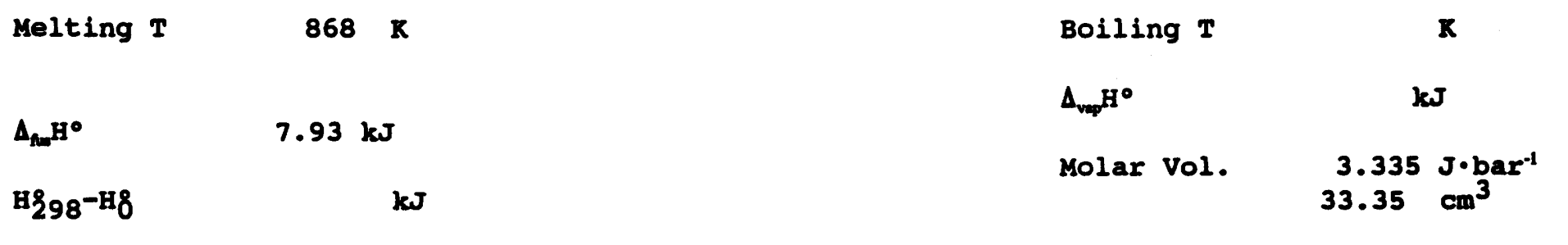


$\mathrm{BaCO}_{3}$ : Orthorhombic crystals 298.15 to $1079 \mathrm{~K}$; tetragonal crystals 1079 to $1241 \mathrm{~K}$; cubic crystals 1241 to $1600 \mathrm{~K}$.

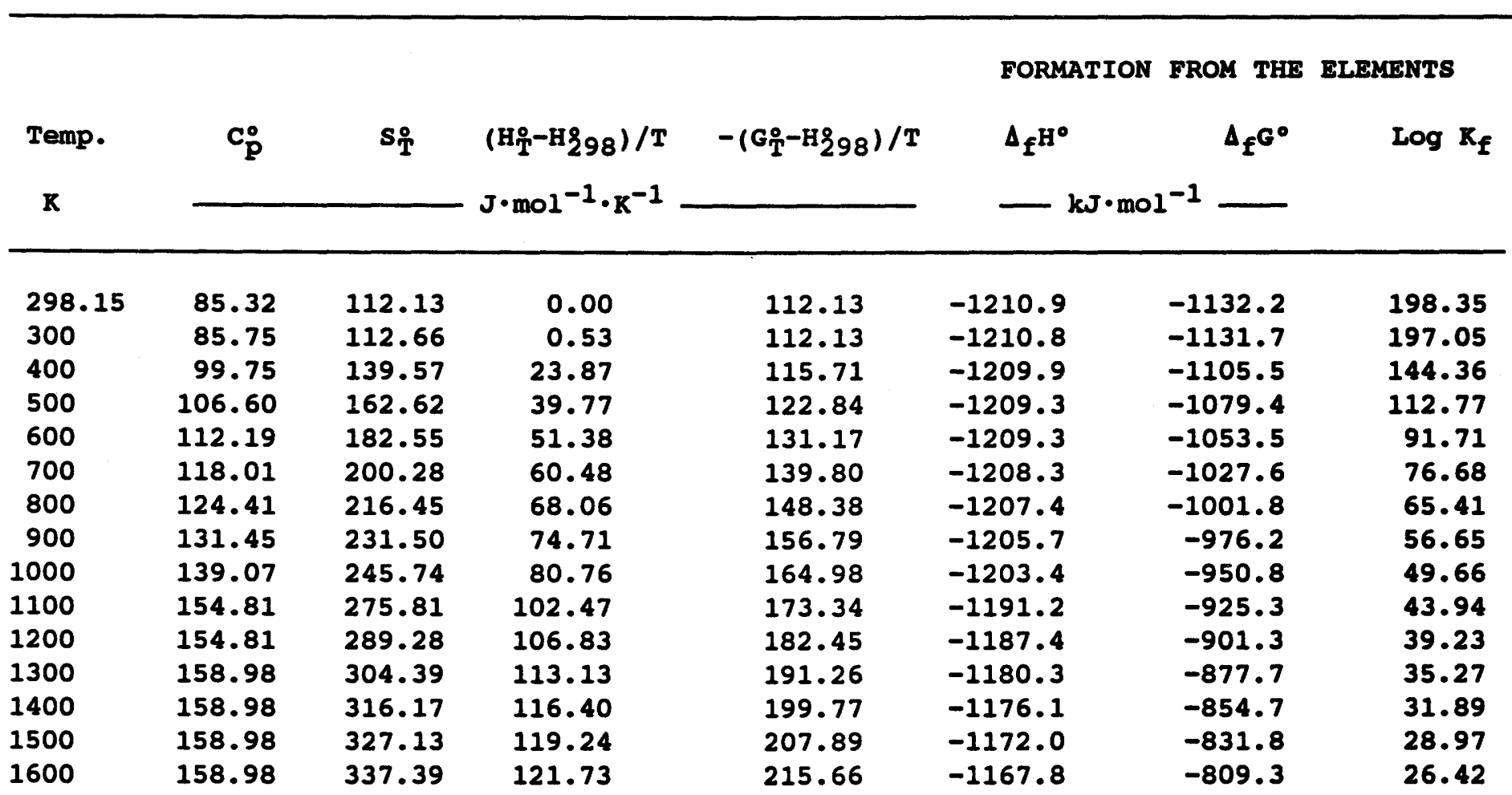

Melting $\mathbf{T}$

$\mathbf{R}$

$\Delta_{\text {fuw }} \mathrm{H}^{\circ}$

$\mathbf{k J}$

H298-
Boiling $T$

$\Delta_{\mathrm{vep}} \mathrm{H}^{\circ}$

Molar Vol.

$B=2.438 \mathrm{E}-01$

$C=-9 \cdot 15 E+05$ 
$\mathrm{CaCO}_{3}$ : Orthorhombic crystals 298.15 to $1000 \mathrm{~K}$.

\begin{tabular}{|c|c|c|c|c|c|c|c|}
\hline Temp. & $\mathbf{c}_{\mathbf{p}}^{\circ}$ & $\mathbf{s}_{\mathbf{T}}$ & \multirow{2}{*}{$\begin{array}{l}\left(\mathrm{H}_{\mathrm{T}}^{\circ}-\mathrm{H}_{298}\right) / \mathrm{T} \\
\mathrm{J} \cdot \mathrm{mol}^{-1} \cdot \mathrm{K}^{-1}\end{array}$} & \multirow[t]{2}{*}{$-\left(G_{T}^{\circ}-H_{298}\right) / T$} & $\begin{array}{l}\text { FORMATION } \\
\Delta_{f} \mathrm{H}^{\circ}\end{array}$ & \multirow[t]{2}{*}{$\begin{array}{r}\text { FROM THE } \\
\Delta_{f^{G^{\circ}}}\end{array}$} & \multirow[t]{2}{*}{$\begin{array}{l}\text { ELEMENTS } \\
\qquad \log \mathbf{k}_{\mathbf{f}}\end{array}$} \\
\hline $\mathbf{R}$ & & & & & $-\mathrm{kJ} \cdot \mathrm{mol}$ & & \\
\hline 298.15 & 82.31 & 87.99 & 0.00 & 87.99 & -1207.4 & -1127.4 & 197.51 \\
\hline 300 & 82.55 & 88.50 & 0.51 & 87.99 & -1207.4 & -1126.9 & 196.21 \\
\hline 400 & 92.67 & 113.75 & 22.38 & 91.37 & -1206.7 & -1100.2 & 143.66 \\
\hline 500 & 99.80 & 135.22 & 37.18 & 98.04 & -1205.8 & -1073.6 & 112.16 \\
\hline 600 & 105.76 & 153.96 & 48.13 & 105.83 & -1204.8 & -1047.3 & 91.17 \\
\hline 700 & 111.17 & 170.67 & 56.75 & 113.92 & -1203.7 & -1021.2 & 76.20 \\
\hline 800 & 116.28 & 185.85 & 63.87 & 121.98 & -1203.4 & -995.0 & 64.97 \\
\hline 900 & 121.22 & 199.84 & 69.97 & 129.86 & -1202.2 & -969.1 & 56.24 \\
\hline 1000 & 126.06 & 212.86 & 75.34 & 137.52 & -1201.0 & -943.2 & 49.27 \\
\hline
\end{tabular}

Melting $\mathbf{T}$

$\mathbf{K}$

$\Delta_{\text {fun }} \mathrm{H}^{\circ}$

$\mathbf{k J}$

H298-H8
Boiling T

$\Delta_{\mathrm{vw}} \mathrm{H}^{\circ}$

Molar Vol.

\section{R}

$\mathbf{k J}$
$3.415 \mathrm{~J} \cdot$ bar $^{-1}$ $34.15 \mathrm{~cm}^{3}$ 
$\mathrm{CaCO}_{3}$ Rhombohedral crystals 298.15 to $1200 \mathrm{~K}$.

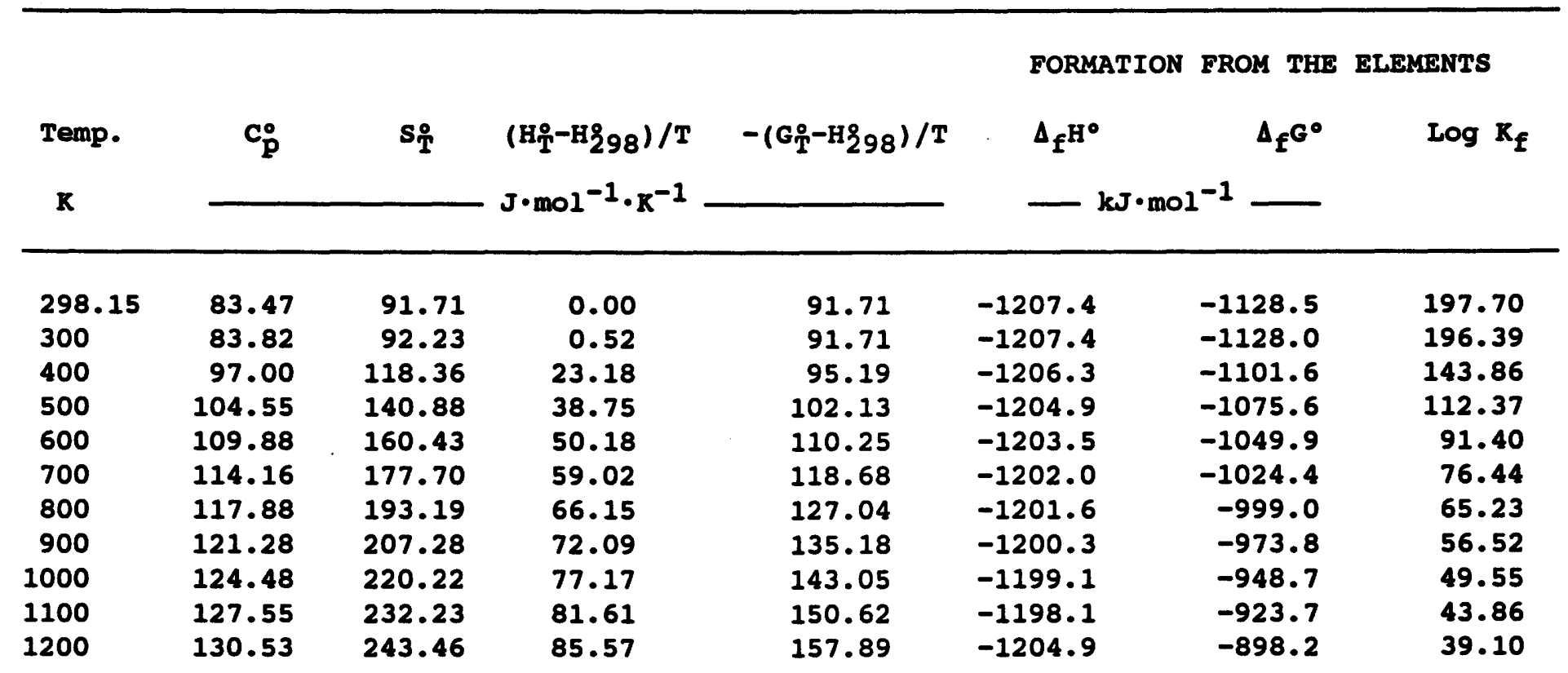

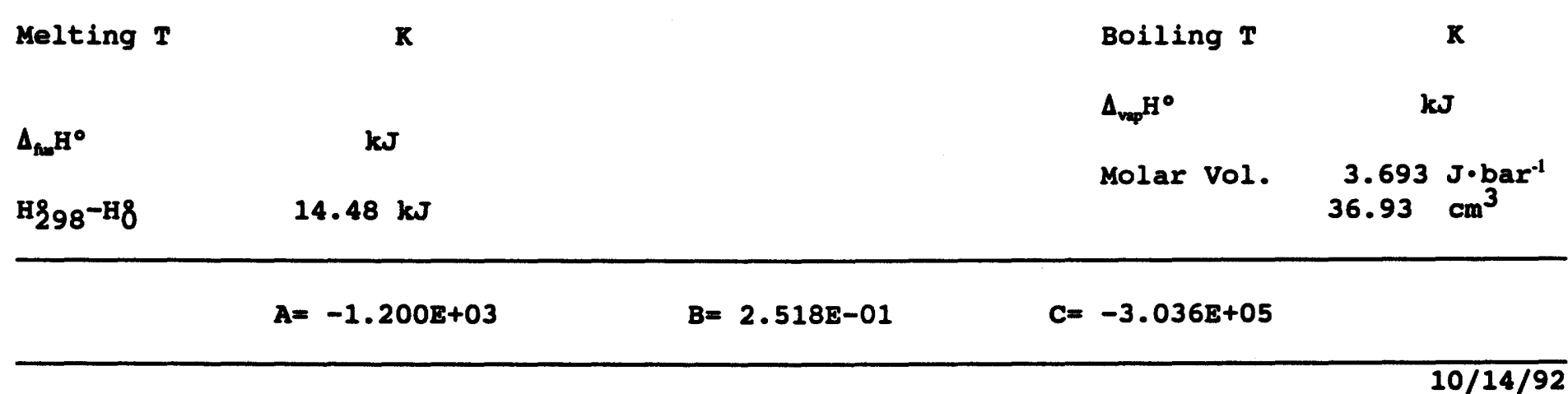


CaMg $\left(\mathrm{CO}_{3}\right)_{2}:$ Rhombohedral crystals 298.15 to $1100 \mathrm{~K}$.

\begin{tabular}{|c|c|c|c|c|c|c|c|}
\hline \multirow[b]{2}{*}{ Temp. } & \multirow[b]{2}{*}{$\mathbf{c}_{\mathbf{p}}^{\circ}$} & \multirow[b]{2}{*}{$\mathbf{s}_{\mathrm{T}}$} & \multirow{3}{*}{$\begin{array}{l}\left(\mathrm{H}_{\mathrm{T}}-\mathrm{H}_{298}\right) / \mathrm{T} \\
\mathrm{J} \cdot \mathrm{mol}^{-1} \cdot \mathrm{K}^{-1}\end{array}$} & \multirow[b]{2}{*}{$-\left(G_{T}^{\circ}-H_{298}\right) / T$} & \multirow{2}{*}{$\begin{array}{l}\text { FORMATION } \\
\Delta_{f^{H}}{ }^{\circ}\end{array}$} & \multirow{2}{*}{$\begin{array}{c}\text { FROM THE } \\
\Delta_{\mathbf{f}} \mathbf{G}^{\circ}\end{array}$} & \multirow{2}{*}{$\begin{array}{l}\text { ELEMENTS } \\
\qquad \log \mathrm{K}_{\mathbf{f}}\end{array}$} \\
\hline & & & & & & & \\
\hline $\mathbf{K}$ & & & & & $-\mathbf{k J} \cdot \mathbf{m o}$ & $1^{-1}$ & \\
\hline 298.15 & 157.51 & 155.18 & 0.00 & 155.18 & -2324.5 & -2161.3 & 378.64 \\
\hline 300 & 158.06 & 156.16 & 0.97 & 155.18 & -2324.5 & -2160.3 & 376.13 \\
\hline 400 & 183.54 & 205.32 & 43.61 & 161.71 & -2323.4 & -2105.7 & 274.97 \\
\hline 500 & 201.89 & 248.35 & 73.52 & 174.83 & -2321.4 & -2051.4 & 214.31 \\
\hline 600 & 215.66 & 286.43 & 96.12 & 190.32 & -2318.8 & -1997.7 & 173.91 \\
\hline 700 & 226.65 & 320.53 & 114.00 & 206.53 & -2316.0 & -1944.4 & 145.09 \\
\hline 800 & 236.08 & 351.42 & 128.69 & 222.74 & -2313.9 & -1891.4 & 123.47 \\
\hline 900 & 244.73 & 379.73 & 141.10 & 238.63 & -2310.8 & -1838.7 & 106.72 \\
\hline 1000 & 253.15 & 405.96 & 151.89 & 254.07 & -2316.2 & -1785.7 & 93.28 \\
\hline 1100 & 261.72 & 430.48 & 161.48 & 269.00 & -2312.9 & -1732.9 & 82.28 \\
\hline
\end{tabular}

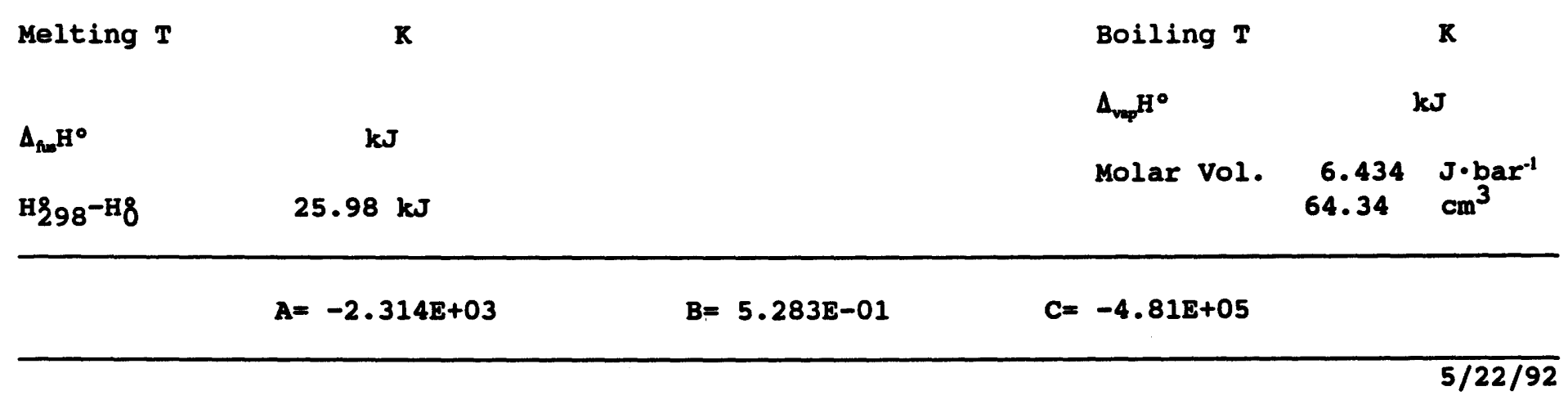


$\mathrm{FeCO}_{3}$ : Rhombohedral crystals 298.15 to $600 \mathrm{~K}$.

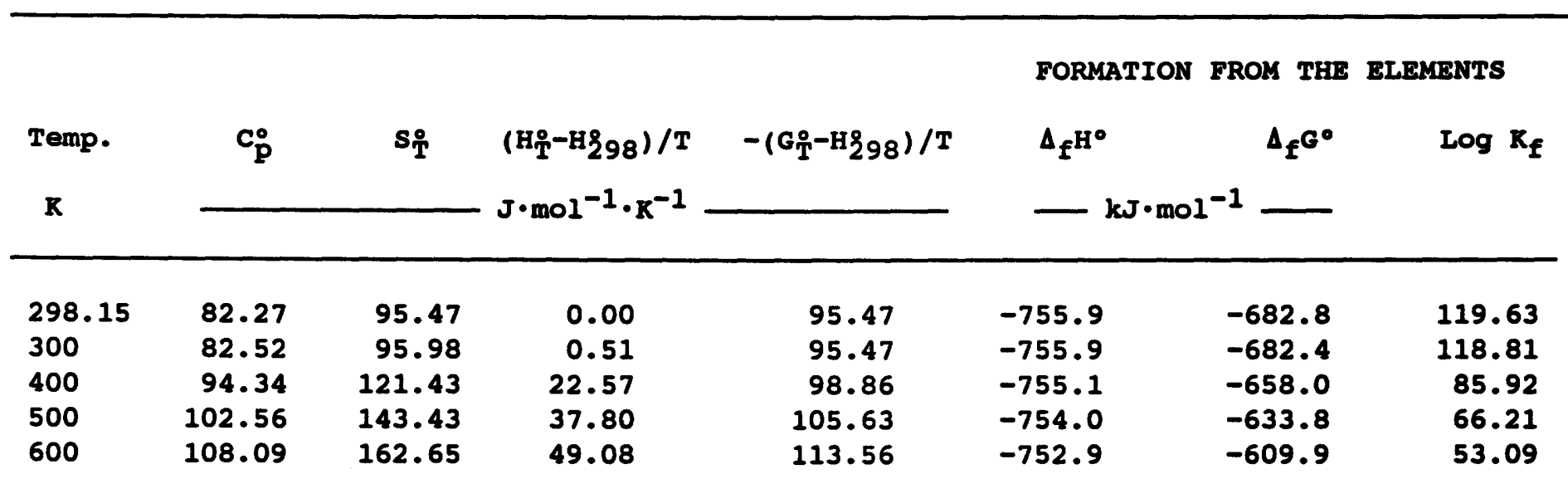

Melting $\mathbf{T}$

$\mathbf{R}$

$\boldsymbol{\Delta}_{\mathrm{fu}} \mathrm{H}^{\circ}$

$\mathbf{k J}$

H298-H8

$14.59 \mathrm{~kJ}$
Boiling T

$\Delta_{\mathrm{vup}} \mathrm{H}^{\circ}$

Molar Vol.
R

kJ
$2.938 \mathrm{~J} \cdot \mathrm{bar}^{-1}$ $29.38 \mathrm{~cm}^{3}$
$A=-7.525 E+02$
$B=2.383 E-01$
$C=-1.23 E+05$ 
$\mathrm{MgCO}_{3}$ : Rhombohedral crystals 298.15 to $1000 \mathrm{~K}$.

\begin{tabular}{|c|c|c|c|c|c|c|c|}
\hline Temp. & $\mathbf{c}_{\mathbf{p}}^{\circ}$ & $\mathbf{s}_{\mathbf{T}}$ & \multirow{2}{*}{$\begin{array}{l}\left(\mathrm{H}_{\mathrm{T}}^{\circ}-\mathrm{H} 298\right) / \mathrm{T} \\
\mathrm{J} \cdot \mathrm{mol}^{-1} \cdot \mathrm{K}^{-1}\end{array}$} & \multirow[t]{2}{*}{$-\left(G_{T}^{\circ}-H_{298}\right) / T$} & \multirow{2}{*}{\multicolumn{2}{|c|}{$\begin{array}{l}\text { FORMATION FROM THE } \\
\Delta_{\mathrm{f}^{\mathrm{H}^{\circ}}}\end{array}$}} & \multirow[t]{2}{*}{$\begin{array}{l}\text { ELEMENTS } \\
\qquad \log k_{f}\end{array}$} \\
\hline $\mathbf{x}$ & & & & & & & \\
\hline 298.15 & 76.09 & 65.09 & 0.00 & 65.09 & -1113.3 & -1029.5 & 180.36 \\
\hline 300 & 76.44 & 65.56 & 0.47 & 65.09 & -1113.3 & -1029.0 & 179.15 \\
\hline 400 & 90.57 & 89.67 & 21.39 & 68.28 & -1112.9 & -1000.9 & 130.70 \\
\hline 500 & 99.92 & 110.94 & 36.20 & 74.73 & -1111.9 & -973.0 & 101.65 \\
\hline 600 & 107.38 & 129.83 & 47.46 & 82.37 & -1110.6 & -945.3 & 82.30 \\
\hline 700 & 113.96 & 146.89 & 56.50 & 90.39 & -1109.1 & -917.9 & 68.49 \\
\hline 800 & 120.06 & 162.51 & 64.07 & 98.44 & -1107.3 & -890.7 & 58.16 \\
\hline 900 & 125.88 & 176.98 & 70.61 & 106.37 & -1105.3 & -863.7 & 50.13 \\
\hline 1000 & 131.54 & 190.54 & 76.42 & 114.12 & -1111.6 & -836.3 & 43.68 \\
\hline
\end{tabular}

Melting T

$\mathbf{R}$

Boiling $\mathbf{T}$

$\mathbf{K}$

\begin{tabular}{lccc}
$\Delta_{\mathrm{fu}} \mathrm{H}^{\circ}$ & $\mathrm{kJ}$ & Molar Vol \\
$\mathrm{H} 298^{-\mathrm{H} 8}$ & $11.63 \mathrm{~kJ}$ & \\
\hline & $\mathrm{A}=-1.108 \mathrm{E}+03$ & $\mathrm{~B}=2.718 \mathrm{E}-01$ & $\mathrm{C}=-2.42 \mathrm{E}+05$
\end{tabular}


$\mathrm{MnCO}_{3}$ : Rhombohedral crystals 298.15 to $600 \mathrm{~K}$.

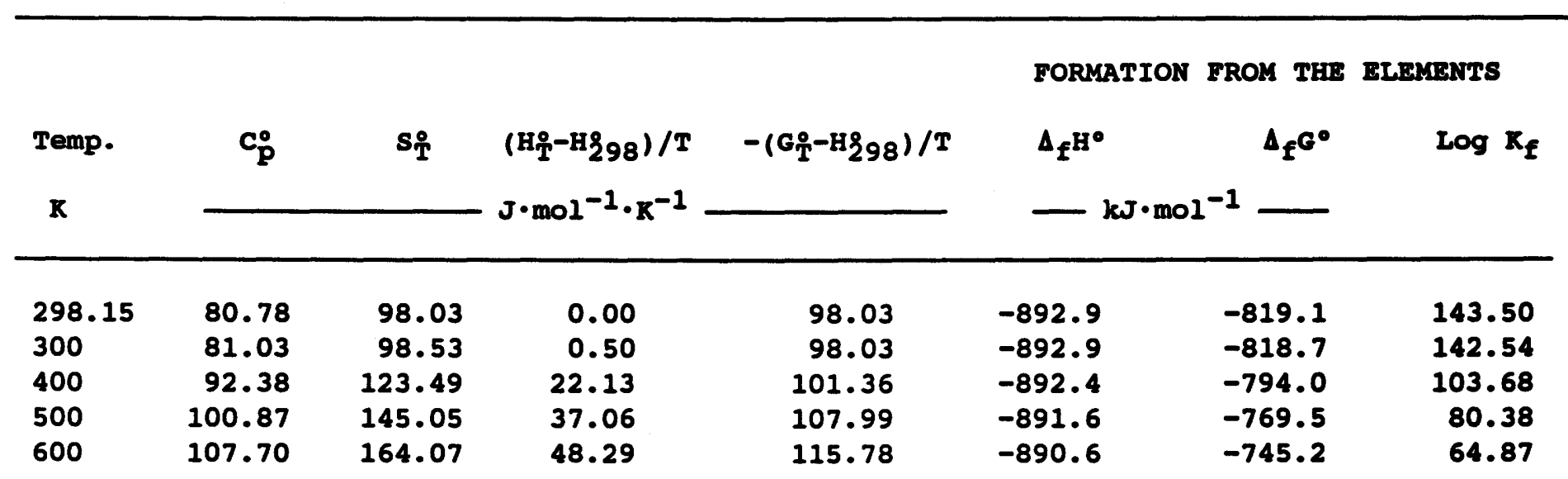

Melting T

Boiling T

$\Delta_{\mathrm{veq}} \mathrm{H}^{\circ}$

$\mathbf{k J}$

$\Lambda_{\text {fun }} \mathrm{H}^{\circ}$

$14.12 \mathrm{~kJ}$
$B=2 \cdot 425 E-01$
$C=-9.44 \mathrm{E}+04$
$A=-8.894 E+02$ 
$\mathrm{NaAlCO}_{3}(\mathrm{OH})_{2}$ : Orthorhombic crystals 298.15 to $500 \mathrm{~K}$.

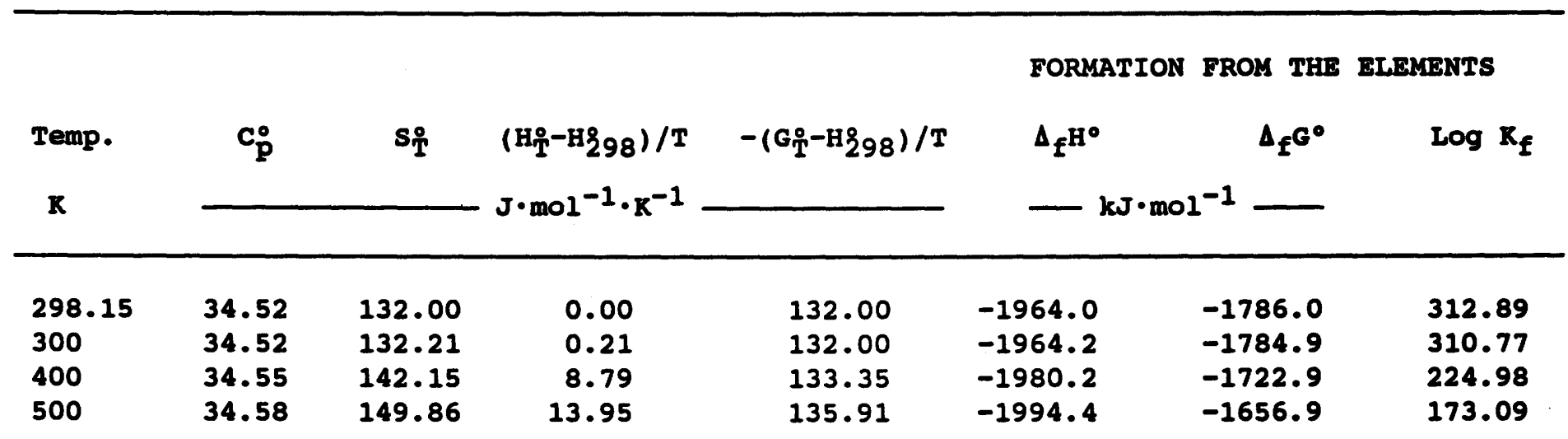

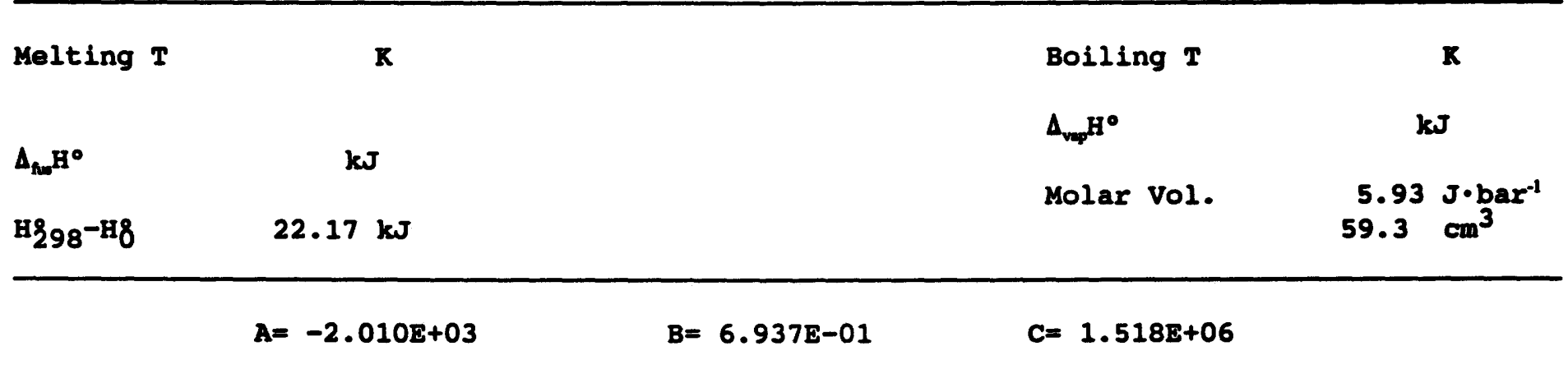


$\mathrm{SrCO}_{3}$ : Orthorhombic crystals 298.15 to $1197 \mathrm{~K}$; hexagonal crystals 1197 to $1400 \mathrm{~K}$.

\begin{tabular}{|c|c|c|c|c|c|c|c|}
\hline Temp. & $c_{p}^{0}$ & $\mathbf{s}_{\mathbf{T}}$ & \multirow{2}{*}{$\begin{array}{l}\left(H_{T}^{\circ}-H_{298}\right) / T \\
J \cdot m^{-1} \cdot K^{-1}\end{array}$} & \multirow[t]{2}{*}{$-\left(G_{\mathrm{T}}^{\circ}-\mathrm{H}_{298}\right) / \mathrm{T}$} & \multirow{2}{*}{\multicolumn{2}{|c|}{$\begin{array}{l}\text { FORMATION FROM THE } \\
\Delta_{f^{H^{\circ}}}\end{array}$}} & \multirow[t]{2}{*}{$\begin{array}{l}\text { ELEMENTS } \\
\qquad \log K_{f}\end{array}$} \\
\hline $\mathbf{K}$ & & & & & & & \\
\hline 298.15 & 81.50 & 97.07 & 0.00 & 97.07 & -1218.7 & -1137.6 & 199.29 \\
\hline 300 & 82.03 & 97.58 & 0.50 & 97.07 & -1218.7 & -1137.1 & 197.97 \\
\hline 400 & 97.72 & 123.80 & 23.26 & 100.54 & -1217.7 & -1110.0 & 144.94 \\
\hline 500 & 102.86 & 146.24 & 38.74 & 107.50 & -1216.4 & -1083.2 & 113.16 \\
\hline 600 & 106.05 & 165.28 & 49.69 & 115.58 & -1215.2 & -1056.6 & 91.99 \\
\hline 700 & 109.46 & 181.87 & 57.98 & 123.89 & -1214.1 & -1030.3 & 76.88 \\
\hline 800 & 113.64 & 196.75 & 64.67 & 132.09 & -1213.0 & -1004.1 & 65.56 \\
\hline 900 & 118.69 & 210.42 & 70.38 & 140.04 & -1212.4 & -978.0 & 56.76 \\
\hline 1000 & 124.54 & 223.22 & 75.50 & 147.72 & -1210.6 & -952.0 & 49.73 \\
\hline 1100 & 131.11 & 235.40 & 80.25 & 155.15 & -1216.3 & -925.9 & 43.97 \\
\hline 1200 & 144.77 & 262.84 & 100.48 & 162.36 & -1195.5 & -899.7 & 39.16 \\
\hline 1300 & 144.77 & 274.42 & 103.89 & 170.53 & -1192.6 & -875.1 & 35.16 \\
\hline 1400 & 144.77 & 285.15 & 106.81 & 178.34 & -1189.9 & -850.8 & 31.74 \\
\hline
\end{tabular}

Melting T

$\mathbf{K}$

Boiling $\mathbf{T}$

$\mathbf{R}$

$\Delta_{\text {nu }} \mathrm{H}^{\circ}$

$\mathbf{k J}$

$\Delta_{\text {vep }} \mathrm{H}^{\circ}$

$\mathbf{k J}$

H298-H8

kJ
Molar Vol. $\quad 3.901 \mathrm{~J} \cdot \mathrm{bar}^{-1}$ $39.01 \mathrm{~cm}^{3}$
$A=-1.210 E+03$
$B=2.577 E-01$
$C=-4.37 \mathrm{E}+05$ 
$\mathrm{ZnCO}_{3}$ : Rhombohedral crystals 298.15 to $1200 \mathrm{~K}$.

\begin{tabular}{|c|c|c|c|c|c|c|c|}
\hline \multirow[b]{2}{*}{ Temp. } & \multirow[b]{2}{*}{$\mathbf{c}_{\mathbf{p}}^{\circ}$} & \multirow[b]{2}{*}{$s_{\text {T }}$} & \multirow{3}{*}{$\begin{array}{l}\left(\mathrm{H}_{\mathrm{T}}-\mathrm{H}_{298}\right) / \mathrm{T} \\
\mathrm{J} \cdot \mathrm{mol}^{-1} \cdot \mathrm{K}^{-1}\end{array}$} & \multirow[b]{2}{*}{$-\left(G_{T}^{\circ}-H_{298}\right) / T$} & \multicolumn{2}{|c|}{ FORMATION FROM THE } & \multirow{2}{*}{$\begin{array}{l}\text { BLAMENTS } \\
\text { Log } \mathrm{K}_{\mathrm{f}}\end{array}$} \\
\hline & & & & & $\Delta_{\mathrm{f}} \mathrm{H}^{\circ}$ & $\Delta_{f} G^{0}$ & \\
\hline $\mathbf{K}$ & & & & & $-\mathbf{k J} \cdot \mathbf{m o}$ & -1 & \\
\hline 298.15 & 80.05 & 81.19 & 0.00 & 81.19 & -817.0 & $-735 \cdot 3$ & 128.82 \\
\hline 300 & 80.29 & 81.69 & 0.49 & 81.19 & -817.0 & -734.8 & 127.94 \\
\hline 400 & 91.77 & 106.42 & 21.93 & 84.49 & -816.4 & -707.5 & 92.39 \\
\hline 500 & 101.01 & 127.92 & 36.86 & 91.07 & $-815 \cdot 4$ & -680.4 & 71.08 \\
\hline 600 & 108.79 & 147.05 & 48.21 & 98.83 & -814.0 & -653.5 & 56.89 \\
\hline 700 & 115.57 & 164.34 & 57.36 & 106.98 & -819.7 & -626.8 & 46.77 \\
\hline 800 & 121.64 & 180.17 & 65.02 & 115.15 & -817.9 & -599.4 & 39.13 \\
\hline 900 & 127.19 & 194.82 & 71.63 & 123.20 & $-815 \cdot 7$ & -572.2 & 33.21 \\
\hline 1000 & 132.34 & 208.49 & 77.44 & 131.05 & -813.2 & $-545 \cdot 3$ & 28.48 \\
\hline 1100 & 137.18 & $221 \cdot 34$ & 82.66 & 138.68 & -810.3 & -518.6 & 24.63 \\
\hline 1200 & 141.77 & 233.47 & 87.39 & 146.08 & $-922 \cdot 2$ & -490.3 & $21 \cdot 34$ \\
\hline
\end{tabular}


$\mathrm{Ba}\left(\mathrm{NO}_{3}\right)_{2}:$ Cubic crystals 298.15 to $800 \mathrm{~K}$.

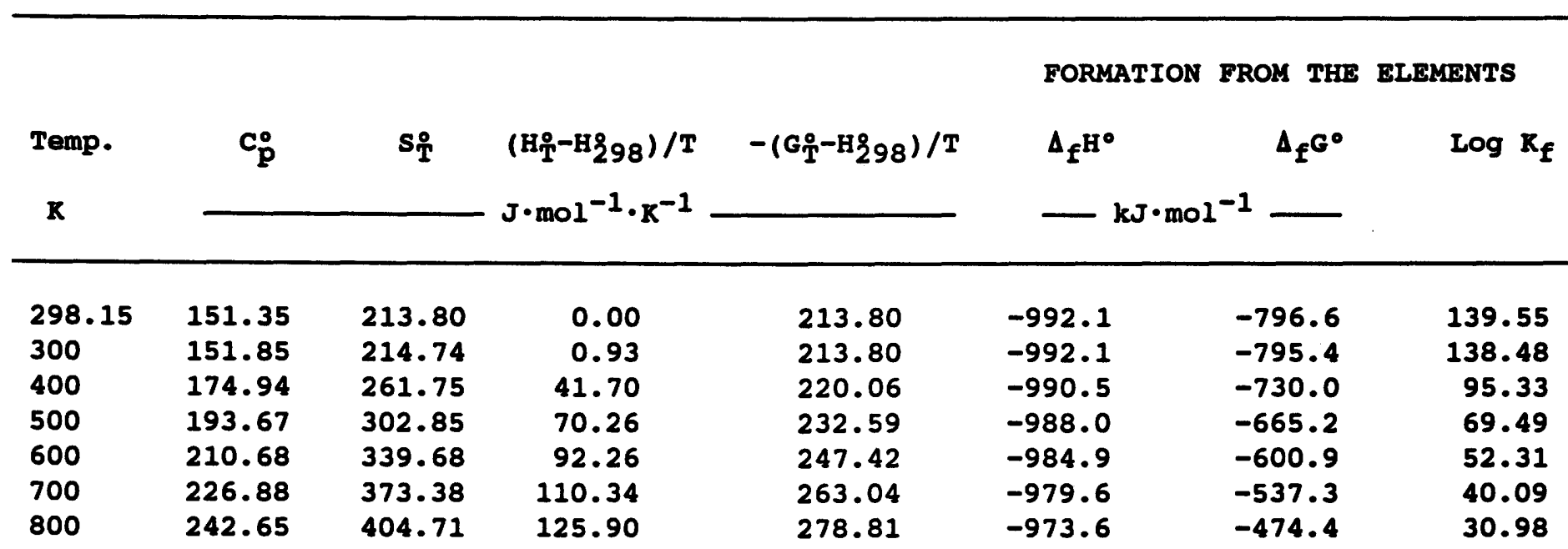

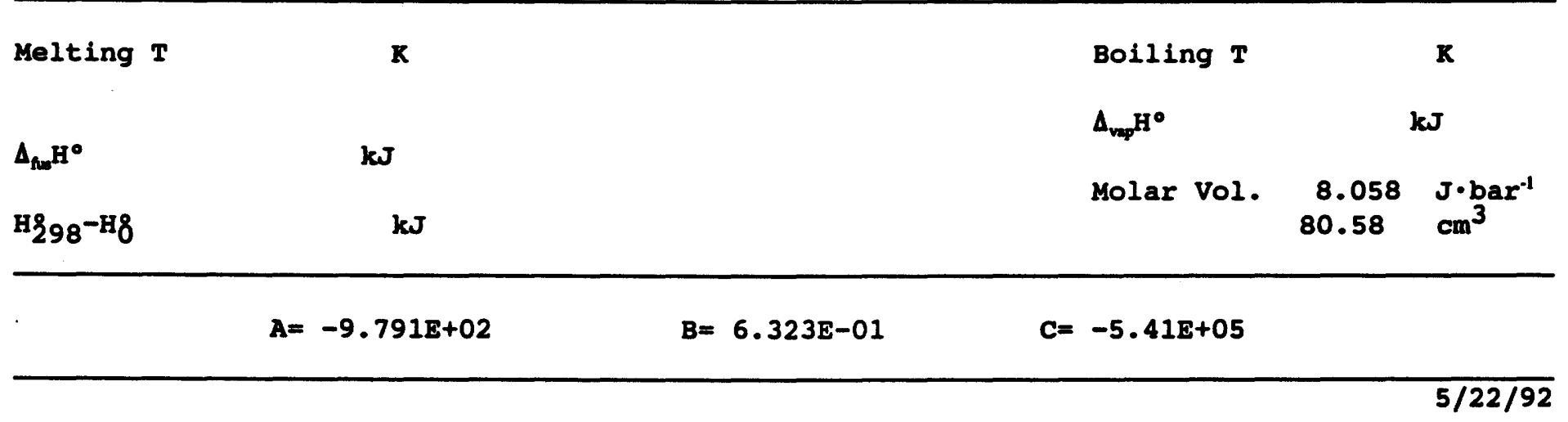


$\mathrm{Ca}\left(\mathrm{NO}_{3}\right)_{2}:$ Cubic crystals 298.15 to $800 \mathrm{~K}$.

\begin{tabular}{|c|c|c|c|c|c|c|c|}
\hline \multirow{3}{*}{$\begin{array}{l}\text { Temp. } \\
\mathbf{K}\end{array}$} & \multirow{3}{*}{$\mathbf{C}_{\mathbf{p}}^{\circ}$} & \multirow{3}{*}{$\mathbf{s}_{\mathrm{T}}$} & \multirow{3}{*}{$\begin{array}{l}\left(\mathrm{H}_{\mathrm{T}}^{\circ}-\mathrm{H}_{298}\right) / \mathrm{T} \\
\mathrm{J} \cdot \mathrm{mol}^{-1} \cdot \mathrm{K}^{-1}\end{array}$} & \multirow{3}{*}{$-\left(G_{T}^{\circ}-H_{298}\right) / T$} & \multicolumn{2}{|c|}{ FORMATION FROM THE } & \multirow{3}{*}{$\begin{array}{l}\text { ELEMENTS } \\
\qquad \log \mathrm{K}_{\mathrm{f}}\end{array}$} \\
\hline & & & & & $\Delta_{f^{H^{\circ}}}$ & $\Delta_{f} G^{0}$ & \\
\hline & & & & & \multicolumn{2}{|c|}{$-\mathrm{kJ} \cdot \mathrm{mol}^{-1}$} & \\
\hline 298.15 & 149.11 & 193.30 & 0.00 & 193.30 & -938.4 & $-742 \cdot 6$ & 130.10 \\
\hline 300 & 149.66 & 194.22 & 0.92 & 193.30 & -938.4 & -741.4 & 129.08 \\
\hline 400 & 173.76 & 240.79 & 41.30 & 199.49 & -936.6 & -675.9 & 88.27 \\
\hline 500 & 192.74 & 281.65 & 69.73 & 211.92 & -933.1 & -611.2 & 63.85 \\
\hline 600 & 210.08 & 318.34 & 91.69 & 226.64 & -928.4 & -547.2 & 47.64 \\
\hline 700 & 226.83 & 351.98 & 109.81 & 242.18 & -922.5 & -484.1 & 36.12 \\
\hline 800 & 243.37 & 383.35 & 125.47 & 257.88 & -916.3 & -421.8 & 27.54 \\
\hline
\end{tabular}

Melting T

$\Delta_{\text {fun }} \mathrm{H}^{\circ}$

H298-H8 $\mathbf{k J}$

$\mathbf{k J}$

\begin{tabular}{|c|c|c|}
\hline Boiling $T$ & & \\
\hline$\Delta_{\mathrm{vap}} \mathrm{H}^{\circ}$ & & $k^{u}$ \\
\hline Molar Vol. & $\begin{array}{c}6.609 \\
66.09\end{array}$ & \\
\hline
\end{tabular}

$C=-6.975+05$ 
$\mathrm{KNO}_{3}$ : Orthorhombic crystals 298.15 to $401 \mathrm{~K}$; Rhombohedral crystals 401 to $610 \mathrm{~K}$; 1 iquid 610 to $700 \mathrm{~K}$.

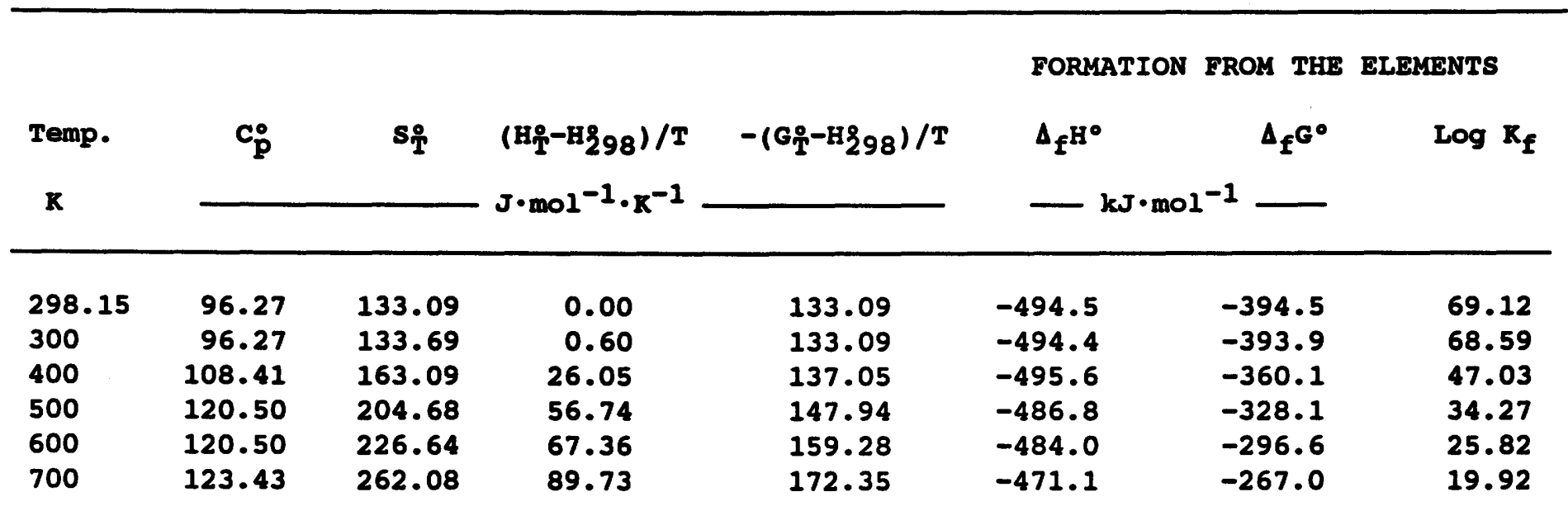

Melting $T$

$610 \mathrm{~K}$

$\Delta_{\text {hum }} \mathrm{H}^{\circ}$

$10.10 \mathrm{~kJ}$

H298-H8

kJ

Boiling $T$

$\Delta_{\mathrm{vep}} \mathrm{H}^{\circ}$

Molar Vol.
R

$\mathbf{k J}$

$$
A=-4.736 \mathrm{E}+02
$$

$B=2.981 \mathrm{E}-01$ 
$\mathrm{Mg}\left(\mathrm{NO}_{3}\right)_{2}:$ Cubic crystals 298.15 to $600 \mathrm{~K}$.

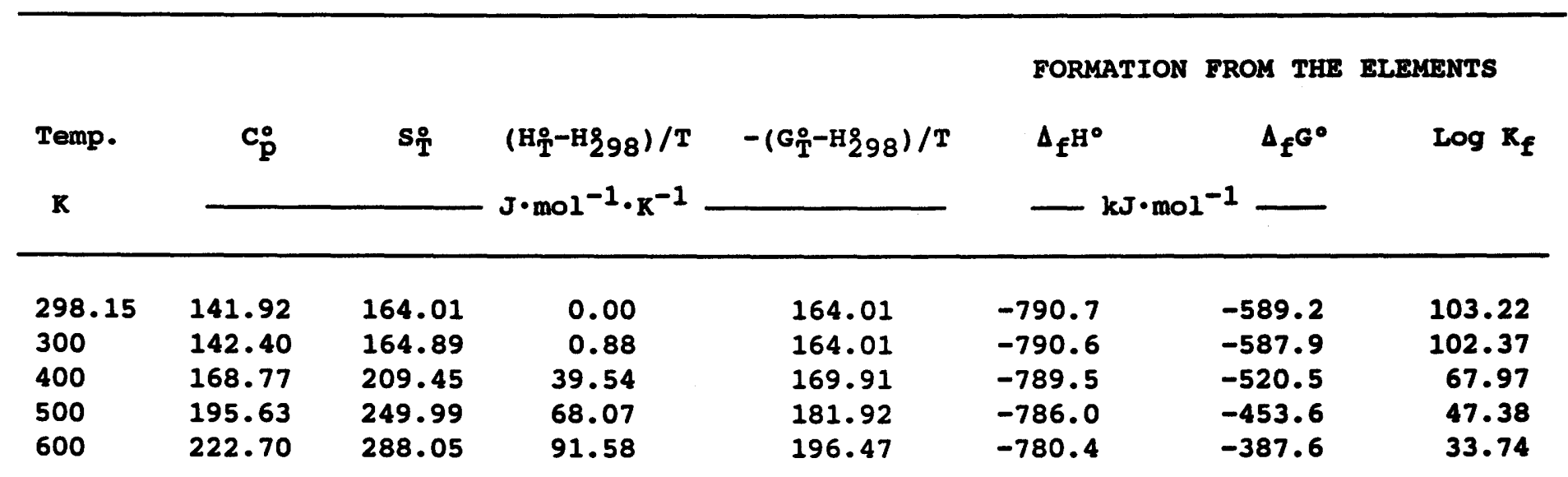

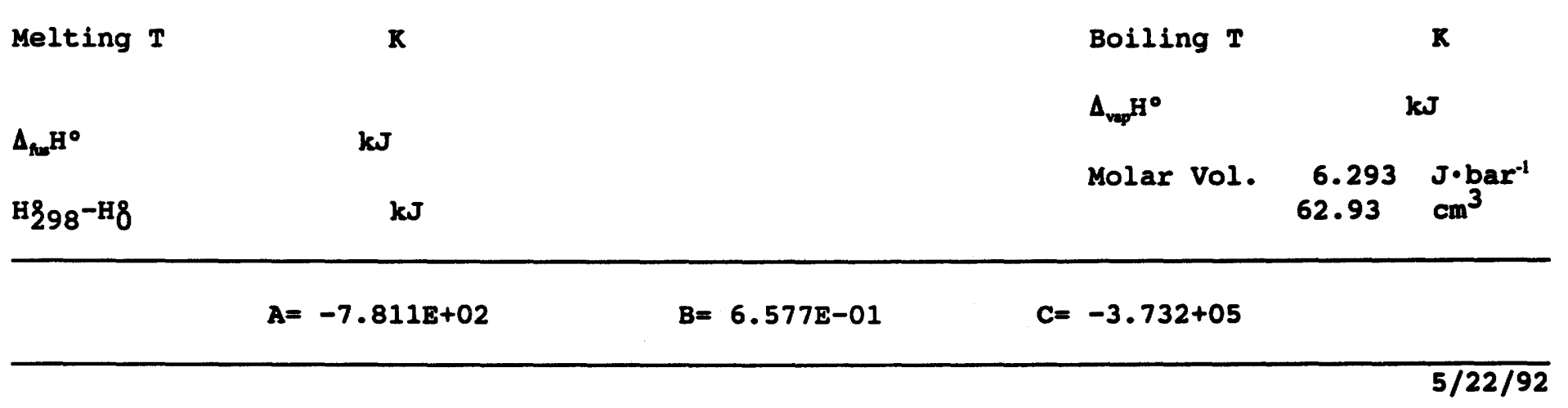


$\mathrm{NH}_{4} \mathrm{NO}_{3}$ : Ammonia-niter undergoes phase changes at $305.3,357.4,398.4 \mathrm{~K}$. It melts at 442.8 K.

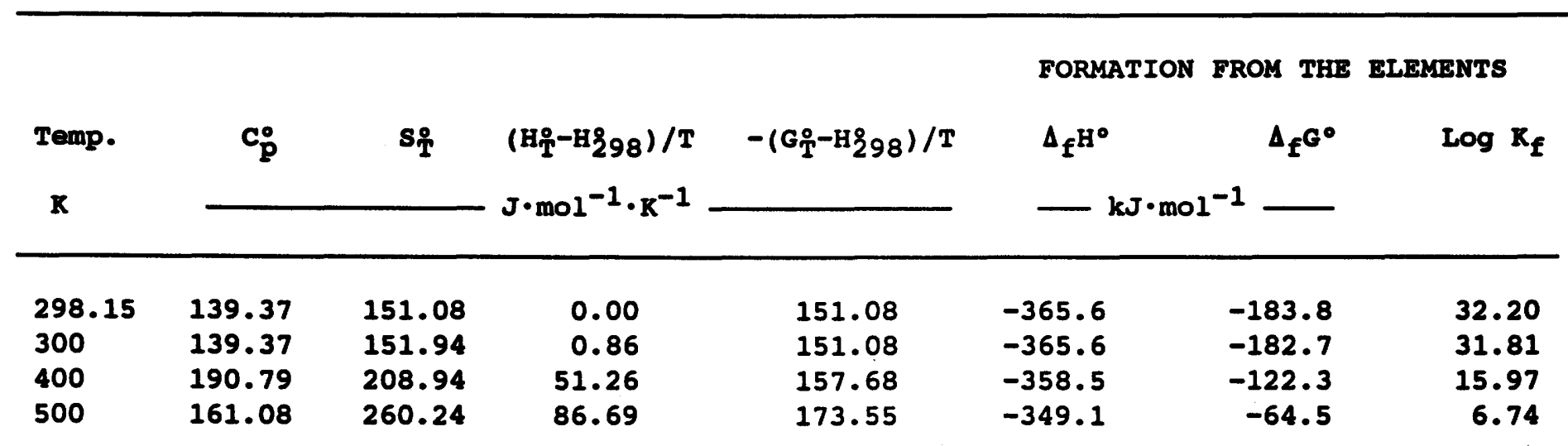

$442.8 \mathrm{~K}$

$\Delta_{\text {du }} \mathrm{H}^{\circ}$

$5.44 k J$

H298-H8
Boiling T

$\Delta_{\mathrm{vq}} \mathrm{H}^{\circ}$

Molar vol.
K

$\mathbf{k J}$
$A=-3 \cdot 384 E+02$
$B=5.556 \mathrm{E}-01$
$C=-9.90 \mathrm{E}+05$ 
$\mathrm{NaNO}_{3}$ : $\alpha$-crystals 298.15 to $549.2 \mathrm{~K}$. $\beta$-crystals 549.2 to $583.2 \mathrm{~K}$. Liquid 583.2 to $700 \mathrm{~K}$.

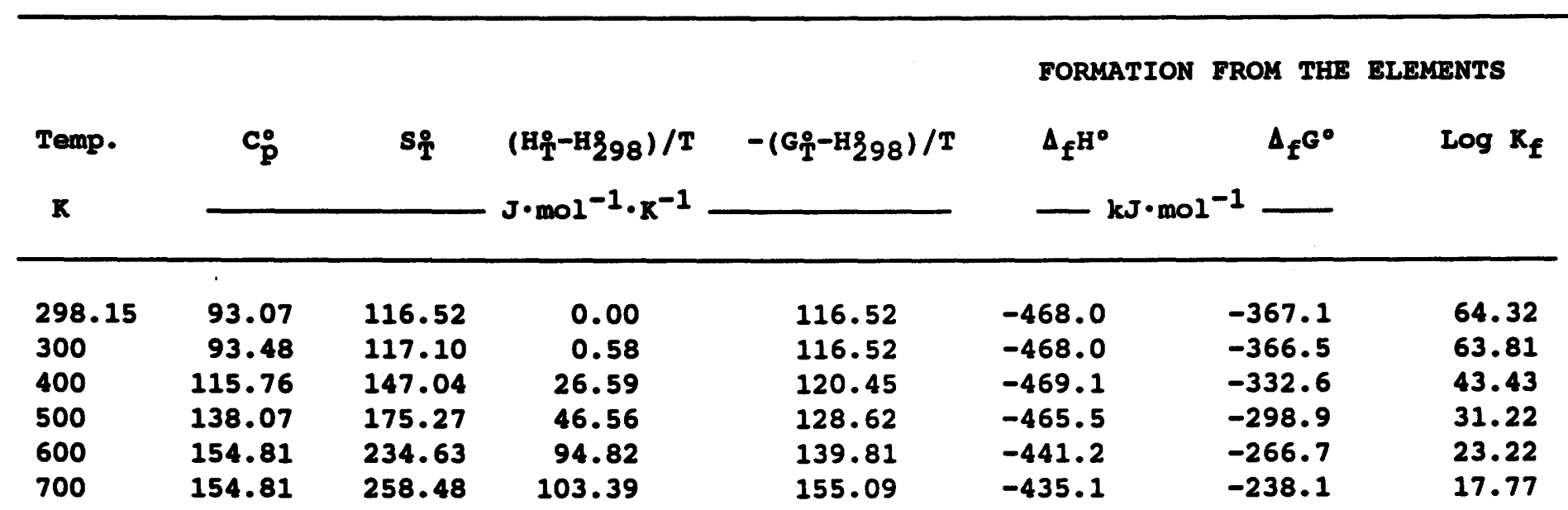

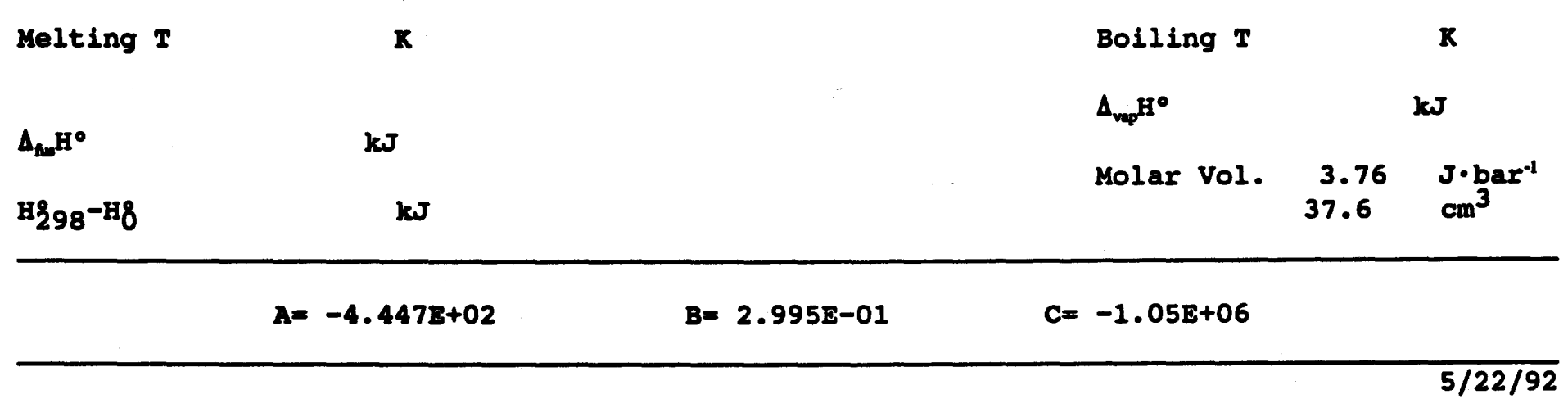


$\operatorname{Sr}\left(\mathrm{NO}_{3}\right)_{2}:$ Cryatals 298.15 to $900 \mathrm{~K}$.

\begin{tabular}{|c|c|c|c|c|c|c|c|}
\hline $\begin{array}{l}\text { Temp. } \\
\mathbf{R}\end{array}$ & $\mathbf{c}_{\mathbf{p}}^{\circ}$ & $\mathbf{s}_{\mathbf{T}}$ & $\begin{array}{l}\left(\mathrm{H}_{\mathrm{T}}-\mathrm{H}_{298}\right) / \mathrm{T} \\
\mathrm{J} \cdot \mathrm{mol}^{-1} \cdot \mathrm{K}^{-1}\end{array}$ & $-\left(G_{T}^{\circ}-H_{2} g 8\right) / T$ & $\Delta_{\mathbf{f}^{H^{\circ}}}$ & $\Delta_{f} G^{0}$ & $\log K_{f}$ \\
\hline 298.15 & 149.93 & 194.56 & 0.00 & 213.80 & -978.2 & -779.0 & 136.47 \\
\hline 300 & 149.88 & 195.49 & 0.92 & 213.80 & -978.2 & -777.8 & 135.42 \\
\hline 400 & 169.18 & 240.55 & 39.98 & 220.06 & -977.0 & -711.1 & 92.86 \\
\hline 500 & 197.81 & 281.44 & 68.73 & 232.59 & -973.6 & -644.9 & 67.37 \\
\hline 600 & 220.32 & 319.61 & 92.23 & 247.42 & -968.0 & -579.7 & 50.47 \\
\hline 700 & 234.85 & 354.76 & 111.66 & 263.04 & -961.1 & -515.5 & 38.47 \\
\hline 800 & 242.20 & 386.68 & 127.58 & 278.81 & -953.4 & -452.4 & 29.54 \\
\hline 900 & 243.53 & 415.33 & 140.44 & 274.89 & -946.4 & -390.1 & 22.64 \\
\hline
\end{tabular}

\begin{tabular}{|c|c|c|c|c|}
\hline Melting $T$ & $\mathbf{K}$ & Boiling $T$ & & $\mathbf{K}$ \\
\hline & & & & $\mathbf{J}$ \\
\hline $\mathrm{H}_{298^{-\mathrm{H}} 8}$ & $\mathbf{k J}$ & Molar Vol. & $\begin{array}{c}7.093 \\
70.93\end{array}$ & $\begin{array}{l}J \cdot \text { bar }^{-1} \\
\mathrm{~cm}^{3}\end{array}$ \\
\hline
\end{tabular}

$A=-9.566 \mathrm{E}+02 \quad \mathrm{~B}=6.317 \mathrm{E}-01 \quad \mathrm{C}=-9.810+05$


$\mathrm{Al}_{2}\left(\mathrm{SO}_{4}\right)_{3}$ : Orthorhombic crystals 298.15 to $1100 \mathrm{~K}$.

\begin{tabular}{|c|c|c|c|c|c|c|c|}
\hline Temp. & $\mathbf{c}_{\mathbf{p}}^{\circ}$ & $\mathbf{s}_{\mathrm{T}}^{\circ}$ & \multirow{2}{*}{$\begin{array}{l}\left(\mathrm{H}_{\mathrm{T}}^{\circ}-\mathrm{H} 298\right) / \mathrm{T} \\
\mathrm{J} \cdot \mathrm{mol}^{-1} \cdot \mathrm{K}^{-1}\end{array}$} & \multirow[t]{2}{*}{$-\left(G_{T}^{\circ}-H 298\right) / T$} & \multirow{2}{*}{\multicolumn{2}{|c|}{$\begin{array}{l}\text { FORMATION FROM THE } \\
\Delta_{f^{H}}{ }^{\circ} \quad \Delta_{f} G^{\circ}\end{array}$}} & \multirow[t]{2}{*}{$\begin{array}{l}\text { ELEMENTS } \\
\qquad \log R_{f}\end{array}$} \\
\hline $\mathbf{K}$ & & & & & & & \\
\hline 298.15 & 259.26 & 239.30 & 0.00 & 239.30 & -3441.8 & -3100.6 & 543.20 \\
\hline 300 & 260.62 & 240.91 & 1.60 & 239.30 & -3441.9 & -3098.5 & 539.48 \\
\hline 400 & 317.35 & 324.34 & 74.05 & 250.30 & -3449.3 & -2983.5 & 389.60 \\
\hline 500 & 352.93 & 399.26 & 126.52 & 272.74 & -3451.1 & -2866.9 & 299.49 \\
\hline 600 & 376.60 & 465.84 & 166.35 & 299.49 & -3449.7 & -2750.1 & 239.41 \\
\hline 700 & 392.79 & 525.19 & 197.62 & 327.57 & -3446.5 & -2633.7 & 196.53 \\
\hline 800 & 403.92 & 578.41 & 222.75 & 355.66 & -3442.2 & -2517.9 & 164.40 \\
\hline 900 & 411.44 & 626.45 & 243.33 & 383.12 & -3596.9 & -2399.4 & 139.25 \\
\hline 1000 & 416.28 & 670.07 & 260.40 & 409.67 & -3609.7 & -2265.3 & 118.32 \\
\hline 1100 & 419.06 & 709.89 & 274.71 & 435.18 & -3600.9 & -2131.3 & 101.20 \\
\hline
\end{tabular}

Melting $\mathbf{T}$

$\mathbf{R}$

$\Delta_{\mathrm{nu}} \mathrm{H}^{\circ}$

$\mathbf{k J}$

H298-H8
Boiling T

$\Delta_{\text {vep }} \mathrm{H}^{\bullet}$

Molar Vol.
$\mathbf{K}$

$\mathbf{k J}$
$B=1.233 E+00$
$C=3.117 \mathrm{E}+06$
$A=-3.501 E+03$
$B=1.233 E+00$ 
$\mathrm{Baso}_{4}$ : Orthorhombic crystals 298.15 to $1300 \mathrm{~K}$.

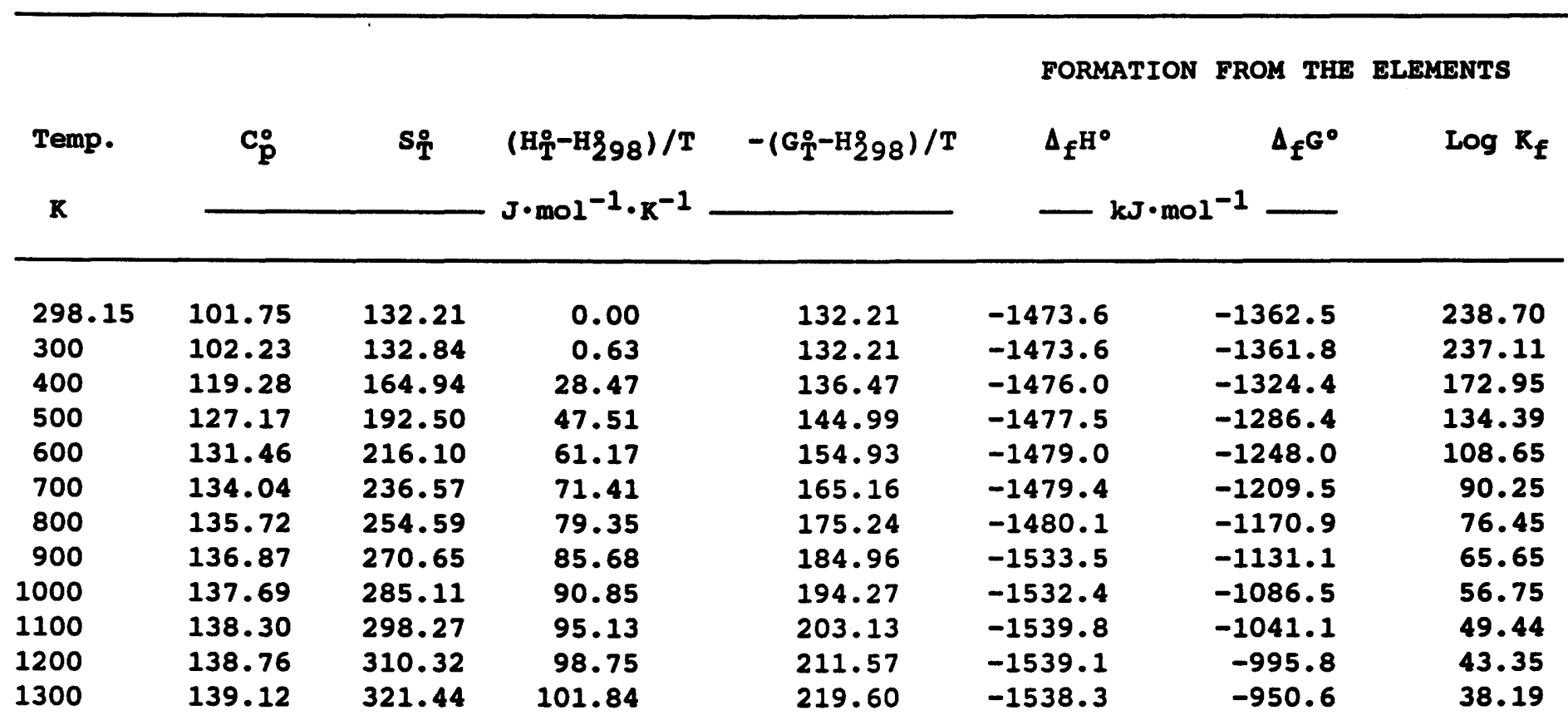

\begin{tabular}{|c|c|c|c|c|}
\hline Melting $T$ & $\mathbf{K}$ & Boiling $T$ & & $\mathbf{K}$ \\
\hline & & $\Delta_{\mathrm{vap}} \mathrm{H}^{\circ}$ & & $\mathbf{k J}$ \\
\hline$\Delta_{\text {fue }} \mathrm{H}^{\circ}$ & $\mathbf{k J}$ & & & \\
\hline Hร98-H8 & $\mathbf{k} \boldsymbol{J}$ & Molar Vol. & $\begin{array}{r}5.21 \\
52.10\end{array}$ & $\begin{array}{l}J \cdot \mathrm{bar}^{-1} \\
\mathrm{~cm}^{3}\end{array}$ \\
\hline
\end{tabular}

$A=-7.496 \mathrm{E}+01 \quad \mathrm{~B}=-9.007 \mathrm{E}-02 \quad \mathrm{C}=4.067 \mathrm{E}-01$


$\mathrm{CaSO}_{4}$ : Orthorhombic crystals 298.15 to $1000 \mathrm{~K}$.

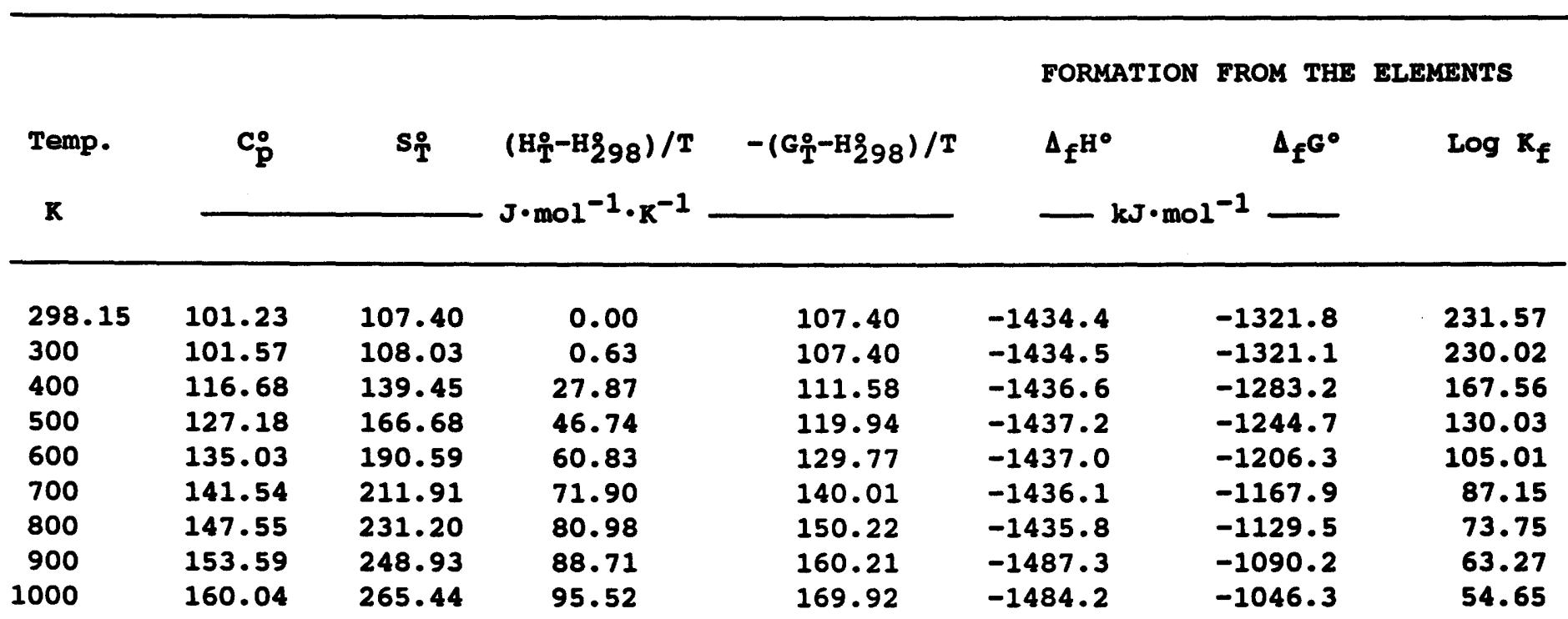

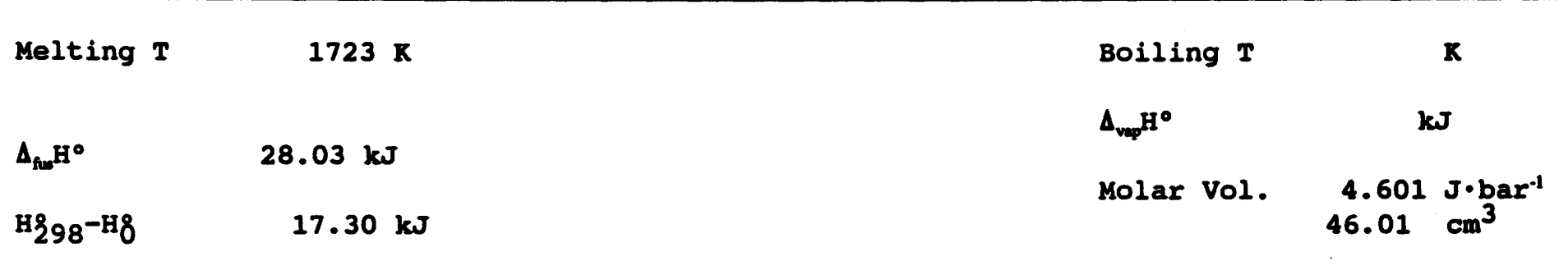
$A=-1.4465 E+02$
$B=3.971 E-01$
$C=5.860 \mathrm{E}+05$ 


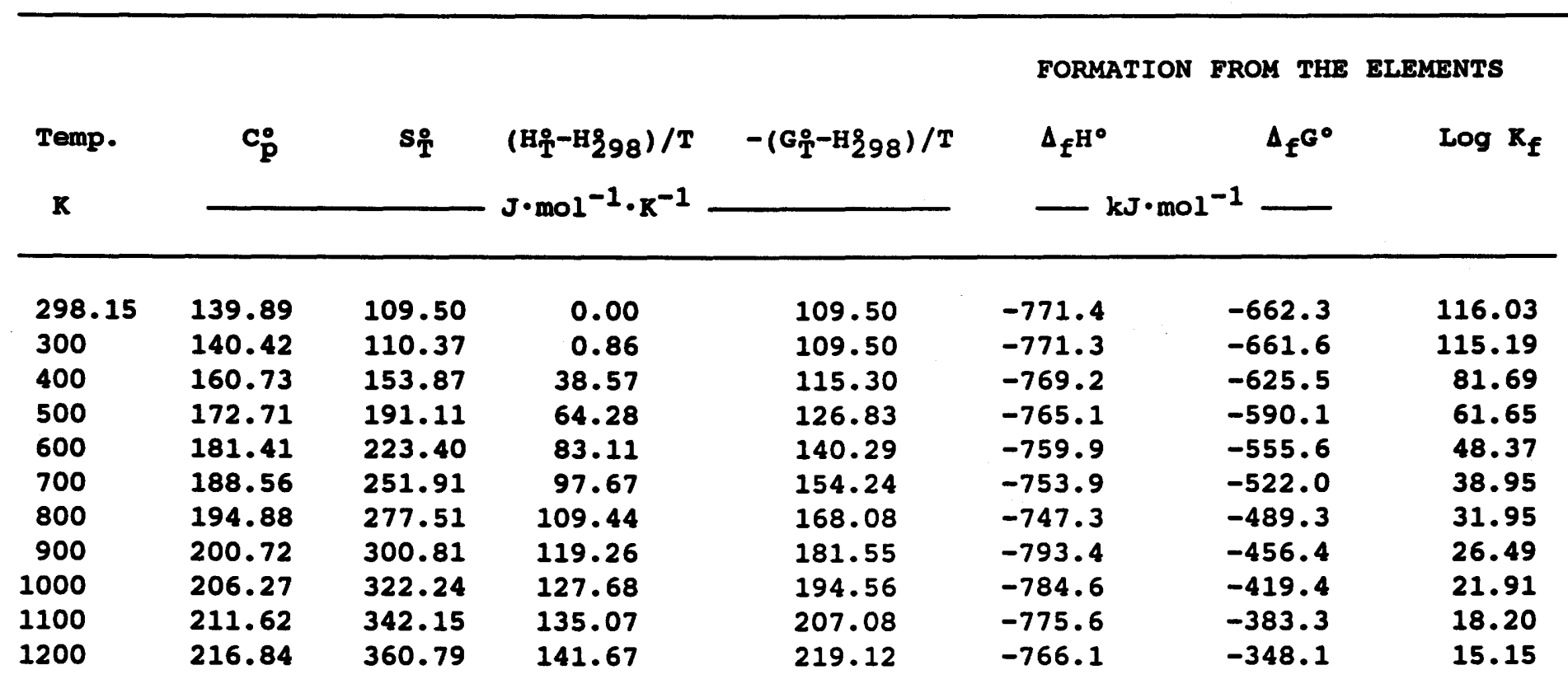

\begin{tabular}{|c|c|c|c|}
\hline Melting $T$ & $\mathbf{K}$ & Boiling $T$ & $\mathbf{R}$ \\
\hline & & $\Delta_{\mathrm{vep}} \mathrm{H}^{\circ}$ & $\mathbf{k} \mathbf{J}$ \\
\hline $\mathrm{H} 298^{-\mathrm{H}}$ & $16.87 \mathrm{~kJ}$ & Molar Vol. & $\begin{array}{ll}4.088 \mathrm{~J} \cdot \mathrm{bar}^{-1} \\
40.88 \mathrm{~cm}^{3}\end{array}$ \\
\hline
\end{tabular}

$A=-7.631 \mathrm{E}+02 \quad \mathrm{~B}=3.445 \mathrm{E}-01 \quad \mathrm{C}=-1.35 \mathrm{E}+05$


$\mathrm{Fe}_{2}\left(\mathrm{SO}_{4}\right)_{3}:$ Orthorhombic crystals 298.15 to $800 \mathrm{~K}$.

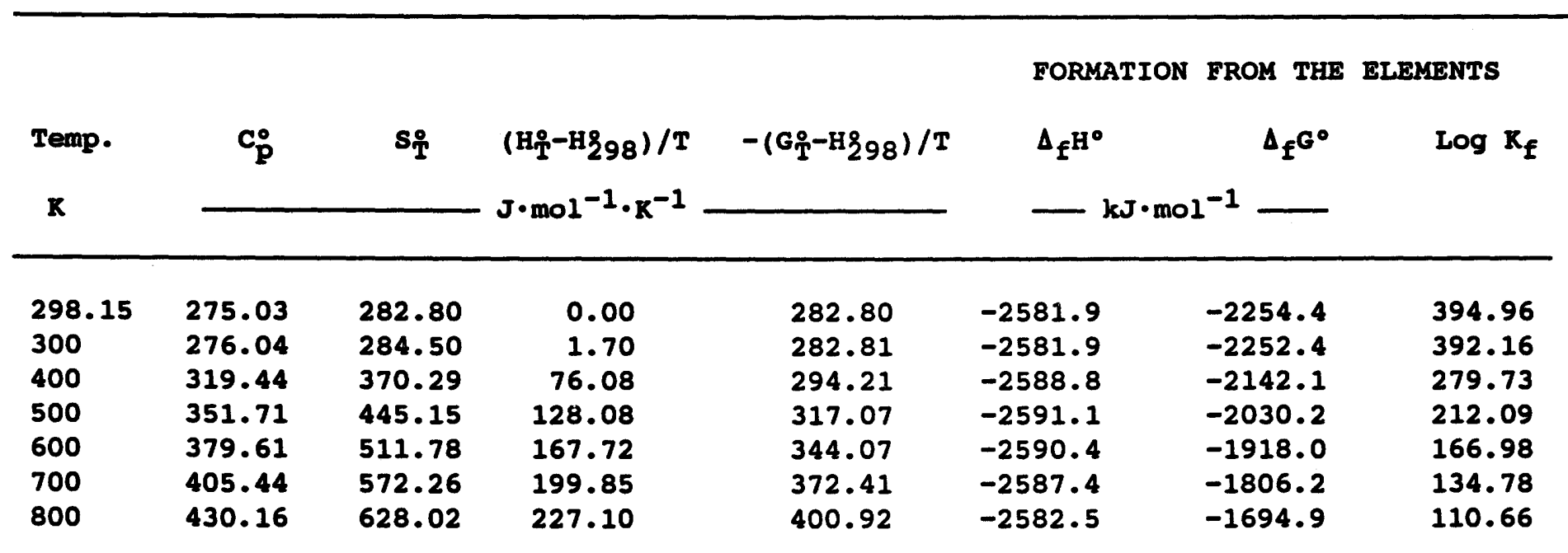

Melting $\mathbf{T}$

$A_{\mathrm{fu}} \mathrm{H}^{\circ}$

н298-H8
R

$\mathbf{k J}$

$\mathbf{k J}$
Boiling T

$\Lambda_{\text {vap }} H^{\circ}$

$\mathbf{k J}$

Molar Vol. $\begin{array}{r}13.08 \mathrm{~J} \cdot \mathrm{bar}^{-1} \\ 130.80 \mathrm{~cm}^{3}\end{array}$ 
$\mathrm{K}_{2} \mathrm{SO}_{4}$ : Orthorhombic crystals $(\alpha) 298.15$ to $856 \mathrm{~K}$. Hexagonal crystals ( $\beta$ ) 856 to melting point $1342 \mathrm{~K}$. Liquid 1342 to $1700 \mathrm{~K}$.

\begin{tabular}{|c|c|c|c|c|c|c|c|}
\hline Temp. & $\mathbf{C}_{\mathbf{p}}^{\circ}$ & $s_{\text {T }}$ & \multirow{2}{*}{$\begin{array}{l}\left(\mathrm{H}_{\mathrm{T}}^{\circ}-\mathrm{H}_{298}\right) / \mathrm{T} \\
\mathrm{J} \cdot \mathrm{mol}^{-1} \cdot \mathrm{K}^{-1}\end{array}$} & \multirow[t]{2}{*}{$-\left(G_{T}^{\circ}-H_{298}\right) / T$} & \multirow{2}{*}{\multicolumn{2}{|c|}{$\begin{array}{l}\text { FORMATION FROM THE } \\
\Delta_{f^{H}}{ }^{\circ}\end{array}$}} & \multirow[t]{2}{*}{$\begin{array}{l}\text { ELEMENTS } \\
\qquad \log R_{f}\end{array}$} \\
\hline $\mathbf{K}$ & & & & & & & \\
\hline 298.15 & 130.01 & 175.56 & 0.00 & 175.56 & -1437.7 & -1319.6 & 231.18 \\
\hline 300 & 130.44 & 176.37 & 0.80 & 175.56 & -1437.7 & -1318.9 & 229.63 \\
\hline 400 & 149.06 & 216.62 & 35.70 & 180.93 & -1445.2 & -1278.2 & 166.91 \\
\hline 500 & 163.03 & 251.43 & 59.81 & 191.62 & -1445.8 & -1236.3 & 129.15 \\
\hline 600 & 175.17 & 282.25 & 78.04 & 204.21 & -1444.9 & -1194.5 & 103.99 \\
\hline 700 & 186.44 & 310.11 & 92.73 & 217.38 & -1442.6 & -1152.9 & 86.03 \\
\hline 800 & 197.25 & 335.71 & 105.12 & 230.59 & -1439.3 & -1111.7 & 72.59 \\
\hline 900 & 203.55 & 368.88 & 125.49 & 243.39 & -1479.5 & -1069.8 & 62.09 \\
\hline 1000 & 189.91 & 389.40 & 132.41 & 256.99 & -1474.8 & -1024.5 & 53.51 \\
\hline 1100 & 197.63 & 407.72 & 137.86 & 269.86 & -1628.5 & -970.4 & 46.08 \\
\hline 1200 & 219.88 & 425.78 & 143.69 & 282.09 & -1620.8 & -910.9 & 39.65 \\
\hline 1300 & 252.38 & 444.61 & 150.77 & 293.84 & -1610.5 & -852.1 & 34.24 \\
\hline 1400 & 197.61 & 485.93 & 180.08 & 305.85 & -1567.7 & -795.0 & 29.66 \\
\hline 1500 & 197.61 & 499.57 & 181.25 & 318.32 & -1561.4 & -740.1 & 25.77 \\
\hline 1600 & 197.61 & 512.32 & 182.28 & 330.04 & -1555.0 & -685.5 & 22.38 \\
\hline 1700 & 197.61 & 524.30 & 183.18 & 341.12 & -1548.8 & -631.3 & 19.40 \\
\hline
\end{tabular}

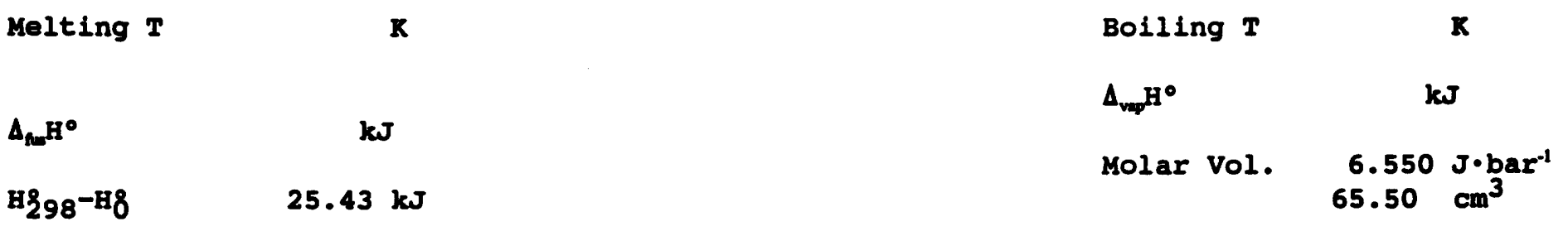
$A=-1.541 E+03$
$B=5.285 \mathrm{E}-02$
$\mathrm{C}=6.26776 \mathrm{E}+06$ 
$\mathrm{KAl}\left(\mathrm{SO}_{4}\right)_{2}:$ Hexagonal crystals 298.15 to $1000 \mathrm{~K}$.

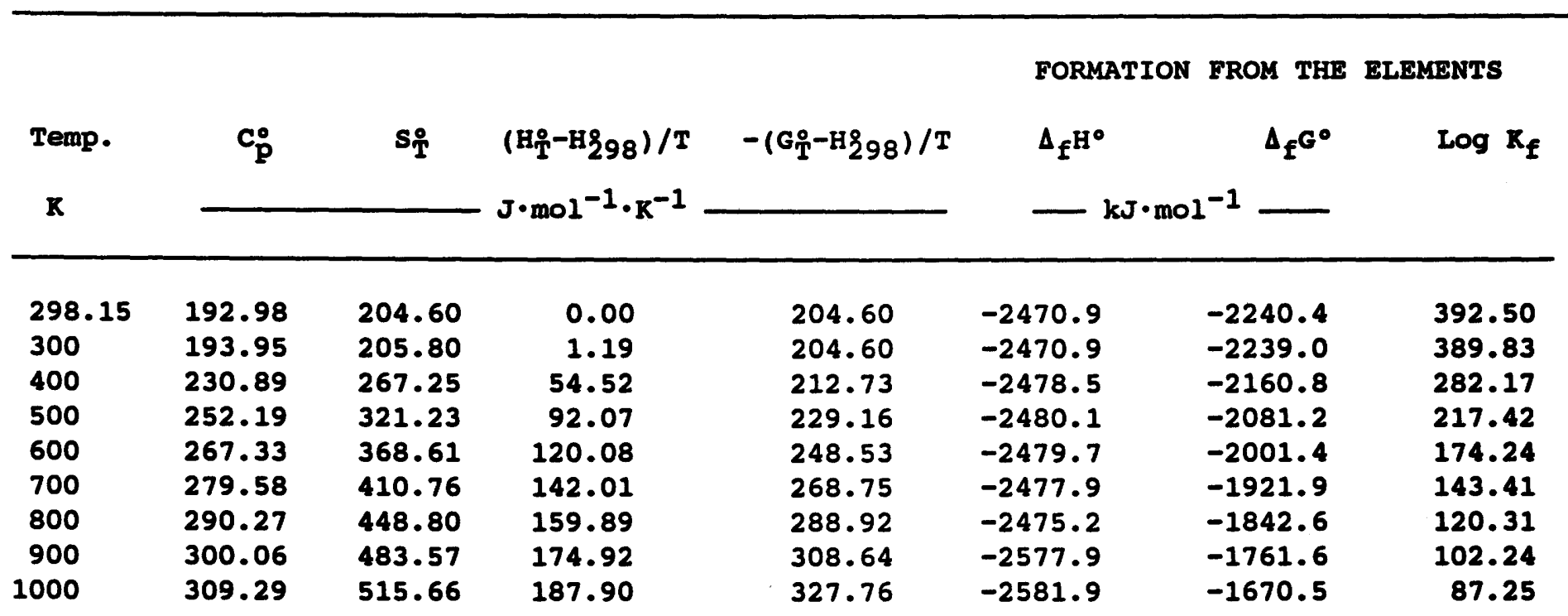

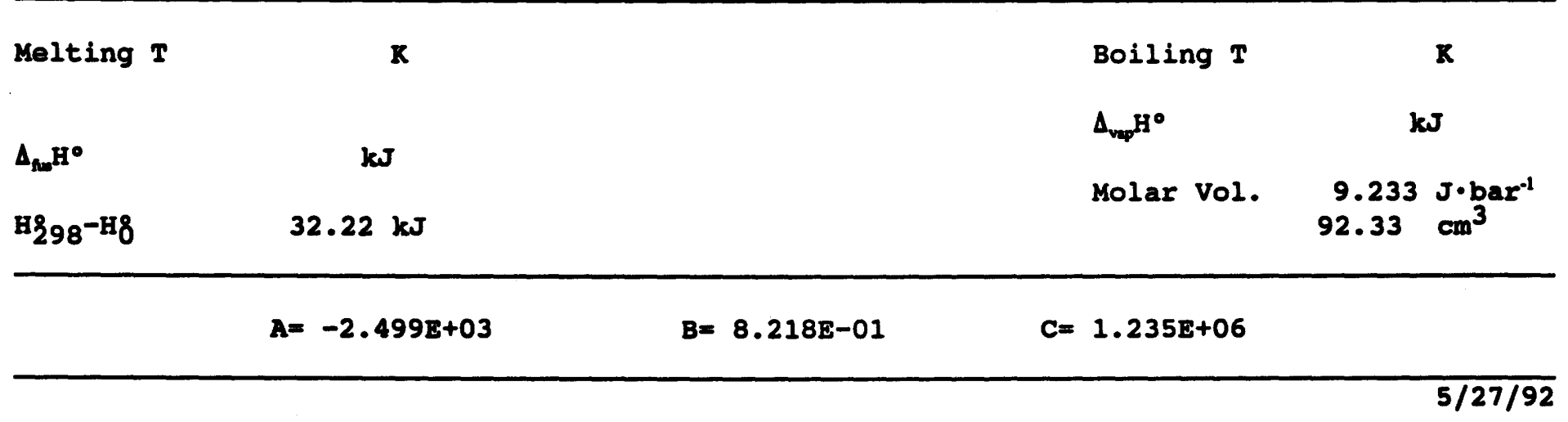


$\mathrm{KAl}_{3}(\mathrm{OH})_{6}\left(\mathrm{SO}_{4}\right)_{2}:$ Crystals 298.15 to $700 \mathrm{~K}$.

\begin{tabular}{|c|c|c|c|c|c|c|c|}
\hline \multirow[b]{2}{*}{ Temp. } & \multirow[b]{2}{*}{$\mathbf{c}_{\mathbf{p}}^{\circ}$} & \multirow[b]{2}{*}{$\mathbf{s}_{\mathbf{T}}^{\circ}$} & \multirow{3}{*}{$\begin{array}{l}\left(\mathrm{H}_{\mathrm{T}}-\mathrm{H}_{298}\right) / \mathrm{T} \\
\mathrm{J} \cdot \mathrm{mol}^{-1} \cdot \mathrm{K}^{-1}\end{array}$} & \multirow[b]{2}{*}{$-\left(G_{T}^{\circ}-H_{298}\right) / T$} & \multirow{2}{*}{$\begin{array}{l}\text { FORMATION } \\
\Delta_{\mathrm{f}} \mathrm{H}^{\circ}\end{array}$} & \multirow{2}{*}{$\begin{array}{c}\text { FROM THE } \\
\Delta_{\mathcal{f}^{G^{\circ}}}\end{array}$} & \multirow{2}{*}{$\begin{array}{l}\text { ELEMENTS } \\
\log \mathbf{x}_{1}\end{array}$} \\
\hline & & & & & & & \\
\hline $\mathbf{R}$ & & & & & $-\mathbf{k J} \cdot \mathbf{m o}$ & $1^{-1}$ & \\
\hline 298.15 & 372.53 & 321.00 & 0.00 & 321.00 & -5176.5 & -4663.5 & 817.00 \\
\hline 300 & 374.90 & 323.31 & 2.30 & 321.01 & -5176.6 & -4660.3 & 811.41 \\
\hline 400 & 462.28 & 444.70 & 107.73 & 336.96 & -5185.9 & -4487.0 & 585.92 \\
\hline 500 & 508.84 & 553.29 & 183.69 & 369.60 & -5186.2 & -4312.1 & 450.48 \\
\hline 600 & 539.32 & 648.92 & 240.57 & 408.35 & -5183.0 & -4137.6 & 360.20 \\
\hline 700 & 562.22 & 733.84 & 284.94 & 448.90 & -5177.7 & -3963.8 & 295.77 \\
\hline
\end{tabular}

Melting $\mathbf{T}$

$\mathbf{K}$

$\mathbf{k J}$

$\Delta_{\text {fun }} \mathrm{H}^{\circ}$

H298-H8

$\mathbf{k J}$
Boiling $T$

$\Lambda_{\text {vap }} \mathrm{H}^{\circ}$

$\mathbf{k J}$ 
$\mathrm{K}_{2} \mathrm{Mg}_{2}\left(\mathrm{SO}_{4}\right)_{3}:$ Cubic crystals 298.15 to $1203 \mathrm{~K}$.

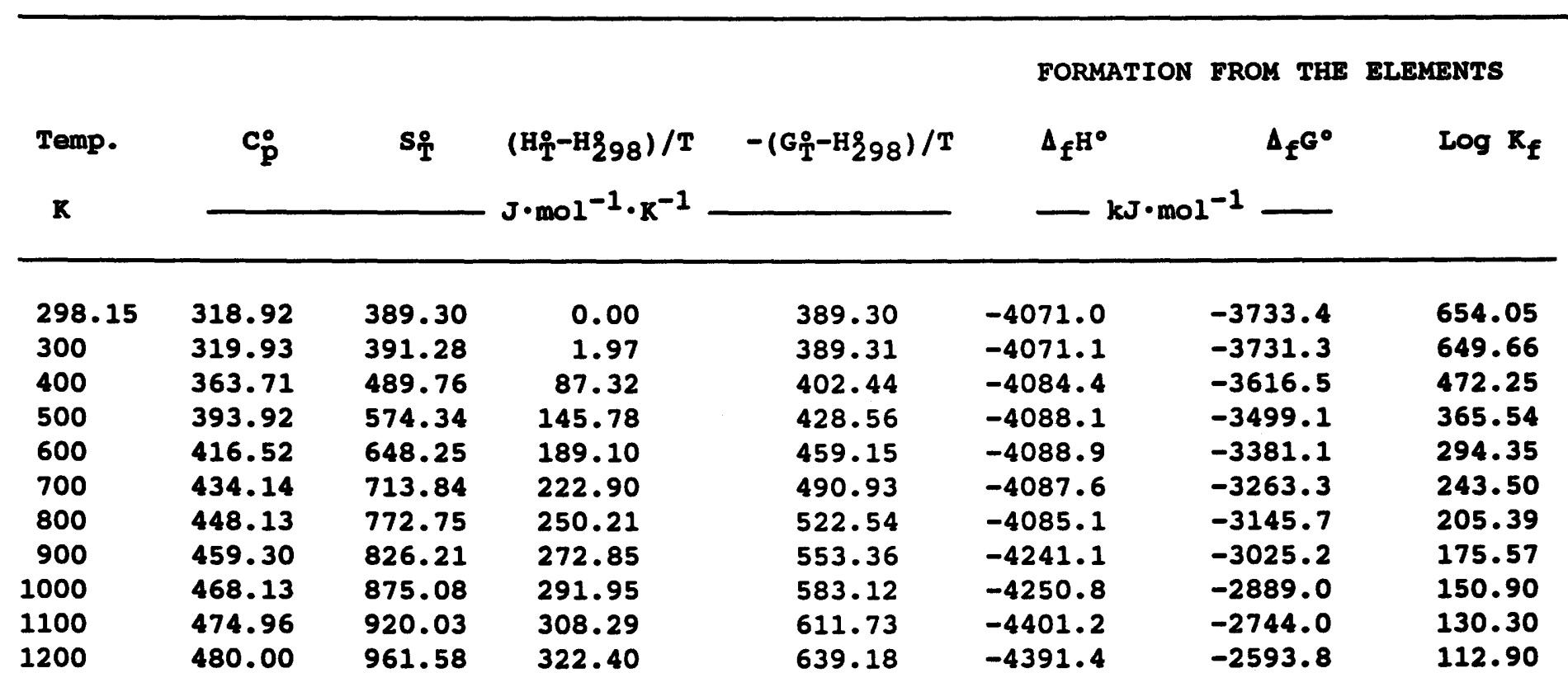

Melting T $1203 \mathrm{~K}$

$\Delta_{\text {fou }} \mathrm{H}^{\bullet}$

$\mathbf{k J}$

H298-H8

$\begin{array}{lc}\text { Boiling T } & \mathrm{K} \\ \Delta_{\mathrm{vep}} \mathrm{H}^{\circ} & \mathrm{kJ} \\ \text { Molar Vol. } & \begin{array}{c}14.695 \mathrm{~J} \cdot \mathrm{bar}^{-1} \\ 146.95 \mathrm{~cm}^{3}\end{array}\end{array}$

$C=6.392 \mathrm{E}+06$ 
$\mathrm{MgSO}_{4}:$ Crystals 298.15 to $1400 \mathrm{~K}$.

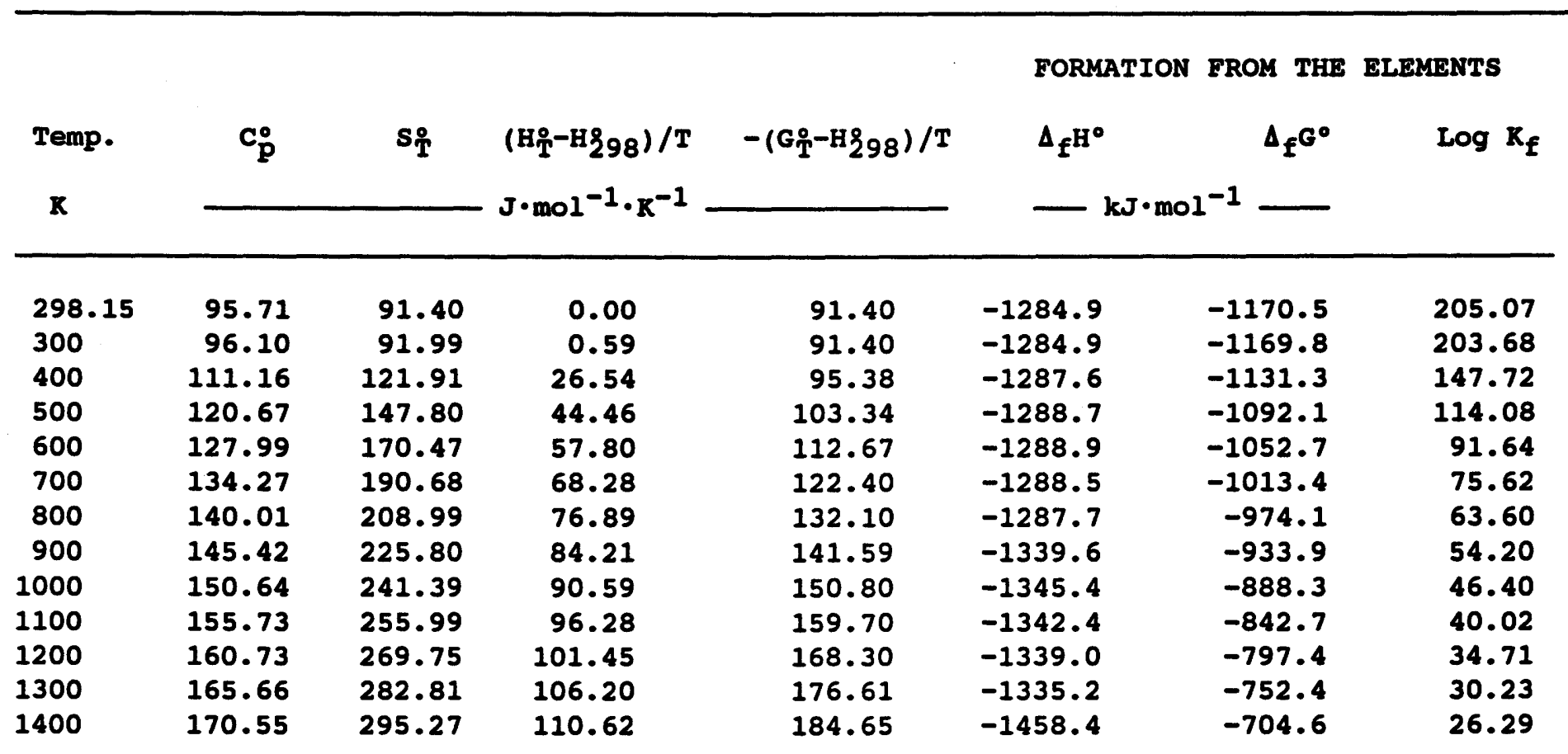

\begin{tabular}{|c|c|c|c|}
\hline Melting $T$ & $1400 \mathrm{~K}$ & Boiling $T$ & $\mathbf{K}$ \\
\hline & & $\Delta_{\mathrm{vep}} \mathrm{H}^{\circ}$ & $\mathbf{k J}$ \\
\hline$\Delta_{\mathrm{fu}} \mathrm{H}^{\circ}$ & $\mathbf{k J}$ & & \\
\hline $\mathrm{H}_{298^{-\mathrm{H}}}$ & $1539 \mathrm{~kJ}$ & Molar Vol. & $\begin{array}{l}J \cdot \mathbf{b} a r^{-1} \\
\mathrm{~cm}^{3}\end{array}$ \\
\hline
\end{tabular}
$A=-1 \cdot 325 \mathrm{E}+03$
$\mathrm{B}=4.385 \mathrm{E}-01$
$C=2.288 E+06$ 
$\mathrm{MnSO}_{4}:$ Orthorhombic crystals 298.15 to $1000 \mathrm{~K}$.

\begin{tabular}{|c|c|c|c|c|c|c|c|}
\hline \multirow[b]{2}{*}{ Temp. } & \multirow[b]{2}{*}{$\mathbf{c}_{\mathbf{p}}^{\circ}$} & \multirow[b]{2}{*}{$\mathbf{s}_{\mathrm{T}}$} & \multirow{3}{*}{$\begin{array}{l}\left(\mathrm{H}_{\mathrm{T}}-\mathrm{H}_{298}\right) / \mathrm{T} \\
\mathrm{J} \cdot \operatorname{mol}^{-1} \cdot \mathrm{K}^{-1}\end{array}$} & \multirow[b]{2}{*}{$-\left(G_{\mathrm{T}}^{\circ}-\mathrm{H}_{298}\right) / \mathrm{T}$} & \multicolumn{2}{|c|}{ FORMATION FROM THE } & \multirow{2}{*}{$\begin{array}{l}\text { ELEMENTS } \\
\qquad \log \mathrm{K}_{\mathbf{f}}\end{array}$} \\
\hline & & & & & $\Delta_{\mathrm{f}^{\mathrm{H}^{\circ}}}$ & $\Delta_{f} G^{\circ}$ & \\
\hline $\mathbf{K}$ & & & & & $-\mathbf{k J} \cdot \mathbf{m o l}$ & -1 & \\
\hline 298.15 & 100.52 & 127.00 & 0.00 & 127.00 & -1065.7 & -962.1 & 168.56 \\
\hline 300 & 100.99 & 127.62 & 0.62 & 127.00 & -1065.7 & -961.5 & 167.40 \\
\hline 400 & 118.68 & 159.38 & 28.17 & 131.21 & -1067.9 & -926.6 & 120.99 \\
\hline 500 & 129.02 & 187.05 & 47.38 & 139.68 & -1068.4 & -891.3 & 93.10 \\
\hline 600 & 136.47 & 211.26 & 61.63 & 149.63 & -1068.2 & -855.8 & 74.49 \\
\hline 700 & $142 \cdot 54$ & 232.77 & 72.77 & 160.00 & $-1067 \cdot 3$ & -820.5 & 61.21 \\
\hline 800 & 147.89 & 252.16 & 81.83 & 170.33 & -1066.1 & -785.3 & 51.26 \\
\hline 900 & 152.81 & 269.86 & 89.45 & 180.42 & -1117.7 & -749.2 & 43.47 \\
\hline 1000 & 157.47 & 286.21 & 96.02 & 190.19 & -1116.9 & -708.4 & 36.99 \\
\hline
\end{tabular}

Melting $\mathbf{T}$

$\mathbf{K}$

$\Delta_{\text {fun }} \mathrm{H}^{\circ}$

$\mathbf{k J}$

H298-H8

kJ
Boiling $\mathbf{T}$

$\Lambda_{\text {vap }} H^{\circ}$

$\mathbf{k J}$

Molar Vol.
$B=3.657 \mathrm{E}-01$

$$
A=-1.077 \mathrm{E}+03
$$

\section{$C=5.563 \mathrm{E}+05$}


$\left(\mathrm{NH}_{4}\right)_{2} \mathrm{SO}_{4}$ : Orthorhombic crystals 298.15 to $600 \mathrm{~K}$.

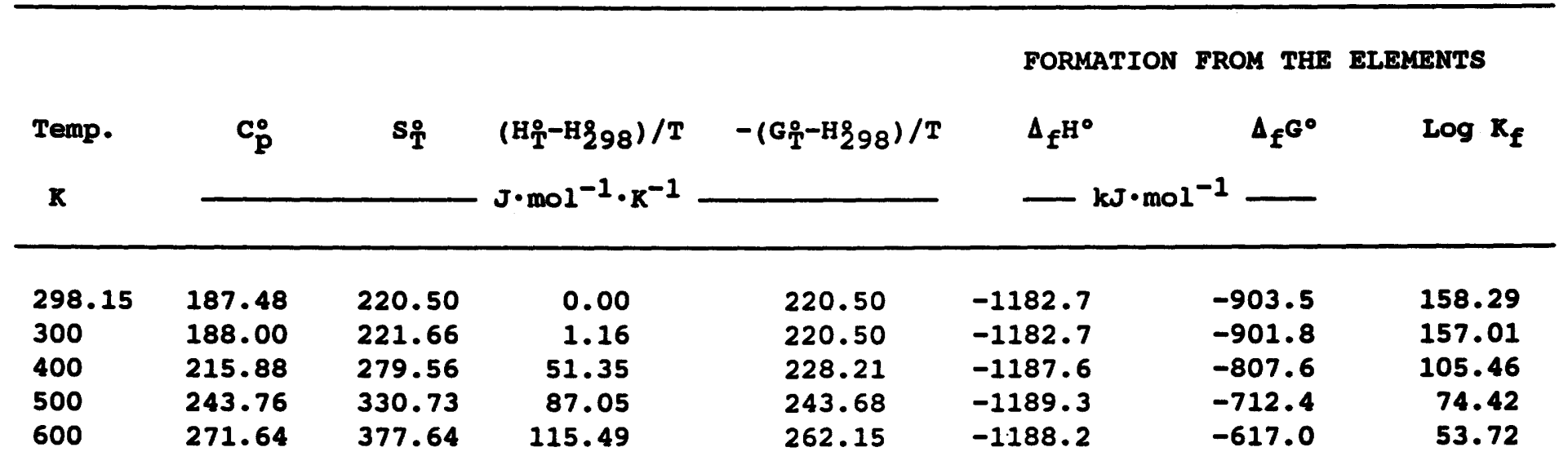

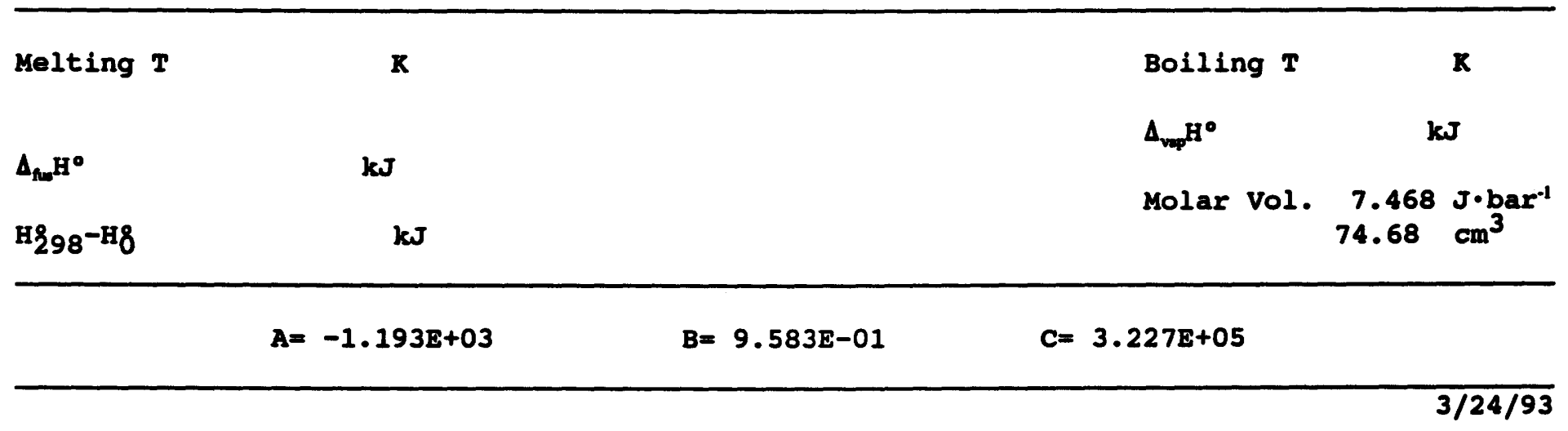


$\mathrm{Na}_{2} \mathrm{SO}_{4}$ : Orthorhombic crystals (V) 298.15 to $450 \mathrm{~K}$. Orthorhombic crystals (III) 450 to 514 R. Hexagonal crystals (I) 514 to $1155 \mathrm{~K}$. Liquid 1155 to $1800 \mathrm{~K}$.

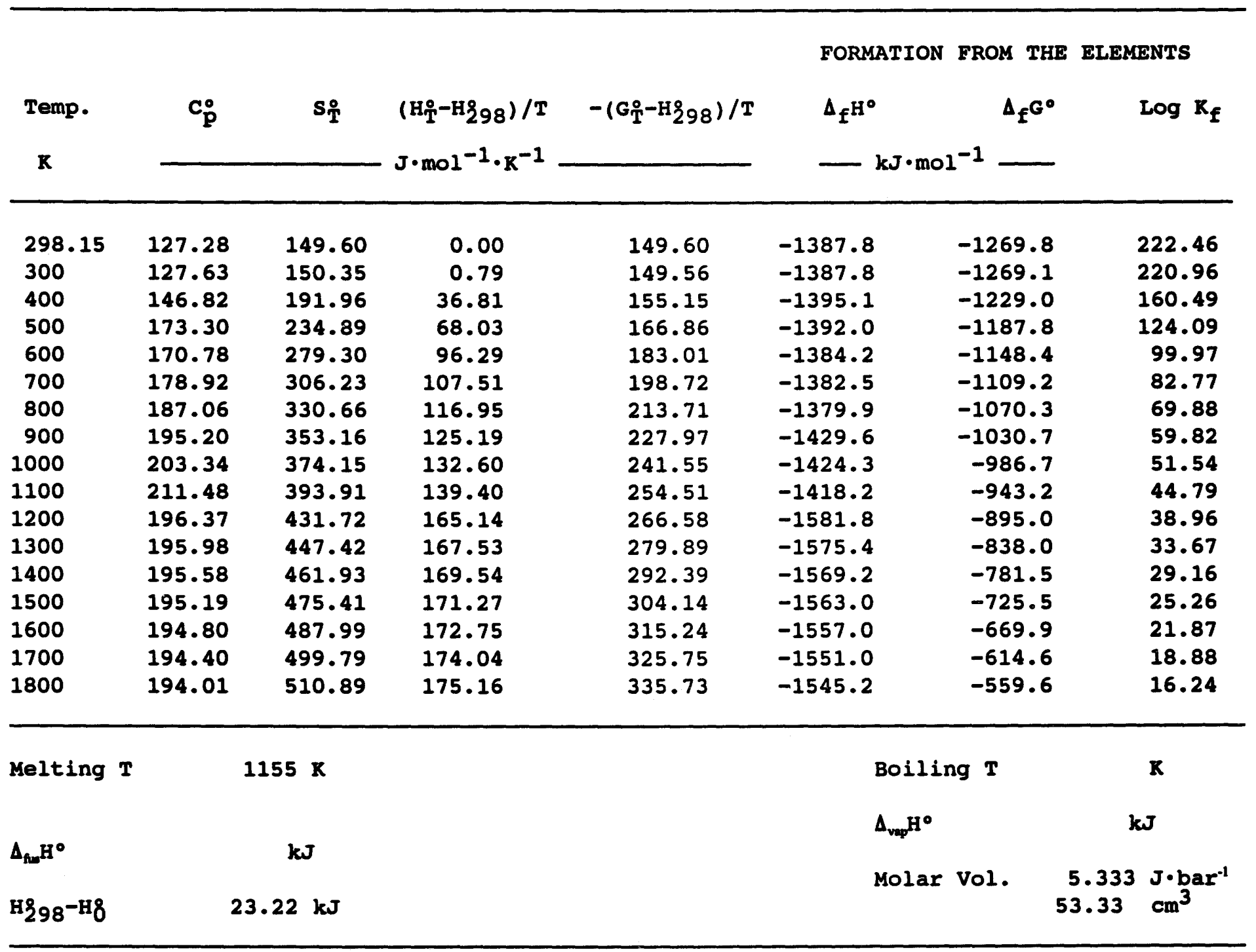
$A=-1 \cdot 482 \mathrm{E}+03$
$\mathrm{B}=5 \cdot 020 \mathrm{E}-01$
$\mathrm{C}=6.251 \mathrm{E}+06$ 
$\mathrm{NiSO}_{4}$ : Orthorhombic crystals 298.15 to $1200 \mathrm{~K}$.

\begin{tabular}{|c|c|c|c|c|c|c|c|}
\hline \multirow{2}{*}{$\begin{array}{l}\text { Temp. } \\
\mathbf{R}\end{array}$} & \multirow[b]{2}{*}{$\mathbf{c}_{\mathbf{p}}^{\circ}$} & \multirow[b]{2}{*}{$\mathbf{s}_{\mathbf{T}}^{\circ}$} & \multirow{2}{*}{$\begin{array}{l}\left(\mathrm{H}_{\mathrm{T}}^{\circ}-\mathrm{H}_{298}\right) / \mathrm{T} \\
\mathrm{J} \cdot \mathrm{mol}^{-1} \cdot \mathrm{K}^{-1}\end{array}$} & \multirow[b]{2}{*}{$-\left(G_{T}^{\circ}-H_{298}\right) / T$} & \multirow{2}{*}{\multicolumn{2}{|c|}{$\begin{array}{l}\text { FORMATION FROM THE } \\
\Delta_{\mathrm{f}^{\mathrm{H}^{\circ}}}\end{array}$}} & \multirow{2}{*}{$\begin{array}{l}\text { ELEMENTS } \\
\qquad \log \mathrm{K}_{\mathrm{f}}\end{array}$} \\
\hline & & & & & & & \\
\hline 298.15 & 97.69 & 101.30 & 0.00 & 101.30 & -873.2 & -762.7 & 133.61 \\
\hline 300 & 98.19 & 101.91 & 0.60 & 101.30 & -873.3 & -762.0 & 132.67 \\
\hline 400 & 116.56 & 132.98 & 27.57 & 105.41 & -875.7 & -724.7 & 94.64 \\
\hline 500 & 126.71 & 160.17 & 46.46 & 113.71 & -876.5 & -686.9 & 71.76 \\
\hline 600 & 133.62 & 183.92 & 60.44 & 123.48 & -876.6 & -648.9 & 56.49 \\
\hline 700 & 139.01 & 204.93 & 71.29 & 133.64 & -876.2 & -611.0 & 45.59 \\
\hline 800 & 143.57 & 223.80 & 80.05 & 143.75 & -875.0 & -573.7 & 37.46 \\
\hline 900 & 147.67 & 240.95 & 87.34 & 153.61 & -926.6 & -534.5 & 31.02 \\
\hline 1000 & 151.47 & 256.71 & 93.56 & 163.14 & -923.7 & -491.1 & 25.65 \\
\hline 1100 & 155.08 & 271.31 & 98.99 & 172.32 & -920.5 & -448.0 & 21.27 \\
\hline 1200 & 158.56 & 284.96 & 103.81 & 181.15 & -917.1 & -405.2 & 17.64 \\
\hline
\end{tabular}

Melting $T$

$$
\Delta_{\text {fun }} \mathrm{H}^{\circ}
$$

$\mathbf{k J}$

Н298-H8

$\begin{array}{lc}\text { Boiling T } & \mathrm{K} \\ \text { Ave }^{\mathrm{H}^{\circ}} & \mathrm{kJ} \\ \text { Molar Vol. } & \begin{array}{c}3.857 \mathrm{~J} \cdot \mathrm{bar}^{-1} \\ 38.57 \mathrm{~cm}^{3}\end{array}\end{array}$

$C=1.381 E+06$ 
$\mathrm{PbsO}_{4}$ : Orthorhombic crystals 298.15 to $1100 \mathrm{~K}$.

\begin{tabular}{|c|c|c|c|c|c|c|c|}
\hline \multirow{3}{*}{$\begin{array}{l}\text { Temp. } \\
\mathbf{X}\end{array}$} & \multirow{3}{*}{$\mathbf{c}_{\mathbf{p}}^{\circ}$} & \multirow{3}{*}{$\mathbf{s}_{\mathrm{T}}^{\circ}$} & \multirow{3}{*}{$\begin{array}{l}\left(\mathrm{H}_{\mathrm{T}}-\mathrm{H} 298\right) / \mathrm{T} \\
\mathrm{J} \cdot \mathrm{mol}^{-1} \cdot \mathrm{K}^{-1}\end{array}$} & \multirow{3}{*}{$-\left(G_{T}^{\circ}-H_{298}\right) / T$} & \multicolumn{2}{|c|}{ FORMATION FROM THE } & \multirow{2}{*}{$\begin{array}{l}\text { BLEMENTS } \\
\qquad \log \mathrm{K}_{\mathrm{f}}\end{array}$} \\
\hline & & & & & $\Delta_{f^{H^{\circ}}}$ & $\Delta_{f} G^{\circ}$ & \\
\hline & & & & & \multicolumn{2}{|c|}{$\mathrm{kJ} \cdot \mathrm{mol}^{-1}$} & \\
\hline 298.15 & 104.33 & 148.50 & 0.00 & 148.50 & -920.0 & -813.1 & 142.44 \\
\hline 300 & 104.33 & 149.15 & 0.64 & 148.50 & -920.0 & $-812 \cdot 4$ & 141.45 \\
\hline 400 & 108.73 & 179.59 & 26.96 & 152.62 & -922.7 & -776.4 & 101.38 \\
\hline 500 & 117.63 & 204.76 & 44.16 & 160.59 & -924.2 & -739.6 & 77.27 \\
\hline 600 & 128.30 & 227.13 & 57.28 & 169.85 & -924.8 & -702.6 & 61.17 \\
\hline 700 & 139.81 & 247.76 & 68.24 & 179.52 & -929.1 & -664.8 & 49.61 \\
\hline 800 & 151.76 & 267.21 & 77.93 & 189.28 & -927.4 & -627.2 & 40.95 \\
\hline 900 & 163.98 & 285.79 & 86.81 & 198.98 & -977.7 & -588.7 & 34.17 \\
\hline 1000 & 176.35 & 303.70 & 95.15 & 208.56 & -972.4 & -545.8 & 28.51 \\
\hline 1100 & 188.83 & $321 \cdot 10$ & 103.09 & 218.00 & -966.0 & -503.4 & 23.90 \\
\hline
\end{tabular}

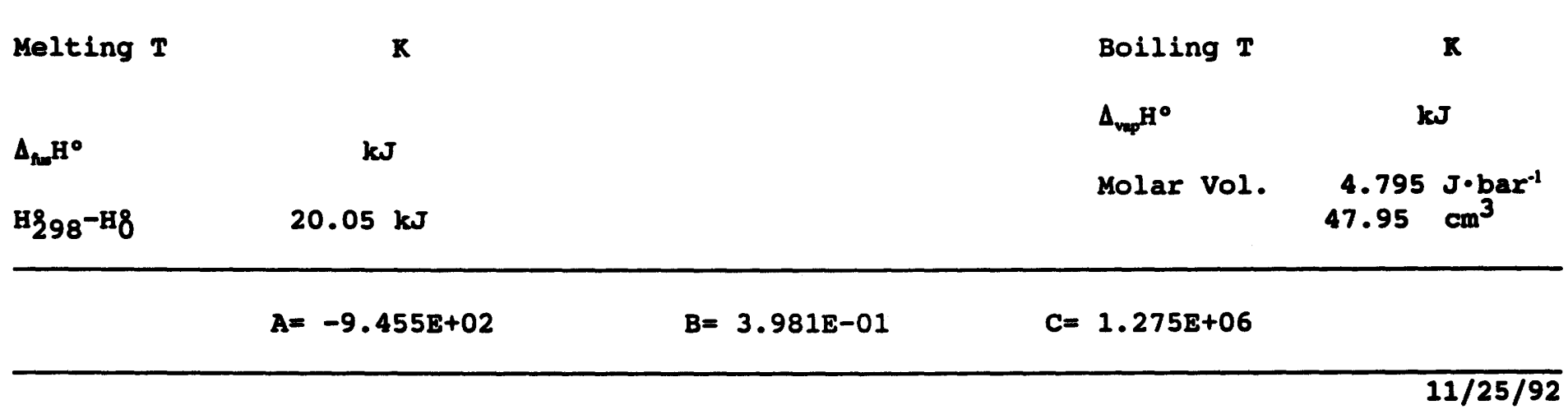


$\mathrm{ZnSO}_{4}$ : Orthorhombic crystals 298.15 to $1100 \mathrm{~K}$.

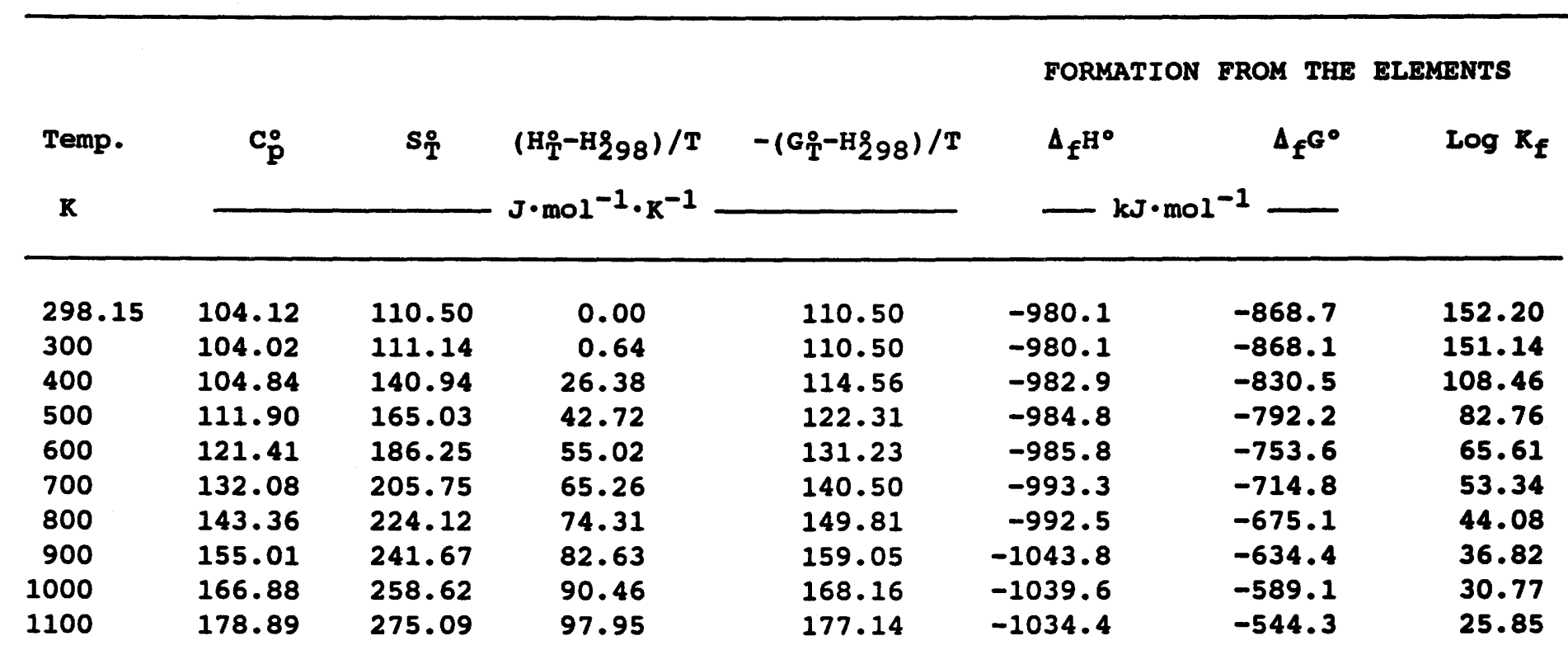

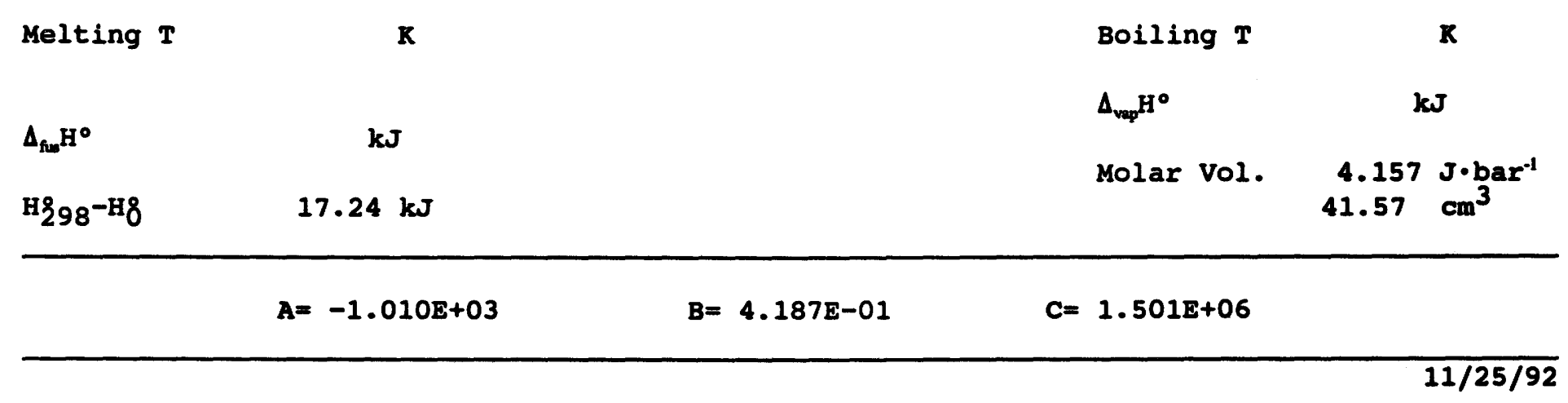


$\mathrm{AlPO}_{4}$ : Rhombohedral crystals 298.15 to $\alpha-\beta$ transition at $855 \mathrm{~K}$. $\beta$-crystals 855 to $1000 \mathrm{~K}$.

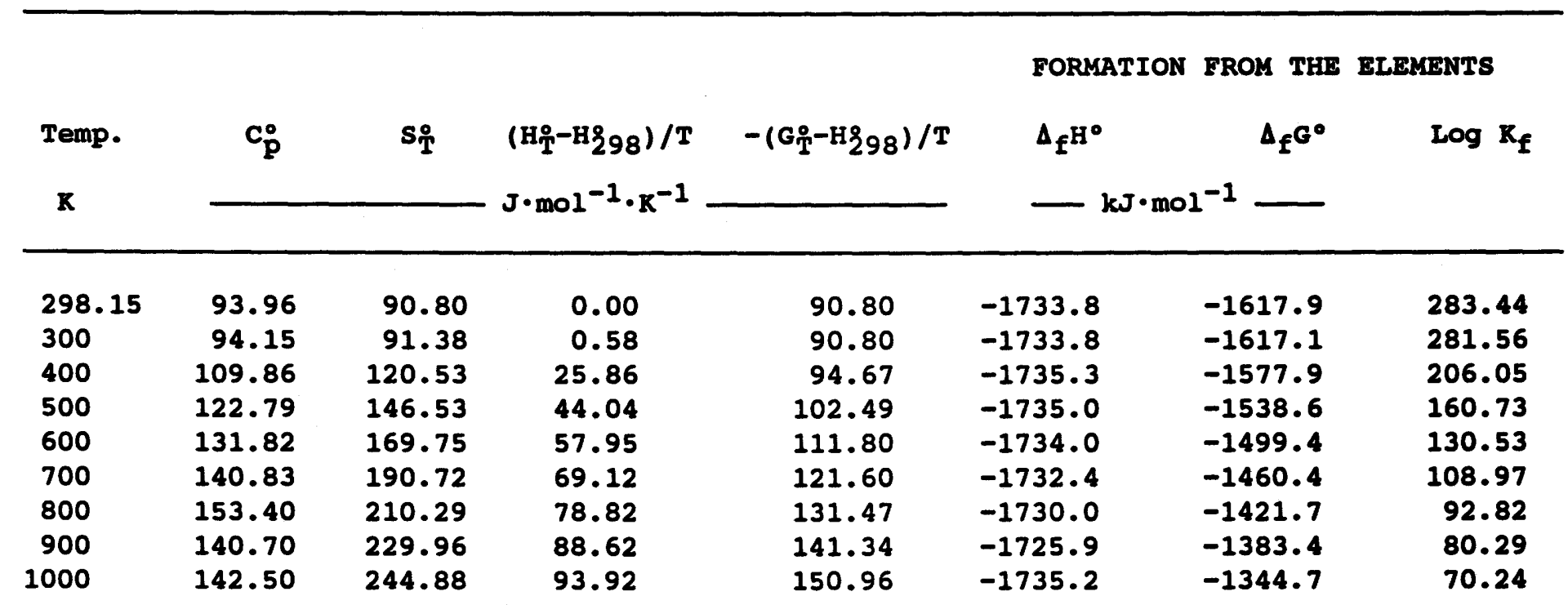

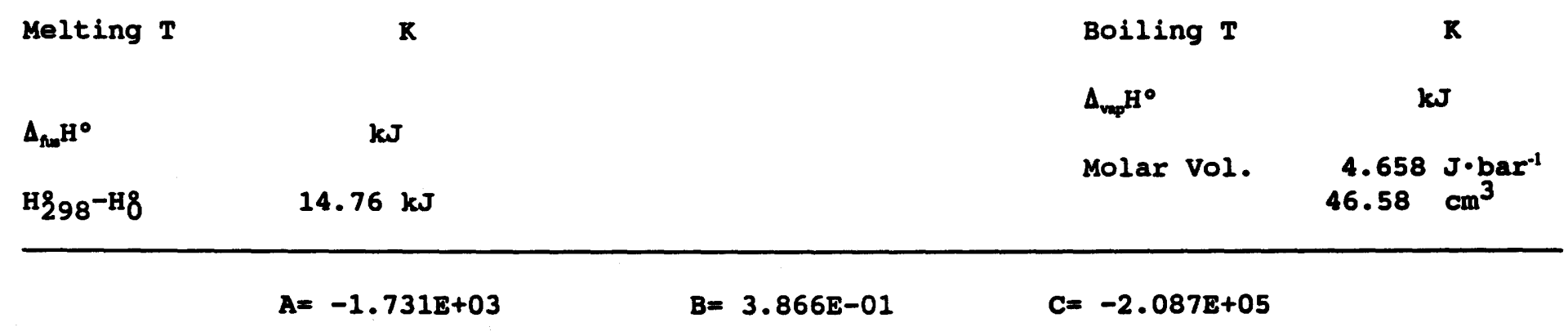


$\mathrm{Ca}_{3}\left(\mathrm{PO}_{4}\right)_{2}$ : Rhombohedral crystals 298.15 to $1373 \mathrm{~K}$. Monoclinic crystals 1373 to $1600 \mathrm{~K}$.

\begin{tabular}{|c|c|c|c|c|c|c|c|}
\hline \multirow{2}{*}{$\begin{array}{l}\text { Temp. } \\
\mathbf{K}\end{array}$} & \multirow[t]{2}{*}{$\mathbf{c}_{\mathbf{p}}^{\circ}$} & \multirow[t]{2}{*}{$s_{\text {T }}^{\circ}$} & \multirow{2}{*}{$\begin{array}{l}\left(\mathrm{H}_{\mathrm{T}}^{\circ}-\mathrm{H} 298\right) / \mathrm{T} \\
\mathrm{J} \cdot \mathrm{mol}^{-1} \cdot \mathrm{K}^{-1}\end{array}$} & \multirow[t]{2}{*}{$-\left(G_{T}^{\circ}-H_{298}\right) / T$} & \multirow{2}{*}{\multicolumn{2}{|c|}{$\begin{array}{l}\text { FORMATION FROM THE } \\
\Delta_{f^{H}} H^{\circ}\end{array}$}} & \multirow[t]{2}{*}{$\begin{array}{l}\text { ELEMENTS } \\
\qquad \log K_{f}\end{array}$} \\
\hline & & & & & & & \\
\hline 298.15 & 231.63 & 236.00 & 0.00 & 236.00 & -4120.8 & -3883.6 & 680.38 \\
\hline 300 & 232.12 & 237.43 & 1.43 & 236.00 & -4120.8 & -3882.2 & 675.93 \\
\hline 400 & 255.24 & 307.49 & 62.09 & 245.40 & -4122.7 & -3802.1 & 496.50 \\
\hline 500 & 275.30 & 366.64 & 102.76 & 263.88 & -4121.9 & -3722.1 & 388.83 \\
\hline 600 & 294.16 & 418.51 & 133.10 & 285.41 & -4120.2 & -3642.3 & 317.08 \\
\hline 700 & 312.44 & 465.23 & 157.42 & 307.82 & -4117.6 & -3562.8 & 265.85 \\
\hline 800 & 330.43 & 508.13 & 177.92 & 330.21 & -4116.6 & -3483.5 & 227.44 \\
\hline 900 & 348.23 & 548.08 & 195.86 & 352.22 & -4112.2 & -3404.6 & 197.59 \\
\hline 1000 & 365.93 & 585.69 & 211.98 & 373.70 & -4107.0 & -3326.2 & 173.74 \\
\hline 1100 & 383.55 & 621.39 & 226.78 & 394.61 & -4101.2 & -3248.4 & 154.25 \\
\hline 1200 & 401.12 & 655.52 & 240.57 & 414.94 & -4245.0 & -3167.2 & 137.86 \\
\hline 1300 & 418.67 & 688.32 & 253.60 & 434.72 & -4232.6 & -3077.8 & 123.67 \\
\hline 1400 & 330.54 & 729.26 & 275.09 & 454.17 & -4205.8 & -2989.8 & 111.55 \\
\hline 1500 & 330.54 & 752.05 & 278.79 & 473.26 & -4201.6 & -2903.0 & 101.09 \\
\hline 1600 & 330.54 & 773.38 & 282.02 & 491.36 & -4197.5 & -2816.6 & 91.95 \\
\hline
\end{tabular}

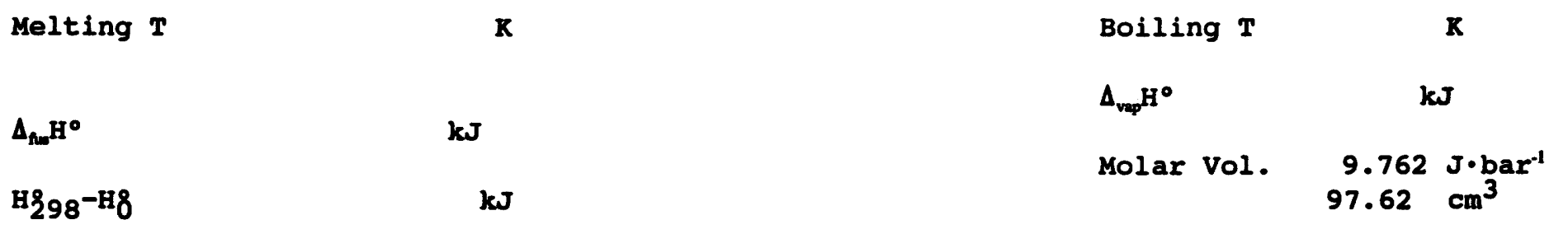

$A=-4.148 \mathrm{E}+03 \quad B=8.253 \mathrm{E}-01 \quad C=1.823 \mathrm{E}+06$ 


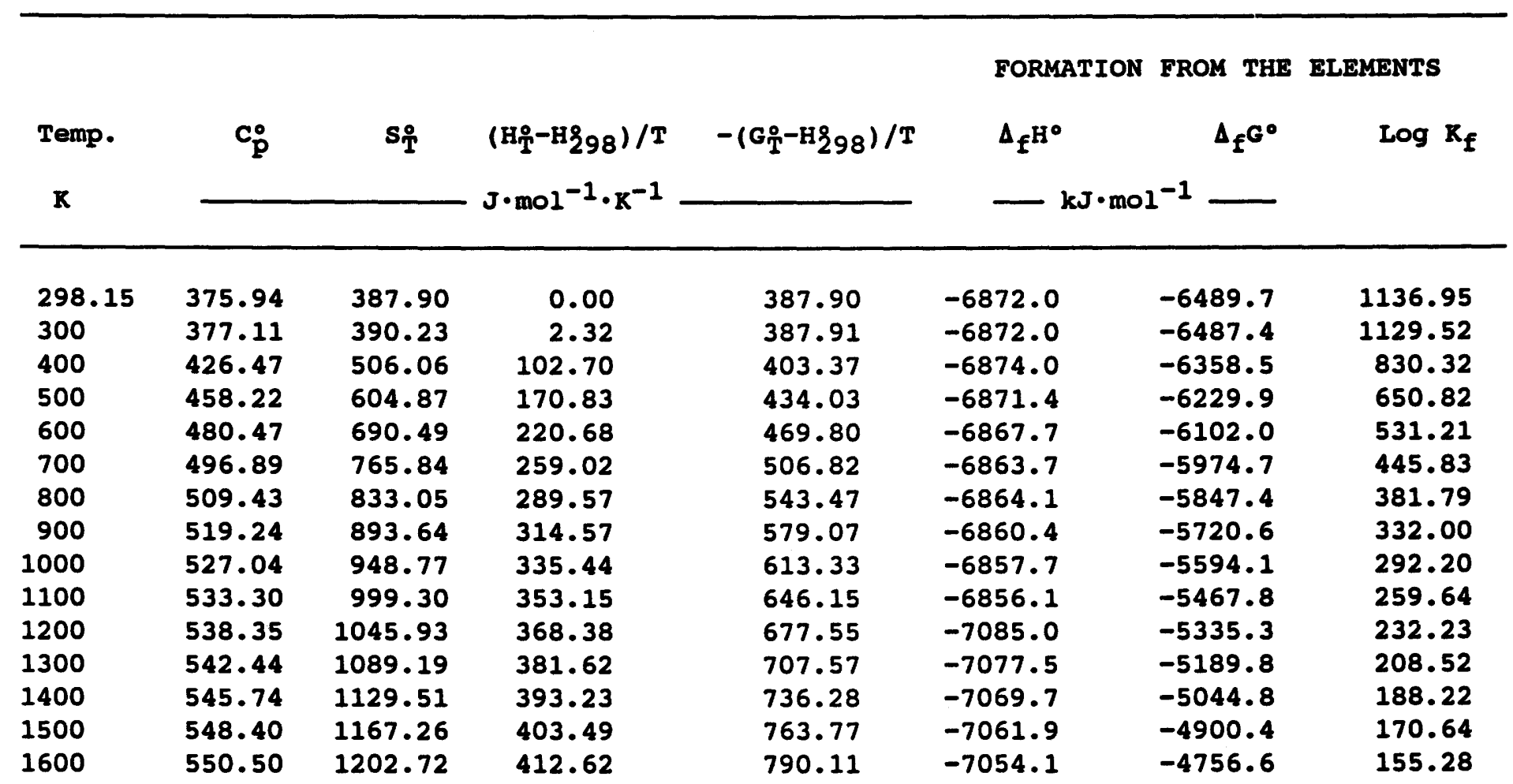

$$
\text { Melting } T
$$$$
\Delta_{\text {fue }} \mathrm{H}^{\circ}
$$

H298-H8$$
\mathbf{k J}
$$

$\mathbf{R}$

$\mathbf{k J}$

$63.47 \mathrm{~kJ}$
Boiling $T$

$\Delta_{\mathrm{veq}} \mathrm{H}^{\circ}$

Molar Vol.
$\mathbf{K}$

$\mathbf{k J}$
$A=-6.931 E+03$
$B=1 \cdot 344 \mathrm{E}+00$
$C=4.048 E+06$ 
$\mathrm{Ca}_{5}\left(\mathrm{PO}_{4}\right)_{3}(\mathrm{OH})$ : Hexagonal crystals 298.15 to $1500 \mathrm{~K}$.

FORMATION FROM THE ELEMENTS

\begin{tabular}{|c|c|c|c|c|c|c|c|}
\hline \multirow{2}{*}{$\begin{array}{l}\text { Temp. } \\
\mathbf{K}\end{array}$} & $\mathbf{c}_{\mathbf{p}}^{\circ}$ & $s_{T}^{\circ}$ & \multirow{2}{*}{$\begin{array}{l}\left(\mathrm{H}_{\mathrm{T}}^{\mathrm{O}}-\mathrm{H}_{298}\right) / \mathrm{T} \\
\mathrm{J} \cdot \mathrm{mol}^{-1} \cdot \mathrm{K}^{-1}\end{array}$} & \multirow[t]{2}{*}{$-\left(G_{T}^{\circ}-H_{298}\right) / T$} & $\Delta_{f^{H}} H^{\circ}$ & \multirow[t]{2}{*}{$\Delta_{f} G^{0}$} & \multirow[t]{2}{*}{$\log R_{f}$} \\
\hline & & & & & 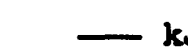 & & \\
\hline 298.15 & 385.17 & 390.40 & 0.00 & 390.40 & -6738.5 & -6337.1 & 1110.22 \\
\hline 300 & 386.83 & 392.79 & 2.38 & 390.41 & -6738.5 & -6334.7 & 1102.94 \\
\hline 400 & 446.42 & 513.36 & 106.92 & 406.44 & -6740.1 & -6199.4 & 809.55 \\
\hline 500 & 477.29 & 616.59 & 178.17 & 438.42 & -6736.9 & -6064.6 & 633.55 \\
\hline 600 & 497.62 & 705.50 & 229.81 & 475.70 & -6732.7 & -5930.6 & 516.29 \\
\hline 700 & 513.35 & 783.43 & 269.23 & 514.21 & -6728.4 & -5797.2 & 432.58 \\
\hline 800 & 526.86 & 852.88 & 300.60 & 552.28 & -6728.4 & -5663.9 & 369.81 \\
\hline 900 & 539.23 & 915.66 & 326.44 & 589.22 & -6724.2 & -5531.1 & 321.01 \\
\hline 1000 & 550.97 & 973.08 & 348.31 & 624.77 & -6720.7 & -5398.8 & 282.00 \\
\hline 1100 & 562.37 & 1026.13 & 367.25 & 658.88 & -6717.9 & -5266.7 & 250.09 \\
\hline 1200 & 573.58 & 1075.54 & 383.98 & 691.57 & -6945.1 & -5128.6 & 223.24 \\
\hline 1300 & 584.69 & 1121.90 & 398.99 & 722.90 & -6935.1 & -4977.6 & 200.00 \\
\hline 1400 & 595.76 & 1165.63 & 412.65 & 752.98 & -6924.2 & -4827.4 & 180.11 \\
\hline 1500 & 606.82 & 1207.11 & 425.23 & 781.88 & -6912.5 & -4678.0 & 162.90 \\
\hline
\end{tabular}

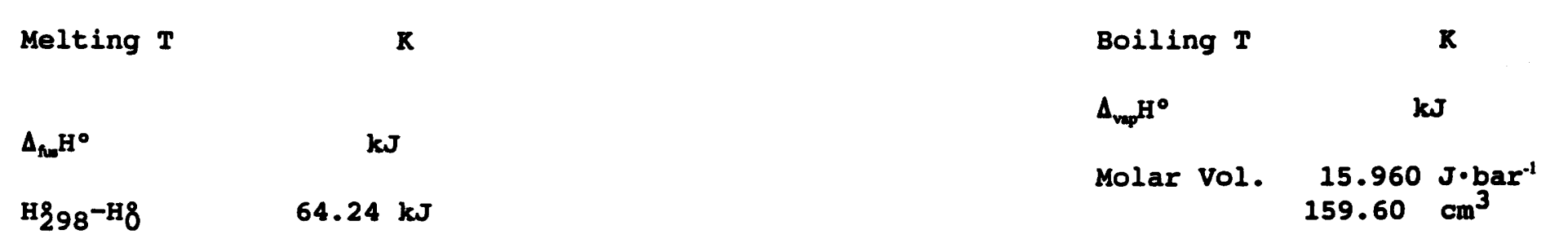
$A=-6.777 E+03$
$B=1 \cdot 386 \mathrm{E}+00$
$C=2.726 \mathrm{E}+06$ 
$\mathrm{Al}_{2} \mathrm{SiO}_{4} \mathrm{~F}_{2}$ : Orthorhombic crystals 298.15 to $1000 \mathrm{~K}$.

\begin{tabular}{|c|c|c|c|c|c|c|c|}
\hline \multirow{3}{*}{$\begin{array}{l}\text { Temp. } \\
\text { R }\end{array}$} & \multirow{3}{*}{$\mathbf{c}_{\mathbf{p}}^{\circ}$} & \multirow{3}{*}{$\mathbf{s}_{\mathrm{T}}$} & \multirow{3}{*}{$\begin{array}{l}\left(\mathrm{H}_{\mathrm{T}}^{\circ}-\mathrm{H}_{298}\right) / \mathrm{T} \\
\mathrm{J} \cdot \mathrm{mol}^{-1} \cdot \mathrm{K}^{-1}\end{array}$} & \multirow{3}{*}{$-\left(G_{T}^{\circ}-H_{298}\right) / T$} & \multirow{3}{*}{\multicolumn{2}{|c|}{$\begin{array}{l}\text { FORMATION FROM THE } \\
\begin{array}{l}\Delta_{\mathrm{f}^{\circ}} \\
\boldsymbol{H}_{\mathrm{f}} \mathrm{G}^{\bullet}\end{array}\end{array}$}} & \multirow{3}{*}{$\begin{array}{l}\text { ELEMENTS } \\
\qquad \log \mathbf{K}_{\mathbf{f}}\end{array}$} \\
\hline & & & & & & & \\
\hline & & & & & & & \\
\hline 298.15 & 143.63 & 105.40 & 0.00 & 105.40 & -3084.5 & -2910.6 & 509.92 \\
\hline 300 & 144.28 & 106.29 & 0.89 & 105.40 & -3084.5 & -2909.6 & 506.59 \\
\hline 400 & $172 \cdot 38$ & 151.96 & 40.52 & 111.44 & -3084.9 & -2851.1 & 372.31 \\
\hline 500 & 190.31 & 192.49 & 68.81 & 123.68 & -3083.7 & -2792.8 & 291.76 \\
\hline 600 & 201.97 & 228.30 & 90.10 & 138.19 & -3081.7 & -2734.8 & 238.08 \\
\hline 700 & 209.49 & 260.04 & 106.66 & 153.38 & -3079.3 & -2677.2 & 199.77 \\
\hline 800 & 214.10 & 288.34 & 119.83 & 168.51 & -3076.9 & -2619.9 & 171.06 \\
\hline 900 & 216.62 & 313.72 & 130.46 & 183.26 & -3074.6 & -2562.9 & 148.75 \\
\hline 1000 & 217.54 & 336.61 & 139.13 & 197.47 & -3093.9 & -2504.7 & 130.83 \\
\hline
\end{tabular}

Melting $\mathbf{T}$

$\mathbf{K}$

$\Delta_{\text {hum }} \mathrm{H}^{\circ}$

$\mathbf{k J}$

H298-H8
Boiling $T$

$\mathbf{K}$

$\mathbf{k J}$

Molar Vol.

\section{$5.153 \mathrm{~J} \cdot$ bar $^{-1}$ $51.53 \mathrm{~cm}^{3}$}


$\mathrm{Al}_{2} \mathrm{SiO}_{5}$ : Triclinic crystals 298.15 to $1800 \mathrm{~K}$.

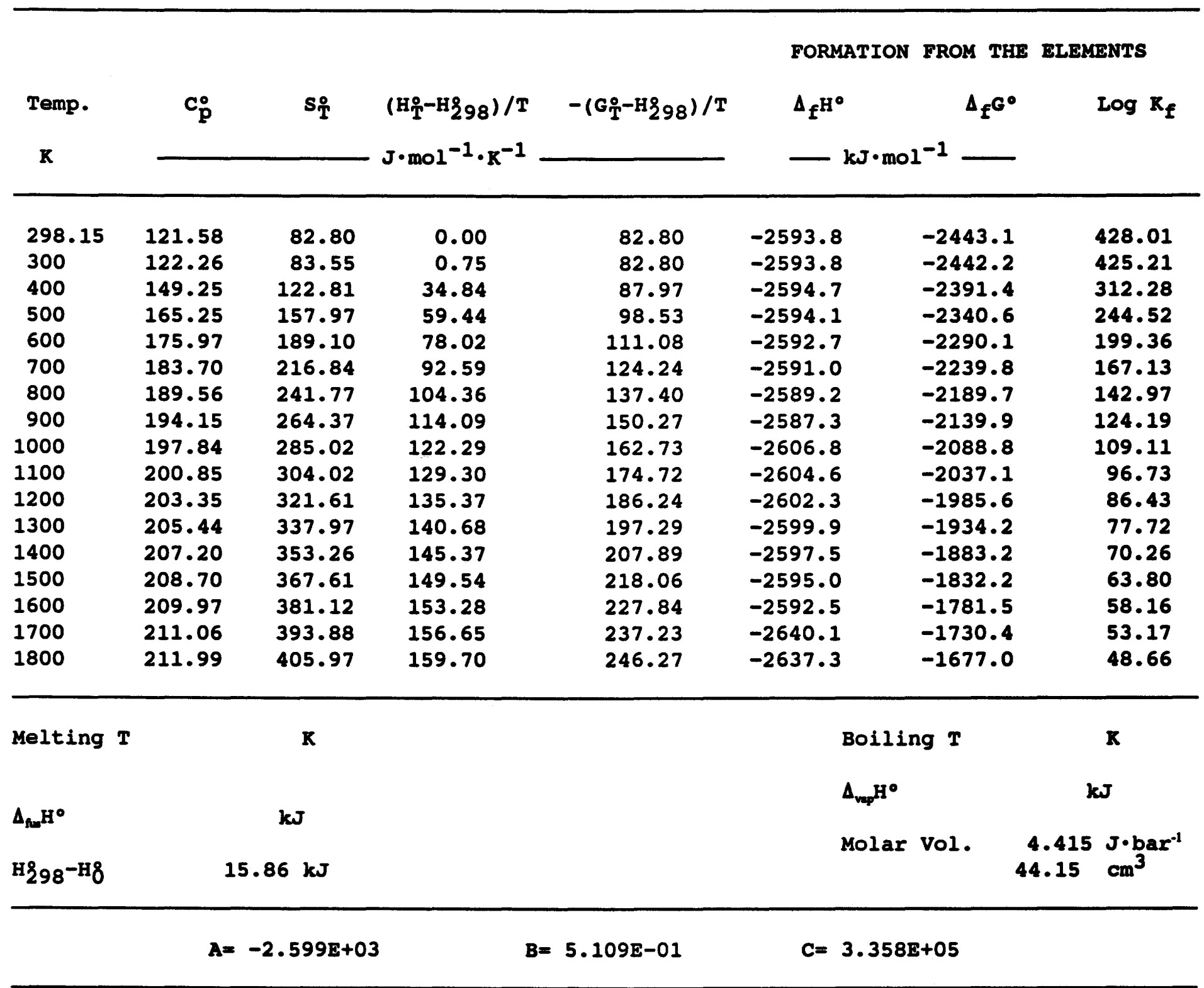


$\mathrm{Al}_{2} \mathrm{SiO}_{5}$ : Orthorhombic crystals 298.15 to $1800 \mathrm{~K}$.

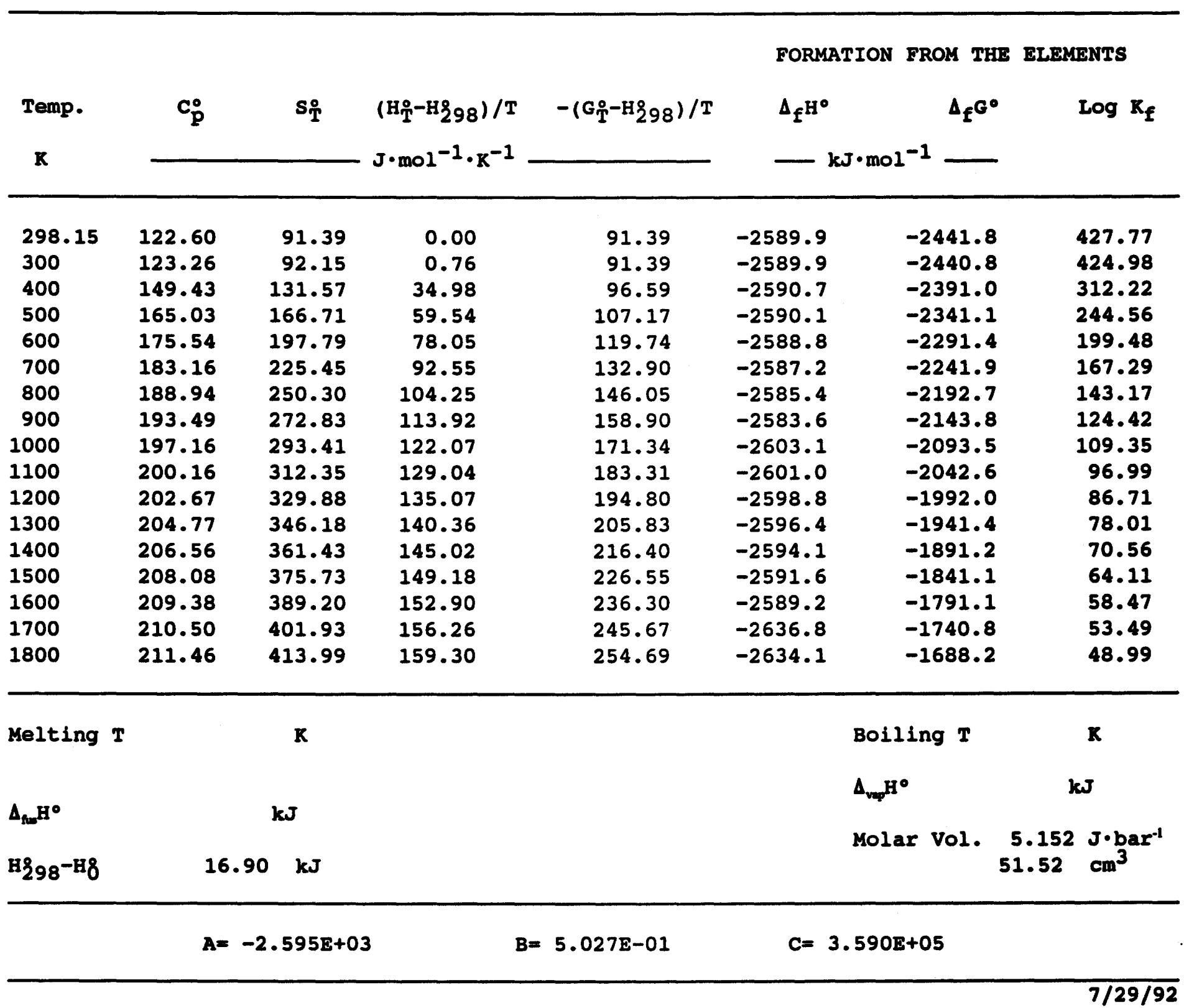


$\mathrm{Al}_{2} \mathrm{SiO}_{5}$ : Orthorhombic crystals 298.15 to $1800 \mathrm{~K}$.

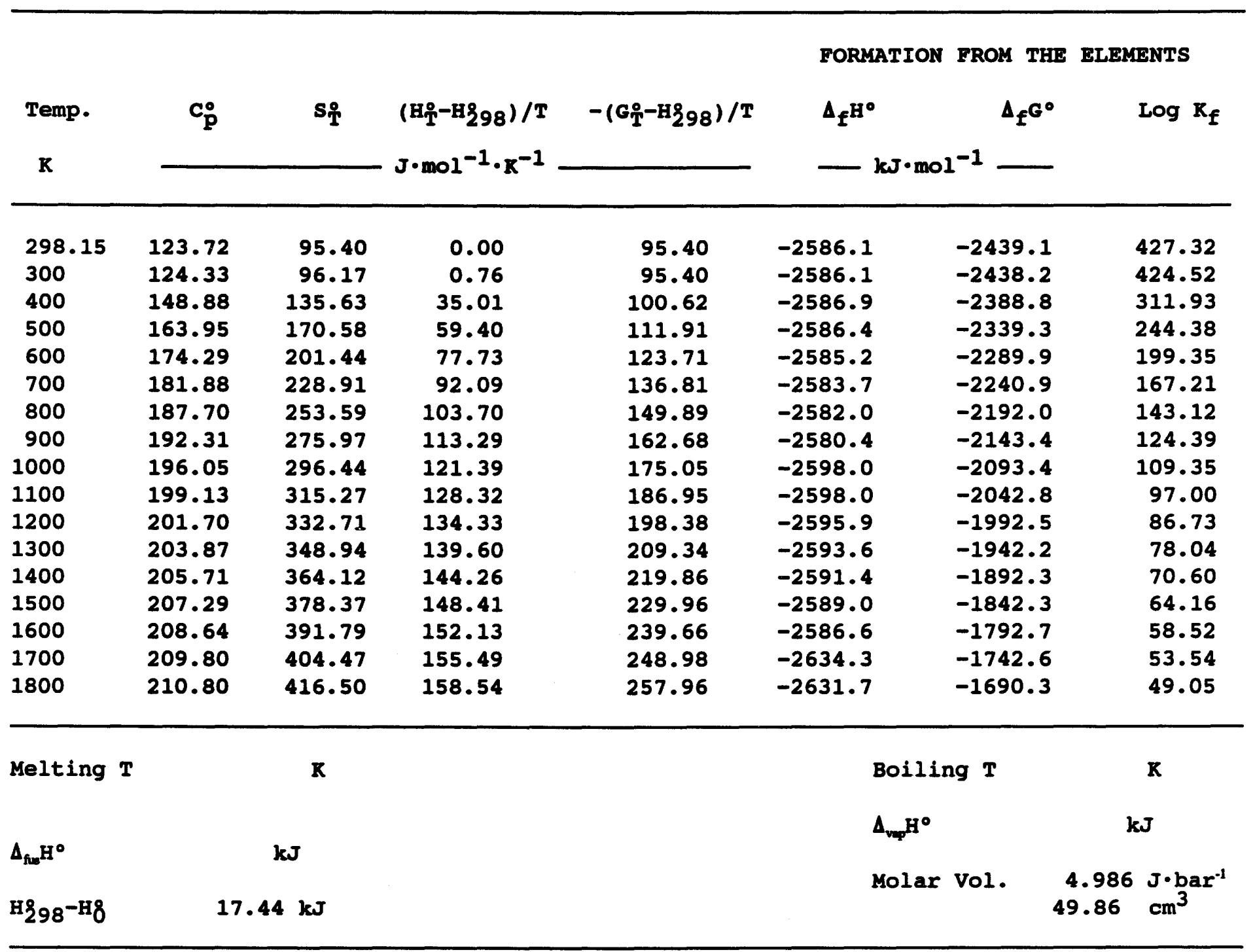


$\mathrm{Al}_{6} \mathrm{Si}_{2} \mathrm{O}_{13}$ : Orthorhombic crystals 298.15 to $1800 \mathrm{~K}$.

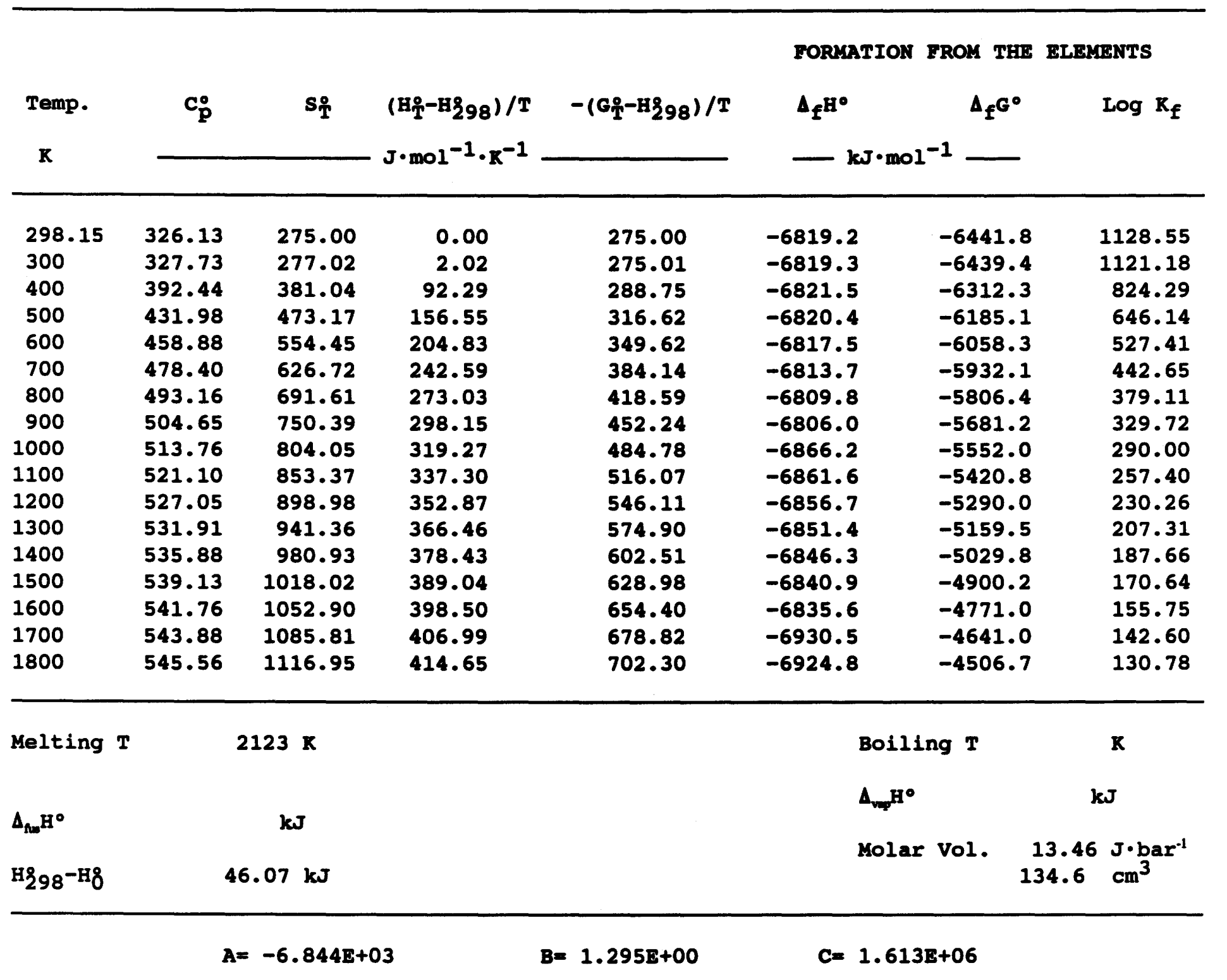


$\left[\mathrm{Al}_{6.75}\right][]_{.25} \mathrm{Si}_{3} \mathrm{BO}_{17.25}(\mathrm{OH}) .75:$ Orthorhombic crystals 298.15 to $1800 \mathrm{~K}$.

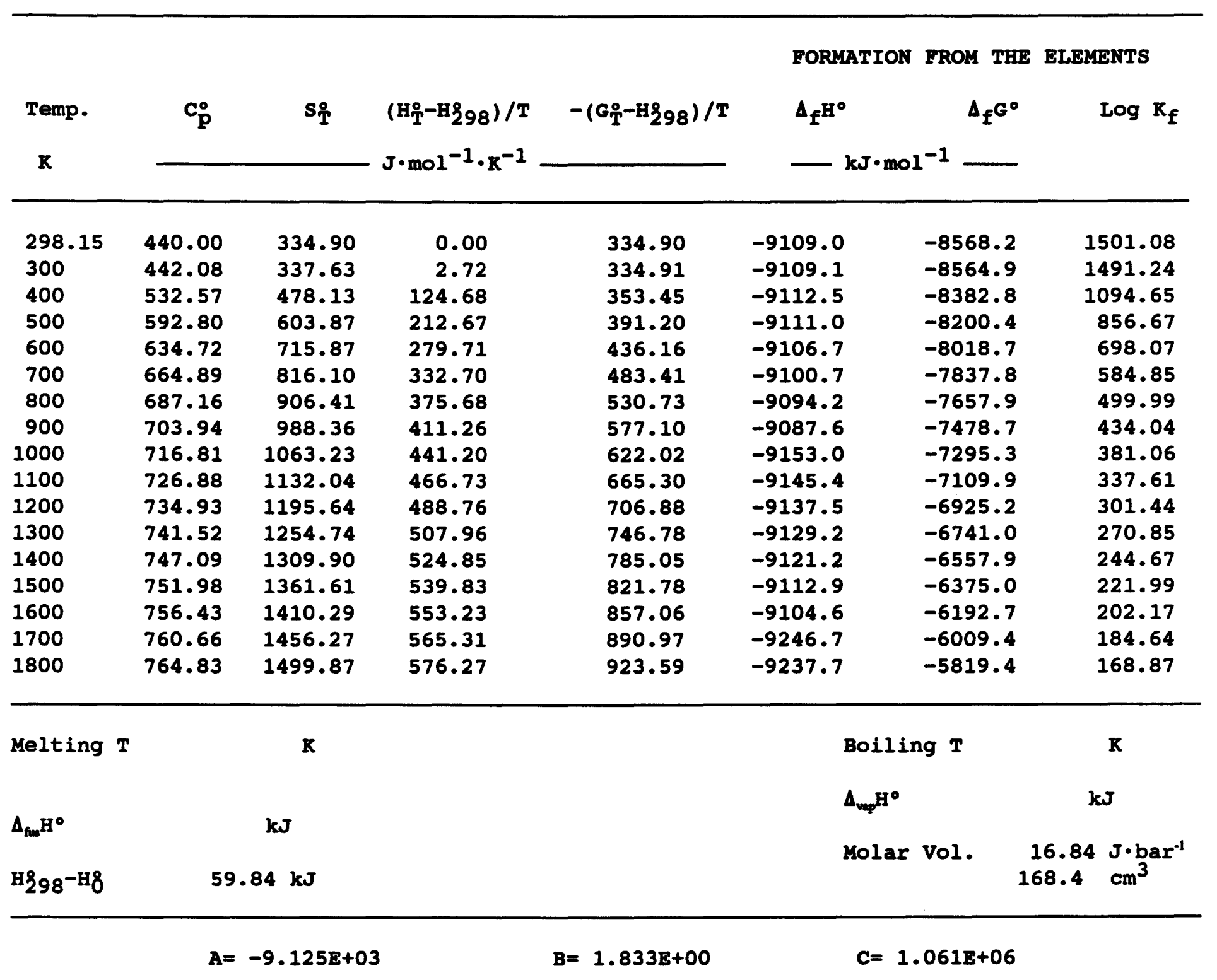


BeAlsio $_{4}(\mathrm{OH}):$ Crystals 298.15 to $1800 \mathrm{~K}$.

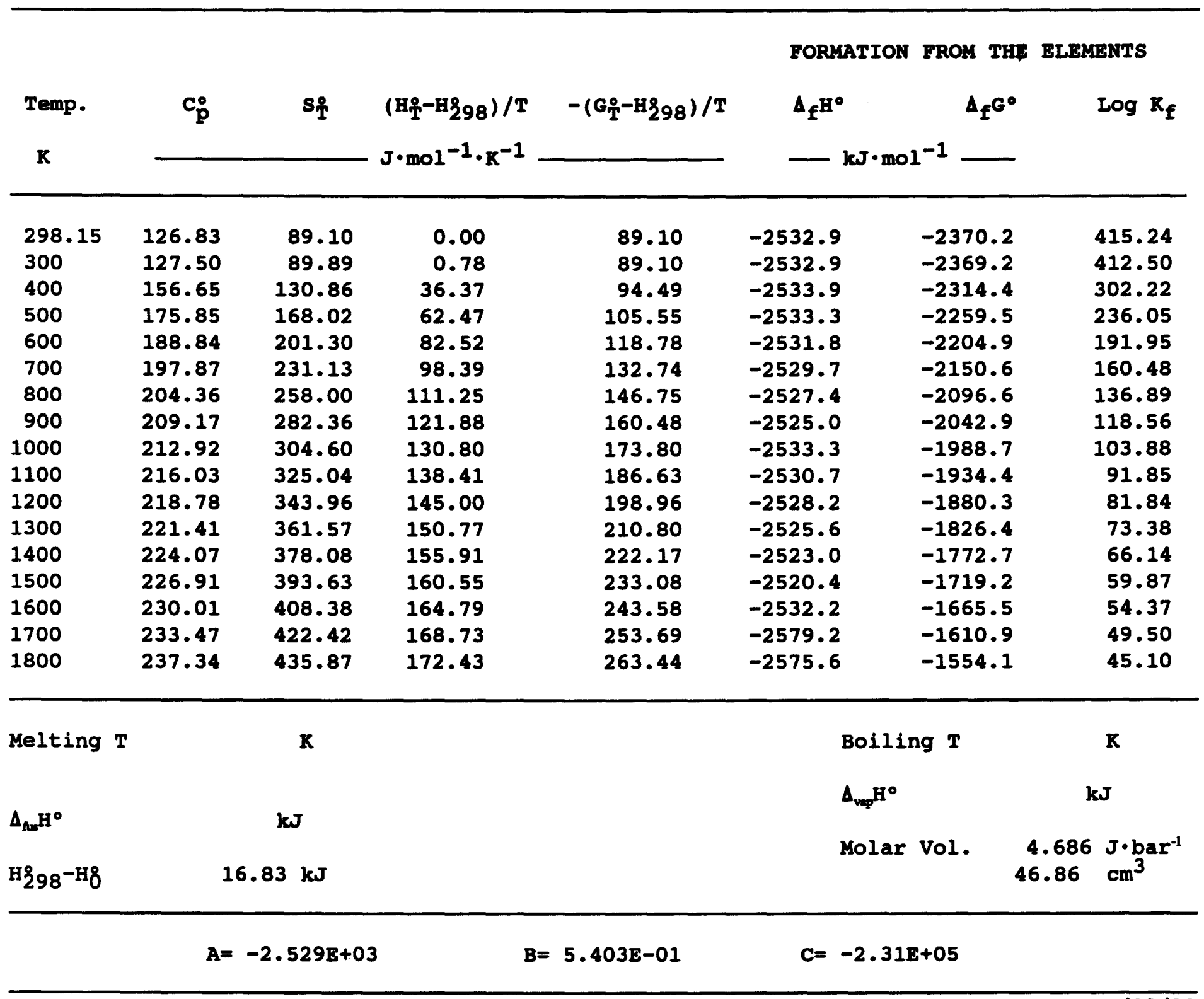


$\mathrm{Be}_{2} \mathrm{SiO}_{4}$ : Hexagonal crystals 298.15 to $1800 \mathrm{~K}$. Decomposes to BeO and $\mathrm{SiO}_{2}$ above $1833 \mathrm{~K}$.

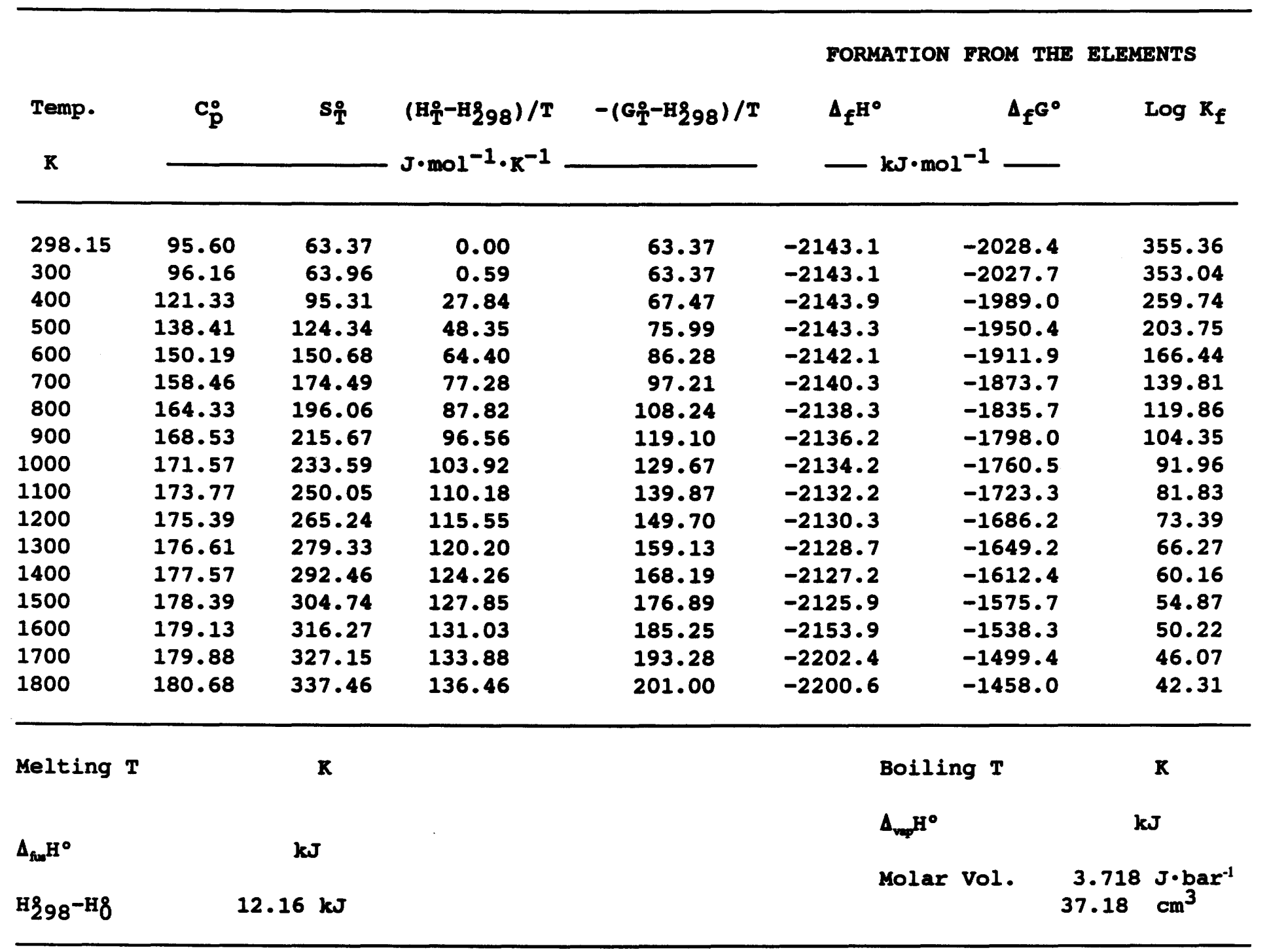


$\mathrm{Be}_{3} \mathrm{Al}_{2}\left(\mathrm{Si}_{6} \mathrm{O}_{18}\right):$ Hexagonal crystals 298.15 to $1800 \mathrm{~K}$.

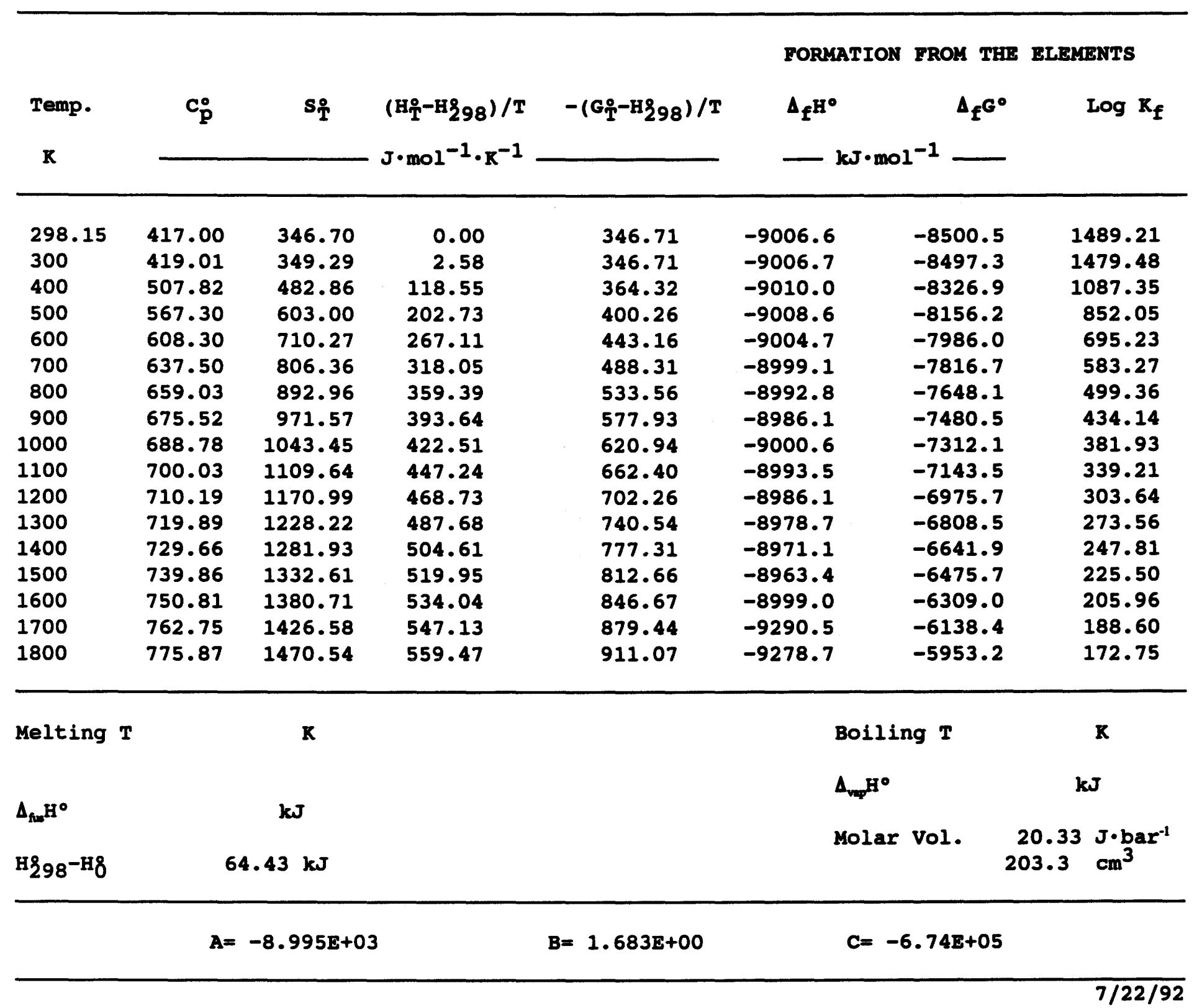


$\mathrm{Be}_{4} \mathrm{Si}_{2} \mathrm{O}_{7}(\mathrm{OH})_{2}$ : Crystals 298.15 to $1400 \mathrm{~K}$.

FORMATION FROM THE ELEMENTS

\begin{tabular}{|c|c|c|c|c|c|c|c|}
\hline $\begin{array}{l}\text { Temp. } \\
\mathrm{K}\end{array}$ & $\mathbf{c}_{\mathbf{p}}^{\circ}$ & sq & $\begin{array}{l}\left(\mathrm{H}_{\mathrm{T}}^{\mathrm{O}}-\mathrm{H} 298\right) / \mathrm{T} \\
\mathrm{J} \cdot \mathrm{mol}^{-1} \cdot \mathrm{K}^{-1}\end{array}$ & $-\left(G_{T}^{\circ}-H_{298}\right) / T$ & $\begin{array}{l}\Delta_{\mathbf{f}^{\mathrm{H}^{\circ}}} \\
-k\end{array}$ & $I^{-1}$ & $\log R_{f}$ \\
\hline 298.15 & 224.64 & 172.10 & 0.00 & 172.10 & -4580.5 & -4295.1 & 752.46 \\
\hline 300 & 225.83 & 173.49 & 1.39 & 172.10 & -4580.6 & -4293.3 & 747.51 \\
\hline 400 & 279.83 & 246.33 & 64.67 & 181.67 & -4582.9 & -4197.1 & 548.07 \\
\hline 500 & 317.42 & 313.06 & 111.68 & 201.38 & -4582.4 & -4100.6 & 428.38 \\
\hline 600 & 344.16 & 373.43 & 148.32 & 225.11 & -4580.2 & -4004.5 & 348.61 \\
\hline 700 & 363.51 & 428.01 & 177.75 & 250.26 & -4576.7 & -3908.8 & 291.67 \\
\hline 800 & 377.60 & 477.52 & 201.90 & 275.63 & -4572.5 & -3813.6 & 249.00 \\
\hline 900 & 387.80 & 522.62 & 222.02 & 300.60 & -4568.1 & -3719.0 & 215.84 \\
\hline 1000 & 395.06 & 563.88 & 238.98 & 324.90 & -4563.5 & -3624.9 & 189.34 \\
\hline 1100 & 400.01 & 601.78 & 253.41 & 348.37 & -4559.1 & -3531.3 & 167.68 \\
\hline 1200 & 403.13 & 636.73 & 265.77 & 370.96 & -4555.0 & -3438.0 & 149.65 \\
\hline 1300 & 404.76 & 669.07 & 276.41 & 392.67 & -4551.4 & -3345.1 & 134.41 \\
\hline 1400 & 405.16 & 699.09 & 285.60 & 413.49 & -4548.3 & -3252.4 & 121.35 \\
\hline
\end{tabular}

Melting $\mathbf{T}$

$\Delta_{\mathrm{fu}} \mathrm{H}^{\circ}$

H298-H8 $\mathbf{k J}$

$\mathbf{R}$

$31.04 \mathrm{~kJ}$
Boiling $T$

$\Delta_{\mathrm{veq}} \mathrm{H}^{\circ}$

Molar Vol.
$\mathbf{K}$

$k J$

$9.179 \mathrm{~J} \cdot \mathrm{bar}^{-1}$ $91.79 \mathrm{~cm}^{3}$
$A=-4.564 E+03$
$B=9.388 E-01$
$C=-1.03 E+06$ 
$\mathrm{CaAl}_{2} \mathrm{Si}_{2} \mathrm{O}_{7}(\mathrm{OH})_{2} \cdot \mathrm{H}_{2} \mathrm{O}$ : Crystals 298.15 to $600 \mathrm{~K}$.

\begin{tabular}{|c|c|c|c|c|c|c|c|}
\hline \multirow{3}{*}{$\begin{array}{l}\text { Temp. } \\
\mathbf{R}\end{array}$} & \multirow{3}{*}{$\mathbf{c}_{\mathbf{p}}^{\circ}$} & \multirow{3}{*}{$\mathbf{s}_{\text {i }}$} & \multirow{3}{*}{$\begin{array}{l}\left(\mathrm{H}_{\mathrm{T}}^{\mathrm{O}}-\mathrm{H}_{298}\right) / \mathrm{T} \\
\mathrm{J} \cdot \mathrm{mol}^{-1} \cdot \mathrm{K}^{-1}\end{array}$} & \multirow{3}{*}{$-\left(G_{T}^{\circ}-H_{298}\right) / T$} & \multicolumn{2}{|c|}{ FORMATION FROM THE } & \multirow{3}{*}{$\begin{array}{l}\text { ELEMENTS } \\
\qquad \log R_{f}\end{array}$} \\
\hline & & & & & \multirow{2}{*}{\multicolumn{2}{|c|}{$\begin{array}{l}\Delta_{\mathrm{f}^{\mathrm{H}^{\circ}}} \\
-\mathrm{kJ} \cdot \mathrm{mol}^{-1}\end{array}$}} & \\
\hline & & & & & & & \\
\hline 298.15 & 275.24 & 230.00 & 0.00 & 230.00 & -4869.0 & -4512.9 & 790.63 \\
\hline 300 & 276.56 & 231.71 & 1.70 & 230.01 & -4869.1 & -4510.7 & 785.37 \\
\hline 400 & 331.03 & 319.37 & 77.78 & 241.59 & -4871.0 & -4390.9 & 573.38 \\
\hline 500 & 368.81 & 397.47 & 132.37 & 265.10 & -4869.7 & -4270.9 & 446.17 \\
\hline 600 & 400.03 & 467.54 & 174.43 & 293.10 & -4866.0 & -4151.5 & 361.41 \\
\hline
\end{tabular}

Melting $T$

$\Delta_{\text {now }} \mathrm{H}^{\circ}$

H298-H8 $\mathbf{k J}$

R

$41.36 \mathrm{~kJ}$
Boiling $T$

$\Delta_{\mathrm{vep}} \mathrm{H}^{\circ}$

Molar Vol. 10.132 101.32
$\mathbf{R}$

$k \boldsymbol{J}$ 
$\mathrm{Ca}_{2} \mathrm{Al}_{2} \mathrm{SiO}_{7}$ : Tetragonal crystals 298.15 to incongruent melting point at $1866 \mathrm{~K}$

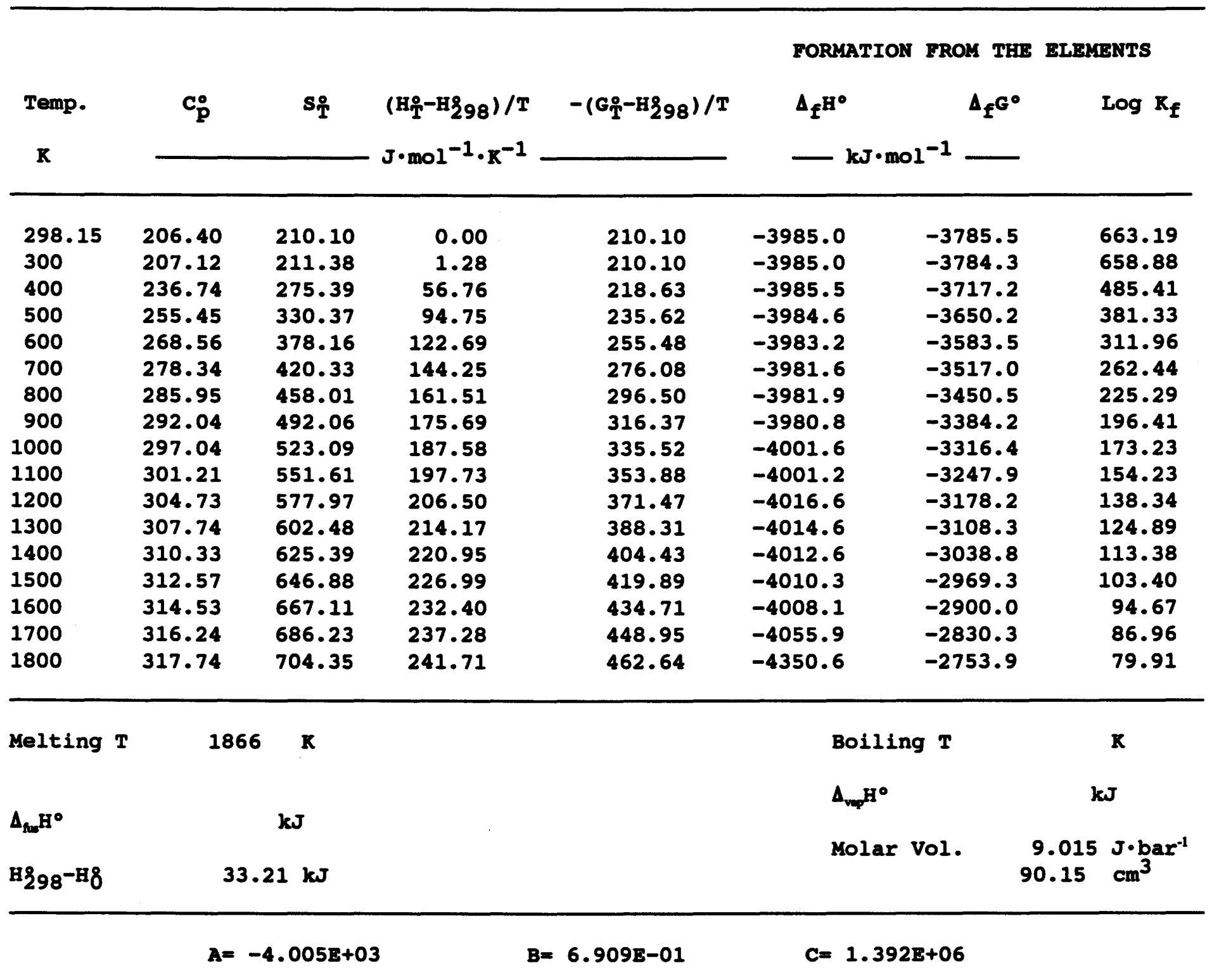


$\mathrm{Ca}_{2} \mathrm{Al}_{3} \mathrm{Si}_{3} \mathrm{O}_{12}(\mathrm{OH}):$ Orthorhombic crystals 298.15 to $1200 \mathrm{~K}$.

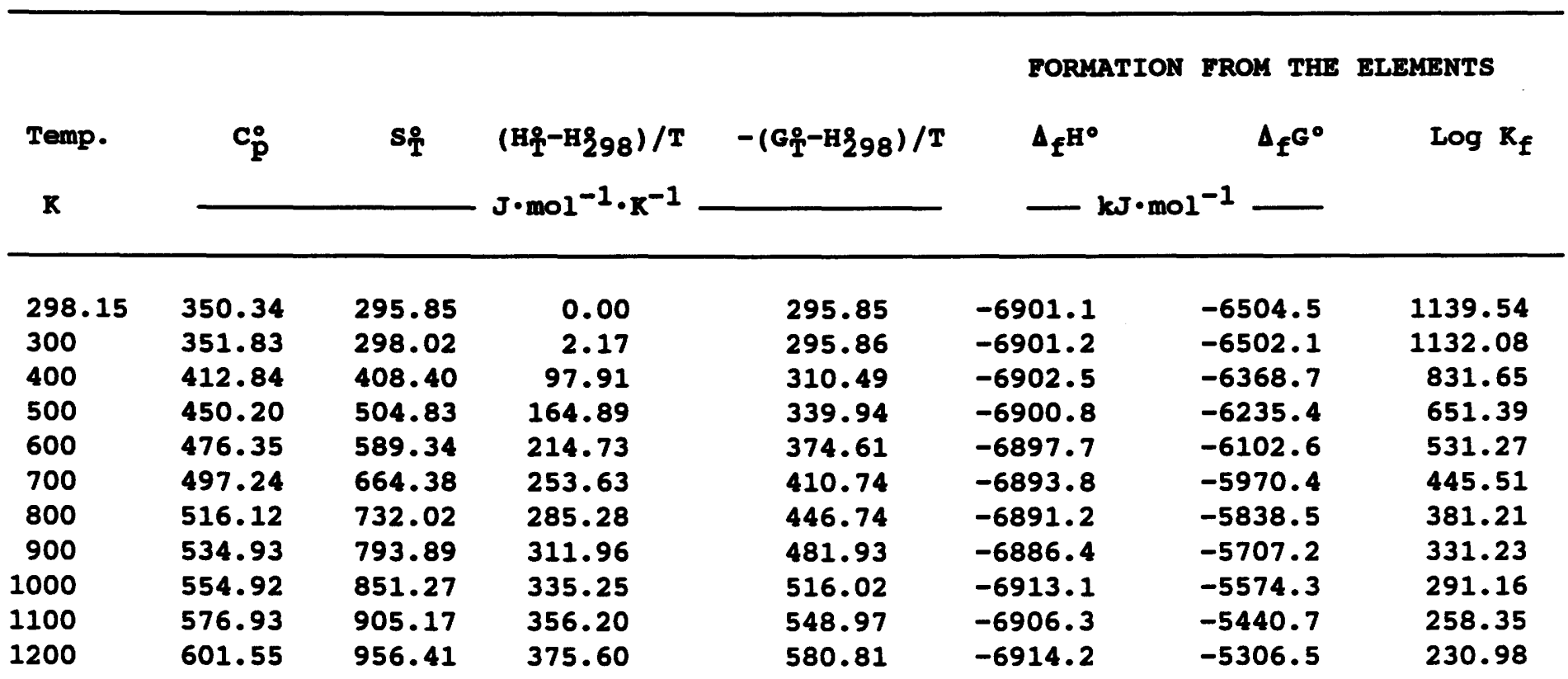

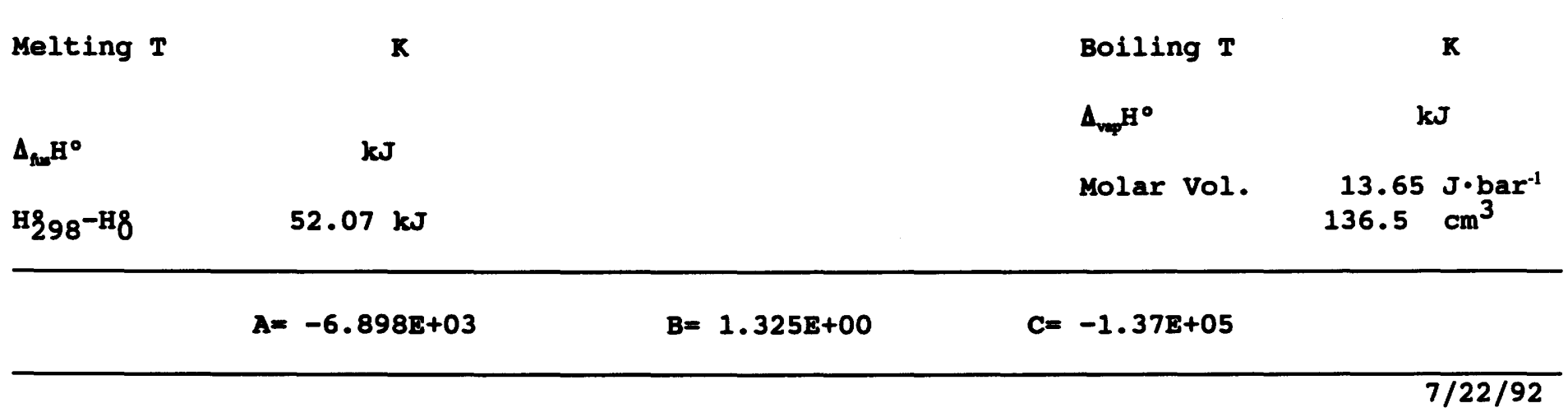


$\mathrm{Ca}_{3} \mathrm{Al}_{2} \mathrm{Si}_{3} \mathrm{O}_{12}:$ Cubic crystals 298.15 to $1200 \mathrm{~K}$.

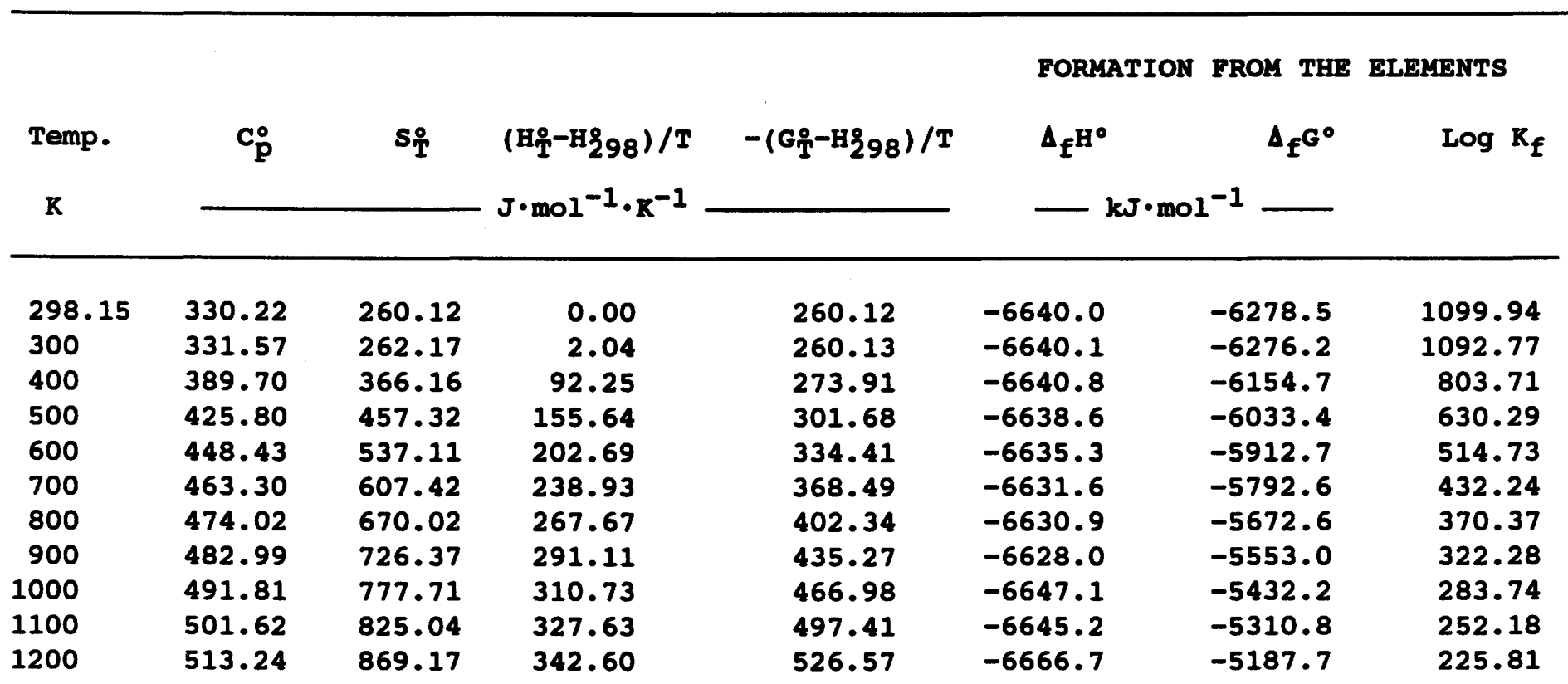

Melting $\mathbf{T}$

$\Delta_{\text {fur }} \mathrm{H}^{\circ}$

H298-H8 $\mathbf{k J}$

$\mathbf{K}$ $47.66 \mathrm{~kJ}$
Boiling $T$

$\Lambda_{\mathrm{vap}} \mathrm{H}^{\circ}$

$\mathbf{k J}$

Molar Vol. $\quad 12.528 \mathrm{~J} \cdot \mathrm{bar}^{-1}$ $125.28 \mathrm{~cm}^{3}$
$A=-6.637 \mathrm{E}+03$
$B=1 \cdot 206 E+00$
$C=-1 \cdot 07 E+05$ 
CaB(Si 4 )OH: Crystals 298.15 to $1000 \mathrm{~K}$. Datolite decomposes in air at about $970 \mathrm{~K}$.

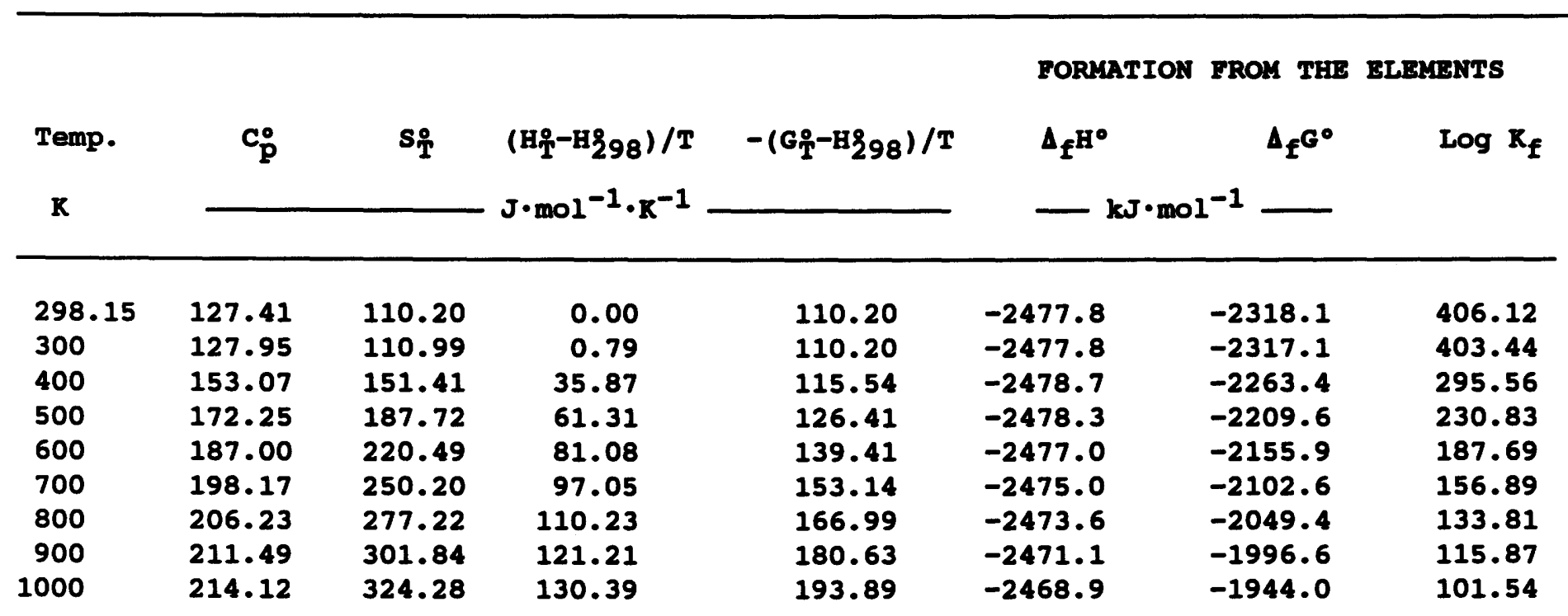

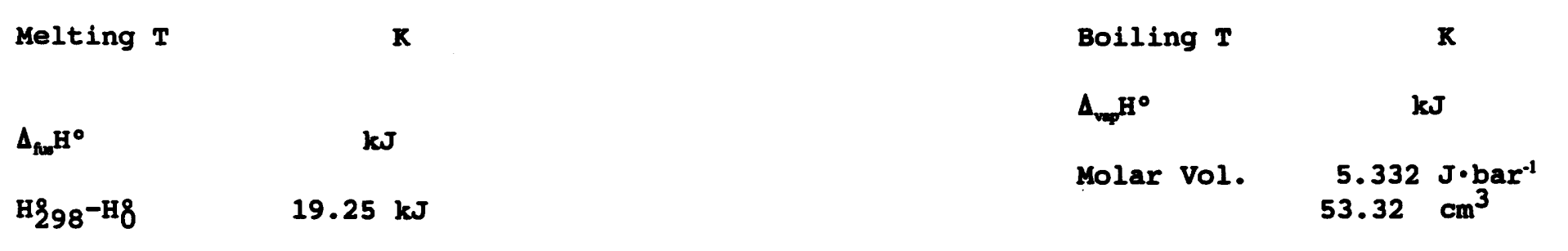
$A=-2.473 E+03$
$B=5.301 \mathrm{E}-01$
$C=-2.53 \mathrm{E}+05$ 
$\mathrm{CaFe}_{3} \mathrm{Si}_{2} \mathrm{O}_{7} \mathrm{O}(\mathrm{OH})$ : Monoclinic crystals 298.15 to $900 \mathrm{~K}$.

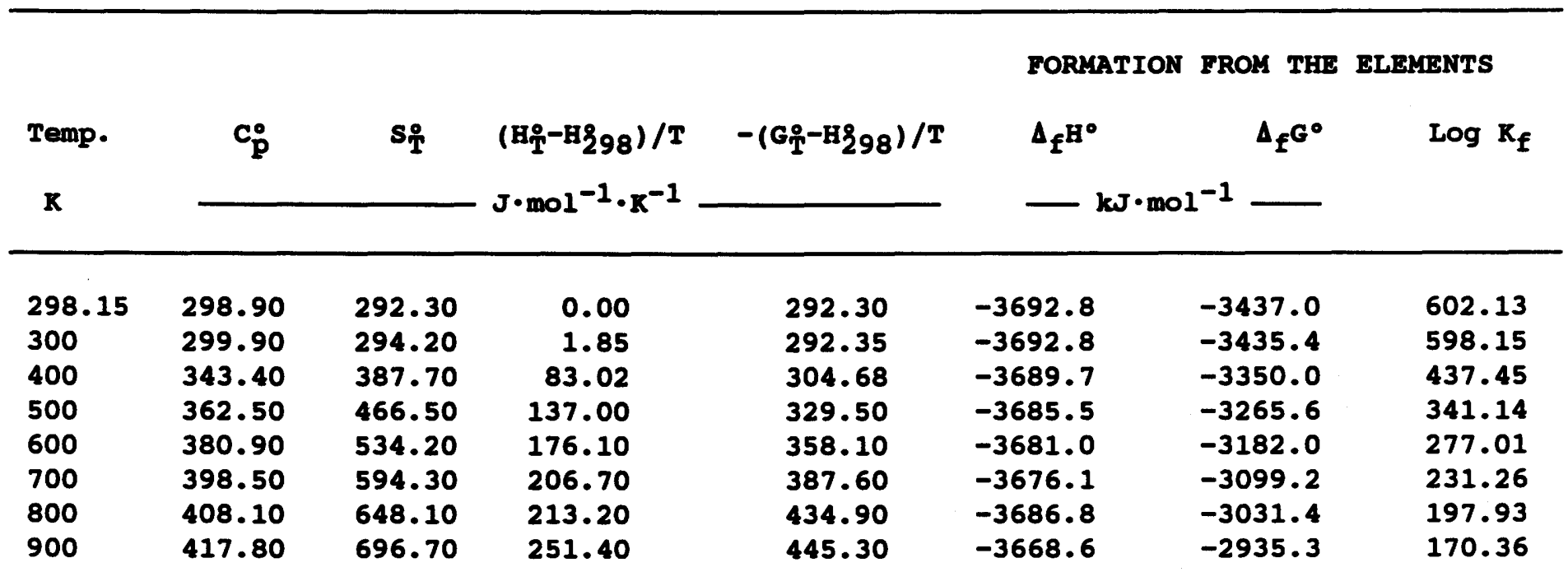

Melting $\mathbf{T}$

$\mathbf{R}$

Boiling $\mathbf{T}$

$\mathbf{R}$

$\Delta_{\text {fud }} \mathbf{H}^{\circ}$

$\mathbf{k J}$

$\Delta_{\mathrm{veq}} \mathrm{H}^{\circ}$

$\mathbf{k J}$

H298-H8

$48.09 \mathrm{~kJ}$

Molar Vol. $\begin{gathered}10.107 \mathrm{~J} \cdot \mathrm{bar}^{-1} \\ 101.07 \mathrm{~cm}^{3}\end{gathered}$

$A=-3.664 E+03$

$B=8.052 \mathrm{E}-01$

$C=-1.208+06$ 


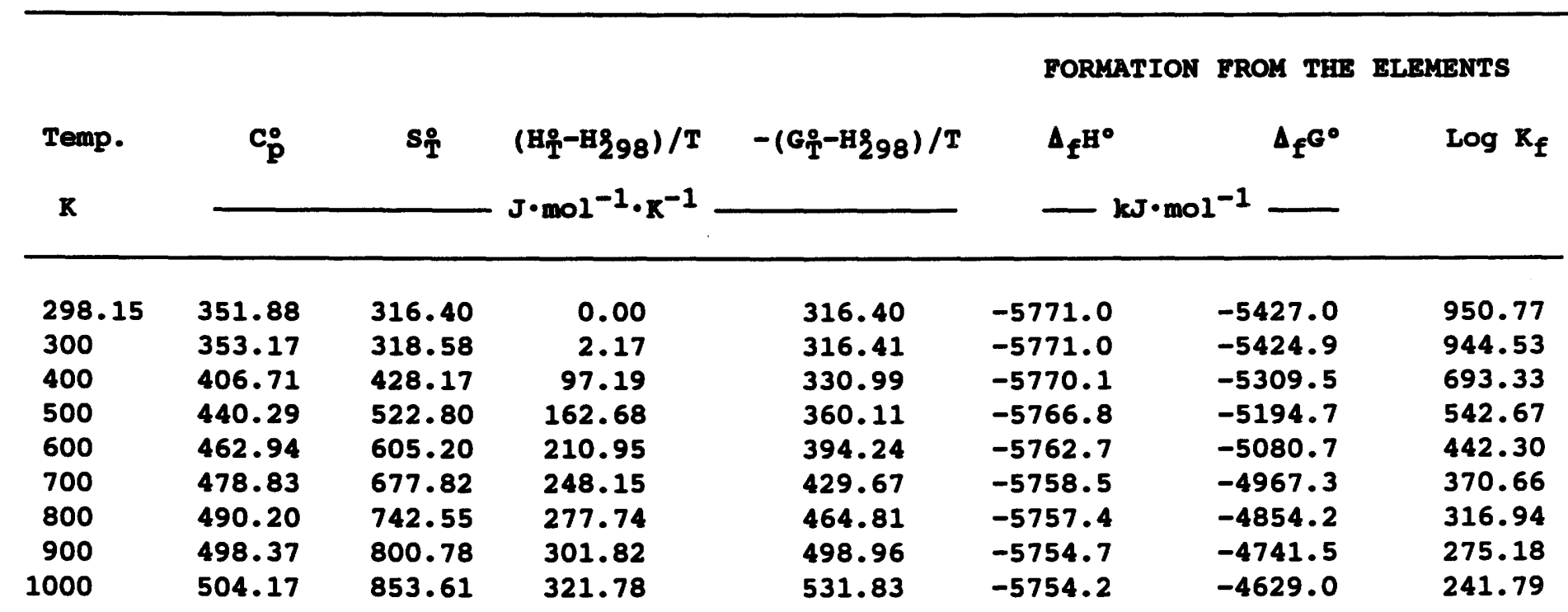

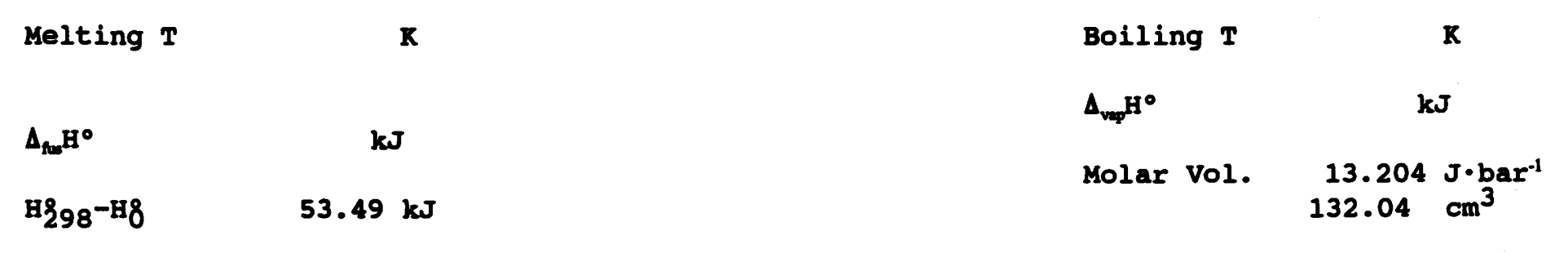

$A=-5.756 \mathrm{E}+03 \quad \mathrm{~B}=1.128 \mathrm{E}+00 \quad \mathrm{C}=-6.85 \mathrm{E}+05$


CaMgsio $_{4}$ : Orthorhombic crystals 298.15 to $1100 \mathrm{~K}$.

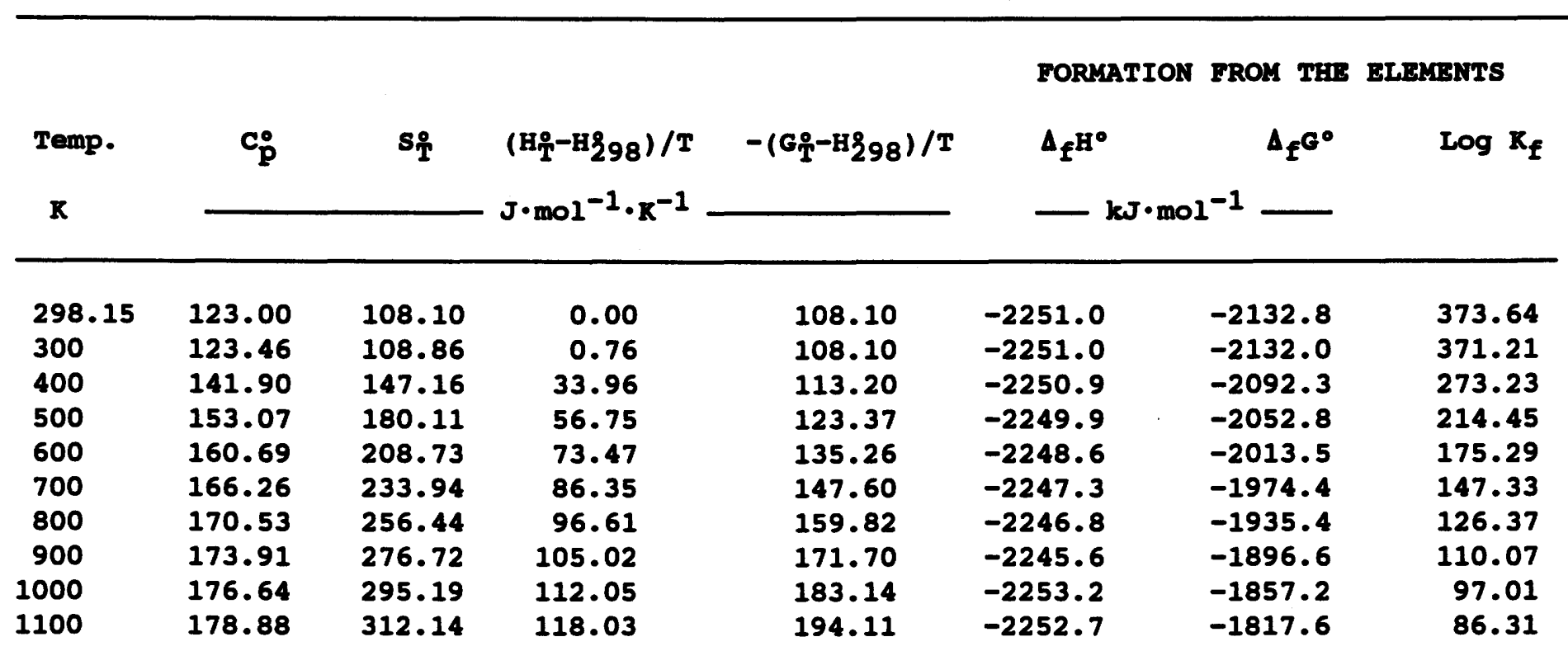

Melting $\mathbf{T}$

X

$\mathbf{w}$

$\Delta_{\operatorname{sun}} \mathrm{H}^{\circ}$

H298-H8
Boiling $T$

$\Delta_{\mathrm{vap}} \mathrm{H}^{\circ}$

Molar vol.
$B=3.910 \mathrm{E}-01$
$C=-1.25 \mathrm{E}+05$
$A=-2 \cdot 248 \mathrm{E}+03$

(

$5.13 \mathrm{~J} \cdot$ bar $^{-1}$ $51.3 \mathrm{~cm}^{3}$ 
$\mathrm{Ca}_{2} \mathrm{MgSi}_{2} \mathrm{O}_{7}$ : Tetragonal crystals 298.15 to melting point at $1731 \mathrm{~K}$. A small transition at $357.9 \mathrm{~K}$ has an enthalpy of $0.68 \mathrm{~kJ}$.

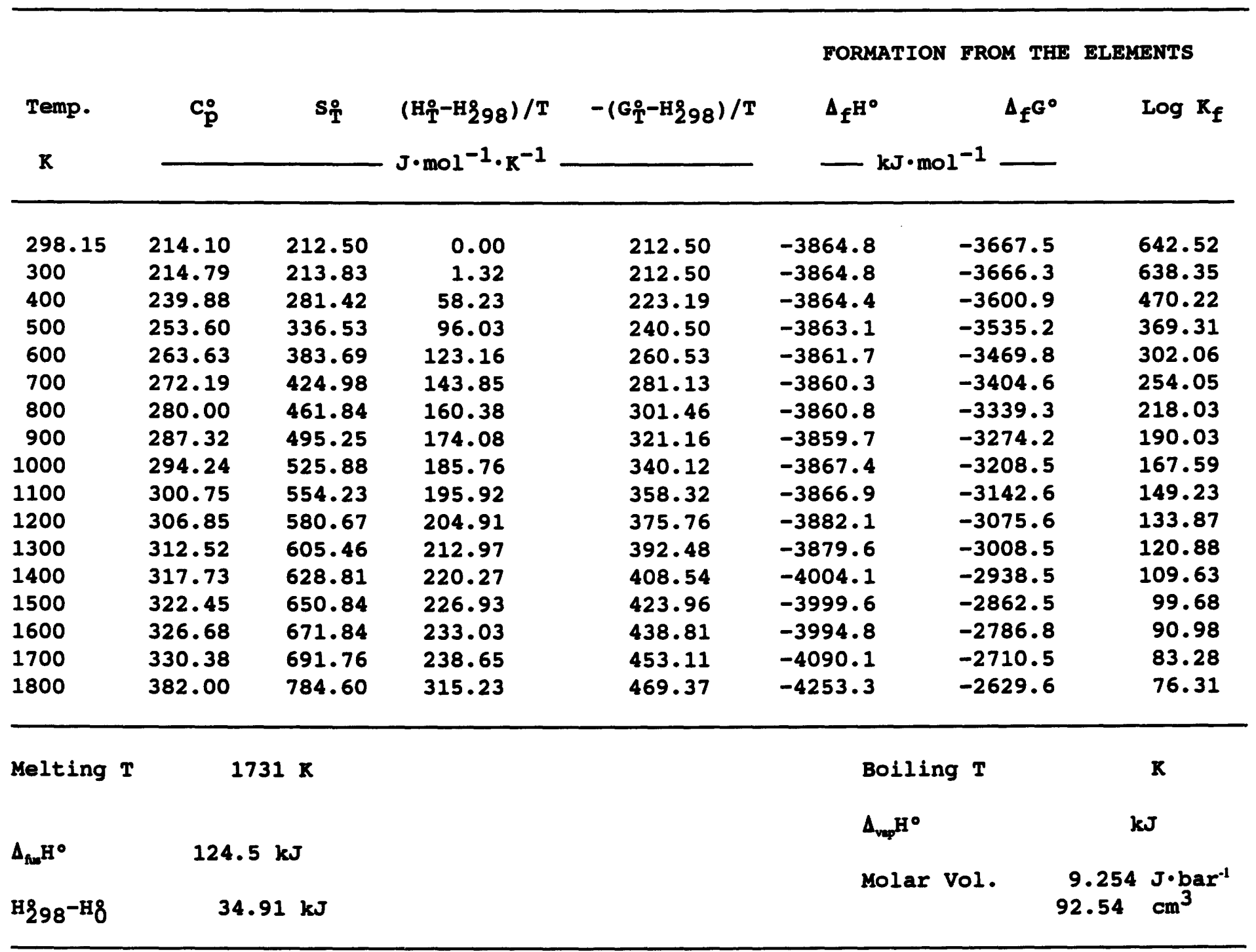


$\mathrm{Ca}_{3} \mathrm{Mg}\left(\mathrm{SiO}_{4}\right)_{2}:$ Monoclinic crystals 298.15 to $1600 \mathrm{~K}$.

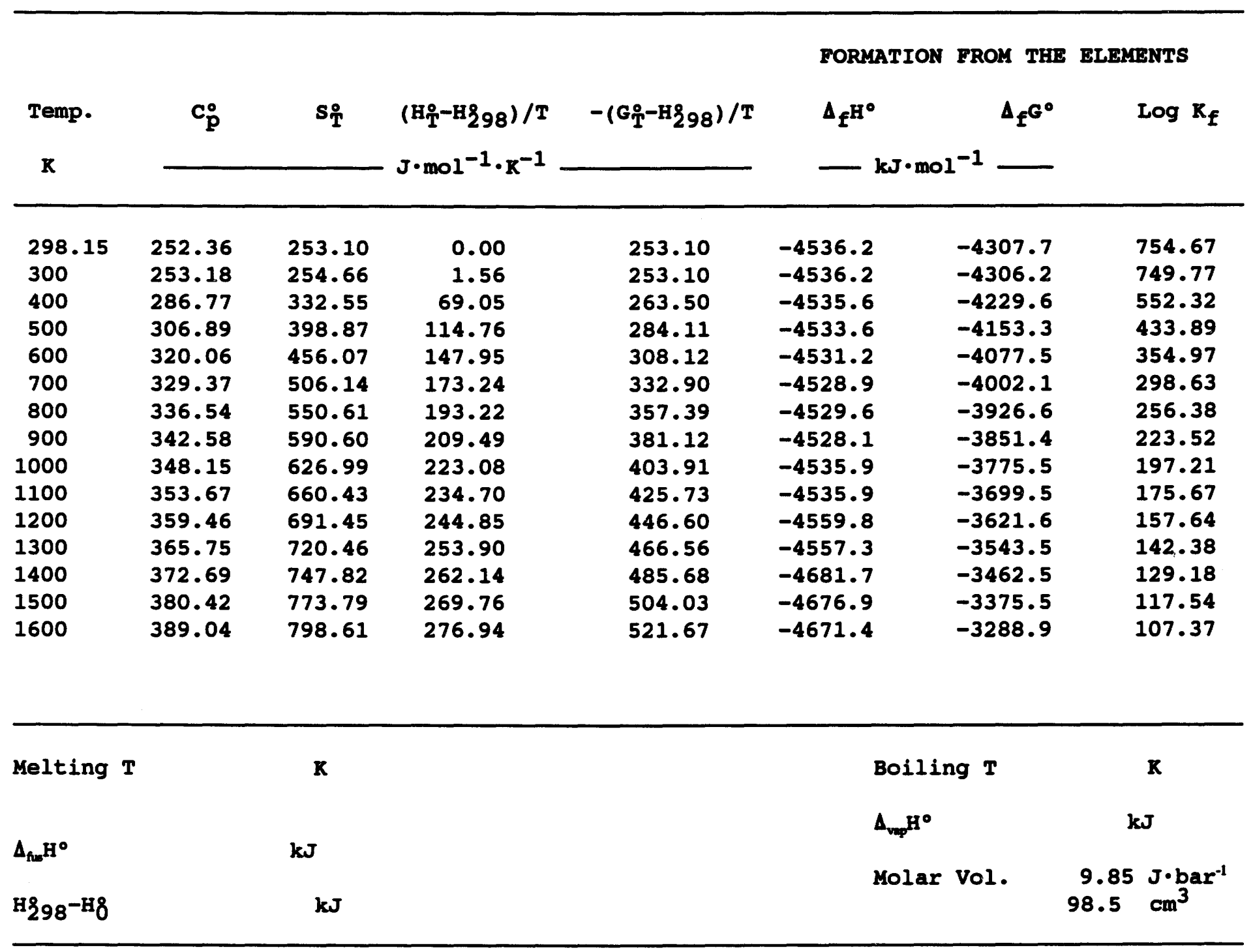
$A=-4.557 \mathrm{E}+03$
$B=7.840 \mathrm{E}-01$
$C=1.573 \mathrm{E}+06$ 
CaTisio : Monoclinic crystals 298.15 to $1670 \mathrm{~K}$.

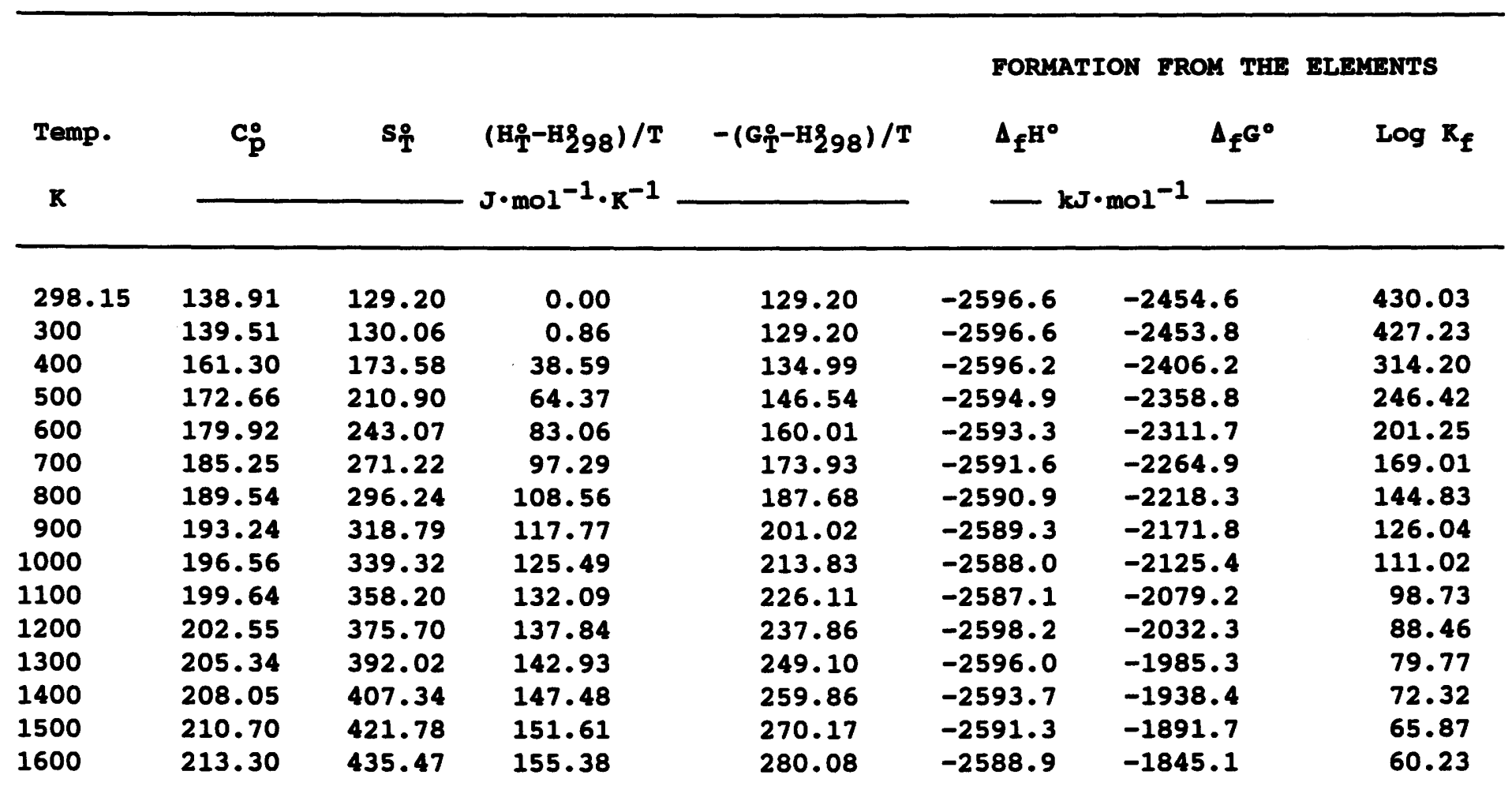

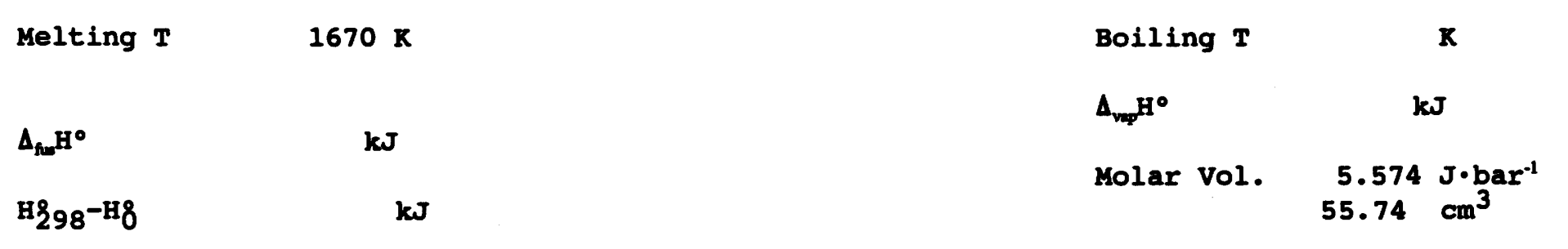

$A=-2.591 \mathrm{E}+03 \quad \mathrm{~B}=4.661 \mathrm{E}-01 \quad \mathrm{C}=-2.27 \mathrm{E}+05$


$\alpha-\mathrm{Ca}_{2} \mathrm{SiO}_{4}$ : Monoclinic crystals 298.15 to $1000 \mathrm{~K}$. Bredigite ( $\left.\alpha^{\prime}\right)$ is the stable modification of $\mathrm{Ca}_{2} \mathrm{SiO}_{4}$ between 970 and $1710 \mathrm{~K}$. $\alpha$-crystals 1710 to $1800 \mathrm{~K}$.

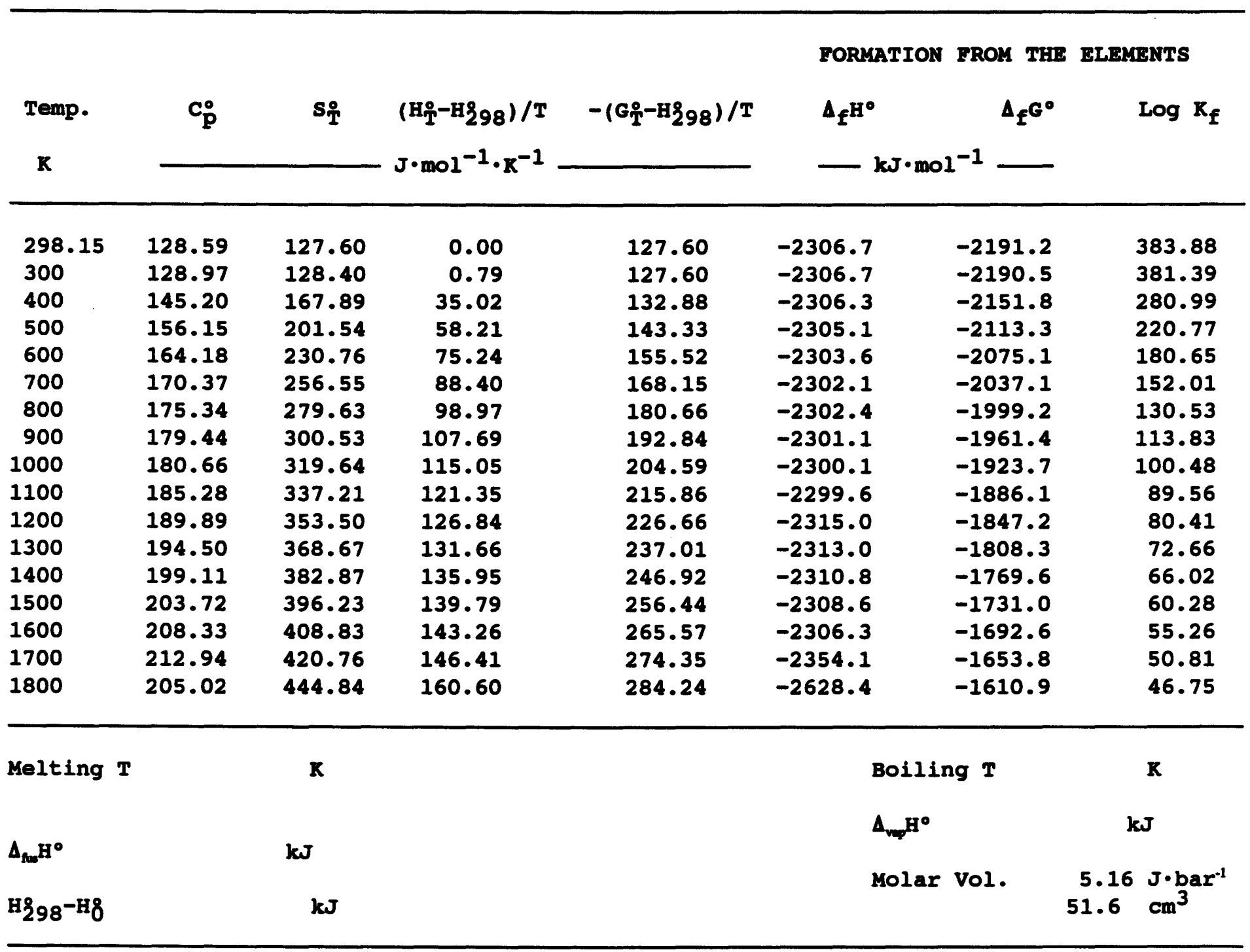
$A=-2 \cdot 308 E+03$
$B=3.850 \mathrm{E}-01$
$C=2.243 \mathrm{E}+05$ 
$\gamma-\mathrm{Ca}_{2} \mathrm{SiO}_{4}$ : Orthorhombic crystals 298.15 to $1200 \mathrm{~K}$.

FORMATION PROM THE ELEMENTS

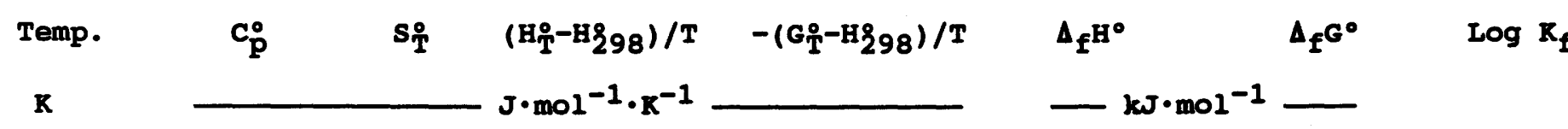

\begin{tabular}{rrrrrrrr}
\hline 298.15 & 126.93 & 120.50 & 0.00 & 120.50 & -2316.5 & -2198.9 & 385.23 \\
300 & 127.29 & 121.29 & 0.78 & 120.50 & -2316.5 & -2198.2 & 382.73 \\
400 & 141.80 & 160.08 & 34.39 & 125.69 & -2316.3 & -2158.7 & 281.90 \\
500 & 151.34 & 192.80 & 56.87 & 135.93 & -2315.5 & -2119.4 & 221.41 \\
600 & 158.91 & 221.08 & 73.26 & 147.82 & -2314.6 & -2080.3 & 181.10 \\
700 & 165.57 & 246.09 & 85.98 & 160.10 & -2313.6 & -2041.3 & 152.32 \\
800 & 171.73 & 268.60 & 96.32 & 172.28 & -2314.3 & -2002.3 & 130.73 \\
900 & 177.61 & 289.17 & 105.03 & 184.14 & -2313.3 & -1963.3 & 113.95 \\
1000 & 183.31 & 308.18 & 112.57 & 195.61 & -2312.4 & -1924.5 & 100.52 \\
1100 & 188.89 & 325.91 & 119.26 & 206.66 & -2311.7 & -1885.7 & 89.54 \\
1200 & 194.39 & 342.58 & 125.29 & 217.30 & -2326.7 & -1845.8 & 80.34
\end{tabular}

Melting $T$

$\mathbf{K}$

$\Delta_{\text {fum }} \mathrm{H}^{\circ}$

H298-H8 $\mathbf{k J}$

$\mathbf{k J}$
Boiling T

$A_{\text {veq }} \mathrm{H}^{\circ}$

Molar vol.
$\mathbf{K}$

kJ

\section{$A=-2 \cdot 314 \mathrm{E}+03$}

$B=3.898 \mathrm{E}-01$ 
$\mathrm{Ca}_{3} \mathrm{SiO}_{5}:$ Triclinic crystals 298.15 to $1800 \mathrm{~K}$.

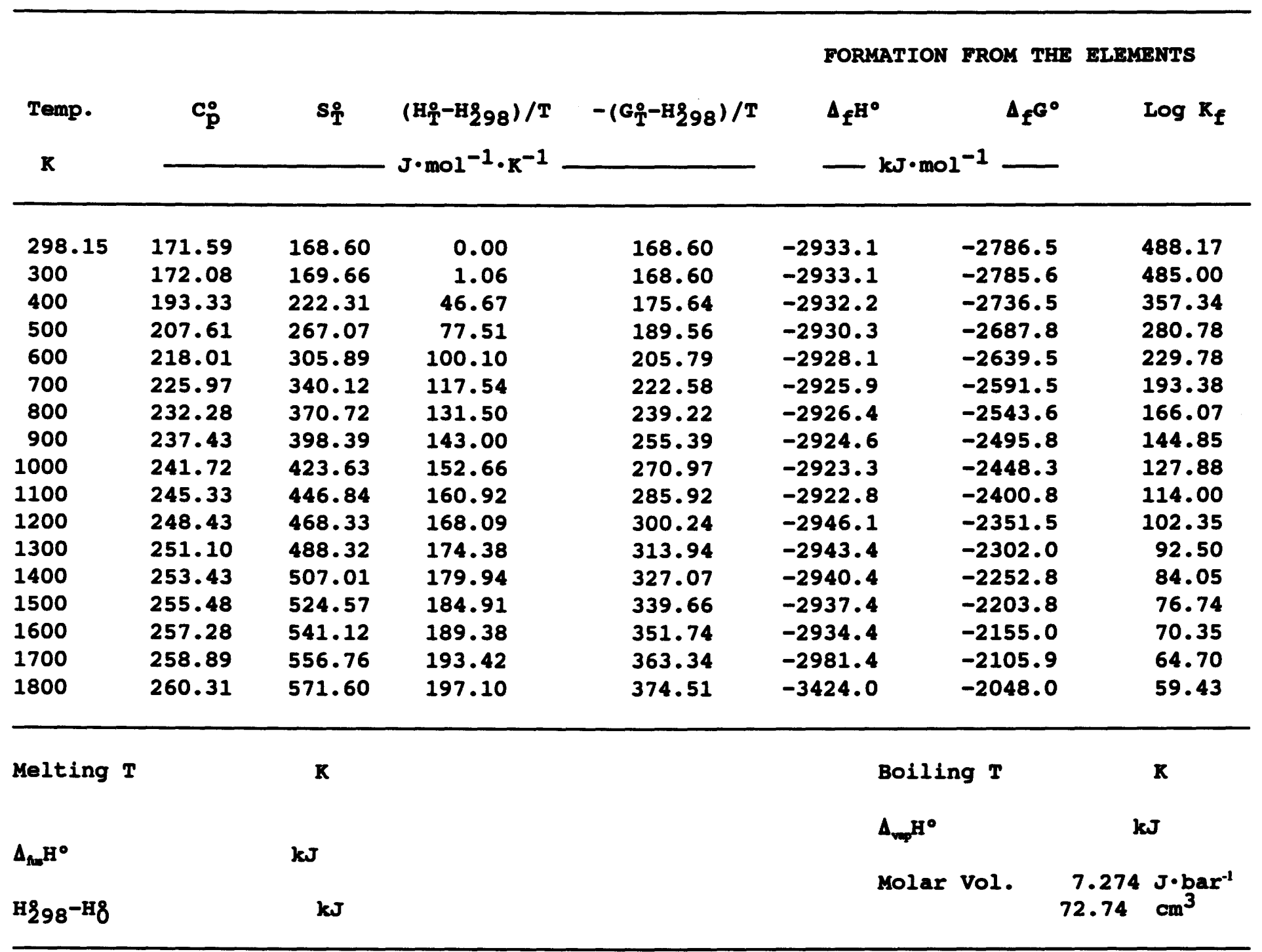


$\mathrm{Ca}_{3} \mathrm{Si}_{2} \mathrm{O}_{7}$ : Monoclinic crystals 298.15 to $1400 \mathrm{~K}$.

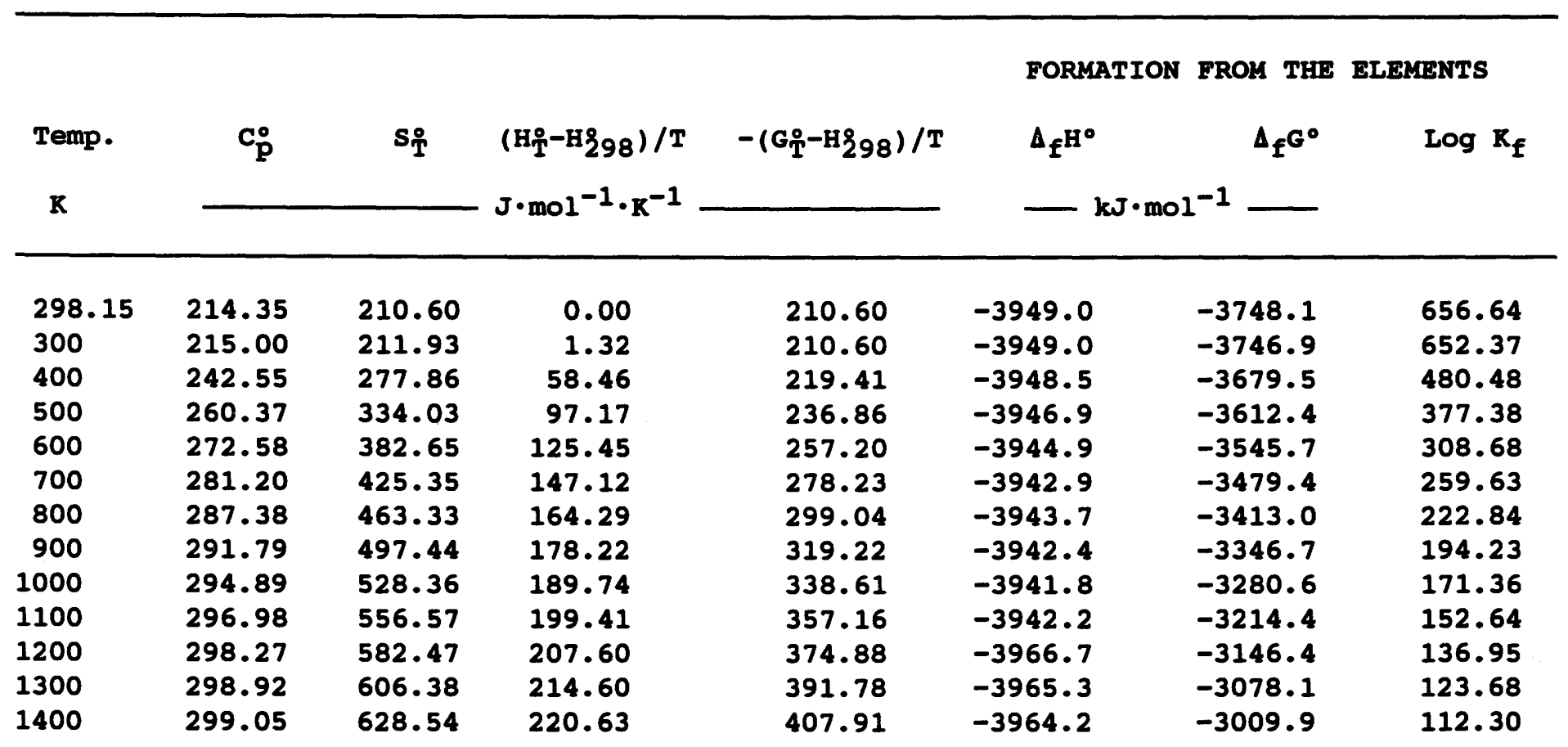

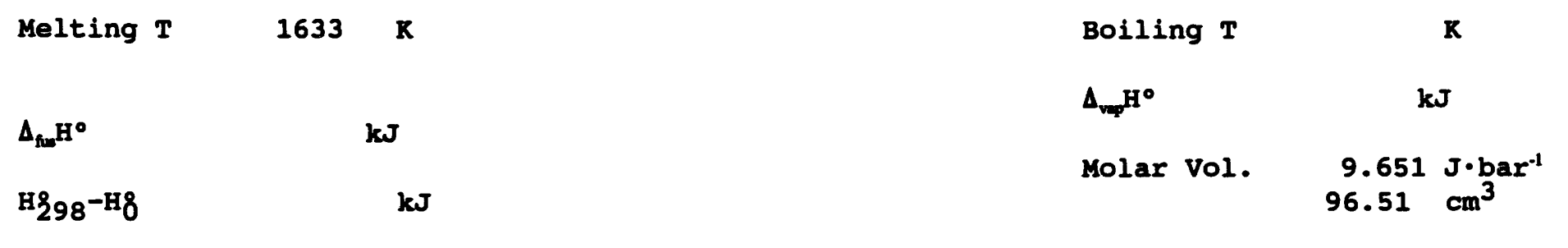
$A=-3.948 E+03$
$B=6.684 \mathrm{E}-01$
$C=5.979 \mathrm{E}+04$ 
$\mathrm{Ca}_{3} \mathrm{Si}_{3} \mathrm{O}_{8}(\mathrm{OH})_{2}:$ Triclinic crystals 298.15 to $1800 \mathrm{~K}$.

FORMATION FROM THE ELEMENTS

\begin{tabular}{|c|c|c|c|c|c|c|c|}
\hline Temp. & $\mathbf{c}_{\mathbf{p}}^{\circ}$ & $\mathbf{s}_{\mathbf{T}}^{\circ}$ & $\left(\mathrm{H}_{\mathrm{T}}-\mathrm{H}_{298}\right) / \mathrm{T}$ & $-\left(G_{T}^{\circ}-H_{298}\right) / T$ & $\Delta_{f^{H^{\circ}}}$ & $\Delta_{f} G^{0}$ & $\log R_{f}$ \\
\hline
\end{tabular}

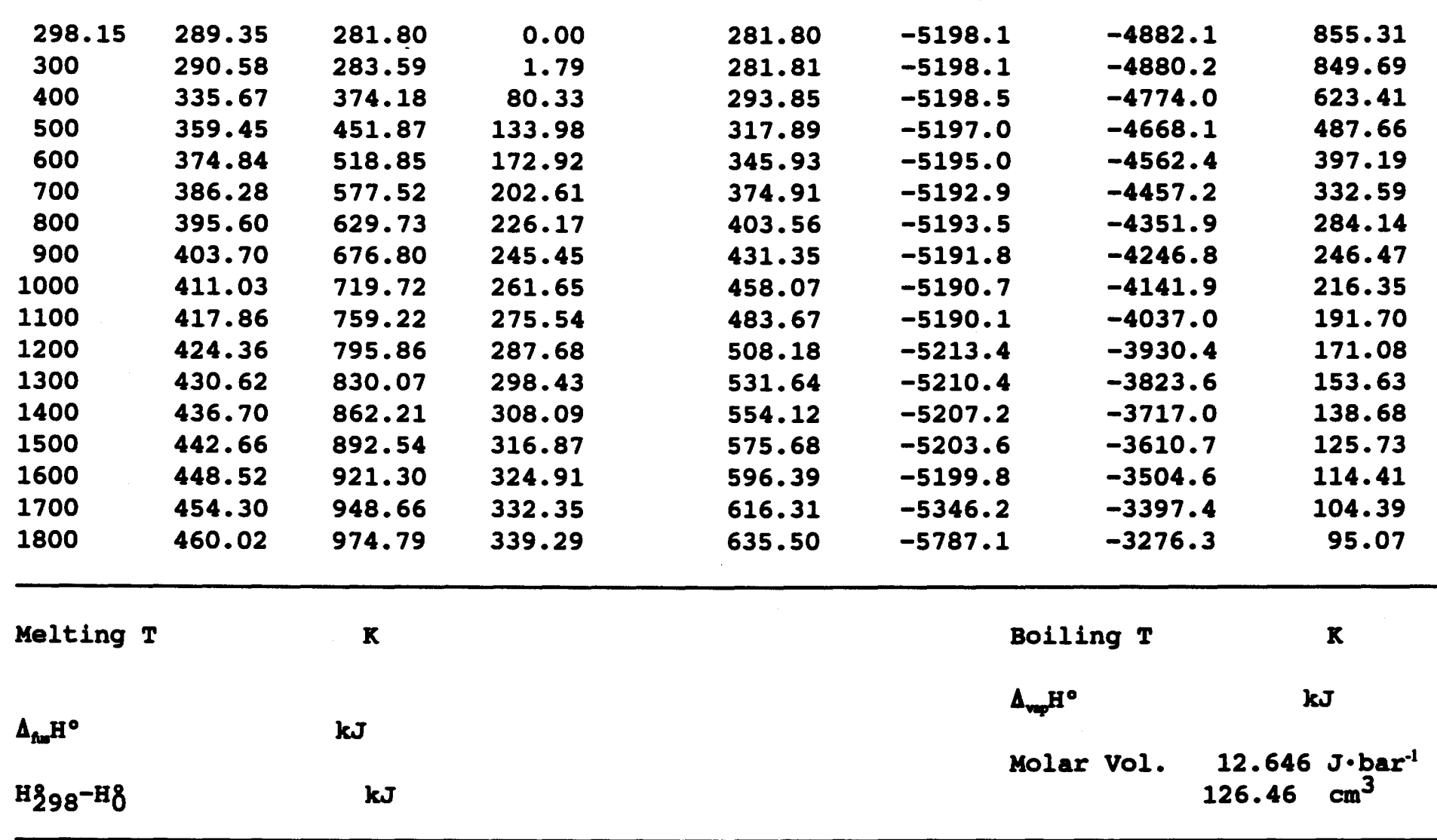


$\mathrm{Ca}_{5}\left(\mathrm{SiO}_{4}\right)_{2} \mathrm{CO}_{3}$ : Crystals 298.15 to $1300 \mathrm{~K}$.

FORMATION FROY THE ELEMENTS

\begin{tabular}{|c|c|c|c|c|}
\hline Temp. & $\mathbf{c}_{\mathbf{p}}^{0}$ & $\mathbf{s}_{\mathbf{T}}$ & (Ho⿱中⿰㇀丶㇀ & $-\left(G_{T}^{0}-\mathrm{H}_{298}\right) / T$ \\
\hline
\end{tabular}

\begin{tabular}{rrrrrrrrr}
\hline 298.15 & 343.62 & 331.00 & 0.00 & 331.00 & -5840.2 & -5525.6 & 968.04 \\
300 & 344.71 & 333.13 & 2.12 & 331.01 & -5840.2 & -5523.6 & 961.73 \\
400 & 388.72 & 438.91 & 93.78 & 345.13 & -5838.1 & -5418.4 & 707.55 \\
500 & 415.98 & 528.78 & 155.67 & 373.11 & -5834.3 & -5313.9 & 555.12 \\
600 & 434.91 & 606.39 & 200.72 & 405.67 & -5830.1 & -5210.2 & 453.58 \\
700 & 449.02 & 674.54 & 235.23 & 439.31 & -5826.2 & -5107.2 & 381.09 \\
800 & 460.04 & $\mathbf{7 3 5 . 2 4}$ & 262.67 & 472.57 & -5826.9 & -5004.2 & 326.73 \\
900 & 468.93 & 789.96 & 285.11 & 504.85 & -5824.0 & -4901.5 & 284.47 \\
1000 & 476.30 & 839.76 & 303.87 & 535.89 & -5822.2 & -4799.1 & 250.68 \\
1100 & 482.51 & 885.45 & 319.84 & 565.62 & -5821.7 & -4696.9 & 223.03 \\
1200 & 487.84 & 927.67 & 333.62 & 594.05 & -5861.1 & -4591.5 & 199.86 \\
1300 & 492.46 & 966.91 & 345.66 & 621.24 & -5857.1 & -4485.9 & 180.24
\end{tabular}

Melting $T$

Boiling T

$\mathbf{K}$

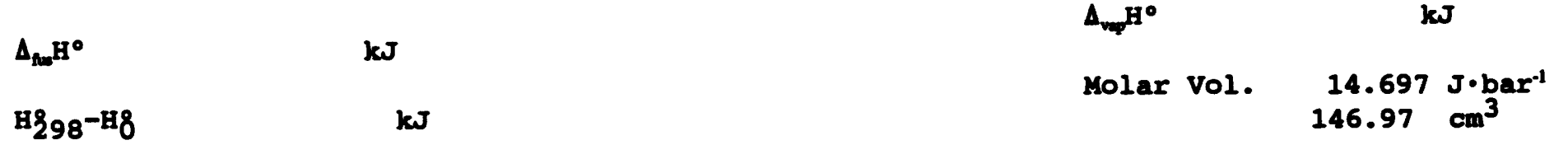


$\mathrm{Ca}_{5} \mathrm{Si}_{2} \mathrm{O}_{7}\left(\mathrm{CO}_{3}\right)_{2}$ : Monoclinic crystals 298.15 to $1200 \mathrm{~K}$.

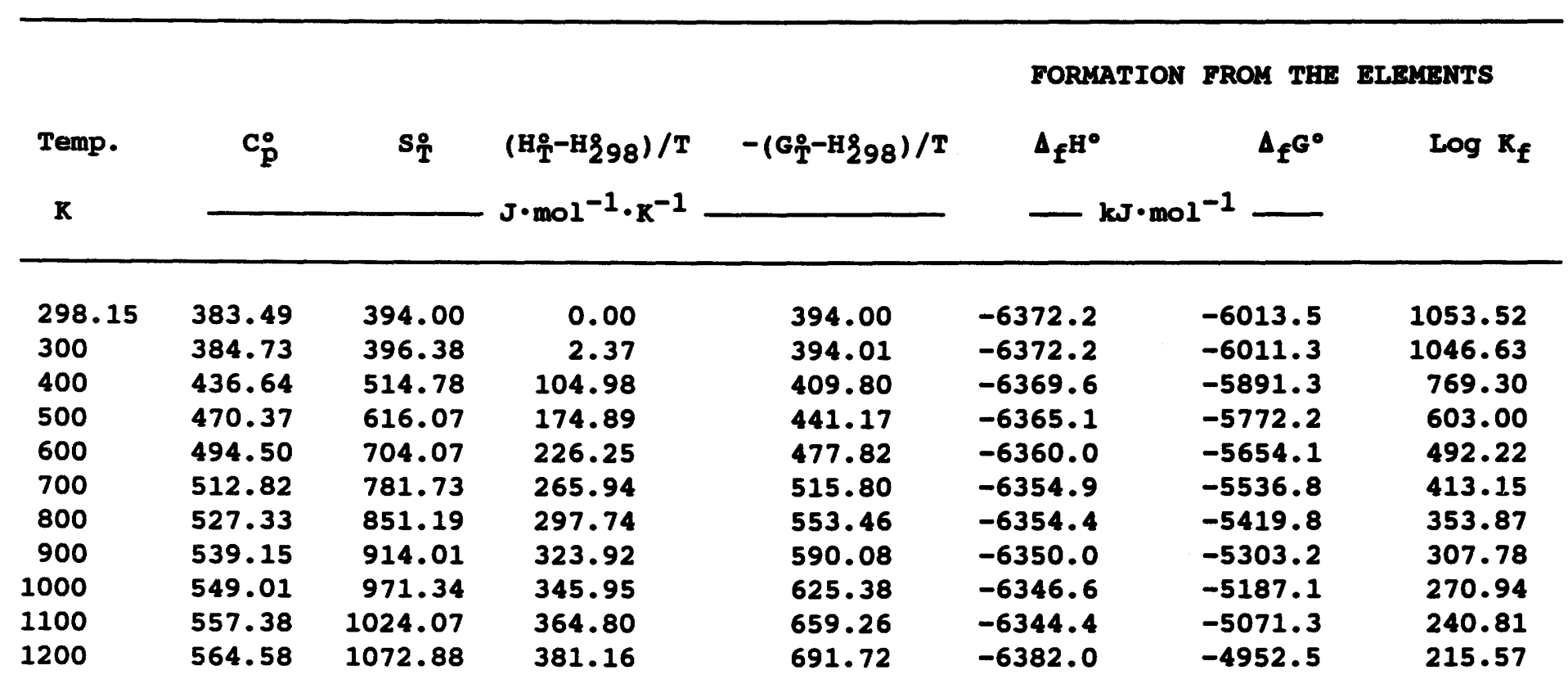

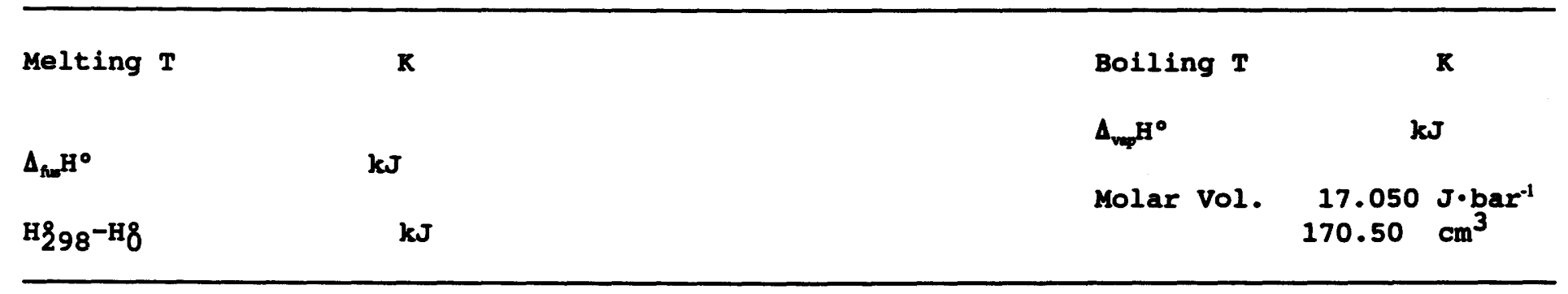
$A=-6.351 \mathrm{E}+03$
$B=1.165 E+00$
$C=-8.90 \mathrm{E}+05$ 
$\mathrm{Co}_{2} \mathrm{SiO}_{4}$ : Orthorhombic crystals 298.15 to $1300 \mathrm{~K}$.

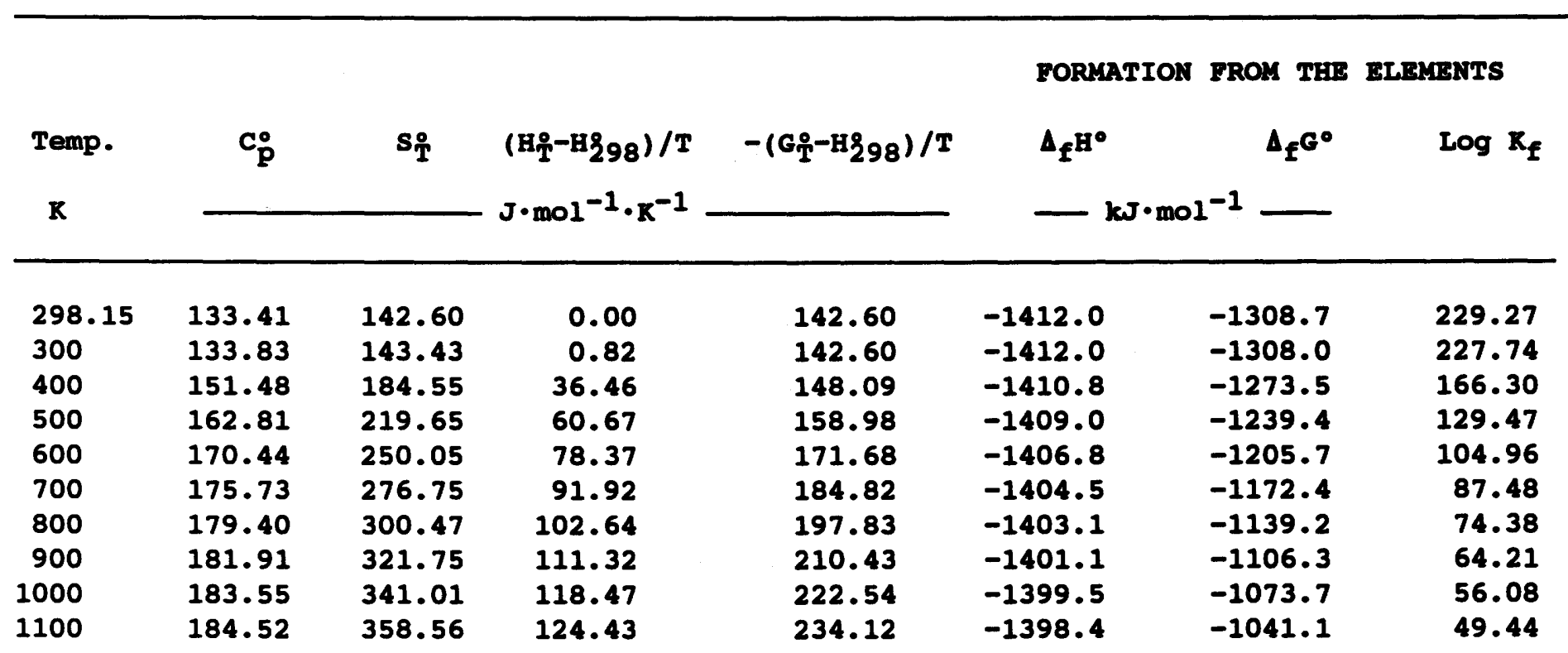

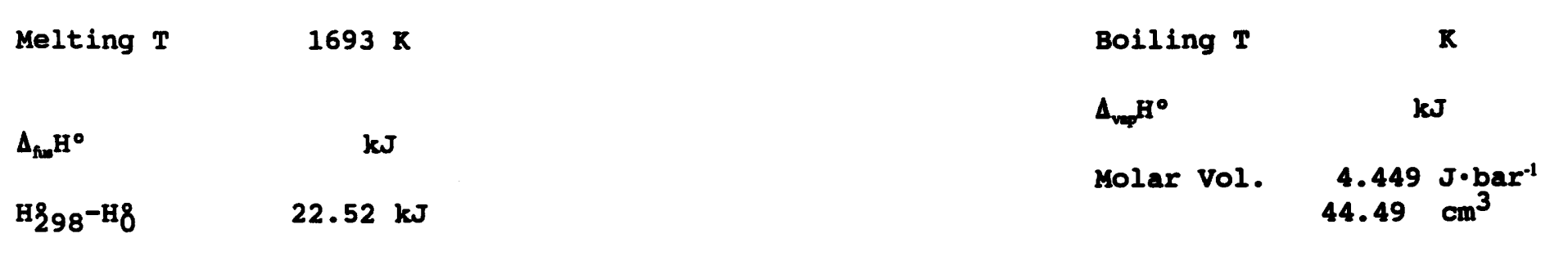

$$
A=-1 \cdot 401 \mathrm{E}+03
$$


$\mathrm{Fe}_{2} \mathrm{SiO}_{4}$ : Orthorhombic crystals 298.15 to $1490 \mathrm{~K}$. Fayalite melts incongruently at $1490 \mathrm{~K}$. Liquid 1490 to $1800 \mathrm{~K}$.

FORMATION FROM THE ELEMENTS

\begin{tabular}{|c|c|c|c|c|c|c|}
\hline Temp. & $\mathbf{c}_{\mathbf{p}}^{0}$ & $\mathbf{s}_{\mathbf{T}}$ & (H年-H298)/T & $-\left(G_{T}^{\circ}-H_{298}\right) / T$ & $\Delta_{f^{H^{\circ}}}$ & $\Delta_{f} G^{0}$ \\
\hline
\end{tabular}

\begin{tabular}{|c|c|c|c|c|c|c|c|c|}
\hline 298.15 & 131.84 & 151.00 & 0.00 & 151.00 & -1478.2 & -1379.1 & & 241.61 \\
\hline 300 & 132.39 & 151.82 & 0.81 & 151.00 & -1478.2 & -1378.5 & & 240.02 \\
\hline 400 & 152.14 & 192.99 & 36.51 & 156.48 & -1477.1 & -1345.4 & & 175.69 \\
\hline 500 & 162.24 & 228.12 & 60.73 & 167.39 & -1475.5 & -1312.7 & & 137.13 \\
\hline 600 & 168.83 & 258.31 & 78.23 & 180.09 & -1473.8 & -1280.3 & & 111.45 \\
\hline 700 & 174.03 & 284.74 & 91.55 & 193.19 & -1472.3 & -1248.1 & & 93.14 \\
\hline 800 & 178.71 & 308.29 & 102.16 & 206.13 & -1471.0 & -1216.2 & & 79.41 \\
\hline 900 & 183.31 & 329.60 & 110.92 & 218.68 & -1470.4 & -1184.4 & & 68.74 \\
\hline 1000 & 188.03 & 349.16 & 118.39 & 230.77 & -1470.9 & -1152.6 & & 60.21 \\
\hline 1100 & 193.02 & 367.31 & 124.95 & 242.36 & -1472.9 & -1120.7 & & 53.21 \\
\hline 1200 & 198.33 & 384.33 & 130.84 & 253.49 & -1473.0 & -1088.7 & & 47.39 \\
\hline 1300 & 204.03 & 400.43 & 136.25 & 264.18 & -1469.6 & -1056.8 & & 42.46 \\
\hline 1400 & 210.14 & 415.77 & 141.30 & 274.46 & -1466.0 & -1025.2 & & 38.25 \\
\hline 1500 & 240.60 & 490.58 & 205.80 & 284.78 & -1372.5 & -994.4 & & 34.63 \\
\hline 1600 & 240.60 & 506.10 & 207.98 & 298.12 & -1366.1 & -969.4 & & 31.65 \\
\hline 1700 & 240.60 & 520.68 & 209.89 & 310.79 & -1412.0 & -944.3 & & 29.01 \\
\hline 1800 & 240.60 & 534.43 & 211.60 & 322.83 & -1406.7 & -916.9 & & 26.61 \\
\hline \multirow[t]{2}{*}{ Melting T } & \multicolumn{2}{|c|}{$1490 \mathrm{~K}$} & & & \multicolumn{2}{|c|}{ Boiling $T$} & \multicolumn{2}{|r|}{$\mathbf{K}$} \\
\hline & & & & & \multicolumn{2}{|c|}{$\Delta_{\mathrm{v}} \mathrm{H}^{\circ}$} & \multicolumn{2}{|c|}{$\mathbf{k J}$} \\
\hline$\Delta_{\text {tros }} \mathrm{H}^{\circ}$ & \multicolumn{2}{|c|}{$89.3 \mathrm{~kJ}$} & & & & \multirow[b]{2}{*}{ vol. } & & \\
\hline H298-H8 & & $49 \mathrm{~kJ}$ & & & & & $\begin{array}{r}4.631 \\
46.31\end{array}$ & $\begin{array}{l}J \cdot \mathrm{bar}^{-1} \\
\mathrm{~cm} 3\end{array}$ \\
\hline
\end{tabular}

$A=-1.457 \mathrm{E}+03$

$B=3.049 \mathrm{E}-01$

$C=-1 \cdot 30 \mathrm{E}+06$ 
$\mathrm{Fe}_{3} \mathrm{Al}_{2} \mathrm{Si}_{3} \mathrm{O}_{12}:$ Cubic crystals 298.15 to $1200 \mathrm{~K}$.

FORMATION FROM THE ELEMENTS

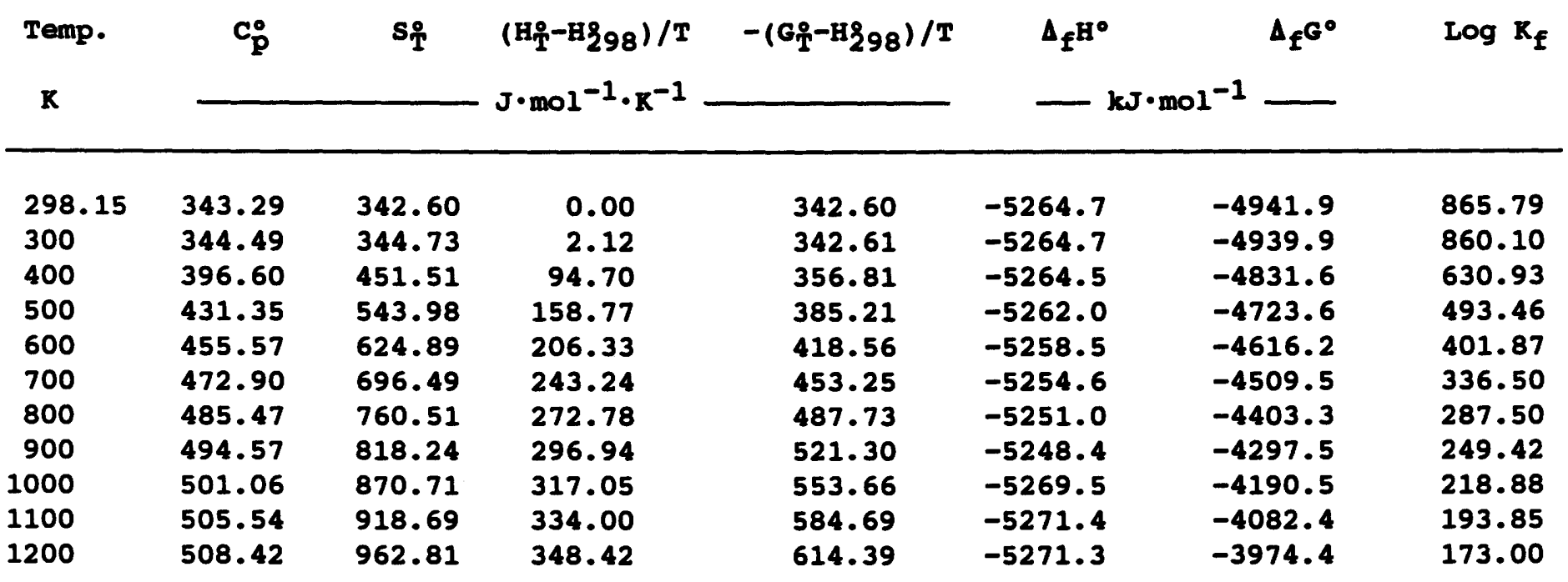

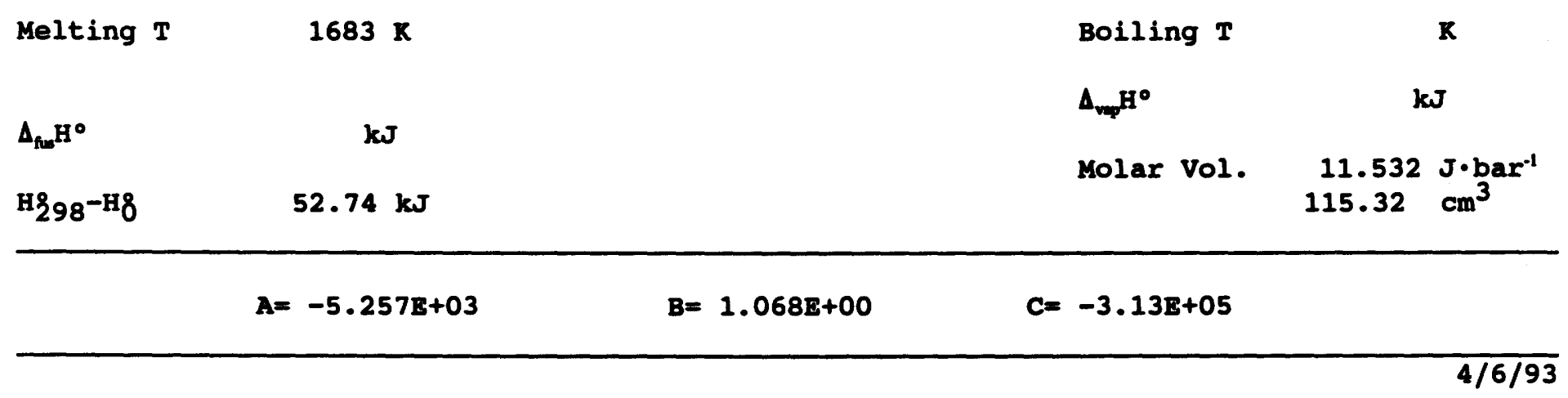


$\mathrm{Mg}_{2} \mathrm{Al}_{3}\left(\mathrm{AlSi}_{5} \mathrm{O}_{18}\right)$ : Orthorhombic crystals 298.15 to $1700 \mathrm{~K}$.

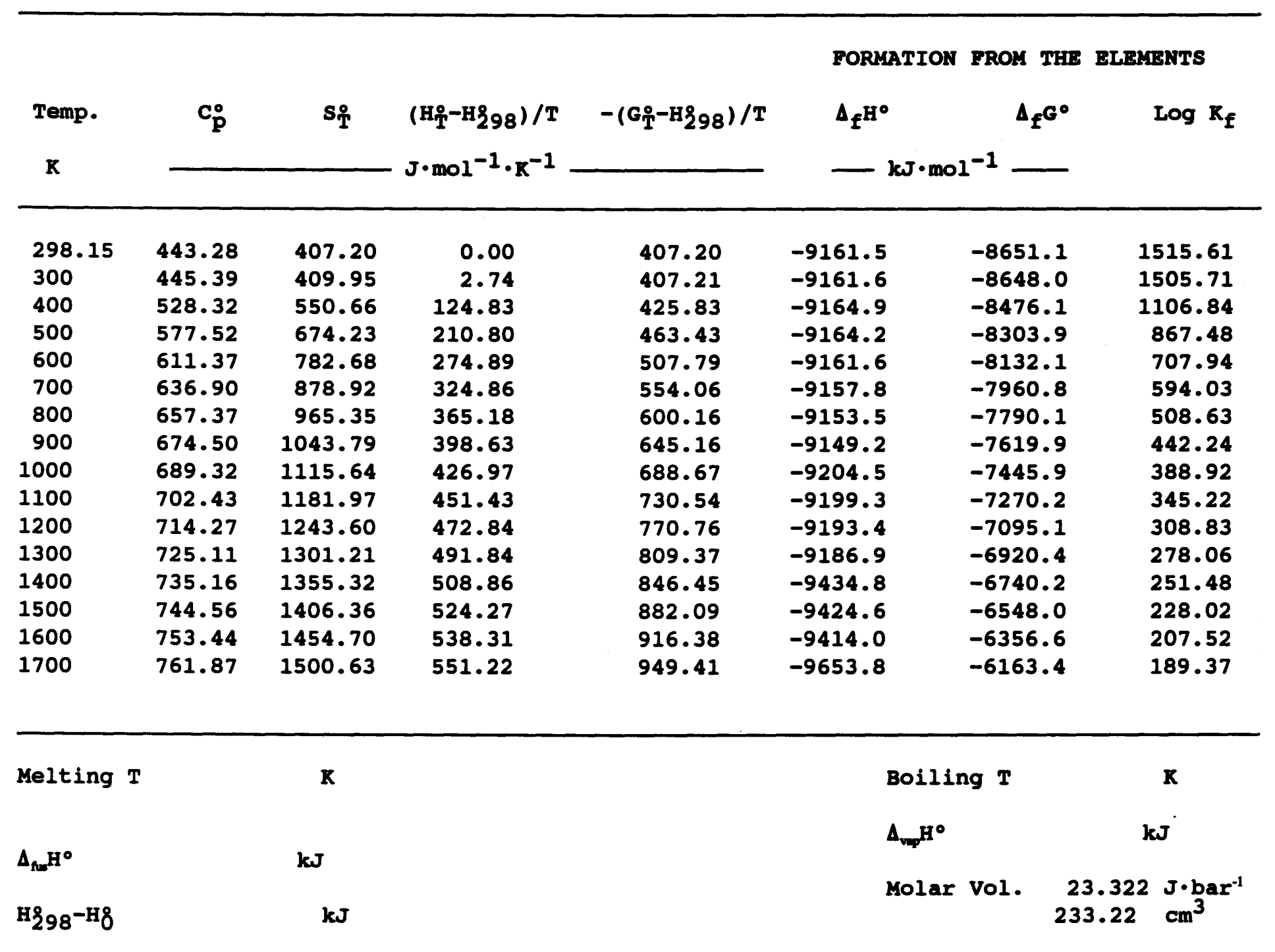


$\mathrm{Mg}_{2} \mathrm{SiO}_{4}$ : Orthorhombic crystals 298.15 to $1800 \mathrm{~K}$.

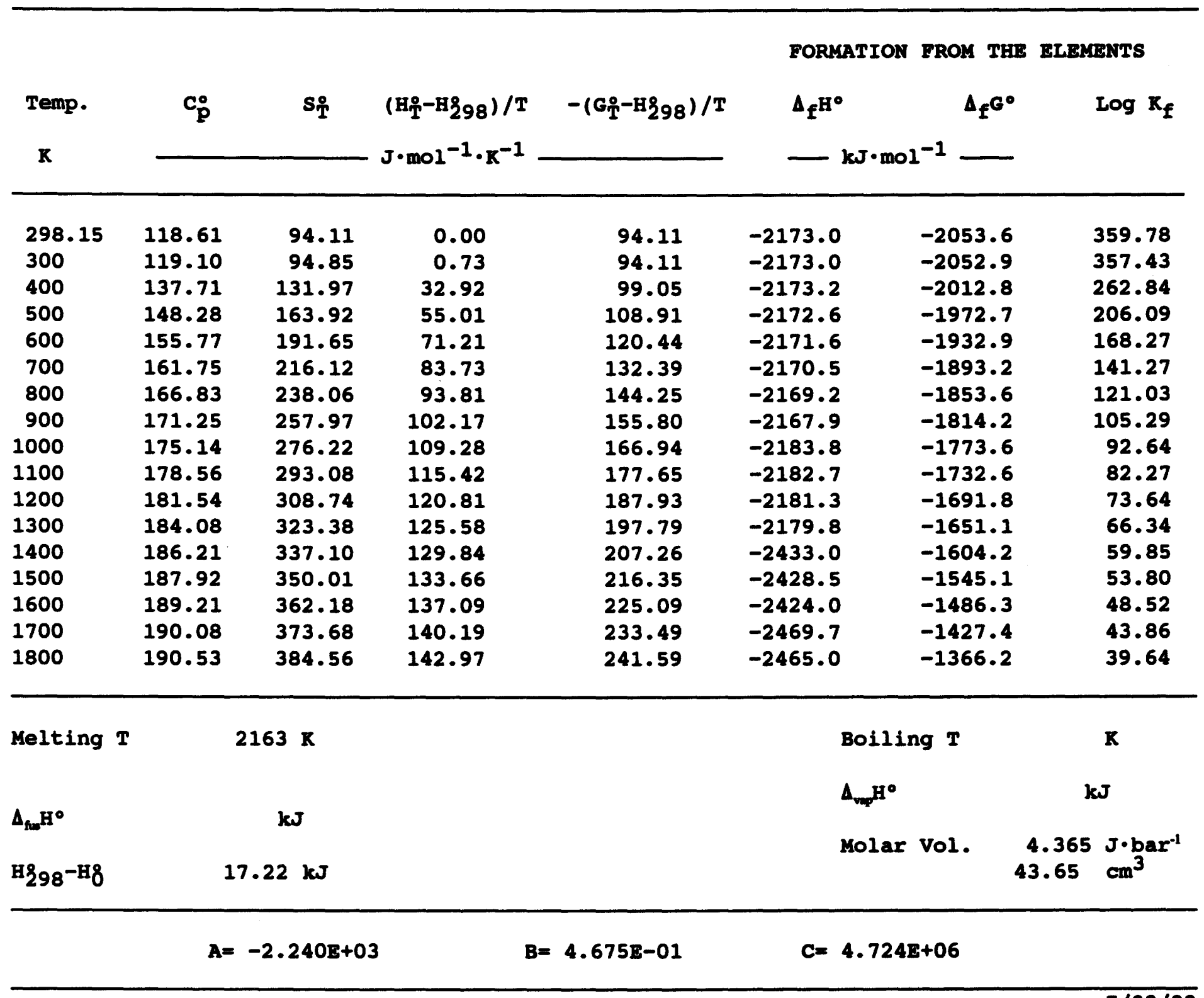


$\mathrm{Mg}_{3} \mathrm{Al}_{2} \mathrm{Si}_{3} \mathrm{O}_{12}:$ Cubic crystals 298.15 to $1570 \mathrm{~K}$.

\begin{tabular}{|c|c|c|c|c|c|c|c|}
\hline Temp. & $\mathbf{c}_{\mathbf{p}}^{\circ}$ & so & \multirow{2}{*}{$\begin{array}{l}\left(\mathrm{H}_{\mathrm{T}}-\mathrm{H}_{298}\right) / \mathrm{T} \\
\mathrm{J} \cdot \mathrm{mol}^{-1} \cdot \mathrm{K}^{-1}\end{array}$} & \multirow[t]{2}{*}{$-\left(G_{T}^{\circ}-H_{298}\right) / T$} & \multirow{2}{*}{\multicolumn{2}{|c|}{$\begin{array}{l}\text { FORMATION FROM THE } \\
\Delta_{f^{H^{\circ}}}\end{array}$}} & \multirow[t]{2}{*}{$\begin{array}{l}\text { ELEMTENTS } \\
\qquad \log \mathrm{K}_{\mathbf{f}}\end{array}$} \\
\hline $\mathbf{K}$ & & & & & & & \\
\hline 298.15 & 325.76 & 266.27 & 0.00 & 266.27 & -6285.0 & -5934.5 & 1039.67 \\
\hline 300 & 327.12 & 268.29 & 2.01 & 266.28 & -6285.1 & -5932.3 & 1032.88 \\
\hline 400 & 383.71 & 370.83 & 90.96 & 279.87 & -6286.1 & -5814.4 & 759.27 \\
\hline 500 & 419.39 & 460.56 & 153.33 & 307.24 & -6284.3 & -5696.7 & 595.12 \\
\hline 600 & 443.59 & 539.30 & 199.81 & 339.49 & -6280.9 & -5579.5 & 485.72 \\
\hline 700 & 460.82 & 609.04 & 235.93 & 373.11 & -6276.9 & -5462.9 & 407.64 \\
\hline 800 & 473.55 & 671.45 & 264.87 & 406.57 & -6272.6 & -5346.9 & 349.11 \\
\hline 900 & 483.27 & 727.81 & 288.62 & 439.19 & -6268.5 & -5231.4 & 303.62 \\
\hline 1000 & 490.92 & 779.14 & 308.49 & 470.65 & -6311.8 & -5112.8 & 267.06 \\
\hline 1100 & 497.14 & 826.23 & 325.36 & 500.87 & -6308.0 & -4993.0 & 237.09 \\
\hline 1200 & 502.37 & 869.72 & 339.90 & 529.82 & -6304.0 & -4873.7 & 212.14 \\
\hline 1300 & 506.94 & 910.11 & 352.58 & 557.54 & -6299.9 & -4754.6 & 191.04 \\
\hline 1400 & 511.10 & 947.84 & 363.75 & 584.08 & -6678.0 & -4626.6 & 172.62 \\
\hline 1500 & 515.01 & 983.23 & 373.71 & 609.52 & -6669.6 & -4480.3 & 156.01 \\
\hline
\end{tabular}

Melting $\mathrm{T} \quad 1570 \mathrm{~K}$

$\Delta_{\text {fun }} \mathrm{H}^{\circ}$

$241.0 \mathrm{~kJ}$

H298-H8
$47.85 \mathrm{~kJ}$

$\begin{array}{lc}\text { Boiling } \mathrm{T} & \mathrm{K} \\ \boldsymbol{\Delta}_{\mathrm{vq}} \mathrm{H}^{\circ} & \mathrm{kJ} \\ \text { Molar vol. } & \begin{array}{c}11.312 \mathrm{~J} \cdot \mathrm{bar}^{-1} \\ 113.12 \mathrm{~cm}^{3}\end{array}\end{array}$
$A=-6 \cdot 316 \mathrm{E}+03$
$B=1 \cdot 208 \mathrm{E}+00$
$C=2 \cdot 167 \mathrm{E}+06$ 
$\mathrm{Mg}_{3} \mathrm{Al}_{2} \mathrm{Si}_{3} \mathrm{O}_{12}$ : Glass 298.15 to glass transition $1020 \mathrm{~K}$.

\begin{tabular}{|c|c|c|c|c|c|c|c|}
\hline Temp. & $\mathbf{c}_{\mathbf{p}}^{\circ}$ & $s_{\text {T }}$ & \multirow{2}{*}{$\begin{array}{l}\left(\mathrm{H}_{\mathrm{T}}-\mathrm{H} 298\right) / \mathrm{T} \\
\mathrm{J} \cdot \mathrm{mol}^{-1} \cdot \mathrm{K}^{-1}\end{array}$} & \multirow[t]{2}{*}{$-\left(G_{T}^{\circ}-H_{298}\right) / T$} & \multirow{2}{*}{\multicolumn{2}{|c|}{$\begin{array}{l}\text { FORMATION FROM THE } \\
\triangle_{\mathrm{f}^{\mathrm{H}}} \mathrm{H}^{\circ}\end{array}$}} & \multirow[t]{2}{*}{$\begin{array}{l}\text { ELELIENTS } \\
\qquad \log \mathbf{k}_{\mathbf{f}}\end{array}$} \\
\hline $\mathbf{K}$ & & & & & & & \\
\hline 298.15 & 387.18 & 346.30 & 0.00 & 346.3 & -6163.0 & -5836.3 & 1022.48 \\
\hline 300 & 387.83 & 348.70 & 2.39 & 346.3 & -6162.9 & -5834.3 & 1015.82 \\
\hline 400 & 416.67 & 464.47 & 102.57 & 361.9 & -6159.5 & -5725.2 & 747.62 \\
\hline 500 & 437.98 & 559.83 & 167.61 & 392.2 & -6155.1 & -5617.2 & 586.81 \\
\hline 600 & 455.32 & 641.26 & 214.16 & 427.1 & -6150.3 & -5510.0 & 479.68 \\
\hline 700 & 470.25 & 712.60 & 249.70 & 462.9 & -6145.2 & -5403.7 & 403.22 \\
\hline 800 & 483.61 & 776.28 & 278.12 & 498.2 & -6140.0 & -5298.2 & 345.93 \\
\hline 900 & 495.85 & 833.95 & 301.64 & 532.3 & -6134.8 & -5193.2 & 301.40 \\
\hline 1000 & 507.28 & 886.79 & 321.64 & 565.2 & -6176.6 & -5085.3 & 265.62 \\
\hline
\end{tabular}

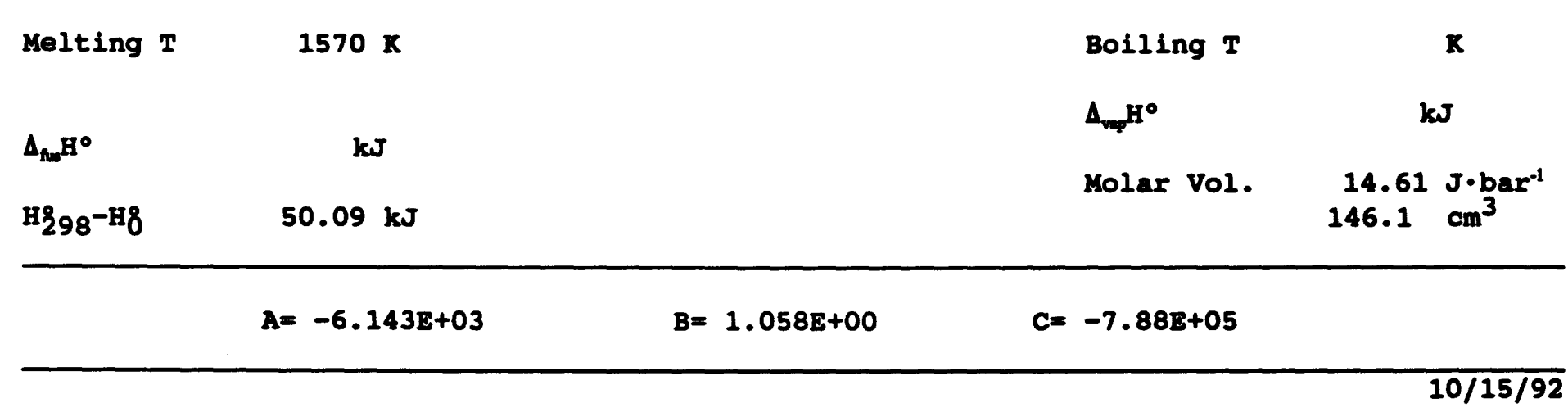


$\mathrm{Mn}_{2} \mathrm{SiO}_{4}$ : Orthorhombic crystals 298.15 to $1524 \mathrm{~K}$. Tephroite melts incongruently at $1524 \mathrm{~K}$.

\begin{tabular}{|c|c|c|c|c|c|c|c|}
\hline \multirow{3}{*}{$\begin{array}{l}\text { Temp. } \\
\mathbf{K}\end{array}$} & \multirow{3}{*}{$\mathbf{c}_{\mathbf{p}}^{\circ}$} & \multirow{3}{*}{$\mathbf{s}_{\mathbf{T}}$} & \multirow{3}{*}{$\begin{array}{l}\left(\mathrm{H}_{\mathrm{T}}^{\circ}-\mathrm{H}_{298}\right) / \mathrm{T} \\
\mathrm{J} \cdot \mathrm{mol}^{-1} \cdot \mathrm{K}^{-1}\end{array}$} & \multirow{3}{*}{$-\left(G_{T}^{0}-H_{298}\right) / T$} & \multirow{3}{*}{\multicolumn{2}{|c|}{$\begin{array}{l}\text { FORMATION FROM THE } \\
\Delta_{f^{H^{\circ}}}\end{array}$}} & \multirow{3}{*}{$\begin{array}{l}\text { ELEMENTS } \\
\qquad \operatorname{LOg} R_{f}\end{array}$} \\
\hline & & & & & & & \\
\hline & & & & & & & \\
\hline 298.15 & 128.74 & 155.90 & 0.00 & 155.90 & -1731.5 & -1631.0 & 285.73 \\
\hline 300 & 129.11 & 156.70 & 0.80 & 155.90 & -1731.5 & -1630.3 & 283.86 \\
\hline 400 & 144.89 & 196.18 & 35.00 & 161.18 & -1731.3 & -1596.6 & 208.49 \\
\hline 500 & 155.22 & 229.69 & 58.08 & 171.62 & -1730.4 & -1563.0 & 163.29 \\
\hline 600 & 162.48 & 258.67 & 74.90 & 183.76 & -1729.5 & -1529.7 & 133.17 \\
\hline 700 & 167.82 & 284.14 & 87.82 & 196.32 & -1728.4 & -1496.4 & 111.66 \\
\hline 800 & 171.86 & 306.82 & 98.08 & 208.74 & -1727.5 & -1463.4 & 95.55 \\
\hline 900 & 174.96 & 327.25 & 106.46 & 220.79 & -1726.6 & -1430.4 & 83.02 \\
\hline 1000 & 177.38 & 345.82 & 113.43 & 232.38 & -1730.3 & -1397.4 & 72.99 \\
\hline 1100 & 179.27 & 362.81 & 119.34 & 243.48 & -1729.8 & -1364.2 & 64.78 \\
\hline 1200 & 180.74 & 378.48 & 124.39 & 254.08 & -1729.2 & -1331.0 & 57.93 \\
\hline 1300 & 181.87 & 392.99 & 128.77 & 264.22 & -1728.7 & -1297.8 & 52.15 \\
\hline 1400 & 182.73 & 406.50 & 132.60 & 273.90 & -1732.9 & -1264.6 & 47.18 \\
\hline 1500 & 183.36 & 419.13 & 135.96 & 283.17 & -1737.6 & -1230.8 & 42.86 \\
\hline
\end{tabular}

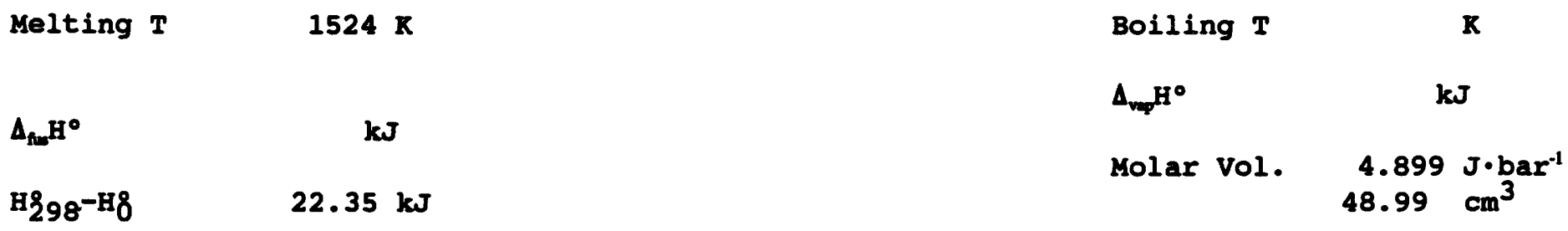

$A=-1.730 \mathrm{E}+03 \quad \mathrm{~B}=3.321 \mathrm{E}-01 \quad \mathrm{C}=-3.65 \mathrm{E}+04$ 
$\mathrm{Ni}_{2} \mathrm{SiO}_{4}:$ Orthorhombic crystals 298.15 to $1300 \mathrm{~K}$.

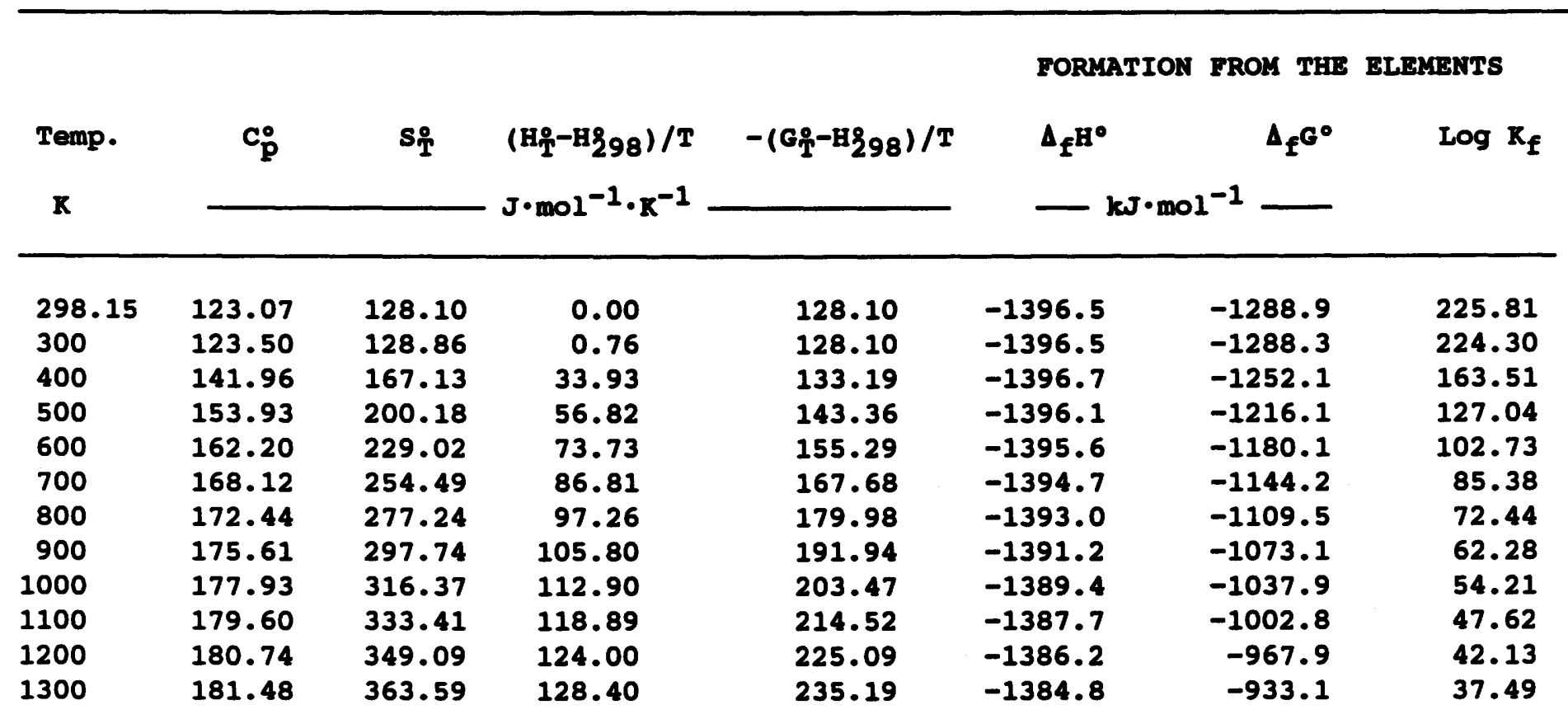

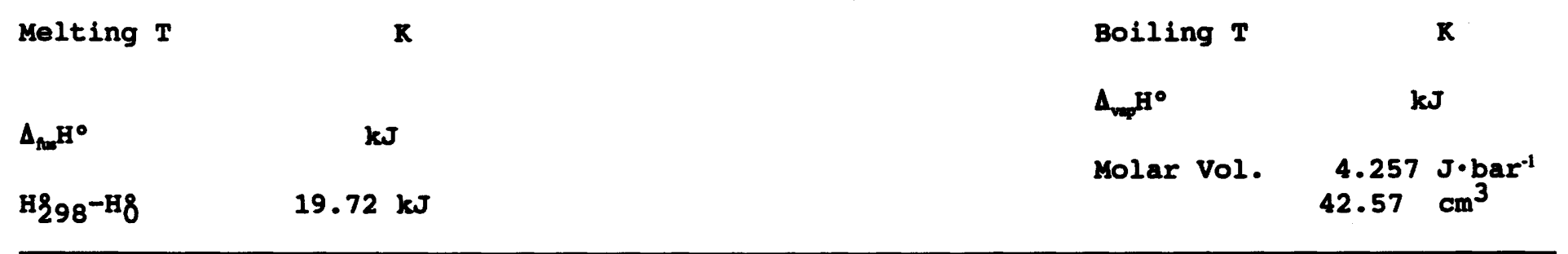
$A=-1.391 \mathrm{E}+03$
$\mathrm{B}=3.528 \mathrm{E}-01$
$C=-3.04 E+05$ 
$\mathrm{Ni}_{2} \mathrm{SiO}_{4}$-SPINEL

Formula wt 209.463

$\mathrm{Ni}_{2} \mathrm{SiO}_{4}:$ Cubic crystals 298.15 to $800 \mathrm{~K}$.

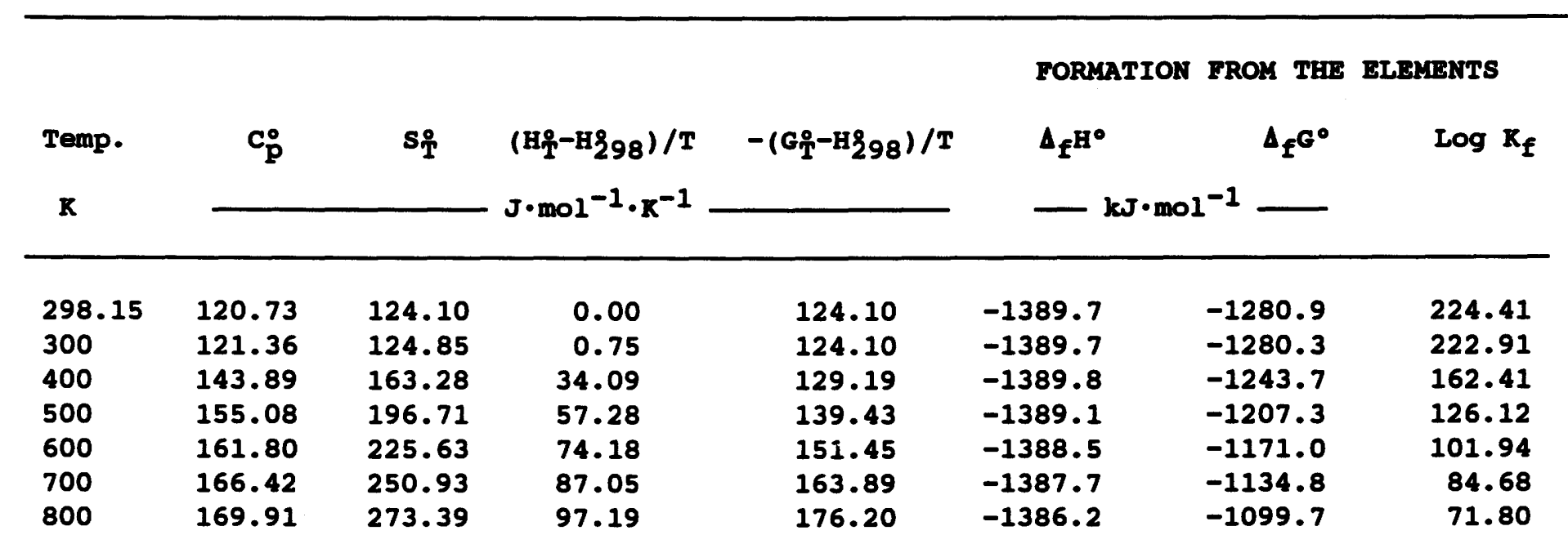

\begin{tabular}{|c|c|c|c|}
\hline Melting $T$ & $\mathbf{R}$ & Bolling $T$ & $\mathbf{K}$ \\
\hline & & $\Delta_{\mathrm{v} e p} \mathrm{H}^{\circ}$ & $\mathbf{k J}$ \\
\hline$\Delta_{\text {fux }} \mathrm{H}^{\circ}$ & $\mathbf{k J}$ & & \\
\hline H298-H8 & $\mathbf{k J}$ & Molar Vol. & $\begin{array}{l}3.981 \mathrm{~J} \cdot \mathrm{bar}^{-1} \\
39.81 \mathrm{~cm}^{3}\end{array}$ \\
\hline
\end{tabular}

$A=-1.385 \mathrm{E}+03 \quad \mathrm{~B}=3.578 \mathrm{E}-01 \quad \mathrm{C}=-2.19 \mathrm{E}+05$


$\mathrm{ZrSiO}_{4}$ : Tetragonal crystals 298.15 to $1600 \mathrm{~K}$.

\begin{tabular}{|c|c|c|c|c|c|c|c|}
\hline \multirow[b]{2}{*}{ Temp. } & \multirow[b]{2}{*}{$\mathbf{c}_{\mathbf{p}}^{\circ}$} & \multirow[b]{2}{*}{$\mathbf{s}_{\mathbf{T}}^{0}$} & \multirow{3}{*}{$\begin{array}{l}\left(\mathrm{H}_{\mathrm{T}}-\mathrm{H}_{298}\right) / \mathrm{T} \\
\mathrm{J} \cdot \mathrm{mol}^{-1} \cdot \mathrm{K}^{-1}\end{array}$} & \multirow[b]{2}{*}{$-\left(G_{T}-H_{298}\right) / T$} & FORMATION & FROY THE & ELMYIBNTS \\
\hline & & & & & $\Delta_{f^{H^{\circ}}}$ & $\Delta_{f} G^{0}$ & $\log \mathbf{K}_{\mathbf{f}}$ \\
\hline $\mathbf{K}$ & & & & & $-\mathbf{k J} \cdot \mathrm{mol}$ & $1^{-1}$ & \\
\hline 298.15 & 98.64 & 84.03 & 0.00 & 84.03 & -2034.2 & -1919.7 & 336.32 \\
\hline 300 & 99.03 & 84.64 & 0.61 & 84.03 & -2034.2 & -1919.0 & 334.12 \\
\hline 400 & 115.51 & 115.58 & 27.45 & 88.14 & -2034.0 & -1880.6 & 245.58 \\
\hline 500 & 126.03 & 142.57 & 46.18 & 96.39 & -2032.9 & -1842.4 & 192.47 \\
\hline 600 & 133.27 & 166.23 & 60.13 & 106.10 & -2031.3 & -1804.4 & 157.08 \\
\hline 700 & 138.46 & 187.18 & 70.97 & 116.21 & -2029.5 & -1766.7 & 131.83 \\
\hline 800 & 142.28 & 205.93 & 79.65 & 126.28 & -2027.4 & -1729.3 & 112.91 \\
\hline 900 & 145.12 & 222.86 & 86.78 & 136.08 & -2025.4 & -1692.2 & 98.21 \\
\hline 1000 & 147.25 & 238.27 & 92.72 & 145.54 & -2023.4 & $-1655 \cdot 3$ & 86.46 \\
\hline 1100 & 148.83 & 252.38 & 97.76 & 154.62 & -2021.4 & -1618.5 & 76.86 \\
\hline 1200 & 149.97 & 265.38 & 102.06 & 163.32 & -2023.2 & -1581.8 & 68.85 \\
\hline 1300 & 150.76 & 277.42 & 105.78 & 171.64 & -2021.0 & -1545.1 & 62.08 \\
\hline 1400 & 151.28 & 288.61 & 109.01 & 179.60 & -2018.8 & -1508.6 & 56.28 \\
\hline 1500 & 151.55 & 299.06 & 111.84 & 187.22 & -2016.7 & -1472.2 & 51.27 \\
\hline 1600 & 151.63 & 308.84 & 114.33 & 194.52 & -2014.8 & -1436.0 & 46.88 \\
\hline
\end{tabular}

Melting $\mathbf{T}$

$$
\Delta_{\text {fum }} \mathrm{H}^{\circ}
$$

$\mathbf{k J}$

H298-H8
R

$\mathbf{k J}$
Boiling $T$

$A_{\text {vep }}$ H $^{\circ}$

Molar Vol.

\section{R}

$\mathbf{k J}$ $\begin{array}{ll}3.926 & \mathrm{~J} \cdot \mathrm{bar}^{-1} \\ 39.26 \mathrm{~cm}^{3}\end{array}$ 
$\mathrm{CaSiO}_{3}$ : Triclinic crystals 298.15 to $1400 \mathrm{~K}$. Pseudowollastonite is stable above $1398 \mathrm{~K}$.

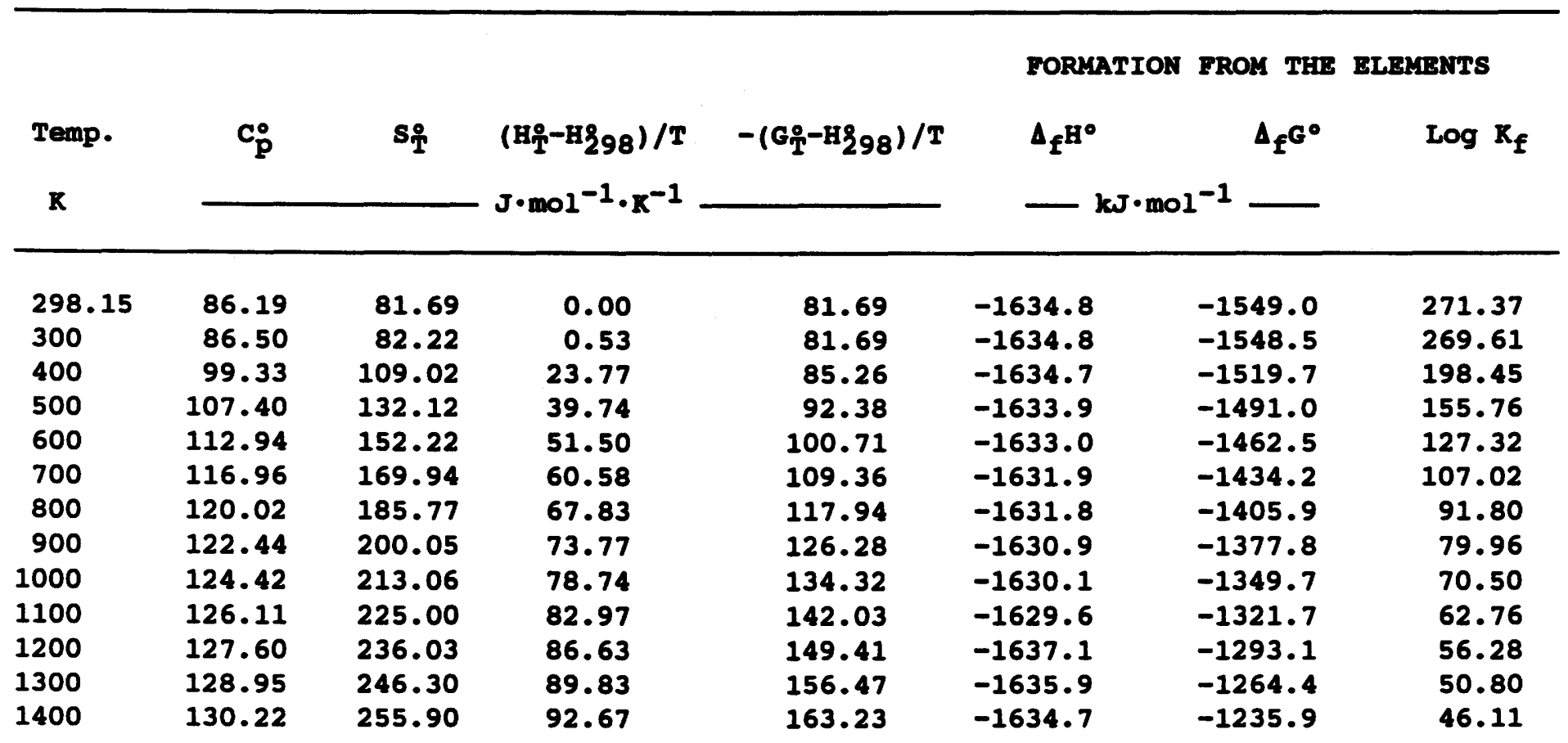

Melting T

$\Delta_{\text {sut }} \mathrm{H}^{\circ}$

H298-H8 $\mathbf{k J}$

$\mathbf{x}$

$13.84 \mathrm{~kJ}$
Boiling $\mathbf{T}$

$\Delta_{\mathrm{vq}} \mathrm{H}^{\circ}$

Molar Vol.
$\mathbf{K}$

$\mathbf{k J}$
$3.990 \mathrm{~J} \cdot$ bar $^{-1}$ $39.90 \mathrm{~cm}^{3}$
$A=-1.632 \mathrm{E}+03$
$B=2.827 \mathrm{E}-01$
$C=-1.12 \mathrm{E}+05$ 
$\mathrm{CaSiO}_{3}$ : Triclinic cryatals 298.15 to melting point $1821 \mathrm{~K}$.

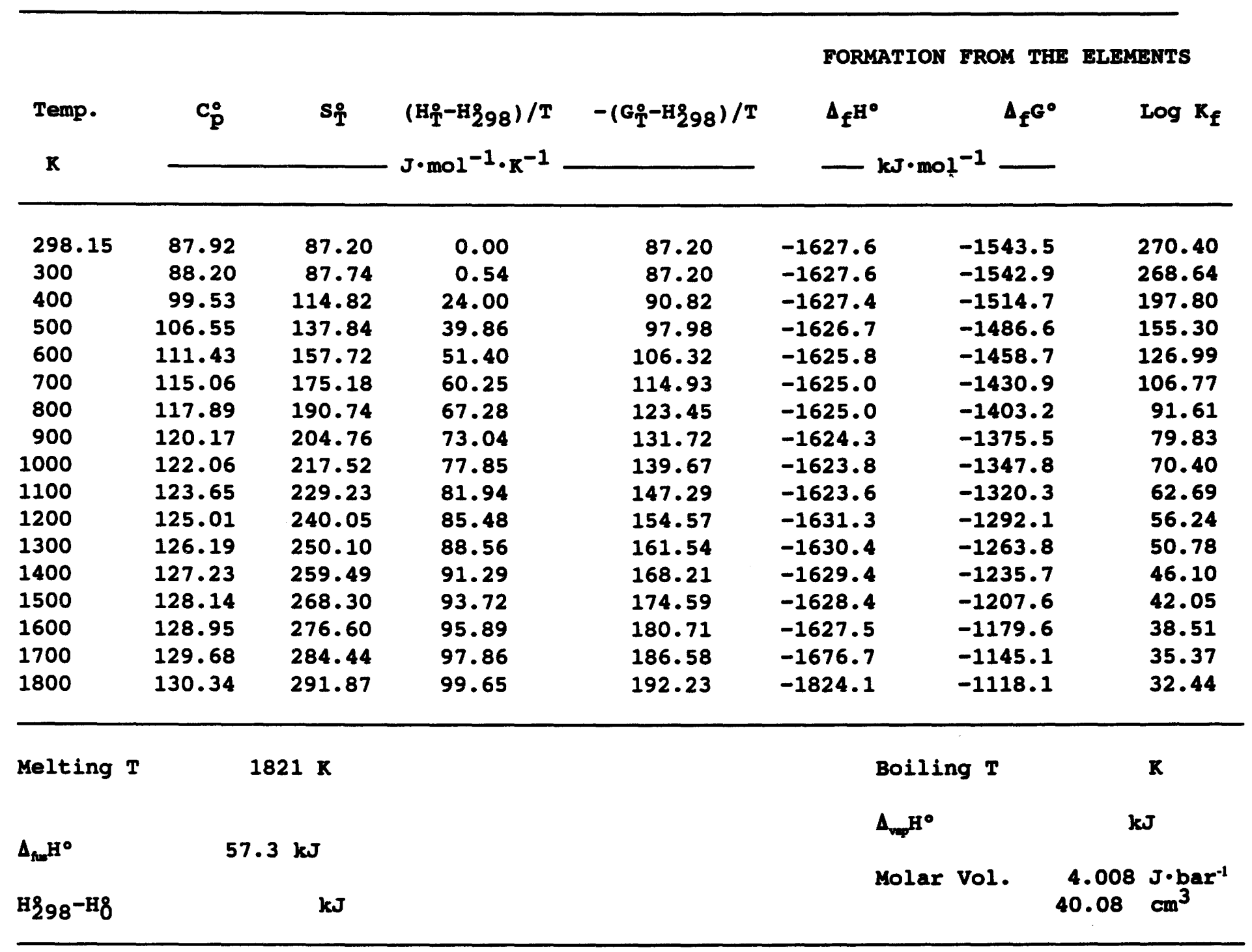


CaSiO $_{3}$ : Glass 298.15 to $1821 \mathrm{~K}$, melting point of pseudowollastonite.

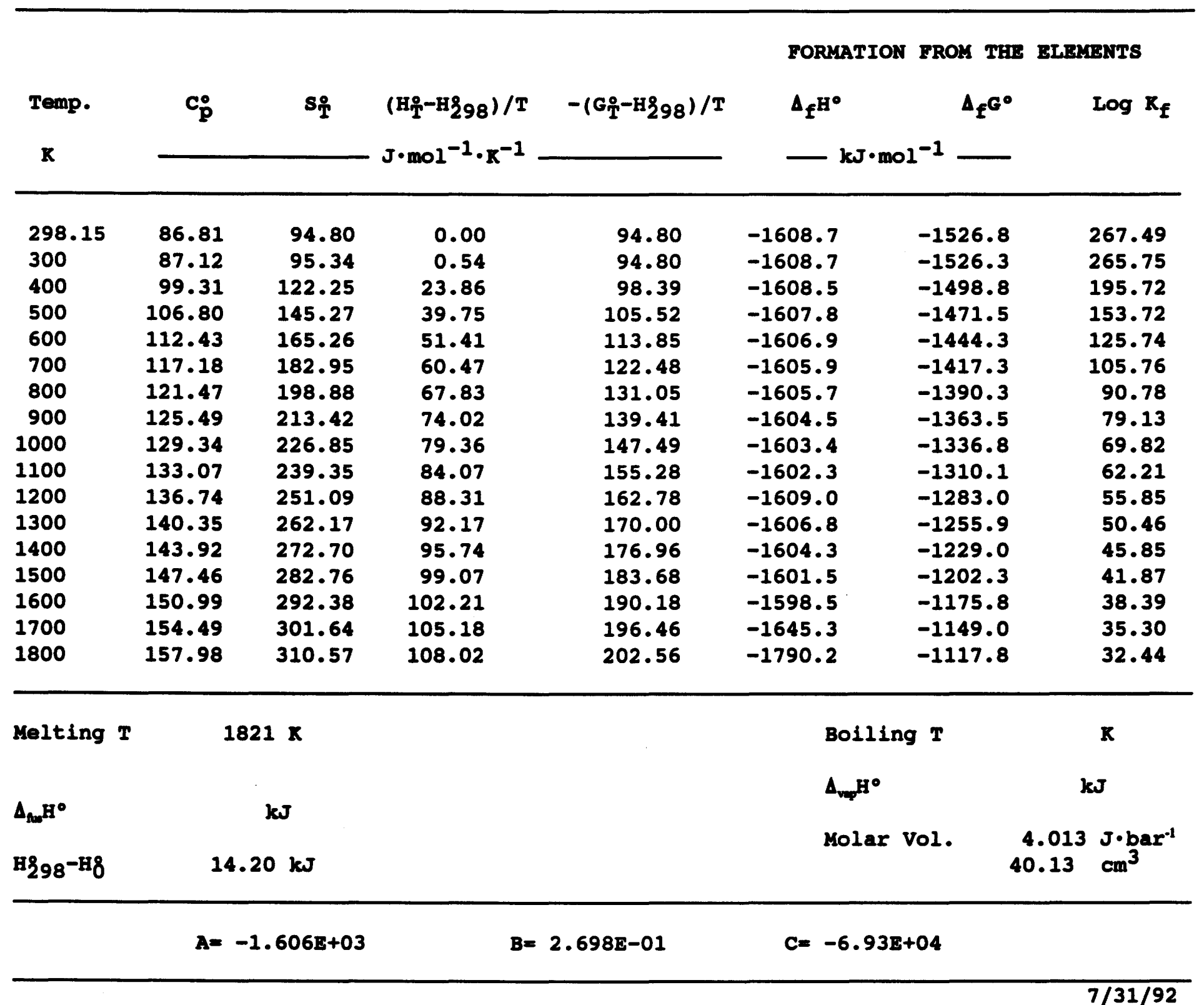


$\mathrm{CaAl}_{2} \mathrm{SiO}_{6}$ : Monoclinic crystals 298.15 to $1000 \mathrm{~K}$.

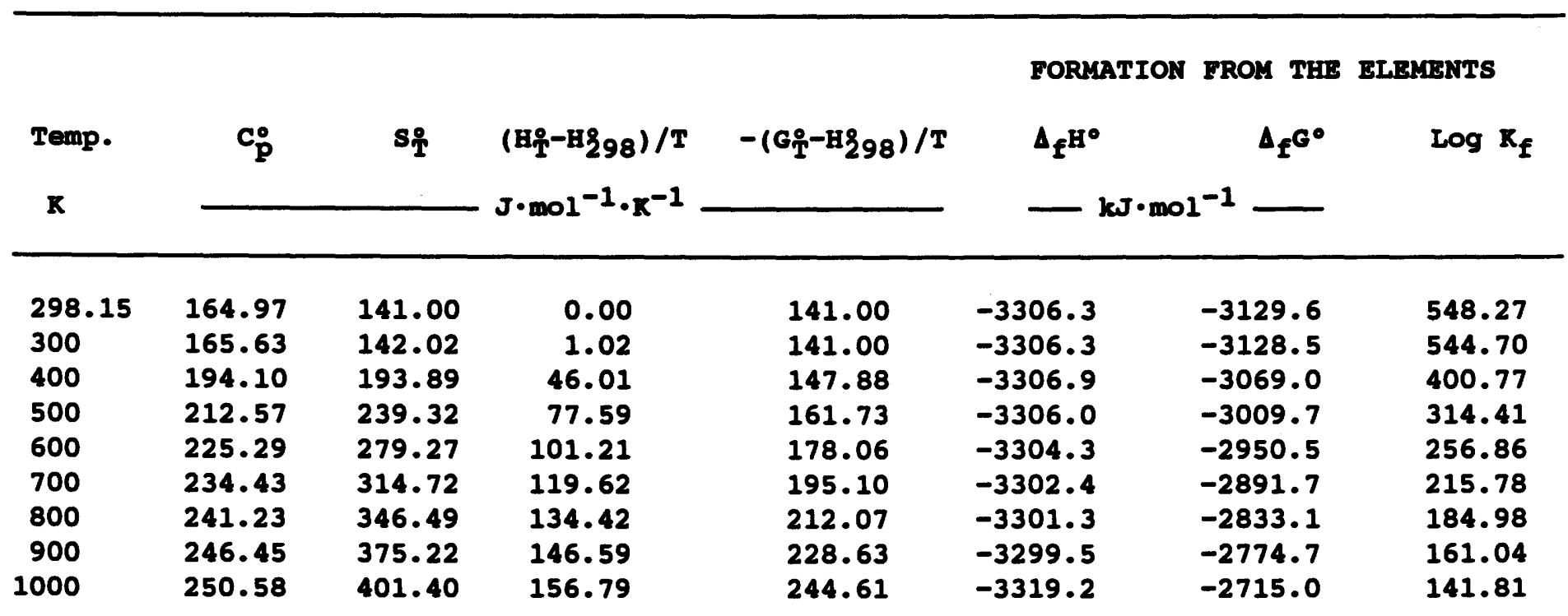

Melting $T$

$\Delta_{\text {fun }} \mathbf{H}^{\circ}$

H298-H8 $\mathbf{k J}$

$\mathbf{R}$

$24.40 \mathrm{~kJ}$
Boiling $T$

$\Delta_{\text {vep }} \mathrm{H}^{\circ}$

Molar Vol. $\mathbf{x}$

$k J$

$6.357 \mathrm{~J} \cdot \mathrm{bar}^{-1}$
$63.57 \mathrm{~cm}^{3}$
$A=-3 \cdot 303 E+03$
$B=\quad 5.877 \mathrm{E}-01$
$C=-1 \cdot 73 E+05$ 
CaFesi ${ }_{2} \mathrm{O}_{6}$ : Monoclinic crystals 298.15 to $1300 \mathrm{~K}$. Decomposes at $1238 \mathrm{~K}$.

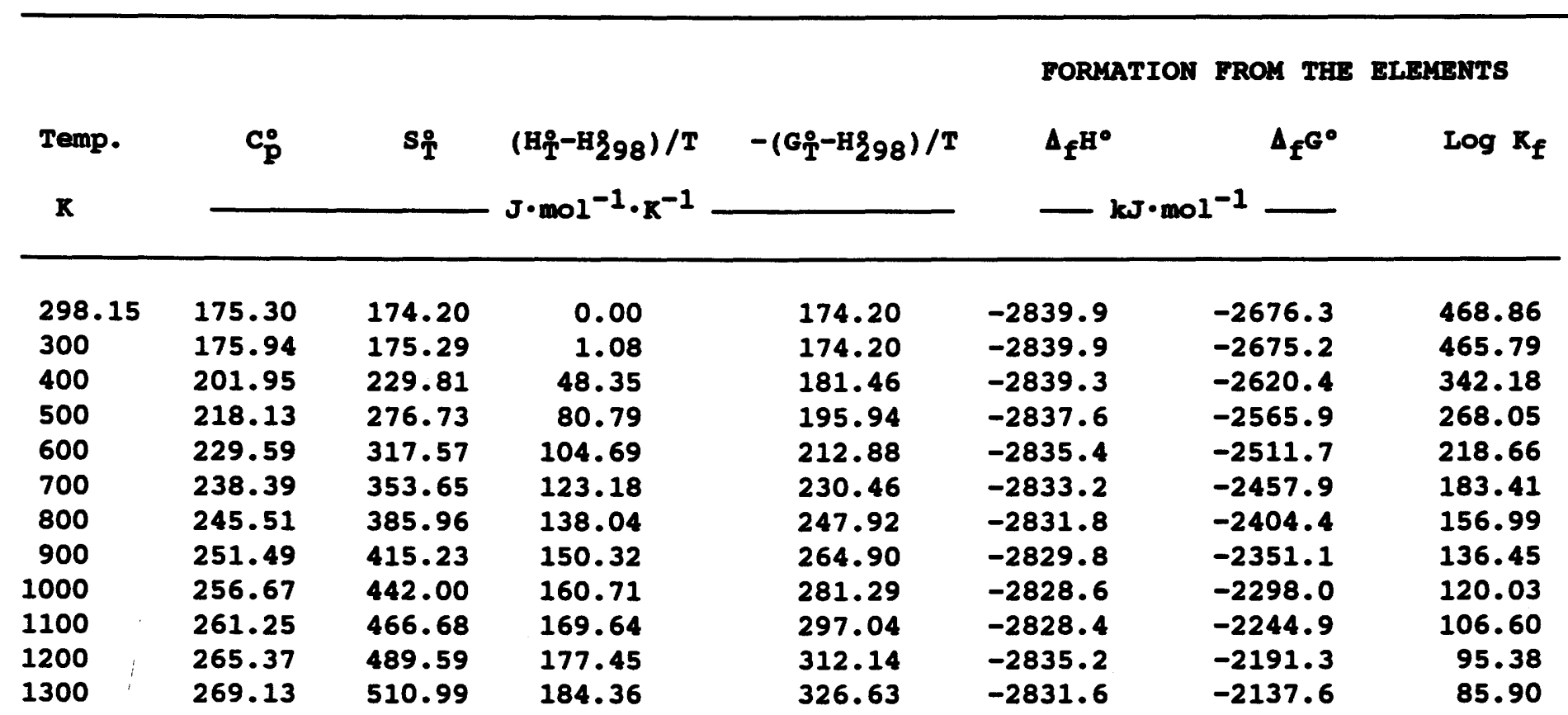

Melting $T$

$\mathbf{K}$

$\Delta_{\text {fun }} \mathrm{H}^{\circ}$

$\mathbf{k J}$

H298-H8
Boiling $\mathbf{T}$

$\Delta_{\mathrm{veq}} \mathrm{H}^{\circ}$

Molar Vol.
$\mathbf{R}$

$\mathbf{k J}$

$6.795 \mathrm{~J} \cdot \mathrm{bar}^{-1}$ $67.95 \mathrm{~cm}^{3}$ 
CaMgsi ${ }_{2} \mathrm{O}_{6}$ : Monoclinic crystals 298.15 to incongruent melting point at $1668 \mathrm{~K}$.

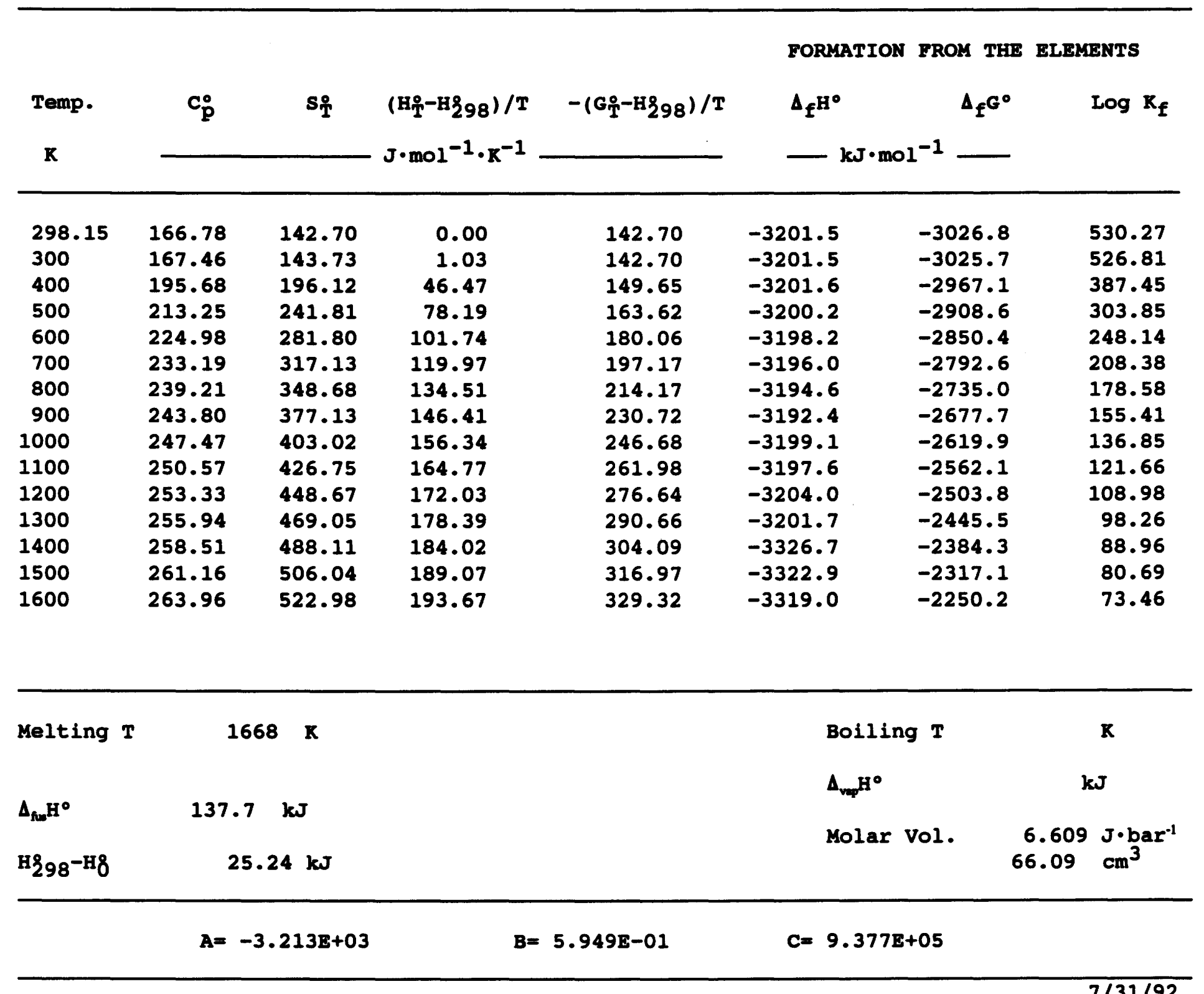


Cakgsi $\mathrm{O}_{6}$ : Glass 298.15 to $1400 \mathrm{~K}$.

\begin{tabular}{|c|c|c|c|c|c|c|c|}
\hline \multirow{2}{*}{$\begin{array}{l}\text { Temp. } \\
\mathrm{K}\end{array}$} & \multirow[t]{2}{*}{$\mathbf{C}_{\mathbf{p}}^{\circ}$} & \multirow[t]{2}{*}{$\mathbf{s}_{\mathbf{T}}^{\circ}$} & \multirow{2}{*}{$\begin{array}{l}\left(\mathrm{H}_{\mathrm{T}}-\mathrm{H}_{298}\right) / \mathrm{T} \\
\mathrm{J} \cdot \mathrm{mol}^{-1} \cdot \mathrm{R}^{-1}\end{array}$} & \multirow[t]{2}{*}{$-\left(G_{T}^{\circ}-\mathrm{H}_{298}\right) / T$} & \multirow{2}{*}{\multicolumn{2}{|c|}{$\begin{array}{l}\text { FORMATION FROM THE } \\
\Delta_{f^{H}}{ }^{\circ} \quad \Delta_{f} G^{\circ}\end{array}$}} & \multirow[t]{2}{*}{$\begin{array}{l}\text { ELEMENTS } \\
\qquad \operatorname{LOg} \mathbf{R}_{f}\end{array}$} \\
\hline & & & & & & & \\
\hline 298.15 & 168.31 & 166.00 & 0.00 & 166.00 & -3156.1 & -2988.3 & 523.53 \\
\hline 300 & 168.93 & 167.04 & 1.04 & 166.00 & -3156.1 & -2987.3 & 520.12 \\
\hline 400 & 196.43 & 219.69 & 46.69 & 172.99 & -3156.1 & -2931.0 & 382.74 \\
\hline 500 & 213.62 & 265.52 & 78.50 & 187.02 & -3154.7 & -2874.9 & 300.33 \\
\hline 600 & 224.44 & 305.50 & 101.99 & 203.51 & -3152.7 & -2819.1 & 245.42 \\
\hline 700 & 232.22 & 340.70 & 120.06 & 220.65 & -3150.5 & -2763.7 & 206.22 \\
\hline 800 & 239.22 & 372.17 & 134.51 & 237.65 & -3149.2 & -2708.4 & 176.84 \\
\hline 900 & 247.01 & 400.78 & 146.57 & 254.21 & -3146.8 & -2653.5 & 154.00 \\
\hline 1000 & 256.65 & 427.28 & 157.07 & 270.21 & -3152.9 & -2598.1 & 135.71 \\
\hline 1100 & 268.92 & 452.30 & 166.66 & 285.63 & -3150.1 & -2542.7 & 120.74 \\
\hline 1200 & 284.36 & 476.33 & 175.80 & 300.53 & -3154.1 & -2487.0 & 108.26 \\
\hline 1300 & 303.39 & 499.82 & 184.86 & 314.96 & -3147.9 & -2431.7 & 97.71 \\
\hline 1400 & 326.34 & 523.12 & 194.12 & 328.99 & -3267.2 & -2373.8 & 88.56 \\
\hline
\end{tabular}

Melting $T$

$\mathbf{K}$

Boiling $T$

$\Delta_{\mathrm{veq}} \mathrm{H}^{\circ}$

$\Delta_{\text {fue }} \mathrm{H}^{\circ}$

$\mathbf{k} \mathbf{J}$

H298-H8

$26.94 \mathrm{~kJ}$

Molar vol.
7.609 76.09

\section{$\mathbf{R}$}

$\mathbf{k J}$ 
$\mathrm{FeSiO}_{3}$ : Orthorhombic cryetals 298.15 to $800 \mathrm{~K}$.

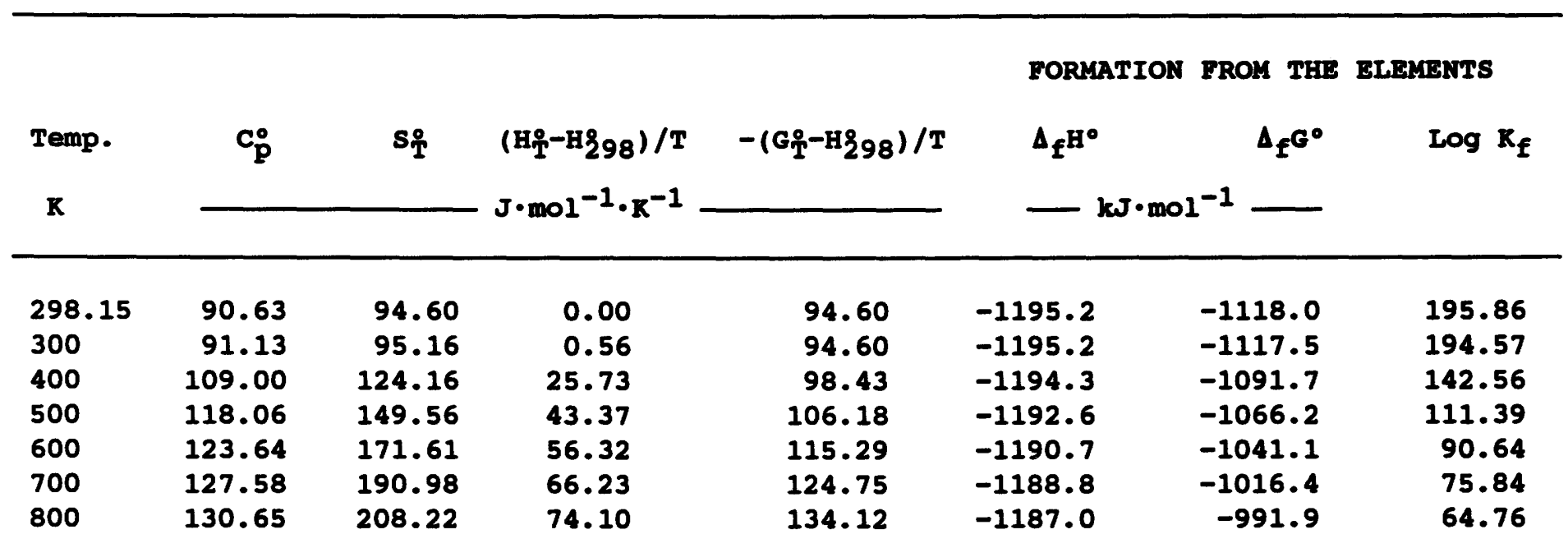

Melting $\mathbf{T}$

$\mathbf{R}$

$\mathbf{k J}$

$\Lambda_{\text {fum }} \mathrm{H}^{\circ}$

H298-H8

$\mathbf{k J}$

Boiling $T$

$A_{\mathrm{vep}} \mathrm{H}^{\circ}$

Molar Vol. 
$\alpha$-SPODUMENE

Formula wt 186.090

LiAlsi ${ }_{2} \mathrm{O}_{6}$ : Monoclinic crystals 298.15 to $1200 \mathrm{~K}$.

FORMATION FROM THE ELEMENTS

\begin{tabular}{|c|c|c|c|c|c|c|c|}
\hline $\begin{array}{l}\text { Temp. } \\
\mathbf{K}\end{array}$ & $\mathbf{c}_{\mathbf{p}}^{\circ}$ & $\mathbf{s}_{\mathrm{T}}$ & $\begin{array}{l}\left(\mathrm{H}_{\mathrm{T}}^{\circ}-\mathrm{H}_{298}\right) / \mathrm{T} \\
\mathrm{J} \cdot \mathrm{mol}^{-1} \cdot \mathrm{K}^{-1}\end{array}$ & $-\left(G_{T}^{\circ}-H_{298}\right) / T$ & $\Delta_{f^{H}} H^{0}$ & $\Delta_{F^{\prime}} G^{0}$ & $\log K_{f}$ \\
\hline 298.15 & 158.93 & 129.30 & 0.00 & 129.30 & -3053.5 & -2880.2 & 504.59 \\
\hline 300 & 159.48 & 130.28 & 0.98 & 129.30 & -3053.5 & -2879.2 & 501.29 \\
\hline 400 & 184.73 & 179.81 & 43.93 & 135.88 & -3054.5 & -2820.8 & 368.36 \\
\hline 500 & 203.25 & 223.13 & 74.03 & 149.09 & -3057.3 & -2762.1 & 288.55 \\
\hline 600 & 217.12 & 261.47 & 96.78 & 164.69 & -3056.3 & -2703.2 & 235.33 \\
\hline 700 & 227.78 & 295.77 & 114.76 & 181.01 & -3054.4 & -2644.5 & 197.33 \\
\hline 800 & 236.12 & 326.75 & 129.43 & 197.32 & -3052.1 & -2586.0 & 168.85 \\
\hline 900 & 242.75 & 354.96 & 141.67 & 213.29 & -3049.6 & -2527.9 & 146.71 \\
\hline 1000 & 248.07 & 380.82 & 152.05 & 228.77 & -3057.4 & -2469.4 & 128.98 \\
\hline 1100 & 252.37 & 404.68 & 160.98 & 243.69 & -3054.3 & -2410.7 & 114.47 \\
\hline 1200 & 255.84 & 426.79 & 168.75 & 258.04 & -3050.9 & -2352.3 & 102.39 \\
\hline
\end{tabular}

Melting T

$\mathbf{R}$

Boiling T

$\mathbf{R}$

$\Delta_{\text {nut }} \mathrm{H}^{\circ}$

$k \boldsymbol{J}$

$\Delta_{\text {vepp }} \mathrm{H}^{\circ}$

$\mathbf{k J}$

H298-H8

$\mathbf{k J}$

Molar Vol.

$5.837 \mathrm{~J} \cdot$ bar $^{-1}$

$58.37 \mathrm{~cm}^{3}$
$A=-3.054 E+03$
$B=5.849 \mathrm{E}-01$
$C=-5 \cdot 34 E+04$ 
LiAlsi ${ }_{2} \mathrm{O}_{6}$ : Tetragonal crystals 298.15 to $1700 \mathrm{~K}$. Melting point is $1696 \mathrm{~K}$.

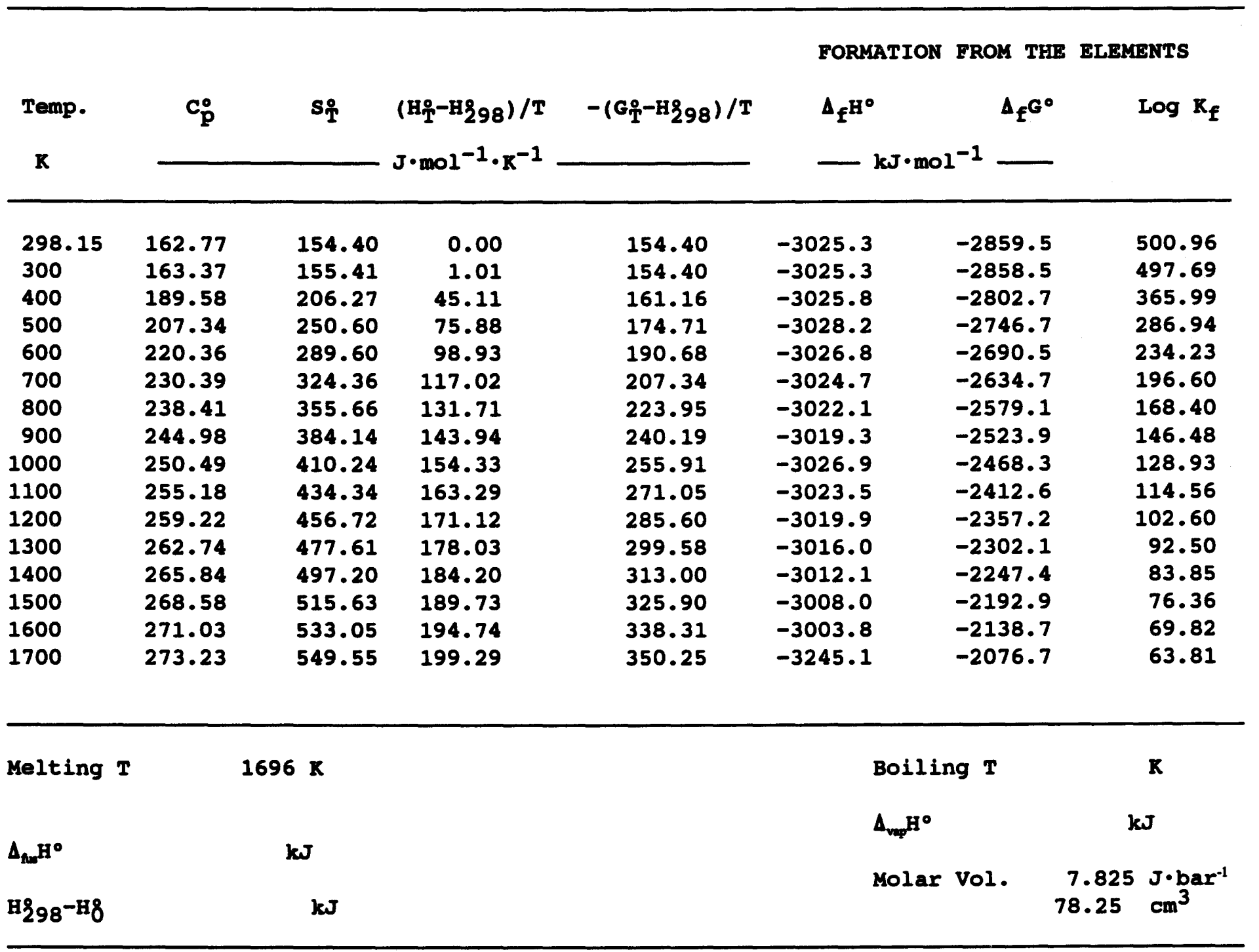


$\mathrm{KgSiO}_{3}$ : Orthorhombic crystals 298.15 to $1000 \mathrm{~K}$.

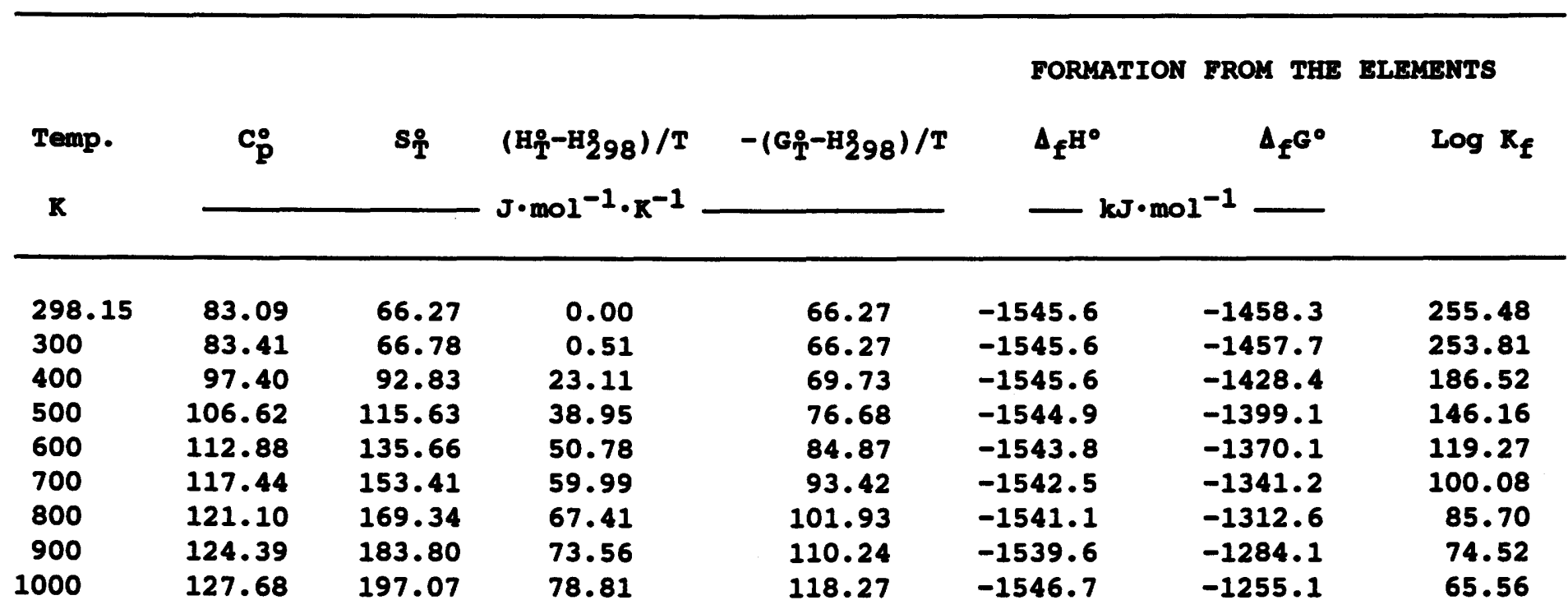

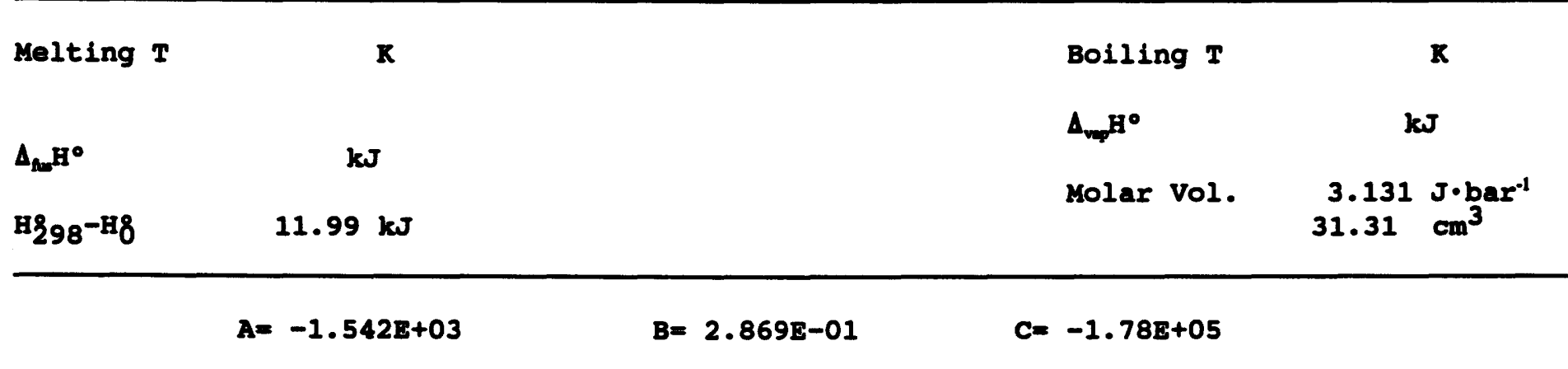


Mgsio 3 : Crystals 298.15 to melting point at $1830 \mathrm{~K}$.

\begin{tabular}{|c|c|c|c|c|c|c|c|}
\hline \multirow{2}{*}{$\begin{array}{l}\text { Temp. } \\
\mathbf{K}\end{array}$} & \multirow[t]{2}{*}{$\mathbf{c}_{\mathbf{p}}^{\circ}$} & \multirow[t]{2}{*}{$s_{\text {T }}^{\circ}$} & \multirow{2}{*}{$\begin{array}{l}\left(\mathrm{H}_{\mathrm{T}}-\mathrm{H}_{298}\right) / \mathrm{T} \\
\mathrm{J} \cdot \mathrm{mol}^{-1} \cdot \mathrm{K}^{-1}\end{array}$} & \multirow[t]{2}{*}{$-\left(G_{T}^{\circ}-H_{298}\right) / T$} & \multirow{2}{*}{\multicolumn{2}{|c|}{$\begin{array}{l}\text { FORMATION FROM THE } \\
\Delta_{f^{\prime}} \mathrm{H}^{\circ}\end{array}$}} & \multirow[t]{2}{*}{$\begin{array}{l}\text { ELERENTS } \\
\qquad \log \mathrm{K}_{\mathbf{f}}\end{array}$} \\
\hline & & & & & & & \\
\hline 298.15 & 82.12 & 67.86 & 0.00 & 67.86 & -1545.0 & -1458.1 & 255.45 \\
\hline 300 & 82.34 & 68.37 & 0.51 & 67.86 & -1545.0 & -1457.6 & 253.78 \\
\hline 400 & 93.04 & 93.59 & 22.36 & 71.23 & -1545.3 & -1428.4 & 186.52 \\
\hline 500 & 101.20 & 115.27 & 37.35 & 77.92 & -1545.1 & -1399.2 & 146.17 \\
\hline 600 & 107.42 & 134.29 & 48.53 & 85.76 & -1544.6 & -1370.0 & 119.27 \\
\hline 700 & 112.22 & 151.23 & 57.30 & 93.92 & -1543.8 & -1341.0 & 100.06 \\
\hline 800 & 115.98 & 166.47 & 64.41 & 102.06 & -1542.9 & -1312.1 & 85.67 \\
\hline 900 & 118.95 & 180.30 & 70.31 & 109.99 & -1541.9 & -1283.3 & 74.48 \\
\hline 1000 & 121.32 & 192.96 & 75.30 & 117.67 & -1549.6 & -1253.9 & 65.49 \\
\hline 1100 & 123.22 & 204.62 & 79.57 & 125.05 & -1548.7 & -1224.3 & 58.14 \\
\hline 1200 & 124.73 & 215.41 & 83.27 & 132.13 & -1547.7 & -1194.9 & 52.01 \\
\hline 1300 & 125.93 & 225.44 & 86.51 & 138.93 & -1546.7 & -1165.5 & 46.83 \\
\hline 1400 & 126.87 & 234.81 & 89.36 & 145.45 & -1673.1 & -1133.1 & 42.28 \\
\hline 1500 & 127.60 & 243.59 & 91.89 & 151.70 & -1670.8 & -1094.6 & 38.12 \\
\hline 1600 & 128.14 & 251.84 & 94.14 & 157.71 & -1668.5 & -1056.3 & 34.48 \\
\hline
\end{tabular}

Melting $\mathrm{T} \quad 1830 \mathrm{~K}$

$\Delta_{\mathrm{fm}} \mathrm{H}^{\circ} \quad 61.50 \mathrm{~kJ}$

H298-H8

kJ
Boiling $\mathbf{T}$

$\Delta_{\mathrm{veq}} \mathrm{H}^{\circ}$

Molar Vol.
$\mathbf{K}$

$\mathbf{k J}$ $\begin{array}{ll}3.128 & \mathrm{~J} \cdot \mathrm{bar}^{-1} \\ 31.28 & \mathrm{~cm}^{3}\end{array}$
$A=-1.562 \mathrm{E}+03$
$B=3.088 \mathrm{E}-01$
$C=1.138 \mathrm{E}+06$ 
$\mathrm{MgSiO}_{3}$ : Ilmenite structure form stable only at high pressure 298.15 to $700 \mathrm{~K}$.

\begin{tabular}{|c|c|c|c|c|c|c|c|}
\hline Temp. & $\mathbf{c}_{\mathbf{p}}^{\circ}$ & $s_{\mathrm{T}}^{\circ}$ & \multirow{2}{*}{$\begin{array}{l}\left(\mathrm{H}_{\mathrm{T}}-\mathrm{H}_{298}\right) / \mathrm{T} \\
\mathrm{J} \cdot \mathrm{mol}^{-1} \cdot \mathrm{K}^{-1}\end{array}$} & \multirow[t]{2}{*}{$-\left(G_{T}^{\circ}-H_{298}\right) / T$} & \multirow{2}{*}{\multicolumn{2}{|c|}{$\begin{array}{l}\text { FORLATION FROM THE } \\
\Delta_{\mathrm{f}^{\mathrm{H}^{\circ}}} \Delta_{\mathrm{f}^{\mathrm{G}^{\circ}}}\end{array}$}} & \multirow[t]{2}{*}{$\begin{array}{l}\text { ELEMENTS } \\
\qquad \operatorname{LOg} \mathbf{K}_{\mathbf{f}}\end{array}$} \\
\hline $\mathbf{K}$ & & & & & & & \\
\hline 298.15 & 102.64 & 60.40 & 0.00 & 60.40 & -1486.6 & -1397.5 & 244.83 \\
\hline 300 & 102.63 & 61.03 & 0.63 & 60.40 & -1486.6 & -1397.0 & 243.23 \\
\hline 400 & 104.92 & 90.77 & 26.33 & 64.44 & -1485.4 & -1367.3 & 178.54 \\
\hline 500 & 109.92 & 114.69 & 42.52 & 72.17 & -1484.2 & -1337.9 & 139.77 \\
\hline 600 & 115.98 & 135.26 & 54.25 & 81.01 & -1482.8 & -1308.8 & 113.94 \\
\hline 700 & 122.55 & 153.62 & 63.53 & 90.09 & -1481.0 & -1279.9 & 95.51 \\
\hline
\end{tabular}

Melting $\mathbf{T}$

$\Delta_{\text {fwo }} \mathrm{H}^{\circ}$

H298-H8 $\mathbf{k}$

$\mathbf{k J}$

$\mathbf{k J}$
Boiling T

$\Delta_{\mathrm{ve}} \mathrm{H}^{\circ}$

Molar vol. 
RHODONITE

Formula wt 131.022

$\mathrm{MnSiO}_{3}$ : Triclinic crystals 298.15 to $1600 \mathrm{~K}$. Melts incongruently at $1564 \mathrm{~K}$.

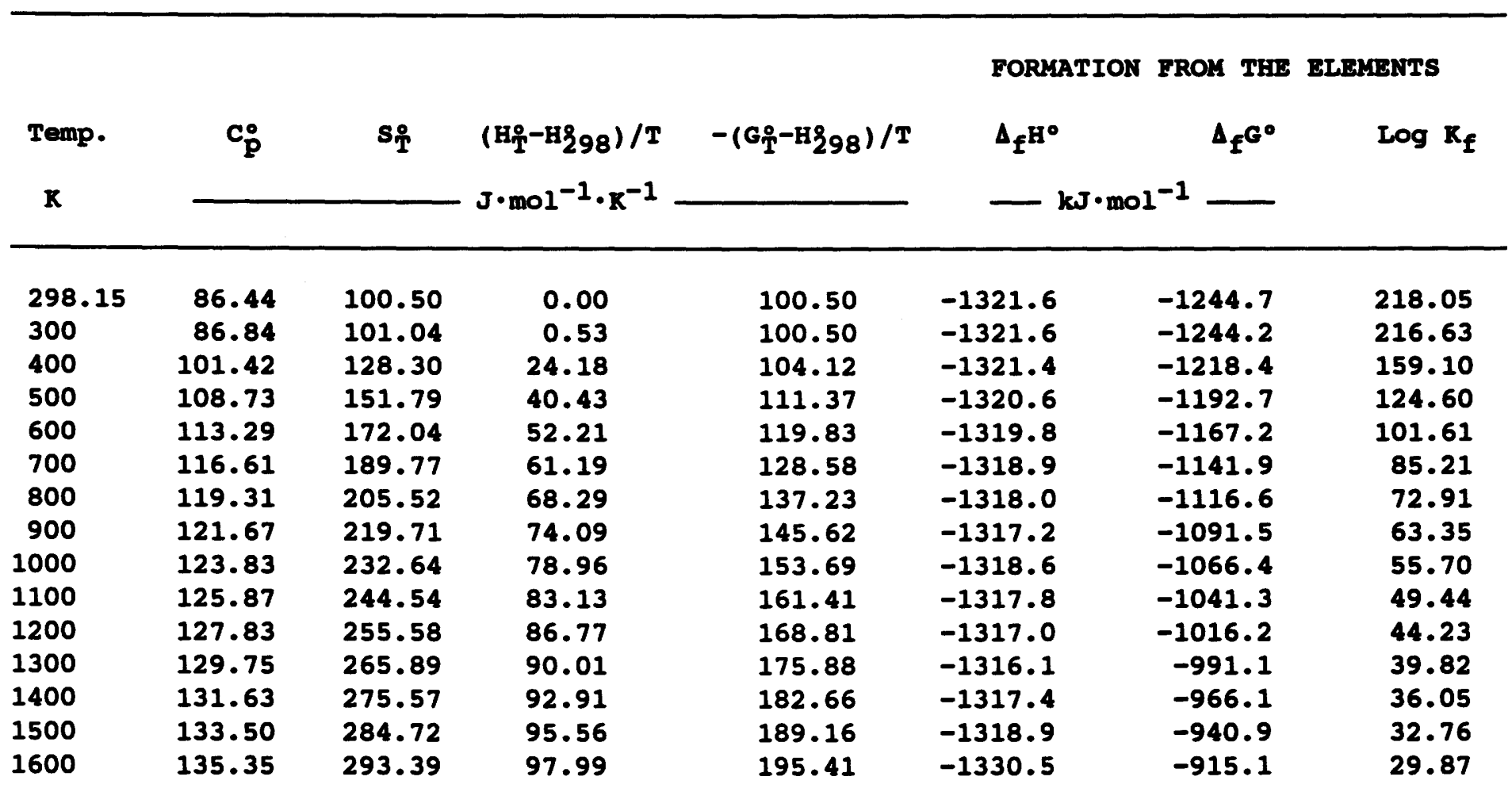

Melting $\mathbf{T}$

$\Delta_{\text {fum }} \mathrm{H}^{\circ}$

H298-H8 $\mathbf{k J}$

\section{$1564 \mathrm{~K}$}

$14.55 \mathrm{~kJ}$
Boiling $T$

$\Delta_{\text {vqp }} \mathbf{H}^{\circ}$

Molar Vol.
$\mathbf{K}$

$\mathbf{k J}$
$A=-1 \cdot 318 \mathrm{E}+03$
$B=2.513 E-01$
$C=-1.87 E+05$ 
$\mathrm{NaAlSi}_{2} \mathrm{O}_{6}$ : Monoclinic crystals 298.15 to $1300 \mathrm{~K}$.

\begin{tabular}{|c|c|c|c|c|c|c|c|}
\hline \multirow{2}{*}{$\begin{array}{l}\text { Temp. } \\
\text { K }\end{array}$} & \multirow[t]{2}{*}{$\mathbf{c}_{\mathbf{p}}^{\circ}$} & \multirow[t]{2}{*}{$\mathbf{s}_{\mathbf{T}}$} & \multirow{2}{*}{$\begin{array}{l}\left(\mathrm{H}_{\mathrm{T}}^{\circ}-\mathrm{H} 298\right) / \mathrm{T} \\
\mathrm{J} \cdot \mathrm{mol}^{-1} \cdot \mathrm{K}^{-1}\end{array}$} & \multirow[t]{2}{*}{$-\left(G_{T}^{\circ}-H_{298}\right) / T$} & \multirow{2}{*}{\multicolumn{2}{|c|}{$\begin{array}{l}\text { FORMATION FROM THE } \\
\Delta_{f^{H^{\circ}}}\end{array}$}} & \multirow[t]{2}{*}{$\begin{array}{l}\text { ELEMENTS } \\
\qquad \log \mathbf{K}_{\mathbf{f}}\end{array}$} \\
\hline & & & & & & & \\
\hline 298.15 & 159.92 & 133.50 & 0.00 & 133.50 & -3029.3 & -2850.6 & 499.40 \\
\hline 300 & 160.62 & 134.49 & 0.99 & 133.50 & -3029.3 & -2849.5 & 496.13 \\
\hline 400 & 188.41 & 184.89 & 44.71 & 140.19 & -3033.0 & -2789.1 & 364.22 \\
\hline 500 & 205.31 & 228.88 & 75.25 & 153.63 & -3032.7 & -2728.2 & 285.01 \\
\hline 600 & 217.07 & 267.41 & 97.96 & 169.45 & -3031.6 & -2667.4 & 232.21 \\
\hline 700 & 225.96 & 301.57 & 115.64 & 185.93 & -3029.9 & -2606.8 & 194.52 \\
\hline 800 & 233.06 & 332.22 & 129.89 & 202.33 & -3027.8 & -2546.5 & 166.27 \\
\hline 900 & 238.96 & 360.02 & 141.69 & 218.33 & -3025.6 & -2486.5 & 144.31 \\
\hline 1000 & 244.02 & 385.46 & 151.67 & 233.79 & -3033.8 & -2426.0 & 126.72 \\
\hline 1100 & 248.44 & 408.93 & 160.27 & 248.66 & -3031.1 & -2365.3 & 112.32 \\
\hline 1200 & 252.39 & 430.72 & 167.79 & 262.94 & -3125.0 & -2302.4 & 100.22 \\
\hline 1300 & 255.96 & 451.07 & 174.44 & 276.63 & -3121.1 & -2234.0 & 89.76 \\
\hline
\end{tabular}

Melting $T$

$\mathbf{R}$

Boiling $\mathbf{T}$

$\mathbf{K}$

$\Delta_{\text {hus }} \mathrm{H}^{\circ}$

$\mathbf{k J}$

H298-H8
$\Delta_{\mathrm{ve}} \mathrm{H}^{\circ}$

Molar vol.
$B=6.146 \mathrm{E}-01$

$A=-3.039 E+03$

(n)

$C=4.741 \mathrm{~s}+05$ 
NaFeSi $_{2} \mathrm{O}_{6}$ : Monoclinic crystals 298.15 to $1300 \mathrm{~K}$.

\begin{tabular}{|c|c|c|c|c|c|c|c|}
\hline \multirow{2}{*}{$\begin{array}{l}\text { Temp. } \\
\mathbf{K}\end{array}$} & \multirow[t]{2}{*}{$\mathbf{C}_{\mathbf{p}}^{\circ}$} & \multirow[t]{2}{*}{$s_{\text {T }}$} & \multirow{2}{*}{$\begin{array}{l}\left(\mathrm{H}_{\mathrm{T}}^{\circ}-\mathrm{H}_{298}\right) / \mathrm{T} \\
\mathrm{J} \cdot \mathrm{mol}^{-1} \cdot \mathrm{K}^{-1}\end{array}$} & \multirow[t]{2}{*}{$-\left(G_{T}^{\circ}-H_{298}\right) / T$} & \multirow{2}{*}{\multicolumn{2}{|c|}{$\begin{array}{l}\text { FORMATION FROM THE } \\
\Delta_{\mathrm{f}^{\mathrm{H}^{\circ}}} \Delta_{\mathrm{f}} \mathrm{G}^{\circ}\end{array}$}} & \multirow[t]{2}{*}{$\begin{array}{l}\text { ELEMENTS } \\
\qquad \log \mathbf{R}_{f}\end{array}$} \\
\hline & & & & & & & \\
\hline 298.15 & 169.88 & 170.57 & 0.00 & 170.57 & -2584.5 & -2417.2 & 423.48 \\
\hline 300 & 170.58 & 171.62 & 1.05 & 170.57 & -2584.5 & -2416.2 & 420.69 \\
\hline 400 & 197.52 & 224.81 & 47.17 & 177.64 & -2587.4 & -2359.8 & 308.15 \\
\hline 500 & 213.32 & 270.70 & 78.93 & 191.78 & -2586.4 & -2303.0 & 240.59 \\
\hline 600 & 224.73 & 310.65 & 102.32 & 208.33 & -2584.9 & -2246.4 & 195.57 \\
\hline 700 & 234.07 & 346.01 & 120.49 & 225.52 & -2582.9 & -2190.2 & 163.43 \\
\hline 800 & 242.31 & 377.81 & 135.21 & 242.60 & -2580.6 & -2134.3 & 139.35 \\
\hline 900 & 249.91 & 406.80 & 147.54 & 259.26 & -2578.2 & -2078.6 & 120.64 \\
\hline 1000 & 257.10 & 433.50 & 158.13 & 275.37 & -2576.2 & -2023.2 & 105.68 \\
\hline 1100 & 264.04 & 458.33 & 167.45 & 290.88 & -2574.5 & -1968.0 & 93.45 \\
\hline 1200 & 270.80 & 481.60 & 175.78 & 305.82 & -2668.5 & -1910.5 & 83.16 \\
\hline 1300 & 277.44 & 503.54 & 183.35 & 320.19 & -2662.8 & -1847.6 & 74.24 \\
\hline
\end{tabular}

Melting $\mathbf{T}$

$\mathbf{R}$

$\Delta_{\text {fuw }} \mathrm{H}^{\circ}$

$\mathbf{k J}$

$\mathrm{H} 298^{-\mathrm{H}} 8$
Boiling $\mathbf{T}$

$\Lambda_{\mathrm{vep}} \mathbf{H}^{\circ}$

Molar vol.
$\mathbf{K}$

$\mathbf{k J}$
$A=-2.587 \mathrm{E}+03$
$B=5.648 \mathrm{E}-01$
$C=9.860 \mathrm{E}+04$ 
$\mathrm{Ca}_{2} \mathrm{Mg}_{5} \mathrm{Si}_{8} \mathrm{O}_{22}(\mathrm{OH})_{2}$ : Monoclinic crystals 298.15 to $1000 \mathrm{~K}$.

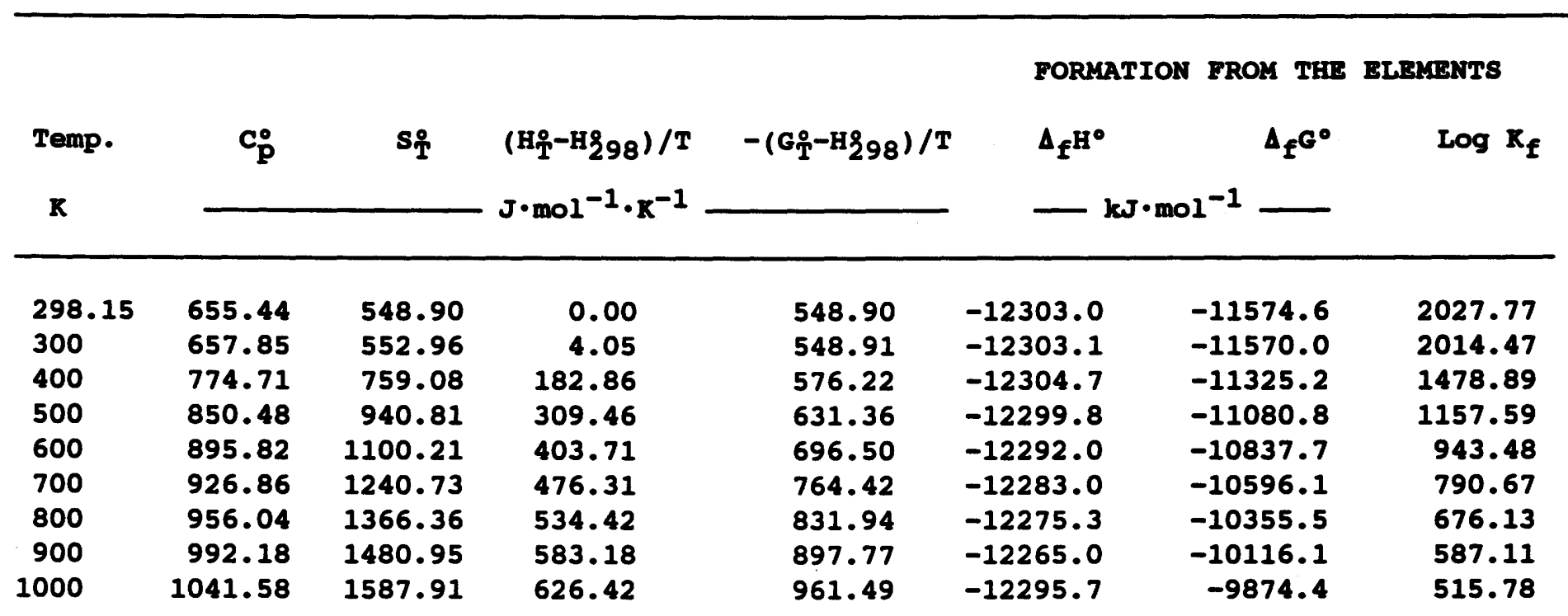

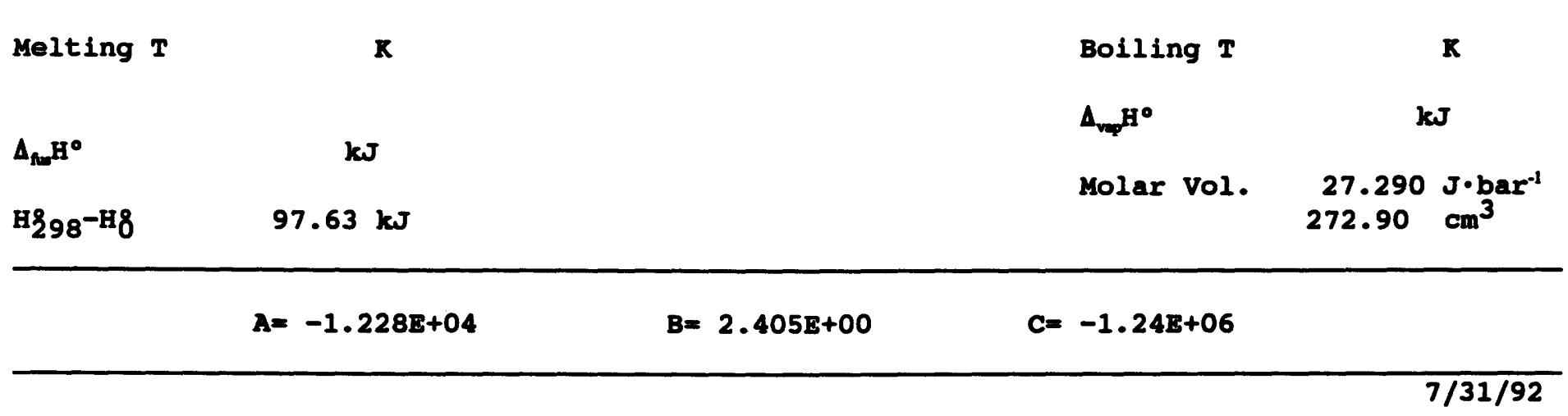


$\mathrm{Fe}_{7} \mathrm{Si}_{8} \mathrm{O}_{22}(\mathrm{OH})_{2}:$ Monoclinic crystals 298.15 to $900 \mathrm{~K}$.

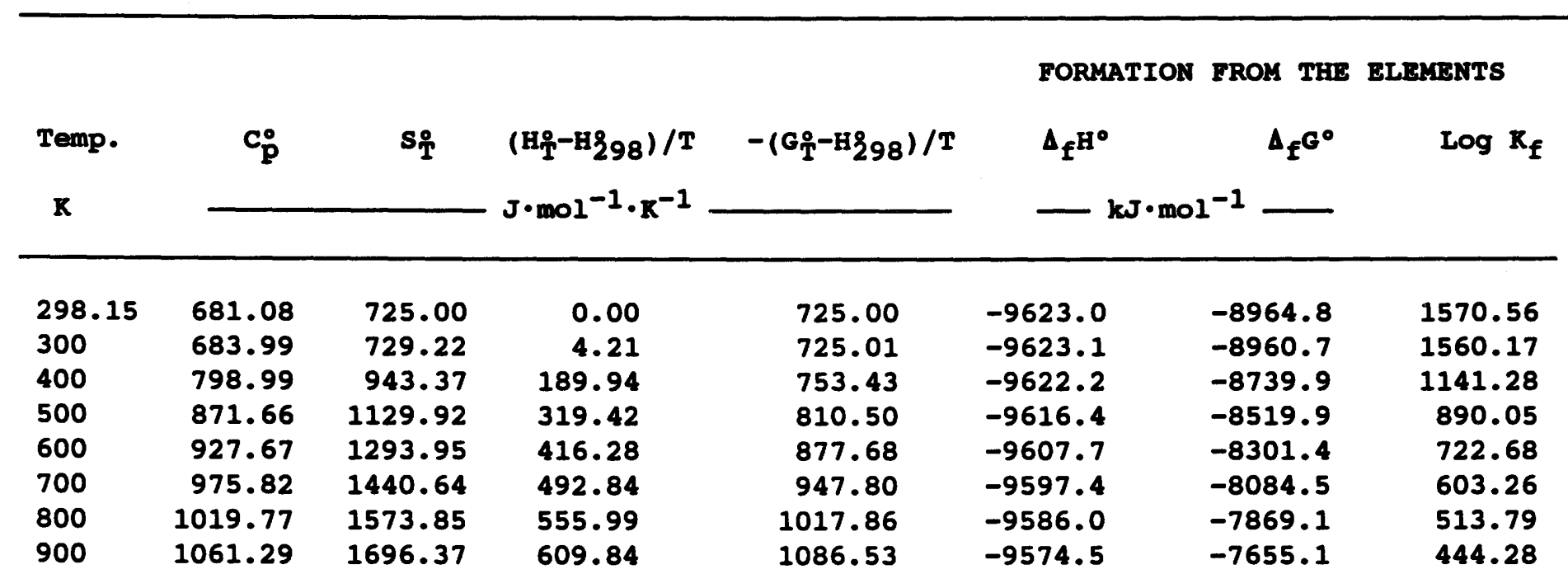

Melting $T$

$\Delta_{\text {fur }} \mathrm{H}^{\circ}$

$\mathrm{H}_{298^{-\mathrm{H}} 8}$
$\mathbf{R}$

$k J$

kJ
Boiling $\mathbf{T}$

$\mathbf{R}$

$\Delta_{\mathrm{vep}} \mathrm{H}^{\circ}$

$\mathbf{k J}$

Molar vol. $\begin{aligned} & 27.870 \mathrm{~J} \cdot \mathrm{bar}^{-1} \\ & 278.70 \mathrm{~cm}^{3}\end{aligned}$ 
$\mathrm{Kg}_{7} \mathrm{Si}_{8} \mathrm{O}_{22}(\mathrm{OH})_{2}:$ Konoclinic crystals 298.15 to $1200 \mathrm{~K}$.

\begin{tabular}{|c|c|c|c|c|c|c|c|c|}
\hline \multirow{2}{*}{$\begin{array}{l}\text { Temp. } \\
\mathbf{K}\end{array}$} & \multirow[t]{2}{*}{$\mathbf{c}_{\mathbf{p}}^{\circ}$} & \multirow[t]{2}{*}{$\mathbf{s}_{\mathbf{T}}^{\circ}$} & \multirow{2}{*}{$\begin{array}{l}\left(\mathrm{H}_{\mathrm{T}}^{\circ}-\mathrm{H} 298\right) / \mathrm{T} \\
\mathrm{J} \cdot \mathrm{mol}^{-1} \cdot \mathrm{K}^{-1}\end{array}$} & \multirow[t]{2}{*}{$-\left(G_{T}^{\circ}-H_{298}\right) / T$} & \multirow{2}{*}{\multicolumn{3}{|c|}{$\begin{array}{l}\text { FORMATION FROM THE } \\
\Delta_{f^{H}}{ }^{\circ} \quad \Delta_{f^{G}}{ }^{\circ}\end{array}$}} & \multirow[t]{2}{*}{$\begin{array}{l}\text { ELEMENTS } \\
\qquad \log K_{\mathbf{f}}\end{array}$} \\
\hline & & & & & & & & \\
\hline 298.15 & 664.02 & 534.50 & 0.00 & 534.50 & -120 & 070.0 & -11343.4 & 1987.26 \\
\hline 300 & 667.12 & 538.62 & 4.10 & 534.51 & -120 & 070.1 & -11338.8 & 1974.22 \\
\hline 400 & 788.45 & 748.97 & 186.60 & 562.37 & -120 & 070.0 & -11094.9 & 1448.81 \\
\hline 500 & 859.43 & 933.12 & 314.60 & 618.53 & -120 & 063.8 & -10851.7 & 1133.64 \\
\hline 600 & 907.21 & 1094.29 & 409.61 & 684.68 & -120 & 054.7 & -10610.1 & 923.67 \\
\hline 700 & 942.29 & 1236.89 & 483.32 & 753.58 & -120 & 043.9 & -10370.2 & 773.82 \\
\hline 800 & 969.58 & 1364.57 & 542.46 & 822.11 & -120 & 032.2 & -10131.9 & 661.53 \\
\hline 900 & 991.72 & 1480.09 & 591.19 & 888.90 & -120 & 020.3 & -9895.0 & 574.28 \\
\hline 1000 & 1010.24 & 1585.56 & 632.19 & 953.37 & -120 & 068.5 & -9654.6 & 504.29 \\
\hline 1100 & 1026.11 & 1682.61 & 667.30 & 1015.31 & -120 & 057.1 & -9413.7 & 447.01 \\
\hline 1200 & 1039.97 & 1772.50 & 697.79 & 1074.71 & -120 & 045.0 & -9174.0 & 399.32 \\
\hline
\end{tabular}

Melting T

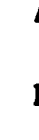

\section{$\mathbf{K}$}

$\mathbf{k J}$
Boiling $\mathbf{T}$

$\Delta_{\mathrm{vq}} \mathrm{H}^{\circ}$

Molar Vol.

\section{$\mathbf{R}$}

ks

H298-H8 $96.78 \mathrm{~kJ}$ $26.540 \mathrm{~J} \cdot \mathrm{bar}^{-1}$
$265.40 \mathrm{~cm}^{3}$

$A=-1.204 E+04$

$B=2.389 E+00$

$C=-1.29 \mathrm{E}+06$ 
$\mathrm{Na}_{2} \mathrm{Mg}_{3} \mathrm{Al}_{2} \mathrm{Si}_{8} \mathrm{O}_{22}(\mathrm{OH})_{2}:$ Monoclinic crystals 298.15 to $1200 \mathrm{~K}$.

\begin{tabular}{|c|c|c|c|c|c|c|c|c|}
\hline \multirow{2}{*}{$\begin{array}{l}\text { Temp. } \\
\mathbf{K}\end{array}$} & \multirow[t]{2}{*}{$\mathbf{c}_{\mathbf{p}}^{\circ}$} & \multirow[t]{2}{*}{$s_{\mathrm{T}}$} & \multirow{2}{*}{$\begin{array}{l}\left(\mathrm{H}_{\mathrm{T}}^{\circ}-\mathrm{H} 298\right) / \mathrm{T} \\
\mathrm{J} \cdot \mathrm{mol}^{-1} \cdot \mathrm{K}^{-1}\end{array}$} & \multirow[t]{2}{*}{$-\left(G_{T}^{\circ}-H_{298}\right) / T$} & \multirow{2}{*}{\multicolumn{3}{|c|}{$\begin{array}{l}\text { FORMATION FROM THE } \\
\Delta_{f^{H}} H^{\circ}\end{array}$}} & \multirow[t]{2}{*}{$\begin{array}{l}\text { ELELENTS } \\
\qquad \log \mathrm{K}_{\mathrm{f}}\end{array}$} \\
\hline & & & & & & & & \\
\hline 298.15 & 645.48 & 541.20 & 0.00 & 541.20 & -119 & 964.0 & -11230.8 & 1967.54 \\
\hline 300 & 647.73 & 545.20 & 3.99 & 541.21 & -119 & 964.1 & -11226.2 & 1954.61 \\
\hline 400 & 750.28 & 746.42 & 178.46 & 567.95 & -119 & 973.3 & -10979.0 & 1433.68 \\
\hline 500 & 823.97 & 922.19 & 300.59 & 621.60 & -119 & 971.7 & -10730.6 & 1120.99 \\
\hline 600 & 878.30 & 1077.46 & 392.56 & 684.89 & -119 & 966.2 & -10482.8 & 912.59 \\
\hline 700 & 919.33 & 1216.07 & 465.02 & 751.05 & -119 & 958.0 & -10236.2 & 763.82 \\
\hline 800 & 950.88 & 1340.98 & 523.86 & 817.12 & -119 & 948.3 & -9990.9 & 652.32 \\
\hline 900 & 975.41 & 1454.45 & 572.73 & 881.73 & -119 & 937.7 & -9746.8 & 565.68 \\
\hline 1000 & 994.60 & 1558.26 & 613.99 & 944.26 & -119 & 973.7 & -9500.4 & 496.24 \\
\hline 1100 & 1009.62 & 1653.79 & 649.31 & 1004.48 & -119 & 962.3 & -9253.5 & 439.40 \\
\hline 1200 & 1021.32 & 1742.16 & 679.84 & 1062.32 & -121 & 144.0 & -9003.0 & 391.88 \\
\hline
\end{tabular}

Melting $T$

$\Delta_{\text {tux }} \mathrm{H}^{\circ}$

H298-H8 $\mathbf{k J}$

K $95.62 \mathrm{~kJ}$
Boiling $T$

$\Delta_{\mathrm{vep}} \mathrm{H}^{\circ}$

Molar Vol. $\begin{aligned} & 26.210 \mathrm{~J} \cdot \mathrm{bar}^{-1} \\ & 262.10 \mathrm{~cm}^{3}\end{aligned}$
$A=-1.196 \mathrm{E}+04$
$B=2.462 \mathrm{E}+00$
$C=-4.11 \mathrm{E}+05$ 


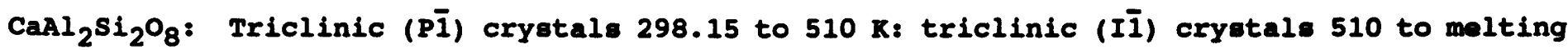
point $1830 \mathrm{~K}$.

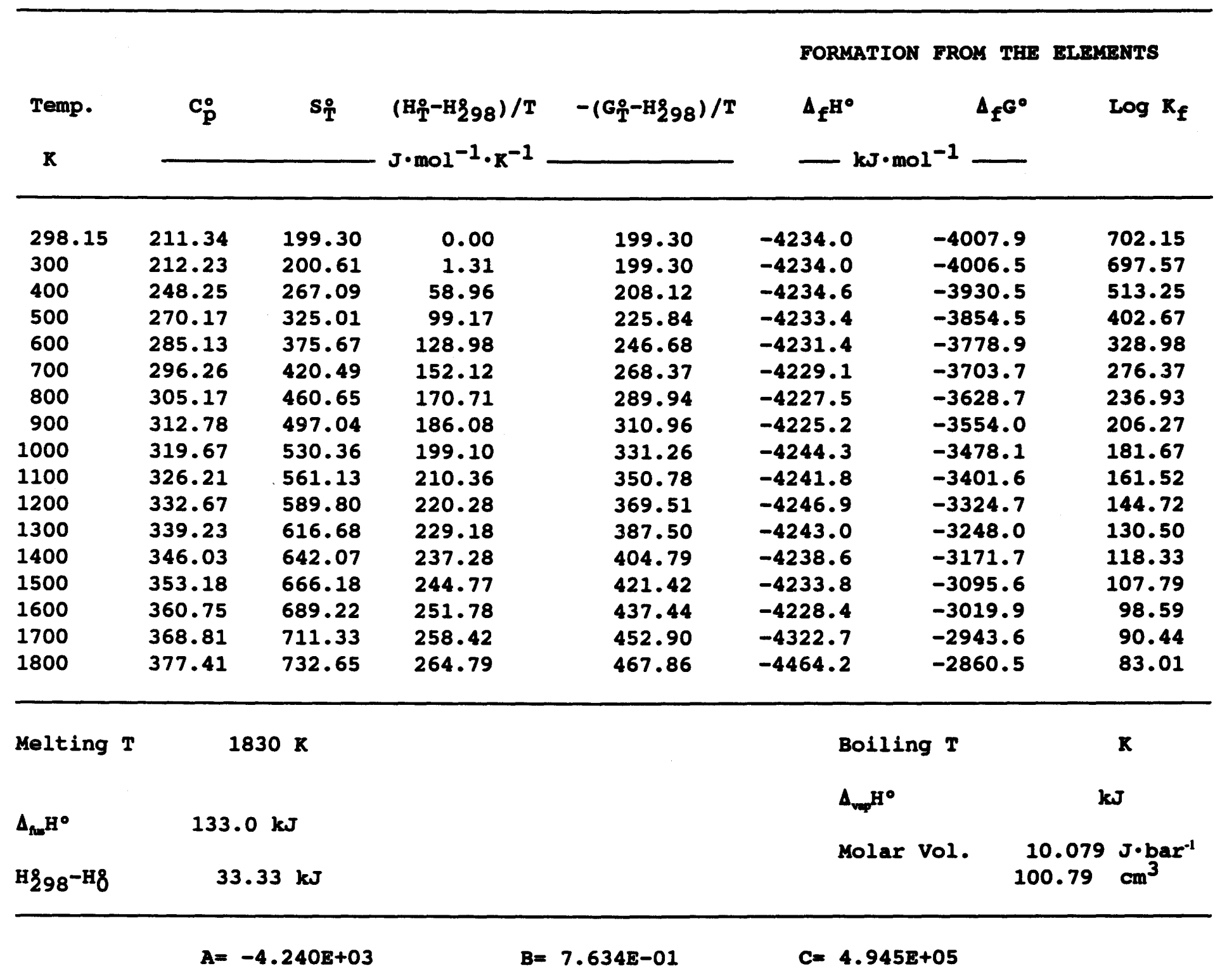


$\mathrm{CaAl}_{2} \mathrm{Si}_{2} \mathrm{O}_{8}$ : Glass 298.15 to $1500 \mathrm{~K}$.

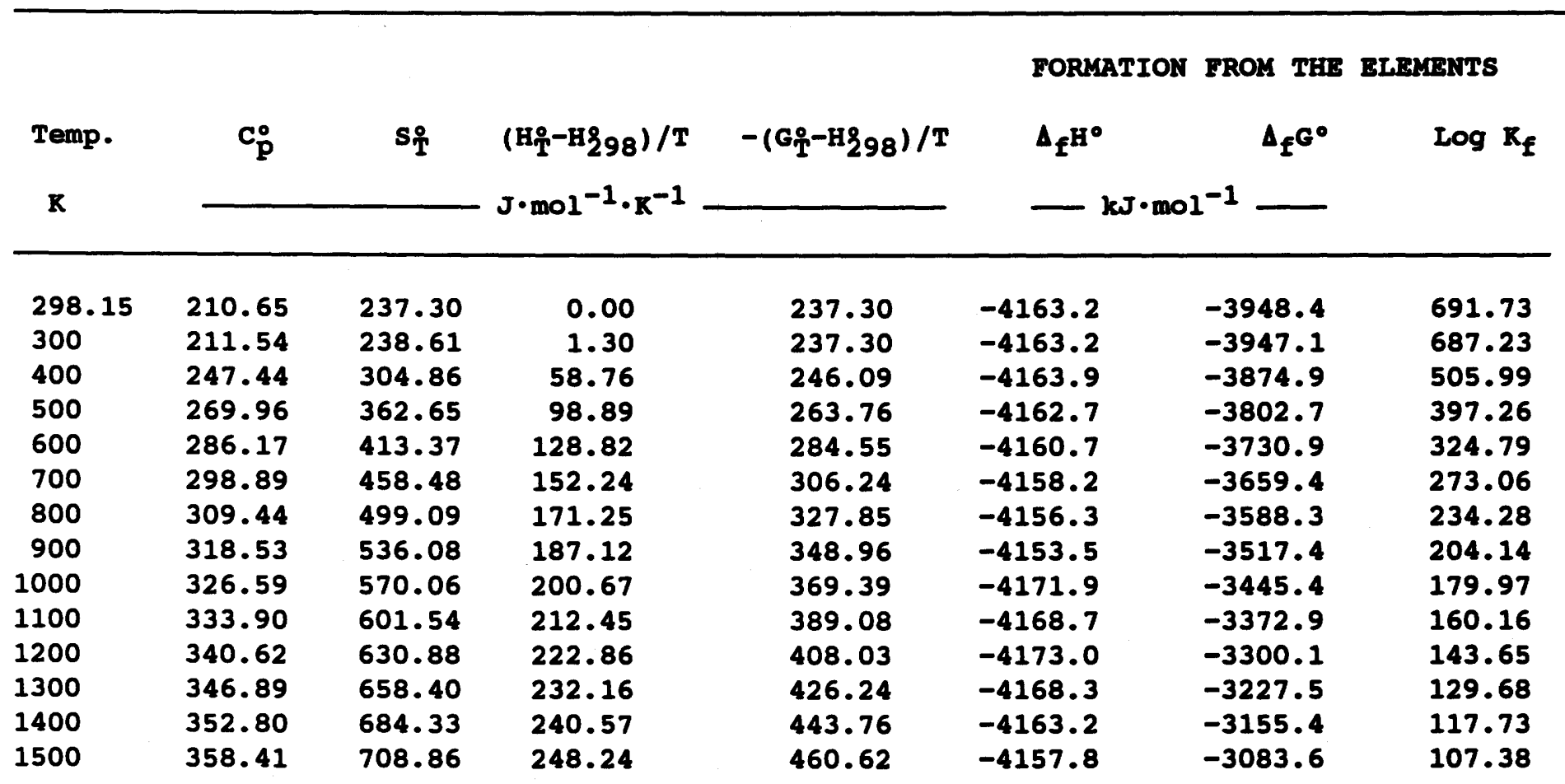

Melting $\mathbf{T}$

$\mathbf{K}$

Boiling $\mathbf{T}$

K

\begin{tabular}{lccc}
$\Delta_{\mathrm{fu}} \mathrm{H}^{\circ}$ & $\mathrm{kJ}$ & $\Delta_{\mathrm{vep}}^{\mathrm{H}^{\circ}}$ \\
$\mathrm{H}_{298^{-\mathrm{H} 8}}$ & $33.15 \mathrm{~kJ}$ & Molar Vol. \\
\hline & $\mathrm{A}=-4.164 \mathrm{E}+03$ & $B=7.199 \mathrm{E}-01$ & $\mathrm{C}=1.207 \mathrm{E}+05$
\end{tabular}


$\mathrm{Ca}_{2} \mathrm{Al}_{2} \mathrm{SiO}_{6}(\mathrm{OH})_{2}$ : Cubic crystals 298.15 to $1800 \mathrm{~K}$.

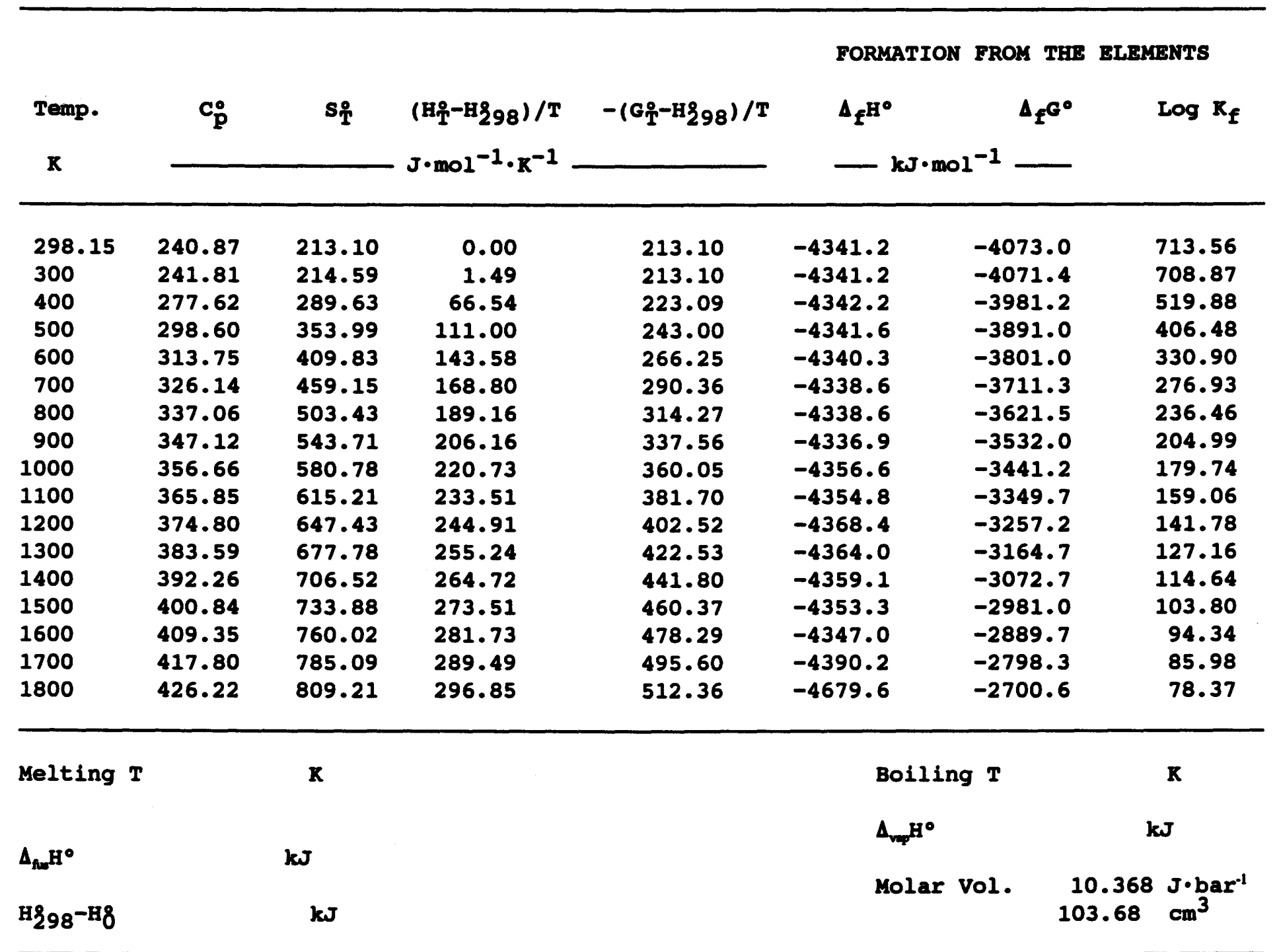


$\mathrm{Ca}_{4} \mathrm{Al}_{6} \mathrm{Si}_{6} \mathrm{O}_{24} \mathrm{CO}_{3}$ : Tetragonal crystals 298.15 to $1200 \mathrm{~K}$.

FORMATION FROM THE ELEMENTS

\begin{tabular}{|c|c|c|c|c|}
\hline Temp. & $\mathbf{c}_{\mathbf{p}}^{0}$ & $\mathbf{s}_{\mathbf{T}}^{\circ}$ & (H\%-H298)/T & $-\left(G_{T}^{\circ}-H_{298}\right) / T$ \\
\hline
\end{tabular}

\begin{tabular}{rrrrrrrr}
\hline 298.15 & 709.51 & 715.20 & 0.00 & 715.20 & -13881.4 & -13131.8 & 2300.58 \\
300 & 713.05 & 719.60 & 4.39 & 715.21 & -13881.5 & -13127.1 & 2285.58 \\
400 & $\mathbf{8 4 4 . 2 9}$ & 945.01 & 199.95 & 745.06 & -13882.2 & -12875.3 & 1681.30 \\
500 & 915.47 & 1141.70 & 336.51 & 805.18 & -13877.0 & -12624.1 & 1318.80 \\
600 & 963.02 & 1313.04 & 437.18 & $\mathbf{8 7 5 . 8 6}$ & -13869.8 & -12374.1 & 1077.24 \\
700 & 999.41 & 1464.33 & 514.99 & 949.33 & -13861.6 & -12125.5 & 904.80 \\
800 & 1029.84 & 1599.81 & $\mathbf{5 7 7 . 4 9}$ & 1022.32 & -13856.8 & -11877.6 & 775.51 \\
900 & 1056.82 & 1722.70 & 629.28 & 1093.42 & -13848.8 & -11630.7 & 675.01 \\
1000 & 1081.65 & 1835.34 & 673.29 & 1162.05 & -13905.1 & -11380.3 & 594.43 \\
1100 & 1105.08 & 1939.54 & 711.49 & 1228.06 & -13896.8 & -11128.1 & 528.42 \\
1200 & 1127.55 & 2036.67 & 745.23 & 1291.44 & -13919.2 & -10874.3 & 473.33
\end{tabular}
$A=-1.387 \mathrm{E}+04$
$B=2.495 \mathrm{E}+00$
$C=-2 \cdot 94 \mathrm{E}+05$ 
KAlsi ${ }_{3} \mathrm{O}_{8}:$ Triclinic cryatals 298.15 to $1400 \mathrm{~K}$.

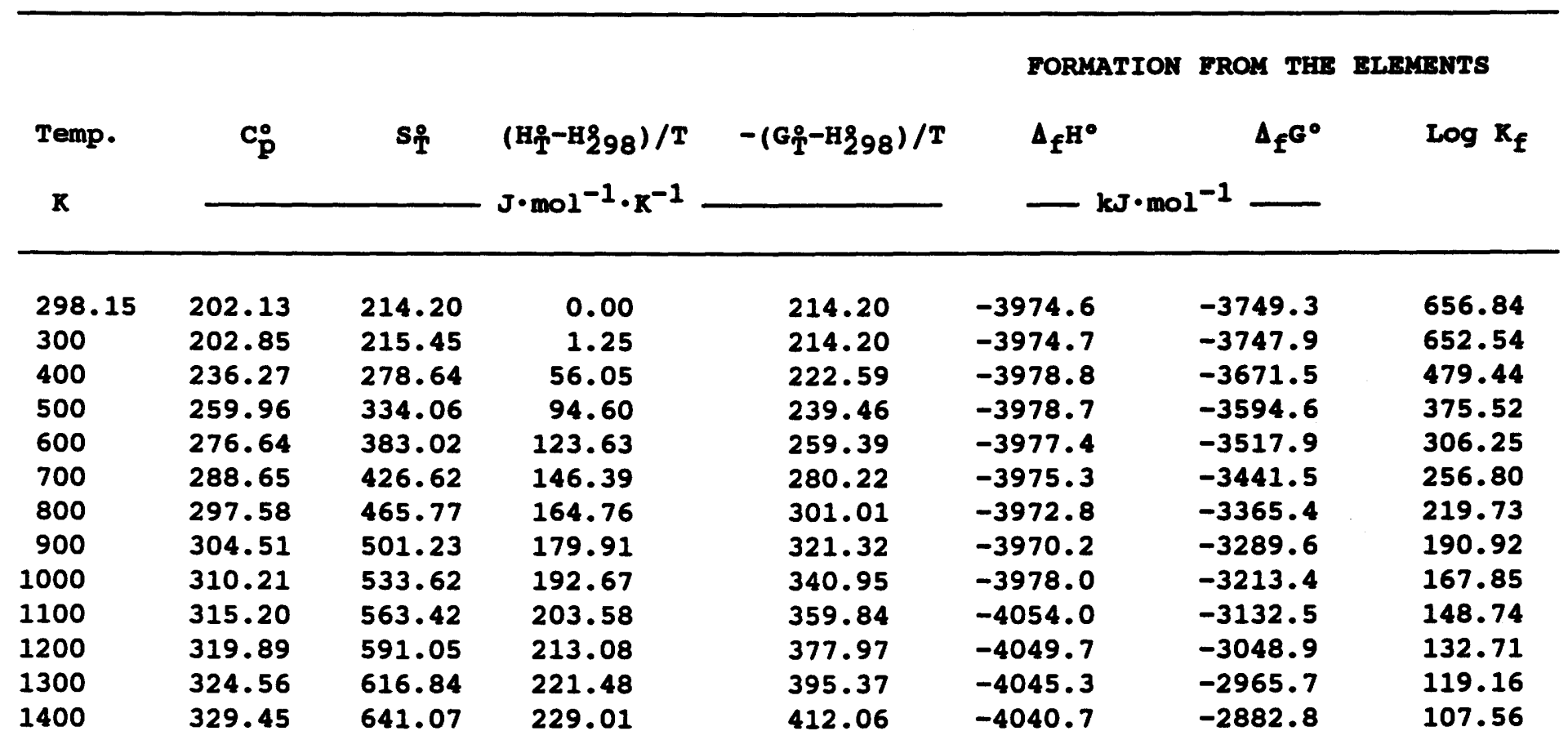

\begin{tabular}{lccc} 
Melting $T$ & $K$ & Boiling T & $K$ \\
$\Delta_{\text {fus }} \mathrm{H}^{\circ}$ & $\mathrm{kJ}$ & $\Delta_{\text {ves }} \mathrm{H}^{\circ}$ & $\mathrm{kJ}$ \\
$\mathrm{H} 298^{-\mathrm{H} 8}$ & $33.99 \mathrm{~kJ}$ & Molar Vol. & $10.872 \mathrm{~J} \cdot \mathrm{bar}^{-1}$ \\
\hline
\end{tabular}
$A=-4.002 \mathrm{E}+03$
$B=7.941 E-01$
$C=1.562 \mathrm{E}+06$ 
$\mathrm{KAlSi}_{3} \mathrm{O}_{8}$ : Monoclinic crystals 298.15 to $1400 \mathrm{~K}$.

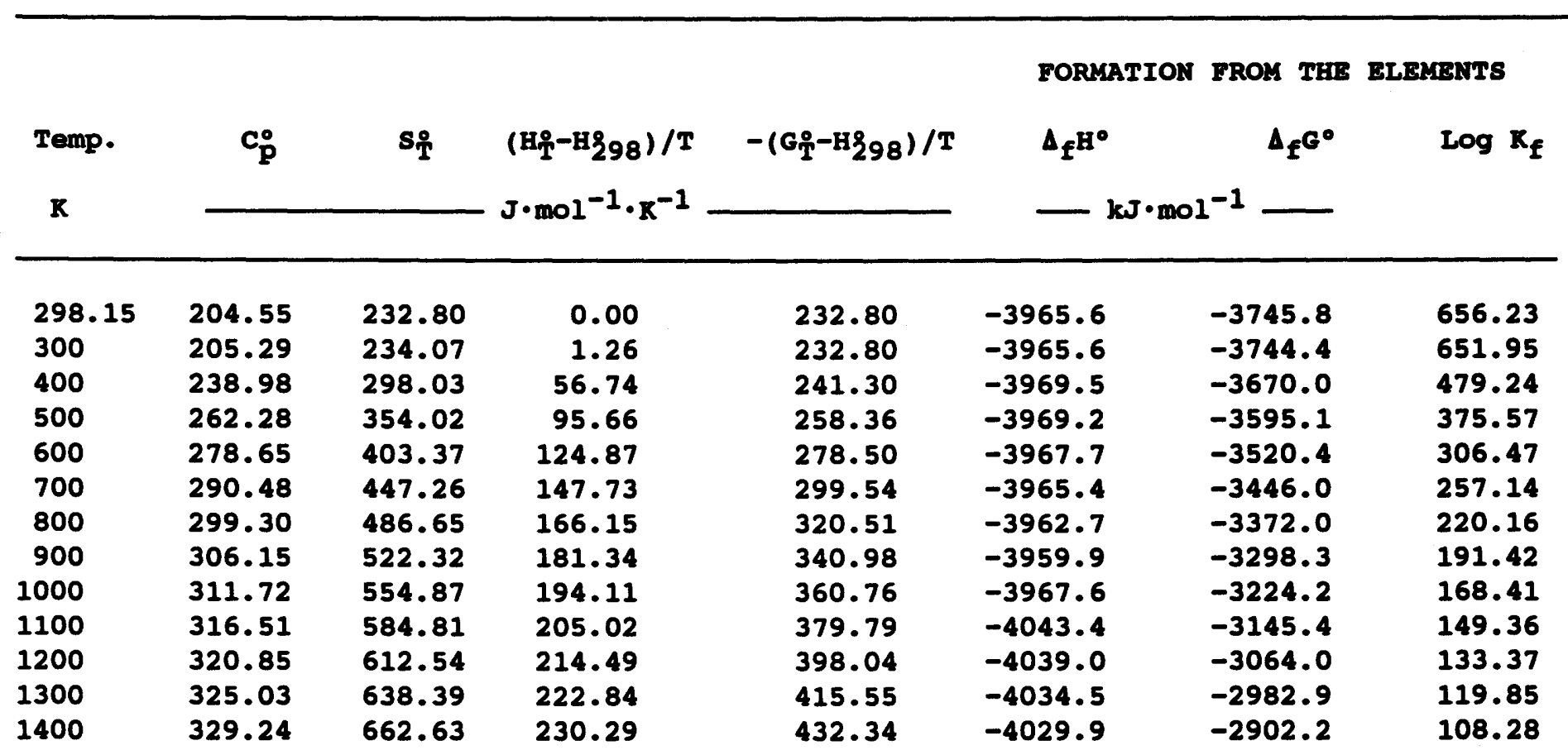

Melting $T \quad 1473 \mathrm{~K}$

$\Delta_{\text {fut }} \mathrm{H}^{\circ}$

$\mathbf{k J}$

H298-H8
Boiling T

$\Delta_{\mathrm{veg}} \mathrm{H}^{\circ}$

Molar Vol.
$\mathbf{R}$

$\mathbf{k J}$ $10.905 \mathrm{~J} \cdot \mathrm{bar}^{-1}$ $109.05 \mathrm{~cm}^{3}$
$A=-3.991 \mathrm{E}+03$
$B=7.728 \mathrm{E}-01$
$C=1.497 \mathrm{E}+06$ 
$\mathrm{KAlSi}_{3} \mathrm{O}_{8}$ : Glass 298.15 to $1300 \mathrm{~K}$.

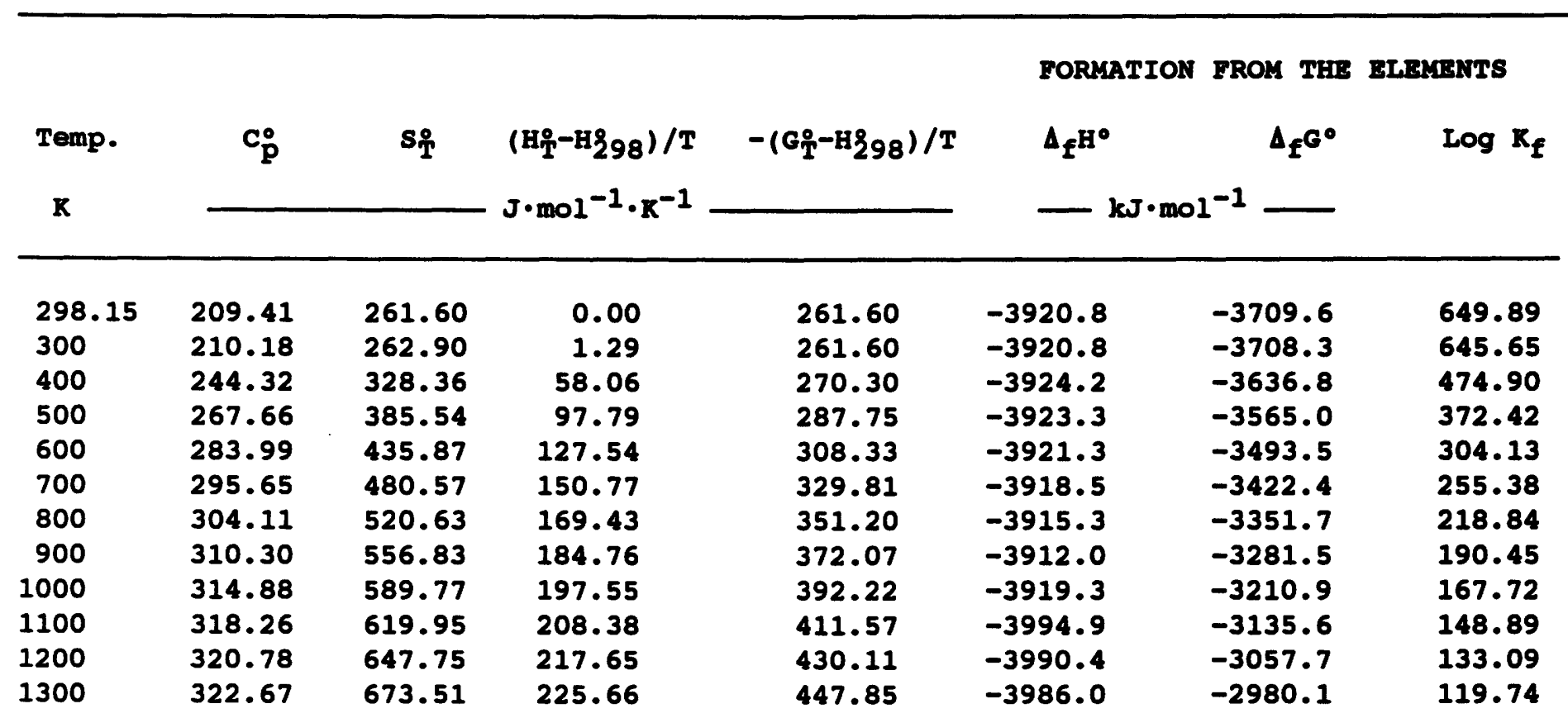

Melting $\mathbf{T}$

$\boldsymbol{\Delta}_{\text {fur }} \mathrm{H}^{\circ}$

H298-H8 $\mathbf{k J}$

$\mathbf{R}$

$34.88 \mathrm{~kJ}$
Boiling $T$

$\Delta_{\mathrm{v}} \mathrm{H}^{\circ}$

Molar vol.
K

$\mathbf{k J}$ 
$\mathrm{KAlsiO}_{4}$ : Hexagonal cryatals 298.15 to $810 \mathrm{~K}$. High kaliophillite 810 to $1800 \mathrm{~K}$.

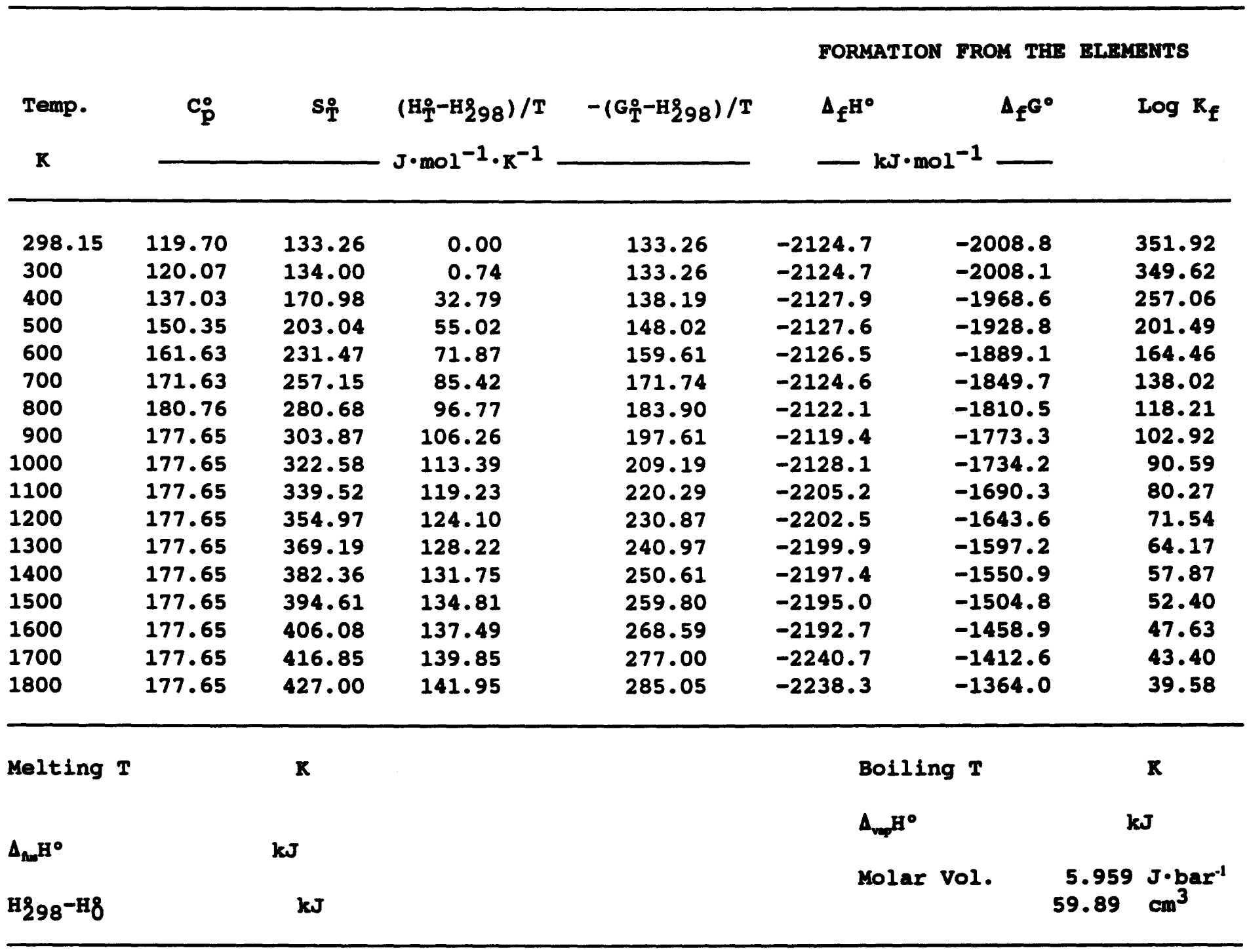


$\mathrm{KAlSi}_{2} \mathrm{O}_{6}$ : Tetragonal crystals 298.15 to $955 \mathrm{~K}$. Cubic crystals 955 to melting point $1959 \mathrm{~K}$.

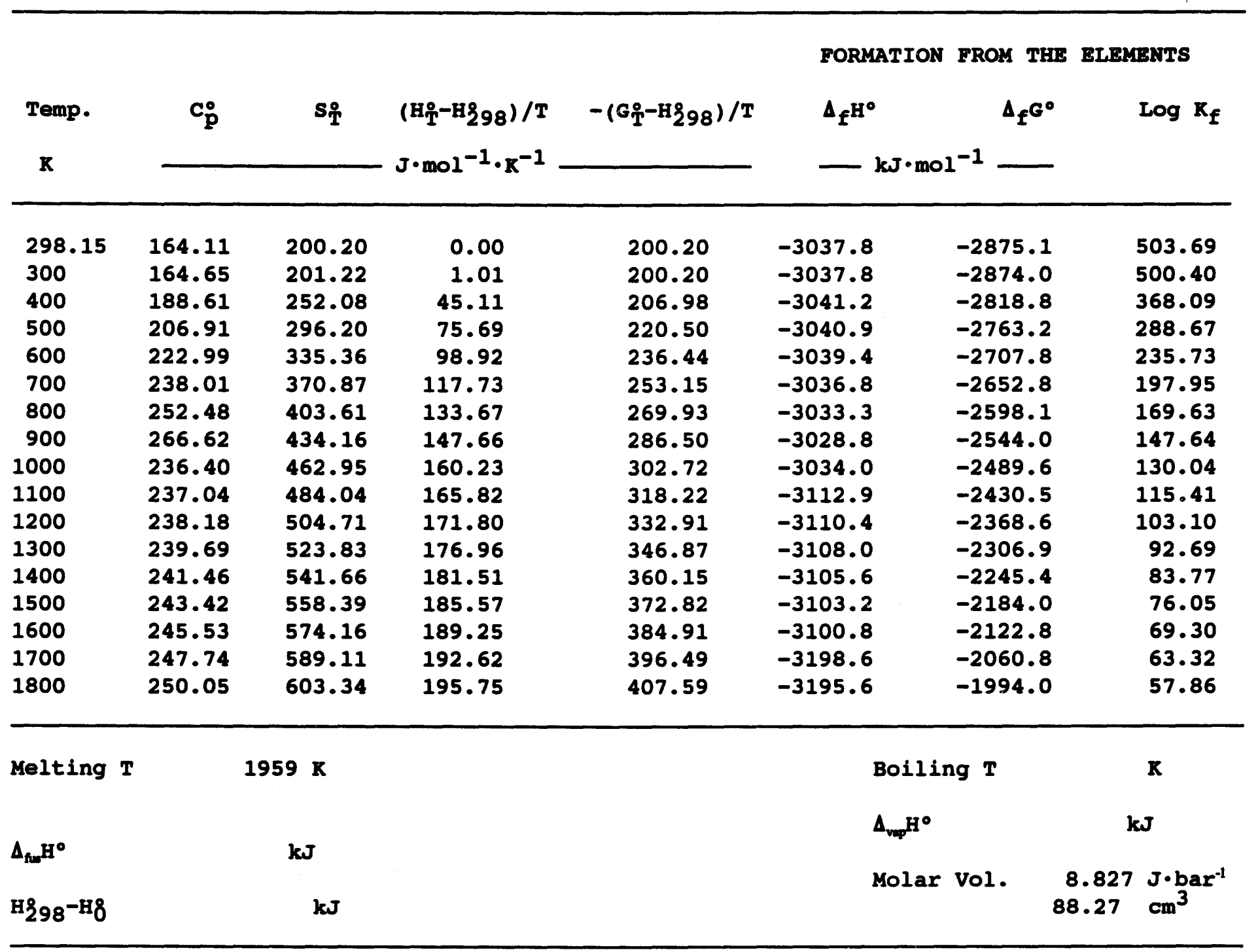


IIAlsio $_{4}: \quad \alpha$-eucryptite 298.15 to $1300 \mathrm{~K}$. B-eucryptite 1300 to $1600 \mathrm{~K}$

\begin{tabular}{|c|c|c|c|c|c|c|c|}
\hline \multirow{2}{*}{$\begin{array}{l}\text { Temp. } \\
\mathbf{K}\end{array}$} & \multirow[t]{2}{*}{$\mathbf{c}_{\mathbf{p}}^{\circ}$} & \multirow[t]{2}{*}{$\mathbf{s}_{\mathrm{I}}$} & \multirow{2}{*}{$\begin{array}{l}\left(\mathrm{H}_{\mathrm{T}}-\mathrm{H}_{298}\right) / \mathrm{T} \\
\mathrm{J} \cdot \mathrm{mol}^{-1} \cdot \mathrm{K}^{-1}\end{array}$} & \multirow[t]{2}{*}{$-\left(G \stackrel{T}{T}-H_{28}\right) / T$} & \multirow{2}{*}{\multicolumn{2}{|c|}{$\begin{array}{l}\text { FORMATION FROM THE } \\
\Delta_{\mathbf{f}^{\mathrm{H}^{\circ}}} \Delta_{\mathbf{f}^{\circ}} \mathrm{G}^{\circ}\end{array}$}} & \multirow[t]{2}{*}{$\begin{array}{l}\text { ELEMENTS } \\
\qquad \log \mathrm{K}_{\mathbf{f}}\end{array}$} \\
\hline & & & & & & & \\
\hline 298.15 & 113.30 & 103.80 & 0.00 & 103.80 & -2123.3 & -2009.2 & 352.00 \\
\hline 300 & 113.85 & 104.50 & 0.70 & 103.80 & -2123.3 & -2008.5 & 349.70 \\
\hline 400 & 136.04 & 140.59 & 32.02 & 108.57 & -2123.9 & -1970.1 & 257.26 \\
\hline 500 & 149.80 & 172.53 & 54.29 & 118.24 & -2126.5 & -1931.4 & 201.76 \\
\hline 600 & 159.40 & 200.73 & 71.05 & 129.68 & -2125.4 & -1892.4 & 164.75 \\
\hline 700 & 166.58 & 225.87 & 84.21 & 141.66 & -2123.9 & -1853.7 & 138.32 \\
\hline 800 & 172.22 & 248.49 & 94.87 & 153.62 & $-2122 \cdot 0$ & -1815.2 & 118.52 \\
\hline 900 & 176.81 & 269.05 & 103.73 & 165.32 & -2119.9 & -1777.0 & 103.13 \\
\hline 1000 & 180.63 & 287.88 & 111.23 & 176.65 & -2128.4 & -1738.3 & 90.80 \\
\hline 1100 & 183.88 & 305.26 & 117.69 & 187.56 & -2125.9 & -1699.4 & 80.70 \\
\hline 1200 & 186.69 & 321.38 & 123.33 & 198.05 & $-2123 \cdot 2$ & -1660.7 & 72.29 \\
\hline 1300 & 189.16 & 336.42 & 128.30 & 208.12 & -2120.3 & -1622.3 & 65.18 \\
\hline 1400 & 191.34 & 350.52 & 132.73 & 217.80 & -2117.4 & -1584.2 & 59.10 \\
\hline 1500 & 193.29 & 363.79 & 136.70 & 227.09 & -2114.3 & -1546.2 & 53.84 \\
\hline 1600 & 195.05 & 376.32 & 140.29 & 236.03 & -2111.1 & -1508.4 & 49.24 \\
\hline
\end{tabular}

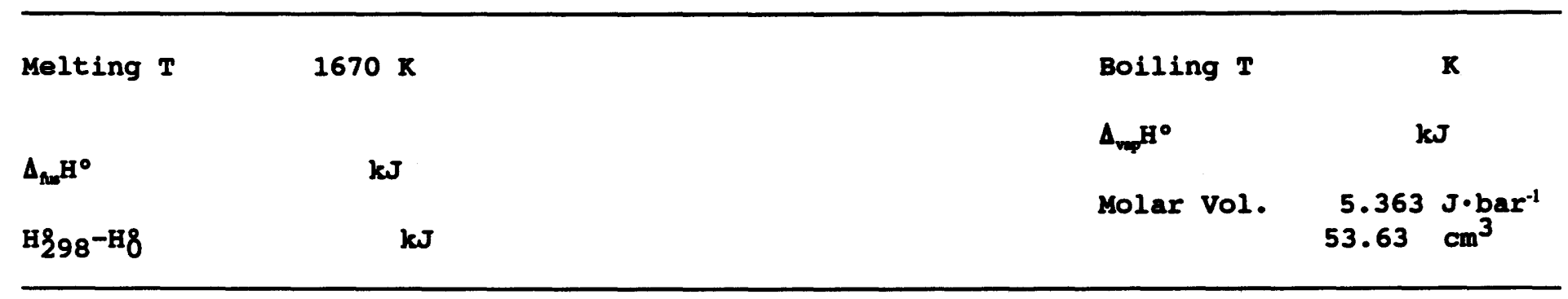
$A=-2 \cdot 123 E+03$
$B=3.846 E-01$
$C=-1 \cdot 10 E+05$ 
$\mathrm{LiAlsi}_{4} \mathrm{O}_{10}$ : Monoclinic crystals 298.15 to $1300 \mathrm{~K}$. Petalite decomposes above about $1100 \mathrm{~K}$.

\begin{tabular}{|c|c|c|c|c|c|c|c|}
\hline \multirow{2}{*}{$\begin{array}{l}\text { Temp. } \\
\mathbf{K}\end{array}$} & \multirow[t]{2}{*}{$\mathbf{c}_{\mathbf{p}}^{\circ}$} & \multirow[t]{2}{*}{$\mathbf{s}_{\text {T }}$} & \multirow{2}{*}{$\begin{array}{l}\left(\mathrm{H}_{\mathrm{T}}-\mathrm{H} 298\right) / \mathrm{T} \\
\mathrm{J} \cdot \mathrm{mol}^{-1} \cdot \mathrm{K}^{-1}\end{array}$} & \multirow[t]{2}{*}{$-\left(G_{T}^{\circ}-H_{298}\right) / T$} & \multirow{2}{*}{\multicolumn{2}{|c|}{$\begin{array}{l}\text { FORMATION FROM THE } \\
\Delta_{f^{\mathrm{H}^{\circ}}} \Delta_{\mathrm{f}^{\circ}}\end{array}$}} & \multirow[t]{2}{*}{$\begin{array}{l}\text { ELEMENTS } \\
\qquad \log k_{f}\end{array}$} \\
\hline & & & & & & & \\
\hline 298.15 & 245.30 & 233.20 & 0.00 & 233.20 & -4886.5 & -4610.7 & 807.75 \\
\hline 300 & 246.32 & 234.72 & 1.52 & 233.20 & -4886.6 & -4608.9 & 802.47 \\
\hline 400 & 292.33 & 312.31 & 68.84 & 243.47 & -4887.9 & -4516.1 & 589.73 \\
\hline 500 & 323.66 & 381.13 & 116.87 & 264.26 & -4889.9 & -4422.9 & 462.05 \\
\hline 600 & 345.43 & 442.18 & 153.25 & 288.92 & -4887.5 & -4329.8 & 376.93 \\
\hline 700 & 360.93 & 496.65 & 181.87 & 314.78 & -4884.0 & -4237.1 & 316.17 \\
\hline 800 & 372.26 & 545.63 & 205.00 & 340.63 & -4879.9 & -4144.9 & 270.63 \\
\hline 900 & 380.77 & 589.99 & 224.08 & 365.91 & -4875.5 & -4053.3 & 235.24 \\
\hline 1000 & 387.40 & 630.46 & 240.09 & 390.37 & -4881.6 & -3961.5 & 206.92 \\
\hline 1100 & 392.77 & 667.64 & 253.73 & 413.91 & -4876.8 & -3869.7 & 183.75 \\
\hline 1200 & 397.36 & 702.02 & 265.52 & 436.50 & -4871.9 & -3778.3 & 164.46 \\
\hline 1300 & 401.52 & 733.99 & 275.82 & 458.17 & -4866.9 & -3687.4 & 148.16 \\
\hline
\end{tabular}

\begin{tabular}{|c|c|c|c|c|}
\hline Melting $T$ & $\mathbf{R}$ & Boiling $T$ & & $\mathbf{R}$ \\
\hline & & $\Delta_{\mathrm{v} \varphi} \mathrm{H}^{0}$ & & $\mathbf{k J}$ \\
\hline $\begin{array}{l}\Delta_{\text {fur }} \mathrm{H}^{\circ} \\
\mathrm{H} 298^{-\mathrm{H}} \mathrm{\delta}\end{array}$ & $\begin{array}{c}\mathrm{kJ} \\
38.31 \mathrm{~kJ}\end{array}$ & Molar vol. & $\begin{array}{c}12.840 \\
128.40\end{array}$ & $\begin{array}{l}J \cdot \text { bar }^{-1} \\
\mathrm{~cm}^{3}\end{array}$ \\
\hline
\end{tabular}

$A=-4.879 \mathrm{E}+03 \quad B=9.174 \mathrm{E}-01 \quad \mathrm{C}=-5.17 \mathrm{E}+05$ 
NaAlsi $\mathrm{O}_{8}:$ Triclinic crystals 298.15 to $1400 \mathrm{~K}$.

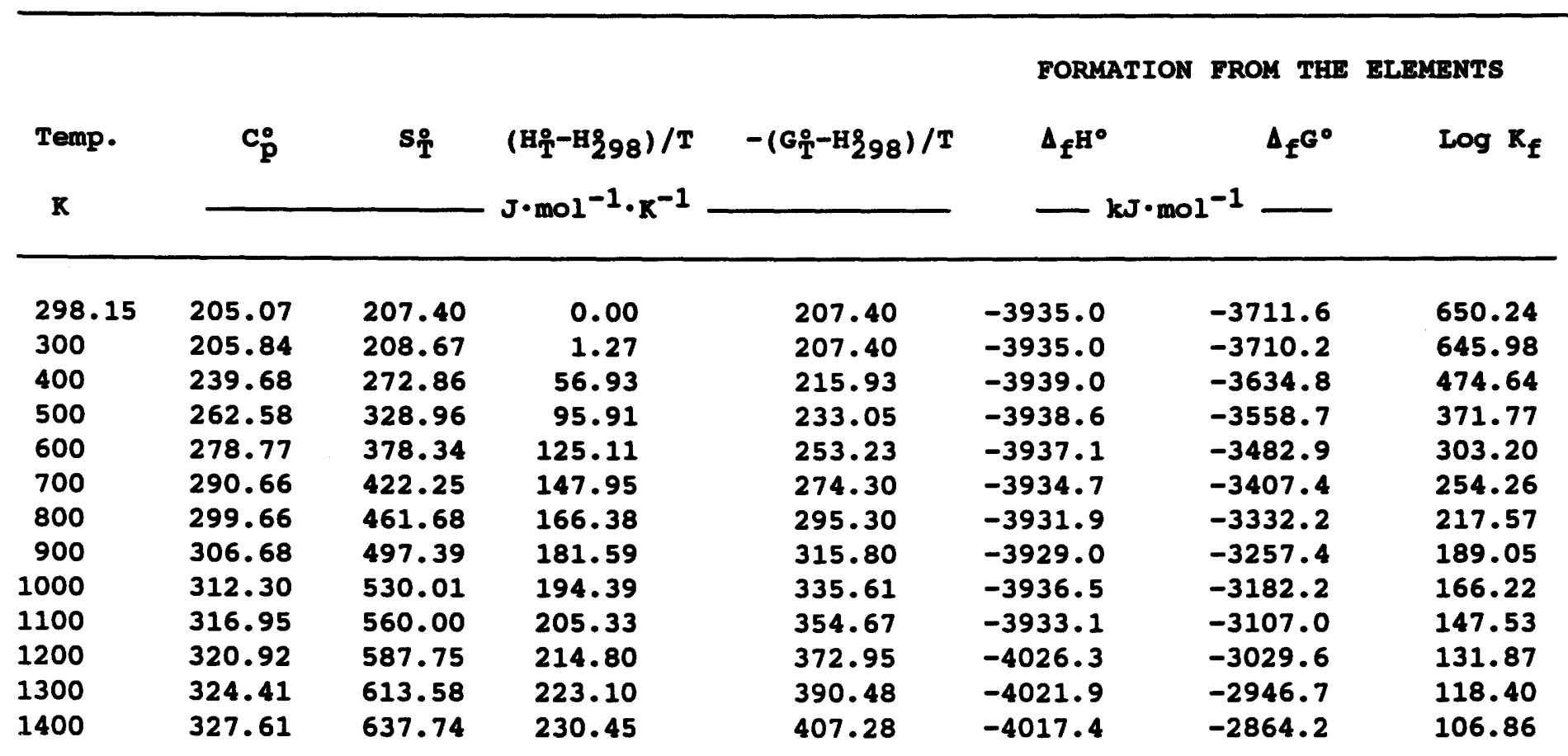

Melting T

$\Delta_{\mathrm{fu}} \mathrm{H}^{\circ}$

$\mathbf{k J}$

H298-H8
Boiling T

$A_{\text {vep }} \mathrm{H}^{\circ}$

Molár Vol.
R

$\mathbf{k J}$ $\begin{array}{ll}10.007 & \mathrm{~J} \cdot \mathrm{bar}^{-1} \\ 100.07 \mathrm{~cm}^{3}\end{array}$ 
$\mathrm{NaAlsi}_{3} \mathrm{O}_{8}:$ Triclinic crystals 298.15 to $1400 \mathrm{~K}$.

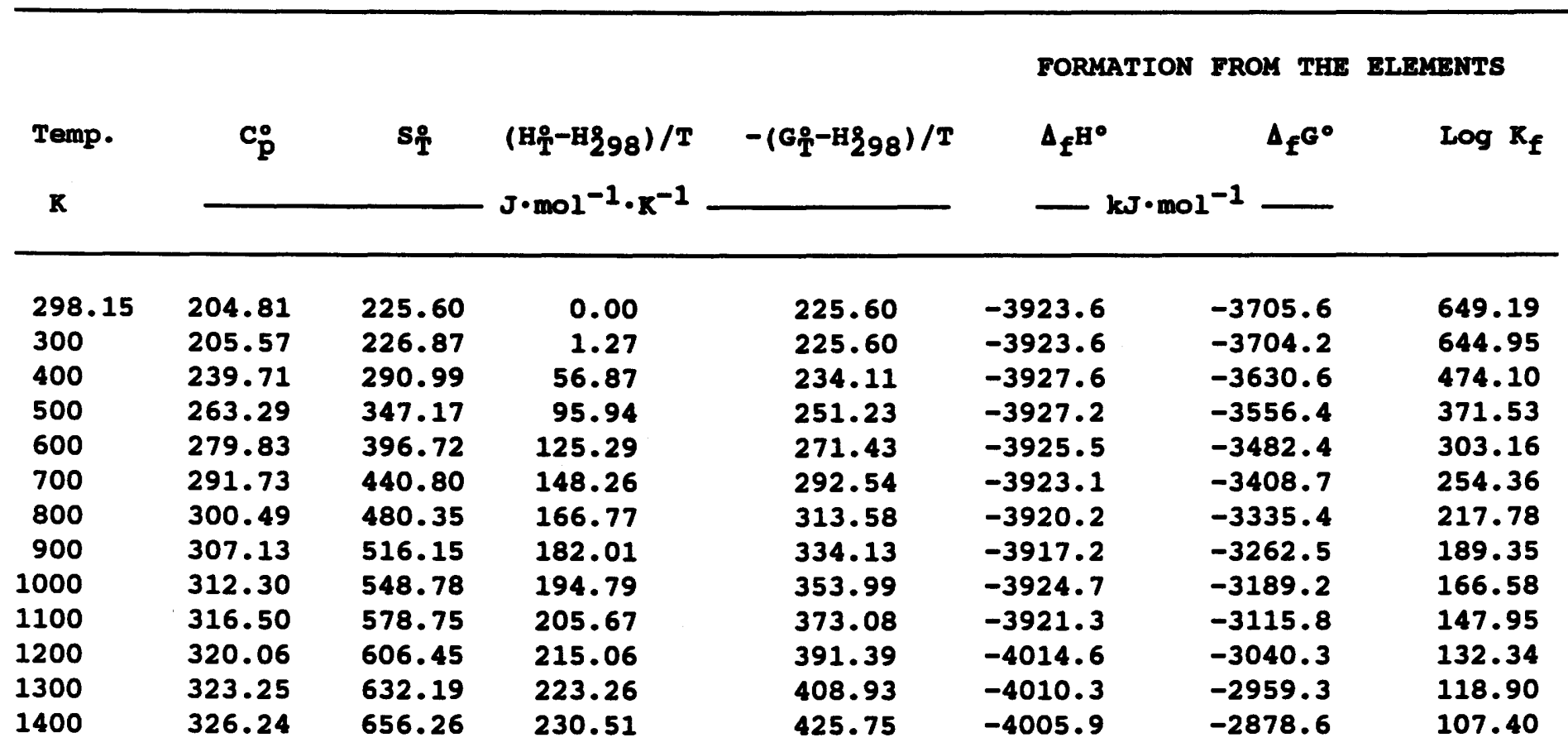

Melting $\mathbf{T} 1391 \mathrm{~K}$

$\Lambda_{\text {fur }} \mathbf{H}^{\circ}$

$\mathbf{W J}$

Hร98-H8
Boiling T

$A_{\text {veg }} \mathrm{H}^{\circ}$

Molar Vol.

\section{R}

kJ

$10.043 \mathrm{~J} \cdot$ bar $^{-1}$ $100.43 \mathrm{~cm}^{3}$
$A=-3.937 \mathrm{E}+03$
$B=7.507 \mathrm{E}-01$
$C=7.781 \mathrm{E}+05$ 
$\mathrm{NaAlsi}_{3} \mathrm{O}_{8}:$ Glass 298.15 to $1200 \mathrm{~K}$

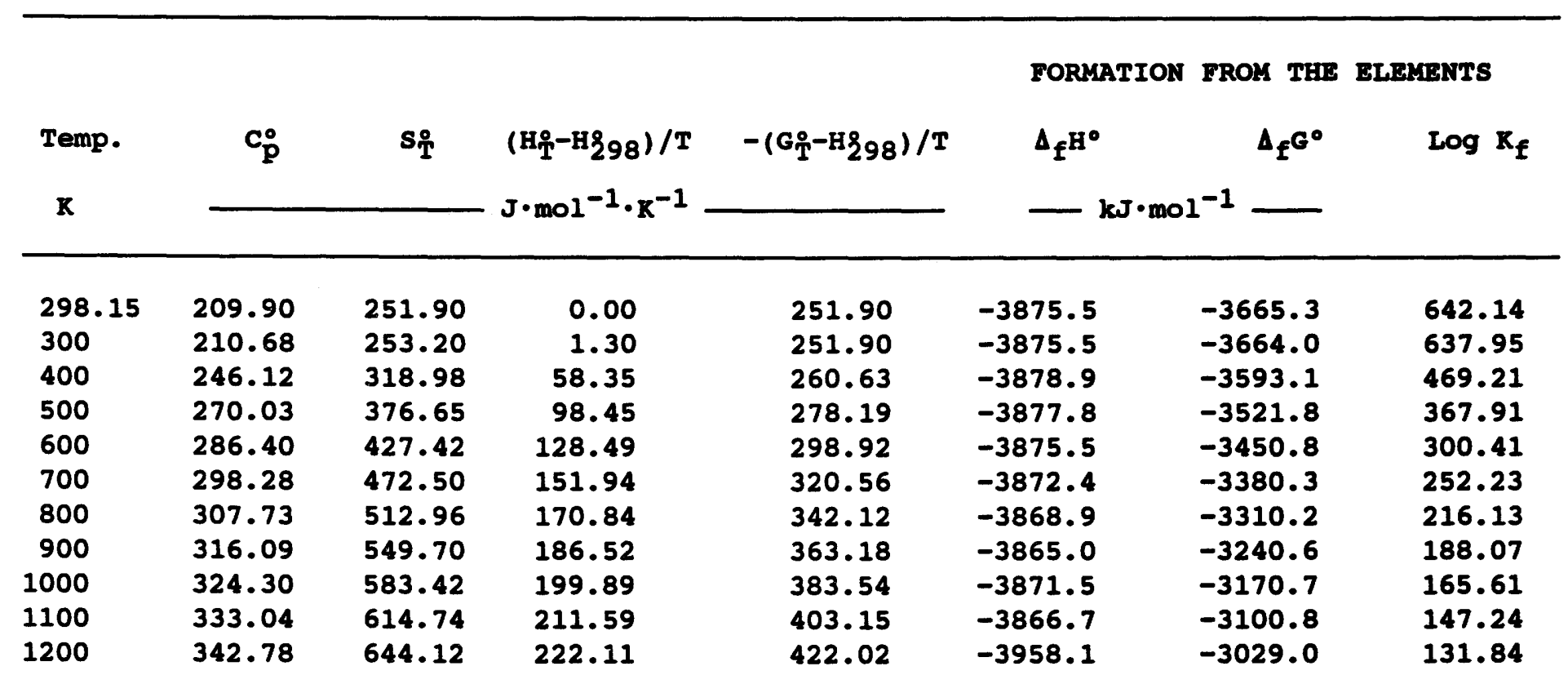

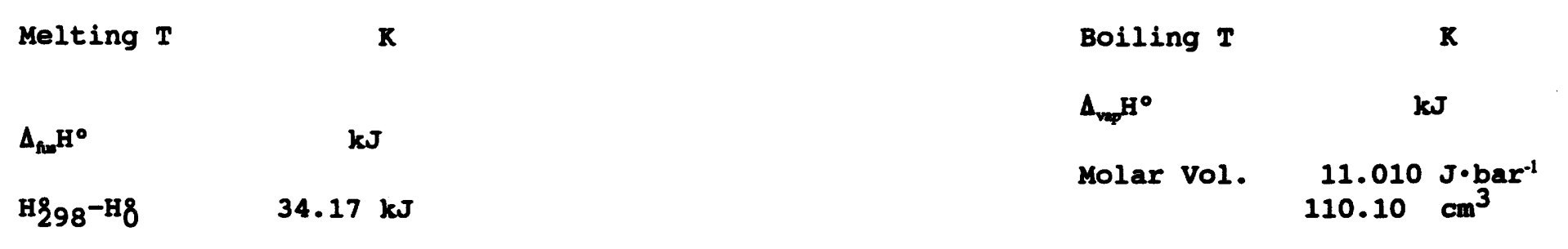
$A=-3.871 \mathrm{E}+03$
$B=7.012 \mathrm{E}-01$
$C=-3.14 E+05$ 
NaAlsio 4 : Orthorhombic(3) crystals 298.15 to $467 \mathrm{~K}$. Hexagonal crystals 467 to $1180 \mathrm{~K}$. Orthorhombic crystals 1180 to $1521 \mathrm{~K}$. Carnegieite is the stable phase of NaAlsio $_{4}$ between 1521 and $1799 \mathrm{~K}$.

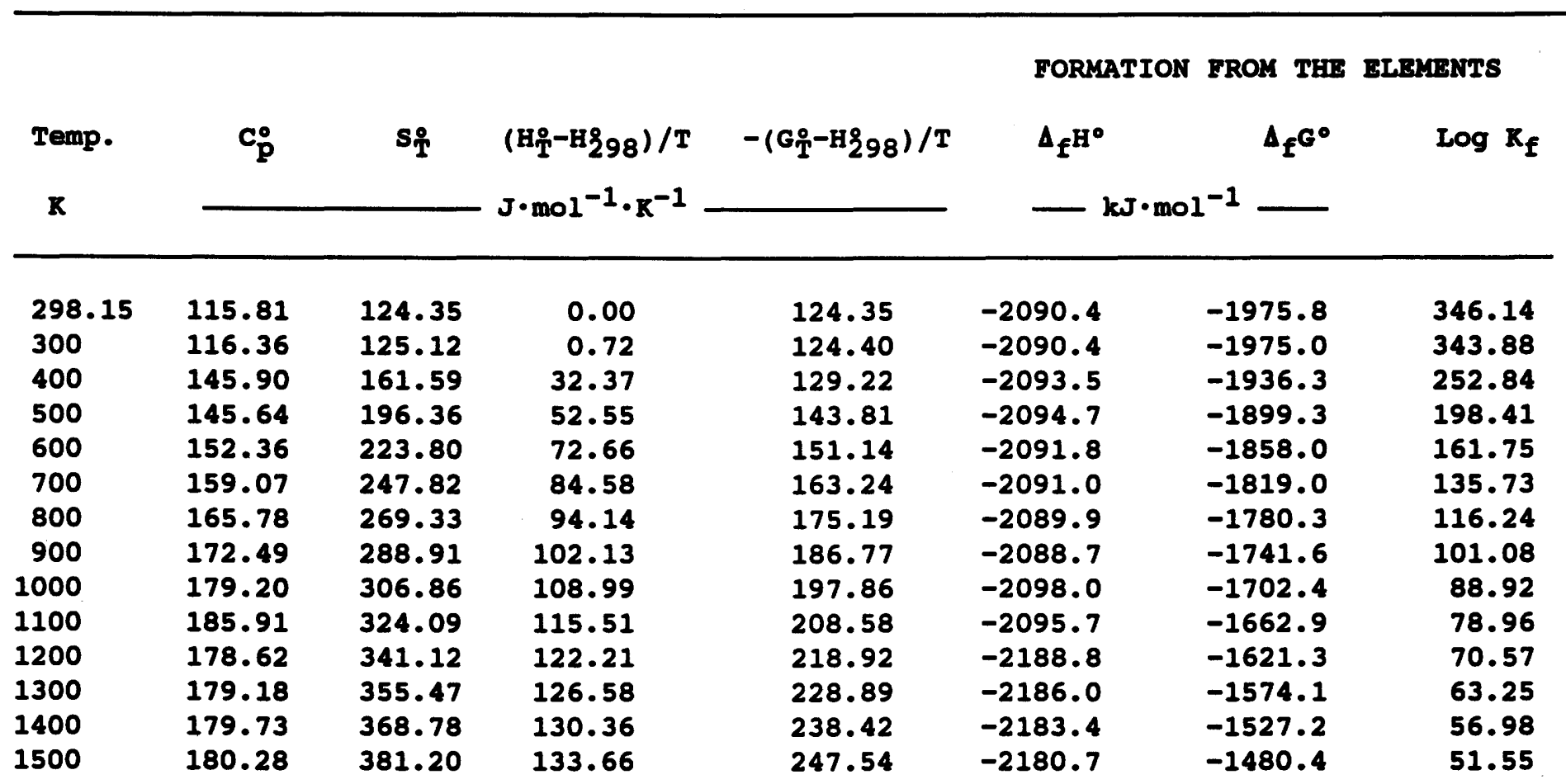

Kelting T

$\Delta_{\mathrm{fu}} \mathrm{H}^{\circ}$

H298-H8 $\mathbf{k J}$

$\mathbf{k J}$
Boiling T

$\Delta_{\mathrm{vep}} \mathrm{H}^{\circ}$

Molar Vol.
$B=4 \cdot 188 \mathrm{E}-01$
$C=1.718 E+06$
$A=-2 \cdot 118 E+03$

$5.419 \mathrm{~J} \cdot$ bar $^{-1}$ $54.19 \mathrm{~cm}^{3}$ 
NaAsio 4 : Triclinic crystals 298.15 to $966 \mathrm{~K}$; cubic crystals 966 to melting point $1799 \mathrm{~K}$.

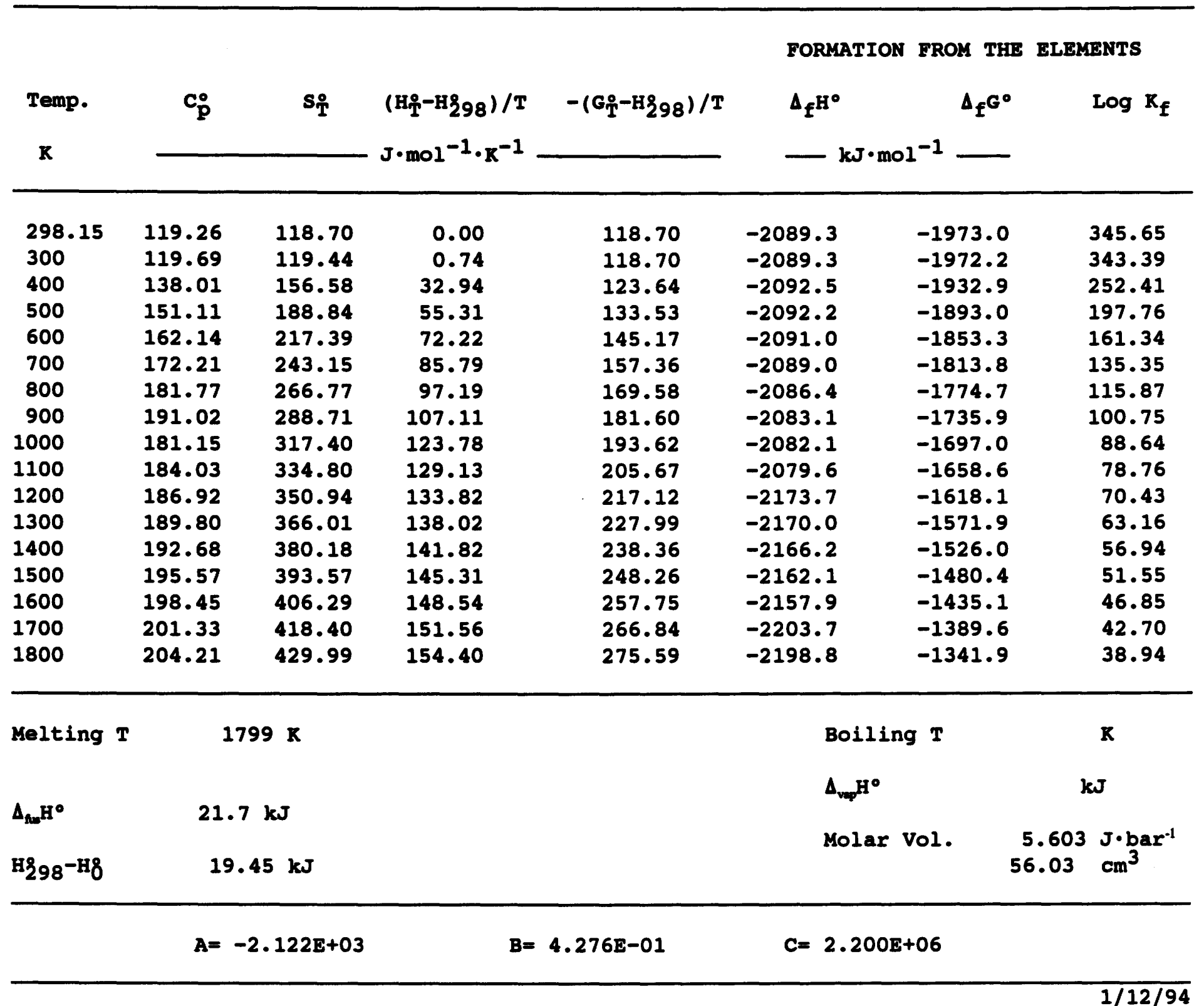


$\mathrm{NaAlsiO}_{4}$ : Glass-liquid 298.15 to glass transition $1000 \mathrm{~K}$.

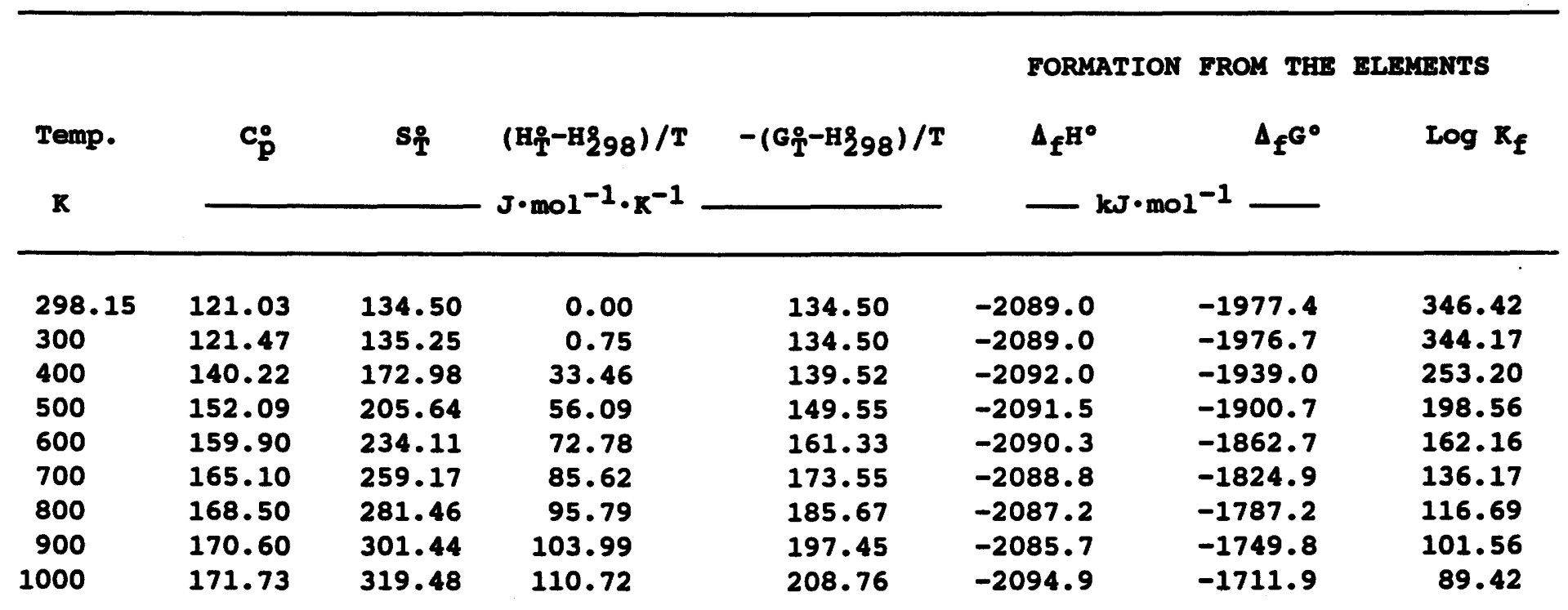

\begin{tabular}{|c|c|c|c|}
\hline Melting $T$ & $\mathbf{R}$ & Boiling T & $\mathbf{K}$ \\
\hline & & $\Delta_{\mathrm{vep}} \mathrm{H}^{\circ}$ & $\mathbf{k J}$ \\
\hline $\begin{array}{l}\Delta_{\text {Rum }} \mathrm{H}^{\circ} \\
\mathrm{H} 298^{-\mathrm{H}}\end{array}$ & $\begin{array}{c}k J J \\
19.99 \mathrm{~kJ}\end{array}$ & Molar Vol. & $\begin{array}{l}5.686 \mathrm{~J} \cdot \mathrm{bar}^{-1} \\
56.86 \mathrm{~cm}^{3}\end{array}$ \\
\hline
\end{tabular}

$A=-2.089 E+03 \quad B=3.771 E-01 \quad C=-9.27 E+04$


NaAlsi ${ }_{2} \mathrm{O}_{6} \cdot \mathrm{H}_{2} \mathrm{O}$ : Cubic crystals 298.15 to $800 \mathrm{~K}$. Analcime starts to lose $\mathrm{H}_{2} \mathrm{O}$ above $500 \mathrm{~K}$.

\begin{tabular}{|c|c|c|c|c|c|c|c|}
\hline \multirow{3}{*}{$\begin{array}{l}\text { Temp. } \\
\text { R }\end{array}$} & \multirow{3}{*}{$\mathbf{c}_{\mathbf{p}}^{\circ}$} & \multirow{3}{*}{$s_{\mathbf{T}}$} & \multirow{3}{*}{$\begin{array}{l}\left(\mathrm{H}_{\mathrm{T}}^{\circ}-\mathrm{H}_{298}\right) / \mathrm{T} \\
\mathrm{J} \cdot \mathrm{mol}^{-1} \cdot \mathrm{K}^{-1}\end{array}$} & \multirow{3}{*}{$-\left(G_{T}^{0}-H_{298}\right) / T$} & \multirow{3}{*}{\multicolumn{2}{|c|}{$\begin{array}{l}\text { FORMATION FROM THE } \\
\Delta_{\mathrm{f}^{\mathrm{H}^{\circ}}} \Delta_{\mathrm{f}^{\mathrm{G}^{\circ}}}\end{array}$}} & \multirow{3}{*}{$\begin{array}{l}\text { ELEMENTS } \\
\qquad \log \mathrm{k}_{\mathrm{f}}\end{array}$} \\
\hline & & & & & & & \\
\hline & & & & & & & \\
\hline 298.15 & 212.23 & 227.70 & 0.00 & 227.70 & -3310.1 & -3090.0 & 541.33 \\
\hline 300 & 212.68 & 229.01 & 1.31 & 227.70 & -3310.1 & -3088.6 & 537.76 \\
\hline 400 & 237.19 & 293.54 & 57.20 & 236.34 & -3313.3 & -3014.4 & 393.63 \\
\hline 500 & 262.19 & 349.14 & 95.69 & 253.45 & -3312.2 & -2939.8 & 307.11 \\
\hline 600 & 287.38 & 399.17 & 125.54 & 273.63 & -3309.3 & -2865.5 & 249.46 \\
\hline 700 & 312.66 & 445.37 & 150.47 & 294.90 & -3304.3 & -2792.0 & 208.33 \\
\hline 800 & 337.99 & 488.77 & 172.32 & 316.45 & -3297.3 & -2719.2 & 177.54 \\
\hline
\end{tabular}

Melting $T$

$\mathbf{K}$

$\mathbf{k J}$

$\Delta_{\text {fun }} \mathrm{H}^{\circ}$

$35.74 \mathrm{~kJ}$

H298-H8
Boiling $T$

$\Delta_{\mathrm{veq}} \mathrm{H}^{\circ}$

wJ

Molar Vol.

$B=7.344 \mathrm{E}-01$

$C=-2.83 E+05$ 
NaAlsi ${ }_{2} \mathrm{O}_{6}:$ Cubic cryetale 298.15 to $1000 \mathrm{~K}$.

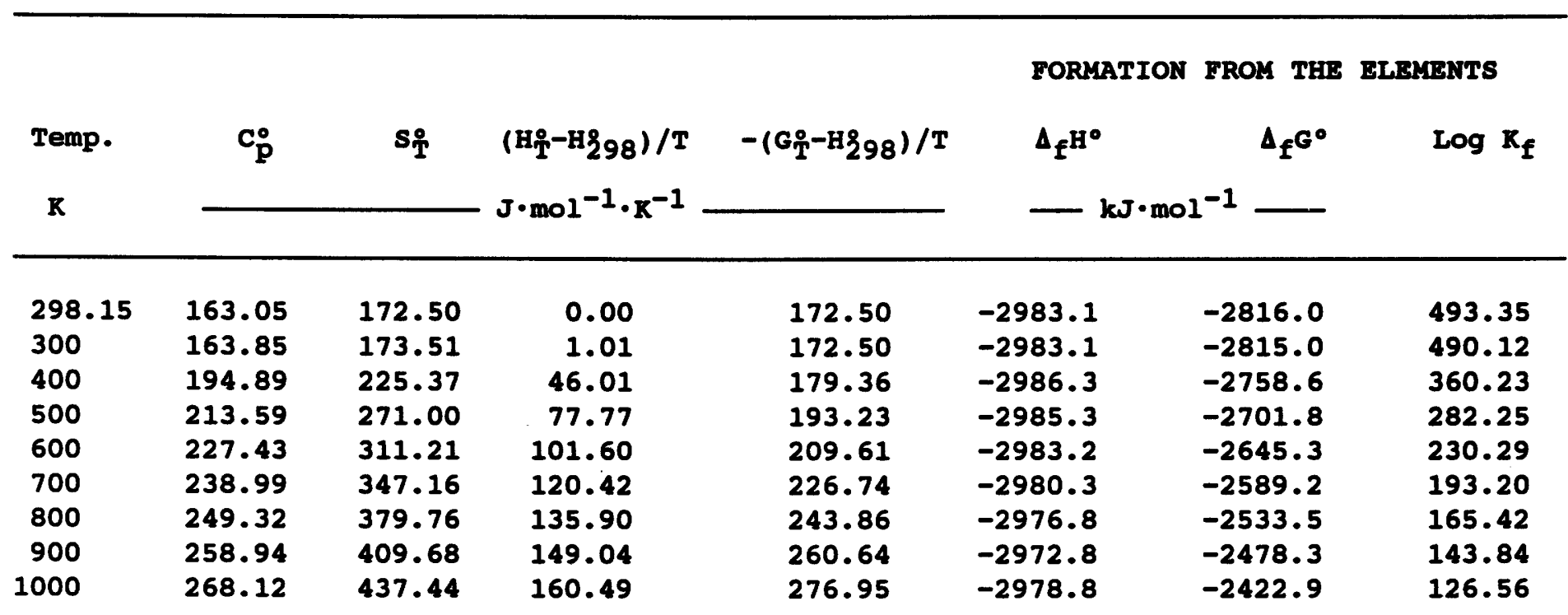

Melting T

$\Delta_{\text {fun }} \mathrm{H}^{\circ}$

$\mathrm{H}_{298^{-H}}$ $\mathbf{k J}$

$\mathbf{K}$

$27.18 \mathrm{~kJ}$
Boiling $T$

$\Delta_{\text {vip }} \mathrm{H}^{\circ}$

Molar Vol.
R

$\mathbf{k J}$

$J \cdot$ bar $^{-1}$
$\mathrm{~cm}^{3}$
$A=-2.978 E+03$
$B=5 \cdot 560 \mathrm{E}-01$
$C=-3 \cdot 36 E+05$ 
$\mathrm{Al}_{2} \mathrm{Si}_{2} \mathrm{O}_{5}(\mathrm{OH})_{2}:$ Triclinic crystals 298.15 to $900 \mathrm{~K}$

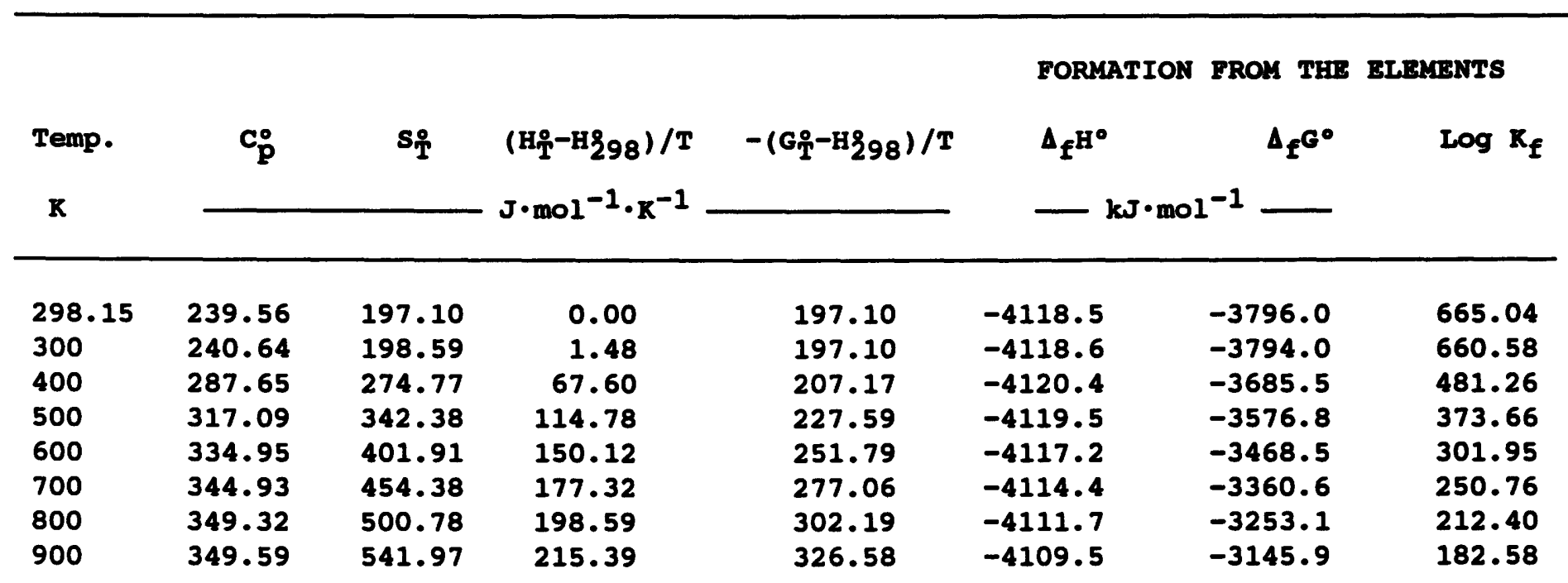

Melting $\mathbf{T}$

$\Delta_{\text {fwo }} \mathrm{H}^{\circ}$

H298-H8 $\mathbf{k J}$

$\mathbf{k J}$
Boiling $\mathbf{T}$

K

$\Lambda_{\text {vep }} \mathrm{H}^{\circ}$

$\mathbf{k J}$

Molar Vol. $\quad 9.856 \mathrm{~J} \cdot \mathrm{bar}^{-1}$ $98.56 \mathrm{~cm}^{3}$
$A=-4.113 E+03$
$B=1.075 \mathrm{E}+00$
$C=-3 \cdot 31 E+05$ 
$\mathrm{Al}_{2} \mathrm{Si}_{2} \mathrm{O}_{5}(\mathrm{OH})_{2}:$ Triclinic crystals 298.15 to $800 \mathrm{~K}$

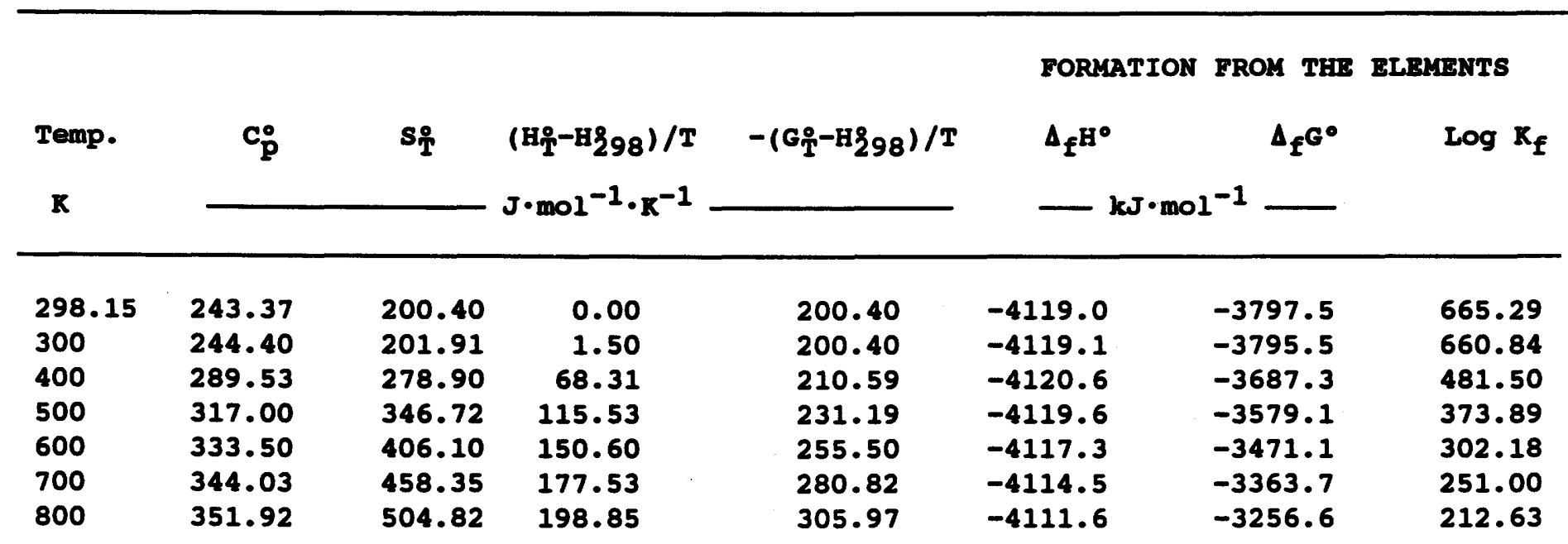

Melting $\mathbf{T}$

$\boldsymbol{A}_{\text {for }} \mathbf{H}^{\circ}$

H298-H8 $\mathbf{k J}$

$\mathbf{R}$

$35.75 \mathrm{~kJ}$
Boiling $T$

$4_{\text {vap }} \mathrm{H}^{\circ}$

Molar Vol.
$\mathbf{K}$

KS

$A=-4.115 E+03$

$B=1.074 \mathrm{E}+00$

$C=-2 \cdot 435+05$ 


\begin{tabular}{|c|c|c|c|c|c|c|c|}
\hline Temp. & $\mathbf{c}_{\mathbf{p}}^{\circ}$ & $\mathbf{s}_{\text {T }}$ & \multirow{2}{*}{$\begin{array}{l}\left(\mathrm{H}_{\mathrm{T}}^{\mathrm{O}}-\mathrm{H} 298\right) / \mathrm{T} \\
\mathrm{J} \cdot \mathrm{mol}^{-1} \cdot \mathrm{K}^{-1}\end{array}$} & \multirow[t]{2}{*}{$-\left(G_{T}^{0}-H_{298}\right) / T$} & \multirow{2}{*}{\multicolumn{2}{|c|}{$\begin{array}{l}\text { FORMATION FROM THE } \\
\Delta_{f^{H}}{ }^{\circ}\end{array}$}} & \multirow[t]{2}{*}{$\begin{array}{l}\text { ELEMGENTS } \\
\qquad \log \mathbf{K}_{\mathbf{f}}\end{array}$} \\
\hline $\mathbf{R}$ & & & & & & & \\
\hline 298.15 & 293.76 & 239.40 & 0.00 & 239.40 & -5640.0 & -5266.1 & 922.58 \\
\hline 300 & 295.04 & 241.22 & 1.82 & 239.41 & -5640.1 & -5263.8 & 916.49 \\
\hline 400 & 349.70 & 334.18 & 82.47 & 251.71 & -5641.8 & -5138.0 & 670.94 \\
\hline 500 & 386.14 & 416.37 & 139.78 & 276.59 & -5640.6 & -5012.1 & 523.60 \\
\hline 600 & 412.51 & 489.22 & 183.14 & 306.08 & -5637.5 & -4886.7 & 425.42 \\
\hline 700 & 432.69 & 554.39 & 217.41 & 336.98 & -5633.1 & -4761.9 & 355.33 \\
\hline 800 & 448.83 & 613.25 & 245.36 & 367.89 & -5628.0 & -4637.8 & 302.81 \\
\hline
\end{tabular}

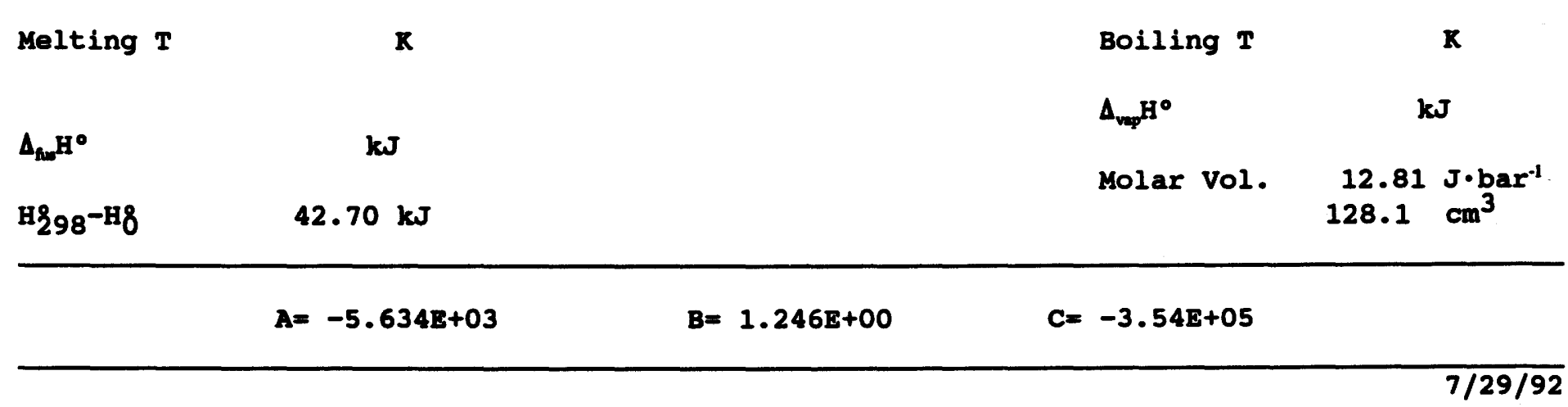


$\mathrm{CaAl}_{2}\left[\mathrm{Al}_{2} \mathrm{Si}_{2}\right]_{10}(\mathrm{OH})_{2}:$ Honoclinic cryetale 298.15 to $1200 \mathrm{~K}$

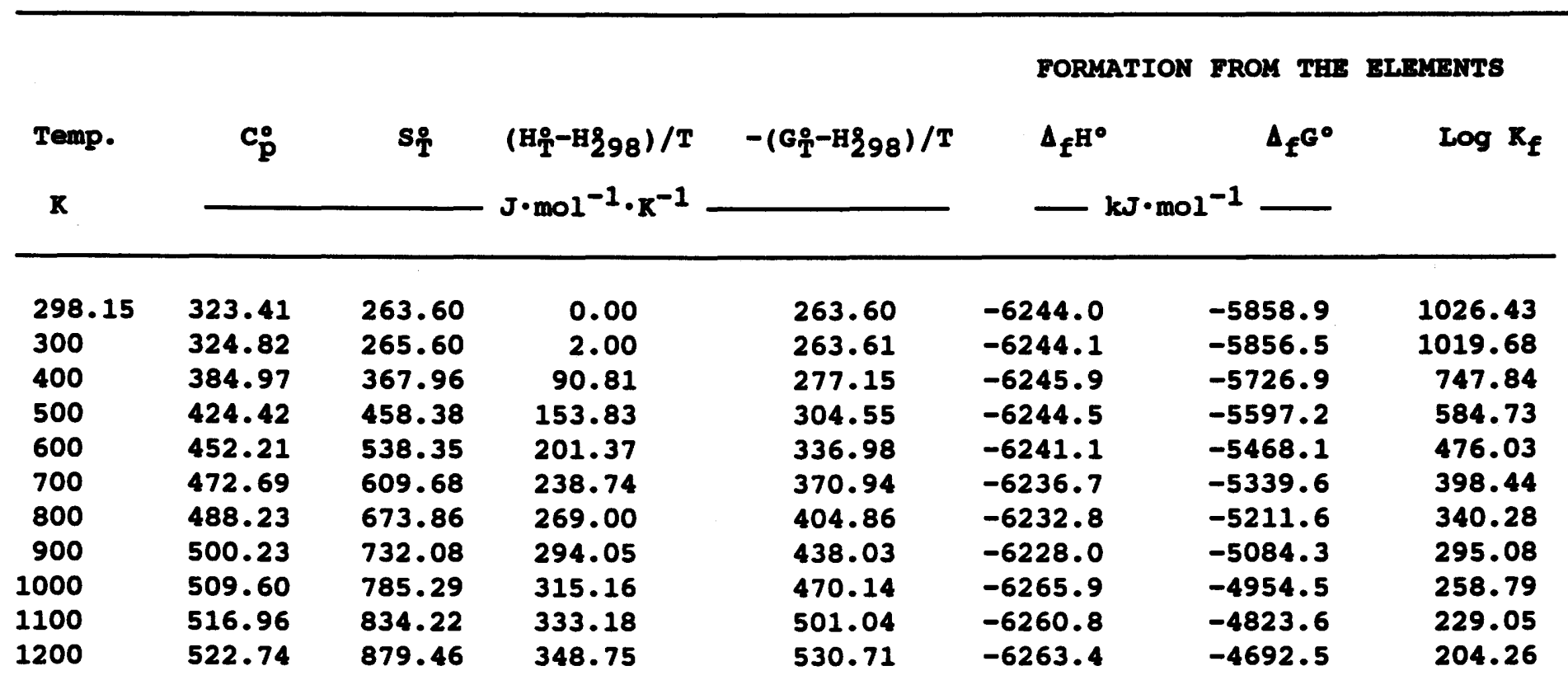

Melting $\mathbf{T}$

$\Lambda_{\text {Aur }} \mathrm{H}^{\circ}$

H898-H8 $\mathbf{k J}$

$\mathbf{R}$

$48.14 \mathrm{~kJ}$
Boiling $T$

$A_{\text {vep }} \mathrm{H}^{\circ}$

Molar Vol. $12.963 \mathrm{~J} \cdot \mathrm{bar}^{-1}$ $129.63 \mathrm{~cm}^{3}$
$A=-6 \cdot 243 B+03$
$B=1.291 E+00$
$C=6.216 \mathrm{E}+02$ 
$\mathrm{Ca}_{2} \mathrm{Al}\left[\mathrm{AlSi} \mathrm{O}_{10} \mathrm{O}_{1}(\mathrm{OH})_{2}\right.$ : Orthorhombic crystals 298.15 to $1200 \mathrm{~K}$

\begin{tabular}{|c|c|c|c|c|c|c|c|}
\hline \multirow{3}{*}{$\begin{array}{l}\text { Temp. } \\
\text { K }\end{array}$} & \multirow{3}{*}{$\mathbf{c}_{\mathbf{p}}^{\circ}$} & \multirow{3}{*}{$s_{\text {T }}^{o}$} & \multirow{3}{*}{$\begin{array}{l}\left(\mathrm{H}_{\mathrm{T}}^{\circ}-\mathrm{H} 298\right) / \mathrm{T} \\
\mathrm{J} \cdot \mathrm{mol}^{-1} \cdot \mathrm{K}^{-1}\end{array}$} & \multirow{3}{*}{$-\left(G_{T}^{\circ}-H_{298}\right) / T$} & \multicolumn{2}{|c|}{ FORMATION FROM THE } & \multirow{3}{*}{$\begin{array}{l}\text { ELEMENTS } \\
\qquad \log \mathrm{k}_{\mathbf{f}}\end{array}$} \\
\hline & & & & & $\Delta_{f} H^{0}$ & $\Delta_{f} G^{0}$ & \\
\hline & & & & & 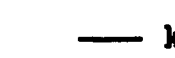 & $1^{-1}$ & \\
\hline 298.15 & 331.12 & 292.80 & 0.00 & 292.80 & -6202.6 & -5824.7 & 1020.43 \\
\hline 300 & 332.41 & 294.85 & 2.05 & 292.81 & -6202.7 & -5822.3 & 1013.73 \\
\hline 400 & 389.19 & 398.83 & 92.23 & 306.60 & -6203.7 & -5695.3 & 743.71 \\
\hline 500 & 427.23 & 490.03 & 155.67 & 334.36 & -6201.7 & -5568.3 & 581.71 \\
\hline 600 & 453.51 & 570.39 & 203.25 & 367.14 & -6198.0 & -5442.0 & 473.76 \\
\hline 700 & 471.95 & 641.76 & 240.39 & 401.37 & -6193.4 & -5316.4 & 396.70 \\
\hline 800 & 484.90 & 705.68 & 270.20 & 435.48 & -6190.4 & -5191.2 & 338.94 \\
\hline 900 & 493.85 & 763.34 & 294.58 & 468.76 & -6185.9 & -5066.5 & 294.05 \\
\hline 1000 & 499.76 & 815.71 & 314.83 & 500.88 & -6203.2 & -4940.9 & 258.08 \\
\hline 1100 & 503.31 & 863.52 & 331.82 & 531.71 & -6199.7 & -4814.8 & 228.63 \\
\hline 1200 & 505.00 & 907.40 & 346.19 & 561.21 & -6212.4 & -4687.7 & 204.05 \\
\hline
\end{tabular}

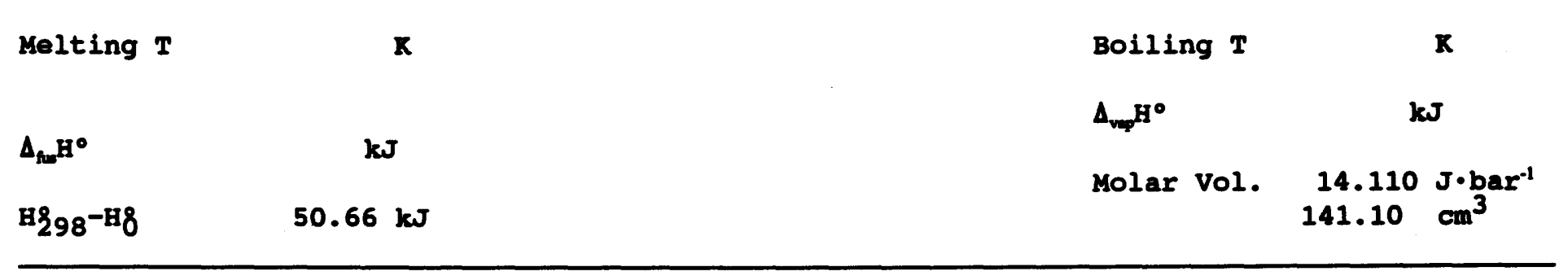
$A=-6.194 \mathrm{E}+03$
$\mathrm{B}=1.255 \mathrm{E}+00$
$C=-3.99 E+05$ 
$\mathrm{KAl}_{2}\left[\mathrm{AlSi}_{3} \mathrm{O}_{10} \mathrm{COH}_{2}\right.$ : Crystals 298.15 to $1000 \mathrm{~K}$.

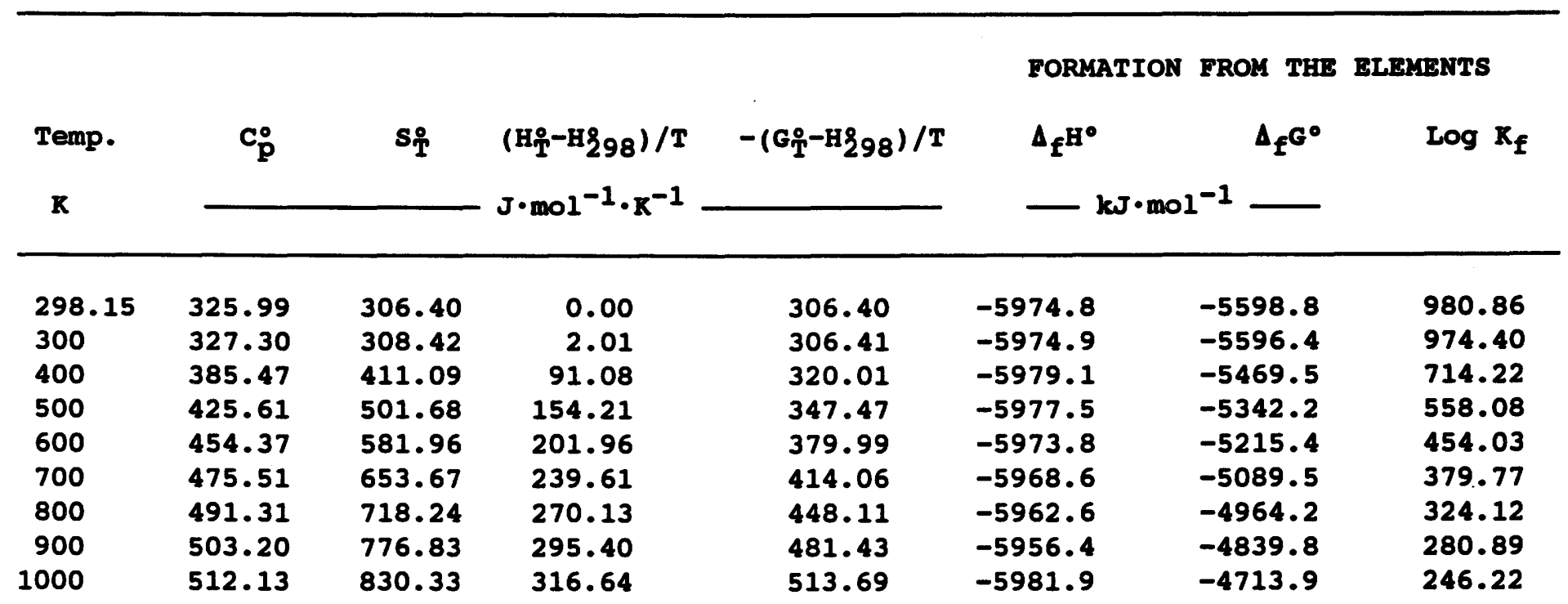

Melting $T$

$\Delta_{\text {fus }} \mathrm{H}^{\circ}$

$\mathbf{k J}$

H298-H8
Boiling $\mathbf{T}$

$\Delta_{\mathrm{vq}} \mathrm{H}^{\circ}$

Molar Vol. $14.081 \mathrm{~J} \cdot$ bar $^{-1}$ $140.81 \mathrm{~cm}^{3}$
$A=-5.966 E+03$
$B=1.253 E+00$
$C=-5 \cdot 87 \mathrm{E}+05$ 
$\mathrm{KAl}_{2}\left[\mathrm{AlSi}_{3} \mathrm{O}_{10}\right.$ ] $(\mathrm{OH})_{2}$ : Monoclinic crystals 298.15 to $1000 \mathrm{~K}$.

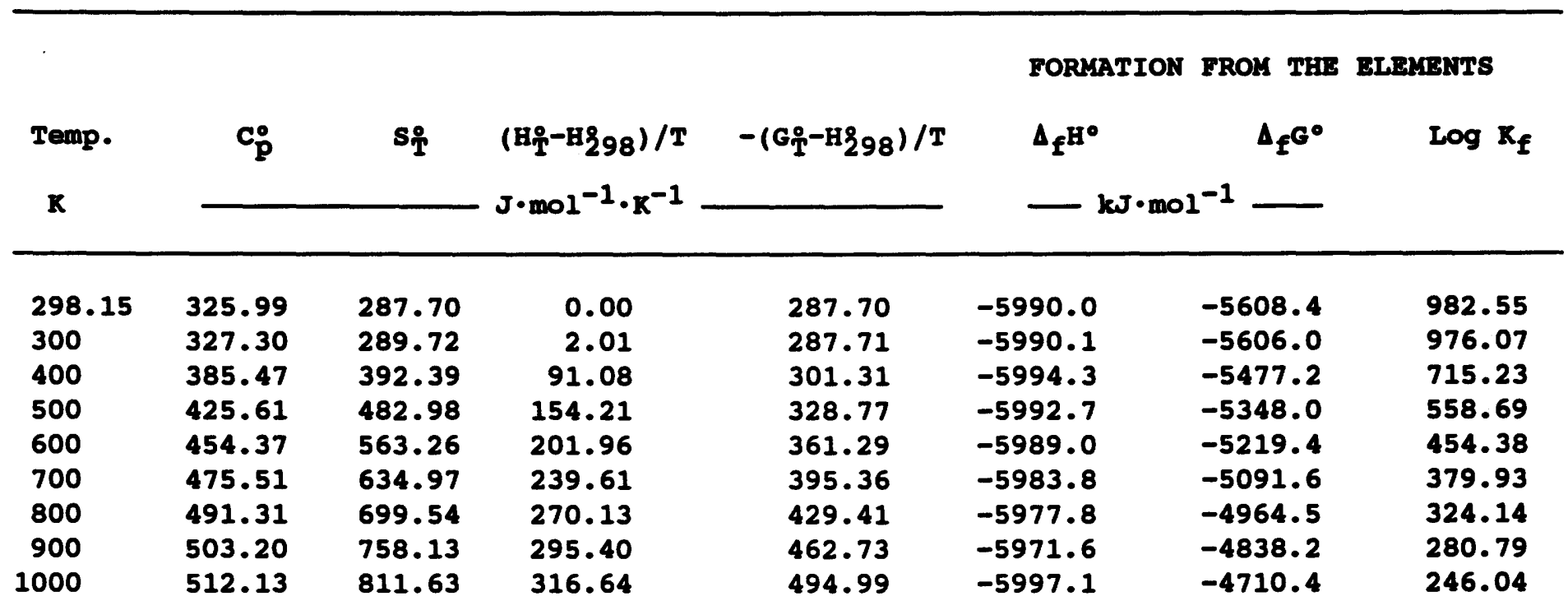

Melting T

$$
\Delta_{\text {fux }} \mathrm{H}^{\circ}
$$

H298-H8 $\mathbf{k J}$

$\mathbf{R}$ $49.41 \mathrm{~kJ}$
Boiling $T$

$\Lambda_{\mathrm{v}} \mathrm{H}^{\circ}$

Molar Vol. $14.081 \mathrm{~J} \cdot \mathrm{bar}^{-1}$ $140.81 \mathrm{~cm}^{3}$
$A=-5.981 \mathrm{E}+03$
$B=1.271 E+00$
$C=-5.87 E+05$ 
$\mathrm{KFe}_{3}\left[\mathrm{AlSi}_{3} \mathrm{O}_{10}\right](\mathrm{OH})_{2}$ : Crystals 298.15 to $1000 \mathrm{~K}$

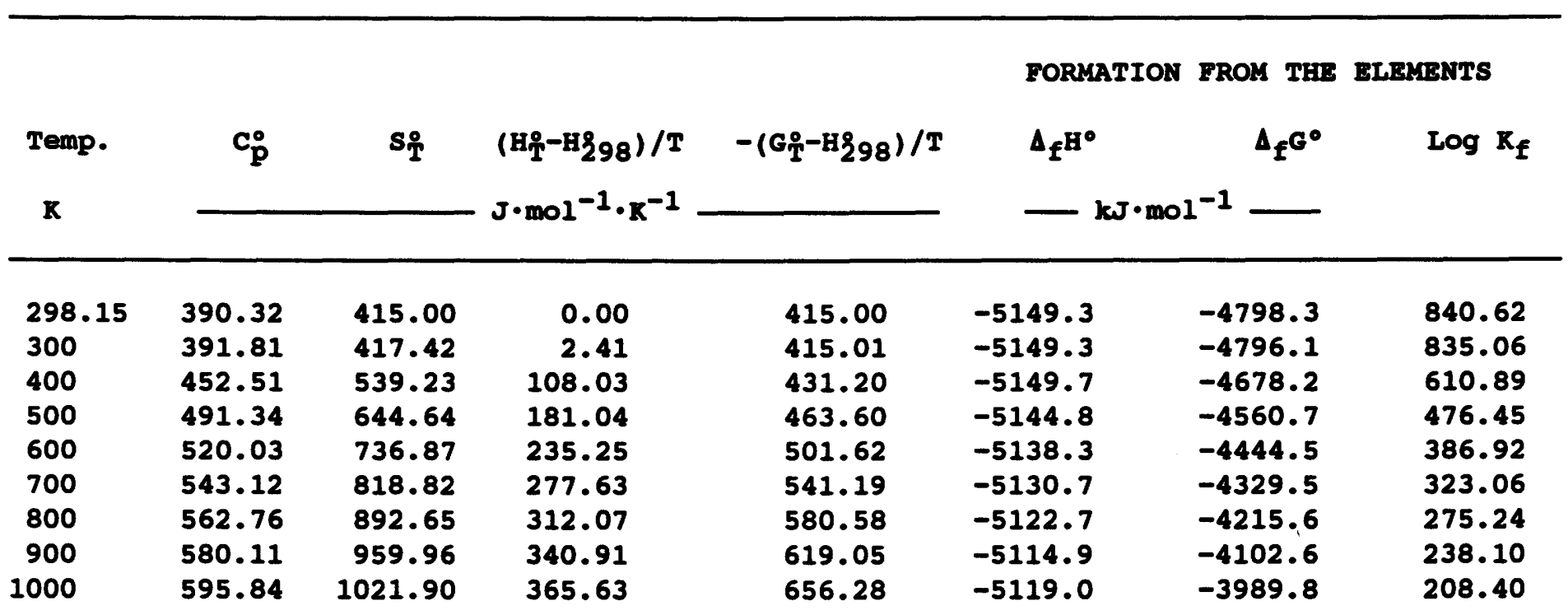

\begin{tabular}{|c|c|c|c|}
\hline Melting $T$ & $\mathbf{K}$ & Boiling $T$ & $\mathbf{K}$ \\
\hline & & $\Delta_{\mathrm{v}} \mathrm{H}^{\circ}$ & $k J$ \\
\hline H298-H8 & $\mathbf{k J}$ & Molar Vol. & $\begin{array}{ll}15.43 & \mathrm{~J} \cdot \mathrm{bar}^{-1} \\
154.3 \mathrm{~cm}^{3}\end{array}$ \\
\hline
\end{tabular}




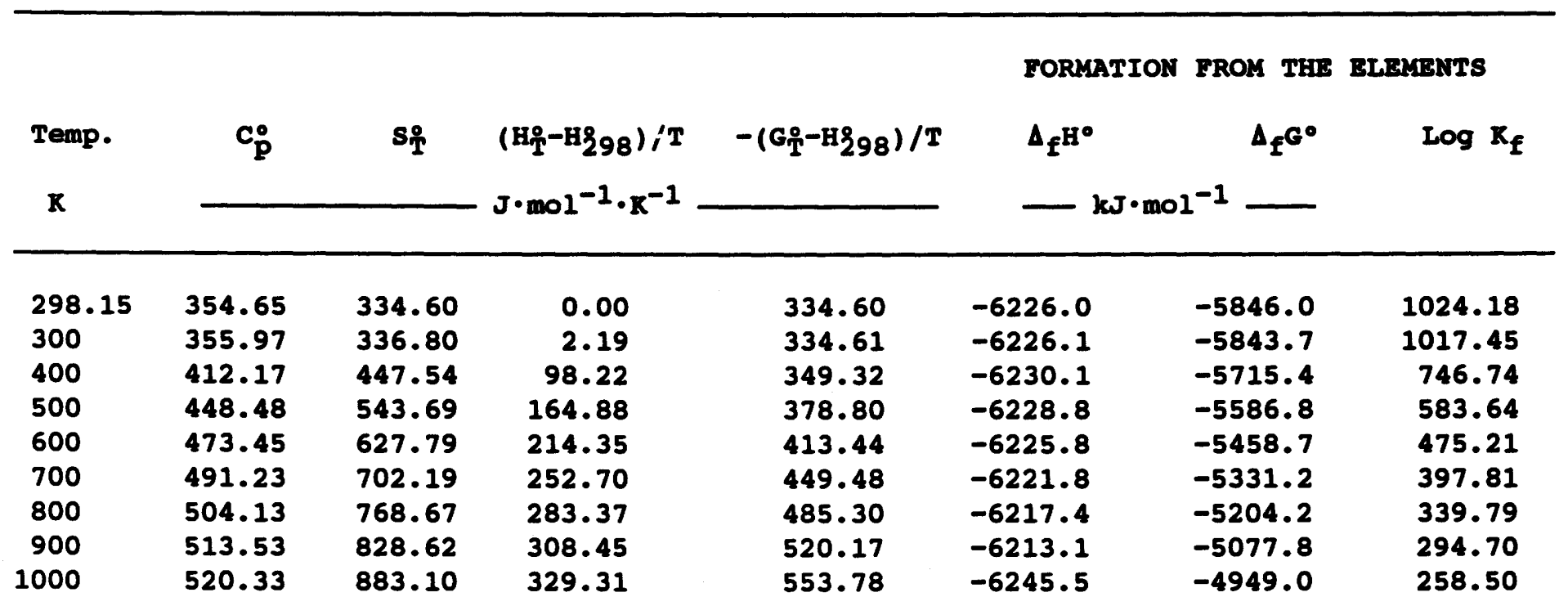

\begin{tabular}{|c|c|c|c|}
\hline Melting $T$ & $\mathbf{K}$ & Boiling $T$ & $\mathbf{K}$ \\
\hline & & $\Delta_{\mathrm{vep}} \mathrm{H}^{\circ}$ & $\mathbf{k J}$ \\
\hline $\begin{array}{l}\Delta_{\text {fwo }} \mathrm{H}^{\circ} \\
\mathrm{H}_{298^{-}}-\mathrm{H} 8\end{array}$ & $\begin{array}{c}k J \\
54.08 k J\end{array}$ & Molar Vol. & $\begin{array}{l}14.965 \mathrm{~J} \cdot \mathrm{bar}^{-1} \\
149.65 \mathrm{~cm}^{3}\end{array}$ \\
\hline
\end{tabular}
$A=-6 \cdot 222 \mathrm{E}+03$
$B=1 \cdot 273 E+00$
$C=-3.45 E+05$ 
$\left.\mathrm{KMg}_{3}\left[\mathrm{AlSi}_{3} \mathrm{O}_{1 \mathrm{O}}\right]_{(\mathrm{OH}}\right)_{2}$ : Monoclinic crystals 298.15 to $1000 \mathrm{~K}$.

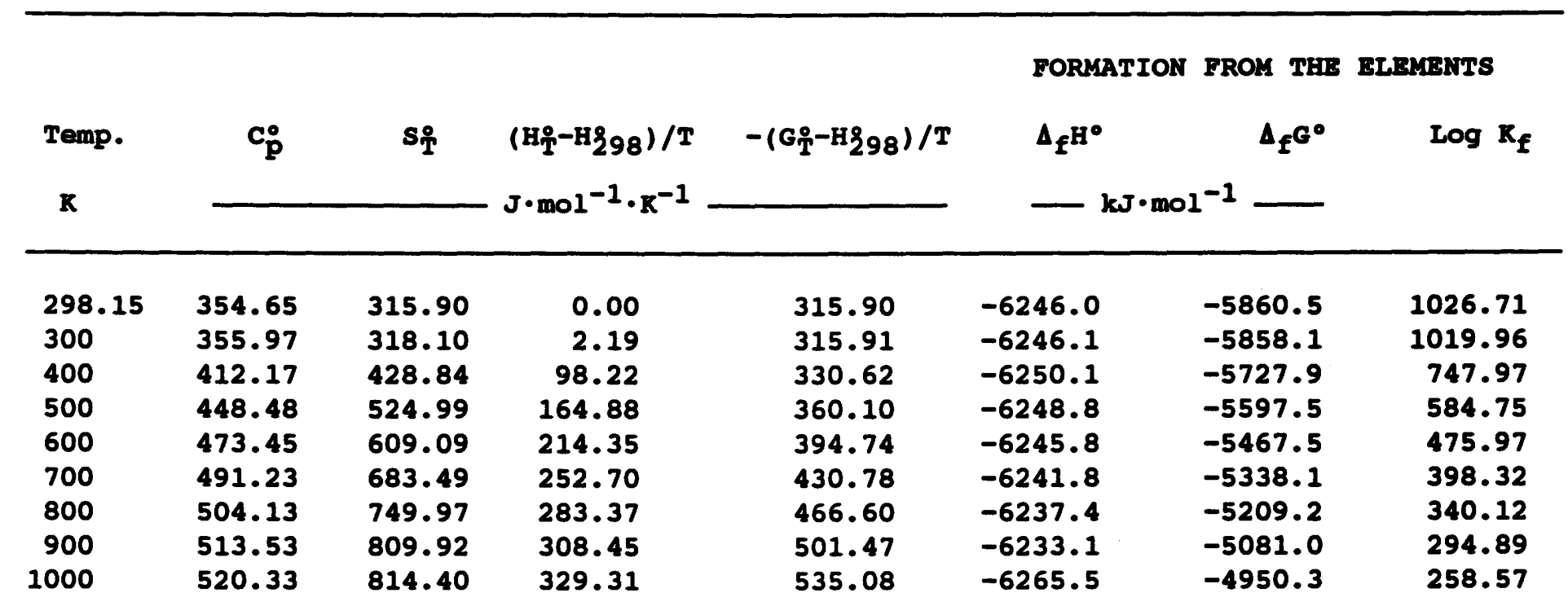

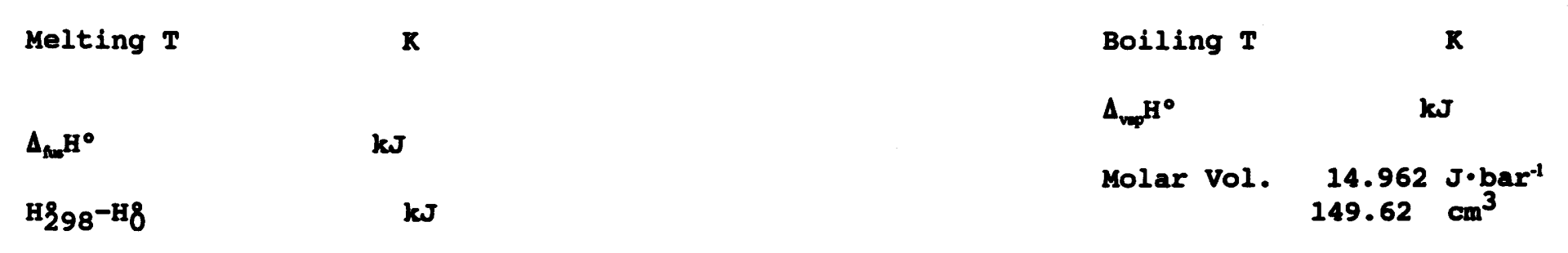


$\mathrm{KMg}_{3}\left[\mathrm{AlSi}_{3} \mathrm{O}_{10}\right]_{2}$ : Crystals 298.15 to $1700 \mathrm{~K}$. Approximate melting point $1670 \mathrm{~K}$.

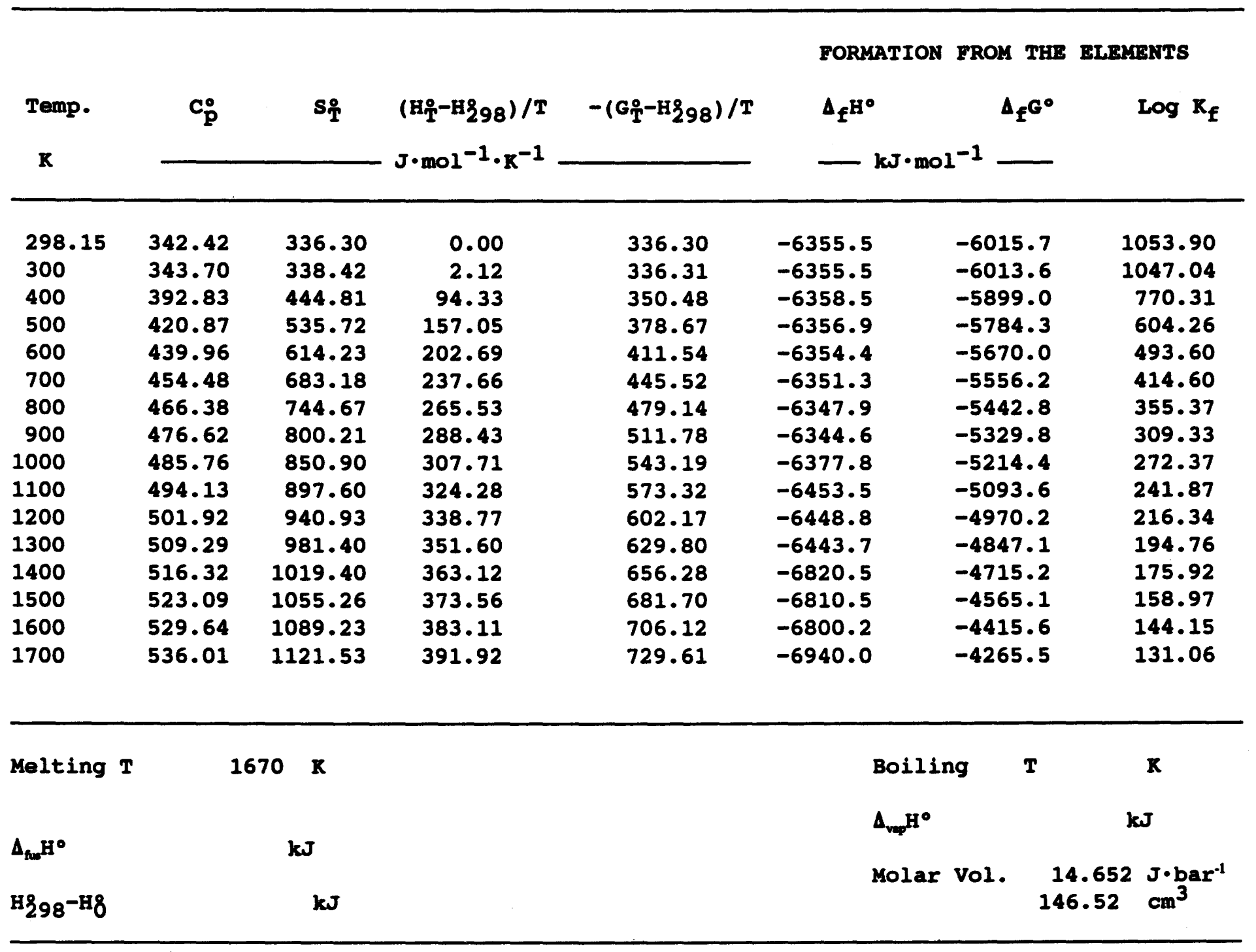
$A=-6.476 E+03$
$B=1.272 E+00$
$\mathrm{C}=8 \cdot 177 \mathrm{E}+06$ 
$\mathrm{KMg}_{3}\left[\mathrm{AlSi}_{3} \mathrm{O}_{10}\right]_{2}$ : Crystals 298.15 to $1700 \mathrm{~K}$. Approximate melting point $1670 \mathrm{~K}$.

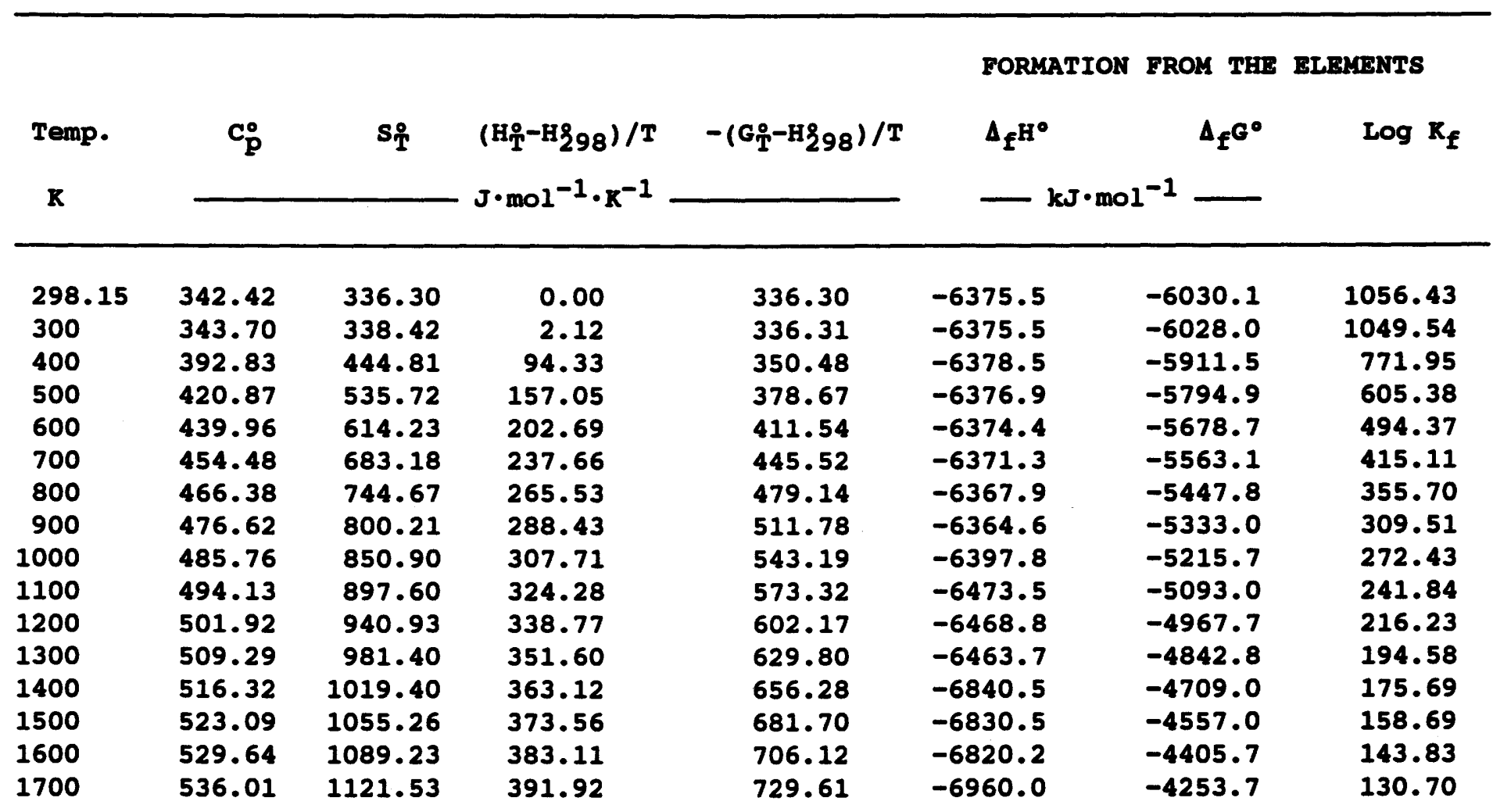

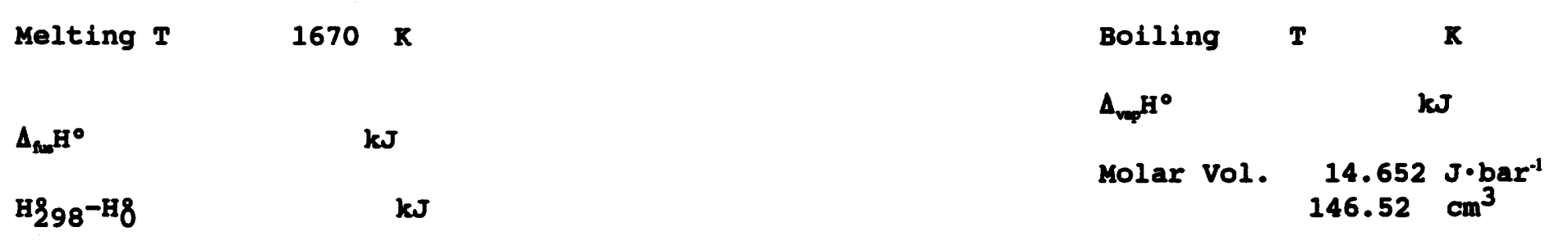

$A=-6.496 \mathrm{E}+03 \quad B=1.291 \mathrm{E}+00 \quad C=8.177 \mathrm{E}+06$


$\mathrm{Mg}_{3} \mathrm{Si}_{4} \mathrm{O}_{10}(\mathrm{OH})_{2}$ : Monoclinic crystals 298.15 to $800 \mathrm{~K}$.

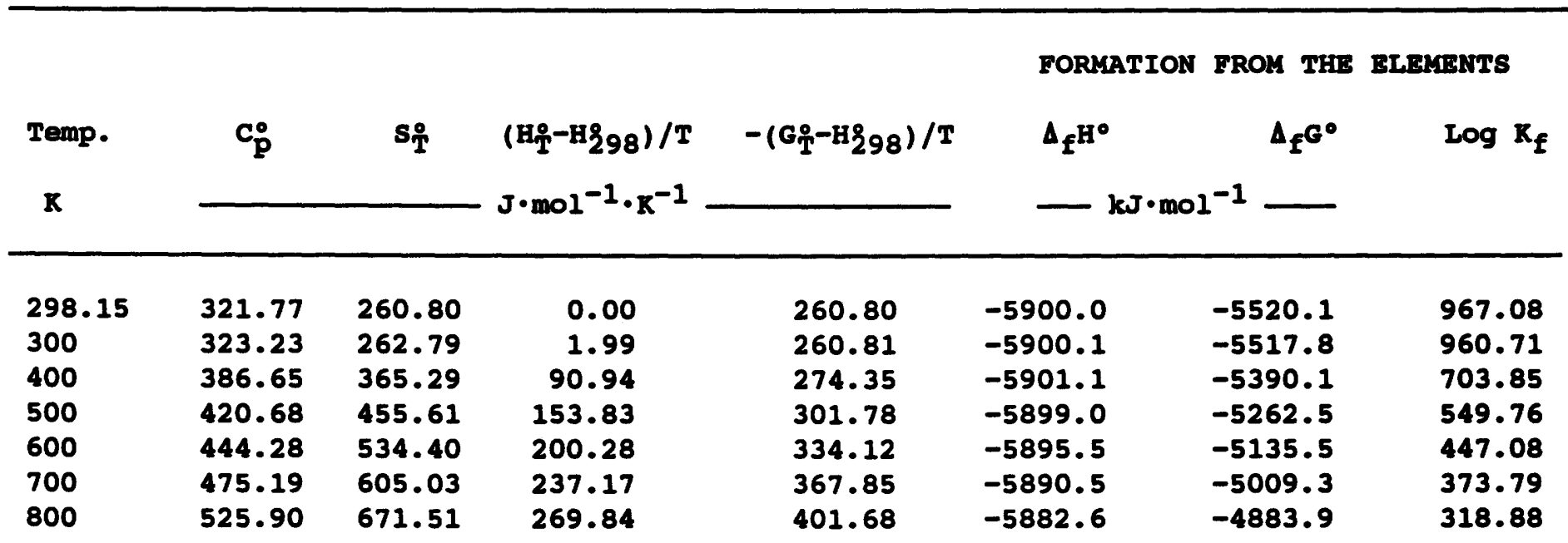

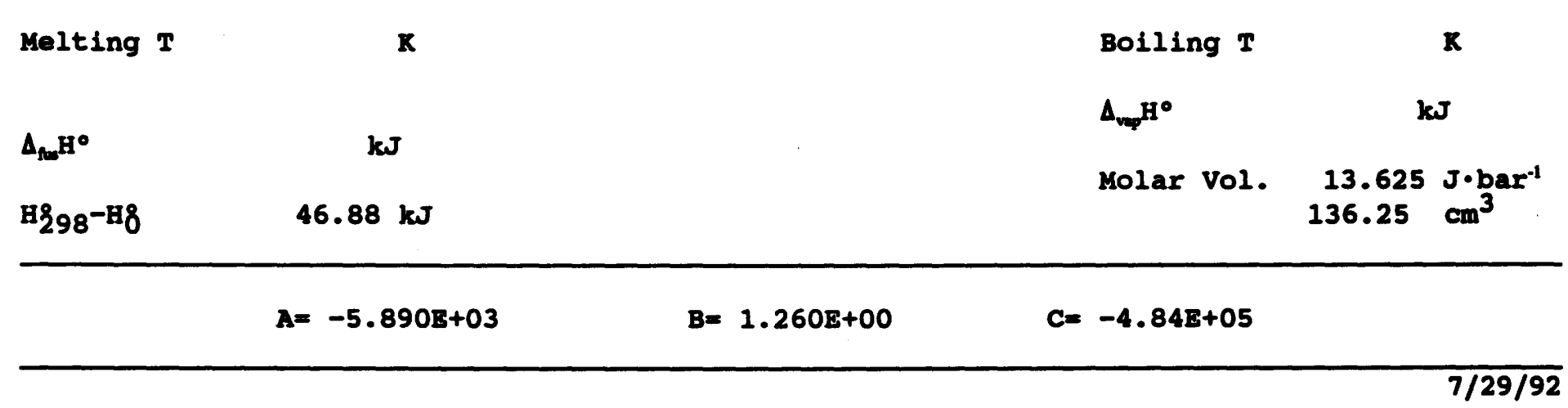


$\mathrm{Mg}_{3} \mathrm{Si}_{2} \mathrm{O5}(\mathrm{OH})_{4}:$ Monoclinic crystals 298.15 to $900 \mathrm{~K}$.

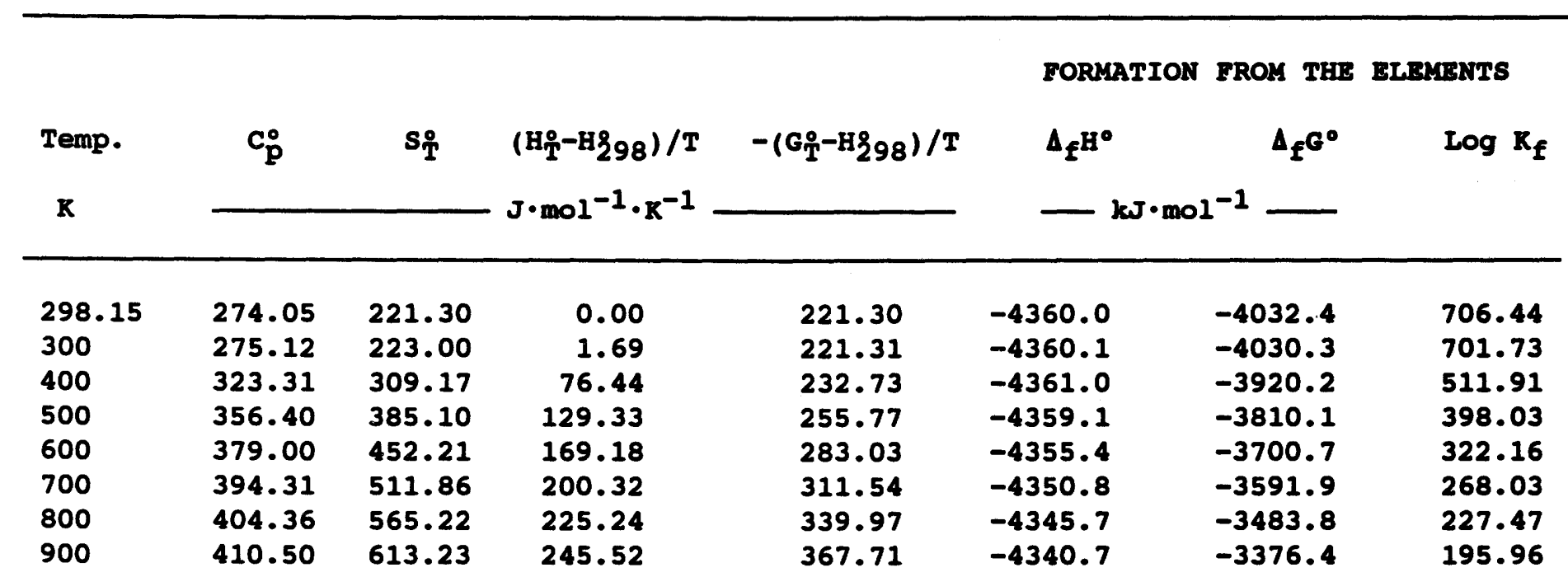

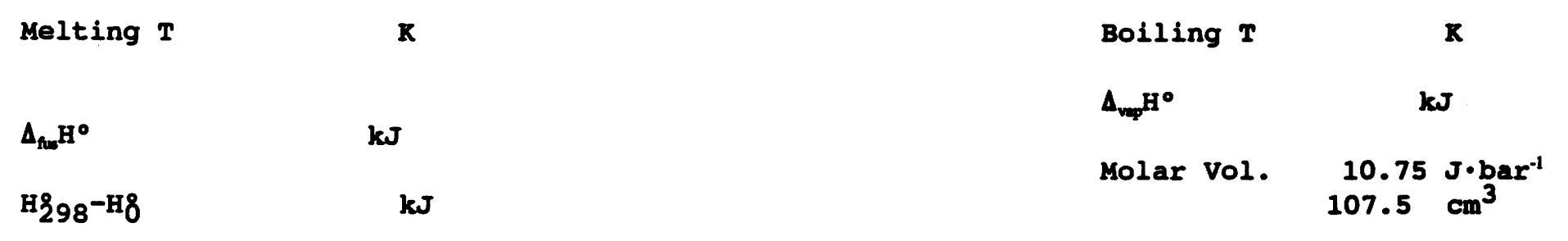
$A=-4 \cdot 348 E+03$
$B=1.081 \mathrm{E}+00$
$C=-6.03 E+05$ 
$\mathrm{NaAl}_{2}\left[\mathrm{AlSi}_{3} \mathrm{O}_{10}\right](\mathrm{OH})_{2}$ : Monoclinic crystals 298.15 to $800 \mathrm{~K}$.

\begin{tabular}{|c|c|c|c|c|c|c|c|}
\hline \multirow{2}{*}{$\begin{array}{l}\text { Temp. } \\
\mathbf{K}\end{array}$} & \multirow[t]{2}{*}{$\mathbf{c}_{\mathbf{p}}^{\circ}$} & \multirow[t]{2}{*}{$\mathbf{s}_{\mathbf{T}}$} & \multirow{2}{*}{$\begin{array}{l}\left(\mathrm{H}_{\mathrm{T}}^{\circ}-\mathrm{H}_{298}\right) / \mathrm{T} \\
\mathrm{J} \cdot \mathrm{mol}^{-1} \cdot \mathrm{K}^{-1}\end{array}$} & \multirow[t]{2}{*}{$-\left(G_{T}^{\circ}-H_{298}\right) / T$} & \multirow{2}{*}{\multicolumn{2}{|c|}{$\begin{array}{l}\text { FORMATION FROY THE } \\
\Delta_{\mathrm{F}^{\mathrm{H}^{\circ}}} \Delta_{\mathrm{F}^{\mathrm{G}^{\circ}}}\end{array}$}} & \multirow[t]{2}{*}{$\begin{array}{l}\text { ELENGNTS } \\
\qquad \log \mathrm{K}_{\mathrm{f}}\end{array}$} \\
\hline & & & & & & & \\
\hline 298.15 & 321.50 & 288.80 & 0.00 & 288.80 & -5933.0 & -5555.7 & 973.31 \\
\hline 300 & 322.86 & 290.79 & 1.99 & 288.81 & -5933.1 & -5553.3 & 966.90 \\
\hline 400 & 380.52 & 392.22 & 89.98 & 302.24 & -5937.9 & -5426.1 & 708.56 \\
\hline 500 & 419.03 & 481.51 & 152.15 & 329.36 & -5936.9 & -5298.2 & 553.49 \\
\hline 600 & 447.59 & 560.55 & 199.12 & 361.43 & -5933.8 & -5170.7 & 450.14 \\
\hline 700 & 470.21 & 631.30 & 236.29 & 395.02 & -5929.2 & -5043.9 & 376.37 \\
\hline 800 & 488.92 & 695.35 & 266.73 & 428.62 & -5923.5 & -4917.8 & 321.09 \\
\hline
\end{tabular}

Melting $T$

$\mathbf{R}$

$\Delta_{\text {hus }} \mathrm{H}^{\circ}$

$\mathbf{k J}$

H298-H8
Boiling T

$\mathbf{A}_{\text {veg }} \mathbf{H}^{\circ}$

$\mathbf{k J}$
Kolar vol. $\quad 13.211 \mathrm{~J} \cdot$ bar $^{-1}$ $132.11 \mathrm{~cm}^{3}$ 
$\mathrm{NaAl}_{2}\left[\mathrm{AlSi}_{3} \mathrm{O}_{10}\right](\mathrm{OH})_{2}$ : Monoclinic crystals 298.15 to $800 \mathrm{~K}$.

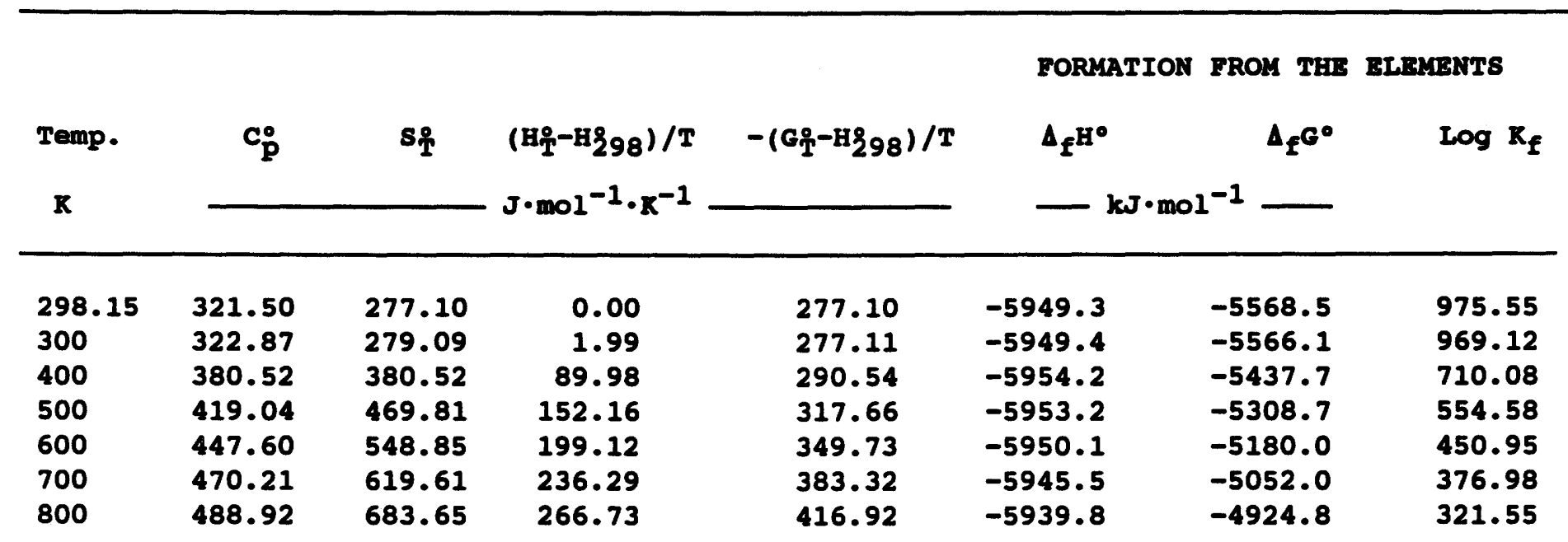

Melting $\mathbf{T}$

$\mathbf{K}$

$\mathbf{k J}$

$\Delta_{\text {fur }} \mathbf{H}^{\circ}$

H298-H8
Boiling $\mathbf{T}$

$\Delta_{\mathrm{vep}} \mathrm{H}^{\mathrm{O}}$

Molar vol.

\section{$\mathbf{K}$}

$\mathbf{k J}$
$13.211 \mathrm{~J} \cdot \mathrm{bar}^{-1}$ $132.11 \mathrm{~cm}^{3}$ 


\section{REFERENCES CITED}

1. Adami, L. H., and Conway, K. C., 1966, Heats and free energies of formation of anhydrous carbonates of barium, strontium, and lead: U.S. Bur. Mines Rept. Inv. 6822, 7 p.

2. Adami, L. H., and Kelley, K. K., 1963, Heats of formation of two crystalline hydrates of ferrous sulfate: U. S. Bur. Mines Rept. Inv. 6260, 7 p.

3. Adami, L. H., and King, E. G., 1964, Heats and free energies of formation of sulfides of manganese, iron, zinc, and cadmium: U.S. Bur. Mines Rept. Inv. 6495, 10 p.

4. Adami, L. H., and King, E. G., 1965, Heats of formation of anhydrous sulfates of cadmium, cobalt, copper, nickel, and zinc: U. S. Bur. Mines Rept. Inv. 6617, 10 p.

5. Agoshkov, V. M., Semenov, Yu. V. Malinko, S. V., and Khodakovskiy, I. L., 1977, Enthalpies of datolite and danburite between 298.15 and $973.15 \mathrm{~K}$ : Geochemistry International, v. 14(6), p. 142-147.

6. Akaogi, M., and Navrotsky, A., 1984, The quartz-cosite-stishovite transformations: new calorimetric measurements and calculations of phase diagrams: Physics of the Earth and Planetary Interiors, v. 36, p. 124-134.

7. Amosee, J. and Mathieu, J.C., 1980, The enthalpies of formation of $\mathrm{FeWO}_{4}, \mathrm{MnWO}_{4}$, and their solid solutions: Jour. Chem. Thermodynamics, v. 12, p. 683-689.

8. Anovitz, L. M., Essene, E. J., Hemingway, B. S., Komada, N. L., and Westrum, E. F., Jr., 1988, The heat-capacities of grunerite and deerite: phase equilibria in the system Fe-Si-C-O-H and implications for the metamorphism of the banded iron formations: EOS, v. 69, p. 515. 
9. Anovitz, L. M., Essene, E. J., Metz, G. W., Bohlen, S. R., Westrum, E. F., Jr., and Hemingway, B. S., 1993, Heat capacity and phase equilibria of almandine, $\mathrm{Fe}_{3} \mathrm{Al}_{2} \mathrm{Si}_{3} \mathrm{O}_{12}:$ Geochim. Cosmochim. Acta, v. 57 , p. $4191-4204$.

10. Anovitz, L. M., Hemingway, B. S., Westrum, E. F., Jr., Metz, G. W., and Essene, E. J., 1987, Heat capacity measurements for cryolite $\left(\mathrm{Na}_{3} \mathrm{AlF}_{8}\right)$ and reactions in the system $\mathrm{Na}-\mathrm{Fe}-\mathrm{Al}-\mathrm{Si}-\mathrm{O}-\mathrm{F}$ : Geochim. Cosmochim. Acta, v. 51, p. 3087-3103.

11. Anovitz, L. M., Treiman, A. H., Essene, E. J., Hemingway, B. S., Westrum, E. F., Jr., Wall, V. J., Burriel, R., and Bohlen, S. R., 1985, The heat-capacity of ilmenite and phase equilibria in the system Fe-Ti-O: Geochim. Cosmochim. Acta, v. 49, p. 2027-2040.

12. Apps, J. A., Neil, J. M., and Jan, C.-H., 1988, Thermochemical properties of gibbsite, bayerite, boehmite, diaspore, and the aluminate ion between 0 and $350^{\circ} \mathrm{C}$ : University of California Lawrence Berkeley Rept., LBL-21482.

13. Ashida, T., Kume, S., Ito, E., and Navrotsky, A., 1988, $\mathrm{MgSiO}_{3}$ ilmenite: heat capacity, thermal expansivity, and enthalpy of transformation: Phys. Chem. Minerals, v. 16, p. 239-245.

14. Baker, F. B., Huber, E. J., Jr., Holley, C. E., Jr., and Krikorian, N. H., 1971, Enthalpies of formation of cerium dioxide, cerium sesquicarbide, and cerium dicarbide: Jour. Chem. Thermodynamics, v. 3, p. 77-84.

15. Bannister, M. J., 1984, The standard molar Gibbs free energy of formation of PbO. Oxygen concentration cell measurements at low temperatures: Jour. Chem. Thermodynamics, v. 16, p. 787-792. 
16. Bannister, M. J., 1986, Oxygen concentration-cell determination of the standard molar Gibbs free energy of formation of $\mathrm{SnO}_{2}$ : Jour. Chem. Thermodynamics, v. 18, p. 455-463.

17. Barany, R., 1962, Heats and free energies of formation of calcium tungstate, calcium molybdate, and magnesium molybdate: U.S. Bur. Mines Rept. Inv. 6143, 11 p.

18. Barany, R., 1965, Heats of formation of goethite, ferrous vanadate, and manganese molybdate: U.S. Bur. Mines Rept. Inv. 6618, 10 p.

19. Barany, R., and Adami, L. H., 1965, Heats of formation of anhydrous ferric sulfate and indium sulfate: U. S. Bur. Mines Rept. Inv. 6687, 8 p.

20. Barany, R., and Adami L. H., 1966, Heats of formation of lithium sulfate and five potassium- and lithium-aluminum silicates: U.S. Bur. Mines Rept. Inv. 6873, 18 p.

21. Bartel, J.J. and Westrum, E.F., Jr., 1975, Heat capacities of $\mathrm{Fe}_{3} \mathrm{O}_{4}$ and $\mathrm{ZnFe}_{2} \mathrm{O}_{4}$ from 300 to $500 \mathrm{~K}$ : Jour. Chem. Thermodynamics, v. 7, p. 706-708.

22. Bartel, J.J. and Westrum, E. F., Jr., 1976, Thermodynamics of Fe(II) Fe(III) oxide systems. II zinc and cadmium-doped $\mathrm{Fe}_{3} \mathrm{O}_{4}$ and crystalline magnetite: Jour. Chem. Thermodynamics, v. 8, p. 583-600.

23. Barton, M. D., 1982, The thermodynamic properties of topaz solid solutions and some petrologic applications: Am. Mineralogist, v. 67, p. 956-974.

24. Barton, M. D., Haselton, H. T., Jr., Hemingway, B. S., Kleppa, O. J., and Robie, R. A., 1982, The thermodynamic properties of fluor-topaz: Am. Mineralogist, v. 67, p. 350-355. 
25. Barton, P. B., Jr., 1969, Thermochemical study of the system Fe-As-S: Geochim. Cosmochim. Acta, v. 33, p. 841-857.

26. Barton, P. B., Jr., and Bethke, P. M., 1960, Thermodynamic properties of some synthetic zinc and copper minerals: Am. Jour. Sci., v. 258-A, (Bradley volume), p. 21-34.

27. Belitskiy, I. A., Gabunda, S. P., Drebushchak, V. A., Naumov, V. N., and Nogteva, V. V., 1984, The specific heat of edingtonite at 5-316 $\mathrm{K}$ and the entropy and enthalpy under standard conditions: Geochemistry International, v. 21(3), p. 21-23.

28. Bennington, K. O., 1982, Stability relationships between petalite $\left(\mathrm{LiAlSi}_{4} \mathrm{O}_{10}\right)$ and spodumene $\left(\mathrm{LiAlSi}_{2} \mathrm{O}_{6}\right)$ : U.S. Bur. Mines Rept. Inv. 8719, 15 p.

29. Bennington, K. O., Beyer, R. P., and Brown, R. R., 1984, Thermodynamic properties of hedenbergite, a complex silicate of $\mathrm{Ca}, \mathrm{Fe}, \mathrm{Mn}$, and $\mathrm{Mg}$ : U.S. Bur. Mines Rept Inv. 8873, 19 p.

30. Bennington, K. O., Beyer, R. P., and Johnson, G. K., 1983, Thermodynamic properties of pollucite la cesium-aluminum silicate): U.S. Bur. Mines Rept. Inv. 8779, 18 p.

31. Bennington, K. O., and Brown, R. R., 1982, Thermodynamic properties of synthetic acmite $\left(\mathrm{NaFe}^{3+} \mathrm{Si}_{2} \mathrm{O}_{6}\right):$ U.S. Bur. Mines Rept Inv. 8621, 12 p.

32. Bennington, K. O., Brown, R. R., Bell, H. E., and Beyer, R. P., 1987, Thermodynamic properties of two manganese silicates, pyroxmangite and fowlerite: U.S. Bur. Mines Rept Inv. 9064, 22 p.

33. Bennington, K. O., Brown, R. R., and Beyer, R. P., 1984, Thermodynamic properties of aegirine: U.S. Bur. Mines Rept. Inv. 8912, v. 16, p. 
34. Bennington, K. O., Ferrante, M. J., and Stuve, J. M., 1978, Thermodynamic data on the amphibole asbestos minerals amosite and crocidolite: U.S. Bur. Mines Rept. Inv. 8265, 30 p.

35. Bennington, K. O., Stuve, J. M., and Ferrante, M. J., 1980, Thermodynamic properties of petalite $\left(\mathrm{Li}_{2} \mathrm{Al}_{2} \mathrm{Si}_{8} \mathrm{O}_{20}\right):$ U.S. U.S. Bur. Mines Rept. Inv. 8451, 20 p.

36. Berg, R. L., and Vanderzee, C. E., 1978, Thermodynamics of carbon dioxide and carbonic acid: (a) the standard enthalpies of solution of $\mathrm{Na}_{2} \mathrm{CO}_{3}(\mathrm{~s}), \mathrm{NaHCO}_{3}(s)$, and $\mathrm{CO}_{2}(\mathrm{~g})$ in water at $298.15 \mathrm{~K}$; (b) the standard enthalpies of formation, standard Gibbs energies of formation, and standard entropies of $\mathrm{CO}_{2}(\mathrm{aq}), \mathrm{HCO}_{3}(\mathrm{aq}), \mathrm{CO}_{3}^{2-}(\mathrm{aq}), \mathrm{NaHCO}_{3}(\mathrm{~s}), \mathrm{Na}_{2} \mathrm{CO}_{3}(\mathrm{~s}), \mathrm{Na}_{2} \mathrm{CO}_{3} \cdot \mathrm{H}_{2} \mathrm{O}(\mathrm{s})$, and $\mathrm{Na}_{2} \mathrm{CO}_{3} \cdot 1 \mathrm{H}_{2} \mathrm{O}(\mathrm{s})$ : Jour. Chem. Thermodynamics, v. 10, p. 1113-1136.

37. Berman, R. G., 1988, Internally consistent thermodynamic data for minerals in the system $\mathrm{Na}_{2} \mathrm{O}-\mathrm{K}_{2} \mathrm{O}-$ $\mathrm{CaO}-\mathrm{MgO}-\mathrm{FeO}-\mathrm{Fe}_{2} \mathrm{O}_{3}-\mathrm{Al}_{2} \mathrm{O}_{3}-\mathrm{SiO}_{2}-\mathrm{TiO}_{2}-\mathrm{H}_{2} \mathrm{O}-\mathrm{CO}_{2}$ : Jour. Petrology, v. 29 pt. 2, p. 445-522.

38. Beyer, R. P., Ferrante, M. J., and Mrazek, R. V., 1983, An automated calorimeter for heat-capacity measurements from 5 to $300 \mathrm{~K}$. The heat capacity of cadmium sulfide from 5.37 to $301.8 \mathrm{~K}$ and the relative enthalpy to $1103.4 \mathrm{~K}$ : Jour. Chem. Thermodynamics, v. 15, p. 827-834.

39. Boerio, J. and Westrum, E.F., Jr., 1978, Heat capacity and thermodynamic properties of strontium from 5 to $350 \mathrm{~K}$ : Jour. Chem. Thermodynamics, v. 10, p. 1-7.

40. Boerio-Goates, J., Artman, J. I., and Woodfield, B. F., 1990, Heat capacity studies of phase transition in langbeinites II. $\mathrm{K}_{2} \mathrm{Mg}_{4}\left(\mathrm{SO}_{4}\right)_{3}$ : Phys. Chem. Minerals, v. 17, p. 173-178.

41. Bohlen, S. R., Metz, G. W., Essene, E. J., Anovitz, L. M., Westrum, E. F., Jr., and Wall, V. J., 1983, Thermodynamics and phase equilibrium of ferrosilite: potential oxygen barometer in mantle rocks: EOS, v. 64, p. 350 . 
42. Bonnickson, K. R., 1954, High-temperature heat contents of calcium and magnesium ferrites: Am. Chem. Soc. Jour., v. 76, p. 1480-1482.

43. Bonnickson, K. R., 1955, High-temperature heat contents of some titanates of aluminum, iron, and zinc: Am. Chem. Soc. Jour., v. 77, p. 2152-2154.

44. Bonnickson, K. R., 1955, High-temperature heat contents of aluminates of calcium and magnesium: Jour. Phys. Chemistry, v. 59, p. 221-221.

45. Boyle, B. J., King, E. G., and Conway, K. C., 1954, Heats of formation of nickel and cobalt oxides (NiO and $\mathrm{CoO}$ ) by combustion calorimetry: Am. Chem. Soc. Jour., v. 76, p. 3835-3837.

46. Brink, I. J., and Holley, C. E., Jr., 1978, The enthalpy of formation of strontium monoxide: Jour. Chem. Thermodynamics, v. 10, p. 259-266.

47. Brodale, G., and Giauque, W. F., 1958, The heat of hydration of sodium sulfate. Low temperature heat capacity and entropy of sodium sulfate decahydrate: Am. Chem. Soc. Jour., v. 80, p. 2042-2044.

48. Brooks, A. A., 1953, A thermodynamic study of the equilibrium $2 \mathrm{Cu}(\mathrm{s})+\mathrm{H}_{2} \mathrm{~S}(\mathrm{~g})=\mathrm{Cu} \mathrm{u}_{2} \mathrm{~S}(r)+\mathrm{H}_{2}(\mathrm{~g})$ : Am. Chem. Soc. Jour., v. 75, p. 2464-2467.

49. Brousse, C., Newton, R. C., and Kleppa, O. J., 1984, Enthalpy of formation of forsterite, enstatite, akermanite, monticellite and merwinite at $1073 \mathrm{~K}$ by alkali borate solution calorimetry: Geochim. Cosmochim. Acta, v. 48, p. 1081-1088.

50. Bryndzia, L. T., and Kleppa, O. J., 1988, Standard molar enthalpies of formation of realgar ( $\alpha$-AsS) and orpiment $\left(\mathrm{As}_{2} \mathrm{~S}_{3}\right)$ by high-temperature direct-synthesis calorimetry: Jour. Chem. Thermodynamics, v. 20, p. $755-764$. 
51. Bryndzia, L. T., and Kleppa, O. J., 1988, High-temperature reaction calorimetry of solid and liquid phases in the quasi-binary system $\mathrm{Ag}_{2} \mathrm{~S}-\mathrm{Sb}_{2} \mathrm{~S}_{3}$ : Geochim. Cosmochim. Acta, v. 52, p. 167-176.

52. Bryndzia, L.T., and Kleppa, O.J., 1988, Standard enthalpies of formation of sulfides and sulfosalts in the Ag-Bi-S system by high-temperature, direct synthisis calorimetry: Economic Geology, v. 83, p. 174-181.

53. Bryndzia, L.T., and Kleppa, O.J., 1988, High-temperature reaction calorimetry of solid and liquid phases in part of the quasi-binary system $\mathrm{Cu}_{2} \mathrm{~S}-\mathrm{Sb}_{2} \mathrm{~S}_{3}$ : Am. Mineralogist, v. 73, p. 707-713.

54. Busenberg, E., and Plummer, L. N., 1986, The solubility of $\mathrm{BaCO}_{3}$ (cr)(witherite) in $\mathrm{CO}_{2}-\mathrm{H}_{2} \mathrm{O}$ solutions between 0 and $90^{\circ} \mathrm{C}$, evaluation of the association constants of $\mathrm{BaHCO}_{3}^{+}(\mathrm{aq})$ and $\mathrm{BaCO}_{3}^{\circ}(\mathrm{aq})$ between 5 and $80^{\circ} \mathrm{C}$, and a preliminary evaluation of the thermodynamic properties of $\mathrm{Ba}^{2+}(\mathrm{aq})$ : Geochim. Cosmochim. Acta, v. 50, p. 2225-2233.

55. Busenberg, E., Plummer, L. N., and Parker, V. B., 1984, The solubility of strontianite $\left(\mathrm{SrCO}_{3}\right)$ in $\mathrm{CO}_{2}-\mathrm{H}_{2} \mathrm{O}$ solutions between 2 and $91^{\circ} \mathrm{C}$, the association constants of $\mathrm{SrHCO}_{3}^{+}(\mathrm{aq})$ and $\mathrm{SrCO}_{3}(\mathrm{aq})^{\circ}$ between 5 and $80^{\circ} \mathrm{C}$, and an evaluation of the thermodynamic properties of $\mathrm{Sr}^{2+}(\mathrm{aq})$ and $\mathrm{SrCO}_{3}(\mathrm{cr})$ at $25^{\circ} \mathrm{C}$ and 1 atm total pressure: Geochim. Cosmochim. Acta, v. 48, p. 2021-2035.

56. Carpenter, M. A., McConnell, J. D. C., and Navrotsky, A., 1985, Enthalpies of ordering in the plagioclase feldspar solid solution: Geochim. Cosmochim. Acta, v. 49, p. 947-966.

57. Castanet, Robert, 1984, Selected data on the thermodynamic properties of alpha-alumina: High Temperatures - High Pressures, v. 16, p. 449-457. 
58. Cemic, L., and Kleppa, O. J., 1986, High temperature calorimetry of sulfide systems. I. Thermochemistry of liquid and solid phases of $\mathrm{Ni}+\mathrm{S}$ : Geochim. Cosmochim. Acta, v. 50, p. $1633-1641$.

59. Cemic, L., and Kleppa, O. J., 1987, High temperature calorimetry of sulfide systems II. Standard enthalpies of formation of pentlandite and violarite: Phys. Chem. Minerals, v. 14, p. 52-57.

60. Cemic, L., and Kleppa, O. J., 1988, High temperature calorimetry of sulfide systems III. Standard enthalpies of formation of phases in the systems Fe-Cu-S and Co-S: Phys. Chem. Minerals, v. 16, p. $172-179$.

61. Chang, S. S., and Bestul, A. B., 1971, Heat capacities of cubic, monoclinic, and vitreous arsenious oxide from 5 to 360 K: Jour. Chem. Physics, v. 55, p. 933-946.

62. Chang, S.S., and Bestul, A.B., 1974, Heat capacities of selenium crystal (trigonal), glass, and liquid from 5 to $360 \mathrm{~K}$ : Jour. Chem. Thermodynamics, v. 6, p. 325-344.

63. Chang, Y. A., and Ahmad, N., 1982, Thermodynamic Data on Metal Carbonates and Related Oxides, (Metallurgical Society of AIME Publ.), 235 p.

64. Charlu, T. V., Newton, R. C., and Kleppa, O. J., 1975, Enthalpies of formation at $970 \mathrm{~K}$ of compounds in the system $\mathrm{MgO}-\mathrm{Al}_{2} \mathrm{O}_{3}-\mathrm{SiO}_{2}$ from high temperature solution calorimetry: Geochim. Cosmochim. Acta, v. 39, p. 1487-1497.

65. Charlu, T. V., Newton, R. C., and Kleppa, O. J., 1978, Enthalpy of formation of some lime silicates by high-temperature solution calorimetry, with discussion of high pressure phase equilibria: Geochim. Cosmochim. Acta, v. 42, p. 367-375. 
66. Charlu, T. V., Newton, R. C., and Kleppa, O. J., 1981, Thermochemistry of synthetic $\mathrm{Ca}_{2} \mathrm{Al}_{2} \mathrm{SiO}_{7}$ (gehlenite) $\mathrm{Ca}_{2} \mathrm{MgSi}_{2} \mathrm{O}_{7}$ (akermanite) melilites: Geochim. Cosmochim. Acta, v. 45, p. 1609-1617.

67. Chase, M. W., Jr., Davies, C. A., Downey, J. R., Jr., Frurip, D. J., McDonald, R. A., and Syverud, A. N.,1985, JANAF Thermochemical Tables Third Edition, Part I, Al-Co; Part II, Cr-Zr: Jour. Phys. Chem. Ref. Data, v. 14, Supplement no. 1, p. 1-1856.

68. Chatterjee, N. D., Johannes, Wilhelm, and Leistner, Hans, 1984, The system $\mathrm{CaO}-\mathrm{Al}_{2} \mathrm{O}_{3}-\mathrm{SiO}_{2}-\mathrm{H}_{2} \mathrm{O}:$ new phase equilibrium data, some calculated phase relations, and their petrological applications: Contributions to Mineralogy and Petrology, v. 88, p. 1-13.

69. Chattilon-Colinet, C., Kleppa, O. J., Newton, R. C., and Perkins, D., 1983, Enthalpy of formation of $\mathrm{Fe}_{3} \mathrm{Al}_{2} \mathrm{Si}_{3} \mathrm{O}_{12}$ (almandine) by high temperature alkali borate solution calorimetry: Geochim. Cosmochim. Acta, v. 47, p. 439-444.

70. Chernosky, J. V., Jr., and Autio, L. K., 1979, The stability of anthophyllite in the presence of quartz: Am. Mineralogist, v. 64, p. 294-303.

71. Chhor, K., Bocquet, J. F., Pommier, C., and Chardon, B., 1986, Heat capacity and thermodynamic behavior of $\mathrm{Mn}_{3} \mathrm{O}_{4}$ and $\mathrm{ZnMn}_{2} \mathrm{O}_{4}$ at low temperatures. Jour. Chem. Thermodynamics, v. 18, p. 88-99.

72. Chhor, K., Pommier, C., Gariner, P. and Abello, L., 1991, Low temperature behavior of $a-\mathrm{PbO}$ and $\mathrm{Pb}_{1-\mathrm{x}} \mathrm{Ti}_{1+\mathrm{x}}$ oxides from calorimetric spectroscopic and neutron diffraction studies: Jour. Phys. Chem. Solids, v. 52, p. 895-902. 
73. Chisholm, R. C., and Stout, J. W., 1962, Heat capacity and entropy of $\mathrm{CoCl}_{2}$ and $\mathrm{MnCl}_{2}$ from $11^{\circ}$ to $300^{\circ} \mathrm{K}$. Thermal anomaly associated with antiferromagnetic ordering in $\mathrm{CoCl}_{2}$ : Jour. Chem. Physics. v. 36, p. 972-979.

74. Cho, M., Maruyama, S., and Liou, J.G., 1987, An experimental investigation of heulandite-laumontite equilibrium at 1000 to 2000 bar $P_{\text {fluid- }}$ Contributions to Mineralogy Petrology, v. 97, p. 43-50.

75. Cohen, E. R., and Taylor, B. N., 1987, The 1986 CODATA recommended values of the fundamental physical constants: U.S. Nat. Bur. Standards, Jour. Research v. 92, p. 85-95.

76. Cook, O. A., 1947, High-temperature heat contents of $\mathrm{V}_{2} \mathrm{O}_{3}, \mathrm{~V}_{2} \mathrm{O}_{4}$, and $\mathrm{V}_{2} \mathrm{O}_{5}$ : Am. Chem. Soc. Jour., v. 69, p. 331-333.

77. Cordfunke, E. H. P., Konings, R. J. M., and Ouweltjes, W., 1990, The standard enthalpies of formation of $\mathrm{MO}(s), \mathrm{MCl}_{2}(s)$, and $\mathrm{M}_{+2}(\mathrm{aq}, \infty)(\mathrm{M}=\mathrm{Ba}, \mathrm{Sr})$ : Jour. Chem. Thermodynamics, v. 22, p. 991-996.

78. Cordfunke, E. H. P., Ouweltjes, W., and Prins, G., 1987, Standard enthalpies of formation of Tellurium compounds. I. Tellurium dioxide: Jour. Chem. Thermodynamics, v. 19, p. 369-375.

79. Coughlin, J. P., 1950, High-temperature heat contents of manganous sulfide, ferrous sulfide, and pyrite: Am. Chem. Soc. Jour., v. 72, p. 5445-5448.

80. Coughlin, J. P., 1951, High-temperature heat contents of nickel chloride: Am. Chem. Soc. Jour., v. 73, p. 5314-5315.

81. Coughlin, J. P., 1955, High-temperature heat contents, heats of transition, and heat of fusion of anhydrous sodium sulfate: Am. Chem. Soc. Jour., v. 77, p. 868-870. 
82. Coughlin, J. P., and King, E. G., 1950, High-temperature heat contents of some zirconium-containing substances: Am. Chem. Soc. Jour., v. 72, p. 2262-2265.

83. Coughlin, J. P., King, E. G., and Bonnickson, K. R., 1951, High-temperature heat contents of ferrous oxide, magnetite and ferric oxide: Am. Chem. Soc. Jour., v. 73, p. 3891-3893.

84. Coughlin, J. P., and O'Brien, C. J., 1957, High temperature heat content of calcium orthosilicate: Jour. Phys. Chemistry., v. 61, p. 767-769.

85. Cox, J. D., Wagman, D. D., and Medvedev, V. A., 1989, CODATA key values for thermodynamics: Hemisphere, New York, 271 p.

86. Dayal, R. R., Johnson, R. E., and Muan, A., 1967, Stability of mullite derived from equilibria in the system $\mathrm{CaO}-\mathrm{Al}_{2} \mathrm{O}_{3}-\mathrm{SiO}_{2}$ : Am. Chem. Soc. Jour., v. 50, p. 537-540.

87. DeKock, C. W., 1982, Thermodynamic properties of selected transition metal sulfates and their hydrates: U. S. Bur. Mines Information Circular 8910, 45 p.

88. DeKock, C. W., 1986, Thermodynamic properties of selected metal sulfates and their hydrates: U. S. Bur. Mines Information Circular 9081, 59 p.

89. Dellien, I., McCurdy, K. G, and Hepler, L. G., 1976, Enthalpies of formation of lead chromate, lead molybdate, and lead tungstate, and the entropy of aqueous tungstate ion: Jour. Chem. Thermodynamics, v. 8, p. 203-207.

90. Desai, P. D., 1986, Thermodynamic properties of iron and silicon: Jour. Phys. Chem. Ref. Data, v. 15, p. 967-983. 
91. Desai, P. D., 1987, Thermodynamic properties of manganese and molybdenum: Jour. Phys. Chem. Reference Data, v. 16, p. 91-108.

92. Desai, P. D., 1987, Thermodynamic properties of aluminum: Int. Jour. Thermophysics, v. 8, p. 621-638.

93. Ditmars, P. D., and Douglas, T. B., 1971, Measurement of the relative enthalpy of pure $\alpha-\mathrm{Al}_{2} \mathrm{O}_{3}$ (NBS heat capacity and enthalpy standard reference material no. 720 ) from 273 to $1173 \mathrm{~K}$ : U. S. Natl. Bur. Standards Jour. Research, v. 75A, p. 401-420.

94. Ditmars, D. A., Isihara, S., Chang, S. S., Bernstein, G., and West, E. D., 1982, Enthalpy and heatcapacity standard reference material: synthetic sapphire $\left(\gamma-\mathrm{Al}_{2} \mathrm{O}_{3}\right)$ from 10 to $2250 \mathrm{~K}$ : U.S. Natl. Bur. Standards Jour. Research, v. 87, p. 159-163.

95. Donahoe, R. J., Hemingway, B. S., and Liou, J. G., 1990, Thermochemical data for merlinoite: 1. Lowtemperature heat capacities, entropies, and enthalpies of formation at $298.15 \mathrm{~K}$ of six synthetic samples having various $\mathrm{Si} / \mathrm{Al}$ and $\mathrm{Na} /(\mathrm{Na}+\mathrm{K})$ ratios: $\mathrm{Am}$. Mineralogist, v. 75, 188-200

96. Donahoe, R. J., Liou, J. G., and Hemingway, B. S., 1990, Thermochemical data for merlinoite: 2. Free energies of formation at $298.15 \mathrm{~K}$ of six synthetic samples having various $\mathrm{Si} / \mathrm{Al}$ and $\mathrm{Na} /(\mathrm{Na}+\mathrm{K})$ ratios and application to saline, alkaline lakes: Am. Mineralogist, v. 75, 201-208

97. Dzhabbarov, A.I., 1985, Heat capacity of the anisotropic antiferromagnetic semiconductor iron antimony sulfide $\left(\mathrm{FeSb}_{2} \mathrm{~S}_{4}\right)$ at low temperature: Zhur. Fiz. Khim., v. 59, p. 202-204.

98. Egami, Akira, Nagakawa, T., Oishi, T., Ono, K., and Moriyama, J., 1986, A thermodynamic study of the systems $\mathrm{Ni}-\mathrm{S}$ and $\mathrm{Co}-\mathrm{S}$ by $\mathrm{CaF}_{2}$ solid electrolyte galvanic cell technique: Trans. Japan Institute of Metals, v. 27, p. 890-897. 
99. Egan, E. P., Jr., Wakefield, Z. T., and Elmore, K. L., 1950, High-temperature heat content of hydroxyapatite: Am. Chem. Soc. Jour., v. 72, p. 2418-2421.

100. Egan, E. P., Jr., Wakefield, Z. T., and Luff, B. B., 1961, Low temperature heat capacity, entropy and heat of formation of crystalline and colloidal ferric phosphate dihydrate: Jour. Phys. Chemistry, v. 65 , p. $1265-1270$.

101. Ellison, A.J.G., and Navrotsky, A. 1992, Enthalpy of formation of zircon. Jour. Am. Ceramic Soc., v. 75, p. $1430-1433$.

102. Eriksson, G. and Pelton, A. D., 1993, Critical evaluation and optimization of the thermodynamic

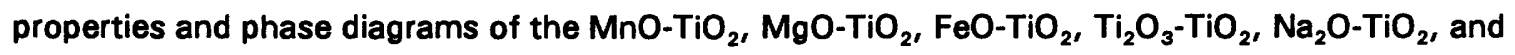
$\mathrm{K}_{2} \mathrm{O}-\mathrm{TiO}_{2}$ systems: Metallurgical Transactions, v. 24B, p. 795-805.

103. Evans, L. G. and Muan, A., 1971, Activity-composition relations of solid solutions and stabilities of manganese and nickel titanates at $1250^{\circ} \mathrm{C}$ as derived from equilibria in the systems $\mathrm{MnO}-\mathrm{CoO}-\mathrm{TiO}_{2}$ and $\mathrm{MnO}-\mathrm{NiO}^{-\mathrm{TiO}_{2}}$ : Thermochimica Acta, v. 2, p. 277-292.

104. Fei, Y., and Saxena, S. K., 1987, An equation for the heat capacity of solids: Geochim. Cosmochim. Acta, v. 51, p. 251-254.

105. Fei, Y., Saxena, S.K. and Navrotsky, A., 1990, Internally consistent thermodynamic data and equilibrium phase relations in the system $\mathrm{MgO}-\mathrm{SiO}_{2}$ at high pressure and high temperature: Jour. Geophys. Research, v. 95B, p. 6915-6928.

106. Ferrante, M. J., 1972, Enthalpies above $298.15 \mathrm{~K}$ for copper sulfate and copper oxysulfate: U. S. Bur. Mines Rept. Inv. 7600, 8 p. 
107. Ferrante, M. J., and Gokcen, N. A., 1982, Relative enthalpies of $\mathrm{Ni}_{3} \mathrm{~S}_{2}$ : U. S. Bur. Mines Rept. Inv., no. 8745,8 p.

108. Ferrante, M.J., Mrazek, R.V., and Brown, R.R., 1987, High-temperature relative enthalpies and related thermodynamic properties of Cul: U.S. Bur. Mines Rept. Inv. 9074, 10 p.

109. Ferrante, M. J., Stuve, J. M., Daut, G. E., and Pankratz, L. B., 1978, Low-temperature heat capacities and high-temperature enthalpies of cuprous and cupric sulfides: U.S. Bur. of Mines Rept. Inv. 8305, p. 1-22. See also High Temp Science, v. 14, p. 77-90.

110. Ferrante, M. J., Stuve, J. M., and Richardson, D.W., 1976, Thermodynamic data for synthetic dawsonite: U.S. Bur. Mines Rept. Inv. 8129, 13 p.

111. Fitzgibbon, G. C., Huber, E. J., Jr., and Holley, C. E., Jr., 1973, Enthalpy of formation of barium monoxide: Jour. Chem. Thermodynamics, v. 5, p. 577-582.

112. Flotow, H. E., and Osborne, D. W., 1974, The heat capacity of dicesium monoxide $\left(\mathrm{Cs}_{2} \mathrm{O}\right)$ from 5 to $350 \mathrm{~K}$ and the Gibbs energy of formation to $763 \mathrm{~K}$ : Jour. Chem. Thermodynamics, v. 6, p. 135-140.

113. Fredrickson, D. R., and Chasanov, M. G., 1970, Enthalpy of uranium dioxide and sapphire to $1500 \mathrm{~K}$ by drop calorimetry: Jour. Chem. Thermodynamics, v. 2, p. 623-630.

114. Fredrickson, D. R., and Chasanov, M. G., 1971, The enthalpy of molybdenum disulfide to $1200^{\circ} \mathrm{K}$ by drop calorimetry: Jour. Chem. Thermodynamics, v. 3, p. 693-696.

115. Fredrickson, D. R., and Chasanov, M. G., 1973, The enthalpy of sodium oxide $\mathrm{Na}_{2} \mathrm{O}$ to $1300 \mathrm{~K}$ by drop calorimetry: Jour. Chem. Thermodynamics, v. 5, p. 485-490. 
116. Furukawa, G. T., and Saba, W. G., 1965, Heat capacity and thermodynamic properties of beryllium aluminate (chrysoberyl), $\mathrm{BeO} \cdot \mathrm{Al}_{2} \mathrm{O}_{3}$, from 16 to $380^{\circ} \mathrm{K}$ : U. S. Natl. Bur. Standards Jour. Research., v. 65, p. 13-18.

117. Garvin, D., Parker, V. B., and White, H. J., 1987, CODATA thermodynamic tables. Selections for some compounds of calcium and related mixtures: A prototype set of tables. Hemisphere, New York, $356 \mathrm{p}$.

118. Gillet, P., Reynard, B., and Tequi, C., 1989, Thermodynamic properties of glaucophane: new data from calorimetric and spectroscopic measurements: Phys. Chem. Minerals, v. 16, p. 659-667.

119. Gillet, P., Richet, P., Guyot, F. and Fiquet, G., 1991, High temperature thermodynamic properties of forsterite: Jour. Geophys. Research, v. 96, p. 11805-11816.

120. Goates, J. R., Cole, A. G., and Gray, E. L., 1951, Free energy of formation and solubility product constant of mercuric sulfide: Amer. Chem. Soc. Jour., v. 73, p. 3596-3597.

121. Goates, J. R., Cole, A. G., Gray, E. L., and Faux, N. D., 1951, Thermodynamic properties of silver sulfide: Am. Chem. Soc. Jour., v. 73, p. 707-708.

122. Goldman, D. T., and Bell, R. J., 1986, The international system of units (SI): U.S. Natl. Bur. Standards Special Publication 330, $51 \mathrm{p}$.

123. Goldsmith, J. R., and Jenkins, D. M., 1985, The high-low albite relations revealed by reversal of degree of order at high pressures: Am. Mineralogist, v. 70, p. 911-923.

124. Good, W. D., Lacina, J. L., and McCullough, J. P., 1960, Sulfuric acid: heat of formation of aqueous solutions by rotating bomb calorimetry: Am. Chem. Soc. Jour., v. 82, p. 5589-5591. 
125. Grimsey, E.J. and Reynolds, K.A., 1986, Equilibrium oxygen pressure of $\left(\mathrm{Co}_{3} \mathrm{O}_{4}+\mathrm{CoO}\right)$ from 1173 to 1228K. Jour. Chem Thermodynamics, v. 18, p. 473-476.

126. Gronvold, F., 1973, Heat capacities and thermodynamic properties of hexagonal and liquid selenium in the range 298 to $1000 \mathrm{~K}$. Enthalpy and temperature of fusion: Jour. Chem. Thermodynamics, v. 5, p. 525-531.

127. Gronvold, F., 1975, Heat capacity and thermodynamic properties of bismuth in the range 300 to 950 K. Fusion characteristics: Acta Chem. Scandinavica, v. 29A, p. 945-955.

128. Gronvold, F., Kveseth, N. J., Sveen, A., and Tichy, J., 1970, Thermodynamics of the UO $2+x$ phase 1. Heat capacities of $\mathrm{UO}_{2.017}$ and $\mathrm{UO}_{2.254}$ from 300 to $1000 \mathrm{~K}$ and electronic contributions: Jour. Chem. Thermodynamics, v. 2, p. 665-680.

129. Gronvold, F., and Meisingset, K. K., 1983, Thermodynamic properties and phase transitions of salt hydrates between 270 and 400 K. II. $\mathrm{Na}_{2} \mathrm{CO}_{3} \cdot \mathrm{H}_{2} \mathrm{O}$ and $\mathrm{Na}_{2} \mathrm{CO}_{3} \cdot 1 \mathrm{OH}_{2} \mathrm{O}$ : Jour. Chem. Thermodynamics, v. 15, p. 881-889.

130. Gronvold, F. and Samuelsen, E.J., 1975, Heat capacity and thermodynamic properties of $a-\mathrm{Fe}_{2} \mathrm{O}_{3}$ in the region 300-1050 K. Antiferromagnetic transition: Jour. Phys. Chem. Solids, v. 36, p. 249-256.

131. Gronvold, F., and Stolen, S., 1992, Thermodynamics of iron sulfides II. Heat capacity and thermodynamic properties of $\mathrm{FeS}$ and of $\mathrm{Fe}_{0.875} \mathrm{~S}$ at temperatures from $298.15 \mathrm{~K}$ to $1000 \mathrm{~K}$, of $\mathrm{Fe}_{0.98} \mathrm{~S}$ from $298.15 \mathrm{~K}$ to $800 \mathrm{~K}$ and of $\mathrm{Fe}_{0.89} \mathrm{~S}$ from $298.15 \mathrm{~K}$ to about $650 \mathrm{~K}$. Thermodynamics of formation: Jour. Chem. Thermodynamics, v. 24, p. 913-916. 
132. Grønvold, F., Stølen, S., Tolmach, P. and Westrum, E. F., Jr., 1993, Heat capacities of the wüstites $\mathrm{Fe}_{0.9379} \mathrm{O}$ and $\mathrm{Fe}_{0.9254} \mathrm{O}$ at temperatures $\mathrm{T}$ from $5 \mathrm{~K}$ to $350 \mathrm{~K}$. Thermodynamics of the reaction $\mathrm{xFe}(\mathrm{s})$ $+(1 / 4) \mathrm{Fe}_{3} \mathrm{O}_{4}(\mathrm{~s})=\mathrm{Fe}_{(0.7500+x)} \mathrm{O}(\mathrm{s})=\mathrm{Fe}_{1-\mathrm{y}} \mathrm{O}(\mathrm{s})$ at $\approx 850 \mathrm{~K}$, and properties of $\mathrm{Fe}_{1-\mathrm{y}} \mathrm{O}(\mathrm{s})$ to $\mathrm{T}=1000$ K. Thermodynamics of formation of wüstite: Jour. Chem. Thermodynamics, v. 25, p. 1089-1117.

133. Gronvold, F. Stolen, S., Westrum, E. F., Jr., and Galeas, C. G., 1987, Thermodynamics of copper sulfides III. Heat capacities and thermodynamic properties of $\mathrm{Cu}_{1.75} \mathrm{~S}, \mathrm{Cu}_{1.80} \mathrm{~S}$ and $\mathrm{Cu}_{1.85} \mathrm{~S}$ from 5 to about $700 \mathrm{~K}$ : Jour. Chem. Thermodynamics, v. 19, p. 1305-1324.

134. Gronvold, F., and Sveen, A., 1974, Heat capacity and thermodynamic properties of synthetic magnetite $\left(\mathrm{Fe}_{3} \mathrm{O}_{4}\right)$ from 300 to $1050 \mathrm{~K}$. Ferrimagnetic transition and zero-point entropy: Jour. Chem. Thermodynamics, v. 6, p. 859-872.

135. Gronvold, F., Svein, S., Labban, A. K., and Westrum, E. F., Jr., 1991, Thermodynamics of iron sulfides I. Heat capacity and thermodynamic properties of $\mathrm{Fe}_{9} \mathrm{~S}_{10}$ at temperatures from $5 \mathrm{~K}$ to $740 \mathrm{~K}$ : Jour. Chem. Thermodynamics, v. 23, p. 261-272.

136. Gronvold, F., Thurmann-Moe, T., and Westrum, E. F., Jr., and Chang, E., 1961, Low-temperature heat capacities and thermodynamic functions of some platinum group chalcogenides. I. Monochalcogenides: PtS, PtTe and PdTe: Jour. Chem. Physics, 35, p. 1665-1669.

137. Gronvold, F. and Westrum, E.F., Jr., 1959, a-ferric oxide: low temperature heat capacity and thermodynamic functions. Am. Chem. Soc. Jour., v. 81, p. 1780-1783.

138. Gronvold, F., and Westrum, E. F., Jr., 1962, Heat capacities and thermodynamic functions of iron disulfide (pyrite), iron-diselenide, and nickel diselenide from 5 to $350^{\circ} \mathrm{K}$. The estimation of standard entropies of transition metal chalcogenides: Inorganic Chemistry, v. 1, p. 36-48. 
139. Gronvold, F., and Westrum, E. F., Jr., 1976, Heat capacities of iron disulfides. Thermodynamics of marcasite from 5 to to $700 \mathrm{~K}$, pyrite from 350 to $770 \mathrm{~K}$, and the transformation of marcasite to pyrite: Jour. Chem. Thermodynamics, v. 8, 1039-1048.

140. Gronvold, F., and Westrum, E. F., Jr., 1980, The anilite/ low-digenite transition: Am. Mineralogist, v. 65, p. $574-575$.

141. Gronvold, F., and Westrum, E. F., Jr., 1986, Silver(I) sulfide: $\mathrm{Ag}_{2}$ S. Heat capacity from 5 to $1000 \mathrm{~K}$, thermodynamic properties, and transitions: Jour. Chem. Thermodynamics, v. 18, p. 381-401.

142. Gronvold, F., and Westrum, E. F., 1987, Thermodynamics of copper sulfides. I. Heat capacity and thermodynamic properties of copper (I) sulfide, $\mathrm{Cu}_{2} \mathrm{~S}$, from 5 to $950 \mathrm{~K}$ : Jour. Chem. Thermodynamics, v. 19, p. 1183-1198.

143. Gronvold, F., Westrum, E. F., Jr., and Chou, C., 1959, Heat capacities and thermodynamic properties of the pyrrhotites FeS and $\mathrm{Fe}_{0.877} \mathrm{~S}$ from 5 to $350^{\circ} \mathrm{K}$ : Jour. Chem. Physics, v. 30, p. 528-531.

144. Gupta, A., and Chatterjee, N. D., 1978, Synthesis, composition, thermal stability and thermodynamic properties of bicchulite, $\mathrm{Ca}_{2}\left[\mathrm{Al}_{2} \mathrm{SiO}_{6}\right](\mathrm{OH})_{2}: \mathrm{Am}$. Mineralogist, v. 63, p. 58-65.

145. Gurevich, V.M., Gorbunov, V.E., Gavrichev, K.S., and Khodakovskii, I.L., 1988, The low-temperature heat capacity of livingstonite $\left(\mathrm{HgSb}_{4} \mathrm{~S}_{8}\right)$ : Russ. Jour. Phys. Chemistry, v. 62, p. 699-700.

146. Gurevich, V. M., Gorbunov, V. E., Gavrichev, K. S., and Khodakovsky, I. L., 1989, Low-temperature heat capacity of proustite $\mathrm{Ag}_{3} \mathrm{AsS}_{3}$ and smithite $\mathrm{AgAsS}_{2}$ : Geokhimiia, p. 132-139. 
147. Gurevich, V. M., Semenov, Yu. V., Sidorov, Yu. I., Gorbunov, V. E., Gavrichev, K. S., Zhananov, V. M., Turdakin, V. A., and Khodakovsky, I. L., 1990, Low-temperature specific heat of epidote $\mathrm{Ca}_{2} \mathrm{FeAl}_{2} \mathrm{Si}_{3} \mathrm{O}_{12}(\mathrm{OH})$. Geochemistry International 27(8), p.111-114.

148. Haas, J. L., Jr., and Hemingway, B. S., 1992, Recommended standard electrochemical potentials and fugacities of oxygen for the solid buffers and thermodynamic data in the systems iron-siliconoxygen, nickel-oxygen, and copper-oxygen / by John L. Hass, Jr with comments and additions by Bruce S. Hemingway: U.S. Geological Survey Open-file Report 92-267.

149. Haas, J. L., Jr., Robinson, G. R., Jr., and Hemingway, B. S., 1981, Thermodynamic tabulations for selected phases in the system $\mathrm{CaO}-\mathrm{Al}_{2} \mathrm{O}_{3}-\mathrm{SiO}_{2}-\mathrm{H}_{2} \mathrm{O}$ at $101.325 \mathrm{kPa}(1 \mathrm{~atm})$ between $273.15 \mathrm{~K}$ and 1800 K: Jour. Phys. Chem. Ref. Data, v. 10, p. 575-669.

150. Halbach, H., and Chatterjee, N. D., 1984, An internally consistent set of thermodynamic data for twenty-one $\mathrm{CaO}-\mathrm{Al}_{2} \mathrm{O}_{3}-\mathrm{SiO}_{2}-\mathrm{H}_{2} \mathrm{O}$ phases by linear parametric programming: Contributions to Mineralogy and Petrology, v. 88, p. 14-23.

151. Harlov, D.E., and Newton, R.C., 1992, Experimental determination of the reaction 2 magnetite + 2 kyanite +4 quartz $=2$ almandine $+\mathrm{O}_{2}$ at high pressure on the magnetite-hematite buffer: Am. Mineralogist, v. 77, p. 558-564.

152. Haselton, H. T., Jr., Cygan, G. L., and Jenkins, D. M., 1994, Experimental study of muscovite stability in pure $\mathrm{H}_{2} \mathrm{O}$ and 1 molal $\mathrm{KCl}-\mathrm{HCl}$ solutions: (unpublished report).

153. Haselton, H. T., and Goldsmith, J. R., 1987, The decarbonation and heat capacity of $\mathrm{ZnCO}_{3}$ : Geochim. Cosmochim. Acta, v. 51, p. 261-265. 
154. Haselton, H. T., Jr., Hemingway, B. S., and Robie, R. A., 1984, Low-temperature heat capacities of $\mathrm{CaAl}_{2} \mathrm{SiO}_{6}$ glass and pyroxene and thermal expansion of $\mathrm{CaAl}_{2} \mathrm{SiO}_{6}$ pyroxene: Am. Mineralogist, v. 69, p. $481-489$.

155. Haselton, H. T., Jr., Hovis, G. L., Hemingway, B. S., and Robie, R. A., 1983, Calorimetric investigation of the excess entropy of mixing in analbite-sanidine solid solutions: Lack of evidence for $\mathrm{Na}$, K short-range order and implications for two-feldspar thermometry: Am. Mineralogist, v. 68, p. 398-413.

156. Haselton, H. R., Jr., Robie, R. A., and Hemingway, B. S., 1987, Heat capacities of synthetic hedenbergite, ferrobustamite, and $\mathrm{CaFeSi}_{2} \mathrm{O}_{0}$ glasses: Geochim. Cosmochim. Acta, v. 51, p. 2211-2217.

157. Haselton, H. T., Jr., and Westrum, E. F., Jr., 1980, Low-temperature heat capacities of synthetic pyrope, grossular, and pyrope $_{80}$ grossular $_{40}:$ Geochim. Cosmochim. Acta, v. 44, p. 701-709.

158. Hatton, W. E., Hildenbrand, D. L., Sinke, G. C., and Stull, D. R., 1959, The chemical thermodynamic properties of calcium hydroxide: Am. Chem. Soc. Jour., v. 81, p. 5028-5030.

159. Helgeson, H. C., Delany, J. M., Nesbitt, H. W., and Bird, D. K., 1978, Summary and critique of the thermodynamic properties of rock-forming minerals: Am. Jour. Science, v.278-A, 220 p.

160. Hemingway, B. S., 1987, Quartz: Heat capacities from 340 to $1000 \mathrm{~K}$ and revised values for the thermodynamic properties: Am. Mineralogist, v. 72, p. 273-279.

161. Hemingway, B. S., 1990, Thermodynamic properties for bunsenite, NiO, magnetite, $\mathrm{Fe}_{3} \mathrm{O}_{4}$, and hematite, $\mathrm{Fe}_{2} \mathrm{O}_{3}$ with comments on selected oxygen buffers: Am. Mineralogist, v. 75, p. 781-790. 
162. Hemingway, B. S., 1991, Thermodynamic properties for anthophyllite and talc: Corrections and discussion of calorimetric data: Am. Mineralogist, v. 76, p. 1589-1596.

163. Hemingway, B. S., Anovitz, L. M., and Robie, R. A., 1990, Thermodynamic properties of dumortierite $\left\langle\mathrm{Si}_{3} \mathrm{~B}(\mathrm{Al})_{6.75}[]_{0.25} \mathrm{O}_{17.25}(\mathrm{OH})_{0.75}:\right.$ Am. Mineralogist, v. 75, 1370-1375.

164. Hemingway, B. S., Barton, M. D., Robie, R. A., and Haselton, H. T., Jr., 1986, Heat capacities and thermodynamic functions for beryl, $\mathrm{Be}_{3} \mathrm{Al}_{2} \mathrm{Si}_{6} \mathrm{O}_{18}$, phenakite, $\mathrm{Be}_{2} \mathrm{SiO}_{4}$, euclase, $\mathrm{BeAlSiO}(\mathrm{OH})$, bertrandite, $\mathrm{Be}_{4} \mathrm{Si}_{2} \mathrm{O}_{7}(\mathrm{OH})_{2}$, and chrysoberyl, $\mathrm{BeAl}_{2} \mathrm{O}_{4}$ : Am. Mineralogist, v. 71, p. 557-568.

165. Hemingway, B. S., Evans, H. T., Jr., Nord, G. L., Haselton, H. T., Jr., Robie, R. A., and McGee, J. J., 1987, Akermanite: phase transitions in heat capacity and thermal expansion, and revised thermodynamic data: Canadian Mineralogist, v. 24, p. 425-434.

166. Hemingway, B. S. and Haselton, H. T., Jr., 1994, A reevaluation of the calorimetric data for the enthalpy of formation of some K- and Na-bearing silicate minerals: U. S. Geological Survey Open-file Report 94-576, 33 p.

167. Hemingway, B. S., Krupka, K. M., and Robie, R. A., 1981, Heat capacities of the alkali feldspars between 350 and $1000 \mathrm{~K}$ by differential scanning calorimetry. The thermodynamic functions of the alkali feldspars from 298.15 to $1400 \mathrm{~K}$, and the reaction quartz + jadeite = analbite: Am. Mineralogist, v. 66, p. $1202-1215.70$ p.

168. Hemingway, B. S., and Robie, R. A., 1972, The heat capacities at low temperatures and entropies at 298.15 $\mathrm{K}$ of huntite, $\mathrm{CaMg}_{3}\left(\mathrm{CO}_{3}\right)_{4}$, and artinite, $\mathrm{Mg}_{2}(\mathrm{OH})_{2} \mathrm{CO}_{3} \cdot 3 \mathrm{H}_{2} \mathrm{O}: \mathrm{Am}$. Mineralogist, v. 57, p. 1754-1767. 
169. Hemingway, B. S., and Robie, R. A., 1973, A calorimetric determination of the standard enthalpies of formation of huntite, $\mathrm{CaMg}_{3}\left(\mathrm{CO}_{3}\right)_{4}$, and artinite, $\mathrm{Mg}_{2}(\mathrm{OH})_{2} \mathrm{CO}_{3} \cdot 3 \mathrm{H}_{2} \mathrm{O}$ and their standard Gibbs free energies of formation: U.S. Geol. Survey Jour. Research, v. 1, p. 535-541.

170. Hemingway, B. S., and Robie, R. A., 1977, The entropy and Gibbs free energy of formation of the aluminum ion: Geochim. Cosmochim. Acta, v. 41, p. 1402-1404.

171. Hemingway, B. S., and Robie, R. A., 1977, Enthalpies of formation of low albite $\left(\mathrm{NaAlSi}_{3} \mathrm{O}_{8}\right)$, gibbsite $\left(\mathrm{Al}(\mathrm{OH})_{3}\right)$, and $\mathrm{NaAlO}_{2} ;$ revised values for $\Delta \mathrm{H}^{\circ}{ }_{\mathrm{f}, 298}$ and $\Delta \mathrm{G}_{\mathrm{f}, 298}$ of alumino-silicates: U.S. Geol. Survey Jour. Research, v. 5, p. 413-429.

172. Hemingway, B. S., and Robie, R. A., 1984, Heat capacity and thermodynamic functions for gehlenite and staurolite: Comments on the Schottky anomaly in staurolite: Am. Mineralogist, v. 69, p. 307-318.

173. Hemingway, B. S., and Robie, R. A., 1984, Thermodynamic properties of zeolites: low temperature heat capacities and thermodynamic functions for phillipsite and clinoptilolite. Estimates of the thermochemical properties of zeolitic water at low temperatures: Am. Mineralogist, v. 69, p. $692-700$.

174. Hemingway, B. S., and Robie, R. A., 1990, Heat capacities and thermodynamic properties of annite (aluminous iron biotite): Am. Mineralogist, v. 75, p. 183-187

175. Hemingway, B. S. and Robie, R. A., 1994, Enthalpy and Gibbs energy of formation of dolomite, $\mathrm{CaMg}\left(\mathrm{CO}_{3}\right)_{2}$, at $298.15 \mathrm{~K}$ from HCl solution calorimetry: U. S. Geological Survey Open-File Report 94-575, 12 p. 
176. Hemingway, B. S., Robie, R. A., and Apps, J. A., 1991, Revised values for the thermodynamic properties of boehmite, $\mathrm{AlO}(\mathrm{OH})$, and comments on the relative stabilities of the aluminum hydroxide and oxyhydroxide phases: Am. Mineralogist, v. 76, p. 445-457.

177. Hemingway, B. S., Robie, R. A., Evans, H. T., Jr., and Kerrick, D. M., 1991, Heat capacities and entropies of sillimanite, fibrolite, andalusite, kyanite, and quartz and the $\mathrm{Al}_{2} \mathrm{SiO}_{5}$ phase diagram. Am. Mineralogist, v. 76, p. 1597-1613.

178. Hemingway, B. S., Robie, R. A., Fisher, J. R., and Wilson, W. H., 1977, The heat capacities of gibbsite, $\mathrm{Al}(\mathrm{OH})_{3}$, between 13 and $480 \mathrm{~K}$ and magnesite, $\mathrm{MgCO}_{3}$, between 13 and $380 \mathrm{~K}$ and their standard entropies at $298.15 \mathrm{~K}$, and the heat capacities of Calorimetry Conference benzoic acid between 12 and 316 K: U.S. Geol. Survey Jour. Research, v. 5, p. 797-806.

179. Hemingway, B. S., Robie, R. A., and Kittrick, J. A., 1978, Revised values for the Gibbs free energy of formation of $\left[\mathrm{Al}(\mathrm{OH})_{4 \times q}^{-}\right]$, diaspore, boehmite and bayerite at $298.15 \mathrm{~K}$ and I bar, the thermodynamic properties of kaolinite to $800 \mathrm{~K}$, and the heats of solution of several gibbsite samples: Geochim. Cosmochim. Acta, v. 42 , p. 1533-1543.

180. Hemingway, B. S., Robie, R. A., Kittrick, J. A., Grew, E. S., Nelen, J. A., and London, D., 1984, The heat capacities of osumilite from 298.15 to $1000 \mathrm{~K}$, the thermodynamics properties of two natural chlorites to $500 \mathrm{~K}$, and the thermodynamic properties of petalite to $1800 \mathrm{~K}$ : Am. Mineralogist, v. 69 , p. $701-710$.

181. Hemingway, B. S., Robie, R. A., and Krupka, K. M., 1992, Thermodynamic properties for berlinite, $\mathrm{AlPO}_{4}$ : U.S. Geol. Survey Open-File Rept. 92-510, 8 p. 
182. Henderson, C. E., Essene, E. J., Anovitz, L. M., Westrum, E. F., Jr., Hemingway, B. S., and Bowman, J.R., 1983, Thermodynamics and phase equilibria of clinochlore $\left(\mathrm{Mg}_{5} \mathrm{Al}\right)\left(\mathrm{Si}_{3} \mathrm{Al}\right) \mathrm{O}_{10}(\mathrm{OH})_{8}$ : EOS, v. 64, p. 466. Final term for $C_{p}$ equation is missing, the value is $+1.4955 \times 10^{5} \mathrm{~T}^{-2}$.

183. Henderson, C.M.B., and Thompson, A.B., 1980, The low-temperature inversion in sub-potassic nephelines: Am. Mineralogist, v. 65, p. 970-980.

184. Holland,T.J.B., 1980, The reaction albite = jadeite + quartz determined experimentally in the range $600-1200^{\circ} \mathrm{C}$ : Am. Mineral., v. 65, p. 129-134.

185. Holland, T. J. B., 1988, Preliminary phase relations involving glaucophane and applications to high pressure petrology: new heat capacity and thermodynamic data: Contributions to Mineralogy and Petrology, v. 99, p. 134-142.

186. Holland, T. J. B., 1989, Dependence of entropy on volume for silicate and oxide minerals: A review and a predictive model: Am. Mineralogist, v. 74, p. 5-13.

187. Holland, T. J. B., and Powell, R., 1985, An internally consistent thermodynamic dataset with uncertainties and correlations: 2. Data and results: Jour. Metamorphic Geology, v. 3, p. 343-370.

188. Holland, T. J. B. and Powell, R., 1990, An enlarged and updated internally consistent thermodynamic dataset with uncertainties and correlation: the system $\mathrm{K}_{2} \mathrm{O}-\mathrm{Na}_{2} \mathrm{O}-\mathrm{CaO}-\mathrm{MgO}-\mathrm{MnO}-\mathrm{FeO}-\mathrm{Fe}_{2} \mathrm{O}_{3}-\mathrm{Al}_{2} \mathrm{O}_{3}-$ $\mathrm{TiO}_{2}-\mathrm{SiO}_{2}-\mathrm{C}-\mathrm{H}_{2}-\mathrm{O}_{2}$ : Jour. Metamorphic Geology. v. 8, p. 89-124.

189. Holm, J. L., Kleppa, O. J., and Westrum, E. F., Jr., 1967, Thermodynamics of polymorphic transformations in silica. Thermal properties from 5 to $1070^{\circ} \mathrm{K}$ and P-T stability fields for coesite and stishovite: Geochim. Cosmochim. Acta, v. 31, p. 2289-2307. 
190. Holmes, R. D., Kersting, A. B., and Arculus, R. J., 1989, Standard molar Gibbs free energy of formation for $\mathrm{Cu}_{2} \mathrm{O}$ : high-resolution electrochemical measurements from 900 to $1300 \mathrm{~K}$ : Jour. Chem. Thermodynamics, v. 21, p. 351-361.

191. Holmes, R.D., O’Neill, H.St.C., and Arculus, R.J., 1986, Standard Gibbs free energy of formation for $\mathrm{Cu}_{2} \mathrm{O}, \mathrm{NiO}, \mathrm{CoO}$, and $\mathrm{Fe}_{x} \mathrm{O}$ : High resolution electrochemical measurements using zirconia solid electrolytes from 900-1400 K: Geochim. Cosmochim. Acta, v. 50, p. 2439-2452.

192. Hosmer, P. K., and Krikorian, O. H., 1980, The high-temperature enthalpies of zinc sulfate and zinc oxysulfate: High Temperatures-High Pressures, v. 12, p. 281-290.

193. Hostetler, P. B., 1963, The stability and surface energy of brucite in water at $25^{\circ} \mathrm{C}$ : Am. Jour. Sci., v. 21, p. 238-258.

194. Hovis, G. L., 1988, Enthalpies and volumes related to K-Na mixing and Al-Si disorder in alkali feldspars: Jour. Petrology, v. 29, p. 731-763

195. Howell, D. A., Johnson, G. K., Tasker, I. R., O’Hare, P. A. G., and Wise, W. S., 1990, Thermodynamic properties of the zeolite stilbite: Zeolites, v. 10, p. 525-531.

196. Huber, E. J., Jr., Head, E. L., and Holley, C. E., Jr., 1964, The heats of formation of zirconium diboride and dioxide: Jour. Phys. Chemistry, v. 689, p. 3040-3042.

197. Hull, H., and Turnbull, A. G., 1973, A thermochemical study of monohydrocalcite: Geochim. Cosmochim. Acta, v. 37, p. 685-694. 
198. Humphrey, G. L., King. E. G., and Kelley, K. K., 1952, Some thermodynamic values for ferrous oxide: U. S. Bur. Mines Rept. Inv. 4870, 16 p.

199. Huntzicker, J. J., and Westrum, E. F., Jr., 1971, The magnetic transition, heat capacity, and thermodynamic properties of uranium dioxide from 5 to $350 \mathrm{~K}$ : Jour. Chem. Thermodynamics, v. 3, p. 61-76.

200. Inaba, H., Miyahara, K., and Naito, K., 1984, Measurements of the molar heat capacities of $\mathrm{MoO}_{2}$ and $\mathrm{MoO}_{3}$ from 350 to $950 \mathrm{~K}$. Jour. Chem. Thermodynamics, v. 16, p. 643-651.

201. Itskevich, Ye. S., 1961, Thermodynamic investigations at low temperatures. XII. Specific heat of bismuth telluride between 1.4 and $65^{\circ} \mathrm{K}$. Enthalpy and entropy of $\mathrm{Bi}_{2} \mathrm{Te}_{3}$ at $298.15 \mathrm{~K}$ : Zhur. Fizicheskoi Khimii, v. 35, no. 8, p. 1813-1815.

202. Jacobs, G. K., Kerrick, D. M., and Krupka, K. M., 1981, The high-temperature heat capacity of natural calcite $\left(\mathrm{CaCO}_{3}\right)$ : Phys. Chem. Minerals, v. 7, p. 55-59.

203. Jeffes, J. H. E., Richardson, F. S., and Pearson, J., 1954, The heats of formation of manganous orthosilicate and manganous sulfide: Faraday Society Transactions, v. 50, p. 364-370.

204. Jenkins, D. M., and Chernosky, J. V., 1986, Phase equilibrium and crystallochemical properties of Mg-chlorite: Am. Mineralogist, v. 71, p. 924-936.

205. Jenkins, D.M., Holland, T.J.B., and Clarke, A., 1991, Experimental determination of the pressure stability and thermochemical properties of synthetic tremolite: Am. Mineralogist, v. 76, p. 458-469. 
206. Johnson, G. K., Flotow, H. E., O'Hare, P. A. G., and Wise, W. S., 1982, Thermodynamic studies of zeolites: analcime and dehydrated analcime: Am. Mineralogist, v. 67, p. 736-748.

207. Johnson, G. K., Flotow, H. E., O'Hare, P. A. G., and Wise, W. S., 1983, Thermodynamic studies of zeolites: natrolite, mesolite, and scolecite: Am. Mineralogist, v. 68, p. 1134-1145.

208. Johnson, G. K., Flotow, H. E., O’Hare, P. A. G., and Wise, W. S., 1985, Thermodynamic studies of zeolites: Heulandite: Am. Mineralogist, v. 70, p. 1065-1071.

209. Johnson, G.K., Grow, R.T. and Hubbard, W.N., 1975, The enthalpy of formation of lithium oxide $\left(\mathrm{Li}_{2} \mathrm{O}\right)$ : Jour. Chem. Thermodynamics, v. 7, p. 781-786.

210. Johnson, G. K., Papatheodorou, G. N., and Johnson, C. E., 1980, The enthalpies of formation and hightemperature thermodynamic functions of $\mathrm{As}_{4} \mathrm{~S}_{4}$ and $\mathrm{As}_{2} \mathrm{~S}_{3}$ : Jour. Chem. Thermodynamics, v. 12, no. 6, p. 545-558.

211. Johnson, G. K., Papatheodorou, G. N., and Johnson, C. E., 1981, The enthalpies of formation of $\mathrm{SbF}_{5}(1)$ and $\mathrm{Sb}_{2} \mathrm{~S}_{3}(c)$ and the high-temperature thermodynamic functions of $\mathrm{Sb}_{2} \mathrm{~S}_{3}(\mathrm{c})$ and $\mathrm{Sb}_{2} \mathrm{~S}_{3}(1)$ : Jour. Chem. Thermodynamics, v. 13, p. 745-754.

212. Johnson, G. K., Tasker, I. R., Flotow, H. E., and Wise, W. S., 1992, Thermodynamic studies of mordenite, dehydrated mordenite, and gibbsite: Am. Mineralogist, v. 77, p. 85-93. Note that quartz was taken as the reference phase for silica instead of silicalite.

213. Johnson, G. K., Tasker, I. R., Howell, D. A., and Smith, J. V., 1987, Thermodynamic properties of silicalite: Jour. Chemical Thermodynamics, v. 19, p. 617-632. 
214. Johnson, G.K., Tasker, I.R., Jurgens, R., and O'Hare, P.A.G., 1991, Thermodynamic studies of zeolites, Clinoptilolite. Jour. Chem. Thermodynamics, v. 23, p. 475-484.

215. Kale, G. M. and Jacob, K. T., 1989, Gibbs energy of formation of $\mathrm{Co}_{2} \mathrm{SiO}_{4}$ and phase relations in the system Co-Si-O: Trans. Instn. Min. Metallurgy. Sect. C: Mineral. Process. Extr. Metall., v. 98, p. $117-122$.

216. Kale, G.M. and Jacob, K.T., 1992, Chemical potential of oxygen for iron-rutile-ilmenite and iron-ilmeniteulvospinel equilibria: Metal. Transactions, v. 23B, p. 57-64.

217. Kelley, K. K., 1960, Contributions to the data on theoretical metallurgy. XIII. High-temperature heatcapacity, and entropy data for the elements and inorganic compounds: U. S. Bureau of Mines Bulletin 584, 232 p.

218. Kelley, K. K., Barany, R., King, E. G., and Christensen, A. U., 1959, Some thermodynamic properties of fluorphlogopite mica: U.S. Bur. Mines Rept. Inv. 5436, 16 p.

219. Kelley, K. K., Shomate, C. H., Young, F. E., Naylor, B. F., Salo, A. E., and Huffman, E. H., 1946, Thermodynamic properties of ammonium and potassium alums and related substances, with reference to extraction of alumina from clay and alunite: U. S. Bur. Mines Tech. Paper 688, 104 p.

220. Kelley, K. K., Todd, S. S., Or, R. L., King, E. G., and Bonnickson, K. R., 1953, Thermodynamic properties of sodium-aluminum and potassium-aluminum silicates: U.S. Bur. Mines Rept. Inv. 4955, $21 \mathrm{p}$.

221. Khodakovsky, I. L. written communication in 1993. 
222. Khriplovich, L. M., Oppermann, H., and Paukov, I. E., 1979, The heat capacity of cobalt oxide $\left(\mathrm{CO}_{3} \mathrm{O}_{4}\right)$ in the range 5.1 - 307.34 K: Zuhr. Fiz Khimii, v. 53, p. 1608-1610.

223. King, E. G., 1951, Heats of formation of crystalline calcium orthosilicate, tricalcium silicate and zinc orthosilicate: Am. Chem. Soc. Jour., v. 73, p. 656-658.

224. King, E. G., 1952, Heats of formation of manganous metasilicate (rhodonite) and ferrous orthosilicate (fayalite): Am. Chem. Soc. Jour., v. 74, p. 4446-4448.

225. King, E.G., 1957, Low-temperature heat capacities and entropies at $298.15^{\circ} \mathrm{K}$. of some crystalline silicates containing calcium. Am. Chem. Soc. Jour., v. 79, p. 5437-5438.

226. King, E. G., Barany, R., Weller, W. W., and Pankratz, L. B., 1967, Thermodynamic properties of forsterite and serpentine: U.S. Bur. Mines Rept. Inv. 6962, 19 p.

227. King, E. G., and Christensen, A. U., 1961, High-temperature heat contents and entropies of cerium dioxide and columbium dioxide: U. S. Bur. Mines Rept. Inv. 5789, 6 p.

228. King, E. G., Ferrante, M. J., and Pankratz, L. B., 1975, Thermodynamic data for $M g(O H)_{2}(b r u c i t e):$ U. S. Bur. Mines Rept. Inv. 8041, 13 p.

229. King, E.G., and Todd, S.S., 1953, Heat capacities at low-temperature and entropies at $298.16^{\circ} \mathrm{K}$ of stannic and stannus sulfides: Am. Chem. Soc. Jour., v. 75, p. 3023-3029.

230. King, E. G., and Weller, W. W., 1961, Low-temperature heat capacities and entropies at $298.15^{\circ} \mathrm{K}$ of diaspore, kaolinite, dickite and halloysite: U.S. Bur. Mines Rept. Inv. 5810, 6 p. 
231. King, E. G., and Weller, W. W., 1961, Low temperature heat capacities and entropies at $298.15^{\circ} \mathrm{K}$ of some sodium- and calcium-aluminum silicates: U.S. Bur. Mines Rept. Inv. 5855, 8 p.

232. King, E. G. and Weller, W. W., 1961, Low-temperature heat capacities and entropies at $298.15^{\circ} \mathrm{K}$ of monotungstates of sodium, magnesium, and calcium: U.S. Bur. Mines Rept. Inv. 5791, 6 p.

233. King, E. G., and Weller, W. W., 1962, Low-temperature heat capacity and entropy at $298.15^{\circ} \mathrm{K}$ of red mercuric sulfide: U.S. Bur. Mines Rept. Inv. 6001, 4 p.

234. King, E. G., and Weller, W. W., 1962, Low-temperature heat capacities and entropies at $298.15^{\circ} \mathrm{K}$ of antimony and indium sulfides: U.S. Bur. mines Rept. Inv. 6040, 5 p.

235. King, E. G., and Weller, W. W., 1970, Low-temperature heat capacities and entropies at $298.15^{\circ} \mathrm{K}$ of goethite and pyrophyllite: U.S. Bur. Mines Rept. Inv. 7369, 6 p.

236. King, E. G., Weller, W. W., and Christensen, A. U., 1960, Thermodynamics of some oxides of molybdenum and tungsten: U. S. Bur. Mines Rept. Inv. 5664, 29 p.

237. Kiseleva, I. A., 1984, The enthalpy of formation of grossular: Geochemistry International, v. 21(5), p. 138-141.

238. Kiseleva, I. A., Mel'chakova, L. V., and Ogorodnikova, L. P., 1986, Thermodynamical properties of beryllium minerals: chrysoberyl, beryl, euclase, and bertrandite: Geokhimiya, n. 5, p. 716-729.

239. Kiseleva, I. A., and Ogorodnikova, L. P., 1986, Calorimetric data on the thermodynamics of epidote, clinozoisite, and zoisite: Geokhimiia, n. 6, p. 846-853. 
240. Kiseleva, I. A., Ogorodnikova, L. P., Topor, N. D., and Chigareva, O. G., 1980, A thermochemical study of the CaO-MgO-SiO ${ }_{2}$ system: Geochemistry International, v. 16(6), p. 122-134

241. Kiseleva, I.A., Ogorodova, L.P., Melchakova, L.V., Bisengalieva, M.R., and Becturganov, N.S., 1992, Thermodynamic properties of copper carbonates-malachite $\mathrm{Cu}_{2}(\mathrm{OH})_{2} \mathrm{CO}_{3}$ and azurite $\mathrm{Cu}_{3}(\mathrm{OH})_{2}\left(\mathrm{CO}_{3}\right)_{2}:$ Phys. Chem. Minerals, v. 19, p. 322-333.

242. Kiseleva,, I. A., Ogorodova, L. P., and Sokolova, E. L., 1989, Enthalpy of formation of andradite: Geokhimiia, n. 1, p. 125-131.

243. Kiseleva, I. A., Topor, N. D., and Andreyenko, E. D., 1974, Thermodynamic parameters of minerals of the epidote group: Geochemistry International, v. 11, p. 389-398.

244. Kiseleva, I. A.;, Topor, N. D., and Melchakova, L. V., 1972, Experimental determination of heat content and heat capacity of andradite and pyrope: Geokhimiia, n. 11, p. 1372-1379.

245. Kiukkola, K., and Wagner, C., 1957, Measurements on galvanic cells involving solid electrolytes: Jour. Electrochem. Soc., v. 104, p. 379-387.

246. Ko, H. C., Ahmad, N., and Chang, Y. A., 1982, Thermodynamics of calcination of calcite: U.S. Bur. Mines Rept. Inv. 8647, 9 p.

247. Ko, H. C., and Daut, G. E., 1980, Enthalpies of formation of $\alpha$ - and $\beta$-magnesium sulfate and magnesium sulfate monohydrate: U. S. Bur. Mines Rept. Inv. 8409, 8p.

248. Ko, H. C., Ferrante, M. J., and Stuve, J. M., 1977, Thermophysical properties of acmite: Proceedings of the Seventh Symposium on Thermophysical Properties, (Am. Soc. Mech. Eng.), p. 392-395. 
249. Koehler, M. F., Barany, R., and Kelley, K. K., 1961, Heats and free energies of formation of ferrites and aluminates of calcium, magnesium, sodium, and lithium: U. S. Bur. Mines Rept. Inv. 5711, 14 p.

250. Koehler, M. F., and Coughlin, J. P., 1959, Heats of formation of ferrous chloride, ferric chloride and manganous chloride: Jour. Phys. Chemistry, v. 63, p. 605-608.

251. Kolesnik, Yu. N., Nogteva, V. V., Arkhipenko, D. K., Orekhov, B. A., and Ye, I., 1979, Thermodynamics of pyrope-grossular solid solutions and the specific heat of grossular at 13-300 K: Geokhimiia, n. 5, p. 713-721.

252. Komada, N., Westrum, E. F., Jr., and Hemingway, B. S., 1994, Thermodynamic properties of two iron silicates: heat capacities of deerite from 10 to $700 \mathrm{~K}$ and grunerite from 10 to $1000 \mathrm{~K}$ : Jour. Chem. Thermodynamics, (in press).

253. Konigsberger, E., and Gamsjager, H., 1987, Solid-solute phase equilibrium in aqueous solution: I solubility constant and free enthalpy of formation of huntite: Ber. Buns. Phys. Chemie., v. 91, p. 785-790.

254. Konings, R. J. M., Cordfunke, E. H. P., and Ouweltjes, W., 1988, The standard enthalpies of formation of hydroxides. II. The alkali hydroxides $\mathrm{CsOH}$ and $\mathrm{KOH}$ : Jour. Chem. Thermodynamics, v. 20, p. 777-780.

255. Kostryakov, V. N., and Kalinkina, I. N., 1964, Heat capacity and entropy for manganese, iron, cobalt, and nickel carbonates at low temperatures: Zhur. Fizicheskoi Khimii, v. 38, p 780-78.

256. Kornilov, A.N., Ushakova, I.M., Huber, E.T., Jr., and Holley, C.E., Jr., 1975, The enthalpy of formation of hafnium dioxide: Jour. Chem. Thermodynamics, v. 7, p. 21-26. 
257. Krupka, K. M., Hemingway, B. S., Robie, R. A., and Kerrick, D. M., 1985, High-temperature heat capacities and derived thermodynamic properties of anthophyllite, diopside, dolomite, enstatite, bronzite, talc, tremolite, and wollastonite: Am. Mineralogist, v. 70, p. 261-271.

258. Krupka, K. M., Robie, R. A., and Hemingway, B. S., 1979, The heat capacities of corundum, periclase, anorthite, $\mathrm{CaAl}_{2} \mathrm{Si}_{2} \mathrm{O}_{8}$ glass, muscovite, pyrophyllite, $\mathrm{KAISi}_{3} \mathrm{O}_{8}$ glass, grossular, and $\mathrm{NaAlSi}_{3} \mathrm{O}_{8}$ glass: Am. Mineralogist, v. 64, p. 86-101.

259. Krupka, K. M., Robie, R. A., Hemingway, B. S., Kerrick, D. M., and Ito, Jun, 1985, Low-temperature heat capacities and derived thermodynamic properties of anthophyllite, diopside, enstatite, and wollastonite: Am. Mineralogist, v. 70, p. 249-260.

260. Landee, C. P., and Westrum, E. F., Jr., 1975, Thermophysical measurements on transition-metal tungstates. I. Heat capacity of zinc tungstate from 5 to $550 \mathrm{~K}$ : Jour. Chem. Thermodynamics, v. 7, p. 973-976.

261. Landee, C. P., and Westrum, E. F., Jr., 1976, Thermophysical measurements on transition metal tungstates. III. Heat capacity of antiferromagnetic manganese tungstate: Jour. Chem. Thermodynamics, v. 8, p. 663-674.

262. Lander, J. J., 1951, Experimental heat contents of $\mathrm{SrO}, \mathrm{BaO}, \mathrm{CaO}, \mathrm{BaCO}_{3}$ and $\mathrm{SrCO}_{3}$ at high temperatures. Dissociation pressures of $\mathrm{BaCO}_{3}$ and $\mathrm{SrCO}_{3}: \mathrm{Am}$. Chem. Soc. Jour., v. 73, p. 5794-5797.

263. Lange, R. A., Carmichael, I. S. E., and Stebbins, J. F., 1986, Phase transitions in leucite $\left(\mathrm{KAISi}_{2} \mathrm{O}_{6}\right)$, orthorhombic $\mathrm{KAISiO}_{4}$, and their iron analogues: Am. Mineralogist, v. 71, p. 937-945. 
264. Lattard, D. and Evans, B.W., 1992, New experiments on the stability of grunerite: Euro., Jour. Mineralogy, v. 4, p. 219-238.

265. Lyon, W. G., and Westrum, E. F., Jr., 1974, Heat capacities of zinc tungstate and ferrous tungstate from 5 to $550^{\circ} \mathrm{K}$ : Jour. Chem. Thermodynamics, v. 6, p. 763-80.

266. Lyon, W. G., and Westrum, E. F., Jr., 1974, High temperature thermal functions and the thermochemistry of zinc tungstate: Jour. Chem. Thermodynamics, v. 6, p. 781-786.

267. Mah, A. D., 1954, Heats of formation of chromium oxide and cadmium oxide from combustion calorimetry: Am. Chem. Soc. Jour., v. 76, p. 3363-3365.

268. Mah, A. D., 1957, Heats of formation of alumina, molybdenum trioxide, and molybenum dioxide: Jour. Phys. Chemistry, v. 61, p. 1572-1573.

269. Mah, A. D., 1961, Heats of formation of cerium sesquioxide and bismuth sesquioxide by combustion calorimetry: U. S. Bur. Mines Rept. Inv. 5676, 7 p.

270. Mah, A. D., 1982, Thermodynamic data for arsenic sulfide reactions: U.S. Bur. Mines Rept. Inv. 8671, $85 \mathrm{p}$.

271. Mah, A. D., and Pankratz, L. B., 1976, Contributions to the data on theoretical metallurgy. XVI. Thermodynamic properties of nickel and its inorganic compounds: U.S. Bur. Mines Bull. 668, 125 p.

272. Mah, A. D., Pankratz, L. B., Weller, W. W., and King. E. G., 1967, Thermodynamic data for cuprous and cupric oxides: U. S. Bur. Mines Rept. Inv. 7026, 20 p. 
273. Marland, G., 1975, The stability of $\mathrm{CaCO}_{3} \cdot 6 \mathrm{H}_{2} \mathrm{O}$ (ikaite). Geochem. Cosmochim. Acta., v. 39, p. 83-91.

274. Mazdab, F. K., Anovitz, L. M., Hemingway, B. S., Robie, R. A., and Navrotsky, A., 1992, Thermodynamic properties of some boron-bearing minerals: Abstracts with Program, 1992, Geological Society of America, p A258.

275. McBride, J. J., and Westrum, E. F., Jr., 1976, Low-temperature heat capacity of anisotropic crystals. Lamellar molybdenum disulfide: Jour. Chem. Thermodynamics, v. 8, p. 37-44.

276. Mehrotra, G. M., Tare, V. B., and Wagner, J. B., Jr., 1985, The Standard Gibbs Energy of Formation of $\mathrm{Ni}_{3+\mathrm{x}} \mathrm{S}_{2}$ : Jour. Electrochem. Soc., v. 132, p. 247-250.

277. Metz, G. W., Anovitz, L. M., Essene, E. J., Bohlen, S. R., Westrum, E. F., and Wall, V. J., 1983, The heat capacity and phase equilibria of almandine: EOS v. 64, p. 346-347.

278. Mills, I., Cuitas, T., Homann, K., Kalloy, N., and Kuchitsu, K., 1988, Quantities, units and symbols in physical chemistry: Blackwell, Oxford, 134 p.

279. Mills, K.C., 1974, Thermodynamic data for inorganic sulfides, selenides and tellurides, Butterworths, London, 845 p.

280. Mizota, T., Tanada, H., Fujii, Y., and Koto, K., 1985, The heat capacity of cubanite and the anomaly in cubic $\mathrm{CuFe}_{2} \mathrm{~S}_{3}$ : Canadian Mineralogist, v. 23, p. 77-82.

281. Moecher, D. P., and Chou, I-Ming, 1990, Experimental investigation of andradite and hedenbergite equilibria employing the hydrogen sensor technique, with revised estimates of $\Delta_{\mathrm{f}} \mathrm{G}_{\mathrm{m}, 298}^{\circ}$ for andradite and hedenbergite: Am. Mineralogist, v. 75, p. 1327-1341. 
282. Moecher, D. P., and Essene, E. J., 1990, Phase equilibria for calcic scapolite and implications of variable Al-Si disorder for P-T, T-X $\mathrm{CO}_{2}$ and a-X relations. Jour. Petrology, v. 31, 997-1024.

283. Moore, G. E., 1943, Heat content of manganese dioxide and carbonate at high temperatures: Am. Chem. Soc. Jour., v. 65, p. 1398-1399.

284. Moore, G. E., 1943, Heat contents at high temperatures of the anhydrous chlorides of calcium, iron, magnesium, and manganese: Am. Chem. Soc. Jour., v. 65, p. 1700-1703.

285. Morey, G. W., Fournier, R. O., and Rowe, J. J., 1962, Solubility of quartz in water in the temperature interval from $25^{\circ}$ to $300^{\circ} \mathrm{C}$ : Geochim. Cosmochim. Acta, v. 26, p. 1029-1043.

286. Mosesman, M. A., and Pitzer, K. S., 1941, Thermodynamic properties of the crystalline forms of silica: Am. Chem. Soc. Jour., v. 63, p. 2348-2356.

287. Nabutouskaya, O.A., Opperman, H., Paukov, I.E., and Khriplovich, L.M., 1985, Thermodynamic properties of cobalt monoxide at 5-328 K: Zhur. Fiz. Khim; v. 59, p. 1253-1254.

288. Navrotsky, Alexandra, 1989, Thermochemistry of perovskites in Navrotsky, Alexandra and Weidner, D.J. (eds.), Perovskite: A structure of great interest to geophysics and materials science: AGU Geophysical Monograph 45, p. 67-80.

289. Navrotsky, Alexandra, and Coons, W. E., 1976, Thermochemistry of some pyroxenes and related compounds: Geochim. Cosmochim. Acta, v. 40, p. 1281-1288.

290. Navrotsky, Alexandra, and Kleppa, O.J., 1968, Thermodynamics of formation of simple spinels: Jour. Inorg. Nucl. Chemistry, v. 30, p. 479-498. 
291. Navrotsky, Alexandra, Pintchovski, F. S., and Akimoto, S. I., 1979, Calorimetric study of the stability of high pressure phases in the systems $\mathrm{CoO}-\mathrm{SiO}_{2}$ and ${ }^{\mathrm{F}} \mathrm{FeO}$ "- $\mathrm{SiO}_{2}$, and calculation of phase diagrams in $\mathrm{MO}-\mathrm{SiO}_{2}$ systems: Physics of the Earth and Planetary Interiors, v. 19, p. 275-292.

292. Naylor, B. F., 1944, High-temperature heat contents of ferrous and magnesium chromites: Ind. Eng. Chemistry, v. 36, p. 933-934.

293. Naylor, B. F, 1945, Heat contents at high temperatures of magnesium and calcium fluorides: Am. Chem. Soc. Jour., v. 67, p. 150-152.

294. Naylor, B. F., 1946, High-temperature heat contents of $\mathrm{TiO}_{1} \mathrm{Ti}_{2} \mathrm{O}_{3}, \mathrm{Ti}_{3} \mathrm{O}_{5}$, and $\mathrm{TiO}_{2}:$ Am. Chem. Soc. Jour. v. 68, p. 1077-1080.

295. Naylor, B. F., and Cook, O. A., 1946, High-temperature heat contents of the metatitanates of calcium, iron, and magnesium: Am. Chem. Soc. Jour., v. 68, p. 1003-1005.

296. Newton, R. C., Charlu, T. V., Anderson, P. A. M., and Kleppa, O. J., 1979, Thermochemistry of synthetic clinopyroxenes on the join $\mathrm{CaMgSi}_{2} \mathrm{O}_{3}-\mathrm{Mg}_{2} \mathrm{Si}_{2} \mathrm{O}_{6}:$ Geochim. Cosmochim. Acta, v. 43, p. 55-60.

297. Newton, R. C., Charlu, T. V., and Kleppa, O. J., 1974, A calorimetric investigation of the stability of anhydrous magnesium cordierite with application to granulite facies metamorphism: Contribution to Mineralogy and Petrology, v. 44, p. 295-311.

298. Newton, R. C., Charlu, T. V., and Kleppa, O. J., 1977, Thermochemistry of high-pressure garnets and clinopyroxenes in the system $\mathrm{CaO}-\mathrm{MgO}-\mathrm{Al}_{2} \mathrm{O}_{3}-\mathrm{SiO}_{2}:$ Geochim. Cosmochim. Acta, v. 41, p. 369-377. 
299. Newton, R. C., Thompson, A. B., and Krupka, K. M., 1977, Heat capacity of synthetic $\mathrm{Mg}_{3} \mathrm{Al}_{2} \mathrm{SiO}_{3} \mathrm{O}_{12}$ from 350 to 1,000 K and the entropy of pyrope: EOS Trans. Am. Geophys. Union, v. 58, p. 523.

300. O'Brien, C. J., and Kelley, K. K., 1957, High temperature heat contents of cryolite, anhydrous aluminum fluoride and sodium fluoride: Am. Chem. Soc. Jour., v. 79, p. 5616-5618.

301. Ogorodova, L. N., Kiseleva, I. A., and Shuriga, T. N., 1989, Enthalpies of formation and phase transformation of cryolite: Geokhimiia, N. 8, p. 1180-1183.

302. O'Hare, P.A.G., 1972, Thermochemical and theoretical investigations of the sodium-oxygen system, Part I. The standard enthalpy of formation of sodium oxide $\left(\mathrm{Na}_{2} \mathrm{O}\right)$ : Jour. Chem. Phys., v. 56, p. 4513-4516.

303. O’Hare, P. A. G., 1987, Inorganic chalcogenides: high-tech materials, low-tech thermodynamics: Jour. Chem. Thermodynamics, v. 19, p. 675-701.

304. O'Hare, P. A. G., Hubbard, W. N., Johnson,G. K., and Flotow, H. E., 1984, Calorimetric mesurements of the low-temperature heat capacity, standard molar enthalpy of formation at $298.15 \mathrm{~K}$, and hightemperature molar enthalpy increments relative to $298.15 \mathrm{~K}$ of tungsten disulfide $\left(\mathrm{WS}_{2}\right)$, and the thermodynamic properties to $1500 \mathrm{~K}$ : Jour. Chem. Thermodynamics, v. 16, p. 45-59.

305. O'Hare, P. A. G., Lewis, B. M., and Parkinson, B. A., 1988, Standard molar enthalpy of formation by fluorine-combustion calorimetry of tungsten diselenide $\left(\mathrm{WSe}_{2}\right)$. Thermodynamics of the hightemperature vaporization of $\mathrm{WSe}_{2}$. Revised value of the standard molar enthalpy of formation of molybdenite $\left(\mathrm{MoS}_{2}\right)$ : Jour. Chem. Thermodynamics, v. 20, p. 681-691. 
306. Okazaki, H. and Takano, A., 1985, The specific heat of $\mathrm{Ag}_{2} \mathrm{~S}$ in alpha-phase: Zeit. Naturforsch., v. 40a, p. 986-988.

307. O'Neill, H. St. C., 1985, Thermodynamics of $\mathrm{Co}_{3} \mathrm{O}_{4}:$ A possible electron spin unpairing transition in $\mathrm{Co}^{3+}$ : Phys. Chem. Minerals, v. 12, p. 149-154.

308. O'Neill, H. St. C., 1987, Free energies of formation of $\mathrm{NiO}, \mathrm{CoO}, \mathrm{Ni}_{2} \mathrm{SiO}_{4}$, and $\mathrm{Co}_{2} \mathrm{SiO}_{4}$ : $\mathrm{Am}^{\mathrm{m}}$ Mineralogist, v. 72, p. 280-291.

309. O'Neill, H. St. C., 1987, Quartz-fayalite-iron and quartz-fayalite-magnetite equilibria and the free energy of formation of fayalite $\left(\mathrm{Fe}_{2} \mathrm{SiO}_{4}\right)$ and magnetite $\left(\mathrm{Fe}_{3} \mathrm{O}_{4}\right)$ : Am. Mineralogist, v. 72, p. 67-75.

310. O'Neill, H. St. C., 1988, Systems Fe-O and Cu-O: Thermodynamic data for the equilibria Fe-"FeO," $\mathrm{Fe}-\mathrm{Fe}_{3} \mathrm{O}_{4}, \quad \mathrm{FeO}^{n}-\mathrm{Fe}_{3} \mathrm{O}_{4}, \mathrm{Fe}_{3} \mathrm{O}_{4}-\mathrm{Fe}_{2} \mathrm{O}_{3}, \mathrm{Cu}-\mathrm{Cu}_{2} \mathrm{O}$, and $\mathrm{Cu}_{2} \mathrm{O}-\mathrm{CuO}$ from emf measurements: Am. Mineralogist, v. 73, p. $470-486$.

311. O'Neill, H. St. C., and Navrotsky, A., 1980, The thermodynamics of the clinopyroxene to pyroxenoid phase transition in the systems $\mathrm{CaO}-\mathrm{MnO}_{\mathrm{SiO}}$ : $\mathrm{EOS}$, v. 61, p. 1147.

312. O'Neill, Hugh St. C., Pownceby, M. I., and Wall, V. J., 1988, Ilmenite-rutile-iron and ulvospinel-ilmeniteiron equilibria and the thermochemistry of ilmenite $\left(\mathrm{FeTiO}_{3}\right)$ and ulvospinel $\left(\mathrm{Fe}_{2} \mathrm{TiO}_{4}\right)$ : Geochim. Cosmochim. Acta, v. 52, p. 2065-2072.

313. Openshaw, R. E., Hemingway, B. S., Robie, R. A., Waldbaum, D. R., and Krupka, K. M., 1976, The heat capacities at low temperatures and entropies at $298.15 \mathrm{~K}$ of low albite, analbite, microcline, and high sanidine: U.S. Geol. Survey Jour. Research, v. 4, p. 195-204. 
314. Orr, R. L., 1953, High temperature heat contents of magnesium orthosilicate and ferrous orthosilicate: Am. Chem. Soc. Jour., v. 75, p. 528-529.

315. Orr, R. L. and Christensen, A. U., 1958, High temperature heat contents of stannous and stannic sulfides: Jour. Phys. Chemistry, v. 62, p. 124-125.

316. Pankratz, L. B., 1964, High temperature heat contents and entropies of muscovite and dehydrated muscovite: U.S. Bur. Mines Rept. Inv. 6371, 6 p.

317. Pankratz, L. B., 1968, High-temperature heat contents and entropies of dehydrated analcite, kaliophilite, and leucite: U.S. Bur. Mines Rept. Inv. 7073, 8 p.

318. Pankratz, L. B., 1970, Thermodynamic data for silver chloride and silver bromide: U. S. Bur. Mines Rept. Inv. 7430, 12 p.

319. Pankratz, L. B., 1982, Thermodynamic properties of elements and oxides: U. S. Bur. Mines Bull. 672 , 509 p.

320. Pankratz, L. B., 1984, Thermodynamic properties of halides: U. S. Bur. Mines Bull. 674, 826 p.

321. Pankratz, L. B., and Kelley, K. K., 1963, Thermodynamic data for magnesium oxide (periclase): U. S. Bur. Mines Rept. Inv. 6295, 5 p.

322. Pankratz, L. B., and Kelley, K. K., 1964, High-temperature heat contents and entropies of andalusite, kyanite, and sillimanite: U.S. Bur. Mines Rept. Inv. 6370, 7 p. 
323. Pankratz, L. B., and Kelley, K. K., 1964, High-temperature heat contents and entropies of akermanite, cordierite, gehlenite, and merwinite: U.S. Bur. Mines Rept. Inv. 6555, 7 p.

324. Pankratz, L. B., and King, E. G., 1965, High-temperature heat contents and entropies of two zinc sulfides and four solid solutions of zinc and iron sulfides: U.S. Bur. Mines Rept. Inv. 6708, 8 p.

325. Pankratz, L. B., and King, E. G., 1970, High-temperature enthalpies and entropies of chalcopyrite and bornite: U.S. Bur. Mines Rept. Inv. 7435, 10 p.

326. Pankratz, L. B., Mah, A. D., and Watson, S. W., 1987, Thermodynamic properties of sulfides: U.S. Bur. Mines Bull., 689, 427 p.

327. Pankratz, L. B., and Weller, W. W., 1967, Thermodynamic properties of three lithium-aluminum silicates: U.S. Bur. Mines Rept. Inv. 7001, 13 p.

328. Pankratz, L. B., and Weller, W. W., 1969, Thermodynamic data for ferric sulfate and indium sulfate: U. S. Bur. Mines Rept. Inv. 7280, 8 p.

329. Pankratz, L. B., Weller, W. W., and Kelley, K. K., 1963, Low-temperature heat capacity and hightemperature heat content of mullite: U.S. Bur.Mines Rept. Inv. 6287, 7 p.

330. Parks, G. A., 1972, Free energies of formation and aqueous solubilities of aluminum hydroxides and oxide hydroxides at $25^{\circ} \mathrm{C}$ : Am. Mineralogist, v. 57, p. 1163-1189.

331. Pashinkin, A.C., Muratova, V.A., Antyukhov, A.M., and Moiseyev, N.V., 1989, Heat capacity and thermodynamic functions of arsenopyrite (in Russian): Izv. Akad. Nauk. SSSR, Neorg. Mater., v. 25, p. 225-227. 
332. Pashinkin, A.C., Muratova, V.A., Moiseyev, N.V. and Bazhenov, J.V., 1991, Heat capacity and thermodynamic functions of iron diarsenide in the temperature range $5 \mathrm{~K}$ to $300 \mathrm{~K}$ : Jour. Chem. Thermodynamics, v. 23, p. 827-830.

333. Pashinkin, A. S., Rabinovich, I. B., Sheiman, M. S., Nistratov, V. P., and Vorobjova, O. I., 1985, Heat capacity and thermodynamic functions of tellurium dioxide in the range 5 to $300 \mathrm{~K}$ : Jour. Chem. Thermodynamics, v. 17, p. 43-47.

334. Perkins, D., III, Essene, E. J., Westrum, E. F., Jr., and Wall, V. J., 1979, New thermodynamic data for diaspore and their implications to the system $\mathrm{Al}_{2} \mathrm{O}_{3}-\mathrm{SiO}_{2}-\mathrm{H}_{2} \mathrm{O}$ : Am. Mineralogist, v. 64, p. $1080-1090$.

335. Perkins, D., III, Westrum, E. F., Jr., and Essene, E. J., 1980, The thermodynamic properties and phase relations of some minerals in the system $\mathrm{CaO}-\mathrm{Al}_{2} \mathrm{O}_{3}-\mathrm{SiO}_{2}-\mathrm{H}_{2} \mathrm{O}$ : Geochim. Cosmochim. Acta, v. 44, p. 61-84.

336. Plummer, L. N., and Busenberg, E., 1982, The solubilities of calcite, aragonite and vaterite in $\mathrm{CO}_{2}-\mathrm{H}_{2} \mathrm{O}$ solutions between $\mathrm{O}$ and $90^{\circ} \mathrm{C}$, and an evaluation of the aqueous model for the system $\mathrm{CaCO}_{3}-\mathrm{CO}_{2}-$ $\mathrm{H}_{2} \mathrm{O}$ : Geochim. Cosmochim. Acta, v. 46, p. 1011-1040.

337. Potter, R.W. II, 1977, An electrochemical investigation of the system copper-sulfur: Economic Geology, v. 72 , p. $1524-1542$.

338. Richards, A. W., 1959, The heats of formation and dissociation of zinc sulfide: Jour. Appl. Chemistry, v. 9, p. $142-145$. 
339. Richardson, D. W., and Brown, R. R., 1974, Enthalpy of formation of malachite $\left[\mathrm{Cu}_{2}\left(\mathrm{CO}_{3}\right)(\mathrm{OH})_{2}\right]$ : U.S. Bur. Mines Rept. Inv. 7851, 5 p.

340. Richet, P., and Bottinga, Y., 1984, Anorthite, andesine, wollastonite, diopside, cordierite and pyrope: thermodynamics of melting, glass transitions, and properties of the amorphous phases: Earth and Planetary Science Letters, v. 67, p. 415-432.

341. Richet, P., Bottingia, Y., Denielou, L., Petitet, J.P., and Tequi, C., 1982, Thermodynamic properties of quartz, cristobalite and amorphous $\mathrm{SiO}_{2}$ : drop calorimetry measurements between 1000 and 1800 $\mathrm{K}$ and a review from 0 to $2000 \mathrm{~K}$. Geochim. Cosmochim. Acta., v. 46, p. 2639-2658.

342. Richet, P., and Fiquet, G., 1991, High-temperature heat capacity and premelting of minerals in the system MgO-CaO- $\mathrm{Al}_{2} \mathrm{O}_{3}-\mathrm{SiO}_{2}$ : Jour. Geophys. Research, v. 96, No. B1, p. 445-456.

343. Richet, P., Robie, R. A., and Hemingway, B. S., 1987, Low-temperature heat capacity of diopside glass $\left(\mathrm{CaMgSi}_{2} \mathrm{O}_{6}\right):$ A calorimetric test of the configurational-entropy theory applied to the viscosity of liquid silicates: Geochm. Cosmochim. Acta, v. 50, p. 1521-1533.

344. Richet, P., Robie, R. A., and Hemingway, B. S., 1991, Thermodynamic properties of wollastonite, pseudowollastonite and $\mathrm{CaSiO}_{3}$ glass and liquid: Europ. Jour. Mineralogy, v. 3, p. 475-484.

445. Richet, P., Robie, R.A., and Hemingway, B.S., 1993, Entropy and structure of silicate glasses and melts: Geochim. Cosmochim. Acta, v. 57, p. 2751-2766.

346. Richet, P., Robie, R. A., Rogez, J., Hemingway, B. S., Courtial, P., and Tequi, C., 1990, Thermodynamics of open networks: ordering and entropy in $\mathrm{NaAlSiO}_{4}$ glass, liquid, and polymorphs: Phys. Chem. Minerals, v. 17, p. 385-394. 
347. Robie, R. A., 1965, Heats and free energies of formation of troilite, herzenbergite, magnesite, and rhodochrosite calculated from equilibrium data: U.S. Geol. Survey Prof. Paper 525-D, p. 65-72.

348. Robie, R. A., Bin, Zhao, Hemingway, B. S., and Barton, M. D., 1987, Heat capacity and thermodynamic properties of andradite garnet, $\mathrm{Ca}_{3} \mathrm{Fe}_{2} \mathrm{Si}_{3} \mathrm{O}_{12}$, between 10 and $1000 \mathrm{~K}$ and revised values for $\Delta_{\mathrm{f}} \mathrm{G}_{m}^{\circ}$ (298.15 K) of hedenbergite and wollastonite: Geochim. Cosmochim. Acta, v. 51, p. 2219-2224.

349. Robie, R. A., Evans, H. T., Jr., and Hemingway, B. S., 1988, Thermophysical properties of ilvaite $\mathrm{CaFe}_{2}^{2+} \mathrm{Fe}^{3+} \mathrm{Si}_{2} \mathrm{O}_{7}(\mathrm{OH}):$ heat capacity from 7 to $920 \mathrm{~K}$ and thermal expansion between 298 and 856 K: Phys. Chem. Minerals, v. 15, p. 390-397.

350. Robie, R. A., Finch, C. B., and Hemingway, B. S., 1982, Heat capacity and entropy of fayalite $\left(\mathrm{Fe}_{2} \mathrm{SiO}_{4}\right)$ between 5.1 and $383 \mathrm{~K}$ : comparison of calorimetric and equilibrium values for the QFM buffer reaction: Am. Mineralogist, v. 67, p. 463-469.

351. Robie, R. A., Haselton, H. T., Jr., and Hemingway, B. S., 1984, Heat capacities and entropies of rhodochrosite $\left(\mathrm{MnCO}_{3}\right)$ and siderite $\left(\mathrm{FeCO}_{3}\right)$ between 5 and $600 \mathrm{~K}$ : Am. Mineralogist, v. 69, p. 349-357.

352. Robie, R. A., Haselton, H. T., Jr., and Hemingway, B. S., 1989, Heat capacities and entropies at 298.15 $\mathrm{K}$ of $\mathrm{MgTiO}_{3}$ (geikielite), $\mathrm{ZnO}$ (zincite), and $\mathrm{ZnCO}_{3}$ (smithsonite): Jour. Chem. Thermodynamics, v. 21, p. 743-749.

353. Robie, R. A., and Hemingway, B. S., 1972, The heat capacities at low-temperatures and entropies at 298.15 $\mathrm{K}$ of nesquehonite, $\mathrm{MgCO}_{3} \cdot \mathrm{H}_{2} \mathrm{O}$, and hydromagnesite: Am. Mineralogist, v. 57, p. 1768-1781. 
354. Robie, R. A., and Hemingway, B. S., 1973, The enthalpies of formation of nesquehonite, $\mathrm{MgCO}_{3} \cdot 3 \mathrm{H}_{2} \mathrm{O}$, and hydromagnesite, $5 \mathrm{MgO} \cdot 4 \mathrm{CO}_{2} \cdot 5 \mathrm{H}_{2} \mathrm{O}$ : U.S. Geol. Survey Jour. Research, v. 1, p. 543-547.

355. Robie, R. A., and Hemingway, B. S., 1984, Entropies of kyanite, andalusite, and sillimanite: additional constraints on the pressure and temperature of the $\mathrm{Al}_{2} \mathrm{SiO}_{5}$ triple point: Am. Mineralogist, v. 69, p. 298-306.

356. Robie, R. A., and Hemingway, B. S., 1984, Heat capacities and entropies of phlogopite $\left(\mathrm{KMg}_{3}\left[\mathrm{AlSi}_{3} \mathrm{O}_{10}\right](\mathrm{OH})_{2}\right)$ and paragonite $\left(\mathrm{NaAl}_{2}\left[\mathrm{AlSi}_{3} \mathrm{O}_{10}\right]\left(\mathrm{OH}_{2}\right)\right.$ between 5 and $900 \mathrm{~K}$ and estimates of the enthalpies and Gibbs free energies of formation: Am. Mineralogist, v. 69, p. 858-868.

357. Robie, R. A., and Hemingway, B. S., 1985, Low-temperature molar heat capacities and entropies of $\mathrm{MnO}_{2}$ (pyrolusite), $\mathrm{Mn}_{3} \mathrm{O}_{4}$ (hausmannite) and $\mathrm{Mn}_{2} \mathrm{O}_{3}$ (bixbyite): Jour. Chem. Thermodynamics, v. 17, p. $165-181$.

358. Robie, R. A., and Hemingway, B. S., 1991, Heat capacities of kaolinite from 7 to $380 \mathrm{~K}$ and of DMSO intercalated kaolinite from 20 to $310 \mathrm{~K}$. The entropy of kaolinite $\mathrm{Al}_{2} \mathrm{Si}_{2} \mathrm{O}_{5}(\mathrm{OH})_{4}$ : Clays and Clay Minerals, v. 39, p. 362-386.

359. Robie, R. A. and Hemingway, B. S., 1994, Heat capacities of synthetic grossular $\left(\mathrm{Ca}_{3} \mathrm{Al}_{2} \mathrm{Si}_{3} \mathrm{O}_{12}\right)$, macrocrystals of magnesite $\left(\left(\mathrm{Mg}_{0.991} \mathrm{Fe}_{0.005} \mathrm{Ca}_{0.003} \mathrm{Mn}_{0.001}\right) \mathrm{CO}_{3}\right)$, high-temperature superconductors $\mathrm{YBa}_{2} \mathrm{Cu}_{3} \mathrm{O}_{6+\mathrm{x}}$ and $\mathrm{BiCaSrCu}_{2} \mathrm{O}_{6+x^{\prime}}$ and type 321 stainless steel: U. S. Geological Survey Open-File Report 94-223, 12 p.

360. Robie, R. A., Hemingway, B. S., and Ditmars, D.A., unpublished data on $\mathrm{C}_{\mathrm{p}}$ between 10 and $360 \mathrm{~K}$ for calcium metal. 
361. Robie, R. A., Hemingway, B. S., and Fisher, J. R., 1978, Thermodynamic properties of minerals and related substances at $298.15 \mathrm{~K}$ and 1 bar $\left(10^{5}\right.$ pascals $)$ pressure and at higher temperatures: U.S. Geol. Survey Bull. 1452, 456 p.

362. Robie, R. A., Hemingway, B. S., Gillet, P., and Reynard, B., 1991, On the entropy of glaucophane $\mathrm{Na}_{2} \mathrm{Mg}_{3} \mathrm{Al}_{2} \mathrm{Si}_{8} \mathrm{O}_{22}(\mathrm{OH})_{2}$ : Contributions to Mineralogy and Petrology, v. 107, p. 484-486.

363. Robie, R. A., Hemingway, B. S., Ito, J., and Krupka, K. M., 1984, Heat capacity and entropy of $\mathrm{Ni}_{2} \mathrm{SiO}_{4}$-olivine from 5 to $1000 \mathrm{~K}$ and of $\mathrm{Co}_{2} \mathrm{SiO}_{4}$ from 360 to $1000 \mathrm{~K}$ : Am. Mineralogist, v. 69, p. $1096-1101$.

364. Robie, R. A., Hemingway, B. S., and Takei, H., 1982, Heat capacities and entropies of $\mathrm{Mg}_{2} \mathrm{SiO}_{4}$, $\mathrm{Mn}_{2} \mathrm{SiO}_{4}$, and $\mathrm{Co}_{2} \mathrm{SiO}_{4}$ between 5 and $380 \mathrm{~K}:$ Am. Mineralogist, v. 67, p. 470-482.

365. Robie, R. A., Hemingway, B. S., and Wilson, W. H., 1976, The heat capacities of Calorimetry Conference copper and muscovite $\mathrm{KAl}_{2}\left(\mathrm{AlSi}_{3}\right) \mathrm{O}_{10}(\mathrm{OH})_{2}$, pyrophyllite $\mathrm{Al}_{2} \mathrm{Si}_{4} \mathrm{O}_{10}(\mathrm{OH})_{2}$, and illite $\mathrm{K}_{3}\left(\mathrm{Al}{ }_{7} \mathrm{Mg}\right)\left(\mathrm{Si}_{14} \mathrm{Al}_{2}\right) \mathrm{O}_{40}(\mathrm{OH})_{8}$ between 15 and $375 \mathrm{~K}$ and their standard entropies at $298.15 \mathrm{~K}$ : U.S. Geol. Survey Jour. Research, v. 4, p. 631-644.

366. Robie, R. A., Hemingway, B. S., and Wilson, W. H., 1978, Low-temperature heat capacities and entropies of $\mathrm{KAISi}_{3} \mathrm{O}_{8}, \mathrm{NaAlSi}_{3} \mathrm{O}_{8}$, and $\mathrm{CaAl}_{2} \mathrm{Si}_{2} \mathrm{O}_{8}$ glasses and of anorthite: Am. Mineralogist, v. 63, p. 110-123.

367. Robie, R.A., Huebner, J.S., and Hemingway, B.S., 1993, Heat capacities and thermodynamic properties of braunite $\left(\mathrm{Mn}_{7} \mathrm{SiO}_{12}\right)$ and rhodonite $\left(\mathrm{MnSiO}_{3}\right)$ : (unpublished report). 
368. Robie, R. A., Russell-Robinson, S., and Hemingway, B. S., 1989, Heat capacities and entropies from 8 to $1000 \mathrm{~K}$ of langbeinite $\left(\mathrm{K}_{2} \mathrm{Mg}_{2}\left(\mathrm{SO}_{4}\right)_{3}\right)$, anhydrite $\left(\mathrm{CaSO}_{4}\right)$ and of gypsum $\left(\mathrm{CaSO}_{4} \cdot 2 \mathrm{H}_{2} \mathrm{O}\right)$ to 325 K: Thermochimica Acta, v. 139, p. 67-81.

369. Robie, R. A., Seal, R. R., II, and Hemingway, B. S., 1994, Heat Capacity and entropy of bornite $\left(\mathrm{Cu}_{5} \mathrm{FeS}_{4}\right)$ between 6 and $760 \mathrm{~K}$ and the thermodynamic properties of phases in the Cu-Fe-S: Canadian Mineralogist, v. 32, 945-956.

370. Robie, R. A., and Stout, J. W., 1963, Heat capacity from 12 to $305^{\circ} \mathrm{K}$ and entropy of talc and tremolite: Jour. Phys. Chemistry., v. 67, p. 2252-2256.

371. Robie, R. A., Wiggins, L. B., Barton, P. B., Jr., and Hemingway, B. S., 1985, Low-temperture heat capacity and entropy of chalcopyrite $\left(\mathrm{CuFeS}_{2}\right)$ : estimates of the standard molar enthalpy and Gibbs free energy of formation of chalcopyrite and bornite $\left(\mathrm{Cu}_{5} \mathrm{FeS}_{4}\right)$ : Jour. Chem. Thermodynamics, v. 17, p. 481-488.

372. Romanovskii, V. A., and Tarasov, V. V., 1960, Heat capacity of the trisulfides of arsenic, antimony, and bismuth in connection with their structural and physical-chemical properties: Sov. Phys.-Solid State, v. 2, p. $1170-1175$.

373. Sack, R.O. and Ghiorso, M.S., 1991, Chromium spinels as petrogenetic indicators: Thermodynamics and petrological applications: Am. Mineralogist, v. 76, p. 827-847.

374. Saxena, S. K., and Chatterjee, N., 1986, Thermochemical data on mineral phases: the system $\mathrm{CaO}-\mathrm{MgO}-\mathrm{Al}_{2} \mathrm{O}_{3}-\mathrm{SiO}_{2}:$ Jour. Petrology, v. 27, p. 827-842.

375. Schaefer, S. C., 1980, Electrochemical determination of Gibbs energies of formation of $\mathrm{MnS}$ and $\mathrm{Fe}_{0.9} \mathrm{~S}$ : U.S. Bur. Mines Rept. Inv., 8486, 17 p. 
376. Schaefer, S. C., 1981, Electrochemical determination of Gibbs energies of formation of cobalt and nickel sulfides: U.S. Bur. Mines Rept. Inv., 8588, 18 p.

377. Schaefer, S. C., 1983, Electrochemical determination of thermodynamic properties of manganese sulfate and cadmium oxysulfate: U. S. Bur. Mines Rept. Inv. 8809, 20 p.

378. Schaefer, S. C., and Gokcen, N. A., 1982, Electrochemical determination of the thermodynamic properties of sphalerite, ZnS (beta): High Temperature Science, v. 15, p. 225-237.

379. Schuiling, R. D., Vergouwen, L., and Van der Rijst, J., 1976, Gibbs energies of formation of zircon $\left(\mathrm{ZrSiO}_{4}\right)$, thorite $\left(\mathrm{ThSiO}_{4}\right)$, and phenacite $\left(\mathrm{Be}_{2} \mathrm{SiO}_{4}\right):$ Am. Mineralogist, v. 61, p. 161-168.

380. Seal, R.R., II, Essene, E.J. and Kelley, W.C., 1990, Tetrahedrite and tennantite: evaluation of thermodynamic data and phase equilibria: Canadian Mineralogist, v. 28, p. 725-738.

381. Seal, R. R., II, Robie, R. A., and Hemingway, B. S., 1991, Unpublished entropy and $C_{p}$ data for enargite.

382. Seal, R. R., II, Robie, R. A., Hemingway, B. S., and Barton, P. B., Jr., 1992, Superambient heat capacities of synthetic stibnite, berthierite and chalcostibite: Revised thermodynamic properties and implications for phase equilibria: Economic Geology, v. 87, p. 1911-1918.

383. Semenov, Yu. V., Malinko, S. V., Kiseleva, I. A., and Khodakovsky, I. L., 1987, The formation conditions of endogenous calcium borates and borosilicates: Geokhimiya, No. 8, p. 1182-1190.

384. Settle, J. L., Johnson, G. K., and Hubbard, W. N., 1974, The enthalpy of formation of dicesium monoxide $\left(\mathrm{Cs}_{2} \mathrm{O}\right)$ : Jour. Chem. Thermodynamics, v. 6, p. 263-269. 
385. Sharp, Z. D., Essene, E. J., Anovitz, L. M., Metz, G. W., Westrum, E. F., Hemingway, B. S., and Valley, J. W., 1983, The heat capacity of natural monticellite and phase equilibria in the system CaO-MgO$\mathrm{SiO}_{2}-\mathrm{CO}_{2}:$ Geochim. Cosmochim. Acta, v. 50, p. 1475-1484.

386. Shearer, J. A., and Kleppa, O. J., 1973, The enthalpies of formation of $\mathrm{MgAl}_{2} \mathrm{O}_{4}, \mathrm{MgSiO}_{3}, \mathrm{Mg}_{2} \mathrm{SiO}_{4}$ and $\mathrm{Al}_{2} \mathrm{SiO}_{5}$ by oxide melt solution calorimetry: Jour. Inorg. Nuc. Chemistry, v. 35, p. 1073-1078.

387. Shomate, C. H., and Naylor, B. B., 1945, High-temperature heat contents of aluminum oxide, aluminum sulfate, potassium sulfate, ammonium sulfate and ammonium bisulfate: Am. Chem. Soc. Jour., v. 67, p. $72-75$.

388. Sirota, N. N., Lubyannikov, E. P., Novikov, V. V., and Sidorov, A. A., 1985, Low-temperture thermodynamic properties of cubic cubanite at temperatures of 5-300 K: Soviet Physics Doklady, v. 30, p. $789-791$.

389. Southard, J. C., 1941, A modified calorimeter for high temperatures. The heat content of silica, wollastonite and thorium dioxide above $25^{\circ}$ : Am. Chem. Soc. Jour., v. 63, p. 3142-3150.

390. Southard, J. C., and Moore, G. E., 1942, High-temperature heat content of $\mathrm{Mn}_{3} \mathrm{O}_{4}, \mathrm{MnSiO}_{3}$ and $\mathrm{Mn}_{3} \mathrm{C}$ : Am. Chem. Soc. Jour., v. 64, p. 1769-1770.

391. Southard, J. C., and Shomate, C. H., 1942, Heat of formation and high-temperature heat content of manganous oxide and manganous sulfate. High-temperature heat content of manganese: Am. Chem. Soc. Jour., v. 64, p. 1770-1774.

392. Staveley, L. A. K., and Linford, R. G., 1969, The heat capacity and entropy of calcite and aragonite and their interpretation: Jour. Chem. Thermodynamics, v. 1, p. 1-11. 
393. Stephenson, C. C., and Smith, D., 1968, Heat capacity of manganese titanate from $30^{\circ}$ to $300^{\circ} \mathrm{K}$ : Jour. Chem. Physics, v. 49, p. 1814-1818.

394. Stolen, S., Gronvold, F., and Westrum, E. F., Jr., 1990, Thermodynamics of copper sulfides. IV. Heat capacity and thermodynamic properties of $\mathrm{Cu}_{1.90} \mathrm{~S}$ from $5 \mathrm{~K}$ to $750 \mathrm{~K}, \mathrm{Cu}_{1.95} \mathrm{~S}$ from $5 \mathrm{~K}$ to 1000 $\mathrm{K}, \mathrm{Cu}_{1.98} \mathrm{~S}$ from $300 \mathrm{~K}$ to $750 \mathrm{~K}$ : Jour. Chem. Thermodynamics, v. 22, p. 1035-1057.

395. Stolen, S., Gronvold, F., Westrum, E. F., Jr., and Kolonin, G. R., 1991, Heat capacities and thermodynamic properties of synthetic heazlewoodite, $\mathrm{Ni}_{3} \mathrm{~S}_{2}$, and of the high-temperature phase $\mathrm{Ni}_{3 \pm x} \mathrm{~S}_{2}$ : Jour. Chem. Thermodynamics, v. 23, p. 77-93.

396. Stout, J. W., and Robie, R. A., 1963, Heat capacity from 11 to $300^{\circ} \mathrm{K}$. Entropy and heat of formation of dolomite: Jour. Phys. Chemistry, v. 67, p. 2248-2252.

397. Stubbles, J. R., and Birchenall, C. E., 1959, Redetermination of the lead-lead sulfide equilibrium between 585 and $920^{\circ} \mathrm{C}$ K: Trans. Metal. Soc. AlME., v. 215, p. 535-539.

398. Stuve, J. M., 1974, Low-temperture heat capacities of sphalerite and wurtzite: U.S. Bur. Mines Rept. Inv., 7940, 8 p.

399. Stuve, J. M., Beyer, R. P., and Brown, R. R., 1985, Thermodynamic properties of $\operatorname{CoS}_{0.89}, \operatorname{CoS}_{1.33}$, and $\mathrm{CoS}_{2}$ : U.S. Bur. Mines Rept. Inv., 8994, 11 p.

400. Stuve, J. M., and Ferrante, M. J., 1980, Low-temperature heat capacities and high-temperature enthalpies of chiolite $\left(\mathrm{Na}_{5} \mathrm{Al}_{3} \mathrm{~F}_{14}\right)$ : U. S. Bur. Mines Rept. Inv. 8442, 8 p.

401. Stuve, J. M., Ferrante, M. J., and Ko, H. C., 1978, Thermodynamic properties of $\mathrm{NiBr}_{2}$ and $\mathrm{NiSO}_{4}$ from 10 to 1,200 K: U.S. Bur. Mines Rept. Inv. 8271, 15 p. 
402. Tequi, C., Robie, R. A., Hemingway, B. S., Neuville, D., and Richet, P., 1991, Melting and thermodynamic properties of pyrope $\left(\mathrm{Mg}_{3} \mathrm{Al}_{2} \mathrm{Si}_{3} \mathrm{O}_{12}\right)$ : Geochim. Cosmochim. Acta, v. 55, p. $1005-1010$.

403. Thompson, A. B., Perkins, D. III, Sonderegger, U., and Newton, R. C., 1978, Heat capacities of synthetic $\mathrm{CaAl}_{2} \mathrm{SiO}_{6}-\mathrm{CaMgSi}_{2} \mathrm{O}_{6}-\mathrm{Mg}_{2} \mathrm{Si}_{2} \mathrm{O}_{6}$ pyroxenes: EOS Transactions, v. 59, p. 395.

404. Thompson, A. B., and Wennemer, M., 1979, Heat capacities and inversions in tridymite, cristobalite, and tridymite-cristobalite mixed phases: Am. Mineralogist, v. 64, p. 1018-1026.

405. Todd, S. S., 1951 , Low-temperature heat capacities and entropies at $298.16^{\circ} \mathrm{K}$ of crystalline calcium orthosilicate, zinc orthosilicate and tricalcium silicate: Am. Chem. Soc. Jour., v. 73, p. 3277-3278.

406. Todd, S. S., and Kelley, K. K., 1956, Heat and free-energy data for tricalcium dititanate, sphene, lithium metatitanate, and zinc-titanium spinel: U.S. Bur. Mines Rept. Inv. 5193, 18 p.

407. Topor, L., Kleppa, O. J., Newton, R. C., and Kerrick, D. M., 1989, Molten salt calorimetry of $\mathrm{Al}_{2} \mathrm{SiO}_{5}$ polymorphs at 1000 K. EOS Transactions (Amer. Geophys. Union), v. 70, p. 493.

408. Torgeson, D. R., and Sahama, T. G., 1948, A hydrofluoric acid solution calorimeter and the determination of the heats of formation of $\mathrm{Mg}_{2} \mathrm{SiO}_{4}, \mathrm{MgSiO}_{3}$, and $\mathrm{CaSiO}_{3}$ : Am. Chem. Soc. Jour., v. 70, p. $2156-2160$.

409. Toulmin, P., III, and Barton, P. B., Jr., 1964, Thermodynamic study of pyrite and pyrrhotite: Geochim. Cosmochim. Acta, v. 28, p. 641-671.

410. Treiman, A. H., and Essene, E. J., 1983, Phase equilibria in the system $\mathrm{CaO}-\mathrm{SiO}_{2}-\mathrm{H}_{2} \mathrm{O}$ : Am. Jour. Science, v. 283-A, p. 97-120. 
411. Tremaine, P.R. and LeBlanc, J.C., 1980, The solubility of magnetite and the hydrolysis and oxidation of $\mathrm{Fe}^{2+}$ in water to $300^{\circ} \mathrm{C}$ : Jour. Sol. Chemistry, v. 9, p. 415-442.

412. Turnbull, A. G., 1973, A thermochemical study of vaterite: Geochim. Cosmochim. Acta, v. 37, p. 1593-1601.

413. Ulbrich, H. H., and Waldbaum, D., R., 1976, Structural and other contributions to the third-law entropies of silicates: Geochim. Cosmochim. Acta, v. 40, p. 1-24.

414. van Lier, J. A., de Bruyn, P. L., and Overbeek, J. Th. G., 1960, The solubility of quartz: Jour. Phys. Chemistry, v. 64, p. 1675-1682.

415. Vanderzee, C. E., Rodenburg, L. N., and Berg, R. L., 1974, Thermochemical studies on red and yellow orthorhombic and on red hexagonal mercuric oxide: Jour. Chem. Thermodynamics, v. 6, p. 17-33.

416. Vanderzee, C. E., and Wigg, D. A., 1981, The standard enthalpies of solution and formation of Wegscheider's salt: $\mathrm{Na}_{2} \mathrm{CO}_{3} \cdot 3 \mathrm{NaHCO}_{3}(\mathrm{~s})$ and of trona: $\mathrm{Na}_{2} \mathrm{CO}_{3} \cdot \mathrm{NaHCO}_{3} \cdot 2 \mathrm{H}_{2} \mathrm{O}(\mathrm{s})$ at $298.15 \mathrm{~K}$ : Jour. Chem. Thermodynamics, v. 13, p. 573-583.

417. Victor, A. C., and Douglas, T. B., 1961, Thermodynamic properties of thorium dioxide from 298 to 1,200K: U. S. Natl. Bur. Standards Jour. Research, v. 65A, p. 105-111.

418. Victor, A. C., and Douglas, T. B., 1963, Thermodynamic properties of magnesium oxide and beryllium oxide from 298 to $1200^{\circ}$ K: U. S. Natl. Bur. Standards Jour. Research, v. 67A, p. 325-329.

419. Wagman, D. D., Evans, W. H., Parker, V. B., Schumm, R. H., Halow, I., Bailey, S. M., Churney, K. L., and Nuttal, R. L., 1982, The NBS tables of chemical thermodynamic properties. Selected values for inorganic and $C_{1}$ and $C_{2}$ organic substances in SI units: Jour. Phys. Chem. Ref. Data, v. 11, supplement no. 2, 392 p. 
420. Waldbaum, D. R., 1973, The configurational entropies of $\mathrm{Ca}_{2} \mathrm{MgSi}_{2} \mathrm{O}_{7}-\mathrm{Ca}_{2} \mathrm{SiAl}_{2} \mathrm{O}_{7}$ melilites and related minerals: Contributions to Mineralogy and Petrology, v. 39, p. 33-54.

421. Waldbaum, D. R., and Robie, R. A., 1971, Calorimetric investigation of Na-K mixing and polymorphism in the alkali feldspars: Zeit. Kristallographie, v. 184, p. 381-420.

422. Walther, J. V., and Helgeson, H. C., 1977, Calculation of the thermodynamic properties of aqueous silica and the solubility of quartz and its polymorphs at high pressures and temperatures: Am. Jour. Sci., v. 227, p. 1315-1351.

423. Watanabe, H., 1982, Thermochemical properties of synthetic high pressure compounds relevant to the earth's mantle. In Akimoto, S., and Manghnani, M. H., eds. High-Pressure Research In Geophysics, p. 441-464, Reidel, Dordrecht.

424. Waterfield, C. G., Linford, R. G., Goalby, B. B., Bates, T. R., Elyard, C. A., and Staveley, L. A. K., 1968, Thermodynamic investigation of disorder in the hydrates of sodium carbonate: Faraday Soc. Trans., v. 69 , p. $868-874$.

425. Wechsler, B.A., and Navrotsky, A., 1984, Thermodynamics and structural chemistry of compounds in the system ${\mathrm{MgO}-\mathrm{TiO}_{2}}_{2}$ J Jour. Solid State Chemistry, v. 55, p. 165-180.

426. Welch, M.D., and Pawley, A.R., 1991, Tremolite: New enthalpy and entropy data from a phase equilibrium study of the reaction tremolite $=2$ diopside +1.5 ortho enstatite $+\beta$-quartz $+\mathrm{H}_{2} \mathrm{O}$ : Am. Mineralogist, v. 76, p. 1931-1939.

427. Weller, W. W., and Kelley, K. K., 1963, Low-temperature heat capacities and entropies at $298.15^{\circ} \mathrm{K}$ of akermanite, cordierite, gehlenite, and merwinite: U.S. Bur. Mines Rept. Inv. 6343, 7 p. 
428. Weller, W. W., and Kelley, K. K., 1964, Low-temperature heat capacities and entropies at $298.15^{\circ} \mathrm{K}$ of lead molybdate and lead tungstates: U.S. Bur. Mines Rept. Inv. 6357, 5 p.

429. Weller, W. W., and Kelley, K. K., 1964, Low-temperature heat capacities and entropies at $298.15^{\circ} \mathrm{K}$ of sulfides of arsenic, germanium and nickel: U.S. Bur. Mines Rept. Inv. 6511, 7 p.

430. Weller, W. W., and Kelley, K. K., 1966, Low-temperature heat capacities and entropies at $298.15^{\circ} \mathrm{K}$ of ferrous molybdate and ferrous tungstate: U.S. Bur. Mines Rept. Inv. 6782, 5 p.

431. Weller, W. W., and King, E. G., 1963, Low-temperature heat capacities and entropies at $298.15^{\circ} \mathrm{K}$ of monomolybdates of sodium, magnesium, and calcium: U.S. Bur. Mines Rept. Inv. 6147, 6 p.

432. Weller, W. W., and King, E. G., 1963, Low-temperature heat capacity and entropy at $298.15^{\circ} \mathrm{K}$ of muscovite: U.S. Bur. Mines Rept. Inv. 6281, 4 p.

433. Westrich, H. R., and Holloway, J. R., 1981, Experimental dehydration of pargasite and calculation of its entropy and Gibbs energy: Am. Jour. Science, v. 281, p. 922-934.

434. Westrich, H. R., and Navrotsky, A., 1981, Some thermodynamic properties of fluorapatite, fluorpargasite, and fluorphlogopite: Am. Jour. Science, v. 281, p. 1091-1103.

435. Westrum, E. F., Jr., written communication, September, 1959.

436. Westrum, E.F., Jr., and Beale, A.F., Jr., 1961, Heat capacities and chemical thermodynamics of cerium (III) fluoride and of cerium (IV) oxide from 5 to $300^{\circ}$ K: Jour. Phys. Chemistry, v. 65, p. 353-355.

437. Westrum, E. F., Jr., Chou, C., and Gronvold, F., 1959, Heat capacities and thermodynamic properties of the iron tellurides $\mathrm{Fe}_{1.11} \mathrm{Te}$ and $\mathrm{FeTe}_{2}$ from 5 to $350^{\circ} \mathrm{K}$ : Jour. Chem. Physics, v. 30, p. 761-764. 
438. Westrum, E. F., Jr., Essene, E. J., and Perkins, D., III, 1977, Thermophysical properties of the garnet, grossular $\left(\mathrm{Ca}_{3} \mathrm{Al}_{2} \mathrm{Si}_{3} \mathrm{O}_{12}\right)$ : Jour. Chem. Thermodynamics, v. 11, p. 57-166.

439. Westrum, E.F., Jr., and Grimes, D.M., 1957, Low temperature heat capacities and thermodynamic properties of zinc ferrite: Jour. Phys. Chem. Solids, v. 3, p. 44-49.

440. Westrum, E.F., Jr., and Gronvold, F., 1969, Magnetite $\left(\mathrm{Fe}_{3} \mathrm{O}_{4}\right)$ heat capacity and thermodynamic properties from 5 to $350^{\circ} \mathrm{K}$ low-temperature transition: Jour. Chem. Thermodynamics, v. 1, p. 543-557.

441. Westrum, E. F., Jr., and Gronvold, F., 1970, Manganese disulfide (hauerite) and manganese ditelluride. Thermal properties from 5 to $350^{\circ} \mathrm{K}$ and antiferromagnetic transitions: Jour. Phys. Chem., v. 52 , p. 3820-3826.

442. Westrum, E. F., Jr., Stolen, S., and Gronvold, F., 1987, Thermodynamics of copper sulfides II. Heat capacity and thermodynamic properties of synthetic covellite, CuS, from 5 to $780.5 \mathrm{~K}$. Enthalpy of decomposition: Jour. Chem. Thermodynamics, v. 19, p. 1199-1208.

443. White, G. K., and Collocott, S. J., 1984, Heat capacity of reference materials: Cu and W: Jour. Phys. Chem. Ref. Data, v. 13, p. 1251-1257.

444. Wise, S. S., Margrave, J. L., Feder, H. M., and Hubbard, W. N., 1963, Fluorine bomb calorimetry. V. The heats of formation of silicon tetrafluoride and silica: Jour. Phys. Chemistry, v. 67, p. 815-821.

445. Woodland, A. B., and Wood, B. J., 1989, Electrochemical measurement of the free energy of almandine $\left(\mathrm{Fe}_{3} \mathrm{Al}_{2} \mathrm{Si}_{3} \mathrm{O}_{12}\right)$ garnet: Geochem. Cosmochim. Acta, v. 53, p. 2277-2282. 
446. Wruck, B., 1987, Thermodynamic behavior of the $\mathrm{PA}$ -11 phase transition in anorthite (abs): Programme Physical Properties and Thermodynamic Behavior of Minerals (NATOI, Cambridge.

447. Yakovlev, B. G., and Vozianova, O. V., 1983, Thermodynamical properties of almandine: Geokimiia, n. 1, p. 64-75.

448. Zhdanov, V. M., Turdakin, V. A., Arutyunov, V. S., Semenov, Yu. V., Malinko, S. V., and Kodakovsky, I. L., 1977, Low-temperature specific heat, standard entropy, and elastic parameters of datolite and danburyite: Geochemistry International, 14(6), p. 135-141.

449. Zhogin, D. Yu., Kosarukina, E. A., and Kolesov, V. P., 1980, Heat capacity in the 10-300 K range and thermodynamic functions of tetragonal tin dioxide: Zhur. Fiz. Khimii, v. 54(4), p. 916-920.

450. Heat capacity of hercynite estimated as $\mathrm{C}_{\mathrm{P}}\left(\mathrm{FeAl} \mathrm{O}_{4}\right)=\sum \mathrm{C}_{\mathrm{P}}(\mathrm{FeO})+\mathrm{C}_{\mathrm{P}}\left(\mathrm{Al}_{2} \mathrm{O}_{3}\right)$ by Robie. Entropy of $\mathrm{MnSO}_{4}$ and other values estimated by Robie.

451. Enthalpies of solution of members of the nepheline-kalsilite series were reported by Hovis and Roux (Hovis, G.L. and Roux, J., 1993, Thermodynamic mixing properties of nepheline-kalsilite crystalline solutions: Am. Jour. Sci., v. 293, p. 1108-1127.). Using enthalpy of solution values calculated from their equations 4 and 5, ancillary data from (166, tables 1-4), and unpublished enthalpy of solution data for $\mathrm{KCl}$ from Hemingway $\left(\Delta \mathrm{H}(\right.$ soln $\left.)=6.045-0.0189 \mathrm{t}\left({ }^{\circ} \mathrm{C}\right)\right)$, we calculate $\Delta_{\mathrm{r}} \mathrm{H}=$ $-2090.4 \pm 2.5$ and $-2124.7 \pm 2.5 \mathrm{~kJ} \cdot \mathrm{mol}^{-1}$, respectively, for nepheline and kalsilite. These results are in good agreement with values given in (361) of $-2092.1 \pm 2.4$ and $-2121.9 \pm 1.4 \mathrm{~kJ} \cdot \mathrm{mol}^{-1}$, respectively, but based on less pure samples $(20,171)$. These results are significantly less negative than the formation properties calculated by (188) from a limited number of phase equilibrium experiments. The calorimetrically determined value of $-3037.8 \pm 2.8 \mathrm{~kJ} \cdot \mathrm{mol}^{-1}$ for leucite $(171,20)$ is preferred to that derived by $(188)$ from a limited number of phase equilibrium data $\left(-3020.0 \pm 2.7 \mathrm{~kJ} \cdot \mathrm{mol}^{-1}\right)$. 
452. Heat capacity measurements on a synthetic alunite are combined with earlier results by Kelley et al. (219) to estimate the entropy at $298.15 \mathrm{~K}$. The reaction scheme used by Kelley et al. (219) is recalculated using modern values for the enthalpy of formation of the reference phases. See, Hemingway, B.S. and Robie, R.A., 1994, Heat capacity and enthalpy of formation of synthetic alunite: U.S. Geological Survey Open-file Report 94-688, 8 p.

453. High temperature heat capacities are derived from 244, $299,402$.

454. The Gibbs energy of livingstonite at 298.15 K is estimated from the study of (Sorokin, V.I., Dadze, T.P., and Pokronskiy, V.A., 1988, The Gibbs free energy of formation of livingstonite: Geokhimiya, No. 6, 897-9001. 
INDEX OF NAMES

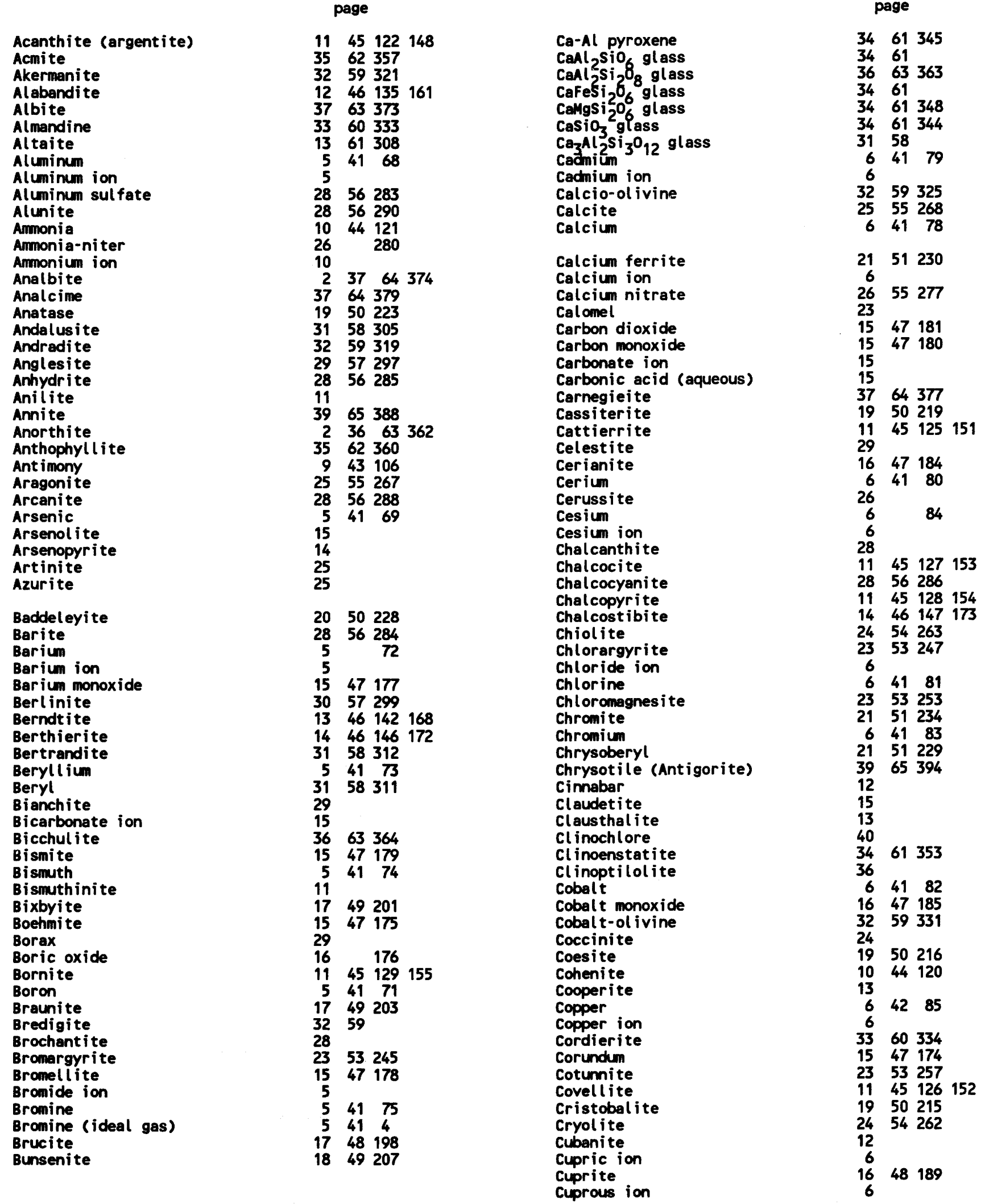




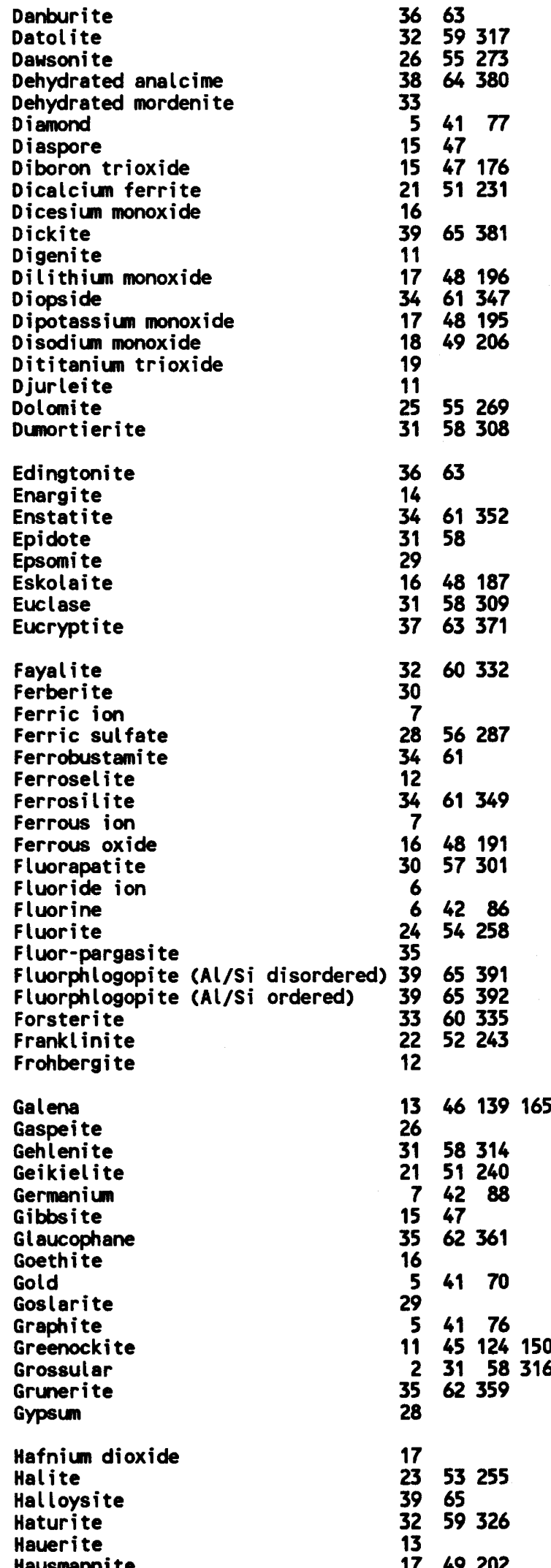

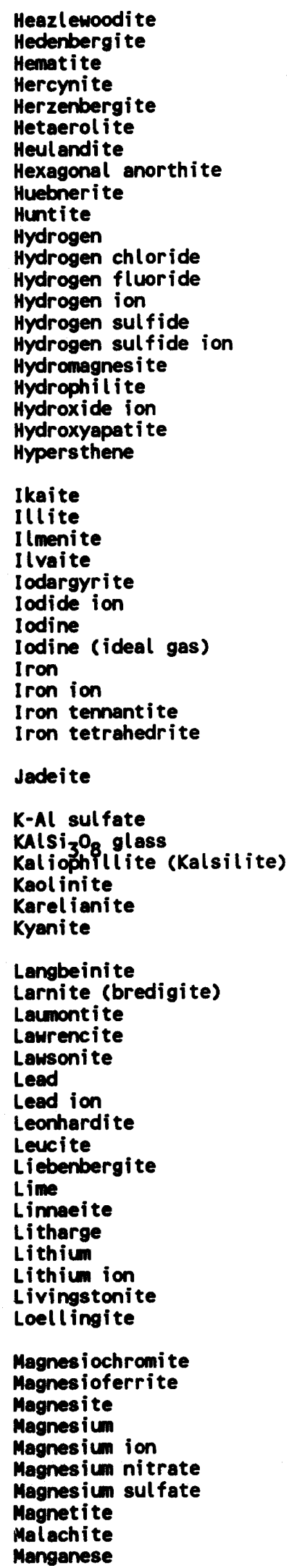

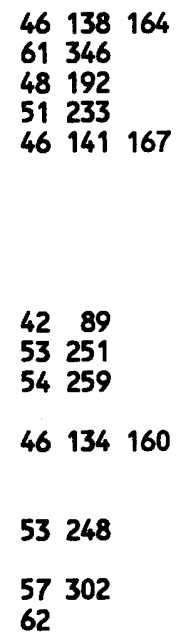


page

Manganese ion

Manganese sulfate

Manganosite

Marcasite

Margarite

Marshite

Mascagnite

Massicot

Matildite

Meionite (Al-Si ordered)

Melanterite

Mercuric ion

Mercurous ion

Mercury

Merl inoite

Merwinite

Mesolite

Metacinnabar

Methane

$\mathrm{MgSiO}_{3}$ glass

$\mathrm{MgSiO}_{3}$-I lmenite

$\mathrm{MgSiO}_{3}$-Perovskite

$\mathrm{Mg}_{3} \mathrm{Al} \mathrm{Si}_{3} \mathrm{O}_{12}$ glass

$\left(\mathrm{Mg}_{1.5} \mathrm{Ca}_{1.5} \mathrm{SAl}_{2} \mathrm{Si}_{3} \mathrm{O}_{12}\right.$ glass

$\left(\mathrm{Mg}_{1.8} \mathrm{Ca}_{1.2}\right)^{2} \mathrm{Al}_{2}^{2} \mathrm{Si}_{3} \mathrm{O}_{12}$ glass

Miargyrité

Microcline

Millerite

Minium

Mirabilite

Molybdenite

Molybdenum

Molybdite

Molysite

Monohydrocalcite

Monteponite

Mont icellite

Montroydi te

Mordenite

Morenosite

Mullite

Muscovite (AL/Si disordered)

Muscovite (Al/Si ordered)

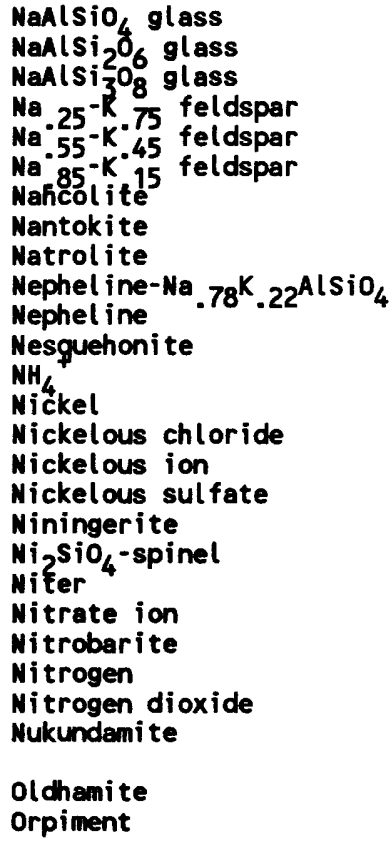

Olchami te

Orpiment

64

11

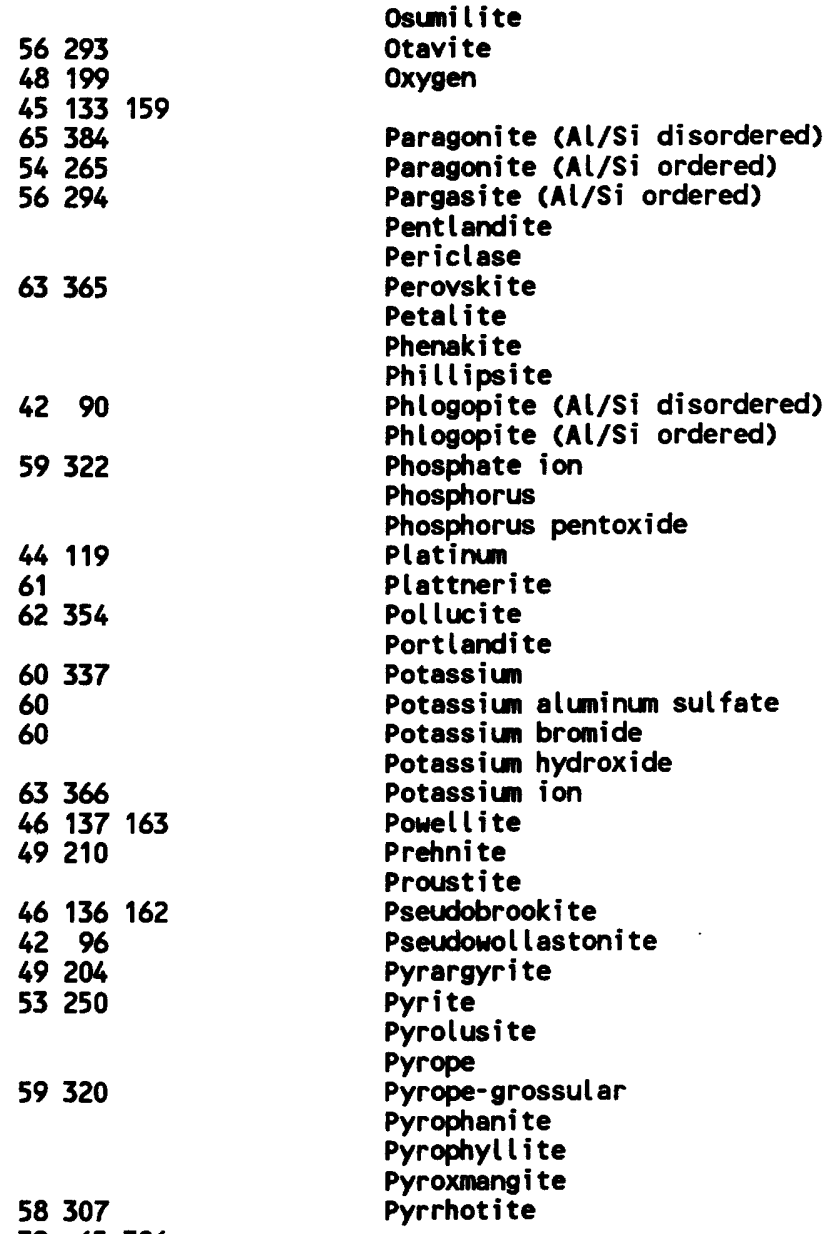

Osumilite
Otavite

Oxygen

Paragonite (Al/Si disordered)

Paragonite (Al/Si ordered)

Pargasite (Al/Si ordered)

Pentlandi te

Periclase

Perovskite

Petalite

Phenakite

Phillipsite

Phlogopite (Al/Si disordered)

Phlogopite (Al/Si ordered)

Phosphate ion

Phosphorus

Phosphorus pentoxide

Platinum

Plattnerite

Pollucite

Portlandite

Potassium

Potassium aluminum sulfate

Potassium bromide

Potassium hydroxide

Potassium ion

Powellite

Prehnite

Proustite

Pseudobrookite

Pseudowoll lastonite

Pyrargyrite

Pyrite

Pyrolusite

Pyrope

Pyrope-grossular

Pyrophanite

Pyrophyllite

Pyroxmangite

Pyrrhotite

64376

4399

53256

56289

60340

278

55276

4297

49205
Quartz

Rankinite

Realgar

Retgersite

Rhodochrosite

Rhodonite

Riebeckite

Rosenhahni te

Rutile

Sal ammoniac

Sanidine

Sanmartinite

Sapphirine

Scacchite

Scheel ite

Scolecite

Seleniun

Sell laite

siderite

silica glass

silicalite

silicic acid (aqueous)

silicon

silicon monoxide

sillimanite

silver

silver ion

Smithite page

3360

25

842100

4066395

4066396

35

$\begin{array}{lll}17 & 48 & 197\end{array}$

$\begin{array}{lll}21 & 51232\end{array}$

$\begin{array}{lll}37 & 63 & 372\end{array}$

$3158 \quad 310$

38

$\begin{array}{lll}39 & 65389\end{array}$

$\begin{array}{lll}39 & 65390\end{array}$

43101

43103

49209

63

$\begin{array}{lll}16 & 47 & 183\end{array}$

$\begin{array}{lll}7 & 42 & 92\end{array}$

$28 \quad 56289$

2353246

17

7

30

39

14

2151

$34 \quad 61343$

$\begin{array}{llll}12 & 45 & 132 & 158\end{array}$

$\begin{array}{lll}17 & 48200\end{array}$

3360336

3360

$\begin{array}{lll}21 & 51 & 241\end{array}$

$3965 \quad 383$

35

12

$\begin{array}{lll}45 & 131 & 157\end{array}$

$18 \quad 49214$

$\begin{array}{lll}32 & 59327\end{array}$

$\begin{array}{llll}11 & 45 & 123 & 149\end{array}$

$\begin{array}{lll}26 & 55272\end{array}$

$\begin{array}{lll}35 & 62 & 355\end{array}$

3562

$\begin{array}{lll}32 & 59 & 328\end{array}$

$19 \quad 50222$

2353

2

$\begin{array}{lll}37 \quad 63 & 367\end{array}$

53254

43107

$24 \quad 54260$

$25 \quad 55270$

$19 \quad 50 \quad 218$

19

919

943108

$\begin{array}{rrr}18 & 49 & 213\end{array}$

3158306

$\begin{array}{lll}5 & 41 & 67\end{array}$

5

14 
page

page

Smi thsonite

Soda-niter

Sodiun

Sodium carbonate

Sodium hydroxide

Sodium ion

Sphalerite

Spinel

a-spodumene

B-spodumene

Spurrite

Staurol ite

Stean

Stibnite

stilbite

Stishovite

Stolzite

Strengi te

Strontianite

Strontium

Strontiun ion

Strontium monoxide

strontium nitrate

sulfate ion

sulfide ion

Sulfite ion

Sulfur

sulfur (monoclinic)

Sulfur (ideal gas)

sulfur dioxide

sulfur trioxide

sulfuric acid

Sylvite

szomolnokite

Talc

Tellurite

Tellurium

Tell lurobismuthite

Tenorite

Tephroite

Thenardi te

Thermonatrite

Thorianite

Thorium

Tialite

Tilleyite

Tin

Titanite (sphene)

Titanium

Topaz

Tremol ite

Trevorite

Tricobalt tetraoxide

Tridymite

Troilite

Trona

Tungsten

Tungsten dioxide

Tungstenite

Tungsten trioxide

Ulvospinel

Uraninite

Uranium

Valent inite

Vanadium

Vaterite

Villiaunite

Violarite

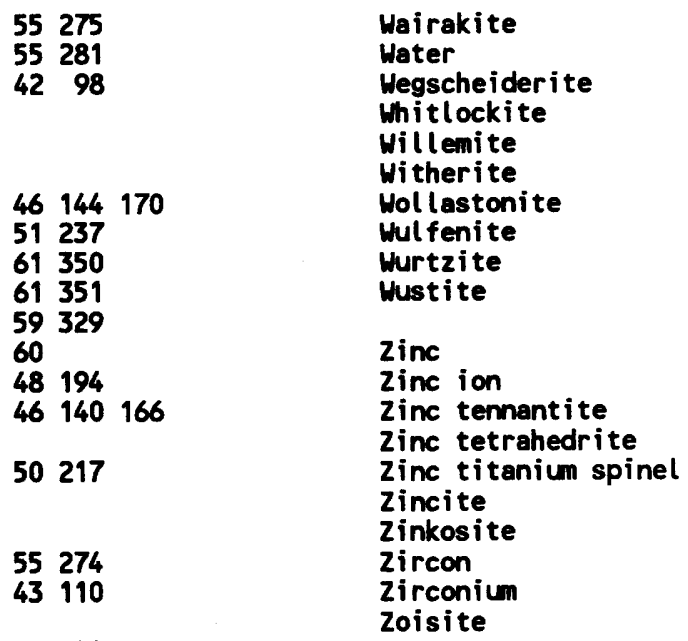

36

26

$\begin{array}{lll}30 & 57300\end{array}$

3360

$\begin{array}{lll}25 & 55 & 266\end{array}$

$\begin{array}{lll}34 & 61342\end{array}$

$\begin{array}{llll}13 & 46 & 145 & 171\end{array}$

$16 \quad 48190$

$10 \quad 44 \quad 117$

50220

55282

843104

943105

49211

49212

53252

65393

43111

48188

60338

56295

55

$50 \quad 221$

43112

51

59330

43109

59323

43113

58303

62358

52242

47186

45130156

44116

50226

46143169

51236

50224

43114

18

44115

54261 


\section{INDEX OF FORMULAS}

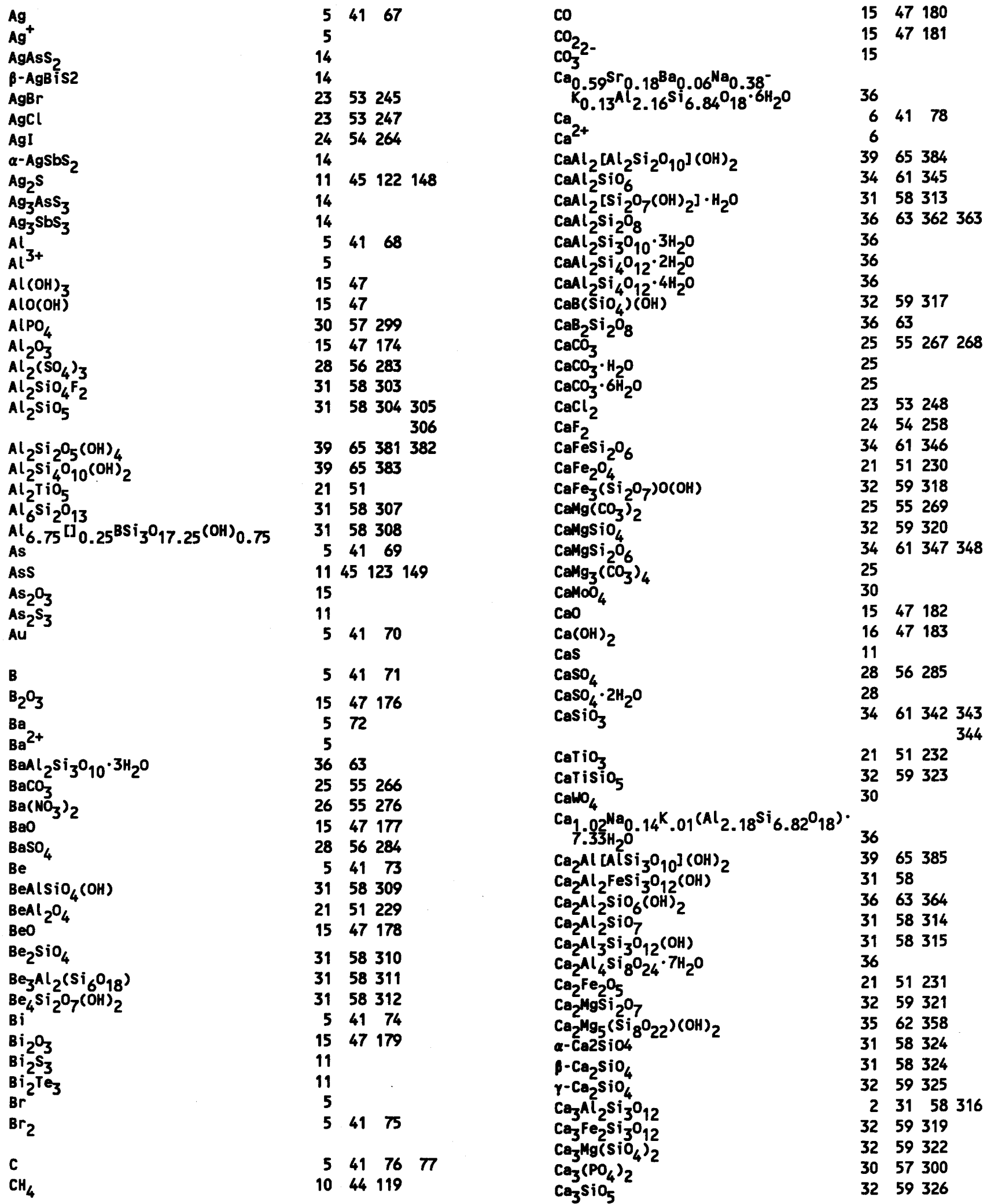




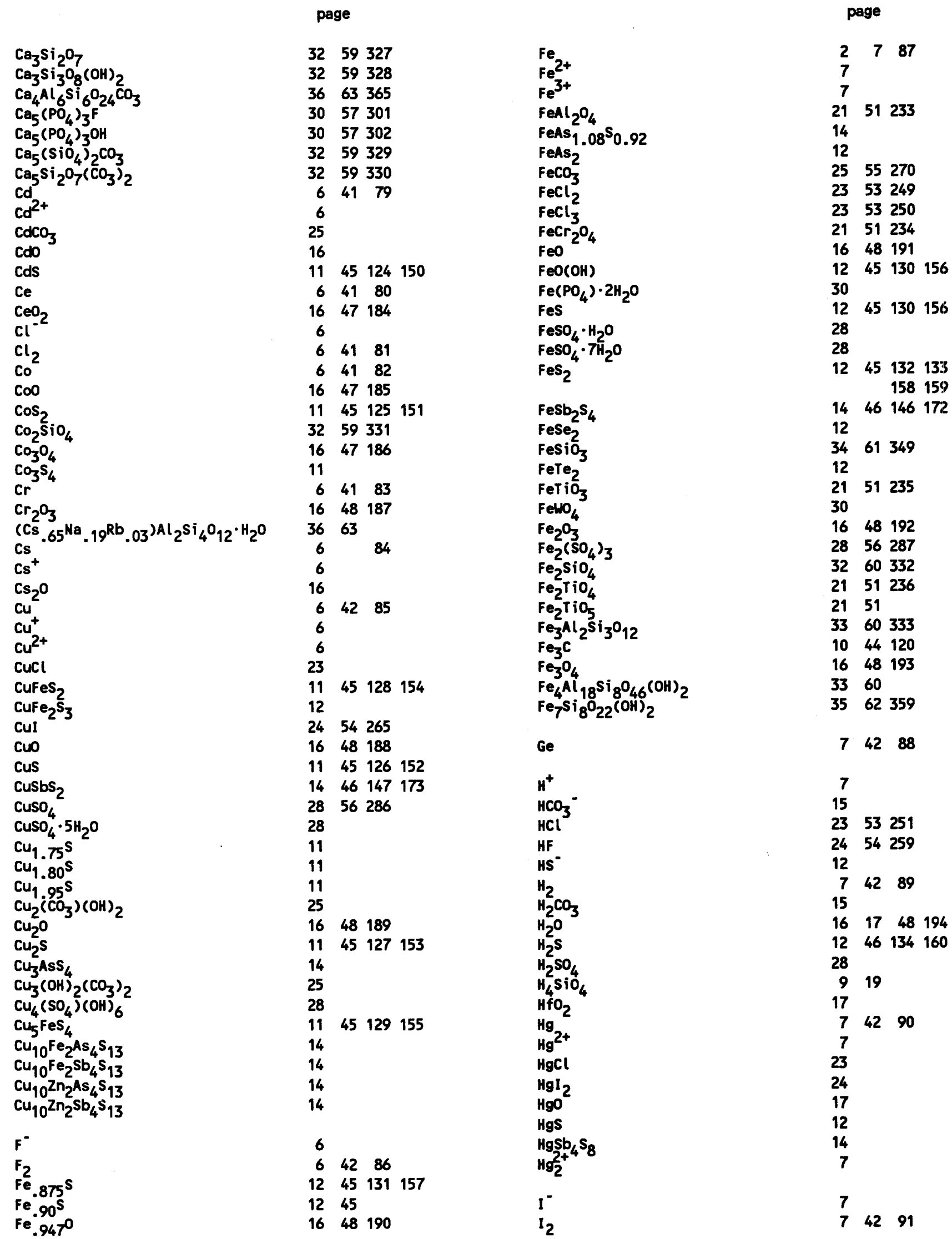




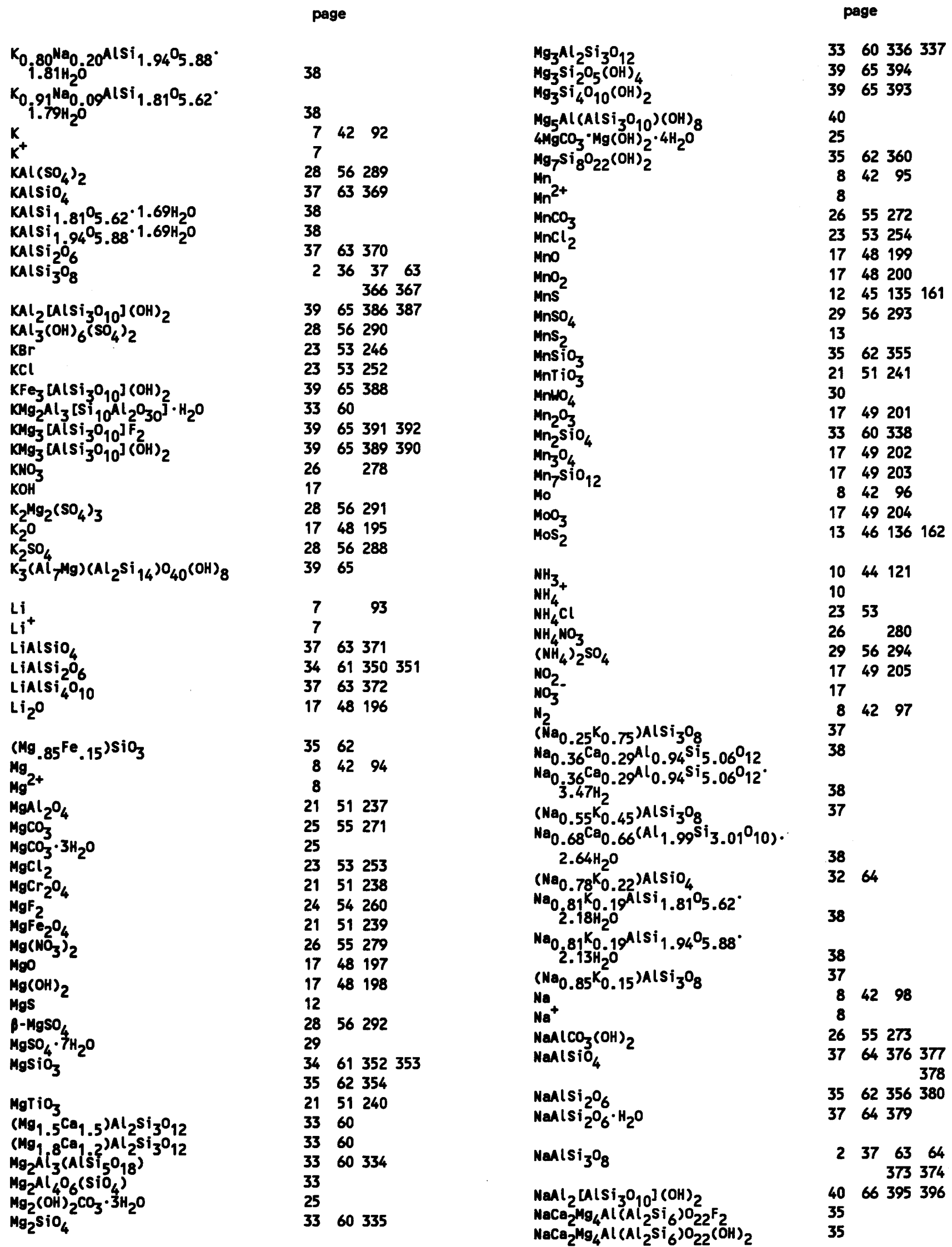




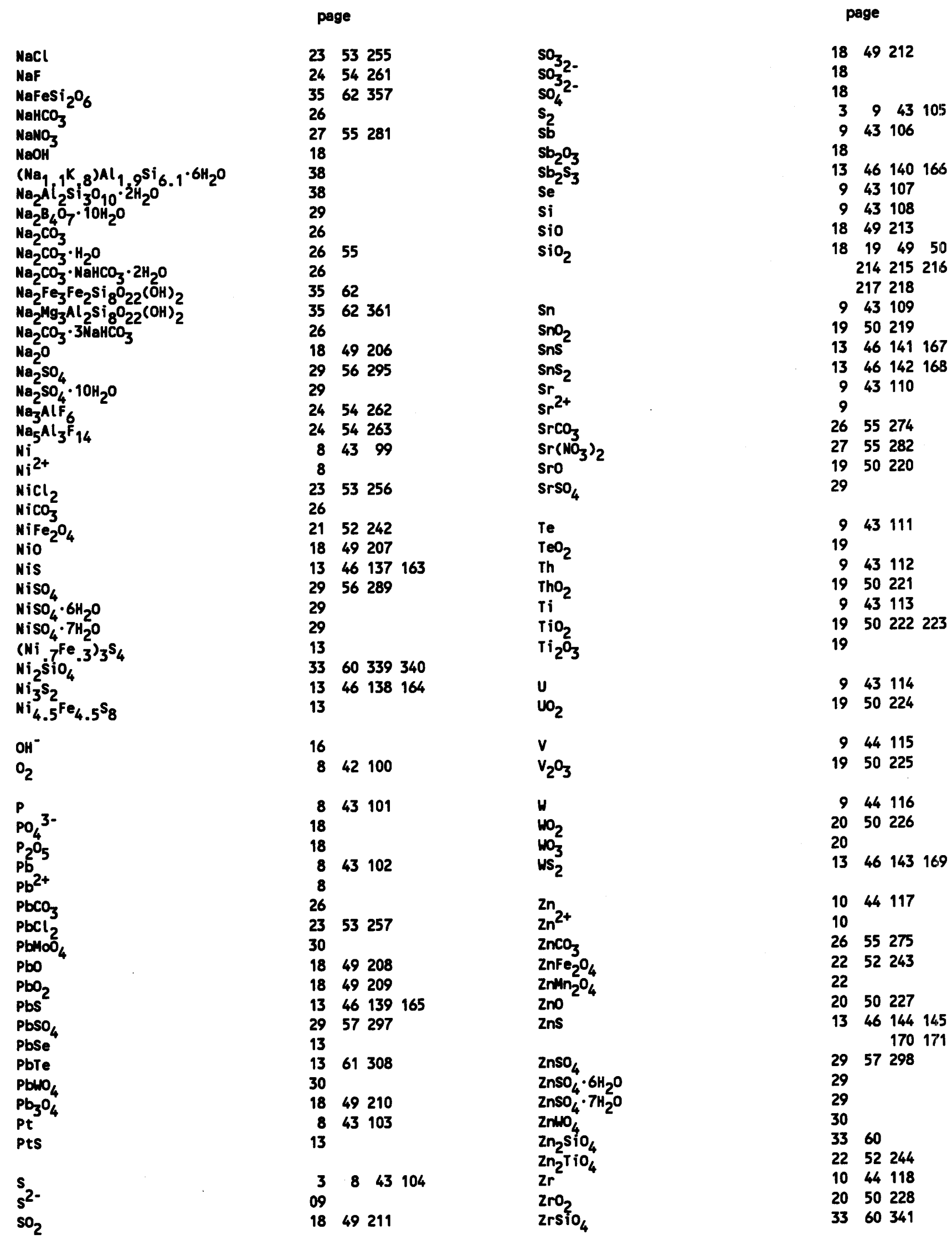



